IOPDARTMENT (OF THE INTERIOR

('NITEI) STATES (BEOLOGIGAL SURVEY

(HAKLES D. WALCOTT, DIRECTOR

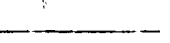

\title{
GEOGRAPHIC
}

\section{TABLES AND FORMULAS}

('OMPILEI) BY

SAMUIEI, S. ('ANNHIT

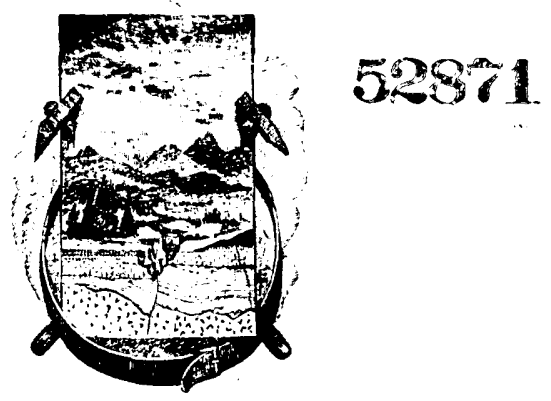

WASIIINGTON

GOVERNMENT PRINTING OFFICE

1903 

DEPARTMENT OF THE INTERIOR .

UNITED STATES GEOLOGICAL SURVEY

CHARLES D. WALCOT'T, DIRECTOR

\section{GEOGRAPHIC}

\section{TABLES AND FORMULAS}

COMPILED BY

SAMUEL S. GANNETT

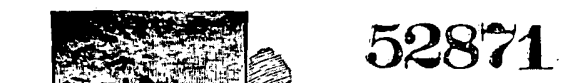

W ASHIN GTON

GOVERNMENT PRINTING OFFICE 1903 



\section{0 N TE E T'S.}

Cinge.

Letter of transmittal .......................................... 7

Rules for solution of right-angled triangles........................ 9

Reduction to center ........................................... 10

Graphic reduction to center .................................... 12

Solution of triangles, two sides and included angle being given .......... 12

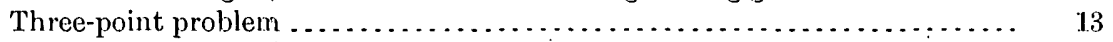

Graphic three-point problem......................................... 15

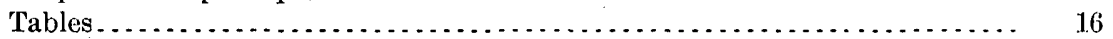

1. Polaris; times of culmination and elongation ................... 16

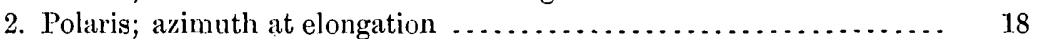

3. Polaris; azimuths and altitudes at different hour angles ........... 19

4. Projections for large areas................................. 29

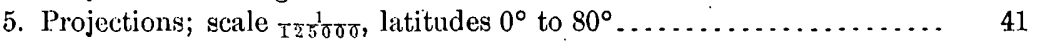

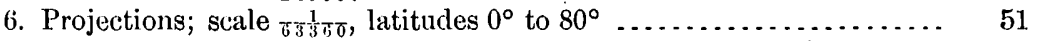

7. Projections; scale $\frac{1}{65 \delta \sigma}$, latitudes $25^{\circ}$ to $50^{\circ} \ldots \ldots \ldots \ldots \ldots \ldots \ldots .63$

8. Projections; scale $\frac{1}{4500}$, latitudes $26^{\circ}$ to $50^{\circ} \ldots \ldots \ldots \ldots \ldots \ldots \ldots .69$

9. Areas of quadrilaterals; $30^{\prime}$ extent, latitudes $0^{\circ}$ to $90^{\circ} \ldots \ldots \ldots \ldots . \quad 77$

10. Areas of quadrilaterals; $15^{\prime}$ extent, latitudes $0^{\circ}$ to $90^{\circ} \ldots \ldots \ldots \ldots . .80$

11. Areas of quadrilaterals; $10^{\prime}$ extent, latitudes $0^{\circ}$ to $90^{\circ} \ldots \ldots \ldots \ldots .86$

12. For conversion of arc into time ........................... 91

13. For conversion of time into arc ......................... 92

14. For conversion of mean time into sidereal time ............... 93

15. For conversion of sidereal time into mean time ................ 94

16. For interconversion of feet and decimals of a mile................. 95

17. For conversion of wheel revolutions into decimals of a mile........ 96

18. Five-place logarithms of natural numbers .................... 97

19. Five-place logarithms of circular functions expressed in arc and time. $\quad 119$

20. Geodetic position computations........................... 164

21. Log. $m$, for use in computing spherical excess ................. 240

22. Mean refraction ....................................... 241

23. Corrections for curvature and refraction ...................... 242

24. For obtaining differences of altitude ....................... 243

25. Horizontal distances and elevations from stadia readings .......... 259

26. For converting metric into United States measures............... 268

27. For converting United States measures into metric............... 269

28. For interconversion of miles and logarithms of meters ............ 270

Convenient equivalents .......................................... 282

Constants ........................................................ 284

Linear expansion of metals...................................... 284 



\section{ILLUSTRATIONS.}

Fic. 1. Solution of right-angle triangles.

2. Reduction to center ...................................... 10

3. Solution of triangles; two sides and included angle given........... 1.2

4. Three-point problem; computation............................. 14

5. Three-point problem; graphic solution ....................... 15 



\title{
LET'TER OF 'TRANSMIT'TAL.
}

\author{
Department of the Intertor, \\ United States Geological:SUrvey, \\ Washington, D. C., April 21, 1903.
}

SrR: I have the honor to transmit herewith, in form for publication, certain geographic tables and formulas pertaining to the work of the topographic branch of this Survey. The endeavor has been to bring together all tables and formulas used by the topographer in the field and office, and it is believed that their publication will be useful, not only to the topographic corps, but to others engaged in similar lines of work. The material has been drawn from various sources, some of it having been prepared from time to time by various members of the Geological Survey and the remaining portions baving been taken principally from publications of the U. S. Coast and Geodetic Survey and the Smithsonian Institution.

The compiling was done by Mr. S. S. Gannett, and the material was revised by Mr. E. M. Douglas and Mr. H. L. Baldwin, jr.

Very respectfully,

R. U. Goope,

Chairman Topographic: Committee.

Hon. Charles D. Walcott,

Director United States Geological Survey. 



\title{
. GEOGRAPHIC TABLES AND FORMULAS.
}

\author{
Compiled by S. S. Ganímt.
}

\section{RUIAS FOR SOLUTION OF RIGHT-ANGIED TIRIANGIES.}

The "parts" of the figures are-

$$
\begin{aligned}
& \mathrm{H}=\text { hypothenuse, } \\
& \mathrm{P}=\text { perpendicular, } \\
& \mathrm{B}=\text { base, }
\end{aligned}
$$

and the six circular functions of the angle $\alpha$ at the base of the triangle.

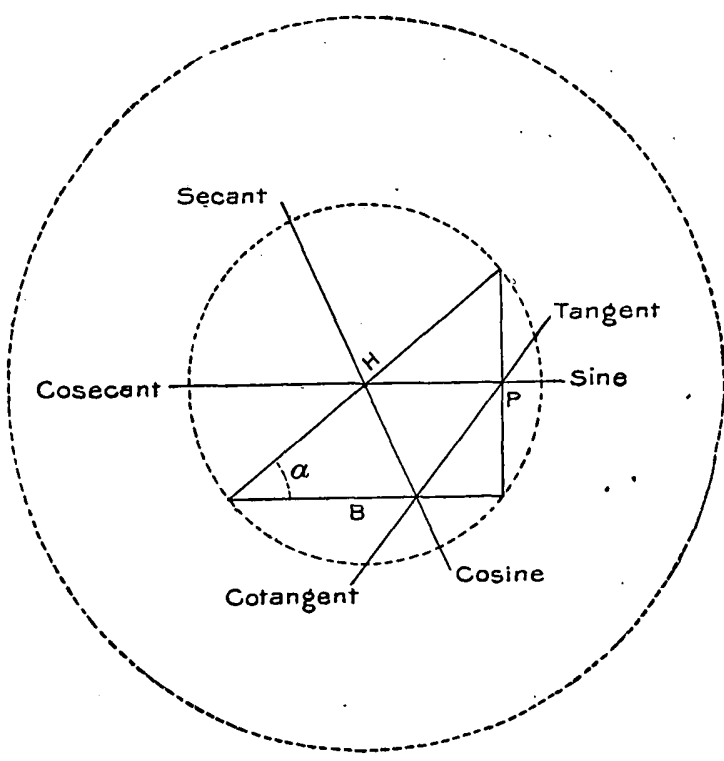

FIG. 1.-Solution of right-angled triangles.

RuLE I. The product of two opposite parts $=1, \therefore$ either is the reciprocal of the other.

Example: Tang $\alpha \times \cot \alpha=1$, tang $\alpha=\frac{1}{\cot \alpha}$.

RuLE II. Each part = adjacent part divided by the following part, $\therefore$ each part $=$ the product of the adjacent parts.

Example: Sine $\alpha=\frac{\cos \alpha}{\cot \alpha}$, sine $\alpha=\frac{\mathrm{P}}{\mathrm{H}}, \mathrm{B}=\mathrm{H} \times \cos \alpha$. 


\section{REDUCIION TO CENTER.}

In fig. 2 let

$\mathrm{P}=$ place of instrument;

$\mathrm{C}=$ center of station;

$\mathrm{Q}=$ measured angle at $\mathbf{P}$ between two objects, $\mathrm{A}$ and $\mathrm{B}$;

$y=$ angle at $\mathrm{P}$ between $\mathrm{C}$ and the left-hand object, $\mathrm{B}$;

$r=$ distance $\mathrm{CP}$;

$\mathrm{C}^{\prime}=$ unknown and required angle at $\mathrm{C}$;

$\mathrm{D}=$ distance $\mathrm{AC}$;

( $r$ and $\mathrm{D}$ must be reduced to same unit, usually meters.).

$\mathrm{G}=$ distance $\mathrm{BC}$; and

$\mathrm{A}=$ angle at $\mathrm{A}$ between $\mathrm{P}$ and $\mathrm{C}$;

$\mathrm{B}=$ angle at $\mathrm{B}$ between $\mathrm{P}$ and $\mathrm{C}$.

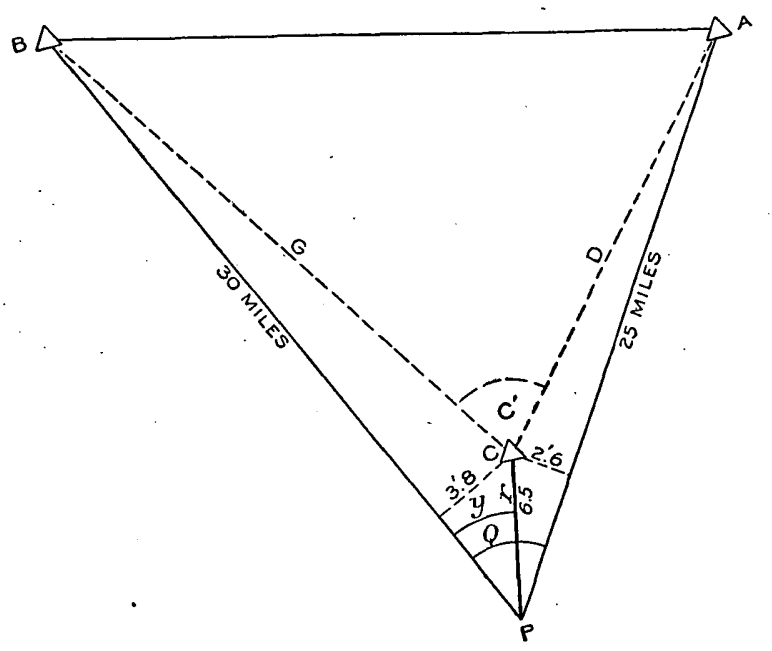

. Frg. 2.-Reduction to center.

Then, from the relation between the parts of the triangle,

hence

$\mathrm{G}: r:: \sin y: \sin \mathrm{B}$

- $\sin \mathrm{B}=\frac{r \sin y}{\mathrm{G}}$.

As the angles at $A$ and $B$ are very small, they may be regarded as equal to $A \sin 1^{\prime \prime}$ and $B \sin 1^{\prime \prime}$; hence

and

$$
\mathrm{B}=(\text { in seconds }) \frac{r \sin y}{\mathrm{G} \sin 1^{\prime \prime}}
$$

$$
\mathrm{C}^{\prime}=\mathrm{Q}+\frac{r \sin (\mathrm{Q} \pm y)}{\mathrm{D} \sin 1^{\prime \prime}}-\frac{r \sin y}{\mathrm{G} \sin 1^{\prime \prime}} \text {. }
$$


In the use of this formula, proper attention should be paid to the signs of $\sin (Q+y)$ and $\sin y$; for the first term will be positive only when $(\mathrm{Q}+y)$ is less than $180^{\circ}$ (the reverse with sin $y$ ); $\mathrm{D}$ being the distance of the right-hand object, the graduation of the instrument running from left to right.

$r$ being relatively small, the lengths of $\mathrm{D}$ and $\mathrm{G}$ are approximately computed with the angle $Q$.

The following quantities must be known in addition to the measured angles in order to find the correction for reducing to center:

1. The angle measured at the instrument, $P$, between the center of the signal or station, $\mathrm{C}$, and the first-observed station to the right of it, A.

2. The distance from the center of the instrument to the center of the station $=r$.

3. The approximate distances, D, G, etc., from the station occupied to the stations observed. The latter may be computed from the uncorrected angles.

- Example: Reduction to center from P to C.

$$
\begin{array}{lr}
\text { Constants: a. c. } \log \sin 1^{\prime \prime} & =5.31443 \\
\log \text { feet to } \log \text { meters } & =9.48402 \\
\log \text { constant (for any station) } & \overline{4.79845} \\
r=6.5 \text { feet: } \log & =0.81291 \\
\log \text { constant for this station } & \overline{5.61136}
\end{array}
$$

\begin{tabular}{|c|c|c|}
\hline & $\begin{array}{l}\text { Angle } \\
Q-Y \\
(\mathrm{CPA}) \\
23^{\circ} 40^{\prime}\end{array}$ & $\begin{array}{c}\text { Angle } \\
\text { Y } \\
\text { (BPC) } \\
37^{\circ} 14^{\prime} \\
\text { or } 322^{\circ}{ }_{46}^{\prime \prime}\end{array}$ \\
\hline log sin angle...$\ldots \ldots \ldots$. & 9.6036 & 9.7818 \\
\hline a. c. $\log$ distance ...... & 5. 3954 & 5. 3162 \\
\hline $\log r+$ constant. & 5.6114 & 5. 6114 \\
\hline $\log$ correction... & 0.6104 & 0.7094 \\
\hline correction to direction....... & $4.08^{\prime \prime}$ & $5.12^{\prime \prime}$ \\
\hline correction to angle B P $\bar{A}=4.08^{\prime \prime}+5.12^{\prime \prime}=9^{\prime \prime} .20$ & & . \\
\hline
\end{tabular}




\section{GRAPITIE REDUCTION TO CENTER.}

Approximate closure errors of triangles may be tested in the field before distances have been computed by scaling from the plot the distances between stations in miles and the perpendicular distance in feet from signal to line joining instrument and distant station.

Then, since 1 foot at a distance of 40 miles subtends an angle of $1^{\prime \prime}$ (nearly),

$$
\frac{\text { length of perpendicular in feet } \times 40}{\text { number of miles }}=\text { correction in seconds. }
$$

Example: Station P. Correction for swing on line B P, 30 miles in length from instrument to signal

$$
=\frac{3.8 \text { feet } \times 40}{30}=" 5.1,
$$

correction for swing on line A P, 25 miles in length,

$$
=\frac{2.6 \text { feet } \times 40}{25}=4.2^{\prime \prime} \text {, }
$$

and correction to angle B P A $=\mathrm{Q}$ to reduce from instrument to signal $=5.1^{\prime \prime}+4.2^{\prime \prime}=9.3^{\prime \prime}$, agreeing closely with the exact computation.

APPROXIMATE SPHERTCAL IEXCESS IN SECONIDS.

This may be obtained by dividing the area of the triangle in square miles by 75.5 .

\section{SOLUTION OF TRIANGLES.}

Given two sides and included angle, to solve the triangle:

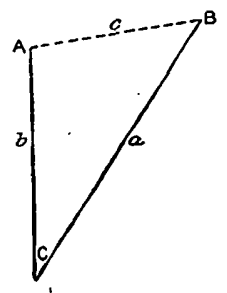

Fra. 3.-Solution of triangles.

Let $x$ be an auxiliary angle; then

$$
\begin{gathered}
\tan x=\frac{b}{a}, \text { or } \log \tan x=\log a-\log b ; \\
\tan \frac{1}{2}(\mathrm{~A}-\mathrm{B})=\tan \left(x-45^{\circ}\right) \tan \frac{1}{2}(\mathrm{~A}+\mathrm{B}) ; \\
\frac{1}{2}(\mathrm{~A}+\mathrm{B})+\frac{1}{2}(\mathrm{~A}-\mathrm{B})=\mathrm{A} ; \\
\frac{1}{2}(\mathrm{~A}+\mathrm{B})-\frac{1}{2}(\mathrm{~A}-\mathrm{B})=\mathrm{B} ;
\end{gathered}
$$

from which remaining parts can be computed. 
Example:

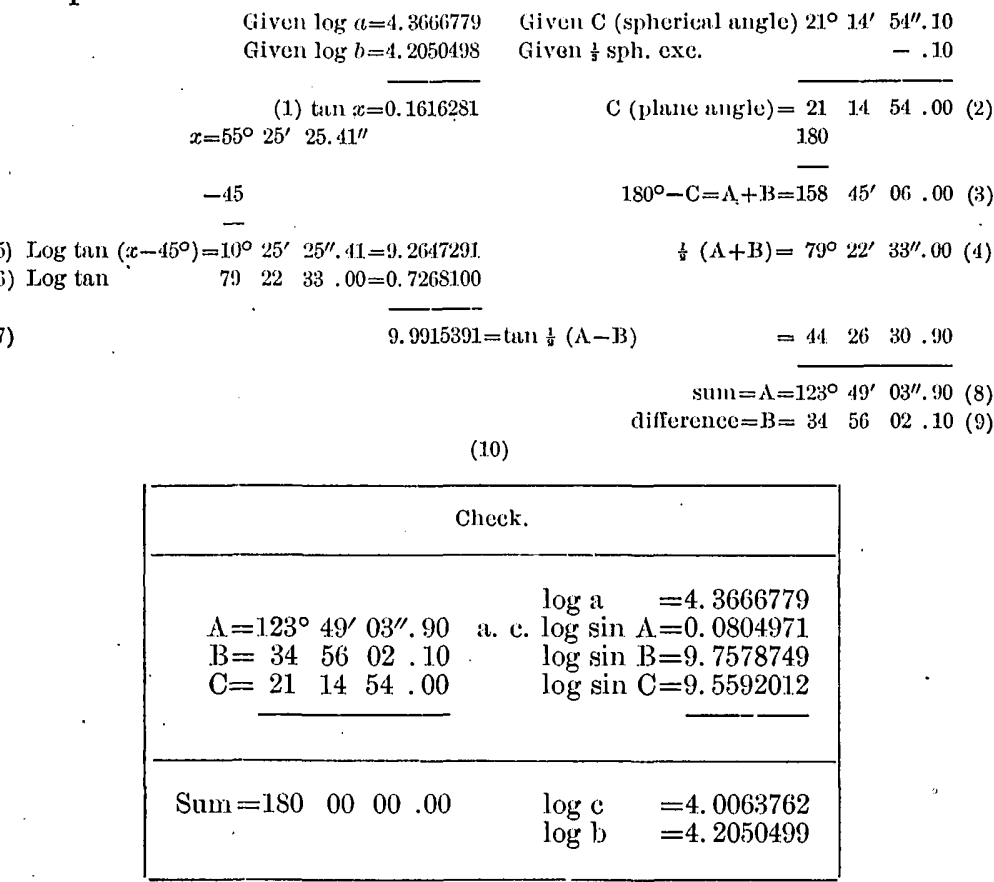

\section{THREF-POINT PIROBJEM.}

If three points, forming a triangle of which the sides and angles are known or can be computed, be visible from it fourth point, $P$, it is required to determine the position of $P$.

Set up the theodolite at $\mathrm{P}$ and measure the two angles subtended by any two of the given sides.

This problem is of use in cases where, the regular triangulation having been completed, additional points are required for the topographic survey, or are needed for special service. The angles should be carefully measured, and in the computations the logarithms should be carried to seven places of decimals.

Three cases of its application are given, as in others, such as when $P$ falls upon one or the other of the sides of the known triangle, or on the prolongation of either, the case resolves itself into the solution of a simple triangle with one side and the angles given; or the problem is indeterminate, as when $\mathrm{P}$ is situated on the circumference of the circle - passing through the three known points-a contingency which rarely occurs. 
Example for each of the three cases.

Given the side $\quad a=11204.5$

Given the side $\quad b=7289.0$

Given the side $\quad c=6273.8$

Given the angle $\mathrm{A}=111^{\circ} 10^{\prime} 54^{\prime \prime}$
Angle observed A P C $=\mathrm{P}^{\prime}$

Angle observed A P B $=\mathrm{P}^{\prime \prime}$

To find

To find

A B $\mathrm{P}=x$

A C P $=y$
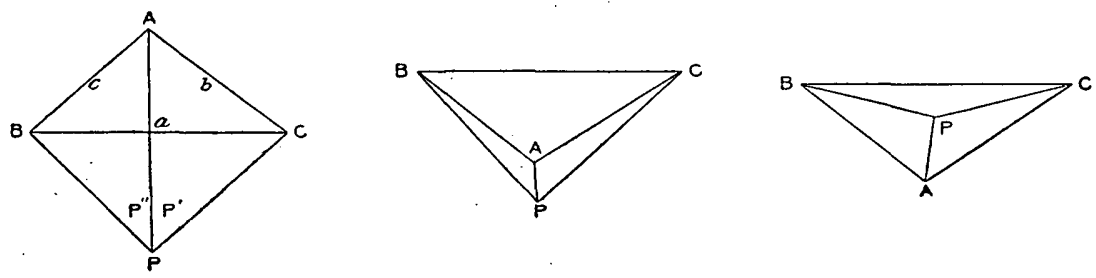

Fig. 4.-Three-point problem; computation.

$$
\begin{aligned}
& \mathrm{P}^{\prime} \ldots 50^{\circ} 06^{\prime} 12^{\prime \prime}
\end{aligned}
$$

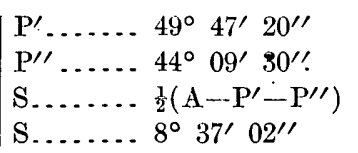

$$
\begin{aligned}
& \mathrm{P}^{\prime} \ldots 104^{\circ} 00^{\prime} 00^{\prime \prime} \\
& \mathrm{P}^{\prime \prime} \ldots 100^{\circ} 20^{\prime} 00^{\prime \prime} \\
& \mathrm{P}^{\prime \prime} \ldots 43^{\circ} 50^{\prime} 38^{\prime \prime} \\
& \text { S. ... } 180^{\circ}-\frac{1}{2}\left(\mathrm{~A}+\mathrm{P}^{\prime}+\mathrm{P}^{\prime \prime}\right) \\
& \text { S.... } 77^{\circ} 26^{\prime} 08^{\prime \prime} \\
& \varepsilon=\frac{1}{2}(x-y) \\
& \mathrm{S} \text {. . . } 180^{\circ}-\frac{1}{2}\left(\mathrm{~A}+\mathrm{P}^{\prime}+\mathrm{P}^{\prime \prime}\right) \\
& \text { S.... } 22^{\circ} 14^{\prime} \cdot 33^{\prime \prime} \\
& \tan Z=\frac{c \sin \mathrm{P}^{\prime}}{b \sin \mathrm{P}^{\prime \prime}} \\
& \tan \varepsilon=\cot \left(Z+45^{\circ}\right) \tan \mathrm{S}
\end{aligned}
$$

$x=\mathrm{S}+\varepsilon \quad y=\mathrm{S}-\varepsilon$, but if $\tan \varepsilon$ be negative, then $x=\mathrm{S}-\varepsilon, \cdot y=\mathrm{S}+\varepsilon$

\section{Computation.}

$\log c$

3. 7975307

$\log c$.

3. 7975397

$\log \sin \mathrm{P}^{\prime}$

9. 8849100

$\log \sin \mathrm{P}^{\prime}$

9.8829061

co. $\log b^{\circ} \ldots \ldots 6.1373320$

co. $\log b \ldots \ldots .6 .1373320$

$\log$ co. $\sin \mathrm{P}^{\prime \prime} \ldots$. 0.1594574

$\log \cos \sin \mathrm{P}^{\prime \prime} \ldots 0.1569894$

$\log \tan \mathrm{Z}$

9.9792301

9.9747583

Z ... $43^{\circ} 37^{\prime} 49^{\prime \prime} .6$

$\log \tan Z$

Z... $43^{\circ} 20^{\prime} \quad 09^{\prime \prime} .2$

$\log \cot \left(\mathrm{Z}+45^{\circ}\right) \quad 8.3785397$

log $\tan$ S....... 0.6519386

$\log \cot \left(Z+45^{\circ}\right) 8.4631818$

$\log \tan \mathrm{S} . \ldots . .9 .9 .1805366$

$\log \tan$

9. 0304783

$\log \tan \varepsilon_{\ldots} \ldots . .7 .6437184$

$\begin{array}{llll}\varepsilon & \ldots & 6^{\circ} 07^{\prime} & 21^{\prime \prime} .7\end{array}$

S.... $77^{\circ} 26^{\prime} 08^{\prime \prime} .0$

$x$.... $83^{\circ} 33^{\prime} 29^{\prime \prime} .7$

$y \ldots . .71^{\circ} 18^{\prime} 46^{\prime \prime} .3$

Hence,

$\log \tan$

8.5239581

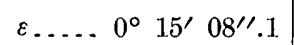

$\varepsilon \ldots 1^{\circ} 54^{\prime} 50^{\prime \prime} .04$

S..... $8^{\circ} 37^{\prime} 02^{\prime \prime} .0$

S... $22^{\circ} 14^{\prime} 33^{\prime \prime} .00$

$x . \ldots .8^{\circ} 52^{\prime} 10^{\prime \prime} .1$

$x \ldots 24^{\circ} 09^{\prime} 23^{\prime \prime} .00$

$y \ldots . .8^{\circ} 21^{\prime} 53^{\prime \prime} .9$

Hence,

$y$... $20^{\circ} 19^{\prime} 43^{\prime \prime} .00$

Hence,

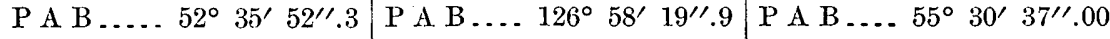

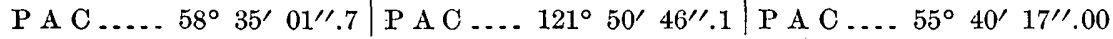


As all the angles and a side in each triangle are now known, the other sides, or the distances from $\mathrm{P}$ to the three given points, can he readily computed.

\begin{tabular}{|c|c|c|}
\hline & & \\
\hline 7194.87 & P B $\ldots \ldots \ldots \ldots \ldots 7194.94$ & 5256. \\
\hline 8999 . & 1388.54 & 2609. \\
\hline 8107 & 8107.91 & $\mathbf{P C}$ \\
\hline 8999.8 & 1388.54 & P A \\
\hline
\end{tabular}

The results are verified when both triangles give the same valce for the line $\mathrm{P} \mathrm{A}$.

\section{GRAPHIC THIREE-POINT PROBLEM.}

1. When new point is on or near the circle passing through the other points, the location is uncertain.

2. When new point is within the triangle formed by the three points, point sought is within the triangle of error.

3. When new point is without the triangle, orient on most distant point; then the point sought is always on the same side of the line from most distant point as the point of intersection of the other two lines.

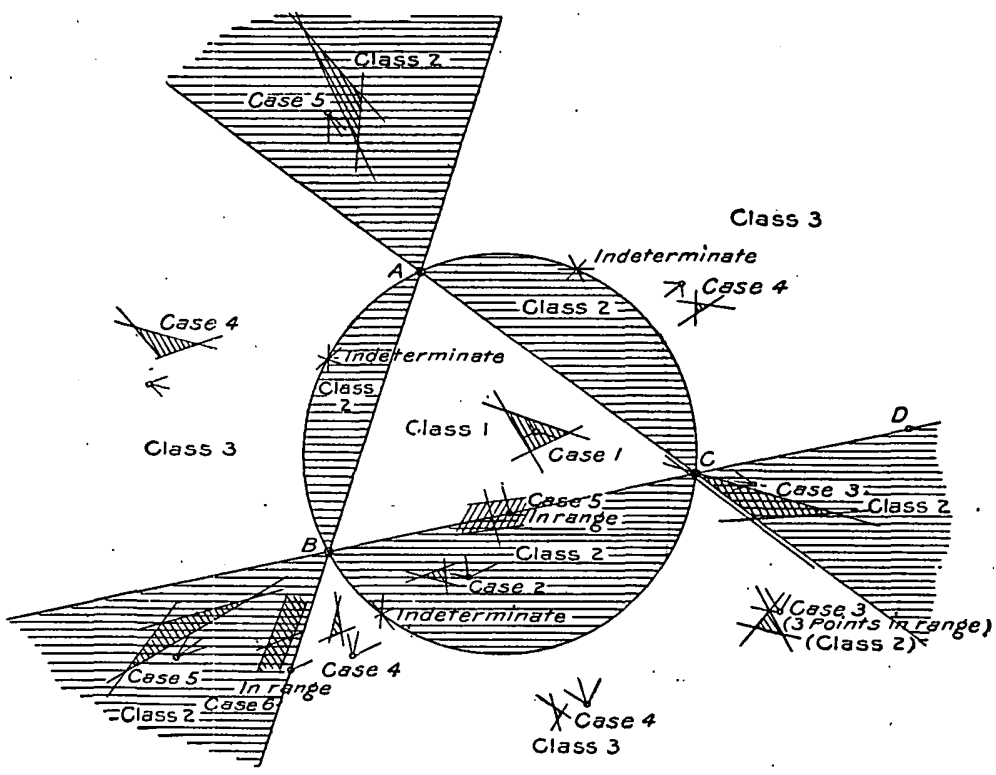

FIG. 5.-Three-point problem; graphic solution: 


\section{TABLES.}

TaBLe 1.-Local mean (astronomical) time of the culminations and elongations of Polaris in the year 1902.

[From Magnetic Declination Tables, U. S. Coast and Geodetic Survey. Computed for ]atitude $40^{\circ}$ north and longitude $90^{\circ}$ or $6^{\mathrm{h}}$,west of Greenwich.]

\begin{tabular}{|c|c|c|c|c|c|c|c|c|}
\hline Date. & \multicolumn{2}{|c|}{$\begin{array}{l}\text { East elonga- } \\
\text { tion. }\end{array}$} & \multicolumn{2}{|c|}{$\begin{array}{l}\text { Upper culmi- } \\
\text { nation. }\end{array}$} & \multicolumn{2}{|c|}{$\begin{array}{l}\text { West elongat- } \\
\text { tion. }\end{array}$} & \multicolumn{2}{|c|}{$\begin{array}{l}\text { Lower culmi- } \\
\text { nation. }\end{array}$} \\
\hline 1902 & : & $\mathrm{m}$ & b & m & h & $\mathrm{n}$ & $\mathrm{h}$ & n \\
\hline January 1. & & 45.8 & & 40.6 & 12 & 35.3 & 1.8 & 38.7 \\
\hline January $15 .$. & & 46.6 & 5 & 45.3 & 1.1. & 40.0 & 17 & 43.4 \\
\hline February 1 & 22 & 39.5 & 4 & 38.2 & 10 & 32.9 & 16 & 36.3 \\
\hline February 15 & 21. & 44.2 & 3 & 42.9 & 9 & 37.7 & 15 & 41.0 \\
\hline March $1 \ldots$ & 20 & 49.0 & 2 & 47.7 & 8 & 42.4 & 14 & 45.8 \\
\hline March 15 & 19 & 54.0 & 1 & 52.7 & 7 & 47.3 & 13 & 50.7 \\
\hline April $1 \ldots$ & 18 & 47.0 & 0 & 45.6 & 6 & 40.3 & 1.2 & 43.7 \\
\hline April 15 & 17 & 52.0 & 23 & 46.7 & 5 & 45.3 & 11 & 48.6 \\
\hline May $1 . .$. & 16 & 49. 1 & 22 & 43.8 & 4 & 42.5 & 10 & 45.7 \\
\hline May $15 \ldots$ & 15 & 54.2 & 21 & 48.9 & 3 & 47.6 & 9 & 50.8 \\
\hline June 1.... & 14 & 47.5 & 20 & 42.3 & 2 & 40.9 & 8 & 44.2 \\
\hline June $15 . .$. & 13 & 52.6 & 19 & 47.4 & 1 & 46.0 & 7 & 49.3 \\
\hline July $1 \ldots$ & 12 & 50.0 & 18 & 44.8 & 0 & 43.4 & 6 & 46.7 \\
\hline July $15 \ldots$ & 11 & 55.1 & 17 & 49.9 & 23 & 44.6 & 5 & 51.8 \\
\hline August 1. & 10 & 48.6 & 16 & 43.4 & 22 & 38.0 & 4 & 45.3 \\
\hline August $15 .$. & 9 & 53.7 & 15 & 48.5 & 21. & 43.1 & 3 & 50.4 \\
\hline September 1 . & 8 & 47.1 & 14 & 41.9 & 20 & 36.5 & 2 & 43.8 \\
\hline September 15 & 7 & 52.2 & 13 & 47.0 & 19 & 41.6 & 1 & 48.9 \\
\hline October $1 \ldots$ & 6 & 49.3 & 12 & 44.1 & 18 & 38.7 & 0 & 46.0 \\
\hline October $15 \ldots$ & 5 & 54.3 & 11 & 49.1 & 17 & 43.7 & 23 & 47.2 \\
\hline November $1 \ldots$ & 4 & 47.5 & 10 & 42.3 & 16 & 36.9 & 22 & 40.4 \\
\hline November 15 & 3 & 52.3 & 9 & 47.1 & 15 & 41.8 & 21 & 45.2 \\
\hline December $1 \ldots$ & 2 & 49.3 & 8 & 44.1 & 14 & 38.8 & 20 & 42.2 \\
\hline December 15 . & & 54.0 & 7 & 48.8 & 13 & 43.6 & 19 & 46.9 \\
\hline
\end{tabular}

A. To refer the above tabular quantities to years subsequent ti. 190\%:

For year 1903 add 1.4 minutes.

\begin{tabular}{|c|c|c|c|c|}
\hline 1904 & add & $\begin{array}{r}2.8 \\
+1.1\end{array}$ & " & $\begin{array}{l}\text { up to March } 1 \\
\text { on and after March } 1\end{array}$ \\
\hline 1905 & add & 0.2 & "، & \\
\hline 1906 & " & 1.5 & ، & \\
\hline 1907 & ،: & 2.9 & "، & \\
\hline 1908 & " & $\left\{\begin{array}{l}4.2 \\
0.3\end{array}\right.$ & "“ & $\begin{array}{l}\text { up to March } 1 \\
\text { on and after March } 1\end{array}$ \\
\hline 0 & "6 & 1.7 & $"$ & \\
\hline 10 & " & 3.0 & " & \\
\hline
\end{tabular}


B. To refer to any calendar day other than the first and fifteenth of each month, sUBTRACT the quantities below from the tabular quantity for the PRECEDING DATE.

\begin{tabular}{|rr|c|c|}
\hline Day of month. & Minutes. & $\begin{array}{c}\text { Number of } \\
\text { days } \\
\text { elapsed. }\end{array}$ \\
\cline { 1 - 2 } 2 or 16 & 3.9 & 1 \\
3 & 17 & .7 .9 & 2 \\
4 & 18 & 11.8 & 3 \\
5 & 19 & 15.8 & 4 \\
6 & 20 & 19.7 & 5 \\
7 & 21 & 23.6 & 6 \\
8 & 22 & 27.6 & 7 \\
9 & 23 & 31.5 & 8 \\
10 & 24 & 35.5 & 9 \\
11 & 25 & 39.4 & 10 \\
12 & 26 & 43.3 & 11 \\
13 & 27 & 47.3 & 12 \\
14 & 28 & 51.2 & 13 \\
& 29 & 55.2 & 14 \\
& 30 & 59.1 & 15 \\
& 31 & 63.0 & 16 \\
\hline
\end{tabular}

C. To refer the table to standard time and to the civil or common method of reckoning:

${ }^{(a)}$ ADD to the tabular quantities four minutes for every degree of longitude the place is west of the standard meridian, and subTrACT when the place is east of the standard meridian.

${ }^{(b)}$ The astronomical day begins twelve hours after the civil day, i. e., begins at noon on the civil day of the same date, and is reckoned from 0 to 24 hours. Consequently an astronomical time less than twelve hours refers to the same civil day, whereas an astronomical time greater than twelve hours refers to the morning of the next civil day.

It will be noticed that for the tabular year two eastern elongations occur on January 12 and two western elongations on July 12. There are also two upper culminations on April 12 and two Jower culminations on October 12. The lower culmination either follows or precedes the upper culmination by $11^{\mathrm{h}} 58^{\mathrm{m}} .1$.

D. To refer to any other than the tabular latitude between the limits of $25^{\circ}$ and $50^{\circ}$ north: ADD to the time of west elongation $0^{\mathrm{m}} .13$ for every degree south of $40^{\circ}$, and sUiBTRACT from the time of west elongation $0^{\mathrm{m}} .18$ for every degree north of $40^{\circ}$. Reverse these operations for correcting times of east elongation.

Bull. $214-03-2$ 
E. To refer to any other than the tabular longitude: ADD $0^{\text {m. }} 16$ for each $15^{\circ}$ east of the ninetieth meridian, and subrracr $0^{\mathrm{m}} .16$ for each $15^{\circ}$ west of the ninetieth meridian.

Table 2.-Azimuth of Polaris when at elongation for any year between 1902 and 1910.

\begin{tabular}{|c|c|c|c|c|c|c|c|c|c|}
\hline Latitude. & 1902.0 & 1903.0 & 1904.0 & 1905.0 & 1906.0 & 1907.0 & 1908.0 & 1909.0 & 1910.0 \\
\hline & o, & $\circ \quad$, & $\circ \quad 1$ & $\circ \quad$, & $0 \quad 1$ & $0 \quad$, & $0 \quad$, & $\circ \quad 1$ & $\circ \quad$, \\
\hline $25^{\circ}$ & 1. 20.5 & 120.1 & 119.8 & 119.4 & 119.1 & 1. 18.7 & 118.4 & 1 1. 18.1. & 117.7 \\
\hline 26 & 21.1 & 20.8 & 20.5 & 20.1 & 19. 8 & 19.4 & 19.1 & 18.7 & 18.4 \\
\hline 27 & 21.9 & 21.5 & 21.2 & 20.8 & 20.5 & 20.1 & 19.8 & 19.4 & 19.1 \\
\hline 28 & 22.6 & 22.2 & 21.9 & 21.6 & 21.3 & 20.9 & 20.5 & 20.1 & 19.8 \\
\hline 29 & 23.4 & 23.0 & 22.7 & 22.4 & 22.1 & 21.7 & 21.3 & 20.9 & 20.5 \\
\hline 30 & 24.2 & 23.9 & 23.5 & 23.1 & 22.8 & 22.4 & 22.1 & 21.7 & 21.3 \\
\hline 31 & 25.1 & 24.7 & 24.4 & 24.0 & 23.6 & 23.2 & 22.9 & 22.5 & 22.2 \\
\hline 32 & 26.0 & 25.6 & 25.3 & 24.9 & 24.5 & 24.1 & 23.8 & 23.4 & 23.1 \\
\hline 33 & 27.0 & 26.6 & 26.2 & 25.9 & 25.5 & 25.1 & 24.7 & 24.3 & 24.0 \\
\hline 34 & 28.0 & 27.6 & 27.2 & 26.9 & 26.5 & 26.1 & 25.7 & . 25.3 & 25.0 \\
\hline 35 & 29.0 & 28.7 & 28.3 & 27.9 & 27.5 & 27.1 & 26.8 & 26.4 & 26.0 \\
\hline 36 & 30.1 & 29,8 & 29.4 & 29.0 & 28. & 28.2 & 27. & 27.5 & 27.1 . \\
\hline 37 & 31.3 & 30.9 & 30.5 & 30.1 & 29. & 29.3 & 29. & 28.6 & 28. 2 \\
\hline 38 & 32.6 & 32.2 & 31.8 & 31.4 & 31.0 & 30.6 & 30.2 & 29.8 & 29.4 \\
\hline 39 & 33.9 & 33.5 & 33.1 & 32.7 & 32.3 & 31.8 & 31.4 & 31.0 & 30.6 \\
\hline 40 & 35.2 & 34.8 & 34.4 & 34.0 & 33.6 & 33.2 & 32.8 & 32.4 & 32.0 \\
\hline 41 & $36 . \overline{7}$ & 36.2 & 35.8 & 35.4 & 35.0 & 34.6 & 34.2 & 33.8 & 33.4 \\
\hline 42 & 38. 2 & 37.7 & 37.3 & 36.9 & 36. & 36.0 & 35.6 & 35.2 & 34.8 \\
\hline 43 & 39.8 & 39.3 & 38.9 & 38.5 & 38.1 & 37.6 & 37.2 & 36.8 & 36.3 \\
\hline 44 & 41.4 & 41.0 & 40.5 & 40.1 & 39.7 & 39.2 & 38.8 & 38.4 & 37.9 \\
\hline 45 & 43.2 & 42.7 & $42: 3$ & 41.8 & 41. & 40.9 & 40.5 & 40.1 & 39.6 \\
\hline 46 & 45.0 & 44.6 & 44.2 & 43.7 & 43. & 42.7 & 42. & 41.9 & 41.4 \\
\hline 47 & 46.9 & 46.5 & 46.0 & 45.6 & 45. 1. & 44.6 & 44.2 & 43.7 & 43. 3 \\
\hline 48 & 49.0 & 48.6 & 48.1 & 47.7 & 47.2 & 46.7 & 46.3 & 45.8 & 45.3 \\
\hline 49 & 51.2 & $50.7^{\circ}$ & 50.2 & 49.8 & 49.3 & 48.8 & 48.4 & 47.9 & 47.4 \\
\hline 50 & 153.5 & 153.0 & 152.5 & 152.0 & 151.5 & 151.0 & 150.6 & 1.50.1 & 1. 49.6 \\
\hline
\end{tabular}

The above table was computed with mean declination of Polaris fo: each year. A more accurate result will be had by applying to the tabular values the following correction, which depends on the difference of the mean and the apparent place of the star. The deduced azimuth will in general be correct within $0^{\prime} .3$.

\begin{tabular}{|c|c|c|c|}
\hline For middle of- & Correction. & For middle of- & Correction. \\
\hline January .. & $\begin{array}{c}\prime \\
-0.4\end{array}$ & Ju!y ..... & $\begin{array}{c}\prime \\
+0.3\end{array}$ \\
\hline February... & -0.3 & August ... & +0.1 \\
\hline March .... & -0.2 & September.. & -0.1 \\
\hline April........ & . 0.0 & October ..... & -0.3 \\
\hline May ..... & +0.2 & November .... & -0.6 \\
\hline June. & +0.3 & December ... & -0.8 \\
\hline
\end{tabular}


Table 3.-Azimuth and Apparent Alitirude or Polaris at' Difreken't Hour Angles.

[From U.S. Coust and Geodetic Survey Report for 1895.]

The accompanying tables are intended for field use, to facilitate placing an instrument in the meridian. They are also suitable for determining the approximate latitude or meridian. They contain the azimuth of Polaris at intervals of fifteen minutes in hour angle for each degree of north latitude from $30^{\circ}$ to $60^{\circ}$, and the apparent altitude at the same intervals and for each fifth degree of latitude. ${ }^{a}$ The tables are computed for the declination of Polaris $88^{\circ}, 46^{\prime}$, but the rate -of change in both arimuth and altitude is given with the argument $1^{\prime}$ increase in declination. ${ }^{b}$ The tables are intended to be used in connection with the American Ephemeris, where are given the apparent right ascension and declination of Polaris for each day in the year. The approximate local time will in general be known with sufficient accuracy from standard time and the approximate. longitude of the place. The following example explains the use of the tables and the derivation of the hour angle of Polaris:

Position, latitude $36^{\circ} 20^{\prime} \mathrm{N}$., longitude $5^{\mathrm{h}} 20^{\mathrm{m}} 30^{\mathrm{s}} \mathrm{W}$. of Grẹenwich.

Time of observation, July 10, 1895, standard (75th mer.) mean time

Reduction to local time

Local mean time

Reduction to sidereal time (Table III, Amer. Ephem.)

Sidereal time mean noon, Greenwich, July 10, 1.895

h. m. s.

Correction for longitude, $5^{\mathrm{h}} 20^{\mathrm{m}} 30^{\mathrm{s}}$ (Table III, Amer. Ephem.)

Local sidereal time

Apparent right ascension of Polaris, July 10, 1895

Hour angle before upper culmination

$852 \quad 40$ p.m.

$-2030$

$8 \quad 32 \quad 10$

$+\quad 124$

$\begin{array}{lll}7 & 12 & 38\end{array}$

$+\quad 0-53$

$\overline{1.5 \quad 47 \quad 05}$

1. $20 \quad 18$

$\begin{array}{lll}9 & 33 & 13\end{array}$

a The tables were computed with the following formulas:

$$
\begin{aligned}
\tan a & =\overline{\cos \phi} \tan ^{-\sin t}-\sin \phi \cos t^{\prime} \\
\sin h & =\sin \phi \sin \delta+\cos \phi \cos \delta \cos t, \\
\sin a_{\mathrm{e}} & =\frac{\cos \delta}{\cos \phi} \\
\cos t_{\mathrm{e}} & =\cot \delta \tan \phi ; \\
\text { where } a & =\text { nzimuth from true north, } \\
t & =\text { hour angle, } \\
\phi & =\text { latitude, } \\
\delta & =\text { declination, } \\
h & =\text { true altitude, } \\
a_{\mathrm{c}} & =\text { azimuth at elongation, } \\
t_{0} & =\text { hour angle at elongation. }
\end{aligned}
$$

$b$ As the corrections are given with proper sign for increase in declination over $88^{\circ} 46^{\prime}$, they are to be applied with reversed sign while the declination is less than $88^{\circ} 46^{\prime}$, as it will be until near the close of the century. 
Declination of table

Apparent declination, July 10, 1895

$$
\begin{array}{lcc}
\circ & \prime & \prime \prime \\
88 & 46 & \\
88 & 44 & 47 \\
\hline-1 & 13 & =-1^{\prime} \cdot 2
\end{array}
$$$$
\text { Increase in declination }
$$

Values from tables (interpolated) azimuth $0 \quad 54 \quad 12$, apparent altitude $35 \quad 21.8$ Correction for $-1^{\prime} \cdot 2$ increase in declination

$$
\begin{aligned}
& \frac{+52}{05504} \\
& \text { East of north }
\end{aligned}
$$

It is to be remembered that Polaris is east of the meridian for twelve hours before upper culmination, and west of the meridian for twelve. hours after. By setting the instrument at the apparent altitude and sweeping near the meridian Polaris can ordinarily be found and the instrument placed in the meridian some time before dark. With transit instruments not provided with horizontal arc, the value of the azimuth adjusting screw may be readily determined and used.

Without the American Ephemeris these tables may be conveniently used for obtaining the approximate meridian or latitude, in connection, with Bulletin 14, United States Coast and Geodetic Survey, ${ }^{a}$ where

\begin{tabular}{|c|c|c|c|c|c|}
\hline & \multicolumn{2}{|c|}{$a$} & \multicolumn{3}{|c|}{$\delta$} \\
\hline $\begin{array}{r}1895 \\
1900 \\
1905 \\
1910\end{array}$ & $\begin{array}{cc}h_{.} & m . \\
1 & 20 \\
1 & 22 \\
1 & 24 \\
1 & 26\end{array}$ & $\begin{array}{c}8 . \\
30.08 \\
33.76 \\
42.48 \\
56.58\end{array}$ & $\begin{array}{l}\circ \\
88 \\
88 \\
88 \\
88\end{array}$ & $\begin{array}{l}\prime \\
44 \\
46 \\
48 \\
49\end{array}$ & $\begin{array}{c}\prime \prime \\
52.68 \\
26.66 \\
00.31 \\
33.61\end{array}$ \\
\hline
\end{tabular}
are given the approximate mean times of culminations of Polaris, and the mean declinations for various epochs.

a Approximate Times of Culminations and Elongations and of the Azimuths at Elongation of Polaris for the Years between 1889 and 1910.

The mean places of Polaris are given as follows: 

TAвне 3.-Azimuth and apparent altitude

\begin{tabular}{|c|c|c|c|c|c|c|}
\hline \multirow{2}{*}{$\begin{array}{l}\text { Hour angle before } \\
\text { or after upper } \\
\text { culmination. }\end{array}$} & \multicolumn{6}{|c|}{ Azimuth of Polaris computed for declination $88^{\circ} 46^{\prime}$. } \\
\hline & $\begin{array}{c}\text { Latitude } \\
30^{\circ} .\end{array}$ & $\begin{array}{l}\text { Latitude } \\
31^{\circ} \text {. }\end{array}$ & $\begin{array}{l}\text { Latitude } \\
32^{\circ} \text {. }\end{array}$ & $\begin{array}{c}\text { Latitude } \\
33^{\circ} .\end{array}$ & $\begin{array}{c}\text { Latitude } \\
34^{\circ} \text {. }\end{array}$ & $\begin{array}{c}\text { Latitude } \\
35^{\circ} .\end{array}$ \\
\hline $\begin{array}{ll}h . & m . \\
0 & 15 \\
0 & 30 \\
0 & 45 \\
1 & 00 \\
1 & 15\end{array}$ & $\begin{array}{lcc}\circ & \prime & \prime \prime \\
0 & 05 & 40 \\
0 & 11 & 18 \\
0 & 16 & 53 \\
0 & 22 & 23 \\
0 & 27 & 48\end{array}$ & $\begin{array}{ccc}\circ & \prime & \prime \prime \\
0 & 05 & 43 \\
0 & 11 & 25 \\
0 & 17 & 04 \\
0 & 22 & 38 \\
0 & 28 & 06\end{array}$ & $\begin{array}{ccc}\circ & , & \prime \prime \\
0 & 05 & 47 \\
0 & 11 & 33 \\
0 & 17 & 15 \\
0 & 22 & 53 \\
0 & 28 & 25\end{array}$ & $\begin{array}{lcc}\circ & \prime & \prime \prime \\
0 & 05 & 51 \\
0 & 11 & 41 \\
0 & 17 & 27 \\
0 & 23 & 09 \\
0 & 28 & 45\end{array}$ & $\begin{array}{ccc}c & \prime & \prime \prime \\
0 & 05 & 55 \\
0 & 11 & 49 \\
0 & 17 & 40 \\
0 & 23 & 26 \\
0 & 29 & 06\end{array}$ & $\begin{array}{ccc}\circ & \prime & \prime \prime \\
0 & 06 & 00 \\
0 & 11 & 58 \\
0 & 17 & 53 \\
0 & 23 & 44 \\
0 & 29 & 28\end{array}$ \\
\hline $\begin{array}{ll}1 & 30 \\
1 & 45 \\
2 & 00 \\
2 & 15 \\
2 & 30\end{array}$ & $\begin{array}{lll}0 & 33 & 05 \\
0 & 38 & 13 \\
0 & 43 & 12 \\
0 & 47 & 58 \\
0 & 52 & 32\end{array}$ & $\begin{array}{lll}0 & 33 & 26 \\
0 & 38 & 38 \\
0 & 43 & 40 \\
0 & 48 & 29 \\
0 & 53 & 06\end{array}$ & $\begin{array}{lll}0 & 33 & 49 \\
0 & 39 & 04 \\
0 & 44 & 09 \\
0 & 49 & 02 \\
0 & 53 & 42\end{array}$ & $\begin{array}{lll}0 & 34 & 13 \\
0 & 39 & 32 \\
0 & 44 & 40 \\
0 & 49 & 36 \\
0 & 54 & 19\end{array}$ & $\begin{array}{lll}0 & 34 & 38 \\
0 & 40 & 00 \\
0 & 45 & 12 \\
0 & 50 & 12 \\
0 & 54 & 59\end{array}$ & $\begin{array}{lll}0 & 35 & 04 \\
0 & 40 & 30 \\
0 & 45 & 46 \\
0 & 50 & 50 \\
0 & 55 & 40\end{array}$ \\
\hline $\begin{array}{ll}2 & 45 \\
3 & 00 \\
3 & 15 \\
3 & 30 \\
3 & 45\end{array}$ & $\begin{array}{lll}0 & 56 & 52 \\
1 & 00 & 58 \\
1 & 04 & 47 \\
1 & 08 & 19 \\
1 & 11 & 33\end{array}$ & $\begin{array}{lll}0 & 57 & 29 \\
1 & 01 & 37 \\
1 & 05 & 28 \\
1 & 09 & 02 \\
1 & 12 & 18\end{array}$ & $\begin{array}{lll}0 & 58 & 07 \\
1 & 02 & 18 \\
1 & 06 & 12 \\
1 & 09 & 48 \\
1 & 13 & 06\end{array}$ & $\begin{array}{lll}0 & 58 & 48 \\
1 & 03 & 01 \\
1 & 06 & 58 \\
1 & 10 & 36 \\
1 & 13 & 56\end{array}$ & $\begin{array}{lll}0 & 59 & 30 \\
1 & 03 & 46 \\
1 & 07 & 46 \\
1 & 11 & 27 \\
1 & 14 & 49\end{array}$ & $\begin{array}{lll}1 & 00 & 15 \\
1 & 04 & 34 \\
1 & 08 & 36 \\
1 & 12 & 20 \\
1 & 15 & 45\end{array}$ \\
\hline $\begin{array}{ll}4 & 00 \\
4 & 15 \\
4 & 30 \\
4 & 45 \\
5 & 00\end{array}$ & $\begin{array}{lll}1 & 14 & 28 \\
1 & 17 & 04 \\
1 & 19 & 19 \\
1 & 21 & 14 \\
1 & 22 & 48\end{array}$ & $\begin{array}{lll}1 & 15 & 15 \\
1 & 17 & 52 \\
1 & 20 & 09 \\
1 & 22 & 05 \\
1 & 23 & 40\end{array}$ & $\begin{array}{lll}1 & 16 & 05 \\
1 & 18 & 44 \\
1 & 21 & 02 \\
1 & 22 & 59 \\
1 & 24 & 35\end{array}$ & $\begin{array}{lll}1 & 16 & 57 \\
1 & 19 & 37 \\
1 & 21 & 57 \\
1 & 23 & 55 \\
1 & 25 & 32\end{array}$ & $\begin{array}{lll}1 & 17 & 52 \\
1 & 20 & 34 \\
1 & 22 & 55 \\
1 & 24 & 55 \\
1 & 26 & 32\end{array}$ & $\begin{array}{lll}1 & 18 & 50 \\
1 & 21 & 34 \\
1 & 23 & 57 \\
1 & 25 & 57 \\
1 & 27 & 36\end{array}$ \\
\hline $\begin{array}{ll}5 & 15 \\
5 & 30 \\
5 & 45 \\
6 & 00 \\
6 & 15\end{array}$ & $\begin{array}{lll}1 & 24 & 00 \\
1 & 24 & 51 \\
1 & 25 & 20 \\
1 & 25 & 27 \\
1 & 25 & 12\end{array}$ & $\begin{array}{lll}1 & 24 & 53 \\
1 & 25 & 44 \\
1 & 26 & 13 \\
1 & 26 & 19 \\
1 & 26 & 04\end{array}$ & $\begin{array}{lll}1 & 25 & 48 \\
1 & 26 & 40 \\
1 & 27 & 09 \\
1 & 27 & 15 \\
1 & 26 & 59\end{array}$ & $\begin{array}{lll}1 & 26 & 46 \\
1 & 27 & 38 \\
1 & 28 & 07 \\
1 & 28 & 14 \\
1 & 27 & 57\end{array}$ & $\begin{array}{lll}1 & 27 & 47 \\
1 & 28 & 39 \\
1 & 29 & 09 \\
1 & 29 & 15 \\
1 & 28 & 59\end{array}$ & $\begin{array}{lll}1 & 28 & 51 \\
1 & 29 & 44 \\
1 & 30 & 14 \\
1 & 30 & 20 \\
1 & 30 & 03\end{array}$ \\
\hline $\begin{array}{ll}6 & 30 \\
6 & 45 \\
7 & 00 \\
7 & 15 \\
7 & 30\end{array}$ & $\begin{array}{lll}1 & 24 & 34 \\
1 & 23 & 36 \\
1 & 22 & 16 \\
1 & 20 & 35 \\
1 & 18 & 34\end{array}$ & $\begin{array}{lll}1 & 25 & 27 \\
1 & 24 & 27 \\
1 & 23 & 06 \\
1 & 21 & 25 \\
1 & 19 & 22\end{array}$ & $\begin{array}{lll}1 & 26 & 21 \\
1 & 25 & 21 \\
1 & 23 & 59 \\
1 & 22 & 16 \\
1 & 20 & 12\end{array}$ & $\begin{array}{lll}1 & 27 & 19 \\
1 & 26 & 18 \\
1 & 24 & 55 \\
1 & 23 & 10 \\
1 & 21 & 05\end{array}$ & $\begin{array}{lll}1 & 28 & 19 \\
1 & 27 & 17 \\
1 & 25 & 53 \\
1 & 24 & 08 \\
1 & 22 & 00\end{array}$ & $\begin{array}{lll}1 & 29 & 23 \\
1 & 28 & 20 \\
1 & 26 & 55 \\
1 & 25 & 08 \\
1 & 22 & 59\end{array}$ \\
\hline $\begin{array}{ll}7 & 45 \\
8 & 00 \\
8 & 15 \\
8 & 30 \\
8 & 45\end{array}$ & $\begin{array}{lll}1 & 16 & 13 \\
1 & 13 & 33 \\
1 & 10 & 34 \\
1 & 07 & 17 \\
1 & 03 & 43\end{array}$ & $\begin{array}{lll}1 & 16 & 59 \\
1 & 14 & 17 \\
1 & 11 & 16 \\
1 & 07 & 57 \\
1 & 04 & 22\end{array}$ & $\begin{array}{lll}1 & 17 & 48 \\
1 & 15 & 04 \\
1 & 12 & 01 \\
1 & 08 & 40 \\
1 & 05 & 02\end{array}$ & $\begin{array}{lll}1 & 18 & 39 \\
1 & 15 & 53 \\
1 & 12 & 48 \\
1 & 09 & 25 \\
1 & 05 & 44\end{array}$ & $\begin{array}{lll}1 & 19 & 33 \\
1 & 16 & 45 \\
1 & 13 & 37 \\
1 & 10 & 12 \\
1 & 06 & 29\end{array}$ & $\begin{array}{lll}1 & 20 & 29 \\
1 & 17 & 39 \\
1 & 14 & 29 \\
1 & 11 & 01 \\
1 & 07 & 15\end{array}$ \\
\hline $\begin{array}{rr}9 & 00 \\
9 & 15 \\
9 & 30 \\
9 & 45 \\
10 & 00\end{array}$ & $\begin{array}{lll}0 & 59 & 54 \\
0 & 55 & 49 \\
0 & 51 & 31 \\
0 & 46 & 59 \\
0 & 42 & 16\end{array}$ & $\begin{array}{lll}1 & 00 & 30 \\
0 & 56 & 23 \\
0 & 52 & 01 \\
0 & 47 & 27 \\
0 & 42 & 42\end{array}$ & $\begin{array}{lll}1 & 01 & 07 \\
0 & 56 & 58 \\
0 & 52 & 34 \\
0 & 47 & 57 \\
0 & 43 & 08\end{array}$ & $\begin{array}{lll}1 & 01 & 47 \\
0 & 57 & 34 \\
0 & 53 & 08 \\
0 & 48 & 28 \\
0 & 43 & 36\end{array}$ & $\begin{array}{rrr}1 & 02 & 29 \\
0 & 58 & 13 \\
0 & 53 & 43 \\
0 & 49 & 00 \\
0 & 44 & 05\end{array}$ & $\begin{array}{ccc}1 & 03 & 12 \\
0 & 58 & 54 \\
0 & 54 & 21 \\
0 & 49 & 34 \\
0 & 44 & 35\end{array}$ \\
\hline $\begin{array}{ll}10 & 15 \\
10 & 30 \\
10 & 45 \\
11 & 00 \\
11 & 15\end{array}$ & $\begin{array}{lll}0 & 37 & 23 \\
0 & 32 & 20 \\
0 & 27 & 09 \\
0 & 21 & 51 \\
0 & 16 & 28\end{array}$ & $\begin{array}{lll}0 & 37 & 45 \\
0 & 32 & 39 \\
0 & 27 & 25 \\
0 & 22 & 04 \\
0 & 16 & 38\end{array}$ & $\begin{array}{lll}0 & 38 & 08 \\
0 & 32 & 59 \\
0 & 27 & 42 \\
0 & 22 & 18 \\
0 & 16 & 48\end{array}$ & $\begin{array}{lll}0 & 38 & 33 \\
0 & 33 & 20 \\
0 & 28 & 00 \\
0 & 22 & 32 \\
0 & 16 & 59\end{array}$ & $\begin{array}{lll}0 & 38 & 59 \\
0 & 33 & 43 \\
0 & 28 & 18 \\
0 & 22 & 47 \\
0 & 17 & 10\end{array}$ & $\begin{array}{lll}0 & 39 & 26 \\
0 & 34 & 06 \\
0 & 28 & 38 \\
0 & 23 & 03 \\
0 & 17 & 22\end{array}$ \\
\hline $\begin{array}{ll}11 & 30 \\
11 . & 45\end{array}$ & $\begin{array}{lll}0 & 111 & 01 \\
0 & 05 & 31\end{array}$ & $\begin{array}{lll}0 & 11 & 08 \\
0 & 05 & 34\end{array}$ & $\begin{array}{lll}0 & 11 & 14 \\
0 & 05 & 38\end{array}$ & $\begin{array}{lll}0 & 11 & 22 \\
0 & 05 & 42\end{array}$ & $\begin{array}{lll}0 & 11 & 29 \\
0 & 05 & 45\end{array}$ & $\begin{array}{lll}0 & 11 & 37 \\
0 & 05 & 49\end{array}$ \\
\hline $\begin{array}{l}\text { Elongation: } \\
\text { Arimuth } \\
\text { Hour angle. }\end{array}$ & $\begin{array}{lll}1 & 25 & 27 \\
\text { h. } & \text { m. } & s . \\
5 & 57 & 09\end{array}$ & $\begin{array}{ccc}1 . & 26 & 20 \\
\text { h. } & \text { m. } & s . \\
5 & 57 & 02\end{array}$ & $\begin{array}{lll}1 & 27 & 16 \\
\text { h. } & m . & s \\
5 & 56 & 55\end{array}$ & $\begin{array}{lll}\text { 1. } & 28 & 14 \\
\text { h. } & \text { m. } & 8 \\
5 & 56 & 48\end{array}$ & $\begin{array}{lll}1 & 29 & 16 \\
\text { h. } & \text { m. } & 8 . \\
5 & 56 & 40\end{array}$ & $\begin{array}{lll}1 & 30 & 20 \\
h . & m . & s . \\
5 & 56 & 33\end{array}$ \\
\hline
\end{tabular}


of Poluris at different hour angles.

\begin{tabular}{|c|c|c|c|c|c|c|c|}
\hline \multicolumn{5}{|c|}{ Azimuth of Polaris computed for declination $88^{\circ} 46^{\prime}$. } & \multicolumn{2}{|c|}{$\begin{array}{l}\text { Correction for } 1^{\prime} \text { in- } \\
\text { crease in declinn- } \\
\text { tion of Polaris. }\end{array}$} & \multirow{2}{*}{$\begin{array}{c}\text { Hour } \\
\text { angle } \\
\text { before } \\
\text { or after } \\
\text { upper } \\
\text { culmi- } \\
\text { nation. }\end{array}$} \\
\hline $\begin{array}{c}\text { Latitude } \\
36^{\circ} \text {. }\end{array}$ & $\begin{array}{l}\text { Latitude } \\
37^{\circ} \text {. }\end{array}$ & $\begin{array}{l}\text { Latitude } \\
38^{\circ} \text {. }\end{array}$ & $\begin{array}{c}\text { Latitude } \\
39^{\circ} .\end{array}$ & $\begin{array}{l}\text { Latitude } \\
40^{\circ} \text {. }\end{array}$ & $\begin{array}{c}\text { Jatitude } \\
30^{\circ} .\end{array}$ & $\begin{array}{l}\text { Latitude } \\
40^{\circ} \text {. }\end{array}$ & \\
\hline $\begin{array}{lcc}\circ & \prime & \prime \prime \\
0 & 06 & 05 \\
0 & 12 & 08 \\
0 & 18 & 07 \\
0 & 24 & 02 \\
0 & 29 & 51\end{array}$ & $\begin{array}{lcc}\circ & \prime & \prime \prime \\
0 & 06 & 10 \\
0 & 12 & 18 \\
0 & 18 & 22 \\
0 & 24 & 22 \\
0 & 30 & 15\end{array}$ & $\begin{array}{lcc}\circ & \prime & \prime \prime \\
0 & 06 & 15 \\
0 & 12 & 28 \\
0 & 18 & 38 \\
0 & 24 & 43 \\
0 & 30 & 41 .\end{array}$ & $\begin{array}{ccc}\circ & \prime & \prime \prime \\
0 & 06 & 20 \\
0 & 12 & 39 \\
0 & 18 & 54 \\
0 & 25 & 04 \\
0 & 31 & 08\end{array}$ & $\begin{array}{lcc}\circ & \prime & \prime \prime \\
0 & 06 & 26 \\
0 & 12 & 50 \\
0 & 19 & 11 \\
0 & 25 & 27 \\
0 & 31 & 36\end{array}$ & $\begin{array}{r}\prime \prime \\
-5 \\
-9 \\
-14 \\
-18 \\
-23\end{array}$ & $\begin{array}{r}\prime \prime \\
-5 \\
-10 \\
-16 \\
-21 \\
-26\end{array}$ & $\begin{array}{ll}h . & m . \\
0 & 15 \\
0 & 30 \\
0 & 45 \\
1 & 00 \\
1 & 15\end{array}$ \\
\hline $\begin{array}{lll}0 & 35 & 31 \\
0 & 41 & 02 \\
0 & 46 & 22 \\
0 & 51 & 29 \\
0 & 56 & 23\end{array}$ & $\begin{array}{lll}0 & 36 & 00 \\
0 & 41 & 35 \\
0 & 47 & 00 \\
0 & 52 & 11 \\
0 & 57 & 09\end{array}$ & $\begin{array}{lll}0 & 36 & 31 \\
0 & 42 & 11 \\
0 & 47 & 39 \\
0 & 52 & 55 \\
0 & 57 & 57\end{array}$ & $\begin{array}{lll}0 & 37 & 02 \\
0 & 42 & 47 \\
0 & 48 & 21 \\
0 & 53 & 41 \\
0 & 58 & 47\end{array}$ & $\begin{array}{lll}0 & 37 & 36 \\
0 & 43 & 26 \\
0 & 49 & 04 \\
0 & 54 & 29 \\
0 & 59 & 40\end{array}$ & $\begin{array}{l}-27 \\
-31 \\
-35 \\
-39 \\
-43\end{array}$ & $\begin{array}{l}-31 \\
-36 \\
-40 \\
-45 \\
-49\end{array}$ & $\begin{array}{ll}\text { 1. } & 30 \\
\text { 1. } 45 \\
2 & 00 \\
2 \curvearrowleft 15 \\
230\end{array}$ \\
\hline $\begin{array}{lll}1 & 01 & 02 \\
1 & 05 & 24 \\
1 & 09 & 29 \\
1 & 13 & 16 \\
1 & 16 & 43\end{array}$ & $\begin{array}{lll}1 & 01 & 51 \\
1 & 06 & 17 \\
1 & 10 & 25 \\
1 & 14 & 14 \\
1 & 17 & 44\end{array}$ & $\begin{array}{lll}1 & 02 & 4.3 \\
1 & 07 & 12 \\
1 & 11 & 24 \\
1 & 15 & 16 \\
1 & 1.8 & 49\end{array}$ & $\begin{array}{lll}1 & 03 & 37 \\
1 & 08 & 10 \\
1 & 12 & 25 \\
1 & 16 & 21 \\
1 & 19 & 57\end{array}$ & $\begin{array}{lll}1 & 04 & 34 \\
1 & 09 & 12 \\
1 & 13 & 30 \\
1 & 17 & 29 \\
1 & 21 & 08\end{array}$ & $\begin{array}{l}-46 \\
-50 \\
-53 \\
-56 \\
-58\end{array}$ & $\begin{array}{l}-53 \\
-57 \\
-60 \\
-63 \\
-66\end{array}$ & $\begin{array}{ll}2 & 45 \\
3 & 00 \\
3 & 15 \\
3 & 30 \\
3 & 45\end{array}$ \\
\hline $\begin{array}{lll}1 & 19 & 50 \\
1 & 22 & 36 \\
1 & 25 & 01 \\
1 & 27 & 03 \\
1 & 28 & 42\end{array}$ & $\begin{array}{lll}\text { 1. } & 20 & 54 \\
1 & 23 & 42 \\
1 . & 26 & 08 \\
1 & 28 & 12 \\
1 . & 29 & 52\end{array}$ & $\begin{array}{lll}1 & 22 & 01 \\
1 & 24 & 51 \\
1 & 27 & 19 \\
1 & 29 & 24 \\
1 & 31 & 06\end{array}$ & $\begin{array}{lll}1 & 23 & 11 \\
1 & 26 & 03 \\
1 & 28 & 33 \\
1 & 30 & 40 \\
1 & 32 & 23\end{array}$ & $\begin{array}{lll}1 & 24 & 25 \\
1 & 27 & 20 \\
1 & 29 & 52 \\
1 & 32 & 00 \\
1 & 33 & 44\end{array}$ & $\begin{array}{l}-61 \\
-63 \\
-64 \\
-66 \\
-68\end{array}$ & $\begin{array}{l}-69 \\
-72 \\
-74 \\
-75 \\
-76\end{array}$ & $\begin{array}{ll}4 & 00 \\
4 & 15 \\
4 & 30 \\
4 & 45 \\
5 & 00\end{array}$ \\
\hline $\begin{array}{lll}1 & 29 & 59 \\
1 & 30 & 52 \\
1 & 31 & 21 \\
1 & 31 & 27 \\
1 & 31 & 10\end{array}$ & $\begin{array}{ccc}1 & 31 & 09 \\
1 & 32 & 03 \\
1 & 32 & 33 \\
1 & 32 & 39 \\
1 & 32 & 21\end{array}$ & $\begin{array}{lll}1 & 32 & 24 \\
1 & 33 & 18 \\
1 & 33 & 48 \\
1 & 33 & 54 \\
1 & 33 & 36\end{array}$ & $\begin{array}{lll}1 & 33 & 42 \\
1 & 34 & 37 \\
1 & 35 & 07 \\
1 & 35 & 13 \\
1 & 34 & 54\end{array}$ & $\begin{array}{lll}1 & 35 & 04 \\
1 & 35 & 59 \\
1 & 36 & 30 \\
1 & 36 & 35 \\
1 & 36 & 16\end{array}$ & $\begin{array}{l}-69 \\
-69 \\
-70 \\
-70 \\
-69\end{array}$ & $\begin{array}{l}-77 \\
-78 \\
-78 \\
-78 \\
-78\end{array}$ & $\begin{array}{ll}5 & 15 \\
5 & 30 \\
5 & 45 \\
6 & 00 \\
6 & 15\end{array}$ \\
\hline $\begin{array}{lll}1 & 30 & 30 \\
1 & 29 & 26 \\
1 & 27 & 59 \\
1 & 26 & 11 \\
1 & 24 & 00\end{array}$ & $\begin{array}{lll}1 & 31 & 40 \\
1 & 30 & 35 \\
1 & 29 & 07 \\
1 & 27 & 17 \\
1 & 25 & 04\end{array}$ & $\begin{array}{lll}1 & 32 & 54 \\
1 & 31 & 48 \\
1 & 30 & 18 \\
1 & 28 & 26 \\
1 & 26 & 12\end{array}$ & $\begin{array}{lll}1 & 34 & 11 \\
1 & 33 & 04 \\
1 & 31 & 33 \\
1 & 29 & 39 \\
1 & 27 & 23\end{array}$ & $\begin{array}{lll}1 & 35 & 32 \\
1 & 34 & 24 \\
1 & 32 & 52 \\
1 & 30 & 56 \\
1 & 28 & 38\end{array}$ & $\begin{array}{l}-68 \\
-67 \\
-66 \\
-65 \\
-64\end{array}$ & $\begin{array}{l}-77 \\
-76 \\
-75 \\
-73 \\
-72\end{array}$ & $\begin{array}{ll}6 & 30 \\
6 & 45 \\
7 & 00 \\
7 & 15 \\
7 & 30\end{array}$ \\
\hline $\begin{array}{lll}1 & 21 & 28 \\
1 & 18 & 36 \\
1 & 15 & 24 \\
1 & 11 & 53 \\
1 & 08 & 04\end{array}$ & $\begin{array}{lll}1 & 22 & 30 \\
1 & 19 & 36 \\
1 & 16 & 21 \\
1 & 12 & 48 \\
1 & 08 & 56\end{array}$ & $\begin{array}{lll}1 & 23 & 36 \\
1 & 20 & 39 \\
1 & 17 & 22 \\
1 & 13 & 45 \\
1 & 09 & 50\end{array}$ & $\begin{array}{lll}1 & 24 & 45 \\
1 & 21 & 45 \\
1 & 18 & 25 \\
1 & 14 & 45 \\
1 & 10 & 47\end{array}$ & $\begin{array}{lll}1 & 25 & 57 \\
1 & 22 & 54 \\
1 & 19 & 31 \\
1 & 15 & 48 \\
1 & 11 & 47\end{array}$ & $\begin{array}{l}-62 \\
-60 \\
-57 \\
-54 \\
-51\end{array}$ & $\begin{array}{l}-69 \\
-66 \\
-64 \\
-61 \\
-58\end{array}$ & $\begin{array}{ll}7 & 45 \\
8 & 00 \\
8 & 15 \\
8 & 30 \\
8 & 45\end{array}$ \\
\hline $\begin{array}{lll}1 & 03 & 58 \\
0 & 59 & 37 \\
0 & 55 & 00 \\
0 & 50 & 10 \\
0 & 45 & 08\end{array}$ & $\begin{array}{lll}1 & 04 & 47 \\
1 & 00 & 22 \\
0 & 55 & 42 \\
0 & 50 & 48 \\
0 & 45 & 42\end{array}$ & $\begin{array}{lll}1 & 05 & 38 \\
1 & 01 & 09 \\
0 & 56 & 25 \\
0 & 51 & 27 \\
0 & 46 & 17\end{array}$ & $\begin{array}{lll}1 & 06 & 31 \\
1 & 01 & 59 \\
0 & 57 & 11 \\
0 & 52 & 09 \\
0 & 46 & 54\end{array}$ & $\begin{array}{lll}1 & 07 & 27 \\
1 & 02 & 51 \\
0 & 57 & 59 \\
0 & 52 & 53 \\
0 & 47 & 34\end{array}$ & $\begin{array}{l}-48 \\
-45 \\
-42 \\
-38 \\
--34\end{array}$ & $\begin{array}{l}-54 \\
-50 \\
-46 \\
-42 \\
-38\end{array}$ & $\begin{array}{rr}9 & 00 \\
9 & 1.5 \\
9 & 30 \\
9 & 45 \\
10 & 00\end{array}$ \\
\hline $\begin{array}{lll}0 & 39 & 54 \\
0 & 34 & 30 \\
0 & 28 & 59 \\
0 & 23 & 19 \\
0 & 17 & 35\end{array}$ & $\begin{array}{lll}0 & 40 & 24 \\
0 & 34 & 57 \\
0 & 29 & 20 \\
0 & 23 & 37 \\
0 & 17 & 48\end{array}$ & $\begin{array}{lll}0 & 40 & 55 \\
0 & 35 & 24 \\
0 & 29 & 43 \\
0 & 23 & 55 \\
0 & 18 & 02\end{array}$ & $\begin{array}{lll}0 & 41 & 28 \\
0 & 35 & 52 \\
0 & 30 & 07 \\
0 & 24 & 14 \\
0 & 18 & 16\end{array}$ & $\begin{array}{lll}0 & 42 & 03 \\
0 & 36 & 22 \\
0 & 30 & 32 \\
0 & 24 & 35 \\
0 & 18 & 31\end{array}$ & $\begin{array}{l}-30 \\
-26 \\
-22 \\
-18 \\
-13\end{array}$ & $\begin{array}{l}-34 \\
-29 \\
-24 \\
-20 \\
-15\end{array}$ & $\begin{array}{ll}10 & 15 \\
10 & 30 \\
10 & 45 \\
11 & 00 \\
1.1 & 15\end{array}$ \\
\hline $\begin{array}{lll}0 & 11 . & 46 \\
0 & 05 & 53\end{array}$ & $\begin{array}{lll}0 & 11 & 54 \\
0 & 05 & 58\end{array}$ & $\begin{array}{lll}0 & 12 & 04 \\
0 & 06 & 02\end{array}$ & $\begin{array}{lll}0 & 12 & 13 \\
0 & 06 & 07\end{array}$ & $\begin{array}{lll}0 & 12 & 23 \\
0 & 06 & 12\end{array}$ & $\begin{array}{l}-9 \\
-4\end{array}$ & $\begin{array}{l}-10 \\
-5\end{array}$ & $\begin{array}{l}1130 \\
1145 .\end{array}$ \\
\hline $\begin{array}{lll}1 & 31 & 28 \\
h . & m . . & s \\
5 & 56 & 25\end{array}$ & $\begin{array}{ccc}1 & 32 & 40 \\
h . & m . \\
5 & 56 & 8.7\end{array}$ & $\begin{array}{ccc}1 & 33 & 55 \\
h . & m . & 8 . \\
5 & 56 & 09\end{array}$ & $\begin{array}{ccc}1 & 35 & 14 \\
h . & m . & s . \\
5 & 56 & 00\end{array}$ & $\begin{array}{lll}1 & 36 & 36 \\
h . & m . & s . \\
5 & 55 & 52\end{array}$ & $\begin{array}{r}-69 \\
8 . \\
+\quad 2\end{array}$ & $\begin{array}{r}-78 \\
+8 \\
+\quad 3\end{array}$ & \\
\hline
\end{tabular}


TABus 3.-Azimuth and apparent altitude

\begin{tabular}{|c|c|c|c|c|c|c|}
\hline \multirow{2}{*}{$\begin{array}{l}\text { Hour angle before } \\
\text { or after upper } \\
\text { culmination. }\end{array}$} & \multicolumn{6}{|c|}{ Azimuth of Polaris computed for declination $88^{\circ} 46^{\prime}$. } \\
\hline & $\begin{array}{l}\text { Latitude } \\
40^{\circ} .\end{array}$ & $\begin{array}{c}\text { Latitude } \\
41^{\circ} .\end{array}$ & $\begin{array}{c}\text { Latitude } \\
42^{\circ} \text {. }\end{array}$ & $\begin{array}{c}\text { Latitude } \\
43^{\circ} .\end{array}$ & $\begin{array}{l}\text { Latitude } \\
\mathbf{4 4}^{\circ} .\end{array}$ & $\begin{array}{l}\text { Latitude } \\
45^{\circ} .\end{array}$ \\
\hline $\begin{array}{cc}h . & m . \\
0 & 15 \\
0 & 30 \\
0 & 45 \\
1 & 00 \\
1 & 15\end{array}$ & $\begin{array}{lcc}\circ & \prime & \prime \prime \\
0 & 06 & 26 \\
0 & 12 & 50 \\
0 & 19 & 11 \\
0 & 25 & 27 \\
0 & 31 & 36\end{array}$ & $\begin{array}{lcc}\circ & \prime & \prime \prime \\
0 & 06 & 32 \\
0 & 13 & 03 \\
0 & 19 & 30 \\
0 & 25 & 51 \\
0 & 32 & 05\end{array}$ & $\begin{array}{lcc}\circ & 1 & \prime \prime \\
0 & 06 & 39 \\
0 & 13 & 15 \\
0 & 19 & 48 \\
0 & 26 & 16 \\
0 & 32 & 36\end{array}$ & $\begin{array}{lcc}\circ & \prime & \prime \prime \\
0 & 06 & 45 \\
0 & 13 & 29 \\
0 & 20 & 08 \\
0 & 26 & 43 \\
0 & 33 & 09\end{array}$ & $\begin{array}{lcc}\circ & \prime & \prime \prime \\
0 & 06 & 52 \\
0 & 13 & 43 \\
0 & 20 & 29 \\
0 & 27 & 10 \\
0 & 33 & 44\end{array}$ & $\begin{array}{lcc}\circ & \prime & \prime \prime \\
0 & 07 & 00 \\
0 & 13 & 58 \\
0 & 20 & 52 \\
0 & 27 & 40 \\
0 & 34 & 21\end{array}$ \\
\hline $\begin{array}{ll}1 & 30 \\
1 & 45 \\
2 & 00 \\
2 & 15 \\
2 & 30\end{array}$ & $\begin{array}{lll}0 & 37 & 36 \\
0 & 43 & 26 \\
0 & 49 & 04 \\
0 & 54 & 29 \\
0 & 59 & 40\end{array}$ & $\begin{array}{lll}0 & 38 & 11 \\
0 & 44 & 07 \\
0 & 49 & 50 \\
0 & 55 & 20 \\
1 & 00 & 35\end{array}$ & $\begin{array}{lll}0 & 38 & 48 \\
0 & 44 & 50 \\
0 & 50 & 39 \\
0 & 56 & 14 \\
1 & 01 & 34\end{array}$ & $\begin{array}{lll}0 & 39 & 27 \\
0 & 45 & 35 \\
0 & 51 & 29 \\
0 & 57 & 10 \\
1 & 02 & 36\end{array}$ & $\begin{array}{lll}0 & 40 & 09 \\
0 & 46 & 22 \\
0 & 52 & 23 \\
0 & 58 & 10 \\
1 & 03 & 41\end{array}$ & $\begin{array}{lll}0 & 40 & 52 \\
0 & 47 & 12 \\
0 & 53 & 19 \\
0 & 59 & 12 \\
1 & 04 & 49\end{array}$ \\
\hline $\begin{array}{rr}2 & 45 \\
3 & 00 \\
-3 & 15 \\
3 & 30 \\
3 & 45\end{array}$ & $\begin{array}{lll}1 & 04 & 34 \\
1 & 09 & 12 \\
1 & 13 & 30 \\
1 & 17 & 29 \\
1 & 21 & 08\end{array}$ & $\begin{array}{ccc}1 & 05 & 34 \\
1 & 10 & 16 \\
1 & 14 & 38 \\
1 & 18 & 41 \\
1 & 22 & 23\end{array}$ & $\begin{array}{lll}1 & 06 & 38 \\
1 & 11 & 24 \\
1 & 15 & 50 \\
1 & 19 & 57 \\
1 & 23 & 42\end{array}$ & $\begin{array}{lll}1 & 07 & 44 \\
1 & 12 & 35 \\
1 & 17 & 06 \\
1 & 21 & 16 \\
1 & 25 & 04\end{array}$ & $\begin{array}{lll}1 & 08 & 54 \\
1 & 13 & 50 \\
1 & 18 & 25 \\
1 & 22 & 39 \\
1 & 26 & 32\end{array}$ & $\begin{array}{lll}1 & 10 & 08 \\
1 & 15 & 09 \\
1 & 19 & 49 \\
1 & 24 & 08 \\
1 & 28 & 04\end{array}$ \\
\hline $\begin{array}{ll}4 & 00 \\
4 & 15 \\
4 & 30 \\
4 & 45 \\
5 & 00\end{array}$ & $\begin{array}{lll}1 & 24 & 25 \\
1 & 27 & 20 \\
1 & 29 & 52 \\
1 & 32 & 00 \\
1 & 33 & 44\end{array}$ & $\begin{array}{lll}1 & 25 & 43 \\
1 & 28 & 40 \\
1 & 31 & 14 \\
1 & 33 & 24 \\
1 & 35 & 10\end{array}$ & $\begin{array}{lll}1 & 27 & 05 \\
1 & 30 & 04 \\
1 & 32 & 41 \\
1 & 34 & 53 \\
1 & 36 & 40\end{array}$ & $\begin{array}{lll}1 & 28 & 31 \\
1 & 31 & 33 \\
1 & 34 & 12 \\
1 & 36 & 25 \\
1 & 38 & 14\end{array}$ & $\begin{array}{lll}1 & 30 & 01 \\
1 & 33 & 07 \\
1 & 35 & 48 \\
1 & 38 & 04 \\
1 & 39 & 54\end{array}$ & $\begin{array}{lll}1 & 31 & 37 \\
1 & 34 & 45 \\
1 & 37 & 29 \\
1 & 39 & 47 \\
1 & 41 & 38\end{array}$ \\
\hline $\begin{array}{ll}5 & 15 \\
5 & 30 \\
5 & 45 \\
6 & 00 \\
6 & 15\end{array}$ & $\begin{array}{lll}1 & 35 & 04 \\
1 & 35 & 59 \\
1 & 36 & 30 \\
1 & 36 & 35 \\
1 & 36 & 16\end{array}$ & $\begin{array}{lll}1 & 36 & 30 \\
1 & 37 & 26 \\
1 & 37 & 57 \\
1 & 38 & 02 \\
1 & 37 & 43\end{array}$ & $\begin{array}{lll}1 & 38 & 02 \\
1 & 38 & 58 \\
1 & 39 & 29 \\
1 & 39 & 34 \\
1 & 39 & 14\end{array}$ & $\begin{array}{lll}1 & 39 & 37 \\
1 & 40 & 34 \\
1 & 41 & 05 \\
1 & 41 & 10 \\
1 & 40 & 49\end{array}$ & $\begin{array}{lll}1 & 41 & 18 \\
1 & 42 & 16 \\
1 & 42 & 47 \\
1 & 42 & 51 \\
1 & 42 & 30\end{array}$ & $\begin{array}{lll}1 & 43 & 04 \\
1 & 44 & 02 \\
1 & 44 & 34 \\
1 & 44 & 38 \\
1 & 44 & 16\end{array}$ \\
\hline $\begin{array}{ll}6 & 30 \\
6 & 45 \\
7 & 00 \\
7 & 15 \\
7 & 30\end{array}$ & $\begin{array}{lll}1 & 35 & 32 \\
1 & 34 & 24 \\
1 & 32 & 52 \\
1 & 30 & 56 \\
1 & 28 & 38\end{array}$ & $\begin{array}{lll}1 & 36 & 58 \\
1 & 35 & 48 \\
1 & 34 & 15 \\
1 & 32 & 17 \\
1 & 29 & 56\end{array}$ & $\begin{array}{lll}1 & 38 & 28 \\
1 & 37 & 17 \\
1 & 35 & 42 \\
1 & 33 & 42 \\
1 & 31 & 19\end{array}$ & $\begin{array}{lll}1 & 40 & 03 \\
1 & 38 & 50 \\
1 & 37 & 13 \\
1 & 35 & 11 \\
1 & 32 & 46\end{array}$ & $\begin{array}{lll}1 & 41 & 42 \\
1 & 40 & 28 \\
1 & 38 & 49 \\
1 & 36 & 45 \\
1 & 34 & 17\end{array}$ & $\begin{array}{lll}1 & 43 & 27 \\
1 & 42 & 12 \\
1 & 40 & 31 \\
1 & 38 & 24 \\
1 & 35 & 53\end{array}$ \\
\hline $\begin{array}{ll}7 & 45 \\
8 & 00 \\
8 & 15 \\
8 & 30 \\
8 & 45\end{array}$ & $\begin{array}{lll}1 & 25 & 57 \\
1 & 22 & 54 \\
1 & 19 & 31 \\
1 & 15 & 48 \\
1 & 11 & 47\end{array}$ & $\begin{array}{lll}1 & 27 & 13 \\
1 & 24 & 07 \\
1 & 20 & 41 \\
1 & 16 & 55 \\
1 & 12 & 49\end{array}$ & $\begin{array}{lll}1 & 28 & 33 \\
1 & 25 & 24 \\
1 & 21 & 55 \\
1 & 18 & 05 \\
1 & 13 & 55\end{array}$ & $\begin{array}{lll}1 & 29 & 56 \\
1 & 26 & 45 \\
1 & 23 & 12 \\
1 & 19 & 18 \\
1 & 15 & 05\end{array}$ & $\begin{array}{lll}1 & 31 & 25 \\
1 & 28 & 10 \\
1 & 24 & 33 \\
1 & 20 & 35 \\
1 & 16 & 18\end{array}$ & $\begin{array}{lll}1 & 32 & 58 \\
1 & 29 & 40 \\
1 & 25 & 59 \\
1 & 21 & 57 \\
1 & 17 & 35\end{array}$ \\
\hline $\begin{array}{rr}9 & 00 \\
9 & 15 \\
9 & 30 \\
9 & 45 \\
10 & 00\end{array}$ & $\begin{array}{lll}1 & 07 & 27 \\
1 & 02 & 51 \\
0 & 57 & 59 \\
0 & 52 & 53 \\
0 & 47 & 34\end{array}$ & $\begin{array}{lll}1 & 08 & 26 \\
1 & 03 & 45 \\
0 & 58 & 49 \\
0 & 53 & 39 \\
0 & 48 & 15\end{array}$ & $\begin{array}{lll}1 & 09 & 28 \\
1 & 04 & 43 \\
0 & 59 & 42 \\
0 & 54 & 27 \\
0 & 48 & 58\end{array}$ & $\begin{array}{lll}1 & 10 & 33 \\
1 & 05 & 43 \\
1 & 00 & 38 \\
0 & 55 & 18 \\
0 & 49 & 44\end{array}$ & $\begin{array}{lll}1 & 11 & 41 \\
1 & 06 & 47 \\
1 & 01 & 37 \\
0 & 56 & 11 \\
0 & 50 & 32\end{array}$ & $\begin{array}{lll}1 & 12 & 54 \\
1 & 07 & 54 \\
1 & 02 & 38 \\
0 & 57 & 07 \\
0 & 51 & 22\end{array}$ \\
\hline $\begin{array}{ll}10 & 15 \\
10 & 30 \\
10 & 45 \\
11 & 00 \\
11 & 15\end{array}$ & $\begin{array}{lll}0 & 42 & 03 \\
0 & 36 & 22 \\
0 & 30 & 32 \\
0 & 24 & 35 \\
0 & \cdot 18 & 31\end{array}$ & $\begin{array}{lll}0 & 42 & 39 \\
0 & 36 & 53 \\
0 & 30 & 58 \\
0 & 24 & 56 \\
0 & 18 & 47\end{array}$ & $\begin{array}{lll}0 & 43 & 18 \\
0 & 37 & 26 \\
0 & 31 & 26 \\
0 & 25 & 18 \\
0 & 19 & 04\end{array}$ & $\begin{array}{lll}0 & -43 & 58 \\
0 & 38 & 01 \\
0 & 31 & 55 \\
0 & 25 & 42 \\
0 & 19 & 22\end{array}$ & $\begin{array}{ccc}0 & 44 & 40 \\
0 & 38 & 38 \\
0 & 32 & 26 \\
0 & 26 & 06 \\
.0 & 19 & 40\end{array}$ & $\begin{array}{lll}0 & 45 & 25 \\
0 & 39 & 16 \\
0 & 32 & 58 \\
0 & 26 & 32 \\
0 & 20 & 00\end{array}$ \\
\hline $\begin{array}{l}11.30 \\
11.45\end{array}$ & $\begin{array}{lll}0 & 12 & 23 \\
0 & 06 & 12\end{array}$ & $\begin{array}{lll}0 & 12 & 34 \\
0 & 06 & 18\end{array}$ & $\begin{array}{lll}0 & 12 & 45 \\
0 & 06 & 23\end{array}$ & $\begin{array}{lll}0 & 12 & 57 \\
0 & 06 & 29\end{array}$ & $\begin{array}{lll}0 & 13 & 09 \\
0 & 06 & 36\end{array}$ & $\begin{array}{lll}0 & 13 & 23 \\
0 & 06 & 42\end{array}$ \\
\hline $\begin{array}{l}\text { Elongation } \\
\text { Azimuth } \\
\text { Hour an }\end{array}$ & $\begin{array}{ccc}1 & 36 & 36 \\
h . & m . & s . \\
5 & 55 & 52\end{array}$ & $\begin{array}{ccc}1 & 38 & 03 \\
h . & m . & s . \\
5 & 55 & 43\end{array}$ & $\begin{array}{ccc}1 & 39 & 35 \\
h . & m . & s \\
5 & 55 & 34\end{array}$ & $\begin{array}{ccc}1 & 41 & 11 \\
h . & m . & s . \\
5 & 55 & 24\end{array}$ & $\begin{array}{ccc}1 & 42 & 53 \\
h . & m . & s . \\
5 & 55 & 14\end{array}$ & $\begin{array}{ccc}1 & 4 \dot{4} & 40 \\
h . & m . & s . \\
5 & 55 & 04\end{array}$ \\
\hline
\end{tabular}


of Polaris at different hour angles-Continued.

\begin{tabular}{|c|c|c|c|c|c|c|c|}
\hline \multicolumn{5}{|c|}{ Azimuth of Poluris computed for declination $88^{\circ} 46^{\prime}$. } & \multicolumn{2}{|c|}{$\begin{array}{l}\text { Correction for } 1^{\prime} \text { in- } \\
\text { crease in declina- } \\
\text { tion of Polaris. }\end{array}$} & \multirow{2}{*}{$\begin{array}{l}\text { Hour } \\
\text { angle } \\
\text { before } \\
\text { or afte } \\
\text { upper } \\
\text { culmi } \\
\text { nation }\end{array}$} \\
\hline $\begin{array}{l}\text { Latitude } \\
46^{\circ} .\end{array}$ & $\begin{array}{l}\text { Latitude } \\
47^{\circ} \text {. }\end{array}$ & $\begin{array}{l}\text { Latitude } \\
48^{\circ} \text {. }\end{array}$ & $\begin{array}{l}\text { Latitude } \\
49^{\circ} .\end{array}$ & $\begin{array}{l}\text { Latitude } \\
50^{\circ} \text {. }\end{array}$ & $\begin{array}{c}\text { Latitude } \\
40^{\circ} .\end{array}$ & $\begin{array}{c}\text { Latitude } \\
50^{\circ} .\end{array}$ & \\
\hline $\begin{array}{lcc}\circ & \prime & \prime \prime \\
0 & 07 & 08 \\
0 & 14 & 13 \\
0 & 21 & 15 \\
0 & 28 & 11 \\
0 & 34 & 59\end{array}$ & $\begin{array}{lcc}\circ & \prime & \prime \prime \\
0 & 07 & 16 \\
0 & 14 & 30 \\
0 & 21 & 40 \\
0 & 28 & 44 \\
0 & 35 & 40\end{array}$ & $\begin{array}{lll}\circ & \prime & \prime \prime \\
0 & 07 & 25 \\
0 & 14 & 48 \\
0 & 22 & 06 \\
0 & 29 & 18 \\
0 & 36 & 23\end{array}$ & $\begin{array}{lcc}\circ & 1 & \prime \prime \\
0 & 07 & 34 \\
0 & 15 & 06 \\
0 & 22 & 33 \\
0 & 29 & 55 \\
0 & 37 & 08\end{array}$ & $\begin{array}{lcc}\circ & \prime & \prime \prime \\
0 & 07 & 44 \\
0 & 15 & 25 \\
0 & 23 & 02 \\
0 & 30 & 33 \\
0 & 37 & 56\end{array}$ & $\begin{array}{r}\prime \prime \\
-5 \\
-10 \\
-16 \\
-21 \\
-26\end{array}$ & $\begin{array}{l}\cdot \quad 1 \\
-6 \\
-13 \\
-19 \\
-25 \\
-32\end{array}$ & $\begin{array}{ll}\text { h. } & m . \\
0 & 15 \\
0 & 30 \\
0 & 45 \\
1 & 00 \\
1 & 15\end{array}$ \\
\hline $\begin{array}{lll}0 & 41 & 38 \\
0 & 48 & 05 \\
0 & 54 & 19 \\
1 & 00 & 18 \\
1 & 06 & 01\end{array}$ & $\begin{array}{lll}0 & 42 & 26 \\
0 & 49 & 01 \\
0 & 55 & 22 \\
1 & 01 & 28 \\
1 & 07 & 17\end{array}$ & $\begin{array}{lll}0 & 43 & 17 \\
0 & 49 & 59 \\
0 & 56 & 28 \\
1 & 02 & 41 \\
1 & 08 & 38\end{array}$ & $\begin{array}{lll}0 & 44 & 11 \\
0 & 51 & 02 \\
0 & 57 & 38 \\
1 & 03 & 59 \\
1 & 10 & 03\end{array}$ & $\begin{array}{lll}0 & 45 & 08 \\
0 & 52 & 07 \\
0 & 58 & 52 \\
1 & 05 & 21 \\
1 & 11 & 32\end{array}$ & $\begin{array}{l}-31 \\
-36 \\
-40 \\
-45 \\
-49\end{array}$ & $\begin{array}{l}-38 \\
-43 \\
-49 \\
-54 \\
-59\end{array}$ & $\begin{array}{ll}1 & 30 \\
1 & 45 \\
2 & 00 \\
2 & 15 \\
2 & 30\end{array}$ \\
\hline $\begin{array}{lll}1 & 11 & 26 \\
1 & 16 & 32 \\
1 & 21 & 17 \\
1 & 25 & 40 \\
1 & 29 & 41\end{array}$ & $\begin{array}{lll}1 & 12 & 48 \\
1 & 18 & 00 \\
1 & 22 & 50 \\
1 & 27 & 18 \\
1 & 31 & 23\end{array}$ & $\begin{array}{lll}1 & 14 & 15 \\
1 & 19 & 33 \\
1 & 24 & 29 \\
1 & 29 & 02 \\
1 & 33 & 11\end{array}$ & $\begin{array}{lll}1 & 1.5 & 47 \\
1 & 21 & 11 \\
1 & 26 & 13 \\
1 & 30 & 51 \\
1 & 35 & 05\end{array}$ & $\begin{array}{lll}1 & 17 & 24 \\
1 & 22 & 54 \\
1 & 28 & 02 \\
1 & 32 & 46 \\
1 & 37 & .06\end{array}$ & $\begin{array}{l}-53 \\
-57 \\
-60 \\
-63 \\
-66\end{array}$ & $\begin{array}{l}-64 \\
-68 \\
-72 \\
-76 \\
-80\end{array}$ & $\begin{array}{ll}2 & 45 \\
3 & 00 \\
3 & 15 \\
3 & 30 \\
3 & 45\end{array}$ \\
\hline $\begin{array}{lll}1 & 33 & 17 \\
1 & 36 & 29 \\
1 & 39 & 15 \\
1 & 41 . & 35 \\
1 & 43 & 29\end{array}$ & $\begin{array}{lll}1 & 35 & 03 \\
1 & 38 & 18 \\
1 & 41 & 08 \\
1 & 43 & 30 \\
1 & 45 & 25\end{array}$ & $\begin{array}{lll}1 & 36 & 55 \\
1 & 40 & 14 \\
1 & 43 & 06 \\
1 & 45 & 31 \\
1 & 47 & 28\end{array}$ & $\begin{array}{lll}1 & 38 & 54 \\
1 & 42 & 16 \\
1 & 45 & 11 \\
1 & 47 & 39 \\
1 & 49 & 38\end{array}$ & $\begin{array}{lll}1 & 40 & 59 \\
1 & 44 & 25 \\
1 & 47 & 24 \\
1 & 49 & 54 \\
1 & 51 & 55\end{array}$ & $\begin{array}{l}-69 \\
-72 \\
-74 \\
-75 \\
-76\end{array}$ & $\begin{array}{l}-83 \\
-86 \\
-88 \\
-90 \\
-91\end{array}$ & $\begin{array}{ll}4 & 00 \\
4 & 15 \\
4 & 30 \\
4 & 45 \\
5 & 00\end{array}$ \\
\hline $\begin{array}{lll}1 & 44 & 55 \\
1 & 45 & 54 \\
1 & 46 & 26 \\
1 & 46 & 31 \\
1 & 46 & 08\end{array}$ & $\begin{array}{lll}1 . & 46 & 53 \\
1 & 47 & 53 \\
1 & 48 & 25 \\
1 & 48 & 29 \\
1 & 48 & 05\end{array}$ & $\begin{array}{lll}1 & 48 & 57 \\
1 & 49 & 58 \\
1 & 50 & 30 \\
1 & 50 & 34 \\
1 & 50 & 10\end{array}$ & $\begin{array}{lll}1 & 51 & 08 \\
1 & 52 & 10 \\
1 & 52 & 43 \\
1 & 52 & 46 \\
1 & 52 & 21\end{array}$ & $\begin{array}{lll}1 & 53 & 27 \\
1 . & 54 & 30 \\
1 & 55 & 03 \\
1 & 55 & 06 \\
1 . & 54 & 40\end{array}$ & $\begin{array}{l}-77 \\
-78 \\
-78 \\
-78 \\
-78\end{array}$ & $\begin{array}{l}-92 \\
-93 \\
-94 \\
-93 \\
-93\end{array}$ & $\begin{array}{ll}5 & 15 \\
5 & 30 \\
5 & 45 \\
6 & 00 \\
6 & 15\end{array}$ \\
\hline $\begin{array}{ccc}1 & 45 & 18 \\
1 & 44 & .01 \\
1 & 42 & 18 \\
1 & 40 & 09 \\
1 & 37 & 35\end{array}$ & $\begin{array}{lll}1 & 47 & 14 \\
1 & 45 & 56 \\
1 & 44 & 10 \\
1 & 41 & 59 \\
1 & 39 & 21\end{array}$ & $\begin{array}{rrr}1 & 49 & 17 \\
1 & 47 & 56 \\
1 . & 46 & 09 \\
.1 & 43 & 54 \\
1 . & 41 & 14\end{array}$ & $\begin{array}{lll}1 & 51 & 27 \\
1 & 50 & 04 \\
1 & 48 & 14 \\
1 & 45 & 57 \\
1 & 43 & 13\end{array}$ & $\begin{array}{lll}1 & 53 & 44 \\
1 & 52 & 20 \\
1 . & 50 & 27 \\
1 & 48 & 06 \\
1 . & 45 & 19\end{array}$ & $\begin{array}{l}-77 \\
-76 \\
-75 \\
-73 \\
-72\end{array}$ & $\begin{array}{l}-92 \\
-91 \\
-89 \\
-87 \\
-85\end{array}$ & $\begin{array}{ll}6 & 30 \\
6 & 45 \\
7 & 00 \\
7 & 15 \\
7 & 30\end{array}$ \\
\hline $\begin{array}{lll}1 & 34 & 36 \\
1 & 31 & 14 \\
1 & 27 & 29 \\
1 & 23 & 28 \\
1 & 18 & 56\end{array}$ & $\begin{array}{lll}1 & 36 & 19 \\
1 & 32 & 53 \\
1 & 29 & 04 \\
1 & 24 & 53 \\
1 & 20 & 21\end{array}$ & $\begin{array}{lll}1 & 38 & 08 \\
1 & 34 & 38 \\
1 & 30 & 44 \\
1 & 26 & 28 \\
1 & 21 & 51\end{array}$ & $\begin{array}{lll}1 & 40 & 03 \\
1 & 36 & 29 \\
1 & 32 & 30 \\
1 & 28 & 09 \\
1 & 23 & 26\end{array}$ & $\begin{array}{lll}1 & 42 & 05 \\
1 & 38 & 26 \\
1 & 34 & 22 \\
1 & 29 & 55 \\
1 & 25 & 07\end{array}$ & $\begin{array}{l}-69 \\
-66 \\
-64 \\
-61 \\
-58\end{array}$ & $\begin{array}{r}-82 \\
-79 \\
-76 \\
-72 \\
-68\end{array}$ & $\begin{array}{ll}7 & 45 \\
8 & 00 \\
8 & 15 \\
8 & 30 \\
8 & 45\end{array}$ \\
\hline $\begin{array}{lll}1 & 14 & 10 \\
1 & 09 & 05 \\
1 & 03 & 44 \\
0 & 58 & 07 \\
0 & 52 & 16\end{array}$ & $\begin{array}{lll}1 & 15 & 30 \\
1 & 10 & 19 \\
1 & 04 & 52 \\
0 & 59 & 09 \\
0 & 53 & 12\end{array}$ & $\begin{array}{lll}1 & 16 & 54 \\
1 & 11 & 38 \\
1 & 06 & 04 \\
1 & 00 & 15 \\
0 & 54 & 11\end{array}$ & $\begin{array}{lll}1 & 18 & 23 \\
1 & 13 & 01 \\
1 & 07 & 21 \\
1 & 01 & 24 \\
0 & 55 & 13\end{array}$ & $\begin{array}{lll}1 & 19 & 57 \\
1 & 14 & 28 \\
1 & 08 & 41 \\
1 & 02 & 38 \\
0 & 56 & 19\end{array}$ & $\begin{array}{l}-54 \\
-50 \\
-46 \\
-42 \\
-38\end{array}$ & $\begin{array}{l}-64 \\
-59 \\
-55 \\
-50 \\
-45\end{array}$ & $\begin{array}{rr}9 & 00 \\
9 & 15 \\
9 & 30 \\
9 & 45 \\
10 & 00\end{array}$ \\
\hline $\begin{array}{lll}0 & 46 & 12 \\
0 & 39 & 57 \\
0 & 33 & 32 \\
0 & 27 & 00 \\
0 & 20 & 20\end{array}$ & $\begin{array}{lll}0 & 47 & 01 \\
0 & 40 & 40 \\
0 & 34 & 08 \\
0 & 27 & 28 \\
0 & 20 & 42\end{array}$ & $\begin{array}{lll}0 & 47 & 53 \\
0 & 41 & 25 \\
0 & 34 & 46 \\
0 & 27 & 59 \\
0 & 21 & 05\end{array}$ & $\begin{array}{lll}0 & 48 & 49 \\
0 & 42 & 12 \\
0 & 35 & 26 \\
0 & 28 & 31 \\
0 & 21 & 29\end{array}$ & $\begin{array}{lll}0 & 49 & 47 \\
0 & 43 & 02 \\
0 & 36 & 08 \\
0 & 29 & 05 \\
0 & 21 & 55\end{array}$ & $\begin{array}{l}-34 \\
-29 \\
-24 \\
-20 \\
-15\end{array}$ & $\begin{array}{l}-40 \\
-34 \\
-29 \\
-23 \\
-18\end{array}$ & $\begin{array}{ll}10 & 15 \\
10 & 30 \\
10 & 45 \\
11 & 00 \\
11 & 15\end{array}$ \\
\hline $\begin{array}{lll}0 & 13 & 36 \\
0 & 06 & 49\end{array}$ & $\begin{array}{lll}0 & 13 & 51 \\
0 & 06 & 56\end{array}$ & $\begin{array}{lll}0 & 14 & 06 \\
0 & 07 & 04\end{array}$ & $\begin{array}{lll}0 & 14 & 22 \\
0 & 07 & 12\end{array}$ & $\begin{array}{lll}0 & 14 & 39 \\
0 & 07 & 21\end{array}$ & $\begin{array}{l}-10 \\
-5\end{array}$ & $\begin{array}{l}-12 \\
-6\end{array}$ & $\begin{array}{ll}11 & 30 \\
11 & 45\end{array}$ \\
\hline $\begin{array}{ccc}1 & 46 & 32 \\
h . & \text { m. } & s . \\
5 & 54 & 53\end{array}$ & $\begin{array}{ccc}1 & 48 & 31 \\
h . & m_{.} & 8 . \\
5 & 54 & 42\end{array}$ & $\begin{array}{ccc}1 & 50 & 36 \\
h . & m . & s \\
5 & 54 & 3 \mathrm{i}\end{array}$ & $\begin{array}{ccc}1 & 52 & 48 \\
h . & m . & 8 . \\
5 & 54 & 20\end{array}$ & $\begin{array}{ccc}1 & 55 & 08 \\
h . & m . & 8 . \\
5 & 54 & 07\end{array}$ & $\begin{array}{r}-78 \\
+3 . \\
+3\end{array}$ & $\begin{array}{r}-93 \\
8 \\
+5\end{array}$ & \\
\hline
\end{tabular}


TABLe 3.-Azimuth and apparent altitude

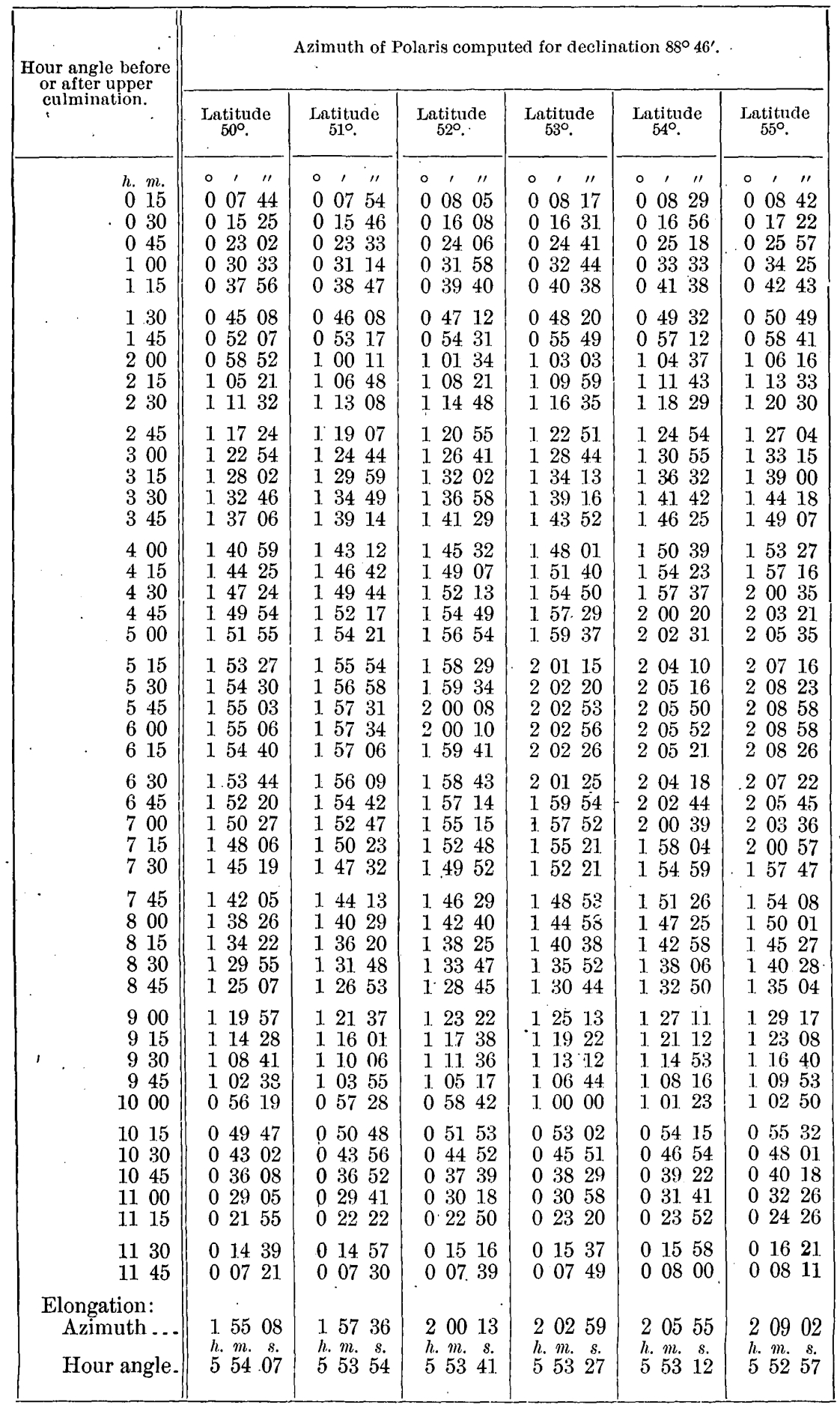


of Polaris al different hour angles-Continued.

\begin{tabular}{|c|c|c|c|c|c|c|c|}
\hline \multicolumn{5}{|c|}{ Azimuth of Polaris computed for declination $88^{\circ} 46^{\prime}$. } & \multicolumn{2}{|c|}{$\begin{array}{l}\text { Correction for } 1^{\prime} \text { in- } \\
\text { crease in declina- } \\
\text { tion of Poluris. }\end{array}$} & \multirow{2}{*}{$\begin{array}{c}\text { Hour } \\
\text { nngle } \\
\text { before } \\
\text { or after } \\
\text { upper } \\
\text { culmi- } \\
\text { nation. }\end{array}$} \\
\hline $\begin{array}{l}\text { Latitude } \\
56^{\circ} .\end{array}$ & $\begin{array}{l}\text { Latitude } \\
57^{\circ} \text {. }\end{array}$ & $\begin{array}{c}\text { Latitude } \\
58^{\circ} \text {. }\end{array}$ & $\begin{array}{c}\text { Latitude } \\
59^{\circ} .\end{array}$ & $\begin{array}{c}\text { Latitude } \\
60^{\circ} .\end{array}$ & \begin{tabular}{|} 
Latitude \\
$50^{\circ}$.
\end{tabular} & $\begin{array}{c}\text { Latitude } \\
60^{\circ} .\end{array}$ & \\
\hline $\begin{array}{lcc}\circ & \prime & \prime \prime \\
0 & 08 & 56 \\
0 & 17 & 50 \\
0 & 26 & 39 \\
0 & 35 & 21 \\
0 & 43 & 52\end{array}$ & $\begin{array}{lcc}\circ & \prime & \prime \prime \\
0 & 09 & 12 \\
0 & 18 & 20 \\
0 & 27 & 24 \\
0 & 36 & 20 \\
0 & 45 & 06\end{array}$ & $\begin{array}{lcc}\circ & \prime & \prime \prime \\
0 & 09 & 28 \\
0 & 18 & 53 \\
0 & 28 & 12 \\
0 & 37 & 23 \\
0 & 46 & 24\end{array}$ & $\begin{array}{lcc}\circ & \prime & \prime \prime \\
0 & 09 & 45 \\
0 & 19 & 27 \\
0 & 29 & 03 \\
0 & 38 & 31 \\
0 & 47 & 48\end{array}$ & $\begin{array}{ccc}\circ & \prime & \prime \prime \\
0 & 10 & 03 \\
0 & 20 & 04 \\
0 & 29 & 58 \\
0 & 39 & 44 \\
0 & 49 & 1.9\end{array}$ & $\begin{array}{r}\prime \prime \\
-6 \\
-13 \\
-19 \\
-25 \\
-32\end{array}$ & $\begin{array}{r}\quad 1 \\
-\quad 8 \\
-17 \\
-\quad 25 \\
-33 \\
-41\end{array}$ & $\begin{array}{ll}\text { h. } & m . \\
0 & 15 \\
0 & 30 \\
0 & 45 \\
1 & 00 \\
1 & 15\end{array}$ \\
\hline $\begin{array}{lll}0 & 52 & 11 \\
1 & 00 & 16 \\
1 & 08 & 03 \\
1 & 15 & 31 \\
1 & 22 & 39\end{array}$ & $\begin{array}{lll}0 & 53 & 39 \\
1 & 01 & 56 \\
1 & 09 & 57 \\
1 & 17 & 37 \\
1 & 24 & 56\end{array}$ & $\begin{array}{lll}0 & 55 & 12 \\
1 & 03 & 44 \\
1 & 11 & 58 \\
1 & 19 & 52 \\
1 & 27 & 24\end{array}$ & $\begin{array}{ccc}0 & 56 & 52 \\
1 & 05 & 40 \\
1 & 14 & 08 \\
1 & 22 & 16 \\
1 & 30 & 01\end{array}$ & $\begin{array}{lll}0 & 58 & 40 \\
1 & 07 & 44 \\
1 & 16 & 28 \\
1 & 24 & 51 \\
1 & 32 & 50\end{array}$ & $\begin{array}{l}-38 \\
-43 \\
-49 \\
-54 \\
-59\end{array}$ & $\begin{array}{l}-49 \\
-57 \\
-64 \\
-71 \\
-78\end{array}$ & $\begin{array}{ll}1 & 30 \\
1 & 45 \\
2 & 00 \\
2 & 15 \\
2 & 30\end{array}$ \\
\hline $\begin{array}{lll}1 & 29 & 23 \\
1 & 35 & 43 \\
1 & 41 & 37 \\
1 & 47 & 03 \\
1 & 52 & 00\end{array}$ & $\begin{array}{lll}1 & 31 & 52 \\
1 & 38 & 22 \\
1 & 44 & 25 \\
1 & 50 & 00 \\
1 & 55 & 04\end{array}$ & $\begin{array}{lll}1 & 34 & 31 \\
1 & 41 & 12 \\
1 & 47 & 25 \\
1 & 53 & 08 \\
1 & 58 & 21\end{array}$ & $\begin{array}{lll}1 & 37 & 21 \\
1 & 44 & 13 \\
1 & 50 & 37 \\
1 & 56 & 30 \\
2 & 01 & 51\end{array}$ & $\begin{array}{lll}1 & 40 & 23 \\
1 & 47 & 28 \\
1 & 54 & 03 \\
2 & 00 & 07 \\
2 & 05 & 37\end{array}$ & $\begin{array}{l}-64 \\
-68 \\
-72 \\
-76 \\
-80\end{array}$ & $\begin{array}{r}-84 \\
-89 \\
-94 \\
-99 \\
-104\end{array}$ & $\begin{array}{ll}2 & 45 \\
3 & 00 \\
3 & 1.5 \\
3 & 30 \\
3 & 45\end{array}$ \\
\hline $\begin{array}{lll}1 & 56 & 26 \\
2 & 00 & 21 \\
2 & 03 & 44 \\
2 & 06 & 34 \\
2 & 08 & 51\end{array}$ & $\begin{array}{lll}1 & 59 & 37 \\
2 & 03 & 38 \\
2 & 07 & 06 \\
2 & 10 & 00 \\
2 & 12 & 20\end{array}$ & $\begin{array}{lll}2 & 03 & 01 \\
2 & 07 & 09 \\
2 & 10 & 42 \\
2 & 13 & 40 \\
2 & 16 & 03\end{array}$ & $\begin{array}{lll}2 & 06 & 40 \\
2 & 10 & 54 \\
2 & 14 & 32 \\
2 & 17 & 35 \\
2 & 20 & 02\end{array}$ & $\begin{array}{lll}2 & 10 & 34 \\
2 & 14 & 55 \\
2 & 18 & 39 \\
2 & 21 & 47 \\
2 & 24 & 17\end{array}$ & $\begin{array}{l}-83 \\
-86 \\
-88 \\
-90 \\
-91\end{array}$ & $\begin{array}{l}-108 \\
-111 \\
-114 \\
-116 \\
-118\end{array}$ & $\begin{array}{ll}4 & 00 \\
4 & 15 \\
4 & 30 \\
4 & 45 \\
5 & 00\end{array}$ \\
\hline $\begin{array}{lll}2 & 10 & 34 \\
2 & 11 & 42 \\
2 & 12 & 17 \\
2 & 12 & 17 \\
2 & 11 & 44\end{array}$ & $\begin{array}{lll}2 & 14 & 05 \\
2 & 15 & 14 \\
2 & 15 & 50 \\
2 & 15 & 49 \\
2 & 15 & 14\end{array}$ & $\begin{array}{lll}2 & 17 & 50 \\
2 & 19 & 01 \\
2 & 19 & 36 \\
2 & 1.9 & 35 \\
2 & 18 & 59\end{array}$ & $\begin{array}{lll}2 & 21 & 51 \\
2 & 23 & 04 \\
2 & 23 & 39 \\
2 & 23 & 37 \\
2 & 22 & 59\end{array}$ & $\begin{array}{lll}2 & 26 & 09 \\
2 & 27 & 23 \\
2 & 27 & 58 \\
2 & 27 & 56 \\
2 & 27 & 15\end{array}$ & $\begin{array}{l}-92 \\
-93 \\
-94 \\
-93 \\
-93\end{array}$ & $\begin{array}{l}-119 \\
-120 \\
-1.20 \\
-1.20 \\
-1.19\end{array}$ & $\begin{array}{ll}5 & 1.5 \\
5 & 30 \\
5 & 45 \\
6 & 00 \\
6 & 1.5\end{array}$ \\
\hline $\begin{array}{lll}2 & 10 & 37 \\
2 & 08 & 57 \\
2 & 06 & 44 \\
2 & 04 & 00 \\
2 & 00 & 45\end{array}$ & $\begin{array}{lll}2 & 14 & 05 \\
2 & 12 & 21 \\
2 & 10 & 05 \\
2 & 07 & 16 \\
2 & 03 & 55\end{array}$ & $\begin{array}{lll}2 & 17 & 47 \\
2 & 16 & 00 \\
2 & 13 & 39 \\
2 & 10 & 45 \\
2 & 07 & 18\end{array}$ & $\begin{array}{lll}2 & 21 & 44 \\
2 & 19 & 53 \\
2 & 17 & 27 \\
2 & 14 & 27 \\
2 & 10 & 54\end{array}$ & $\begin{array}{lll}2 & 25 & 57 \\
2 & 24 & 03 \\
2 & 21 & 32 \\
2 & 18 & 26 \\
2 & 14 & 46\end{array}$ & $\begin{array}{l}-92 \\
-91 \\
-89 \\
-87 \\
-85\end{array}$ & $\begin{array}{l}-118 \\
-1.16 \\
-1.14 \\
-111 \\
-1.108\end{array}$ & $\begin{array}{ll}6 & 30 \\
6 & 45 \\
7 & 00 \\
7 & 15 \\
7 & 30\end{array}$ \\
\hline $\begin{array}{lll}1 & 57 & 00 \\
1 & 52 & 47 \\
1 & 48 & 06 \\
1 & 42 & 58 \\
1 & 37 & 26\end{array}$ & $\begin{array}{lll}2 & 00 & 04 \\
1 & 55 & 43 \\
1 & 50 & 54 \\
1 & 45 & 39 \\
1 & 39 & 57\end{array}$ & $\begin{array}{lll}2 & 03 & 20 \\
1 & 58 & 52 \\
1 & 53 & 54 \\
1 & 48 & 30 \\
1 & 42 & 39\end{array}$ & $\begin{array}{lll}2 & 06 & 49 \\
2 & 02 & 12 \\
1 & 57 & 06 \\
1 & 51 & 32 \\
1 & 45 & 31\end{array}$ & $\begin{array}{lll}2 & 1.0 & 32 \\
2 & 05 & 47 \\
2 & 00 & 32 \\
1 & 54 & 47 \\
1 & 48 & 35\end{array}$ & $\begin{array}{l}-82 \\
-79 \\
-76 \\
-72 \\
-68\end{array}$ & $\begin{array}{r}-104 \\
-100 \\
-96 \\
-91 \\
-86\end{array}$ & $\begin{array}{ll}7 & 45 \\
8 & 00 \\
8 & 15 \\
8 & 30 \\
8 & 45\end{array}$ \\
\hline $\begin{array}{lll}1 & 31 & 30 \\
1 & 25 & 12 \\
1 & 18 & 34 \\
1 & 11 & 37 \\
1 & 04 & 23\end{array}$ & $\begin{array}{lll}1 & 33 & 51 \\
1 & 27 & 24 \\
1 & 20 & 36 \\
1 & 13 & 28 \\
1 & 06 & 03\end{array}$ & $\begin{array}{lll}1 & 36 & 23 \\
1 & 29 & 44 \\
1 & 22 & 45 \\
1 & 15 & 25 \\
1 & 07 & 48\end{array}$ & $\begin{array}{lll}1 & 39 & 05 \\
1 & 32 & 14 \\
1 & 25 & 03 \\
1 & 17 & 31 \\
1 & 09 & 41\end{array}$ & $\begin{array}{lll}1 & 41 & 57 \\
1 & 34 & 55 \\
1 & 27 & 30 \\
1 & 19 & 45 \\
1 & 11 & 41\end{array}$ & $\begin{array}{l}-64 \\
-59 \\
-55 \\
-50 \\
-45\end{array}$ & $\begin{array}{l}-80 \\
-75 \\
-69 \\
-63 \\
-56\end{array}$ & $\begin{array}{rr}9 & 00 \\
9 & 15 \\
9 & 30 \\
9 & 45 \\
10 & 00\end{array}$ \\
\hline $\begin{array}{lll}0 & 56 & 54 \\
0 & 49 & 1.2 \\
0 & 41 & 18 \\
0 & 33 & 14 \\
0 & 25 & 02\end{array}$ & $\begin{array}{lll}0 & 58 & 22 \\
0 & 50 & 27 \\
0 & 42 & 21 \\
0 & 34 & 05 \\
0 & 25 & 41\end{array}$ & $\begin{array}{llll}0 & 59 & 55 \\
0 & 51 & 48 \\
0 & 43 & 28 \\
0 & 34 & 59 \\
0 & 26 & 21\end{array}$ & $\begin{array}{lll}1 & 01 & 34 \\
0 & 53 & 14 \\
0 & 44 & 40 \\
0 & 35 & 57 \\
0 & 27 & 05\end{array}$ & $\begin{array}{lll}1 & 03 & 20 \\
0 & 54 & 45 \\
0 & 45 & 57 \\
0 & 36 & 59 \\
0 & 27 & 51\end{array}$ & $\begin{array}{l}-40 \\
-34 \\
-29 \\
-23 \\
-18\end{array}$ & $\begin{array}{l}-50 \\
-43 \\
-36 \\
-29 \\
-22\end{array}$ & $\begin{array}{ll}10 & 15 \\
10 & 30 \\
10 & 45 \\
11 & 00 \\
11 & 1.5\end{array}$ \\
\hline $\begin{array}{lll}0 & 16 & 45 \\
0 & 08 & 23\end{array}$ & $\begin{array}{lll}0 & 17 & 10 \\
0 & 08 & 36\end{array}$ & $\begin{array}{lll}0 & 17 & 38 \\
0 & 08 & 50\end{array}$ & $\begin{array}{lll}0 & 18 & 07 \\
0 & 09 & 04\end{array}$ & $\begin{array}{lll}0 & 18 & 38 \\
0 & 09 & 20\end{array}$ & $\begin{array}{l}-12 \\
-6\end{array}$ & $\begin{array}{l}-14 \\
-\quad 7\end{array}$ & $\begin{array}{l}1130 \\
1145\end{array}$ \\
\hline $\begin{array}{llr}2 & 12 & 21 \\
h . & m . & s . \\
5 & 52 & 41\end{array}$ & $\begin{array}{lcc}2 & 15 & 54 \\
h . & m . & s . \\
5 & 52 & 24\end{array}$ & $\begin{array}{ccc}2 & 19 & 40 \\
h . & m . & s . \\
5 & 52 & 06\end{array}$ & $\begin{array}{ccc}2 & 23 & 43 \\
h . & \text { m. } \\
5 & 51 & 4 \\
4\end{array}$ & $\begin{array}{ccc}2 & 28 & 02 \\
h . & m . & s \\
5 & 51 & 27\end{array}$ & $\begin{array}{r}-93 \\
+8 \\
+5\end{array}$ & $\begin{array}{r}-120 \\
+\quad 8\end{array}$ & \\
\hline
\end{tabular}


TABLE 3.-Azimuth and apparent altitude of Palaris at different hour angles-Continued.

\begin{tabular}{|c|c|c|c|c|c|c|c|c|c|}
\hline \multirow{2}{*}{\begin{tabular}{|c|} 
Hour \\
angle \\
before \\
orafter \\
upper \\
culmi- \\
nation.
\end{tabular}} & \multicolumn{7}{|c|}{$\begin{array}{l}\text { Apparent altitude of Polaris, computed for declination } 88^{\circ} 46^{\prime} \text { and } \\
\text { mean refraction. }\end{array}$} & \multirow{2}{*}{$\begin{array}{c}\text { Correc- } \\
\text { tion } \\
\text { for 1' } \\
\text { in- } \\
\text { crease } \\
\text { in dec- } \\
\text { lination } \\
\text { of Po- } \\
\text { laris. }\end{array}$} & \multirow{2}{*}{$\begin{array}{l}\text { Hour } \\
\text { angle } \\
\text { before } \\
\text { or after } \\
\text { upper } \\
\text { culmi- } \\
\text { nation. }\end{array}$} \\
\hline & $\begin{array}{c}\text { Latitude } \\
30^{\circ} .\end{array}$ & $\begin{array}{c}\text { Latitude } \\
35^{\circ} .\end{array}$ & $\begin{array}{c}\text { Latitude } \\
40^{\circ} .\end{array}$ & $\begin{array}{c}\text { Latitude } \\
45^{\circ} .\end{array}$ & atitude & $\begin{array}{c}\text { Latitude } \\
55^{\circ} .\end{array}$ & $\begin{array}{c}\text { Latitude } \\
60^{\circ} .\end{array}$ & & \\
\hline 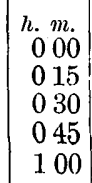 & $\begin{array}{cc}\circ & \\
31 & 15.6 \\
31 & 15.4 \\
31 & 14.9 \\
31 & 14.2 \\
31 & 13.0\end{array}$ & $\mid \begin{array}{cc}\circ & \prime \\
36 & 15.3 \\
36 & 15.2 \\
36 & 14.7 \\
36 & 13.9 \\
35 & 12.8\end{array}$ & $\begin{array}{cc}\circ & \prime \\
41 & 15.1 \\
41 & 14.9 \\
41 & 14.5 \\
41 & 13.7 \\
41 & 12.5\end{array}$ & $\begin{array}{cc}\circ & \prime \\
46 & 14.9 \\
46 & 14.8 \\
46 & 14.3 \\
46 & 13.5 \\
46 & 12.3\end{array}$ & $\begin{array}{cc}\circ & \prime \\
51 & 14.8 \\
51 & 14.6 \\
51 & 14.2 \\
51 & 13.3 \\
51 & 12.2\end{array}$ & $\begin{array}{cc}\circ & \prime \\
56 & 14.6 \\
56 & 14.4 \\
56 & 14.0 \\
56 & 13.2 \\
56 & 12.0\end{array}$ & $\begin{array}{cc}\circ & \prime \\
61 & 14.5 \\
61 & 14.3 \\
61 & 13.8 \\
61 & 13.0 \\
61 & 11.9\end{array}$ & $\begin{array}{r}1 \\
-1.0 \\
-1.0 \\
-1.0 \\
-1.0 \\
-1.0\end{array}$ & $\begin{array}{rr}h . & m . \\
0 & 00 \\
0 & 1.5 \\
0 & 30 \\
0 & 45 \\
1 & 00\end{array}$ \\
\hline $\begin{array}{ll}1 & 15 \\
1 & 30 \\
1 & 45 \\
2 & 00 \\
2 & 15\end{array}$ & $\begin{array}{ll}31 & 11.6 \\
31 & 09.9 \\
31 & 07.9 \\
31 & 05.6 \\
31 & 03.0\end{array}$ & $\begin{array}{|ll|}36 & 11.3 \\
36 & 09.6 \\
36 & 07.6 \\
36 & 05.3 \\
36 & 02.7\end{array}$ & $\begin{array}{ll}41 & 11.1 \\
41 & 09.4 \\
41 & 07.3 \\
41 & 05.0 \\
41 & 02.4\end{array}$ & $\begin{array}{ll}46 & 10.9 \\
46 & 09.2 \\
46 & 07.2 \\
46 & 04.8 \\
46 & 02.2\end{array}$ & $\begin{array}{ll}51 & 10.8 \\
51 & 09.0 \\
51 & 07.0 \\
51 & 04.6 \\
51 & 02.0\end{array}$ & $\begin{array}{ll}56 & 10.6 \\
56 & 08.8 \\
56 & 06.8 \\
56 & 04.4 \\
56 & 01.8\end{array}$ & $\begin{array}{ll}61 & 10.4\end{array}$ & $\begin{array}{l}-0.9 \\
-0.9 \\
-0.9 \\
-0.8 \\
-0.8\end{array}$ & $\begin{array}{ll}1 & 15 \\
1 & 30 \\
1 & 45 \\
2 & 00 \\
2 & 15\end{array}$ \\
\hline \begin{tabular}{ll|}
2 & 30 \\
2 & 45 \\
3 & 00 \\
3 & 15 \\
3 & 30
\end{tabular} & $\mid \begin{array}{ll}31 & 00.1 \\
30 & 57.0 \\
30 & 53.7 \\
30 & 50.1 \\
30 & 46.4\end{array}$ & $\begin{array}{ll}35 & 59.8 \\
35 & 56.7 \\
35 & 53.4 \\
35 & 49.8 \\
35 & 46.0\end{array}$ & \begin{tabular}{|ll}
40 & 59.5 \\
40 & 56.5 \\
40 & 53.1 \\
40 & 49.5 \\
40 & 45.7
\end{tabular} & $\begin{array}{ll}45 & 59.3 \\
45 & 56.2 \\
45 & 52.9 \\
45 & 49.2 \\
45 & 45.5\end{array}$ & $\begin{array}{ll}50 & 59.1 \\
50 & 56.0 \\
50 & 52.6 \\
50 & 49.0 \\
50 & 45.2\end{array}$ & & & $\begin{array}{l}-0.8 \\
-0.7 \\
-0.7 \\
-0.6 \\
-0.6\end{array}$ & $\begin{array}{ll}2 & 30 \\
2 & 45 \\
3 & 00 \\
3 & 15 \\
3 & 30\end{array}$ \\
\hline $\begin{array}{l}345 \\
400 \\
415 \\
430 \\
445\end{array}$ & $\begin{array}{ll}30 & 42.4 \\
30 & 38.3 \\
30 & 34.0 \\
30 & 29.6 \\
30 & 25.0\end{array}$ & $\begin{array}{ll}35 & 42.1 \\
35 & 38.0 \\
35 & 33.6 \\
35 & 29.2 \\
35 & 24.6\end{array}$ & $\begin{array}{ll}40 & 41.8 \\
40 & 37.6 \\
40 & 33.3 \\
40 & 28.9 \\
40 & 24.3\end{array}$ & $\begin{array}{ll}45 & 41.5 \\
45 & 37.4 \\
45 & 33.0 \\
45 & 28.5 \\
45 & 24.0\end{array}$ & $\begin{array}{ll}50 & 41.3 \\
50 & 37.1 \\
50 & 32.8 \\
50 & 28.3 \\
50 & 23.7\end{array}$ & $\begin{array}{ll}55 & 28.0 \\
55 & 23.4\end{array}$ & & $\begin{array}{l}-0.5 \\
-0.5 \\
-0.4 \\
-0.4 \\
-0.3\end{array}$ & $\begin{array}{ll}3 & 45 \\
4 & 00 \\
4 & 15 \\
4 & 30 \\
4 & 45\end{array}$ \\
\hline $\begin{array}{ll}5 & 00 \\
5 & 15 \\
5 & 30 \\
5 & 45 \\
6 & 00\end{array}$ & $\begin{array}{ll}30 & 20.4 \\
30 & 15.6 \\
30 & 10.8 \\
30 & 06.0 \\
30 & 01.2\end{array}$ & \begin{tabular}{|ll}
35 & 20.0 \\
35 & 15.3 \\
35 & 10.4 \\
35 & 05.6 \\
35 & 00.8
\end{tabular} & $\begin{array}{ll}40 & 19.7 \\
40 & 14.9 \\
40 & 10.1 \\
40 & 05.3 \\
40 & 00.5\end{array}$ & $\begin{array}{ll}45 & 19.4 \\
45 & 14.6 \\
45 & 09.9 \\
45 & 05.0 \\
45 & 00.2\end{array}$ & $\begin{array}{lll}50 & 19.1 \\
50 & 14.3 \\
50 & 09.6 \\
50 & 04.7 \\
49 & 59.9\end{array}$ & 5459.5 & & $\begin{array}{r}-0.1 \\
0.0 \\
0.0\end{array}$ & $\begin{array}{l}530 \\
545 \\
600\end{array}$ \\
\hline $\begin{array}{ll}6 & 15 \\
6 & 30 \\
6 & 45 \\
7 & 00 \\
7 & 15\end{array}$ & $\mid \begin{array}{ll}29 & 56.4 \\
29 & 51.6 \\
29 & 46.8 \\
39 & 42.1 \\
29 & 37.5\end{array}$ & $\begin{array}{ll}34 & 56.0 \\
34 & 51.2 \\
34 & 46.4 \\
34 & 41.7 \\
34 & 37.1\end{array}$ & $\begin{array}{ll}39 & 55.6 \\
39 & 50.8 \\
39 & 46.0 \\
39 & 41.4 \\
39 & 36.8\end{array}$ & $\begin{array}{ll}44 & 55.3 \\
44 & 50.5 \\
44 & 45.7 \\
44 & 41.1 \\
44 & 36.4\end{array}$ & $\begin{array}{ll}49 & 55.0 \\
49 & 50.2 \\
49 & 45.5 \\
49 & 40.8 \\
49 & 36.2\end{array}$ & 043 & & $\begin{array}{l}+0.3 \\
+0.4\end{array}$ & $\begin{array}{l}630 \\
645 \\
700 \\
715\end{array}$ \\
\hline $\begin{array}{ll}7 & 30 \\
7 & 45 \\
8 & 00 \\
8 & 15 \\
8 & 30\end{array}$ & $\begin{array}{ll}29 & 33.0 \\
29 & 28.6 \\
29 & 24.4 \\
29 & 20.3 \\
29 & 16.4\end{array}$ & $\begin{array}{ll}34 & 32.6 \\
34 & 28.2 \\
34 & 24.0 \\
34 & 19.9 \\
34 & 16.0\end{array}$ & $\begin{array}{ll}39 & 32.3 \\
39 & 27.9 \\
39 & 23.7 \\
39 & 19.6 \\
39 & 15.7\end{array}$ & $\begin{array}{ll}44 & 32.0 \\
44 & 27.6 \\
44 & 23.4 \\
44 & 19.3 \\
44 & 15.4\end{array}$ & $\begin{array}{ll}49 & 31.7 \\
49 & 27.3 \\
49 & 23.1 \\
49 & 19.0 \\
49 & 1.5 .2\end{array}$ & & & & $\begin{array}{l}800 \\
815 \\
830\end{array}$ \\
\hline $\begin{array}{ll}8 & 45 \\
9 & 00 \\
9 & 15 \\
9 & 30 \\
9 & 45\end{array}$ & $\begin{array}{ll}29 & 12.7 \\
29 & 09.2 \\
29 & 05.9 \\
29 & 02.8 \\
29 & 00.0\end{array}$ & $\begin{array}{ll}34 & 12.3 \\
34 & 08.8 \\
34 & 05.5 \\
34 & 02.5 \\
33 & 59.7\end{array}$ & $\begin{array}{ll}39 & 12.0 \\
39 & 08.5 \\
39 & 05.3 \\
39 & 02.2 \\
38 & 59.4\end{array}$ & $\begin{array}{ll}44 & 05.0 \\
44 & 02.0 \\
43 & 59.2\end{array}$ & $\begin{array}{ll}49 & 1.1 .5 \\
49 & 08.1 \\
49 & 04.8 \\
49 & 01.8 \\
48 & 59.0\end{array}$ & 0300.0 & & & $\begin{array}{l}915 \\
930 \\
945\end{array}$ \\
\hline $\begin{array}{l}1000 \\
1015 \\
1030 \\
1045\end{array}$ & $\begin{array}{ll}28 & 57.5 \\
28 & 55.3 \\
28 & 53.3 \\
28 & 51.6 \\
28 & 50.2\end{array}$ & $\begin{array}{ll}33 & 57.2 \\
33 & 55.0 \\
33 & 53.0 \\
33 & 51.3 \\
33 & 49.9\end{array}$ & $\begin{array}{ll}38 & 56.9 \\
38 & 54.7 \\
38 & 52.8 \\
38 & 51.1 \\
38 & 49.7\end{array}$ & $\begin{array}{ll}43 & 56.7 \\
43 & 54.5 \\
43 & 52.5 \\
43 & 50.8 \\
43 & 49.5\end{array}$ & $\begin{array}{ll}48 & 50.7 \\
48.4\end{array}$ & 5349.1 & $100 . x^{3}$ & $\begin{array}{l}+0.9 \\
+0.9 \\
+0.9 \\
+0.9 \\
+1.0\end{array}$ & $\begin{array}{ll}10 & 30 \\
10 & 45 \\
11 & 00\end{array}$ \\
\hline $\begin{array}{l}1145 \\
1200\end{array}$ & $\mid \begin{array}{ll}28 & 49.2 \\
28 & 48.4 \\
28 & 47.9 \\
28 & 47.7\end{array}$ & $\begin{array}{ll}33 & 48.9 \\
33 & 48.1 \\
33 & 47.6 \\
33 & 47.4\end{array}$ & $\begin{array}{ll}38 & 48.6 \\
38 & 47.8 \\
38 & 47.4 \\
38 & 47.2\end{array}$ & $\begin{array}{ll}43 & 48.4 \\
43 & 47.6 \\
43 & 47.1 \\
43 & 47.0\end{array}$ & $\begin{array}{ll}48 & 48.2 \\
48 & 47.5 \\
48 & 47.0 \\
48 & 46.8\end{array}$ & 5346.7 & 5846.6 & $\begin{array}{l}+1.0 \\
+1.0 \\
+1.0 \\
+1.0\end{array}$ & $\begin{array}{l}1145 \\
1200\end{array}$ \\
\hline
\end{tabular}


TABLE 4.-For projection of maps of large areas.

[The ratio of the yard to the meter us stated by Clarke, namely, 1 meter $=1.093623$ yards $=39.370432$ inches, is that used in the table.]

LENGTHS OF DEGREES OF THE MERIDIAN.

\begin{tabular}{|c|c|c|c|c|c|}
\hline Latitude. & Meters. $a$ & $\begin{array}{l}\text { Statute } \\
\text { miles. }\end{array}$ & Latitude. & Meters. $\iota$ & $\begin{array}{c}\text { Statute } \\
\text { miles. }\end{array}$ \\
\hline$\circ$ & & & 0 & & \\
\hline 0 & $110,567.2$ & 68.704 & 45 & $111,130.9$ & 69.054 \\
\hline 1 & $110,567.6$ & 68.704 & 46 & $111,150.6$ & 69.066 \\
\hline 2 & $110,568.6$ & 68.705 & 47 & $111,170.4$ & 69.079 \\
\hline 3 & 1.10,570. 3 & 68.706 & 48 & $111,190.1$ & 69.091. \\
\hline 4 & $1.10,572.7$ & 68.708 & 49 & $1.11,209.7$ & 69.103 \\
\hline 5 & $110,575.8$ & 68.710 & 50 & $111,229.3$ & 69.115 \\
\hline 6 & $.110,579.5$ & 68.712 & 51 & $111,248.7$ & 69.127 \\
\hline .7 & $110,583.9$ & 68.715 & 52 & $111,268.0$ & 69.139 \\
\hline 8 & $110,589.0$ & 68.718 & 53 & $111,287.1$ & 69.151 \\
\hline 9 & $110,594.7$ & 68.721 & 54 & $111,306.0$ & 69.163 \\
\hline 10 & $110,601.1$ & 68.725 & 55 & $111,324.8$ & 69.175 \\
\hline 11 & $1] 0,608.1$ & 68.730 & 56 & $111,343.3$ & $69.186^{\circ}$ \\
\hline 12 & $110,615.8$ & 68.734 & 57 & 1.11, 361. 5 & 69.197 \\
\hline 13 & $110,624.1$ & 68.739 & 58 & $111,379.5$ & 69.209 \\
\hline 14 & $110,633.0$ & 68.744 & 59 & $111,397.2$ & 69.220 \\
\hline 15 & $110,642.5$ & 68.751 & 60 & $111,414.5$ & 69.230 \\
\hline 16 & $110,652.6$ & 68.757 & 61. & 1111., 431.. 5 & 69.241 \\
\hline 17 & $110,663.3$ & 68.764 & 62 & $111,448.2$ & 69.251 \\
\hline 18 & $110,674.5$ & 68.771 & 63 & $111,464.4$ & 69.261. \\
\hline 19 & $110,686.3$ & 68.778 & 64 & $111,480.3$ & 69.271 \\
\hline 20 & $110,698.7$ & 68.786 & 65 & $111,495.7$ & 69.281 \\
\hline 21. & $110,711.6$ & 68.794 & 66 & $111,510.7$ & 69.290 \\
\hline 22 & $110,725.0$ & 68.802 & 67 & $111,525.3$ & 69.299 \\
\hline 23 & $110,738.8$ & 68.811 & 68 & $111,539.3$ & 69.308 \\
\hline 24 & $110,753.2$ & 68.820 & 69 & $111 ., 552.9$ & 69.316 \\
\hline 25 & $110,768.0$ & 68.829 & 70 & $111,565.9$ & 69.324 \\
\hline 26 . & $110,783.3$ & 68.839 & 71. & $111,578.4$ & 69.332 \\
\hline 27 & $110,799.0$ & 68.848 & 72 & $111,590.4$ & 69.340 \\
\hline 28 & $110,815.1$ & 68.858 & 73 & $111,601.8$ & 69.347 \\
\hline 29 & $110,831.6$ & 68.869 & 74 & $111,612.7$ & 69.354 \\
\hline 30 & $110,848.5$ & 68.879 & 75 & $111,622.9$ & 69.360 \\
\hline 31. & $110,865.7$ & 68.890 & 76 & $111,632.6$ & 69.366 \\
\hline 32 & $110,883.2$ & 68. 901 & 77 & $111,641.6$ & 69.372 \\
\hline 33 & $110,901.1$ & 68.912 & 78 & $111,650.0$ & 69.377 \\
\hline 34 & $110,919.2$ & 68.923 & 79 & $111,657.8$ & 69.382 \\
\hline 35 & $110,937.6$ & 68.935 & 80 & $111,664.9$ & 69.386 \\
\hline 36 & $11.0,956.2$ & 68.946 & 81. & $111,671.4$ & 69.390 \\
\hline 37 & $110,975.1$ & 68.958 & 82 & $111,677.2$ & 69.394 \\
\hline 38 & $110,994.1$ & 68.969 & 83 & $111,682.4$ & 69.397 \\
\hline 39 & $111,013.3$ & 68.981. & 84 & 1.11, 686.9 & 69.400 \\
\hline 40 & $111,032.7$ & 68.993 & 85 & $111,690.7$ & 69.402 \\
\hline 41. & $111,052.2$ & 69.006 & 86 & $111,693.8$ & 69.404 \\
\hline 42 & $111,071.7$ & 69.018 & 87 & $111,696.2$ & 69.405 \\
\hline 43 & $111,091.4$ & 69.030 & 88 & $111,697.9$ & 69.407 \\
\hline 44 & $111,111.1$ & 69.042 & 89 & 11.1. 699.0 & 69.407 \\
\hline 45 & $111,130.9$ & 69.054 & 90 & $111,699.3$ & 69.407 \\
\hline
\end{tabular}

a'These quantities express the number of meters and statute miles contained within an arc of which the degree of latitude named is the middle; thus, the quantity $111,032.7$, opposite latitude $40^{\circ}$, is the number of meters between latitude $39^{\circ} 30^{\prime}$ and latitude $40^{\circ} 30^{\prime}$. 
TABLE 4.-For projection of maps of large areas-Continued.

[Extracted from Appendix No. 6, U. S. Coast and Geodetic Survey Report for 18\$4.]

LENGTHS OF DEGREES OF THE PARALLEL.

\begin{tabular}{|c|c|c|c|c|c|}
\hline Iatitude. & Meters. & $\begin{array}{l}\text { Statute. } \\
\text { miles. }\end{array}$ & Latitude. & Meters. & $\begin{array}{l}\text { Strtute } \\
\text { miles. }\end{array}$ \\
\hline o & & & 。 & & \\
\hline 0 & 111,321 & 69.172 & 45 & 78,849 & 48.995 \\
\hline 1. & 111,304 & 69.162 & 46 & 77,466 & 48. 136 \\
\hline 2 & 111,253 & 69.130 & 47 & 76,058 & 47.261 \\
\hline 3 & 111,169 & 69.078 & 48 & 74,628 & 46.372 \\
\hline 4 & 111,051 & 69.005 & 49 & 73,174 & 45.469 \\
\hline 5 & 110,900 & 68.911 & 50 & 71,698 & 44.552 \\
\hline 6 & 110,715 & 68.795 & 51. & 70,200 & 43.621 \\
\hline 7 & $1.10,497$ & 68.660 & 52 & 68,680 & 42.676 \\
\hline 8 & 110,245 & 68.504 & 53 & 67,140 & 41.719 \\
\hline 9 & 109,959 & 68.326 & 54 & 65,578 & 40.749 \\
\hline 10 & 109,641 & 68.129 & 55 & 63,996 & 39.766 \\
\hline 11 & 109,289 & 67.910 & 56 & 62,395 & 38.771 \\
\hline 12 & 108,904 & 67.670 & 57 & 60,774 & 37.764 \\
\hline 13 & 108,486 & 67.410 & 58 & 59,135 & 36.745 \\
\hline 14 & 108,036 & 67.131 & 59 & 57,478 & 35.716 \\
\hline 15 & 107,553 & 66.830 & 60 & 55,802 & 34.674 \\
\hline 16 & 107,036 & 66.510 & 61 & 54,110 & 33.623 \\
\hline 17 & 106,487 & 66.169 & 62 & 52,400 & 32.560 \\
\hline 18 & 105,906 & 65.808 & 63 & 50,675 & 31.488 \\
\hline 19 & 105,294 & 65.427 & $64:$ & 48,934 & 30.406 \\
\hline 20 & 104,649 & 65.026 & 65 & 47,177 & 29.315 \\
\hline 21 . & 103,972 & 64.606 & 66 & 45,407 & 28.215 \\
\hline 22 & 103,264 & 64.166 & 67 & 43,622 & 27.106 \\
\hline 23 & 102,524 & 63.706 & 68 & 41,823 & 25.988 \\
\hline 24 & 101,754 & 63.228 & 69 & 40,012 & 24.862 \\
\hline 25 & 100,952 & 62.729 & 70 & 38,188 & 23.729 \\
\hline 26 & 100,119 & 62.212 & 71 & 36,353 & 22.589 \\
\hline 27 & 99,257 & 61.676 & 72 & 34,506 & 21.441 \\
\hline 28 & 98,364 & 61.122 & 73 & 32,648 & 20.287 \\
\hline 29 & 97,441 & 60.548 & 74 & 30,781 & 19.127 \\
\hline 30 & 96,488 & 59.956 & 75 & 28,903 & 17.960 \\
\hline 31 & 95,506 & 59.345 & 76 & 27,017 & 16.788 \\
\hline 32 & 94,495 & 58.716 & 77 & 25,123 & 15,611 \\
\hline 33 & 93,455 & 58.071 & 78 & 23,220 & 14.428 \\
\hline 34 & 92,387 & 57.407 & 79 & 21,311 & 13. 242 \\
\hline 35 & 91,290 & 56.725 & 80 & 19,394 & 12.051 \\
\hline 36 & 90,166 & 56.027 & 81. & 17,472 & 10.857 \\
\hline 37 & 89,014 & 55.311 & 82 & 15,545 & 9.659 \\
\hline 38 & 87,835 & 54.579 & 83 & 13,612 & 8. 458 \\
\hline 39 & 86,629 & 53.829 & 84 & 11,675 & 7.255 \\
\hline 40 & 85,396 & 53.063 & 85 & 9,735 & 6. 049 . \\
\hline 41 & 84,137 & 52. 281 & 86 & 7,792 & 4. 842 \\
\hline 42 & $82 ; 853$ & 51.483 & 87 & 5,846 & 3.632 \\
\hline 43 & 81,543 & 50.669 & 88 & 3,898 & 2.422 \\
\hline 44 & 80,208 & 49.840 & 89 & 1,949 & 1. 211 \\
\hline 45 & 78,849 & 48.995 & 90 & 0 & 0.000 \\
\hline
\end{tabular}


TABLE 4.-For projection of maps of large areas-Continued.

[Extracted from Appendix No. 6, U. S. Coast and Geodetic Survey Report for 1884.]

ARCS OF THE PARALLEL IN METERS.

\begin{tabular}{|c|c|c|c|c|c|c|c|c|}
\hline \multicolumn{2}{|c|}{ Iatitude. } & \multirow[t]{2}{*}{ Value of $1^{\prime}$. } & \multicolumn{2}{|c|}{ Latitude. } & \multirow[t]{2}{*}{ Value of $1^{\prime}$. } & \multicolumn{2}{|c|}{ Latitude. } & \multirow[t]{2}{*}{ Value of $11^{\prime}$} \\
\hline$\circ$ & , & & $\circ$ & ' & & $\circ$ & ' & \\
\hline \multirow{6}{*}{24} & 00 & 1695.9 & 33 & 00 & 1557.6 & 42 & 00 & 1380.9 \\
\hline & 10 & 1693.7 & & 10 & 1554.7 & & 10 & 1377.3 \\
\hline & 20 & 1691.5 & & 20 & 1.551 .7 & & 20 & 1373.7 \\
\hline & 30 & 1689.3 & & 30 & 1548.7 & & 30 & 1370.0 \\
\hline & 40 & 1.687 .0 & & 40 & 1545.8 & & 40 & 1366.4 \\
\hline & 50 & 1684.8 & & 50 & $1.542: 8$ & & 50 & 1.362 .7 \\
\hline \multirow[t]{6}{*}{25} & 00 & 1682.5 & 34 & 00 & 1539.8 & 43 & 00 & 1359. 1 \\
\hline & 10 & 1680.3 & & 10 & 1536.8 & & 10 & 1355.4 \\
\hline & 20 & 1678.0 & & 20 & 1533.7 & & 20 & 1351. 7 \\
\hline & 30 & 1675.7 & & 30 & 1530.7 & & 30 & 1348.0 \\
\hline & 40 & 1673.3 & & 40 & 1527.6 & & 40 & 1344.3 \\
\hline & 50 & 1671.0 & & 50 & 1524.6 & & 50 & 1340.5 \\
\hline \multirow[t]{6}{*}{26} & 00 & 1668.7 & 35 & 00 & 1521.5 & 44 & 00 & 1336.8 \\
\hline & 10 & 1666.3 & & 10 & 1518.4 & & 10 & 1333.1 \\
\hline & 20 & 1663.9 & & 20 & 1515.3 & & 20 & 1329.3 \\
\hline & 30 & 1661.5 & & 30 & 1512. 2 & & 30 & 1325.5 \\
\hline & 40 & 1659.1 & & 40 & 1509.1 & . & 40 & 1321. 7 \\
\hline & 50 & 1656.7 & & 50 & 1505.9 & & 50 & 1318.0 \\
\hline \multirow[t]{6}{*}{27} & 00 & 1654.3 & 36 & 00 & 1502.8 & 45 & 00 & 1314. 2 \\
\hline & 10 & 1651.8 & & 10 & 1499.6 & & 10 & 1310.3 \\
\hline & 20 & 1649.4 & & 20 & 1496.4 & & 20 & 1306.5 \\
\hline & 30 & 1646.9 & & 30 & 1493.2 & & 30 & 1302.7 \\
\hline & 40 & 1644.4 & & 40 & 1490.0 & & 40 & 1298.8 \\
\hline & 50 & 1641.9 & & 50 & 1486.8 & & 50 & 1295.0 \\
\hline \multirow[t]{6}{*}{28} & 00 & 1639.4 & 37 & 00 & 1483.6 & 46 & $\mathrm{CO}$ & 1291.0 \\
\hline & 10 & 1636.9 & & 10 & 1480.3 & & 10 & 1287.2 \\
\hline & 20 & 1634.3 & & 20 & 1477.1 & & 20 & 1283.3 \\
\hline & 30 & 1631.8 & & 30 & 1473.8 & & 30 & 1279.4 \\
\hline & 40 & 1629.2 & & 40 & 1470.5 & & 40 & 1275.5 \\
\hline & 50 & 1626.6 & & 50 & 1467.2 & & 50 & 1271.6 \\
\hline \multirow[t]{6}{*}{29} & 00 & 1624.0 & 38 & 00 & 1463.9 & 47 & 00 & 1267.6 \\
\hline & 10 & 1621.4 & & 10 & 1460.6 & & 10 & 1263.7 \\
\hline & 20 & 1618.8 & & 20 & 1457.3 & & 20 & 1259.7 \\
\hline & 30 & 1616.1 & & 30 & 1453.9 & & 30 & 1255.8 \\
\hline & 40 & 1613.5 & & 40 & 1450.6 & & 40 & 1251.8 \\
\hline & 50 & 1.610 .8 & & 50 & 1447.2 & & 50 & 1247.8 \\
\hline \multirow[t]{6}{*}{30} & 00 & I608. 1 & 39 & 00 & 1443.8 & 48 & 00 & 1243.8 \\
\hline & 10 & 1605.4 & & 10 & 1440.4 & & 10 & 1239.8 \\
\hline & 20 & 1602.7 & & 20 & 1437.0 & & 20 & 1235.8 \\
\hline & 30 & 1600.0 & & 30 & 1433.6 & & 30 & 1231. 7 \\
\hline & 40 & 1597.3 & & 40 & 1430.2 & & 40 & 1227.7 \\
\hline & 50 & 1594.5 & & 50 & 1426.7 & & 50 & 1223.6 \\
\hline \multirow[t]{6}{*}{31} & 00 & 1591.8 & 40 & 00 & 1423.3 & 49 & 00 & 1219.6 \\
\hline & 10 & 1589.0 & & 10 & $14] 9.8$ & & 10 & 1215.5 \\
\hline & 20 & 1586.2 & & 20 & 1416. 3 & & 20 & 1211.4 \\
\hline & 30 & 1583.4 & & 30 & 1412.8 & & 30 & 1207.3 \\
\hline & 40 & 1580.6 & & 40 & 1409.3 & & 40 & 1203.2 \\
\hline & 50 & 1577.8 & & 50 & 1405.8 & & 50 & 1199.1 \\
\hline \multirow{6}{*}{$\begin{array}{l}32 \\
-\end{array}$} & 00 & 1574.9 & 41 & 00 & 1402.3 & 50 & 00 & 1195.0 \\
\hline & 10 & 1572.1 & & 10 & 1398.8 & & 10 & 1190.8 \\
\hline & 20 & 1569.2 & & 20 & 1395.2 & & 20 & 1186.7 \\
\hline & 30 & 1566.3 & & 30 & 1391.6 & & 30 & 1182.5 \\
\hline & 40 & 1563.4 & & 40 & 1388.1 & & 40 & 1.178 .4 \\
\hline & 50 & 1560.5 & & 50 & -1384.5 & & 50 & 1174.2 \\
\hline
\end{tabular}


TABLE 4.-For projections of mapss of large areas-Continued. COORDINATES OF CURVATURE.

\begin{tabular}{|c|c|c|c|c|c|c|c|c|}
\hline \multicolumn{9}{|c|}{ Natural scale. - Values of $\mathrm{X}$ and $\mathrm{Y}$ in meters. } \\
\hline \multicolumn{3}{|c|}{ Latitude $24^{\circ}$. } & \multicolumn{3}{|c|}{ Latitude $25^{\circ}$. } & \multicolumn{3}{|c|}{ Latitude $26^{\circ}$. } \\
\hline $\begin{array}{l}\text { Longi- } \\
\text { tude. }\end{array}$ & $\mathrm{x}$ & $Y$ & $\begin{array}{l}\text { Longi- } \\
\text { tude. }\end{array}$ & $\mathrm{x}$ & $\mathrm{Y}$ & $\begin{array}{l}\text { Longi- } \\
\text { tude. }\end{array}$ & $x$ & $\mathrm{Y}$ \\
\hline $\begin{array}{ll}\circ & , \\
1 & 00 \\
2 & 00 \\
3 & 00 \\
4 & 00\end{array}$ & $\begin{array}{l}101,753 \\
203,500 \\
305,237 \\
406,959\end{array}$ & $\begin{array}{r}361 \\
1,445 \\
3,250 \\
5,778\end{array}$ & $\begin{array}{ll}\circ & 1 \\
1 & 00 \\
2 & 00 \\
3 & 00 \\
4 & 00\end{array}$ & $\begin{array}{l}100,951 \\
201,896 \\
302,831 \\
403,749\end{array}$ & $\begin{array}{r}372 \\
1,489 \\
3,351 \\
5,957\end{array}$ & $\begin{array}{ll}\circ & 1 \\
1 & 00 \\
2 & 00 \\
3 & 00 \\
4 & 00\end{array}$ & $\begin{array}{l}100,118 \\
200,231 \\
300,332 \\
400,416\end{array}$ & $\begin{array}{r}383 \\
1,532 \\
3,447 \\
6,128\end{array}$ \\
\hline $\begin{array}{l:l}5 & 00 \\
6 & 00 \\
7 & 00 \\
8 & 00 \\
9 & 00\end{array}$ & $\begin{array}{l}508,660 \\
610,336 \\
711,981 \\
813,590 \\
915,159\end{array}$ & $\begin{array}{r}9,028 \\
13,001 \\
17,695 \\
23,109 \\
29,245\end{array}$ & $\begin{array}{ll}5 & 00 \\
6 & 00 \\
7 & 00 \\
8 & 00 \\
9 & 00\end{array}$ & $\begin{array}{r}504,645 \\
605,514 \\
706,349 \\
807,146 \\
.907,899\end{array}$ & $\begin{array}{r}9,307 \\
13,401 \\
18,239 \\
23,821 \\
30,146\end{array}$ & $\begin{array}{ll}5 & 00 \\
6 & 00 \\
7 & 00 \\
8 & 00 \\
9 & 00\end{array}$ & $\begin{array}{l}500,476 \\
600,506 \\
700,501 \\
800 ; 456 \\
900,364\end{array}$ & $\begin{array}{r}9,574 \\
13,786 \\
18,763 \\
24,505 \\
31,011\end{array}$ \\
\hline $\begin{array}{ll}10 & 00 \\
11 & 00 \\
12 & 00 \\
13 & 00 \\
14 & 00\end{array}$ & $\begin{array}{l}1,016,681 \\
1,118,152 \\
1,219,566 \\
1,320,919 \\
1,422,205\end{array}$ & $\begin{array}{l}36,102 \\
43,679 \\
51,977 \\
60,994 \\
70,731\end{array}$ & $\begin{array}{ll}10 & 00 \\
11 & 00 \\
12 & 00 \\
13 & 00 \\
14 & 00\end{array}$ & $\begin{array}{l}1,008,603 \\
1,109,252 \\
1,209,841 \\
1,310,364 \\
1, \mathbf{4 1 0}, 815\end{array}$ & $\begin{array}{l}37,215 \\
45,026 \\
53,578 \\
62,873 \\
72,909\end{array}$ & $\begin{array}{ll}10 & 00 \\
11 . & 00 \\
12 & 00 \\
13 & 00 \\
14 & 00\end{array}$ & $\begin{array}{l}1,000,218 \\
1,100,015 \\
1,199,747 \\
1,299,409 \\
1,398,994\end{array}$ & $\begin{array}{l}38,282 \\
46,316 \\
55,114 \\
64,675 \\
74,998\end{array}$ \\
\hline $\begin{array}{ll}15 & 00 \\
16 & 00 \\
17 & 00 \\
18 & 00 \\
19 & 00\end{array}$ & $\begin{array}{l}1,523,420 \\
1,624,558 \\
1,725,614 \\
1,826,583 \\
1,927,460\end{array}$ & $\begin{array}{r}81,186 \\
92,360 \\
104,251 \\
116,859 \\
130,184\end{array}$ & $\begin{array}{ll}15 & 00 \\
16 & 00 \\
17 & 00 \\
18 & 00 \\
19 & 00\end{array}$ & $\begin{array}{l}1,511,190 \\
1,611,483 \\
1,711,688 \\
1,811,800 \\
1,911,813\end{array}$ & $\begin{array}{r}83,685 \\
9 \overline{5}, 202 \\
107,458 \\
120,453 \\
134,186\end{array}$ & $\begin{array}{ll}15 & 00 \\
16 & 00 \\
17 & 00 \\
18 & 00 \\
19 & 00\end{array}$ & $\begin{array}{l}1,498,498 \\
1,597,914 \\
1,697,237 \\
1,796,460 \\
1,895,578\end{array}$ & $\begin{array}{r}86,082 \\
97,928 \\
110,534 \\
123,899 \\
138,023\end{array}$ \\
\hline $\begin{array}{ll}20 & 00 \\
21 & 00 \\
22 & 00 \\
23 & 00 \\
24 & 00\end{array}$ & $\begin{array}{l}2,028,240 \\
2,128,918 \\
2,229,488 \\
2,329,946 \\
2,430,287\end{array}$ & $\begin{array}{l}144,225 \\
158,981 \\
174,451 \\
190,634 \\
207,530\end{array}$ & $\begin{array}{ll}20 & 00 \\
21 & 00 \\
22 & 00 \\
23 & 00 \\
24 & 00\end{array}$ & $\begin{array}{l}2,011,722 \\
2,111,522 \\
2,211,207 \\
2,310,771 . \\
2,410,210\end{array}$ & $\begin{array}{l}148,656 \\
163,862 \\
179,805 \\
196,482 \\
213,894\end{array}$ & $\begin{array}{ll}20 & 00 \\
21 & 00 \\
22 & 00 \\
23 & 00 \\
24 & 00\end{array}$ & $\begin{array}{l}1,994,585 \\
2,093,475 \\
2,192,243 \\
2,290,882 \\
2,389,387\end{array}$ & $\begin{array}{l}152,905 \\
168,544 \\
184,939 \\
202,089 \\
219,993\end{array}$ \\
\hline $\begin{array}{ll}25 & 00 \\
26 & 00 \\
27 & 00 \\
28 & 00 \\
29 & 00 \\
30 & 00\end{array}$ & $\begin{array}{l}2,530,505 \\
2,630,596 \\
2,730,554 \\
2,830,374 \\
2,930,052 \\
3,029,582\end{array}$ & $\begin{array}{l}225,158 \\
243,458 \\
262,487 \\
282,225 \\
302,671 \\
323,825\end{array}$ & $\begin{array}{ll}25 & 00 \\
26 & 00 \\
27 & 00 \\
28 & 00 \\
29 & 00 \\
30 & 00\end{array}$ & $\begin{array}{l}2,509,518 \\
2,608,689 \\
2,707,718 \\
2,806,600 \\
2,905,329 \\
3,003,900\end{array}$ & $\begin{array}{l}232,038 \\
250,914 \\
270,521 \\
290,859 \\
311,925 \\
333,718\end{array}$ & $\begin{array}{ll}25 & 00 \\
26 & 00 \\
27 & 00 \\
28 & 00 \\
29 & 00 \\
30 & 00\end{array}$ & $\begin{array}{l}2,487,753 \\
2,585,973 \\
2,684,042 \\
2,781,953 \\
2,879,702 \\
2,977,281\end{array}$ & $\begin{array}{l}238,650 \\
258,061 \\
278,222 \\
299,132 \\
320,788 \\
343,197\end{array}$ \\
\hline
\end{tabular}


TABLE 4.-For projections of maps of large areas-Continued.

COORDINATES OF CURVATURE.

\begin{tabular}{|c|c|c|c|c|c|c|c|c|}
\hline \multicolumn{9}{|c|}{ Natural scale. - Values of $\mathrm{X}$ and $\mathrm{Y}$ in meters. } \\
\hline \multicolumn{3}{|c|}{ Latitude $27^{\circ}$. } & \multicolumn{3}{|c|}{ - Latitude $28^{\circ}$. } & \multicolumn{3}{|c|}{ Latitude $29^{\circ}$. } \\
\hline $\begin{array}{l}\text { Longi- } \\
\text { tude. }\end{array}$ & $\mathbf{x}$ & $\mathrm{Y}$ & $\begin{array}{l}\text { Longi- } \\
\text { tude. }\end{array}$ & $\mathbf{x}$ & $\mathrm{Y}$ & $\begin{array}{l}\text { Longi- } \\
\text { tude. }\end{array}$ & $\mathbf{x}$ & $\mathbf{Y}$ \\
\hline $\begin{array}{ll}\circ & , \\
1 & 00 \\
2 & 00 \\
3 & 00 \\
4 & 00\end{array}$ & $\begin{array}{r}99,256 \\
198,505 \\
297 ; 742 \\
396,960\end{array}$ & $\begin{array}{r}393 \\
1,573 \\
3,539 \\
6,291\end{array}$ & $\begin{array}{lc}\circ & \prime \\
1 & 00 \\
2 & 00 \\
3 & 00 \\
4 & 00\end{array}$ & $\begin{array}{r}98,363 \\
196,719 \\
295,062 \\
393,385\end{array}$ & $\begin{array}{r}403 \\
1,612 \\
3,627 \\
6,447\end{array}$ & $\begin{array}{ll}\circ & , \\
1 & 00 \\
2 & 00 \\
3 & 00 \\
4 & 00\end{array}$ & $\begin{array}{r}97,439 \\
194,872 \\
292,291 \\
389,689\end{array}$ & $\begin{array}{r}412 \\
1,649 \\
3,710 \\
6,595\end{array}$ \\
\hline $\begin{array}{ll}5 & 00 \\
6 & 00 \\
7 & 00 \\
8 & 00 \\
9 & 00\end{array}$ & $\begin{array}{l}496,154 \\
595,316 \\
694,440 \\
793,522 \\
892,554\end{array}$ & $\begin{array}{r}9,829 \\
14,154 \\
19,264 \\
25,159 \\
31,839\end{array}$ & $\begin{array}{ll}5 & 00 \\
6 & 00 \\
7 & 00 \\
8 & 00 \\
9 & 00\end{array}$ & $\begin{array}{l}491,682 \\
589,945 \\
688,168 \\
786,347 \\
884,472\end{array}$ & $\begin{array}{l}10,073 \\
14,505 \\
19,741 \\
25,782 \\
32,627\end{array}$ & $\begin{array}{ll}5 & 00 \\
6 & 00 \\
7 & 00 \\
8 & 00 \\
9 & 00\end{array}$ & $\begin{array}{r}487,059 \\
584,394 \\
. \quad 681,687 \\
778,931 \\
.876,120\end{array}$ & $\begin{array}{r}10,305 \\
14,838 \\
20,194 \\
26,374 \\
. \quad 33,376\end{array}$ \\
\hline $\begin{array}{ll}10 & 00 \\
11 & 00 \\
12 & 00 \\
13 & 00 \\
14 & 00\end{array}$ & $\begin{array}{r}991,529 \\
1,090,442 \\
1,189,287 \\
1,288,057 \\
1,386,746\end{array}$ & $\begin{array}{l}39,303 \\
47,551 \\
56,583 \\
66,398 \\
76,995\end{array}$ & $\begin{array}{ll}10 & 00 \\
11 & 00 \\
12 & 00 \\
13 & 00 \\
14 & 00\end{array}$. & $\begin{array}{r}982,537 \\
1,080,537 \\
1,178,464 \\
1,276,312 \\
1,374,075\end{array}$ & $\begin{array}{l}40,276 \\
48,728 \\
57,983 \\
68,040 \\
78,899\end{array}$ & $\begin{array}{ll}10 & 00 \\
11 & 00 \\
12 & 00 \\
13 & 00 \\
14 & 00\end{array}$ & $\begin{array}{r}973,246 \\
1,070,302 \\
1,167,282 \\
1,264,178 \\
1,360,983\end{array}$ & $\begin{array}{l}41,199 \\
49,845 \\
59,313 \\
69,601 \\
80,706\end{array}$ \\
\hline $\begin{array}{ll}15 & 00 \\
16 & 00 \\
17 & 00 \\
18 & 00 \\
19 & 00\end{array}$ & $\begin{array}{l}1,485,348 \\
1,583,857 \\
1,682,267 \\
1,780,570 \\
1,878,762\end{array}$ & $\begin{array}{r}88,374 \\
100,534 \\
113,474 \\
127,193 \\
141,690\end{array}$ & $\begin{array}{ll}15 & 00 \\
16 & 00 \\
17 & 00 \\
18 & 00 \\
19 & 00\end{array}$ & $\begin{array}{l}1,471,745 \\
1,569,315 \\
1,666,781 \\
1,764,135 \\
.1,861,371\end{array}$ & $\begin{array}{r}90,558 \\
103,017 \\
116,275 \\
130,331 \\
145,185\end{array}$ & $\begin{array}{ll}15 & 00 \\
16 & 00 \\
17 & 00 \\
18 & 00 \\
19 & 00\end{array}$ & $\begin{array}{l}1,457,691 \\
1,654,295 \\
1,650,787 \\
1,747,161 \\
1,843,410\end{array}$ & $\begin{array}{r}92,631 \\
105,375 \\
118,935 \\
133,311 \\
148,502\end{array}$ \\
\hline $\begin{array}{ll}20 & 00 \\
21 & 00 \\
22 & 00 \\
23 & 00 \\
24 & 00\end{array}$ & $\begin{array}{r}1,976,836 \\
2,074,786 \\
2,172, \text { ti06 } \\
2,270,289 \\
2,367,830\end{array}$ & $\begin{array}{l}156,966 \\
173,018 \\
189,845 \\
207,447 \\
225,823\end{array}$ & $\begin{array}{ll}20 & 00 \\
21 & 00 \\
22 & 00 \\
23 & 00 \\
24 & 00\end{array}$ & $\begin{array}{l}1,958,481 \\
2,055,460 \\
2,152,302 \\
2,248,998 \\
2,345,544\end{array}$ & $\begin{array}{l}160,835 \\
177.280 \\
194,518 \\
212,550 \\
231,374\end{array}$ & $\begin{array}{ll}20 & 00 \\
21 & 00 \\
22 & 00 \\
23 & 00 \\
24 & 00\end{array}$ & $\begin{array}{l}1,939,527 \\
2,035,505 \\
2,131,338 \\
2,227,020 \\
2,322,539\end{array}$ & $\begin{array}{l}164,506 \\
181,324 \\
198,963 \\
217,892 \\
236,640\end{array}$ \\
\hline $\begin{array}{ll}25 & 00 \\
26 & 00 \\
27 & 00 \\
28 & 00 \\
29 & 00 \\
30 & 00\end{array}$ & $\begin{array}{l}2,465,222 \\
2,562,459 \\
2,659,535 \\
2,756,445 \\
2,853,181 \\
2,949,739\end{array}$ & $\begin{array}{l}244,970 \\
264,889 \\
285,577 \\
307,035 \\
329,259 \\
352,249\end{array}$ & $\begin{array}{ll}25 & 00 \\
26 & 00 \\
27 & 00 \\
28 & 00 \\
29 & 00 \\
30 & 00\end{array}$ & $\begin{array}{l}2,441,932 \\
2,538,156 \\
2,634,210 \\
2,730,087 \\
2,825,779 \\
2,921,284\end{array}$ & $\begin{array}{l}250,988 \\
271,391 \\
292,582 \\
314,559 \\
337,321 \\
360,866\end{array}$ & $\begin{array}{ll}25 & 00 \\
26 & 00 \\
27 & 00 \\
28 & 00 \\
29 & 00 \\
30 & 00\end{array}$ & $\begin{array}{l}2,417,893 \\
2,513,074 \\
2,608,075 \\
2,702,890 \\
2,797,511 \\
2,891,931\end{array}$ & $\begin{array}{l}256,695 \\
277,558 \\
299,224 \\
321,694 \\
344,964 \\
369,036\end{array}$ \\
\hline
\end{tabular}

Bull. 214-03-3 
TABLE 4.-For projections of maps of large areas-Continued.

COORDINATES OF CURVATURE.

\begin{tabular}{|c|c|c|c|c|c|c|c|c|}
\hline \multicolumn{9}{|c|}{ Natural scale.-Values of $\mathrm{X}$ and $\mathrm{Y}$ in meters. } \\
\hline \multicolumn{3}{|c|}{ Latitude $30^{\circ}$. } & \multicolumn{3}{|c|}{ Latitude $31^{\circ}$. } & \multicolumn{3}{|c|}{ Làtitude $32^{\circ}$. } \\
\hline $\begin{array}{l}\text { Longi- } \\
\text { tude. }\end{array}$ & $\mathrm{x}$ & $Y$ & $\begin{array}{l}\text { Longi- } \\
\text { tude. }\end{array}$ & $\mathrm{x}$ & $Y$ & $\begin{array}{l}\text { Longi- } \\
\text { tude. }\end{array}$ & $\mathrm{X}$ & $Y$ \\
\hline $\begin{array}{ll}\circ & , \\
1 & 00 \\
2 & 00 \\
3 & 00 \\
4 & 00\end{array}$ & $\begin{array}{r}96,487 \\
192,967 \\
289,432 \\
385,875\end{array}$ & $\begin{array}{r}421 \\
1,684 \\
3,789 \\
6,735\end{array}$ & $\begin{array}{ll}\circ & , \\
1 & 00 \\
2 & 00 \\
3 & 00 \\
4 & 00\end{array}$ & $\begin{array}{r}95,505 \\
191,002 \\
286,484 \\
381,943\end{array}$ & $\begin{array}{r}429 \\
1,717 \\
3,863 \\
6,867\end{array}$ & $\begin{array}{ll}\circ & 1 \\
1 & 00 \\
2 & 00 \\
3 & 00 \\
4 & 00\end{array}$ & $\begin{array}{r}94,494 \\
188,980 \\
283,449 \\
377,894\end{array}$ & $\begin{array}{r}437 \\
1,748 \\
3,933 \\
6,991\end{array}$ \\
\hline $\begin{array}{ll}5 & 00 \\
6 & 00 \\
7 & 00 \\
8 & 00 \\
9 & 00\end{array}$ & $\begin{array}{l}482,288 \\
578,665 \\
674,998 \\
771,279 \\
867,502\end{array}$ & $\begin{array}{l}10,523 \\
15,153 \\
20,623 \\
26,934 \\
34,084\end{array}$ & $\begin{array}{ll}5 & 00 \\
6 & 00 \\
7 & 00 \\
8 & 00 \\
9 & 00\end{array}$ & $\begin{array}{l}477,371 \\
572,760 \\
668,103 \\
763,392 \\
858,619\end{array}$ & $\begin{array}{l}10,729 \\
15,450 \\
21,027 \\
27,461 \\
34,751\end{array}$ & $\begin{array}{ll}5 & 00 \\
6 & 00 \\
7 & 00 \\
8 & 00 \\
9 & 00\end{array}$ & $\begin{array}{l}472,307 \\
566,680 \\
661,004 \\
755,272 \\
849,475\end{array}$ & $\begin{array}{l}10,922 \\
15,727 \\
21,404 \\
27,954 \\
35,375\end{array}$ \\
\hline $\begin{array}{ll}10 & 00 \\
11 & 00 \\
12 & 00 \\
13 & 00 \\
14 & 00\end{array}$ & $\begin{array}{r}963,658 \\
1,059,741 \\
1,155,744 \\
1,251,658 \\
1 ; 347,477\end{array}$ & $\begin{array}{l}42,074 \\
50,903 \\
60,570 \\
71,074 \\
82,415\end{array}$ & $\begin{array}{ll}10 & 00 \\
11 & 00 \\
12 & 00 \\
13 & 00 \\
14 & 00\end{array}$ & $\begin{array}{r}953,777 \\
1,048,858 \\
1,143,854 \\
1,238,758 \\
1,333,561\end{array}$ & $\begin{array}{l}42,897 \\
51,898 \\
61,753 \\
72,462 \\
84,024\end{array}$ & $\begin{array}{ll}10 & 00 \\
11 & 00 \\
12 & 00 \\
13 & 00 \\
14 & 00\end{array}$ & $\begin{array}{r}943,605 \\
1,037,655 \\
1,131,616 \\
1,225,480 \\
1,319,239\end{array}$ & $\begin{array}{l}43,667 \\
52,829 \\
62,861 \\
73,761 \\
85,529\end{array}$ \\
\hline $\begin{array}{ll}15 & 00 \\
16 & 00 \\
17 & 00 \\
18 & 00 \\
19 & 00\end{array}$ & $\begin{array}{l}1,443,193 \\
1,538,800 \\
1,634,290 \\
1,729,654 \\
1,824,887\end{array}$ & $\begin{array}{r}94,591 \\
107,603 \\
121,449 \\
136,127 \\
151,637\end{array}$ & $\begin{array}{ll}15 & 00 \\
16 & 00 \\
17 & 00 \\
18 & 00 \\
19 & 00\end{array}$ & $\begin{array}{l}1,428,257 \\
1,522,837 \\
1,617,294 \\
1,711,621 \\
1,805,810\end{array}$ & $\begin{array}{r}96,437 \\
109,701 \\
123,815 \\
138,777 \\
154,586\end{array}$ & $\begin{array}{ll}15 & 00 \\
16 & 00 \\
17 & 00 \\
18 & 00 \\
19 & 00\end{array}$ & $\begin{array}{l}1,412,885 \\
1,506,411 \\
1,599,808 \\
1,693,067 \\
1,786,182\end{array}$ & $\begin{array}{r}98,164 \\
111,664 \\
126,029 \\
141,256 \\
157,346\end{array}$ \\
\hline $\begin{array}{ll}20 & 00 \\
21 & 00 \\
22 & 00 \\
23 & 00 \\
24 & 00\end{array}$ & $\begin{array}{l}1,919,982 \\
2,014,930 \\
2,109,725 \\
2,204,359 \\
2,298,825\end{array}$ & $\begin{array}{l}167,977 \\
185,147 \\
203,143 \\
221,966 \\
241,616\end{array}$ & $\begin{array}{ll}20 & 00 \\
21 & 00 \\
22 & 00 \\
23 & 00 \\
24 & 00\end{array}$ & $\begin{array}{l}1,899,852 \\
1,993,740 \\
2,087,468 \\
2,181,027 \\
2,274,411\end{array}$ & $\begin{array}{l}171,241 \\
188,741 \\
207,08 \tilde{0} \\
226,270 \\
246,29 \tilde{0}\end{array}$ & $\begin{array}{ll}20 & 00 \\
21 & 00 \\
22 & 00 \\
23 & 00 \\
24 & 00\end{array}$ & $\begin{array}{l}1,879,144 \\
1,971,946 \\
2,064,579 \\
2,157,035 \\
2,249,305\end{array}$ & $\begin{array}{l}174,296 \\
192,105 \\
210,772 \\
230,295 \\
250,672\end{array}$ \\
\hline $\begin{array}{ll}25 & 00 \\
26 & 00 \\
27 & 00 \\
28 & 00 \\
29 & 00 \\
30 & 00\end{array}$ & $\begin{array}{l}2,393,116 \\
2,487,224 \\
2,581,144 \\
2,674,867 \\
2,768,385 \\
2,861,694\end{array}$ & $\begin{array}{l}262,089 \\
283,383 \\
305,498 \\
328,432 \\
352,183 \\
376,749\end{array}$ & $\begin{array}{ll}25 & 00 \\
26 & 00 \\
27 & 00 \\
28 & 00 \\
29 & 00 \\
30 & 00\end{array}$ & $\begin{array}{l}2,367,610 \\
2,460,618 \\
2,553,427 \\
2,646,029 \\
2,738,418 \\
2,830,585\end{array}$ & $\begin{array}{l}267,159 \\
288,860 \\
311,396 \\
334,765 \\
358,966 \\
383,-997\end{array}$ & $\begin{array}{ll}25 & 00 \\
26 & 00 \\
27 & 00 \\
28 & 00 \\
29 & 00 \\
30 & 00\end{array}$ & $\begin{array}{l}2,341,385 \\
2,433,264 \\
2,524,935 \\
2,616,390 \\
2,707,621 \\
2,798,621\end{array}$ & $\begin{array}{l}271,901 \\
293,981 \\
316,910 \\
340,686 \\
365,307 \\
390,770\end{array}$ \\
\hline
\end{tabular}


TAB.LE 4.-For projections of maps of large areces-Continued.

COORDINATES OF CURVATURE.

\begin{tabular}{|c|c|c|c|c|c|c|c|c|}
\hline \multicolumn{8}{|c|}{ Natural seale.-Values of $\mathrm{X}$ and $\mathrm{Y}$ in meters. } & \\
\hline \multicolumn{3}{|c|}{ Latitude $33^{\circ}$. } & \multicolumn{3}{|c|}{ Latitude $34^{\circ}$. } & \multicolumn{3}{|c|}{ Latitude $35^{\circ}$. } \\
\hline $\begin{array}{l}\text { Longi- } \\
\text { tude. }\end{array}$ & $\mathbf{X}$ & $\mathrm{Y}$ & $\begin{array}{l}\text { Longi- } \\
\text { tude. }\end{array}$ & $\mathbf{X}$ & $\mathbf{Y}$ & $\begin{array}{l}\text { Longi- } \\
\text { tude. }\end{array}$ & $\mathbf{x}$ & $Y$ \\
\hline $\begin{array}{cc}\circ & 1 \\
1 & 00 \\
2 & 00 \\
3 & 00 \\
4 & 00\end{array}$ & $\begin{array}{r}93,454 \\
186,899 \\
280,328 \\
373,731\end{array}$ & $\begin{array}{r}444 \\
1,777 \\
3,997 \\
7,106\end{array}$ & $\begin{array}{ll}\circ & \prime \\
1 & 00 \\
2 & 00 \\
3 & 00 \\
4 & 00\end{array}$ & $\begin{array}{r}92,385 \\
184,762 \\
277,121 \\
369,454\end{array}$ & $\begin{array}{r}451 \\
1,803 \\
4,057 \\
7,212\end{array}$ & $\begin{array}{lc}\circ & \prime \\
1 & 00 \\
2 & 00 \\
3 & 00 \\
4 & 00\end{array}$ & $\begin{array}{r}91,289 \\
182,568 \\
273,830 \\
365,064\end{array}$ & $\begin{array}{r}457 \\
1,828 \\
4,112 \\
7,310\end{array}$ \\
\hline $\begin{array}{ll}5 & 00 \\
6 & 00 \\
7 & 00 \\
8 & 00 \\
9 & 00\end{array}$ & $\begin{array}{l}467,100 \\
560,428 \\
653,704 \\
746,922 \\
840,072\end{array}$ & $\begin{array}{l}11,102 \\
15,986 \\
21,757 \\
28,414 \\
35,957\end{array}$ & $\begin{array}{ll}5 & 00 \\
6 & 00 \\
7 & 00 \\
8 & 00 \\
9 & 00\end{array}$ & $\begin{array}{l}461,751 \\
554,004 \\
646,205 \\
738,344 \\
830,413\end{array}$ & $\begin{array}{l}11,268 \\
16,225 \\
22,082 \\
28,839 \\
36,494\end{array}$ & $\begin{array}{ll}5 & 00 \\
6 & 00 \\
7 & 00 \\
8 & 00 \\
9 & 00\end{array}$ & $\begin{array}{l}456,261 \\
547,412 \\
638,509 \\
729,542 \\
820,501\end{array}$ & $\begin{array}{l}11,421 \\
16,445 \\
22,381 \\
29,229 \\
36,987\end{array}$ \\
\hline $\begin{array}{ll}10 & 00 \\
11 & 00 \\
12 & 00 \\
13 & 00 \\
14 & 00\end{array}$ & $\begin{array}{r}933,146 \\
1,026,136 \\
1,119,033 \\
1,211,829 \\
1,304,515\end{array}$ & $\begin{array}{l}44,385 \\
53,697 \\
63,893 \\
74,971 \\
86,931\end{array}$ & $\begin{array}{ll}10 & 00 \\
11 & 00 \\
12 & 00 \\
13 & 00 \\
14 & 00\end{array}$ & $\begin{array}{r}922,403 \\
1,014,305 \\
1,106,110 \\
1,197,809 \\
, 1,289,395\end{array}$ & $\begin{array}{l}45,048 \\
54,499 \\
64,846 \\
76,089 \\
88,227\end{array}$ & $\begin{array}{ll}10 & 00 \\
11 & 00 \\
12 & 00 \\
13 & 00 \\
14 & 00\end{array}$ & $\begin{array}{r}911,379 \\
1,002,165 \\
1,092,850 \\
1,183,426 \\
1,273,884\end{array}$ & $\begin{array}{l}45,656 \\
55,234 \\
65,721 \\
77,115 \\
89,415\end{array}$ \\
\hline $\begin{array}{ll}15 & 00 \\
16 & 00 \\
17 & 00 \\
18 & 00 \\
19 & 00\end{array}$ & $\begin{array}{l}1,397,083 \\
1,489,526 \\
1,581,834 \\
1,673,998 \\
1,766,011\end{array}$ & $\begin{array}{r}99,771 \\
113,491 \\
128,089 \\
143,564 \\
159,914\end{array}$ & $\begin{array}{ll}15 & 00 \\
16 & 00 \\
17 & 00 \\
18 & 00 \\
19 & 00\end{array}$ & $\begin{array}{l}1,380,858 \\
1,472,190 \\
1,563,381 \\
1,654,423 \\
1,745,308\end{array}$ & $\begin{array}{l}101,258 \\
115,180 \\
129,993 \\
145,696 \\
162,287\end{array}$ & $\begin{array}{ll}15 & 00 \\
16 & 00 \\
17 & 00 \\
18 & 00 \\
19 & 00\end{array}$ & $\begin{array}{l}1,364,214 \\
1,454,407 \\
1,544,451 \\
1,634,347 \\
1,724,076\end{array}$ & $\begin{array}{l}102,619 \\
116,728 \\
131,738 \\
147,650 \\
164,460\end{array}$ \\
\hline $\begin{array}{ll}20 & 00 \\
2.1 & 00 \\
22 & 00 \\
23 & 00 \\
24 & 00\end{array}$ & $\begin{array}{l}1,857,866 \\
1,949,553 \\
2,041,062 \\
2,132,387 \\
2,223,521\end{array}$ & $\begin{array}{l}177,138 \\
195,234 \\
214,201 \\
234,037 \\
.554,740\end{array}$ & $\begin{array}{ll}20 & 00 \\
21 & 00 \\
22 & 00 \\
23 & 00 \\
24 & 00\end{array}$ & $\begin{array}{l}1,836,026 \\
1,926,569 \\
2,016,929 \\
2,107,097 \\
2,197,065\end{array}$ & $\begin{array}{l}179,763 \\
198,124 \\
217,368 \\
237,493 \\
258,497\end{array}$ & $\begin{array}{ll}20 & 00 \\
21 & 00 \\
22 & 00 \\
23 & 00 \\
24 & 00\end{array}$ & $\begin{array}{l}1,813,632 \\
1,903,006 \\
1,992,190 \\
2,081,174 \\
2,169,949\end{array}$ & $\begin{array}{l}182,168 \\
200,772 \\
220,268 \\
240,657 \\
261,936\end{array}$ \\
\hline $\begin{array}{ll}25 & 00 \\
26 & 00 \\
27 & 00 \\
28 & 00 \\
29 & 00 \\
30 & 00\end{array}$ & $\begin{array}{l}2,314,453 \\
2,405,175 \\
2,495,680 \\
2,585,961 \\
2,676,007 \\
2,765,812\end{array}$ & $\begin{array}{l}276,309 \\
298,741 \\
322,034 \\
346,187 \\
371,197 \\
397,061\end{array}$ & $\begin{array}{ll}25 & 00 \\
26 & 00 \\
27 & 00 \\
28 & 00 \\
24 & 00 \\
30 & 00\end{array}$ & $\begin{array}{l}2,286,823 \\
2,376,363 \\
2,465,677 \\
2,554,756 \\
2,643,591 \\
2,732,175\end{array}$ & $\begin{array}{l}230,378 \\
303,134 \\
326,763 \\
351,262 \\
376,629 \\
402,863\end{array}$ & $\begin{array}{ll}25 & 00 \\
26 & 00 \\
27 & 00 \\
28 & 00 \\
29 & 0 C \\
30 & 00\end{array}$ & $\begin{array}{l}2,258,507 \\
2,346,838 \\
2,434,934 \\
2,522,787 \\
2,610,386 \\
2,697,724\end{array}$ & $\begin{array}{l}284,102 \\
307,154 \\
331,089 \\
355,905 \\
381,598 \\
408,168\end{array}$ \\
\hline
\end{tabular}


$\mathrm{T}_{\mathrm{ABLE}}$ 4.-For projections of maps of large areas-Continued. COORDINATES OF CURVATURE.

\begin{tabular}{|c|c|c|c|c|c|c|c|c|}
\hline \multicolumn{9}{|c|}{ Natural scale.-Values of $\mathrm{X}$ and $\mathrm{Y}$ meters. } \\
\hline \multicolumn{3}{|c|}{ Latitude $36^{\circ}$. } & \multicolumn{3}{|c|}{ Latitude $37^{\circ}$. } & \multicolumn{3}{|c|}{ Latitude $38^{\circ}$. } \\
\hline $\begin{array}{l}\text { Longi- } \\
\text { tude. }\end{array}$ & $\mathbf{X}$ & $\mathbf{Y}$ & $\begin{array}{l}\text { Longi- } \\
\text { tude. }\end{array}$ & $\mathbf{x}$ & $Y$ & $\begin{array}{l}\text { Longi- } \\
\text { tude. }\end{array}$ & $\mathbf{X}$ & $Y$ \\
\hline $\begin{array}{ll}\circ & 1 \\
1 & 00 . \\
2 & 00 \\
3 & 00 \\
4 & 00\end{array}$ & $\begin{array}{r}90,164 \\
180,319 \\
270,455 \\
360,562\end{array}$ & $\begin{array}{r}462 \\
1,850 \\
4,162 \\
7,399\end{array}$ & $\begin{array}{ll}\circ & 1 \\
1 & 00 \\
2 & 00 \\
3 & 00 \\
4 & 00\end{array}$ & $\begin{array}{r}89,012 \\
178,015 \\
266,997 \\
355,951\end{array}$ & $\begin{array}{r}467 \\
1,870 \\
4,207 \\
7,479\end{array}$ & $\begin{array}{ll}\circ & 1 \\
1 & 00 \\
2 & 00 \\
3 & 00 \\
4 & 00\end{array}$ & $\begin{array}{r}87,833 \\
175,656 \\
263,458 \\
351,230\end{array}$ & $\begin{array}{r}472 \\
1,888 \\
4,247 \\
7,549\end{array}$ \\
\hline $\begin{array}{ll}5 & 00 \\
6 & 00 \\
7 & 00 \\
8 & 00 \\
9 & 00\end{array}$ & $\begin{array}{l}450,631 \\
540,653 \\
630,618 \\
720,517 \\
810,340\end{array}$ & $\begin{array}{l}11,560 \\
16,645 \\
22,652 \\
29,583 \\
37,435\end{array}$ & $\begin{array}{ll}5 & 00 \\
6 & 00 \\
7 & 00 \\
8 & 00 \\
9 & 00\end{array}$ & $\begin{array}{l}444,865 \\
533,730 \\
622,536 \\
711,273 \\
799,932\end{array}$ & $\begin{array}{l}11,685 \\
16,824 \\
22,896 \\
29,901 \\
37,838\end{array}$ & $\begin{array}{ll}5 & 00 \\
6 & 00 \\
7 & 00 \\
8 & 00 \\
9 & 00\end{array}$ & $\begin{array}{l}438,962 \\
526,643 \\
614,263 \\
701,812 \\
789,280\end{array}$ & $\begin{array}{l}11,795 \\
16,983 \\
23,112 \\
30,183 \\
38,195\end{array}$ \\
\hline $\begin{array}{ll}10 & 00 \\
11 & 00 \\
12 & 00 \\
13 & 0 j \\
14 & 00\end{array}$ & $\begin{array}{r}900,078 \\
989,720 \\
1,079,259 \\
1,168,684 \\
1,257,987\end{array}$ & $\begin{array}{l}46,209 \\
55,903 \\
66,515 \\
78,046 \\
90,494\end{array}$ & $\begin{array}{ll}10 & 00 \\
11 & 00 \\
12 & 00 \\
13 & 00 \\
14 & 00\end{array}$ & $\begin{array}{r}888,503 \\
976,975 \\
1,065,340 \\
1,153,587 \\
1,241,707\end{array}$ & $\begin{array}{r}46,706 \\
56,503 \\
67,229 \\
78,882 \\
.91,462\end{array}$ & $\begin{array}{ll}10 & 00 \\
11 & 00 \\
12 & 00 \\
13 & 00 \\
14 & 00\end{array}$ & $\begin{array}{r}876,657 \\
963,933 \\
1,051,098 \\
1,138,141 \\
1,225,053\end{array}$ & $\begin{array}{l}47,145 \\
57,034 \\
67,860 \\
79,622 \\
92,319\end{array}$ \\
\hline $\begin{array}{ll}15 & 00 \\
16 & 00 \\
17 & 00 \\
18 & 00 \\
19 & 00\end{array}$ & $\begin{array}{l}1,347,156 \\
1,436,184 \\
1,525,061 \\
1,613,777 \\
1,702,324\end{array}$ & $\begin{array}{l}103,856 \\
118,133 \\
133,323 \\
149,423 \\
166,433\end{array}$ & $\begin{array}{ll}15 & 00 \\
16 & 00 \\
17 & 00 \\
18 & 00 \\
19 & 00\end{array}$ & $\begin{array}{l}1,329,690 \\
1,417,526 \\
1,505,206 \\
1,592,721 \\
1,680,059\end{array}$ & $\begin{array}{l}104,967 \\
119,395 \\
134,745 \\
151,015 \\
168,203\end{array}$ & $\begin{array}{ll}15 & 00 \\
16 & 00 \\
17 & 00 \\
18 & 00 \\
19 & 00\end{array}$ & $\begin{array}{l}1,311,823 \\
1,398,441 \\
1,484,899 \\
1,571,185 \\
1,657,289\end{array}$ & $\begin{array}{l}105,949 \\
120,511 \\
136,002 \\
152,421 \\
169,767\end{array}$ \\
\hline $\begin{array}{ll}20 & 00 \\
21 & 00 \\
22 & 00 \\
23 & 00 \\
24 & 00\end{array}$ & $\begin{array}{l}1,790,691 \\
1,878,870 \\
1,966,851 \\
2,054,625 \\
2,142,183\end{array}$ & $\begin{array}{l}184,350 \\
203,173 \\
222,899 \\
243,527 \\
265,055\end{array}$ & $\begin{array}{ll}20 & 00 \\
21 & 00 \\
22 & 00 \\
23 & 00 \\
24 & 00\end{array}$ & $\begin{array}{l}1,767,211 \\
1,854,169 \\
1,940,922 \\
2,027,462 \\
2,113,777\end{array}$ & $\begin{array}{l}186,307 \\
205,326 \\
225,258 \\
246,099 \\
267,849\end{array}$ & $\begin{array}{ll}20 & 00 \\
21 & 00 \\
22 & 00 \\
23 & 00 \\
24 & 00\end{array}$ & $\begin{array}{l}1,743,202 \\
1,828,914 \\
1,914,415 \\
1,999,694 \\
2,084,743\end{array}$ & $\begin{array}{l}188,037 \\
207,229 \\
227,341 \\
248,370 \\
270,315\end{array}$ \\
\hline $\begin{array}{ll}25 & 00 \\
26 & 00 \\
27 & 00 \\
28 & 00 \\
29 & 00 \\
30 & 00\end{array}$ & $\begin{array}{r}2,229,516 \\
2,316,613 \\
-2,403,467 \\
2,490,068 \\
2,576,407 \\
2,662,475\end{array}$ & $\begin{array}{l}287,479 \\
310,798 \\
335,009 \\
360,111 \\
386,099 \\
412,971\end{array}$ & $\begin{array}{ll}25 & 00 \\
26 & 00 \\
27 & 00 \\
28 & 00 \\
29 & 00 \\
30 & 00\end{array}$ & $\begin{array}{l}2,199,860 \\
2,285,699 \\
2,371,287 \\
2,456,612 \\
2,541,667 \\
2,626,441\end{array}$ & $\begin{array}{l}290,503 \\
314,061 \\
338,519 \\
363,874 \\
390,125 \\
417,267\end{array}$ & $\begin{array}{ll}25 & 00 \\
26 & 00 \\
27 & 00 \\
28 & 00 \\
29 & 00 \\
30 & 00\end{array}$ & $\begin{array}{l}2,169,551 \\
2,254,109 \\
2,338,406 \\
2,422,433 \\
2,506,181 \\
2,589,639\end{array}$ & $\begin{array}{l}293,172 \\
316,939 \\
341,613 \\
367,192 \\
393,672 \\
421,050\end{array}$ \\
\hline
\end{tabular}


Table 4.-For projections of maps of large areas-Continued.

COORDINATES OF CURVATURE.

\begin{tabular}{|c|c|c|c|c|c|c|c|c|}
\hline \multicolumn{9}{|c|}{ Natural scale.-Values of $\mathrm{X}$ and $\mathrm{Y}$ in meters. } \\
\hline \multicolumn{3}{|c|}{ Latitude $39^{\circ}$. } & \multicolumn{3}{|c|}{ Latitude $40^{\circ}$ : } & \multicolumn{3}{|c|}{ Latitude $41^{\circ}$} \\
\hline $\begin{array}{l}\text { Longi- } \\
\text { tude. }\end{array}$ & $\mathbf{x}$ & $\mathbf{Y}$ & $\begin{array}{l}\text { Longi- } \\
\text { tude. }\end{array}$ & $\mathbf{x}$ & $\mathbf{Y}$ & $\begin{array}{l}\text { Longi- } \\
\text { tude. }\end{array}$ & $\mathbf{x}$ & $\mathbf{Y}$ \\
\hline $\begin{array}{ll}0 & 1 \\
1 & 00 \\
2 & 00 \\
3 & 00 \\
4 & 00\end{array}$ & $\begin{array}{r}86,627 \\
173,243 \\
259,859 \\
346,403\end{array}$ & $\begin{array}{r}476 \\
1,903 \\
4,281 \\
7,611\end{array}$ & $\begin{array}{ll}\circ & 1 \\
1 & 00 \\
2 & 00 \\
3 & 00 \\
4 & 00\end{array}$ & $\begin{array}{r}85,394 \\
170,778 \\
256,140 \\
341,470\end{array}$ & $\begin{array}{r}479 \\
1,916 \\
4,311 \\
7,663\end{array}$ & $\begin{array}{ll}\circ & 1 \\
1 & 00 \\
2 & 00 \\
3 & 00 \\
4 & 00\end{array}$ & $\begin{array}{r}84,136 \\
168,260 \\
252,363 \\
336,432\end{array}$ & $\begin{array}{r}482 \\
1,927 \\
4,335 \\
7,706\end{array}$ \\
\hline $\begin{array}{ll}5 & 00 \\
6 & 00 \\
7 & 00 \\
8 & 00 \\
9 & 00\end{array}$ & $\begin{array}{l}432,925 \\
519,396 \\
605,803 \\
692,138 \\
778,388\end{array}$ & $\begin{array}{l}11,891 \\
17,121 \\
23,300 \\
30,428 \\
38,504\end{array}$ & $\begin{array}{ll}5 & 00 \\
6 & 00 \\
7 & 00 \\
8 & 00 \\
9 & 00\end{array}$ & $\begin{array}{l}426,757 \\
511,990 \\
597,158 \\
682,252 \\
767,260\end{array}$ & $\begin{array}{l}11,972 \\
17,238 \\
23,460 \\
30,637 \\
38,768\end{array}$ & $\begin{array}{ll}5 & 00 \\
6 & 00 \\
7 & 00 \\
8 & 00 \\
9 & 00\end{array}$ & $\begin{array}{l}420,457 \\
504,428 \\
588,332 \\
672,159 \\
755,897\end{array}$ & $\begin{array}{l}12,039 \\
17,335 \\
23,591 \\
30,807 \\
38,983\end{array}$ \\
\hline $\begin{array}{ll}10 & 00 \\
11 & 00 \\
12 & 00 \\
13 & 00 \\
14 & 00\end{array}$ & $\begin{array}{r}864,545 \\
950,598 \\
1,036,536 \\
1,122,349 \\
1,208,027\end{array}$ & $\begin{array}{l}47,527 \\
57,496 \\
68,409 \\
80,266 \\
93,064\end{array}$ & $\begin{array}{ll}10 & 00 \\
11 & 00 \\
12 & 00 \\
13 & 00 \\
14 & 00\end{array}$ & $\begin{array}{r}852,171 \\
936,975 \\
1,021,661 \\
1,106,218 \\
1,190,636\end{array}$ & $\begin{array}{l}47,852 \\
57,888 \\
68,875 \\
80,811 \\
93,695\end{array}$ & $\begin{array}{ll}10 & 00 \\
11 & 00 \\
12 & 00 \\
13 & 00 \\
14 & 00\end{array}$ & $\begin{array}{r}839,537 \\
923,067 \\
1,006,475 \\
1,089,752 \\
1,172,886\end{array}$ & $\begin{array}{l}48,118 \\
58,209 \\
69,256 \\
81,258 \\
94,212\end{array}$ \\
\hline $\begin{array}{ll}15 & 00 \\
16 & 00 \\
17 & 00 \\
18 & 00 \\
19 & 00\end{array}$ & $\begin{array}{l}1,293,559 \\
1,378,934 \\
1,464,144 \\
1,549,177 \\
1 ; 634,023\end{array}$ & $\begin{array}{l}106,802 \\
121,479 \\
137,093 \\
153,642 \\
171,124\end{array}$ & $\begin{array}{ll}15 & 00 \\
16 & 00 \\
17 & 00 \\
18 & 00 \\
19 & 00\end{array}$ & $\begin{array}{l}1,274,904 \\
1,359,012 \\
1,442,949 \\
1,526,704 \\
1,610,267\end{array}$ & $\begin{array}{l}107,525 \\
122,300 \\
138,017 \\
154,675 \\
172,272\end{array}$ & $\begin{array}{ll}15 & 00 \\
16 & 00 \\
17 & 00 \\
18 & 00 \\
19 & 00\end{array}$ & $\begin{array}{l}1,255,866 \\
1,338,681 \\
1,421,321 \\
1,503,775 \\
1,686,031\end{array}$ & $\begin{array}{l}108,117 \\
122,971 \\
138,773 \\
155,520 \\
173,210\end{array}$ \\
\hline $\begin{array}{ll}20 & 00 \\
21 & 00 \\
22 & 00 \\
23 & 00 \\
24 & 00\end{array}$ & $\begin{array}{l}1,718,671 \\
1,803,113 \\
1,887,337 \\
1,971,333 \\
2,055,091\end{array}$ & $\begin{array}{l}189,587 \\
208,878 \\
229,146 \\
250,337 \\
272,450\end{array}$ & $\begin{array}{ll}20 & 00 \\
21 & 00 \\
22 & 00 \\
23 & 00 \\
24 & 00\end{array}$ & $\begin{array}{l}1,693,628 \\
1,776,775 \\
1,859,698 \\
1,942,387 \\
2,024,833\end{array}$ & $\begin{array}{l}190,805 \\
210,272 \\
230,671 \\
251,998 \\
274,252\end{array}$ & $\begin{array}{ll}20 & 00 \\
21 & 00 \\
22 & 00 \\
23 & 00 \\
24 & 00\end{array}$ & $\begin{array}{r}1,668,079 \\
1,749,909 \\
1,831,509 \\
1,912,869 \\
1,993,978\end{array}$ & $\begin{array}{l}191,841 \\
211,409 \\
231,914 \\
253,352 \\
275,719\end{array}$ \\
\hline $\begin{array}{ll}25 & 00 \\
26 & 00 \\
27 & 00 \\
28 & 00 \\
29 & 00 \\
30 & 00\end{array}$ & $\begin{array}{l}2,138,602 \\
2,221,854 \\
2,304,838 \\
2,387,545 \\
2,469,963 \\
2,552,084\end{array}$ & $\begin{array}{l}295,481 \\
319,429 \\
344,289 \\
370,059 \\
396,736 \\
424,317\end{array}$ & $\begin{array}{ll}25 & 00 \\
26 & 00 \\
27 & 00 \\
28 & 00 \\
29 & 00 \\
30 & 00\end{array}$ & $\begin{array}{l}2,107,023 \\
2,188,948 \\
2,270,597 \\
2,351,961 \\
2,433,029 \\
2,513,790\end{array}$ & $\begin{array}{l}297,430 \\
321,528 \\
346,543 \\
372,473 \\
399,314 \\
427,063\end{array}$ & $\begin{array}{ll}25 & 00 \\
26 & 00 \\
27 & 00 \\
28 & 00 \\
29 & 00 \\
30 & 00\end{array}$ & $\begin{array}{l}2,074,826 \\
2,155,402 \\
2,235,695 \\
2,315,695 \\
2,395,392 \\
2,474,774\end{array}$ & $\begin{array}{l}299,014 \\
323,233 \\
348,374 \\
374,432 \\
401,404 \\
429,287\end{array}$ \\
\hline
\end{tabular}


TABLE 4.-For projections of maps of large areas-Continued. COORDINATES OF CURVATURE.

\begin{tabular}{|c|c|c|c|c|c|c|c|c|}
\hline \multicolumn{9}{|c|}{ Natural scale. - Values of $\mathrm{X}$ and $\mathrm{Y}$ in meters. } \\
\hline \multicolumn{3}{|c|}{, Latitude $42^{\circ}$} & \multicolumn{3}{|c|}{ Latitude $43^{\circ}$. } & \multicolumn{3}{|c|}{ Latitude $44^{\circ}$. } \\
\hline $\begin{array}{l}\text { Longi- } \\
\text { tude. }\end{array}$ & $\mathbf{x}$ & $\mathbf{Y}$ & $\begin{array}{l}\text { Longi- } \\
\text { tude. }\end{array}$ & $\mathbf{x}$ & Y & $\begin{array}{l}\text { Longi- } \\
\text { tude. }\end{array}$ & $\mathbf{x}$ & Y \\
\hline $\begin{array}{lc}\circ & , \\
1 & 00 \\
2 & 00 \\
3 & 00 \\
4 & 00\end{array}$ & $\begin{array}{r}82,851 \\
165,691 \\
248,508 \\
331,292\end{array}$ & $\begin{array}{r}484 \\
1,935 \\
4,354 \\
7,739\end{array}$ & $\begin{array}{ll}\circ & , \\
1 & 00 \\
2 & 00 \\
3 & 00 \\
4 & 00\end{array}$ & $\begin{array}{r}81,541 \\
163,071 \\
244,578 \\
326,050\end{array}$ & $\begin{array}{r}485 \\
1,941 \\
4,367 \\
7,763\end{array}$ & $\begin{array}{ll}\circ & , \\
1 & 00 \\
2 & 00 \\
3 & 00 \\
4 & 00\end{array}$ & $\begin{array}{r}80,206 \\
160,401 \\
240,572 \\
320,708\end{array}$ & $\begin{array}{r}486 \\
1,945 \\
4,375 \\
7,778\end{array}$ \\
\hline $\begin{array}{ll}5 & 00 \\
6 & 00 \\
7 & 00 \\
8 & 00 \\
9 & 00\end{array}$ & $\begin{array}{l}414,030 \\
496,712 \\
579,325 \\
661,861 \\
744,305\end{array}$ & $\begin{array}{l}12,092 \\
17,410 \\
23,693 \\
30,941 \\
39,152\end{array}$ & $\begin{array}{ll}5 & 00 \\
6 & 00 \\
7 & 00 \\
8 & 00 \\
9 & 00\end{array}$ & $\begin{array}{l}407,476 \\
488,844 \\
570,143 \\
651,361 \\
732,486\end{array}$ & $\begin{array}{l}12,129 \\
17,464 \\
23,766 \\
31,036 \\
39,272\end{array}$ & $\begin{array}{rr}5 & 00 \\
6 & 00 \\
7 & 00 \\
8 & 00 \\
9 & 00\end{array}$ & $\begin{array}{l}400,797 \\
480,827 \\
560,786 \\
640,662 \\
720,445\end{array}$ & $\begin{array}{l}12,152 \\
17,496 \\
23,811 \\
31,094 \\
39,345\end{array}$ \\
\hline $\begin{array}{ll}10 & 00 \\
11 & 00 \\
12 & 00 \\
13 & 00 \\
14 & 00\end{array}$ & $\begin{array}{r}826,648 \\
908,879 \\
990,985 \\
1,072,956 \\
1,154,781\end{array}$ & $\begin{array}{l}.48,325 \\
58,459 \\
69,553 \\
81,605 \\
94,614\end{array}$ & $\begin{array}{ll}10 & 00 \\
11 & 00 \\
12 & 00 \\
13 & 00 \\
14 & 00\end{array}$ & $\begin{array}{r}813,508 \\
894,415 \\
975,195 \\
1,055,837 \\
1,136,329\end{array}$ & $\begin{array}{l}48,474 \\
58,639 \\
69,766 \\
81,854 \\
94,901\end{array}$ & $\begin{array}{ll}10 & 00 \\
11 & 00 \\
12 & 00 \\
13 & 00 \\
14 & 00\end{array}$ & $\begin{array}{r}800,122 \\
879,681 \\
959,110 \\
1,038,399 \\
1,117,535\end{array}$ & $\begin{array}{l}48,568 \\
58,746 \\
69,893 \\
82,002 \\
95,072\end{array}$ \\
\hline $\begin{array}{ll}15 & 00 \\
16 & 00 \\
17 & 00 \\
18 & 00 \\
19 & 00\end{array}$ & $\begin{array}{l}1,236,449 \\
1,317,948 \\
1,399,267 \\
1,480,395 \\
1,561,321\end{array}$ & $\begin{array}{l}108,577 \\
123,493 \\
139,360 \\
156,175 \\
173,937\end{array}$ & $\begin{array}{ll}15 & 00 \\
16 & 00 \\
17 & 00 \\
18 & 00 \\
19 & 00\end{array}$ & $\begin{array}{l}1,216,661 \\
1,296,820 \\
1,376,795 \\
1,456,575 \\
1,536,148\end{array}$ & $\begin{array}{l}108,905 \\
123,864 \\
139,777 \\
156,640 \\
174,-151\end{array}$ & $\begin{array}{ll}15 & 00 \\
16 & 00 \\
17 & 09 \\
18 & 00 \\
19 & 00\end{array}$ & $\begin{array}{l}1,196,507 \\
1,275,303 \\
1,353,911 \\
1,432,320 \\
1,510,519\end{array}$ & $\begin{array}{l}109,100 \\
124,084 \\
140,023 \\
156,913 \\
174,753\end{array}$ \\
\hline $\begin{array}{ll}20 & 00 \\
21 & 00 \\
22 & 00 \\
23 & 00 \\
24 & 00\end{array}$ & $\begin{array}{l}1,642,035 \\
1,722,524 \\
1,802,779 \\
1,882,788 \\
1,962,540\end{array}$ & $\begin{array}{l}192,642 \\
212,289 \\
232,874 \\
254,396 \\
276,850 .\end{array}$ & $\begin{array}{ll}20 & 00 \\
21 & 00 \\
22 & 00 \\
23 & 00 \\
24 & 00\end{array}$ & $\begin{array}{l}1,615,505 \\
1,694,632 \\
1,773,519 \\
1,852,155 \\
1,930,528\end{array}$ & $\begin{array}{l}193,209 \\
212,909 \\
233,551 \\
255,129 \\
277,642\end{array}$ & $\begin{array}{ll}20 & 00 \\
21 & 00 \\
22 & 00 \\
23 & 00 \\
24 & 00\end{array}$ & $\begin{array}{l}1,588,496 \\
1,666,240 \\
1,743,738 \\
1,820,980 \\
1,897,955\end{array}$ & $\begin{array}{l}193,540 \\
213,270 \\
233,942 \\
255,552 \\
278,096\end{array}$ \\
\hline $\begin{array}{ll}25 & 00 \\
26 & 00 \\
27 & 00 \\
28 & 00 \\
29 & 00 \\
30 & 00\end{array}$ & $\begin{array}{l}2,042,024 \\
2,121,230 \\
2,200,146 \\
2,278,762 \\
2,357,067 \\
2,435,052\end{array}$ & $\begin{array}{l}300,234 \\
324,544 \\
349,778 \\
375,932 \\
403,002 \\
430,985\end{array}$ & $\begin{array}{ll}25 & 00 \\
26 & 00 \\
.27 & 00 \\
28 & 00 \\
29 & 00 \\
30 & 00\end{array}$ & $\begin{array}{l}2,008,628 \\
2,086,443 \\
2,163,963 \\
2,241,176 \\
2,318,071 \\
2,394,639\end{array}$ & $\begin{array}{r}301,087 \\
325,459 \\
350,750 \\
376,974 \\
404,109 \\
432,157\end{array}$ & $\begin{array}{ll}25 & 00 \\
26 & 00 \\
27 & 00 \\
28 & 00 \\
29 & 00 \\
30 & 00\end{array}$ & $\begin{array}{l}1,974,650 \\
2,051,055 \\
2,127,159 \\
2,202,950 \\
2,278,417 \\
2,353,550\end{array}$ & $\begin{array}{l}301,572 \\
325,977 \\
351,306 \\
377,555 \\
404,722 \\
432,801\end{array}$ \\
\hline
\end{tabular}


TABLE 4.-For projections of maps of large areas-Continued. COORDINATES OF CURVATURIE.

\begin{tabular}{|c|c|c|c|c|c|c|c|c|}
\hline \multicolumn{9}{|c|}{ Natural scale.-Values of $\mathrm{X}$ and $\mathrm{Y}$ in meters. } \\
\hline \multicolumn{3}{|c|}{ Latitude $45^{\circ}$. } & \multicolumn{3}{|c|}{ Latitude $46^{\circ}$. } & \multicolumn{3}{|c|}{ Latitude $47^{\circ}$. } \\
\hline $\begin{array}{l}\text { Longi- } \\
\text { tude. }\end{array}$ & $\mathbf{x}$ & $Y$ & $\begin{array}{l}\text { Longi- } \\
\text { tude. }\end{array}$ & $\mathrm{x}$ & $\mathrm{Y}$ & $\begin{array}{l}\text { Longi- } \\
\text { tude. }\end{array}$ & $\mathbf{x}$ & $\mathrm{Y}$ \\
\hline $\begin{array}{lc}\circ & 1 \\
1 & 00 \\
2 & 00 \\
3 & 00 \\
4 & 00\end{array}$ & $\begin{array}{r}78,847 \\
157,682 \\
236,493 \\
315,269\end{array}$ & $\begin{array}{r}486 \\
1,946 \\
4,378 \\
7,783\end{array}$ & $\begin{array}{ll}\circ & , \\
1 & 00 \\
2 & 00 \\
3 & 00 \\
4 & 00\end{array}$ & $\begin{array}{r}77,464 \\
154,915 \\
232,342 \\
309,732\end{array}$ & $\begin{array}{r}486 \\
1,945 \\
4,376 \\
7,779\end{array}$ & $\begin{array}{ll}0 & , \\
1 & 00 \\
2 & 00 \\
3 & 00 \\
4 & 00\end{array}$ & $\begin{array}{r}76,056 \\
152,100 \\
228,119 \\
304,101\end{array}$ & $\begin{array}{r}485 \\
. \quad 1,942 \\
4,368 \\
7,765\end{array}$ \\
\hline $\begin{array}{ll}5 & 00 \\
6 & 00 \\
7 & 00 \\
8 & 00 \\
9 & 00\end{array}$ & $\begin{array}{l}393,996 \\
472,663 \\
551,258 \\
629,769 \\
708,184\end{array}$ & $\begin{array}{l}12,160 \\
17,508 \\
23,826 \\
31,114 \\
39,370\end{array}$ & $\begin{array}{ll}5 & 00 \\
6 & 00 \\
7 & 00 \\
8 & 00 \\
9 & 00\end{array}$ & $\begin{array}{l}387,074 \\
464,354 \\
541,562 \\
618,684 \\
695,708\end{array}$ & $\begin{array}{r}12,153 \\
17,498 \\
23,813 \\
31,096 \\
39,347\end{array}$ & $\begin{array}{ll}5 & 00 \\
6 & 00 \\
7 & 00 \\
8 & 00 \\
9 & 00\end{array}$ & $\begin{array}{l}380,034 \\
455,904 \\
531,700 \\
607,410 \\
683,020\end{array}$ & $\begin{array}{l}12,131 \\
17,467 \\
23,770 \\
31,040 \\
39,276\end{array}$ \\
\hline $\begin{array}{ll}10 & 00 \\
11 & 00 \\
12 & 00 \\
13 & 00 \\
14 & 00\end{array}$ & $\begin{array}{r}786,492 \\
864,679 \\
942,735 \\
1,020,647 \\
-1,098,404\end{array}$ & $\begin{array}{l}48,594 \\
58,782 \\
69,936 \\
82,051 \\
95,127\end{array}$ & $\begin{array}{ll}10 & 00 \\
11 & 00 \\
12 & 00 \\
13 & 00 \\
14 & 00\end{array}$ & $\begin{array}{r}772,623 \\
849,416 \\
926,075 \\
1,002,588 \\
1,078,943\end{array}$ & $\begin{array}{l}48,565 \\
58,747 \\
69,893 \\
82,000 \\
95,067\end{array}$ & $\begin{array}{ll}10 & 00 \\
11 & 00 \\
12 & 00 \\
13 & 00 \\
14 & 00\end{array}$ & $\begin{array}{r}758,520 \\
833,895 \\
909,135 \\
984,227 \\
1,059,158\end{array}$ & $\begin{array}{l}48,477 \\
58,640 \\
69,765 \\
81,849 \\
94,890\end{array}$ \\
\hline $\begin{array}{ll}15 & 00 \\
16 & 00 \\
17 & 00 \\
18 & 00 \\
19 & 00\end{array}$ & $\begin{array}{l}1,175,994 \\
1,253,404 \\
1,330,624 \\
1,407,640 \\
1,484,443\end{array}$ & $\begin{array}{l}109,162 \\
124,153 \\
\mathbf{1 4 0}, 099 \\
156,996 \\
\mathbf{1 7 4 , 8 4 2}\end{array}$ & $\begin{array}{ll}15 & 00 \\
15 & 00 \\
17 & 00 \\
18 & 00 \\
19 & 00\end{array}$ & $\begin{array}{l}1,155,128 \\
1,231,131 \\
1,306,940 \\
1,382,543 \\
1,457,928\end{array}$ & $\begin{array}{l}109,091 \\
124,071 \\
140,003 \\
156,887 \\
174,718\end{array}$ & $\begin{array}{ll}15 & 00 \\
16 & 00 \\
17 & 00 \\
18 & 00 \\
19 & 00\end{array}$ & $\begin{array}{l}1,133,917 \\
1,208,491 \\
1,282,868 \\
1,357,036 \\
1,430,984\end{array}$ & $\begin{array}{l}108,887 \\
123,837 \\
139,738 \\
156,587 \\
174,381\end{array}$ \\
\hline $\begin{array}{ll}20 & 00 \\
21 & 00 \\
22 & 00 \\
23 & 00 \\
24 & 00\end{array}$ & $\begin{array}{l}1,561,019 \\
1,637,358 \\
1,713,447 \\
1,789,276 \\
1,864,831\end{array}$ & $\begin{array}{l}193,635 \\
213,371 \\
234,048 \\
255,663 \\
278,211\end{array}$ & $\begin{array}{ll}20 & 00 \\
21 & 00 \\
22 & 00 \\
23 & 00 \\
24 & 00\end{array}$ & $\begin{array}{l}1,533,083 \\
1,607,997 \\
1,682,657 \\
1,757,052 \\
1,831,170\end{array}$ & $\begin{array}{l}193,494 \\
213,212 \\
233,869 \\
255,462 \\
277,987\end{array}$ & $\begin{array}{ll}20 & 00 \\
21 & 00 \\
22 & 00 \\
23 & 00 \\
24 & 00\end{array}$ & $\begin{array}{l}1,504,697 \\
1,578,166 \\
1,651,377 \\
1,724,320 \\
1,796,982\end{array}$ & $\begin{array}{l}193,118 \\
212,793 \\
233,405 \\
254,950 \\
277,425\end{array}$ \\
\hline $\begin{array}{ll}25 & 00 \\
26 & 00 \\
27 & 00 \\
28 & 00 \\
29 & 00 \\
30 & 00\end{array}$ & $\begin{array}{l}1,940,103 \\
2,015,079 \\
2,089,749 \\
2,164,100 \\
2,238,121 \\
2,311,802\end{array}$ & $\begin{array}{l}301,690 \\
326,097 \\
351,427 \\
377,676 \\
404,841 \\
432,918\end{array}$ & $\begin{array}{ll}25 & 00 \\
26 & 00 \\
27 & 00 \\
28 & 00 \\
29 & 00 \\
30 & 00\end{array}$ & $\begin{array}{l}1,904,999 \\
1,978,528 \\
2,051,745 \\
2,124,639 \\
2,197,197 \\
2,269,410\end{array}$ & $\begin{array}{l}301,441 \\
325,820 \\
351,120 \\
377,337 \\
404,468 \\
432,507\end{array}$ & $\begin{array}{ll}25 & 00 \\
26 & 00 \\
27 & 00 \\
28 & 00 \\
29 & 00 \\
30 & 00\end{array}$ & $\begin{array}{l}1,869,351 \\
1,941,415 \\
2,013,163 \\
2,084,583 \\
2,155,663 \\
2,226,392\end{array}$ & $\begin{array}{l}300,824 \\
325,146 \\
350,386 \\
376,539 \\
403,602 \\
431,569\end{array}$ \\
\hline
\end{tabular}


TABLE 4.-For projections of maps of large areas-Continued. COORDINATES OF CURVATURE.

\begin{tabular}{|c|c|c|c|c|c|c|c|c|}
\hline \multicolumn{9}{|c|}{ Natural scale.-Values of $X$ and $Y$ in meters. } \\
\hline \multicolumn{3}{|c|}{ Latitude $48^{\circ}$} & \multicolumn{3}{|c|}{ Latitude $49^{\circ}$. } & \multicolumn{3}{|c|}{ Latitude $50^{\circ}$. } \\
\hline $\begin{array}{l}\text { Longi- } \\
\text { tude. }\end{array}$ & $\mathbf{x}$ & $Y$ & $\begin{array}{l}\text { Longi- } \\
\text { tude. }\end{array}$ & $\mathbf{x}$ & $\mathbf{Y}$ & $\begin{array}{l}\text { Longi- } \\
\text { tude. }\end{array}$ & $\mathbf{X}$ & - $\mathbf{Y}$ \\
\hline $\begin{array}{ll}\circ & 1 \\
1 & 00 \\
2 & 00 \\
3 & 00 \\
4 & 00\end{array}$ & $\begin{array}{r}74,626 \\
-\quad 149,239 \\
223,827 \\
298,377\end{array}$ & $\begin{array}{r}484 \\
1,936 \\
4,355 \\
7,742\end{array}$ & $\begin{array}{lc}\circ & , \\
1 & 00 \\
2 & 00 \\
3 & 00 \\
4 & 00\end{array}$ & $\begin{array}{r}73,172 \\
146,331 \\
219,465 \\
292,561\end{array}$ & $\begin{array}{r}482 \\
1,928 \\
4,337 \\
7,709\end{array}$ & $\begin{array}{ll}\circ & , \\
1 & 00 \\
2 & 00 \\
3 & 00 \\
4 & 00\end{array}$ & $\begin{array}{r}71,696 \\
143,379 \\
215,037 \\
286,656\end{array}$ & $\begin{array}{r}479 \\
1,917 \\
4,313 \\
7,667\end{array}$ \\
\hline $\begin{array}{ll}5 & 00 \\
6 & 00 \\
7 & 00 \\
8 & 00 \\
9 & 00\end{array}$ & $\begin{array}{l}372,877 \\
447,314 \\
521,677 \\
595,951 \\
670,125\end{array}$ & $\begin{array}{l}12,095 \\
17,414 \\
23,698 \\
30,946 \\
39,157\end{array}$ & $\begin{array}{ll}5 & 00 \\
6 & 00 \\
7 & 00 \\
8 & 00 \\
9 & 00\end{array}$ & $\begin{array}{r}365,606 \\
438,588 \\
511,493 \\
584,310 \\
657,026\end{array}$ & $\begin{array}{l}12,044 \\
17,340 \\
23,598 \\
30,815 \\
38,991\end{array}$ & $\begin{array}{ll}5 & 00 \\
6 & 00 \\
7 & 00 \\
8 & 00 \\
9 & 00\end{array}$ & $\begin{array}{l}358,224 \\
429,727 \\
501,154 \\
572,492 \\
643,727\end{array}$ & $\begin{array}{l}11,978 \\
17,246 \\
23,469 \\
30,646 \\
38,777\end{array}$ \\
\hline $\begin{array}{ll}10 & 00 \\
11 & 00 \\
12 & 00 \\
13 & 00 \\
14 & 00\end{array}$ & $\begin{array}{r}744,186 \\
818,123 \\
891,921 \\
965,570 \\
1,039,056\end{array}$ & $\begin{array}{l}48,329 \\
58,461 \\
69,552 \\
81,598 \\
94,598\end{array}$ & $\begin{array}{ll}10 & 00 \\
11 & 00 \\
12 & 00 \\
13 & 00 \\
14 & 00\end{array}$ & $\begin{array}{r}729,627 \\
802,102 \\
874,438 \\
946,622 \\
1,018,642\end{array}$ & $\begin{array}{l}48,123 \\
58,212 \\
69,254 \\
81,248 \\
94,191\end{array}$ & $\begin{array}{ll}10 & 00 \\
11 & 00 \\
12 & 00 \\
13 & 00 \\
14 & 00\end{array}$ & $\begin{array}{l}714,847 \\
785,839 \\
856,691 \\
927,389 \\
997,922\end{array}$ & $\begin{array}{l}47,859 \\
57,891 \\
68,872 \\
80,798 \\
93,669\end{array}$ \\
\hline $\begin{array}{ll}15 & 00 \\
16 & 00 \\
17 & 00 \\
18 & 00 \\
19 & 00\end{array}$ & $\begin{array}{l}1,112,367 \\
1,185,491 \\
1,258,416 \\
1,331,129 \\
1,403,618\end{array}$ & $\begin{array}{l}108,551 \\
123,453 \\
139,302 \\
156,096 \\
173,832\end{array}$ & $\begin{array}{ll}15 & 00 \\
16 & 00 \\
17 & 00 \\
18 & 00 \\
19 & 00\end{array}$ & $\begin{array}{l}1,090,485 \\
1,162,138 \\
1,233,591 \\
1,304,829 \\
1,375,840\end{array}$ & $\begin{array}{l}108,082 \\
122,918 \\
138,697 \\
155,416 \\
173,071\end{array}$ & $\begin{array}{ll}15 & 00 \\
16 & 00 \\
17 & 00 \\
18 & 00 \\
19 & 00\end{array}$ & $\begin{array}{l}1,068,277 \\
1,138,440 \\
1,208,400 \\
1,278,144 \\
1,347,660\end{array}$ & $\begin{array}{l}107,482 \\
122,234 \\
137,923 \\
154,546 \\
172,099\end{array}$ \\
\hline $\begin{array}{ll}20 & 00 \\
21 & 00 \\
22 & 00 \\
23 & 00 \\
24 & 00\end{array}$ & $\begin{array}{l}1,475,871 \\
1,547,876 \\
1,619,620 \\
1,691,091 \\
1,762,279\end{array}$ & $\begin{array}{l}192,506 \\
212,116 \\
232,658 \\
254,128 \\
276,524\end{array}$ & $\begin{array}{ll}20 & 00 \\
21 & 00 \\
22 & 00 \\
23 & 00 \\
24 & 00\end{array}$ & $\begin{array}{r}1,446,613 \\
1,517,135 \\
1,587,394 \\
1,657,378 \\
1,727,073\end{array}$ & $\begin{array}{l}191,660 \\
211,160 \\
231,627 \\
252,998 \\
275,288\end{array}$ & $\begin{array}{ll}20 & 00 \\
21 & 00 \\
22 & 00 \\
23 & 00 \\
24 & 00\end{array}$ & $\begin{array}{l}1,416,934 \\
1,485,956 \\
1,554,711 \\
1,623,189 \\
1,691,377\end{array}$ & $\begin{array}{l}190,581 \\
209,987 \\
230,314 \\
251,559 \\
273,717\end{array}$ \\
\hline $\begin{array}{ll}25 & 00 \\
26 & 00 \\
27 & 00 \\
28 & 00 \\
29 & 00 \\
30 & 00\end{array}$ & $\begin{array}{l}1,833,170 \\
1,903,752 \\
1,974,015 \\
2,043,945 \\
2,113,531 \\
2,182,762\end{array}$ & $\begin{array}{l}299,842 \\
324,077 \\
349,225 \\
375,283 \\
402,245 \\
430,107\end{array}$ & $\begin{array}{ll}25 & 00 \\
26 & 00 \\
27 & 00 \\
28 & 00 \\
29 & 00 \\
30 & 00\end{array}$ & $\begin{array}{l}1,796,470 \\
1,865,554 \\
1,934,315 \\
2,002,740 \\
2,070,817 \\
2,138,536\end{array}$ & $\begin{array}{l}298,495 \\
322,614 \\
347,640 \\
373,570 \\
400,399 \\
428,123\end{array}$ & $\begin{array}{ll}25 & 00 \\
26 & 00 \\
27 & 00 \\
28 & 00 \\
29 & 00 \\
30 & 00\end{array}$ & $\begin{array}{l}1,759,262 \\
1,826,833 \\
1,894,077 \\
1,960,983 \\
2,028,538 \\
2,093,731\end{array}$ & $\begin{array}{l}296,785 \\
320,758 \\
345,633 \\
371,404 \\
398,063 \\
425,619\end{array}$ \\
\hline
\end{tabular}


ТАВце 5.-Coordinates for projection of maps (scale т5รобо).

[From Smithsonian Geographical Tables.]

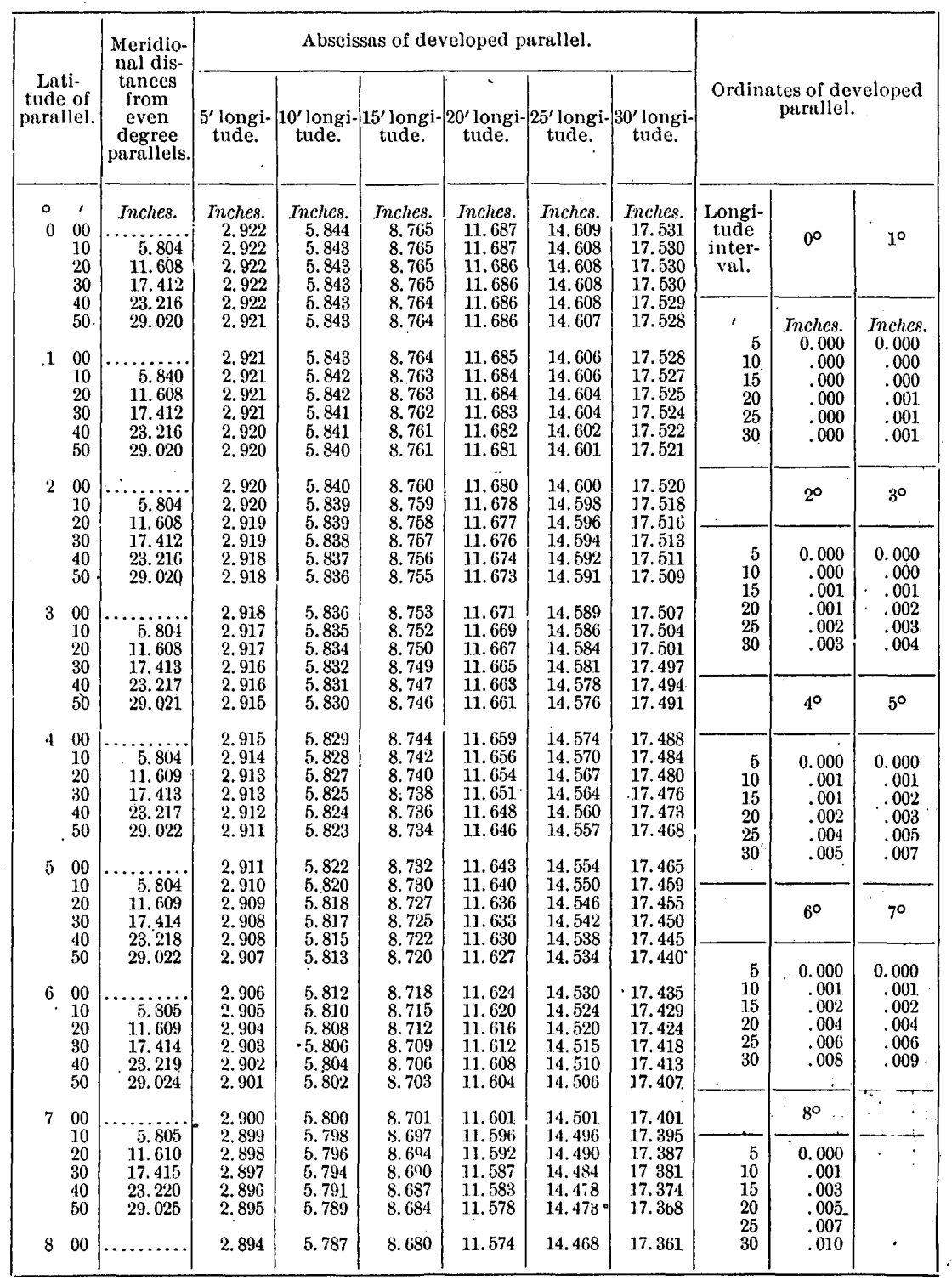




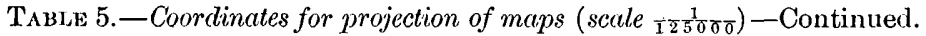

[From Smithsonian Geographical Tubles.]

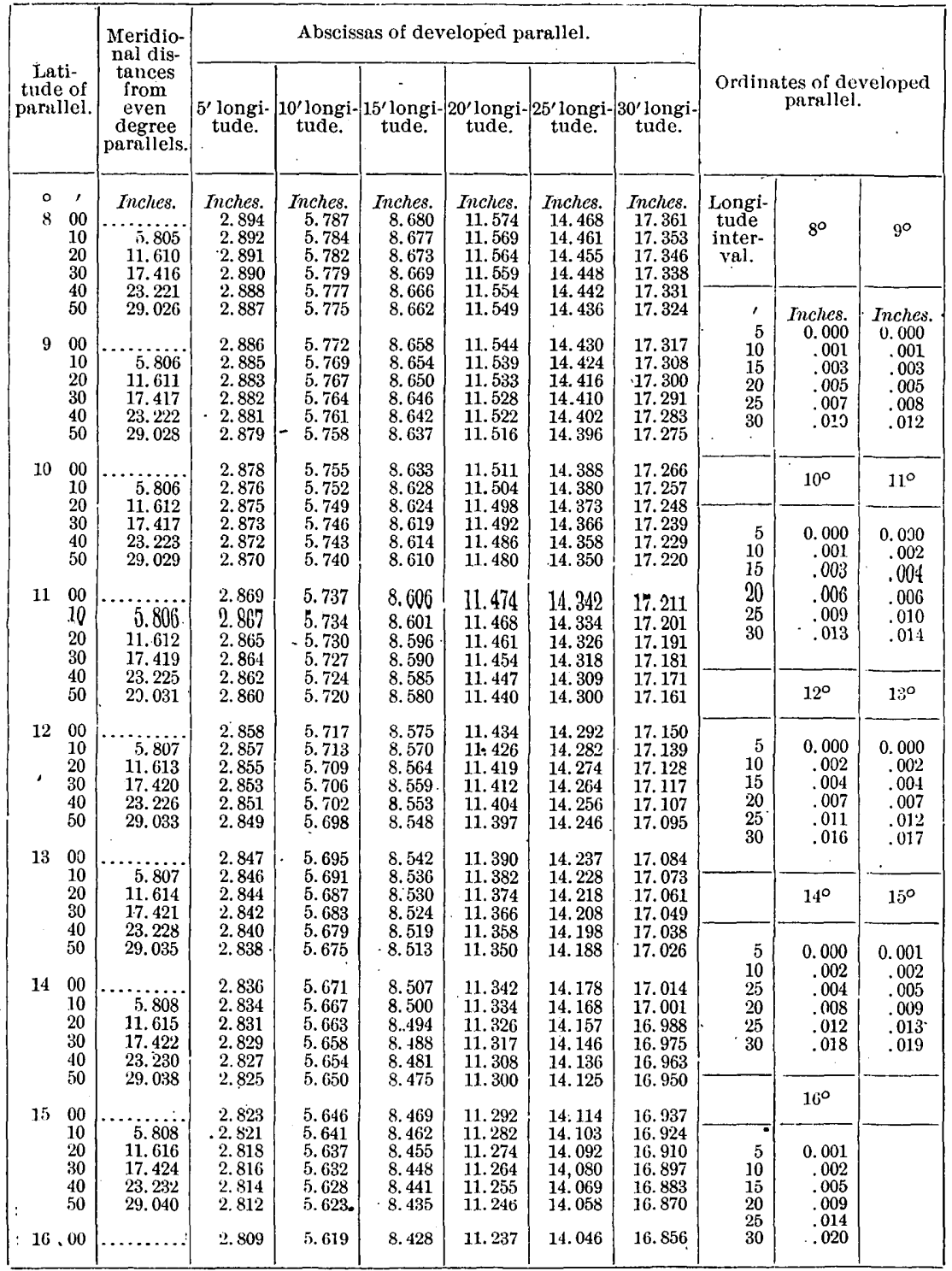




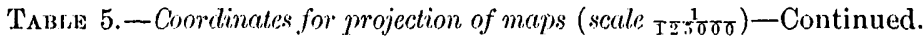

[From Smithsonian Geographicul Tables.]

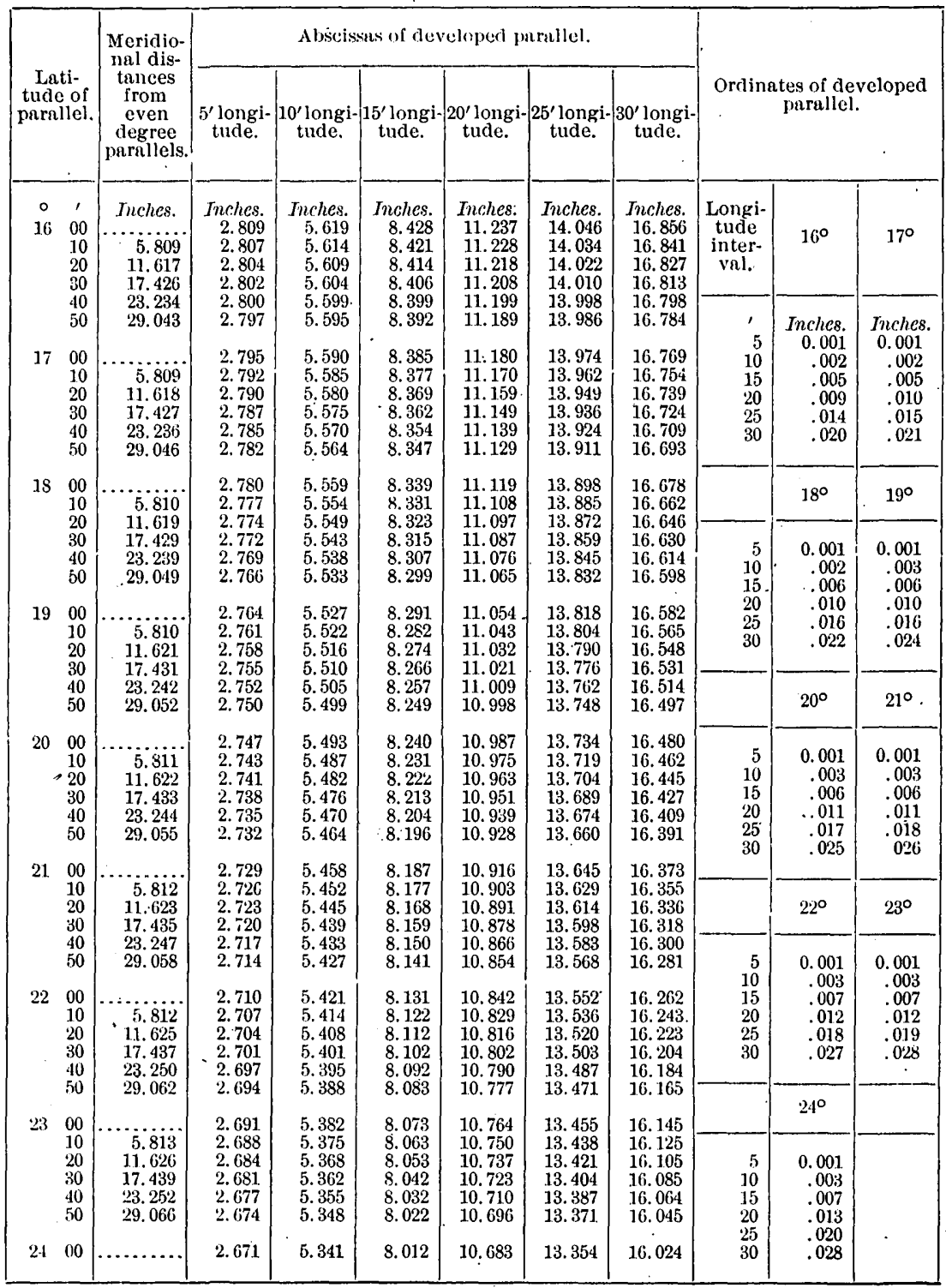




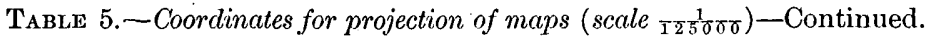

[From Smithsonian Geographical Tables.]

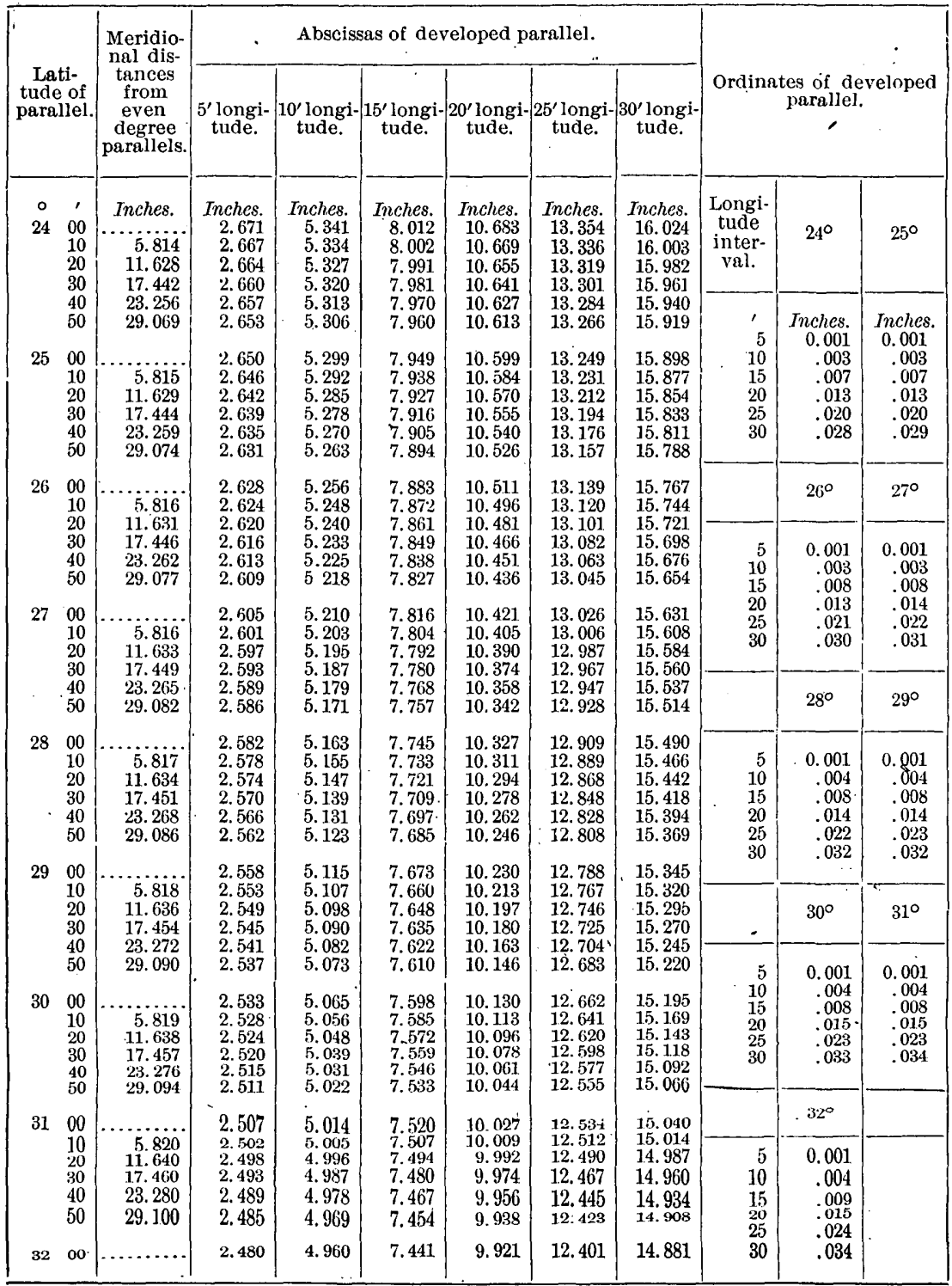


TABLE 5.-Coordinates for projection of maps (scale $\left.\frac{1}{125000}\right)$-Continued.

[From Smithsonian Geographical Tables.]

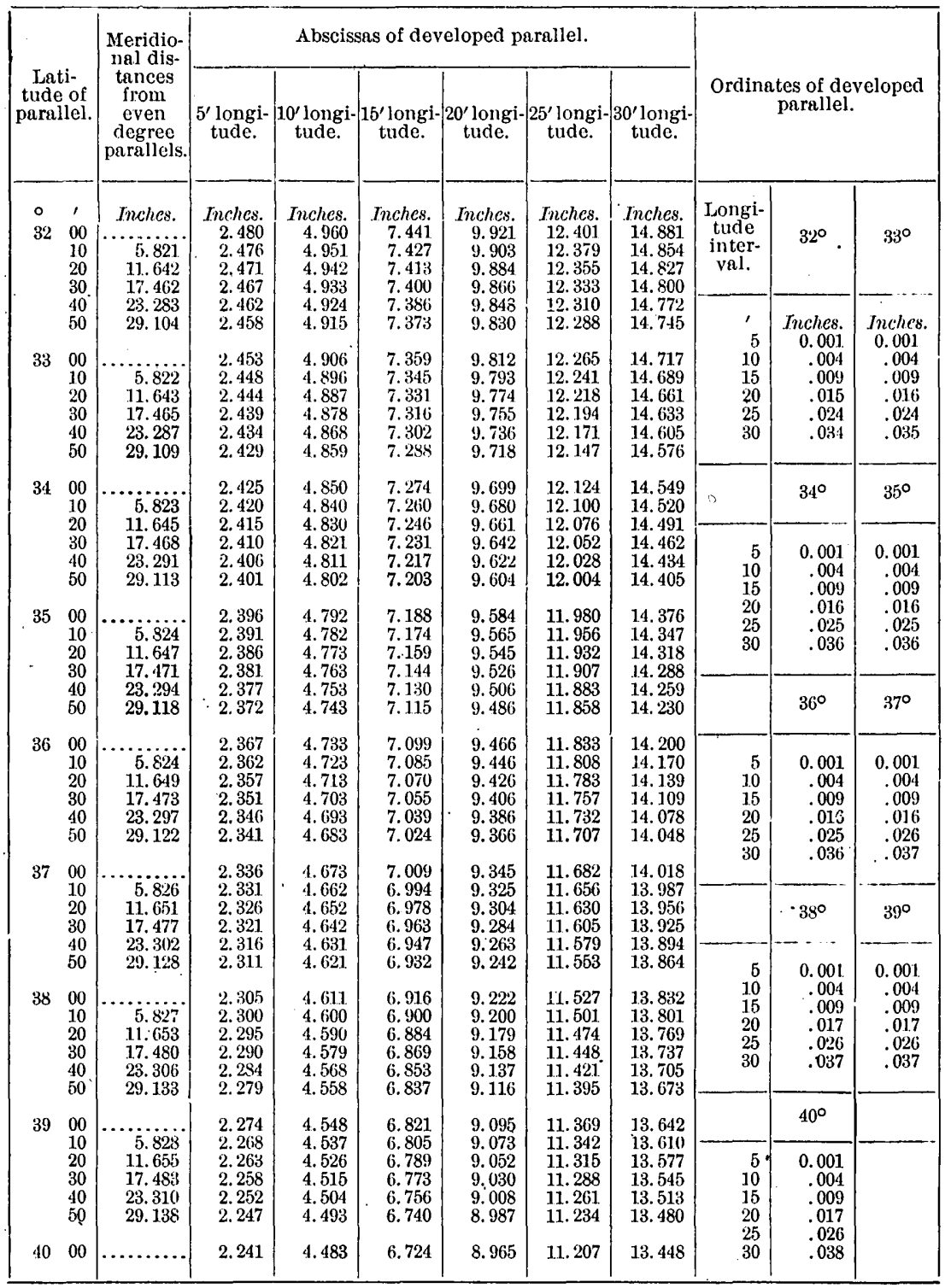


TABLE 5.-Coordinates for projećtion of maps (scale $\left.\frac{1}{15500}\right)$-Continued.

[From Smithsonian Geographical Tables.]

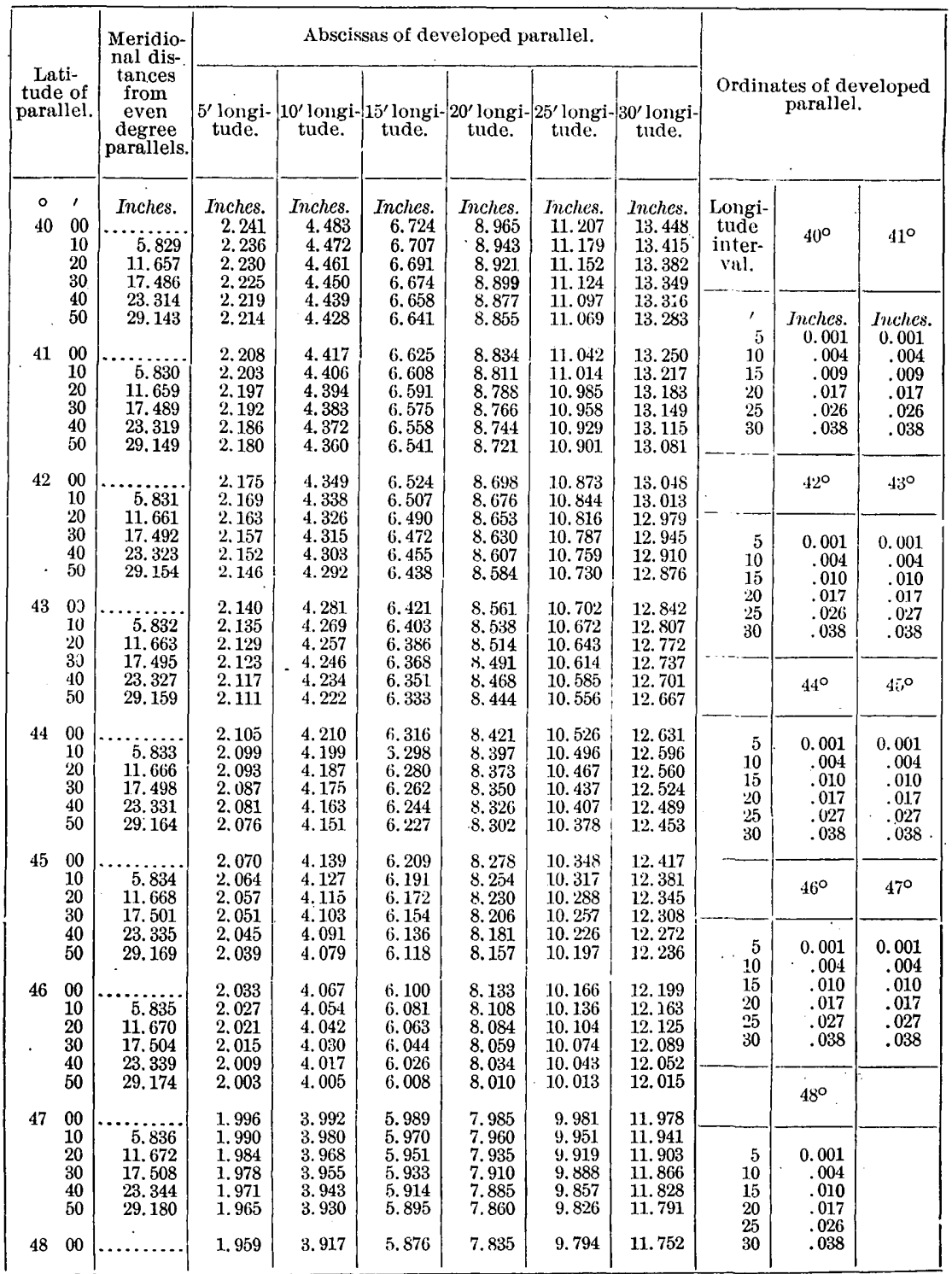




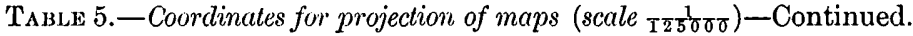

[From Smithsonian Geographical Tables.]

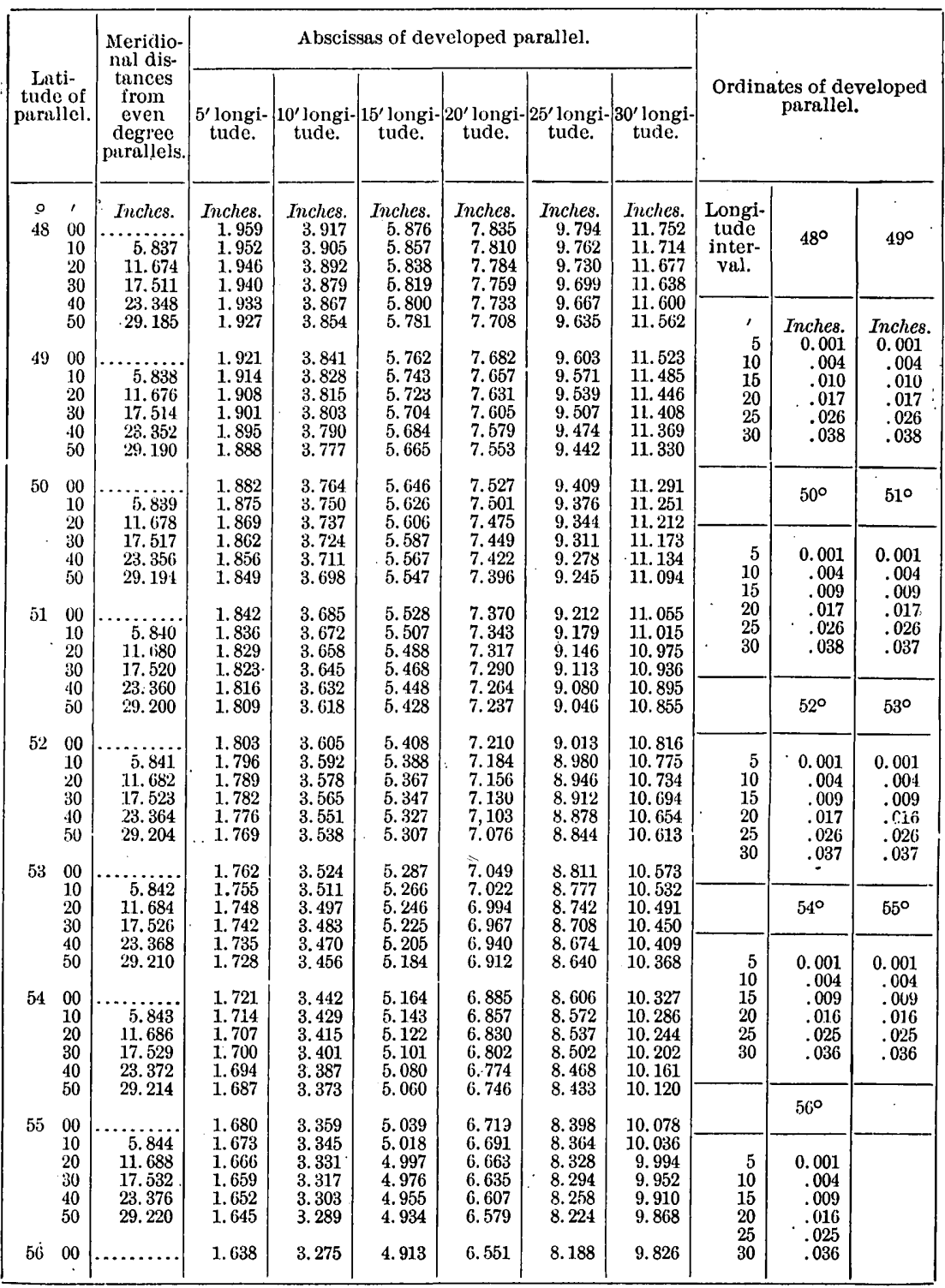


TABLE 5.-Coordinates for projection of maps (scale $\frac{15500}{1250}$ ).-Continued.

[From Smithsonian Geographical Tables.]

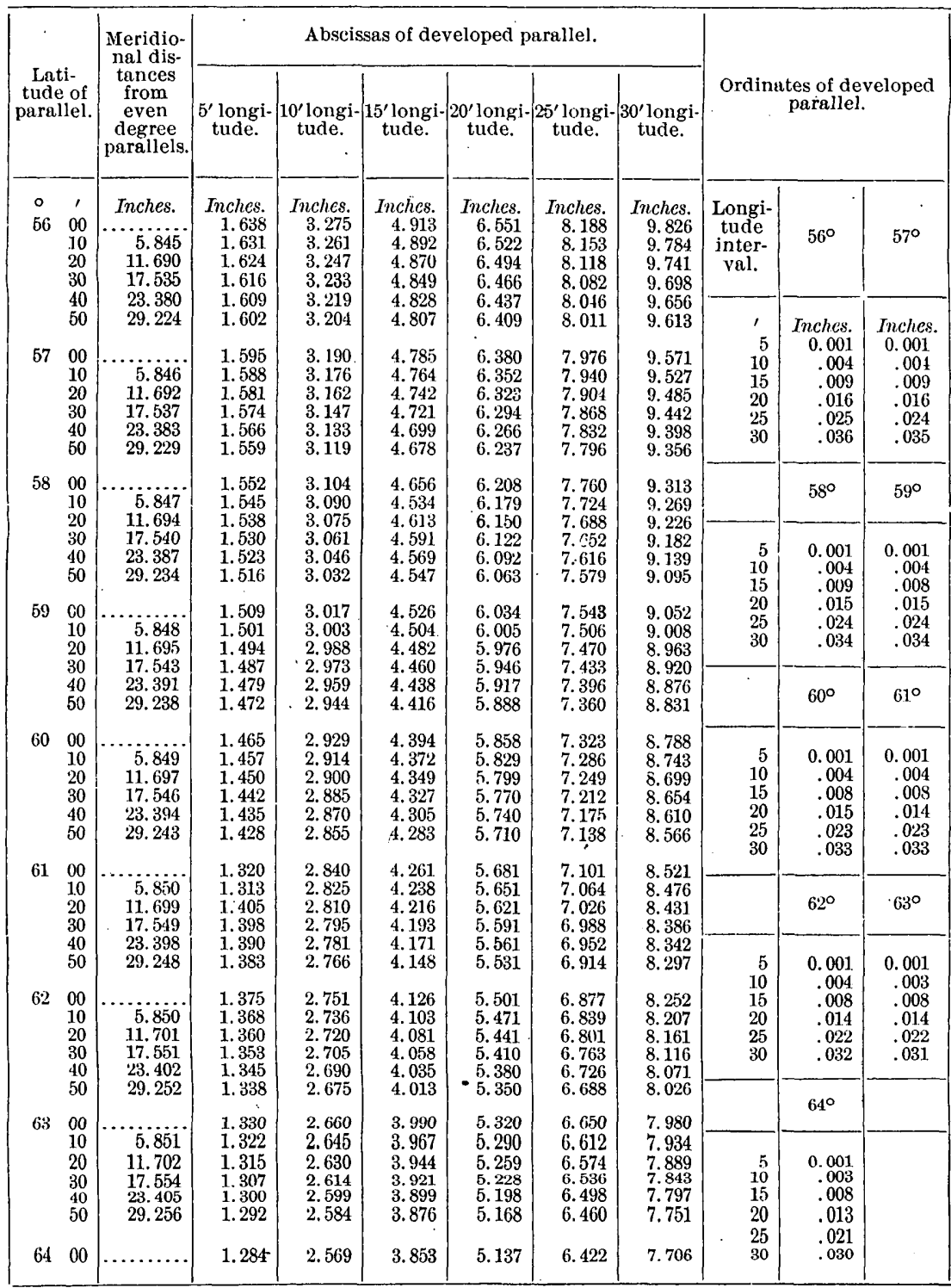




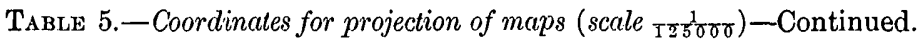

[Erom Smithsonian Geographical Tables.]

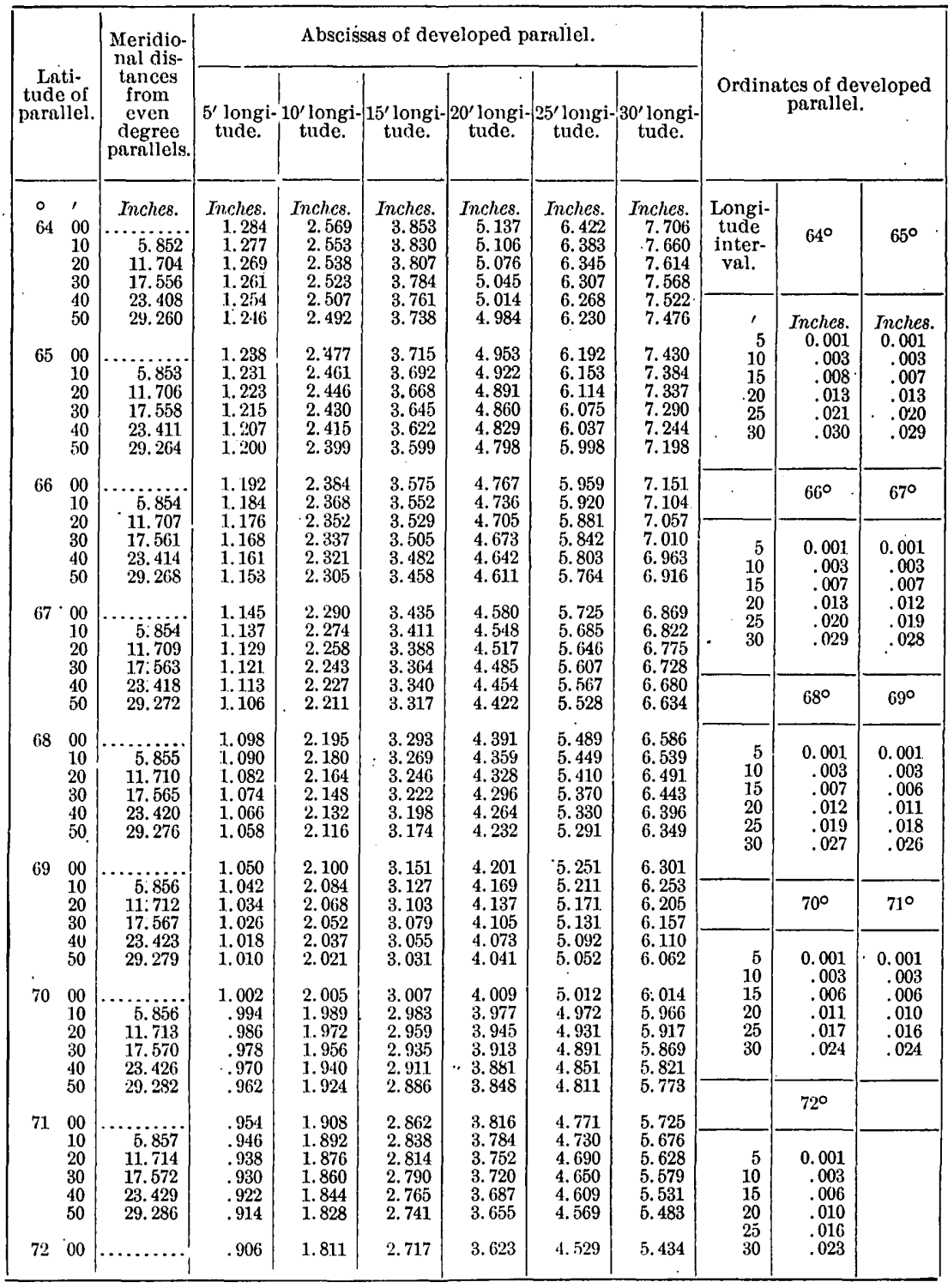

Bull. $214-03-4$ 
TaBLE 5.-Coordinates for projection of maps (scale ${ }_{\left.\text {I } \frac{1}{5} \frac{1}{5 \sigma 0}\right)}$-Continued.

[From Smithsonian Geographical Tables.]

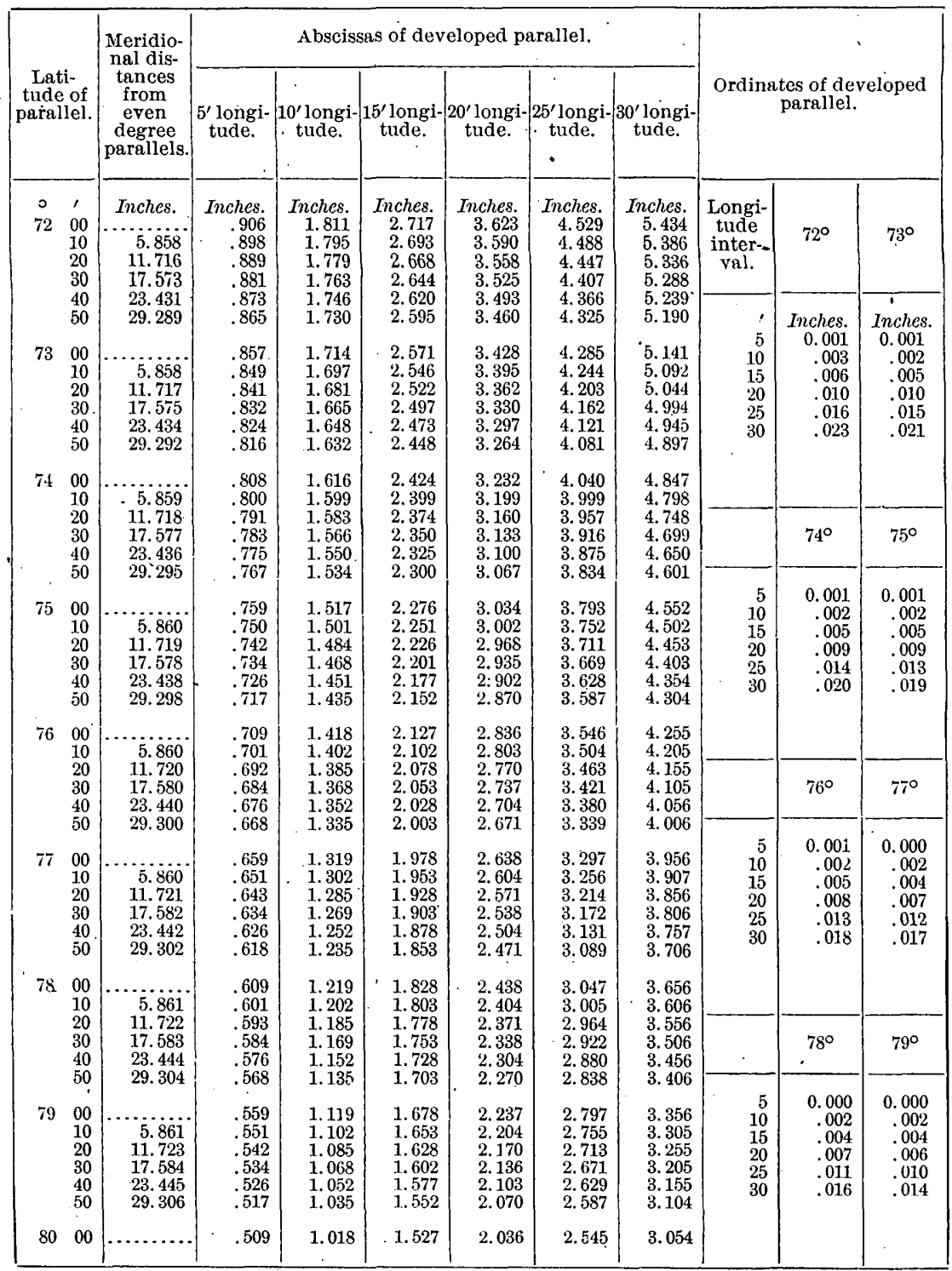


TABLE 6.-Coordinates for projection of maps (scale $\frac{1}{63} \frac{1}{36}$ ).

[From Smithsonian Geographical Tables.]

\begin{tabular}{|c|c|c|c|c|c|c|c|c|c|c|}
\hline \multirow{2}{*}{$\begin{array}{c} \\
\text { Lati- } \\
\text { tude o } \\
\text { paralle }\end{array}$} & \multirow{2}{*}{\begin{tabular}{|c} 
Meridio- \\
nal dis- \\
tances \\
from \\
even \\
degree \\
parallels
\end{tabular}} & \multicolumn{6}{|c|}{ Abscissas of developed parallel. } & \multirow{2}{*}{\multicolumn{3}{|c|}{$\begin{array}{l}\text { Ordinates of developed } \\
\text { parallel. }\end{array}$}} \\
\hline & & $5^{\prime}$ longi- & 10 & Uing & 20 & git & -30'longi- & & & \\
\hline \multirow[t]{4}{*}{$\begin{array}{lll}\circ & 1 \\
0 & 0 & \end{array}$} & Inches. & $\begin{array}{l}\text { Inches. } \\
5.764\end{array}$ & \begin{tabular}{|l} 
Inches. \\
11.529
\end{tabular} & $\begin{array}{l}\text { Inches. } \\
17.293\end{array}$ & $\begin{array}{l}\text { Inches. } \\
23.058\end{array}$ & $\begin{array}{l}\text { Inches. } \\
28.822\end{array}$ & $\begin{array}{l}\text { Inches. } \\
34.586\end{array}$ & \multirow{2}{*}{$\begin{array}{l}\text { Longi- } \\
\text { tufe } \\
\text { inter- } \\
\text { val. }\end{array}$} & \multirow{2}{*}{$0^{\circ}$} & \multirow{2}{*}{$1^{\mathrm{o}^{\prime}}$} \\
\hline & 11. 451 & 5.764 & 11.528 & $\begin{array}{l}17.293 \\
17.292\end{array}$ & $\begin{array}{l}23.057 \\
23.056\end{array}$ & $\begin{array}{l}28.821 \\
28.821\end{array}$ & $\begin{array}{l}34.585 \\
34.585\end{array}$ & & & \\
\hline & 34. 352 & 5. 764 & $\begin{array}{l}11.528 \\
11.528\end{array}$ & $\begin{array}{l}17.292 \\
17.292\end{array}$ & $\begin{array}{l}25.000 \\
23.056\end{array}$ & $\begin{array}{l}28.821 \\
28.820\end{array}$ & $\begin{array}{l}34.580 \\
34.583 \\
0.502\end{array}$ & & \multirow{7}{*}{$\begin{array}{r}\text { Inch. } \\
0.000 \\
.000 \\
.000 \\
.000 \\
.000 \\
.000\end{array}$} & \multirow{7}{*}{$\begin{array}{r}\text { Inch. } \\
0.000 \\
.000 \\
.001 \\
.001 \\
.002 \\
.003\end{array}$} \\
\hline & $\begin{array}{l}45.803 \\
57.254\end{array}$ & $\begin{array}{l}5.764 \\
5.764\end{array}$ & $\begin{array}{l}11 . .528 \\
11.527\end{array}$ & $\begin{array}{l}\text { 17. } 291 \\
\text { 17. } 291\end{array}$ & $\begin{array}{r}23.055 \\
23.054\end{array}$ & $\begin{array}{r}28.819 \\
28.818\end{array}$ & $\begin{array}{r}34.583 \\
34.582\end{array}$ & \multirow{6}{*}{$\begin{array}{r}5 \\
10 \\
15 \\
20 \\
25 \\
30\end{array}$} & & \\
\hline \multirow[t]{5}{*}{1.} & 68.704 & 5.764 & 11.527 & 17.291 & 23.054 & 28.818 & 34.581 & & & \\
\hline & 11.451 & 5.763 & 11. 526 & 17.289 & 23.052 & 28.816 & 34.579 & & & \\
\hline & & $76 \%$ & - & 17.288 & 23.050 & 28.813 & & & & \\
\hline & & 67 & 11.524 & 17. 287 & $\begin{array}{r}23.049 \\
23047-30\end{array}$ & 28.811. & 34. & & & \\
\hline & $\begin{array}{l}45.803 \\
57.254\end{array}-10$ & 5.762 & $\begin{array}{l}11.524 \\
11.523\end{array}$ & 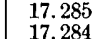 & 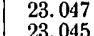 & $\begin{array}{l}28.809 \\
2807\end{array}-10$ & $\begin{array}{l}34.571 \\
34.568\end{array}$ & & & \\
\hline \multirow{5}{*}{200} & 68.704 & 5.761 & 11. 522 & 17.283 & 23. 044 & 28.805 & 34.565 & \multirow{9}{*}{$\begin{array}{r}5 \\
10 \\
15 \\
20 \\
25 \\
30\end{array}$} & \multirow{2}{*}{$2^{\circ}$} & \multirow{2}{*}{$3^{\circ}$} \\
\hline & 11.451 & 5.760 & 1.1. 520 & 17.281 & 23.041 & 28.801 & 34.561. & & & \\
\hline & & 5. 759 & & & $\begin{array}{l}23.038 \\
23.035\end{array}-\mathrm{l} \mathrm{l}$ & & & & \multirow{7}{*}{$\begin{array}{l}0.000 \\
.001 \\
.001 \\
.002 \\
.004 \\
.005\end{array}$} & \multirow{7}{*}{$\begin{array}{r}0.000 \\
.001 \\
.002 \\
.003 \\
.005 \\
.008\end{array}$} \\
\hline & & & 11.517 & 17. 276 & 23.035 & 28 & & & & \\
\hline & $\begin{array}{l}45.204 \\
57.254\end{array}$ & 5.757 & 11. 514 & 17. 272 & 23.029 & 28.786 & 34.543 & & & \\
\hline \multirow[t]{5}{*}{ 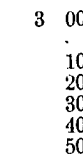 } & 68.705 & 5.756 & 11.513 & 17.270 & 23.026 & 28.783 & 34.639 & & & \\
\hline & 11.451 & 5.756 & 11.511 & 17. 267 & 23.022 & 28.778 & 34. 533 & & & \\
\hline & & 5.754 & 11. 509 & 17. & & & & & & \\
\hline & $\begin{array}{l}34.833 \\
45.804\end{array}-130$ & $\begin{array}{l}5.753 \\
5.752\end{array}$ & $\begin{array}{l}11.507 \\
11.505\end{array}$ & $\begin{array}{l}17.260 \\
17.257\end{array}$ & $\begin{array}{l}23.014 \\
23.010\end{array}-14$ & 28. & & & & \\
\hline & 57.2 & 5.751 & 11.503 & 17.254 & 23.006 & 28.757 & 34. 508 & & \multirow{2}{*}{4} & \\
\hline \multirow[t]{5}{*}{40} & 68.706 & 5. 750 & 11.501 & 17.251 & 23.002 & 28.752 & 34. 502 & & & \\
\hline & 451 & 5.749 & 11 & 17. 247 & 22. & 28. & 34 & \multirow{6}{*}{$\begin{array}{r}5 \\
10 \\
15 \\
20 \\
25 \\
30\end{array}$} & & 0.000 \\
\hline & & & & & & & & & .001 & .001 \\
\hline & & & & & & & & & .003 & .003 \\
\hline & $\begin{array}{l}\begin{array}{l}45.800 \\
57.256\end{array} \\
57\end{array}$ & $\begin{array}{l}5.745 \\
5.744\end{array}$ & $\begin{array}{l}\begin{array}{l}11.490 \\
11.488\end{array} \\
\text { lo }\end{array}$ & $\begin{array}{l}77.236 \\
17.232\end{array}$ & $\begin{array}{l}22.981 \\
22.976\end{array}$ & $\begin{array}{r}28.726 \\
28.720\end{array}$ & $\begin{array}{l}34.471 \\
34.463\end{array}$ & & $\begin{array}{l}.005 \\
.007\end{array}$ & .006 \\
\hline 50 & 68.708 & 5.743 & 11.485 & 17.228 & 22.970 & 28.713 & 34.456 & & & \\
\hline 1 & 11.452 & 5. 741 & 11.482 & 17. 223 & 22. 964 & 28.705 & 34.446 & & & \\
\hline 2 & & $\begin{array}{l}5.7 \\
5.7\end{array}$ & $\begin{array}{l}11.479 \\
11.476\end{array}$ & 17.213 & $\begin{array}{l}22.908 \\
22.951\end{array}$ & $\begin{array}{l}28.097 \\
28.689\end{array}$ & $\begin{array}{l}34.4500 \\
34.427\end{array}$ & & & \\
\hline 4 & & & & & & & & & $6^{\circ}$. & $7^{\circ}$ \\
\hline & & & & & & & & & & \\
\hline $\begin{array}{ll}6 & 0\end{array}$ & 68.710 & 5.733 & 11. 466 & 17.199 & 22. 932 & 28.665 & 34.398 & 5 & & \\
\hline 1 & & & 11. & 17. & 22. & 28. & & 15 & .004 & .005 \\
\hline & & & & & & & & 0 & & 008 \\
\hline & & & 11.4 & 17. & 22. 910 & & & & & 3 \\
\hline & 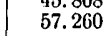 & 5. 724 & $\begin{array}{l}11.447 \\
11.491\end{array}$ & 17.171 & $\begin{array}{l}22.892 \\
22.894\end{array}$ & $\begin{array}{l}28.618 \\
28.618\end{array}$ & $\begin{array}{l}34.353 \\
34.342 \\
34.35\end{array}$ & 30 & & .018 \\
\hline 70 & 68.712 & 5.722 & i1. 443 & 17.165 & 22.887 & 28.609 & 34.330 & & & \\
\hline
\end{tabular}


TABLE 6.-Coordinates for projection of maps (scale $\frac{{ }_{63} \frac{1}{36}}{6}$ )-Continued.

[From Smithsonian Geographical Tables.]

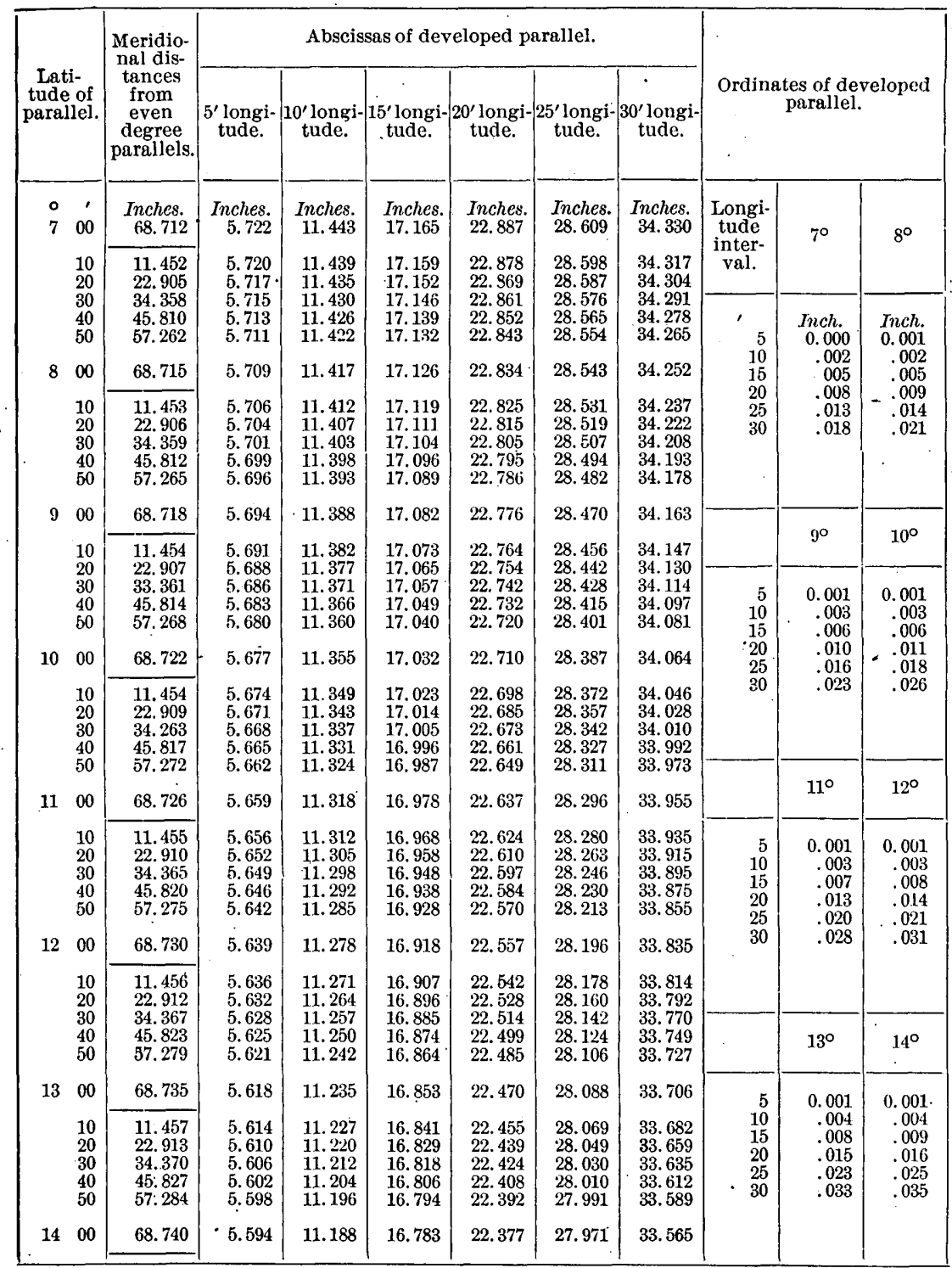




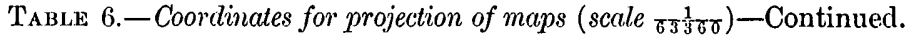

[From Smithsonian Geographical Tubles.]

\begin{tabular}{|c|c|c|c|c|c|c|c|c|c|c|c|}
\hline \multirow{2}{*}{\multicolumn{2}{|c|}{$\begin{array}{l}\text { Iati- } \\
\text { tude of } \\
\text { parallel. }\end{array}$}} & \multirow{3}{*}{$\begin{array}{c}\text { Meridio- } \\
\text { nal dis- } \\
\text { tances } \\
\text { from } \\
\text { even } \\
\text { degree } \\
\text { parallels. } \\
\text { Inches. } \\
68.740\end{array}$} & \multicolumn{6}{|c|}{ Abscissas of developed parallel. } & \multirow{2}{*}{\multicolumn{3}{|c|}{$\begin{array}{c}\text { Ordinates of developed } \\
\text { parallel. }\end{array}$}} \\
\hline & & & \multirow{2}{*}{$\begin{array}{c}5 \text { 'longi- } \\
\text { tude. }\end{array}$} & $\begin{array}{c}10^{\prime} \text { longi- } \\
\text { tude. }\end{array}$ & \multirow{2}{*}{$\frac{\begin{array}{c}15 \text { longi- } \\
\text { tude. }\end{array}}{\text { Inches. }}$} & $\begin{array}{c}20^{\prime} \text { longi- } \\
\text { tude. }\end{array}$ & $-\begin{array}{c}25 \text { longi- } \\
\text { tude. } \\
\text { Inches. }\end{array}$ & \multirow{2}{*}{$\begin{array}{c}30 \text { longi- } \\
\text { tude. }\end{array}$} & & & \\
\hline $\begin{array}{l}\circ \\
14\end{array}$ & & & & $\begin{array}{c}\text { Inches. } \\
11.188\end{array}$ & & $\begin{array}{r}\text { Inches. } \\
22.377\end{array}$ & $\begin{array}{c}\text { Inches. } \\
27.971\end{array}$ & & \multirow{2}{*}{$\begin{array}{l}\text { Longi- } \\
\text { tude } \\
\text { inter- } \\
\text { val. }\end{array}$} & \multirow{2}{*}{$14^{\circ}$} & \multirow[t]{2}{*}{$15^{\circ}$} \\
\hline & $\begin{array}{l}10 \\
20\end{array}$ & $\begin{array}{l}11.458 \\
22.915\end{array}$ & $\begin{array}{l}5.590 \\
5.586\end{array}$ & $\begin{array}{l}11.180 \\
11.172\end{array}$ & $\begin{array}{l}16.770 \\
16.758\end{array}$ & $\begin{array}{l}22.360 \\
22.344\end{array}$ & $\begin{array}{l}27.950 \\
27.930\end{array}$ & $\begin{array}{l}33.540 \\
33.515\end{array}$ & & & \\
\hline & 30 & 34.373 & 5.582 & 11.163 & 16.745 & 22.327 & 27.909 & 33.490 & & & \\
\hline & $\begin{array}{l}40 \\
50\end{array}$ & $\begin{array}{l}45.830 \\
57.288\end{array}$ & $\begin{array}{l}5.578 \\
5.573\end{array}$ & $\begin{array}{l}11.155 \\
11.147\end{array}$ & $\begin{array}{l}16.733 \\
16.720\end{array}$ & $\begin{array}{l}22.310 \\
22.294\end{array}$ & $\begin{array}{l}27.888 \\
27.867\end{array}$ & $\begin{array}{l}33.465 \\
33.440\end{array}$ & & Inches. & Tnches. \\
\hline \multirow[t]{2}{*}{15} & 00 & 68.746 & 5.569 & 11.138 & 16.708 & 22.277 & 27.846 & 33.415 & $\begin{array}{l}10 \\
15\end{array}$ & $\begin{array}{l}.004 \\
.009\end{array}$ & $\begin{array}{l}.004 \\
.009\end{array}$ \\
\hline & $\begin{array}{l}10 \\
20 \\
30 \\
40 \\
50\end{array}$ & $\begin{array}{r}11.459 \\
22.917 \\
34.376 \\
45.834 \\
57.293\end{array}$ & $\begin{array}{l}5.565 \\
5.560 \\
5.556 \\
5.551 \\
5.547\end{array}$ & $\begin{array}{l}11.130 \\
11.121 \\
11.112 \\
11.103 \\
11.094\end{array}$ & $\begin{array}{l}16.694 \\
16.681 \\
16.667 \\
16.654 \\
16.641\end{array}$ & $\begin{array}{l}22.259 \\
22.241 \\
22.223 \\
22.206 \\
22.188\end{array}$ & $\begin{array}{l}27.824 \\
27.802 \\
27.779 \\
27.757 \\
27.735\end{array}$ & $\begin{array}{l}33.389 \\
33.362 \\
33.335 \\
33.308 \\
33.282\end{array}$ & $\begin{array}{l}25 \\
30\end{array}$ & $\begin{array}{l}.010 \\
.025 \\
.035\end{array}$ & $\begin{array}{l}.026 \\
.038\end{array}$ \\
\hline \multirow[t]{3}{*}{16} & 00 & 68.752 & 5.542 & 11.085 & 16.628 & 22.170 & $\grave{2} 7.713$ & 33.255 & & \multirow{2}{*}{$16^{\circ}$} & \multirow{2}{*}{$17^{\circ}$} \\
\hline & 10 & 11. 460 & 5.538 & 11.076 & 16.613 & 22.151 & 27.689 & 33.227 & & & \\
\hline & $\begin{array}{l}20 \\
30 \\
40 \\
50\end{array}$ & $\begin{array}{l}22.919 \\
34.379 \\
45.838 \\
57.298\end{array}$ & $\begin{array}{l}5.533 \\
5.528 \\
5.524 \\
5.519\end{array}$ & $\begin{array}{l}11.066 \\
11.057 \\
11.047 \\
11.038\end{array}$ & $\begin{array}{l}16.599 \\
16.585 \\
16.571 \\
16.556\end{array}$ & $\begin{array}{l}22.132 \\
22.113 \\
22.094 \\
22.075\end{array}$ & $\begin{array}{l}27.665 \\
27.642 \\
27.618 \\
27.594\end{array}$ & $\begin{array}{l}33.198 \\
33.170 \\
33.142 \\
33.113\end{array}$ & $\begin{array}{r}5 \\
10 \\
15\end{array}$ & $\begin{array}{r}0.001 \\
.004 \\
.010\end{array}$ & $\begin{array}{r}0.001 \\
.005 \\
.011\end{array}$ \\
\hline \multirow[t]{3}{*}{17} & 00 & 68.758 & 5.514 & 11.028 & 16.542 & 22.056 & 27.571 & 33.085 & \multirow{2}{*}{$\begin{array}{r}20 \\
25 \\
30\end{array}$} & \multirow{2}{*}{$\begin{array}{l}.018 \\
.028 \\
.040\end{array}$} & \multirow{2}{*}{$\begin{array}{l}.010 \\
.029 \\
.042\end{array}$} \\
\hline & $\begin{array}{l}10 \\
20 \\
30 \\
40\end{array}$ & $\begin{array}{l}11.461 \\
22.921 \\
34.382 \\
45.843\end{array}$ & $\begin{array}{l}5.509 \\
5.504 \\
5.499 \\
5.494\end{array}$ & $\begin{array}{l}11.018 \\
11.008 \\
10.998 \\
10.988\end{array}$ & $\begin{array}{l}16.527 \\
16.512 \\
16.497 \\
16.482\end{array}$ & $\begin{array}{l}22.036 \\
22.016 \\
21.996 \\
21.976\end{array}$ & $\begin{array}{l}27.546 \\
27.521 \\
27.495 \\
27.470\end{array}$ & $\begin{array}{l}33.055 \\
33.025 \\
32.994 \\
32.964\end{array}$ & & & \\
\hline & 50 & 57.304 & 5.489 & 10.978 & 16.467 & 21.956 & 27.445 & 32.934 & & $18^{\circ}$ & $19^{\circ}$ \\
\hline \multirow[t]{2}{*}{18} & 00 & 68.764 & 5.484 & 10.968 & 16.452 & 21.936 & 27.420 & 32.904 & & & \\
\hline & $\begin{array}{l}10 \\
20 \\
30 \\
40 \\
50\end{array}$ & $\begin{array}{l}11.462 \\
22.924 \\
34.386 \\
45.848 \\
57.310\end{array}$ & $\begin{array}{l}5.479 \\
5.473 \\
5.468 \\
5.463 \\
5.458\end{array}$ & $\begin{array}{l}10.957 \\
10.947 \\
10.936 \\
10.926 \\
10.915\end{array}$ & $\begin{array}{l}16.436 \\
16.420 \\
16.404 \\
16.389 \\
16.373\end{array}$ & $\begin{array}{l}21.915 \\
21.894 \\
21.872 \\
21.852 \\
21.830\end{array}$ & $\begin{array}{l}27.394 \\
27.367 \\
27.341 \\
27.315 \\
27.288\end{array}$ & $\begin{array}{l}32.872 \\
32.840 \\
32.809 \\
32.777 \\
32.746\end{array}$ & \multirow[t]{4}{*}{$\begin{array}{r}5 \\
10 \\
15 \\
20 \\
25 \\
30\end{array}$} & \multirow[t]{3}{*}{$\begin{array}{l}0.001 \\
.005 \\
.011 \\
.020 \\
.031 \\
.044\end{array}$} & \multirow[t]{3}{*}{$\begin{array}{r}0.001 \\
.005 \\
.012 \\
.021 \\
.032 \\
.046\end{array}$} \\
\hline \multirow[t]{3}{*}{19} & 00 & 68.771 & 5. 452 & 10.905 & 16.357 & 21.809 & 27.262 & 32.714 & & & \\
\hline & $\begin{array}{l}10 \\
20\end{array}$ & $\begin{array}{l}11.463 \\
22.926\end{array}$ & $\begin{array}{l}5.447 \\
5.441\end{array}$ & $\begin{array}{l}10.893 \\
10.882\end{array}$ & $\begin{array}{l}16.340 \\
16.324\end{array}$ & $\begin{array}{l}21.787 \\
21.765\end{array}$ & $\begin{array}{l}27.234 \\
27.206\end{array}$ & $\begin{array}{l}32.680 \\
32.647\end{array}$ & & & \\
\hline & $\begin{array}{l}30 \\
40 \\
50\end{array}$ & $\begin{array}{l}34.390 \\
45.853 \\
57.316\end{array}$ & $\begin{array}{l}5.436 \\
5.430 \\
5.424\end{array}$ & $\begin{array}{l}10.871 \\
10.860 \\
10.849\end{array}$ & $\begin{array}{l}16.307 \\
16.290 \\
16.274\end{array}$ & $\begin{array}{l}21.742 \\
21.720 \\
21.698\end{array}$ & $\begin{array}{l}27.178 \\
27.150 \\
27.123\end{array}$ & $\begin{array}{l}32.614 \\
32.580 \\
32.547\end{array}$ & & $20^{\circ}$ & $21^{\circ}$ \\
\hline \multirow[t]{2}{*}{20} & 00 & 68.779 & 5.419 & 10.838 & 16.257 & $21.676^{\circ}$ & 27.095 & 32.513 & \multirow{2}{*}{$\begin{array}{r}5 \\
10 \\
15 \\
20 \\
25 \\
30\end{array}$} & \multirow{3}{*}{$\begin{array}{r}0.001 \\
.005 \\
.012 \\
.022 \\
.034 \\
.049\end{array}$} & \multirow{2}{*}{$\begin{array}{r}0.001 \\
.006 \\
.013 \\
.022 \\
.035 \\
.051\end{array}$} \\
\hline & $\begin{array}{l}10 \\
20 \\
30 \\
40 \\
50\end{array}$ & $\begin{array}{l}11.464 \\
22.929 \\
34.394 \\
45.858 \\
57.322\end{array}$ & $\begin{array}{l}5.413 \\
5.407 \\
5.401 \\
5.396 \\
5.390\end{array}$ & $\begin{array}{l}10.826 \\
10.814 \\
10.803 \\
10.791 \\
10.779\end{array}$ & $\begin{array}{l}16.239 \\
16.222 \\
16.204 \\
16.187 \\
16.169\end{array}$ & $\begin{array}{l}21.652 \\
21.629 \\
21.605 \\
21.582 \\
21.558\end{array}$ & $\begin{array}{l}27.065 \\
27.036 \\
27.007 \\
26.978 \\
26.948\end{array}$ & $\begin{array}{l}32.478 \\
32.443 \\
32.408 \\
32.373 \\
32.338\end{array}$ & & & \\
\hline 21 & 00 & 68.787 & 5.384 & 10.768 & 16. 151 & 21.535 & 26.919 & 32.303 & & & $!$ \\
\hline
\end{tabular}


TABLE 6.-Coordinates for projection of maps $\left(\right.$ scale $\left.\frac{1}{6 \frac{1}{3} 6 \sigma}\right)$-Continued.

[From Smithsonian Geographical Tables.]

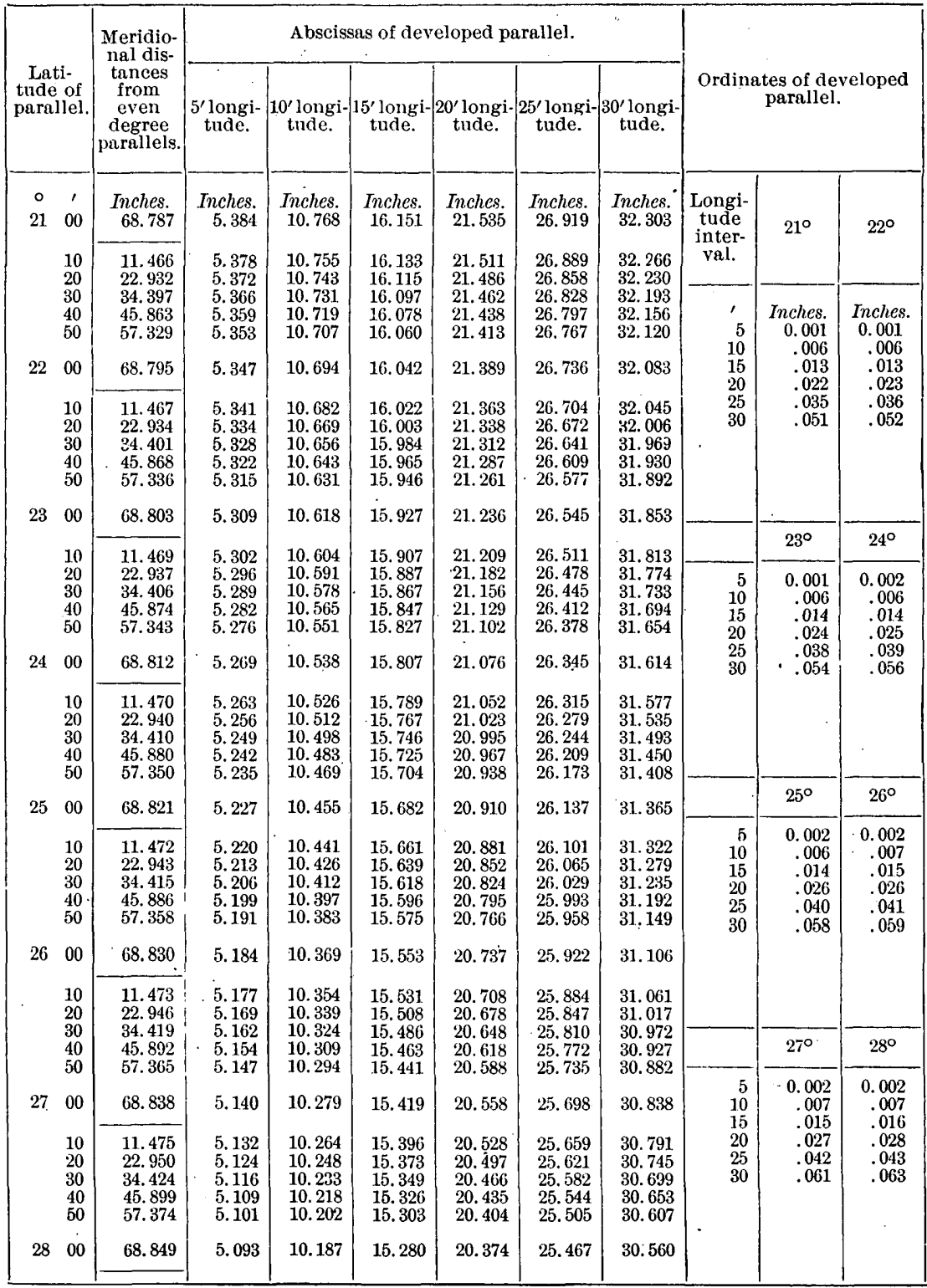




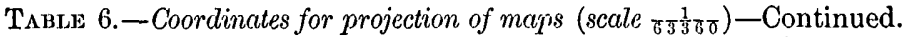

[From Smithsonian Geographical Tables.]

\begin{tabular}{|c|c|c|c|c|c|c|c|c|c|c|c|}
\hline \multirow{2}{*}{\multicolumn{2}{|c|}{$\begin{array}{l}\text { Lati- } \\
\text { tude of } \\
\text { parallel. }\end{array}$}} & \multirow{2}{*}{$\begin{array}{l}\text { Meridio- } \\
\text { nal dis- } \\
\text { tances } \\
\text { from } \\
\text { even } \\
\text { degree } \\
\text { parnllels. }\end{array}$} & \multicolumn{6}{|c|}{ A.bscissas of developed parallel. } & \multirow{2}{*}{\multicolumn{3}{|c|}{$\begin{array}{l}\text { Ordinates of developed } \\
\text { parallel. }\end{array}$}} \\
\hline & & & $\begin{array}{c}5^{\prime} \text { longi- } \\
\text { tude. }\end{array}$ & $\begin{array}{c}10^{\prime} \text { longi- } \\
\text { tude. }\end{array}$ & $\begin{array}{c}15^{\prime} \text { longi- } \\
\text { tude. }\end{array}$ & $\begin{array}{l}20 \text { 'longi- } \\
\text { tude. }\end{array}$ & \multirow{2}{*}{$\mid \begin{array}{c}25 \text { 'longi- } \\
\text { tude. }\end{array}$} & \multirow{2}{*}{$\frac{\begin{array}{c}30 \\
\text { longi- }\end{array}}{\begin{array}{r}\text { Inches. } \\
30.560\end{array}}$} & & & \\
\hline \multirow[t]{4}{*}{$\begin{array}{c}\circ \\
28\end{array}$} & $\stackrel{1}{00}$ & $\begin{array}{l}\text { Inches. } \\
\quad 68.849\end{array}$ & $\begin{array}{l}\text { Inches. } \\
5.093\end{array}$ & $\begin{array}{l}\text { Inches. } \\
.10 .187\end{array}$ & $\begin{array}{l}\text { Inches. } \\
15.280\end{array}$ & $\begin{array}{l}\text { Inches. } \\
20.374\end{array}$ & & & \multirow{2}{*}{$\begin{array}{c}\text { Longi- } \\
\text { tude } \\
\text { inter- } \\
\text { val. }\end{array}$} & \multirow[t]{2}{*}{$28^{\circ}$} & \multirow[t]{2}{*}{$29^{\circ}$} \\
\hline & $\begin{array}{l}10 \\
20\end{array}$ & $\begin{array}{l}11.476 \\
22.953\end{array}$ & $\begin{array}{r}5.085 \\
5.077\end{array}$ & $\begin{array}{l}10.171 \\
10.155\end{array}$ & $\begin{array}{l}15.256 \\
15.232\end{array}$ & $\begin{array}{l}20.342 \\
20.310\end{array}$ & $\begin{array}{l}25.427 \\
25.387\end{array}$ & $\begin{array}{l}30.513 \\
30.465\end{array}$ & & & \\
\hline & $\begin{array}{l}30 \\
40\end{array}$ & $\begin{array}{l}34.430 \\
45.906\end{array}$ & $\begin{array}{l}5.069 \\
5.061\end{array}$ & $\begin{array}{l}10.139 \\
10.123\end{array}$ & $\begin{array}{l}15.208 \\
15.185\end{array}$ & $\begin{array}{l}20.278 \\
20.246\end{array}$ & $\begin{array}{l}25.347 \\
25.308\end{array}$ & $\begin{array}{l}30.417 \\
30.369\end{array}$ & \multirow{4}{*}{$\begin{array}{r}5 \\
10 \\
15 \\
20 \\
25 \\
30\end{array}$} & \multirow{4}{*}{$\begin{array}{r}\text { Inches. } \\
0.002 \\
.007 \\
.016 \\
.028 \\
.043 \\
.063\end{array}$} & \multirow{4}{*}{$\begin{array}{c}\text { Inches. } \\
0.002 \\
.007 \\
.016 \\
.028 \\
.044 \\
.064\end{array}$} \\
\hline & 50 & 57.383 & 5.054 & 10.107 & 15.161 & 20.214 & 25.268 & 30.321 & & & \\
\hline \multirow[t]{2}{*}{29} & 00 & 68.859 & 5.046 & 10.091 & 15.137 & 20.182 & 25.228 & 30.274 & & & \\
\hline & $\begin{array}{l}10 \\
20 \\
30 \\
40 \\
50\end{array}$ & $\begin{array}{l}11.478 \\
22.957 \\
34.435 \\
45.913 \\
57.391\end{array}$ & $\begin{array}{l}5.037 \\
5.029 \\
5.021 \\
5.013 \\
5.004\end{array}$ & $\begin{array}{l}10.075 \\
10.058 \\
10.042 \\
10.025 \\
10.009\end{array}$ & $\begin{array}{l}15.112 \\
15.087 \\
15.063 \\
15.038 \\
15.013\end{array}$ & $\begin{array}{l}20.150 \\
20.117 \\
20.084 \\
20.051 \\
20.018\end{array}$ & $\begin{array}{l}25.187 \\
25.146 \\
25.105 \\
25.064 \\
25.022\end{array}$ & $\begin{array}{l}30.224 \\
30.175 \\
30.126 \\
30.076 \\
30.027\end{array}$ & & & \\
\hline \multirow[t]{5}{*}{30} & 00 & 68.870 & 4.996 & 9.993 & 14.989 & 19.985 & 24.981 & 29.978 & & \multirow{2}{*}{$30^{\circ}$} & \multirow{2}{*}{$31^{\circ}$} \\
\hline & 10 & $\begin{array}{l}11.480 \\
22.960\end{array}$ & $\begin{array}{l}4.988 \\
4.979\end{array}$ & & & & & 29.927 & & & \\
\hline & 30 & 34.440 & 4.971 & 9.942 & 14.912 & 19.883 & 24.854 & $\begin{array}{l}29.805 \\
29.825\end{array}$ & \multirow{7}{*}{$\begin{array}{r}5 \\
10 \\
15 \\
20 \\
25 \\
30\end{array}$} & \multirow{5}{*}{$\begin{array}{l}0.002 \\
.007 \\
.016 \\
.029 \\
.045 \\
.065\end{array}$} & \multirow{6}{*}{$\begin{array}{r}0.002 \\
.007 \\
.017 \\
.030 \\
.046 \\
.067\end{array}$} \\
\hline & 40 & 45.920 & 4.962 & 9.925 & 14.887 & 19.849 & 24.812 & 29.774 & & & \\
\hline & 50 & 400 & 4.954 & 9.908 & 862 & 815 & 24.769 & 29.723 & & & \\
\hline \multirow[t]{3}{*}{31} & 00 & 68.880 & 4.945 & 9.891 & 14.836 & 19. 782 & 24.727 & $29.67 \% 2$ & & & \\
\hline & $\begin{array}{l}10 \\
20 \\
30 \\
40\end{array}$ & $\begin{array}{l}11.482 \\
22.964 \\
34.446 \\
45.927\end{array}$ & $\begin{array}{l}4.937 \\
4.928 \\
4.919 \\
4.910\end{array}$ & $\begin{array}{l}9.873 \\
9.856 \\
9.838 \\
9.821\end{array}$ & $\begin{array}{l}14.810 \\
14.784 \\
14.758 \\
14.731\end{array}$ & $\begin{array}{l}19.747 \\
19.712 \\
19.677 \\
19.642\end{array}$ & $\begin{array}{l}24.683 \\
24.640 \\
24.596 \\
24.552\end{array}$ & $\begin{array}{l}29.620 \\
29.568 \\
29.515 \\
29.463\end{array}$ & & & \\
\hline & 5 & & .902 & 9. 804 & 705 & 19.607 & 24.509 & 29.411 & & & \\
\hline \multirow[t]{2}{*}{32} & 00 & 68.891 & 4. 893 & 9.786 & 14. 679 & 19.572 & 24.465 & 29.358 & & & \\
\hline & $\begin{array}{l}10 \\
20 \\
30 \\
40 \\
50\end{array}$ & $\begin{array}{l}11.484 \\
22.967 \\
34.451 \\
45.934 \\
57.418\end{array}$ & $\begin{array}{l}4.884 \\
4.875 \\
4.866 \\
4.857 \\
4.348\end{array}$ & $\begin{array}{l}9.768 \\
9.750 \\
0.732 \\
9.714 \\
9.696\end{array}$ & $\begin{array}{l}14.652 \\
14.625 \\
14.598 \\
14.572 \\
14.545\end{array}$ & $\begin{array}{l}19.536 \\
19.500 \\
19.465 \\
19.429 \\
19.393\end{array}$ & $\begin{array}{l}24.420 \\
24.376 \\
24.331 \\
24.286 \\
24.241\end{array}$ & $\begin{array}{l}29.305 \\
29.251 \\
29.197 \\
29.143 \\
29.089\end{array}$ & \multirow[t]{3}{*}{$\begin{array}{r}5 \\
10 \\
15 \\
20 \\
25 \\
30\end{array}$} & \multirow[t]{3}{*}{$\begin{array}{r}0.002 \\
.007 \\
.017 \\
.030 \\
.047 \\
.068\end{array}$} & \multirow[t]{3}{*}{$\begin{array}{r}0.002 \\
.008 \\
.017 \\
.031 \\
.048 \\
.069\end{array}$} \\
\hline \multirow[t]{3}{*}{33} & 00 & 68.902 & 4.839 & 9.679 & 14.518 & 19.357 & 24.196 & 29.036 & & & \\
\hline & $\begin{array}{l}10 \\
20\end{array}$ & $\begin{array}{l}185 \\
971\end{array}$ & $\begin{array}{l}4.830 \\
4.821\end{array}$ & $\begin{array}{l}9.660 \\
9.642\end{array}$ & $\begin{array}{l}14.490 \\
14.462\end{array}$ & $\begin{array}{l}19.320 \\
19.283\end{array}$ & $\begin{array}{l}24.150 \\
24.104\end{array}$ & $\begin{array}{l}28.980 \\
28.925\end{array}$ & & & \\
\hline & $\begin{array}{l}30 \\
40 \\
50\end{array}$ & $\begin{array}{l}34.456 \\
45.942 \\
57.427\end{array}$ & $\begin{array}{l}4.812 \\
4.802 \\
4.793\end{array}$ & $\begin{array}{l}9.623 \\
9.605 \\
9.586\end{array}$ & $\begin{array}{l}14.435 \\
14.407 \\
14.379\end{array}$ & $\begin{array}{l}19.246 \\
19.210 \\
19.173\end{array}$ & $\begin{array}{l}24.058 \\
24.012 \\
23.966\end{array}$ & $\begin{array}{l}28.870 \\
28.814 \\
28.759\end{array}$ & I & $34^{\circ}$ & $35^{\circ}$ \\
\hline 34 & 00 & 68.913 & 4.784 & 9.568 & 352 & .136 & 920 & 704 & 5 & 0.002 & 0.002 \\
\hline & $\begin{array}{l}10 \\
20 \\
30 \\
40 \\
50\end{array}$ & $\begin{array}{l}11.487 \\
22.975 \\
34.462 \\
45.949 \\
57.437\end{array}$ & $\begin{array}{l}4.774 \\
4.765 \\
4.755 \\
4.746 \\
4.737\end{array}$ & $\begin{array}{l}9.549 \\
9.530 \\
9.511 \\
9.492 \\
9.473\end{array}$ & $\begin{array}{l}14.323 \\
14.295 \\
14.267 \\
14.238 \\
14.210\end{array}$ & $\begin{array}{l}19.098 \\
19.060 \\
19.022 \\
18.984 \\
18.946\end{array}$ & $\begin{array}{l}23.872 \\
23.825 \\
23.778 \\
23.730 \\
23.683\end{array}$ & $\begin{array}{l}28.647 \\
28.590 \\
28.533 \\
28.476 \\
28.420\end{array}$ & $\begin{array}{l}10 \\
15 \\
20 \\
25 \\
30\end{array}$ & $\begin{array}{l}.008 \\
.017 \\
.031 \\
.049 \\
.070\end{array}$ & $\begin{array}{r}.008 \\
.018 \\
.031 \\
.049 \\
.071\end{array}$ \\
\hline 35 & 00 & 68.924 & 4. 727 & 9.454 & 14.181 & 18.908 & 23.636 & 28.363 & & & \\
\hline
\end{tabular}


TABLE 6.-Coordinates for projection of maps (scale $\left.\frac{1}{6 \frac{1}{3} 5 \sigma}\right)$-Continued.

[From Smithsonian Geographical Tables.]

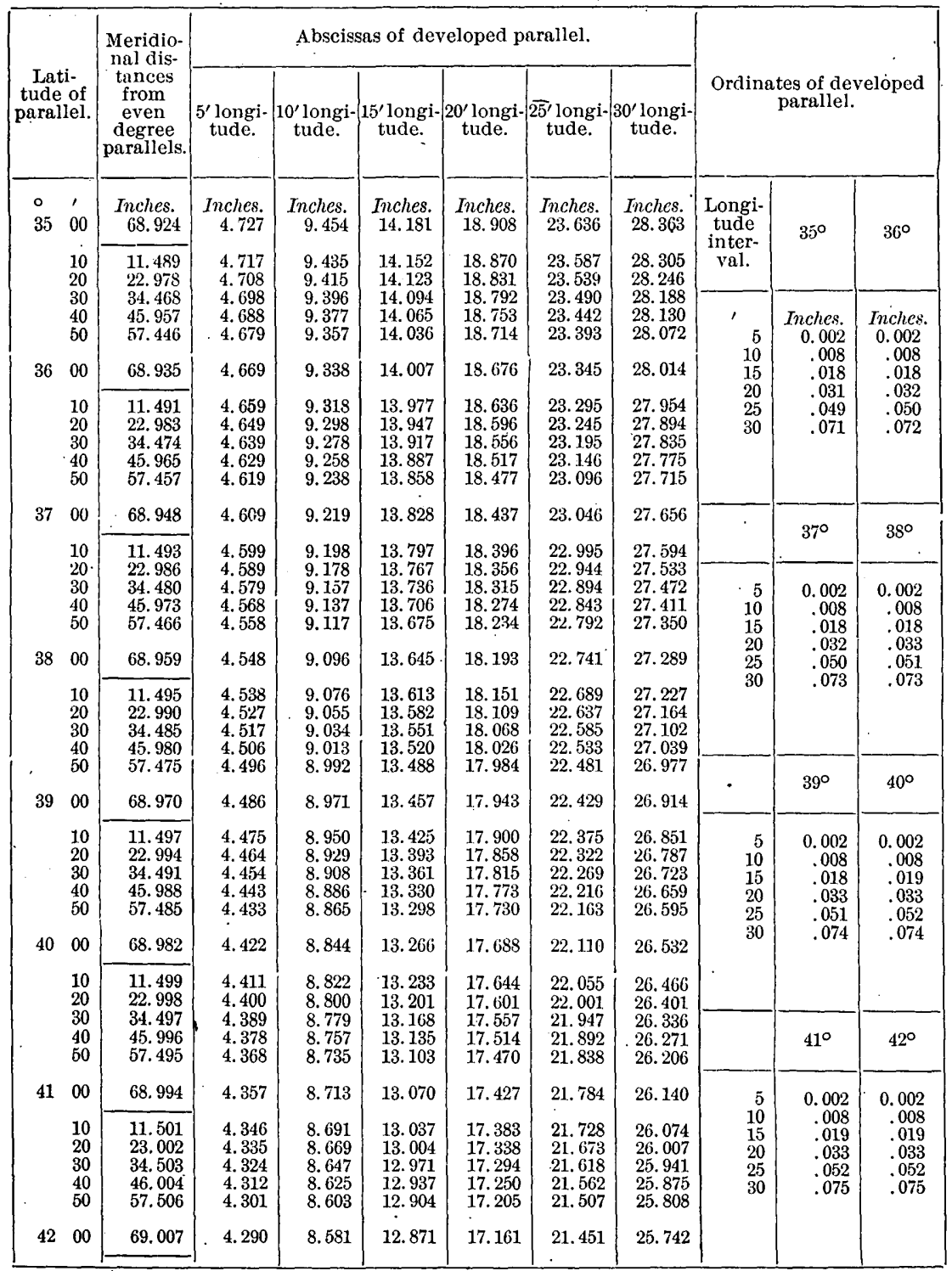


TABLE 6.-Coordinates for projection of maps (scale $\frac{1}{63} \frac{1}{5 \sigma \gamma}$ )-Continued.

[From Smithsonian Geographical Tables.]

\begin{tabular}{|c|c|c|c|c|c|c|c|c|c|c|c|}
\hline \multirow{2}{*}{\multicolumn{2}{|c|}{$\begin{array}{c}\text { Lati- } \\
\text { tude of } \\
\text { parallel. }\end{array}$}} & \multirow{2}{*}{$\begin{array}{c}\text { Meridio- } \\
\text { nal dis- } \\
\text { tances } \\
\text { from } \\
\text { even } \\
\text { degree } \\
\text { parallels. }\end{array}$} & \multicolumn{6}{|c|}{ Abscissas of developed parallel. } & \multirow{2}{*}{\multicolumn{3}{|c|}{$\begin{array}{l}\text { Ordinates of developed } \\
\text { parnllel. }\end{array}$}} \\
\hline & & & \multirow{2}{*}{$\begin{array}{r}\begin{array}{c}5^{\prime} \text { longi- } \\
\text { tude. }\end{array} \\
\begin{array}{r}\text { Inches. } \\
4.290\end{array}\end{array}$} & \multirow{2}{*}{$\mid \begin{array}{c}10^{\prime} \text { longi- } \\
\text { tude. } \\
\text { Inches. }\end{array}$} & \multirow{2}{*}{ 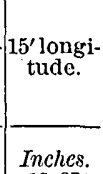 } & \multirow{2}{*}{$\frac{\begin{array}{c}20 \\
\text { tude. }\end{array}}{\text { Inches. }}$} & \multirow{2}{*}{$\begin{array}{c}25 \text { 'longi- } \\
\text { tude. }\end{array}$} & \multirow{2}{*}{$\begin{array}{c}30 \text { 'longi- } \\
\text { tude. } \\
\\
\begin{array}{c}\text { Inches. } \\
25.742\end{array}\end{array}$} & & & \\
\hline \multirow{5}{*}{\multicolumn{2}{|c|}{4200}} & $\begin{array}{c}\text { Inches. } \\
69.007\end{array}$ & & & & & & & \multirow{2}{*}{$\begin{array}{c}\text { Longi- } \\
\text { tude } \\
\text { inter- } \\
\text { val. }\end{array}$} & \multirow{2}{*}{$42^{\circ}$} & \multirow[t]{2}{*}{$43^{\circ}$} \\
\hline & & 11.503 & 4. 279 & 8.558 & 12.837 & 17.116 & 21.395 & 25.674 & & & \\
\hline & & $\begin{array}{l}23.006 \\
34.510\end{array}$ & $\begin{array}{l}4.268 \\
4.256\end{array}$ & $\begin{array}{l}8.535 \\
8.513\end{array}$ & $\begin{array}{l}12.803 \\
12.769\end{array}$ & $\begin{array}{l}17.071 \\
17.025\end{array}$ & $\begin{array}{l}21.338 \\
21.282\end{array}$ & $\begin{array}{l}25.606 \\
25.538\end{array}$ & \multirow{8}{*}{$\begin{array}{r}1 \\
5 \\
10 \\
15 \\
20 \\
25 \\
30\end{array}$} & \multirow{8}{*}{$\begin{array}{r}\text { Inches. } \\
0.002 \\
.008 \\
.019 \\
.033 \\
.052 \\
.075\end{array}$} & \multirow{8}{*}{$\begin{array}{c}\text { Inches. } \\
0.002 \\
.008 \\
.019 \\
.033 \\
.052 \\
.075\end{array}$} \\
\hline & & 46.013 & 4.245 & 8.490 & 12.735 & 16.980 & 21.. 225 & 25.470 & & & \\
\hline & 00 & 57.516 & 4.234 & 8.467 & 12.701 & 16.935 & 21.169 & 25.402 & & & \\
\hline \multirow{5}{*}{.43} & 00 & 69.019 & 4.222 & 8.445 & 12.667 & 16.890 & 21.112 & 25.334 & & & \\
\hline & 10 & 11.505 & 4.211 & $\begin{array}{l}8.422 \\
8.399\end{array}$ & $\begin{array}{l}12.633 \\
12.598\end{array}$ & 16.844 & 21.054 & 25.265 & & & \\
\hline & $\begin{array}{l}20 \\
30\end{array}$ & $\begin{array}{l}23.010 \\
34.515\end{array}$ & $\begin{array}{l}4.199 \\
4.188\end{array}$ & $\begin{array}{l}8.399 \\
8: 376\end{array}$ & $\begin{array}{l}12.598 \\
12.564\end{array}$ & $\begin{array}{l}16.798 \\
16.751\end{array}$ & $\begin{array}{l}20.997 \\
20.939\end{array}$ & $\begin{array}{l}25.196 \\
25.127\end{array}$ & & & \\
\hline & 45 & 46.020 & 4.176 & 8. 353 & 12.529 & 16.705 & 20.882 & 25.058 & & & \\
\hline & 50 & 57.525 & 4.165 & 8.330 & 12.494 & 16.659 & 20.824 & 24.989 & & & \\
\hline \multirow[t]{5}{*}{44} & 00 & 69.030 & 4.153 & 8.307 & 12.460 & 16.613 & 20.767 & 24.920 & \multirow{10}{*}{$\begin{array}{r}5 \\
10 \\
15 \\
20 \\
25 \\
30\end{array}$} & \multirow{2}{*}{$44^{\circ}$} & \multirow{2}{*}{$45^{\circ}$} \\
\hline & \multirow{4}{*}{$\begin{array}{l}10 \\
20 \\
30 \\
40 \\
50\end{array}$} & 11.507 & 4. 142 & 8.283 & 12.425 & 16.566 & 20.708 & 24.849 & & & \\
\hline & & $\begin{array}{l}23.014 \\
34.522\end{array}$ & $\begin{array}{l}4.130 \\
4.118\end{array}$ & $\begin{array}{l}8.260 \\
8.236\end{array}$ & $\begin{array}{l}12.390 \\
12.354\end{array}$ & $\begin{array}{l}16.519 \\
16.473\end{array}$ & $\begin{array}{l}20.649 \\
20.591\end{array}$ & $\begin{array}{l}24.779 \\
24.709\end{array}$ & & & \\
\hline & & $\begin{array}{l}46.029 \\
\end{array}$ & $\begin{array}{l}4.110 \\
4.106\end{array}$ & $\begin{array}{l}8.200 \\
8.213\end{array}$ & 12.319 & $\begin{array}{l}10.470 \\
16.426\end{array}$ & 20.532 & 24.638 & & $\begin{array}{l}0.002 \\
.008\end{array}$ & .008 \\
\hline & & 57.536 & 4.095 & 8.189 & 12.284 & 16.379 & 20.473 & 24.568 & & .019 & .019 \\
\hline 45 & 00 & 69.043 & 4.083 & 8.166 & 12.249 & 16. 332 & $20.41 \bar{\jmath}$ & 24.498 & & .052 & .053 \\
\hline & 10 & 11.509 & 4.071 & 8.142 & 12.213 & 16.284 & 20.355 & 24,426 & & 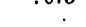 & \\
\hline & 20 & 23.018 & 4.059 & 8.118 & $12.17 \bar{\zeta}$ & 16.236 & 20.295 & 24.354 & & 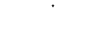 & \\
\hline & 30 & 34.528 & 4.047 & 8.094 & 12.141 & 16. 188 & 20.236 & 24.283 & & & \\
\hline & 40 & 46.037 & 4. 035 & 8.070 & 12. 105 & 16.141 & 20.176 & 24.211 & & & \\
\hline & 50 & 57.546 & 4.023 & 8.016 & 12.070 & 16.093 & 20.116 & 24.139 & & $46^{\circ}$ & $47^{\circ}$ \\
\hline 46 & 00 & 69.055 & 4.011 & 8.023 & 12.034 & 16.045 & 20.056 & 24.068 & & & \\
\hline & 10 & 11.511 & 3.999 & 7. 998 & 11. 997 & 15.997 & 19.996 & 23.995 & 5 & 0.002 & 0.002 \\
\hline & 20 & & & 7.974 & 11.9 & & & & 10 & .008 & .008 \\
\hline & 30 & 34.534 & 3.975 & 7.950 & 11.925 & 15. 899 & 19.974 & & & & .019 \\
\hline & 40 & 46.045 & 3. 9 & 7.925 & 11. 888 & 15.851 & 19.813 & 23.776 & 20 & .034 & .034 \\
\hline & 50 & 57.557 & 3.951 & 7.901 & 11.852 & 15.802 & 19.753 & 23.703 & 25 & .053 & .052 \\
\hline 47 & 00 & 69.068 & 3.988 & 7.877 & 11.815 & 15.754 & 19. 692 & 23.630 & & & \\
\hline & 10 & 11. 513 & 3. 926 & 7.852 & 11.778 & 15. 704 & 19.630 & $\begin{array}{l}23.556 \\
\end{array}$ & & & \\
\hline & $\begin{array}{l}20 \\
30\end{array}$ & $\begin{array}{l}23.027 \\
34.540\end{array}$ & $\begin{array}{l}3.914 \\
3.901\end{array}$ & $\begin{array}{l}7.827 \\
7.803\end{array}$ & $\begin{array}{l}11.741 \\
11.704\end{array}$ & $\begin{array}{l}15.655 \\
15.606\end{array}$ & $\begin{array}{l}19.569 \\
19.507\end{array}$ & $\begin{array}{l}23.482 \\
23.408\end{array}$ & & & \\
\hline & 40 & 46.053 & 3. 889 & 7.778 & 11.667 & 15.556 & 19.445 & 23.334 & & $48^{\circ}$ & $49^{\circ}$ \\
\hline & 50 & 57.567 & 3.877 & 7.753 & $11.630^{\circ}$ & 15.507 & 19.383 & 23.260 & & & \\
\hline 48 & 00 & 69.080 & 3.864 & 7.729 & 11.593 & 15.457 & 19.322 & 23.186 & 5 & 0.002 & 0.002 \\
\hline & 10 & 11.516 & 3. 852 & 7. 704 & 11.555 & 15.4 & 19.259 & 23. & 15 & .019 & .019 \\
\hline & 20 & & 3.839 & 7.679 & 11.518 & & 19.196 & & 20 & .033 & .033 \\
\hline & 30 & 34.546 & 3.827 & 7.653 & 11.480 & 15.307 & 19.134 & 22.960 & 25 & .052 & .052 \\
\hline & 40 & 46. 062 & 3.814 & 7.628 & 11.442 & 15.257 & 19.071 & 22.885 & 30 & .075 & .075 \\
\hline & 50 & 57.577 & 3.802 & 7.603 & 11.405 & 15.206 & 19.008 & 22.810 & & & \\
\hline 49 & 00 & 69.093 & 3.789 & 7.578 & 11.367 & 15.156 & 18.945 & 22.734 & & & \\
\hline
\end{tabular}


TABLE 6.-Coordinates for projection of maps (scale $\frac{-1}{6} \frac{1}{3} \frac{1}{6}$ ) -Continued.

[From Smithsonian Geographical Tables.]

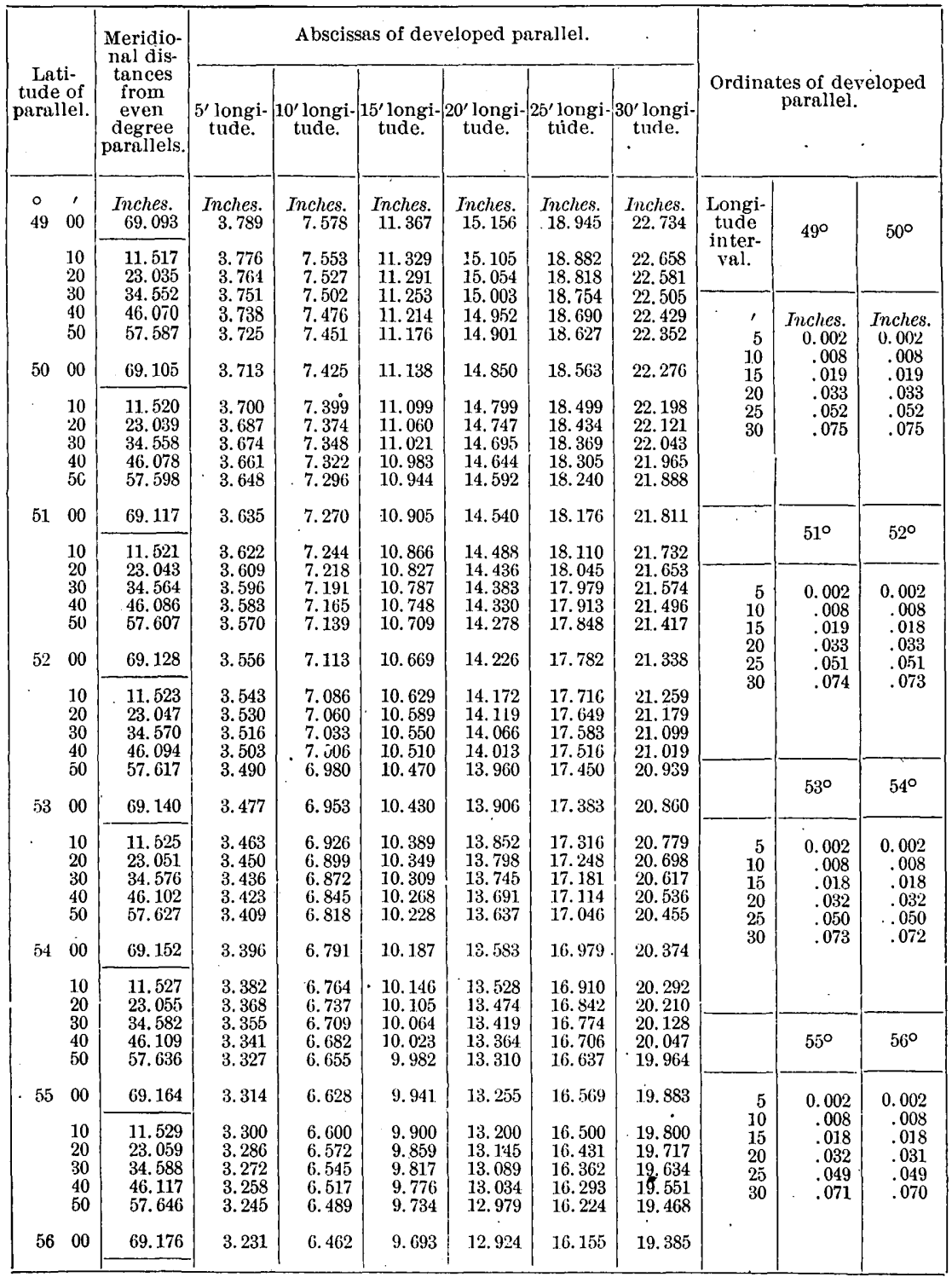


TABLE 6.-Coordinates for projection of maps (scale $\frac{15}{6360}$ ) -Continued.

[From Smithsonian Geographical Tables.]

\begin{tabular}{|c|c|c|c|c|c|c|c|c|c|c|c|}
\hline \multirow{2}{*}{\multicolumn{2}{|c|}{$\begin{array}{l}\text { Lati- } \\
\text { tude of } \\
\text { parallel. }\end{array}$}} & \multirow{3}{*}{$\begin{array}{c}\text { Meridio- } \\
\text { nal dis- } \\
\text { tances } \\
\text { from } \\
\text { even } \\
\text { degree } \\
\text { parnllels. } \\
\text { Inches. }\end{array}$} & \multicolumn{6}{|c|}{ Abscissas of developed parallel. } & \multirow{2}{*}{\multicolumn{3}{|c|}{$\begin{array}{l}\text { Ordinates of developed } \\
\text { parallel. }\end{array}$}} \\
\hline & & & \multirow{2}{*}{$\frac{\begin{array}{c}5 \text { ' longi- } \\
\text { tude. }\end{array}}{\text { Inches. }}$} & \multirow{2}{*}{$\frac{\begin{array}{c}10^{\prime} \text { longi- } \\
\text { tude. }\end{array}}{\text { Inches. }}$} & \multirow{2}{*}{$\begin{array}{c}15^{\prime} \text { longi- } \\
\text { tude. }\end{array}$} & \multirow{2}{*}{$-\begin{array}{c}20^{\prime} \text { longi } \\
\text { tude. }\end{array}$} & \multirow{2}{*}{$\frac{\begin{array}{c}25^{\prime} \text { longi- } \\
\text { tude. }\end{array}}{\text { Inches. }}$} & \multirow{2}{*}{$\begin{array}{c}30^{\prime} \text { longi- } \\
\text { tude. }\end{array}$} & & & \\
\hline$\circ$ & ' & & & & & & & & \multirow{3}{*}{$\begin{array}{l}\text { Longi- } \\
\text { tude } \\
\text { inter- } \\
\text { val. }\end{array}$} & \multirow{4}{*}{$56^{\circ}$} & \multirow{4}{*}{$57^{\circ}$} \\
\hline 56 & 00 & 69.176 & 3.231 & 6.462 & 9.693 & 12.924 & 16.155 & 19.385 & & & \\
\hline & 10 & 11.531 & 3.217 & 6.434 & 9.651 & 12.868 & 16.085 & 19. 301 & & & \\
\hline & 20 & 23.063 & 3.203 & 6.406 & 9.609 & 12.812 & 16.015 & 19.217 & & & \\
\hline & 30 & 34.594 & 3. 189 & .6 .378 & 9.567 & 12. 756 & 15.945 & 19.134 & \multirow{8}{*}{$\begin{array}{r}\prime \\
5 \\
10 \\
15 \\
20 \\
25 \\
30\end{array}$} & \multirow{8}{*}{$\begin{array}{r}\text { Inchcs. } \\
0.002 \\
.008 \\
.018 \\
.031 \\
.049 \\
.070\end{array}$} & \multirow{8}{*}{$\begin{array}{r}\text { Inches. } \\
0.002 \\
.008 \\
.017 \\
.031 \\
.048 \\
.069\end{array}$} \\
\hline & 40 & 46. 125 & 3.175 & 6.350 & 9.525 & 12.700 & 15.875 & 19.050 & & & \\
\hline & 50 & 57. 656 & 3.161 & 6.322 & 9.483 & 12.644 & 15.805 & 18.966 & & & \\
\hline \multirow[t]{5}{*}{57} & 00 & $\cdot 69.188$ & 3.147 & 6.294 & 9.441. & 12.588 & 15. 735 & 18.882 & & & \\
\hline & 10 & 11.533 & 3.133 & 6.266 & 9.398 & 12.531 & 15.664 & 18.797 & & & \\
\hline & 20 & 23.066 & 3.119 & 6.237 & 9.356 & 12.475 & 15.594 & 18. 712 & & & \\
\hline & 30 & 34.599 & 3.104 & 6. 209 & 9.314 & 12. 418 & 15.523 & 18. 627 & & & \\
\hline & $\begin{array}{l}40 \\
50\end{array}$ & $\begin{array}{l}\mathbf{4 6 .} 132 \\
\mathbf{5 7 . 6 6 6}\end{array}$ & $\begin{array}{l}3.090 \\
3.076\end{array}$ & $\begin{array}{l}6.181 \\
6.152\end{array}$ & $\begin{array}{l}9.271 \\
9.229\end{array}$ & $\begin{array}{l}12.362 \\
12.305\end{array}$ & $\begin{array}{l}15.452 \\
15.381\end{array}$ & $\begin{array}{l}18.542 \\
18.457\end{array}$ & & & \\
\hline \multirow[t]{6}{*}{58} & 00 & 69.199 & 3.062 & 6.124 & 9.186 & 12.248 & 15. 311 & 18.373 & & \multirow{2}{*}{$58^{\circ}$} & \multirow{2}{*}{$59^{\circ}$} \\
\hline & 10 & 11.535 & 3.048 & 6.096 & 9.143 & 12.191 & 15. 239 & 18. 287 & & & \\
\hline & 20 & 23.070 & 3.034 & 6.067 & 9.101 & 12.134 & 15.168 & 18.201 . & & & \\
\hline & 30 & 34.605 & 3.019 & 6.038 & 9.058 & 12.077 & 15.096 & 18.115 & 5 & 0.002 & 0.002 \\
\hline & 40 & 46.140 & 3.005 & 6.010 & 9.015 & 12.020 & 15.025 & 18.029 & 10 & .008 & .007 \\
\hline & 50 & 57.675 & 2.991 & 5.981 & 8.972 & 11.962 & 14. 953 & 17. 944 & 15 & .017 & .017 \\
\hline \multirow[t]{6}{*}{59} & 00 & 69.210 & 2.976 & 5.953 & 8.929 & 11.905 & 14.882 & 17.858 & 25 & .030 & .046 \\
\hline & 10 & 11.537 & 2.962 & 5.924 & 8.885 & 11.847 & 14.809 & 17.771 & & & \\
\hline & 20 & 23.074 & 2.947 & 5.895 & 8.842 & 11.790 & 1.4. 737 & 17. 684 & & & \\
\hline & 30 & 34.610 & 2.933 & 5. 866 & 8.799 & 11. 732 & 14.665 & 17.597 & & & \\
\hline & 40 & 46.147 & 2.918 & 5.837 & 8.755 & 11.674 & 14.592 & 17.510 & & & \\
\hline & 50 & 57.684 & 2.904 & 5.808 & 8.712 & 11.616 & 14.520 & 17.424 & & & \\
\hline 60 & 00 & 69.221 & 2.890 & 5,779 & 8.669 & 11.558 & 14.448 & 17. 337 & & 60 & $61^{\circ}$ \\
\hline & 10 & 11.539 & 2.875 & 5. 750 & 8. 625 & 11. 500 & 14.375 & 17. 249 & 5 & 0.002 & 0.002 \\
\hline & 20 & 23.077 & 2.860 & 5.721 & 8. 581 & 11.441 & 14.302 & 17. 162 & 10 & $\begin{array}{r}.002 \\
.007\end{array}$ & $\begin{array}{l}0.004 \\
.007\end{array}$ \\
\hline & 30 & 34.616 & 2.846 & 5. 691 & 8.537 & 11.383 & 14.229 & 17.074 & 15 & .016 & .016 \\
\hline & 40 & 46.154 & 2.831 & 5. 662 & 8.493 & 11.324 & 14. 156 & 16.987 & 20 & .029 & .029 \\
\hline & 50 & 57.693 & 2.816 & 5.633 & 8.450 & 11.266 & 14.083 & 16. 899 。 & 25 & .045 & .045 \\
\hline 61 & 00 & 69.232 & 2.802 & 5.604 & 8.406 & 11. 208 & 14.010 & 16.811 & & & 064 \\
\hline & 10 & 11.540 & 2.787 & 5.574 & 8.361. & 11.148 & 13.936 & 16. 723 & & & \\
\hline & 20 & 23.081 & 2.772 & 5.545 & 8.317 & 11.090 & 13.862 & 16.634 & & & \\
\hline & 30 & 34.621 & 2.758 & 5.115 & 8.273 & 11.030 & 13.788 & 16.546 & & & \\
\hline & 40 & 46.162 & 2.743 & 5. 486 & 8.229 & 10.972 & 13.715 & 16.457 & & $62^{\circ}$ & $63^{\circ}$ \\
\hline & 50 & 57.702 & 2.728 & 5. 456 & 8,184 & 10.912 & 13.641 & 16. 369 & & $02^{\circ}$ & \\
\hline 62 & 00 & 69.242 & $2.7 \mathrm{i3}$ & 5.427 & 8.140 & 10.854 & 13.567 & 16.280 & 5 & 0.002 & 0.002 \\
\hline & 10 & 11.542 & 2. 699 & 5. 397 & 8.096 & 10.794 & 13.493 & 16.191. & 10 & .007 & .007 \\
\hline & 20 & 23.084 & 2.684 & 5. 367 & 8.051 & 10.734 & 13.418 & 16.102 & $\begin{array}{l}10) \\
20\end{array}$ & $\begin{array}{r}.010 \\
.028\end{array}$ & $\begin{array}{r}.015 \\
027\end{array}$ \\
\hline & 30 & 34.626 & 2.669 & 5.337 & 8.006 & 10.675 & 13.344 & 16.012 & 25 & 044 & 043 \\
\hline & 40 & 46.168 & 2.654 & 5. 308 & 7. 961 & 10.615 & 13. 269 & 15. 923 & 30 & .045 & .061 \\
\hline & 50 & 57.710 & 2.639 & 5. 278 & 7.917 & 10.556 & 13.195 & 15.833 & & & \\
\hline 63 & 00 & 69.253 & 2.624 & 5.248 & 7.872 & 10.496 & 13.120 & 15. 744 & & & \\
\hline
\end{tabular}


TABLE 6.-Coordinates for projection of maps (scale $\left.\frac{6}{6} \frac{1}{360}\right)$-Continued.

[From Smithsonian Geographical Tables.]

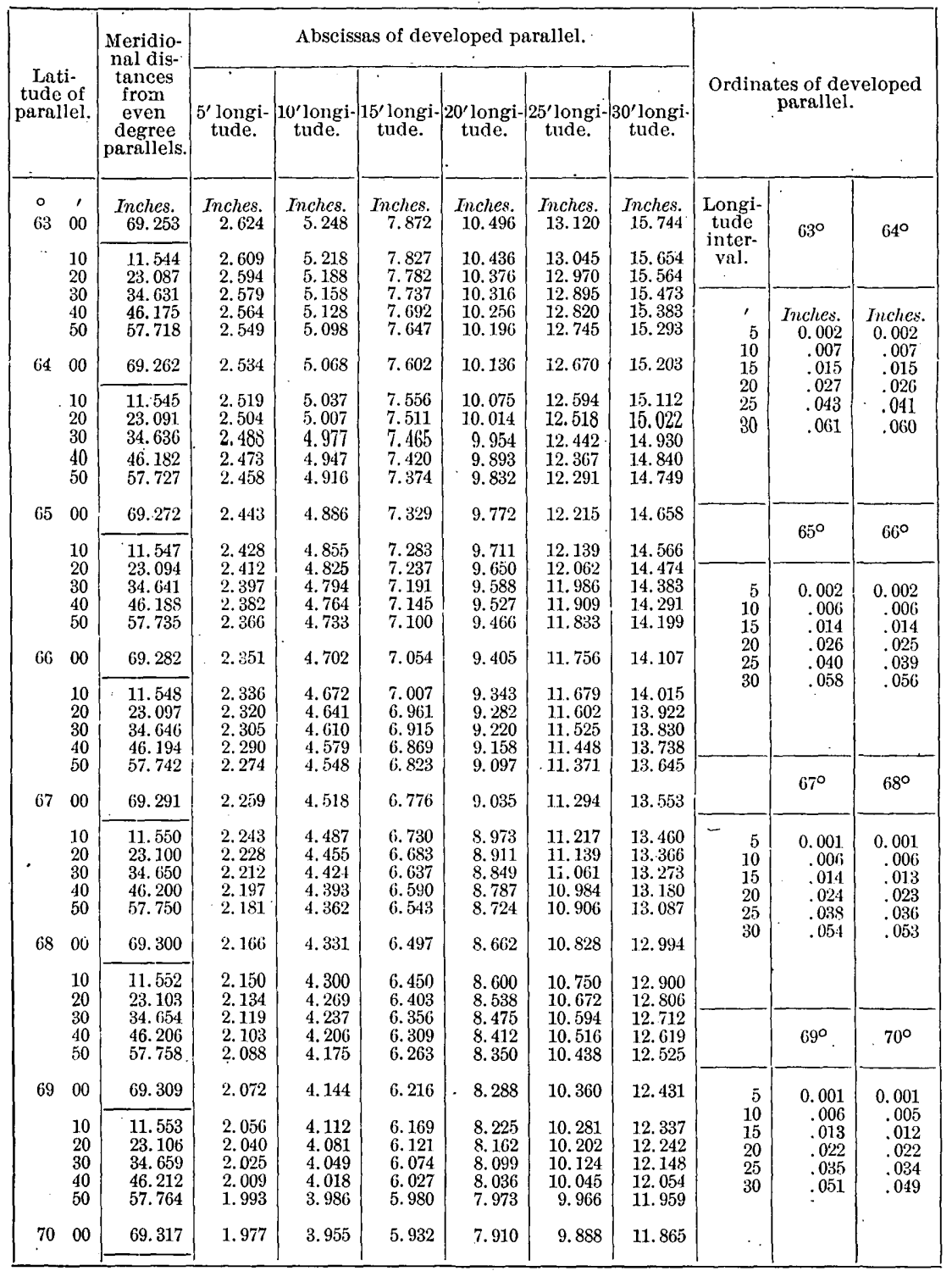


TABLE 6.-Coordinates for projection of maps $\left(\right.$ scale $\left._{5 \frac{1}{3} \frac{1}{3} \sigma 0}\right)$-Continued.

[From Smithsonian Geographical Tables.]

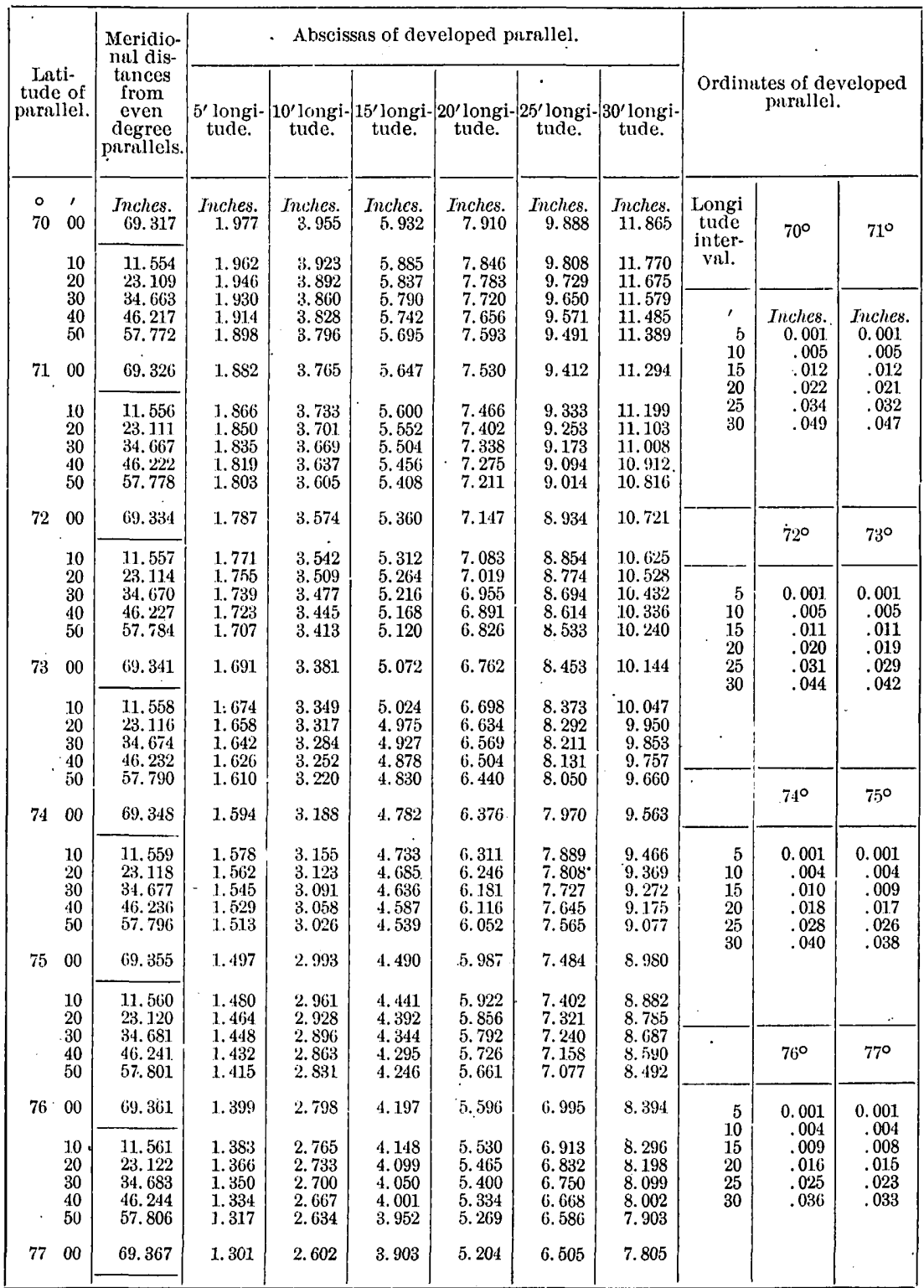


TABLE 6.-Coordinates for projection of maps (scale $\frac{{ }_{6} \frac{1}{360}}{360}$ )-Continued.

[From Smithsonian Geographical Tables.]

\begin{tabular}{|c|c|c|c|c|c|c|c|c|c|c|c|}
\hline \multirow{2}{*}{\multicolumn{2}{|c|}{$\begin{array}{c}\text { Lati- } \\
\text { tude of } \\
\text { parallel. }\end{array}$}} & \multirow{3}{*}{\begin{tabular}{|c|} 
Meridio- \\
nal dis- \\
tances \\
from \\
even \\
degree \\
parallels. \\
\\
Inches. \\
69.367
\end{tabular}} & \multicolumn{6}{|c|}{ Abscissas of developed parallel. } & \multirow{2}{*}{\multicolumn{3}{|c|}{$\begin{array}{l}\text { Ordinates of developed } \\
\text { parallel. }\end{array}$}} \\
\hline & & & \multirow{2}{*}{$\begin{array}{c}5 \text { 'longi- } \\
\text { tude. } \\
\text { Inches. }\end{array}$} & $\frac{\begin{array}{c}10^{\prime} \text { longi- } \\
\text { tude. }\end{array}}{\text { Inches. }}$ & $\begin{array}{c}15^{\prime} \text { longi- } \\
\text { tude. }\end{array}$ & $\begin{array}{c}20^{\prime} \text { longi- } \\
\text { tude. }\end{array}$ & $\underbrace{}_{\begin{array}{c}25 \\
\text { lude. }\end{array}}$ & $\left\{\begin{array}{c}30^{\prime} \text { longi- } \\
\text { tude. }\end{array}\right.$ & & & \\
\hline $\begin{array}{l}\circ \\
77\end{array}$ & $\frac{1}{00}$ & & & $\begin{array}{r}\text { Inches. } \\
2.602\end{array}$ & $\begin{array}{l}\text { Inches. } \\
\quad 3.903\end{array}$ & $\begin{array}{r}\text { Inches. } \\
5.204\end{array}$ & $\begin{array}{l}\text { Inches. } \\
6.505\end{array}$ & $\begin{array}{r}\text { Inches. } \\
7.805\end{array}$ & \multirow{2}{*}{$\begin{array}{c}\text { Longi- } \\
\text { tude } \\
\text { inter- } \\
\text { val. }\end{array}$} & \multirow[t]{2}{*}{$77^{\circ}$} & \multirow[t]{2}{*}{$78^{\circ}$} \\
\hline & $\begin{array}{l}10 \\
20\end{array}$ & $\begin{array}{l}11.562 \\
23.124\end{array}$ & $\begin{array}{l}1.284 \\
1.268\end{array}$ & $\begin{array}{l}2.569 \\
2.536\end{array}$ & $\begin{array}{l}3.854 \\
3.804\end{array}$ & $\begin{array}{l}5.138 \\
5.072\end{array}$ & $\begin{array}{l}6.423 \\
6.341\end{array}$ & $\begin{array}{l}7.707 \\
7.609\end{array}$ & & & \\
\hline & 30 & 34.686 & 1. 252 & 2.503 & 3.755 & 5.006 & 6.258 & 7.510 & & Inches. & Jnches \\
\hline & 50 & $\begin{array}{l}46.248 \\
57.810\end{array}$ & $\begin{array}{l}1.235 \\
1.219\end{array}$ & $\begin{array}{l}2.470 \\
2.438\end{array}$ & $\begin{array}{l}3.706 \\
3.656\end{array}$ & $\begin{array}{l}4.941 \\
4.875\end{array}$ & $\begin{array}{l}6.176 \\
6.094\end{array}$ & $\begin{array}{l}7.411 \\
7.313\end{array}$ & $\begin{array}{r}5 \\
10\end{array}$ & $\begin{array}{r}0.001 \\
.004\end{array}$ & $\begin{array}{c}0.001 \\
.003\end{array}$ \\
\hline \multirow[t]{2}{*}{78} & 00 & 69.373 & 1. 202 & 2.405 & 3.607 & 4. 810 & 6.012 & 7.214 & $\begin{array}{l}15 \\
20\end{array}$ & $\begin{array}{l}.008 \\
.015\end{array}$ & $\begin{array}{l}.008 \\
.014\end{array}$ \\
\hline & $\begin{array}{l}10 \\
20 \\
30 \\
40 \\
50\end{array}$ & $\begin{array}{l}11.563 \\
23.126 \\
34.689 \\
46.252 \\
57.814\end{array}$ & $\begin{array}{l}1.186 \\
1.169 \\
1.153 \\
1.136 \\
1.120\end{array}$ & $\begin{array}{l}2.372 \\
2.339 \\
2.306 \\
2.273 \\
2.240\end{array}$ & $\begin{array}{l}3.558 \\
3.508 \\
3.459 \\
3.410 \\
3.360\end{array}$ & $\begin{array}{l}4.744 \\
4.678 \\
4.612 \\
4.546 \\
4.480\end{array}$ & $\begin{array}{l}5.930 \\
5.847 \\
5.765 \\
5.683 \\
5.600\end{array}$ & $\begin{array}{l}7.115 \\
7.016 \\
6.918 \\
6.819 \\
6.720\end{array}$ & $\begin{array}{l}25 \\
30\end{array}$ & $\begin{array}{l}.023 \\
.033\end{array}$ & $\begin{array}{l}.021 \\
.031\end{array}$ \\
\hline 79 & 00 & 69.377 & 1.104 & 2.207 & 3.311 & 4. 414 & 5.518 & 6.621 & & \multirow{2}{*}{$79^{\circ}$} & \multirow{2}{*}{$80^{\circ}$} \\
\hline & .10 & 11. 564 & 1,087 & 2.174 & 3.261 & 4. 348 & 5.435 & 6.522 & & & \\
\hline & 20 & $23: 127$ & 1.070 & 2.141 & 3.211. & 4. 282 & 5. 352 & 6.422 & & & \\
\hline & 30 & 34.691 & 1.054 & 2.108 & 3.162 & 4. 216 & 5.270 & 6. 323 & $\begin{array}{r}5 \\
10\end{array}$ & $\begin{array}{r}0.001 \\
.003\end{array}$ & $\begin{array}{r}0.001 \\
.003\end{array}$ \\
\hline & $\begin{array}{l}40 \\
50\end{array}$ & $\begin{array}{l}46.255 \\
57.818\end{array}$ & 1.021 & $\begin{array}{l}2.075 \\
2.042\end{array}$ & $\begin{array}{l}3.112 \\
3.062\end{array}$ & $\begin{array}{l}4.150 \\
4.083\end{array}$ & $\begin{array}{l}5.187 \\
5.104\end{array}$ & $\begin{array}{l}6.224 \\
6.125\end{array}$ & $\begin{array}{l}15 \\
20\end{array}$ & .007 & .006 \\
\hline 80 & 00 & 69.382 & 1. 004 & 2.009 & 3.013 & 4.017 & 5.022 & 6.026 & $\begin{array}{l}25 \\
30\end{array}$ & $\begin{array}{l}.020 \\
.028\end{array}$ & $\begin{array}{l}.018 \\
.018 \\
.026\end{array}$ \\
\hline
\end{tabular}


TABLE. 7.-Coordinates for projection of maps (scale $\left.\frac{1}{62} \frac{1}{500}\right)$.

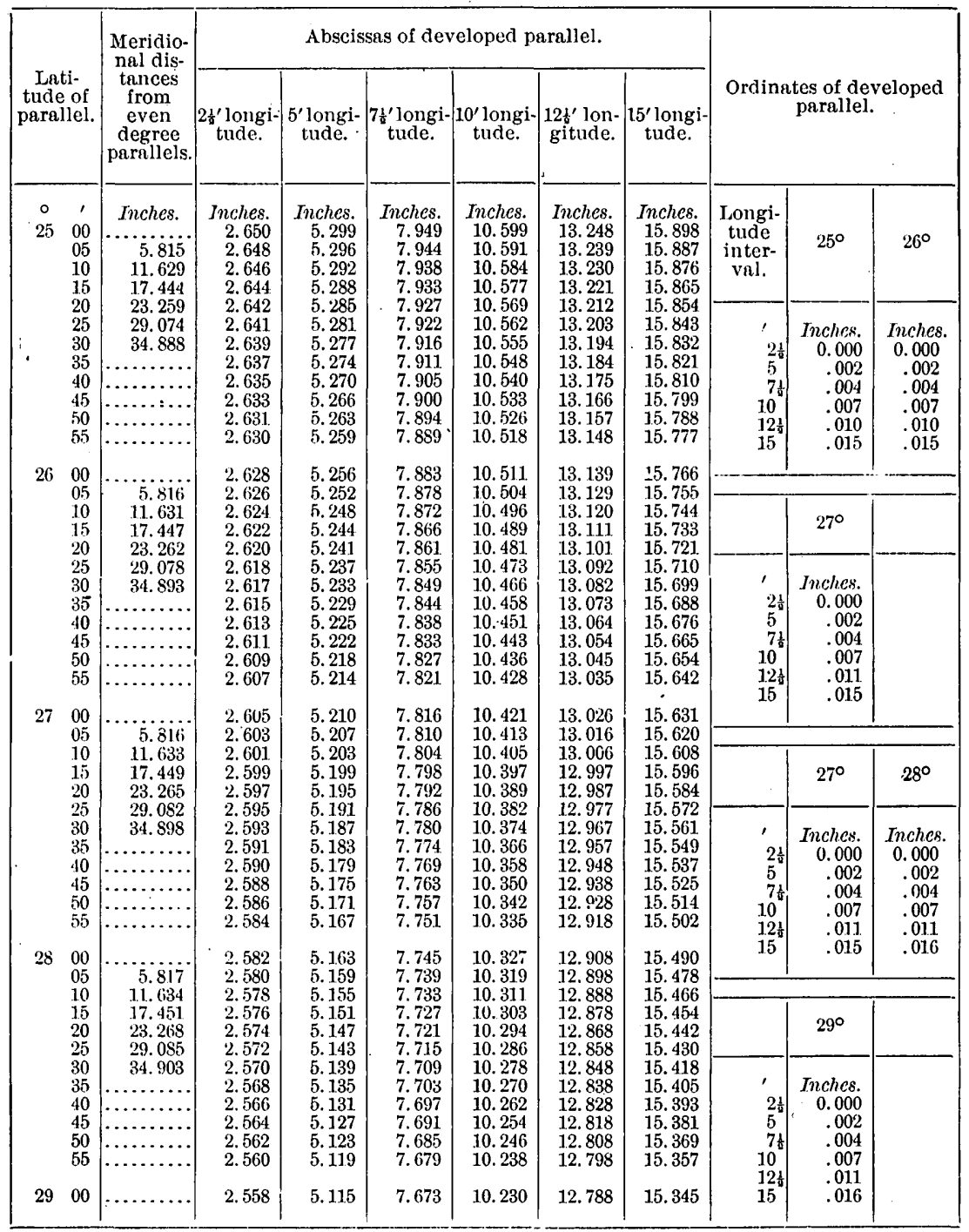




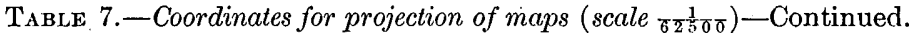

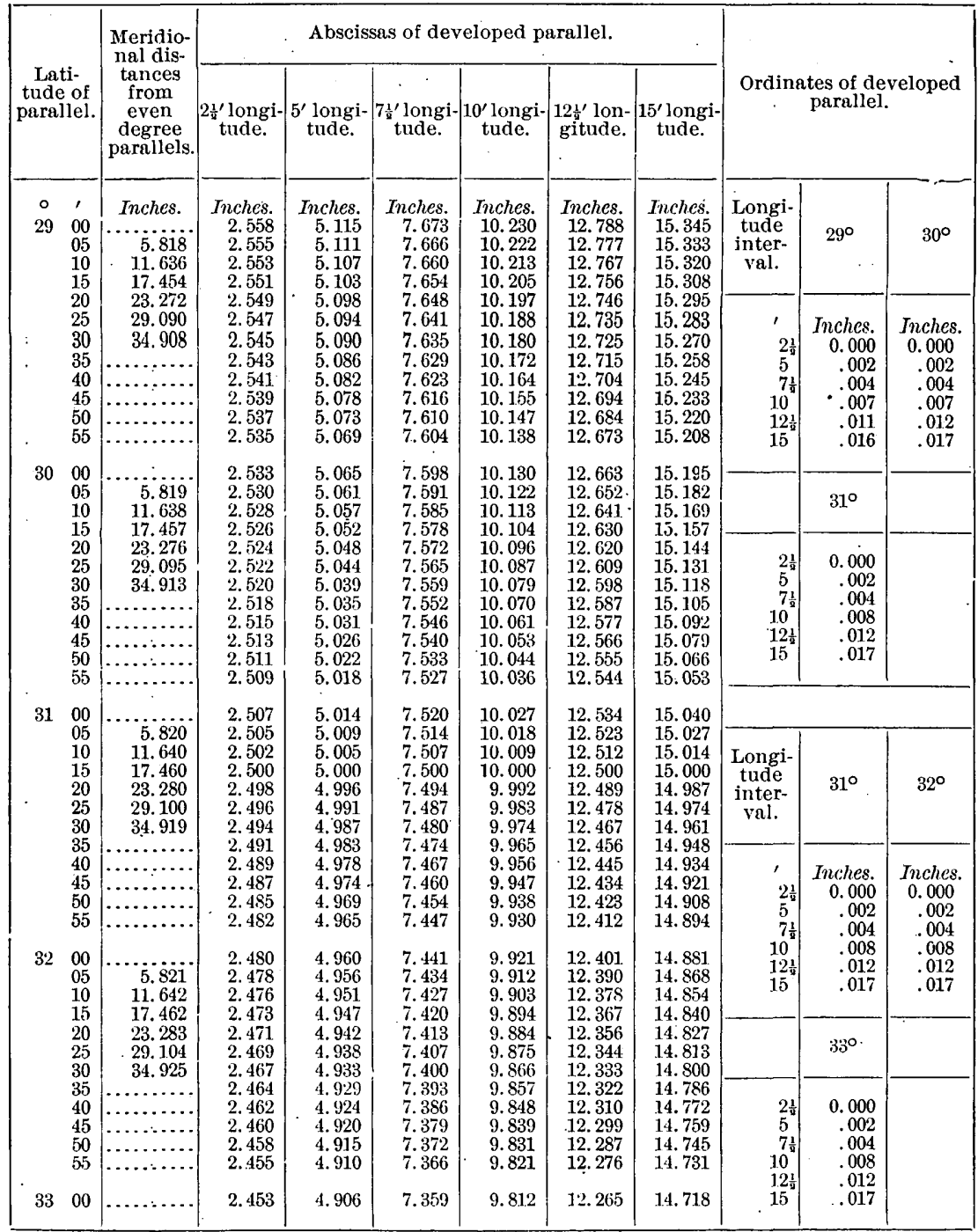


TABLE 7.-Coordinales for projection of maps (scale $\left.\frac{1}{2500}\right)$-Continued.

[From Smithsonian Geographical Tables.]

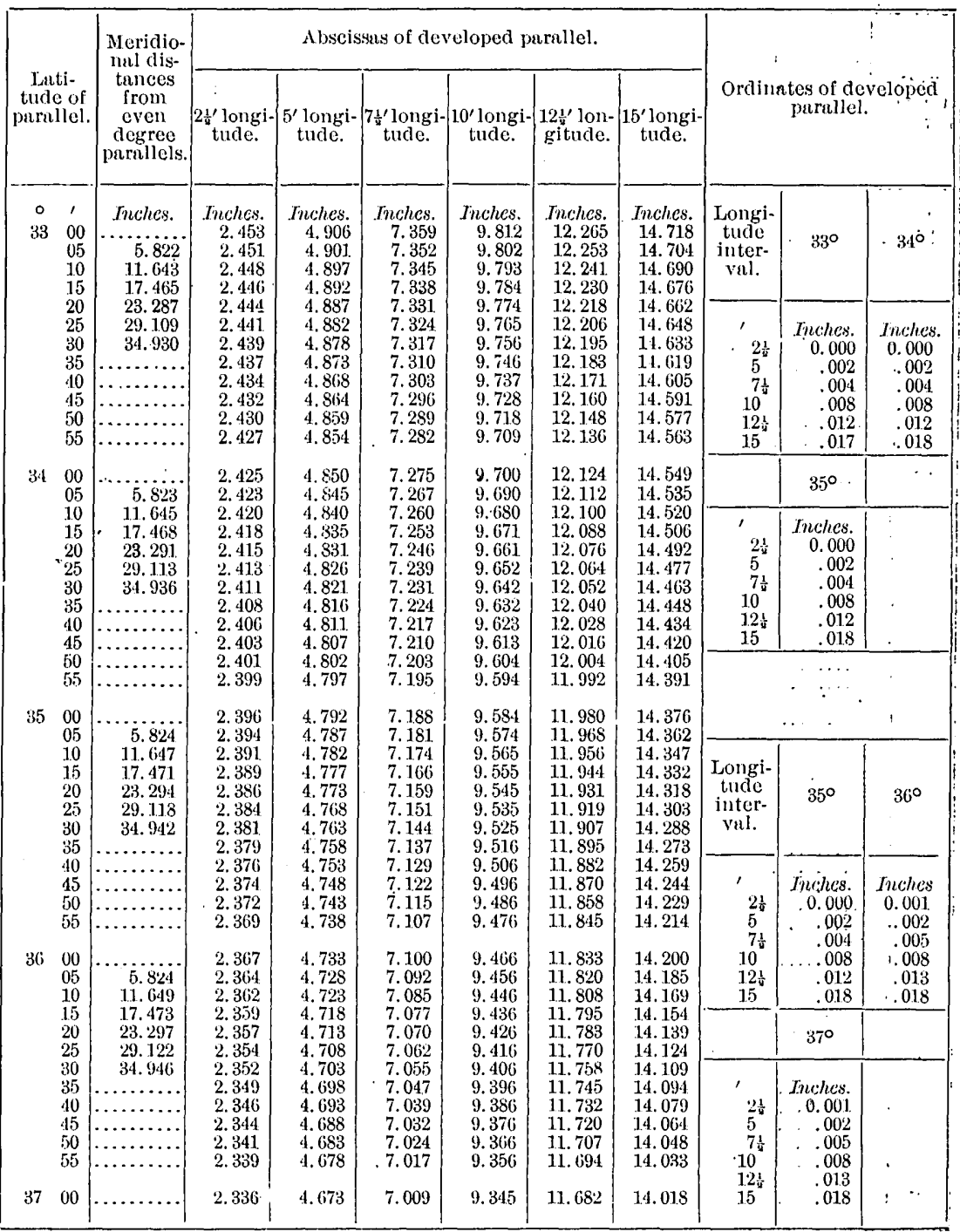

Bull. 214-03-5 
TABLE 7.-Coordinates for projection of maps (scale $\frac{1}{6250}$ )-Continued.

[From Smithsonian Geographical Tables.]

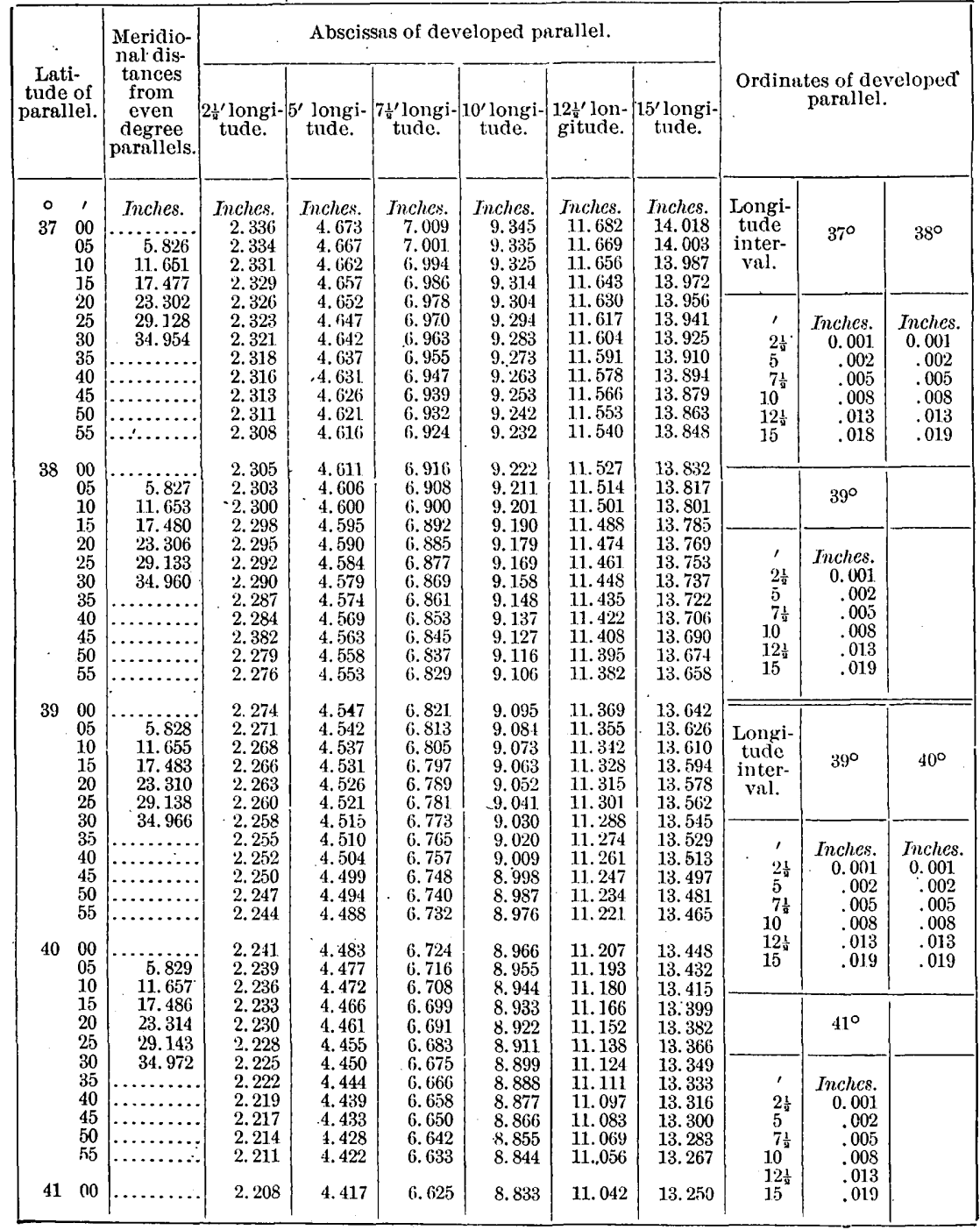


TABLE 7.-Coordinates for projection of maps (scale $\left.\frac{1}{2} \frac{1}{50 \pi}\right)$-Continued.

[From Smithsonian Geographical Tables.]

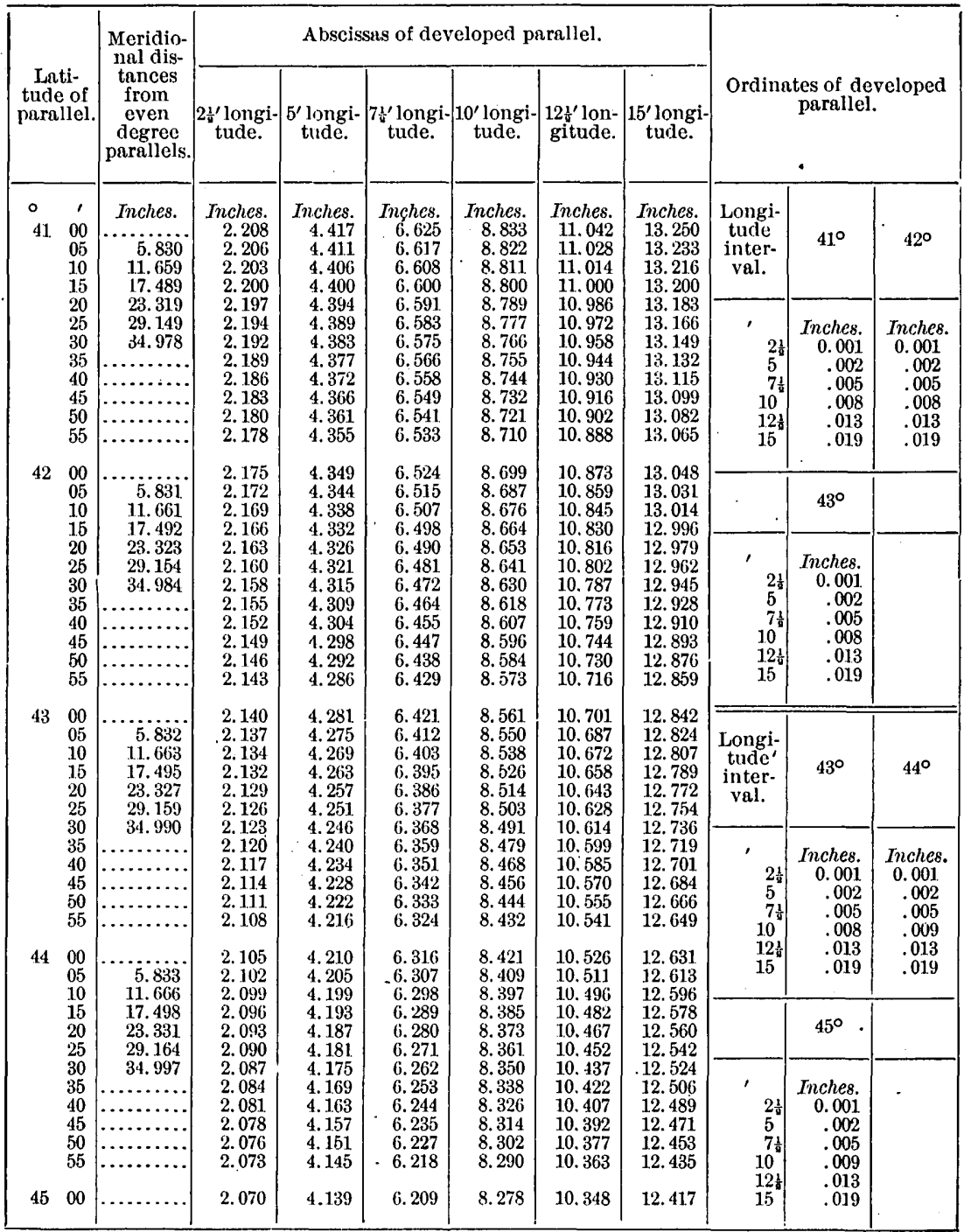


TABLE 7.-Coordinates for projection of maps (scale $\left.\frac{15}{50 \sigma}\right)$-Continued.

[From Smithsonian Geographical Tables.]

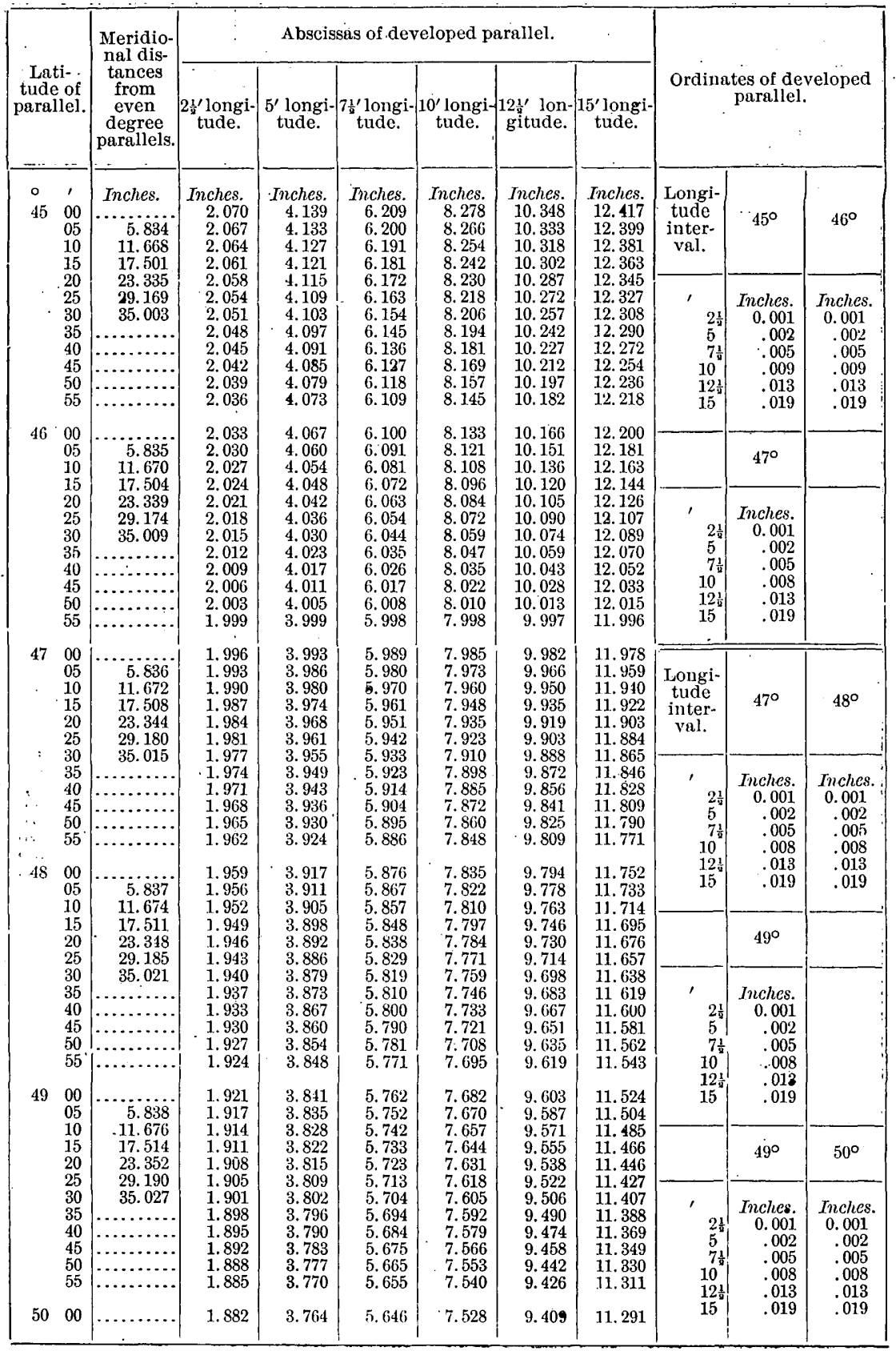


TABLE 8.-Coordinates for projection of maps (scale $\frac{1}{4500 \sigma}$ ).

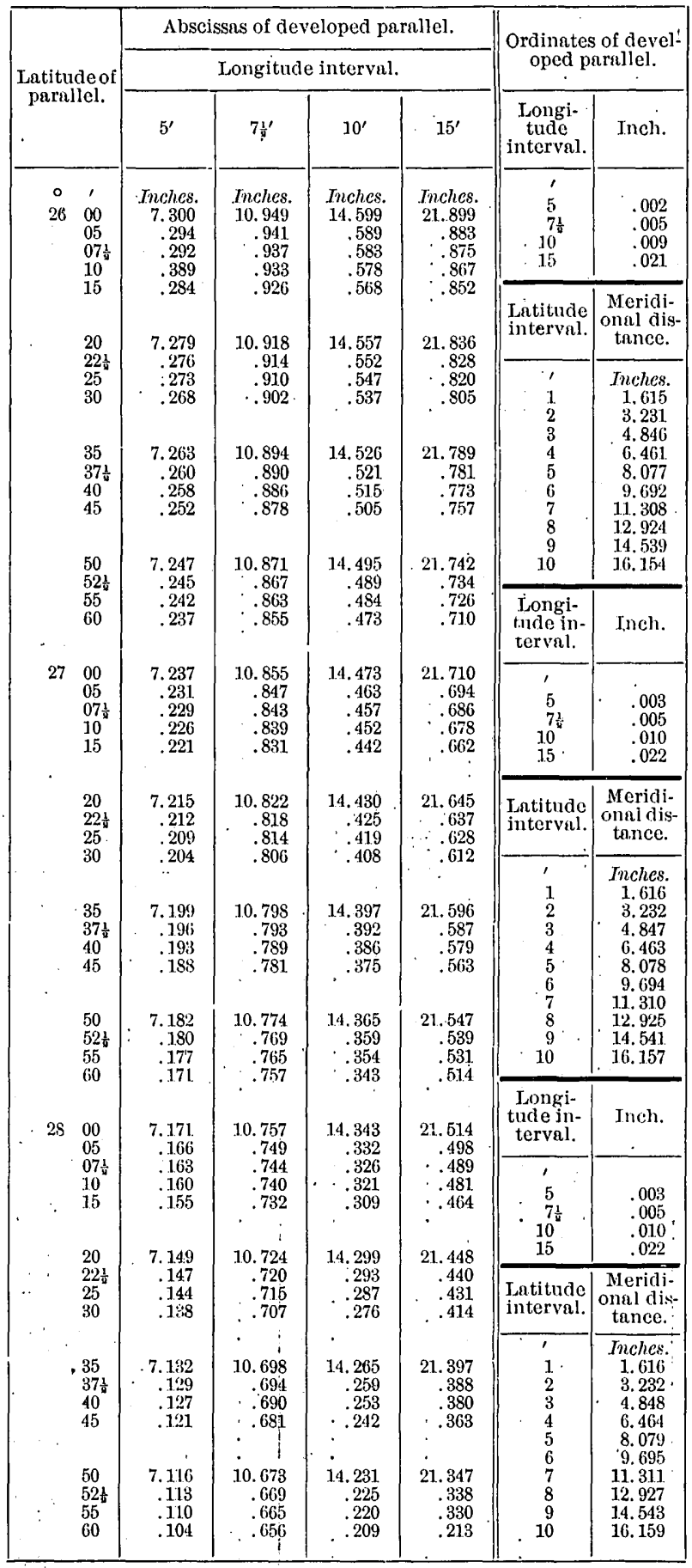


TABLE 8.-Coordinates for projection of maps (scale $\left.{ }_{4}{ }_{3}^{\frac{1}{0}}{ }_{0 \gamma}.\right)$-Continued.

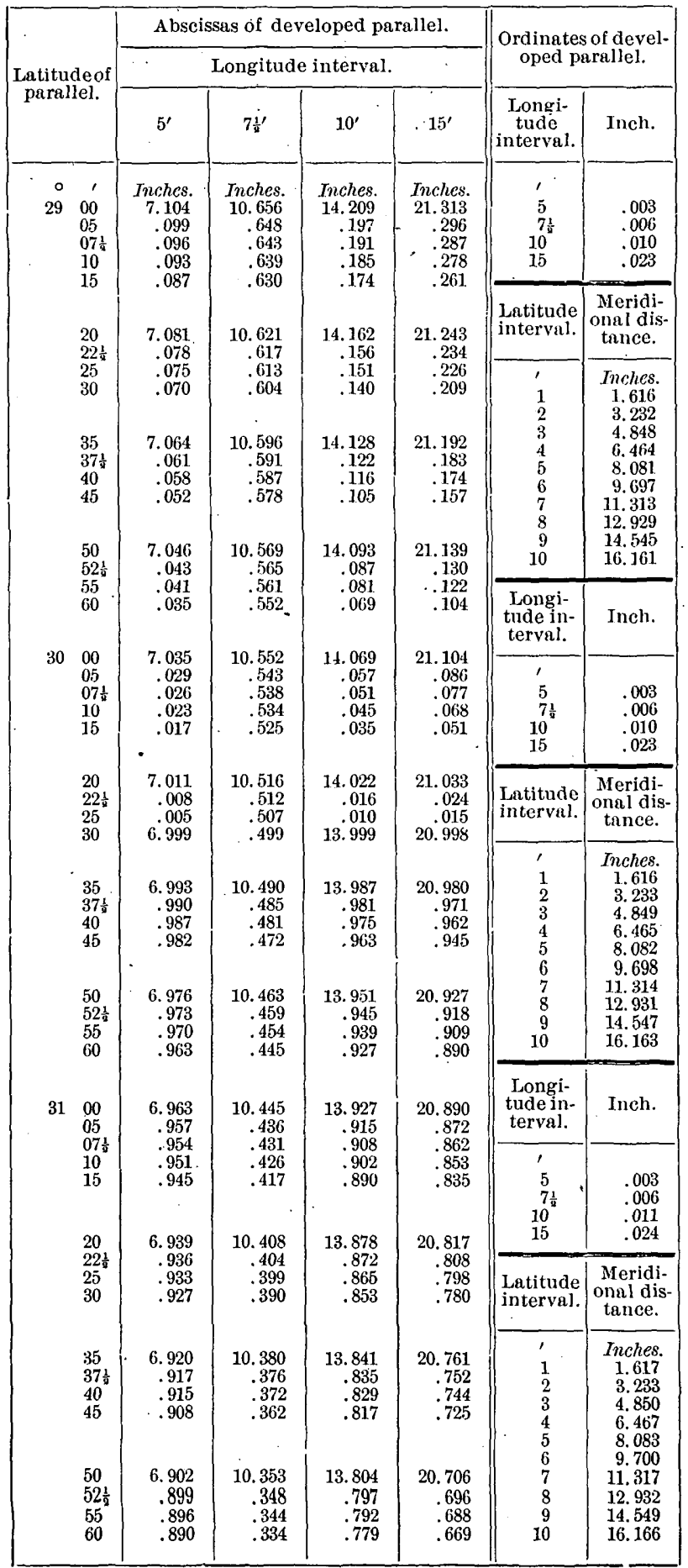


Taвte 8.-Coordinates for projection of maps (scale $\left.{ }_{450} \frac{1}{5 \sigma}\right)$-Continued.

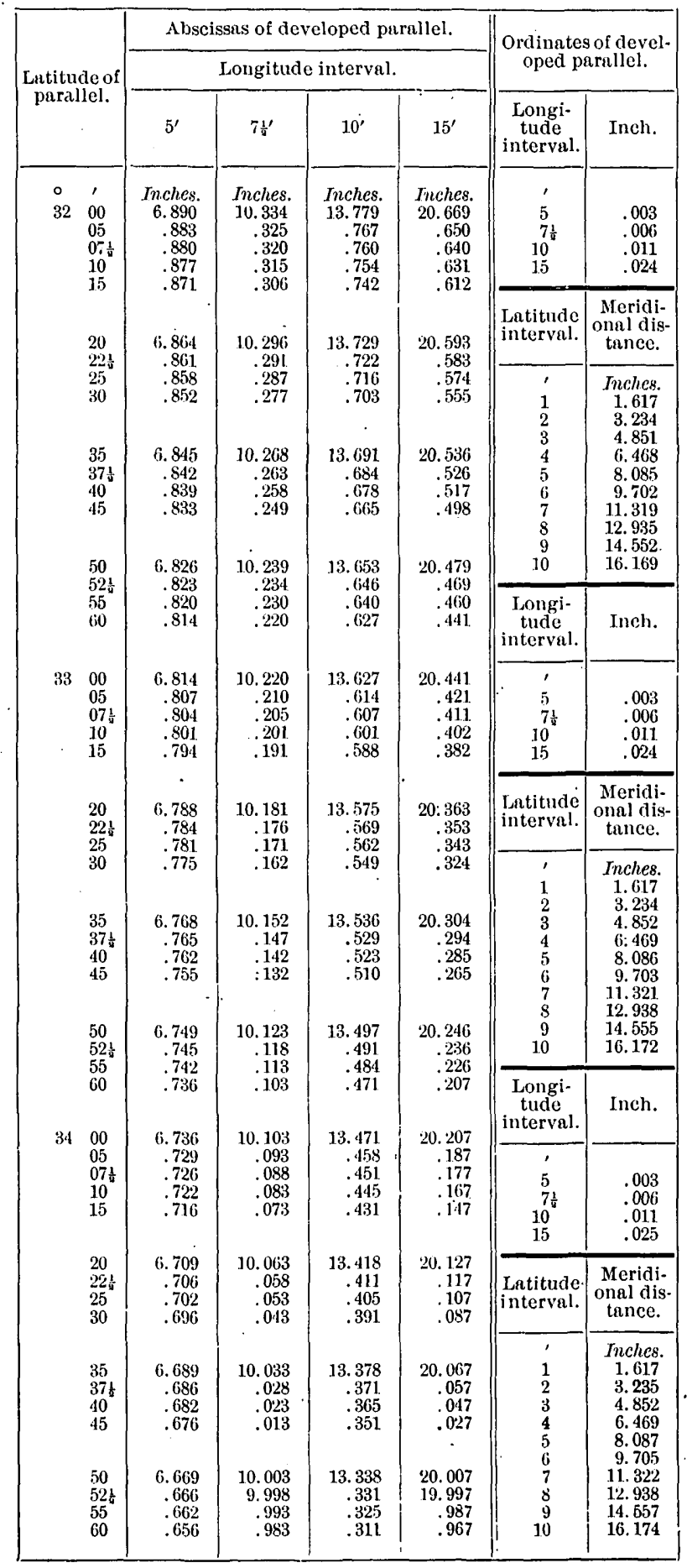


TABLe 8.-Coordinates for projection of maps (scale, $\left.{ }_{45} \frac{1}{0 \sigma \sigma}\right)$-Continued.

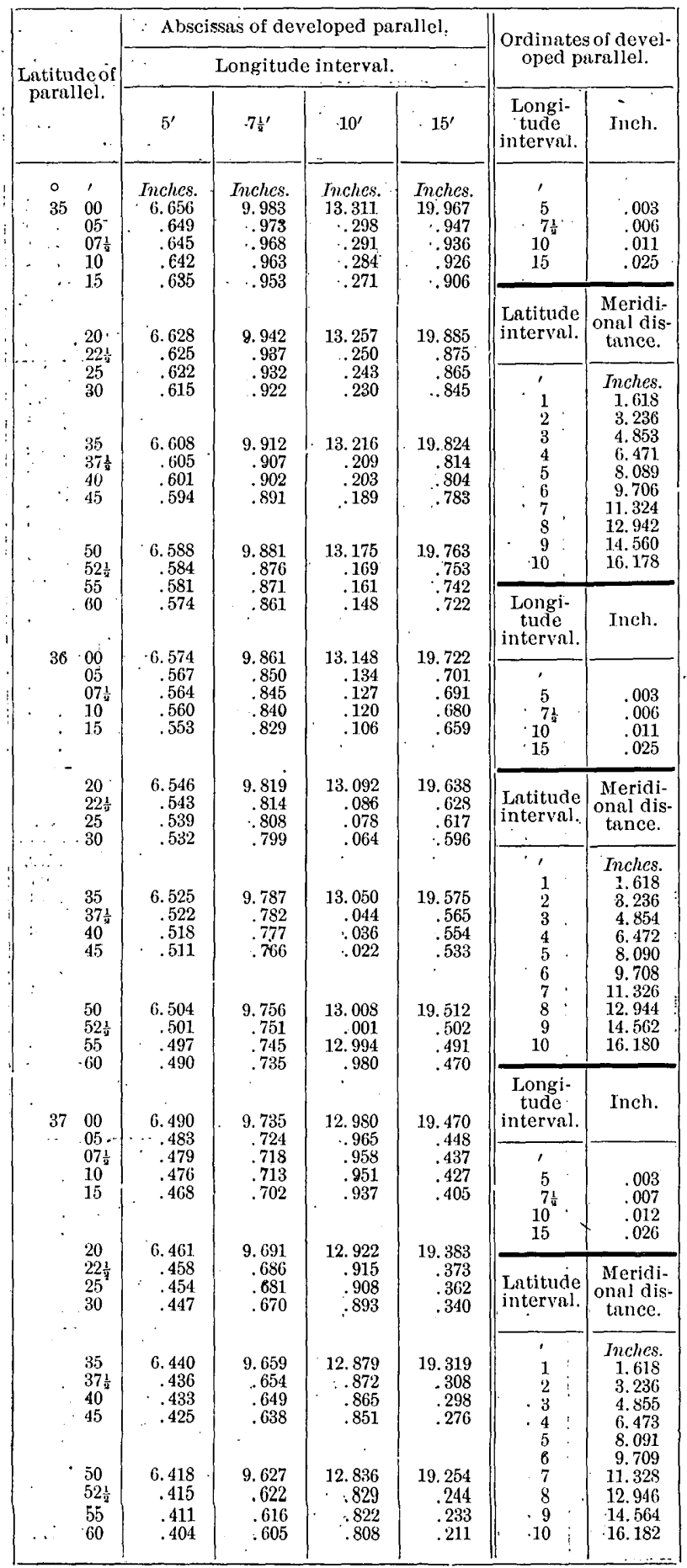




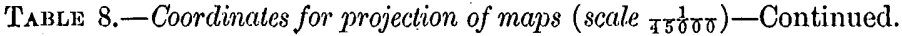

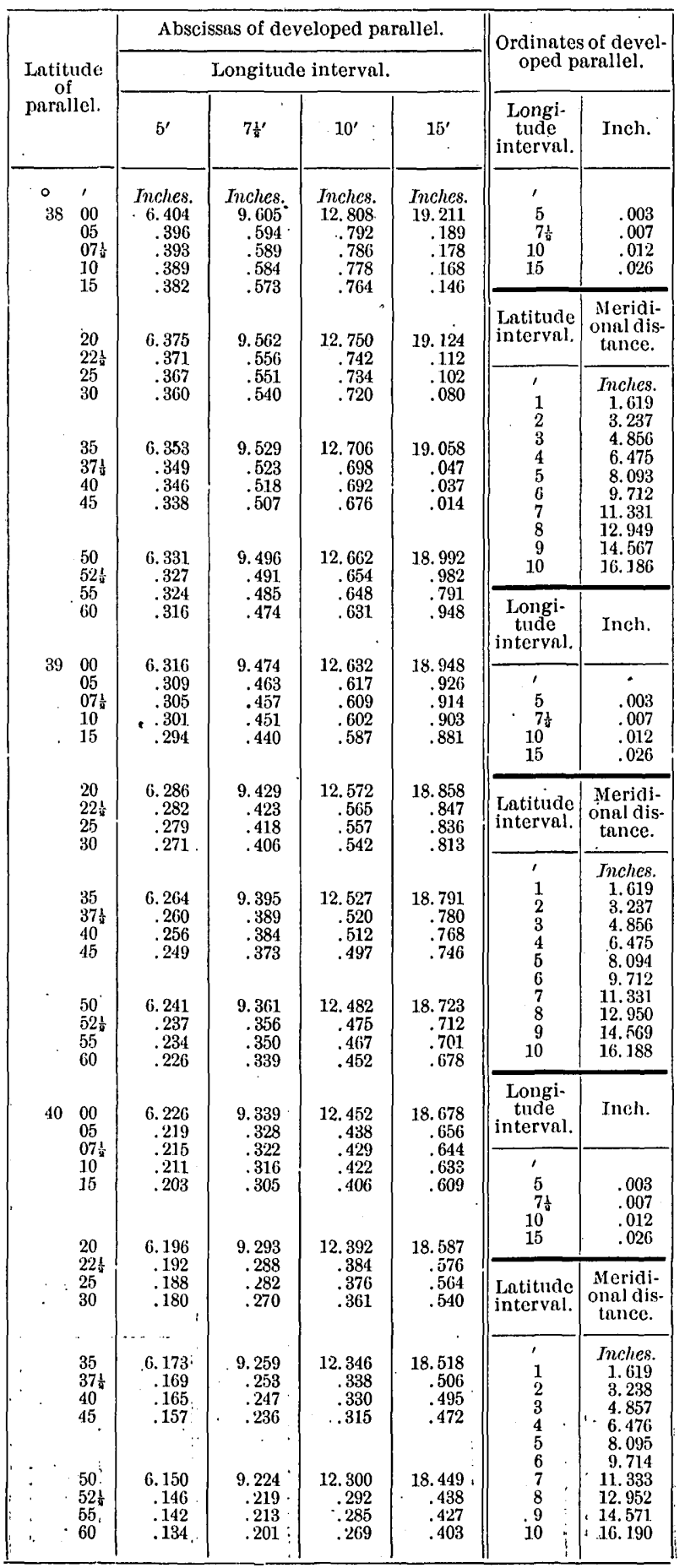


TABLE 8.-Coordinates for projection of maps (scale ${ }_{4500 \sigma}$ )-Continued.

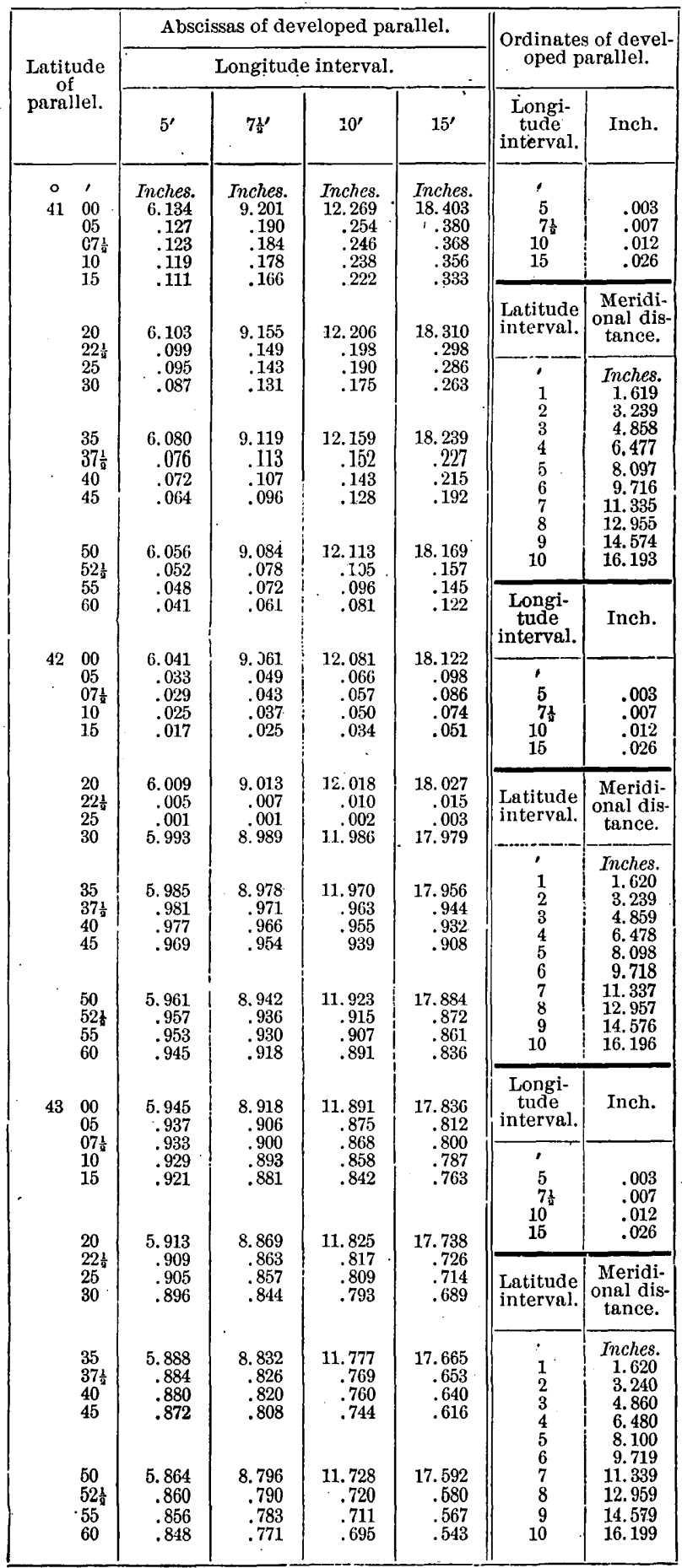


TABLe 8.-Coordinates for projection of maps (scale $\left.\frac{1}{450 \delta \delta}\right)$-Continued. .

\begin{tabular}{|c|c|c|c|c|c|c|c|}
\hline \multirow{3}{*}{\multicolumn{2}{|c|}{$\begin{array}{c}\text { Latitude } \\
\text { of } \\
\text { parallel. }\end{array}$}} & \multirow{2}{*}{\multicolumn{4}{|c|}{ Abscissas of developed parallel. }} & \multirow{2}{*}{\multicolumn{2}{|c|}{$\begin{array}{l}\text { Ordinates of devel- } \\
\text { oped parallel. }\end{array}$}} \\
\hline & & & & & & & \\
\hline & & $5^{\prime}$ & $7 \frac{1}{1}^{\prime}$. & $10^{\prime}$. & $15^{\prime}$. & $\begin{array}{c}\text { Longi- } \\
\text { tude } \\
\text { interval. }\end{array}$ & Inch. \\
\hline \multirow[t]{6}{*}{$\begin{array}{c}\circ \\
44\end{array}$} & \multirow{3}{*}{$\begin{array}{l}\prime \\
00 \\
05 \\
07 \frac{1}{8} \\
10 \\
15 \\
\\
20 \\
22 \frac{1}{4} \\
25 \\
30\end{array}$} & $\begin{array}{r}\text { Inches. } \\
5.848 \\
.839 \\
.835 \\
.831 \\
.823\end{array}$ & $\begin{array}{r}\text { Inches. } \\
8.771 \\
.759 \\
.753 \\
.746 \\
.734\end{array}$ & $\begin{array}{r}\text { Inches. } \\
11.695 \\
.679 \\
.670 \\
.662 \\
.646\end{array}$ & \multirow[t]{2}{*}{$\begin{array}{r}\text { Tnches. } \\
17.543 \\
.518 \\
.505 \\
493 \\
.469\end{array}$} & $\begin{array}{c}1 \\
-5 \\
7 \frac{1}{9} \\
10 \\
15\end{array}$ & $\begin{array}{l}.003 \\
.007 \\
.012 \\
.027 \\
\end{array}$ \\
\hline & & \multirow{2}{*}{$\begin{array}{r}6.815 \\
.810 \\
.806 \\
.798\end{array}$} & \multirow{2}{*}{$\begin{array}{r}8.722 \\
.715 \\
.709 \\
.697\end{array}$} & \multirow{2}{*}{$\begin{array}{r}11.629 \\
.621 \\
.613 \\
.596\end{array}$} & & $\begin{array}{l}\text { Latitude } \\
\text { interval. }\end{array}$ & $\begin{array}{c}\text { Meridi- } \\
\text { onal dis- } \\
\text { tance. }\end{array}$ \\
\hline & & & & & $\begin{array}{r}17.444 \\
.431 \\
.419 \\
.394\end{array}$ & $\begin{array}{l}1 \\
1 \\
2 \\
3\end{array}$ & $\begin{array}{c}\text { Inches. } \\
1.620 \\
3.240 \\
4.861\end{array}$ \\
\hline & $\begin{array}{l}35 \\
37 \frac{1}{8} \\
40 \\
45\end{array}$ & $\begin{array}{r}5.790 \\
.786 \\
.782 \\
.773\end{array}$ & $\begin{array}{r}8.685 \\
.678 \\
.672 \\
.660\end{array}$ & $\begin{array}{r}11.580 \\
.571 \\
.563 \\
.547\end{array}$ & $\begin{array}{r}17.370 \\
.357 \\
.345 \\
.320\end{array}$ & $\begin{array}{r}4 \\
5 \\
6 \\
7 \\
8 \\
9 \\
10\end{array}$ & $\begin{array}{r}6.481 \\
8.101 \\
9.721 \\
11.341 \\
12.962 \\
14.582 \\
16.202\end{array}$ \\
\hline & \multirow{2}{*}{$\begin{array}{l}50 \\
52 \frac{1}{2} \\
55 \\
60\end{array}$} & \multirow{2}{*}{$\begin{array}{l}6.765 \\
.761 \\
.757 \\
.749\end{array}$} & \multirow{2}{*}{$\begin{array}{r}8.647 \\
.641 \\
.635 \\
.623\end{array}$} & \multirow{2}{*}{$\begin{array}{r}11.530 \\
.523 \\
.514 \\
.497\end{array}$} & \multirow{2}{*}{$\begin{array}{r}17.295 \\
.284 \\
.271 \\
.246\end{array}$} & & \\
\hline & & & & & & $\begin{array}{c}\text { Longi- } \\
\text { tude } \\
\text { interval. }\end{array}$ & Inch. \\
\hline \multirow[t]{6}{*}{45} & \multirow[t]{3}{*}{$\begin{array}{l}00 \\
05 \\
07 \frac{1}{9} \\
10 \\
15\end{array}$} & $\begin{array}{r}5.749 \\
.740 \\
.736 \\
.732 \\
.724\end{array}$ & $\begin{array}{r}8.623 \\
.610 \\
.604 \\
.598 \\
.585\end{array}$ & $\begin{array}{r}11.497 \\
.481 \\
.472 \\
.464 \\
.447\end{array}$ & $\begin{array}{r}17.246 \\
.221 \\
.208 \\
.196 \\
.171\end{array}$ & $\begin{array}{c}1 \\
5 \\
7 \frac{1}{4} \\
10 \\
15\end{array}$ & $\begin{array}{l}.003 \\
.007 \\
.012 \\
.027\end{array}$ \\
\hline & & \multirow{2}{*}{$\begin{array}{r}5.715 \\
.711 \\
.707 \\
.699\end{array}$} & \multirow{2}{*}{$\begin{array}{r}8.573 \\
.567 \\
.560 \\
.548\end{array}$} & \multirow{2}{*}{$\begin{array}{r}11.431 \\
.423 \\
.414 \\
.397\end{array}$} & \multirow{2}{*}{$\begin{array}{r}17.146 \\
.134 \\
.121 \\
.096\end{array}$} & $\begin{array}{l}\text { Latitude } \\
\text { interval. }\end{array}$ & $\begin{array}{c}\text { Meridi- } \\
\text { onal dis- } \\
\text { tance. }\end{array}$ \\
\hline & & & & & & $\begin{array}{l}1 \\
1 \\
2\end{array}$ & $\begin{array}{c}\text { Inches. } \\
1.621 \\
3.241\end{array}$ \\
\hline & $\begin{array}{l}35 \\
37 \frac{1}{6} \\
40 \\
45\end{array}$ & $\begin{array}{r}5.690 \\
.686 \\
.682 \\
.673\end{array}$ & $\begin{array}{r}8.535 \\
.528 \\
.522 \\
.510\end{array}$ & $\begin{array}{r}11.380 \\
.371 \\
.363 \\
.347\end{array}$ & $\begin{array}{r}17.070 \\
.057 \\
.045 \\
.020\end{array}$ & $\begin{array}{l}3 \\
4 \\
5 \\
6 \\
7 \\
8\end{array}$ & $\begin{array}{r}4.862 \\
6.483 \\
8.103 \\
9.723 \\
11.345 \\
12.964\end{array}$ \\
\hline & \multirow{2}{*}{$\begin{array}{l}50 \\
52 \frac{1}{8} \\
55 \\
60\end{array}$} & \multirow{2}{*}{$\begin{array}{r}5.665 \\
.661 \\
.657 \\
.648\end{array}$} & \multirow{2}{*}{$\begin{array}{r}8.497 \\
.491 \\
.485 \\
.472\end{array}$} & \multirow{2}{*}{$\begin{array}{r}11.330 \\
.321 \\
.313 \\
.296\end{array}$} & $\begin{array}{r}16.995 \\
.982\end{array}$ & $\begin{array}{r}9 \\
10\end{array}$ & $\begin{array}{l}14.585 \\
16.206\end{array}$ \\
\hline & & & & & .944 & $\begin{array}{c}\text { Longi- } \\
\text { tude } \\
\text { interval. }\end{array}$ & Inch. \\
\hline 46 & $\begin{array}{l}00 \\
05 \\
07 \frac{1}{6} \\
10 \\
15\end{array}$ & $\begin{array}{r}5.648 \\
.639 \\
.635 \\
.631 \\
.622\end{array}$ & $\begin{array}{r}8.472 \\
.459 \\
.453 \\
.446 \\
.433\end{array}$ & $\begin{array}{r}11.296 \\
.278 \\
.271 \\
.262 \\
.245\end{array}$ & $\begin{array}{r}16.944 \\
.918 \\
.906 \\
.893 \\
.867\end{array}$ & $\begin{array}{c}\text { interval. } \\
5 \\
5 \\
7 \frac{1}{9} \\
10 \\
16\end{array}$ & $\begin{array}{l}.003 \\
.007 \\
.012 \\
.027\end{array}$ \\
\hline & $\begin{array}{l}20 \\
22 f \\
25 \\
30\end{array}$ & $\begin{array}{r}5.614 \\
.609 \\
.605 \\
.597\end{array}$ & $\begin{array}{r}8.420 \\
.414 \\
.408 \\
.395\end{array}$ & $\begin{array}{r}11.227 \\
.219 \\
.211 \\
.193\end{array}$ & $\begin{array}{r}16.841 \\
.828 \\
.816 \\
.790\end{array}$ & $\begin{array}{l}\text { Latitude } \\
\text { interval. }\end{array}$ & $\begin{array}{l}\text { Meridi- } \\
\text { onal dis- } \\
\text { tance. }\end{array}$ \\
\hline & $\begin{array}{l}35 \\
37 \frac{1}{4} \\
40 \\
45\end{array}$ & $\begin{array}{r}5.588 \\
.584 \\
.579 \\
.571\end{array}$ & $\begin{array}{r}8.382 \\
.376 \\
.369 \\
.356\end{array}$ & $\begin{array}{r}11.176 \\
.167 \\
.159 \\
.142\end{array}$ & $\begin{array}{r}16.764 \\
.751 \\
.738 \\
.713\end{array}$ & $\begin{array}{l} \\
1 \\
2 \\
3 \\
4 \\
5 \\
6\end{array}$ & $\begin{array}{r}\text { Inches. } \\
1.621 \\
3.242 \\
4.863 \\
6.484 \\
8.105 \\
9.725\end{array}$ \\
\hline & $\begin{array}{l}50 \\
52 \frac{1}{6} \\
55 \\
60\end{array}$ & $\begin{array}{r}5.562 \\
.558 \\
.554 \\
.545\end{array}$ & $\begin{array}{r}8.343 \\
.337 \\
.330 \\
.318\end{array}$ & $\begin{array}{r}11.125 \\
.117 \\
.107 \\
.091\end{array}$ & $\begin{array}{r}16.687 \\
.675 \\
.661 \\
.636\end{array}$ & $\begin{array}{r}7 \\
8 \\
9 \\
10\end{array}$ & $\begin{array}{l}11.347 \\
12.968 \\
14.588 \\
16.209\end{array}$ \\
\hline
\end{tabular}


TABLE 8.-Coordinates for projection of maps (scale $\left.\frac{150}{450 \sigma}\right)$-Continued.

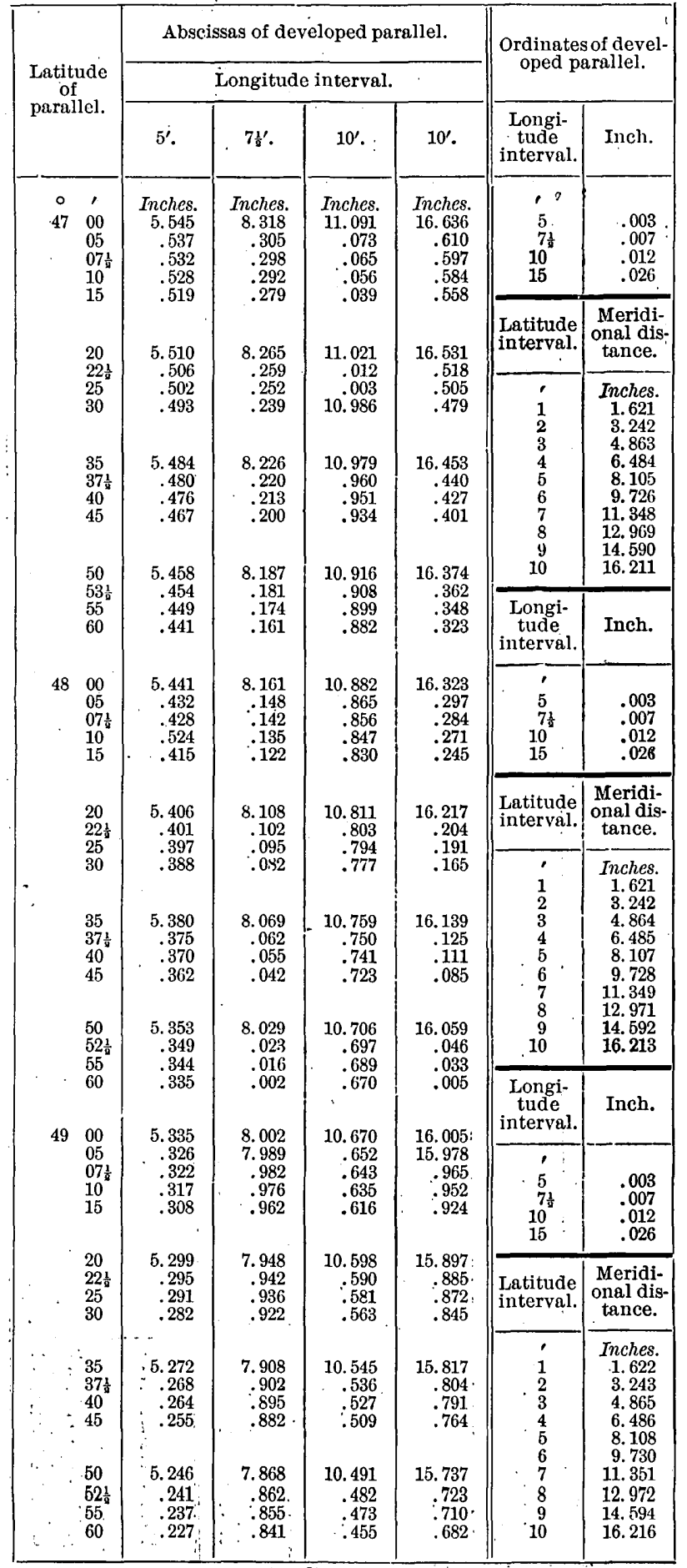


TABLE 9.-Areas of quadrilaterals of earth's surface of $30^{\prime}$ extent in latitude and longitude.

[From Smithsonian Geographical Tables.]

\begin{tabular}{|c|c|c|c|c|c|c|c|c|}
\hline \multicolumn{2}{|c|}{$\begin{array}{l}\text { Middle lati- } \\
\text { tude of } \\
\text { quadrilateral. }\end{array}$} & \multirow[t]{2}{*}{$\begin{array}{c}\text { Area in } \\
\text { square miles. }\end{array}$} & \multicolumn{2}{|c|}{$\begin{array}{l}\text { Middle lati- } \\
\text { tude of } \\
\text { quadrilateral. }\end{array}$} & \multirow[t]{2}{*}{$\begin{array}{c}\text { Area in } \\
\text { square miles. }\end{array}$} & \multicolumn{2}{|c|}{$\begin{array}{l}\text { Middle lati- } \\
\text { tude of } \\
\text { quadrilateral. }\end{array}$} & \multirow[t]{2}{*}{$\begin{array}{c}\text { Area in } \\
\text { square miles. }\end{array}$} \\
\hline 0 & 1 & & $\circ$ & ' & & 0 & 1 & \\
\hline 0 & 00 & $1,188,10$ & 11 & 00 & $1,166.84$ & 22 & 00 & $1,103.68$ \\
\hline 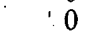 & 15 & $1,188.08$ & 11 & 15 & $1,165.86$ & 22 & 15 & $1,101.77$ \\
\hline 0 & 30 & $1,188.05$ & 11 & 30 & $1,164.86$ & 22 & 30 & $1,099.84$ \\
\hline 0 & 45 & $1,188.00$ & 11 & 45 & $1,163.85$ & 22 & 45 & $1,097.88$ \\
\hline 1 & 00 & $1,187.92$ & 12 & 00 & $1,162.81$ & 23 & 00 & $1,095.91$ \\
\hline 1 & 15 & $1,187.82$ & 12 & 15 & $1,161.75$ & 23 & 15 & $1,093.92$ \\
\hline 1 & 30 & $1,187.70$ & 12 & 30 & $1,160.67$ & 23 & 30 & $1,091.90$ \\
\hline 1 & 45 & $1,187.56$ & 12 & 45 & $1,159.56$ & 23 & 45 & $1,089.87$ \\
\hline 2 & 00 & $1,187.39$ & 13 & 00 & $1,158.44$ & 24 & 00 & $1,087.81$ \\
\hline 2 & 15 & $1,187.20$ & 13 & 15 & $1,157.29$ & 24 & 15 & $1,085.74$ \\
\hline 2 & 30 & $1,186.09$ & 13 & 30 & $1,156.12$ & 24 & 30 & $1,083.64$ \\
\hline 2 & 45 & $1,186.76$ & 13 & 45 & $1,154.93$ & 24 & 45 & $1,081.52$ \\
\hline 3 & 00 & $1,186.51$ & 14 & 00 & $1,153.72$ & 25 & 00 & $1,079.39$ \\
\hline 3 & 15 & $1,186.24$ & 14 & 15 & $1,152.48$ & 25 & 15 & $1,077.23$ \\
\hline 3 & 30 & $1,185.95$ & 14 & 30 & $1,151.23$ & 25 & 30 & $1,075.05$ \\
\hline 3 & 45 & $1,185.62$ & 14 & 45 & $1,149.95$ & 25 & 45 & $1,072.85$ \\
\hline 4 & 00 & $1,185.28$ & 15 & 00 & $1,148.65$ & 26 & 00 & $1,070,64$ \\
\hline 4 & 15 & $1,184.92$ & 15 & 15 & $1,147.33$ & 26 & 15 & $1,068.40$ \\
\hline 4 & 30 & $1,184.53$ & 15 & 30 & $1,145.99$ & 26 & 30 & $1,066.14$ \\
\hline 4 & 45 & $1,184.13$ & 15 & 45 & $1,144.63$ & 26 & 45 & $1,063.86$ \\
\hline 5 & 00 & $1,183.70$ & 16 & 00 & $1,143.25$ & 27 & 00 & $1,061.56$ \\
\hline 5 & 15 & 1183.24 & 16 & 15 & 114184 & 27 & 15 & 1.059 .24 \\
\hline 5 & 30 & $1,182.77$ & 16 & 30 & 1. 140.41 & 27 & 30 & $1,056.90$ \\
\hline 5 & 45 & $1,182.28$ & 16 & 45 & $1,138.96$ & 27 & 45 & $1,054.54$ \\
\hline 6 & 00 & $1,181.76$ & 17 & 00 & $1,137.50$ & 28 & 00 & $1,052.16$ \\
\hline 6 & 15 & $1,181.22$ & 17 & 15 & $1,136.00$ & 28 & 15 & $1,049.76$ \\
\hline 6 & 30 & $1,180.66$ & 17 & 30 & $1,104.49$ & 28 & $30^{\circ}$ & $1,047.34$ \\
\hline 6 & 45 & $1,180.08$ & 17 & 45 & $1,132.96$ & 28 & 45 & $1,044.90$ \\
\hline 7 & 00 & $1,179.48$ & 18 & 00 & $1,131.41$ & 29 & 00 & $1,042.44$ \\
\hline 7 & 15 & $1,178.85$ & 18 & 15 & $1,129.83$ & 29 & 15 & $1,039.97$ \\
\hline 7 & 30 & $1,178.20$ & 18 & 30 & $1,128.24$ & 29 & 30 & $1,037.47$ \\
\hline 7 & 45 & $1,177.53$ & 18 & 45 & $1,126.62$ & 29 & 45 & $1,034.95$ \\
\hline 8 & 00 & $1,176.84$ & 19 & 00 & $1,124.98$ & 30 & 00 & $1,032.41$ \\
\hline 8 & 15 & $1,176.13$ & 19 & 15 & $1,123.32$ & 30 & 15 & $1,029.85$ \\
\hline 8 & 30 & $1,175.39$ & 19 & 30 & $1,121.64$ & 30 & 30 & $1,027.27$ \\
\hline 8 & 45 & $1,174.63$ & 19 & 45 & $1,119.93$ & 30 & 45 & $1,024.68$ \\
\hline 9 & 00 & $1,173.86$ & 20 & 00 & $1,118.21$ & 31 & 00 & $1,022.06$ \\
\hline 9 & 15 & $1,173.06$ & 20 & 15 & $1,116.47$ & 31 & 15 & $1 ; 019: 43$ \\
\hline 9 & 30 & $1,172.23$ & 20 & 30 & $1,114.71$ & 31 & 30 & $1,016.77$ \\
\hline 9 & 45 & $1,171.39$ & 20 & 45 & $1,112.92$ & 31 & 45 & $1,014.10$ \\
\hline 10 & .00 & $1,170.52$ & 21 & 00 & $1,111.11$ & 32 & 00 & $1,011.40$ \\
\hline 10 & .15 & $1,169.63$ & 21 & 15 & $1,109.28$ & 32 & 15 & $1,008.69$ \\
\hline 10 & 30 & $1,168.73$ & 21 & 30 & $1,107.44$ & 32 & 30 & 1., 005.96 \\
\hline 10 & 45 & $1,167.80$ & 21 & 45 & $1,105.57$ & 32 & 45 & $1,003.20$ \\
\hline
\end{tabular}


TABLE 9.-Areas of quadrilaterals of earth's surface of $30^{\prime}$ extent in latitude and longitude-Continued.

[From Smithsonian Geographical Tables.]

\begin{tabular}{|c|c|c|c|c|c|c|c|c|}
\hline \multicolumn{2}{|c|}{$\begin{array}{l}\text { Middle lati- } \\
\text { tude of } \\
\text { quadrilateral. }\end{array}$} & \multirow[t]{2}{*}{$\begin{array}{c}\text { Area in } \\
\text { square miles. }\end{array}$} & \multicolumn{2}{|c|}{$\begin{array}{l}\text { Middle lati- } \\
\text { tude of } \\
\text { quadrilateral. }\end{array}$} & \multirow[t]{2}{*}{$\begin{array}{l}\text { Arẹa in } \\
\text { square miles. }\end{array}$} & \multicolumn{2}{|c|}{$\begin{array}{l}\text { Middle lati- } \\
\text { tude of } \\
\text { quadrilateral. }\end{array}$} & \multirow[t]{2}{*}{$\begin{array}{c}\text { Area in } \\
\text { square miles. }\end{array}$} \\
\hline$\circ$ & ' & & & $!$ & & $\circ$ & 1 & \\
\hline 33 & 00 & $1,000,43$ & & 00 & 860.25 & 55 & 00 & 687.70 \\
\hline 33 & 15 & 997.64 & 44 & 15 & 856.67 & 55 & 15 & 683.44 \\
\hline 33 & 30 & 994.83 & 44 & 30 & 853.07 & 55 & 30 & 679.17 \\
\hline 33 & 45 & 992.00 & 44 & 45 & 849.46 & 55 & 45 & 674.89 \\
\hline 34 & 00 & 989.16 & 45 & 00 & 845.82 & 56 & 00 & 670.60 \\
\hline 34 & 15 & 986.29 & 45 & 15 & 842.18 & 56 & 15 & 666.29 \\
\hline 34 & 30 & 983.41 & 45 & 30 & 838.51 & 56 & 30 & 661.97 \\
\hline 34 & 45 & 980.50 & 45 & 45 & 834.83 & 56 & 45 & 657.64 \\
\hline 35 & 00 & 977.58 & 46 & 00 & 831.13 & 57 & 00 & 653.29 \\
\hline 35 & 15. & 974.64 & 46 & 15 & 827.42 & 57 & 15 & 648.93 \\
\hline 35 & 30 & 971.68 & 46 & .30 & 823.68 & 57 & 30 & 644.55 \\
\hline 35 & 45 & 968.70 & 46 & 45 & 819.94 & 57 & 45 & 640.17 \\
\hline 36 & 00 & 965.70 & 47 & 00 & 816. 18 & 58 & 00 & 635.77 \\
\hline 36 & 15 & 962.68 & 47 & 15 & 812.40 & 58 & 15 & 631.36 \\
\hline 36 & 30 & 959.65 & 47 & 30 & 808.60 & 58 & 30 & 626.93 \\
\hline 36 & 45 & 956.60 & 47 & 45 & 804.79 & 58 & 45 & 622.49 \\
\hline 37 & 00 & 953.52 & 48 & 00 & 800.97 & 59 & 00 & 618.05 \\
\hline 37 & 15 & 950.43 & 48 & 15 & 797.13 & 59 & 15 & 613.59 \\
\hline 37 & 30 & 947.32 & 48 & 30 & 793.27 & 59 & 30 & 609.11 \\
\hline 37 & 45 & 944.21 & 48 & 45 & 789.39 & 59 & 45 & 604.62 \\
\hline 38 & 00 & 941.05 & 49 & 00 & 785.50 & 60 & 00 & 600.13 \\
\hline 38 & 15. & 937.88 & 49 & 15 & 781.60 & 60 & 15 & 595.62 \\
\hline 38 & 30 & 934.71 & 49 & 30 & 777.68 & 60 & 30 & 591.09 \\
\hline 38 & 45 & 931.51 & 49 & 45 & 773.74 & 60 & 45 & 586.56 \\
\hline 39 & 00 & 928.29 & 50 & 00 & 769.79 & 61 & 00 & 582.01 \\
\hline 39 & 15 & 925.06 & 50 & 15 & 765.83 & 61. & 15 & 577.45 \\
\hline 39 & 30 & 921.80 & 50 & 30 & 761.85 & 61 & 30 & 572.88 \\
\hline 39 & 45 & 918.53 & 50 & 45 & 757.85 & 61 & 45 & 568.30 \\
\hline 40 & 00 & 915.25 & 51 & 00 & 753.84 & 62 & 00 & 563.71 \\
\hline 40 & 15 & 911.94 & 51 & 15 & 749.82 & 62 & 15 & 559.11 \\
\hline 40 & 30 & 908.61 & 51 & 30 & 745.78 & 62 & 30 & 554.49 \\
\hline 40 & 45 & 905.27 & 51 & 45 & 741.72 & 62 & 45 & 549.86 \\
\hline 41 & 00 & 901.91 & 52 & 00 & 737.65 & 63 & 00 & 545.23 \\
\hline 41 & 15 & 898.54 & 52 & 15 & 733.57 & 63 & 15 & 540.58 \\
\hline 41 & 30 & 895.14 & 52 & 30 & 729.47 & 63 & 30 & 5.35 .92 \\
\hline 41 & $4 \tilde{5}$ & 891.73 & 52 & 45 & 725.36 & 63 & 45 & 531.25 \\
\hline 42 & 00 & 888.30 & 53 & 00 & 721.23 & 64 & 00 & 526.57 \\
\hline 42 & 15 & 884.85 & 53 & 15 & 717.08 & 64 & 15 & 521.88 \\
\hline 42 & 30 & 881.39 & 53 & 30 & 712.93 & 64 & 30 & 517.17 \\
\hline 42 & 45 & 877.91 & 53 & 45 & 708.76 & 64 & 45 & 512.46 \\
\hline 43 & 00 & 874.41 & 54 & 00 & 704.57 & 65. & 00 & 507.74 \\
\hline 43 & 15 & 870.90 & 54. & 15 & 700.38 & 65 & 15 & 503.01 \\
\hline 43 & 30 & 867.37 & 54 & 30 & 696.16 & 65 & 30 & 498.26 \\
\hline 43 & 45 & 863.82 & 54 & 45 & 691.94 & 65 & 45 & 493.51 \\
\hline
\end{tabular}


TAвтs 9.-Areas of quadrilaterals of earth's surface of $30^{\prime}$ extent in latitude and longitude-Continued.

[From Smithsonian Geographical Tables.]

\begin{tabular}{|c|c|c|c|c|c|c|c|c|}
\hline \multicolumn{2}{|c|}{$\begin{array}{l}\text { Middle lntitude } \\
\text { of quadrilat- } \\
\text { eral. }\end{array}$} & \multirow[t]{2}{*}{$\begin{array}{c}\text { Area in square } \\
\text { miles. }\end{array}$} & \multicolumn{2}{|c|}{$\begin{array}{l}\text { Middle latitude } \\
\text { of quadrilat- } \\
\text { eral. }\end{array}$} & \multirow[t]{2}{*}{$\begin{array}{c}\text { Area in square } \\
\text { miles. }\end{array}$} & \multicolumn{2}{|c|}{$\begin{array}{c}\text { Middle latitude } \\
\text { of quadrilat- } \\
\text { eral. }\end{array}$} & \multirow[t]{2}{*}{$\begin{array}{c}\text { Area in square } \\
\text { miles. }\end{array}$} \\
\hline$\circ$ & , & & 。 & , & & ० & , & \\
\hline 66 & 00 & 488.75 & 74 & 00 & 331.62 & 82 & 00 & 167.57 \\
\hline 66 & 15 & 483.97 & 74 & 15 & 326.58 & 82 & 15 & 162.37 \\
\hline 66 & 30 & 479.19 & 74 & 30 & 321.53 & 82 & 30 & 157.16 \\
\hline 66 & 45 & 474.40 & 74 & 45 & 316.48 & 82 & 45 & 151.95 \\
\hline 67 & 00 & 469.60 & 75 & 00 & 311.42 & 83 & 00 & 146.74 \\
\hline 67 & 15 & 464.78 & 75 & 15 & 306.36 & 83 & 15 & 141.53 \\
\hline 67 & 30 & 459.96 & 75 & 30 & 301.28 & 83 & 30 & 1.36. 31 \\
\hline 67 & 45 & 455.13 & 75 & 45 & 296.21 & 83 & 45 & 131. 09 \\
\hline 68 & 00 & 450.29 & 76 & 00 & 291. 12 & 84 & 00 & 125.87 \\
\hline 68 & 15 & 445.45 & 76 & 15 & 286.04 & 84 & 15 & 120.64 \\
\hline 68 & 30 & 440.59 & 76 & 30 & 280.94 & 84 & 30 & 115. 42 \\
\hline 68 & 45 & 435.72 & 76 & 45 & 275.84 & 84 & 45 & 110.18 \\
\hline 69 & 00 & 430.84 & 77 & 00 & 270.73 & 85 & 00 & 104.95 \\
\hline 69 & 15 & 425.96 & 77 & 15 & 265.62 & 85. & 15 & 99.72 \\
\hline 69 & 30 & 421.06 & 77 & 30 & 260.50 & 85 & 30 & 94.48 \\
\hline 69 & 45 & 416. 16 & 77 & 45 & 255.38 & 85 & 45 & 89.25 \\
\hline 70 & 00 & 411.25 & 78 & 00 & 250.25 & 86 & 00 & 84.01 . \\
\hline 70 & 15 & 406.34 & 78 & 15 & 245.12 & 86 & 15 & 78.76 \\
\hline 70 & 30 & 401.41 & 78 & 30 & 239.98 & 86 & 30 & 73.52 \\
\hline 70 & 45 & 396.47 & 78 & 45 & 234.83 & 86 & 45 & 68.27 \\
\hline 71 & 00 & 391.53 & 79 & 00 & 229.68 & 87 & 00 & 63.03 \\
\hline 71. & 15 & 386.58 & 79 & 15 & 224.53 & 87 & 15 & 57.78 \\
\hline 71 & 30 & 381.62 & 79 & 30 & 219.37 & $87^{\circ}$ & 30 & 52.53 \\
\hline 71 & 45 & 376.65 & 79 & 45 & 214.21 & 87 & 45 & 47.28 \\
\hline 72 & 00 & 371.68 & 80 & 00 & 209.05 & 88 & 00 & 42.03 \\
\hline 72 & 15 & 366.70 & 80 & 15 & 203.88 & 88 & 15 & 36.78 \\
\hline 72 & 30 & 361.71 & 80 & 30 & 198.70 & 88 & 30 & 31.53 \\
\hline 72 & 45 & 356.71 & 80 & 45 & 193.52 & 88 & 45 & 26. 27 \\
\hline 73 & 00 & 351.71 & 81 & 00 & 188.34 & 89 & 00 & 21.02 \\
\hline 73 & 15 & 346.69 & 81 & 15 & 183.15 & 89 & 15 & 15.76 \\
\hline 73 & 30 & 341.68 & 81. & 30 & 177.96 & 89 & 30 & 10.51 \\
\hline 73 & 45 & 336.65 & 81 & 45 & 172.77 & 89 & 45 & 5.26 \\
\hline
\end{tabular}


TABLE 10.- Areas of quadrilaterals of earth's surface of $15^{\prime}$ extent in latitude and longitude.

[From Smithsonian Geographical Tables.]

\begin{tabular}{|c|c|c|c|c|c|c|c|c|c|c|c|}
\hline \multicolumn{3}{|c|}{$\begin{array}{l}\text { Middle latitude } \\
\text { of quadrilateral. }\end{array}$} & \multirow[t]{2}{*}{$\begin{array}{c}\text { Area in } \\
\text { square miles: }\end{array}$} & \multicolumn{3}{|c|}{$\begin{array}{l}\text { Middle latitude } \\
\text { of quadrilateral. }\end{array}$} & \multirow[t]{2}{*}{$\begin{array}{c}\text { Area in } \\
\text { squaremiles. }\end{array}$} & \multicolumn{3}{|c|}{$\begin{array}{l}\text { Middle latitude } \\
\text { of quadrilateral. }\end{array}$} & \multirow[t]{2}{*}{$\begin{array}{l}\text { Area in } \\
\text { squaremiles }\end{array}$} \\
\hline$\circ$ & $i$ & $\prime \prime$ & & $\circ$ & 1 & $\prime \prime$ & & $\circ$ & , & "I & \\
\hline 0 & 07 & 30 & 297.02 & 5 & 37 & 30 & 295.63 & 11 & 07 & 30 & 291.59 \\
\hline 0 & 15 & 00 & 297.02 & 5 & 45 & 00 & 295.57 & 11 & 15 & 00 & 291.47 \\
\hline 0 & 22 & 30 & 297.02 & 5 & 52 & 30 & 295.51 & 11 & 22 & 30 & 291. 34 \\
\hline 0 & 30 & 00 & 297.01 & 6 & 00 & 00 & 295.44 & 11 & 30 & 00 & 291.22 \\
\hline 0 & 37 & 30 & 297.01 & 6 & 07 & 30 & 295.37 & 11 & 37 & 30 & 291.09 \\
\hline 0 & 45 & 00 & 297.00 & 6 & 15 & 00 & 295.31 & 11 & 45 & 00 & 290.96 \\
\hline 0 & 52 & 30 & 296.99 & 6 & 22 & 30 & 295.24 & 11 & 52 & 30 & 290.83 \\
\hline 1 & 00 & 00 & 296.98 & 6 & 30 & 00 & 295.17 & 12 & 00 & 00 & 290.70 \\
\hline 1 & 07 & 30 & 296.97 & 6 & 37 & 30 & 295.09 & 12 & 07 & 30 & 290.57 \\
\hline 1 & 15 & 00 & 296.96 & 6 & 45 & 00 & 295.02 & 12 & 15 & 00 & 290.44 \\
\hline 1 & 22 & 30 & 296.94 & 6 & 52 & 30 & 294.95 & 12 & 22 & 30 & 290.30 \\
\hline 1 & 30 & 00 & 296.93 & 7 & 00 & 00 & 294.87 & 12 & 30 & 00 & 290.17 \\
\hline 1 & 37 & 30 & 296. 91 & 7 & 07 & 30 & 294.79 & 12 & 37 & 30 & 290.03 \\
\hline 1 & 45 & 00 & 296.89 & 7 & 15 & 00 & 294.71 & 12 & 45 & 00 & 289.89 \\
\hline 1. & 52 & 30 & 296.87 & 7 & 22 & 30 & 294.63 & 12 & 52 & 30 & 289.75 \\
\hline 2 & 00 & 00 & 296.85 & 7 & 30 & 00 & 294.55 & 13 & 00 & 00 & 289.61 \\
\hline 2 & 07 & 30 & 296.82 & 7 & 37 & 30 & 294.47 & 13 & 07 & 30 & 289.47 \\
\hline 2 & 15 & 00 & 296.80 & 7 & 45 & 00 . & 294.39 & 13 & 15 & 00 & 289.33 \\
\hline 2 & 22 & 30 & 296.77 & 7 & 52 & 30 & 294.30 & 13 & 22 & 30 & 289.18 \\
\hline 2 & 30 & 00 & 296.75 & 8 & 00 & 00 & 294.21 & 13 & 30 & 00 & 289.03 \\
\hline 2 & 37 & 30. & 296. 72 & 8 & 07 & $30^{\circ}$ & 294.12 & 13 & 37 & 30 & 288.88 \\
\hline 2 & 45 & 00 & 296.69 & 8 & 15 & 00 & 294.03 & 13 & 45 & 00 & 288.73 \\
\hline 2 & 52 & 30 & 296.66 & 8 & 22 & 30 & 293.94 & 13 & 52 & 30 & 288.58 \\
\hline 3 & 00 & 00 & 296.63 & 8 & 30 & 00 & 293.85 & 14 & 00 & 00 & 288.43 \\
\hline 3 & 07 & 30 & 296.60 & 8 & 37 & 30 & 293.75 & 14 & 07 & 30 & 288.28 \\
\hline 3 & 15 & 00 & 296.56 & 8 & 45 & 00 & 293.66 & 14 & 15 & 00 & 288.12 \\
\hline 3 & 22 & 30 & 296.53 & 8 & 52 & 30 & 293.56 & 14 & 22 & 30 & 287.96 \\
\hline 3 & 30 & 00 & 296.49 & 9 & 00 & 00 & 293.47 & 14 & 30 & 00 & 287.81 \\
\hline 3 & 37 & 30 & 296.45 & 9 & 07 & 30 & 293.37 & 14 & 37 & 30 & 287.65 \\
\hline 3 & 45 & 00 & 296.41 & 9 & 1.5 & 00 & 293. 27 & 14 & 45 & 00 & 287.49 \\
\hline 3 & 52 & 30 & 296.36 & 9 & 22 & $30:$ & 293.16 & 14 & 52 & 30 & 287.33 \\
\hline 4 & 00 & 00 . & 296.32 & 9 & 30 & 00 & 293.06 & 15 & 00 & 00 & 287.17 \\
\hline 4 & 07 & 30 & 296.28 & 9 & 37 & 30 & 292.95 & 15 & 07 & 30 & 287.00 \\
\hline 4 & 15 & 01) & 296. 23 & 9 & 45 & 00 & 292.85 & 15 & 15 & 00 & 286.83 \\
\hline 4 & 22 & 30 & 296.18 & 9 & 52 & 30 & 292.74 & 15 & 22 & 30 & 286.67 \\
\hline 4 & 30 & 00 & 296.13 & 10 & 00 & 00 & 292.63 & 15 & 30 & 00 & 286.50 \\
\hline 4 & 37 & 30 & 296.08 & 10 & 07 & 30 & 292.52 & 15 & 37 & 30 & 286.33 \\
\hline 4 & 45 & 00 & 296.03 & 10 & 15 & 00 & 292.41 & 15 & 45 & 00 & 286.16 \\
\hline 4 & 52 & 30 & 295.98 & 10 & 22 & 30 & 292.30 & 15 & 52 & 30 & 285.99 \\
\hline 5 & 00 & 00 & 295.93 & 10 & 30 & 00 & 292.19 & 16 & 00 & 00 & 285.82 \\
\hline 5 & 07 & 30 & 295.87 & 10 & 37 & 30 & 292.07 & 16 & $07^{\circ}$ & 30 & 285.64 \\
\hline 5 & 15 & 00 & 295.81 & 10 & 45 & 00 & 291.95 & 16 & 15 & 00 & 285.46 \\
\hline 5 & 22 & 30 & 295.75 & 10 & 52 & 30 & 291.83 & 16 & 22 & 30 & 285.28 \\
\hline 5 & 30 & 00 & 295.69 & 11 & 00 & 00 & 291.71 & 16 & 30 & 00 & 285.10 \\
\hline
\end{tabular}


TABLE 10.-Areas of quadrilaterals of earth's surface of $15^{\prime}$ extent in latitude and longitude-Continued.

[From Smithsonian Geographical Tables.]

\begin{tabular}{|c|c|c|c|c|c|c|c|c|c|c|c|}
\hline \multicolumn{3}{|c|}{$\begin{array}{l}\text { Middle latitude } \\
\text { of quadrilateral. }\end{array}$} & \multirow[t]{2}{*}{$\begin{array}{c}\text { Area in } \\
\text { square miles. }\end{array}$} & \multicolumn{3}{|c|}{$\begin{array}{l}\text { Middle latitude } \\
\text { of quadrilateral. }\end{array}$} & \multirow[t]{2}{*}{$\begin{array}{c}\text { Area in } \\
\text { squaremiles. }\end{array}$} & \multicolumn{3}{|c|}{$\begin{array}{l}\text { Middle latitude } \\
\text { of quadrilateral. }\end{array}$} & \multirow[t]{2}{*}{$\begin{array}{l}\text { Area in } \\
\text { quare miles }\end{array}$} \\
\hline 。 & 1 & " & & 。 & ' & " & & 。 & ' & $" \prime$ & \\
\hline 16 & 37 & 30 & 284.92 & 22 & 07 & 30 & 275.68 & 27 & 37 & 30 & 263.93 \\
\hline 16 & 45 & 00 & 284.74 & 22 & 15 & 00 & 275.44 & 27 & 45 & 00 & 263.64 \\
\hline 16 & 52 & 30 & 284.56 & 22 & 22 & 30 & 275.20 & 27 & 52 & 30 & 263.34 \\
\hline 17 & 00 & 00 & 284.38 & 22 & 30 . & .00 & 274.96 & 28 & 00 & 00 & 263.04 \\
\hline 17 & 07 & 30 & 284.19 & 22 & 37 & 30 & 274.72 & 28 & 07 & 30 & 262.74 \\
\hline 17 & 15 & 00 & 284.00 & 22 & 45 & 00 & 274.47 & 28 & 15 & 00 & 262.44 \\
\hline 17 & 22 & 30 & 283.81 & 22 & 52 & 30 & 274.22 & 28 & 22 & 30 & 262.14 \\
\hline 17 & 30 & 00 & 283.62 & 23 & 00 & 00 & 273. 98 & 28 & 30 & 00. & 261.84 \\
\hline 17 & 37 & 30 & 283.43 & 23 & .07 & 30 & 273.73 & 28 & 37 & 30 & 261.53 \\
\hline 17 & 45 & 00 & 283.24 & 23 & 15 & 00 & 273.48 & 28 & 45 & 00 & 261.23 \\
\hline 17 & 52 & 30 & 283.05 & 23 & 22 & 30 & 273.23 & 28 & 52 & 30 . & 260.92 \\
\hline 18 & 00 & 00 & 282.86 & 23 & 30 & 00 & 272.98 & 29 & 00 & $00^{\circ}$ & 260.61 \\
\hline 18 & 07 & 30 & 282.66 & 23 & 37 & 30 & 272.72 & 29 & 07 & 30 & 260.30 \\
\hline 18 & 15 & 00 & 282.46 & 23 & 45 & 00 & 272.47 & 29 & 15 & 00 & 259.99 \\
\hline 18 & 22 & 30 & 282.26 & 23 & 52 & 30 & 272.21 & 29 & 22 & 30 & 259.68 \\
\hline 18 & 30 & 00 & 282.06 & 24 & 00 & 00 & 271.95 & 29 & 30 & 00 & 259.37 \\
\hline 18 & 37 & 30 & 281.86 & 24 & 07 & 30 & 371.69 & 29 & 37 & 30 & 25 \\
\hline 18 & 45 & 00 & 281.66 & 24 & 15 & $00^{\circ}$ & 271.44 . & 29 & 45 & 00 & 258.74 \\
\hline 18 & 52 & 30 & 281.45 & 24 & 22 & 30 & 271.17 & 29 & 52 & 30 & 258.42 \\
\hline 19 & 00 & 00 & 281.25 & 24 & 30 & 00 & 270.91 & 30 & 00 & 00 & 258.10 \\
\hline 19 & 07 & 30 & 281.04 & 24 & 37 & 30 & 270.65 & 30 & 07 & 30 & 257.78 \\
\hline 19 & 15 & 00 & 280 & 24 & 45 & 00 & 270 & 30 & 1.5 & 0 & 257.46 \\
\hline 19 & 22 & 30 & 280.62 & 24 & 52 & 30 & 270.11 & 30 & 22 & 30 & 357.14 \\
\hline 19 & 30 & 00 & 280.41 & 25 & 00 & 00 & 269.85 & 30 & 30 & 00 & 256.82 \\
\hline 19 & 37 & 30 & 280 & 25 & 07 & 30 & 269 & 30 & 37 & 30 & 25 \\
\hline 19 & 45 & 00 & & 25 & 15 & 00 & & 30 & 45 & 0 & 17 \\
\hline 19 & 52 & 30 & 279.77 & 25 & 22 & 30 & 269.04 & 30 & 52 & 30 & 255. 84 \\
\hline 20 & 00 & 00 & 279.55 & 25 & 30 & 00 . & 268.76 & 31 & 00 & 00 & 255.52 \\
\hline 20 & 07 & 30 & 27 & 25 & 37 & 30 & 268 & 31 & 07 & 30 & 255. \\
\hline 20 & 15 & 00 & & 25 & 45 & 00 & & 31. & 15 & 00 & \\
\hline 20 & 22 & 30 & 278.90 & 25 & 52 & 30 & 267.94 & 31. & 22 & 30 & 254.53 \\
\hline 20 & 30 & 00 & 278.68 & 26 & 00 & 00 & 267.66 & 31 & 30 & 00 & 254.19 \\
\hline 20 & 37 & 30 & $27 \varepsilon$ & 26 & 07 & 30 & 26 & 31. & 37 & 30 & 25 \\
\hline 20 & 45 & 00 & & 26 & 15 & 00 & & 31 & 45 & 00 & \\
\hline 20 & 52 & 30 & 278.00 & 26 & 22 & 30 & 266. & 31 & 52 & 30 & 253.19 \\
\hline 21 & 00 & 00 & 277.78 & 26 & 30 & 00 & 266.54 & 32 & 00 & 00 & 252.85 \\
\hline 21 & 07 & 30 & 277 & 26 & 37 & 30 & 26 & 32 & 07 & 3 & 25 \\
\hline 21 & 15 & 00 & & 26 & 45 & 00 & & 32 & 15 & 0 & 252.17 \\
\hline 21 & 22 & 30 & 277.09 & 26 & 52 & 30 & & 32 & 22 & 30 & 251.83 \\
\hline 21 & 30 & 00 & 276.86 & 27 & 00 & 00 & 265.39 & 32 & 30 & 00 & 251.49 \\
\hline 21 & 37 & 30 & 276 & 27 & 07 & 30 & 265. & 32 & 37 & 3 & 251. \\
\hline 21 & 45 & 00 & 276 & 27 & 15 & 00 & 264 & 32 & 45 & 00 & 250.80 \\
\hline 21 & 52 & 30 & 276.16 & 27 & 22 & 30 & 264.52 & 32 & 52 & 30 & 250.45 \\
\hline 22 & 00 & 00 & 275.92 & 27 & 30 & 00 & 264.23 & 33 & 00 & 00 & 250.11 \\
\hline
\end{tabular}

- Bull. 214-03-6 
TABLE 10.-Areas of quadrilaterals of earth's surface of $15^{\prime}$ extent in latitude and longitude-Continued.

[From Smithsonian Geographical Tables.]

\begin{tabular}{|c|c|c|c|c|c|c|c|c|c|c|c|}
\hline \multicolumn{3}{|c|}{$\begin{array}{l}\text { Middle latitude } \\
\text { of quadrilateral. }\end{array}$} & \multirow[t]{2}{*}{$\begin{array}{l}\text { Area in } \\
\text { squaremiles. }\end{array}$} & \multicolumn{3}{|c|}{$\begin{array}{l}\text { Middle latitude } \\
\text { of quadrilateral. }\end{array}$} & \multirow[t]{2}{*}{$\begin{array}{c}\text { Area in } \\
\text { square miles. }\end{array}$} & \multicolumn{3}{|c|}{$\begin{array}{l}\text { Middle latitude } \\
\text { of quadrilateral. }\end{array}$} & \multirow[t]{2}{*}{$\begin{array}{l}\text { Area in } \\
\text { squaremiles }\end{array}$} \\
\hline$\circ$ & , & 11 & & $\circ$ & , & "I & & ○ & 1 & "I & \\
\hline 33 & 07 & 30 & 249.76 & 38 & 37 & 30 & 233. 28 & 44 & 07 & 30 & 214.61 \\
\hline 33 & 15 & 00 & 249.41 & 38 & 45 & 00 & 232.88 & 44 & 15 & 00 & 214.17 \\
\hline 33 & 22 & 30 & 249.06 & 38 & 52 & 30 & 232.48 & 44 & 22 & 30 & 213.72 \\
\hline 33 & 30 & 00 & 248.71 & 39 & 00 & 00 & 232.07 & 44 & 30 & 00 & 213.27 \\
\hline 33 & 37 & 30 & 248.36 & 39 & 07 & 30 & 231.67 & 44 & 37 & 30 & 212.82 \\
\hline 33 & 45 & 00 & 248.00 & 39 & 15 & 00 & 231.27 & 44 & 45 & 00 & 212.37 \\
\hline 33 & 52 & 30 & 247.65 & 39 & 22 & 30 & 230.86 & 44 & 52 & 30 & 211.91 \\
\hline 34 & 00 & 00 & 247.29 & 39 & 30 & 00 & 230.45 & 45 & 00 & 00 & 211.46 \\
\hline 34 & 07 & 30 & 246.93 & 39 & 37 & 30 & 230.04 & 45 & 07 & 30 & 211.00 \\
\hline 34 & 15 & 00 & 246.57 & 39 & 45 & 00 & 229.63 & 45 & 15 & 00 & 210.55 \\
\hline 34 & 22 & 30 & 246.21 & 39 & 52 & 30 & 229.22 & 45 & 22 & 30 & 210.09 \\
\hline 34 & 30 & 00 & 245.85 & 40 & 00 & 00 & 228.81 & 45 & 30 & 00 & 209.63 \\
\hline 34 & 37 & 30 & 245.49 & 40 & 07 & 30 & 228.40 & 45 & 37 & 30 & 209.17 \\
\hline 34 & 45 & 00 & 245.13 & 40 & 15 & 00 & 227.99 & 45 & 45 & 00 & 208.71 \\
\hline 34 & 52 & 30 & 244.76 & 40 & 22 & 30 & 227.57 & 45 & 52 & 30 & 208.25 \\
\hline 35 & 00 & 00 & 244.40 & 40 & 30 & 00 & 227.15 & 46 & 00 & 00 & 207.78 \\
\hline 35 & 07 & 30 & 244.03 & 40 & 37 & 30 & 226.73 & 46 & 07 & 30 & 207. 32 \\
\hline 35 & 15 & 00 & 243.66 & 40 & 45 & 00 & 226.32 & 46 & 15 & 00 & 206.86 \\
\hline 35 & 22 & 30 & 243.29 & 40 & 52 & 30 & 225.90 & 46 & 22 & 30 & 206. 39 \\
\hline 35 & 30 & 00 & 242.92 & 41 & 00 & 00 & 225.48 & 46 & 30 & 00 & 205.92 \\
\hline 35 & 37 & 30 & 242.55 & 41 & 07 & 30 & 225.06 & 46 & 37 & 30 & 205.45 \\
\hline 35 & 45 & 00 & 242.18 & 41 & 15 & 00 & 224.64 & 46 & 45 & 00 & 204.99 \\
\hline 35 & 52 & 30 & 241.80 & 41 & 22 & 30 & 224.21 & 46 & 52 & 30 & 204.52 \\
\hline 36 & 00 & 00 & 241.43 & 41 & 30 & 00 & 223.79 & 47 & 00 & 00 & 204.05 \\
\hline 36 & 07 & 30 & 241.05 & 41 & 37 & 30 & 223.36 & 47 & 07 & 30 & 203.57 \\
\hline 36 & 15 & 00 & 240.67 & 41 & 45 & 00 & 222.93 & 47 & 15 & 00 & 203.10 \\
\hline 36 & 22 & 30 & 240.29 & 41 & 52 & 30 & 222.50 & 47 & 22 & 30 & 202.63 \\
\hline 36 & 30 & 00 & 239.91 & 42 & 00 & 00 & 222.08 & 47 & 30 & 00 & 202.15 \\
\hline 36 & 37 & 30 & 239.53 & 42 & 07 & 30 & 221.65 & 47 & 37 & 30 & 201.67 \\
\hline 36 & 45 & 00 & 239.15 & 42 & 15 & 00 & 221.21 & 47 & 45 & 00 & 201. 20 \\
\hline 36 & 52 & 30 & 238.77 & 42 & 22 & 30 & 220.78 & 47 & 52 & 30 & 200.72 \\
\hline 37 & 00 & 00 & 238.38 & 42 & 30 & 00 & 220.35 & 48 & 00 & 00 & 200.24 \\
\hline 37 & 07 & 30 & 237.99 & 42 & 37 & 30 & 219.91 & 48 & 07 & 30 & 199.76 \\
\hline 37 & 15 & 00 & 237.61 & 42 & 45 & 00 & 219.48 & 48 & 15 & 00 & 199.28 \\
\hline 37 & 22 & 30 & 237.22 & 42 & 52 & 30 & 219.04 & 48 & 22 & 30 & 198.80 \\
\hline 37 & 30 & 00 & 236.83 & 43 & 00 & 00 & $2] 8.60$ & 48 & 30 & 00 & 198. 32 \\
\hline 37 & 37 & 30 & 236.44 & 43 & 07 & 30 & 218.16 & 48 & 37 & 30 & 197.83 \\
\hline 37 & 45 & 00 & 236.05 & 43 & 15 & 00 & 217.73 & 48 & 45 & 00 & 197.35 \\
\hline 37 & 52 & 30 & 235.66 & 43 & 22 & 30 & 217.28 & 48 & 52 & 30 & 196.86 \\
\hline 38 & 00 & 00 & 235.26 & 43 & 30 & 00 & 216.84 & 49 & 00 & 00 & 196.38 \\
\hline 38 & 07 & 30 & 234.87 & 43 & 37 & 30 & 216.40 & 49 & 07 & 30 & 195.89 \\
\hline 38 & 15 & 00 & 234.47 & 43 & 45 & 00 & 215.96 & 49 & 15 & 00 & 195.40 \\
\hline 38 & 22 & 30 & 234.07 & 43 & 52 & 30 & 215.51 & 49 & 22 & 30 & 194.91 \\
\hline 38 & 30 & 00 & 233.68 & 44 & 00 & 00 & 215.06 & 49 & 30 & 00 & 194.42 \\
\hline
\end{tabular}


TABLE 10.-Areas of quadrilaterals of earth's surface of $15^{\prime}$ extent in latitude and longitude-Continued.

[From Smithsonian Geographical Tables.]

\begin{tabular}{|c|c|c|c|c|c|c|c|c|c|c|c|}
\hline \multicolumn{3}{|c|}{$\begin{array}{l}\text { Middle latitude } \\
\text { of quadrilateral. }\end{array}$} & \multirow[t]{2}{*}{$\begin{array}{l}\text { Area in } \\
\text { square miles. }\end{array}$} & \multicolumn{3}{|c|}{$\begin{array}{l}\text { Middle latitude } \\
\text { of quadrilateral. }\end{array}$} & \multirow[t]{2}{*}{$\begin{array}{c}\text { Area in } \\
\text { square miles. }\end{array}$} & \multicolumn{3}{|c|}{$\begin{array}{l}\text { Middle latitude } \\
\text { of quadrilateral. }\end{array}$} & \multirow[t]{2}{*}{$\begin{array}{c}\text { Area in } \\
\text { square miles. }\end{array}$} \\
\hline$\circ$ & , & "I & & $\circ$ & , & $" \prime$ & & $\circ$ & , & 11 & \\
\hline 49 & 37 & 30 & 193.93 & 55 & 07 & 30 & 171. 39 & 60 & 37 & 30 & 147.21 \\
\hline 49 & 45 & 00 & 193.44 & 55 & 15 & 00 & 170.86 & 60 & 45 & 00 & 146.64 \\
\hline 49 & 52 & 30 & 192.94 & 55 & 22 & 30 & 170.33 & 60 & 52 & 30 & 146.07 \\
\hline 50 & 00 & 00 & 192.45 & 55 & 30 & 00 & 169.79 & 61 & 00 & 00 & 145.50 \\
\hline 50 & 07 & 30 & 191.95 & 55 & 37 & 30 & 169.26 & 61 & 07 & 30 & 144.93 \\
\hline 50 & 15 & 00 & 191.46 & 55 & 45 & 00 & 168.72 & 61 & 15 & 00 & 144.36 \\
\hline 50 & 22 & 30 & 190.96 & 55 & 52 & 30 & 168.19 & 61 & 22 & 30 & 143. 79 \\
\hline 50 & 30 & 00 & 190.46 & 56 & 00 & 00 & 167.65 & 61 & 30 & 00 & 143.22 \\
\hline 50 & 37 & 30 & 189.96 & 56 & 07 & 30 & 167.11 & 61. & 37 & 30 & 142.65 \\
\hline 50 & 45 & 00 & 189.46 & 56 & 15 & 00 & 166.57 & 61 & 45 & 00 & 142.08 \\
\hline 50 & 52 & 30 & 188.96 & 56 & 22 & 30 & 166.03 & 61 & 52 & 30 & 141.50 \\
\hline 51 & 00 & 00 & 188.46 & 56 & 30 & 00 & 165.49 & 62 & 00 & 00 & 140.93 \\
\hline 51 & 07 & 30 & 187.96 & 56 & 37 & 30 & 164.95 & 62 & 07 & 30 & 140.35 \\
\hline 51 & 15 & 00 & 187.46 & 56 & 45 & 00 & 164.41 & 62 & 15 & 00 & 139.78 \\
\hline 51 & 22 & 30 & 186.95 & 56 & 52 & 30 & 163.87 & 62 & 22 & 30 & 139.20 \\
\hline 51 & 30 & 00 & 186.45 & 57 & 00 & 00 & 163.32 & 62 & 30 & 00 & 138. 62 \\
\hline 51 & 37 & 30 & 185 & 57 & 07 & 30 & 162.78 & 62 & 37 & 30 & 138.04 \\
\hline 51 & 45 & 00 & 185.43 & 57 & 15 & 00 & 162.23 & 62 & 45 & 00 & 137.47 \\
\hline 51 & 52 & 30 & 184.92 & 57 & 22 & 30 & 161.68 & 62 & 52 & 30 & 136.89 \\
\hline 52 & 00 & 00 & 184.41 & 57 & 30 & 00 & 161.14 & 63 & 00 & 00 & 136.31 \\
\hline 52 & 07 & 30 & 18 & 57 & 37 & 30 & 160.59 & 63 & 07 & 30 & 135.73 \\
\hline 52 & 15 & 00 & 183.39 & 57 & 45 & 00 & 160.04 & 63 & 15 & 00 & 135.15 \\
\hline 52 & 22 & 30 & 182.88 & 57 & 52 & 30 & 159.49 & 63 & 22 & 30 & 134.56 \\
\hline 52 & 30 & 00 & 182.37 & 58 & 00 & 00 & 158.94 & 63 & 30 & 00 & $133.98^{\circ}$ \\
\hline 52 & 37 & 30 & 5 & 58 & 07 & 30 & 15 & 63 & 37 & 30 & 133.40 \\
\hline 52 & 45 & 00 & 181.34 & 58 & 15 & 00 & 157.84 & 63 & 45 & 00 & 132.81 \\
\hline 52 & 52 & 30 & 180.82 & 58 & 22 & 30 & 157.29 & 63 & 52 & 30 & 132.23 \\
\hline 53 & 00 & 00 & 180.31 & 58 & 30 & 00 & 156.73 & 64 & 00 & 00 & 131.64 \\
\hline 53 & 07 & 30 & 179. 79 & 58 & 37 & 30 & 15 & 64 & 07 & 30 & 131.06 \\
\hline 53 & 15 & 00 & 179.27 & 58 & 45 & 00 & 155.62 & 64 & 15 & 00 & 130.47 \\
\hline 53 & 22 & 30 & 178.75 & 58 & 52 & 30 & 155.07 & 64 & 22 & 30 & 129.88 \\
\hline 53 & 30 & 00 & 178.23 & 59 & 00 & 00 & 154.51 & 64 & 30 & 00 & 129.29 \\
\hline 53 & 37 & 30 & 17 & 59 & 07 & 30 & & 64 & 37 & 30 & 70 \\
\hline 53 & 45 & 00 & 177.19 & 59 & 15 & 00 & 153.40 & 64 & 45 & 00 & 128.12 \\
\hline 53 & 52 & 30 & 176.67 & 59 & 22 & 30 & 152.84 & 64 & 52 & 30 & 127.53 \\
\hline 54 & 00 & 00 & 176.14 & 59 & 30 & 00 & 152.28 & 65 & 00 & 00 & 126.94 \\
\hline 54 & 07 & 30 & 2 & 59 & 37 & 30 & & 65 & 07 & 30 & 126.34 \\
\hline 54 & 15 & 00 & 175.10 & 59 & 45 & 00 & 151.16 & 65 & 15 & 00 & 125.75 \\
\hline 54 & 22 & 30 & 174.57 & 59 & 52 & 30 & 150.60 & 65 & 22 & 30 & 125.16 \\
\hline 54 & 30 & 00 & 174.04 & 60 & 00 & 00 & 150.03 & 65 & 30 & 00 & 124.57 \\
\hline 54 & 37 & 30 & 17 & 60 & 07 & 30 & 14 & 65 & 37 & 30 & $123: 97$ \\
\hline 54 & 45 & 00 & 172.99 & 60 & 15 & 00 & 148.91 & 65 & 45 & 00 & 123.38 \\
\hline 54 & 52 & 30 & 172.46 & 60 & 22 & 30 & 148.34 & 65 & 52 & 30 & 122.78 \\
\hline 55 & 00 & 00 & 171.93 & 60 & 30 & 00 & 147.77 & 66 & 00 & 00 & 122.19 \\
\hline
\end{tabular}


TABLE 10.-Areas of quadrilaterals of earth's surface of $15^{\prime}$ extent in latitude and longitude-Continued.

[From Smithsonian Geographical Tables.]

\begin{tabular}{|c|c|c|c|c|c|c|c|c|c|c|c|}
\hline \multicolumn{3}{|c|}{$\begin{array}{l}\text { Middle latitude } \\
\text { of quadrilateral. }\end{array}$} & \multirow{2}{*}{$\begin{array}{c}\text { Area in. } \\
\text { square miles. }\end{array}$} & \multicolumn{3}{|c|}{$\begin{array}{l}\text { Middle latitude } \\
\text { of quadrilateral. }\end{array}$} & \multirow{2}{*}{$\begin{array}{c}\text { Area in } \\
\text { square miles. }\end{array}$} & \multicolumn{3}{|c|}{$\begin{array}{l}\text { Middle latitude } \\
\text { of quadrilateral. }\end{array}$} & \multirow{2}{*}{$\begin{array}{c}\text { Area in } \\
\text { square miles. } \\
\end{array}$} \\
\hline$\circ$ & , & $\prime \prime$ & & ० & 1 & $" \prime$ & & $\circ$ & ' & "I & \\
\hline 66 & 07 & 30 & 121.59 & 71 & 37 & 30 & 94.78 & 77 & 07 & 30 & 67.04 \\
\hline 66 & 15 & 00 & 120.99 & 71 & 45 & 00 & $94.16^{-}$ & 77 & 15. & 00 & 66.41 \\
\hline 66 & 22 & 30 & 120.40 & 71 & 52 & 30 & 93.54 & 77 & 22 & 30 & 65.77 \\
\hline 66 & 30 & 00 & 119.80 & 72 & $0 \overline{0}$ & 00 & 92.92 & 77 & 30 & 00 & 65.13 \\
\hline 66 & 37 & 30 & 119.20 & 72 & 07 & 30 & 92.30 & 77 & 37 & 30 & 64.49 \\
\hline 66 & 45 & 00 & 118.60 & 72 & 15 & 00 & 91.68 & 77 & 45 & 00 & 63.85 \\
\hline 66 & 52 & 30 & 118.00 & 72 & 22 & 30 & 91.05 & 77 & 52 & 30 & 63.20 \\
\hline 67 & 00 & 00 & 117.40 & 72 & 30 & 00 & 90.43 & 78 & 00 & 00 & 62.56 \\
\hline 67 & 07 & 30 & 116.80 & 72 & 37 & 30 & 89.80 & 78 & 07 & 30 & 61.92 \\
\hline 67 & 15 & 00 & 116.20 & 72 & 45 & 00 & 89.18 & 78 & 15 & 00 & 61.28 \\
\hline 67 & 22 & 30 & 115.59 & 72 & 52 & 30 & 88.55 & 78 & 22 & 30 & 60.64 \\
\hline 67 & 30 & 00 & 114.99 & 73 & 00 & 00 & 87.93 & 78 & 30 & 00 & 60.00 \\
\hline 67 & 37 & 30 & 114.39 & 73 & 07 & 30 & 87.30 & 78 & 37 & 30 & 59.35 \\
\hline 67 & 45 & 00 & 113.78 & 73 & 15 & 00 & 86.67 & 78 & 45 & 00 & 58. 71 \\
\hline 67 & 52 & 30 & 113.18 & 73 & 22 & 30 & 86.05 & 78 & 52 & 30 & 58.06 \\
\hline 68 & 00 & 00 & 112.57 & 73 & 30 & 00 & 85.42 & 79 & 00 & 00 & 57.42 \\
\hline 68 & 07 & 30 & 111.97 & 73 & 37 & 30 & 84.79 & 79 & 07 & 30 & 56. 78 \\
\hline 68 & 15 & 00 & 111.36 & 73 & 45 & 00 & 84. 16 . & 79 & 15. & 00 & 56.13 \\
\hline 68 & 22 & 30 & 110.76 & 73 & 52 & 30 & 83.53 & 79 & 22 & 30 & 55.49 \\
\hline 68 & 30 & 00 & 110.15 & $74^{\circ}$ & 00 & 00 & 82.91 & 79 & 30 & 00 & 54.84 \\
\hline 68 & 37 & 30 & 109.54 & 74 & 07 & 30 & 82.28 & 79 & 37 & 30 & 54.20 \\
\hline 68 & 45 & 00 & 108.93 & 74 & 15 & 00 & 81.65 & 79 & 45 & 00 & 53.55 \\
\hline 68 & 52 & 30 & 108.32 & 74 & 22 & 30 & 81.01 & 79 & 52 & 30 & 52.91 \\
\hline 69 & 00 & 00 & 107.71 & 74 & 30 . & 00 & 80.38 & 80 & 00 & 00 & 52.26 \\
\hline 69 & 07 & 30 & 107.10 & 74 & 37 & 30 & 79.75 & 80 & 07 & 30 & 51.62 \\
\hline 69 & 15 & 00 & 106.49 & 74 & 45 & 00 & 79.12 & 80 & 15 & 00 & 50.97 \\
\hline 69 & 22 & 30 & 105.88 & 74 & 52 & 30 & 78.49 & 80 & 22 & 30 & 50.32 \\
\hline 69 & 30 & 00 & 105.27 & 75 & 00 & 00 & 77.86 & 80 & 30 & 00 & 49.68 \\
\hline 69 & 37 & 30 & 104.65 & 75 & 07 & 30 & 77.22 & $80^{\circ}$ & 37 & 30 & 49.03 \\
\hline 69 & $45^{\circ}$ & 00 & 104.04 & 75 & 15 & 00 & 76.59 & 80 & 45 & 00 & 48.38 \\
\hline 69 & 52 & 30 & 103.43 & 75 & 22 & 30 & 75.95 & 80 & 52 & 30 & 47. 73 \\
\hline 70 & 00 & 00 & 102.81 & 75 & 30 & 00 & 75.32 & 81 & 00 & 00 & 47.08 \\
\hline 70 & 07 & 30 & 102.20 & 75 & 37 & 30 & 74.69 & 81 & 07 & 30 & 46.44 \\
\hline 70 & 15 & 00 & 101.59 & 75 & 45 & 00 & 74.05 & 81 . & 15 & 00 & 45.79 \\
\hline 70 & 22 & 30 & 100.97 & 75 & 52. & 30 & 73.42 & 81 & 22 & 30 & 45.14 \\
\hline 70 & 30 & 00 & 100.35 & 76 & 00 & 00 & 72.78 & 81 & 30 & 00 & 44.49 \\
\hline 70 & 37 & 30 & 99.74 & 76 & 07 & 30 & 72.14 & 81 & 37 & 30 & 43.84 \\
\hline 70 & 45 & 00 & 99.12 & 76 & 15 & 00 & 71.51 & 81 & 45 & 00 & 43.19 \\
\hline 70 & 52 & 30 & 98.50 & 76 & 22 & 30 & 70.87 & 81 & 52 & 30 & 42.54 \\
\hline 7.1 & 00 & 00 & 97.88 & 76 & 30 & 00 & 70.24 & 82 & 00 & 00 & 41.89 \\
\hline 71 & 07 & 30 & 97.26 & 76 & 37 & 30 & 69.60 & 82 & 07 & $30^{\circ}$ & 41.24 \\
\hline 71 & 15 & 00 & 96.65 & 76 & 45 & 00 & 68.96 & 82 & 15 & 00 & 40.59 \\
\hline 71 & 22 & 30 & 96.03 & 76 & 52 & 30 & 68.32 & 82 & 22 & 30 & 39.94 \\
\hline 71 & $\cdot 30$ & 00 & 95.41 & 77 & 00 & 00 & 67.68 & 82 & 30 & .00 & 39.29 \\
\hline
\end{tabular}


TABLe 10.-Areas of quadrilaterals of earth's surface of $15^{\prime}$ extent in latitude and longitude-Continued.

[From Smithsonian Geographical Tables.]

\begin{tabular}{|c|c|c|c|c|c|c|c|c|c|c|c|}
\hline \multicolumn{3}{|c|}{$\begin{array}{l}\text { Middle latitude } \\
\text { of quadrilateral. }\end{array}$} & \multirow[t]{2}{*}{$\begin{array}{l}\text { Area in } \\
\text { square miles. }\end{array}$} & \multicolumn{3}{|c|}{$\begin{array}{l}\text { Middle latitude } \\
\text { of quadrilateral. }\end{array}$} & \multirow[t]{2}{*}{$\begin{array}{c}\text { Area in } \\
\text { squaremiles. }\end{array}$} & \multicolumn{3}{|c|}{$\begin{array}{l}\text { Middle latitude } \\
\text { of quadrilateral. }\end{array}$} & \multirow[t]{2}{*}{$\begin{array}{c}\text { Area in } \\
\text { square miles }\end{array}$} \\
\hline$\circ$ & 1 & "I & & $\circ$ & 1 & "I & & $\circ$ & 1 & "I & \\
\hline 82 & 37 & 30 & 38.64 & 85 & 07 & 30 & 25.58 & 87 & 37 & 30 & 12.48 \\
\hline 82 & 45 & 00 & 37.99 & 85 & 15 & 00 & 24. 93 & 87 . & 45 & 00 & 11.82 \\
\hline 82 & 52 & 30 & 37.34 & 85 & 22 & 30 & 24.27 & 87 & 52 & 30 & 11. 16 \\
\hline 83 & 00 & 00 & 36.69 & 85 & 30 & 00 & 23.62 & 88 & 00 & 00 & 10.51 \\
\hline 83 & 07 & 30 & 36.03 & 85 & 37 & 30 & 22.97 & 88 & 07 & 30 & 9.85 \\
\hline 83 & 15 & 00 & 35.38 & 85 & 45 & 00 & 22.31 & 88 & 15 & 00 & 9.20 \\
\hline 83 & 22 & 30 & 34.73 & 85 & 52 & 30 & 21.66 & 88 & 22 & 30 & 8.54 \\
\hline 83 & 30 . & 00 & 34.08 & 86 & 00 & 00 & 21.00 . & 88 & 30 & 00 & 7.88 \\
\hline 83 & 37 & 30 & 33.42 & 86 & 07 & 30 & 20.35 & 88 & 37 & 30 & 7.22 \\
\hline 83 & 45 & 00 & 32.77 & 86 & 15 & 00 & 19.69 & 88 & 45 & 00 & 6. 57 \\
\hline 83 & 52 & 30 & 32.12 & 86 & 22 & 30 & 19.04 & 88 & 52 & 30 & 5.91 \\
\hline 84 & 00 & 00 & 31.47 & 86 & 30 & 00 & 18.38 & 89 & 00 & 00 & 5.26 \\
\hline 84 & 07 & 30 & 30.81 & 86 & 37 & 30 & 17.72 & 89 & 07 & 30 & 4. 60 \\
\hline 84 & 15 & 00 & 30.16 & 86 & 45 & 00 & 17.07 & 89 & 15 & 00 & 3.94 \\
\hline 84 & 22 & 30 & 29.51 & 86 & 52 & 30 & 16. 41 & 89 & 22 & 30 & 3. 28 \\
\hline 84 & 30 & 00 & 28.86 & 87 & 00 & 00 & 15.76 & 89 & 30 & 00 & 2. 63 \\
\hline 84 & 37 & 30 & 28.20 & 87 & 07 & 30 & 15. 10 & 89 & 37 & 30 & 1. 97 \\
\hline 84 & 45 & 00 & 27.54 & 87 & 15 & 00 & 14.44 & 89 & 45 & 00 & 1. 31 \\
\hline 84 & 52 & 30 & 26.89 & 87 & 22 & 30 & 13.79 & 89 & 52 & 30 & 0.66 \\
\hline 85 & 00 & 00 & 26.24 & 87 & 30 & 00 & 13.13 & & & & \\
\hline
\end{tabular}


'TaBLe 11.-Areas of quadrilaterals of earth's surface of $10^{\prime}$ extent in latitude and iongitude.

[From Smithsonian Geographical Tables.]

\begin{tabular}{|c|c|c|c|c|c|c|c|c|}
\hline \multicolumn{2}{|c|}{$\begin{array}{l}\text { Middle lati- } \\
\text { tude of } \\
\text { quadrilateral. }\end{array}$} & \multirow[t]{2}{*}{$\begin{array}{l}\text { Area in square } \\
\text { miles. }\end{array}$} & \multicolumn{2}{|c|}{$\begin{array}{l}\text { Middle lati- } \\
\text { tude of } \\
\text { quadrilateral. }\end{array}$} & \multirow[t]{2}{*}{$\begin{array}{c}\text { Area in square } \\
\text { miles. }\end{array}$} & \multicolumn{2}{|c|}{$\begin{array}{l}\text { Middle lati- } \\
\text { tudde of } \\
\text { quadrilateral. }\end{array}$} & \multirow{2}{*}{$\begin{array}{c}\text { Area in square } \\
\text { miles. }\end{array}$} \\
\hline$\circ$ & , & & $\circ$ & . & & $\circ$ & , & \\
\hline 0 & 05 & 132.01 & 7 & 25 & 130.93 & 14 & 45 & 127.77 \\
\hline 0 & 15 & 132.01 & 7 & 35 & 130.88 & 14 & 55 & 127.67 \\
\hline 0 & 25 & 132.01 & 7 & 45 & 130.84 & 15 & 05 & 127.58 \\
\hline 0 & 35 & 132.00 & 7 & 55 & 130.79 & 15 & 15 & 127.48 \\
\hline 0 & 45 & 132.00 & 8 & 05 & 130.73 & 15 & 25 & 127.38 \\
\hline 0 & 55 & 131.99 & 8 & 15 & 130.68 & 15 & 35 & 127.28 \\
\hline 1 & 05 & 131.99 & 8 & 25 & 130.63 & 15 & 45 & 127.18 \\
\hline 1 & 15 & 131.98 & 8 & 35 & 130.57 & 15 & 55 & 127.08 \\
\hline 1 & 25 & 131.97 & 8 & 45 & 130.51 & 16 & 05 & 126.98 \\
\hline 1 & 35 & 131.96 & 8 & 55 & 130.46 & 16 & 15 & 126.87 \\
\hline 1. & 45. & 131.95 & 9 & 05 & 130.40 & 16 & 25 & 126.77 \\
\hline 1 & 55 & 131.94 & 9 & 15 & 130.34 & 16 & 35 & 126.66 \\
\hline 2 & 05 & 131. 93 & 9 & 25 & 130.28 & 16 & 45 & 126.55 \\
\hline 2 & 15 & 131.91 & 9 & 35 & 130.22 & 16 & 55 & 126.44 \\
\hline 2 & 25 & 131.90 & 9 & 45 & 130.15 & 17 & 05 & 126.33 \\
\hline 2 & 35 & 131.88 & 9 & 55 & 130.09 & 17 & 15 & 126.22 \\
\hline 2 & 45 & 131.86 & 10 & 05 & 130.02 & 17 & 25 & 126.11 \\
\hline 2 & 55 & 1.31. 84 & 10 & 15 & 129.96 & 17 & 35 & 126.00 \\
\hline 3 & 05 & 131.82 & 10 & 25 & 129.89 & 17 & 45 & 125.88 \\
\hline 3 & 15 & 131.80 & 10 & 35 & 129.82 & 17 & 55 & 125.77 \\
\hline 3 & 25 & 131.78 & 10 & 45 & 129.76 & 18 & 05 & 125.65 \\
\hline 3 & 35 & 131.76 & 10 & 55 & 129.68 & 18 & 15 & 125.54 \\
\hline 3 & 45 & 131.74 & 11 & 05 & 129.61 & 18 & 25 & 125.42 \\
\hline 3 & 55 & 131.71 & 11 & 15 & 129.54 & 18 & 35 & 125.30 \\
\hline 4 & 05 & 131. 68 & 11 & 25 & 129.47 & 18 & 45 & 125.18 \\
\hline 4 & 15 & 131. 66 & 11 & 35 & 129.39 & 18 & 55 & 125.06 \\
\hline 4 & 25 & 131.63 & 11 & 45 & 129.32 & 19 & 05 & 124.94 \\
\hline 4 & 35 & 131.60 & 11 & 55 & 129.24 & 19 & 15 & 124.81 \\
\hline 4 & 45 & 131.57 & 12 & 05 & 129.16 & 19 & 25 & 124.69 \\
\hline 4 & 55 & 131.54 & 12 & 15 & 129.08 & 19 & 35 & 124.56 \\
\hline 5 & 05 & 131.50 & 12 & 25 & 129.00 & 19 & 45 & 124.44 \\
\hline 5 & 15 & 131.47 & 12 & 35 & 128.92 & 19 & 55 & 124.31 \\
\hline 5 & 25 & 131.44 & 12 & 45 & 128.84 & 20 & 05 & 124.18 \\
\hline 5 & 35 & 131. 40 & 12 & 55 & 128.76 & 20 & 15 & 124.05 \\
\hline 5 & 45 & 131.36 & 13 & 05 & 128.67 & 20 & 25 & 123. 92 \\
\hline 5 & 55 & 131.33 & 13 & 15 & 128.59 & 20 & 35 & 123.79 \\
\hline 6 & 05 & 131. 29 & 13 & 25 & 128.50 & 20 & 45 & 123.66 \\
\hline 6 & 15 & 131.25 & 13 & 35 & 128. 41 & 20 & 55 & 123.52 \\
\hline 6 & 25 & 131. 21 & 13 & 45 & 128. 33 & 21 & 05 & 123.39 \\
\hline 6 & 35 & 131.16 & 13 & 55 & 128.24 & 21 & 15 & 123.25 \\
\hline 6 & 45 & 131.12 & 14 & 05 & 128. 14 & 21 & 25 & 123.12 \\
\hline 6 & 55 & 131.07 & 14 & 15 & 128.05 & 21 & 35 & 122.98 \\
\hline 7 & 05 & 131.03 & 14 & 25 & 127.96 & 21 & 45 & 122.84 \\
\hline 7 & 15 & 130.98 & 14 & 35 & 127.87 & 21 & 55 & 122.70 \\
\hline
\end{tabular}


TABLE 11.-Areas of quadrilaterals of earth's surface of $10^{\prime}$ extent in latitude and longitude-Continued.

[From Smithsonian Geographical Tables.]

\begin{tabular}{|c|c|c|c|c|c|c|c|c|}
\hline \multicolumn{2}{|c|}{$\begin{array}{l}\text { Middle lati- } \\
\text { tude of } \\
\text { quadrilateral. }\end{array}$} & \multirow[t]{2}{*}{$\begin{array}{l}\text { Area in square } \\
\text { miles. }\end{array}$} & \multicolumn{2}{|c|}{$\begin{array}{l}\text { Middle lati- } \\
\text { tude of } \\
\text { quadrilateral. }\end{array}$} & \multirow[t]{2}{*}{$\begin{array}{c}\text { A rea in square } \\
\text { miles. }\end{array}$} & \multicolumn{2}{|c|}{$\begin{array}{l}\text { Middle lati- } \\
\text { tude of } \\
\text { quadrilateral. }\end{array}$} & \multirow[t]{2}{*}{$\begin{array}{c}\text { Area in square } \\
\text { miles. }\end{array}$} \\
\hline$\circ$ &, 1 & & 0 & ' & & 。 & , & \\
\hline 22 & 05 & 122.56 & 29 & 25 & 115.37 & 36 & 45 & 106. 29 \\
\hline 22 & 15 & 122.42 & 29 & 35 & 115.18 & 36 & 55 & 106.06 \\
\hline 22 & 25 & .122 .28 & 29 & 45 & 114.99 & 37 & 05 & 105.83 \\
\hline 22 & 35 & 122.13 & 29 & 55 & 114.81 & 37 & 15 & 105.60 \\
\hline 22 & 45 & 121.99 & 30 & 05 & 114.62 & 37 & 25 & 105.37 \\
\hline 22 & 55 & 121.84 & 30 & 15 & 114.43 & 37 & 35 & 1.05. 14 \\
\hline 23 & 05 & 121.69 & 30 & 25 & 114.24 & 37 & 45 & 104.91 \\
\hline 23 & 15 & 121.55 & 30 & 35 & 114.04 & 37 & 55 & 104.68 \\
\hline 23 & 25 & 121.40 & 30 & 45 & 113.85 & 38 & 05 & 104.44 \\
\hline 23 & 35 & 121.25 & 30 & 55 & 113.66 & 38 & 15 & 104.21 \\
\hline 23 & 45 & 121.10 & 31. & 05 & 113.47 & 38 & 25 & 103.97 \\
\hline 23 & 55 & 120.94 & 31 & 15 & 113.27 & 38 & 35 & 103.74 \\
\hline 24 & 05 & 120.79 & 31 & 25 & 113.07 & 38 & 45 & 103.50 \\
\hline 24 & 15 & 120.64 & 31 & 35 & 112.88 & 38 & 55 & 103. 26 \\
\hline 24 & 25 & 120.48 & 31. & 45 & 112.68 & 39 & 05 & 103.02 \\
\hline 24 & 35 & 120.33 & 31 & 55 & 112.48 & 39 & 15 & 102.78 \\
\hline 24 & 45 & 120.17 & 32 & 05 & 112.28 & 39 & 25 & 102.54 \\
\hline 24 & 55 & 120.01 & 32 & 15 & 112.08 & 39 & 35 & 102.30 \\
\hline 25 & 05 & 119.85 & 32 & 25 & 111.87 & 39 & 45 & 102.06 \\
\hline 25 & 15 & 119.69 & 32 & 35 & 111.67 & 39 & 55 & 101.82 \\
\hline 25 & 25 & 119.53 & 32 & 45 & 111.47 & 40 & 05 & 101.57 \\
\hline 25 & 35 & 119.37 & 32 & 55 & 111.26 & 40 & 15 & 101.33 \\
\hline 25 & 45 & 119. 21 & 33 & 05 & 111: 06 & 40 & 25 & 101.08 \\
\hline 25 & 55 & 119.04 & 33 & 15 & 110.85 & 40 & 35 & 100.83 \\
\hline 26 & 05 & 118.87 & 33 & 25 & 110.64 & 40 & 45 & 100.59 \\
\hline 26 & 15 & 118.71 & 33 & 35 & 110.43 & 40 & 55 & 100.34 \\
\hline 26 & 25 & 118.54 & 33 & 45 & 110.22 & 41 & 05 & 100.09 \\
\hline 26 & 35 & 118.37 & 33 & 55 & 110.01 & 41 & 15 & 99.84 \\
\hline 26 & 45 & 118. 21 & 34 & 05 & 109.80 & 41 & 25 & 99.59 \\
\hline 26 & 55 & 118.04 & 34 & 15 & 109.59 & 41 & 35 & 99.33 \\
\hline 27 & 05 & 117.87 & 34 & 25 & 109.37 & 41 & 45 & 99.08 \\
\hline 27 & 15 & $\cdot 117.69$ & 34 & 35 & 109.16 & 41. & 55 & 98.83 \\
\hline 27 & 25 & 117.52 & 34 & 45 & 108.94 & 42 & 05 & 98.57 \\
\hline 27 & 35 & - 117.35 & 34 & 55 & 108.73 & 42 & 15 & 98.32 \\
\hline 27 & 45 & 117.17 & 35 & 05 & .108 .51 & 42 & 25 & 98.06 \\
\hline 27 & 55 & 116. 99 & 35 & 1.5 & 108.29 & 42 & 35 & 97.80 \\
\hline 28 & 05 & 116.82 & 35 & 25 & $108.07 \cdot$ & 42 & 45 & 97.55 \\
\hline 28 & 15 & 116.64 & 35 & 35 & 107.85 & 42 & 55 & 97.29 \\
\hline 28 & 25 & 116.46 & 35 & 45 & 107.63 & - 43 & 05 & 97.03 \\
\hline 28 & 35 & 116.28 & 35 & 55 & 107.41 & 43 & 15 & 96.77 \\
\hline 28 & 45 & 116. 10 & 36 & 05 & 107.19 & 43 & 25 & 96.50 \\
\hline 28 & 55 & 115. 92 & 36 & 15 & 106.96 & 43 & 35 & 96.24 \\
\hline 29 & 05 & 115.73 & 36 & 25 & 106.74 & 43 & 45 & 95.98 \\
\hline 29 & 15 & 115.55 & 36 & 35 & 106.51 & 43 & 55 & 95.71 \\
\hline
\end{tabular}


TABLE 1.1.-Areas of quadrilaterals of earth's surface of $10^{\prime}$ extent in latitude and longitude-Continued.

[From Smithsonian Geographical Tables.]

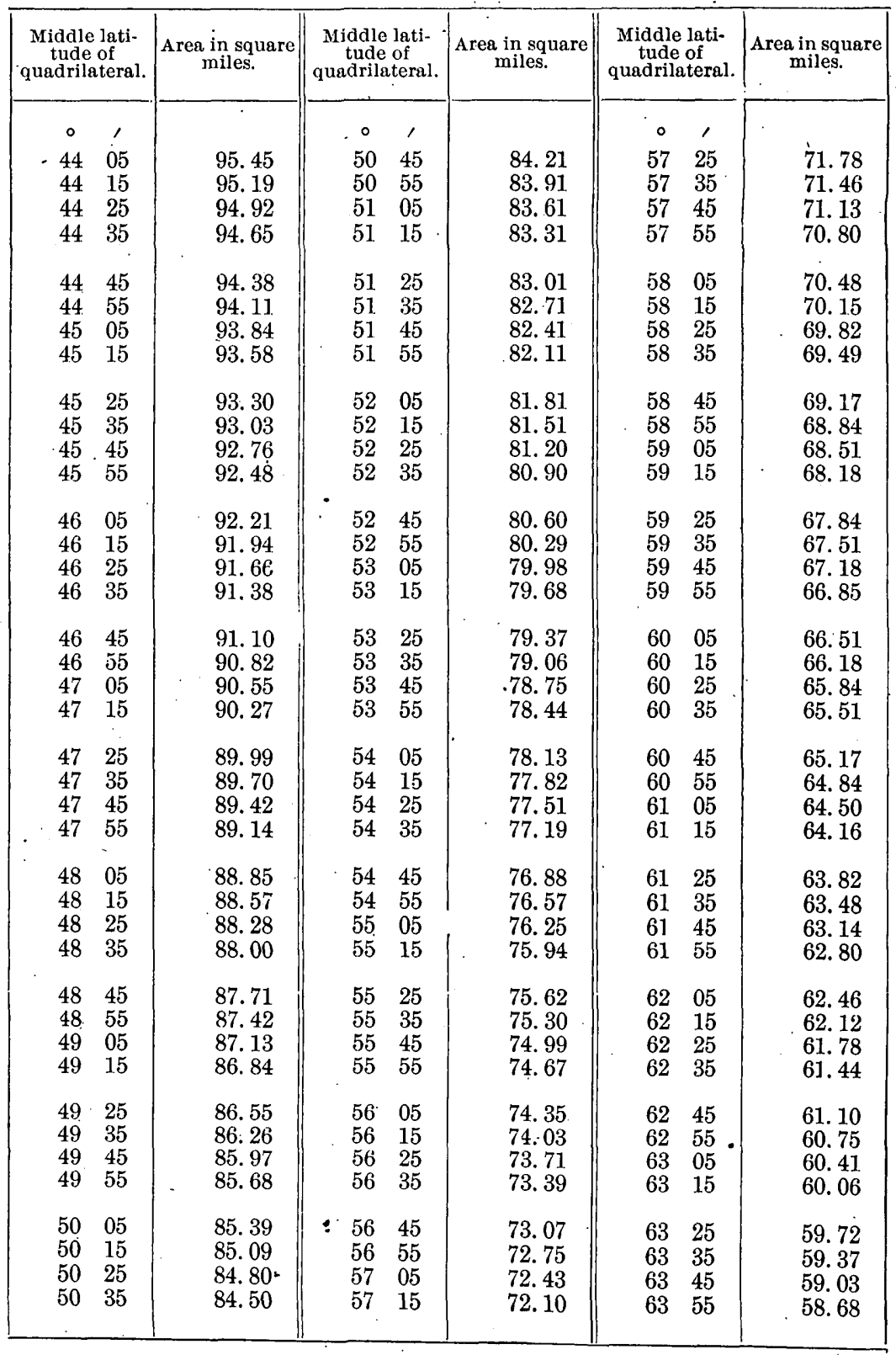


TABLE 11:-Areas of quadrilaterals of earth's surface of $10^{\prime}$ extent in latitude and longitude-Continued.

[From Smithsonian Geographical Tables.]

\begin{tabular}{|c|c|c|c|c|c|c|c|c|}
\hline \multicolumn{2}{|c|}{$\begin{array}{l}\text { Middle lati- } \\
\text { tude of } \\
\text { quadrilateral. }\end{array}$} & \multirow[t]{2}{*}{$\begin{array}{c}\text { Area in square } \\
\text { miles. }\end{array}$} & \multicolumn{2}{|c|}{$\begin{array}{l}\text { Middle lati- } \\
\text { tude of } \\
\text { quadrilateral. }\end{array}$} & \multirow{2}{*}{$\begin{array}{c}\begin{array}{c}\text { Area in square } \\
\text { miles. }\end{array} \\
\end{array}$} & \multicolumn{2}{|c|}{$\begin{array}{l}\text { Middle lati- } \\
\text { tude of } \\
\text { quadrilateral. }\end{array}$} & \multirow[t]{2}{*}{$\begin{array}{c}\text { Area in square } \\
\text { miles. }\end{array}$} \\
\hline 0 & , & & 。 & , & & 0 & , & \\
\hline 64 & 05 & 58.33 & 70 & 45 & 44.05 & 77 & 25 & 29.13 \\
\hline 64 & 15 & 57.99 & 70 & 55 & 43. 69 & 77 & 35 & 28.76 \\
\hline 64 & 25 & 57. 64 & 71 & 05 & 43. 32 & 77 & 45 & 28.37 \\
\hline 64 & 35 & 57.29 & 71 & 15 & 42.95 & 77 & 55 & 27.99 \\
\hline 64 & 45 & 56.94 & 71 & 25 & 42.58 & .78 & 05 & 27.62 \\
\hline 64 & 55 & 56.59 & 71 & 35 & 42.22 & 78 & 15 & 27.24 \\
\hline 65 & 05 & 56.24 & 71 & 45 & 41.85 & 78 & 25 & 26.85 \\
\hline 65 & 15 & 55.89 & 71 & 55 & 41.48 & 78 & 35 & 26.47 \\
\hline 65 & 25 & 55.54 & 72 & 05 & 41. 11 & 78 & 45 & 26.09 \\
\hline 65 & 35 & 55. 19 & 72 & 15 & 40.74 & 78 & 55 & 25.71 \\
\hline 65 & 45 & 54.83 & 72 & 25 & 40.37 & 79 & 05 & 25.33 \\
\hline 65 & 55 & 54.48 & 72 & 35 & 40.00 & 79 & 15 & 24.95 \\
\hline 66 & 05 & 54.13 & 72 & 45 & 39.63 & 79 & 25 & 24.57 \\
\hline 66 & 15 & 53.78 & 72 & 55 & 39.26 & 79 & 35 & 24.18 \\
\hline 66 & 25 & 53.42 & 73 & 05 & 38.89 & 79 & 45 & 23.80 \\
\hline 66 & 35 & 53.06 & 73 & 15 & 38.52 & 79 & 55 & 23. 42 \\
\hline 66. & 45 & 52.71 & 73 & 25 & 38.15 & 80 & 05 & 23.04 \\
\hline 66 & 55 & 52.35 & 73 & 35 & 37.78 & 80 & 15 & 22.65 \\
\hline 67 & 05 & 52.00 & 73 & 45 & 37.41 & 80 & 25 & 22.27 \\
\hline 67 & 15 & 51.64 & 73 & 55 & 37.03 & 80 & 35 & 21.89 \\
\hline 67 & 25 & 51.28 & 74 & 05 & 36.66 & 80 & 45 & 21.50 \\
\hline 67 & 35 & 50.93 & 74 & 15 & 36.29 & 80 & 55 & 21. 12 \\
\hline 67 & 45 & 50.57 & 74 & 25 & 35.91 & 81 & 05 & 20.73 \\
\hline 67 & 55 & 50.21 & 74 & 35 & 35.54 & 81 & 15 & 20.35 \\
\hline 68 & 05 & 49.85 & 74 & 45 & 35.17 & 81 & 25 & 19.97 \\
\hline 68 & 15 & 49.49 & 74 & 55 & 34.79 & 81 & 35 & 19.58 \\
\hline 68 & 25 & 49.13 & 75 & 05 & 34.42 & 81 & 45 & 19.20 \\
\hline 68 & 35 & 48.77 & 75 & 15 & 34.04 & 81 & 55 & 18. 81 \\
\hline 68 & 45 & 48.41 & 75 & 25 & 33.66 & 82 & 05 & 18. 43 \\
\hline 68 & 55 & 48.05 & 75 & 35 & 33.29 & 82. & 15 & 18.04 \\
\hline 69 & 05 & 47.69 & 75 & 45 & 32.91 & 82 & 25 & 17.65 \\
\hline 69 & 15 & 47.33 & 75 & 55 & 32.53 & 82 & 35 & 17.27 \\
\hline 69 & 25 & 46.97 & 76 & 05 & 32.16 & 82 & 45 & 16.88 \\
\hline 69 & 35 & 46.60 & 76 & 15 & 31.78 & 82 & 55 & 16.50 \\
\hline 69 & 45 & 46. 24 & 76 & 25 & 31.40 & 83 & 05 & 16. 11 \\
\hline 69 & 55 & 45.88 & 76 & 35 & 31.03 & 83 & 15 & 15.73 \\
\hline 70 & 05 & 45.51 & 76 & 45 & 30.65 & 83 & 25 & 15. 34 \\
\hline 70 & 15 & 45.15 & 76 & 55 & 30.27 & 83 & 35 & 14.95 \\
\hline 70 & 25 & 44. 78 & 77 & 05 & 29.89 & 83 & 45 & 14.57 \\
\hline 70 & 35 & 44.42 & 77 & 15 & 29.51 & 83 & 55 & 14.18 \\
\hline
\end{tabular}


TABLe 11.-Area of quadrilaterals of earth's surface of $10^{\prime}$ extent in latitude and longitude-Continued.

[From Smithsonian Geographical Tables.]

\begin{tabular}{|c|c|c|c|c|c|c|c|c|}
\hline \multicolumn{2}{|c|}{$\begin{array}{c}\text { Middle lati- } \\
\text { tude of } \\
\text { quadrilateral. }\end{array}$} & \multirow[t]{2}{*}{$\begin{array}{l}\text { Area in square } \\
\text { miles. }\end{array}$} & \multicolumn{2}{|c|}{$\begin{array}{l}\text { Middle lati- } \\
\text { tude of } \\
\text { quadrilateral. }\end{array}$} & \multirow[t]{2}{*}{$\begin{array}{l}\text { Area in square } \\
\text { miles. }\end{array}$} & \multicolumn{2}{|c|}{$\begin{array}{l}\text { Middle lati- } \\
\text { tude of } \\
\text { quadrilateral. }\end{array}$} & \multirow[t]{2}{*}{$\begin{array}{l}\text { Area in square } \\
\text { miles. }\end{array}$} \\
\hline$\circ$ & , & & $\circ$ & , & & 。 & , & \\
\hline 84 & 05 & 13. 79 & 86 & 05 & 9.14 & 88 & 05 & 4. 47 \\
\hline 84 & 15 & 13.40 & 86 & 15 & 8.75 & 88 & 15 & 4. 09 \\
\hline 84 & 25 & 13.02 & 86 & 25 & 8. 36 & 88 & 25 & 3. 70 \\
\hline 84 & 35 & 12.63 & 86 & 35 & 7. 97 & 88 & 35 & 3. 31 \\
\hline 84 & 45 & 12.24 & 86 & 45 & 7.59 & 88 & 45 & 2.92 \\
\hline 84 & 55 & 11. 86 & 86 & 55 & 7.20 & 88 & 55 & 2.53 \\
\hline 85 & 05 & 11.47 & 87 & 05 & 6.81 & 89 & 05 & 2.14 \\
\hline 85 & 15 & 11.08 & 87 & 15 & 6.42 & 89 & 15 & 1.75 \\
\hline 85 & 25 & 10.69 & 87 & 25 & 6.03 & 89 & 25 & 1. 36 \\
\hline 85 & 35 & 10. 30 & 87 & 35 & 5. 64 & 89 & 35 & 0.97 \\
\hline 85 & 45 & 9.92 & 87 & 45 & 5.25 & 89 & 45 & 0.58 \\
\hline 85 & 55 & 9.53 & 87 & 55 & 4. 86 & 89 & 55 & 0.19 \\
\hline
\end{tabular}


'TABLe 12.-For conversion of arc into time.

\begin{tabular}{|c|c|c|c|c|c|c|c|c|c|c|c|c|c|c|c|}
\hline ○ & h. m. & 。 & h. m. & $\circ$ & h. m. & $\circ$ & h. m. & $\circ$ & h. m. & $\circ$ & h. m. & $'$ & m. s. & $\prime \prime$ & s. \\
\hline $\begin{array}{l}0 \\
1 \\
2 \\
3 \\
4 \\
5 \\
6 \\
7 \\
8 \\
9\end{array}$ & $\begin{array}{lr}0 & 0 \\
0 & 4 \\
0 & 8 \\
0 & 12 \\
0 & 16 \\
0 & 20 \\
0 & 24 \\
0 & 28 \\
0 & 32 \\
0 & 36\end{array}$ & $\begin{array}{l}\mathbf{6 0} \\
61 \\
62 \\
63 \\
64 \\
\mathbf{6 5} \\
66 \\
67 \\
68 \\
69\end{array}$ & $\begin{array}{ll}4 & 0 \\
4 & 4 \\
4 & 8 \\
4 & 12 \\
4 & 16 \\
4 & 20 \\
4 & 24 \\
4 & 28 \\
4 & 32 \\
4 & 36\end{array}$ & $\begin{array}{r}\mathbf{1 2 0} \\
121 \\
122 \\
123 \\
124 \\
\mathbf{1 2 5} \\
126 \\
127 \\
.128 \\
129\end{array}$ & $\begin{array}{lr}8 & 0 \\
8 & 4 \\
8 & 8 \\
8 & 12 \\
8 & 16 \\
8 & 20 \\
8 & 24 \\
8 & 28 \\
8 & 32 \\
8 & 36\end{array}$ & $\begin{array}{l}180 \\
181 \\
182 \\
183 \\
184 \\
185 \\
186 \\
187 \\
188 \\
189\end{array}$ & $\begin{array}{rr}12 & 0 \\
12 & 4 \\
12 & 8 \\
12 & 12 \\
12 & 16 \\
12 & 20 \\
12 & 24 \\
12 & 28 \\
12 & 32 \\
12 & 36\end{array}$ & $\begin{array}{l}\mathbf{2 4 0} \\
241 \\
242 \\
243 \\
244 \\
\mathbf{2 4 5} \\
246 \\
247 \\
248 \\
249\end{array}$ & $\begin{array}{rr}16 & 0 \\
16 & 4 \\
16 & 8 \\
16 & 12 \\
16 & 16 \\
16 & 20 \\
16 & 24 \\
16 & 28 \\
16 & 32 \\
16 & 36\end{array}$ & \begin{tabular}{|l}
$\mathbf{3 0 0}$ \\
301 \\
302 \\
303 \\
304 \\
$\mathbf{3 0 5}$ \\
306 \\
307 \\
308 \\
309
\end{tabular} & $\begin{array}{rr}20 & 0 \\
20 & 4 \\
20 & 8 \\
20 & 12 \\
20 & 16 \\
20 & 20 \\
20 & 24 \\
20 & 28 \\
20 & 32 \\
20 & 36\end{array}$ & $\begin{array}{l}0 \\
1 \\
2 \\
3 \\
4 \\
6 \\
6 \\
7 \\
8 \\
9\end{array}$ & $\begin{array}{lr}0 & 0 \\
0 & 4 \\
0 & 8 \\
0 & 82 \\
0 & 12 \\
0 & 16 \\
0 & 20 \\
0 & 24 \\
0 & 28 \\
0 & 32 \\
0 & 36\end{array}$ & $\begin{array}{l}0 \\
1 \\
2 \\
3\end{array}$ & $\begin{array}{l}0.000 \\
0.067 \\
0.133 \\
0.200 \\
0.267 \\
0.333 \\
0.400 \\
0.467 \\
0.533 \\
0.600\end{array}$ \\
\hline 10 & 040 & 70 & 440 & 130 & 840 & 100 & 1240 & 250 & 1640 & 310 & 2040 & 10 & 040 & 10 & 0.667 \\
\hline $\begin{array}{l}11 \\
12 \\
13 \\
14 \\
15 \\
16 \\
17 \\
18 \\
19\end{array}$ & $\begin{array}{lr}0 & 44 \\
0 & 48 \\
0 & 52 \\
0 & 56 \\
1 & 0 \\
1 & 4 \\
1 & 8 \\
1 & 12 \\
1 & 16\end{array}$ & $\begin{array}{l}71 \\
72 \\
73 \\
74 \\
75 \\
76 \\
77 \\
78 \\
79\end{array}$ & $\begin{array}{rr}4 & 44 \\
4 & 48 \\
4 & 52 \\
4 & 56 \\
5 & 0 \\
5 & 4 \\
5 & 8 \\
5 & 12 \\
5 & 16\end{array}$ & \begin{tabular}{|l}
131 \\
132 \\
133 \\
134 \\
$\mathbf{1 3 5}$ \\
$\mathbf{1 3 6}$ \\
137 \\
138 \\
139
\end{tabular} & $\begin{array}{lr}8 & 44 \\
8 & 48 \\
8 & 52 \\
8 & 56 \\
9 & 0 \\
9 & 4 \\
9 & 8 \\
9 & 12 \\
9 & 16\end{array}$ & $\begin{array}{r}191 \\
192 \\
193 \\
194 \\
195 \\
196 \\
197 \\
198 \\
199\end{array}$ & $\begin{array}{rr}12 & 44 \\
12 & 48 \\
12 & 52 \\
12 & 56 \\
13 & 0 \\
13 & 4 \\
13 & 8 \\
13 & 12 \\
13 & 16\end{array}$ & \begin{tabular}{|l|}
251 \\
252 \\
253 \\
254 \\
$\mathbf{2 5 5}$ \\
256 \\
257 \\
258 \\
259 \\
\end{tabular} & $\begin{array}{rr}16 & 44 \\
16 & 48 \\
16 & 52 \\
16 & 56 \\
17 & 0 \\
17 & 4 \\
17 & 8 \\
17 & 12 \\
17 & 16\end{array}$ & \begin{tabular}{|r}
311 \\
312 \\
313 \\
314 \\
$\mathbf{3 1 5}$ \\
316 \\
317 \\
318 \\
319 \\
\end{tabular} & $\begin{array}{rr}20 & 44 \\
20 & 48 \\
20 & 52 \\
20 & 56 \\
21 & 0 \\
21 & 4 \\
21 & 8 \\
21 & 12 \\
21 & 16\end{array}$ & $\begin{array}{l}11 \\
12 \\
13 \\
14 \\
15 \\
16 \\
17 \\
18 \\
19\end{array}$ & $\begin{array}{rr}0 & 44 \\
0 & 48 \\
0 & 52 \\
0 & 56 \\
1 & 0 \\
1 & 4 \\
1 & 8 \\
1 & 12 \\
1 & 16\end{array}$ & $\begin{array}{l}11 \\
12 \\
13 \\
14 \\
16 \\
16 \\
17 \\
18 \\
19\end{array}$ & $\begin{array}{l}0.733 \\
0.800 \\
0.867 \\
0.933 \\
1.000 \\
1.067 \\
1.133 \\
1.200 \\
1.267\end{array}$ \\
\hline 20 & 120 & 80 & 520 & 140 & 920 & 1200 & $\overline{1320}$ & 260 & 1720 & 320 & 2120 & 20 & 120 & 20 & 7.333 \\
\hline $\begin{array}{r}21 \\
22 \\
23 \\
24 \\
\mathbf{2 5} \\
26 \\
27 \\
28 \\
29\end{array}$ & $\begin{array}{ll}1 & 24 \\
1 & 28 \\
1 & 32 \\
1 & 36 \\
1 & 40 \\
1 & 44 \\
1 & 48 \\
1 & 52 \\
1 & 56\end{array}$ & $\begin{array}{l}81 \\
82 \\
83 \\
84 \\
85 \\
86 \\
87 \\
88 \\
89\end{array}$ & $\begin{array}{ll}5 & 24 \\
5 & 28 \\
5 & 32 \\
5 & 36 \\
5 & 40 \\
5 & 44 \\
5 & 48 \\
5 & 52 \\
5 & 56\end{array}$ & $\begin{array}{l}141 \\
142 \\
143 \\
144 \\
145 \\
146 \\
147 \\
148 \\
149\end{array}$ & $\begin{array}{ll}9 & 24 \\
9 & 28 \\
9 & 32 \\
9 & 36 \\
9 & 40 \\
9 & 44 \\
9 & 48 \\
9 & 52 \\
9 & 56\end{array}$ & $\begin{array}{r}201 \\
202 \\
203 \\
204 \\
\mathbf{2 0 5} \\
206 \\
207 \\
208 \\
209\end{array}$ & $\begin{array}{ll}13 & 24 \\
13 & 28 \\
13 & 32 \\
13 & 30 \\
13 & 40 \\
13 & 44 \\
13 & 48 \\
13 & 52 \\
13 & 56\end{array}$ & \begin{tabular}{|}
261 \\
262 \\
263 \\
264 \\
$\mathbf{2 6 5}$ \\
266 \\
267 \\
268 \\
269
\end{tabular} & $\begin{array}{ll}17 & 24 \\
17 & 28 \\
17 & 32 \\
17 & 36 \\
17 & 40 \\
17 & 44 \\
17 & 48 \\
17 & 52 \\
17 & 56\end{array}$ & \begin{tabular}{|c}
321 \\
322 \\
323 \\
324 \\
$\mathbf{3 2 5}$ \\
326 \\
327 \\
328 \\
329
\end{tabular} & $\begin{array}{ll}21 & 24 \\
21 & 28 \\
21 & 32 \\
21 & 36 \\
21 & 40 \\
21 & 44 \\
21 & 48 \\
21 & 52 \\
21 & 56\end{array}$ & $\begin{array}{l}21 \\
22 \\
23 \\
24 \\
25 \\
26 \\
27 \\
28 \\
29\end{array}$ & $\begin{array}{ll}1 & 24 \\
1 & 28 \\
1 & 32 \\
1 & 36 \\
1 & 40 \\
1 & 44 \\
1 & 48 \\
1 & 52 \\
1 & 56\end{array}$ & $\begin{array}{l}21 \\
22 \\
23 \\
24 \\
25 \\
26 \\
27 \\
28 \\
29\end{array}$ & $\begin{array}{l}1.400 \\
1.467 \\
1.533 \\
1.600 \\
1.667 \\
1.733 \\
1.800 \\
1.867 \\
1.933\end{array}$ \\
\hline 30 & \begin{tabular}{l|l|}
2 & 0 \\
\end{tabular} & 90 & $\begin{array}{ll}6 & 0\end{array}$ & 150 & 100 & 210 & $\begin{array}{ll}140 \\
\end{array}$ & $\overline{270}$ & $\overline{180}$ & 330 & $\begin{array}{ll}220 \\
\end{array}$ & 30 & \begin{tabular}{ll|}
2 & 0 \\
\end{tabular} & 30 & 2.000 \\
\hline $\begin{array}{l}31 \\
32 \\
33 \\
34 \\
\mathbf{3 5} \\
36 \\
37 \\
38 \\
39\end{array}$ & $\begin{array}{rr}2 & 4 \\
2 & 8 \\
2 & 12 \\
2 & 16 \\
2 & 20 \\
2 & 24 \\
2 & 28 \\
2 & 32 \\
2 & 36\end{array}$ & $\begin{array}{c}91 \\
92 \\
93 \\
94 \\
95 \\
96 \\
97 \\
98 \\
99\end{array}$ & $\begin{array}{ll}6 & 4 \\
6 & 8 \\
6 & 12 \\
6 & 16 \\
6 & 20 \\
6 & 24 \\
6 & 28 \\
6 & 32 \\
6 & 36\end{array}$ & \begin{tabular}{|l}
151 \\
152 \\
153 \\
154 \\
155 \\
156 \\
157 \\
158 \\
159
\end{tabular} & $\begin{array}{rr}10 & 4 \\
10 & 8 \\
10 & 12 \\
10 & 16 \\
10 & 20 \\
10 & 24 \\
10 & 28 \\
10 & 32 \\
10 & 36\end{array}$ & $\begin{array}{l}211 \\
212 \\
213 \\
214 \\
215 \\
216 \\
217 \\
218 \\
219\end{array}$ & $\begin{array}{rr}14 & 4 \\
14 & 8 \\
14 & 12 \\
14 & 16 \\
14 & 20 \\
14 & 24 \\
14 & 28 \\
14 & 32 \\
14 & 36\end{array}$ & $\begin{array}{r}271 \\
272 \\
273 \\
274 \\
275 \\
276 \\
277 \\
278 \\
279\end{array}$ & \begin{tabular}{|rr}
18 & 4 \\
18 & 8 \\
18 & 12 \\
18 & 16 \\
18 & 20 \\
18 & 24 \\
18 & 28 \\
18 & 32 \\
18 & 36
\end{tabular} & \begin{tabular}{|r}
331 \\
332 \\
333 \\
334 \\
335 \\
336 \\
337 \\
338 \\
339
\end{tabular} & $\begin{array}{rr}22 & 4 \\
22 & 8 \\
22 & 12 \\
22 & 16 \\
22 & 20 \\
22 & 24 \\
22 & 28 \\
22 & 32 \\
22 & 36\end{array}$ & $\begin{array}{l}31 \\
32 \\
33 \\
34 \\
35 \\
36 \\
37 \\
38 \\
39\end{array}$ & $\begin{array}{ll}2 & 4 \\
2 & 8 \\
2 & 12 \\
2 & 16 \\
2 & 20 \\
2 & 24 \\
2 & 28 \\
2 & 32 \\
2 & 36\end{array}$ & $\begin{array}{l}31 \\
32 \\
33 \\
34 \\
\mathbf{3 5} \\
36 \\
37 \\
38 \\
39\end{array}$ & $\begin{array}{l}2.067 \\
2.133 \\
2.200 \\
2.267 \\
2.333 \\
2.400 \\
2.467 \\
2.533 \\
2.600\end{array}$ \\
\hline 40 & 240 & 100 & 640 & 160 & 1040 & 220 & $\overline{1440}$ & 280 & 1840 & 340 & 2240 & 40 & 240 & 40 & 2.667 \\
\hline $\begin{array}{l}41 \\
42 \\
43 \\
44 \\
45 \\
46 \\
47 \\
48 \\
49\end{array}$ & $\begin{array}{rr}2 & 44 \\
2 & 48 \\
2 & 52 \\
2 & 56 \\
3 & 0 \\
3 & 4 \\
3 & 8 \\
3 & 12 \\
3 & 16\end{array}$ & $\begin{array}{l}101 \\
102 \\
103 \\
104 \\
\mathbf{1 0 5} \\
106 \\
107 \\
108 \\
109\end{array}$ & $\begin{array}{rr}6 & 44 \\
6 & 48 \\
6 & 58 \\
6 & 56 \\
7 & 0 \\
7 & 4 \\
7 & 8 \\
7 & 15 \\
7 & 16 \\
7 & 16\end{array}$ & $\begin{array}{l}161 \\
162 \\
163 \\
164 \\
105 \\
166 \\
167 \\
168 \\
169\end{array}$ & $\begin{array}{rr}10 & 44 \\
10 & 48 \\
10 & 52 \\
10 & 56 \\
11 & 0 \\
11 & 4 \\
11 & 8 \\
11 & 12 \\
11 & 16\end{array}$ & $\begin{array}{r}221 \\
222 \\
223 \\
224 \\
\mathbf{2 2 5} \\
226 \\
227 \\
228 \\
229\end{array}$ & $\begin{array}{rr}14 & 44 \\
14 & 48 \\
14 & 52 \\
14 & 56 \\
15 & 0 \\
15 & 4 \\
15 & 8 \\
15 & 12 \\
15 & 16\end{array}$ & $\begin{array}{r}281 \\
282 \\
283 \\
284 \\
\mathbf{2 8 5} \\
286 \\
287 \\
288 \\
289\end{array}$ & $\begin{array}{rr}18 & 44 \\
18 & 48 \\
18 & 52 \\
18 & 56 \\
19 & 0 \\
19 & 4 \\
19 & 8 \\
19 & 12 \\
19 & 16\end{array}$ & \begin{tabular}{|l}
341 \\
342 \\
343 \\
344 \\
$\mathbf{3 4 5}$ \\
346 \\
347 \\
348 \\
349
\end{tabular} & $\begin{array}{rr}22 & 44 \\
22 & 48 \\
22 & 52 \\
22 & 56 \\
23 & 0 \\
23 & 4 \\
23 & 8 \\
23 & 12 \\
23 & 16\end{array}$ & $\begin{array}{l}41 \\
42 \\
43 \\
44 \\
45 \\
46 \\
47 \\
48 \\
49\end{array}$ & $\begin{array}{rr}2 & 44 \\
2 & 48 \\
2 & 52 \\
2 & 56 \\
3 & 0 \\
3 & 4 \\
3 & 8 \\
3 & 12 \\
3 & 16\end{array}$ & $\begin{array}{l}41 \\
42 \\
43 \\
44 \\
45 \\
46 \\
47 \\
48 \\
49\end{array}$ & $\begin{array}{l}2.733 \\
2.800 \\
2.867 \\
2.933 \\
3.000 \\
3.067 \\
3.133 \\
3.200 \\
3.267\end{array}$ \\
\hline 50 & 320 & $\overline{110}$ & 720 & $\overline{170}$ & 1120 & 230 & $\begin{array}{lll}15 & 20\end{array}$ & 290 & \begin{tabular}{|lll}
19 & 20
\end{tabular} & 350 & $23 \quad 20$ & 50 & 320 & 50 & 3.333 \\
\hline $\begin{array}{l}51 \\
52 \\
53 \\
54 \\
\mathbf{5 5} \\
56 \\
57 \\
58 \\
59\end{array}$ & $\begin{array}{lll}3 & 24 \\
3 & 28 \\
3 & 32 \\
3 & 36 \\
3 & 40 \\
3 & 44 \\
3 & 48 \\
3 & 52 \\
3 & 56\end{array}$ & $\begin{array}{l}111 \\
112 \\
113 \\
114 \\
115 \\
116 \\
117 \\
118 \\
119\end{array}$ & $\begin{array}{ll}7 & 24 \\
7 & 28 \\
7 & 39 \\
7 & 31 \\
7 & 40 \\
7 & 44 \\
7 & 48 \\
7 & 52 \\
7 & 56\end{array}$ & $\begin{array}{l}171 \\
172 \\
173 \\
174 \\
175 \\
176 \\
177 \\
178 \\
179\end{array}$ & $\begin{array}{ll}11 & 24 \\
11 & 28 \\
11 & 32 \\
11 & 36 \\
11 & 40 \\
11 & 44 \\
11 & 48 \\
11 & 52 \\
11 & 56\end{array}$ & $\begin{array}{r}231 \\
232 \\
233 \\
234 \\
235 \\
236 \\
237 \\
238 \\
239\end{array}$ & $\begin{array}{ll}15 & 24 \\
15 & 28 \\
15 & 32 \\
15 & 36 \\
15 & 40 \\
15 & 44 \\
15 & 48 \\
15 & 52 \\
15 & 56\end{array}$ & $\begin{array}{r}291 \\
292 \\
293 \\
294 \\
\mathbf{2 0 5} \\
296 \\
297 \\
298 \\
299\end{array}$ & $\begin{array}{ll}19 & 24 \\
19 & 28 \\
19 & 32 \\
19 & 36 \\
19 & 40 \\
19 & 44 \\
19 & 48 \\
19 & 52 \\
19 & 56\end{array}$ & $\begin{array}{l}351 \\
352 \\
353 \\
354 \\
\mathbf{3 5 5} \\
356 \\
357 \\
358 \\
359\end{array}$ & $\begin{array}{ll}23 & 24 \\
23 & 28 \\
23 & 32 \\
23 & 36 \\
23 & 40 \\
23 & 44 \\
23 & 48 \\
23 & 52 \\
23 & 56\end{array}$ & $\begin{array}{l}51 \\
52 \\
53 \\
54 \\
55 \\
56 \\
57 \\
58 \\
59\end{array}$ & $\begin{array}{lll}3 & 24 \\
3 & 28 \\
3 & 32 \\
3 & 36 \\
3 & 40 \\
3 & 44 \\
3 & \mathbf{4 8} \\
3 & 52 \\
3 & 56\end{array}$ & $\begin{array}{l}51 \\
52 \\
63 \\
54 \\
\mathbf{5 5} \\
56 \\
57 \\
58 \\
59\end{array}$ & $\begin{array}{l}3.400 \\
3.467 \\
3.533 \\
3.600 \\
3.667 \\
3.733 \\
3.800 \\
3.867 \\
3.933\end{array}$ \\
\hline 60 & 40 & 120 & 80 & $\overline{180}$ & 120 & 240 & 160 & $\overline{300}$ & 200 & 360 & 240 & 60 & $\begin{array}{ll}4 & 0\end{array}$ & 60 & 4.000 \\
\hline
\end{tabular}


TABIE 13.-For conversion of time into arc.

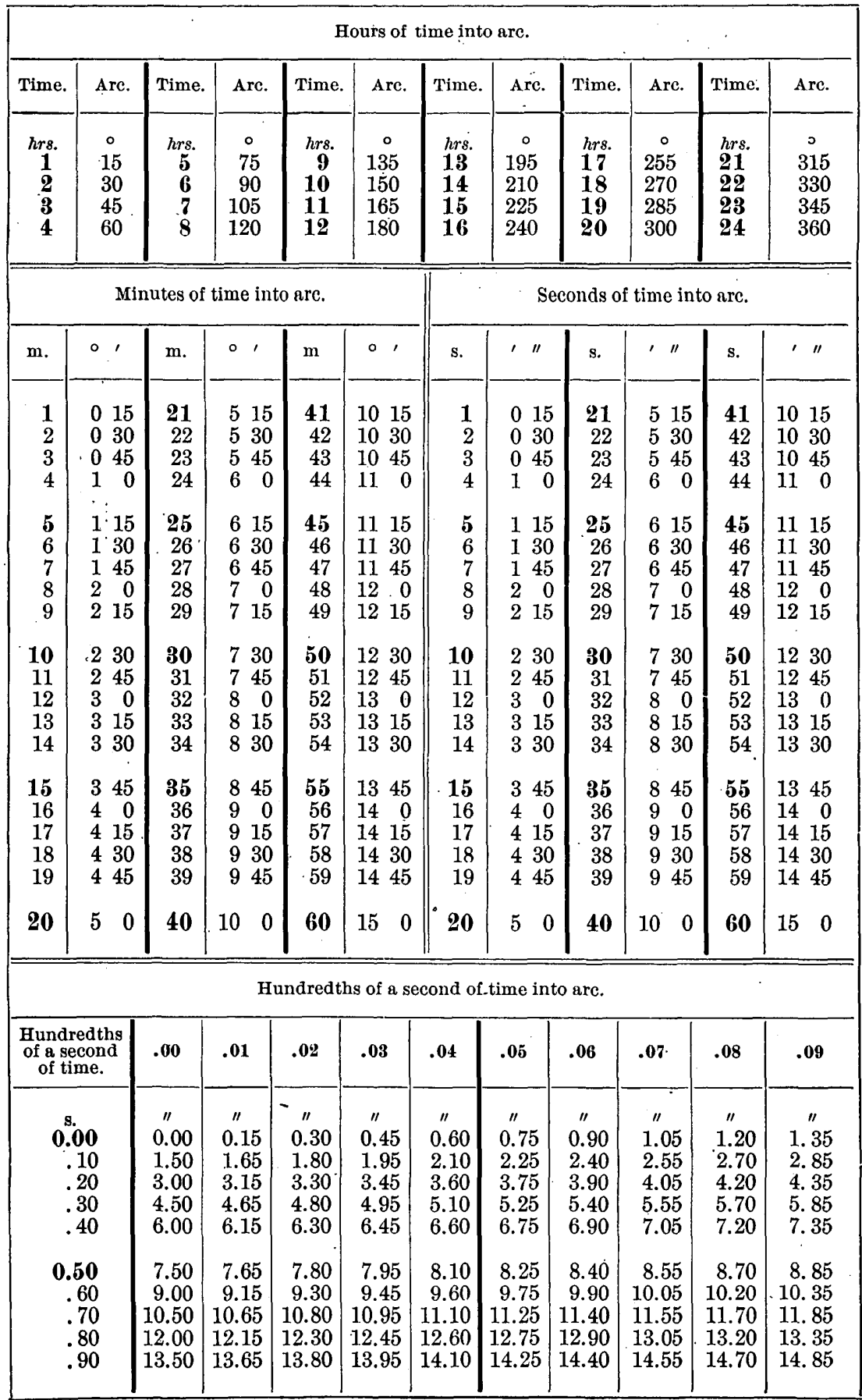


TABLE 14.-For conversion of mean time into sidereal time.

\begin{tabular}{|c|c|c|c|c|c|c|c|c|c|c|c|c|c|c|c|c|c|c|}
\hline $\mathbf{s}$ & & $\underset{0}{\mathrm{~m}}$ & & & $\frac{\mathrm{m}}{1}$ & & & $\mathrm{~m}_{2}$ & & & $\frac{\mathrm{m}}{3}$ & & & & & & & \\
\hline 0 & $\begin{array}{c}\mathrm{h} \\
0\end{array}$ & $\mathrm{~m}_{0}$ & $\begin{array}{l}\mathbf{s} \\
0\end{array}$ & $\begin{array}{r}\mathbf{h} \\
6\end{array}$ & $\underset{5}{\mathrm{~m}}$ & $\begin{array}{c}\mathrm{s} \\
15\end{array}$ & $\begin{array}{c}\mathrm{h} \\
12\end{array}$ & $\mathrm{~m}_{10}$ & $\begin{array}{c}\mathrm{s} \\
29\end{array}$ & $\underset{18}{h}$ & $\frac{m}{15}$ & $\begin{array}{c}s \\
44\end{array}$ & $\stackrel{s}{0.00}$ & $\underset{0}{\mathrm{~m}}$ & $\begin{array}{l}\mathbf{s} \\
\mathbf{0}\end{array}$ & $\stackrel{8}{0.50}$ & & $\begin{array}{ll}3 & 8 \\
3\end{array}$ \\
\hline $\begin{array}{l}1 \\
2 \\
3 \\
4 \\
5 \\
6 \\
7 \\
8 \\
9\end{array}$ & $\begin{array}{l}0 \\
0 \\
0 \\
0 \\
0 \\
0 \\
0 \\
0 \\
0\end{array}$ & $\begin{array}{r}6 \\
12 \\
18 \\
24 \\
30 \\
36 \\
42 \\
48 \\
54\end{array}$ & $\begin{array}{r}5 \\
10 \\
16 \\
21 \\
26 \\
31 \\
37 \\
42 \\
47\end{array}$ & $\begin{array}{l}6 \\
6 \\
6 \\
6 \\
6 \\
6 \\
6 \\
6 \\
7\end{array}$ & $\begin{array}{r}11 \\
17 \\
23 \\
29 \\
35 \\
41 \\
47 \\
53 \\
0\end{array}$ & $\begin{array}{r}20 \\
25 \\
30 \\
36 \\
41 \\
46 \\
51 \\
56 \\
2\end{array}$ & $\begin{array}{l}12 \\
12 \\
12 \\
12 \\
12 \\
12 \\
12 \\
12 \\
13\end{array}$ & $\begin{array}{r}16 \\
22 \\
28 \\
34 \\
40 \\
47 \\
53 . \\
59 \\
5\end{array}$ & $\begin{array}{r}34 \\
40 \\
45 \\
50 \\
55 \\
1 \\
6 \\
11 \\
16\end{array}$ & $\begin{array}{l}18 \\
18 \\
18 \\
18 \\
18 \\
18 \\
18 \\
19 \\
19\end{array}$ & $\begin{array}{r}21 \\
27 \\
33 \\
40 \\
46 \\
52 \\
58 \\
4 \\
10\end{array}$ & $\begin{array}{r}49 \\
54 \\
59 \\
5 \\
10 \\
15 \\
20 \\
26 \\
31 .\end{array}$ & $\begin{array}{l}0.01 \\
0.02 \\
0.03 \\
0.04 \\
0.05 \\
0.06 \\
0.07 \\
0.08 \\
0.09\end{array}$ & $\begin{array}{l}0 \\
0 \\
0 \\
0 \\
0 \\
0 \\
0 \\
0 \\
0\end{array}$ & $\begin{array}{r}4 \\
7 \\
11 \\
15 \\
18 \\
22 \\
26 \\
29 \\
33\end{array}$ & $\begin{array}{l}0.51 \\
0.52 \\
0.53 \\
0.54 \\
0.55 \\
0.56 \\
0.57 \\
0.58 \\
0.59\end{array}$ & & $\begin{array}{r}6 \\
10 \\
14 \\
17 \\
21 \\
25 \\
28 \\
32 \\
35\end{array}$ \\
\hline 10 & 1 & 0 & 52 & 7 & 6 & 7 & 13 & 11 & 21 & 19 & 16 & 36 & 0.10 & 0 & 37 & 0.60 & & 39 \\
\hline $\begin{array}{l}11 \\
12 \\
13 \\
14 \\
15 \\
16 \\
17 \\
18 \\
19\end{array}$ & $\begin{array}{l}1 \\
1 \\
1 \\
1 \\
1 \\
1 \\
1 \\
1 \\
1\end{array}$ & $\begin{array}{r}6 \\
13 \\
19 \\
25 \\
31 \\
37 \\
43 \\
49 \\
55\end{array}$ & $\begin{array}{r}58 \\
3 \\
8 \\
13 \\
19 \\
24 \\
29 \\
34 \\
40\end{array}$ & $\begin{array}{l}7 \\
7 \\
7 \\
7 \\
7 \\
7 \\
7 \\
7 \\
3\end{array}$ & $\begin{array}{r}12 \\
18 \\
24 \\
30 \\
36 \\
42 \\
48 \\
54 \\
0\end{array}$ & $\begin{array}{l}12 \\
17 \\
23 \\
28 \\
33 \\
38 \\
44 \\
49 \\
54\end{array}$ & $\begin{array}{l}13 \\
13 \\
13 \\
13 \\
13 \\
13 \\
13 \\
14 \\
14\end{array}$ & $\begin{array}{r}17 \\
23 \\
29 \\
35 \\
41 \\
47 \\
53 \\
0 \\
6\end{array}$ & $\begin{array}{r}27 \\
32 \\
37 \\
42 \\
48 \\
53 \\
58 \\
3 \\
9\end{array}$ & $\begin{array}{l}19 \\
19 \\
19 \\
19 \\
19 \\
19 \\
19 \\
20 \\
20\end{array}$ & $\begin{array}{r}22 \\
28 \\
34 \\
40 \\
47 \\
53 \\
59 \\
5 \\
11\end{array}$ & $\begin{array}{r}41 \\
47 \\
52 \\
57 \\
2 \\
7 \\
13 \\
18 \\
23\end{array}$ & $\begin{array}{l}0.11 \\
0.12 \\
0.13 \\
0.14 \\
0.15 \\
0.16 \\
0.17 \\
0.18 \\
0.19\end{array}$ & $\begin{array}{l}0 \\
0 \\
0 \\
0 \\
0 \\
0 \\
1 \\
1 \\
1\end{array}$ & $\begin{array}{r}40 \\
44 \\
47 \\
51 \\
55 \\
58 \\
2 \\
6 \\
9\end{array}$ & $\begin{array}{l}0.61 \\
0.62 \\
0.63 \\
0.64 \\
0.65 \\
0.66 \\
0.67 \\
0.68 \\
0.69\end{array}$ & & $\begin{array}{r}43 \\
46 \\
50 \\
54 \\
57 \\
1 \\
5 \\
8 \\
12\end{array}$ \\
\hline 20 & 2 & 1 & 45 & 8 & 6 & 59 & 14 & 12 & 14 & 20 & 17 & 28 & 0.20 & 1 & 13 & 0.70 & & 16 \\
\hline $\begin{array}{l}21 \\
22 \\
23 \\
24 \\
25 \\
26 \\
27 \\
28 \\
29\end{array}$ & $\begin{array}{l}2 \\
2 \\
2 \\
2 \\
2 \\
2 \\
2 \\
2 \\
2\end{array}$ & $\begin{array}{r}7 \\
13 \\
20 \\
26 \\
32 \\
38 \\
44 \\
50 \\
56 \\
\end{array}$ & $\begin{array}{r}50 \\
55 \\
1 \\
6 \\
11 \\
16 \\
22 \\
27 \\
32\end{array}$ & $\begin{array}{l}8 \\
8 \\
8 \\
8 \\
8 \\
8 \\
8 \\
8 \\
9\end{array}$ & $\begin{array}{l}13 \\
19 \\
25 \\
31 \\
37 \\
43 \\
49 \\
55 \\
1\end{array}$ & $\begin{array}{r}5 \\
10 \\
15 \\
20 \\
26 \\
31 \\
36 \\
41 \\
47\end{array}$ & $\begin{array}{l}14 \\
14 \\
14 \\
14 \\
14 \\
14 \\
14 \\
15 \\
15\end{array}$ & $\begin{array}{r}18 \\
24 \\
30 \\
36 \\
42 \\
48 \\
54 \\
0 \\
7\end{array}$ & $\begin{array}{l}19 \\
24 \\
30 \\
35 \\
40 \\
45 \\
51 \\
56 \\
1\end{array}$ & $\begin{array}{l}20 \\
20 \\
20 \\
20 \\
20 \\
20 \\
21 \\
21 \\
21 \\
\end{array}$ & $\begin{array}{r}23 \\
29 \\
35 \\
41 \\
47 \\
54 \\
0 \\
6 \\
12\end{array}$ & $\begin{array}{r}34 \\
39 \\
44 \\
49 \\
55 \\
0 \\
5 \\
10 \\
16 \\
\end{array}$ & $\begin{array}{l}0.21 \\
0.22 \\
0.23 \\
0.24 \\
0.25 \\
0.26 \\
0.27 \\
0.28 \\
0.29\end{array}$ & $\begin{array}{l}1 \\
1 \\
1 \\
1 \\
1 \\
1 \\
1 \\
1 \\
1\end{array}$ & $\begin{array}{l}17 \\
20 \\
24 \\
28 \\
31 \\
35 \\
39 \\
42 \\
46\end{array}$ & $\begin{array}{l}0.71 \\
0.72 \\
0.73 \\
0.74 \\
0.75 \\
0.76 \\
0.77 \\
0.78 \\
0.79\end{array}$ & & $\begin{array}{l}19 \\
23 \\
27 \\
30 \\
34 \\
38 \\
41 \\
45 \\
49\end{array}$ \\
\hline 30 & 3 & 2 & 37 & 9 & 7 & 52 & 15 & 13 & 6 & 21 & 18 & 21 & 0.30 & 1 & 50 & 0.80 & & 52 \\
\hline $\begin{array}{l}31 \\
32 \\
33 \\
34 \\
35 \\
36 \\
37 \\
38 \\
39\end{array}$ & $\begin{array}{l}3 \\
3 \\
3 \\
3 \\
3 \\
3 \\
3 \\
3 \\
3\end{array}$ & $\begin{array}{r}8 \\
14 \\
20 \\
26 \\
33 \\
39 \\
45 \\
51 \\
57\end{array}$ & $\begin{array}{r}43 \\
48 \\
53 \\
58 \\
3 \\
9 \\
14 \\
19 \\
24\end{array}$ & $\begin{array}{r}9 \\
9 \\
9 \\
9 \\
9 \\
9 \\
9 \\
9 \\
10\end{array}$ & $\begin{array}{r}13 \\
20 \\
26 \\
32 \\
38 \\
44 \\
50 \\
56 \\
2\end{array}$ & $\begin{array}{r}57 \\
2 \\
8 \\
13 \\
18 \\
23 \\
28 \\
34 \\
39\end{array}$ & $\begin{array}{l}15 \\
15 \\
15 \\
15 \\
15 \\
15 \\
15 \\
16 \\
16\end{array}$ & $\begin{array}{r}19 \\
25 \\
31 \\
37 \\
43 \\
49 \\
55 \\
1 \\
7\end{array}$ & $\begin{array}{l}12 \\
17 \\
22 \\
27 \\
33 \\
38 \\
43 \\
48 \\
54\end{array}$ & $\begin{array}{l}21 \\
21 \\
21 \\
21 \\
21 \\
21 \\
22 \\
22 \\
22\end{array}$ & $\begin{array}{r}24 \\
30 \\
36 \\
42 \\
48 \\
54 \\
0 \\
7 \\
13\end{array}$ & $\begin{array}{r}26 \\
31 \\
37 \\
42 \\
47 \\
52 \\
58 \\
3 \\
8\end{array}$ & $\begin{array}{l}0.31 \\
0.32 \\
0.33 \\
0.34 \\
0.35 \\
0.36 \\
0.37 \\
0.38 \\
0.39\end{array}$ & $\begin{array}{l}1 \\
1 \\
2 \\
2 \\
2 \\
2 \\
2 \\
2 \\
2\end{array}$ & $\begin{array}{r}53 \\
57 \\
1 \\
4 \\
8 \\
11 \\
15 \\
19 \\
22\end{array}$ & $\begin{array}{l}0.81 \\
0.82 \\
0.83 \\
0.84 \\
0.85 \\
0.86 \\
0.87 \\
0.88 \\
0.89\end{array}$ & & $\begin{array}{r}56 \\
59 \\
3 \\
7 \\
10 \\
14 \\
18 \\
21 \\
25\end{array}$ \\
\hline 40 & 4 & 3 & 30 & 10 & 8 & 44 & 16 & 13 & 59 & 22 & 19 & 13 & 0.40 & & 26 & 0.90 & & 29 \\
\hline $\begin{array}{l}41 \\
42 \\
43 \\
44 \\
45 \\
46 \\
47 \\
48 \\
49\end{array}$ & $\begin{array}{l}4 \\
4 \\
4 \\
4 \\
4 \\
4 \\
4 \\
4 \\
4\end{array}$ & $\begin{array}{r}9 \\
15 \\
21 \\
27 \\
33 \\
40 \\
46 \\
52 \\
58\end{array}$ & $\begin{array}{r}35 \\
40 \\
45 \\
51 \\
56 \\
1 \\
6 \\
12 \\
17\end{array}$ & $\begin{array}{l}10 \\
10 \\
10 \\
10 \\
10 \\
10 \\
10 \\
10 \\
11\end{array}$ & $\begin{array}{r}14 \\
20 \\
27 \\
33 \\
39 \\
45 \\
51 \\
57 \\
3\end{array}$ & $\begin{array}{r}49 \\
55 \\
0 \\
5 \\
10 \\
16 \\
21 \\
26 \\
31\end{array}$ & $\begin{array}{l}16 \\
16 \\
16 \\
16 \\
16 \\
16 \\
16 \\
17 \\
17\end{array}$ & $\begin{array}{r}20 \\
26 \\
32 \\
38 \\
44 \\
50 \\
56 \\
2 \\
8\end{array}$ & $\begin{array}{r}4 \\
9 \\
14 \\
20 \\
25 \\
30 \\
35 \\
41 \\
46\end{array}$ & $\begin{array}{l}22 \\
22 \\
22 \\
22 \\
22 \\
22 \\
23 \\
23 \\
23\end{array}$ & $\begin{array}{r}25 \\
31 \\
37 \\
43 \\
49 \\
55 \\
1 \\
7 \\
14\end{array}$ & $\begin{array}{r}19 \\
24 \\
29 \\
34 \\
39 \\
45 \\
50 \\
55 \\
0\end{array}$ & $\begin{array}{l}0.41 \\
0.42 \\
0.43 \\
0.44 \\
0.45 \\
0.46 \\
0.47 \\
0.48 \\
0.49\end{array}$ & $\begin{array}{l}2 \\
2 \\
2 \\
2 \\
2 \\
2 \\
2 \\
2 \\
2 \\
2\end{array}$ & $\begin{array}{l}30 \\
33 \\
37 \\
41 \\
44 \\
48 \\
52 \\
55 \\
59\end{array}$ & $\begin{array}{l}0.91 \\
0.92 \\
0.93 \\
0.94 \\
0.95 \\
0.96 \\
0.97 \\
0.98 \\
0.99\end{array}$ & & $\begin{array}{r}32 \\
36 \\
40 \\
43 \\
47 \\
51 \\
54 \\
58 \\
2\end{array}$ \\
\hline 50 & 5 & 4 & 22 & 11 & 9 & 37 & 17 & 14 & 51 & 23 & 20 & 6 & 0.50 & 3 & 3 & 1.00 & & 5 \\
\hline $\begin{array}{l}51 \\
52 \\
53 \\
54 \\
55 \\
56 \\
57 \\
58 \\
59\end{array}$ & $\begin{array}{l}5 \\
5 \\
5 \\
5 \\
5 \\
5 \\
5 \\
5 \\
5\end{array}$ & $\begin{array}{l}10 \\
16 \\
22 \\
28 \\
34 \\
40 \\
46 \\
53 \\
59\end{array}$ & $\begin{array}{r}27 \\
33 \\
38 \\
43 \\
48 \\
54 \\
59 \\
4 \\
9\end{array}$ & $\begin{array}{l}11 \\
11 \\
11 \\
11 \\
11 \\
11 \\
11 \\
11 \\
12\end{array}$ & $\begin{array}{r}15 \\
21 \\
27 \\
33 \\
40 \\
46 \\
52 \\
58 \\
4\end{array}$ & $\begin{array}{r}42 \\
47 \\
52 \\
58 \\
3 \\
8 \\
13 \\
19 \\
24\end{array}$ & $\begin{array}{l}17 \\
17 \\
17 \\
17 \\
17 \\
17 \\
17 \\
18 \\
18\end{array}$ & $\begin{array}{r}20 \\
27 \\
33 \\
39 \\
45 \\
51 \\
57 \\
3 \\
9\end{array}$ & $\begin{array}{r}56 \\
2 \\
7 \\
12 \\
17 \\
23 \\
28 \\
33\end{array}$ & $\begin{array}{l}23 \\
23 \\
23 \\
23 \\
23 \\
23 \\
24 \\
24 \\
24\end{array}$ & $\begin{array}{r}26 \\
32 \\
38 \\
44 \\
50 \\
56 \\
2 \\
8 \\
14\end{array}$ & $\begin{array}{l}11 \\
16 \\
21 \\
27 \\
32 \\
37 \\
42 \\
48\end{array}$ & \multirow{2}{*}{\multicolumn{6}{|c|}{ 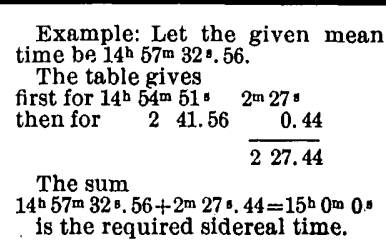 }} \\
\hline 60 & 6 & 5 & 15 & 12 & 10 & 29 & 18 & 15 & 94 & 24 & 20 & 08 & & & & & & \\
\hline
\end{tabular}


TABLE 15.-For conversion of sidereal time into mean time.

\begin{tabular}{|c|c|c|c|c|c|c|c|c|c|c|c|c|c|c|c|c|c|}
\hline s & & $\underset{0}{\mathrm{~m}}$ & & & $\frac{m}{I}$ & & & $\frac{\mathrm{m}}{2}$ & & & $\underset{3}{\mathrm{~m}}$ & & & & & & \\
\hline$: 0$ & $\begin{array}{l}\mathbf{h} \\
0\end{array}$ & $\underset{0}{\mathrm{~m}}$ & $\begin{array}{l}\mathbf{s} \\
0\end{array}$ & $\begin{array}{l}\mathrm{h} \\
6\end{array}$ & $\frac{\mathrm{m}}{6}$ & $\begin{array}{c}\mathbf{s} \\
15\end{array}$ & $\begin{array}{r}\mathrm{h} \\
12\end{array}$ & $\begin{array}{l}\mathrm{m} \\
12\end{array}$ & $\begin{array}{c}\mathrm{s} \\
29\end{array}$ & $\begin{array}{l}\mathrm{h} \\
18\end{array}$ & $\mathrm{~m}_{18}$ & $\begin{array}{c}\mathrm{s} \\
44\end{array}$ & $\begin{array}{c}\mathrm{s} \\
0.00\end{array}$ & $\begin{array}{cc}\mathrm{m} & \mathrm{s} \\
0 & 0\end{array}$ & $\begin{array}{c}\mathrm{s} \\
0.50\end{array}$ & & $\begin{array}{l}\mathrm{s} \\
3\end{array}$ \\
\hline $\begin{array}{l}1 \\
2 \\
3 \\
4 \\
5 \\
6 \\
7 \\
8 \\
9\end{array}$ & $\begin{array}{l}0 \\
0 \\
0 \\
0 \\
0 \\
0 \\
0 \\
0 \\
0 \\
0 \\
0\end{array}$ & $\begin{array}{r}6 \\
12 \\
18 \\
24 \\
30 \\
36 \\
42 \\
48 \\
54\end{array}$ & $\begin{array}{r}6 \\
12 \\
19 \\
25 \\
31 \\
37 \\
44 \\
50 \\
56\end{array}$ & $\begin{array}{l}6 \\
6 \\
6 \\
6 \\
6 \\
6 \\
6 \\
6 \\
7\end{array}$ & $\begin{array}{c}12 \\
18 \\
24 \\
30 \\
36 \\
42 \\
48 \\
55 \\
1\end{array}$ & $\begin{array}{r}21 \\
27 \\
33 \\
40 \\
46 \\
52 \\
58 \\
4 \\
11\end{array}$ & $\begin{array}{l}12 \\
12 \\
12 \\
12 \\
12 \\
12 \\
12 \\
13 \\
13\end{array}$ & $\begin{array}{r}18 \\
24 \\
30 \\
36 \\
43 \\
49 \\
55 \\
1 \\
7\end{array}$ & $\begin{array}{r}35 \\
42 \\
48 \\
54 \\
0 \\
7 \\
13 \\
19 \\
25\end{array}$ & $\begin{array}{l}18 \\
18 \\
18 \\
18 \\
18 \\
18 \\
19 \\
19 \\
19\end{array}$ & $\begin{array}{r}24 \\
30 \\
37 \\
43 \\
49 \\
55 \\
1 \\
7 \\
13\end{array}$ & $\begin{array}{r}50 \\
56 \\
2 \\
9 \\
15 \\
21 \\
27 \\
34 \\
40\end{array}$ & $\begin{array}{l}0.01 \\
0.02 \\
0.03 \\
0.04 \\
0.05 \\
0.06 \\
0.07 \\
0.08 \\
0.09\end{array}$ & $\begin{array}{lr}0 & 4 \\
0 & 7 \\
0 & 11 \\
0 & 15 \\
0 & 18 \\
0 & 22 \\
0 & 26 \\
0 & 29 \\
0 & 33\end{array}$ & $\begin{array}{l}0.51 \\
0.52 \\
0.53 \\
0.54 \\
0.55 \\
0.56 \\
0.57 \\
0.58 \\
0.59\end{array}$ & & $\begin{array}{r}7 \\
10 \\
14 \\
18 \\
21 \\
25 \\
29 \\
32 \\
36\end{array}$ \\
\hline 10 & 1 & 1 & 2 & 7 & 7 & 17 & 13 & 13 & 31 & 19 & 19 & 46 & 0.10 & $0 \quad 37$ & 0.60 & 3 & 40 \\
\hline $\begin{array}{l}11 \\
12 \\
13 \\
14 \\
15 \\
16 \\
17 \\
18 \\
19\end{array}$ & $\begin{array}{ll}1 & \\
1 & 1 \\
1 & 1 \\
1 & 2 \\
1 & 3 \\
1 & 3 \\
1 & 5 \\
1 & 4 \\
1 & 5\end{array}$ & $\begin{array}{r}7 \\
13 \\
19 \\
25 \\
31 \\
37 \\
43 \\
49 \\
55\end{array}$ & $\begin{array}{r}9 \\
15 \\
21 \\
27 \\
34 \\
40 \\
46 \\
52 \\
59\end{array}$ & $\begin{array}{l}7 \\
7 \\
7 \\
7 \\
7 \\
7 \\
7 \\
7 \\
8\end{array}$ & $\begin{array}{r}13 \\
19 \\
25 \\
31 \\
37 \\
43 \\
50 \\
56 \\
2\end{array}$ & $\begin{array}{r}23 \\
29 \\
36 \\
42 \\
48 \\
54 \\
1 \\
7 \\
13\end{array}$ & $\begin{array}{l}13 \\
13 \\
13 \\
13 \\
13 \\
13 \\
13 \\
14 \\
14\end{array}$ & $\begin{array}{r}19 \\
25 \\
31 \\
37 \\
44 \\
50 \\
56 \\
2 \\
8\end{array}$ & $\begin{array}{r}38 \\
44 \\
50 \\
56 \\
3 \\
9 \\
15 \\
21 \\
28\end{array}$ & $\begin{array}{l}19 \\
19 \\
19 \\
19 \\
19 \\
19 \\
20 \\
20 \\
20\end{array}$ & $\begin{array}{r}25 \\
31 \\
38 \\
44 \\
50 \\
56 \\
2 \\
8 \\
14\end{array}$ & $\begin{array}{r}52 \\
59 \\
5 \\
11 \\
17 \\
23 \\
30 \\
36 \\
42\end{array}$ & $\begin{array}{l}0.11 \\
0.12 \\
0.13 \\
0.14 \\
0.15 \\
0.16 \\
0.17 \\
0.18 \\
0.19\end{array}$ & $\begin{array}{rr}0 & 40 \\
0 & 44 \\
0 & 42 \\
0 & 51 \\
0 & 55 \\
0 & 59 \\
1 & 2 \\
1 & 6 \\
1 & 10\end{array}$ & $\begin{array}{l}0.61 \\
0.62 \\
0.63 \\
0.64 \\
0.65 \\
0.66 \\
0.67 \\
0.68 \\
0.69\end{array}$ & $\begin{array}{l}3 \\
3 \\
3 \\
3 \\
4\end{array}$ & $\begin{array}{r}43 \\
47 \\
51 \\
54 \\
58 \\
2 \\
5 \\
9 \\
13\end{array}$ \\
\hline 20 & 2 & 2 & 5 & 8 & 8 & 19 & 14 & 14 & 34 & 20 & 20 & 48 & 0.20 & 113 & 0.70 & 4 & 16 \\
\hline $\begin{array}{l}21 \\
22 \\
23 \\
24 \\
25 \\
26 \\
27 \\
28 \\
29\end{array}$ & $\begin{array}{ll}2 & \\
2 & 1 \\
2 & 2 \\
2 & 2 \\
2 & 3 \\
2 & 3 \\
2 & 4 \\
2 & 5 \\
2 & 5\end{array}$ & $\begin{array}{r}8 \\
14 \\
20 \\
26 \\
32 \\
38 \\
44 \\
50 \\
57\end{array}$ & $\begin{array}{l}11 \\
17 \\
24 \\
30 \\
36 \\
42 \\
49 \\
55 \\
1\end{array}$ & $\begin{array}{l}8 \\
8 \\
8 \\
8 \\
8 \\
8 \\
8 \\
8 \\
9\end{array}$ & $\begin{array}{l}14 \\
20 \\
26 \\
32 \\
38 \\
44 \\
51 \\
57 \\
3\end{array}$ & $\begin{array}{r}26 \\
32 \\
38 \\
44 \\
51 \\
67 \\
3 \\
9 \\
16\end{array}$ & $\begin{array}{l}14 \\
14 \\
14 \\
14 \\
14 \\
14 \\
14 \\
15 \\
15\end{array}$ & $\begin{array}{r}20 \\
26 \\
32 \\
38 \\
45 \\
51 \\
57 \\
3 \\
9\end{array}$ & $\begin{array}{r}40 \\
46 \\
53 \\
59 \\
5 \\
11 \\
18 \\
24 \\
30\end{array}$ & $\begin{array}{l}20 \\
20 \\
20 \\
20 \\
20 \\
20 \\
21 \\
21 \\
21\end{array}$ & $\begin{array}{r}26 \\
33 \\
39 \\
45 \\
51 \\
57 \\
3 \\
9 \\
15\end{array}$ & $\begin{array}{r}55 \\
1 \\
7 \\
13 \\
20 \\
26 \\
32 \\
38 \\
45\end{array}$ & $\begin{array}{l}0.21 \\
0.22 \\
0.23 \\
0.24 \\
0.25 \\
0.26 \\
0.27 \\
0.28 \\
0.29\end{array}$ & $\begin{array}{ll}1 & 17 \\
1 & 21 \\
1 & 24 \\
1 & 28 \\
1 & 32 \\
1 & 35 \\
1 & 39 \\
1 & 43 \\
1 & 46\end{array}$ & $\begin{array}{l}0.71 \\
0.72 \\
0.73 \\
0.74 \\
0.75 \\
0.76 \\
0.77 \\
0.78 \\
0.79\end{array}$ & $\begin{array}{l}4 \\
4 \\
4 \\
4 \\
4 \\
4\end{array}$ & $\begin{array}{l}20 \\
24 \\
27 \\
31 \\
35 \\
38 \\
42 \\
46 \\
49\end{array}$ \\
\hline 30 & 3 & 3 & 7 & 9 & 9 & 22 & 15 & 15 & 36 & 21 & 21 & 51 & 0.30 & 150 & 0.80 & 4 & 53 \\
\hline $\begin{array}{l}31 \\
32 \\
33 \\
34 \\
35 \\
36 \\
37 \\
38 \\
39\end{array}$ & $\begin{array}{ll}3 & \\
3 & 1 \\
3 & 2 \\
3 & 2 \\
3 & 2 \\
3 & 3 \\
3 & 4 \\
3 & 5 \\
3 & 5\end{array}$ & $\begin{array}{r}9 \\
15 \\
21 \\
27 \\
33 \\
39 \\
45 \\
51 \\
58\end{array}$ & $\begin{array}{r}14 \\
20 \\
26 \\
32 \\
38 \\
45 \\
51 \\
57 \\
3\end{array}$ & $\begin{array}{r}9 \\
9 \\
9 \\
9 \\
9 \\
9 \\
9 \\
9 \\
10\end{array}$ & $\begin{array}{r}15 \\
21 \\
27 \\
33 \\
39 \\
45 \\
52 \\
58 \\
4\end{array}$ & $\begin{array}{r}28 \\
34 \\
41 \\
47 \\
53 \\
59 \\
5 \\
12 \\
18\end{array}$ & $\begin{array}{l}15 \\
15 \\
15 \\
15 \\
15 \\
15 \\
15 \\
16 \\
16\end{array}$ & $\begin{array}{r}21 \\
27 \\
33 \\
40 \\
46 \\
52 \\
58 \\
4 \\
10\end{array}$ & $\begin{array}{r}43 \\
49 \\
55 \\
1 \\
8 \\
14 \\
20 \\
26 \\
33\end{array}$ & $\begin{array}{l}21 \\
21 \\
21 \\
21 \\
21 \\
21 \\
22 \\
22 \\
22\end{array}$ & $\begin{array}{r}27 \\
34 \\
40 \\
46 \\
52 \\
58 \\
4 \\
10 \\
16\end{array}$ & $\begin{array}{r}57 \\
3 \\
10 \\
16 \\
22 \\
28 \\
35 \\
41 \\
47\end{array}$ & $\begin{array}{l}0.31 \\
0.32 \\
0.33 \\
0.34 \\
0.35 \\
0.36 \\
0.37 \\
0.38 \\
0.39\end{array}$ & $\begin{array}{rr}1 & 54 \\
1 & 57 \\
2 & 1 \\
2 & 5 \\
2 & 8 \\
2 & 12 \\
2 & 16 \\
2 & 19 \\
2 & 23\end{array}$ & $\begin{array}{l}0.81 \\
0.82 \\
0.83 \\
0.84 \\
0.85 \\
0.86 \\
0.87 \\
0.88 \\
0.89\end{array}$ & & $\begin{array}{r}57 \\
0 \\
4 \\
8 \\
11 \\
15 \\
19 \\
22 \\
26\end{array}$ \\
\hline 40 & 4 & 4 & 10 & 10 & 10 & 24 & 16 & 16 & 39 & 22 & 22 & 53 & 0.40 & 226 & 0.90 & 5 & 30 \\
\hline $\begin{array}{l}41 \\
42 \\
43 \\
44 \\
45 \\
46 \\
47 \\
48 \\
49\end{array}$ & $\begin{array}{ll}4 & 1 \\
4 & 1 \\
4 & 2 \\
4 & 2 \\
4 & 3 \\
4 & 5 \\
4 & 4 \\
4 & 5 \\
4 & 5\end{array}$ & $\begin{array}{l}10 \\
16 \\
22 \\
28 \\
34 \\
40 \\
46 \\
53 \\
59\end{array}$ & $\begin{array}{r}16 \\
22 \\
28 \\
35 \\
41 \\
47 \\
53 \\
0 \\
6\end{array}$ & $\begin{array}{l}10 \\
10 \\
10 \\
10 \\
10 \\
10 \\
10 \\
10 \\
11\end{array}$ & $\begin{array}{r}16 \\
22 \\
28 \\
34 \\
40 \\
47 \\
53 \\
59 \\
5\end{array}$ & $\begin{array}{r}30 \\
37 \\
43 \\
49 \\
55 \\
2 \\
8 \\
14 \\
20\end{array}$ & $\begin{array}{l}16 \\
16 \\
16 \\
16 \\
16 \\
16 \\
16 \\
17 \\
17\end{array}$ & $\begin{array}{r}22 \\
28 \\
34 \\
41 \\
47 \\
53 \\
59 \\
5 \\
11\end{array}$ & $\begin{array}{r}45 \\
51 \\
57 \\
4 \\
10 \\
16 \\
22 \\
29 \\
35\end{array}$ & $\begin{array}{l}22 \\
22 \\
22 \\
22 \\
22 \\
22 \\
23 \\
23 \\
23\end{array}$ & $\begin{array}{r}29 \\
35 \\
41 \\
47 \\
53 \\
59 \\
5 \\
11 \\
17\end{array}$ & $\begin{array}{r}0 \\
6 \\
12 \\
18 \\
24 \\
31 \\
37 \\
43 \\
49\end{array}$ & $\begin{array}{l}0.41 \\
0.42 \\
0.43 \\
0.44 \\
0.45 \\
0.46 \\
0.47 \\
0.48 \\
0.49\end{array}$ & $\begin{array}{ll}2 & 30 \\
2 & 34 \\
2 & 37 \\
2 & 41 \\
2 & 45 \\
2 & 48 \\
2 & 52 \\
2 & 56 \\
2 & 59\end{array}$ & $\begin{array}{l}0.91 \\
0.92 \\
0.93 \\
0.94 \\
0.95 \\
0.96 \\
0.97 \\
0.98 \\
0.99\end{array}$ & $\begin{array}{l}0 \\
5 \\
6\end{array}$ & $\begin{array}{r}33 \\
37 \\
41 \\
44 \\
48 \\
52 \\
55 \\
59 \\
3\end{array}$ \\
\hline 60 & 5 & 5 & 12 & 11 & 11 & 27 & 17 & 17 & 41 & 23 & 23 & 56 & 0.50 & 33 & 1.00 & 6 & 6 \\
\hline $\begin{array}{l}51 \\
52 \\
53 \\
54 \\
55 \\
56 \\
57 \\
58 \\
59 \\
\end{array}$ & $\begin{array}{l}5 \\
5 \\
5 \\
5 \\
5 \\
5 \\
5 \\
5 \\
6 \\
\end{array}$ & $\begin{array}{r}11 \\
17 \\
23 \\
29 \\
35 \\
41 \\
47 \\
54 \\
0 \\
\end{array}$ & $\begin{array}{r}18 \\
25 \\
31 \\
37 \\
43 \\
50 \\
56 \\
2 \\
8 \\
\end{array}$ & $\begin{array}{l}11 \\
11 \\
11 \\
11 \\
11 \\
11 \\
11 \\
12 \\
12 \\
\end{array}$ & $\begin{array}{r}17 \\
23 \\
29 \\
35 \\
41 \\
48 \\
54 \\
0 \\
6 \\
\end{array}$ & $\begin{array}{r}33 \\
39 \\
45 \\
52 \\
58 \\
4 \\
10 \\
17 \\
23 \\
\end{array}$ & $\begin{array}{l}17 \\
17 \\
17 \\
17 \\
17 \\
17 \\
18 \\
18 \\
18 \\
\end{array}$ & $\begin{array}{r}23 \\
29 \\
36 \\
42 \\
48 \\
54 \\
0 \\
6 \\
12 \\
\end{array}$ & $\begin{array}{r}47 \\
54 \\
0 \\
6 \\
12 \\
19 \\
25 \\
31 \\
37\end{array}$ & $\begin{array}{l}23 \\
23 \\
23 \\
23 \\
23 \\
24 \\
24 \\
24 \\
24 \\
\end{array}$ & $\begin{array}{r}30 \\
36 \\
42 \\
48 \\
54 \\
0 \\
6 \\
12 \\
18 \\
\end{array}$ & $\begin{array}{r}2 \\
8 \\
14 \\
21 \\
27 \\
33 \\
39 \\
46 \\
52 \\
\end{array}$ & \multirow{2}{*}{\multicolumn{5}{|c|}{ 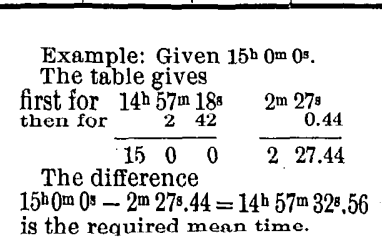 }} \\
\hline 60 & 6 & 6 & 15 & .12 & 12 & 29 & 18 & 18 & 44 & 24 & 24 & 58 & & & & & \\
\hline
\end{tabular}


TABLE 16.--For intérconversion of feet and decimals of a mile.

\begin{tabular}{|c|c|c|c|c|c|c|c|}
\hline Feet. & Miles. & Feet. & Miles. & Feet. & Miles. & Feet. & Miles. \\
\hline 53 & .01 & 1373 & 26 & 2693 & .51 & 4013 & .76 \\
\hline 106 & .02 & 1426 & .27 & 2746 & .52 & 4066 & .77 \\
\hline 158 & .03 & 1478 & 28 & 2798 & .53 & 4118 & 78 \\
\hline 211. & .04 & 1531 & 29 & 2851 & .54 & 4171 & .79 \\
\hline 264 & .05 & 1584 & .30 & 2904 & .55 & 4224 & .80 \\
\hline 317 & .06 & 1637 & .31 & 2957 & .56 & 4277 & .81 \\
\hline 370 & .07 & 1690 & .32 & 3010 & .57 & 4330 & 82 \\
\hline 422 & .08 & 1742 & .33 & 3062 & .58 & 4382 & .83 \\
\hline 475 & .09 & 1795 & .34 & 3115 & .59 & 4435 & 84 \\
\hline 528 . & .10 & 1848 & .35 & 3168 & .60 & 4488 & .85 \\
\hline 581 & .11 & 1901 & .36 & 3221 & .61 & 4541 & 86 \\
\hline 634 & .12 & 1954 & .37 & 3274 & .62 & 4594 & .87 \\
\hline 686 & .13 & 2006 & . 38 & 3326 & .63 & 4646 & .88 \\
\hline 739 & .14 & 2059 & . 39 & 3379 & .64 & 4699 & .89 \\
\hline 792 & .15 & 2112 & .40 & 3432 & .65 & 4752 & .90 \\
\hline 845 & .16 & 2165 & .41 & 3485 & .66 & 4805 & .91 \\
\hline 898 & .17 & 2218 & .42 & 3538 & .67 & 4858 & .92 \\
\hline 950 & .18 & 2270 & .43 & 3590 & . 68 & 4910 & .93 \\
\hline 1003 & .19 & 2323 & .44 & 3643 & . 69 & 4963 & . 94 \\
\hline 1056 & .20 & 2376 & .45 & 3696 & .70 & 5016 & .95 \\
\hline 1109 & .21 & 2429 & .46 & 3749 & .71 & 5069 & .96 \\
\hline 1162 & .22 & 2482 & .47 & 3802 & .72 & 5122 & .97 \\
\hline 1214 & .23 & 2534 & .48 & 3854 & .73 & 5174 & .98 \\
\hline 1267 & .24 & 2587 & . 49 & 3907 & .74 & 5227 & . 99 \\
\hline 1320 & .25 & 2640 & .50 & 3960 & .75 & 5280 & 1.00 \\
\hline
\end{tabular}




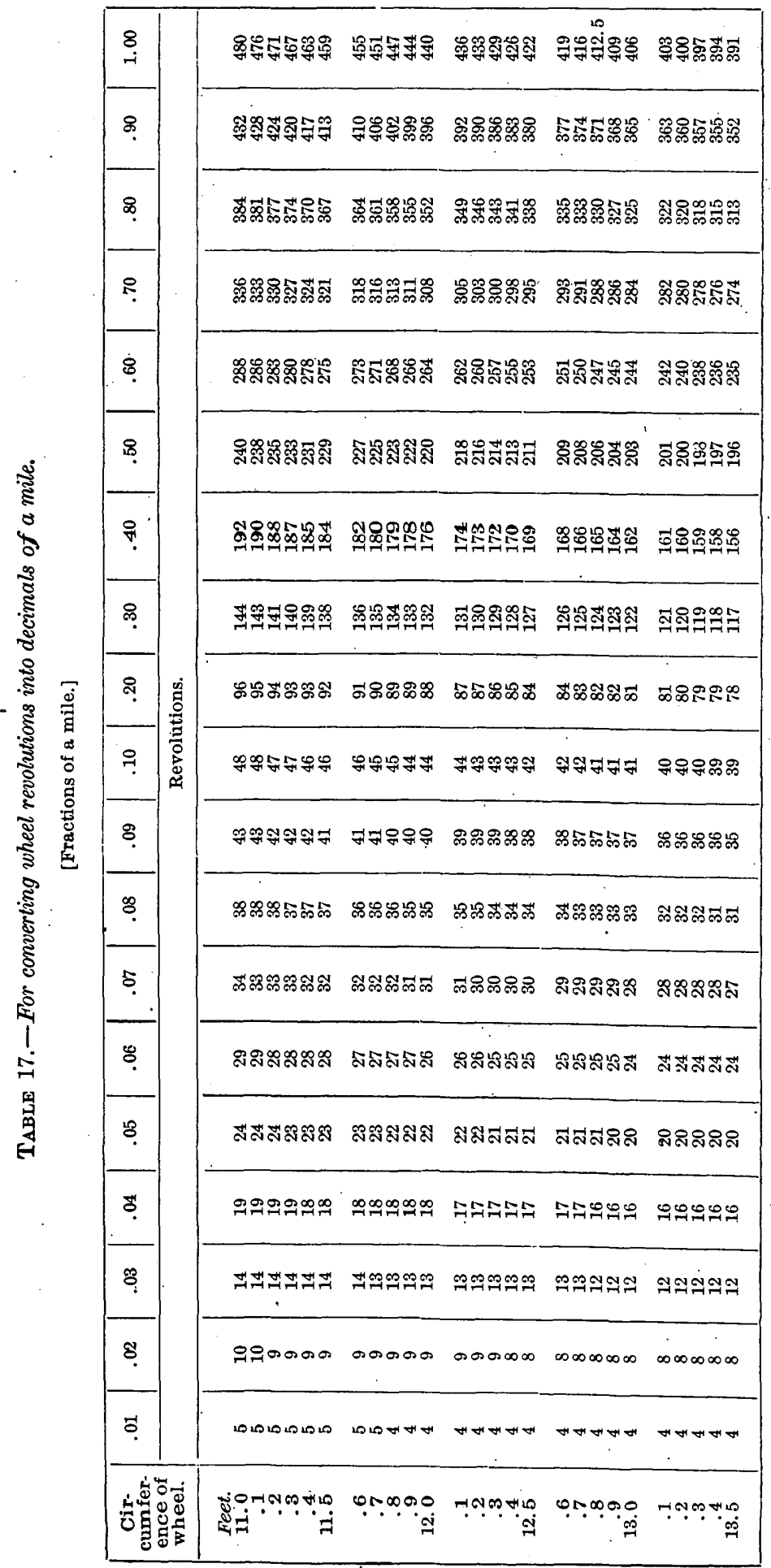


TABLE 18.-Five-place logarithms of natural numbers.

[Fractional change in a number corresponding to a change in its logarithm.]

Computed from the formula,

$$
\frac{\Delta N}{N}=\frac{\Delta \log N}{\mu}
$$

$\mu=$ modulus of common logarithms $=0.43429448$.

\begin{tabular}{|c|c|c|c|}
\hline $\begin{aligned} & \text { For } \\
= & \log N \\
= & \text { unit in }\end{aligned}$ & $\frac{\Delta N}{N}$ & $\begin{aligned} & \text { For } \\
&= \Delta \log N \\
& 4 \text { units in }\end{aligned}$ & $\begin{array}{c}\frac{\Delta N}{N} \\
\text { (in round } \\
\text { numbers) }\end{array}$ \\
\hline $\begin{array}{l}\text { Fourth place } . . . \ldots \ldots \\
\text { Fifth place } \ldots \ldots \ldots \\
\text { Sixth place } \ldots \ldots \ldots \\
\text { Seventh place........ }\end{array}$ & $\begin{array}{c}\frac{1}{4343} \\
\frac{1}{43429} \\
\frac{1}{434294} \\
434^{\frac{1}{2} 945}\end{array}$ & $\begin{array}{l}\text { Fourth place } . . . \ldots \ldots \\
\text { Fifth place } . . . \ldots \ldots \\
\text { Sixth place } . . . \ldots \ldots \\
\text { Seventh place } . . .\end{array}$ & $\begin{array}{l}\frac{1}{1000} \\
10800 \\
100000 \\
1000000\end{array}$ \\
\hline
\end{tabular}

Bull. $214-03-7$ 
TABLE 18.-Five-place logarithms of natural numbers-Continued.

\begin{tabular}{|c|c|c|c|c|c|c|c|c|c|c|}
\hline N. & L. 0 & 1 & 2 & 3 & 4 & 5 & 6 & 7 & 8 & 9 \\
\hline $\mathbf{0}$ & & 00000 & 30103 & 47712 & 60206 & 69897 & 77815 & 84510 & 90309 & $95 \quad 424$ \\
\hline $\begin{array}{l}1 \\
2 \\
3\end{array}$ & $\begin{array}{lll}00 & 000 \\
30 & 103 \\
47 & 712\end{array}$ & $\begin{array}{ll}04 & 139 \\
32 & 222 \\
49 & 136\end{array}$ & $\begin{array}{ll}07 & 918 \\
34 & 242 \\
50 & 515\end{array}$ & $\begin{array}{ll}11 & 394 \\
36 & 173 \\
51 & 851\end{array}$ & $\begin{array}{ll}14 & 613 \\
38 & 021 \\
53 & 148\end{array}$ & $\begin{array}{ll}17 & 609 \\
39 & 794 \\
54 & 407\end{array}$ & $\begin{array}{ll}20 & 412 \\
41 & 497 \\
55 & 630\end{array}$ & $\begin{array}{ll}23 & 045 \\
43 & 136 \\
56 & 820\end{array}$ & $\begin{array}{ll}25 & 527 \\
44 & 716 \\
57 & 978\end{array}$ & $\begin{array}{ll}27 & 875 \\
46 & 240 \\
59 & 106\end{array}$ \\
\hline $\begin{array}{l}4 \\
5 \\
6\end{array}$ & $\begin{array}{ll}60 & 206 \\
69 & 897 \\
77 & 815\end{array}$ & $\begin{array}{ll}61 & 278 \\
70 & 757 \\
.78 \quad 533\end{array}$ & $\begin{array}{ll}62 & 325 \\
71 & 600 \\
79 & 239\end{array}$ & $\begin{array}{ll}63 & 347 \\
72 & 428 \\
79 & 934\end{array}$ & $\begin{array}{ll}64 & 345 \\
7 \mathrm{~s} & 239 \\
80 & 618\end{array}$ & $\begin{array}{ll}65 & 321 \\
74 & 036 \\
81 & 291\end{array}$ & $\begin{array}{ll}66 & 276 \\
74 & 819 \\
81 & 954\end{array}$ & $\begin{array}{ll}67 & 210 \\
75 & 587 \\
82 & 607\end{array}$ & $\begin{array}{ll}68 & 124 \\
76 & 343 \\
83 & 251\end{array}$ & $\begin{array}{ll}69 & 020 \\
77 & 085 \\
83 & 885\end{array}$ \\
\hline $\begin{array}{l}7 \\
8 \\
9\end{array}$ & $\begin{array}{ll}84 & 510 \\
90 & 309 \\
95 & 424\end{array}$ & $\begin{array}{ll}85 & 126 \\
90 & 849 \\
95 & 904\end{array}$ & $\begin{array}{ll}85 & 733 \\
91 & 381 \\
96 & 379\end{array}$ & $\begin{array}{l}86332 \\
91908 \\
96 \quad 848\end{array}$ & $\begin{array}{ll}86 & 923 \\
92 & 428 \\
97 & 313\end{array}$ & $\begin{array}{ll}87 & 506 \\
92 & 942 \\
97 & 772\end{array}$ & $\begin{array}{ll}88 & 081 \\
93 & 450 \\
98 & 227\end{array}$ & 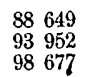 & $\begin{array}{ll}89 & 209 \\
94 & 448 \\
99 & 123\end{array}$ & $\begin{array}{ll}89 & 763 \\
94 & 939 \\
99 & 564\end{array}$ \\
\hline 10 & 00000 & 00432 & 00860 & 01284 & 01708 & 02119 & 02531 & 02938 & 03342 & 03743 \\
\hline $\begin{array}{l}11 \\
12 \\
13\end{array}$ & $\begin{array}{ll}04 & 139 \\
07 & 918 \\
11 & 394\end{array}$ & $\begin{array}{ll}04 & 532 \\
08 & 279 \\
11 & 727\end{array}$ & $\begin{array}{ll}04 & 922 \\
08 & 636 \\
12 & 057\end{array}$ & $\begin{array}{ll}05 & 308 \\
08 & 991 \\
12 & 385\end{array}$ & $\begin{array}{ll}05 & 690 \\
09 & 342 \\
12 & 710\end{array}$ & $\begin{array}{ll}06 & 070 \\
09 & 691 \\
13 & 033\end{array}$ & $\begin{array}{lll}06 & 446 \\
10 & 037 \\
13 & 354\end{array}$ & $\begin{array}{ll}06 & 819 \\
10 & 380 \\
13 & 672\end{array}$ & $\begin{array}{ll}07 & 188 \\
10 & 721 \\
13 & 988\end{array}$ & $\begin{array}{ll}07 & 555 \\
11 & 059 \\
14 & 301\end{array}$ \\
\hline $\begin{array}{l}14 \\
15 \\
16\end{array}$ & $\begin{array}{ll}14 & 613 \\
17 & 609 \\
20 & 412\end{array}$ & $\begin{array}{l}14922 \\
17898 \\
20683\end{array}$ & $\begin{array}{ll}15 & 229 \\
18 & 184 \\
20 & 952\end{array}$ & $\begin{array}{ll}15 & 534 \\
18 & 469 \\
21 & 219\end{array}$ & $\begin{array}{ll}15 & 836 \\
18 & 752 \\
21 & 484\end{array}$ & $\begin{array}{ll}16 & 137 \\
19 & 033 \\
21 & 748\end{array}$ & $\begin{array}{ll}16 & 435 \\
19 & 312 \\
22 & 011\end{array}$ & $\begin{array}{ll}16 & 732 \\
19 & 590 \\
22 & 272\end{array}$ & $\begin{array}{ll}17 & 026 \\
19 & 866 \\
22 & 531\end{array}$ & $\begin{array}{ll}17 & 319 \\
20 & 140 \\
22 & 789\end{array}$ \\
\hline $\begin{array}{l}17 \\
18 \\
19\end{array}$ & $\begin{array}{ll}23 & 045 \\
25 & 527 \\
27 & 875\end{array}$ & $\begin{array}{ll}23 & 300 \\
25 & 768 \\
28 & 103\end{array}$ & $\begin{array}{ll}23 & 553 \\
26 & 007 \\
28 & 330\end{array}$ & $\begin{array}{ll}23 & 805 \\
26 & 245 \\
28 & 556\end{array}$ & $\begin{array}{ll}24 & 055 \\
26 & 482 \\
28 & 780\end{array}$ & $\begin{array}{ll}24 & 304 \\
26 & 717 \\
29 & 003\end{array}$ & $\begin{array}{ll}24 & 551 \\
26 & 951 \\
29 & 226\end{array}$ & $\begin{array}{ll}24 & 797 \\
27 & 184 \\
29 & 447\end{array}$ & $\begin{array}{ll}25 & 042 \\
27 & 416 \\
29 & 667\end{array}$ & $\begin{array}{ll}25 & 285 \\
27 & 646 \\
29 & 885\end{array}$ \\
\hline 20 & 30103 & 30320 & $3053 \dot{5}$ & $307 \overline{50}$ & $30^{\circ} 963$ & $31 \quad 175$ & 31387 & 31.597 & 31806 & $3201 \overline{5}$ \\
\hline $\begin{array}{l}21 \\
22 \\
23\end{array}$ & \begin{tabular}{|ll}
32 & 222 \\
34 & 242 \\
36 & 173
\end{tabular} & $\begin{array}{lll}32 & 428 \\
34 & 439 \\
36 & 361\end{array}$ & $\begin{array}{ll}32 & 634 \\
34 & 635 \\
36 & 549\end{array}$ & $\begin{array}{ll}32 & 838 \\
34 & 830 \\
36 & 736\end{array}$ & $\begin{array}{ll}33 & 041 \\
35 & 025 \\
36 & 922\end{array}$ & $\begin{array}{ll}33 & 244 \\
35 & 218 \\
37 & 107\end{array}$ & $\begin{array}{ll}33 & 445 \\
35 & 411 \\
37 & 291\end{array}$ & $\begin{array}{ll}33 & 646 \\
35 & 603 \\
37 & 475\end{array}$ & $\begin{array}{ll}33 & 846 \\
35 & 793 \\
37 & 658\end{array}$ & $\begin{array}{ll}34 & 044 \\
35 & 984 \\
37 & 840\end{array}$ \\
\hline $\begin{array}{l}24 \\
25 \\
26\end{array}$ & $\begin{array}{ll}38 & 021 \\
39 & 794 \\
41 & 497\end{array}$ & $\begin{array}{ll}38 & 202 \\
39 & 967 \\
41 & 664\end{array}$ & $\begin{array}{ll}38 & 382 \\
40 & 140 \\
41 & 830\end{array}$ & $\begin{array}{ll}38 & 561 \\
40 & 312 \\
41 & 996\end{array}$ & 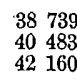 & $\begin{array}{ll}38 & 917 \\
40 & 654 \\
42 & 325\end{array}$ & $\begin{array}{ll}39 & 094 \\
40 & 824 \\
42 & 488\end{array}$ & $\begin{array}{ll}39 & 270 \\
40 & 993 \\
42 & 651\end{array}$ & $\begin{array}{ll}39 & 445 \\
41 & 162 \\
42 & 813\end{array}$ & $\begin{array}{ll}39 & 620 \\
41 & 330 \\
42 & 975\end{array}$ \\
\hline $\begin{array}{l}27 \\
28 \\
29\end{array}$ & $\begin{array}{ll}43 & 136 \\
44 & 716 \\
46 & 240\end{array}$ & $\begin{array}{ll}43 & 297 \\
44 & 871 \\
46 & 389\end{array}$ & $\begin{array}{ll}43 & 457 \\
45 & 025 \\
46 & 538\end{array}$ & $\begin{array}{l}43616 \\
\mathbf{4 5} \mathbf{1 7 9} \\
\mathbf{4 6} 687\end{array}$ & $\begin{array}{l}43775 \\
45332 \\
46835\end{array}$ & $\begin{array}{l}43933 \\
45484 \\
46982\end{array}$ & $\begin{array}{ll}44 & 091 \\
45 & 637 \\
47 & 129\end{array}$ & $\begin{array}{ll}44 & 248 \\
45 & 788 \\
47 & 276\end{array}$ & $\begin{array}{l}44404 \\
45939 \\
47 \quad 422\end{array}$ & $\begin{array}{ll}44 & 560 \\
46 & 090 \\
47 & 567\end{array}$ \\
\hline $\mathbf{3 0}$ & 47712 & 47857 & 48001 & 48144 & 48287 & 48430 & 48572 & 48714 & 48855 & 48996 \\
\hline $\begin{array}{l}31 \\
32 \\
33\end{array}$ & $\begin{array}{lll}49 & 136 \\
50 & 515 \\
51 & 851\end{array}$ & $\begin{array}{ll}49 & 276 \\
50 & 651 \\
51 & 983\end{array}$ & $\begin{array}{ll}49 & 41 \dot{5} \\
50 & 786 \\
52 & 114\end{array}$ & $\begin{array}{ll}49 & 554 \\
50 & 920 \\
52 & 244\end{array}$ & $\begin{array}{ll}49 & 693 \\
51 & 055 \\
52 & 37 \overline{5}\end{array}$ & $\begin{array}{ll}49 & 831 \\
51 & 188 \\
52 & 504\end{array}$ & $\begin{array}{l}49969 \\
51322 \\
52 \quad 634\end{array}$ & $\begin{array}{ll}50 & 106 \\
51 & 455 \\
52 & 763\end{array}$ & $\begin{array}{ll}50 & 243 \\
51 & 587 \\
52 & 892\end{array}$ & $\begin{array}{ll}50 & 379 \\
51 & 720 \\
53 & 020\end{array}$ \\
\hline $\begin{array}{l}34 \\
35 \\
36\end{array}$ & $\begin{array}{ll}53.148 \\
54 \quad 407 \\
55 \quad 630\end{array}$ & $\begin{array}{ll}53 & 275 \\
54 & 531 \\
55 & 751\end{array}$ & $\begin{array}{ll}53 & 403 \\
54 & 654 \\
55 & 871\end{array}$ & $\begin{array}{ll}53 & 529 \\
54 & 777 \\
55 & 991\end{array}$ & $\begin{array}{ll}53 & 656 \\
54 & 900 \\
56 & 110\end{array}$ & $\begin{array}{ll}53 & 782 \\
55 & 023 \\
56 & 229\end{array}$ & $\begin{array}{ll}53 & 908 \\
55 & 145 \\
56 & 348\end{array}$ & $\begin{array}{ll}54 & 033 \\
55 & 267 \\
56 & 467\end{array}$ & $\begin{array}{l}54.158 \\
55.388 \\
56 \quad 585\end{array}$ & $\begin{array}{ll}54 & 283 \\
55 & 509 \\
56 & 703\end{array}$ \\
\hline $\begin{array}{l}37 \\
38 \\
39\end{array}$ & $\begin{array}{ll}56 & 820 \\
57 & 978 \\
59 & 106\end{array}$ & $\begin{array}{ll}56 & 937 \\
58 & 092 \\
59 & 218\end{array}$ & $\begin{array}{ll}57 & 054 \\
58 & 206 \\
59 & 329\end{array}$ & $\begin{array}{ll}57 & 171 \\
58 & 320 \\
59 & 439\end{array}$ & $\begin{array}{ll}57 & 287 \\
58 & 433 \\
59 & 550\end{array}$ & $\begin{array}{ll}57 & 403 \\
58 & 546 \\
59 & 660\end{array}$ & $\begin{array}{ll}57 & 519 \\
58 & 659 \\
59 & 770\end{array}$ & $\begin{array}{ll}57 & 634 \\
58 & 771 \\
59 & 879\end{array}$ & $\begin{array}{ll}57 & 749 \\
58 & 883 \\
59 & 988\end{array}$ & $\begin{array}{ll}57 & 864 \\
58 & 995 \\
60 & 097\end{array}$ \\
\hline 40 & 60206 & 60314 & 60423 & 60531 & 60638 & $\overline{60745}$ & 60853 & 60959 & 61066 & 61172 \\
\hline $\begin{array}{l}41 \\
42 \\
43\end{array}$ & $\begin{array}{ll}61 & 278 \\
62 & 325 \\
63 & 347\end{array}$ & $\begin{aligned} 61384 \\
62428 \\
63448\end{aligned}$ & $\begin{array}{ll}61 & 490 \\
62 & 531 \\
63 & 548\end{array}$ & $\begin{array}{ll}61 & 59 \dot{5} \\
62 & 634 \\
63 & 649\end{array}$ & $\begin{array}{ll}61 & 700 \\
62 & 737 \\
63 & 749\end{array}$ & $\begin{array}{ll}61 & 80 \overline{5} \\
62 & 839 \\
63 & 849\end{array}$ & $\begin{array}{ll}61 & 909 \\
62 & 941 \\
63 & 949\end{array}$ & $\begin{array}{ll}62 & 014 \\
63 & 043 \\
64 & 048\end{array}$ & $\begin{array}{ll}62 & 118 \\
63 & 144 \\
64 & 147\end{array}$ & $\begin{array}{ll}62 & 221 \\
63 & 246 \\
64 & 246\end{array}$ \\
\hline $\begin{array}{l}44 \\
45 \\
46\end{array}$ & $\begin{array}{ll}64 & 345 \\
65 & 321 \\
66 & 276\end{array}$ & $\begin{array}{ll}64 & 444 \\
65 & 418 \\
66 & 370\end{array}$ & $\begin{array}{ll}64 & 542 \\
65 & 514 \\
66 & 464\end{array}$ & $\begin{array}{ll}64 & 640 \\
65 & 610 \\
66 & 558\end{array}$ & $\begin{array}{l}64738 \\
65706 \\
66 \quad 652\end{array}$ & $\begin{array}{ll}64836 \\
65 & 801 \\
66 & 745\end{array}$ & $\begin{array}{ll}64 & 933 \\
65 & 896 \\
66 & 839\end{array}$ & $\begin{array}{ll}65 & 031 \\
65 & 992 \\
66 & 932\end{array}$ & $\begin{array}{l}65128 \\
66 \quad 087 \\
67 \quad 025\end{array}$ & $\begin{array}{ll}65 & 225 \\
66 & 181 \\
67 & 117\end{array}$ \\
\hline $\begin{array}{l}47 \\
48 \\
49\end{array}$ & \begin{tabular}{|ll}
67 & 210 \\
68 & 124 \\
69 & 020
\end{tabular} & $\begin{array}{ll}67 & 302 \\
68 & 215 \\
69 & 108\end{array}$ & $\begin{array}{ll}67 & 394 \\
68 & 305 \\
69 & 197\end{array}$ & $\begin{array}{l}67486 \\
68395 \\
69285\end{array}$ & $\begin{array}{ll}67 & 578 \\
68 & 485 \\
69 & 373\end{array}$ & $\begin{array}{ll}67 & 669 \\
68 & 574 \\
69 & 461\end{array}$ & $\begin{array}{r}67761 \\
68664 \\
69548\end{array}$ & $\begin{array}{ll}67 & 852 \\
68 & 753 \\
69 & 636\end{array}$ & $\begin{array}{l}67943 \\
68842 \\
69723\end{array}$ & $\begin{array}{ll}68 & 034 \\
68 & 931 \\
69 & 810\end{array}$ \\
\hline 50 & $\begin{array}{ll}69 & 897\end{array}$ & 69984 & $70 \quad 070$ & 70157 & 70243 & 70329 & $7041 \dot{5}$ & 70501 & 70586 & 70672 \\
\hline N. & L. 0 & 1. & 2 & 3 & 4. & 5 & 6 & 7 & 8. & 9 \\
\hline \multicolumn{2}{|c|}{$0^{\circ} 1^{\prime}=60^{\prime \prime}$} & \multicolumn{2}{|c|}{ S. 4.68557} & \multicolumn{2}{|c|}{ T. 4.68557} & \multicolumn{2}{|c|}{$0^{\circ} 5^{\prime}=300^{\prime \prime}$} & 4. $68 \quad 557$ & \multicolumn{2}{|c|}{ T. 4.68558} \\
\hline \multicolumn{2}{|l|}{0} & \multicolumn{2}{|c|}{ 4. 68557} & \multicolumn{2}{|c|}{ 4. 68557} & \multicolumn{2}{|c|}{$0 \quad 6=360$} & 4. 68557 & \multicolumn{2}{|c|}{ - 4. 68558} \\
\hline \multirow{2}{*}{\multicolumn{2}{|c|}{$\begin{array}{ll}0 & 3 \\
0 & 4\end{array}$}} & 4. 68 & 3557 & 4. 68 & 557 & \multicolumn{2}{|c|}{$7=420$} & 4. 68557 & & 68558 \\
\hline & & 4. 68 & 857 & 4. 68 & 558 & $0 \quad 8=48$ & & 4. $68 \quad 557$ & & 68558 \\
\hline
\end{tabular}


TABLE 18. - Five-place logarithms of natural numbers-Continued.

\begin{tabular}{|c|c|c|c|c|c|c|c|c|c|c|}
\hline N. & L. 0 & 1 & 2 & 3 & 4 & 5 & 6 & 7 & 8 & 9 \\
\hline 50 & 69897 & 69984 & $70 \quad 070$ & $\begin{array}{ll}70 & 157\end{array}$ & 70243 & 70329 & $7041 \dot{5}$ & 70501 & 70586 & 70672 \\
\hline $\begin{array}{l}51 \\
52 \\
53\end{array}$ & $\begin{array}{lll}70 & 757 \\
71 & 600 \\
72 & 428\end{array}$ & $\begin{array}{ll}70 & 842 \\
71 & 634 \\
72 & 509\end{array}$ & $\begin{array}{ll}70 & 927 \\
71 & 767 \\
72 & 591\end{array}$ & $\begin{array}{ll}71 & 012 \\
71 & 850 \\
72 & 673\end{array}$ & $\begin{array}{ll}71 & 096 \\
71 & 933 \\
72 & .754\end{array}$ & $\begin{array}{ll}71 & 181 \\
72 & 016 \\
72 & 835\end{array}$ & $\begin{array}{ll}71 & 265 \\
72 & 099 \\
72 & 916\end{array}$ & $\begin{array}{ll}71 & 349 \\
72 & 181 \\
72 & 997\end{array}$ & $\begin{array}{ll}71 & 433 \\
72 & 263 \\
73 & 078\end{array}$ & $\begin{array}{ll}71 & 517 \\
72 & 346 \\
73 & 159\end{array}$ \\
\hline $\begin{array}{l}54 \\
55 \\
56\end{array}$ & \begin{tabular}{|ll}
73 & 239 \\
74 & 036 \\
74 & 819
\end{tabular} & $\begin{array}{ll}73 & 320 \\
74 & 115 \\
74 & 896\end{array}$ & $\begin{array}{ll}73 & 400 \\
74 & 194 \\
74 & 974\end{array}$ & $\begin{array}{ll}73 & 480 \\
74 & 273 \\
75 & 051\end{array}$ & $\begin{array}{ll}73 & 560 \\
74 & 351 \\
75 & 128\end{array}$ & $\begin{array}{ll}73 & 640 \\
74 & 429 \\
75 & 205\end{array}$ & $\begin{array}{ll}73 & 719 \\
74 & 507 \\
75 & 282\end{array}$ & $\begin{array}{ll}73 & 799 \\
74 & 586 \\
75 & 358\end{array}$ & $\begin{array}{ll}73 & 878 \\
74 & 663 \\
75 & 435\end{array}$ & $\begin{array}{ll}73 & 957 \\
74 & 741 \\
75 & 511\end{array}$ \\
\hline $\begin{array}{l}57 \\
58 \\
59\end{array}$ & $\begin{array}{ll}75 & 587 \\
76 & 343 \\
77 & 085\end{array}$ & $\begin{array}{ll}75 & 664 \\
76 & 418 \\
77 & 159\end{array}$ & $\begin{array}{ll}75 & 740 \\
76 & 492 \\
77 & 232\end{array}$ & $\begin{array}{ll}75 & 815 \\
76 & 567 \\
77 & 305\end{array}$ & $\begin{array}{ll}75 & 891 \\
76 & 641 \\
77 & 379\end{array}$ & $\begin{array}{ll}75 & 967 \\
76 & 716 \\
77 & 452\end{array}$ & $\begin{array}{l}76042 \\
76790 \\
77525\end{array}$ & $\begin{array}{ll}76 & 118 \\
76 & 864 \\
77 & 597\end{array}$ & $\begin{array}{ll}76 & 193 \\
76 & 938 \\
77 & 670\end{array}$ & $\begin{array}{ll}76 & 268 \\
77 & 012 \\
77 & 743\end{array}$ \\
\hline 60 & 77815 & 77887 & 77960 & 78032 & $78 \quad 104$ & $78 \quad 176$ & 78247 & 78319 & 78390 & 78462 \\
\hline $\begin{array}{l}61 \\
62 \\
63\end{array}$ & $\begin{array}{ll}78 & 533 \\
79 & 239 \\
79 & 934\end{array}$ & $\begin{array}{ll}78 & 604 \\
79 & 309 \\
80 & 003\end{array}$ & $\begin{array}{ll}78 & 675 \\
79 & 379 \\
80 & 072\end{array}$ & $\begin{array}{cc}78 & 746 \\
79 & 449 \\
80 & 140\end{array}$ & $\begin{array}{ll}78 & 817 \\
79 & 518 \\
80 & 209\end{array}$ & $\begin{array}{ll}78 & 888 \\
79 & 588 \\
80 & 277\end{array}$ & $\begin{array}{ll}78 & 958 \\
79 & 657 \\
80 & 346\end{array}$ & $\begin{array}{ll}79 & 029 \\
79 & 727 \\
80 & 414\end{array}$ & $\begin{array}{ll}79 & 099 \\
79 & 796 \\
80 & 482\end{array}$ & $\begin{array}{ll}79 & 169 \\
79 & 865 \\
80 & 550\end{array}$ \\
\hline $\begin{array}{l}64 \\
65 \\
66\end{array}$ & $\begin{array}{ll}80 & 618 \\
81 & 291 \\
81 & 954\end{array}$ & 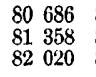 & $\begin{array}{ll}80 & 754 \\
81 . & 425 \\
82 & 086\end{array}$ & $\begin{array}{ll}80 & 821 \\
81 & 491 \\
82 & 1.51\end{array}$ & $\begin{array}{ll}80 & 889 \\
81 & 558 \\
82 & 217\end{array}$ & $\begin{array}{ll}80 & 956 \\
81 & 624 \\
82 & 282\end{array}$ & $\begin{array}{ll}81 & 023 \\
81 & 690 \\
82 & 347\end{array}$ & $\begin{array}{ll}81 & 090 \\
81 & 757 \\
82 & 413\end{array}$ & $\begin{array}{ll}81 & 158 \\
81 & 823 \\
82 & 478\end{array}$ & $\begin{array}{ll}81 & 224 \\
81 . & 889 \\
82 & 543\end{array}$ \\
\hline $\begin{array}{l}67 \\
68 \\
69\end{array}$ & $\begin{array}{ll}82 & 607 \\
83 & 251 \\
83 & 885 \\
\end{array}$ & $\begin{array}{ll}82 & 672 \\
83 & 315 \\
83 & 948\end{array}$ & $\begin{array}{ll}82 & 737 \\
83 & 378 \\
84 & 011\end{array}$ & $\begin{array}{ll}82 & 802 \\
83 & 442 \\
84 & 073\end{array}$ & $\begin{array}{ll}82 & 866 \\
83 & 506 \\
84 & 136\end{array}$ & $\begin{array}{ll}82 & 930 \\
83 & 569 \\
84 & 198\end{array}$ & $\begin{array}{ll}82 & 99 \overline{5} \\
83 & 632 \\
84 & 261\end{array}$ & $\begin{array}{ll}83 & 059 \\
83 & 69 i \\
84 & 323\end{array}$ & $\begin{array}{ll}83 & 123 \\
83 & 759 \\
84 & 386\end{array}$ & $\begin{array}{ll}83 & 187 \\
83 & 822 \\
84 & 448\end{array}$ \\
\hline 70 & 84510 & 84572 & 84634 & 84696 & 84757 & 84819 & 84880 & 84942 & 85003 & 85065 \\
\hline $\begin{array}{l}71 \\
72 \\
.73\end{array}$ & $\begin{array}{ll}85 & 126 \\
85 & 733 \\
86 & 332\end{array}$ & $\begin{array}{ll}85 & 187 \\
85 & 794 \\
86 & 392\end{array}$ & $\begin{array}{ll}85 & 248 \\
85 & 854 \\
86 & 451\end{array}$ & $\begin{array}{ll}85 & 309 \\
85 & 914 \\
86 & 510\end{array}$ & $\begin{array}{ll}85 & 370 \\
85 & 974 \\
86 & 570\end{array}$ & $\begin{array}{lll}85 & 431 \\
86 & 034 \\
86 & 629\end{array}$ & $\begin{array}{ll}85 & 491 \\
86 & 094 \\
86 & 688\end{array}$ & $\begin{array}{ll}85 & 552 \\
86 & 153 \\
86 & 747\end{array}$ & $\begin{array}{ll}85 & 612 \\
86 & 213 \\
86 & 806\end{array}$ & $\begin{array}{ll}85 & 673 \\
86 & 273 \\
86 & 864\end{array}$ \\
\hline $\begin{array}{l}74 \\
75 \\
76\end{array}$ & $\begin{array}{ll}86 & 923 \\
87 & 506 \\
88 & 081\end{array}$ & $\begin{array}{ll}86 & 982 \\
87 & 564 \\
88 & 1.38\end{array}$ & $\begin{array}{ll}87 & 040 \\
87 & 622 \\
88 & 195\end{array}$ & $\begin{array}{ll}87 & 099 \\
87 & 679 \\
88 & 252\end{array}$ & $\begin{array}{ll}87 & 157 \\
87 & 737 \\
88 & 309\end{array}$ & $\begin{array}{ll}87 & 216 \\
87 & 795 \\
88 & 366\end{array}$ & $\begin{array}{ll}87 & 274 \\
87 & 852 \\
88 & 423\end{array}$ & $\begin{array}{ll}87 & 332 \\
87 & 910 \\
88 & 480\end{array}$ & $\begin{array}{l}87390 \\
87967 \\
88536\end{array}$ & $\begin{array}{ll}87 & 448 \\
88 & 024 \\
88 & 593\end{array}$ \\
\hline $\begin{array}{l}77 \\
78 \\
79\end{array}$ & $\begin{array}{ll}88 & 649 \\
89 & 209 \\
89 & 763\end{array}$ & $\begin{array}{l}88705 \\
89 \\
8965 \\
818\end{array}$ & $\begin{array}{ll}88 & 762 \\
89 & 321 \\
89 & 873\end{array}$ & $\begin{array}{ll}88 & 818 \\
89 & 376 \\
89 & 927\end{array}$ & $\begin{array}{l}88874 \\
89432 \\
89982\end{array}$ & $\begin{array}{ll}88 & 930 \\
89 & 487 \\
90 & 037\end{array}$ & $\begin{array}{ll}88 & 986 \\
89 & 542 \\
90 & 091\end{array}$ & $\begin{array}{ll}89 & 042 \\
89 & 597 \\
90 & 146\end{array}$ & $\begin{array}{l}89098 \\
89653 \\
90200\end{array}$ & $\begin{array}{ll}89 & 154 \\
89 & 708 \\
90 & 255\end{array}$ \\
\hline 80 & 90309 & 90863 & 90417 & 90472 & 90526 & 90580 & 90634 & 90687 & 90741 & $9079 \overline{\overline{5}}$ \\
\hline $\begin{array}{l}81 \\
82 \\
83\end{array}$ & $\begin{array}{ll}90 & 849 \\
91 & 381 \\
91 & 908\end{array}$ & $\begin{array}{ll}90 & 902 \\
91 & 434 \\
91 & 960\end{array}$ & $\begin{array}{ll}90 & 956 \\
91 & 487 \\
92 & 012\end{array}$ & $\begin{array}{ll}91 & 009 \\
91 & 540 \\
92 & 06 \overline{5}\end{array}$ & $\begin{array}{ll}91 & 062 \\
91 & 593 \\
92 & 117\end{array}$ & $\begin{array}{ll}91 & 116 \\
91 & 645 \\
92 & 169\end{array}$ & $\begin{array}{ll}91 & 169 \\
91 & 698 \\
92 & 221\end{array}$ & $\begin{array}{ll}91 & 222 \\
91 & 751 \\
92 & 273\end{array}$ & $\begin{array}{ll}91 & 275 \\
91 & 803 \\
92 & 324\end{array}$ & $\begin{array}{ll}91 & 328 \\
91 & 855 \\
92 & 376\end{array}$ \\
\hline $\begin{array}{l}84 \\
85 \\
86\end{array}$ & $\begin{array}{ll}92 & 428 \\
92 & 942 \\
93 & 450\end{array}$ & $\begin{array}{l}92480 \\
92 \quad 993 \\
93 \quad 500\end{array}$ & $\begin{array}{ll}92 & 531 \\
93 & 044 \\
93 & 551\end{array}$ & $\begin{array}{ll}92 & 583 \\
93 & 095 \\
93 & 601\end{array}$ & $\begin{array}{ll}92 & 634 \\
93 & 146 \\
93 & 651\end{array}$ & $\begin{array}{lll}92 & 686 \\
93 & 197 \\
93 & 702\end{array}$ & $\begin{array}{ll}92 & 737 \\
93 & 247 \\
93 & 752\end{array}$ & $\begin{array}{ll}92 & 788 \\
93 & 298 \\
93 & 802\end{array}$ & $\begin{array}{ll}92 & 840 \\
93 & 349 \\
93 & 852\end{array}$ & $\begin{array}{ll}92 & 891 \\
93 & 399 \\
93 & 902\end{array}$ \\
\hline $\begin{array}{l}87 \\
88 \\
89\end{array}$ & $\begin{array}{ll}93 & 952 \\
94 & 448 \\
94 & 939\end{array}$ & $\begin{array}{ll}94 & 002 \\
94 & 498 \\
94 & 988\end{array}$ & $\begin{array}{ll}94 & 052 \\
94 & 547 \\
95 & 036\end{array}$ & $\begin{array}{ll}94 & 101 \\
94 & 596 \\
95 & 085\end{array}$ & $\begin{array}{ll}94 & 151 \\
94 & 645 \\
95 & 134\end{array}$ & $\begin{array}{ll}94 & 201 \\
94 & 694 \\
95 & 182\end{array}$ & $\begin{array}{ll}94 & 250 \\
94 & 743 \\
95 & 231\end{array}$ & $\begin{array}{ll}94 & 300 \\
94 & 792 \\
95 & 279\end{array}$ & $\begin{array}{ll}94 & 349 \\
94 & 841 \\
95 & 328\end{array}$ & $\begin{array}{ll}94 & 399 \\
94 & 890 \\
95 & 376\end{array}$ \\
\hline 90 & 95424 & 95472 & 95521 & 95569 & 95617 & $9566 \overline{5}$ & 95713 & 95761 & 95809 & 95856 \\
\hline $\begin{array}{l}91 \\
92 \\
93\end{array}$ & $\begin{array}{ll}95 & 904 \\
96 & 379 \\
96 & 848\end{array}$ & $\begin{array}{ll}95 & 952 \\
96 & 426 \\
96 & 395\end{array}$ & $\begin{array}{ll}95 & 999 \\
96 & 473 \\
96 & 942\end{array}$ & $\begin{array}{ll}96 & 047 \\
96 & 520 \\
96 & 988\end{array}$ & $\begin{array}{ll}96 & 095 \\
96 & 567 \\
97 & 035\end{array}$ & \begin{tabular}{|lll}
96 & 142 \\
96 & 614 \\
97 & 081
\end{tabular} & $\begin{array}{ll}96 & 190 \\
96 & 661 \\
97 & 128\end{array}$ & $\begin{array}{ll}96 & 237 \\
96 & 708 \\
97 & 174\end{array}$ & $\begin{array}{l}96284 \\
96 \quad 755 \\
97.220\end{array}$ & $\begin{array}{ll}96 & 332 \\
96 & 802 \\
97 & 267\end{array}$ \\
\hline $\begin{array}{l}94 \\
95 \\
96\end{array}$ & $\begin{array}{ll}97 & 313 \\
97 & 772 \\
98 & 227\end{array}$ & $\begin{array}{ll}97 & 359 \\
97 & 818 \\
98 & 272\end{array}$ & $\begin{array}{ll}97 & 405 \\
97 & 864 \\
98 & 318\end{array}$ & $\begin{array}{ll}97 & 451 \\
97 & 909 \\
98 & 363\end{array}$ & $\begin{array}{ll}97 & 497 \\
97 & 955 \\
98 & 408\end{array}$ & $\begin{array}{ll}97 & 543 \\
98 & 000 \\
98 & 453\end{array}$ & $\begin{array}{ll}97 & 589 \\
98 & 046 \\
98 & 498 .\end{array}$ & $\begin{array}{r}9763 \tilde{5} \\
98091 \\
.98543\end{array}$ & $\begin{array}{ll}97 & 681 \\
98 & 137 \\
98 & 588\end{array}$ & $\begin{array}{ll}97 & 727 \\
98 & 182 \\
98 & 632\end{array}$ \\
\hline $\begin{array}{l}97 \\
98 \\
99\end{array}$ & $\begin{array}{ll}98 & 677 \\
99 & 123 \\
99 & 564\end{array}$ & $\begin{array}{ll}98 & 722 \\
99 & 167 \\
99 & 607\end{array}$. & $\begin{array}{rr}98 & 767 \\
-99 & 211 \\
99 & 651\end{array}$ & $\begin{array}{ll}98 & 811 \\
99 & 255 \\
99 & 695\end{array}$ & $\begin{array}{ll}98 & 856 \\
99 & 300 \\
99 & 739\end{array}$ & $\begin{array}{ll}98 & 900 \\
99 & 344 \\
99 & 782\end{array}$ & $\begin{array}{l}9894 \overline{5} \\
99388 \\
99 \\
826\end{array}$ & $\begin{array}{ll}98 & 989 \\
99 & 432 \\
99 & 870\end{array}$ & $\begin{array}{l}99034 \\
99 \\
996 \\
99\end{array}$ & $\begin{array}{ll}99 & 078 \\
99 & 520 \\
99 & 957\end{array}$ \\
\hline 100 & 00000 & 00043 & $00 \quad 087$ & 00130 & 00173 & 00217 & 00260 & 00303 & 00346 & 00389 \\
\hline N. & L. 0 & 1 & 2 & 3 & 4 & 5 & 6 & 7 & 8 & 9 \\
\hline \multicolumn{2}{|c|}{$0^{\circ} \quad 9^{\prime}=540^{\prime \prime}$} & \multicolumn{4}{|c|}{$\begin{array}{ll}\text { S. 4. } 68557 & \text { T. } 4.68558\end{array}$} & \multicolumn{5}{|c|}{$0^{\circ} 13^{\prime}=780^{\prime \prime} \quad$ S. $4.68557 \quad$ T. 4.68558} \\
\hline \multicolumn{2}{|c|}{$0 \quad 10=600$} & \multicolumn{2}{|c|}{ 4. 68557} & 4. 68 & 558 & \multicolumn{2}{|c|}{$14=840$} & 4. 68657 & & 68558 \\
\hline \multicolumn{2}{|c|}{$0 \quad 11=660$} & \multicolumn{2}{|c|}{ 4. 68557} & 4. 68 & 558 & \multicolumn{2}{|c|}{$15=900$} & 4. 68557 & 7 & 68558 \\
\hline \multicolumn{2}{|l|}{$0 \quad 12$} & \multicolumn{2}{|c|}{ 4. 68557} & 4. 68 & 558 & \multicolumn{2}{|c|}{$16=960$} & 4. 68557 & 7 & 68558 \\
\hline
\end{tabular}


TABLE 18.-Five-place logarithms of naturat numbers-Continued.

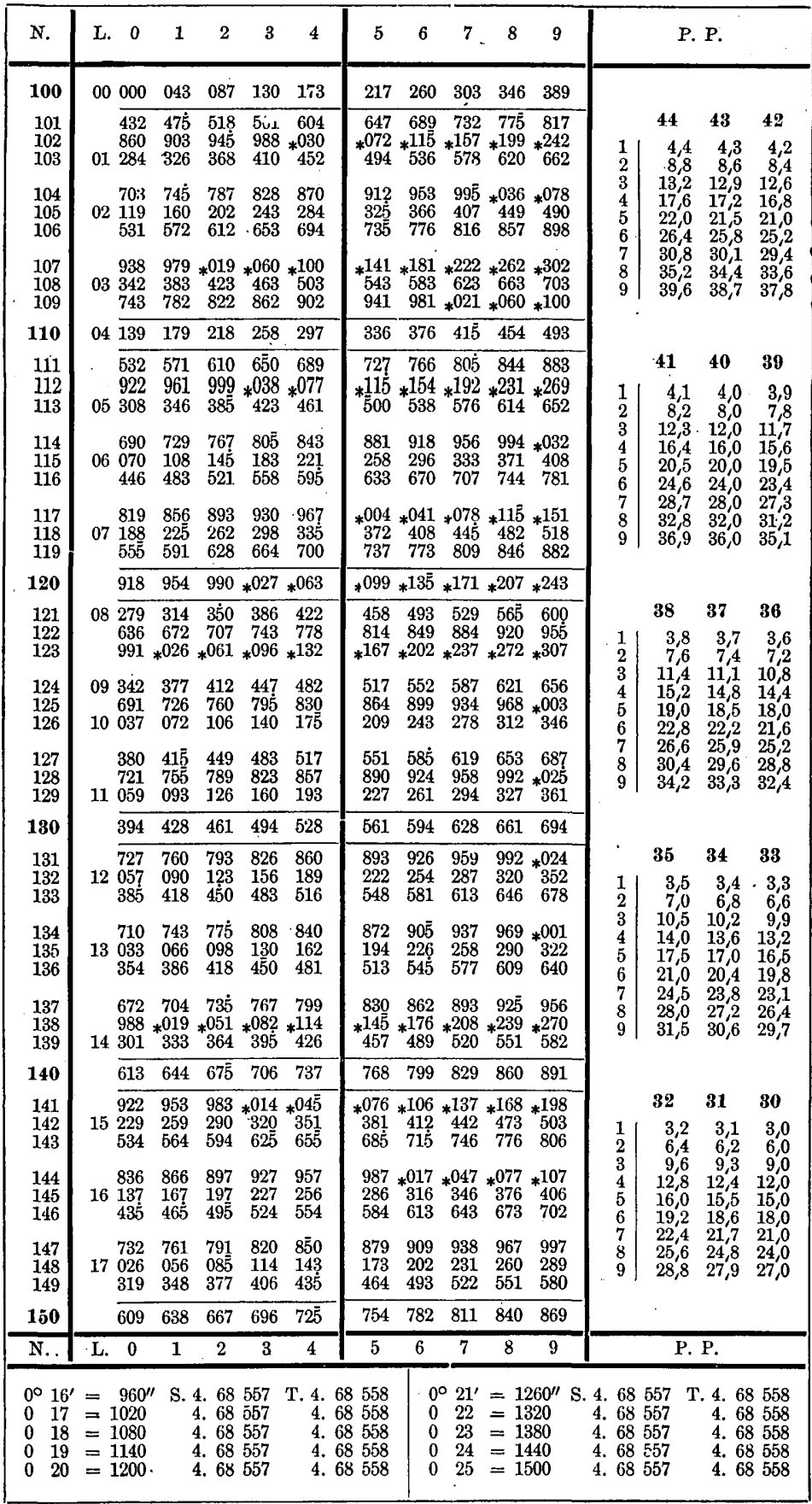


TABBE 18. - Five-place logarithms of natural numbers-Continued.

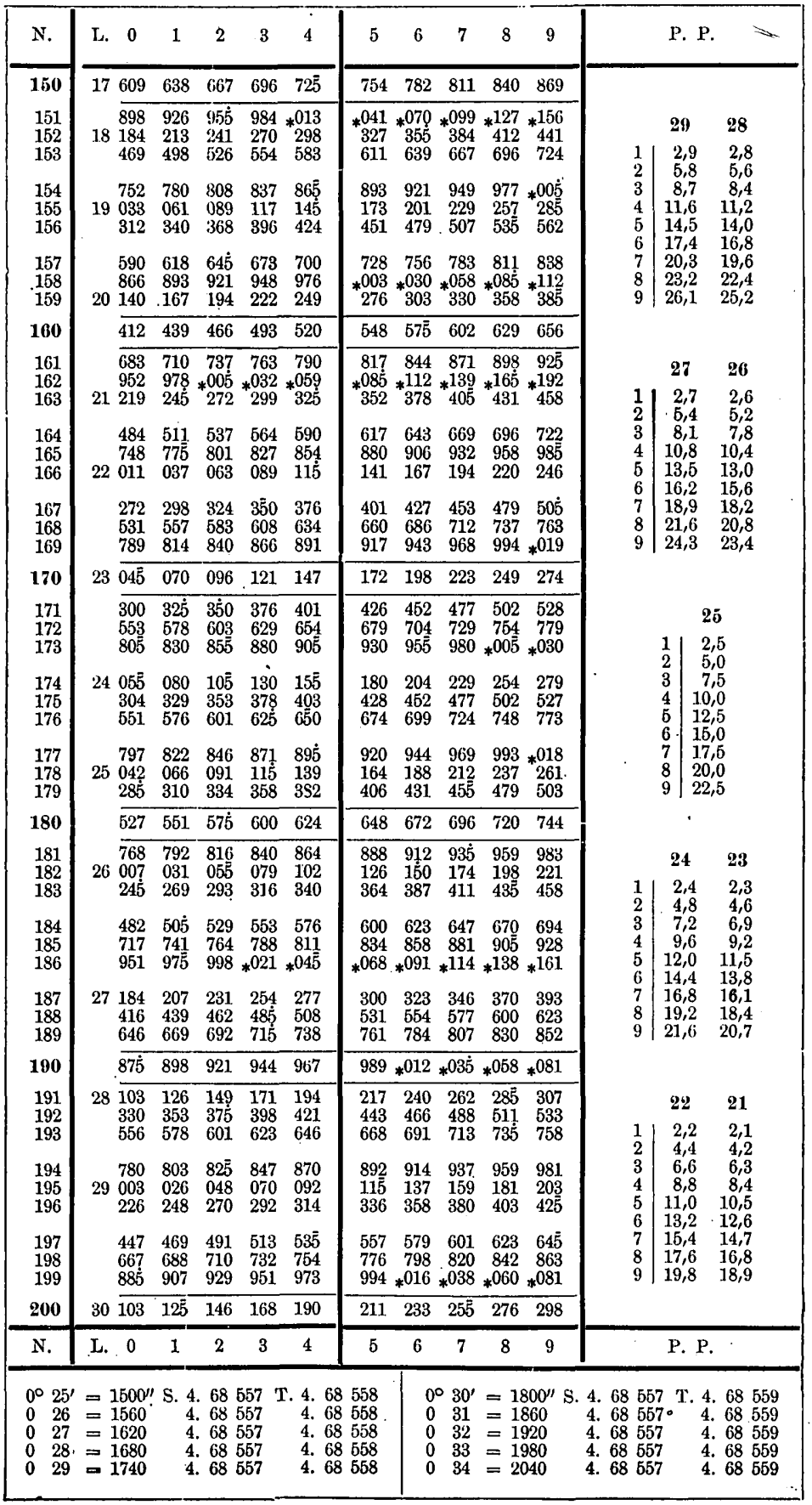


TABLE 18.-Five-place logarithms of natural numbers-Continued.

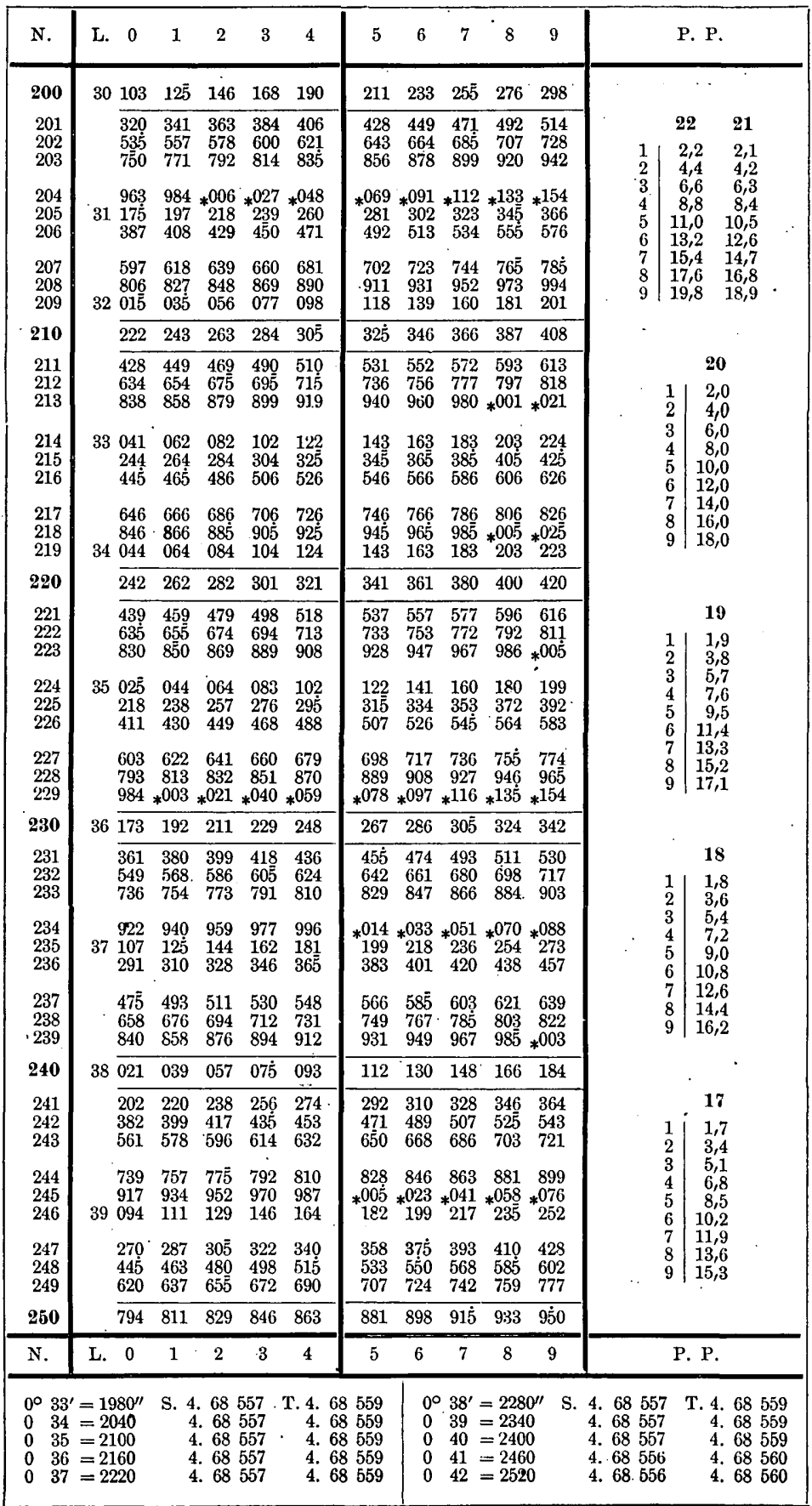


TABLE 18. -Five-place logarithms of natural numbers-Continued.

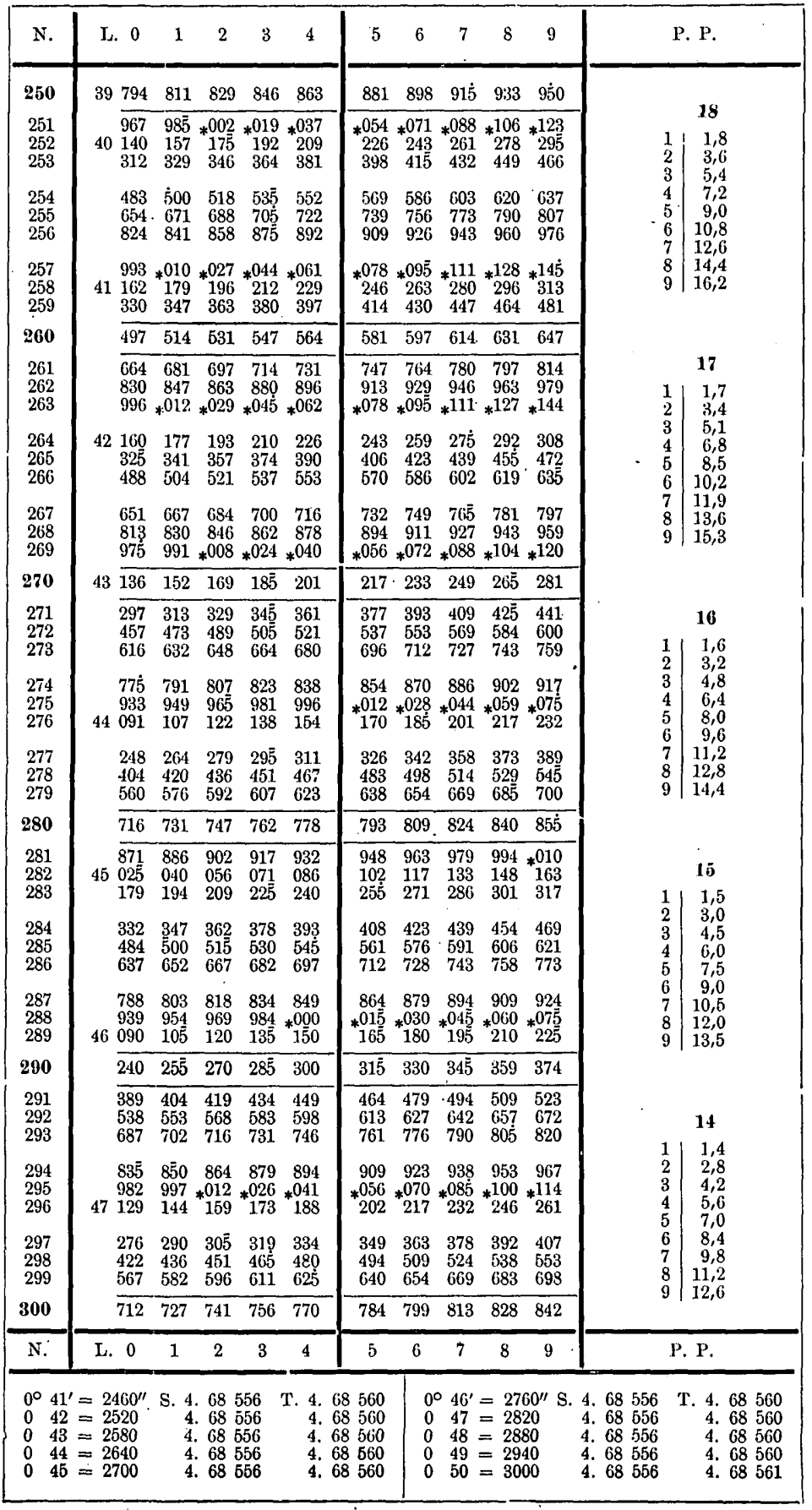


TABLE 18.-Five-place logarithms of natural numbers-Continued.

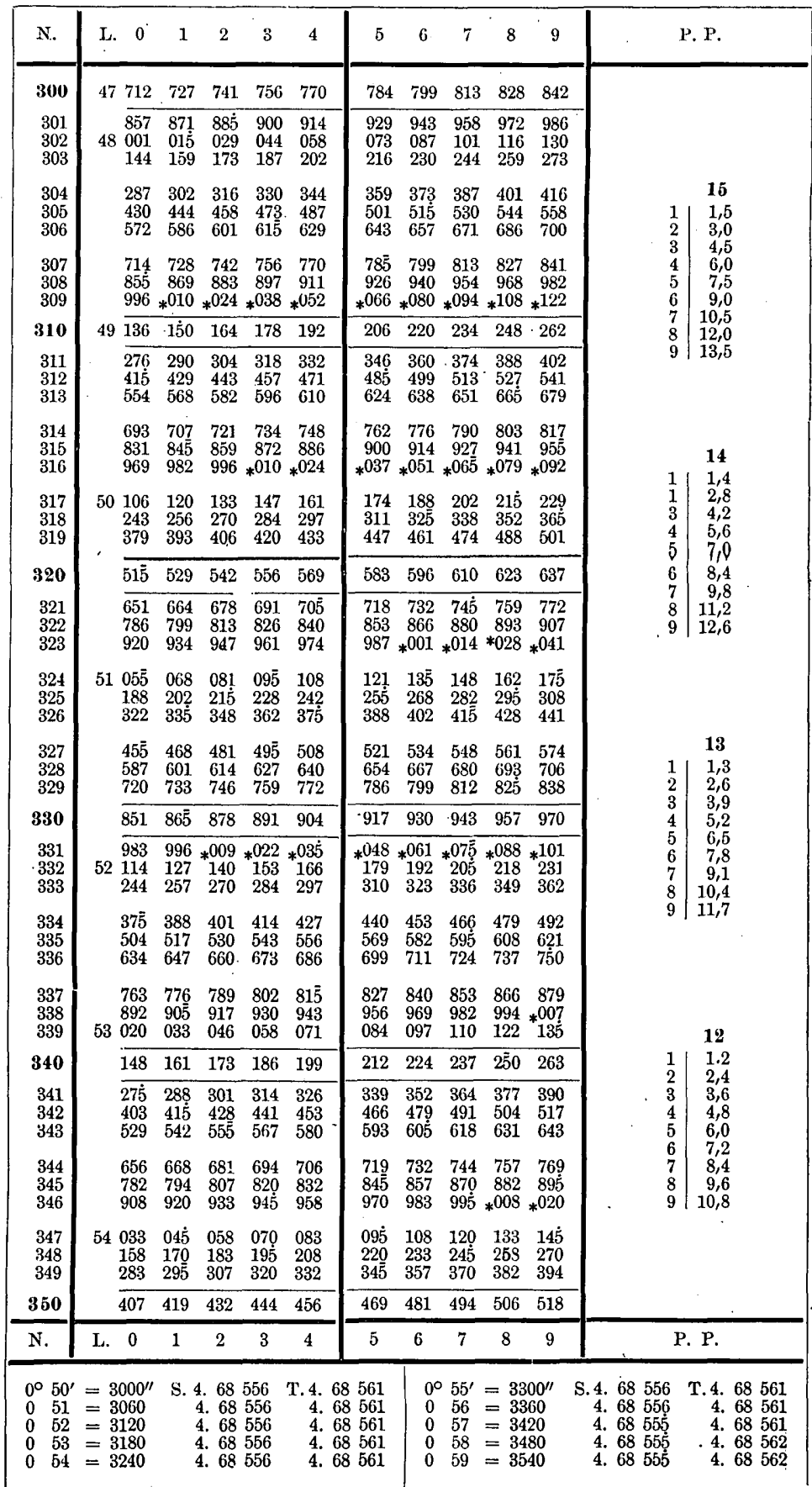


TABLE 18.-Five-place logarithms of natural numbers-Continued.

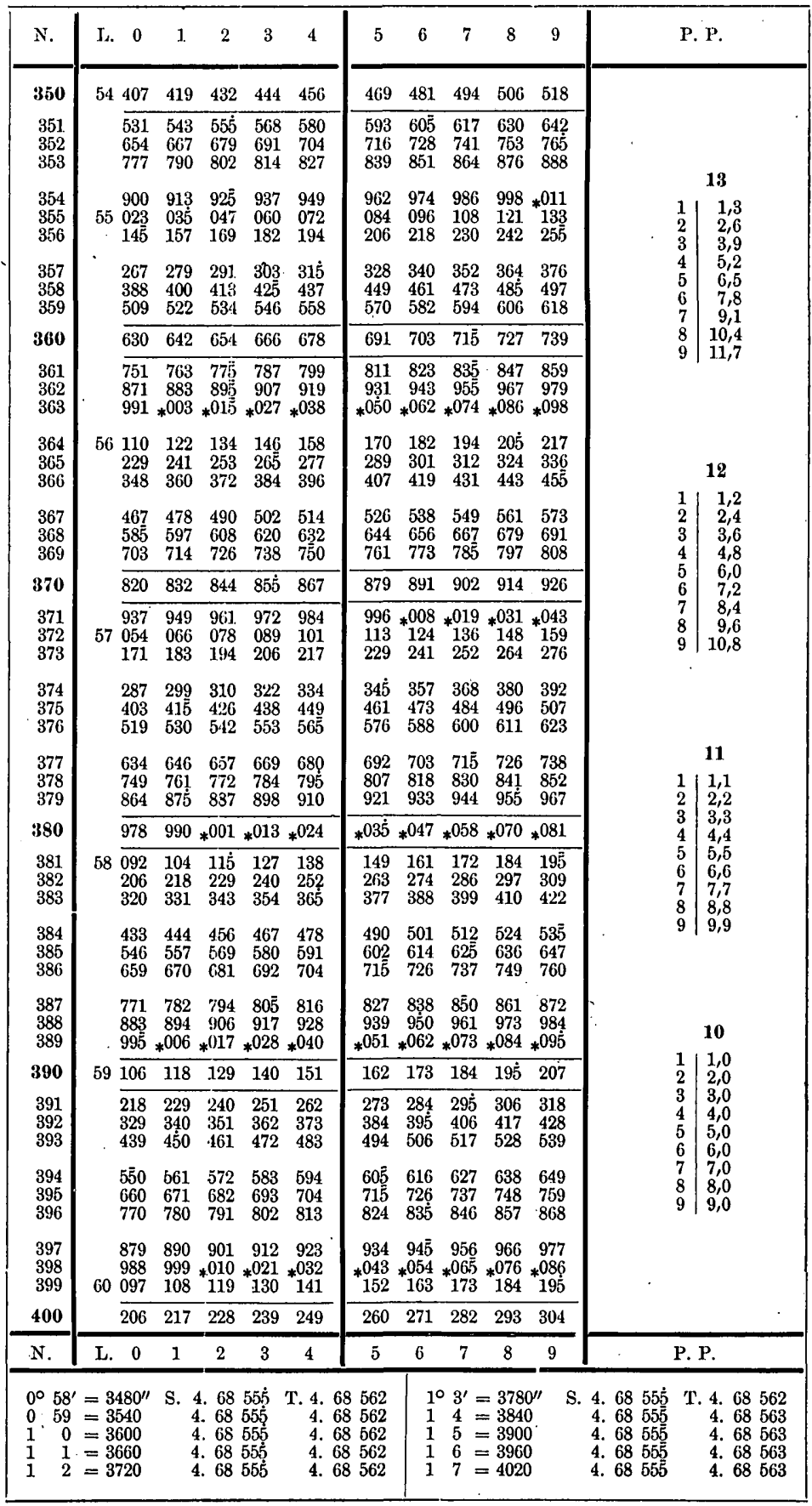


TABLE 18.-Five-place logarithms of natural numbers-Conitinued.

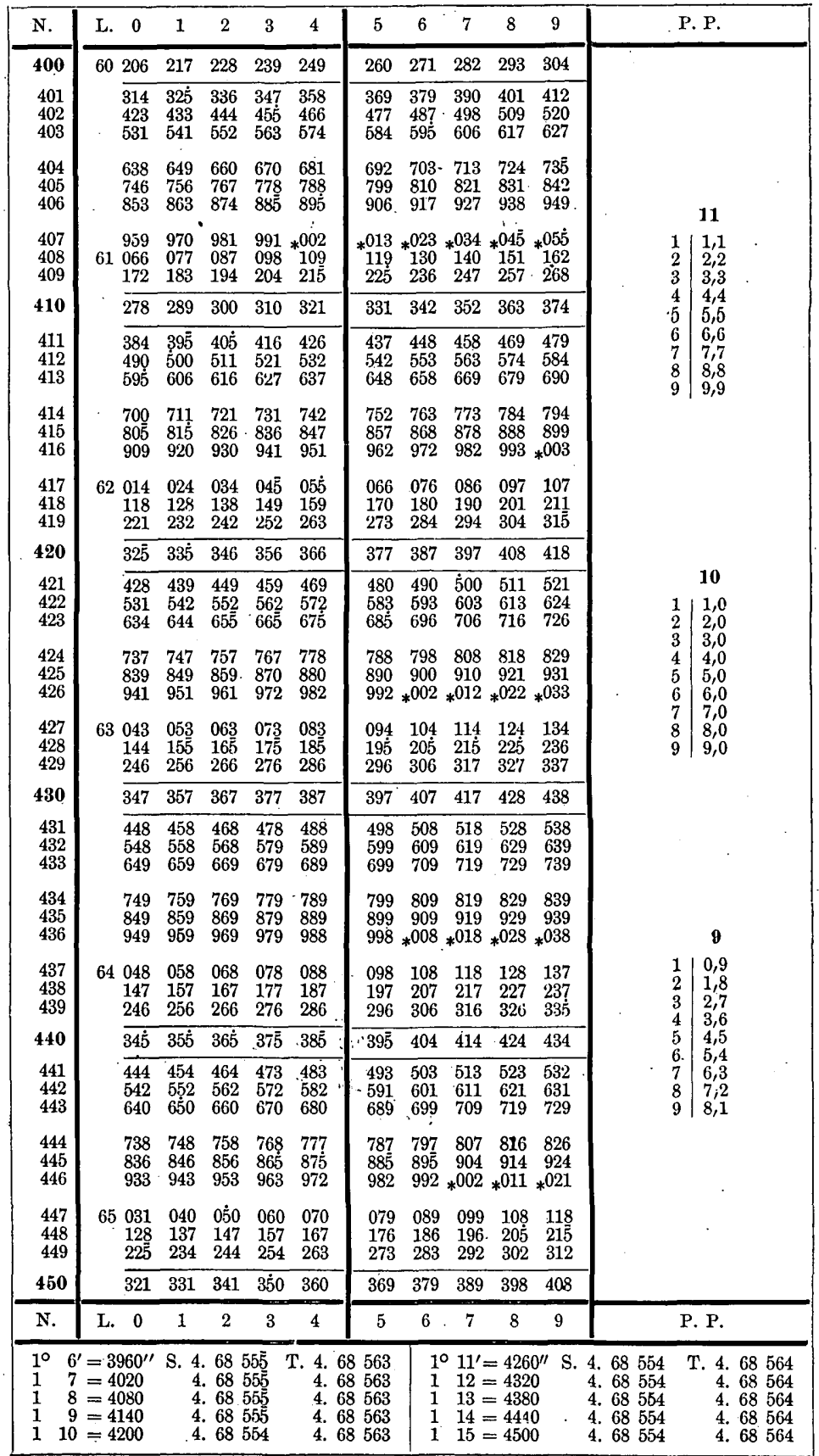


TABLe 18. -Five-place logarithms of natural numbers-Continued.

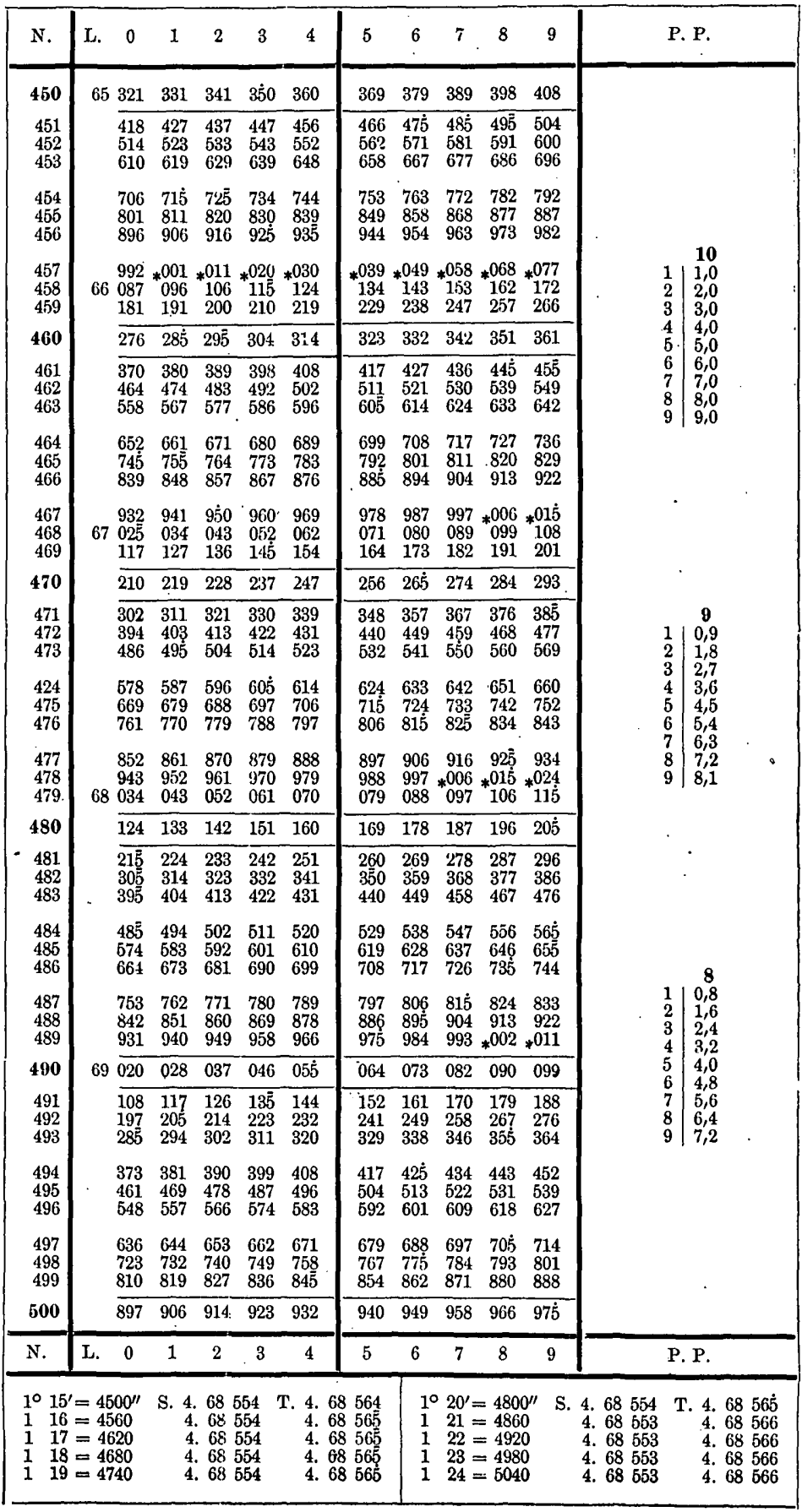


TABLE 18.-Five-place logarithms of natural numbers-Continued.

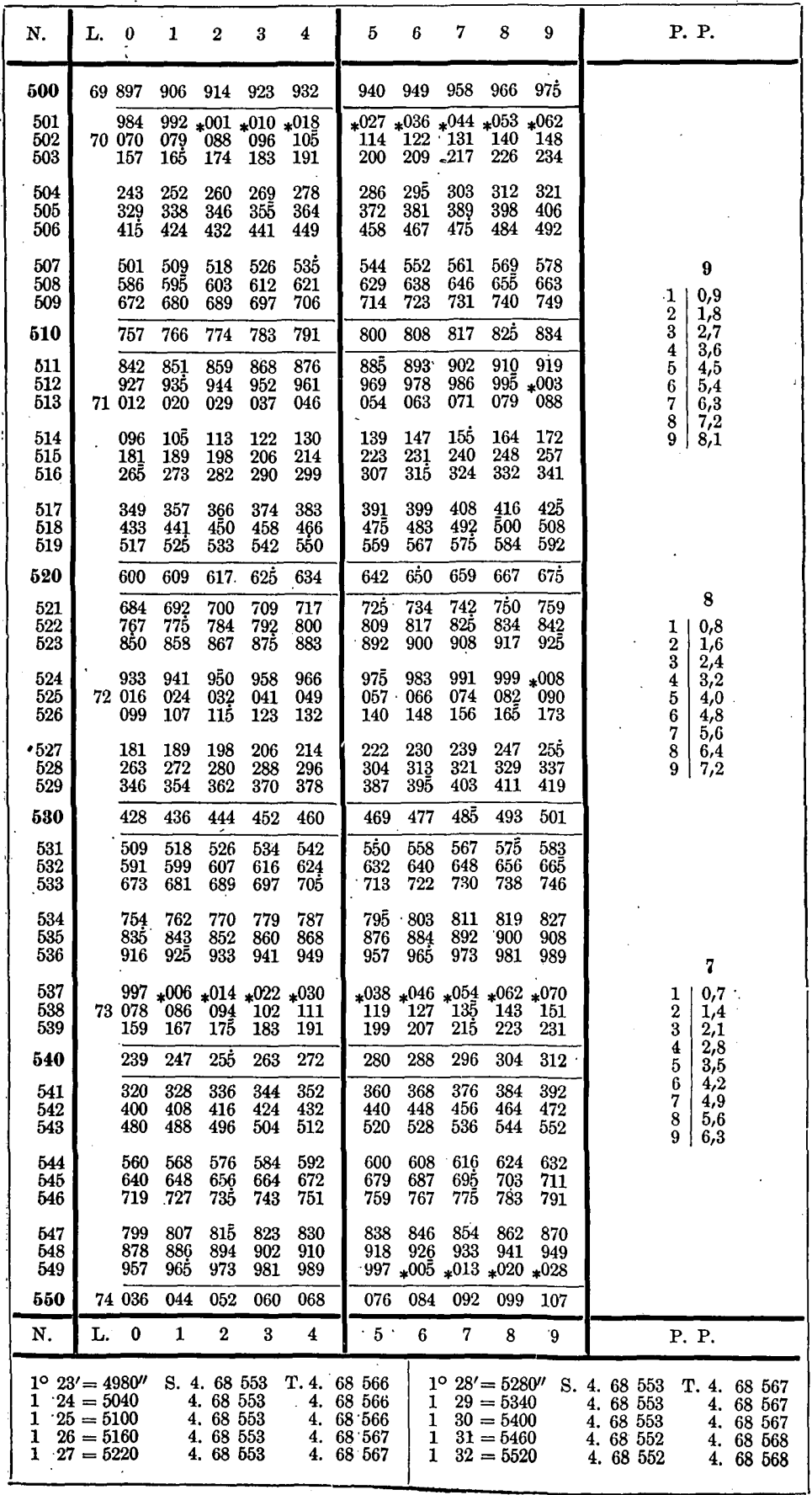


TABLE 18.-Five-place Togarithms of natural numbers-Continued.

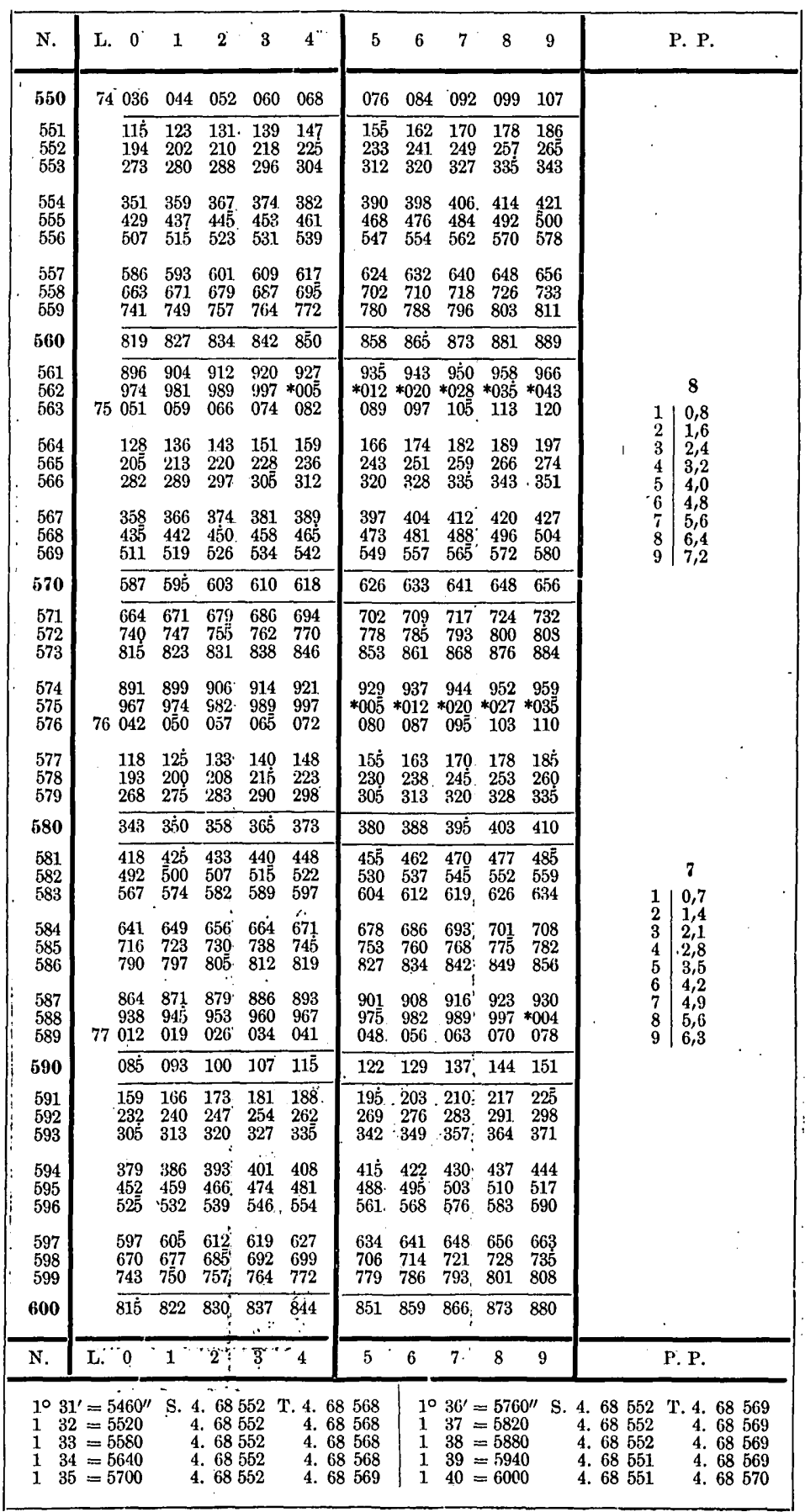


TABLE 18.-Five-place logarithms of natural numbers-Continued.

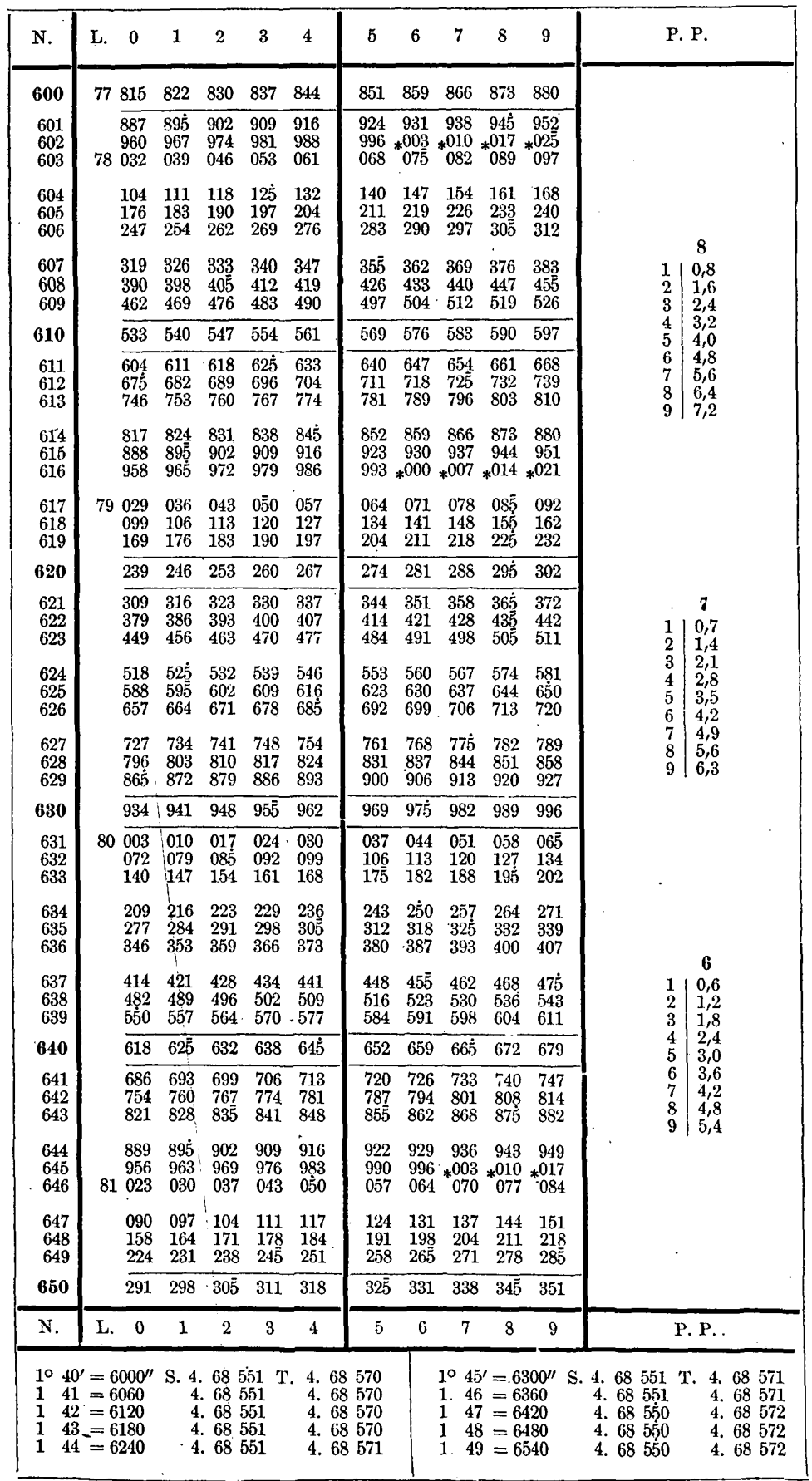


TaBLe 18.-Five-place logarithms of natural numbers-Continued.

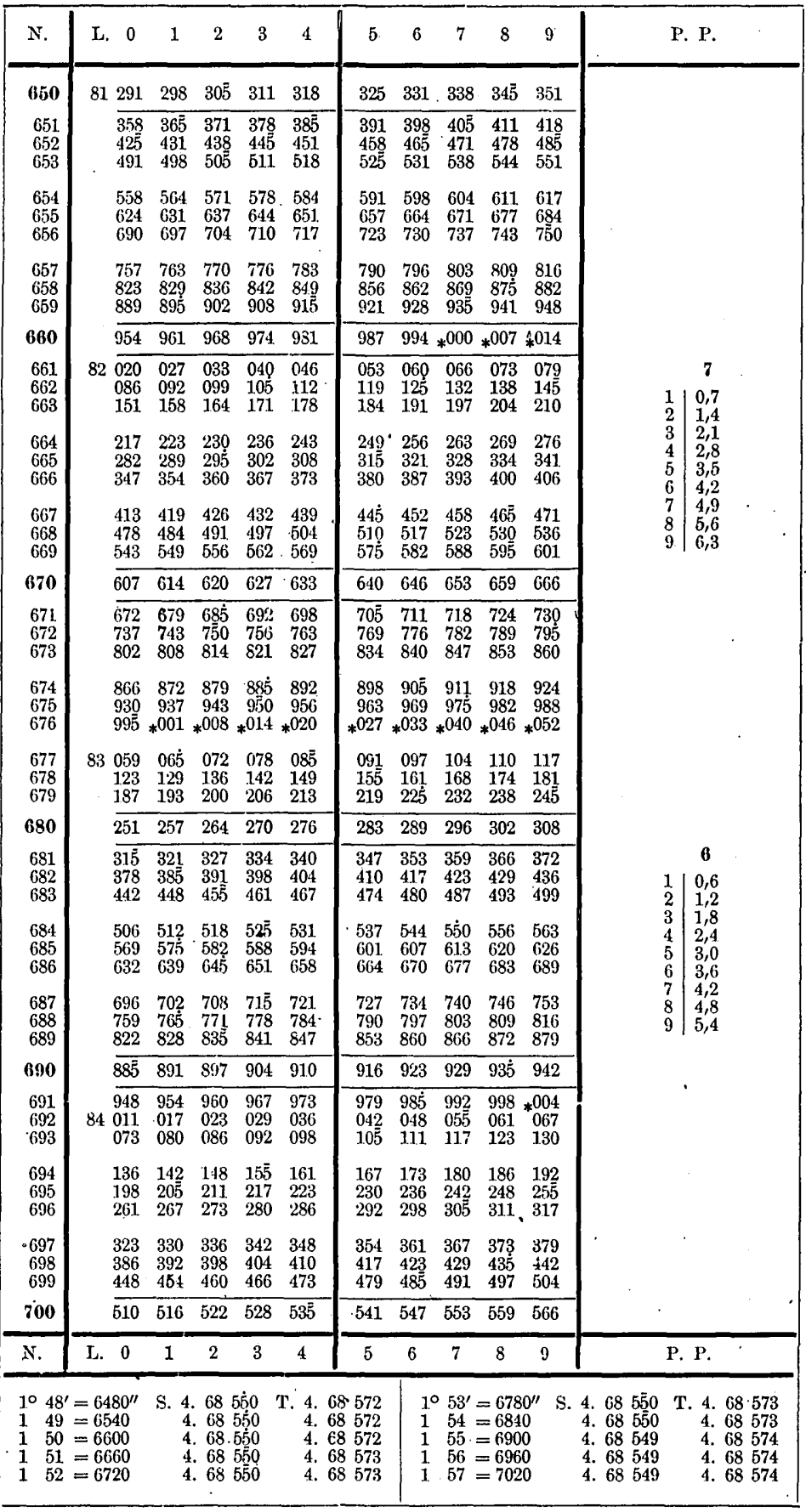


TABLE 18.-Five-place logarithms of natural numbers-Continued.

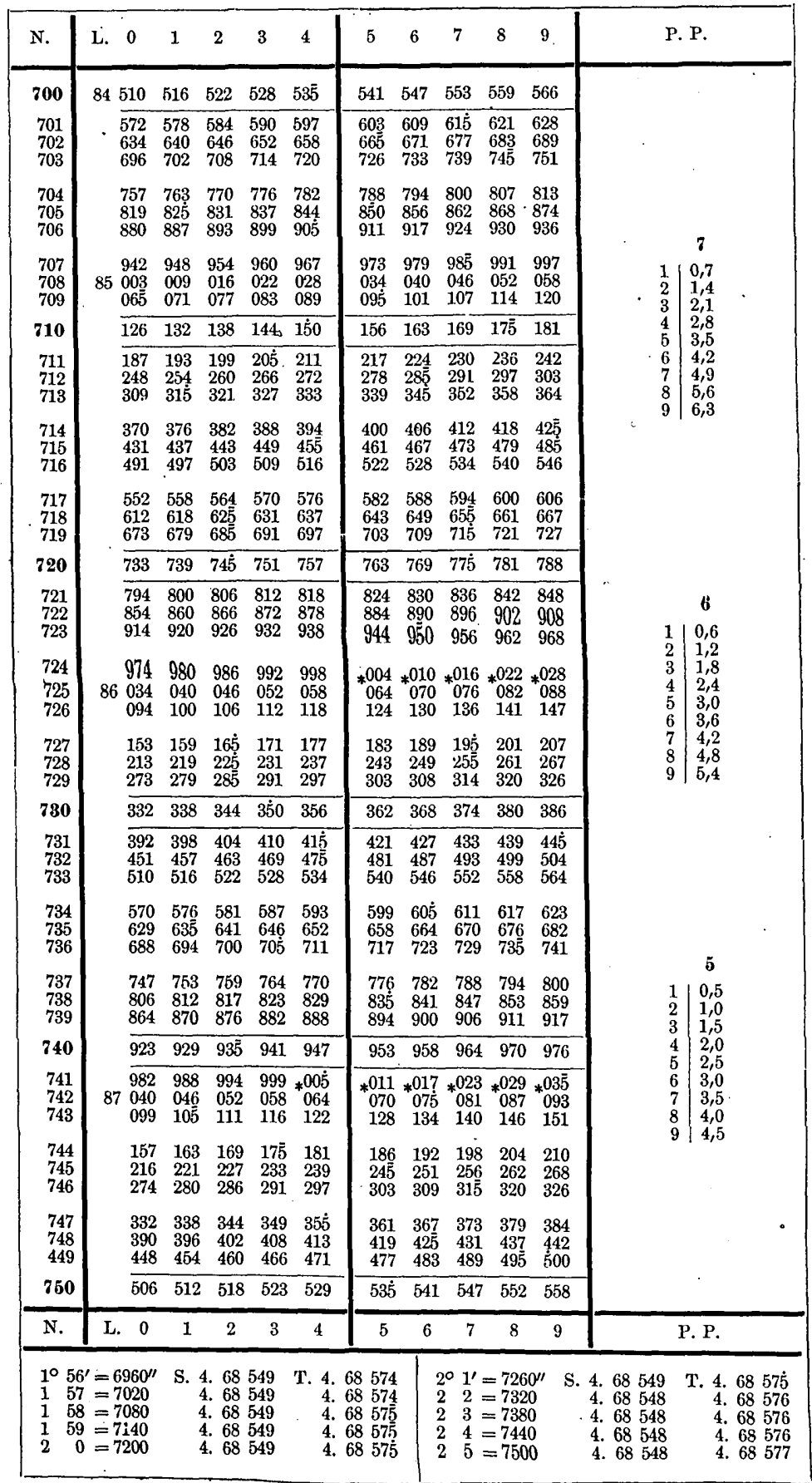


TABLE 18.-Five-place logarithms of natural numbers-Continued.

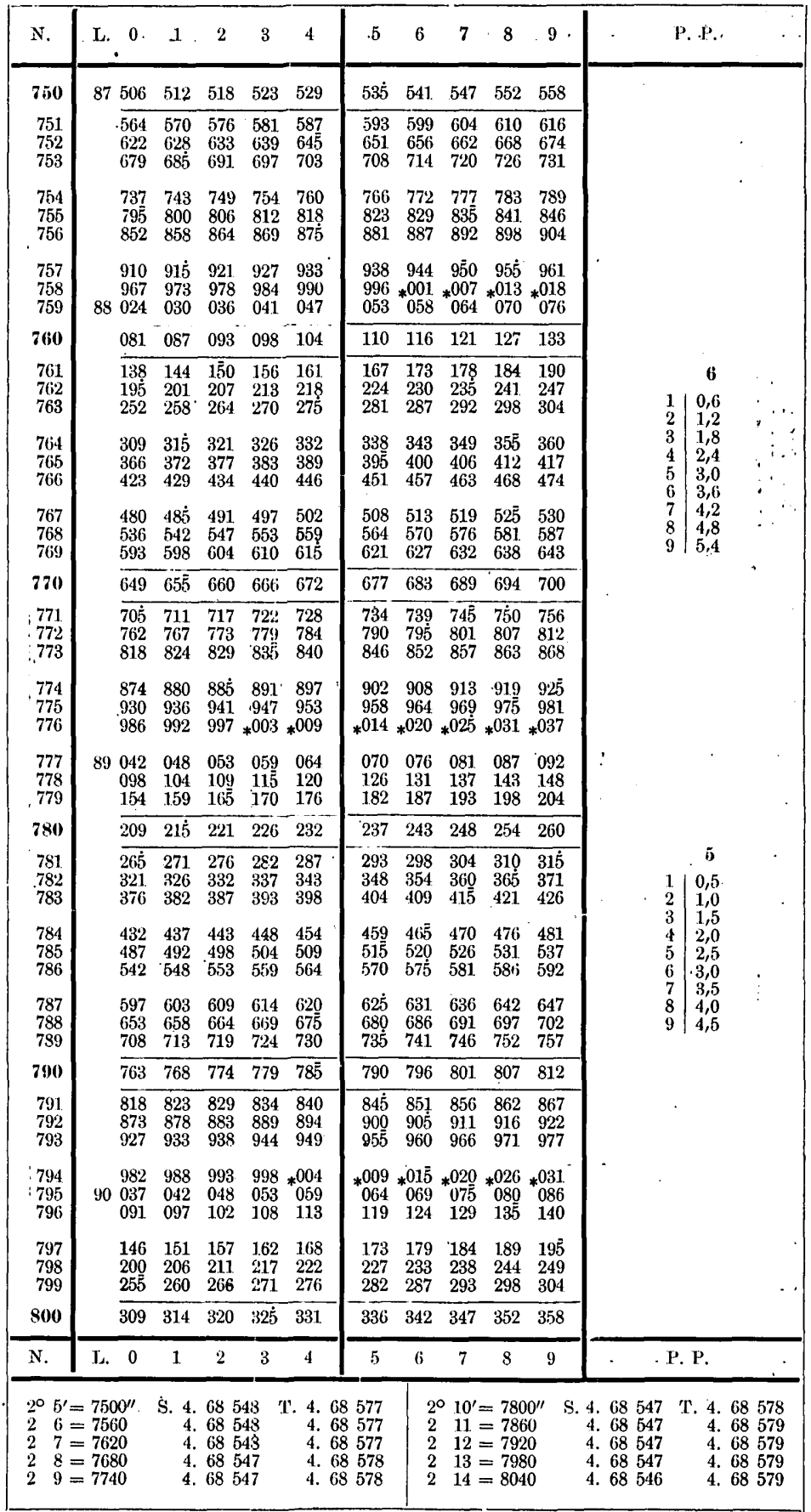

Bull. 214-03-8 
TABLE 18.-Five-place logarithms of natural numbers-Continued.

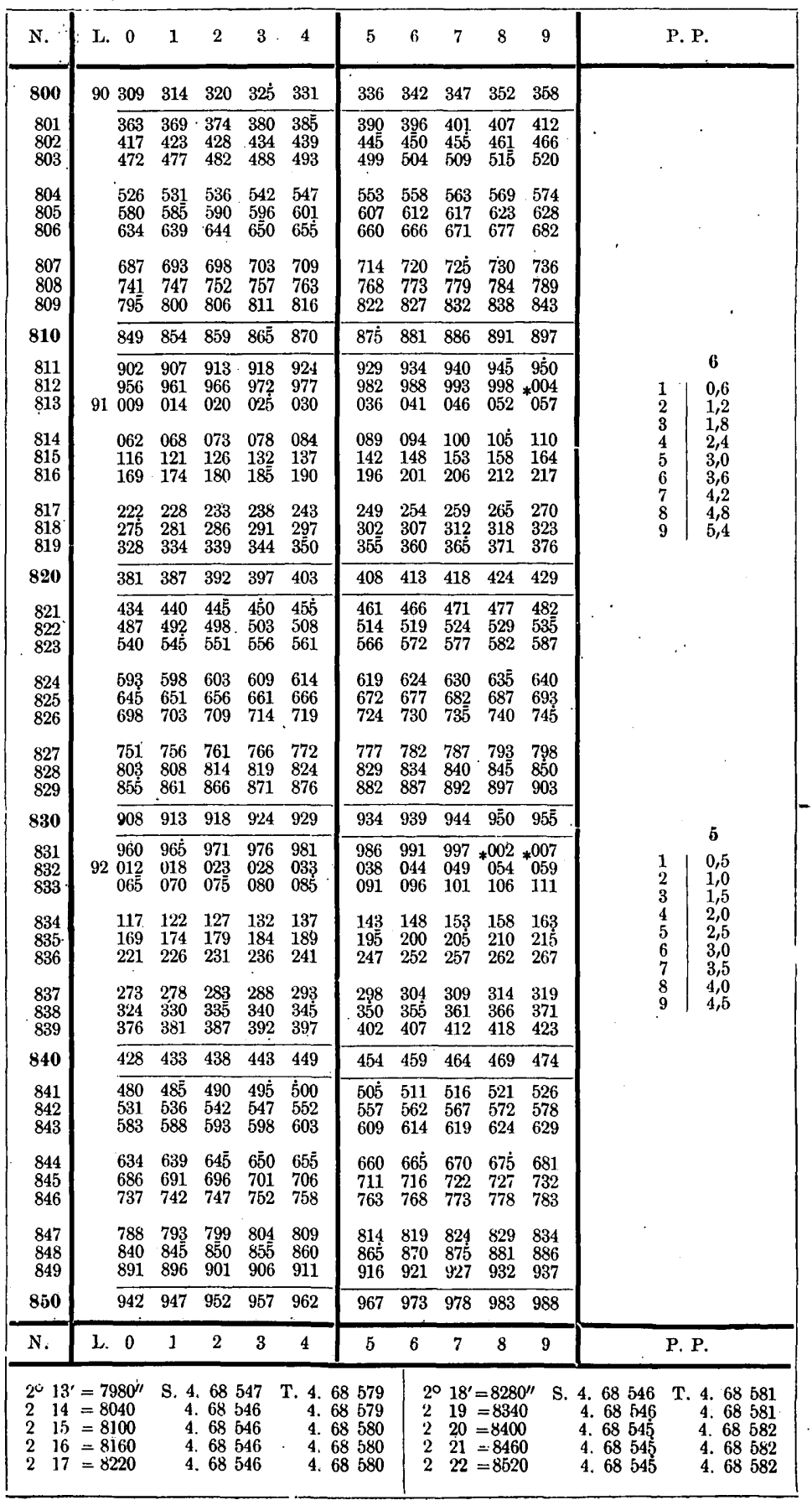


TABLE 18.-Five-place logarithms of natural numbers-Continued.

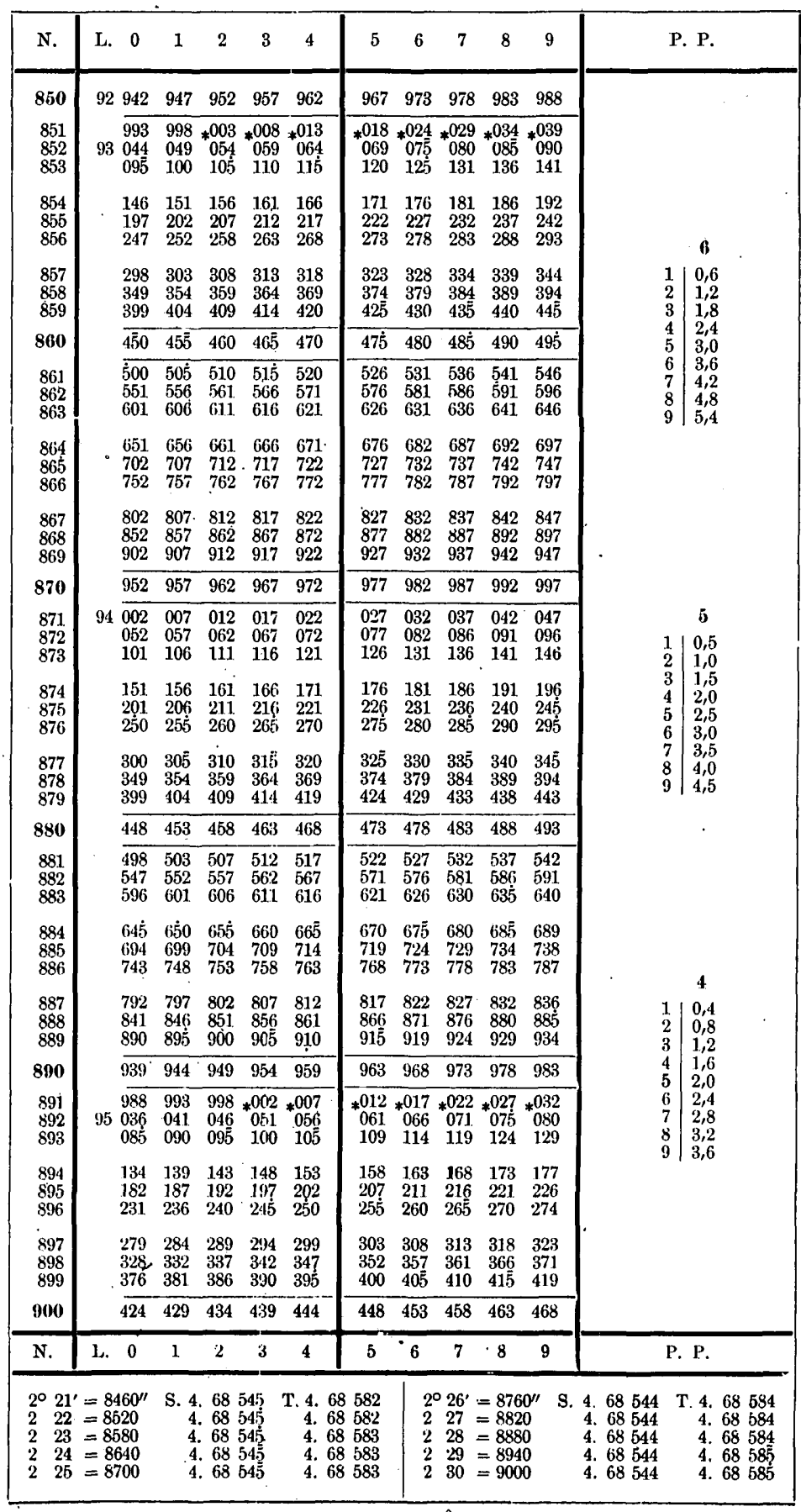


TABLE 18.-Five-place logarithms of natural numbers-Continued.

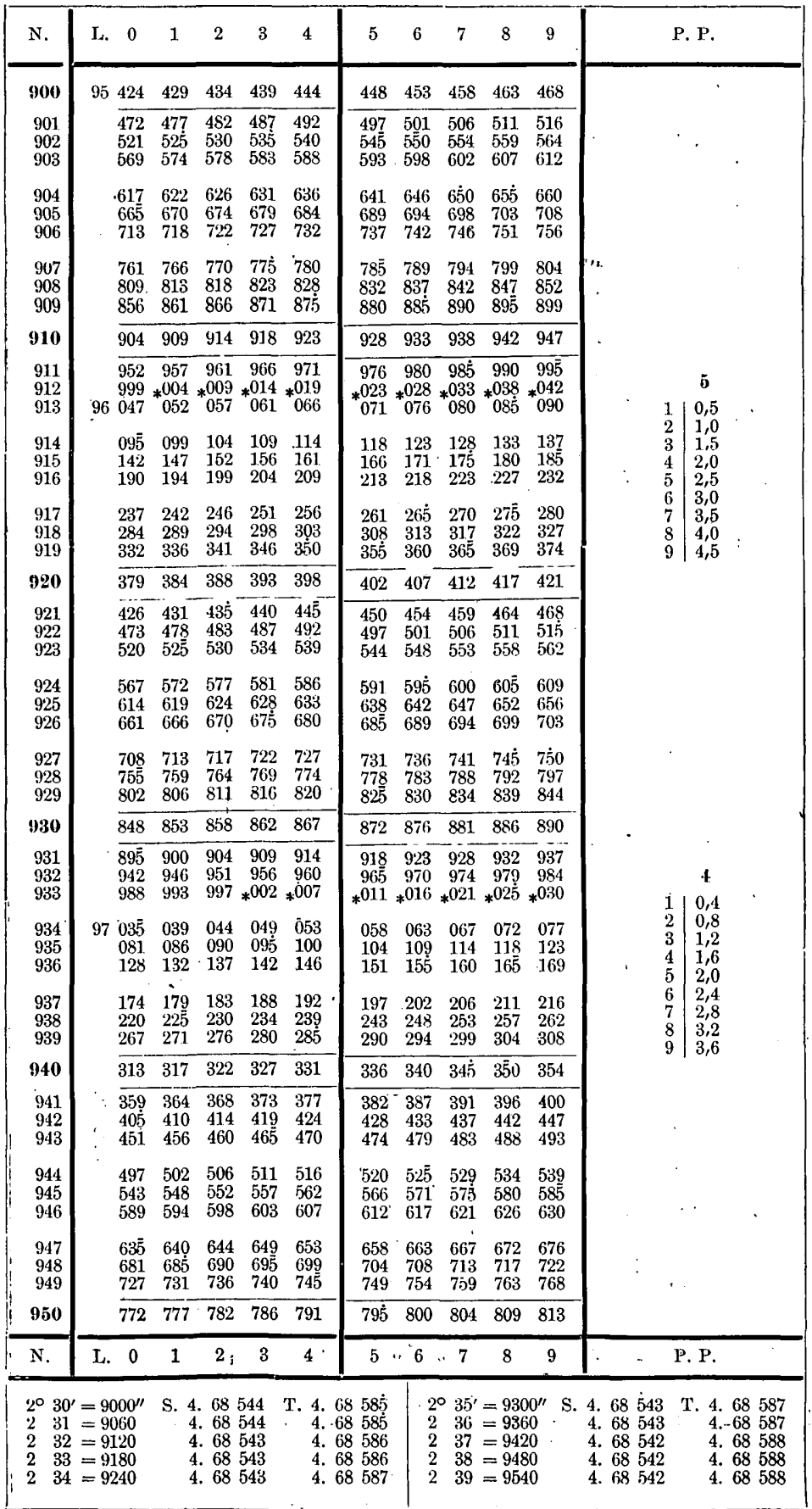


ТАвцE 18.-Five-place logarithms of natural numbers-Continued.

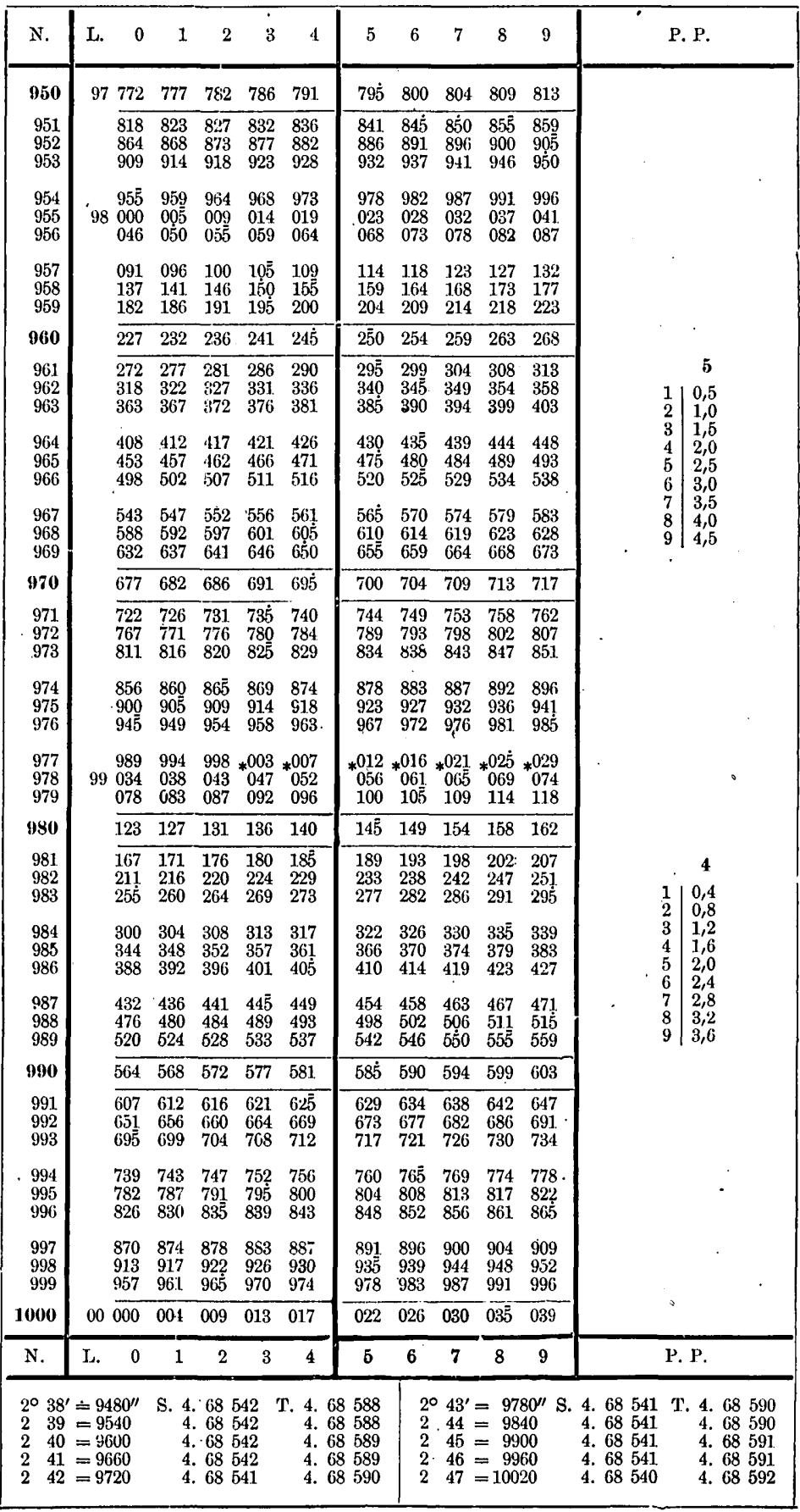


Formula for using quantities $S$ and $T$ :

$\log \sin a=\quad \log a^{\prime \prime}+S$.

$\log \tan a=\quad \log a^{\prime \prime}+T$.

$\log \cot a=$ a. c. $\log a^{\prime \prime}+$ a. c. $\log T$.

$\log a^{\prime \prime}=\log \sin a-S=\log \tan a-T$.

$\log \cos a=\quad \log \left(90^{\circ}-a\right)^{\prime \prime}+S$.

$\log \cot a=\log \left(90^{\circ}-a\right)^{\prime \prime}+T$.

$\log \tan a=$ a. c. $\log \left(90^{\circ}-a\right)^{\prime \prime}+$ a. c. $\log T$.

$\log \left(90^{\circ}-a\right)^{\prime \prime}=\log \cos a-S=\log \cot a-T$. 
TАвце 19.-Five-place logarithms of circular functions, expressed in arc and time.

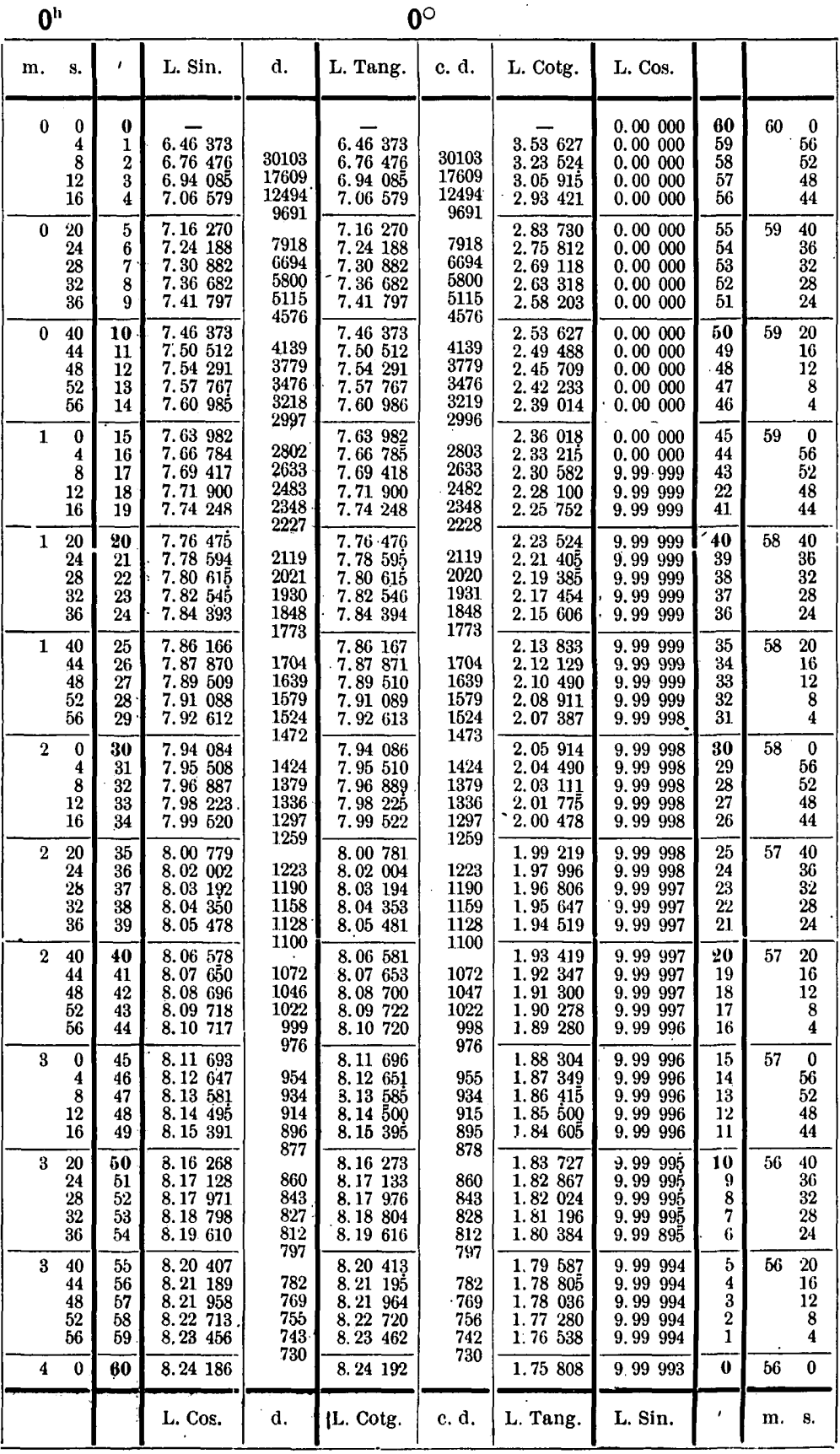


TABLE 19.-Five-place logarithms of circular functions, etc.-Continued.

$0^{\text {h }}$

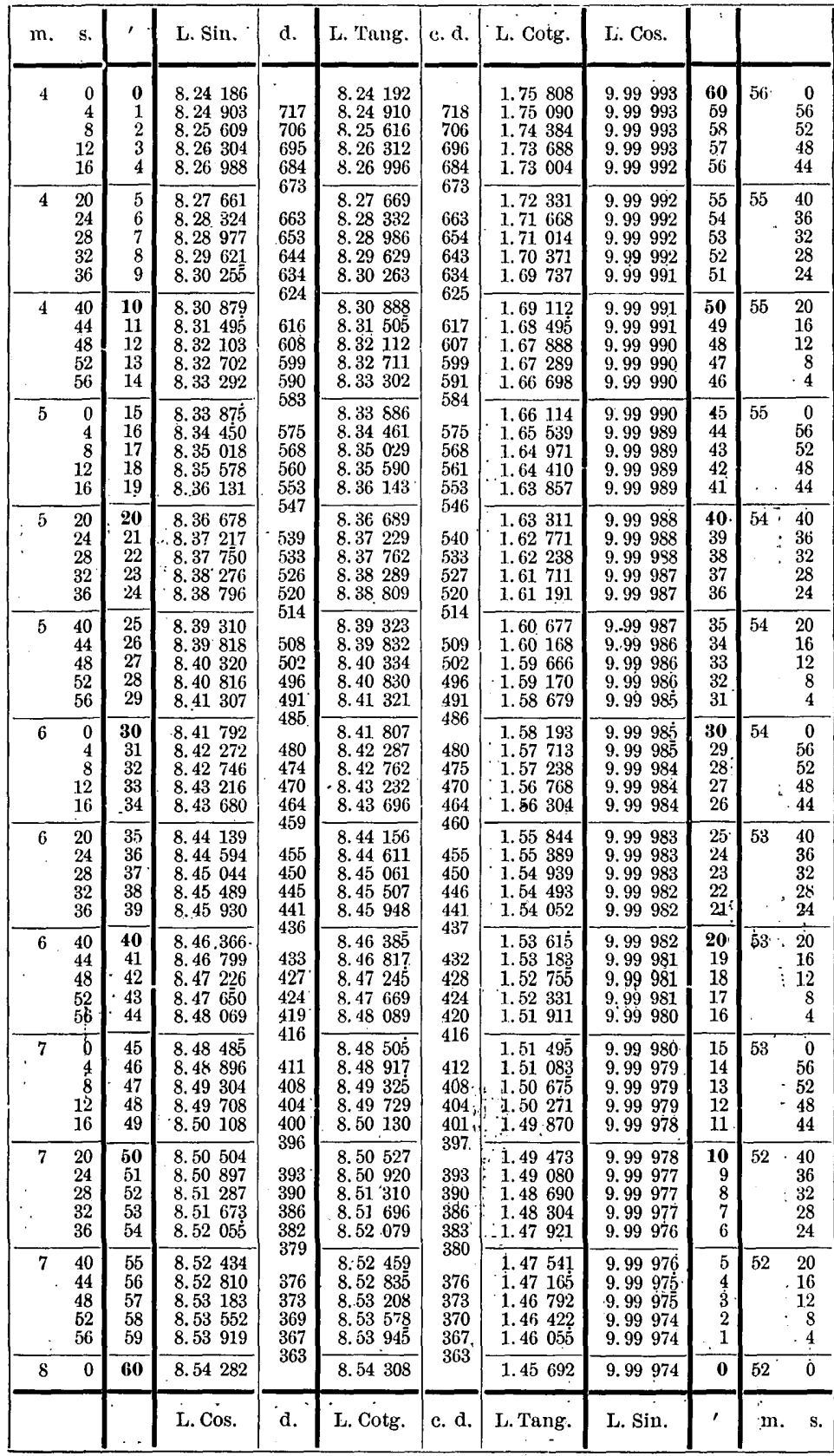

$88^{\circ}$

$1^{\circ}$ 
TАвье 19.-Five-place logarithms of circular functions, elc.-Continued.

$0^{\mathrm{h}} \quad 2^{\circ}$

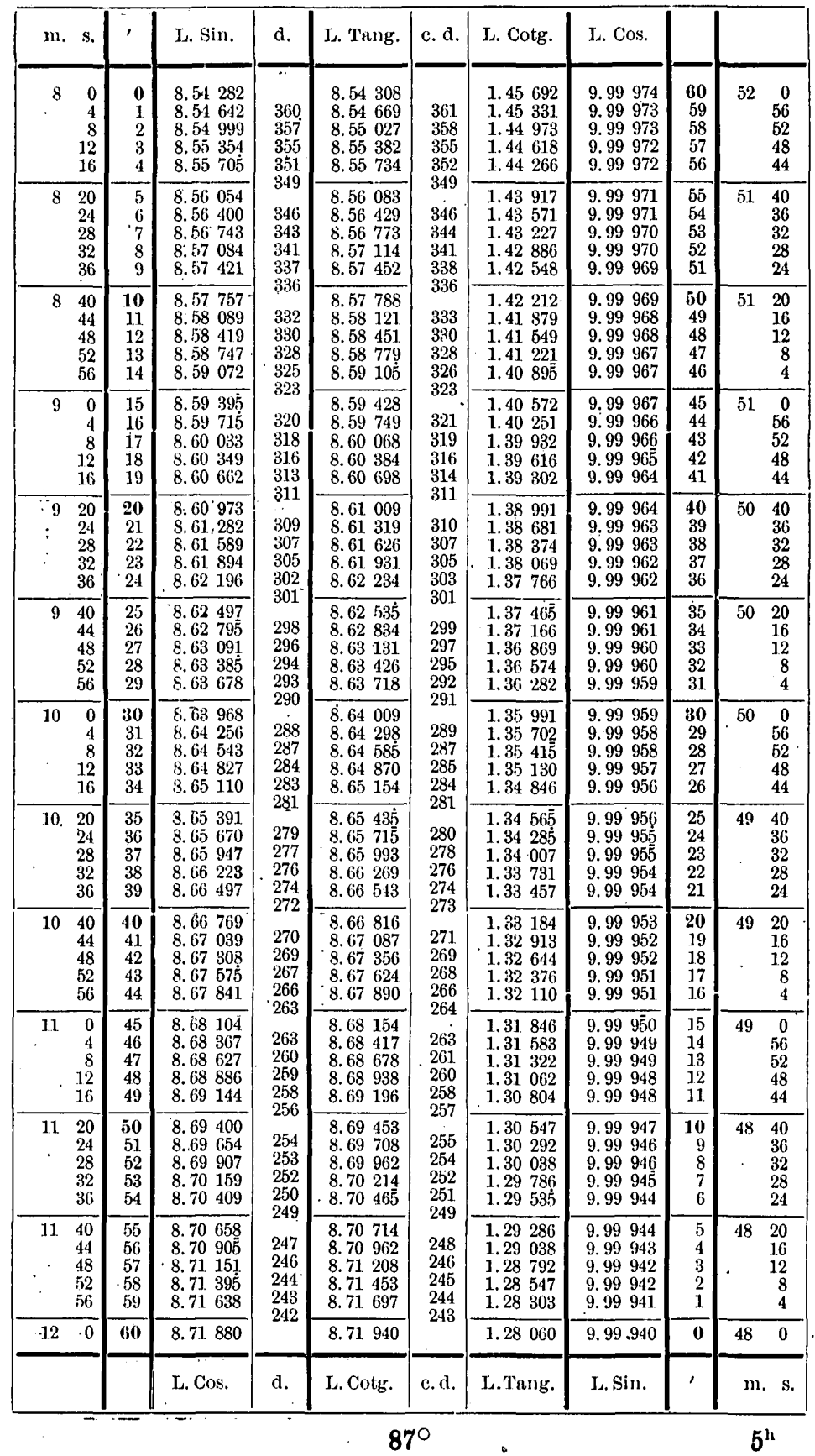


TABLe 19.-Five-place logarithms of circular functions, etc.-Continued.

\begin{tabular}{|c|c|c|c|c|c|c|c|c|c|c|c|}
\hline $\mathrm{m}$ & s. & ' & L. Sin. & $\mathrm{d}$ & L. Tang. & c. d. & L. Cotg. & L. Cos. & & & \\
\hline 12 & $\begin{array}{r}0 \\
4 \\
8 \\
12 \\
16\end{array}$ & $\begin{array}{l}0 \\
1 \\
2 \\
3 \\
4\end{array}$ & $\begin{array}{ll}8.71 & 880 \\
8.72 & 120 \\
8.72 & 359 \\
8.72 & 597 \\
8.72 & 834\end{array}$ & \multirow{2}{*}{$\begin{array}{l}240 \\
239 \\
\cdot 238 \\
237 \\
235 \\
- \\
234 \\
232 \\
232 \\
230 \\
229\end{array}$} & $\begin{array}{l}8.71940 \\
8.72181 \\
8.72 \quad 420 \\
8.72659 \\
8.72 \quad 896\end{array}$ & $\begin{array}{l}241 \\
239 \\
239 \\
237 \\
236\end{array}$ & $\begin{array}{l}1.28060 \\
1.27819 \\
1.27580 \\
1.27341 \\
1.27104\end{array}$ & 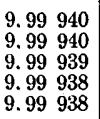 & $\begin{array}{l}60 \\
59 \\
58 \\
57 \\
56\end{array}$ & & $\begin{array}{r}0 \\
56 \\
52 \\
48 \\
44\end{array}$ \\
\hline 12 & $\begin{array}{l}20 \\
24 \\
28 \\
32 \\
36\end{array}$ & $\begin{array}{l}5 \\
6 \\
7 \\
8 \\
9\end{array}$ & $\begin{array}{ll}8.73 & 069 \\
8.73 & 303 \\
8.73 & 535 \\
8.73 & 767 \\
8.73 & 997\end{array}$ & & $\begin{array}{lll}8.73 & 132 \\
8.73 & 366 \\
8.73 & 600 \\
8.73 & 832 \\
8.74 & 063\end{array}$ & $\begin{array}{l}236 \\
234 \\
234 \\
232 \\
231\end{array}$ & 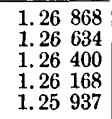 & 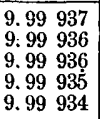 & $\begin{array}{l}55 \\
54 \\
53 \\
52 \\
51\end{array}$ & 47 & $\begin{array}{l}40 \\
36 \\
32 \\
28 \\
24\end{array}$ \\
\hline 12 & $\begin{array}{l}40 \\
44 \\
48 \\
52 \\
56\end{array}$ & $\begin{array}{l}10 \\
11 \\
12 \\
13 \\
14\end{array}$ & $\begin{array}{ll}8.74 & 226 \\
8.74 & 454 \\
8.74 & 680 \\
8.74 & 906 \\
8.75 & 130\end{array}$ & $\begin{array}{l}229 \\
228 \\
226 \\
226 \\
224\end{array}$ & $\begin{array}{ll}8.74 & 292 \\
8.74 & 521 \\
8.74 & 748 \\
8.74 & 974 \\
8.75 & 199\end{array}$ & $\begin{array}{l}229 \\
227 \\
226 \\
225\end{array}$ & $\begin{array}{ll}1.25 & 708 \\
1.25 & 479 \\
1.25 & 252 \\
1.25 & 026 \\
1.24 & 801\end{array}$ & 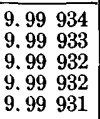 & $\begin{array}{l}50 \\
49 \\
48 \\
47 \\
46\end{array}$ & 47 & $\begin{array}{r}20 \\
16 \\
12 \\
8 \\
4\end{array}$ \\
\hline 13 & $\begin{array}{r}0 \\
4 \\
8 \\
12 \\
16\end{array}$ & $\begin{array}{l}15 \\
16 \\
17 \\
18 \\
19\end{array}$ & $\begin{array}{ll}8.75 & 353 \\
8.75 & 575 \\
8.75 & 795 \\
8.76 & 015 \\
8.76 & 234\end{array}$ & $\begin{array}{l}222 \\
220 \\
220 \\
219\end{array}$ & $\begin{array}{ll}8.75 & 423 \\
8.75 & 645 \\
8.75 & 867 \\
8.76 & 087 \\
8.76 & 306\end{array}$ & $\begin{array}{l}222 \\
222 \\
220 \\
219\end{array}$ & 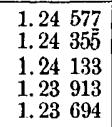 & 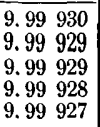 & $\begin{array}{l}45 \\
44 \\
43 \\
42 \\
41\end{array}$ & 47 & $\begin{array}{r}0 \\
56 \\
52 \\
48 \\
44\end{array}$ \\
\hline 13 & $\begin{array}{l}20 \\
24 \\
28 \\
32 \\
36\end{array}$ & $\begin{array}{l}20 \\
21 \\
22 \\
23 \\
24\end{array}$ & $\begin{array}{ll}8.76 & 451 \\
8.76 & 667 \\
8.76 & 883 \\
8.77 & 097 \\
8.77 & 310\end{array}$ & $\begin{array}{l}216 \\
216 \\
214 \\
213\end{array}$ & $\begin{array}{ll}8.76 & 525 \\
8.76 & 742 \\
8.76 & 958 \\
8.77 & 173 \\
8.77 & 387\end{array}$ & $\begin{array}{l}217 \\
216 \\
215 \\
214\end{array}$ & 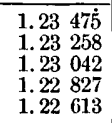 & 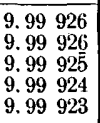 & $\begin{array}{l}\mathbf{4 0} \\
39 \\
38 \\
37 \\
36\end{array}$ & 46 & $\begin{array}{l}40 \\
36 \\
32 \\
28 \\
24\end{array}$ \\
\hline 13 & $\begin{array}{l}40 \\
44 \\
48 \\
52 \\
56\end{array}$ & $\begin{array}{l}25 \\
26 \\
27 \\
28 \\
29\end{array}$ & $\begin{array}{ll}8.77 & 522 \\
8.77 & 733 \\
8.77 & 943 \\
8.78 & 152 \\
8.78 & 360\end{array}$ & $\begin{array}{l}212 \\
211 \\
210 \\
209 \\
208\end{array}$ & $\begin{array}{ll}8.77 & 600 \\
8.77 & 811 \\
8.78 & 022 \\
8.78 & 232 \\
8.78 & 441\end{array}$ & $\begin{array}{l}215 \\
211 \\
211 \\
210 \\
209\end{array}$ & $\begin{array}{ll}1.22 & 400 \\
1.22 & 189 \\
1.21 & 978 \\
1.21 & 768 \\
1.21 & 559\end{array}$ & 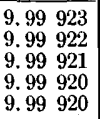 & $\begin{array}{l}35 \\
34 \\
33 \\
32 \\
31\end{array}$ & 46 & $\begin{array}{r}20 \\
16 \\
12 \\
8 \\
4\end{array}$ \\
\hline 14 & $\begin{array}{r}0 \\
4 \\
8 \\
12 \\
16\end{array}$ & $\begin{array}{l}30 \\
31 \\
32 \\
33 \\
34\end{array}$ & $\begin{array}{ll}8.78 & 568 \\
8.78 & 774 \\
8.78 & 979 \\
8.79 & 183 \\
8.79 & 386\end{array}$ & $\begin{array}{l}206 \\
205 \\
204 \\
203\end{array}$ & $\begin{array}{ll}8.78 & 649 \\
8.78 & 855 \\
8.79 & 061 \\
8.79 & 266 \\
8.79 & 470\end{array}$ & $\begin{array}{l}208 \\
206 \\
206 \\
205 \\
204\end{array}$ & $\begin{array}{ll}1.21 & 351 \\
1.21 & 145 \\
1.20 & 939 \\
1.20 & 734 \\
1.20 & 530\end{array}$ & 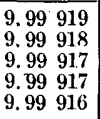 & $\begin{array}{l}\mathbf{3 0} \\
29 \\
28 \\
27 \\
26\end{array}$ & 46 & $\begin{array}{r}0 \\
56 \\
52 \\
48 \\
44\end{array}$ \\
\hline 14 & $\begin{array}{l}20 \\
24 \\
28 \\
32 \\
36\end{array}$ & $\begin{array}{l}35 \\
36 \\
37 \\
38 \\
39\end{array}$ & 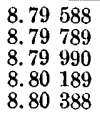 & $\begin{array}{l}2112 \\
201 \\
201 \\
199 \\
199\end{array}$ & $\begin{array}{ll}8.79 & 673 \\
8.79 & 875 \\
8.80 & 076 \\
8.80 & 277 \\
8.80 & 476\end{array}$ & $\begin{array}{l}205 \\
202 \\
201 \\
201 \\
199\end{array}$ & 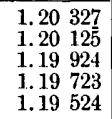 & 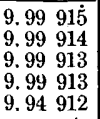 & $\begin{array}{l}25 \\
24 \\
23 \\
22 \\
21\end{array}$ & 45 & $\begin{array}{l}40 \\
36 \\
32 \\
28 \\
24\end{array}$ \\
\hline 14 & $\begin{array}{l}40 \\
44 \\
48 \\
52 \\
56\end{array}$ & $\begin{array}{l}40 \\
41 \\
42 \\
43 \\
44\end{array}$ & 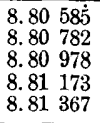 & $\begin{array}{l}197 \\
196 \\
195 \\
194\end{array}$ & $\begin{array}{ll}8.80 & 674 \\
8.80 & 872 \\
8.81 & 068 \\
8.81 & 264 \\
8.81 & 459\end{array}$ & $\begin{array}{l}198 \\
196 \\
196 \\
1.95\end{array}$ & $\begin{array}{ll}1.19 & 326 \\
1.19 & 128 \\
1.18 & 932 \\
1.18 & 736 \\
1.18 & 541\end{array}$ & 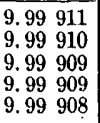 & $\begin{array}{l}20 \\
19 \\
18 \\
17 \\
16\end{array}$ & 45 & $\begin{array}{r}20 \\
16 \\
12 \\
8 \\
4\end{array}$ \\
\hline 15 & $\begin{array}{r}0 \\
4 \\
8 \\
12 \\
16\end{array}$ & $\begin{array}{l}45 \\
46 \\
47 \\
48 \\
49\end{array}$ & $\begin{array}{ll}8.81 & 560 \\
8.81 & 752 \\
8.81 & 944 \\
8.82 & 134 \\
8.82 & 324\end{array}$ & $\begin{array}{l}192 \\
192 \\
190 \\
190\end{array}$ & $\begin{array}{ll}8.81 & 653 \\
8.81 & 846 \\
8.82 & 038 \\
8.82 & 230 \\
8.82 & 420\end{array}$ & $\begin{array}{l}193 \\
1.92 \\
192 \\
190\end{array}$ & 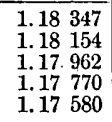 & 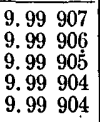 & $\begin{array}{l}15 \\
14 \\
13 \\
12 \\
11\end{array}$ & 45 & $\begin{array}{r}0 \\
56 \\
52 \\
48 \\
44\end{array}$ \\
\hline 15 & $\begin{array}{l}20 \\
24 \\
28 \\
32 \\
36\end{array}$ & $\begin{array}{l}50 \\
51 \\
52 \\
53 \\
54\end{array}$ & $\begin{array}{ll}8.82 & 513 \\
8.82 & 701 \\
8.82 & 888 \\
8.83 & 075 \\
8.83 & 261\end{array}$ & $\begin{array}{l}188 \\
187 \\
187 \\
186\end{array}$ & $\begin{array}{ll}8.82 & 610 \\
8.82 & 799 \\
8.82 & 987 \\
8.83 & 175 \\
8.83 & 361\end{array}$ & $\begin{array}{l}189 \\
188 \\
188 \\
186\end{array}$ & $\begin{array}{ll}1.17 & 390 \\
1.17 & 201 \\
1.17 & 013 \\
1.16 & 825 \\
1.16 & 639\end{array}$ & 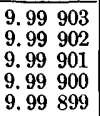 & $\begin{array}{r}\mathbf{1 0} \\
9 \\
8 \\
7 \\
6\end{array}$ & 44 & $\begin{array}{l}40 \\
36 \\
32 \\
28 \\
24\end{array}$ \\
\hline 15 & $\begin{array}{l}40 \\
44 \\
48 \\
52 \\
56\end{array}$ & $\begin{array}{l}55 \\
56 \\
\mathbf{5 7} \\
\mathbf{5 8} \\
59\end{array}$ & $\begin{array}{ll}8.83 & 446 \\
8.83 & 630 \\
8.83 & 813 \\
8.83 & 996 \\
8.84 & 177\end{array}$ & $\begin{array}{l}184 \\
183 \\
183 \\
181\end{array}$ & $\begin{array}{ll}8.83 & 547 \\
8.83 & 732 \\
8.83 & 916 \\
8.84 & 100 \\
8.84 & 282\end{array}$ & $\begin{array}{l}185 \\
184 \\
184 \\
182\end{array}$ & 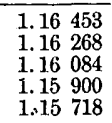 & 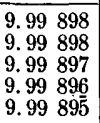 & $\begin{array}{l}5 \\
4 \\
3 \\
2\end{array}$ & 44 & $\begin{array}{r}20 \\
16 \\
12 \\
8 \\
4\end{array}$ \\
\hline 16 & 0 & 60 & 8.84358 & & 8.84464 & & 1.15536 & 9.99894 & 0 & 44 & 0 \\
\hline & & & L. Cos. & d. & L. Cotg. & c. $\mathrm{d}$. & L. Tang. & E. Sin. & ' & II. & \\
\hline
\end{tabular}


TiвLs 19.-Five-place logurilloms of circular functions, etc.-Continued.

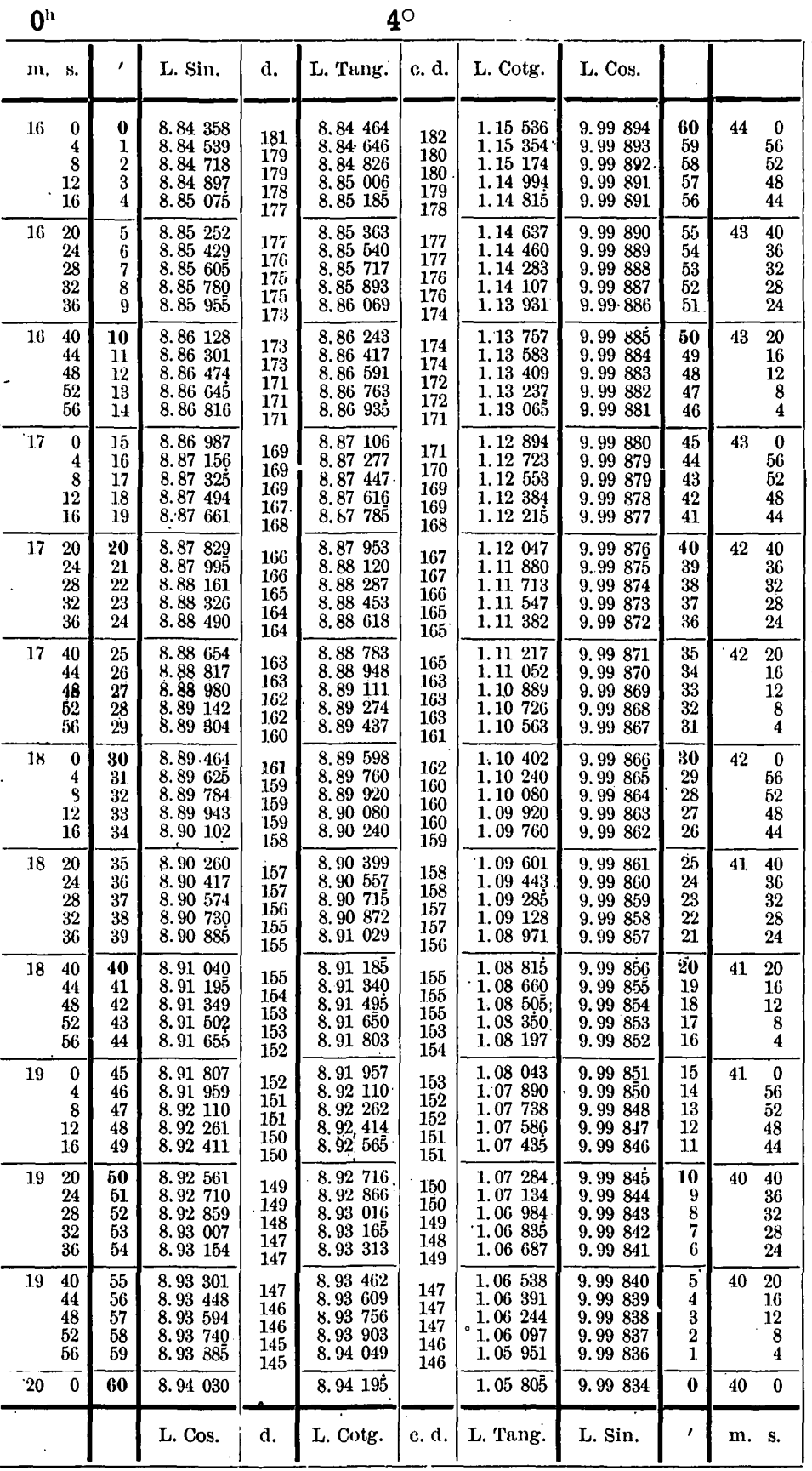


TABLE 19.-Five-place logarithms of circular functions, etc.-Continued.

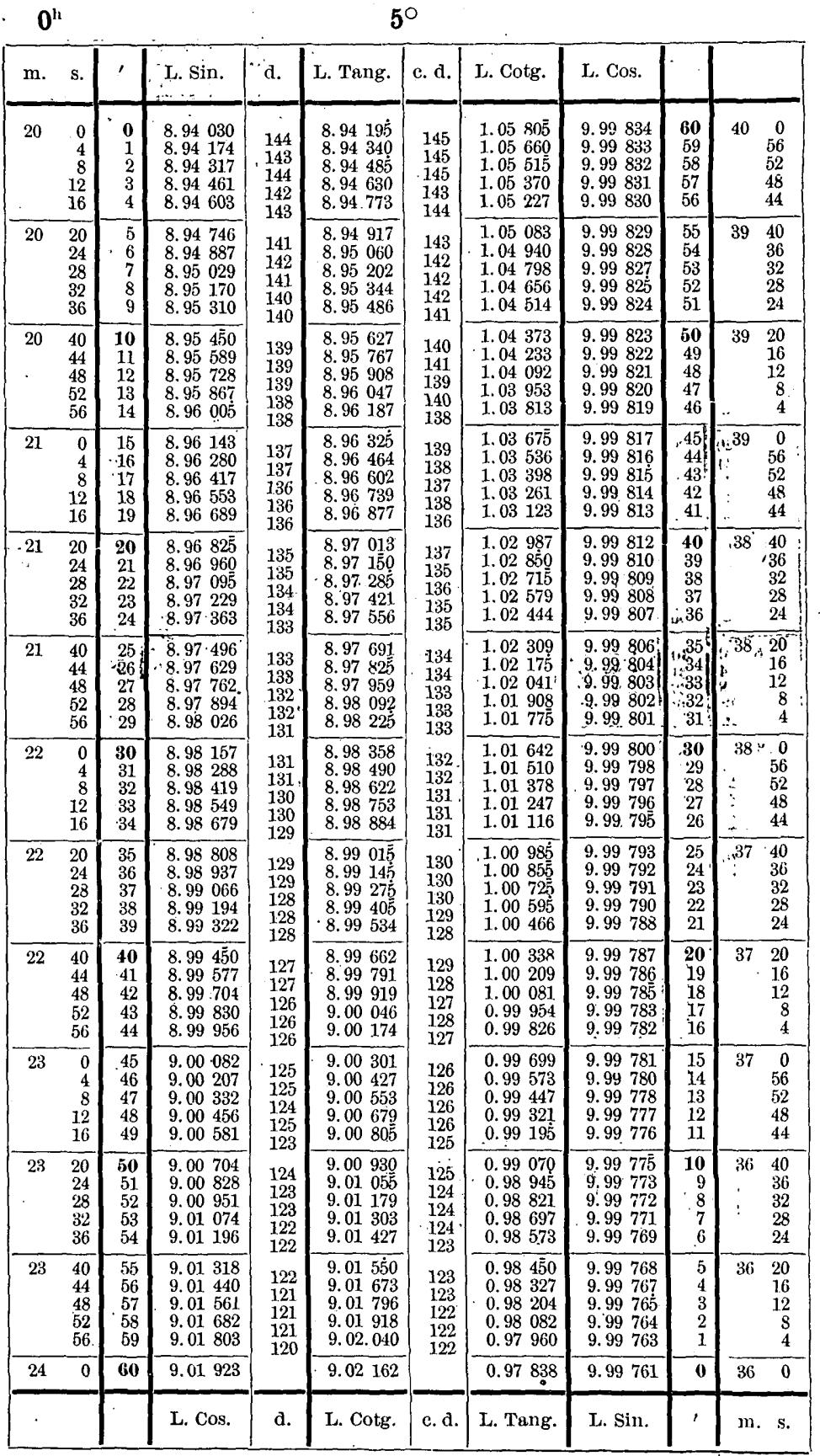


TABLE 19.-Five-place logarithms of circular functions, etc.-Continued.

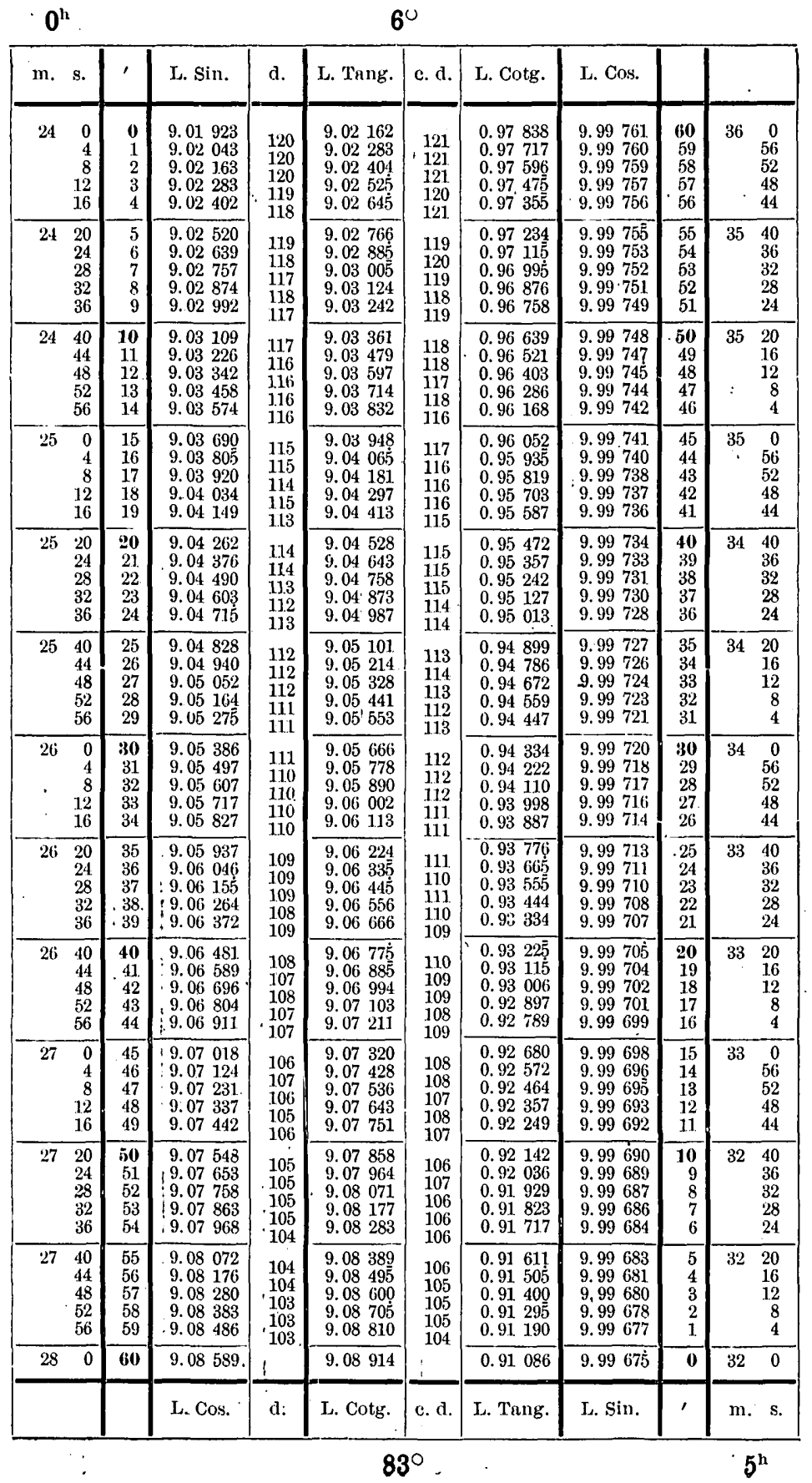


TABLE 19.-Five-place logarithmis of circular functions, etc.-Continued.

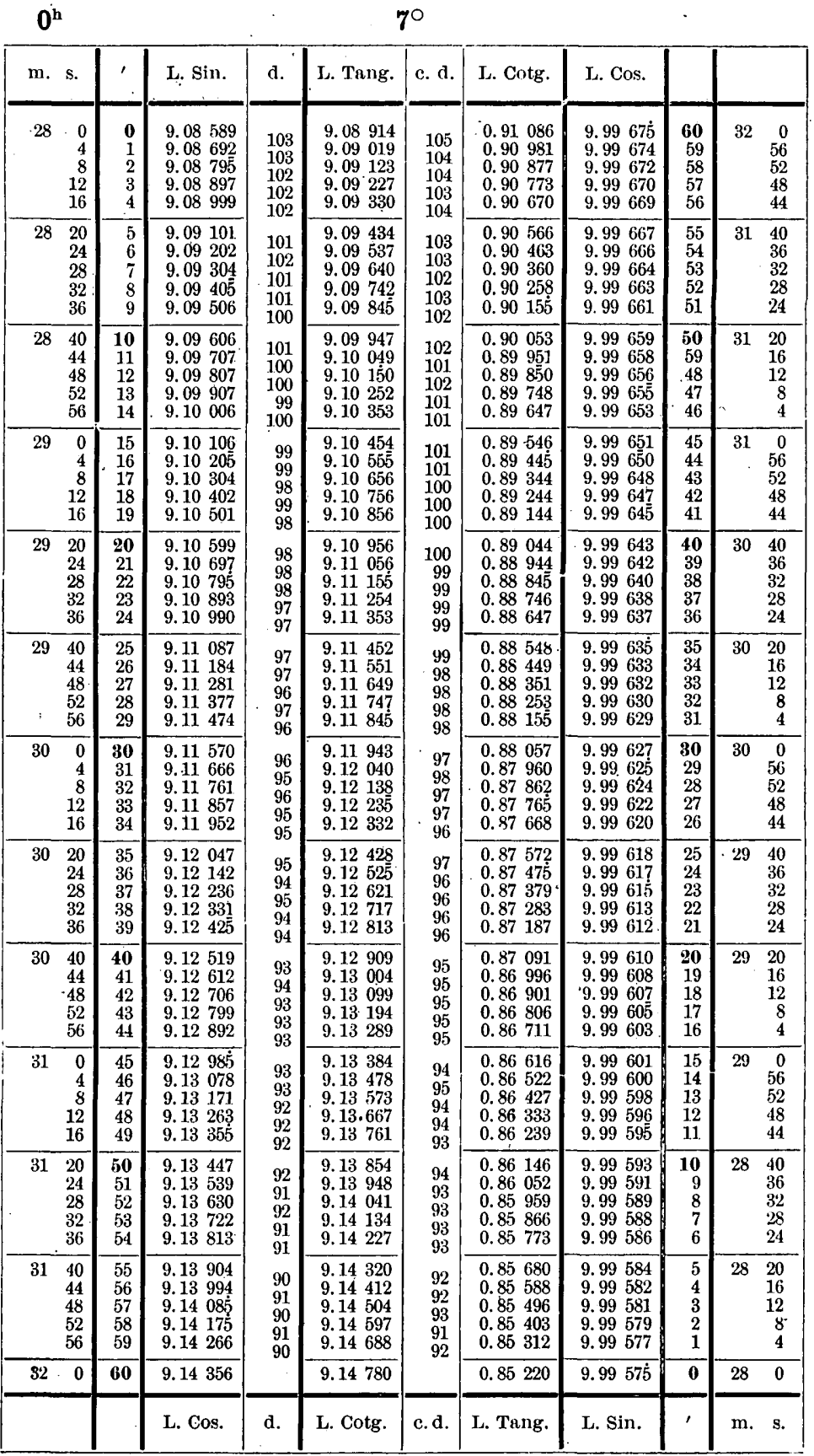


TABLe 19.-Five-place logarithms of circular functions, etc.-Continued.

$0^{\text {h }}$

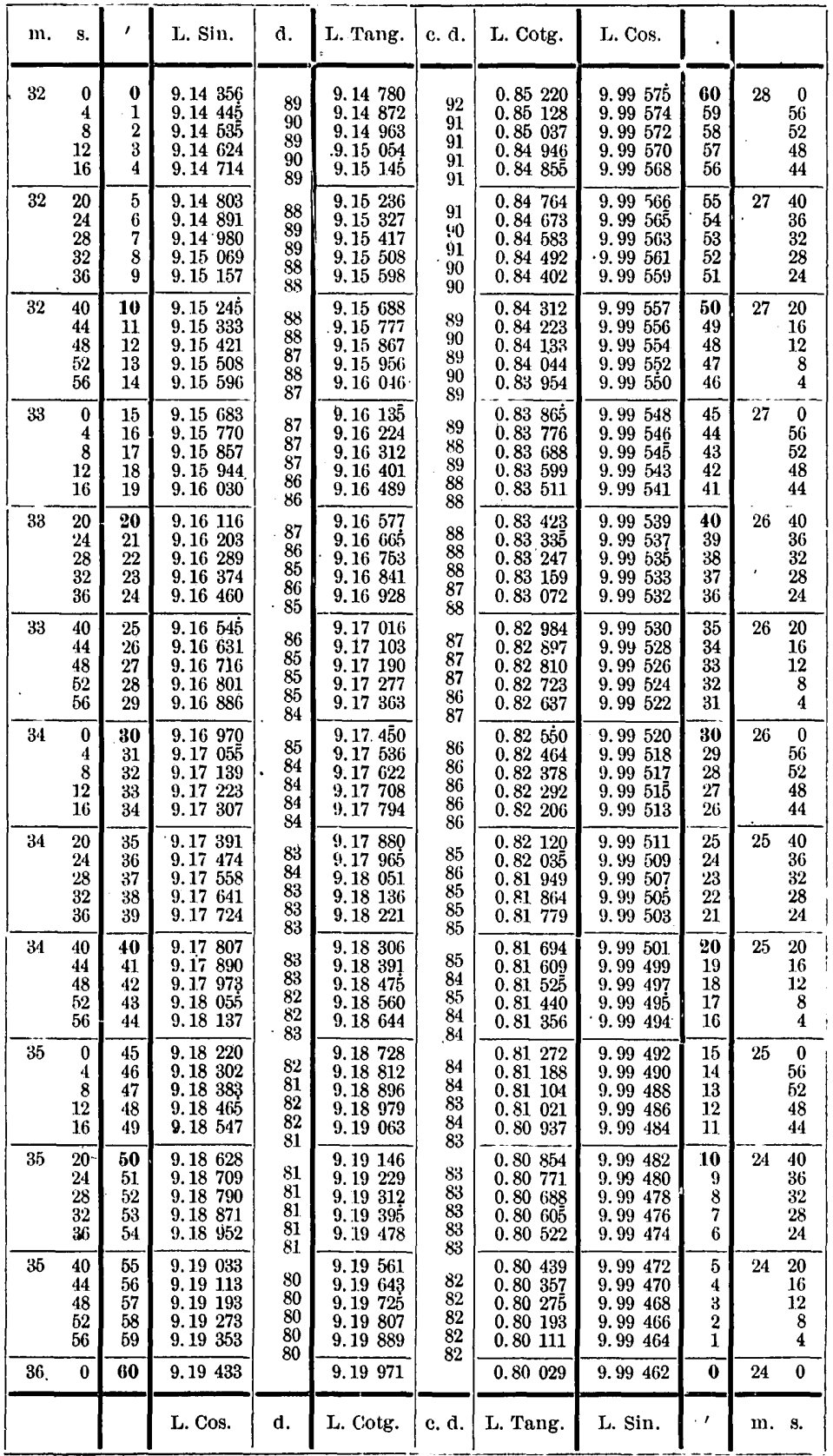


TABLE 19.-Five-place logarithms of circular functions, etc.-Continued.

\begin{tabular}{|c|c|c|c|c|c|c|c|c|c|c|c|}
\hline $\mathrm{m}$. & s. & ' & I. Sin. & d. & L. Tang. & c. d. & L. Cotg. & L. Cos. & 、 & & \\
\hline 36 & $\begin{array}{r}0 \\
4 \\
8 \\
12 \\
16\end{array}$ & $\begin{array}{l}0 \\
1 \\
2 \\
3 \\
4\end{array}$ & 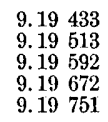 & $\begin{array}{l}80 \\
79 \\
80 \\
79 \\
79\end{array}$ & $\begin{array}{lll}9.19 & 971 \\
9.20 & 053 \\
9.20 & 134 \\
9.20 & 216 \\
9.20 & 297\end{array}$ & $\begin{array}{l}82 \\
81 \\
82 \\
81 \\
81\end{array}$ & 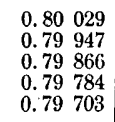 & 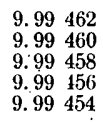 & $\begin{array}{r}\mathbf{6 0} \\
59 \\
58 \\
57 \\
56\end{array}$ & 24 & $\begin{array}{r}0 \\
56 \\
52 \\
48 \\
44\end{array}$ \\
\hline 36 & $\begin{array}{l}20 \\
24 \\
28 \\
32 \\
36\end{array}$ & $\begin{array}{l}5 \\
6 \\
7 \\
8 \\
9\end{array}$ & $\begin{array}{l}9.19830 \\
9.19909 \\
9.19988 \\
9.20067 \\
9.20145\end{array}$ & $\begin{array}{l}79 \\
79 \\
79 \\
78 \\
78\end{array}$ & $\begin{array}{ll}9.20 & 378 \\
9.20 & 459 \\
9.20 & 540 \\
9.20 & 621 \\
9.20 & 701 \\
\end{array}$ & $\begin{array}{l}81 \\
81 \\
81 \\
80 \\
81\end{array}$ & 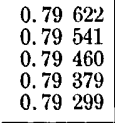 & $\begin{array}{l}9.99452 \\
9.99450 \\
9.99448 \\
9.99 \\
9.946 \\
9.944\end{array}$ & $\begin{array}{l}55 \\
54 \\
53 \\
52 \\
51\end{array}$ & 23 & $\begin{array}{l}40 \\
36 \\
32 \\
28 \\
24\end{array}$ \\
\hline 36 & $\begin{array}{l}40 \\
44 \\
48 \\
52 \\
56\end{array}$ & $\begin{array}{l}10 \\
11 \\
12 \\
13 \\
14\end{array}$ & $\begin{array}{lll}9.20 & 223 \\
9.20 & 302 \\
9.20 & 380 \\
9.20 & 458 \\
9.20 & 535\end{array}$ & $\begin{array}{l}79 \\
78 \\
78 \\
77 \\
78\end{array}$ & 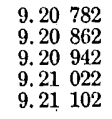 & $\begin{array}{l}80 \\
80 \\
80 \\
80 \\
80\end{array}$ & $\begin{array}{ll}0.79 & 218 \\
0.79 & 138 \\
0.79 & 058 \\
0.78 & 978 \\
0.78 & 898\end{array}$ & 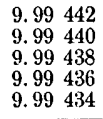 & $\begin{array}{l}50 \\
49 \\
48 \\
47 \\
46\end{array}$ & 23 & $\begin{array}{r}20 \\
16 \\
12 \\
8 \\
4\end{array}$ \\
\hline 37 & $\begin{array}{r}0 \\
4 \\
8 \\
12 \\
16\end{array}$ & $\begin{array}{l}15 \\
16 \\
17 \\
18 \\
19\end{array}$ & 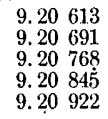 & $\begin{array}{l}78 \\
77 \\
77 \\
77 \\
77\end{array}$ & $\begin{array}{ll}9.21 & 182 \\
9.21 & 261 \\
9.21 & 341 \\
9.21 & 420 \\
9.21 & 499\end{array}$ & $\begin{array}{l}79 \\
80 \\
79 \\
79 \\
79\end{array}$ & $\begin{array}{ll}0.78 & 818 \\
0.78 & 739 \\
0.78 & 659 \\
0.78 & 580 \\
0.78 & 501 .\end{array}$ & $\begin{array}{r}9.99432 \\
9.99429 \\
9.99427 \\
9.99425 \\
-9.99423\end{array}$ & $\begin{array}{l}45 \\
44 \\
43 \\
42 \\
41\end{array}$ & 23 & $\begin{array}{r}0 \\
56 \\
52 \\
48 \\
44\end{array}$ \\
\hline 37 & $\begin{array}{l}20 \\
24 \\
28 \\
32 \\
36\end{array}$ & $\begin{array}{l}\mathbf{2 0} \\
21 \\
22 \\
23 \\
24\end{array}$ & 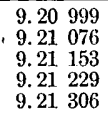 & $\begin{array}{r}77 \\
\cdot 77 \\
76 \\
77 \\
76\end{array}$ & $\begin{array}{ll}9.21 & 578 \\
9.21 & 657 \\
9.21 & 736 \\
9.21 & 814 \\
9.21 & 893\end{array}$ & $\begin{array}{l}79 \\
79 \\
78 \\
79 \\
78\end{array}$ & $\begin{array}{ll}0.78 & 422 \\
0.78 & 343 \\
0.78 & 264 \\
0.78 & 186 \\
0.78 & 107\end{array}$ & 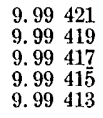 & $\begin{array}{l}\mathbf{4 0} \\
39 \\
38 \\
37 \\
36\end{array}$ & 22 & $\begin{array}{l}40 \\
36 \\
32 \\
28 \\
24\end{array}$ \\
\hline 37 & $\begin{array}{l}40 \\
44 \\
48 \\
52 \\
56\end{array}$ & $\begin{array}{l}25 \\
26 \\
27 \\
28 \\
29\end{array}$ & $\begin{array}{ll}9.21 & 382 \\
9.21 & 458 \\
9.21 & 534 \\
9.21 & 610 \\
9.21 & 685\end{array}$ & $\begin{array}{l}76 \\
76 \\
76 \\
75 \\
76\end{array}$ & $\begin{array}{ll}9.21 & 971 \\
9.22 & 049 \\
9.22 & 127 \\
9.22 & 205 \\
9.22 & 283 \\
\end{array}$ & $\begin{array}{l}78 \\
78 \\
78 \\
78 \\
78\end{array}$ & $\begin{array}{ll}0.78 & 029 \\
0.77 & 951 \\
0.77 & 873 \\
0.77 & 795 \\
0.77 & 717 \\
\end{array}$ & 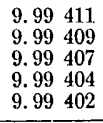 & $\begin{array}{l}35 \\
34 \\
33 \\
32 \\
31 \\
\end{array}$ & 22 & $\begin{array}{r}20 \\
16 \\
12 \\
8 \\
4\end{array}$ \\
\hline 38 & $\begin{array}{r}0 \\
4 \\
8 \\
12 \\
16\end{array}$ & $\begin{array}{r}\mathbf{3 0} \\
31 \\
32 \\
33 \\
34\end{array}$ & $\begin{array}{ll}9.21 & 761 \\
9.21 & 836 \\
9.21 & 912 \\
9.21 & 987 \\
9.22 & 062 \\
\end{array}$ & $\begin{array}{l}75 \\
76 \\
75 \\
75 \\
75\end{array}$ & $\begin{array}{ll}9.22 & 361 \\
9.22 & 438 \\
9.22 & 516 \\
9.22 & 593 \\
9.22 & 670 \\
\end{array}$ & $\begin{array}{l}77 \\
78 \\
77 \\
77 \\
77\end{array}$ & $\begin{array}{ll}0.77 & 639 \\
0.77 & 562 \\
0.77 & 484 \\
0.77 & 407 \\
0.77 & 330\end{array}$ & 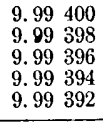 & $\begin{array}{l}30 \\
29 \\
28 \\
27 \\
26\end{array}$ & 22 & $\begin{array}{r}0 \\
56 \\
52 \\
48 \\
44 \\
\end{array}$ \\
\hline 38 & $\begin{array}{l}20 \\
24 \\
28 \\
32 \\
36\end{array}$ & $\begin{array}{l}35 \\
36 \\
37 \\
38 \\
39\end{array}$ & $\begin{array}{ll}9.22 & 137 \\
9.22 & 211 \\
9.22 & 286 \\
9.22 & 361 \\
9.22 & 435\end{array}$ & $\begin{array}{l}74 \\
75 \\
75 \\
74 \\
74\end{array}$ & $\begin{array}{ll}9.22 & 747 \\
9.22 & 824 \\
9.22 & 901 \\
9.22 & 977 \\
9.23 & 054 \\
\end{array}$ & $\begin{array}{l}77 \\
77 \\
76 \\
77 \\
76\end{array}$ & $\begin{array}{ll}0.77 & 253 \\
0.77 & 176 \\
0.77 & 099 \\
0.77 & 023 \\
0.76 & 946\end{array}$ & 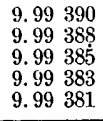 & $\begin{array}{l}25 \\
24 \\
23 \\
22 \\
21\end{array}$ & 21 & $\begin{array}{l}40 \\
36 \\
32 \\
28 \\
24\end{array}$ \\
\hline 38 & $\begin{array}{l}40 \\
44 \\
48 \\
52 \\
56\end{array}$ & $\begin{array}{l}40 \\
41 \\
42 \\
43 \\
44\end{array}$ & $\begin{array}{ll}9.22 & 509 \\
9.22 & 583 \\
9.22 & 657 \\
9.22 & 731 \\
9.22 & 805 .\end{array}$ & $\begin{array}{l}74 \\
74 \\
74 \\
74 \\
73\end{array}$ & $\begin{array}{ll}9.23 & 130 \\
9.23 & 206 \\
9.23 & 283 \\
9.23 & 359 \\
9.23 & 435\end{array}$ & $\begin{array}{l}76 \\
77 \\
76 \\
76 \\
75\end{array}$ & $\begin{array}{ll}0.76 & 870 \\
0.76 & 794 \\
0.76 & 717 \\
0.76 & 641 \\
0.76 & 565\end{array}$ & 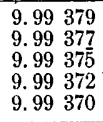 & $\begin{array}{l}\mathbf{2 0} \\
19 \\
18 \\
17 \\
16\end{array}$ & 21. & $\begin{array}{r}20 \\
16 \\
12 \\
8 \\
4\end{array}$ \\
\hline 39 & $\begin{array}{r}0 \\
4 \\
8 \\
12 \\
16\end{array}$ & $\begin{array}{l}45 \\
46 \\
47 \\
48 \\
49\end{array}$ & $\begin{array}{ll}9.22 & 878 \\
9.22 & 952 \\
9.23 & 025 \\
9.23 & 008 \\
9.23 & 171 \\
\end{array}$ & $\begin{array}{l}74 \\
73 \\
73 \\
73 \\
73\end{array}$ & $\begin{array}{ll}9.23 & 510 \\
9.23 & 586 \\
9.23 & 661 \\
9.23 & 737 \\
9.23 & 812 \\
\end{array}$ & $\begin{array}{l}76 \\
75 \\
76 \\
75 \\
75\end{array}$ & $\begin{array}{ll}0.76 & 490 \\
0.76 & 414 \\
0.76 & 339 \\
0.76 & 263 \\
0.76 & 188 \\
\end{array}$ & 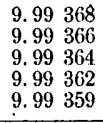 & $\begin{array}{l}15 \\
14 \\
13 \\
12 \\
11 \\
\end{array}$ & 21. & $\begin{array}{r}0 \\
56 \\
52 \\
48 \\
44 \\
\end{array}$ \\
\hline 39 & $\begin{array}{l}20 \\
24 \\
28 \\
32 \\
36\end{array}$ & $\begin{array}{c}50 \\
51 \\
52 \\
53 \\
54\end{array}$ & $\begin{array}{ll}9.23 & 244 \\
9.23 & 31.7 \\
9.23 & 390 \\
9.23 & 462 \\
9.23 & 535\end{array}$ & $\begin{array}{l}73 \\
73 \\
72 \\
73 \\
72\end{array}$ & $\begin{array}{ll}9.23 & 887 \\
9.23 & 962 \\
9.24 & 037 \\
9.24 & 112 \\
9.24 & 186\end{array}$ & $\begin{array}{l}75 \\
75 \\
75 \\
74\end{array}$ & $\begin{array}{ll}0.76 & 113 \\
0.76 & 038 \\
0.75 & 963 \\
0.75 & 888 \\
0.75 & 814\end{array}$ & 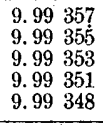 & $\begin{array}{r}10 \\
9 \\
8 \\
7 \\
6\end{array}$ & -20 & $\begin{array}{l}40 \\
36 \\
32 \\
28 \\
24\end{array}$ \\
\hline 39 & $\begin{array}{l}40 \\
44 \\
48 \\
52 \\
56\end{array}$ & $\begin{array}{l}55 \\
56 \\
57 \\
58 \\
59\end{array}$ & 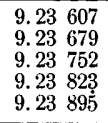 & $\begin{array}{l}72 \\
73 \\
71 \\
72 \\
72\end{array}$ & $\begin{array}{ll}9.24 & 261 \\
9.24 & 335 \\
9.24 & 410 \\
9.24 & 484 \\
9.24 & 558\end{array}$ & $\begin{array}{l}74 \\
75 \\
74 \\
74 \\
74\end{array}$ & $\begin{array}{ll}0.75 & 739 \\
0.75 & 665 \\
0.75 & 590 \\
0.75 & 516 \\
0.75 & 442\end{array}$ & 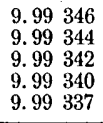 & $\begin{array}{l}5 \\
4 \\
3 \\
2 \\
2 \\
1\end{array}$ & 20 & $\begin{array}{r}20 \\
16 \\
12 \\
8 \\
4\end{array}$ \\
\hline 40 & 0 & 60 & 9.23967 & & 9.24632 & & 0.75368 & 9.99335 & 0 & 20 & 0 \\
\hline & & & Cos. & c & Cotg. & d. & L. Ta & Sin. & ' & 1. & 3. \\
\hline
\end{tabular}


TABLE 19.-Five-place logarithms of circular functions, etc.-Continued.

$0^{\text {h }}$

\begin{tabular}{|c|c|c|c|c|c|c|c|c|c|c|c|c|}
\hline m. & s. & ' & J. $\operatorname{Sin}$. & d. & I. Tang. & c. d. & L. Cotg. & l. Cos. & d. & & & \\
\hline 40 & \begin{tabular}{r|}
0 \\
4 \\
8 \\
12 \\
16
\end{tabular} & $\begin{array}{l}0 \\
1 \\
2 \\
3 \\
4\end{array}$ & 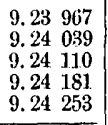 & $\begin{array}{l}72 \\
71 \\
71 \\
72\end{array}$ & $\begin{array}{r}9.24632 \\
.9 .24706 \\
9.24779 \\
9.24853 \\
9.24926\end{array}$ & $\begin{array}{l}74 \\
73 \\
74 \\
73\end{array}$ & 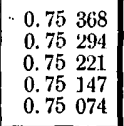 & $\begin{array}{l}9.99335 \\
9.99333 \\
9.99331 \\
9.99328 \\
9.99326\end{array}$ & \begin{tabular}{l|l}
2 \\
2 \\
3 \\
2 \\
2
\end{tabular} & $\begin{array}{r}\mathbf{6 0} \\
59 \\
58 \\
57 \\
56 \\
\end{array}$ & 20 & $\begin{array}{r}0 \\
56 \\
52 \\
48 \\
44\end{array}$ \\
\hline 40 & $\begin{array}{l}20 \\
24 \\
28 \\
32 \\
36\end{array}$ & $\begin{array}{l}5 \\
6 \\
7 \\
8 \\
9\end{array}$ & 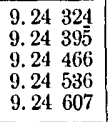 & $\begin{array}{l}71 \\
71 \\
71 \\
70 \\
71\end{array}$ & $\begin{array}{ll}9.25 & 000 \\
9.25 & 073 \\
7.25 & 146 \\
9.25 & 219 \\
9.25 & 292\end{array}$ & $\begin{array}{l}74 \\
73 \\
73 \\
73 \\
73 \\
79\end{array}$ & $\begin{array}{ll}0.75 & 000 \\
0.74 & 927 \\
0.74 & 854 \\
0.74 & 781 \\
0.74 & 708\end{array}$ & 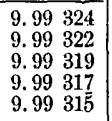 & $\begin{array}{l}2 \\
3 \\
2 \\
2\end{array}$ & $\begin{array}{l}55 \\
54 \\
53 \\
52 \\
51\end{array}$ & 19 & $\begin{array}{l}40 \\
36 \\
32 \\
28 \\
24\end{array}$ \\
\hline 40 & $\begin{array}{l}40 \\
44 \\
48 \\
52 \\
56\end{array}$ & $\begin{array}{l}\mathbf{1 0} \\
11 \\
12 \\
13 \\
14\end{array}$ & $\begin{array}{ll}9.24 & 677 \\
9.24 & 748 \\
9.24 & 818 \\
9.24 & 888 \\
9.24 & 958\end{array}$ & $\begin{array}{l}70 \\
71 \\
70 \\
70 \\
70\end{array}$ & $\begin{array}{ll}9.25 & 36 \overline{5} \\
9.25 & 437 \\
9.25 & 510 \\
9.25 & 582 \\
9.25 & 655\end{array}$ & $\begin{array}{l}73 \\
72 \\
73 \\
72 \\
73\end{array}$ & $\begin{array}{ll}0.74 & 635 \\
0.74 & 563 \\
0.74 & 490 \\
0.74 & 418 \\
0.74 & 345\end{array}$ & 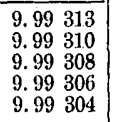 & $\begin{array}{l}3 \\
2 \\
2 \\
2\end{array}$ & $\begin{array}{l}\mathbf{5 0} \\
49 \\
48 \\
\mathbf{4 7} \\
\mathbf{4 6}\end{array}$ & 19 & $\begin{array}{r}20 \\
16 \\
12 \\
8 \\
4\end{array}$ \\
\hline 41 & \begin{tabular}{r|}
0 \\
4 \\
5 \\
12 \\
16
\end{tabular} & $\begin{array}{l}15 \\
16 \\
17 \\
18 \\
19\end{array}$ & $\begin{array}{ll}9.25 & 028 \\
9.25 & 098 \\
9.25 & 168 \\
9.25 & 237 \\
9.25 & 307\end{array}$ & $\begin{array}{l}70 \\
70 \\
69 \\
70\end{array}$ & $\begin{array}{ll}9.25 & 727 \\
9.25 & 799 \\
9.25 & 871 \\
9.25 & 943 \\
9.26 & 015\end{array}$ & $\begin{array}{l}72 \\
72 \\
72 \\
72\end{array}$ & $\begin{array}{ll}0.74 & 273 \\
0.74 & 201 \\
0.74 & 129 \\
0.74 & 057 \\
0.73 & 985\end{array}$ & 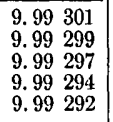 & $\begin{array}{l}2 \\
2 \\
3 \\
2\end{array}$ & $\begin{array}{l}45 \\
44 \\
43 \\
42 \\
41\end{array}$ & 19 & $\begin{array}{r}0 \\
56 \\
52 \\
48 \\
44\end{array}$ \\
\hline 41 & $\begin{array}{l}20 \\
24 \\
28 \\
32 \\
36\end{array}$ & $\begin{array}{l}\mathbf{2 0} \\
21 \\
22 \\
23 \\
24\end{array}$ & 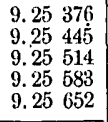 & $\begin{array}{l}69 \\
69 \\
69 \\
69\end{array}$ & $\begin{array}{ll}9.26 & 086 \\
9.26 & 158 \\
9.26 & 229 \\
9.26 & 301 \\
9.26 & 372\end{array}$ & $\begin{array}{l}72 \\
71 \\
72 \\
71\end{array}$ & $\begin{array}{ll}0.73 & 914 \\
0.73 & 842 \\
0.73 & 771 \\
0.73 & 699 \\
0.73 & 628\end{array}$ & 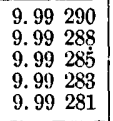 & \begin{tabular}{l|}
2 \\
3 \\
2 \\
2
\end{tabular} & $\begin{array}{l}\mathbf{4 0} \\
39 \\
38 \\
37 \\
36\end{array}$ & 18 & $\begin{array}{l}40 \\
36 \\
32 \\
28 \\
24\end{array}$ \\
\hline 41 & $\begin{array}{l}40 \\
44 \\
48 \\
52 \\
56\end{array}$ & $\begin{array}{l}25 \\
26 \\
27 \\
28 \\
29\end{array}$ & $\begin{array}{ll}9.25 & 721 \\
9.25 & 790 \\
9.25 & 858 \\
9.25 & 927 \\
9.25 & 995 \\
\end{array}$ & $\begin{array}{l}69 \\
68 \\
69 \\
68\end{array}$ & $\begin{array}{ll}9.26 & 443 \\
9.26 & 514 \\
9.26 & 585 \\
9.26 & 655 \\
9.26 & 726\end{array}$ & $\begin{array}{l}71 \\
71 \\
70 \\
71\end{array}$ & $\begin{array}{ll}0.73 & 557 \\
0.73 & 486 \\
0.73 & 415 \\
0.73 & 345 \\
0.73 & 274\end{array}$ & 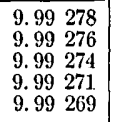 & $\begin{array}{l}2 \\
2 \\
3 \\
2\end{array}$ & $\begin{array}{l}35 \\
34 \\
33 \\
32 \\
31\end{array}$ & 18 & $\begin{array}{r}20 \\
16 \\
12 \\
8 \\
4\end{array}$ \\
\hline $\begin{array}{r}42 \\
\text {. }\end{array}$ & $\begin{array}{r}0 \\
4 \\
8 \\
12 \\
16\end{array}$ & $\begin{array}{l}30 \\
31 \\
32 \\
33 \\
34\end{array}$ & $\begin{array}{ll}9.26 & 063 \\
9.26 & 131 \\
9.26 & 199 \\
9.26 & 267 \\
9.26 & 335 \\
\end{array}$ & $\begin{array}{l}00 \\
68 \\
68 \\
68 \\
68\end{array}$ & $\begin{array}{ll}9.26 & 797 \\
9.26 & 867 \\
9.26 & 937 \\
9.27 & 008 \\
9.27 & 078\end{array}$ & $\begin{array}{l}71 \\
70 \\
70 \\
71 \\
70\end{array}$ & $\begin{array}{ll}0.73 & 203 \\
0.73 & 133 \\
0.73 & 063 \\
0.72 & 992 \\
0.72 & 922\end{array}$ & 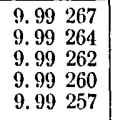 & \begin{tabular}{l|}
2 \\
3 \\
2 \\
2 \\
3
\end{tabular} & \begin{tabular}{r|}
30 \\
29 \\
28 \\
27 \\
26
\end{tabular} & 18 & $\begin{array}{r}0 \\
56 \\
52 \\
48 \\
44\end{array}$ \\
\hline 42 & $\begin{array}{l}20 \\
24 \\
28 \\
32 \\
36\end{array}$ & $\begin{array}{l}35 \\
36 \\
37 \\
38 \\
39\end{array}$ & $\begin{array}{ll}9.26 & 403 \\
9.26 & 470 \\
9.26 & 538 \\
9.26 & 605 \\
9.26 & 672\end{array}$ & $\begin{array}{l}67 \\
68 \\
67 \\
67\end{array}$ & $\begin{array}{ll}9.27 & 148 \\
9.27 & 218 \\
9.27 & 288 \\
9.27 & 357 \\
9.27 & \mathbf{4 2 7}\end{array}$ & $\begin{array}{l}70 \\
70 \\
70 \\
69 \\
70\end{array}$ & $\begin{array}{ll}0.72 & 852 \\
0.72 & 782 \\
0.72 & 712 \\
0.72 & 643 \\
0.72 & 573\end{array}$ & 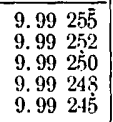 & \begin{tabular}{l|}
2 \\
3 \\
2 \\
2 \\
3
\end{tabular} & $\begin{array}{l}25 \\
24 \\
23 \\
22 \\
21\end{array}$ & 17 & $\begin{array}{l}40 \\
36 \\
32 \\
28 \\
24\end{array}$ \\
\hline 42 & $\begin{array}{l}40 \\
44 \\
48 \\
52 \\
56 \\
\end{array}$ & $\begin{array}{l}40 \\
41 \\
42 \\
43 \\
44 \\
\end{array}$ & $\begin{array}{ll}9.26 & 739 \\
9.26 & 806 \\
9.26 & 873 \\
9.26 & 940 \\
9.27 & 007 \\
\end{array}$ & $\begin{array}{l}67 \\
67 \\
67 \\
67\end{array}$ & $\begin{array}{ll}9.27 & 496 \\
9.27 & 566 \\
9.27 & 635 \\
9.27 & 704 \\
9.27 & 773 \\
\end{array}$ & $\begin{array}{r}70 \\
69 \\
69 \\
69\end{array}$ & $\begin{array}{ll}0.72 & 504 \\
0.72 & 434 \\
0.72 & 365 \\
0.72 & 296 \\
0.72 & 227\end{array}$ & $\begin{array}{ll}9.99 & 243 \\
9.99 & 241 \\
9.99 & 238 \\
9.99 & 236 \\
9.99 & 233\end{array}$ & $\begin{array}{l}2 \\
3 \\
2 \\
3 \\
3\end{array}$ & $\begin{array}{l}\mathbf{6 0} \\
19 \\
18 \\
17 \\
1.6\end{array}$ & 17 & $\begin{array}{r}20 \\
16 \\
12 \\
8 \\
4\end{array}$ \\
\hline 43 & $\begin{array}{r}0 \\
4 \\
8 \\
12 \\
16\end{array}$ & $\begin{array}{l}45 \\
46 \\
47 \\
48 \\
49\end{array}$ & $\begin{array}{ll}9.27 & 073 \\
9.27 & 140 \\
9.27 & 206 \\
9.27 & 273 \\
9.27 & 339 \\
\end{array}$ & $\begin{array}{l}67 \\
66 \\
67 \\
66\end{array}$ & $\begin{array}{ll}9.27 & 842 \\
9.27 & 911 \\
9.27 & 980 \\
9.28 & 049 \\
9.28 & 117 \\
\end{array}$ & $\begin{array}{l}69 \\
69 \\
69 \\
68\end{array}$ & $\begin{array}{lll}0.72 & 158 \\
0.72 & 089 \\
0.72 & 020 \\
0.71 & 951 \\
0.71 & 883\end{array}$ & $\begin{array}{ll}9.99 & 231 \\
9.99 & 229 \\
9.99 & 226 \\
9.99 & 224 \\
9.99 & 221\end{array}$ & $\begin{array}{l}2 \\
2 \\
3 \\
2 \\
3\end{array}$ & $\begin{array}{l}15 \\
14 \\
13 \\
12 \\
11\end{array}$ & 17 & $\begin{array}{r}0 \\
56 \\
52 \\
48 \\
44\end{array}$ \\
\hline 43 & $\begin{array}{l}20 \\
24 \\
28 \\
32 \\
36\end{array}$ & $\begin{array}{l}50 \\
51 . \\
52 \\
53 \\
54\end{array}$ & 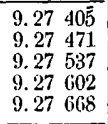 & $\begin{array}{l}66 \\
66 \\
65 \\
66\end{array}$ & $\begin{array}{lll}9.28 & 186 \\
9.28 & 254 \\
9.28 & 323 \\
9.28 & 391 \\
9.28 & 459\end{array}$ & $\begin{array}{l}68 \\
69 \\
68 \\
68\end{array}$ & $\begin{array}{ll}0.71 & 814 \\
0.71 & 746 \\
0.71 . & 677 \\
0.71 & 609 \\
0.71 . & 541\end{array}$ & $\begin{array}{lll}9.99 & 219 \\
9.99 & 217 \\
9.99 & 214 \\
9.99 & 212 \\
9.99 & 209\end{array}$ & $\begin{array}{l}2 \\
2 \\
3 \\
2 \\
3\end{array}$ & $\begin{array}{r}10 \\
9 \\
8 \\
7 \\
6\end{array}$ & 16 & $\begin{array}{l}40 \\
36 \\
32 \\
28 \\
24\end{array}$ \\
\hline 43 & $\begin{array}{l}40 \\
44 \\
48 \\
52 \\
56\end{array}$ & $\begin{array}{r}55 \\
56 \\
57 \\
58 \\
59\end{array}$ & 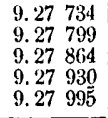 & $\begin{array}{l}65 \\
65 \\
66 \\
65\end{array}$ & $\begin{array}{ll}9.28 & 527 \\
9.28 & 595 \\
9.28 & 662 \\
9.28 & 730 \\
9,28 & 798\end{array}$ & $\begin{array}{l}68 \\
67 \\
68 \\
68\end{array}$ & $\begin{array}{ll}0.71 & 473 \\
0.71 & 405 \\
0.71 & 338 \\
0.71 & 270 \\
0.71 & 202\end{array}$ & $\begin{array}{lll}9.99 & 207 \\
9.99 & 204 \\
9.99 & 202 \\
9.99 & 200 \\
9.99 & 197\end{array}$ & $\begin{array}{l}3 \\
2 \\
2 \\
3\end{array}$ & $\begin{array}{l}5 \\
4 \\
3 \\
2 \\
1\end{array}$ & 16 & $\begin{array}{r}20 \\
16 \\
12 \\
8 \\
4\end{array}$ \\
\hline 44 & 0 & 60 & 9.28060 & 00 & $9.2886 \dot{5}$ & b & $0.71,135$ & $9.99 \quad 195$ & 2 & 0 & 16 & 0 \\
\hline & & & Cos. & d. & I. Cotg. & c. d. & I. Tang. & L. Sin. & d. & , & m. & \\
\hline
\end{tabular}

$79^{\circ}$

$10^{\circ}$ 
TABLE 19.-Five-place logarithms of circular functions, etc.-Continued.

$\mathbf{0}^{\mathrm{h}}$

\begin{tabular}{|c|c|c|c|c|c|c|c|c|c|c|c|c|}
\hline $\mathrm{m}$. & s. & ' & L. Sin. & d. & L. Tang. & c. d. & L. Cotg. & L. Cos. & d. & & \\
\hline 44 & $\begin{array}{r}0 \\
4 \\
8 \\
12 \\
16\end{array}$ & $\begin{array}{l}0 \\
1 \\
2 \\
3 \\
4\end{array}$ & $\begin{array}{ll}9.28 & 060 \\
9.28 & 125 \\
9.28 & 190 \\
9.28 & 254 \\
9.28 & 319\end{array}$ & \multirow{2}{*}{$\begin{array}{l}65 \\
65 \\
64 \\
65 \\
65 \\
64 \\
64 \\
65 \\
64 \\
64\end{array}$} & 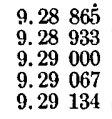 & \multirow{2}{*}{$\begin{array}{l}68 \\
67 \\
67 \\
67 \\
67 \\
67 \\
67 \\
67 \\
66 \\
67\end{array}$} & $\begin{array}{ll}0.71 & 135 \\
0.71 & 067 \\
0.71 & 000 \\
0.70 & 933 \\
0.70 & 866\end{array}$ & $\begin{array}{ll}9.99 & 19 \overline{5} \\
9.99 & 192 \\
9.99 & 190 \\
9.99 & 187 \\
9.99 & 185\end{array}$ & \multirow{2}{*}{$\begin{array}{l}3 \\
2 \\
3 \\
2 \\
3 \\
2 \\
3 \\
2 \\
3 \\
2\end{array}$} & $\begin{array}{l}\mathbf{6 0} \\
\mathbf{5 9} \\
58 \\
57 \\
56\end{array}$ & 16 & $\begin{array}{r}0 \\
56 \\
52 \\
48 \\
44\end{array}$ \\
\hline 44 & $\begin{array}{l}20 \\
24 \\
28 \\
32 \\
36\end{array}$ & $\begin{array}{l}5 \\
6 \\
7 \\
8 \\
9\end{array}$ & $\begin{array}{ll}9.28 & 384 \\
9.28 & 448 \\
9.28 & 512 \\
9.28 & 577 \\
9.28 & 641\end{array}$ & & $\begin{array}{ll}9.29 & 201 \\
9.29 & 268 \\
9.29 & 335 \\
9.29 & 402 \\
9.29 & 468\end{array}$ & & $\begin{array}{lll}0.70 & 799 \\
0.70 & 732 \\
0.70 & 665 \\
0.70 & 598 \\
0.70 & 532\end{array}$ & $\begin{array}{ll}9.99 & 182 \\
9.99 & 180 \\
9.99 & 177 \\
9.99 & 175 \\
9.99 & 172\end{array}$ & & $\begin{array}{l}55 \\
54 \\
53 \\
52 \\
51\end{array}$ & 15 & $\begin{array}{l}40 \\
36 \\
32 \\
28 \\
24\end{array}$ \\
\hline 44 & $\begin{array}{l}40 \\
44 \\
48 \\
52 \\
56\end{array}$ & $\begin{array}{l}10 \\
11 \\
12 \\
13 \\
14\end{array}$ & 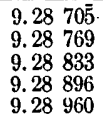 & $\begin{array}{l}04 \\
64 \\
64 \\
63 \\
64\end{array}$ & 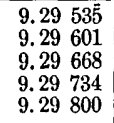 & $\begin{array}{l}67 \\
66 \\
67 \\
66 \\
66\end{array}$ & $\begin{array}{lll}0.70 & 465 \\
0.70 & 399 \\
0.70 & 332 \\
0.70 & 266 \\
0.70 & 200\end{array}$ & $\begin{array}{ll}9.99 & 170 \\
9.99 & 167 \\
9.99 & 165 \\
9.99 & 162 \\
9.99 & 160\end{array}$ & $\begin{array}{l}2 \\
3 \\
2 \\
3 \\
2\end{array}$ & $\begin{array}{r}50 \\
49 \\
48 \\
47 \\
46\end{array}$ & 15 & $\begin{array}{r}20 \\
16 \\
12 \\
8 \\
4\end{array}$ \\
\hline 45 & $\begin{array}{r}0 \\
4 \\
8 \\
12 \\
16\end{array}$ & $\begin{array}{l}15 \\
16 \\
17 \\
18 \\
19\end{array}$ & $\begin{array}{ll}9.29 & 024 \\
9.29 & 087 \\
9.29 & 150 \\
9.29 & 214 \\
9.29 & 277\end{array}$ & $\begin{array}{l}6 x \\
63 \\
63 \\
64 \\
63\end{array}$ & 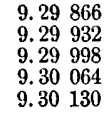 & $\begin{array}{l}66 \\
66 \\
66 \\
66\end{array}$ & $\begin{array}{ll}0.70 & 134 \\
0.70 & 068 \\
0.70 & 002 \\
0.69 & 936 \\
0.69 & 870\end{array}$ & $\begin{array}{ll}9.99 & 157 \\
9.99 & 155 \\
9.99 & 152 \\
9.99 & 150 \\
9.99 & 147\end{array}$ & $\begin{array}{l}2 \\
3 \\
2 \\
3\end{array}$ & $\begin{array}{l}45 \\
44 \\
43 \\
42 \\
41\end{array}$ & 15 & $\begin{array}{r}0 \\
56 \\
52 \\
48 \\
44\end{array}$ \\
\hline 45 & $\begin{array}{l}20 \\
24 \\
28 \\
32 \\
36\end{array}$ & $\begin{array}{l}20 \\
21 \\
22 \\
23 \\
24\end{array}$ & 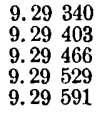 & $\begin{array}{l}63 \\
63 \\
63 \\
62\end{array}$ & $\begin{array}{lll}9.30 & 195 \\
9.30 & 261 \\
9.30 & 326 \\
9.30 & 391 \\
9.30 & 457\end{array}$ & $\begin{array}{l}66 \\
65 \\
65 \\
66\end{array}$ & 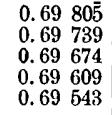 & $\begin{array}{ll}9.99 & 145 \\
9.99 & 142 \\
9.99 & 140 \\
9.99 & 137 \\
9.99 & 135\end{array}$ & $\begin{array}{l}3 \\
2 \\
3 \\
2\end{array}$ & $\begin{array}{l}\mathbf{4 0} \\
39 \\
38 \\
37 \\
36\end{array}$ & 14 & $\begin{array}{l}40 \\
36 \\
32 \\
28 \\
24\end{array}$ \\
\hline 45 & $\begin{array}{l}40 \\
44 \\
48 \\
52 \\
56\end{array}$ & $\begin{array}{l}25 \\
26 \\
27 \\
28 \\
29\end{array}$ & $\begin{array}{ll}9.29 & 654 \\
9.29 & 716 \\
9.29 & 779 \\
9.29 & 841 \\
9.29 & 903\end{array}$ & $\begin{array}{l}62 \\
63 \\
62 \\
62\end{array}$ & $\begin{array}{ll}9.30 & 522 \\
9.30 & 587 \\
9.30 & 652 \\
9.30 & 717 \\
9.30 & 782\end{array}$ & $\begin{array}{l}65 \\
65 \\
65 \\
65\end{array}$ & $\begin{array}{ll}0.69 & 478 \\
0.69 & 413 \\
0.69 & 348 \\
0.69 & 283 \\
0.69 & 218\end{array}$ & $\begin{array}{ll}9.99 & 132 \\
9.99 & 130 \\
9.99 & 127 \\
9.99 & 124 \\
9.99 & 122\end{array}$ & $\begin{array}{l}2 \\
3 \\
3 \\
2\end{array}$ & $\begin{array}{l}35 \\
34 \\
33 \\
32 \\
31\end{array}$ & 14 & $\begin{array}{r}20 \\
16 \\
12 \\
8 \\
4\end{array}$ \\
\hline 46 & $\begin{array}{r}0 \\
4 \\
8 \\
12 \\
16\end{array}$ & $\begin{array}{l}\mathbf{3 0} \\
31 \\
32 \\
33 \\
34\end{array}$ & $\begin{array}{ll}9.29 & 966 \\
9.30 & 028 \\
9.30 & 090 \\
9.30 & 151 \\
9.30 & 213\end{array}$ & $\begin{array}{l}62 \\
62 \\
61 \\
62\end{array}$ & $\begin{array}{ll}9.30 & 846 \\
9.30 & 911 \\
9.30 & 975 \\
9.31 & 040 \\
9.31 & 104\end{array}$ & $\begin{array}{l}65 \\
64 \\
65 \\
64\end{array}$ & $\begin{array}{ll}0.69 & 154 \\
0.69 & 089 \\
0.69 & 025 \\
0.68 & 960 \\
0.68 & 896\end{array}$ & $\begin{array}{ll}9.99 & 119 \\
9.99 & 117 \\
9.99 & 114 \\
9.99 & 112 \\
9.99 & 109\end{array}$ & $\begin{array}{l}2 \\
3 \\
2 \\
3\end{array}$ & $\begin{array}{r}\mathbf{3 0} \\
29 \\
28 \\
27 \\
26\end{array}$ & 14 & $\begin{array}{r}0 \\
56 \\
52 \\
48 \\
44\end{array}$ \\
\hline 46 & $\begin{array}{l}20 \\
24 \\
28 \\
32 \\
36\end{array}$ & $\begin{array}{l}35 \\
36 \\
37 \\
38 \\
39\end{array}$ & $\begin{array}{lll}9.30 & 275 \\
9.30 & 336 \\
9.30 & 398 \\
9.30 & 459 \\
9.30 & 521\end{array}$ & $\begin{array}{l}61 \\
62 \\
61 \\
62\end{array}$ & $\begin{array}{ll}9.31 & 168 \\
9.31 & 233 \\
9.31 & 297 \\
9.31 & 361 \\
9.31 & 425\end{array}$ & $\begin{array}{l}65 \\
64 \\
64 \\
64\end{array}$ & $\begin{array}{ll}0.68 & 832 \\
0.68 & 767 \\
0.68 & 703 \\
0.68 & 639 \\
0.68 & 575\end{array}$ & $\begin{array}{ll}9.99 & 106 \\
9.99 & 104 \\
9.99 & 101 \\
9.99 & 099 \\
9.99 & 096\end{array}$ & $\begin{array}{l}2 \\
3 \\
2 \\
3\end{array}$ & $\begin{array}{l}25 \\
24 \\
23 \\
22 \\
21\end{array}$ & 13 & $\begin{array}{l}40 \\
36 \\
32 \\
28 \\
24\end{array}$ \\
\hline 46 & $\begin{array}{l}40 \\
44 \\
48 \\
52 \\
56\end{array}$ & $\begin{array}{l}40 \\
41 \\
42 \\
43 \\
44\end{array}$ & $\begin{array}{ll}9.30 & 582 \\
9.30 & 643 \\
9.30 & 704 \\
9.30 & 76 \dot{5} \\
9.30 & 826\end{array}$ & $\begin{array}{l}61 \\
61 \\
61 \\
61\end{array}$ & $\begin{array}{ll}9.31 & 489 \\
9.31 & 552 \\
9.31 & 616 \\
9.31 & 679 \\
9.31 & 743\end{array}$ & $\begin{array}{l}63 \\
64 \\
63 \\
64\end{array}$ & $\begin{array}{ll}0.68 & 511 \\
0.68 & 448 \\
0.68 & 384 \\
0.68 & 321 \\
0.68 & 257\end{array}$ & $\begin{array}{ll}9.99 & 093 \\
9.99 & 091 \\
9.99 & 088 \\
9.99 & 086 \\
9.99 & 083\end{array}$ & $\begin{array}{l}2 \\
3 \\
2 \\
3\end{array}$ & $\begin{array}{l}\mathbf{2 0} \\
19 \\
18 \\
17 \\
16\end{array}$ & 13 & $\begin{array}{r}20 \\
16 \\
12 \\
8 \\
4\end{array}$ \\
\hline 47 & $\begin{array}{r}0 \\
4 \\
8 \\
12 \\
16\end{array}$ & $\begin{array}{l}45 \\
46 \\
47 \\
48 \\
49\end{array}$ & $\begin{array}{ll}9.30 & 887 \\
9.30 & 947 \\
9.31 & 008 \\
9.31 & 068 \\
9.31 & 129\end{array}$ & $\begin{array}{l}60 \\
61 \\
60 \\
61\end{array}$ & $\begin{array}{ll}9.31 & 806 \\
9.31 & 870 \\
9.31 & 933 \\
9.31 & 996 \\
9.32 & 059\end{array}$ & $\begin{array}{l}64 \\
63 \\
63 \\
63\end{array}$ & $\begin{array}{ll}0.68 & 194 \\
0.68 & 130 \\
0.68 & 067 \\
0.68 & 004 \\
0.67 & 941\end{array}$ & 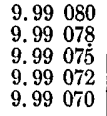 & $\begin{array}{l}2 \\
3 \\
3 \\
2\end{array}$ & $\begin{array}{l}15 \\
14 \\
13 \\
12 \\
11\end{array}$ & 13 & $\begin{array}{r}0 \\
56 \\
52 \\
48 \\
44\end{array}$ \\
\hline 47 & $\begin{array}{l}20 \\
24 \\
28 \\
32 \\
36\end{array}$ & $\begin{array}{l}\mathbf{5 0} \\
51 \\
52 \\
53 \\
54\end{array}$ & $\begin{array}{ll}9.31 & 189 \\
9.31 .250 \\
9.31 & 310 \\
9.31 & 370 \\
9.31 & 430\end{array}$ & $\begin{array}{l}61 \\
60 \\
60 \\
60\end{array}$ & $\begin{array}{ll}9.32 & 122 \\
9.32 & 185 \\
9.32 & 248 \\
9.32 & 311 \\
9.32 & 373\end{array}$ & $\begin{array}{l}63 \\
63 \\
63 \\
62\end{array}$ & $\begin{array}{ll}0.67 & 878 \\
0.67 & 815 \\
0.67 & 752 \\
0.67 & 689 \\
0.67 & 627\end{array}$ & $\begin{array}{ll}9.99 & 067 \\
9.99 & 064 \\
9.99 & 062 \\
9.99 & 059 \\
9.99 & 056\end{array}$ & $\begin{array}{l}3 \\
2 \\
3 \\
3\end{array}$ & $\begin{array}{r}\mathbf{1 0} \\
9 \\
8 \\
7 \\
6\end{array}$ & 12 & $\begin{array}{l}40 \\
36 \\
32 \\
28 \\
24\end{array}$ \\
\hline 47 & $\begin{array}{l}40 \\
44 \\
48 \\
52 \\
56\end{array}$ & $\begin{array}{l}55 \\
56 \\
57 \\
58 \\
59\end{array}$ & $\begin{array}{ll}9.31 & 490 \\
\mathbf{9 . 3 1} & 549 \\
\mathbf{9 . 3 1} & 609 \\
\mathbf{9 . 3 1} & 669 \\
\mathbf{9 . 3 1} & \mathbf{7 2 8}\end{array}$ & $\begin{array}{l}59 \\
60 \\
60 \\
59\end{array}$ & $\begin{array}{ll}9.32 & 436 \\
9.32 & 498 \\
9.32 & 561 \\
9.32 & 623 \\
9.32 & 685\end{array}$ & $\begin{array}{l}62 \\
63 \\
62 \\
62\end{array}$ & $\begin{array}{ll}0.67 & 564 \\
0.67 & 502 \\
0.67 & 439 \\
0.67 & 377 \\
0.67 & 315\end{array}$ & $\begin{array}{ll}9.99 & 054 \\
9.99 & 051 \\
9.99 & 048 \\
9.99 & 046 \\
9.99 & 043\end{array}$ & $\begin{array}{l}3 \\
3 \\
2 \\
3\end{array}$ & $\begin{array}{l}5 \\
4 \\
3 \\
2 \\
1\end{array}$ & 12 & $\begin{array}{r}20 \\
16 \\
12 \\
8 \\
4\end{array}$ \\
\hline 48 & 0 & 60 & 9.31788 & & 9.32747 & & 0.67253 & 9.99040 & J & 0 & 12 & 0 \\
\hline & & & Cos. & d. & I. Cotg. & c. d. & L. Tang. & L. $\operatorname{Sin}$. & d. & $'$ & $\mathrm{~m}$ & s. \\
\hline
\end{tabular}


TABLe 19.-Five-place logarithms of circular functions, etc.-Continued.

$0^{\text {h }}$

\begin{tabular}{|c|c|c|c|c|c|c|c|c|c|c|c|c|}
\hline m. & s. & ' & I. Sin. & d. & L. Tung. & c. d. & L. Cotg. & L. $\operatorname{Cos}$. & d. & & & \\
\hline 48 & $\begin{array}{r}0 \\
4 \\
8 \\
12 \\
16\end{array}$ & $\begin{array}{l}0 \\
1 \\
2 \\
3 \\
4\end{array}$ & $\begin{array}{ll}9.31 & 788 \\
9.31 & 847 \\
9.31 & 907 \\
9.31 & 966 \\
9.32 & 025\end{array}$ & $\begin{array}{l}59 \\
60 \\
59 \\
59 \\
59\end{array}$ & 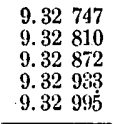 & $\begin{array}{l}63 \\
62 \\
61 \\
62 \\
62\end{array}$ & $\begin{array}{ll}0.67 & 253 \\
0.67 & 190 \\
0.67 & 128 \\
0.67 & 067 \\
0.67 & 005\end{array}$ & 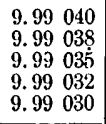 & $\begin{array}{l}2 \\
3 \\
3 \\
2 \\
3\end{array}$ & $\begin{array}{l}60 \\
59 \\
58 \\
57 \\
56\end{array}$ & 12 & $\begin{array}{r}0 \\
56 \\
52 \\
48 \\
44\end{array}$ \\
\hline 48 & $\begin{array}{l}20 \\
24 \\
28 \\
32 \\
36\end{array}$ & $\begin{array}{l}5 \\
6 \\
7 \\
8 \\
9\end{array}$ & $\begin{array}{ll}9.32 & 084 \\
9.32 & 143 \\
9.32 & 202 \\
9.32 & 261 \\
9.32 & 319 \\
\end{array}$ & $\begin{array}{l}59 \\
59 \\
59 \\
58 \\
59\end{array}$ & $\begin{array}{ll}9.33 & 057 \\
9.33 & 119 \\
9.33 & 130 \\
9.33 & 242 \\
9.33 & 303\end{array}$ & $\begin{array}{l}62 \\
61 \\
62 \\
61 \\
62\end{array}$ & $\begin{array}{ll}0.66 & 943 \\
0.66 & 881 \\
0.66 & 820 \\
0.66 & 758 \\
0.66 & 697\end{array}$ & 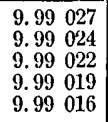 & $\begin{array}{l}3 \\
2 \\
3 \\
3 \\
3\end{array}$ & $\begin{array}{l}55 \\
54 \\
53 \\
52 \\
51\end{array}$ & 11 & $\begin{array}{l}40 \\
36 \\
32 \\
28 \\
24\end{array}$ \\
\hline 48 & $\begin{array}{l}40 \\
44 \\
48 \\
52 \\
56\end{array}$ & $\begin{array}{l}10 \\
11 . \\
12 \\
13 \\
14\end{array}$ & $\begin{array}{ll}9.32 & 378 \\
9.32 & 437 \\
9.32 & 495 \\
9.32 & 553 \\
9.32 & 612\end{array}$ & $\begin{array}{l}59 \\
58 \\
58 \\
59 \\
58\end{array}$ & $\begin{array}{ll}9.33 & 365 \\
9.33 & 426 \\
9.33 & 487 \\
9.33 & 548 \\
9.33 & 609\end{array}$ & $\begin{array}{l}61 \\
61 \\
61 \\
61 \\
61\end{array}$ & 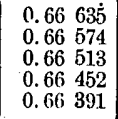 & $\begin{array}{ll}\mathbf{9 . 9 9} & 013 \\
\mathbf{9 . 9 9} & 011 \\
\mathbf{9 . 9 9} & 008 \\
9.99 & 005 \\
\mathbf{9 . 9 9} & 002\end{array}$ & $\begin{array}{l}2 \\
3 \\
3 \\
3 \\
2\end{array}$ & $\begin{array}{c}50 \\
49 \\
48 \\
47 \\
46\end{array}$ & 11 & $\begin{array}{r}20 \\
16 \\
12 \\
8 \\
4\end{array}$ \\
\hline 49 & $\begin{array}{r}0 \\
4 \\
5 \\
12 \\
16\end{array}$ & $\begin{array}{l}15 \\
16 \\
17 \\
18 \\
19\end{array}$ & 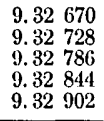 & $\begin{array}{l}58 \\
58 \\
58 \\
58 \\
58\end{array}$ & $\begin{array}{ll}9.33 & 670 \\
9.33 & 731 \\
9.33 & 792 \\
9.33 & 353 \\
9.33 & 913\end{array}$ & $\begin{array}{l}61 \\
61 \\
61 \\
60 \\
61\end{array}$ & $\begin{array}{ll}0.66 & 330 \\
0.66 & 269 \\
0.66 & 208 \\
0.66 & 147 \\
0.66 & 087\end{array}$ & 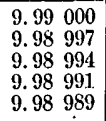 & $\begin{array}{l}3 \\
3 \\
3 \\
2 \\
3\end{array}$ & $\begin{array}{l}45 \\
44 \\
43 \\
42 \\
41\end{array}$ & 11 & $\begin{array}{r}0 \\
56 \\
52 \\
48 \\
44\end{array}$ \\
\hline 49 & $\begin{array}{l}20 \\
24 \\
28 \\
32 \\
36\end{array}$ & $\begin{array}{l}\mathbf{9 0} \\
21 \\
22 \\
23 \\
24\end{array}$ & $\begin{array}{ll}9.32 & 960 \\
9.33 & 018 \\
9.33 & 075 \\
9.33 & 133 \\
9.33 & 1.90\end{array}$ & $\begin{array}{r}58 \\
57 \\
58 \\
.57 \\
58\end{array}$ & $\begin{array}{ll}9.33 & 974 \\
9.34 & 034 \\
9.34 & 095 \\
9.34 & 155 \\
9.34 & 215\end{array}$ & $\begin{array}{l}60 \\
61 \\
60 \\
60 \\
61\end{array}$ & $\begin{array}{ll}0.66 & 026 \\
0.65 & 966 \\
0.65 & 905 \\
0.65 & 84 \overline{5} \\
0.65 & 785 \\
\end{array}$ & 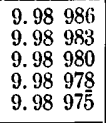 & $\begin{array}{l}3 \\
3 \\
2 \\
3 \\
3 .\end{array}$ & $\begin{array}{l}\mathbf{4 0} \\
39 \\
38 \\
37 \\
36\end{array}$ & 10 & $\begin{array}{l}40 \\
36 \\
32 \\
28 \\
24 \\
\end{array}$ \\
\hline 49 & $\begin{array}{l}40 \\
44 \\
48 \\
52 \\
56\end{array}$ & $\begin{array}{l}25 \\
26 \\
27 \\
28 \\
29 \\
\end{array}$ & $\begin{array}{lll}9.33 & 248 \\
9.33 & 305 \\
9.33 & 362 \\
9.33 & 420 \\
9.33 & 477 \\
\end{array}$ & $\begin{array}{l}57 \\
57 \\
58 \\
57 \\
57\end{array}$ & $\begin{array}{ll}9.34 & 276 \\
9.34 & 336 \\
9.34 & 396 \\
9.34 & 456 \\
9.34 & 516 \\
\end{array}$ & $\begin{array}{r}60 \\
60 \\
60 \\
60 \\
60\end{array}$ & $\begin{array}{ll}0.65 & 724 \\
0.65 & 664 \\
0.65 & 604 \\
0.65 & 544 \\
0.65 & 484 \\
\end{array}$ & 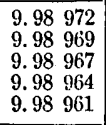 & $\begin{array}{l}3 \\
2 \\
3 \\
3 \\
3\end{array}$ & $\begin{array}{l}35 \\
34 \\
33 \\
32 \\
31\end{array}$ & 10 & $\begin{array}{r}20 \\
16 \\
12 \\
8 \\
4 \\
\end{array}$ \\
\hline 50 & $\begin{array}{r}0 \\
4 \\
8 \\
1.2 \\
16 \\
\end{array}$ & $\begin{array}{l}\mathbf{3 0} \\
31 \\
32 \\
33 \\
34 \\
\end{array}$ & $\begin{array}{ll}9.33 & 534 \\
9.33 & 591 \\
9.33 & 647 \\
9.33 & 704 \\
9.33 & 761 \\
\end{array}$ & $\begin{array}{l}57 \\
56 \\
57 \\
57 \\
57\end{array}$ & $\begin{array}{ll}9.34 & 576 \\
9.34 & 635 \\
9.34: & 695 \\
9.34 & 755 \\
9.34 & 814 \\
\end{array}$ & $\begin{array}{l}59 \\
60 \\
60 \\
59 \\
60\end{array}$ & $\begin{array}{ll}0.65 & 424 \\
0.65 & 365 \\
0.65 & 305 \\
0.65 & 245 \\
0.65 & 186\end{array}$ & $\begin{array}{l}9.98958 \\
9.98955 \\
9.98953 \\
9.98950 \\
9.98947 \\
\end{array}$ & $\begin{array}{l}3 \\
2 \\
3 \\
3 \\
3\end{array}$ & $\begin{array}{l}30 \\
29 \\
28 \\
27 \\
26 \\
\end{array}$ & 10 & $\begin{array}{r}0 \\
56 \\
52 \\
48 \\
44\end{array}$ \\
\hline 50 & $\begin{array}{l}20 \\
24 \\
28 \\
32 \\
36 \\
\end{array}$ & $\begin{array}{l}35 \\
36 \\
37 \\
38 \\
39\end{array}$ & $\begin{array}{ll}9.33 & 81.8 \\
9.33 & 874 \\
9.33 & 931 \\
9.33 & 987 \\
9.34 & 043 \\
\end{array}$ & $\begin{array}{l}56 \\
57 \\
56 \\
56 \\
57\end{array}$ & $\begin{array}{lll}9.34 & 874 \\
9.34 & 933 \\
9.34 & 992 \\
9.35 & 051 \\
9.35 & 111 \\
\end{array}$ & $\begin{array}{l}59 \\
59 \\
59 \\
60 \\
59\end{array}$ & $\begin{array}{ll}0.65 & 126 \\
0.65 & 067 \\
0.65 & 008 \\
0.64 & 949 \\
0.64 & 889 \\
\end{array}$ & 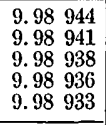 & $\begin{array}{l}3 \\
3 \\
2 \\
3 \\
3 \\
3\end{array}$ & $\begin{array}{l}25 \\
24 \\
23 \\
22 \\
21\end{array}$ & 9 & $\begin{array}{l}40 \\
36 \\
32 \\
28 \\
24 \\
\end{array}$ \\
\hline 50 & $\begin{array}{l}40 \\
44 \\
48 \\
52 \\
56 \\
\end{array}$ & $\begin{array}{l}40 \\
41 \\
42 \\
43 \\
44\end{array}$ & $\begin{array}{ll}9.34 & 100 \\
9.34 & 156 \\
9.34 & 212 \\
9.34 & 268 \\
9.34 & 324 \\
\end{array}$ & $\begin{array}{l}56 \\
56 \\
56 \\
56 \\
56\end{array}$ & $\begin{array}{ll}9.35 & 170 \\
9.35 & 229 \\
9.35 & 288 \\
9.35 & 347 \\
9.35 & 405 \\
\end{array}$ & $\begin{array}{l}59 \\
59 \\
59 \\
58 \\
59\end{array}$ & $\begin{array}{ll}0.64 & 830 \\
0.64 & 771 \\
0.64 & 712 \\
0.64 & 653 \\
0.64 & 595 \\
\end{array}$ & $\begin{array}{rr}9.98 & 930 \\
9.98 & 927 \\
9.98 & 924 \\
9.98 & 921 \\
9.98 & 919 \\
\end{array}$ & \begin{tabular}{l|}
3 \\
3 \\
3 \\
2 \\
3
\end{tabular} & $\begin{array}{l}20 \\
19 \\
18 \\
17 \\
16 \\
\end{array}$ & 9 & $\begin{array}{r}20 \\
16 \\
12 \\
8 \\
4\end{array}$ \\
\hline 51 & $\begin{array}{r}0 \\
4 \\
8 \\
12 \\
16 \\
\end{array}$ & $\begin{array}{l}45 \\
46 \\
47 \\
48 \\
49 \\
\end{array}$ & $\begin{array}{ll}9.34 & 380 \\
9.34 & 436 \\
9.34 & 491 \\
9.34 & 547 \\
9.34 & 602 \\
\end{array}$ & $\begin{array}{l}56 \\
55 \\
56 \\
55 \\
56\end{array}$ & 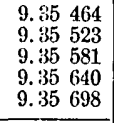 & $\begin{array}{l}59 \\
58 \\
59 \\
58 \\
59\end{array}$ & $\begin{array}{ll}0.64 & 536 \\
0.64 & 477 \\
0.64 & 419 \\
0.64 & 360 \\
0.64 & 302 \\
\end{array}$ & 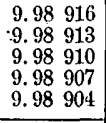 & \begin{tabular}{l|}
3 \\
3 \\
3 \\
3 \\
3
\end{tabular} & $\begin{array}{l}15 \\
14 \\
13 \\
12 \\
11 . \\
\end{array}$ & 9 & $\begin{array}{r}0 \\
56 \\
52 \\
48 \\
44 \\
\end{array}$ \\
\hline 51 & $\begin{array}{l}20 \\
24 \\
28 \\
32 \\
36\end{array}$ & $\begin{array}{l}50 \\
51 \\
52 \\
53 \\
54\end{array}$ & $\begin{array}{ll}9.34 & 658 \\
9.34 & 713 \\
9.34 & 769 \\
9.34 & 824 \\
9.34 & 879 \\
\end{array}$ & $\begin{array}{l}55 \\
55 \\
55 \\
55 \\
55\end{array}$ & $\begin{array}{ll}9.35 & 757 \\
9.35 & 81 \overline{5} \\
9.35 & 873 \\
9.35 & 931 \\
9.35 & 989\end{array}$ & $\begin{array}{l}58 \\
58 \\
58 \\
58 \\
58\end{array}$ & $\begin{array}{ll}0.64 & 243 \\
0.64 & 185 \\
0.64 & 127 \\
0.64 & 069 \\
0.64 & 011\end{array}$ & $\begin{array}{ll}9.98 & 901 \\
9.98 & 898 \\
9.98 & 896 \\
9.98 & 893 \\
9.98 & 890 \\
\end{array}$ & \begin{tabular}{l|}
3 \\
2 \\
3 \\
3 \\
3
\end{tabular} & $\begin{array}{r}10 \\
9 \\
8 \\
7 \\
6\end{array}$ & 8 & $\begin{array}{l}40 \\
36 \\
32 \\
28 \\
24\end{array}$ \\
\hline 51 & $\begin{array}{l}40 \\
44 \\
48 \\
52 \\
56\end{array}$ & $\begin{array}{l}55 \\
56 \\
57 \\
58 \\
59\end{array}$ & $\begin{array}{ll}9.34 & 934 \\
9.34 & 989 \\
9.35 & 044 \\
9.35 & 099 \\
9.35 & 154\end{array}$ & $\begin{array}{l}55 \\
55 \\
55 \\
55 \\
55\end{array}$ & $\begin{array}{ll}9.36 & 047 \\
9.36 & 105 \\
9.36 & 163 \\
9.36 & 221 \\
9.36 & 279 \\
\end{array}$ & $\begin{array}{l}58 \\
58 \\
58 \\
58 \\
.57\end{array}$ & $\begin{array}{ll}0.63 & 953 \\
0.63 & 895 \\
0.63 & 837 \\
0.63 & 779 \\
0.63 & 721\end{array}$ & 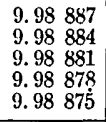 & $\begin{array}{l}3 \\
3 \\
3 \\
3 \\
3\end{array}$ & $\begin{array}{l}5 \\
4 \\
3 \\
2 \\
1\end{array}$ & 8 & $\begin{array}{r}20 \\
16 \\
12 \\
8 \\
4\end{array}$ \\
\hline 52 & 0 & 60 & 9.35209 & & 9.36336 & & 0.63664 & 9.98872 & & $\mathbf{0}$ & 8 & 0 \\
\hline & & & I. Cọs. & d. & L. Cotg. & c. d. & L. Tang. & L. Sin. & d. & 1 & m. & \\
\hline
\end{tabular}

$12^{\circ}$ 
TABLE 19.-Five-place logaritlms of circular functions, etc.-Continued.

$0^{\text {h }}$

\begin{tabular}{|c|c|c|c|c|c|c|c|c|c|c|c|}
\hline $\mathrm{m}$. & s. & ' & I. $\operatorname{Sin}$. & d. & L. Tang. & c. $\mathrm{d}$. & L. Cotg. & L. Cos. & d. & & \\
\hline 52 & $\begin{array}{r}0 \\
4 \\
8 \\
12 \\
16\end{array}$ & $\begin{array}{l}0 \\
1 \\
2 \\
3 \\
4\end{array}$ & $\begin{array}{ll}9.35 & 209 \\
9.35 & 263 \\
9.35 & 318 \\
9.35 & 373 \\
9.35 & 427\end{array}$ & $\begin{array}{l}54 \\
55 \\
55 \\
54 \\
54\end{array}$ & 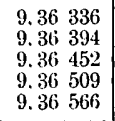 & $\begin{array}{l}58 \\
58 \\
57 \\
57 \\
58\end{array}$ & $\begin{array}{ll}0.63 & 664 \\
0.63 & 606 \\
0.63 & 548 \\
0.63 & 491 \\
0.63 & 434\end{array}$ & 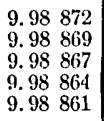 & $\begin{array}{l}3 \\
2 \\
3 \\
3 \\
3\end{array}$ & $\begin{array}{c}60 \\
59 \\
58 \\
57 \\
56\end{array}$ & $\begin{array}{r}0 \\
56 \\
52 \\
48 \\
44\end{array}$ \\
\hline 52 & $\begin{array}{l}20 \\
24 \\
28 \\
32 \\
36\end{array}$ & $\begin{array}{l}5 \\
6 \\
7 \\
8 \\
9\end{array}$ & 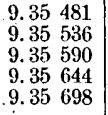 & $\begin{array}{l}55 \\
54 \\
54 \\
54 \\
54\end{array}$ & $\begin{array}{ll}9.36 & 624 \\
9.36 & 681 \\
9.36 & 738 \\
9.36 & 795 \\
9.36 & 852\end{array}$ & $\begin{array}{l}57 \\
57 \\
57 \\
57 \\
57\end{array}$ & $\begin{array}{ll}0.63 & 376 \\
0.63 & 319 \\
0.63 & 262 \\
0.63 & 205 \\
0.63 & 148\end{array}$ & 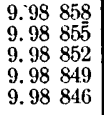 & $\begin{array}{l}3 \\
3 \\
3 \\
3 \\
3\end{array}$ & $\begin{array}{l}55 \\
54 \\
53 \\
52 \\
51\end{array}$ & 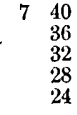 \\
\hline 52 & $\begin{array}{l}40 \\
44 \\
48 \\
52 \\
56\end{array}$ & $\begin{array}{l}10 \\
11 \\
12 \\
13 \\
14\end{array}$ & 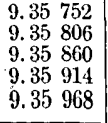 & $\begin{array}{l}54 \\
54 \\
54 \\
54 \\
54\end{array}$ & $\begin{array}{ll}9.36 & 909 \\
9.36 & 966 \\
9.37 & 023 \\
9.37 & 080 \\
9.37 & 137\end{array}$ & $\begin{array}{l}57 \\
57 \\
57 \\
57 \\
56\end{array}$ & $\begin{array}{ll}0.63 & 091 \\
0.63 & 034 \\
0.62 & 977 \\
0.62 & 920 \\
0.62 & 863\end{array}$ & 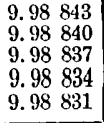 & $\begin{array}{l}3 \\
3 \\
3 \\
3 \\
3\end{array}$ & $\begin{array}{r}\mathbf{5 0} \\
49 \\
48 \\
47 \\
46 \\
\end{array}$ & 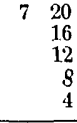 \\
\hline 53 & $\begin{array}{r}0 \\
4 \\
8 \\
12 \\
16\end{array}$ & $\begin{array}{l}15 \\
16 \\
17 \\
18 \\
19\end{array}$ & $\begin{array}{lll}9.36 & 022 \\
9.36 & 075 \\
9.36 & 129 \\
9.36 & 182 \\
9.36 & 236 \\
\end{array}$ & $\begin{array}{l}53 \\
54 \\
53 \\
54 \\
53\end{array}$ & $\begin{array}{ll}9.37 & 193 \\
9.37 & 250 \\
9.37 & 306 \\
9.37 & 363 \\
9.37 & 419\end{array}$ & $\begin{array}{l}57 \\
56 \\
57 \\
56 \\
57\end{array}$ & 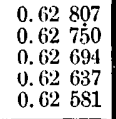 & 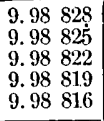 & $\begin{array}{l}3 \\
3 \\
3 \\
3 \\
3\end{array}$ & $\begin{array}{l}45 \\
44 \\
43 \\
42 \\
41\end{array}$ & $\begin{array}{lr}7 & 0 \\
& 56 \\
& 52 \\
& 48 \\
& 44\end{array}$ \\
\hline 53 & $\begin{array}{l}20 \\
24 \\
28 \\
32 \\
36\end{array}$ & $\begin{array}{l}20 \\
21 \\
22 \\
23 \\
24\end{array}$ & $\begin{array}{lll}9.36 & 289 \\
9.36 & 342 \\
9.36 & 395 \\
9.36 & 449 \\
9.36 & 502\end{array}$ & $\begin{array}{l}53 \\
53 \\
54 \\
53 \\
53\end{array}$ & $\begin{array}{ll}9.37 & 476 \\
9.37 & 532 \\
9.37 & 588 \\
9.37 & 644 \\
9.37 & 700\end{array}$ & $\begin{array}{l}56 \\
56 \\
56 \\
56 \\
56\end{array}$ & $\begin{array}{ll}0.62 & 524 \\
0.62 & 468 \\
0.62 & 412 \\
0.62 & 356 \\
0.62 & 300\end{array}$ & 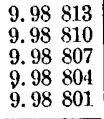 & $\begin{array}{l}3 \\
3 \\
3 \\
3 \\
3 \\
3\end{array}$ & $\begin{array}{l}40 \\
39 \\
38 \\
37 \\
36\end{array}$ & $\begin{array}{l}6 \quad 40 \\
\quad 36 \\
\\
32 \\
\\
\\
\\
\\
\\
28 \\
24\end{array}$ \\
\hline 53 & $\begin{array}{l}40 \\
44 \\
48 \\
52 \\
56\end{array}$ & $\begin{array}{l}25 \\
26 \\
27 \\
28 \\
29\end{array}$ & 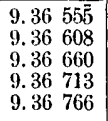 & $\begin{array}{l}53 \\
52 \\
53 \\
53\end{array}$ & 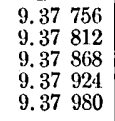 & $\begin{array}{l}56 \\
56 \\
56 \\
56 \\
55\end{array}$ & $\begin{array}{ll}0.62 & 244 \\
0.62 & 188 \\
0.62 & 132 \\
0.62 & 076 \\
0.62 & 020\end{array}$ & $\begin{array}{ll}9.98 & 798 \\
9.98 & 795 \\
9.98 & 792 \\
9.98 & 789 \\
9.98 & 786\end{array}$ & $\begin{array}{l}3 \\
3 \\
3 \\
3 \\
3\end{array}$ & $\begin{array}{l}35 \\
34 \\
33 \\
32 \\
31\end{array}$ & 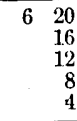 \\
\hline 54 & $\begin{array}{r}0 \\
4 \\
8 \\
12 \\
16 \\
\end{array}$ & $\begin{array}{l}\mathbf{3 0} \\
31 \\
32 \\
33 \\
34 \\
\end{array}$ & $\begin{array}{ll}9.36 & 819 \\
9.36 & 871 \\
9.36 & 924 \\
9.36 & 976 \\
9.37 & 028 \\
\end{array}$ & $\begin{array}{l}52 \\
53 \\
52 \\
52 \\
53\end{array}$ & $\begin{array}{ll}9.38 & 035 \\
9.38 & 091 \\
9.38 & 147 \\
9.38 & 202 \\
9.38 & 257 \\
\end{array}$ & $\begin{array}{l}56 \\
56 \\
55 \\
55 \\
56\end{array}$ & $\begin{array}{ll}0.61 & 965 \\
0.61 & 909 \\
0.61 & 853 \\
0.61 & 798 \\
0.61 & 743 \\
\end{array}$ & $\begin{array}{ll}9.98 & 783 \\
9.98 & 780 \\
9.98 & 777 \\
9.98 & 774 \\
9.98 & 771 \\
\end{array}$ & $\begin{array}{l}3 \\
3 \\
3 \\
3 \\
3\end{array}$ & $\begin{array}{r}30 \\
29 \\
28 \\
\cdot 27 \\
26 \\
\end{array}$ & $\begin{array}{rr}6 & 0 \\
& 56 \\
& 52 \\
& 48 \\
& 44 \\
& \end{array}$ \\
\hline .54 & $\begin{array}{l}20 \\
24 \\
28 \\
32 \\
36\end{array}$ & $\begin{array}{l}35 \\
36 \\
37 \\
38 \\
39\end{array}$ & $\begin{array}{ll}9.37 & 081 \\
9.37 & 133 \\
9.37 & 185 \\
9.37 & 237 \\
9.37 & 289 \\
\end{array}$ & $\begin{array}{l}52 \\
52 \\
52 \\
52 \\
52\end{array}$ & 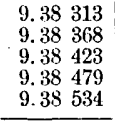 & $\begin{array}{l}55 \\
55 \\
56 \\
55 \\
55\end{array}$ & $\begin{array}{ll}0.61 .687 \\
0.61 & 632 \\
0.61 . & 577 \\
0.61 & 521 . \\
0.61 & 466\end{array}$ & 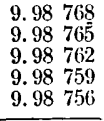 & $\begin{array}{l}3 \\
3 \\
3 \\
3 \\
3\end{array}$ & $\begin{array}{l}25 \\
24 \\
23 \\
22 \\
21\end{array}$ & $\begin{array}{l}5 \quad 40 \\
36 \\
32 \\
\\
28 \\
\\
24\end{array}$ \\
\hline 54 & $\begin{array}{l}40 \\
44 \\
48 \\
52 \\
56 \\
\end{array}$ & $\begin{array}{r}40 \\
41 \\
42 \\
43 \\
44 \\
\end{array}$ & 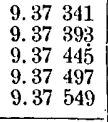 & $\begin{array}{r}52 \\
52 \\
52 \\
52 \\
.51\end{array}$ & $\begin{array}{ll}9.38 & 589 \\
9.38 & 644 \\
9.38 & 699 \\
9.38 & 754 \\
9.38 & 808 \\
\end{array}$ & $\begin{array}{l}55 \\
55 \\
55 \\
54 \\
55\end{array}$ & $\begin{array}{ll}0.61 & 411 \\
0.61 .356 \\
0.61 . & 301 \\
0.61 & 246 \\
0.61 & 192\end{array}$ & 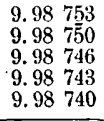 & $\begin{array}{l}3 \\
4 \\
3 \\
3 \\
3\end{array}$ & $\begin{array}{l}20 \\
19 \\
18 \\
17 \\
16\end{array}$ & $\begin{array}{r}5 \quad 20 \\
16 \\
12 \\
. \quad 8 \\
4\end{array}$ \\
\hline 55 & $\begin{array}{r}0 \\
4 \\
8 \\
12 \\
16 \\
\end{array}$ & $\begin{array}{l}45 \\
46 \\
47 \\
48 \\
49 \\
\end{array}$ & 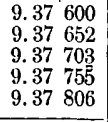 & $\begin{array}{l}52 \\
51 \\
52 \\
51 \\
52\end{array}$ & $\begin{array}{ll}9.38 & 863 \\
9.38 & 918 \\
9.38 & 972 \\
9.39 & 027 \\
9.39 & 082 \\
\end{array}$ & $\begin{array}{l}55 \\
54 \\
55 \\
55 \\
54\end{array}$ & $\begin{array}{ll}0.61 & 137 \\
0.61 & 082 \\
0.61 & 028 \\
0.60 & 973 \\
0.60 & 918\end{array}$ & 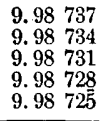 & $\begin{array}{l}3 \\
3 \\
3 \\
3 \\
3\end{array}$ & $\begin{array}{l}15 \\
14 \\
13 \\
12 \\
11\end{array}$ & $\begin{array}{rr}5 & 0 \\
& 56 \\
& 52 \\
& 48 \\
& 44\end{array}$ \\
\hline 55 & $\begin{array}{l}20 \\
24 \\
28 \\
32 \\
36 \\
\end{array}$ & $\begin{array}{l}50 \\
51 \\
52 \\
53 \\
54 \\
\end{array}$ & $\begin{array}{ll}9.37 & 858 \\
9.37 & 909 \\
9.37 & 960 \\
9.38 & 011 \\
9.38 & 062 . \\
\end{array}$ & $\begin{array}{l}51 \\
51 \\
51 \\
51 \\
51\end{array}$ & $\begin{array}{ll}9.39 & 136 \\
9.39 & 190 \\
9.39 & 245 \\
9.39 & 299 \\
9.39 & 353 \\
\end{array}$ & $\begin{array}{l}54 \\
55 \\
54 \\
54 \\
54\end{array}$ & 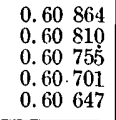 & $\begin{array}{ll}9.98 & 722 \\
9.98 & 719 \\
9.98 & 715 \\
9.98 & 712 \\
9.98 & 709\end{array}$ & $\begin{array}{l}3 \\
4 \\
3 \\
3 \\
3\end{array}$ & $\begin{array}{r}10 \\
9 \\
8 \\
7 \\
6\end{array}$ & $\begin{array}{r}4 \quad 40 \\
36 \\
32 \\
\\
28 \\
\\
24\end{array}$ \\
\hline 55 & $\begin{array}{l}40 \\
44 \\
48 \\
52 \\
56\end{array}$ & $\begin{array}{l}55 \\
56 \\
57 \\
58 \\
59\end{array}$ & $\begin{array}{ll}9.38 & 113 \\
9.38 & 164 \\
9.38 & 215 \\
9.38 & 266 \\
9.38 & 317 \\
\end{array}$ & $\begin{array}{l}51 \\
51 \\
51 \\
51 \\
51\end{array}$ & $\begin{array}{ll}9.39 & 407 \\
9.39 & 461 \\
9.39 & 515 \\
9.39 & 569 \\
9.39 & 623\end{array}$ & $\begin{array}{l}54 \\
54 \\
54 \\
54 \\
54\end{array}$ & $\begin{array}{ll}0.60 & 593 \\
0.60 & 539 \\
0.60 & 485 \\
0.60 & 431 \\
0.60 & 377\end{array}$ & 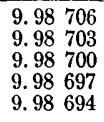 & $\begin{array}{l}3 \\
3 \\
3 \\
3 \\
4\end{array}$ & $\begin{array}{l}5 \\
4 \\
3 \\
2 \\
1\end{array}$ & $\begin{array}{rr}4 \quad 20 \\
16 \\
\\
12 \\
\\
\\
& 8 \\
& 4\end{array}$ \\
\hline 56 & 0 & 60 & 9.38368 & & 9.39677 & & 0.60323 & 9.98690 & & 0 & 4 \\
\hline & & & L. Cos. & d. & L. Cotg. & c. d. & L. Tang. & L. Sin. . & d. & ' & m. \\
\hline
\end{tabular}


TAüL 19.-Five-place logarilhms of circular functions, etc.-Continued.

$0^{\text {h }}$

\begin{tabular}{|c|c|c|c|c|c|c|c|c|c|c|c|c|}
\hline $\mathrm{m}$ & s. & ' & I. Sin: & d. & L. Tang. & c. di. & I. Cotg. & I. Cos. & d. & & & \\
\hline 66 & $\begin{array}{r}0 \\
4 \\
8 \\
12 \\
16\end{array}$ & $\begin{array}{l}0 \\
1 \\
2 \\
3 \\
4\end{array}$ & 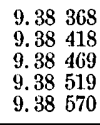 & \multirow{3}{*}{$\begin{array}{l}50 \\
51 \\
50 \\
51 \\
50 \\
50 \\
51 \\
50 \\
50 \\
50 \\
50 \\
50 \\
50 \\
50 \\
50\end{array}$} & $\begin{array}{ll}9.39 & 677 \\
9.39 & 731 \\
9.39 & 785 \\
9.39 & 838 \\
9.39 & 892\end{array}$ & \multirow{3}{*}{$\begin{array}{l}54 \\
54 \\
53 \\
54 \\
53 \\
54 \\
53 \\
54 \\
63 \\
53 \\
54 \\
53 \\
53 \\
53 \\
53\end{array}$} & $\begin{array}{ll}0.60 & 323 \\
0.60 & 269 \\
0.60 & 215 \\
0.60 & 162 \\
0.60 & 108\end{array}$ & $\begin{array}{l}9.98690 \\
9.98687 \\
9.98684 \\
9.98681 \\
9.98678\end{array}$ & \multirow{2}{*}{$\begin{array}{l}3 \\
3 \\
3 \\
3 \\
3 \\
4 \\
3 \\
3 \\
3 \\
3\end{array}$} & $\begin{array}{l}\mathbf{6 0} \\
59 \\
58 \\
57 \\
56\end{array}$ & 4 & $\begin{array}{r}0 \\
.56 \\
52 \\
4 \dot{8} \\
44\end{array}$ \\
\hline 56 & $\begin{array}{l}20 \\
24 \\
28 \\
32 \\
36\end{array}$ & $\begin{array}{l}5 \\
6 \\
7 \\
8 \\
9\end{array}$ & $\begin{array}{ll}9.38 & 620 \\
9.38 & 670 \\
9.38 & 721 \\
9.38 & 771 \\
9.38 & 821\end{array}$ & & $\begin{array}{ll}9.39 & 94 \dot{5} \\
9.39 & 999 \\
9.40 & 052 \\
9.40 & 106 \\
9.40 & 159\end{array}$ & & $\begin{array}{lll}0.60 & 05 \overline{5} \\
0.60 & 001 \\
0.59 & 948 \\
0.59 & 894 \\
0.59 & 841\end{array}$ & $\begin{array}{l}9.9867 \overline{5} \\
9.98671 \\
9.98668 \\
9.98665 \\
9.98662\end{array}$ & & $\begin{array}{l}55 \\
54 \\
53 \\
52 \\
51\end{array}$ & 3 & $\begin{array}{l}40 \\
36 \\
32 \\
28 \\
24\end{array}$ \\
\hline 56 & $\begin{array}{l}40 \\
44 \\
48 \\
52 \\
56\end{array}$ & $\begin{array}{l}\mathbf{1 0} \\
11 \\
12 \\
13 \\
14\end{array}$ & $\begin{array}{ll}9.38 & 871 \\
9.38 & 921 \\
9.38 & 971 \\
9.39 & 021 \\
9.39 & 071\end{array}$ & & $\begin{array}{ll}9.40 & 212 \\
9.40 & 266 \\
9.40 & 319 \\
9.40 & 372 \\
9.40 & 425\end{array}$ & & 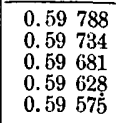 & $\begin{array}{ll}9.98 & 659 \\
9.98 & 656 \\
9.98 & 652 \\
9.98 & 649 \\
9.98 & 646\end{array}$ & $\begin{array}{l}3 \\
3 \\
4 \\
3 \\
3\end{array}$ & $\begin{array}{l}\mathbf{5 0} \\
\mathbf{4 9} \\
48 \\
\mathbf{4 7} \\
\mathbf{4 6}\end{array}$ & 3 & $\begin{array}{r}20 \\
16 \\
12 \\
8 \\
4\end{array}$ \\
\hline 57 & $\begin{array}{r}0 \\
4 \\
8 \\
12 \\
16\end{array}$ & $\begin{array}{l}15 \\
16 \\
17 \\
18 \\
19\end{array}$ & $\begin{array}{ll}9.39 & 121 \\
9.39 & 170 \\
9.39 & 220 \\
9.39 & 270 \\
9.39 & 319\end{array}$ & $\begin{array}{l}49 \\
50 \\
50 \\
49\end{array}$ & 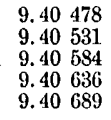 & $\begin{array}{l}63 \\
63 \\
63 \\
612 \\
63\end{array}$ & 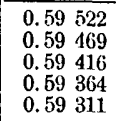 & $\begin{array}{l}9.98643 \\
9.98640 \\
9.98636 \\
9.98633 \\
9.98630\end{array}$ & $\begin{array}{l}3 \\
4 \\
3 \\
3\end{array}$ & $\begin{array}{l}45 \\
44 \\
43 \\
42 \\
41\end{array}$ & 3 & $\begin{array}{r}0 \\
56 \\
52 \\
48 \\
44\end{array}$ \\
\hline 57 & $\begin{array}{l}20 \\
24 \\
28 \\
32 \\
36\end{array}$ & $\begin{array}{l}\mathbf{2 0} \\
21 \\
22 \\
23 \\
24\end{array}$ & 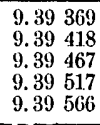 & $\begin{array}{l}49 \\
49 \\
60 \\
49\end{array}$ & 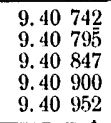 & $\begin{array}{l}63 \\
62 \\
53 \\
52\end{array}$ & $\begin{array}{ll}0.59 & 258 \\
0.59 & 205 \\
0.59 & 153 \\
0.59 & 100 \\
0.59 & 048\end{array}$ & $\begin{array}{l}9.98627 \\
9.98623 \\
9.98620 \\
9.98617 \\
9.98614\end{array}$ & $\begin{array}{l}4 \\
3 \\
3 \\
3\end{array}$ & $\begin{array}{r}40 \\
399 \\
38 \\
37 \\
36\end{array}$ & 2 & $\begin{array}{l}40 \\
36 \\
32 \\
28 \\
24\end{array}$ \\
\hline 57 & $\begin{array}{l}40 \\
44 \\
48 \\
52 \\
56\end{array}$ & $\begin{array}{l}25 \\
26 \\
27 \\
28 \\
29\end{array}$ & 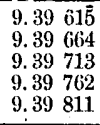 & $\begin{array}{l}49 \\
49 \\
49 \\
49\end{array}$ & $\begin{array}{lll}9.41 & 005 \\
9.41 & 057 \\
9.41 & 109 \\
9.41 & 161 \\
9.41 & 214\end{array}$ & $\begin{array}{r}52 \\
52 \\
52 \\
.53\end{array}$ & $\begin{array}{ll}0.58 & 995 \\
0.58 & 943 \\
0.58 & 891 \\
0.58 & 839 \\
0.58 & 786\end{array}$ & $\begin{array}{l}9.98610 \\
9.98607 \\
9.98604 \\
9.98601 \\
9.98597\end{array}$ & $\begin{array}{l}3 \\
3 \\
3 \\
4\end{array}$ & $\begin{array}{l}35 \\
34 \\
33 \\
32 \\
31\end{array}$ & 2 & $\begin{array}{r}20 \\
16 \\
12 \\
8 \\
4\end{array}$ \\
\hline 58 & $\begin{array}{r}0 \\
4 \\
8 \\
12 \\
16\end{array}$ & $\begin{array}{l}\mathbf{3 0} \\
31 \\
32 \\
33 \\
34\end{array}$ & 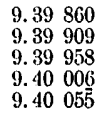 & $\begin{array}{l}49 \\
49 \\
48 \\
49\end{array}$ & $\begin{array}{ll}9.11 & 266 \\
9.41 & 318 \\
9.41 & 370 \\
9.41 & 422 \\
9.41 & 474\end{array}$ & $\begin{array}{r}52 \\
52 \\
52 \\
52\end{array}$ & 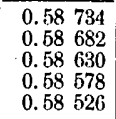 & 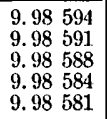 & $\begin{array}{l}3 \\
3 \\
4 \\
3\end{array}$ & $\begin{array}{r}\mathbf{3 0} \\
29 \\
28 \\
27 \\
26\end{array}$ & 2 & $\begin{array}{r}0 \\
56 \\
52 \\
48 \\
44\end{array}$ \\
\hline 58 & $\begin{array}{l}20 \\
24 \\
28 \\
32 \\
36\end{array}$ & $\begin{array}{l}35 \\
36 \\
37 \\
38 \\
39\end{array}$ & 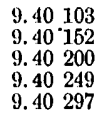 & $\begin{array}{l}49 \\
48 \\
49 \\
48\end{array}$ & $\begin{array}{ll}9.41 & 526 \\
9.41 & 578 \\
9.41 & 629 \\
9.41 & 681 \\
9.41 & 733 \\
\end{array}$ & $\begin{array}{l}52 \\
51 \\
52 \\
52 \\
51\end{array}$ & $\begin{array}{ll}0.58 & 474 \\
0.58 & 422 \\
0.58 & 371 \\
0.58 & 319 \\
0.58 & 267\end{array}$ & 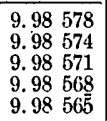 & $\begin{array}{l}4 \\
3 \\
3 \\
3\end{array}$ & $\begin{array}{l}25 \\
24 \\
23 \\
22 \\
21\end{array}$ & 1 & $\begin{array}{l}40 \\
36 \\
32 \\
28 \\
24\end{array}$ \\
\hline 58 & $\begin{array}{l}40 \\
44 \\
48 \\
62 \\
56\end{array}$ & $\begin{array}{r}40 \\
41 \\
42 \\
43 \\
44\end{array}$ & 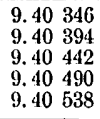 & $\begin{array}{l}48 \\
48 \\
48 \\
48\end{array}$ & $\begin{array}{ll}9.41 & 784 \\
9.41 & 836 \\
9.41 & 887 \\
9.41 & 939 \\
9.41 & 990\end{array}$ & $\begin{array}{l}52 \\
51 \\
52 \\
51\end{array}$ & $\begin{array}{ll}0.58 & 216 \\
0.58 & 164 \\
0.58 & 113 \\
0.58 & 061 \\
0.58 & 01.0\end{array}$ & $\begin{array}{ll}9.98 & 561 \\
9.98 & 558 \\
9.98 & 555 \\
9.98 & 551 \\
9.98 & 548\end{array}$ & $\begin{array}{l}3 \\
3 \\
4 \\
3\end{array}$ & $\begin{array}{l}20 \\
19 \\
18 \\
17 \\
16\end{array}$ & 1 & $\begin{array}{r}20 \\
16 \\
12 \\
8 \\
4\end{array}$ \\
\hline .59 & $\begin{array}{r}0 \\
4 \\
8 \\
12 \\
16\end{array}$ & $\begin{array}{l}45 \\
46 \\
47 \\
48 \\
49\end{array}$ & 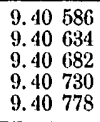 & $\begin{array}{l}48 \\
48 \\
48 \\
48\end{array}$ & $\begin{array}{ll}9.42 & 041 \\
9.42 & 093 \\
9.42 & 144 \\
9.42 & 195 \\
9.42 & 246\end{array}$ & $\begin{array}{l}52 \\
51 \\
51 \\
51 .\end{array}$ & $\begin{array}{ll}0.57 & 959 \\
0.57 & 907 \\
0.57 & 856 \\
0.57 & 805 \\
0.57 & 754\end{array}$ & 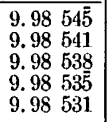 & $\begin{array}{l}4 \\
3 \\
3 \\
4\end{array}$ & $\begin{array}{l}15 \\
14 \\
13 \\
12 \\
11\end{array}$ & 1 & $\begin{array}{r}0 \\
56 \\
52 \\
48 \\
44\end{array}$ \\
\hline 59 & $\begin{array}{l}20 \\
24 \\
28 \\
32 \\
86\end{array}$ & $\begin{array}{l}50 \\
51 \\
52 \\
53 \\
54\end{array}$ & 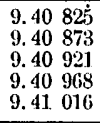 & $\begin{array}{l}48 \\
48 \\
47 \\
48\end{array}$ & $\begin{array}{ll}9.42 & 297 \\
9.42 & 348 \\
9.42 & 399 \\
9.42 & 450 \\
9.42 & 501 \\
\end{array}$ & $\begin{array}{l}61 . \\
51 \\
51 \\
51\end{array}$ & $\begin{array}{ll}0.57 & 703 \\
0.57 & 652 \\
0.57 & 601 \\
0.57 & 550 \\
0.57 & 499\end{array}$ & 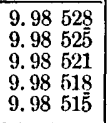 & $\begin{array}{l}3 \\
4 \\
3 \\
3 \\
4\end{array}$ & $\begin{array}{r}10 \\
9 \\
8 \\
7 \\
6\end{array}$ & 0 & $\begin{array}{l}40 \\
36 \\
32 \\
28 \\
24\end{array}$ \\
\hline 59 & $\begin{array}{l}40 \\
44 \\
48 \\
52 \\
56\end{array}$ & $\begin{array}{l}55 \\
56 \\
57 \\
58 \\
59\end{array}$ & $\begin{array}{ll}9.41 & 063 \\
9.41 & 111 \\
9.41 & 158 \\
9.41 & 205 \\
9.41 & 252\end{array}$ & $\begin{array}{l}48 \\
47 \\
47 \\
47\end{array}$ & 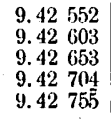 & $\begin{array}{l}51 . \\
50 \\
51 . \\
51 \\
50\end{array}$ & $\begin{array}{ll}0.57 & 448 \\
0.57 & 397 \\
0.57 & 347 \\
0.57 & 296 \\
0.57 & 245\end{array}$ & $\begin{array}{ll}9.98 & 511 \\
9.98 & 508 \\
9.98 & 505 \\
9.98 & 501 \\
9.98 & 498\end{array}$ & $\begin{array}{l}3 \\
3 \\
4 \\
3\end{array}$ & $\begin{array}{l}5 \\
4 \\
3 \\
2 \\
1 .\end{array}$ & 0 & $\begin{array}{r}20 \\
16 \\
12 \\
8 \\
4\end{array}$ \\
\hline 60 & 0 & 60 & 9.41300 & & $9.42 \dot{805}$ & & $0.5719 \overrightarrow{5}$ & $9.98 \quad 494$ & & $\mathbf{0}$ & 0 & 0 \\
\hline & & & I. Cos. & d. & L. Cotg. & c. $d$. & L. Tang. & L. Sin. & $\mathrm{d}$. & ' & & \\
\hline
\end{tabular}


TABLe 19.-Five-place logarithms of circular functions, etc.-Continued.

$1^{\text {h }}$

\begin{tabular}{|c|c|c|c|c|c|c|c|c|c|c|c|c|}
\hline $\mathbf{m}$ & s. & ' & I. Sin. & d. & I. Tang. & c. d. & I. Cotg. & L. Cos. & d. & & & \\
\hline 0 & $\begin{array}{r}0 \\
4 \\
8 \\
12 \\
16\end{array}$ & $\begin{array}{l}0 \\
1 \\
2 \\
3 \\
4\end{array}$ & 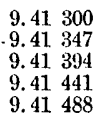 & $\begin{array}{l}47 \\
47 \\
47 \\
47\end{array}$ & 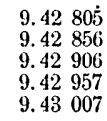 & $\begin{array}{l}51 \\
50 \\
51 \\
50\end{array}$ & $\begin{array}{ll}0.57 & 195 \\
0.57 & 144 \\
0.57 & 094 \\
0.57 & 043 \\
0.56 & 993\end{array}$ & 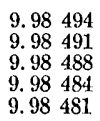 & $\begin{array}{l}3 \\
3 \\
4 \\
3\end{array}$ & $\begin{array}{l}\mathbf{6 0} \\
59 \\
58 \\
57 \\
56\end{array}$ & 60 & $\begin{array}{r}0 \\
56 \\
52 \\
48 \\
44\end{array}$ \\
\hline 0 & $\begin{array}{l}20 \\
24 \\
28 \\
32 \\
36\end{array}$ & $\begin{array}{l}5 \\
6 \\
7 \\
8 \\
9\end{array}$ & 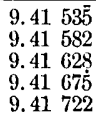 & $\begin{array}{l}47 \\
46 \\
47 \\
47\end{array}$ & $\begin{array}{ll}9.43 & 0.57 \\
9.43 & 108 \\
9.43 & 158 \\
9.43 & 208 \\
9.43 & 258\end{array}$ & $\begin{array}{l}50 \\
51 \\
50 \\
50 \\
50\end{array}$ & $\begin{array}{ll}0.56 & 943 \\
0.56 & 892 \\
0.56 & 842 \\
0.56 & 792 \\
0.56 & 742\end{array}$ & 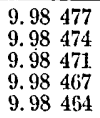 & $\begin{array}{l}4 \\
3 \\
3 \\
4 \\
3\end{array}$ & $\begin{array}{l}55 \\
54 \\
53 \\
52 \\
51\end{array}$ & 59 & $\begin{array}{l}40 \\
36 \\
32 \\
28 \\
24\end{array}$ \\
\hline 0 & $\begin{array}{l}40 \\
44 \\
48 \\
53 \\
56\end{array}$ & $\begin{array}{l}10 \\
11 \\
12 \\
13 \\
14\end{array}$ & $\begin{array}{ll}9.41 & 768 \\
9.41 & 815 \\
9.41 & 861 \\
9.41 & 908 \\
9.41 & 954\end{array}$ & $\begin{array}{l}47 \\
46 \\
47 \\
46\end{array}$ & 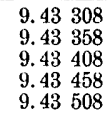 & $\begin{array}{l}50 \\
50 \\
50 \\
50\end{array}$ & $\begin{array}{ll}0.56 & 692 \\
0.56 & 642 \\
0.56 & 592 \\
0.56 & 542 \\
0.56 & 492\end{array}$ & 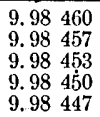 & $\begin{array}{l}3 \\
4 \\
3 \\
3\end{array}$ & $\begin{array}{l}50 \\
49 \\
48 \\
47 \\
46\end{array}$ & 59 & $\begin{array}{r}20 \\
16 \\
12 \\
8 \\
4\end{array}$ \\
\hline 1 & $\begin{array}{r}0 \\
4 \\
8 \\
12 \\
16\end{array}$ & $\begin{array}{l}15 \\
16 \\
17 \\
18 \\
19\end{array}$ & $\begin{array}{ll}9.42 & 001 \\
9.42 & 047 \\
9.42 & 093 \\
9.42 & 140 \\
9.42 & 186\end{array}$ & $\begin{array}{l}46 \\
46 \\
47 \\
46\end{array}$ & 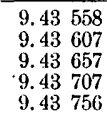 & $\begin{array}{l}49 \\
50 \\
50 \\
49\end{array}$ & $\begin{array}{ll}0.56 & 442 \\
0.56 & 393 \\
0.56 & 343 \\
0.56 & 293 \\
0.56 & 244\end{array}$ & 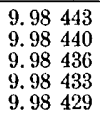 & $\begin{array}{l}3 \\
4 \\
3 \\
4\end{array}$ & $\begin{array}{l}45 \\
44 \\
43 \\
42 \\
41\end{array}$ & 59 & $\begin{array}{r}0 \\
56 \\
52 \\
48 \\
44\end{array}$ \\
\hline 1 & $\begin{array}{l}20 \\
24 \\
28 \\
32 \\
36\end{array}$ & $\begin{array}{l}\mathbf{2 0} \\
21 \\
22 \\
23 \\
24\end{array}$ & 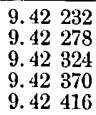 & $\begin{array}{l}46 \\
46 \\
46 \\
46\end{array}$ & 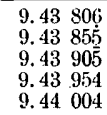 & $\begin{array}{l}49 \\
50 \\
49 \\
50\end{array}$ & $\begin{array}{ll}0.56 & 194 \\
0.56 & 145 \\
0.56 & 095 \\
0.56 & 046 \\
0.55 & 996\end{array}$ & 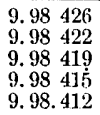 & $\begin{array}{l}4 \\
3 \\
4 \\
3\end{array}$ & $\begin{array}{l}\mathbf{4 0} \\
39 \\
38 \\
37 \\
36\end{array}$ & 58 & $\begin{array}{l}40 \\
36 \\
32 \\
28 \\
24\end{array}$ \\
\hline 1 & $\begin{array}{l}40 \\
44 \\
48 \\
52 \\
56\end{array}$ & $\begin{array}{l}25 \\
26 \\
27 \\
28 \\
29\end{array}$ & 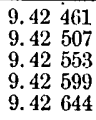 & $\begin{array}{l}46 \\
46 \\
46 \\
45\end{array}$ & $\begin{array}{ll}9.44 & 053 \\
9.44 & 102 \\
9.44 & 151 \\
9.44 & 201 \\
9.44 & 250\end{array}$ & $\begin{array}{l}49 \\
49 \\
50 \\
49\end{array}$ & $\begin{array}{ll}0.55 & 947 \\
0.55 & 898 \\
0.55 & 849 \\
0.55 & 799 \\
0.55 & 750\end{array}$ & $\begin{array}{ll}9.98 & 409 \\
9.98 & 405 \\
9.98 & 402 \\
9.98 & 398 \\
9.98 & 395\end{array}$ & $\begin{array}{l}4 \\
3 \\
4 \\
3\end{array}$ & $\begin{array}{l}35 \\
34 \\
33 \\
32 \\
31\end{array}$ & 58 & $\begin{array}{r}20 \\
16 \\
12 \\
8 \\
4\end{array}$ \\
\hline 2 & $\begin{array}{r}0 \\
4 \\
8 \\
12 \\
16\end{array}$ & $\begin{array}{l}\mathbf{3 0} \\
31 \\
32 \\
33 \\
34\end{array}$ & 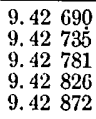 & $\begin{array}{l}45 \\
46 \\
45 \\
46\end{array}$ & 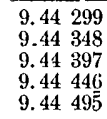 & $\begin{array}{l}49 \\
49 \\
49 \\
49 \\
49\end{array}$ & $\begin{array}{ll}0.55 & 701 \\
0.55 & 652 \\
0.55 & 603 \\
0.55 & 554 \\
0.55 & 505\end{array}$ & $\begin{array}{ll}9.98 & 391 . \\
9.98 & 388 \\
9.98 & 384 \\
9.98 & 381 \\
9.98 & 377\end{array}$ & $\begin{array}{l}3 \\
4 \\
3 \\
4\end{array}$ & $\begin{array}{l}30 \\
29 \\
28 \\
27 \\
26\end{array}$ & 58 & $\begin{array}{r}0 \\
56 \\
52 \\
48 \\
44\end{array}$ \\
\hline 2 & $\begin{array}{l}20 \\
24 \\
28 \\
32 \\
36\end{array}$ & $\begin{array}{l}35 \\
36 \\
37 \\
38 \\
39\end{array}$ & $\begin{array}{ll}9.42 & 917 \\
9.42 & 962 \\
9.43 & 008 \\
9.43 & 053 \\
9.43 & 098\end{array}$ & $\begin{array}{l}45 \\
46 \\
45 \\
45\end{array}$ & 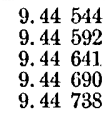 & $\begin{array}{r}48 \\
49 \\
49 \\
. \quad 48\end{array}$ & $\begin{array}{ll}0.55 & 456 \\
0.55 & 408 \\
0.55 & 359 \\
0.55 & 310 \\
0.55 & 262\end{array}$ & 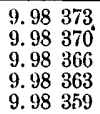 & $\begin{array}{l}3 \\
4 \\
3 \\
4\end{array}$ & $\begin{array}{l}25 \\
24 \\
23 \\
22 \\
21\end{array}$ & 57 & $\begin{array}{l}40 \\
36 \\
32 \\
28 \\
24\end{array}$ \\
\hline 2 & $\begin{array}{l}40 \\
44 \\
48 \\
52 \\
56\end{array}$ & $\begin{array}{l}40 \\
41 \\
42 \\
43 \\
44\end{array}$ & $\begin{array}{ll}9.43 & 143 \\
9.43 & 188 \\
9.43 & 233 \\
9.43 & 278 \\
9.43 & 323\end{array}$ & $\begin{array}{l}45 \\
45 \\
45 \\
45\end{array}$ & 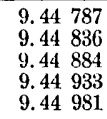 & $\begin{array}{l}49 \\
48 \\
49 \\
48\end{array}$ & $\begin{array}{ll}0.55 & 213 \\
0.55 & 164 \\
0.55 & 116 \\
0.55 & 067 \\
0.55 & 019\end{array}$ & 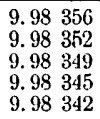 & $\begin{array}{l}4 \\
3 \\
4 \\
3\end{array}$ & $\begin{array}{l}\mathbf{3 0} \\
19 \\
18 \\
17 \\
16\end{array}$ & 57 & $\begin{array}{r}20 \\
16 \\
12 \\
8 \\
4\end{array}$ \\
\hline 3 & $\begin{array}{r}0 \\
4 \\
8 \\
12 \\
16\end{array}$ & $\begin{array}{l}45 \\
46 \\
47 \\
48 \\
49\end{array}$ & 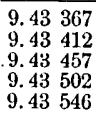 & $\begin{array}{l}45 \\
45 \\
45 \\
44\end{array}$ & $\begin{array}{ll}9.45 & 029 \\
9.45 & 078 \\
9.45 & 126 \\
\mathbf{9 . 4 5} & 174 \\
9.45 & 222\end{array}$ & $\begin{array}{l}49 \\
48 \\
48 \\
48\end{array}$ & $\begin{array}{ll}0.54 & 971 \\
0.54 & 922 \\
0.54 & 874 \\
0.54 & 826 \\
0.54 & 778\end{array}$ & 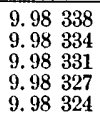 & $\begin{array}{l}4 \\
3 \\
4 \\
3\end{array}$ & $\begin{array}{l}15 \\
14 \\
13 \\
12 \\
11\end{array}$ & 57 & $\begin{array}{r}0 \\
56 \\
52 \\
48 \\
44\end{array}$ \\
\hline 3 & $\begin{array}{l}20 \\
24 \\
28 \\
32 \\
36\end{array}$ & $\begin{array}{r}\mathbf{5 0} \\
51 \\
-52 \\
53 \\
54\end{array}$ & $\begin{array}{ll}9.43 & 591 \\
9.43 & 635 \\
9.43 & 680 \\
9.43 & 724 \\
9.43 & 769 \\
\end{array}$ & $\begin{array}{l}44 \\
45 \\
44 \\
45\end{array}$ & $\begin{array}{ll}9.45 & 271 \\
9.45 & 319 \\
9.45 & 367 \\
9.45 & 415 \\
9.45 & 463\end{array}$ & $\begin{array}{l}48 \\
48 \\
48 \\
48\end{array}$ & 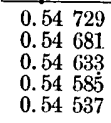 & 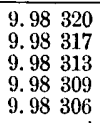 & $\begin{array}{l}3 \\
4 \\
4 \\
3\end{array}$ & $\begin{array}{r}\mathbf{1 0} \\
9 \\
8 \\
7 \\
6\end{array}$ & 56 & $\begin{array}{l}40 \\
36 \\
32 \\
28 \\
24\end{array}$ \\
\hline 3 & $\begin{array}{l}40 \\
44 \\
48 \\
52 \\
56\end{array}$ & $\begin{array}{l}55 \\
56 \\
57 \\
58 \\
59\end{array}$ & $\begin{array}{ll}9.43 & 813 \\
9.43 & 857 \\
9.43 & 901 \\
9.43 & 946 \\
9.43 & 990\end{array}$ & $\begin{array}{l}44 \\
44 \\
45 \\
44\end{array}$ & $\begin{array}{ll}9.45 & 511 \\
9.45 & 559 \\
9.45 & 606 \\
9.45 & 654 \\
9.45 & 702\end{array}$ & $\begin{array}{l}48 \\
47 \\
48 \\
48\end{array}$ & $\begin{array}{ll}0.54 & 489 \\
0.54 & 441 \\
0.54 & 394 \\
0.54 & 346 \\
0.54 & 298\end{array}$ & $\begin{array}{ll}9.98 & 302 \\
9.98 & 299 \\
9.98 & 295 \\
9.98 & 291 \\
9.98 & 288\end{array}$ & $\begin{array}{l}3 \\
4 \\
4 \\
3\end{array}$ & $\begin{array}{l}5 \\
4 \\
3 \\
2 \\
1\end{array}$ & 56 & $\begin{array}{r}20 \\
16 \\
12 \\
8 \\
4\end{array}$ \\
\hline 4 & 0 & 60 & 9.44034 & & $9.457 \overline{50}$ & 48 & $0.542 \dot{5} 0$ & 9.98284 & 4 & O & 56 & 0 \\
\hline & & & L. Cos. & d. & L. Cotg. & c. d. & L. Tang. & L. Sin. & d. & ' & $\mathrm{m}$. & s. \\
\hline
\end{tabular}


Table 19.-Five-place logarillms of circular functions, etc.-Continued.

$1^{\text {h }}$

\begin{tabular}{|c|c|c|c|c|c|c|c|c|c|c|c|c|}
\hline m. & s. & ' & L. $\operatorname{Sin}$. & d. & L. Tang. & c. d. & L. Cotg. & L. $\operatorname{Cos}$. & d. & & & \\
\hline 4 & $\begin{array}{r}0 \\
4 \\
8 \\
12 \\
16\end{array}$ & $\begin{array}{l}0 \\
1 \\
2 \\
3 \\
4\end{array}$ & $\begin{array}{ll}9.44 & 034 \\
9.44 & 078 \\
9.44 & 122 \\
9.44 & 166 \\
9.44 & 210\end{array}$ & \multirow{3}{*}{$\begin{array}{l}44 \\
44 \\
44 \\
44 \\
43 \\
44 \\
44 \\
44 \\
43 \\
44 \\
44 \\
43 \\
43 \\
44\end{array}$} & 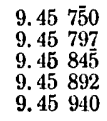 & \multirow{2}{*}{$\begin{array}{l}47 \\
48 \\
47 \\
48 \\
47 \\
48 \\
47 \\
48 \\
47 \\
47\end{array}$} & $\begin{array}{ll}0.54 & 250 \\
0.54 & 203 \\
0.54 & 155 \\
0.54 & 108 \\
0.54 & 060\end{array}$ & $\begin{array}{ll}9.98 & 284 \\
9.98 & 281 \\
9.98 & 277 \\
9.98 & 273 \\
9.98 & 270\end{array}$ & \multirow{2}{*}{$\begin{array}{l}3 \\
4 \\
4 \\
3 \\
4 \\
4 \\
3 \\
4 \\
4\end{array}$} & $\begin{array}{l}60 \\
59 \\
58 \\
57 \\
56\end{array}$ & 56 & $\begin{array}{r}0 \\
56 \\
52 \\
48 \\
44\end{array}$ \\
\hline 4 & $\begin{array}{l}20 \\
24 \\
28 \\
32 \\
36\end{array}$ & $\begin{array}{l}5 \\
6 \\
7 \\
8 \\
9\end{array}$ & $\begin{array}{ll}9.44 & 253 \\
9.44 & 297 \\
9.44 & 341 \\
9.44 & 385 \\
9.44 & 428\end{array}$ & & $\begin{array}{ll}9.45 & 987 \\
9.46 & 035 \\
9.46 & 082 \\
9.46 & 130 \\
9.46 & 177\end{array}$ & & $\begin{array}{ll}0.54 & 013 \\
0.53 & 965 \\
0.53 & 918 \\
0.53 & 870 \\
0.53 & 823\end{array}$ & $\begin{array}{ll}9.98 & 266 \\
9.98 & 262 \\
9.98 & 259 \\
9.98 & 255 \\
9.98 & 251\end{array}$ & & $\begin{array}{l}55 \\
54 \\
53 \\
52 \\
51\end{array}$ & 55 & $\begin{array}{l}40 \\
36 \\
32 \\
28 \\
24\end{array}$ \\
\hline 4 & $\begin{array}{l}40 \\
44 \\
48 \\
52 \\
56\end{array}$ & $\begin{array}{l}10 \\
11 \\
12 \\
13 \\
14\end{array}$ & 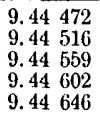 & & $\begin{array}{ll}9.46 & 224 \\
9.46 & 271 \\
9.46 & 319 \\
9.46 & 366 \\
9.46 & 413\end{array}$ & $\begin{array}{l}47 \\
48 \\
47 \\
47\end{array}$ & $\begin{array}{ll}0.53 & 776 \\
0.53 & 729 \\
0.53 & 681 \\
0.53 & 634 \\
0.53 & 587\end{array}$ & $\begin{array}{ll}9.98 & 248 \\
9.98 & 244 \\
9.98 & 240 \\
9.98 & 237 \\
9.98 & 233\end{array}$ & $\begin{array}{l}4 \\
4 \\
3 \\
4\end{array}$ & $\begin{array}{l}\mathbf{5 0} \\
49 \\
48 \\
47 \\
46\end{array}$ & 55 & $\begin{array}{r}20 \\
16 \\
12 \\
8 \\
4\end{array}$ \\
\hline 5 & $\begin{array}{r}0 \\
.4 \\
8 \\
12 \\
16\end{array}$ & $\begin{array}{l}15 \\
16 \\
17 \\
18 \\
19\end{array}$ & $\begin{array}{l}9.44689 \\
9.44733 \\
9.44776 \\
9.44819 \\
9.44862\end{array}$ & $\begin{array}{l}44 \\
43 \\
43 \\
43\end{array}$ & 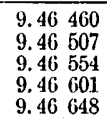 & $\begin{array}{l}47 \\
47 \\
47 \\
47\end{array}$ & $\begin{array}{ll}0.53 & 540 \\
0.53 & 493 \\
0.53 & 446 \\
0.53 & 399 \\
0.53 & 352\end{array}$ & $\begin{array}{ll}9.98 & 229 \\
9.98 & 226 \\
9.98 & 222 \\
9.98 & 218 \\
9.98 & 215\end{array}$ & $\begin{array}{l}3 \\
4 \\
4 \\
3 \\
4\end{array}$ & $\begin{array}{l}45 \\
44 \\
43 \\
42 \\
41\end{array}$ & 55 & $\begin{array}{r}0 \\
56 \\
52 \\
48 \\
44\end{array}$ \\
\hline 5 & $\begin{array}{l}20 \\
24 \\
28 \\
32 \\
36\end{array}$ & $\begin{array}{l}\mathbf{2 0} \\
21 \\
22 \\
23 \\
24\end{array}$ & 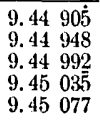 & $\begin{array}{l}43 \\
44 \\
43 \\
42\end{array}$ & 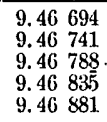 & $\begin{array}{l}47 \\
47 \\
47 \\
46\end{array}$ & $\begin{array}{ll}0.53 & 306 \\
0.53 & 259 \\
0.53 & 212 \\
0.53 & 165 \\
0.53 & 119\end{array}$ & $\begin{array}{ll}9.98 & 211 \\
9.98 & 207 \\
9.98 & 204 \\
9.98 & 200 \\
9.98 & 196\end{array}$ & $\begin{array}{l}4 \\
3 \\
4 \\
4\end{array}$ & $\begin{array}{l}\mathbf{4 0} \\
39 \\
38 \\
37 \\
36\end{array}$ & 54 & $\begin{array}{l}40 \\
36 \\
32 \\
28 \\
24\end{array}$ \\
\hline 5 & $\begin{array}{l}40 \\
44 \\
48 \\
52 \\
56\end{array}$ & $\begin{array}{l}25 \\
26 \\
27 \\
28 \\
29\end{array}$ & $\begin{array}{ll}9.45 & 120 \\
9.45 & 163 \\
9.45 & 206 \\
9.45 & 249 \\
9.45 & 292\end{array}$ & $\begin{array}{l}43 \\
43 \\
43 \\
43\end{array}$ & $\begin{array}{ll}9.46 & 928 \\
9.46 & 975 \\
9.47 & 021 \\
9.47 & 068 \\
9.47 & 114\end{array}$ & $\begin{array}{l}47 \\
46 \\
47 \\
46\end{array}$ & $\begin{array}{ll}0.53 & 072 \\
0.53 & 025 \\
0.52 & 979 \\
0.52 & 932 \\
0.52 & 886\end{array}$ & $\begin{array}{ll}9.98 & 192 \\
9.98 & 189 \\
9.98 & 185 \\
9.98 & 181 \\
9.98 & 177\end{array}$ & $\begin{array}{l}3 \\
4 \\
4 \\
4\end{array}$ & $\begin{array}{l}35 \\
34 \\
33 \\
32 \\
31\end{array}$ & 54 & $\begin{array}{r}20 \\
16 \\
12 \\
8 \\
4\end{array}$ \\
\hline .6 & $\begin{array}{r}0 \\
4 \\
8 \\
12 \\
16\end{array}$ & $\begin{array}{l}30 \\
31 \\
32 \\
33 \\
34\end{array}$ & 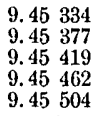 & $\begin{array}{l}43 \\
42 \\
43 \\
42\end{array}$ & $\begin{array}{ll}9.47 & 160 \\
9.47 & 207 \\
9.47 & 253 \\
9.47 & 299 \\
9.47 & 346\end{array}$ & $\begin{array}{l}47 \\
46 \\
46 \\
47\end{array}$ & $\begin{array}{ll}0.52 & 840 \\
0.52 & 793 \\
0.52 & 747 \\
0.52 & 701 \\
0.52 & 654\end{array}$ & $\begin{array}{ll}9.98 & 174 \\
9.98 & 170 \\
9.98 & 166 \\
9.98 & 162 \\
9.98 & 159\end{array}$ & $\begin{array}{l}4 \\
4 \\
4 \\
3\end{array}$ & $\begin{array}{l}\mathbf{3 0} \\
29 \\
28 \\
27 \\
26\end{array}$ & 54 & $\begin{array}{r}0 \\
56 \\
52 \\
48 \\
44\end{array}$ \\
\hline 6 & $\begin{array}{l}20 \\
24 \\
28 \\
32 \\
36\end{array}$ & $\begin{array}{l}35 \\
36 \\
37 \\
38 \\
39\end{array}$ & 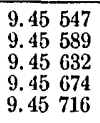 & $\begin{array}{l}42 \\
43 \\
42 \\
42\end{array}$ & $\begin{array}{ll}9.47 & 392 \\
9.47 & 438 \\
9.47 & 484 \\
9.47 & 530 \\
9.47 & 576\end{array}$ & $\begin{array}{l}46 \\
46 \\
46 \\
46\end{array}$ & $\begin{array}{ll}0.52 & 608 \\
0.52 & 562 \\
0.52 & 516 \\
0.52 & 470 \\
0.52 & 424\end{array}$ & $\begin{array}{lll}9.98 & 155 \\
9.98 & 15.1 \\
9.98 & 147 \\
9.98 & 144 \\
9.98 & 140\end{array}$ & $\begin{array}{l}4 \\
4 \\
3 \\
4 \\
4\end{array}$ & $\begin{array}{l}25 \\
24 \\
23 \\
22 \\
21\end{array}$ & 53 & $\begin{array}{l}40 \\
36 \\
32 \\
28 \\
24\end{array}$ \\
\hline 6 & $\begin{array}{l}40 \\
44 \\
48 \\
52 \\
56\end{array}$ & $\begin{array}{l}40 \\
41 \\
42 \\
43 \\
44\end{array}$ & 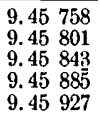 & $\begin{array}{l}43 \\
42 \\
42 \\
42\end{array}$ & 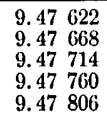 & $\begin{array}{l}46 \\
46 \\
46 \\
46\end{array}$ & $\begin{array}{ll}0.52 & 378 \\
0.52 & 332 \\
0.52 & 286 \\
0.52 & 240 \\
0.52 & 194\end{array}$ & $\begin{array}{ll}9.98 & 136 \\
9.98 & 132 \\
9.98 & 129 \\
9.98 & 125 \\
9.98 & 121\end{array}$ & $\begin{array}{l}4 \\
3 \\
4 \\
4 \\
4\end{array}$ & $\begin{array}{l}\mathbf{2 0} \\
19 \\
18 \\
17 \\
16\end{array}$ & 53 & $\begin{array}{r}20 \\
16 \\
12 \\
8 \\
4\end{array}$ \\
\hline 7 & $\begin{array}{r}0 \\
4 \\
8 \\
12 \\
16\end{array}$ & $\begin{array}{l}45 \\
46 \\
47 \\
48 \\
49\end{array}$ & $\begin{array}{ll}9.45 & 969 \\
9.46 & 011 \\
9.46 & 053 \\
9.46 & 095 \\
9.46 & 136\end{array}$ & $\begin{array}{l}42 \\
42 \\
42 \\
41\end{array}$ & 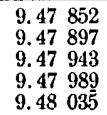 & $\begin{array}{l}46 \\
46 \\
46 \\
46\end{array}$ & $\begin{array}{ll}0.52 & 148 \\
0.52 & 103 \\
0.52 & 057 \\
0.52 & 011 \\
0.51 & 965\end{array}$ & $\begin{array}{ll}9.98 & 117 \\
9.98 & 113 \\
9.98 & 110 \\
9.98 & 106 \\
9.98 & 102\end{array}$ & $\begin{array}{l}4 \\
3 \\
4 \\
4\end{array}$ & $\begin{array}{l}15 \\
14 \\
13 \\
12 \\
11\end{array}$ & 63 & $\begin{array}{r}0 \\
56 \\
52 \\
48 \\
44\end{array}$ \\
\hline 7 & $\begin{array}{l}20 \\
24 \\
28 \\
32 \\
36\end{array}$ & $\begin{array}{l}50 \\
51 \\
52 \\
53 \\
54\end{array}$ & $\begin{array}{ll}\mathbf{9 . 4 6} & \mathbf{1 7 8} \\
\mathbf{9 . 4 6} & 220 \\
\mathbf{9 . 4 6} & 262 \\
\mathbf{9 . 4 6} & 303 \\
\mathbf{9 . 4 6} & 345\end{array}$ & $\begin{array}{l}42 \\
42 \\
41 \\
42\end{array}$ & 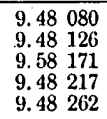 & $\begin{array}{l}46 \\
45 \\
46 \\
45\end{array}$ & $\begin{array}{ll}0.51 & 920 \\
0.51 & 874 \\
0.51 & 829 \\
0.51 & 783 \\
0.51 & 738\end{array}$ & $\begin{array}{ll}9.98 & 098 \\
9.98 & 094 \\
9.98 & 090 \\
9.98 & 087 \\
9.98 & 083\end{array}$ & $\begin{array}{l}4 \\
4 \\
3 \\
4 \\
4\end{array}$ & $\begin{array}{r}10 \\
9 \\
8 \\
7 \\
6\end{array}$ & 52 & $\begin{array}{l}40 \\
36 \\
32 \\
28 \\
24\end{array}$ \\
\hline 7 & $\begin{array}{l}40 \\
44 \\
48 \\
52 \\
56\end{array}$ & $\begin{array}{l}55 \\
56 \\
57 \\
58 \\
59\end{array}$ & $\begin{array}{ll}9.46 & 386 \\
9.46 & 428 \\
9.46 & 469 \\
9.46 & 511 \\
9.46 & 552\end{array}$ & $\begin{array}{l}42 \\
41 \\
42 \\
41\end{array}$ & 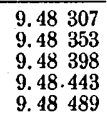 & $\begin{array}{l}46 \\
45 \\
45 \\
46\end{array}$ & $\begin{array}{ll}0.51 & 693 \\
0.51 & 647 \\
0.51 & 602 \\
0.51 & 557 \\
0.51 & 511\end{array}$ & $\begin{array}{lll}9.98 & 079 \\
9.98 & 075 \\
9.98 & 071 \\
9.98 & 067 \\
9.98 & 063\end{array}$ & $\begin{array}{l}4 \\
4 \\
4 \\
4 \\
3\end{array}$ & $\begin{array}{l}5 \\
4 \\
3 \\
2 \\
1\end{array}$ & 52 & $\begin{array}{r}20 \\
16 \\
12 \\
8 \\
4\end{array}$ \\
\hline 8 & $\overline{0}$ & 60 & 9.46594 & & 9.48534 & & $0.51: 466$ & 9.98060 & & $\overline{0}$ & 52 & 0 \\
\hline & & & L. $\operatorname{Cos}$ & d. & L. Cotg. & c.d. & L. Tang & L. $\operatorname{Sin}$. & d. & , & $\mathrm{m}$ & s. \\
\hline
\end{tabular}


TaвLe 19. - Five-place logarithms of circular functions, etc.-Continued.

$1^{\text {h }}$

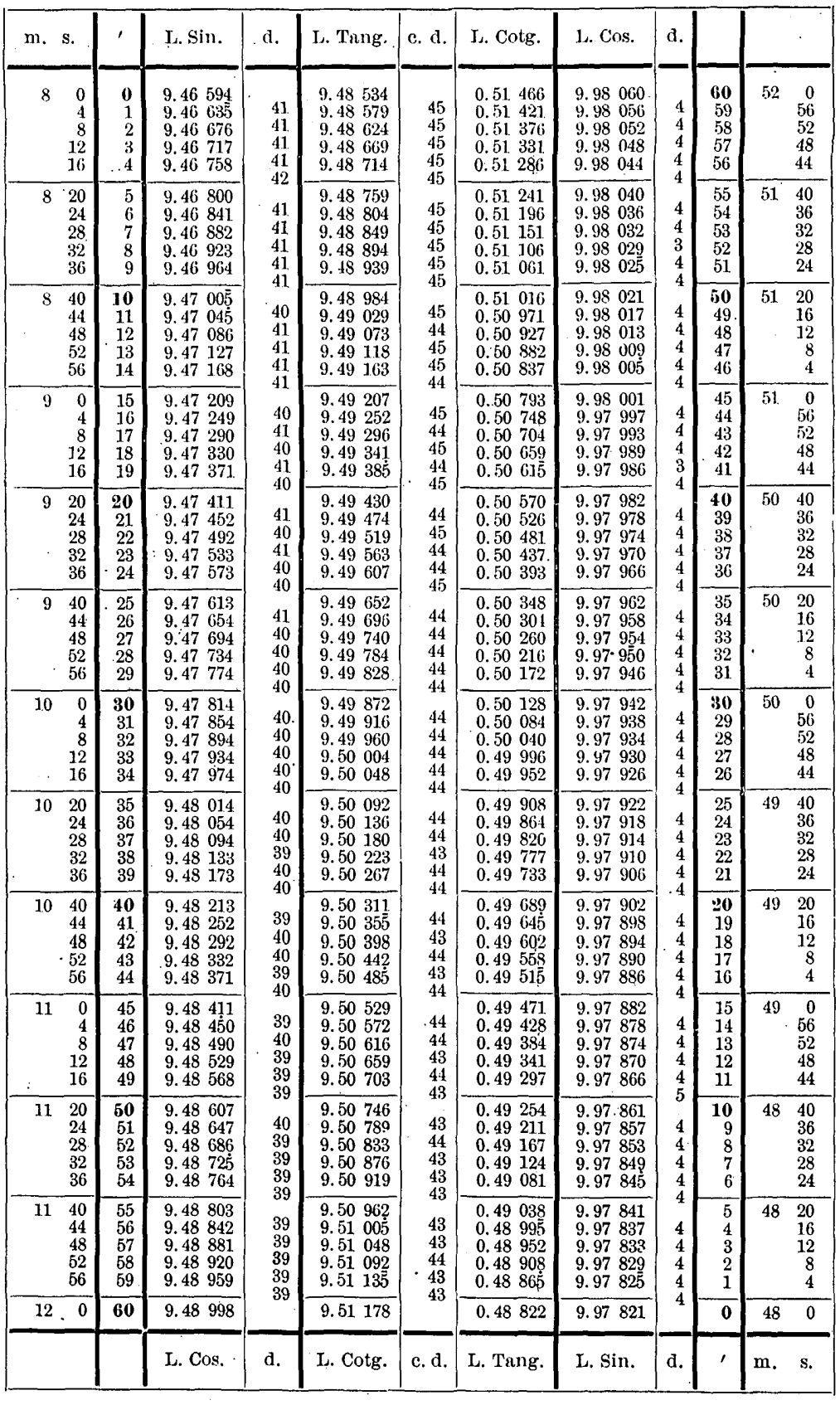


TABLE 19.-Five-place logarithms of circular functions, etc.-Continued.

$1^{\mathrm{h}}$

\begin{tabular}{|c|c|c|c|c|c|c|c|c|c|c|c|c|}
\hline m. s & s. & 1 & I. Sin. & d. & L. Tang. & c. $\mathrm{d}$. & I. Cotg. & I. Cos. & d. & & & \\
\hline 12 & $\begin{array}{r}0 \\
4 \\
8 \\
\cdot .12 \\
16\end{array}$ & $\begin{array}{l}0 \\
1 \\
2 \\
3 \\
4\end{array}$ & $\begin{array}{ll}9.48 & 998 \\
9.49 & 037 \\
9.49 & 076 \\
9.49 & 11 \overline{5} \\
9.49 & 153\end{array}$ & $\begin{array}{l}39 \\
39 \\
39 \\
38 \\
39\end{array}$ & $\begin{array}{ll}9.51 & 178 \\
9.51 & 221 \\
9.51 & 264 \\
9: 51 & 306 \\
9.51 & 349\end{array}$ & $\begin{array}{l}43 \\
43 \\
42 \\
43 \\
43\end{array}$ & $\begin{array}{ll}0.48 & 822 \\
0.48 & 779 \\
0.48 & 736 \\
0.48 & 694 \\
0.48 & 651\end{array}$ & $\begin{array}{l}9.97821 \\
9.97817 \\
9.97812 \\
9.97808 \\
9.97804\end{array}$ & $\begin{array}{l}4 \\
5 \\
4 \\
4\end{array}$ & $\begin{array}{l}\mathbf{6 0} \\
59 \\
58 \\
57 \\
56\end{array}$ & 48 & $\begin{array}{r}0 \\
56 \\
52 \\
48 \\
44\end{array}$ \\
\hline 12 & $\begin{array}{l}20 \\
24 \\
28 \\
32 \\
36\end{array}$ & $\begin{array}{l}5 \\
6 \\
7 \\
8 \\
9\end{array}$ & $\begin{array}{ll}9.49 & 192 \\
9.49 & 231 \\
9.49 & 269 \\
9.49 & 308 \\
9.49 & 347\end{array}$ & $\begin{array}{l}39 \\
38 \\
39 \\
39 \\
38\end{array}$ & $\begin{array}{ll}9.51 & 392 \\
9.51 & 435 \\
9.51 & 478 \\
9.51 & 520 \\
9.51 & 563\end{array}$ & $\begin{array}{l}43 \\
43 \\
42 \\
43 \\
43\end{array}$ & $\begin{array}{ll}0.48 & 608 \\
0.48 & 565 \\
0.48 & 522 \\
0.48 & 480 \\
0.48 & 437\end{array}$ & 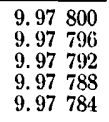 & $\begin{array}{l}4 \\
4 \\
4 \\
4 \\
5\end{array}$ & $\begin{array}{l}55 \\
54 \\
53 \\
52 \\
51\end{array}$ & 47 & $\begin{array}{l}40 \\
36 \\
32 \\
28 \\
24\end{array}$ \\
\hline 12 & $\begin{array}{l}40 \\
44 \\
48 \\
52 \\
56\end{array}$ & $\begin{array}{l}10 \\
11 \\
12 \\
13 \\
14\end{array}$ & 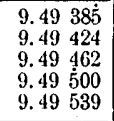 & $\begin{array}{l}39 \\
33 \\
38 \\
39 \\
38\end{array}$ & $\begin{array}{ll}9.51 & 606 \\
9.51 & 648 \\
9.51 & 691 \\
9.51 & 734 \\
9.51 & 776\end{array}$ & $\begin{array}{l}42 \\
43 \\
43 \\
42 \\
43\end{array}$ & $\begin{array}{ll}0.48 & 394 \\
0.48 & 352 \\
0.48 & 309 \\
0.48 & 266 \\
0.48 & 224\end{array}$ & 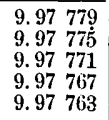 & $\begin{array}{l}4 \\
4 \\
4 \\
4 \\
4\end{array}$ & $\begin{array}{l}50 \\
49 \\
48 \\
47 \\
46\end{array}$ & 47 & $\begin{array}{r}20 \\
16 \\
12 \\
8 \\
4\end{array}$ \\
\hline 13 & $\begin{array}{r}0 \\
4 \\
8 \\
12 \\
16\end{array}$ & $\begin{array}{l}15 \\
16 \\
17 \\
18 \\
19\end{array}$ & $\begin{array}{ll}9.49 & 577 \\
9.49 & 615 \\
9.49 & 654 \\
9.49 & 692 \\
9.49 & 730\end{array}$ & $\begin{array}{l}38 \\
39 \\
38 \\
38 \\
38\end{array}$ & $\begin{array}{ll}9.51 & 819 \\
9.51 & 861 \\
9.51 & 903 \\
9.51 & 946 \\
9.51 & 988\end{array}$ & $\begin{array}{l}42 \\
42 \\
43 \\
42 \\
43\end{array}$ & $\begin{array}{ll}0.48 & 181 \\
0.48 & 139 \\
0.48 & 097 \\
0.48 & 054 \\
0.48 & 012\end{array}$ & 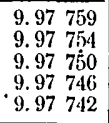 & $\begin{array}{l}5 \\
4 \\
4 \\
4 \\
4\end{array}$ & $\begin{array}{l}45 \\
44 \\
43 \\
42 \\
41\end{array}$ & 47 & $\begin{array}{r}0 \\
56 \\
52 \\
48 \\
44\end{array}$ \\
\hline 13 & $\begin{array}{l}20 \\
24 \\
28 \\
32 \\
36\end{array}$ & $\begin{array}{l}\mathbf{2 0} \\
21 \\
22 \\
23 \\
24\end{array}$ & 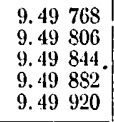 & $\begin{array}{l}38 \\
38 \\
38 \\
38 \\
38\end{array}$ & $\begin{array}{ll}9.52 & 031 \\
9.52 & 073 \\
9.52 & 115 \\
9.52 & 157 \\
9.52 & 200\end{array}$ & $\begin{array}{l}42 \\
42 \\
42 \\
43 \\
42\end{array}$ & $\begin{array}{ll}0.47 & 969 \\
0.47 & 927 \\
0.47 & 885 \\
0.47 & 843 \\
0.47 & 800\end{array}$ & 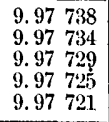 & $\begin{array}{l}4 \\
5 \\
4 \\
4 \\
4\end{array}$ & $\begin{array}{l}40 \\
39 \\
38 \\
37 \\
36\end{array}$ & 46 & $\begin{array}{l}40 \\
36 \\
32 \\
28 \\
24\end{array}$ \\
\hline 13 & $\begin{array}{l}40 \\
44 \\
48 \\
52 \\
56\end{array}$ & $\begin{array}{l}25 \\
26 \\
27 \\
28 \\
29\end{array}$ & 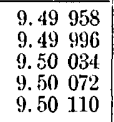 & $\begin{array}{l}38 \\
38 \\
38 \\
38 \\
38\end{array}$ & $\begin{array}{ll}9.52 & 242 \\
9.52 & 284 \\
9.52 & 326 \\
9.52 & 368 \\
9.52 & 410\end{array}$ & $\begin{array}{l}42 \\
42 \\
42 \\
42 \\
42\end{array}$ & $\begin{array}{ll}0.47 & 758 \\
0.47 & 716 \\
0.47 & 674 \\
0.47 & 632 \\
0.47 & 590\end{array}$ & $\begin{array}{ll}9.97 & 717 \\
9.97 & 713 \\
9.97 & 708 \\
9.97 & 704 \\
9.97 & 700\end{array}$ & $\begin{array}{l}4 \\
5 \\
4 \\
4 \\
4\end{array}$ & $\begin{array}{l}35 \\
34 \\
33 \\
32 \\
31\end{array}$ & 46 & $\begin{array}{r}20 \\
16 \\
12 \\
8 \\
4\end{array}$ \\
\hline 14 & $\begin{array}{r}0 \\
4 \\
8 \\
12 \\
16\end{array}$ & $\begin{array}{l}30 \\
31 \\
32 \\
33 \\
34\end{array}$ & $\begin{array}{lll}9.50 & 148 \\
9.50 & 185 \\
9.50 & 223 \\
9.50 & 261 \\
9.50 & 298\end{array}$ & $\begin{array}{l}37 \\
38 \\
38 \\
37 \\
38\end{array}$ & $\begin{array}{ll}9.52 & 452 \\
9.52 & 494 \\
9.52 & 536 \\
9.52 & 578 \\
9.52 & 620\end{array}$ & $\begin{array}{l}42 \\
42 \\
42 \\
42 \\
41\end{array}$ & $\begin{array}{ll}0.47 & 548 \\
0.47 & 506 \\
0.47 & 464 \\
0.47 & 422 \\
0.47 & 380\end{array}$ & $\begin{array}{l}9.97696 \\
9.97691 \\
9.97687 \\
9.97683 \\
9.97679\end{array}$ & $\begin{array}{l}5 \\
4 \\
4 \\
4 \\
5\end{array}$ & $\begin{array}{l}\mathbf{3 0} \\
29 \\
28 \\
27 \\
26\end{array}$ & 46 & $\begin{array}{r}0 \\
56 \\
52 \\
48 \\
44\end{array}$ \\
\hline 14 & $\begin{array}{l}20 \\
24 \\
28 \\
32 \\
36\end{array}$ & $\begin{array}{l}35 \\
36 \\
37 \\
38 \\
39\end{array}$ & $\begin{array}{lll}9.50 & 336 \\
9.50 & 374 \\
9.50 & 411 \\
9.50 & 449 \\
9.50 & 486\end{array}$ & $\begin{array}{l}38 \\
37 \\
38 \\
37 \\
37\end{array}$ & 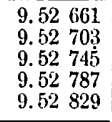 & $\begin{array}{l}42 \\
42 \\
42 \\
42 \\
41\end{array}$ & $\begin{array}{ll}0.47 & 339 \\
0.47 & 297 \\
0.47 & 255 \\
0.47 & 213 \\
0.47 & 171 .\end{array}$ & $\begin{array}{l}9.97674 \\
9.97670 \\
9.97666 \\
9.97662 \\
9.97657\end{array}$ & $\begin{array}{l}4 \\
4 \\
4 \\
5 \\
4\end{array}$ & $\begin{array}{l}25 \\
24 \\
23 \\
22 \\
21\end{array}$ & 45 & $\begin{array}{l}40 \\
30 \\
32 \\
28 \\
24\end{array}$ \\
\hline 14 & $\begin{array}{l}40 \\
44 \\
48 \\
52 \\
56\end{array}$ & $\begin{array}{l}40 \\
41 \\
42 \\
43 \\
44\end{array}$ & $\begin{array}{lll}9.50 & 523 \\
9.50 & 561 \\
9.50 & 593 \\
9.50 & 635 \\
9.50 & 673\end{array}$ & $\begin{array}{l}38 \\
37 \\
37 \\
38 \\
37\end{array}$ & $\begin{array}{ll}9.52 & 870 \\
9.52 & 912 \\
9.52 & 953 \\
9.52 & 995 \\
9.53 & 037\end{array}$ & $\begin{array}{l}42 \\
41 \\
42 \\
42 \\
41\end{array}$ & $\begin{array}{ll}0.47 & 130 \\
0.47 & 088 \\
0.47 & 047 \\
0.47 & 005 \\
0.46 & 963\end{array}$ & $\begin{array}{l}9.97653 \\
9.97649 \\
9.97645 \\
9.97640 \\
9.97636\end{array}$ & $\begin{array}{l}4 \\
4 \\
5 \\
4 \\
4\end{array}$ & $\begin{array}{l}\mathbf{2 0} \\
19 \\
18 \\
17 \\
16\end{array}$ & 45 & $\begin{array}{r}.20 \\
16 \\
12 \\
8 \\
4\end{array}$ \\
\hline 15 & $\begin{array}{r}0 \\
4 \\
8 \\
12 \\
16\end{array}$ & $\begin{array}{l}45 \\
46 \\
47 \\
48 \\
49 \\
\end{array}$ & $\begin{array}{lll}9.50 & 710 \\
9.50 & 747 \\
9.50 & 784 \\
9.50 & 821 \\
9.50 & 858 \\
\end{array}$ & $\begin{array}{l}37 \\
37 \\
37 \\
37 \\
38\end{array}$ & $\begin{array}{ll}9.53 & 078 \\
9.53 & 120 \\
9.53 & 161 \\
9.53 & 202 \\
9.53 & 244 \\
\end{array}$ & $\begin{array}{l}42 \\
41 \\
41 \\
42 \\
41\end{array}$ & $\begin{array}{ll}0.46 & 922 \\
0.46 & 880 \\
0.46 & 839 \\
0.46 & 798 \\
0.46 & 756\end{array}$ & $\begin{array}{ll}9.97 & 632 \\
9.97 & 628 \\
9.97 & 623 \\
9.97 & 619 \\
9.97 & 615\end{array}$ & $\begin{array}{l}4 \\
5 \\
4 \\
4 \\
5\end{array}$ & $\begin{array}{l}15 \\
14 \\
13 \\
12 \\
11\end{array}$ & 45 & $\begin{array}{r}0 \\
56 \\
52 \\
48 \\
44\end{array}$ \\
\hline 15 & $\begin{array}{l}20 \\
24 \\
28 \\
32 \\
36\end{array}$ & $\begin{array}{l}\mathbf{5 0} \\
51 \\
52 \\
53 \\
54\end{array}$ & $\begin{array}{lll}9.50 & 896 \\
9.50 & 933 \\
9.50 & 970 \\
9.51 & 007 \\
9.51 & 043\end{array}$ & $\begin{array}{l}37 \\
37 \\
37 \\
36 \\
37\end{array}$ & $\begin{array}{ll}9.53 & 285 \\
9.53 & 327 \\
9.53 & 368 \\
9.53 & 409 \\
9.53 & 450\end{array}$ & $\begin{array}{l}42 \\
41 \\
41 \\
41 \\
42\end{array}$ & $\begin{array}{ll}0.46 & 715 \\
0.46 & 673 \\
0.46 & 632 \\
0.46 & 591 \\
0.46 & 550\end{array}$ & $\begin{array}{ll}9.97 & 610 \\
9.97 & 606 \\
9.97 & 602 \\
9.97 & 597 \\
9.97 & 593\end{array}$ & $\begin{array}{l}4 \\
4 \\
5 \\
4 \\
4\end{array}$ & $\begin{array}{r}10 \\
9 \\
8 \\
7 \\
6\end{array}$ & 44 & $\begin{array}{l}40 \\
36 \\
32 \\
28 \\
24\end{array}$ \\
\hline 15 & $\begin{array}{l}40 \\
44 \\
48 \\
52 \\
56\end{array}$ & $\begin{array}{l}55 \\
56 \\
57 \\
58 \\
59\end{array}$ & $\begin{array}{ll}9.51 & 080 \\
9.51 & 117 \\
9.51 & 154 \\
9.51 & 191 \\
9.51 & 227\end{array}$ & $\begin{array}{l}37 \\
37 \\
37 \\
36 \\
37\end{array}$ & $\begin{array}{ll}9.53 & 492 \\
9.53 & 533 \\
9.53 & 5.4 \\
9.53 & 615 \\
9.53 & 656\end{array}$ & $\begin{array}{l}41 \\
41 \\
41 \\
41 \\
41\end{array}$ & $\begin{array}{ll}0.46 & 508 \\
0.46 & 467 \\
0.46 & 426 \\
0.46 & 385 \\
0.46 & 344\end{array}$ & $\begin{array}{l}9.97589 \\
9.97584 \\
9.97580 \\
9.97576 \\
9.97571\end{array}$ & $\begin{array}{l}5 \\
4 \\
4 \\
5 \\
4\end{array}$ & $\begin{array}{l}5 \\
4 \\
3 \\
2 \\
1\end{array}$ & 44 & $\begin{array}{r}20 \\
16 \\
12 \\
8 \\
4\end{array}$ \\
\hline 16 & 0 & 60 & 9.51264 & & $\dot{9} .53697$ & & 0.46303 & 9.97567 & & 0 & 44 & 0 \\
\hline & & & L. Cos. & d. & L. Cotg. & c. d. & L. 'Tang. & I. Sin. & d. & ' & $\mathrm{m}$. & s. \\
\hline
\end{tabular}

$18^{\circ}$ 
TABLe 19.-Five-place logarithms of circular functions, etc.-Continued. $1^{\mathbf{h}}$ $19^{\circ}$

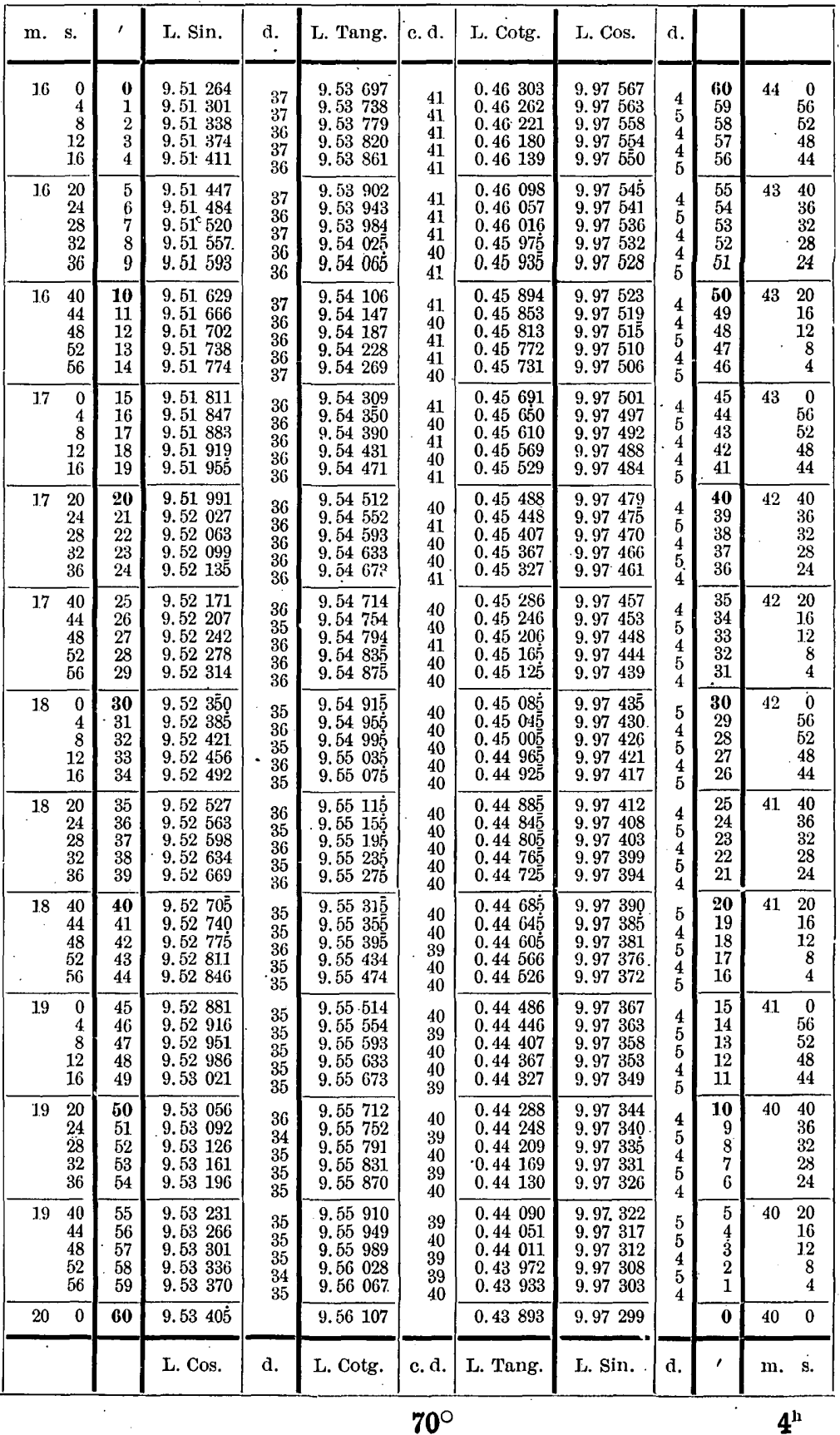


TAвте 19.-Five-place logarithms of circular functions, etc.-Continued.

$1^{\text {h }}$

\begin{tabular}{|c|c|c|c|c|c|c|c|c|c|c|c|c|}
\hline $\mathrm{m}$. & $\mathrm{s}$. & ' & I. Sin. & d. & L. Tang. & c. di. & I. Cotg. & I. Cos. & d. & & & \\
\hline 20 & $\begin{array}{r}0 \\
4 \\
8 \\
12 \\
16\end{array}$ & $\begin{array}{l}0 \\
1 \\
2 \\
3 \\
4\end{array}$ & 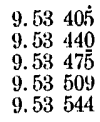 & \multirow{3}{*}{$\begin{array}{l}35 \\
35 \\
34 \\
35 \\
34 \\
35 \\
34 \\
35 \\
34 \\
35 \\
34 \\
34 \\
35 \\
34 \\
34\end{array}$} & $\begin{array}{ll}9.56 & 107 \\
9.56 & 146 \\
9.56 & 185 \\
9.56 & 224 \\
9.56 & 264\end{array}$ & \multirow{2}{*}{$\begin{array}{l}39 \\
39 \\
39 \\
40 \\
39 \\
39 \\
39 \\
39 \\
39 \\
39\end{array}$} & 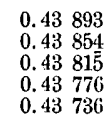 & 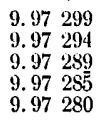 & $\begin{array}{l}5 \\
5 \\
4 \\
5 \\
4\end{array}$ & $\begin{array}{r}\mathbf{6 0} \\
59 \\
58 \\
57 \\
56\end{array}$ & 40 & $\begin{array}{r}0 \\
56 \\
52 \\
48 \\
44\end{array}$ \\
\hline 20 & $\begin{array}{l}20 \\
24 \\
28 \\
32 \\
36\end{array}$ & $\begin{array}{l}5 \\
6 \\
7 \\
8 \\
9\end{array}$ & 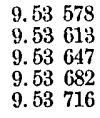 & & $\begin{array}{ll}9.56 & 303 \\
9.56 & 342 \\
9.56 & 381 \\
9.56 & 420 \\
9.56 .459\end{array}$ & & $\begin{array}{ll}0.43 & 697 \\
0.43 & 658 \\
0.43 & 619 \\
0.43 & 580 \\
0.43 & 541\end{array}$ & $\begin{array}{ll}9.97 & 276 \\
9.97 & 271 \\
9.97 & 266 \\
9.97 & 262 \\
9.97 & 257\end{array}$ & \multirow[b]{2}{*}{$\begin{array}{l}4 \\
5 \\
5 \\
4 \\
5\end{array}$} & $\begin{array}{l}55 \\
54 \\
53 \\
52 \\
51\end{array}$ & 39 & $\begin{array}{l}40 \\
36 \\
32 \\
28 \\
24\end{array}$ \\
\hline 20 & $\begin{array}{l}40 \\
44 \\
48 \\
52 \\
56\end{array}$ & $\begin{array}{l}10 \\
11 . \\
12 \\
13 \\
14\end{array}$ & 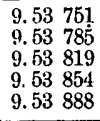 & & $\begin{array}{ll}9.56 & 498 \\
9.56 & 537 \\
9.56 & 576 \\
9.56 & 615 \\
9.56 & 654\end{array}$ & $\begin{array}{l}39 \\
39 \\
39 \\
39 \\
39\end{array}$ & $\begin{array}{ll}0.43 & 502 \\
0.43 & 463 \\
0.43 & 424 \\
0.43 & 385 \\
0.43 & 346\end{array}$ & $\begin{array}{ll}9.97 & 252 \\
9.97 & 245 \\
9.97 & 243 \\
9.97 & 238 \\
9.97 & 234\end{array}$ & & $\begin{array}{l}50 \\
49 \\
48 \\
47 \\
46\end{array}$ & 39 & $\begin{array}{r}20 \\
1.6 \\
1.2 \\
8 \\
4\end{array}$ \\
\hline 21. & $\begin{array}{r}0 \\
4 \\
8 \\
12 \\
16\end{array}$ & $\begin{array}{l}15 \\
16 \\
17 \\
18 \\
19\end{array}$ & 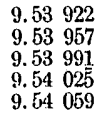 & \multirow{2}{*}{$\begin{array}{l}35 \\
34 \\
34 \\
34 \\
34 \\
34 \\
34 \\
34 \\
34 \\
34\end{array}$} & 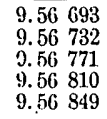 & $\begin{array}{l}39 \\
39 \\
39 \\
39 \\
39\end{array}$ & $\begin{array}{ll}0.43 & 307 \\
0.43 & 268 \\
0.43 & 229 \\
0.43 & 190 \\
0.43 & 151\end{array}$ & $\begin{array}{ll}9.97 & 229 \\
9.97 & 224 \\
9.97 & 220 \\
9.97 & 215 \\
9.97 & 210\end{array}$ & $\begin{array}{l}5 \\
4 \\
5 \\
5 \\
4\end{array}$ & $\begin{array}{l}45 \\
44 \\
43 \\
42 \\
41\end{array}$ & 39 & $\begin{array}{r}0 \\
56 \\
52 \\
48 \\
44\end{array}$ \\
\hline 21 & $\begin{array}{l}20 \\
24 \\
28 \\
32 \\
36\end{array}$ & $\begin{array}{l}\mathbf{2 0} \\
21 \\
22 \\
23 \\
24\end{array}$ & 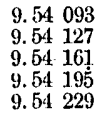 & & $\begin{array}{ll}9.56 & 887 \\
9.56 & 926 \\
9.56 & 965 \\
9.57 & 004 \\
9.57 & 042\end{array}$ & $\begin{array}{l}39 \\
39 \\
39 \\
38\end{array}$ & $\begin{array}{ll}0.43 & 113 \\
0.43 & 074 \\
0.43 & 035 \\
0.42 & 996 \\
0.42 & 958\end{array}$ & $\begin{array}{lll}9.97 & 206 \\
9.97 & 201 \\
9.97 & 196 \\
9.97 & 192 \\
9.97 & 187\end{array}$ & $\begin{array}{l}5 \\
5 \\
4 \\
5\end{array}$ & $\begin{array}{l}40 \\
39 \\
38 \\
37 \\
36\end{array}$ & 38 & $\begin{array}{l}40 \\
36 \\
32 \\
28 \\
24\end{array}$ \\
\hline $\begin{array}{l}21 \\
.\end{array}$ & $\begin{array}{l}40 \\
44 \\
48 \\
52 \\
56\end{array}$ & $\begin{array}{l}25 \\
26 \\
27 \\
28 \\
29\end{array}$ & 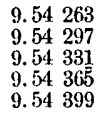 & \multirow{2}{*}{$\begin{array}{l}34 \\
34 \\
34 \\
34 \\
34 \\
33 \\
34 \\
34 \\
33 \\
34\end{array}$} & $\begin{array}{ll}9.57 & 081 \\
9.57 & 120 \\
9.57 & 158 \\
9.57 & 197 \\
9.57 & 235\end{array}$ & $\begin{array}{l}39 \\
38 \\
39 \\
38\end{array}$ & $\begin{array}{ll}0.42 & 919 \\
0.42 & 880 \\
0.42 & 842 \\
0.42 & 803 \\
0.42 & 765\end{array}$ & $\begin{array}{ll}9.97 & 182 \\
9.97 & 178 \\
9.97 & 173 \\
9.97 & 168 \\
9.97 & 163\end{array}$ & $\begin{array}{l}4 \\
5 \\
5 \\
5\end{array}$ & $\begin{array}{l}35 \\
34 \\
33 \\
32 \\
31\end{array}$ & 38 & $\begin{array}{r}20 \\
16 \\
12 \\
8 \\
4\end{array}$ \\
\hline 22 & $\begin{array}{r}0 \\
4 \\
8 \\
12 \\
16\end{array}$ & $\begin{array}{l}30 \\
31 \\
32 \\
33 \\
34\end{array}$ & 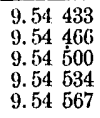 & & $\begin{array}{ll}9.57 & 274 \\
9.57 & 312 \\
9.57 & 351 \\
9.57 & 389 \\
9.57 & 428\end{array}$ & $\begin{array}{l}38 \\
39 \\
38 \\
39\end{array}$ & $\begin{array}{ll}0.42 & 726 \\
0.42 & 688 \\
0.42 & 649 \\
0.42 & 611 \\
0.42 & 572\end{array}$ & $\begin{array}{ll}9.97 & 159 \\
9.97 & 154 \\
9.97 & 149 \\
9.97 & 145 \\
9.97 & 140\end{array}$ & $\begin{array}{l}5 \\
5 \\
4 \\
5\end{array}$ & $\begin{array}{l}30 \\
29 \\
28 \\
27 \\
26\end{array}$ & 38 & $\begin{array}{r}0 \\
56 \\
52 \\
48 \\
44\end{array}$ \\
\hline 22 & $\begin{array}{l}20 \\
24 \\
28 \\
32 \\
36\end{array}$ & $\begin{array}{l}35 \\
36 \\
37 \\
38 \\
39\end{array}$ & $\begin{array}{l}9.54601 \\
9.54635 \\
9.54668 \\
9.54702 \\
9.54 \\
935\end{array}$ & $\begin{array}{l}34 \\
33 \\
34 \\
33\end{array}$ & $\begin{array}{ll}9.57 & 466 \\
9.57 & 504 \\
9.57 & 543 \\
9.57 & 581 \\
9.57 & 619\end{array}$ & $\begin{array}{l}38 \\
39 \\
38 \\
38 \\
39\end{array}$ & $\begin{array}{ll}0.42 & 534 \\
0.42 & 496 \\
0.42 & 457 \\
0.42 & 419 \\
0.42 & 381\end{array}$ & $\begin{array}{ll}9.97 & 135 \\
9.97 & 130 \\
9.97 & 126 \\
9.97 & 121 \\
9.97 & 116\end{array}$ & $\begin{array}{l}5 \\
4 \\
5 \\
5 \\
5\end{array}$ & $\begin{array}{l}25 \\
24 \\
23 \\
22 \\
21\end{array}$ & 37 & $\begin{array}{l}40 \\
36 \\
32 \\
28 \\
24\end{array}$ \\
\hline . & $\begin{array}{l}40 \\
44 \\
48 \\
52 \\
56\end{array}$ & $\begin{array}{l}40 \\
41 \\
42 \\
43 \\
44\end{array}$ & $\begin{array}{l}9.54769 \\
9.54802 \\
9.54836 \\
9.54869 \\
9.54903\end{array}$ & \multirow{2}{*}{$\begin{array}{l}83 \\
34 \\
33 \\
34 \\
33 \\
33 \\
34 \\
33 \\
33 \\
33\end{array}$} & 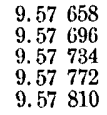 & $\begin{array}{l}38 \\
38 \\
38 \\
38 \\
39\end{array}$ & $\begin{array}{ll}0.42 & 342 \\
0.42 & 304 \\
0.42 & 266 \\
0.42 & 228 \\
0.42 & 190\end{array}$ & $\begin{array}{ll}9.97 & 111 \\
9.97 & 107 \\
9.97 & 102 \\
9.97 & 097 \\
9.97 & 092\end{array}$ & $\begin{array}{l}4 \\
5 \\
5 \\
5 \\
5\end{array}$ & $\begin{array}{l}20 \\
19 \\
18 \\
17 \\
16\end{array}$ & 37 & $\begin{array}{r}20 \\
16 \\
12 \\
8 \\
4\end{array}$ \\
\hline 23 & $\begin{array}{r}0 \\
4 \\
8 \\
12 \\
16\end{array}$ & $\begin{array}{l}45 \\
46 \\
47 \\
48 \\
49\end{array}$ & $\begin{array}{ll}9.54 & 936 \\
9.54 & 969 \\
9.55 & 003 \\
9.55 & 036 \\
9.55 & 069\end{array}$ & & $\begin{array}{l}9.57849 \\
9.57887 \\
9.57925 \\
9.57963 \\
9.58001\end{array}$ & $\begin{array}{l}38 \\
38 \\
38 \\
38 \\
38\end{array}$ & $\begin{array}{ll}0.42 & 151 \\
0.42 & 113 \\
0.42 & 075 \\
0.42 & 037 \\
0.41 & 999\end{array}$ & $\begin{array}{ll}9.97 & 087 \\
9.97 & 083 \\
9.97 & 078 \\
9.97 & 073 \\
9.97 & 068\end{array}$ & $\begin{array}{l}4 \\
5 \\
5 \\
5 \\
5\end{array}$ & $\begin{array}{l}15 \\
14 \\
13 \\
12 \\
11 .\end{array}$ & 37 & $\begin{array}{r}0 \\
56 \\
52 \\
48 \\
44\end{array}$ \\
\hline 23 & $\begin{array}{l}20 \\
24 \\
28 \\
32 \\
36\end{array}$ & $\begin{array}{l}50 \\
51 \\
52 \\
53 \\
54\end{array}$ & $\begin{array}{ll}9.55 & 102 \\
9.55 & 136 \\
9.55 & 169 \\
9.55 & 202 \\
9.55 & 235\end{array}$ & $\begin{array}{l}34 \\
33 \\
33 \\
33 \\
39\end{array}$ & $\begin{array}{lll}9.58 & 039 \\
9.58 & 077 \\
9.58 & 115 \\
9.5 \$ & 153 \\
9.58 & 191 .\end{array}$ & $\begin{array}{l}38 \\
38 \\
38 \\
38\end{array}$ & $\begin{array}{ll}0.41 & 961 \\
0.41 & 923 \\
0.41 & 885 \\
0.41 & 847 \\
0.41 & 809\end{array}$ & $\begin{array}{ll}9.97 & 063 \\
9.97 & 059 \\
9.97 & 054 \\
9.97 & 049 \\
9.97 & 044\end{array}$ & $\begin{array}{l}4 \\
5 \\
5 \\
5\end{array}$ & $\begin{array}{r}10 \\
9 \\
8 \\
\cdot 7 \\
6\end{array}$ & 36 & $\begin{array}{l}40 \\
36 \\
32 \\
28 \\
24\end{array}$ \\
\hline 23 & $\begin{array}{l}40 \\
44 \\
48 \\
52 \\
56\end{array}$ & $\begin{array}{l}55 \\
56 \\
57 \\
58 \\
59\end{array}$ & 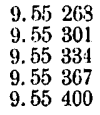 & $\begin{array}{l}33 \\
33 \\
33 \\
33 \\
\end{array}$ & 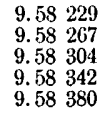 & $\begin{array}{l}38 \\
37 . \\
38 \\
38\end{array}$ & $\begin{array}{ll}0.41 & 771 \\
0.41 & 733 \\
0.41 & 696 \\
0.41 & 658 \\
0.41 & 62.0\end{array}$ & 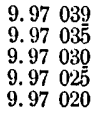 & $\begin{array}{l}4 \\
5 \\
5 \\
5\end{array}$ & $\begin{array}{l}\mathbf{5} \\
4 \\
\mathbf{3} \\
2 \\
1\end{array}$ & 36 & $\begin{array}{r}20 \\
16 \\
12 \\
8 \\
4\end{array}$ \\
\hline 24 & 0 & 60 & 9.55433 & & 9.58418 & & 0.41582 & $9.9701 \dot{5}$ & & 0 & 36 & 0 \\
\hline & & & L. Cos. & d. & I. Cotg. & c. d. & I. Tang. & L. $\operatorname{Sin}$. & d. & 1 & $\mathrm{~m}$. & .. $\mathrm{s}$ \\
\hline
\end{tabular}

$20^{\circ}$

$69^{\circ}$ 
TABLE 19.-Five-place logarithms of circular functions, elc.-Continued.

$1^{\mathrm{h}}$ $21^{\circ}$

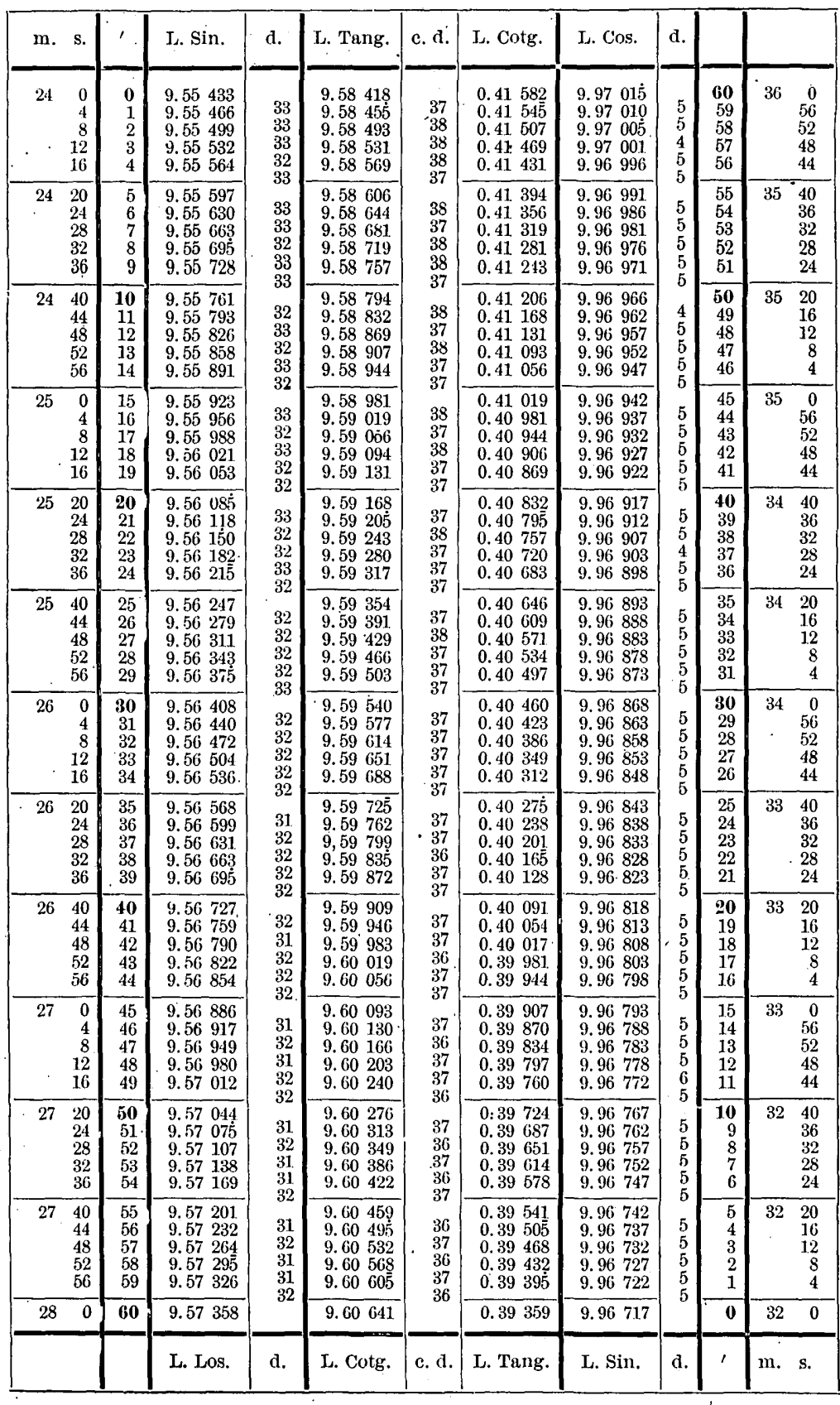


TaBLe 19.-Five-place logarithms of circular functions, etc.-Continued.

$1^{\text {h }}$

\begin{tabular}{|c|c|c|c|c|c|c|c|c|c|c|c|c|}
\hline $\mathrm{m}$. & s. & ' & I. $\operatorname{Sin}$. & d. & L. 'Tang. & c. d. & L. Cotg. & L. Cos. & d. & & & \\
\hline 28 & $\begin{array}{r}0 \\
4 \\
8 \\
12 \\
16\end{array}$ & $\begin{array}{l}0 \\
1 \\
2 \\
3 \\
4\end{array}$ & 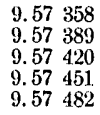 & $\begin{array}{l}31 \\
31 \\
31 \\
31\end{array}$ & $\begin{array}{lll}9.60 & 641 \\
9.60 & 677 \\
9.60 & 714 \\
9.60 & 750 \\
9.60 & 786\end{array}$ & $\begin{array}{l}36 \\
37 \\
36 \\
36 \\
37\end{array}$ & 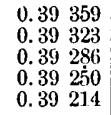 & 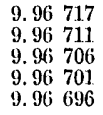 & $\begin{array}{l}6 \\
5 \\
5 \\
5 \\
5\end{array}$ & $\begin{array}{l}\mathbf{6 0} \\
59 \\
58 \\
57 \\
56\end{array}$ & & $\begin{array}{r}0 \\
56 \\
52 \\
48 \\
44\end{array}$ \\
\hline 28 & $\begin{array}{l}20 \\
24 \\
28 \\
32 \\
36\end{array}$ & $\begin{array}{l}5 \\
6 \\
7 \\
8 \\
9\end{array}$ & 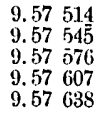 & $\begin{array}{l}31 \\
31 \\
31 \\
31\end{array}$ & 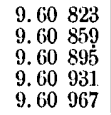 & $\begin{array}{l}36 \\
36 \\
36 \\
36\end{array}$ & $\begin{array}{ll}0.39 & 177 \\
0.39 & 141 \\
0.39 & 105 \\
0.39 & 069 \\
0.39 & 033\end{array}$ & 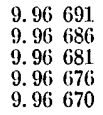 & $\begin{array}{l}5 \\
5 \\
5 \\
6 \\
5\end{array}$ & $\begin{array}{l}55 \\
54 \\
53 \\
52 \\
51\end{array}$ & 31 & $\begin{array}{l}40 \\
36 \\
32 \\
28 \\
24\end{array}$ \\
\hline 28 & $\begin{array}{l}40 \\
44 \\
48 \\
52 \\
56\end{array}$ & $\begin{array}{l}10 \\
11 \\
12 \\
13 \\
14\end{array}$ & $\begin{array}{ll}9.57 & 669 \\
9.57 & 700 \\
9.57 & 731 \\
9.57 & 762 \\
9.57 & 793\end{array}$ & $\begin{array}{l}31 \\
31 \\
31 \\
31\end{array}$ & $\begin{array}{ll}9.61 & 004 \\
9.61 & 040 \\
9.61 & 076 \\
9.61 & 112 \\
9.61 & 148\end{array}$ & $\begin{array}{l}36 \\
36 \\
36 \\
36\end{array}$ & $\begin{array}{ll}0.38 & 996 \\
0.38 & 960 \\
0.38 & 924 \\
0.38 & 888 \\
0.38 & 852\end{array}$ & 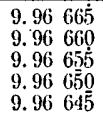 & $\begin{array}{l}5 \\
5 \\
5 \\
5 \\
5\end{array}$ & $\begin{array}{l}50 \\
49 \\
48 \\
47 \\
46\end{array}$ & 31 & $\begin{array}{r}20 \\
16 \\
12 \\
8 \\
4\end{array}$ \\
\hline 29 & $\begin{array}{r}0 \\
4 \\
8 \\
1.2 \\
16\end{array}$ & $\begin{array}{l}15 \\
16 \\
17 \\
18 \\
19\end{array}$ & $\begin{array}{l}9.57824 \\
9.57855 \\
9.57885 \\
9.57916 \\
9.57947\end{array}$ & $\begin{array}{l}31 \\
30 \\
31 \\
31\end{array}$ & $\begin{array}{ll}9.61 & 184 \\
9.61 & 220 \\
9.61 & 256 \\
9.61 . & 292 \\
9.61 & 328\end{array}$ & $\begin{array}{l}36 \\
36 \\
36 \\
36 \\
36\end{array}$ & 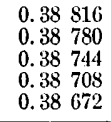 & 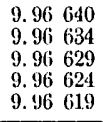 & $\begin{array}{l}6 \\
5 \\
5 \\
5 \\
5\end{array}$ & $\begin{array}{l}45 \\
44 \\
43 \\
42 \\
41\end{array}$ & 31 & $\begin{array}{r}0 \\
50 \\
52 \\
48 \\
44\end{array}$ \\
\hline 29 & $\begin{array}{l}20 \\
24 \\
28 \\
32 \\
36\end{array}$ & $\begin{array}{r}\mathbf{2 0} \\
21 \\
22 \\
23 \\
24\end{array}$ & 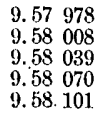 & $\begin{array}{l}30 \\
31 \\
31 \\
31\end{array}$ & $\begin{array}{ll}9.61 . & 364 \\
9.61 & 400 \\
9.61 & 436 \\
9.61 & 472 \\
9.61 & 508\end{array}$ & $\begin{array}{l}36 \\
36 \\
36 \\
36 \\
36\end{array}$ & $\begin{array}{l}0.38636 \\
0.38600 \\
0.38564 \\
0.38528 \\
0.38 \quad 492\end{array}$ & 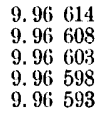 & $\begin{array}{l}6 \\
5 \\
5 \\
5 \\
5\end{array}$ & $\begin{array}{l}\mathbf{4 0} \\
39 \\
38 \\
37 \\
36\end{array}$ & 30 & $\begin{array}{l}40 \\
36 \\
32 \\
28 \\
24\end{array}$ \\
\hline 29 & $\begin{array}{l}40 \\
44 \\
48 \\
52 \\
56\end{array}$ & $\begin{array}{l}25 \\
26 \\
27 \\
28 \\
29\end{array}$ & $\begin{array}{ll}9.58 & 131 \\
9.58 & 162 \\
9.58 & 192 \\
9.58 & 223 \\
9.58 & 253\end{array}$ & $\begin{array}{l}31 \\
30 \\
31 \\
30 \\
31\end{array}$ & $\begin{array}{ll}9,61 & 544 \\
9.61 & 579 \\
9.61 & 615 \\
9.61 & 651 \\
9.61 & 687\end{array}$ & $\begin{array}{l}35 \\
36 \\
36 \\
36 \\
35\end{array}$ & $\begin{array}{ll}0.38 & 456 \\
0.38 & 421 \\
0.38 & 385 \\
0.38 & 349 \\
0.38 & 313\end{array}$ & 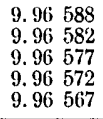 & $\begin{array}{l}6 \\
5 \\
5 \\
5 \\
5\end{array}$ & $\begin{array}{l}35 \\
34 \\
33 \\
32 \\
31\end{array}$ & 30 & $\begin{array}{r}20 \\
16 \\
12 \\
8 \\
4\end{array}$ \\
\hline 30 & $\begin{array}{r}0 \\
4 \\
8 \\
12 \\
16\end{array}$ & $\begin{array}{l}30 \\
31 \\
32 \\
33 \\
34\end{array}$ & 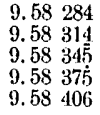 & $\begin{array}{l}30 \\
31 \\
30 \\
31\end{array}$ & $\begin{array}{ll}9.61 & 722 \\
9.61 & 758 \\
9.61 & 794 \\
9.61 & 830 \\
9.61 . & 865\end{array}$ & $\begin{array}{l}36 \\
36 \\
36 \\
35 \\
36\end{array}$ & $\begin{array}{ll}0.38 & 278 \\
0.38 & 242 \\
0.38 & 206 \\
0.38 & 170 \\
0.38 & 135\end{array}$ & 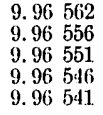 & $\begin{array}{l}6 \\
5 \\
5 \\
5 \\
6\end{array}$ & $\begin{array}{l}30 \\
29 \\
28 \\
27 \\
26\end{array}$ & 30 & $\begin{array}{r}0 \\
56 \\
52 \\
48 \\
44\end{array}$ \\
\hline 30 & $\begin{array}{l}20 \\
24 \\
28 \\
32 \\
36\end{array}$ & $\begin{array}{l}35 \\
36 \\
37 \\
38 \\
39\end{array}$ & 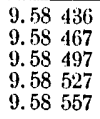 & $\begin{array}{l}31 \\
30 \\
30 \\
30 \\
31\end{array}$ & $\begin{array}{ll}9.61 & 901 \\
9.61 & 936 \\
9.61 & 972 \\
9.62 & 008 \\
9.62 & 043\end{array}$ & $\begin{array}{r}35 \\
36 \\
36 \\
35 \\
36\end{array}$ & $\begin{array}{ll}0.38 & 099 \\
0.38 & 064 \\
0.38 & 028 \\
0.37 & 992 \\
0.37 & 957\end{array}$ & 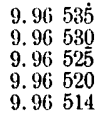 & $\begin{array}{l}5 \\
5 \\
5 \\
6 \\
5\end{array}$ & $\begin{array}{l}25 \\
24 \\
23 \\
22 \\
21\end{array}$ & 29 & $\begin{array}{l}40 \\
36 \\
32 \\
28 \\
24\end{array}$ \\
\hline 30 & $\begin{array}{l}40 \\
44 \\
48 \\
52 \\
56\end{array}$ & $\begin{array}{r}40 \\
41 \\
42 \\
43 \\
44\end{array}$ & 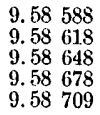 & $\begin{array}{l}30 \\
30 \\
30 \\
31\end{array}$ & $\begin{array}{ll}9.62 & 079 \\
9.62 & 114 \\
9.62 & 150 \\
9.62 & 185 \\
9.62 & 221\end{array}$ & $\begin{array}{l}35 \\
36 \\
35 \\
36 \\
35\end{array}$ & $\begin{array}{ll}0.37 & 921 \\
0.37 & 886 \\
0.37 & 850 \\
0.37 & 815 \\
0.37 & 779\end{array}$ & 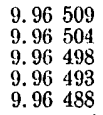 & $\begin{array}{l}5 \\
6 \\
5 \\
5 \\
5\end{array}$ & $\begin{array}{l}20 \\
19 \\
1.8 \\
17 \\
16\end{array}$ & 29 & $\begin{array}{r}20 \\
16 \\
12 \\
8 \\
4\end{array}$ \\
\hline 31 & $\begin{array}{r}0 \\
4 \\
8 \\
12 \\
16\end{array}$ & $\begin{array}{l}45 \\
46 \\
47 \\
48 \\
49\end{array}$ & 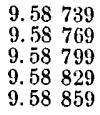 & $\begin{array}{l}30 \\
30 \\
30 \\
30 \\
30\end{array}$ & $\begin{array}{ll}9,62 & 256 \\
9.62 & 292 \\
9.62 & 327 \\
9.62 & 362 \\
9.62 & 398\end{array}$ & $\begin{array}{l}36 \\
35 \\
35 \\
36 \\
35\end{array}$ & $\begin{array}{ll}0.37 & 744 \\
0.37 & 708 \\
0.37 & 673 \\
0.37 & 638 \\
0.37 & 602\end{array}$ & 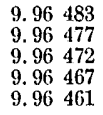 & $\begin{array}{l}6 \\
5 \\
5 \\
6 \\
5\end{array}$ & $\begin{array}{l}15 \\
14 \\
13 \\
12 \\
11\end{array}$ & 29 & $\begin{array}{r}0 \\
56 \\
52 \\
48 \\
44\end{array}$ \\
\hline 31 & $\begin{array}{l}20 \\
24 \\
28 \\
32 \\
36\end{array}$ & $\begin{array}{c}50 \\
51 \\
52 \\
53 \\
54\end{array}$ & 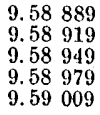 & $\begin{array}{l}30 \\
30 \\
30 \\
30 \\
30\end{array}$ & 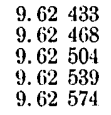 & $\begin{array}{l}35 \\
36 \\
35 \\
35 \\
35\end{array}$ & $\begin{array}{ll}0.37 & 567 \\
0.37 & 532 \\
0.37 & 496 \\
0.37 & 461 \\
0.37 & 426\end{array}$ & 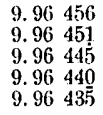 & $\begin{array}{l}5 \\
6 \\
5 \\
5 \\
6\end{array}$ & $\begin{array}{r}10 \\
9 \\
8 \\
7 \\
6\end{array}$ & 28 & $\begin{array}{l}40 \\
36 \\
32 \\
28 \\
24\end{array}$ \\
\hline 31 & $\begin{array}{l}40 \\
44 \\
48 \\
52 \\
56\end{array}$ & $\begin{array}{l}55 \\
56 \\
57 \\
58 \\
59\end{array}$ & 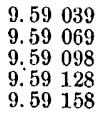 & $\begin{array}{l}30 \\
29 \\
30 \\
30 \\
30\end{array}$ & 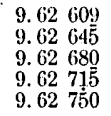 & $\begin{array}{l}36 \\
35 \\
35 \\
35 \\
35\end{array}$ & $\begin{array}{ll}0.37 & 391 \\
0.37 & 355 \\
0.37 & 320 \\
0.37 & 285 \\
0.37 & 250\end{array}$ & 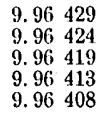 & $\begin{array}{l}5 \\
5 \\
6 \\
5 \\
5\end{array}$ & $\begin{array}{l}5 \\
4 \\
3 \\
2 \\
1\end{array}$ & 28 & $\begin{array}{r}20 \\
16 \\
12 \\
8 \\
4\end{array}$ \\
\hline 32 & 0 & 60 & 9.59188 & & $9.6278 \dot{5}$ & & $0.3721 \overline{5}$ & 9.96403 & & 0 & 28 & 0 \\
\hline & & & L. $\operatorname{Cos}$. & d. & L. Cotg. & c. d. & I. Tang. & L. $\operatorname{Sin}$. & d. & ' & $\mathrm{m}$ & s. \\
\hline
\end{tabular}


TABLE 19.-Five-place logarithms of circular functions, etc.-Continued.

$1^{\text {h }}$

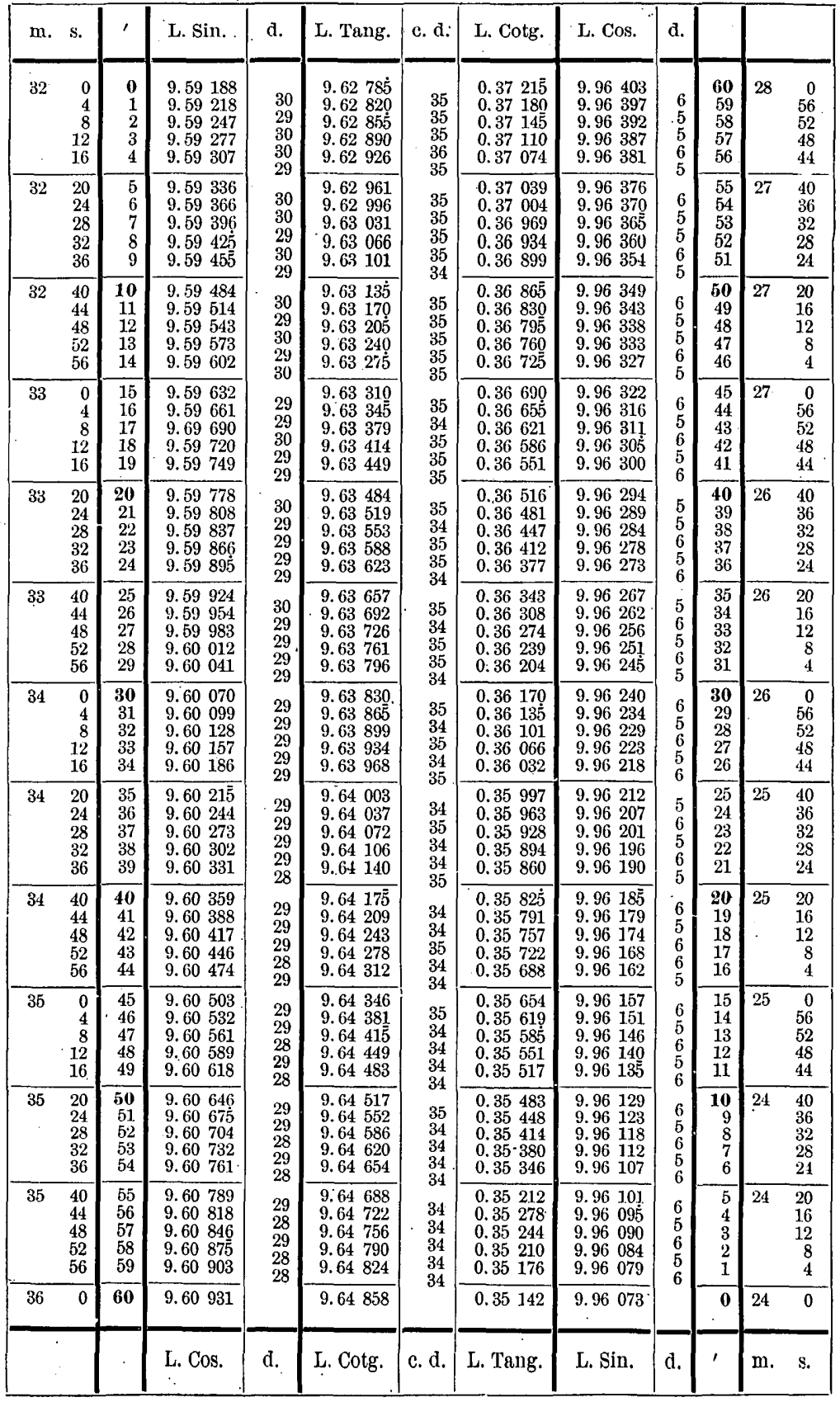

$23^{\circ}$ 
TABLE 19.-Five-place logarithms of circular functions, etc.-Continied.

$1^{\mathrm{h}}$

$24^{\circ}$

\begin{tabular}{|c|c|c|c|c|c|c|c|c|c|c|c|c|}
\hline $\mathrm{m}$. & s. & ' & L. Sin. & d. & L. Tang. & c. d. & L. Cotg. & L. Cos. & d. & & & \\
\hline 36 & $\begin{array}{r}0 \\
4 \\
8 \\
12 \\
16\end{array}$ & $\begin{array}{l}0 \\
1 \\
2 \\
3 \\
4\end{array}$ & $\begin{array}{ll}9.60 & 931 \\
9.60 & 960 \\
9.60 & 988 \\
9.61 & 016 \\
9.61 & 045\end{array}$ & $\begin{array}{l}29 \\
28 \\
28 \\
29\end{array}$ & $\begin{array}{l}9.64858 \\
9.64892 \\
9.64926 \\
9.64960 \\
9.64994\end{array}$ & $\begin{array}{l}34 \\
34 \\
34 \\
34\end{array}$ & $\begin{array}{ll}0.35 & 142 \\
0.35 & 108 \\
0.35 & 074 \\
0.35 & 040 \\
0.35 & 006\end{array}$ & 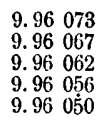 & $\begin{array}{l}6 \\
5 \\
6 \\
6\end{array}$ & $\begin{array}{l}60 \\
59 \\
58 \\
57 \\
56\end{array}$ & & $\begin{array}{r}0 \\
56 \\
52 \\
48 \\
44\end{array}$ \\
\hline 36 & $\begin{array}{l}20 \\
24 \\
28 \\
32 \\
36\end{array}$ & $\begin{array}{l}5 \\
6 \\
7 \\
8 \\
9\end{array}$ & $\begin{array}{ll}9.61 & 073 \\
9.61 & 101 \\
9.61 & 129 \\
9.61 & 158 \\
9.61 & 186\end{array}$ & $\begin{array}{l}28 \\
28 \\
29 \\
28\end{array}$ & $\begin{array}{ll}9.65 & 028 \\
9.65 & 062 \\
9.65 & 096 \\
9.65 & 130 \\
\mathbf{9 . 6 5} & \mathbf{1 6 4}\end{array}$ & $\begin{array}{l}34 \\
34 \\
34 \\
34\end{array}$ & $\begin{array}{l}0.34972 \\
0.34938 \\
0.34904 \\
0.34870 \\
0.34836\end{array}$ & 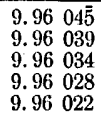 & $\begin{array}{l}6 \\
5 \\
6 \\
6\end{array}$ & $\begin{array}{l}55 \\
54 \\
53 \\
52 \\
51\end{array}$ & 23 & $\begin{array}{l}40 \\
36 \\
32 \\
28 \\
24\end{array}$ \\
\hline 36 & $\begin{array}{l}40 \\
44 \\
48 \\
52 \\
56\end{array}$ & $\begin{array}{r}10 \\
11 \\
12 \\
13 \\
14\end{array}$ & $\begin{array}{ll}9.61 & 214 \\
9.61 & 242 \\
9.61 & 270 \\
9.61 & 298 \\
9.61 & 326\end{array}$ & $\begin{array}{l}28 \\
28 \\
28 \\
28\end{array}$ & $\begin{array}{ll}9.65 & 197 \\
9.65 & 231 \\
9.65 & 265 \\
9.65 & 299 \\
9.65 & 333\end{array}$ & $\begin{array}{l}34 \\
34 \\
34 \\
34\end{array}$ & $\begin{array}{ll}0.34 & 803 \\
0.34 & 769 \\
0.34 & 735 \\
0.34 & 701 \\
0.34 & 667\end{array}$ & $\begin{array}{ll}9.96 & 017 \\
9.96 & 011 \\
9.96 & 005 \\
9.96 & 000 \\
9.95 & 994\end{array}$ & $\begin{array}{l}6 \\
6 \\
5 \\
6\end{array}$ & $\begin{array}{l}\mathbf{5 0} \\
49 \\
48 \\
47 \\
46\end{array}$ & 23 & $\begin{array}{r}20 \\
16 \\
12 \\
8 \\
4\end{array}$ \\
\hline 37. & $\begin{array}{r}0 \\
4 \\
8 \\
12 \\
16\end{array}$ & $\begin{array}{l}15 \\
16 \\
17 \\
18 \\
19\end{array}$ & $\begin{array}{ll}9.61 & 354 \\
9.61 & 382 \\
9.61 & 411 \\
9.61 & 438 \\
9.61 & 466\end{array}$ & $\begin{array}{l}28 \\
29 \\
27 \\
28\end{array}$ & $\begin{array}{ll}9.65 & 366 \\
9.65 & 400 \\
9.65 & 434 \\
9.65 & 467 \\
9.65 & 501\end{array}$ & $\begin{array}{l}34 \\
34 \\
33 \\
34\end{array}$ & $\begin{array}{ll}0.34 & 634 \\
0.34 & 600 \\
0.34566 \\
0.34 & 533 \\
0.34 & 499\end{array}$ & $\begin{array}{ll}9.95 & 988 \\
9.95 & 982 \\
9.95 & 977 \\
9.95 & 971 . \\
9.95 & 965\end{array}$ & $\begin{array}{l}6 \\
5 \\
6 \\
6\end{array}$ & $\begin{array}{l}45 \\
44 \\
43 \\
42 \\
41 .\end{array}$ & 23 & $\begin{array}{r}0 \\
56 \\
.52 \\
48 \\
44\end{array}$ \\
\hline 37 & $\begin{array}{l}20 \\
24 \\
28 \\
32 \\
36\end{array}$ & $\begin{array}{l}\mathbf{2 0} \\
21 \\
22 \\
23 \\
24\end{array}$ & $\begin{array}{ll}9.61 & 494 \\
9.61 & 522 \\
9.61 & 550 \\
9.61 & 578 \\
9.61 & 606\end{array}$ & $\begin{array}{l}28 \\
28 \\
28 \\
28\end{array}$ & $\begin{array}{ll}9.65 & 535 \\
9.65 & 568 \\
9.65 & 602 \\
9.65 & 636 \\
9.65 & 669\end{array}$ & $\begin{array}{l}33 \\
34 \\
34 \\
33\end{array}$ & $\begin{array}{ll}0.34 & 465 \\
0.34 & 432 \\
0.34 & 398 \\
0.34 & 364 \\
0.34 & 331\end{array}$ & 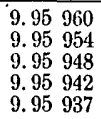 & $\begin{array}{l}6 \\
6 \\
6 \\
5\end{array}$ & $\begin{array}{l}40 \\
39 \\
38 \\
37 \\
36\end{array}$ & 22 & $\begin{array}{l}40 \\
36 \\
32 \\
28 \\
24\end{array}$ \\
\hline 37 & $\begin{array}{l}40 \\
44 \\
48 \\
52 \\
56\end{array}$ & $\begin{array}{l}25 \\
26 \\
27 \\
28 \\
29\end{array}$ & $\begin{array}{ll}9.61 & 634 \\
9.61 & 662 \\
9.61 & 689 \\
9.61 & 717 \\
9.61 & 745\end{array}$ & $\begin{array}{l}28 \\
27 \\
28 \\
28\end{array}$ & $\begin{array}{ll}9.65 & 703 \\
9.65 & 736 \\
9.65 & 770 \\
9.65 & 803 \\
9.65 & 837\end{array}$ & $\begin{array}{l}33 \\
34 \\
33 \\
34\end{array}$ & $\begin{array}{ll}0.34 & 297 \\
0.34 & 264 \\
0.34 & 230 \\
0.34 & 197 \\
0.34 & 163\end{array}$ & 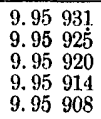 & $\begin{array}{l}6 \\
5 \\
6 \\
6\end{array}$ & $\begin{array}{l}35 \\
34 \\
33 \\
32 \\
31\end{array}$ & 22 & $\begin{array}{r}20 \\
16 \\
12 \\
8 \\
4\end{array}$ \\
\hline 38 & $\begin{array}{r}0 \\
4 \\
8 \\
12 \\
16\end{array}$ & $\begin{array}{l}\mathbf{3 0} \\
31 \\
32 \\
33 \\
34\end{array}$ & $\begin{array}{ll}9.61 & 773 \\
9.61 & 800 \\
9.61 & 828 \\
9.61 & 856 \\
9.61 & 883\end{array}$ & $\begin{array}{l}27 \\
28 \\
28 \\
27\end{array}$ & $\begin{array}{ll}9.65 & 870 \\
9.65 & 904 \\
9.65 & 937 \\
9.65 & 971 \\
9.66 & 004\end{array}$ & $\begin{array}{l}34 \\
33 \\
34 \\
33\end{array}$ & $\begin{array}{ll}0.34 & 130 \\
0.34 & 096 \\
0.34 & 063 \\
0.34 & 029 \\
0.33 & 996\end{array}$ & 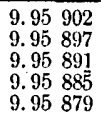 & $\begin{array}{l}5 \\
6 \\
6 \\
6\end{array}$ & $\begin{array}{r}\mathbf{3 0} \\
29 \\
28 \\
27 \\
26\end{array}$ & 22 & $\begin{array}{r}0 \\
56 \\
52 \\
48 \\
44\end{array}$ \\
\hline 38 & $\begin{array}{l}20 \\
24 \\
28 \\
32 \\
36\end{array}$ & $\begin{array}{l}35 \\
36 \\
37 \\
38 \\
39\end{array}$ & $\begin{array}{ll}9.61 & 911 \\
9.61 & 939 \\
9.61 & 966 \\
9.61 & 994 \\
9.62 & 021\end{array}$ & $\begin{array}{l}28 \\
27 \\
28 \\
27\end{array}$ & $\begin{array}{ll}9.66 & 038 \\
9.66 & 071 \\
9.66 & 104 \\
9.66 & 138 \\
9.66 & 1.71\end{array}$ & $\begin{array}{l}33 \\
33 \\
34 \\
33\end{array}$ & $\begin{array}{ll}0.33 & 962 \\
0.33 & 929 \\
0.33 & 896 \\
0.33 & 862 \\
0.33 & 829\end{array}$ & 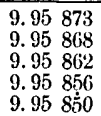 & $\begin{array}{l}5 \\
6 \\
6 \\
6\end{array}$ & $\begin{array}{l}25 \\
24 \\
23 \\
22 \\
21\end{array}$ & 21 & $\begin{array}{l}40 \\
36 \\
32 \\
28 \\
24\end{array}$ \\
\hline 38 & $\begin{array}{l}40 \\
44 \\
48 \\
52 \\
56\end{array}$ & $\begin{array}{l}40 \\
41 \\
42 \\
43 \\
44\end{array}$ & $\begin{array}{ll}9.62 & 049 \\
9.62 & 076 \\
9.62 & 104 \\
9.62 & 131 \\
9.62 & 159\end{array}$ & $\begin{array}{l}27 \\
28 \\
27 \\
28\end{array}$ & $\begin{array}{ll}9.66 & 204 \\
9.66 & 238 \\
9.66 & 271 \\
9.66 & 304 \\
9.66 & 337\end{array}$ & $\begin{array}{l}34 \\
33 \\
33 \\
33\end{array}$ & $\begin{array}{ll}0.33 & 796 \\
0.33 & 762 \\
0.33 & 729 \\
0.33 & 696 \\
0.33 & 663\end{array}$ & $\begin{array}{ll}9.95 & 844 \\
9.95 & 839 \\
9.95 & 833 \\
9.95 & 827 \\
9.95 & 821\end{array}$ & $\begin{array}{l}5 \\
6 \\
6 \\
6\end{array}$ & $\begin{array}{l}20 \\
19 \\
18 \\
17 \\
16\end{array}$ & & $\begin{array}{r}20 \\
16 \\
12 \\
8 \\
4\end{array}$ \\
\hline 39 & $\begin{array}{r}0 \\
4 \\
8 \\
12 \\
16\end{array}$ & $\begin{array}{l}45 \\
46 \\
47 \\
48 \\
49\end{array}$ & $\begin{array}{ll}9.62 & 186 \\
9.62 & 214 \\
9.62 & 241 \\
9.62 & 268 \\
9.62 & 296\end{array}$ & $\begin{array}{l}28 \\
27 \\
27 \\
28\end{array}$ & $\begin{array}{ll}9.66 & 371 \\
9.66 & 404 \\
9.66 & 437 \\
9.66 & 470 \\
9.66 & 503\end{array}$ & $\begin{array}{l}33 \\
33 \\
33 \\
33\end{array}$ & $\begin{array}{ll}0.33 & 629 \\
0.33 & 596 \\
0.33 & 563 \\
0.33 & 530 \\
0.33 & 497\end{array}$ & $\begin{array}{ll}9.95 & 81 \tilde{5} \\
9.95 & 810 \\
9.95 & 804 \\
9.95 & 798 \\
9.95 & 792\end{array}$ & $\begin{array}{l}5 \\
6 \\
6 \\
6\end{array}$ & $\begin{array}{l}15 \\
14 \\
13 \\
12 \\
11\end{array}$ & & $\begin{array}{r}0 \\
56 \\
52 \\
48 \\
44\end{array}$ \\
\hline 39 & $\begin{array}{l}20 \\
24 \\
28 \\
32 \\
36\end{array}$ & $\begin{array}{l}50 \\
51 \\
52 \\
53 \\
54\end{array}$ & $\begin{array}{ll}9.62 & 323 \\
9.62 & 350 \\
9.62 & 377 \\
9.62 & 405 \\
9.62 & 432\end{array}$ & $\begin{array}{l}27 \\
27 \\
28 \\
27\end{array}$ & $\begin{array}{ll}9.66 & 537 \\
9.66 & 570 \\
9.66 & 603 \\
9.66 & 636 \\
9.66 & 669\end{array}$ & $\begin{array}{l}33 \\
33 \\
33 \\
33\end{array}$ & $\begin{array}{ll}0.33 & 463 \\
0.33 & 430 \\
0.33 & 397 \\
0.33 & 364 \\
0.33 & 331\end{array}$ & $\begin{array}{ll}9.95 & 786 \\
9.95 & 780 \\
9.95 & 775 \\
9.95 & 769 \\
9.95 & 763\end{array}$ & $\begin{array}{l}6 \\
5 \\
6 \\
6\end{array}$ & $\begin{array}{r}10 \\
9 \\
8 \\
7 \\
6\end{array}$ & 20 & $\begin{array}{l}40 \\
36 \\
32 \\
28 \\
24\end{array}$ \\
\hline 39 & $\begin{array}{l}40 \\
44 \\
48 \\
52 \\
56\end{array}$ & $\begin{array}{l}55 \\
56 \\
57 \\
58 \\
59\end{array}$ & 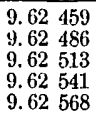 & $\begin{array}{l}27 \\
27 \\
28 \\
27\end{array}$ & $\begin{array}{ll}9.66 & 702 \\
9.66 & 735 \\
9.66 & 768 \\
9.66 & 801 \\
9.66 & 834\end{array}$ & $\begin{array}{l}33 \\
33 \\
33 \\
33\end{array}$ & $\begin{array}{ll}0.33 & 298 \\
0.33 & 265 \\
0.33 & 232 \\
0.33 & 199 \\
0.33 & 166\end{array}$ & $\begin{array}{ll}9.95 & 757 \\
9.95 & 751 \\
9.95 & 745 \\
9.95 & 739 \\
9.95 & 733\end{array}$ & $\begin{array}{l}6 \\
6 \\
6 \\
6\end{array}$ & $\begin{array}{l}5 \\
4 \\
3 \\
2 \\
1\end{array}$ & 20 & $\begin{array}{r}20 \\
16 \\
12 \\
8 \\
4\end{array}$ \\
\hline 40 & 0 & 60 & $9.6259 \overline{5}$ & & 9.66867 . & & 0.33133 & 9.95728 & 5 & 0 & 20 & 0 \\
\hline & & & Cos. & d. & L. Cotg. & c. d. & L. Tang. & L. Sin. & d. & 1 & m. & s. \\
\hline
\end{tabular}


TABLE 19.-Five-place logarithms of circular functions, etc.-Continued. $1^{\mathrm{h}}$

\begin{tabular}{|c|c|c|c|c|c|c|c|c|c|c|c|c|}
\hline $\mathrm{m}$. & s. & ' & L. Sin. & d. & L. Tang. & c. d. & L. Cotg. & I. Cos. & d. & & & \\
\hline 40 & $\begin{array}{r}0 \\
4 \\
8 \\
12 \\
16\end{array}$ & $\begin{array}{l}0 \\
1 \\
2 \\
3 \\
4\end{array}$ & 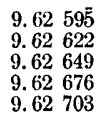 & $\begin{array}{l}27 \\
27 \\
27 \\
27\end{array}$ & 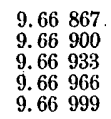 & $\begin{array}{l}33 \\
33 \\
33 \\
33\end{array}$ & $\begin{array}{ll}0.33 & 133 \\
0.33 & 100 \\
0.33 & 067 \\
0.33 & 034 \\
0.33 & 001\end{array}$ & 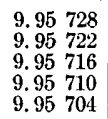 & $\begin{array}{l}6 \\
6 \\
6 \\
6\end{array}$ & $\begin{array}{l}60 \\
59 \\
58 \\
57 \\
56\end{array}$ & 20 & $\begin{array}{r}0 \\
56 \\
52 \\
48 \\
44\end{array}$ \\
\hline 40 & $\begin{array}{l}20 \\
24 \\
28 \\
32 \\
36\end{array}$ & $\begin{array}{l}5 \\
6 \\
7 \\
8 \\
9\end{array}$ & $\begin{array}{ll}9.62 & 730 \\
9.62 & 757 \\
9.62 & 784 \\
9.62 & 811 \\
9.62 & 838\end{array}$ & $\begin{array}{l}27 \\
27 \\
27 \\
27\end{array}$ & $\begin{array}{ll}9.67 & 032 \\
9.67 & 065 \\
9.67 & 098 \\
9.67 & 131 \\
9.67 & 163\end{array}$ & $\begin{array}{r}33 \\
33 \\
.33 \\
32\end{array}$ & 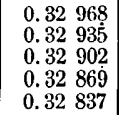 & $\begin{array}{l}9.95698 \\
9.95692 \\
9.95686 \\
9.95680 \\
9.95674\end{array}$ & $\begin{array}{l}6 \\
6 \\
6 \\
6\end{array}$ & $\begin{array}{l}55 \\
54 \\
53 \\
52 \\
51\end{array}$ & 19 & $\begin{array}{l}40 \\
36 \\
32 \\
28 \\
24\end{array}$ \\
\hline 40 & $\begin{array}{l}40 \\
44 \\
48 \\
52 \\
56\end{array}$ & $\begin{array}{l}10 \\
11 \\
12 \\
13 \\
14\end{array}$ & 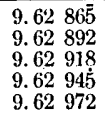 & $\begin{array}{l}27 \\
26 \\
27 \\
27\end{array}$ & $\begin{array}{ll}9.67 & 196 \\
9.67 & 229 \\
9.67 & 262 \\
9.67 & 295 \\
9.67 & 327\end{array}$ & $\begin{array}{l}33 \\
33 \\
33 \\
32\end{array}$ & $\begin{array}{ll}0.32 & 804 \\
0.32 & 771 \\
0.32 & 738 \\
0.32 & 705 \\
0.32 & 673\end{array}$ & $\begin{array}{ll}9.95 & 668 \\
9.95 & 663 \\
9.95 & 657 \\
9.95 & 651 \\
9.95 & 645\end{array}$ & $\begin{array}{l}5 \\
6 \\
6 \\
6\end{array}$ & $\begin{array}{l}50 \\
49 \\
48 \\
47 \\
46\end{array}$ & 19 & $\begin{array}{r}20 \\
16 \\
12 \\
8 \\
4\end{array}$ \\
\hline 41 & $\begin{array}{r}0 \\
4 \\
8 \\
12 \\
16\end{array}$ & $\begin{array}{l}15 \\
16 \\
17 \\
18 \\
19\end{array}$ & $\begin{array}{ll}9.62 & 999 \\
9.63 & 026 \\
9.63 & 052 \\
9.63 & 079 \\
9.63 & 106\end{array}$ & $\begin{array}{l}27 \\
27 \\
26 \\
27 \\
27\end{array}$ & 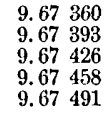 & $\begin{array}{l}33 \\
33 \\
32 \\
33\end{array}$ & $\begin{array}{ll}0.32 & 640 \\
0.32 & 607 \\
0.32 & 574 \\
0.32 & 542 \\
0.32 & 509\end{array}$ & 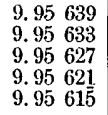 & $\begin{array}{l}6 \\
6 \\
6 \\
6\end{array}$ & $\begin{array}{l}45 \\
44 \\
43 \\
42 \\
41\end{array}$ & 19 & $\begin{array}{r}0 \\
56 \\
52 \\
48 \\
44\end{array}$ \\
\hline 41 & $\begin{array}{l}20 \\
24 \\
28 \\
32 \\
36\end{array}$ & $\begin{array}{l}\mathbf{2 0} \\
21 \\
22 \\
23 \\
24\end{array}$ & $\begin{array}{ll}9.63 & 133 \\
9.63 & 159 \\
9.63 & 186 \\
9.63 & 213 \\
9.63 & 239\end{array}$ & $\begin{array}{l}26 \\
27 \\
27 \\
26\end{array}$ & 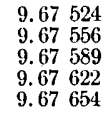 & $\begin{array}{l}00 \\
32 \\
33 \\
33 \\
32\end{array}$ & $\begin{array}{ll}0.32 & 476 \\
0.32 & 444 \\
0.32 & 411 \\
0.32 & 378 \\
0.32 & 346\end{array}$ & $\begin{array}{ll}9.95 & 609 \\
9.95 & 603 \\
9.95 & 597 \\
9.95 & 591 \\
9.95 & 585\end{array}$ & $\begin{array}{l}6 \\
6 \\
6 \\
6\end{array}$ & $\begin{array}{l}\mathbf{4 0} \\
39 \\
38 \\
37 \\
36\end{array}$ & 18 & $\begin{array}{l}40 \\
36 \\
32 \\
28 \\
24\end{array}$ \\
\hline 41 & $\begin{array}{l}40 \\
44 \\
48 \\
52 \\
56\end{array}$ & $\begin{array}{l}25 \\
26 \\
27 \\
28 \\
29\end{array}$ & $\begin{array}{ll}9.63 & 266 \\
9.63 & 292 \\
9.63 & 319 \\
9.63 & 345 \\
9.63 & 372\end{array}$ & $\begin{array}{l}26 \\
27 \\
26 \\
27\end{array}$ & $\begin{array}{ll}9.67 & 687 \\
9.67 & 719 \\
9.67 & 752 \\
9.67 & 785 \\
9.67 & 81.7\end{array}$ & $\begin{array}{l}32 \\
33 \\
33 \\
32\end{array}$ & $\begin{array}{ll}0.32 & 313 \\
0.32 & 281 \\
0.32 & 248 \\
0.32 & 215 \\
0.32 & 183\end{array}$ & $\begin{array}{ll}9.95 & 579 \\
9.95 & 573 \\
9.95 & 567 \\
9.95 & 551 \\
9.95 & 555\end{array}$ & $\begin{array}{l}6 \\
6 \\
6 \\
6\end{array}$ & $\begin{array}{l}35 \\
34 \\
33 \\
32 \\
31\end{array}$ & 18 & $\begin{array}{r}20 \\
16 \\
12 \\
8 \\
4\end{array}$ \\
\hline 42 & $\begin{array}{r}0 \\
4 \\
8 \\
12 \\
16\end{array}$ & $\begin{array}{l}\mathbf{3 0} \\
31 \\
32 \\
33 \\
34\end{array}$ & 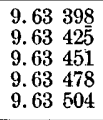 & $\begin{array}{l}27 \\
26 \\
27 \\
26\end{array}$ & 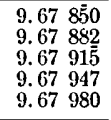 & $\begin{array}{l}32 \\
33 \\
32 \\
33\end{array}$ & $\begin{array}{ll}0.32 & 150 \\
0.32 & 118 \\
0.32 & 085 \\
0.32 & 053 \\
0.32 & 020\end{array}$ & $\begin{array}{ll}9.95 & 549 \\
9.95 & 543 \\
9.95 & 537 \\
9.95 & 531 \\
9.95 & 525\end{array}$ & $\begin{array}{l}6 \\
6 \\
6 \\
6\end{array}$ & $\begin{array}{l}30 \\
29 \\
28 \\
27 \\
26\end{array}$ & 18 & $\begin{array}{r}0 \\
56 \\
52 \\
48 \\
.44\end{array}$ \\
\hline 42 & $\begin{array}{l}20 \\
24 \\
28 \\
32 \\
36\end{array}$ & $\begin{array}{l}35 \\
36 \\
37 \\
38 \\
39\end{array}$ & 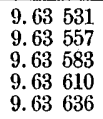 & $\begin{array}{l}26 \\
26 \\
27 \\
26\end{array}$ & $\begin{array}{ll}9.68 & 012 \\
9.68 & 044 \\
9.68 & 077 \\
9.68 & 109 \\
9.68 & 142\end{array}$ & $\begin{array}{l}32 \\
33 \\
32 \\
33\end{array}$ & $\begin{array}{ll}0.31 & 988 \\
0.31 & 956 \\
0.31 & 923 \\
0.31 & 891 \\
0.31 & 858\end{array}$ & $\begin{array}{ll}9.95 & 519 \\
9.95 & 513 \\
9.95 & 507 \\
9.95 & 500 \\
9.95 & 494\end{array}$ & $\begin{array}{l}6 \\
6 \\
7 \\
6\end{array}$ & $\begin{array}{l}25 \\
24 \\
23 \\
22 \\
21\end{array}$ & 17 & $\begin{array}{l}40 \\
36 \\
32 \\
28 \\
24\end{array}$ \\
\hline 42 & $\begin{array}{l}40 \\
44 \\
48 \\
52 \\
56\end{array}$ & $\begin{array}{l}40 \\
41 \\
42 \\
43 \\
44\end{array}$ & 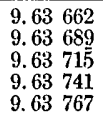 & $\begin{array}{l}27 \\
26 \\
26 \\
26\end{array}$ & $\begin{array}{ll}9.68 & 174 \\
9.68 & 206 \\
9.68 & 239 \\
9.68 & 271 \\
9.68 & 303\end{array}$ & $\begin{array}{l}32 \\
33 \\
32 \\
32\end{array}$ & $\begin{array}{ll}0.31 & 826 \\
0.31 & 794 \\
0.31 & 761 \\
0.31 & 729 \\
0.31 & 697\end{array}$ & $\begin{array}{ll}9.95 & 488 \\
9.95 & 482 \\
9.95 & 476 \\
9.95 & 470 \\
9.95 & 464\end{array}$ & $\begin{array}{l}6 \\
6 \\
6 \\
6\end{array}$ & $\begin{array}{l}\mathbf{2 0} \\
19 \\
18 \\
17 \\
16\end{array}$ & 17 & $\begin{array}{r}20 \\
16 \\
12 \\
8 \\
4\end{array}$ \\
\hline 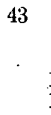 & $\begin{array}{r}0 \\
4 \\
8 \\
12 \\
16\end{array}$ & $\begin{array}{l}45 \\
46 \\
47 \\
48 \\
49\end{array}$ & $\begin{array}{l}9.63794 \\
9.63820 \\
9.63 .846 \\
9.63872 \\
9.63898\end{array}$ & $\begin{array}{l}26 \\
26 \\
26 \\
26\end{array}$ & $\begin{array}{ll}9.68 & 336 \\
9.68 & .368 \\
9.68 & 400 \\
9.68 & 432 \\
9.68 & 465\end{array}$ & $\begin{array}{l}32 \\
32 \\
32 \\
33\end{array}$ & $\begin{array}{ll}0.31 & 664 \\
0.31 & 632 \\
0.31 & 600 \\
0.31 & 568 \\
0.31 & 535\end{array}$ & 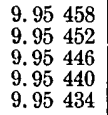 & $\begin{array}{l}6 \\
6 \\
6 \\
6\end{array}$ & $\begin{array}{l}15 \\
14 \\
13 \\
12 \\
11\end{array}$ & 17 & $\begin{array}{r}0 \\
56 \\
52 \\
48 \\
44\end{array}$ \\
\hline 43 & $\begin{array}{l}20 \\
24 \\
28 \\
32 \\
36\end{array}$ & $\begin{array}{l}\mathbf{5 0} \\
51 \\
52 \\
53 \\
54\end{array}$ & $\begin{array}{ll}9.63 & 924 \\
9.63 & 950 \\
9.63 & 976 \\
9.64 & 002 \\
9.64 & 028\end{array}$ & $\begin{array}{l}26 \\
26 \\
26 \\
26\end{array}$ & $\begin{array}{ll}9.68 & 497 \\
9.68 & 529 \\
9.68 & 561 \\
9.68 & 593 \\
9.68 & 626\end{array}$ & $\begin{array}{l}32 \\
32 \\
32 \\
33\end{array}$ & $\begin{array}{ll}0.31 & 503 \\
0.31 & 471 \\
0.31 & 439 \\
0.31 & 407 \\
0.31 & 374\end{array}$ & $\begin{array}{ll}9.95 & 427 \\
9.95 & 421 \\
9.95 & 415 \\
9.95 & 409 \\
9.95 & 403\end{array}$ & $\begin{array}{l}6 \\
6 \\
6 \\
6\end{array}$ & $\begin{array}{r}\mathbf{1 0} \\
9 \\
8 \\
7 \\
6\end{array}$ & 16 & $\begin{array}{l}40 \\
36 \\
32 \\
28 \\
24\end{array}$ \\
\hline 43 & $\begin{array}{l}40 \\
44 \\
48 \\
52 \\
56\end{array}$ & $\begin{array}{l}55 \\
56 \\
57 \\
58 \\
59\end{array}$ & $\begin{array}{l}9.64054 \\
9.64080 \\
9.64106 \\
9.64132 \\
9.64158\end{array}$ & $\begin{array}{l}26 \\
26 \\
26 \\
26\end{array}$ & $\begin{array}{ll}9.68 & 658 \\
9.68 & 690 \\
9.68 & 722 \\
9.68 & 754 \\
9.68 & 786\end{array}$ & $\begin{array}{l}32 \\
32 \\
32 \\
32\end{array}$ & $\begin{array}{ll}0.31 & 342 \\
0.31 & 310 \\
0.31 & 278 \\
0.31 & 246 \\
0.31 & 214\end{array}$ & $\begin{array}{ll}9.95 & 397 \\
9.95 & 391 \\
9.95 & 384 \\
9.95 & 378 \\
9.95 & 372\end{array}$ & $\begin{array}{l}6 \\
7 \\
6 \\
6\end{array}$ & $\begin{array}{l}5 \\
4 \\
3 \\
2 \\
1\end{array}$ & 16 & $\begin{array}{r}20 \\
16 \\
12 \\
8 \\
4\end{array}$ \\
\hline 44 & 0 & 60 & 9.64184 & & 9.68818 & & $0.31 \quad 182$ & 9.95366 & 0 & 0 & 16 & 0 \\
\hline & & & L. Cos. & d. & L. Cotg. & c. d. & L. Tang. & L. $\operatorname{Sin}$. & d. & ' & m. & 3. \\
\hline
\end{tabular}


'TABLE 19.- Five-place logarithms of circular functions, etc.-Continued.

\section{$1^{\text {h }}$}

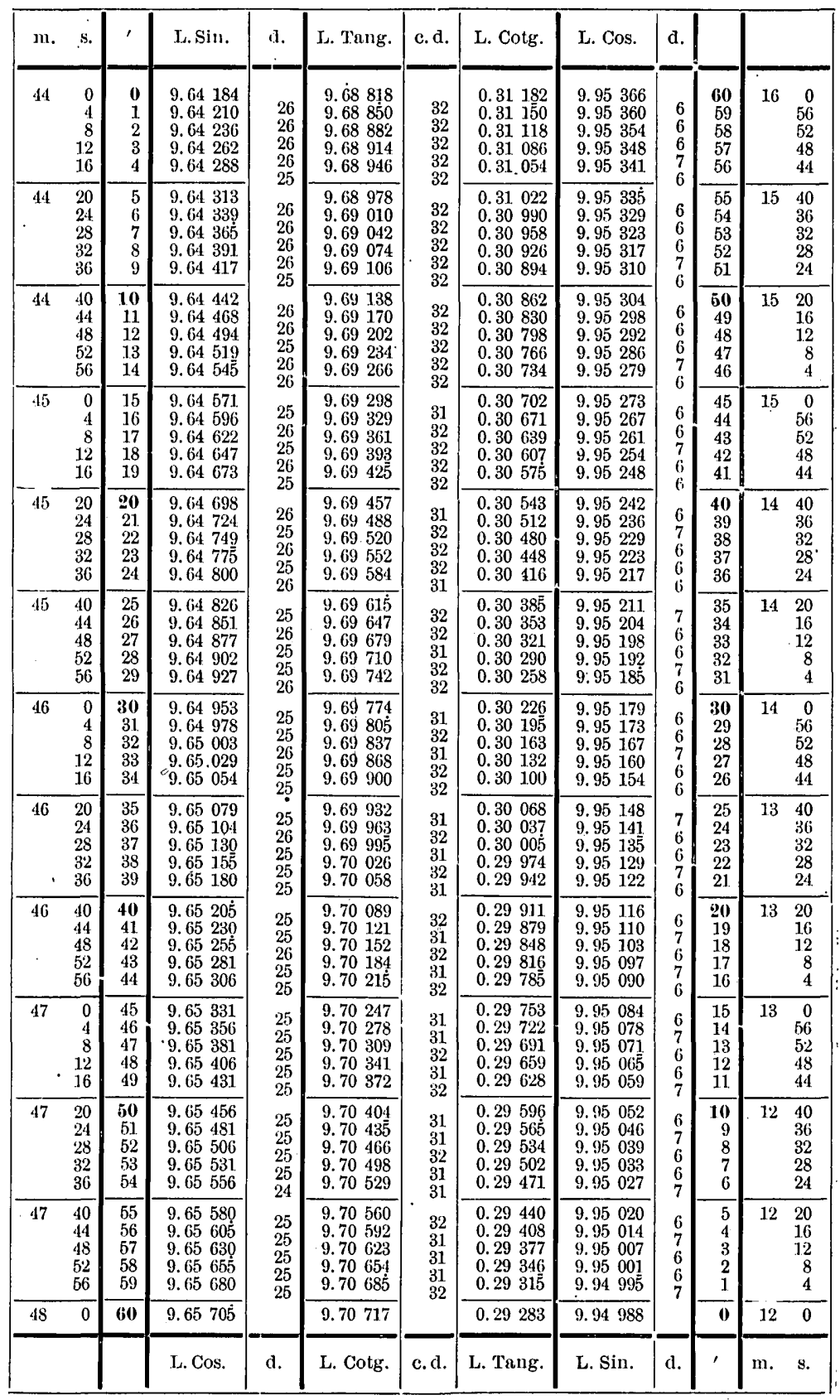

$63^{\circ}$

$26^{\circ}$ 
TABLE 19.-Five-place logarithms of circular functions, etc.-Continued.

$1^{\text {h }}$

\begin{tabular}{|c|c|c|c|c|c|c|c|c|c|c|c|c|}
\hline $\mathrm{m}$. & & 1 & L. Sin. & d. & L. Tang. & c. d. & L. Cotg. & L. Cos. & d. & & & \\
\hline 48 & $\begin{array}{r}0 \\
4 \\
8 \\
12 . \\
16\end{array}$ & $\begin{array}{l}0 \\
1 \\
2 \\
3 \\
4\end{array}$ & 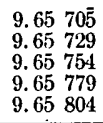 & $\begin{array}{l}24 \\
25 \\
25 \\
25\end{array}$ & 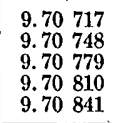 & $\begin{array}{l}31 \\
31 \\
31 \\
31\end{array}$ & $\begin{array}{ll}0.29 & 283 \\
0.29 & 252 \\
0.29 & 221 \\
0.29 & 190 \\
0.29 & 159\end{array}$ & 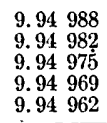 & $\begin{array}{l}6 \\
7 \\
6 \\
7\end{array}$ & $\begin{array}{l}\mathbf{6 0} \\
59 \\
58 \\
57 \\
56\end{array}$ & 12 & $\begin{array}{r}0 \\
56 \\
52 \\
48 \\
44\end{array}$ \\
\hline 48 & $\begin{array}{l}20 \\
24 \\
28 \\
32 \\
36\end{array}$ & $\begin{array}{l}5 \\
6 \\
7 \\
8 \\
9\end{array}$ & $\begin{array}{l}9.65828 \\
9.65853 \\
9.65878 \\
9.65902 \\
9.65927\end{array}$ & $\begin{array}{l}24 \\
25 \\
25 \\
24 \\
25\end{array}$ & 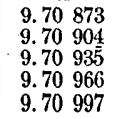 & $\begin{array}{l}32 \\
31 \\
31 \\
31 \\
31\end{array}$ & $\begin{array}{ll}0.29 & 127 \\
0.29 & 096 \\
0.29 & 065 \\
0.29 & 034 \\
0.29 & 003\end{array}$ & $\begin{array}{l}9.94956 \\
9.94949 \\
9.94943 \\
9.94936 \\
9.94930\end{array}$ & $\begin{array}{l}7 \\
6 \\
7 \\
6\end{array}$ & $\begin{array}{l}55 \\
54 \\
53 \\
52 \\
51\end{array}$ & 11 & $\begin{array}{l}40 \\
36 \\
32 \\
28 \\
24\end{array}$ \\
\hline 48 & $\begin{array}{l}40 \\
44 \\
48 \\
52 \\
56\end{array}$ & $\begin{array}{l}10 \\
11 \\
12 \\
13 \\
14\end{array}$ & $\begin{array}{ll}9.65 & 952 \\
9.65 & 976 \\
9.66 & 001 \\
9.66 & 025 \\
9.66 & 050\end{array}$ & $\begin{array}{l}24 \\
25 \\
24 \\
25\end{array}$ & $\begin{array}{ll}9.71 & 028 \\
9.71 & 059 \\
9.71 & 090 \\
9.71 & 121 \\
9.71 & 153\end{array}$ & $\begin{array}{l}31 \\
31 \\
31 \\
32\end{array}$ & 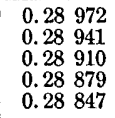 & 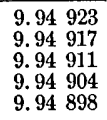 & $\begin{array}{l}6 \\
6 \\
7 \\
6\end{array}$ & $\begin{array}{l}\mathbf{5 0} \\
49 \\
48 \\
47 \\
46\end{array}$ & 11 & $\begin{array}{r}20 \\
16 \\
12 \\
8 \\
4\end{array}$ \\
\hline 49 & $\begin{array}{r}0 \\
4 \\
8 \\
12 \\
16\end{array}$ & $\begin{array}{l}15 \\
16 \\
17 \\
18 \\
19\end{array}$ & $\begin{array}{ll}9.66 & 07 \overline{5} \\
9.66 & 099 \\
9.66 & 124 \\
9.66 & 148 \\
9.66 & 173\end{array}$ & $\begin{array}{l}24 \\
25 \\
24 \\
25\end{array}$ & $\begin{array}{ll}9.71 & 184 \\
9.71 & 215 \\
9.71 & 246 \\
9.71 & 277 \\
9.71 & 308\end{array}$ & $\begin{array}{l}31 \\
31 \\
31 \\
31\end{array}$ & $\begin{array}{ll}0.28 & 816 \\
0.28 & 785 \\
0.28 & 754 \\
0.28 & 723 \\
0.28 & 692\end{array}$ & $\begin{array}{l}9.94891 \\
9.94885 \\
9.94878 \\
9.94871 \\
9.94865\end{array}$ & $\begin{array}{l}6 \\
7 \\
7 \\
6\end{array}$ & $\begin{array}{l}45 \\
44 \\
43 \\
42 \\
41\end{array}$ & 11 & $\begin{array}{r}0 \\
56 \\
52 \\
48 \\
44\end{array}$ \\
\hline 49 & $\begin{array}{l}20 \\
24 \\
28 \\
32 \\
36\end{array}$ & $\begin{array}{l}20 \\
21 \\
22 \\
23 \\
24\end{array}$ & $\begin{array}{ll}9.66 & 197 \\
9.66 & 221 \\
9.66 & 246 \\
9.66 & 270 \\
9.66 & 295\end{array}$ & $\begin{array}{l}24 \\
24 \\
25 \\
24 \\
25\end{array}$ & $\begin{array}{ll}9.71 & 339 \\
9.71 & 370 \\
9.71 & 401 \\
9.71 & 431 \\
9.71 & 462\end{array}$ & $\begin{array}{l}31 \\
31 \\
31 \\
31 \\
31\end{array}$ & $\begin{array}{ll}0.28 & 661 \\
0.28 & 630 \\
0.28 & 599 \\
0.28 & 569 \\
0.28 & 538\end{array}$ & $\begin{array}{l}9.94858 \\
9.94852 \\
9.94845 \\
9.94839 \\
9.94832\end{array}$ & $\begin{array}{l}6 \\
7 \\
6 \\
7\end{array}$ & $\begin{array}{l}\mathbf{4 0} \\
39 \\
38 \\
37 \\
36\end{array}$ & 10 & $\begin{array}{l}40 \\
36 \\
32 \\
28 \\
24\end{array}$ \\
\hline 49 & $\begin{array}{l}40 \\
44 \\
48 \\
52 \\
56\end{array}$ & $\begin{array}{l}25 \\
26 \\
27 \\
28 \\
29\end{array}$ & $\begin{array}{ll}9.66 & 319 \\
9.66 & 343 \\
9.66 & 368 \\
9.66 & 392 \\
9.66 & 416\end{array}$ & $\begin{array}{l}24 \\
25 \\
24 \\
24\end{array}$ & $\begin{array}{ll}9.71 & 493 \\
9.71 & 524 \\
9.71 & 555 \\
9.71 & 586 \\
9.71 & 617\end{array}$ & $\begin{array}{l}31 \\
31 \\
31 \\
31\end{array}$ & $\begin{array}{ll}0.28 & 507 \\
0.28 & 476 \\
0.28 & 445 \\
0.28 & 414 \\
0.28 & 383\end{array}$ & $\begin{array}{ll}9.94 & 826 \\
9.94 & 819 \\
9.94 & 813 \\
9.94 & 806 \\
9.94 & 799\end{array}$ & $\begin{array}{l}7 \\
6 \\
7 \\
7\end{array}$ & $\begin{array}{l}35 \\
34 \\
33 \\
32 \\
31\end{array}$ & 10 & $\begin{array}{r}20 \\
16 \\
12 \\
8 \\
4\end{array}$ \\
\hline 50 & $\begin{array}{r}0 \\
4 \\
8 \\
12 \\
16\end{array}$ & $\begin{array}{l}\mathbf{3 0} \\
31 \\
32 \\
33 \\
34\end{array}$ & $\begin{array}{ll}9.66 & 441 \\
9.66 & 465 \\
9.66 & 489 \\
9.66 & 513 \\
9.66 & 537\end{array}$ & $\begin{array}{l}20 \\
24 \\
24 \\
24 \\
24\end{array}$ & $\begin{array}{ll}9.71 & 648 \\
9.71 & 679 \\
9.71 & 709 \\
9.71 & 740 \\
9.71 & 771\end{array}$ & $\begin{array}{l}31 \\
30 \\
31 \\
31\end{array}$ & $\begin{array}{ll}0.28 & 352 \\
0.28 & 321 \\
0.28 & 291 \\
0.28 & 260 \\
0.28 & 229\end{array}$ & 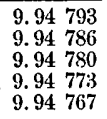 & $\begin{array}{l}7 \\
6 \\
7 \\
6\end{array}$ & $\begin{array}{l}\mathbf{3 0} \\
29 \\
28 \\
27 \\
26\end{array}$ & 10 & $\begin{array}{r}0 \\
56 \\
52 \\
48 \\
44\end{array}$ \\
\hline 50 & $\begin{array}{l}20 \\
24 \\
28 \\
32 \\
36\end{array}$ & $\begin{array}{l}35 \\
36 \\
37 \\
38 \\
39\end{array}$ & $\begin{array}{ll}9.66 & 562 \\
9.66 & 586 \\
9.66 & 610 \\
9.66 & 634 \\
9.66 & 658\end{array}$ & $\begin{array}{l}24 \\
24 \\
24 \\
24\end{array}$ & $\begin{array}{ll}9.71 & 802 \\
9.71 & 833 \\
9.71 & 863 \\
9.71 & 894 \\
9.71 & 925\end{array}$ & $\begin{array}{l}31 \\
30 \\
31 \\
31\end{array}$ & $\begin{array}{ll}0.28 & 198 \\
0.28 & 167 \\
0.28 & 137 \\
0.28 & 106 \\
0.28 & 075\end{array}$ & 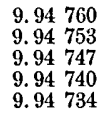 & $\begin{array}{l}7 \\
6 \\
7 \\
6\end{array}$ & $\begin{array}{l}25 \\
24 \\
23 \\
22 \\
21\end{array}$ & 9 & $\begin{array}{l}40 \\
36 \\
32 \\
28 \\
24\end{array}$ \\
\hline 50 & $\begin{array}{l}40 \\
44 \\
48 \\
52 \\
56\end{array}$ & $\begin{array}{l}40 \\
41 \\
42 \\
43 \\
44\end{array}$ & $\begin{array}{ll}9.66 & 682 \\
9.66 & 706 \\
9.66 & 731 \\
9.66 & 755 \\
9.66 & 779\end{array}$ & $\begin{array}{l}24 \\
25 \\
24 \\
24\end{array}$ & $\begin{array}{ll}9.71 & 95 \dot{5} \\
9.71 & 986 \\
9.72 & 017 \\
9.72 & 048 \\
9.72 & 078\end{array}$ & $\begin{array}{l}31 \\
31 \\
31 \\
30\end{array}$ & $\begin{array}{ll}0.28 & 04 \overline{5} \\
0.28 & 014 \\
0.27 & 983 \\
0.27 & 952 \\
0.27 & 922\end{array}$ & 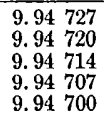 & $\begin{array}{l}7 \\
7 \\
6 \\
7 \\
7\end{array}$ & $\begin{array}{l}20 \\
19 \\
18 \\
17 \\
16\end{array}$ & $\overline{9}$ & $\begin{array}{r}20 \\
16 \\
12 \\
8 \\
4\end{array}$ \\
\hline 51 & $\begin{array}{r}0 \\
4 \\
8 \\
12 \\
16\end{array}$ & $\begin{array}{l}45 \\
46 \\
47 \\
48 \\
49\end{array}$ & $\begin{array}{ll}9.66 & 803 \\
9.66 & 827 \\
9.66 & 851 \\
9.66 & 875 \\
9.66 & 899\end{array}$ & $\begin{array}{l}24 \\
24 \\
24 \\
24\end{array}$ & $\begin{array}{lll}9.72 & 109 \\
9.72 & 140 \\
9.72 & 170 \\
9.72 & 201 \\
9.72 & 231\end{array}$ & $\begin{array}{l}31 \\
30 \\
31 \\
30\end{array}$ & 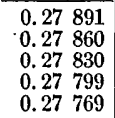 & $\begin{array}{l}9.94694 \\
9.94687 \\
9.94680 \\
9.94674 \\
9.94667\end{array}$ & $\begin{array}{l}7 \\
7 \\
6 \\
7\end{array}$ & $\begin{array}{l}15 \\
14 \\
13 \\
12 \\
11\end{array}$ & 9 & $\begin{array}{r}0 \\
56 \\
52 \\
48 \\
44\end{array}$ \\
\hline 51 & $\begin{array}{l}20 \\
24 \\
28 \\
32 \\
36\end{array}$ & $\begin{array}{l}\mathbf{5 0} \\
51 \\
52 \\
53 \\
54\end{array}$ & $\begin{array}{l}9.66922 \\
9.66946 \\
9.66970 \\
9.66994 \\
9.67018\end{array}$ & $\begin{array}{l}24 \\
24 \\
24 \\
24\end{array}$ & $\begin{array}{ll}9.72 & 262 \\
9.72 & 293 \\
9.72 & 323 \\
9.72 & 354 \\
9.72 & 384\end{array}$ & $\begin{array}{l}31 \\
30 \\
31 \\
30\end{array}$ & 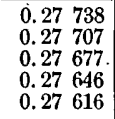 & 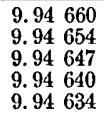 & $\begin{array}{l}6 \\
7 \\
7 \\
6\end{array}$ & $\begin{array}{r}10 \\
9 \\
8 \\
7 \\
6\end{array}$ & 8 & $\begin{array}{l}40 \\
36 \\
32 \\
28 \\
24\end{array}$ \\
\hline 51 & $\begin{array}{l}40 \\
44 \\
48 \\
52 \\
56\end{array}$ & $\begin{array}{l}\mathbf{5 5} \\
56 \\
\mathbf{5 7} \\
58 \\
59\end{array}$ & $\begin{array}{ll}9.67 & 042 \\
9.67 & 066 \\
9.67 & 090 \\
9.67 & 113 \\
9.67 & 137\end{array}$ & $\begin{array}{l}24 \\
24 \\
23 \\
24\end{array}$ & $\begin{array}{ll}9.72 & 41 \overline{5} \\
9.72 & 44 \overline{5} \\
9.72 & 476 \\
9.72 & 506 \\
9.72 & 537\end{array}$ & $\begin{array}{l}30 \\
31 \\
30 \\
31\end{array}$ & $\begin{array}{ll}0.27 & 585 \\
0.27 & 555 \\
0.27 & 524 \\
5.27 & 494 \\
0.27 & 463\end{array}$ & 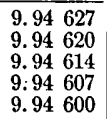 & $\begin{array}{l}7 \\
6 \\
7 \\
7\end{array}$ & $\begin{array}{l}5 \\
4 \\
3 \\
2 \\
1\end{array}$ & 8 & $\begin{array}{r}20 \\
16 \\
12 \\
8 \\
4\end{array}$ \\
\hline 52 & 0 & 60 & 9.67161 & & 9.72567 & & 0.27433 & 9.94593 & 7 & 0 & 8 & 0 \\
\hline & & & L. Cos. & d. & L. Cotg. & c. d. & L. Tang. & L. Sin. & d. & ' & $\mathrm{m}$ & s. \\
\hline
\end{tabular}

$27^{\circ}$ 
TABLe 19.-Five-place logarithms of circular functions, etc.-Continued.

$1^{\mathrm{h}}$

\begin{tabular}{|c|c|c|c|c|c|c|c|c|c|c|c|c|}
\hline m.' & s. & , & I. Sin. & d. & L. Tang. & c. $d$. & L. Cotg. & L. Cos. & d. & & & \\
\hline 52 & $\begin{array}{r}0 \\
4 \\
8 \\
12 \\
16\end{array}$ & $\begin{array}{l}0 \\
1 \\
2 \\
3 \\
4\end{array}$ & $\begin{array}{ll}9.67 & 161 \\
9.67 & 185 \\
9.67 & 208 \\
9.67 & 232 \\
9.67 & 256\end{array}$ & \multirow{3}{*}{$\begin{array}{l}24 \\
23 \\
24 \\
24 \\
24 \\
23 \\
24 \\
23 \\
24 \\
24 \\
23 \\
24 \\
23 \\
24 \\
23\end{array}$} & 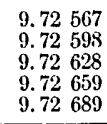 & \multirow{2}{*}{$\begin{array}{l}31 \\
30 \\
31 \\
30 \\
31 \\
\\
30 \\
30 \\
31 \\
30 \\
31\end{array}$} & $\begin{array}{ll}0.27 & 433 \\
0.27 & 402 \\
0.27 & 372 \\
0.27 & 341 \\
0.27 & 311\end{array}$ & $\begin{array}{l}9.94593 \\
\mathbf{9 . 9 4} 587 \\
\mathbf{9 . 9 4} 580 \\
9.94573 \\
9.94567\end{array}$ & \multirow{2}{*}{$\begin{array}{l}6 \\
7 \\
7 \\
6 \\
7 \\
7 \\
7 \\
6 \\
7 \\
7\end{array}$} & $\begin{array}{l}60 \\
59 \\
58 \\
57 \\
56\end{array}$ & & $\begin{array}{r}0 \\
56 \\
52 \\
48 \\
44\end{array}$ \\
\hline 52 & $\begin{array}{l}20 \\
24 \\
28 \\
32 \\
36\end{array}$ & $\begin{array}{l}5 \\
6 \\
7 \\
8 \\
9\end{array}$ & 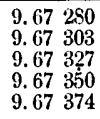 & & 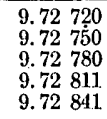 & & $\begin{array}{ll}0.27 & 280 \\
0.27 & 250 \\
0.27 & 220 \\
0.27 & 189 \\
0.27 & 159\end{array}$ & $\begin{array}{l}\mathbf{9 . 9 4} 560 \\
\mathbf{9 . 9 4} 553 \\
9.94 \\
\mathbf{5 4 6} \\
\mathbf{9 . 9 4} 540 \\
\mathbf{9 . 9 4} 533\end{array}$ & & $\begin{array}{l}55 \\
54 \\
53 \\
52 \\
51\end{array}$ & 7 & $\begin{array}{l}40 \\
36 \\
32 \\
28 \\
24\end{array}$ \\
\hline 52 & $\begin{array}{l}40 \\
44 \\
48 \\
52 \\
56\end{array}$ & $\begin{array}{l}10 \\
11 \\
12 \\
13 \\
14\end{array}$ & 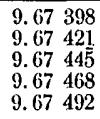 & & $\begin{array}{ll}9.72 & 872 \\
9.72 & 902 \\
9.72 & 932 \\
9.72 & 963 \\
9.72 & 993\end{array}$ & $\begin{array}{l}30 \\
30 \\
31 \\
30\end{array}$ & $\begin{array}{ll}0.27 & 128 \\
0.27 & 098 \\
0.27 & 068 \\
0.27 & 037 \\
0.27 & 007\end{array}$ & $\begin{array}{ll}9.94 & 526 \\
9.94 & 519 \\
9.94 & 513 \\
9.94 & 506 \\
9.94 & 499\end{array}$ & $\begin{array}{l}7 \\
6 \\
7 \\
7 \\
7\end{array}$ & $\begin{array}{r}50 \\
49 \\
48 \\
47 \\
46\end{array}$ & 7 & $\begin{array}{r}20 \\
16 \\
12 \\
8 \\
4\end{array}$ \\
\hline 53 & $\begin{array}{r}0 \\
4 \\
8 \\
12 \\
16\end{array}$ & $\begin{array}{l}15 \\
16 \\
17 \\
18 \\
19\end{array}$ & $\begin{array}{ll}9.67 & 51 \dot{5} \\
9.67 & 539 \\
9.67 & 562 \\
9.67 & 586 \\
9.67 & 609\end{array}$ & \multirow{3}{*}{$\begin{array}{l}24 \\
23 \\
24 \\
23 \\
24 \\
23 \\
24 \\
23 \\
23 \\
24 \\
23 \\
23 \\
24 \\
23 \\
23\end{array}$} & $\begin{array}{lll}9.73 & 023 \\
9.73 & 054 \\
9.73 & 084 \\
9.73 & 114 \\
9.73 & 144\end{array}$ & $\begin{array}{l}31 \\
30 \\
30 \\
30\end{array}$ & $\begin{array}{ll}0.26 & 977 \\
0.26 & 946 \\
0.26 & 916 \\
0.26 & 886 \\
0.26 & 856\end{array}$ & 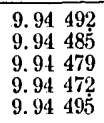 & $\begin{array}{r}7 \\
6 \\
7 \\
7 \\
7\end{array}$ & $\begin{array}{l}45 \\
44 \\
43 \\
42 \\
41\end{array}$ & 7 & $\begin{array}{r}0 \\
56 \\
52 \\
48 \\
44\end{array}$ \\
\hline 53 & $\begin{array}{l}20 \\
24 \\
28 \\
32 \\
36\end{array}$ & $\begin{array}{l}\mathbf{2 0} \\
21 \\
22 \\
23 \\
24\end{array}$ & 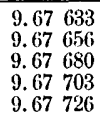 & & $\begin{array}{lll}9.73 & 175 \\
9.73 & 205 \\
9.73 & 235 \\
9.73 & 265 \\
9.73 & 295\end{array}$ & $\begin{array}{l}30 \\
30 \\
30 \\
30 \\
30 \\
31\end{array}$ & 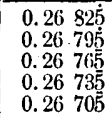 & 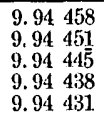 & $\begin{array}{l}7 \\
6 \\
7 \\
7 \\
7\end{array}$ & $\begin{array}{l}\mathbf{4 0} \\
39 \\
38 \\
37 \\
36\end{array}$ & 6 & $\begin{array}{l}40 \\
36 \\
32 \\
28 \\
24\end{array}$ \\
\hline 53. & $\begin{array}{l}40 \\
44 \\
48 \\
52 \\
56\end{array}$ & $\begin{array}{l}25 \\
26 \\
27 \\
28 \\
29\end{array}$ & 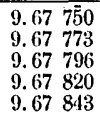 & & 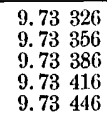 & $\begin{array}{l}30 \\
30 \\
30 \\
30\end{array}$ & $\begin{array}{ll}0.26 & 674 \\
0.26 & 644 \\
0.26 & 614 \\
0.26 & 584 \\
0.26 & 554\end{array}$ & 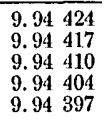 & $\begin{array}{l}7 \\
7 \\
6 . \\
7\end{array}$ & $\begin{array}{l}35 \\
34 \\
33 \\
32 \\
31\end{array}$ & & $\begin{array}{r}20 \\
16 \\
12 \\
8 \\
4\end{array}$ \\
\hline 54 & $\begin{array}{r}0 \\
4 \\
8 \\
12 \\
16\end{array}$ & $\begin{array}{l}30 \\
31 \\
32 \\
33 \\
34\end{array}$ & 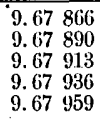 & $\begin{array}{l}24 \\
23 \\
28 \\
28\end{array}$ & $\begin{array}{lll}9.73 & 476 \\
9.73 & 507 \\
9.73 & 537 \\
9.73 & 567 \\
9.73 & 597 .\end{array}$ & $\begin{array}{l}31 \\
30 \\
30 \\
30\end{array}$ & $\begin{array}{ll}0.26 & 524 \\
0.26 & 493 \\
0.26 & 463 \\
0.26 & 433 \\
0.26 & 403\end{array}$ & 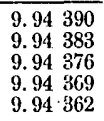 & $\begin{array}{l}7 \\
7 \\
7 \\
7\end{array}$ & $\begin{array}{l}30 \\
29 \\
28 \\
27 \\
26\end{array}$ & 6 & $\begin{array}{r}0 \\
56 \\
52 \\
48 \\
44\end{array}$ \\
\hline 54 & $\begin{array}{l}20 \\
24 \\
28 \\
32 \\
36\end{array}$ & $\begin{array}{l}35 \\
36 \\
37 \\
38 \\
39\end{array}$ & $\begin{array}{ll}9.67 & 982 \\
9.68 & 006 \\
9.68 & 029 \\
9.68 & 052 \\
9.68 & 075\end{array}$ & $\begin{array}{l}24 \\
23 \\
23 \\
23 \\
23\end{array}$ & $\begin{array}{lll}9.73 & 627 \\
9.73 & 657 \\
9.73 & 687 \\
9.73 & 717 \\
9.73 & 747\end{array}$ & $\begin{array}{l}30 \\
30 \\
30 \\
30 \\
30\end{array}$ & $\begin{array}{ll}0.26 & 373 \\
0.26 & 343 \\
0.26 & 313 \\
0.26 & 283 \\
0.26 & 253\end{array}$ & $\begin{array}{l}9.94355 \\
9.94349 \\
9.94342 \\
9.94335 \\
9.94328\end{array}$ & $\begin{array}{l}6 \\
7 \\
7 \\
7 \\
7\end{array}$ & $\begin{array}{l}25 \\
24 \\
23 \\
22 \\
21\end{array}$ & 5 & $\begin{array}{l}40 \\
36 \\
32 \\
28 \\
24\end{array}$ \\
\hline 54 & $\begin{array}{l}40 \\
44 \\
48 \\
52 \\
56\end{array}$ & $\begin{array}{l}40 \\
41 \\
42 \\
43 \\
44\end{array}$ & $\begin{array}{ll}9.68 & 098 \\
9.68 & 121 \\
9.68 & 144 \\
9.68 & 167 \\
9.68 & 190\end{array}$ & $\begin{array}{l}23 \\
23 \\
23 \\
23\end{array}$ & 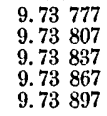 & $\begin{array}{l}30 \\
30 \\
30 \\
30\end{array}$ & $\begin{array}{ll}0.26 & 223 \\
0.26 & 193 \\
0.26 & 163 \\
0.26 & 133 \\
0.26 & 103\end{array}$ & 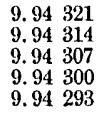 & $\begin{array}{l}7 \\
7 \\
7 \\
7\end{array}$ & $\begin{array}{l}20 \\
19 \\
18 \\
17 \\
16\end{array}$ & 5 & $\begin{array}{r}20 \\
16 \\
12 \\
8 \\
4\end{array}$ \\
\hline 55 & $\begin{array}{r}0 \\
4 \\
8 \\
12 \\
16\end{array}$ & $\begin{array}{l}45 \\
46 \\
47 \\
48 \\
49\end{array}$ & 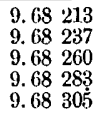 & $\begin{array}{l}24 \\
24 \\
.3 \\
22 \\
92\end{array}$ & $\begin{array}{ll}9.73 & 927 \\
9.73 & 957 \\
9.73 & 987 \\
9.74 & 017 \\
9.74 & 047\end{array}$ & $\begin{array}{l}30 \\
30 \\
30 \\
30\end{array}$ & $\begin{array}{ll}0.26 & 073 \\
0.26 & 043 \\
0.26 & 013 \\
0.25 & 983 \\
0.25 & 953\end{array}$ & 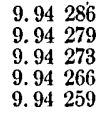 & $\begin{array}{l}7 \\
6 \\
7 \\
7\end{array}$ & $\begin{array}{l}15 \\
14 \\
13 \\
12 \\
11\end{array}$ & 5 & $\begin{array}{r}0 \\
56 \\
52 \\
48 \\
\mathbf{4 4}\end{array}$ \\
\hline 55 & $\begin{array}{l}20 \\
24 \\
28 \\
32 \\
36\end{array}$ & $\begin{array}{l}\mathbf{5 0} \\
51 \\
52 \\
53 \\
54\end{array}$ & $\begin{array}{ll}9.68 & 328 \\
9.68 & 351 \\
9.68 & 374 \\
9.68 & 397 \\
9.68 & 420\end{array}$ & $\begin{array}{l}23 \\
23 \\
23 \\
23\end{array}$ & $\begin{array}{lll}9.74 & 077 \\
9.74 & 107 \\
9.74 & 137 \\
9.74 & 166 \\
9.74 & 196\end{array}$ & $\begin{array}{l}30 \\
30 \\
29 \\
30\end{array}$ & $\begin{array}{ll}0.25 & 923 \\
0.25 & 893 \\
0.25 & 863 \\
0.25 & 834 \\
0.25 & 804\end{array}$ & $\begin{array}{ll}9.94 & 252 \\
9.94 & 245 \\
9.94 & 238 \\
9.94 & 231 \\
9.94 & 224\end{array}$ & $\begin{array}{l}7 \\
7 \\
7 \\
7 \\
7\end{array}$ & $\begin{array}{r}10 \\
9 \\
8 \\
7 \\
6\end{array}$ & 4 & $\begin{array}{l}40 \\
36 \\
32 \\
28 \\
24\end{array}$ \\
\hline 55 & $\begin{array}{l}40 \\
44 \\
48 \\
52 \\
56\end{array}$ & $\begin{array}{l}55 \\
56 \\
57 \\
58 \\
59\end{array}$ & 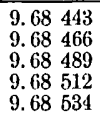 & $\begin{array}{l}23 \\
23 \\
23 \\
22\end{array}$ & $\begin{array}{ll}9.74 & 226 \\
9.74 & 256 \\
9.74 & 286 \\
9.74 & 316 \\
9.74 & 345\end{array}$ & $\begin{array}{l}30 \\
30 \\
30 \\
29\end{array}$ & $\begin{array}{ll}0.25 & 774 \\
0.25 & 744 \\
0.25 & 714 \\
0.25 & 684 \\
0.25 & 655\end{array}$ & 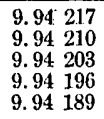 & $\begin{array}{l}7 \\
7 \\
7 \\
7 \\
7\end{array}$ & $\begin{array}{l}5 \\
4 \\
3 \\
2 \\
1\end{array}$ & 4 & $\begin{array}{r}20 \\
16 \\
12 \\
8 \\
4\end{array}$ \\
\hline 56 & 0 & $\overline{60}$ & $9.68 \quad 557$ & & $9.7437 \dot{5}$ & & $0.2562 \overline{5}$ & 9.94182 & & $\overline{0}$ & 4 & 0 \\
\hline & & & L. Cos. & $a$ & L. Cotg. & c.d. & L. Tang. & L. $\operatorname{Sin}$. & d. & ' & $\mathrm{m}$. & \\
\hline
\end{tabular}


TABLE 19.-Five-place logarithms of circular functions, etc.-Continued.

$1^{\mathrm{h}}$

\begin{tabular}{|c|c|c|c|c|c|c|c|c|c|c|c|c|}
\hline $\mathrm{m}$. & s. & ' & I. Sin. & d. & L. Tang. & c. d. & L. Cotg. & L. Cos. & d. & & & \\
\hline 56 & $\begin{array}{r}0 \\
4 \\
8 \\
12 \\
16\end{array}$ & $\begin{array}{r}0 \\
1 \\
2 \\
3 \\
4\end{array}$ & $\begin{array}{l}9.68557 \\
9.68580 \\
9.68603 \\
9.68625 \\
9.68648\end{array}$ & $\begin{array}{l}23 \\
23 \\
22 \\
23 \\
23\end{array}$ & $\begin{array}{l}9.74375 \\
9.74405 \\
9.74435 \\
9.74465 \\
9.74494\end{array}$ & $\begin{array}{l}30 \\
30 \\
30 \\
29\end{array}$ & $\begin{array}{ll}0.25 & 62 \overline{5} \\
0.25 & 595 \\
0.25 & 565 \\
0.25 & 535 \\
0.25 & 506\end{array}$ & 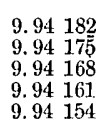 & $\begin{array}{l}7 \\
7 \\
7 \\
7\end{array}$ & $\begin{array}{l}\mathbf{6 0} \\
59 \\
58 \\
57 \\
56\end{array}$ & & $\begin{array}{r}0 \\
56 \\
52 \\
48 \\
44\end{array}$ \\
\hline 56 & $\begin{array}{l}20 \\
24 \\
28 \\
32 \\
36\end{array}$ & $\begin{array}{l}5 \\
6 \\
7 \\
8 \\
9\end{array}$ & $\begin{array}{ll}9.68 & 671 \\
9.68 & 694 \\
9.68 & 716 \\
9.68 & 739 \\
9.68 & 762\end{array}$ & $\begin{array}{l}23 \\
22 \\
23 \\
23 \\
00\end{array}$ & 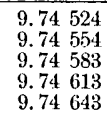 & $\begin{array}{l}30 \\
29 \\
30 \\
30\end{array}$ & $\begin{array}{ll}0.25 & 476 \\
0.25 & 446 \\
0.25 & 417 \\
0.25 & 387 \\
0.25 & 357\end{array}$ & 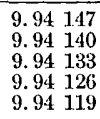 & $\begin{array}{l}7 \\
7 \\
7 \\
7\end{array}$ & $\begin{array}{l}55^{\circ} \\
54 \\
53 \\
52 \\
51\end{array}$ & 3 & $\begin{array}{l}40 \\
36 \\
32 \\
28 \\
24\end{array}$ \\
\hline 56 & $\begin{array}{l}40 \\
44 \\
48 \\
52 \\
56\end{array}$ & $\begin{array}{l}10 \\
11 \\
12 \\
13 \\
14\end{array}$ & 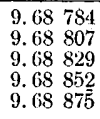 & $\begin{array}{l}23 \\
22 \\
23 \\
23\end{array}$ & 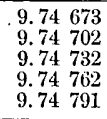 & $\begin{array}{l}29 \\
30 \\
30 \\
29\end{array}$ & $\begin{array}{ll}0.25 & 327 \\
0.25 & 298 \\
0.25 & 268 \\
0.25 & 238 \\
0.25 & 209\end{array}$ & $\begin{array}{ll}9.94 & 112 \\
9.94 & 105 \\
9.94 & 098 \\
9.94 & 090 \\
9.94 & 083\end{array}$ & $\begin{array}{l}7 \\
7 \\
8 \\
7\end{array}$ & $\begin{array}{l}50 \\
49 \\
48 \\
47 \\
46\end{array}$ & 3 & $\begin{array}{r}20 \\
16 \\
12 \\
8 \\
4\end{array}$ \\
\hline 57 & $\begin{array}{r}0 \\
4 \\
8 \\
12 \\
16\end{array}$ & $\begin{array}{l}15 \\
16 \\
17 \\
18 \\
19\end{array}$ & 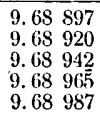 & $\begin{array}{l}23 \\
22 \\
23 \\
22\end{array}$ & $\begin{array}{ll}9.74 & 821 \\
9.74 & 851 \\
9.74 & 880 \\
9.74 & 910 \\
9.74 & 939\end{array}$ & $\begin{array}{l}30 \\
29 \\
30 \\
29\end{array}$ & $\begin{array}{ll}0.25 & 179 \\
0.25 & 149 \\
0.25 & 120 \\
0.25 & 090 \\
0.25 & 061\end{array}$ & 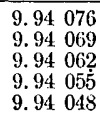 & $\begin{array}{l}7 \\
7 \\
7 \\
7\end{array}$ & $\begin{array}{r}45 \\
44 \\
43 \\
42 \\
.41\end{array}$ & 3 & $\begin{array}{r}0 \\
56 \\
52 \\
48 \\
44\end{array}$ \\
\hline 57 & $\begin{array}{l}20 \\
24 \\
28 \\
32 \\
36\end{array}$ & $\begin{array}{l}\mathbf{2 0} \\
21 \\
22 \\
23 \\
24\end{array}$ & $\begin{array}{ll}9.69 & 010 \\
9.69 & 032 \\
9.69 & 055 \\
9.69 & 077 \\
9.69 & 100\end{array}$ & $\begin{array}{l}22 \\
23 \\
22 \\
23\end{array}$ & $\begin{array}{ll}9.74 & 969 \\
9.74 & 998 \\
9.75 & 028 \\
9.75 & 058 \\
9.75 & 087\end{array}$ & $\begin{array}{l}29 \\
30 \\
30 \\
29\end{array}$ & $\begin{array}{ll}0.25 & 031 \\
0.25 & 002 \\
0.24 & 972 \\
0.24 & 942 \\
0.24 & 913\end{array}$ & $\begin{array}{ll}9.94 & 041 \\
9.94 & 034 \\
9.94 & 027 \\
9.94 & 020 \\
9.94 & 012\end{array}$ & $\begin{array}{l}7 \\
7 \\
7 \\
8\end{array}$ & $\begin{array}{l}\mathbf{4 0} \\
39 \\
38 \\
37 \\
36\end{array}$ & 2 & $\begin{array}{l}40 \\
36 \\
32 \\
28 \\
24\end{array}$ \\
\hline 57 & $\begin{array}{l}40 \\
44 \\
48 \\
52 \\
56\end{array}$ & $\begin{array}{l}25 \\
26 \\
27 \\
28 \\
29\end{array}$ & $\begin{array}{ll}9.69 & 122 \\
9.69 & 144 \\
9.69 & 167 \\
9.69 & 189 \\
9.69 & 212 \\
\end{array}$ & $\begin{array}{l}22 \\
23 \\
22 \\
23\end{array}$ & $\begin{array}{ll}9.75 & 117 \\
9.75 & 146 \\
9.75 & 176 \\
9.75 & 205 \\
9.75 & 235\end{array}$ & $\begin{array}{l}29 \\
30 \\
29 \\
30\end{array}$ & 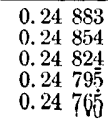 & 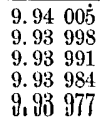 & $\begin{array}{l}7 \\
7 \\
7 \\
7\end{array}$ & $\begin{array}{l}35 \\
34 \\
33 \\
32 \\
31\end{array}$ & 2 & $\begin{array}{r}20 \\
16 \\
12 \\
8 \\
4\end{array}$ \\
\hline 58 & $\begin{array}{r}0 \\
4 \\
.8 \\
12 \\
16\end{array}$ & $\begin{array}{l}30 \\
31 \\
32 \\
33 \\
34\end{array}$ & $\begin{array}{ll}9.69 & 234 \\
9.69 & 256 \\
9.69 & 279 \\
9.69 & 301 \\
9.69 & 323\end{array}$ & $\begin{array}{l}22 \\
23 \\
22 \\
22\end{array}$ & 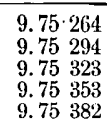 & $\begin{array}{l}30 \\
29 \\
30 \\
29\end{array}$ & 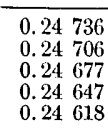 & 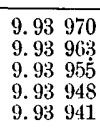 & $\begin{array}{l}7 \\
8 \\
7 \\
7\end{array}$ & $\begin{array}{l}30 \\
29 \\
28 \\
27 \\
26\end{array}$ & 2 & $\begin{array}{r}0 \\
56 \\
52 \\
48 \\
44\end{array}$ \\
\hline 58 & $\begin{array}{l}20 \\
24 \\
28 \\
32 \\
36\end{array}$ & $\begin{array}{l}35 \\
36 \\
37 \\
38 \\
39\end{array}$ & 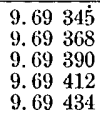 & $\begin{array}{l}23 \\
22 \\
22 \\
22\end{array}$ & $\begin{array}{ll}9.75 & 411 \\
9.75 & 441 \\
9.75 & 470 \\
9.75 & 500 \\
9.75 & 529\end{array}$ & $\begin{array}{l}30 \\
29 \\
30 \\
29\end{array}$ & $\begin{array}{ll}0.24 & 589 \\
0.24 & 559 \\
0.24 & 530 \\
0.24 & 500 \\
0.24 & 471\end{array}$ & 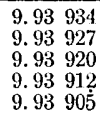 & $\begin{array}{l}7 \\
7 \\
8 \\
7\end{array}$ & $\begin{array}{l}25 \\
24 \\
23 \\
22 \\
21\end{array}$ & 1 & $\begin{array}{l}40 \\
36 \\
32 \\
28 \\
24\end{array}$ \\
\hline 58 & $\begin{array}{l}40 \\
44 \\
48 \\
52 \\
56\end{array}$ & $\begin{array}{l}\mathbf{4 0} \\
41 \\
42 \\
43 \\
44\end{array}$ & 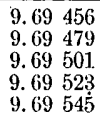 & $\begin{array}{l}23 \\
22 \\
22 \\
22\end{array}$ & $\begin{array}{ll}9.75 & 558 \\
9.75 & 588 \\
9.75 & 617 \\
9.75 & 647 \\
9.75 & 676\end{array}$ & $\begin{array}{l}30 \\
29 \\
30 \\
29\end{array}$ & 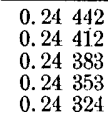 & 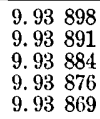 & $\begin{array}{l}7 \\
7 \\
8 \\
7\end{array}$ & $\begin{array}{l}20 \\
19 \\
18 \\
17 \\
16\end{array}$ & 1 & $\begin{array}{r}20 \\
16 \\
12 \\
8 \\
4\end{array}$ \\
\hline 59 & $\begin{array}{r}0 \\
4 \\
8 \\
12 \\
16\end{array}$ & $\begin{array}{l}45 \\
46 \\
47 \\
48 \\
49\end{array}$ & $\begin{array}{ll}9.69 & 567 \\
9.69 & 589 \\
9.69 & 611 \\
9.69 & 633 \\
9.69 & 655\end{array}$ & $\begin{array}{l}22 \\
22 \\
22 \\
22\end{array}$ & $\begin{array}{ll}9.75 & 705 \\
9.75 & 735 \\
9.75 & 764 \\
9.75 & 793 \\
9.75 & 822\end{array}$ & $\begin{array}{l}30 \\
29 \\
29 \\
29\end{array}$ & $\begin{array}{ll}0.24 & 29 \overline{5} \\
0.24 & 265 \\
0.24 & 236 \\
0.24 & 207 \\
0.24 & 178\end{array}$ & 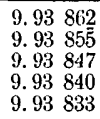 & $\begin{array}{l}7 \\
8 \\
7 \\
7\end{array}$ & $\begin{array}{l}15 \\
14 \\
13 \\
12 \\
11\end{array}$ & 1 & $\begin{array}{r}0 \\
56 \\
52 \\
48 \\
44\end{array}$ \\
\hline 59 & $\begin{array}{l}20 \\
24 \\
28 \\
32 \\
36\end{array}$ & $\begin{array}{l}50 \\
51 \\
52 \\
53 \\
54\end{array}$ & $\begin{array}{l}9.69677 \\
9.69699 \\
9.69721 \\
9.69743 \\
9.69765\end{array}$ & $\begin{array}{l}22 \\
22 \\
22 \\
22\end{array}$ & $\begin{array}{ll}9.75 & 852 \\
9.75 & 881 \\
9.75 & 910 \\
9.75 & 939 \\
9.75 & 969\end{array}$ & $\begin{array}{l}29 \\
29 \\
29 \\
30\end{array}$ & $\begin{array}{ll}0.24 & 148 \\
0.24 & 119 \\
0.24 & 090 \\
0.24 & 061 \\
0.24 & 031\end{array}$ & 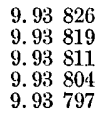 & $\begin{array}{l}7 \\
8 \\
7 \\
7\end{array}$ & $\begin{array}{r}\mathbf{1 0} \\
9 \\
8 \\
7 \\
6\end{array}$ & 0 & $\begin{array}{l}40 \\
36 \\
32 \\
28 \\
24\end{array}$ \\
\hline 59 & $\begin{array}{l}40 \\
44 \\
48 \\
52 \\
56\end{array}$ & $\begin{array}{l}55 \\
56 \\
57 \\
58 \\
59\end{array}$ & $\begin{array}{r}9.69787 \\
9.69809 \\
9.69831 \\
9.69853 \\
9.69875\end{array}$ & $\begin{array}{l}22 \\
22 \\
22 \\
22\end{array}$ & $\begin{array}{ll}9.75 & 998 \\
9.76 & 027 \\
9.76 & 056 \\
9.76 & 086 \\
9.76 & 115\end{array}$ & $\begin{array}{l}29 \\
29 \\
30 \\
29\end{array}$ & $\begin{array}{ll}0.24 & 002 \\
0.23 & 973 \\
0.23 & 944 \\
0.23 & 914 \\
0.23 & 885\end{array}$ & $\begin{array}{ll}9.93 & 789 \\
9.93 & 782 \\
9.93 & 775 \\
9.93 & 768 \\
9.93 & 760\end{array}$ & $\begin{array}{l}7 \\
7 \\
7 \\
8\end{array}$ & $\begin{array}{l}5 \\
4 \\
3 \\
2 \\
1\end{array}$ & 0 & $\begin{array}{r}20 \\
16 \\
12 \\
8 \\
4\end{array}$ \\
\hline 60 & 0 & 60 & 9.69897 & & $9.76 \quad 144$ & & 0.23856 & 9.93753 & & 0 & 0 & 0 \\
\hline & & & os. & d. & L. Cotg. & c. d. & L. Tang. & L. Sin. & d. & ' & m. & \\
\hline
\end{tabular}

$60^{\circ}$ 
Thuse 19.-Five-place logarithms of circular functions, etc.-Continued.

$2^{\text {h }}$

$30^{\circ}$

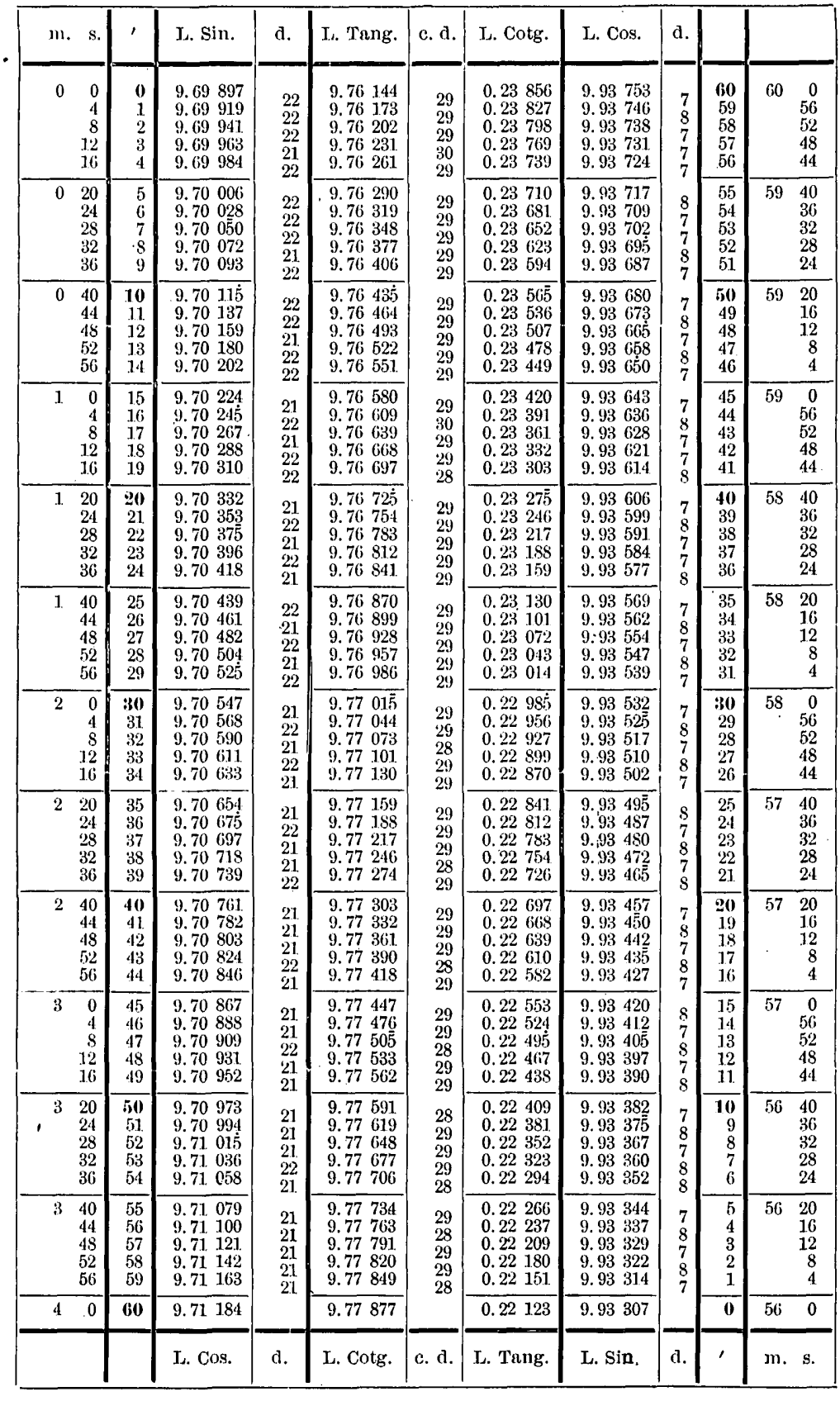


TABLE 19.-Five-place logarithms of circular functions, etc.-Continued.

$2^{\mathrm{h}}$

\begin{tabular}{|c|c|c|c|c|c|c|c|c|c|c|c|c|}
\hline m. & s. & , & L. Sin. & d. & L. Tang. & c. $d$. & L. Cotg. & L. Cos. & d. & & & \\
\hline 4 & $\begin{array}{r}0 \\
4 \\
8 \\
12 \\
16\end{array}$ & $\begin{array}{l}\mathbf{0} \\
\mathbf{1} \\
2 \\
3 \\
3 \\
4\end{array}$ & $\begin{array}{ll}9.71 & 184 \\
9.71 & 205 \\
9.71 & 226 \\
9.71 & 247 \\
9.71 & 268\end{array}$ & \multirow{3}{*}{$\begin{array}{l}21 \\
21 \\
21 \\
21 \\
21 \\
21 \\
21 \\
21 \\
21 \\
20 \\
21 \\
21 \\
21 \\
21 \\
21\end{array}$} & 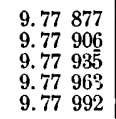 & \multirow{3}{*}{$\begin{array}{l}29 \\
29 \\
28 \\
29 \\
28 \\
29 \\
28 \\
29 \\
29 \\
28 \\
29 \\
28 \\
29 \\
28 \\
29\end{array}$} & $\begin{array}{ll}0.22 & 123 \\
0.22 & 094 \\
0.22 & 065 \\
0.22 & 037 \\
0.22 & 008\end{array}$ & $\begin{array}{ll}9.93 & 307 \\
9.93 & 299 \\
9.93 & 291 \\
9.93 & 284 \\
9.93 & 276\end{array}$ & \multirow{2}{*}{$\begin{array}{l}8 \\
8 \\
7 \\
8 \\
7 \\
8 \\
8 \\
7 \\
8\end{array}$} & $\begin{array}{r}\mathbf{6 0} \\
59 \\
58 \\
57 \\
56\end{array}$ & 56 & $\begin{array}{r}0 \\
56 \\
52 \\
48 \\
44\end{array}$ \\
\hline 4 & $\begin{array}{l}20 \\
24 \\
28 \\
32 \\
36\end{array}$ & $\begin{array}{l}5 \\
6 \\
7 \\
8 \\
9\end{array}$ & $\begin{array}{ll}9.71 & 289 \\
9.71 & 310 \\
9.71 & 331 \\
9.71 & 352 \\
9.71 & 373\end{array}$ & & 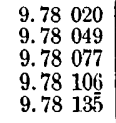 & & $\begin{array}{ll}0.21 & 980 \\
0.21 & 951 \\
0.21 & 923 \\
0.21 & 894 \\
0.21 & 865\end{array}$ & $\begin{array}{ll}9.93 & 269 \\
9.93 & 261 \\
9.93 & 253 \\
9.93 & 246 \\
9.93 & 238\end{array}$ & & $\begin{array}{l}55 \\
54 \\
53 \\
52 \\
51\end{array}$ & 55 & $\begin{array}{l}40 \\
36 \\
32 \\
28 \\
24\end{array}$ \\
\hline 4 & $\begin{array}{l}40 \\
44 \\
48 \\
52 \\
56\end{array}$ & $\begin{array}{l}10 \\
11 \\
12 \\
13 . \\
14\end{array}$ & $\begin{array}{ll}9.71 & 393 \\
9.71 & 414 \\
9.71 & 435 \\
9.71 & 456 \\
9.71 & 477\end{array}$ & & $\begin{array}{lll}9.78 & 163 \\
9.78 & 192 \\
9.78 & 220 \\
9.78 & 249 \\
9.78 & 277\end{array}$ & & $\begin{array}{ll}0.21 & 837 \\
0.21 & 808 \\
0.21 & 780 \\
0.21 & 751 \\
0.21 & 723\end{array}$ & $\begin{array}{ll}9.93 & 230 \\
9.93 & 223 \\
9.93 & 215 \\
9.93 & 207 \\
9.93 & 200\end{array}$ & $\begin{array}{l}7 \\
8 \\
8 \\
7 \\
8\end{array}$ & $\begin{array}{l}\mathbf{5 0} \\
\mathbf{4 9} \\
48 \\
47 \\
46\end{array}$ & 55 & $\begin{array}{r}20 \\
16 \\
12 \\
8 \\
4\end{array}$ \\
\hline 5 & $\begin{array}{r}0 \\
4 \\
8 \\
12 \\
16\end{array}$ & $\begin{array}{l}15 \\
16 \\
17 \\
18 \\
19\end{array}$ & $\begin{array}{ll}9.71 & 498 \\
9.71 & 519 \\
9.71 & 539 \\
9.71 & 560 \\
9,71 & 581\end{array}$ & $\begin{array}{l}21 \\
20 \\
21 \\
21\end{array}$ & 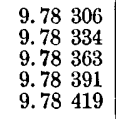 & $\begin{array}{l}28 \\
29 \\
28 \\
28 \\
29\end{array}$ & $\begin{array}{ll}0.21 & 694 \\
0.21 & 666 \\
0.21 & 637 \\
0.21 & 609 \\
0.21 & 581\end{array}$ & $\begin{array}{ll}9.93 & 192 \\
9.93 & 184 \\
9.93 & 177 \\
9.93 & 169 \\
9.93 & 161\end{array}$ & $\begin{array}{l}8 \\
7 \\
8 \\
8 \\
7\end{array}$ & $\begin{array}{l}45 \\
44 \\
43 \\
42 \\
41\end{array}$ & 55 & $\begin{array}{r}0 \\
56 \\
52 \\
48 \\
44\end{array}$ \\
\hline 5 & $\begin{array}{l}20 \\
24 \\
28 \\
32 \\
36\end{array}$ & $\begin{array}{l}\mathbf{2 0} \\
21 \\
22 \\
23 \\
24\end{array}$ & $\begin{array}{ll}9.71 & 602 \\
9.71 & 622 \\
9.71 & 643 \\
9.71 & 664 \\
9.71 & 685\end{array}$ & $\begin{array}{l}20 \\
21 \\
21 \\
21\end{array}$ & 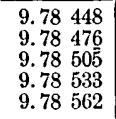 & $\begin{array}{l}28 \\
29 \\
28 \\
29 \\
28\end{array}$ & $\begin{array}{ll}0.21 & 552 \\
0.21 & 524 \\
0.21 & 495 \\
0.21 & 467 \\
0.21 & 438\end{array}$ & $\begin{array}{ll}9.93 & 154 \\
9.93 & 146 \\
9.93 & 138 \\
9.93 & 131 \\
9.93 & 123\end{array}$ & $\begin{array}{l}8 \\
8 \\
7 \\
8 \\
8\end{array}$ & $\begin{array}{l}\mathbf{4 0} \\
39 \\
38 \\
37 \\
36\end{array}$ & 54 & $\begin{array}{l}40 \\
36 \\
32 \\
28 \\
24\end{array}$ \\
\hline 5 & $\begin{array}{l}40 \\
44 \\
48 \\
52 \\
56\end{array}$ & $\begin{array}{l}25 \\
26 \\
27 \\
28 \\
29\end{array}$ & $\begin{array}{ll}9.71 & 705 \\
9.71 & 726 \\
9.71 & 747 \\
9.71 & 767 \\
9.71 & 788\end{array}$ & $\begin{array}{l}21 \\
21 \\
20 \\
21\end{array}$ & $\begin{array}{ll}9.78 & 590 \\
9.78 & 618 \\
9.78 & 647 \\
9.78 & 675 \\
9.78 & 704 \\
\end{array}$ & $\begin{array}{l}28 \\
29 \\
28 \\
29 \\
28\end{array}$ & $\begin{array}{ll}0.21 & 410 \\
0.21 & 382 \\
0.21 & 353 \\
0.21 & 325 \\
0.21 & 296\end{array}$ & $\begin{array}{ll}9.93 & 115 \\
9.93 & 108 \\
9.93 & 100 \\
9.93 & 092 \\
9.93 & 084 \\
\end{array}$ & $\begin{array}{l}7 \\
8 \\
8 \\
8\end{array}$ & $\begin{array}{l}35 \\
34 \\
33 \\
32 \\
31\end{array}$ & 54 & $\begin{array}{r}20 \\
16 \\
12 \\
8 \\
4\end{array}$ \\
\hline 6 & \begin{tabular}{r|}
0 \\
4 \\
8 \\
12 \\
16
\end{tabular} & $\begin{array}{l}\mathbf{3 0} \\
31 \\
32 \\
33 \\
34 \\
\end{array}$ & $\begin{array}{ll}9.71 & 809 \\
9.71 & 829 \\
9.71 & 850 \\
9.71 & 870 \\
9.71 & 891 \\
\end{array}$ & $\begin{array}{l}20 \\
21 \\
20 \\
21\end{array}$ & $\begin{array}{ll}9.78 & 732 \\
9.78 & 760 \\
9.78 & 789 \\
9.78 & 817 \\
9.78 & 845 \\
\end{array}$ & $\begin{array}{l}28 \\
29 \\
28 \\
28 \\
29\end{array}$ & $\begin{array}{ll}0.21 & 268 \\
0.21 & 240 \\
0.21 & 211 \\
0.21 & 183 \\
0.21 & 155\end{array}$ & $\begin{array}{ll}9.93 & 077 \\
9.93 & 069 \\
9.93 & 061 . \\
9.93 & 053 \\
9.93 & 046 \\
\end{array}$ & $\begin{array}{l}8 \\
8 \\
8 \\
7\end{array}$ & $\begin{array}{l}\mathbf{3 0} \\
29 \\
28 \\
27 \\
26\end{array}$ & 54 & $\begin{array}{r}0 \\
56 \\
52 \\
48 \\
44\end{array}$ \\
\hline .6 & $\begin{array}{l}20 \\
24 \\
28 \\
32 \\
36 \\
\end{array}$ & $\begin{array}{l}35 \\
36 \\
37 \\
38 \\
39 \\
\end{array}$ & $\begin{array}{ll}9.71 & 911 \\
9.71 & 932 \\
9.71 & 952 \\
9.71 & 973 \\
9.71 & 994 \\
\end{array}$ & $\begin{array}{l}21 \\
20 \\
21 \\
21\end{array}$ & 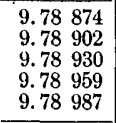 & $\begin{array}{l}28 \\
28 \\
29 \\
28\end{array}$ & $\begin{array}{ll}0.21 & 126 \\
0.21 & 098 \\
0.21 & 070 \\
0.21 & 041 \\
0.21 & 013 \\
\end{array}$ & $\begin{array}{ll}9.93 & 038 \\
9.93 & 030 \\
9.93 & 022 \\
9.93 & 014 \\
9.93 & 007 \\
\end{array}$ & $\begin{array}{l}8 \\
8 \\
8 \\
7\end{array}$ & $\begin{array}{l}25 \\
24 \\
23 \\
22 \\
21\end{array}$ & 53 & $\begin{array}{l}40 \\
36 \\
32 \\
28 \\
24\end{array}$ \\
\hline 6 & $\begin{array}{l}40 \\
44 \\
48 \\
52 \\
56\end{array}$ & $\begin{array}{l}40 \\
41 \\
42 \\
43 \\
44\end{array}$ & $\begin{array}{lll}y .72 & 014 \\
9.72 & 034 \\
9.72 & 05 \overline{5} \\
9.72 & 075 \\
9.72 & 096\end{array}$ & $\begin{array}{l}20 \\
21 \\
20 \\
21\end{array}$ & $\begin{array}{ll}9.79 & 015 \\
9.79 & 043 \\
9.79 & 072 \\
9.79 & 100 \\
9.79 & 128\end{array}$ & $\begin{array}{l}28 \\
29 \\
28 \\
28 \\
28\end{array}$ & 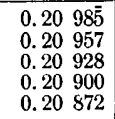 & 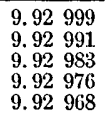 & $\begin{array}{l}8 \\
8 \\
7 \\
8\end{array}$ & $\begin{array}{l}\mathbf{2 0} \\
19 \\
18 \\
17 \\
16\end{array}$ & 53 & $\begin{array}{r}20 \\
16 \\
12 \\
8 \\
4\end{array}$ \\
\hline 7 & $\begin{array}{r}0 \\
4 \\
8 \\
12 \\
16\end{array}$ & $\begin{array}{l}45 \\
46 \\
47 \\
48 \\
49\end{array}$ & $\begin{array}{lll}9.72 & 116 \\
9.72 & 137 \\
9.72 & 157 \\
9.72 & 177- \\
9.72 & 198\end{array}$ & $\begin{array}{l}21 \\
20 \\
20 \\
21 \\
20\end{array}$ & $\begin{array}{lll}9.79 & 156 \\
9.79 & 185 \\
9.79 & 213 \\
9.79 & 241 \\
9.79 & 269\end{array}$ & $\begin{array}{l}29 \\
28 \\
28 \\
28 \\
28\end{array}$ & $\begin{array}{ll}0.20 & 844 \\
0.20 & 815 \\
0.20 & 787 \\
0.20 & 759 \\
0.20 & 731\end{array}$ & $\begin{array}{ll}9.92 & 960 \\
9.92 & 952 \\
9.92 & 944 \\
9.92 & 936 \\
9.92 & 929\end{array}$ & $\begin{array}{l}8 \\
8 \\
8 \\
7 \\
8\end{array}$ & $\begin{array}{r}15 \\
14 \\
13 \\
12 \\
11\end{array}$ & $\begin{array}{l}53 \\
.\end{array}$ & $\begin{array}{r}0 \\
56 \\
52 \\
48 \\
44\end{array}$ \\
\hline 7 & $\begin{array}{l}20 \\
24 \\
28 \\
32 \\
36\end{array}$ & $\begin{array}{l}\mathbf{5 0} \\
51 \\
52 \\
53 \\
54 \\
\end{array}$ & $\begin{array}{lll}9.72 & 218 \\
9.72 & 238 \\
9.72 & 259 \\
9.72 & 279 \\
9.72 & 299\end{array}$ & $\begin{array}{l}20 \\
21 \\
20 \\
20 \\
21\end{array}$ & $\begin{array}{ll}9.79 & 297 \\
9.79 & 326 \\
9.79 & 354 \\
9.79 & 382 \\
9.79 & 410\end{array}$ & $\begin{array}{l}29 \\
28 \\
28 \\
28 \\
28\end{array}$ & $\begin{array}{ll}0.20 & 703 \\
0.20 & 674 \\
0.20 & 646 \\
0.20 & 618 \\
0.20 & 590\end{array}$ & $\begin{array}{ll}9.92 & 921 \\
9.92 & 913 \\
9.92 & 905 \\
9.92 & 897 \\
9.92 & 889\end{array}$ & $\begin{array}{l}8 \\
8 \\
8 \\
8 \\
8\end{array}$ & $\begin{array}{r}\mathbf{1 0} \\
9 \\
8 \\
7 \\
6\end{array}$ & & $\begin{array}{l}40 \\
36 \\
32 \\
28 \\
24\end{array}$ \\
\hline 7 & $\begin{array}{l}40 \\
44 \\
48 \\
52 \\
56 \\
\end{array}$ & $\begin{array}{l}55 \\
56 \\
57 \\
58 \\
59\end{array}$ & 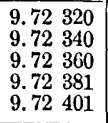 & $\begin{array}{l}20 \\
20 \\
21 \\
20 \\
20\end{array}$ & $\begin{array}{ll}9.79 & 438 \\
9.79 & 466 \\
9.79 & 495 \\
9.79 & 523 \\
9.79 & 551\end{array}$ & $\begin{array}{l}28 \\
29 \\
28 \\
28 \\
28\end{array}$ & $\begin{array}{ll}0.20 & 562 \\
0.20 & 534 \\
0.20 & 505 \\
0.20 & 477 \\
0.20 & 449\end{array}$ & $\begin{array}{l}9.92881 \\
9.92874 \\
9.92866 \\
9.92858 \\
9.92850\end{array}$ & $\begin{array}{l}7 \\
8 \\
8 \\
8 \\
8\end{array}$ & $\begin{array}{l}5 \\
4 \\
3 \\
2 \\
1\end{array}$ & $\begin{array}{c}52 \\
.\end{array}$ & $\begin{array}{r}20 \\
16 \\
12 \\
8 \\
4\end{array}$ \\
\hline 8 & 0 & 60 & 9.72421 & & 9.79579 & & 0.20421 & 9.92842 & & 0 & 52 & 0 \\
\hline & & & L. Cos. & d. & L. Cotg. & c. d. & L. Tang. & L. Sin. & d. & ' & m. & S. \\
\hline
\end{tabular}


TABLE 19.-Five-place logarithms of circular functions, etc.-Continued.

$2^{\text {h }}$

\begin{tabular}{|c|c|c|c|c|c|c|c|c|c|c|c|c|}
\hline $\mathrm{m}$. & s. & ' & L. Sin. & d. & L. Tang. & c. d. & L. Cotg. & L. Cos. & d. & & & \\
\hline 8 & $\begin{array}{r}0 \\
4 \\
8 \\
12 \\
16\end{array}$ & $\begin{array}{l}0 \\
1 \\
2 \\
3 \\
4\end{array}$ & 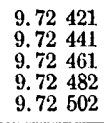 & \multirow{3}{*}{$\begin{array}{l}20 \\
20 \\
21 \\
20 \\
20 \\
\\
20 \\
20 \\
20 \\
20 \\
20 \\
21 \\
20 \\
20 \\
20 \\
20\end{array}$} & 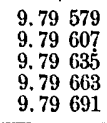 & \multirow{3}{*}{$\begin{array}{l}28 \\
28 \\
28 \\
28 \\
28 \\
28 \\
29 \\
28 \\
28 \\
28 \\
28 \\
28 \\
28 \\
28 \\
28\end{array}$} & $\begin{array}{ll}0.20 & 421 \\
0.20 & 393 \\
0.20 & 365 \\
0.20 & 337 \\
0.20 & 309\end{array}$ & 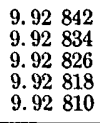 & \multirow{2}{*}{$\begin{array}{l}8 \\
8 \\
8 \\
8 \\
7 \\
8 \\
8 \\
8 \\
8 \\
8\end{array}$} & $\begin{array}{c}60 \\
59 \\
58 \\
57 \\
56 \\
\end{array}$ & 52 & $\begin{array}{r}0 \\
56 \\
52 \\
48 \\
44\end{array}$ \\
\hline 8 & $\begin{array}{l}20 \\
24 \\
28 \\
32 \\
36\end{array}$ & $\begin{array}{l}5 \\
6 \\
7 \\
8 \\
9\end{array}$ & $\begin{array}{ll}9.72 & 522 \\
9.72 & 542 \\
9.72 & 562 \\
9.72 & 582 \\
9.72 & 602\end{array}$ & & 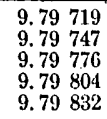 & & $\begin{array}{ll}0.20 & 281 \\
0.20 & 253 \\
0.20 & 224 \\
0.20 & 196 \\
0.20 & 168\end{array}$ & $\begin{array}{ll}9.92 & 803 \\
9.92 & 795 \\
9.92 & 787 \\
9.92 & 779 \\
9.92 & 771\end{array}$ & & $\begin{array}{l}55 \\
54 \\
53 \\
52 \\
51\end{array}$ & 51 & $\begin{array}{l}40 \\
36 \\
32 \\
28 \\
24\end{array}$ \\
\hline 8 & $\begin{array}{l}40 \\
44 \\
48 \\
52 \\
56\end{array}$ & $\begin{array}{l}10 \\
11 \\
12 \\
13 \\
14\end{array}$ & 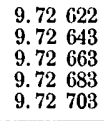 & & 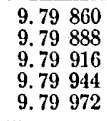 & & $\begin{array}{lll}0.20 & 140 \\
0.20 & 112 \\
0.20 & 084 \\
0.20 & 056 \\
0.20 & 028\end{array}$ & 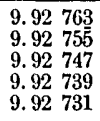 & $\begin{array}{l}8 \\
8 \\
8 \\
8\end{array}$ & $\begin{array}{r}50 \\
49 \\
48 \\
47 \\
46\end{array}$ & 51 & $\begin{array}{r}20 \\
16 \\
12 \\
8 \\
4\end{array}$ \\
\hline 9 & $\begin{array}{r}0 \\
4 \\
8 \\
12 \\
16\end{array}$ & $\begin{array}{l}15 \\
16 \\
17 \\
18 \\
19\end{array}$ & $\begin{array}{ll}9.72 & 723 \\
9.72 & 743 \\
9.72 & 763 \\
9.72 & 783 \\
9.72 & 803\end{array}$ & $\begin{array}{l}20 \\
20 \\
20 \\
20 \\
20\end{array}$ & $\begin{array}{ll}9.80 & 000 \\
9.80 & 028 \\
9.80 & 056 \\
9.80 & 084 \\
9.80 & 1.12\end{array}$ & $\begin{array}{l}28 \\
28 \\
28 \\
28 \\
28\end{array}$ & $\begin{array}{l}0.20000 \\
0.19972 \\
0.19944 \\
0.19916 \\
0.19888\end{array}$ & 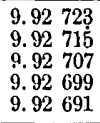 & $\begin{array}{l}8 \\
8 \\
8 \\
8 \\
8\end{array}$ & $\begin{array}{l}45 \\
44 \\
43 \\
42 \\
41\end{array}$ & 51 & $\begin{array}{r}0 \\
56 \\
52 \\
48 \\
44\end{array}$ \\
\hline 9 & $\begin{array}{l}20 \\
24 \\
28 \\
32 \\
36\end{array}$ & $\begin{array}{l}20 \\
21 \\
22 \\
23 \\
24\end{array}$ & 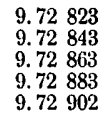 & \multirow{2}{*}{$\begin{array}{l}20 \\
20 \\
20 \\
19 \\
20 \\
20 \\
20 \\
20 \\
20 \\
20\end{array}$} & $\begin{array}{lll}9.80 & 140 \\
9.80 & 168 \\
9.80 & 195 \\
9.80 & 223 \\
9.80 & 251\end{array}$ & $\begin{array}{l}28 \\
27 \\
28 \\
28 \\
28\end{array}$ & 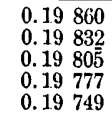 & 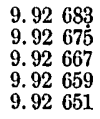 & $\begin{array}{l}8 \\
8 \\
8 \\
8 \\
8\end{array}$ & $\begin{array}{r}.40 \\
39 \\
38 \\
37 \\
36 .\end{array}$ & 50 & $\begin{array}{l}40 \\
36 \\
32 \\
28 \\
24\end{array}$ \\
\hline 9 & $\begin{array}{l}40 \\
44 \\
48 \\
52 \\
56\end{array}$ & $\begin{array}{l}25 \\
26 \\
27 \\
28 \\
29\end{array}$ & 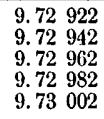 & & $\begin{array}{ll}9.80 & 279 \\
9.80 & 307 \\
9.30 & 335 \\
9.80 & 363 \\
9.30 & 391\end{array}$ & $\begin{array}{l}28 \\
28 \\
28 \\
28 \\
28\end{array}$ & $\begin{array}{l}0.19721 \\
0.19693 \\
0.19665 \\
0.19637 \\
0.19609\end{array}$ & 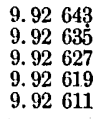 & \begin{tabular}{l|}
8 \\
8 \\
8 \\
8 \\
8
\end{tabular} & $\begin{array}{l}35 \\
34 \\
33 \\
32 \\
31\end{array}$ & 50 & $\begin{array}{r}20 \\
16 \\
12 \\
8 \\
4\end{array}$ \\
\hline 10 & $\begin{array}{r}0 \\
4 \\
8 \\
12 \\
16\end{array}$ & $\begin{array}{l}30 \\
31 \\
32 \\
33 \\
34\end{array}$ & $\begin{array}{lll}9.73 & 022 \\
9.73 & 041 \\
9.73 & 061 \\
9.73 & 081 . \\
9.73 & 101\end{array}$ & $\begin{array}{l}19 \\
20 \\
20 \\
20 \\
20\end{array}$ & 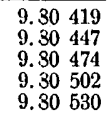 & $\begin{array}{l}28 \\
27 \\
28 \\
28\end{array}$ & $\begin{array}{ll}0.19 & 581 \\
0.19 & 553 \\
0.19 & 426 \\
0.19 & 498 \\
0.19 & 470\end{array}$ & $\begin{array}{ll}9.92 & 603 \\
9.92 & 595 \\
9.92 & 587 \\
9.92 & 579 \\
9.92 & 571\end{array}$ & \begin{tabular}{l|}
8 \\
8 \\
8 \\
8 \\
8
\end{tabular} & $\begin{array}{r}\mathbf{3 0} \\
29 \\
28 \\
27 \\
26\end{array}$ & 50 & $\begin{array}{r}0 \\
56 \\
52 \\
48 \\
44\end{array}$ \\
\hline 10 & $\begin{array}{l}20 \\
24 \\
28 \\
32 \\
36\end{array}$ & $\begin{array}{l}35 \\
36 \\
37 \\
38 \\
39\end{array}$ & $\begin{array}{ll}9.73 & 121 \\
9.73 & 140 \\
9.73 & 160 \\
9.73 & 180 \\
9.73 & 200\end{array}$ & $\begin{array}{l}19 \\
20 \\
20 \\
20 \\
10\end{array}$ & 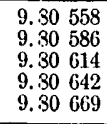 & $\begin{array}{l}28 \\
28 \\
28 \\
27\end{array}$ & 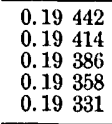 & $\begin{array}{ll}9.92 & 563 \\
9.92 & 555 \\
9.92 & 546 \\
9.92 & 538 \\
9.92 & 530 \\
\end{array}$ & $\begin{array}{l}8 \\
9 \\
3 \\
8 \\
8\end{array}$ & $\begin{array}{l}25 \\
24 \\
23 \\
22 \\
21\end{array}$ & 49 & $\begin{array}{l}40 \\
36 \\
32 \\
28 \\
24\end{array}$ \\
\hline 10 & $\begin{array}{l}40 \\
44 \\
48 \\
52 \\
56\end{array}$ & $\begin{array}{l}40 \\
41 \\
42 \\
43 \\
44\end{array}$ & $\begin{array}{ll}9.73 & 219 \\
9.73 & 239 \\
9.73 & 259 \\
9.73 & 278 \\
9.73 & 298\end{array}$ & $\begin{array}{l}20 \\
20 \\
19 \\
20 \\
20\end{array}$ & 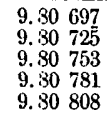 & $\begin{array}{l}28 \\
28 \\
28 \\
27\end{array}$ & $\begin{array}{r}0.19303 \\
0.19275 \\
0.19247 \\
0.19219 \\
0.19192\end{array}$ & $\begin{array}{ll}9.92 & 522 \\
9.92 & 514 \\
9.92 & 506 \\
9.92 & 498 \\
9.92 & 490\end{array}$ & $\begin{array}{l}8 \\
8 \\
8 \\
8 \\
8\end{array}$ & $\begin{array}{l}20 \\
19 \\
18 \\
17 \\
16\end{array}$ & 49 & $\begin{array}{r}20 \\
16 \\
12 \\
8 \\
4\end{array}$ \\
\hline 11 & $\begin{array}{r}0 \\
4 \\
8 \\
12 \\
16\end{array}$ & $\begin{array}{l}45 \\
46 \\
47 \\
48 \\
49\end{array}$ & 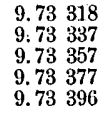 & $\begin{array}{l}19 \\
20 \\
20 \\
19 \\
20\end{array}$ & 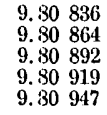 & $\begin{array}{l}28 \\
28 \\
27 \\
28\end{array}$ & $\begin{array}{ll}0.19 & 164 \\
0.19 & 136 \\
0.19 & 108 \\
0.19 & 081 \\
0.19 & 053\end{array}$ & 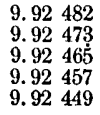 & $\begin{array}{l}9 \\
8 \\
8 \\
8 \\
8\end{array}$ & $\begin{array}{l}15 \\
14 \\
.13 \\
12 \\
11\end{array}$ & 49 & $\begin{array}{r}0 \\
56 \\
52 \\
48 \\
44\end{array}$ \\
\hline 11 & $\begin{array}{l}20 \\
24 \\
28 \\
32 \\
36\end{array}$ & $\begin{array}{l}50 \\
51 \\
52 \\
53 \\
54\end{array}$ & $\begin{array}{lll}9.73 & 416 \\
9.73 & 435 \\
9.73 & 455 \\
9.73 & 474 \\
9.73 & 494\end{array}$ & \multirow{3}{*}{$\begin{array}{l}19 \\
20 \\
19 \\
20 \\
19 \\
20 \\
19 \\
20 \\
19 \\
20\end{array}$} & $\begin{array}{lll}9.80 & 97 \overline{5} \\
9.81 & 003 \\
9.81 & 030 \\
9.81 & 058 \\
9.81 & 086\end{array}$ & \multirow{3}{*}{$\begin{array}{l}28 \\
27 \\
28 \\
28 \\
27 \\
28 \\
28 \\
27 \\
28 \\
28\end{array}$} & $\begin{array}{l}0.19025 \\
0.18997 \\
0.18970 \\
0.18942 \\
0.18 \\
\end{array}$ & 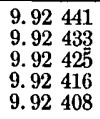 & $\begin{array}{l}8 \\
8 \\
9 \\
8 \\
8\end{array}$ & $\begin{array}{r}\mathbf{1 0} \\
9 \\
8 \\
7 \\
6\end{array}$ & 48 & $\begin{array}{l}40 \\
36 \\
32 \\
28 \\
24\end{array}$ \\
\hline 11 & $\begin{array}{l}40 \\
44 \\
48 \\
52 \\
56\end{array}$ & $\begin{array}{l}55 \\
56 \\
57 \\
58 \\
59\end{array}$ & 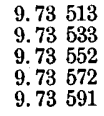 & & $\begin{array}{ll}9.81 & 113 \\
9.81 & 141 \\
9.81 & 169 \\
9.81 & 196 \\
9.81 & 224\end{array}$ & & 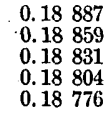 & 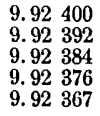 & \multirow[t]{2}{*}{$\begin{array}{l}8 \\
8 \\
8 \\
9 \\
8\end{array}$} & $\begin{array}{l}5 \\
4 \\
3 \\
2 \\
1\end{array}$ & 48 & $\begin{array}{r}20 \\
16 \\
12 \\
8 \\
4\end{array}$ \\
\hline 12 & 0 & 60 & 9.73611 & & 9.81252 & & 0.18748 & 9.92359 & & 0 & 48 & 0 \\
\hline & & & L. Cos. & d. & L. Cotg. & c. $d$. & L. Tang. & L. Sin. & d. & ' & $\mathrm{m}$. & s. \\
\hline
\end{tabular}


TaBLe 19.-Five-place logarithms of circular functions, elc.-Continued.

$2^{11}$

\begin{tabular}{|c|c|c|c|c|c|c|c|c|c|c|c|c|}
\hline $\mathrm{m}$. & s. & ' & L. $\operatorname{Sin}$. & d. & I. Tang. & c. d. & L. Cotg. & I. $\operatorname{Cos}$. & d. & & & \\
\hline 12 & $\begin{array}{r}0 \\
4 \\
8 \\
1.2 \\
1.6\end{array}$ & $\begin{array}{l}0 \\
1 \\
2 \\
3 \\
4\end{array}$ & 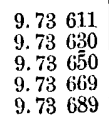 & $\begin{array}{l}19 \\
20 \\
19 \\
20\end{array}$ & $\begin{array}{ll}9.81 & 252 \\
9.81 & 279 \\
9.81 & 307 \\
9.81 & 335 \\
9.81 & 362\end{array}$ & $\begin{array}{l}27 \\
28 \\
28 \\
27\end{array}$ & 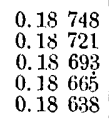 & $\begin{array}{ll}9.92 & 359 \\
9.92 & 351 \\
9.92 & 343 \\
9.92 & 335 \\
9.92 & 326\end{array}$ & $\begin{array}{l}8 \\
8 \\
8 \\
9\end{array}$ & $\begin{array}{r}\mathbf{6 0} \\
59 \\
58 \\
57 \\
56\end{array}$ & & $\begin{array}{r}0 \\
56 \\
52 \\
48 \\
44\end{array}$ \\
\hline 12 & $\begin{array}{l}20 \\
24 \\
28 \\
32 \\
36\end{array}$ & $\begin{array}{l}5 \\
6 \\
7 \\
8 \\
9\end{array}$ & $\begin{array}{ll}9.73 & 708 \\
9.73 & 727 \\
9.73 & 747 \\
9.73 & 766 \\
9.73 & 785\end{array}$ & $\begin{array}{l}19 \\
20 \\
19 \\
19\end{array}$ & $\begin{array}{ll}9.81 & 390 \\
9.81 & 418 \\
9.81 & 445 \\
9.81 & 473 \\
9.81 & 500\end{array}$ & $\begin{array}{l}28 \\
27 \\
28 \\
27\end{array}$ & $\begin{array}{ll}0.18 & 610 \\
0.18 & 582 \\
0.18 & 555 \\
0.18 & 527 \\
0.18 & 500\end{array}$ & 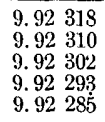 & $\begin{array}{l}8 \\
8 \\
9 \\
8\end{array}$ & $\begin{array}{l}55 \\
54 \\
53 \\
52 \\
51\end{array}$ & 47 & $\begin{array}{l}40 \\
36 \\
32 \\
28 \\
24\end{array}$ \\
\hline 12 & $\begin{array}{l}40 \\
14 \\
48 \\
52 \\
56\end{array}$ & $\begin{array}{l}10 \\
11 \\
12 \\
13 \\
14\end{array}$ & $\begin{array}{ll}9.73 & 80 \overline{\overline{5}} \\
9.73 & 824 \\
9.73 & 843 \\
9.73 & 863 \\
9.73 & 882\end{array}$ & $\begin{array}{l}19 \\
19 \\
20 \\
19\end{array}$ & $\begin{array}{ll}9.81 & 528 \\
9.81 & 556 \\
9.81 & 583 \\
9.81 & 611 \\
9.81 & 638\end{array}$ & $\begin{array}{l}28 \\
27 \\
28 \\
27\end{array}$ & $\begin{array}{ll}0.18 & 472 \\
0.18 & 444 \\
0.18 & 417 \\
0.18 & 389 \\
0.18 & 362\end{array}$ & $\begin{array}{ll}9.92 & 277 \\
9.92 & 269 \\
9.92 & 260 \\
9.92 & 252 \\
9.92 & 244\end{array}$ & $\begin{array}{l}8 \\
9 \\
8 \\
8\end{array}$ & $\begin{array}{l}50 \\
49 \\
48 \\
47 \\
46\end{array}$ & 47 & $\begin{array}{r}20 \\
16 \\
12 \\
8 \\
4\end{array}$ \\
\hline 13 & $\begin{array}{r}0 \\
4 \\
8 \\
12 \\
16\end{array}$ & $\begin{array}{l}15 \\
16 \\
17 \\
18 \\
19\end{array}$ & $\begin{array}{r}9.73901 \\
9.73921 \\
9.73940 \\
9.73959 \\
9.73978\end{array}$ & $\begin{array}{l}20 \\
19 \\
19 \\
19\end{array}$ & $\begin{array}{ll}9.81 & 666 \\
9.81 & 693 \\
9.81 & 721 \\
9.81 & 748 \\
9.81 & 776\end{array}$ & $\begin{array}{l}27 \\
28 \\
27 \\
28\end{array}$ & $\begin{array}{ll}0.18 & 334 \\
0.18 & 307 \\
0.18 & 279 \\
0.18 & 252 \\
0.18 & 224\end{array}$ & $\begin{array}{ll}9.92 & 235 \\
9.92 & 227 \\
9.92 & 219 \\
9.92 & 211 \\
9.92 & 202\end{array}$ & $\begin{array}{l}8 \\
8 \\
8 \\
9\end{array}$ & $\begin{array}{l}45 \\
44 \\
43 \\
42 \\
41\end{array}$ & 47 & $\begin{array}{r}0 \\
56 \\
52 \\
48 \\
44\end{array}$ \\
\hline $\begin{array}{l}13 \\
. \\
\end{array}$ & $\begin{array}{l}20 \\
24 \\
28 \\
32 \\
36\end{array}$ & $\begin{array}{l}20 \\
21 \\
22 \\
23 \\
24\end{array}$ & $\begin{array}{lll}9.73 & 997 \\
9.74 & 017 \\
9.74 & 036 \\
9.74 & 055 \\
9.74 & 074\end{array}$ & $\begin{array}{l}20 \\
19 \\
19 \\
19\end{array}$ & $\begin{array}{ll}9.81 & 803 \\
9.81 & 831 \\
9.81 & 858 \\
9.81 & 886 \\
9.81 & 913\end{array}$ & $\begin{array}{l}28 \\
27 \\
28 \\
27\end{array}$ & $\begin{array}{ll}0.18 & 197 \\
0.18 & 169 \\
0.18 & 142 \\
0.18 & 114 \\
0.18 & 087\end{array}$ & $\begin{array}{ll}9.92 & 194 \\
9.92 & 186 \\
9.92 & 177 \\
9.92 & 169 \\
9.92 & 161 .\end{array}$ & $\begin{array}{l}8 \\
9 \\
8 \\
8\end{array}$ & $\begin{array}{l}40 \\
39 \\
38 \\
37 \\
36\end{array}$ & 46 & $\begin{array}{l}40 \\
36 \\
32 \\
28 \\
24\end{array}$ \\
\hline 13 & $\begin{array}{l}40 \\
44 \\
48 \\
52 \\
56\end{array}$ & $\begin{array}{l}25 \\
26 \\
27 \\
28 \\
29\end{array}$ & $\begin{array}{lll}9.74 & 093 \\
9.74 & 113 \\
9.74 & 132 \\
9.74 & 151 \\
9.74 & 170\end{array}$ & $\begin{array}{l}20 \\
19 \\
19 \\
19\end{array}$ & $\begin{array}{ll}9.81 & 941 \\
9.81 & 968 \\
9.81 & 996 \\
9.82 & 023 \\
9.82 & 051\end{array}$ & $\begin{array}{l}27 \\
28 \\
27 \\
28\end{array}$ & $\begin{array}{ll}0.18 & 059 \\
0.18 & 032 \\
0.18 & 004 \\
0.17 & 977 \\
0.17 & 949\end{array}$ & $\begin{array}{ll}9.92 & 152 \\
9.92 & 144 \\
9.92 & 186 \\
9.92 & 127 \\
9.92 & 119\end{array}$ & $\begin{array}{l}8 \\
8 \\
9 \\
8\end{array}$ & $\begin{array}{l}35 \\
34 \\
33 \\
32 \\
31\end{array}$ & 46 & $\begin{array}{r}20 \\
16 \\
12 \\
8 \\
4\end{array}$ \\
\hline 14 & $\begin{array}{r}0 \\
4 \\
8 \\
12 \\
16\end{array}$ & $\begin{array}{l}\mathbf{3 0} \\
31 \\
32 \\
33 \\
34\end{array}$ & $\begin{array}{lll}9.74 & 189 \\
9.74 & 208 \\
9.74 & 227 \\
9.74 & 246 \\
9.74 & 265\end{array}$ & $\begin{array}{l}19 \\
19 \\
19 \\
19\end{array}$ & $\begin{array}{ll}9.82 & 078 \\
9.82 & 106 \\
9.82 & 133 \\
9.82 & 161 \\
9.82 & 188\end{array}$ & $\begin{array}{l}28 \\
27 \\
28 \\
27\end{array}$ & $\begin{array}{ll}0.17 & 922 \\
0.17 & 894 \\
0.17 & 867 \\
0.17 & 839 \\
0.17 & 812\end{array}$ & $\begin{array}{ll}9.92 & 111 \\
9.92 & 102 \\
9.92 & 094 \\
9.92 & 086 \\
9.92 & 077\end{array}$ & $\begin{array}{l}9 \\
8 \\
8 \\
9\end{array}$ & $\begin{array}{l}\mathbf{3 0} \\
29 \\
28 \\
27 \\
26\end{array}$ & 46 & $\begin{array}{r}0 \\
56 \\
52 \\
48 \\
44\end{array}$ \\
\hline 14 & $\begin{array}{l}20 \\
24 \\
28 \\
32 \\
36\end{array}$ & $\begin{array}{l}35 \\
36 \\
37 \\
38 \\
39\end{array}$ & $\begin{array}{l}9.74284 \\
9.74303 \\
9.74322 \\
9.74341 \\
9.74360\end{array}$ & $\begin{array}{l}19 \\
19 \\
19 \\
19\end{array}$ & $\begin{array}{ll}9.82 & 21 \dot{5} \\
9.82 & 243 \\
9.82 & 270 \\
9.82 & 298 \\
9.82 & 325\end{array}$ & $\begin{array}{l}28 \\
27 \\
28 \\
27\end{array}$ & $\begin{array}{ll}0.17 & 785 \\
0.17 & 757 \\
0.17 & 730 \\
0.17 & 702 \\
0.17 & 675\end{array}$ & $\begin{array}{ll}9.92 & 069 \\
9.92 & 060 \\
9.92 & 052 \\
9.92 & 044 \\
9.92 & 035\end{array}$ & $\begin{array}{l}9 \\
8 \\
8 \\
9\end{array}$ & $\begin{array}{l}25 \\
24 \\
23 \\
22 \\
21\end{array}$ & 45 & $\begin{array}{l}40 \\
36 \\
32 \\
28 \\
24\end{array}$ \\
\hline 14 & $\begin{array}{l}40 \\
44 \\
48 \\
52 \\
56\end{array}$ & $\begin{array}{l}40 \\
41 \\
42 \\
43 \\
44\end{array}$ & 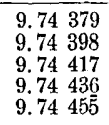 & $\begin{array}{l}19 \\
19 \\
19 \\
19\end{array}$ & $\begin{array}{ll}9.82 & 352 \\
9.82 & 380 \\
9.82 & 407 \\
9.82 & 435 \\
9.82 & 462\end{array}$ & $\begin{array}{l}28 \\
27 \\
28 \\
27\end{array}$ & $\begin{array}{ll}0.17 & 648 \\
0.17 & 620 \\
0.17 & 593 \\
0.17 & 565 \\
0.17 & 538\end{array}$ & $\begin{array}{ll}9.92 & 027 \\
9.92 & 018 \\
9.92 & 010 \\
9.92 & 002 \\
9.91 & 993\end{array}$ & $\begin{array}{l}9 \\
8 \\
8 \\
9\end{array}$ & $\begin{array}{l}\mathbf{2 0} \\
19 \\
18 \\
17 \\
16\end{array}$ & 45 & $\begin{array}{r}20 \\
16 \\
12 \\
8 \\
4\end{array}$ \\
\hline 15 & $\begin{array}{r}0 \\
4 \\
8 \\
12 \\
16\end{array}$ & $\begin{array}{l}45 \\
46 \\
47 \\
48 \\
49\end{array}$ & 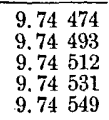 & $\begin{array}{l}19 \\
19 \\
19 \\
18\end{array}$ & $\begin{array}{ll}9.82 & 489 \\
9.82 & 517 \\
9.82 & 544 \\
9.82 & 571 \\
9.82 & 599\end{array}$ & $\begin{array}{l}28 \\
27 \\
27 \\
28\end{array}$ & $\begin{array}{ll}0.17 & 511 \\
0.17 & 483 \\
0.17 & 456 \\
0.17 & 429 \\
0.17 & 401\end{array}$ & 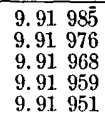 & $\begin{array}{l}9 \\
8 \\
9 \\
8\end{array}$ & $\begin{array}{l}15 \\
14 \\
13 \\
12 \\
11\end{array}$ & 45 & $\begin{array}{r}0 \\
56 \\
52 \\
48 \\
44\end{array}$ \\
\hline 15 & $\begin{array}{l}20 \\
24 \\
28 \\
32 \\
36\end{array}$ & $\begin{array}{l}\mathbf{5 0} \\
51 \\
52 \\
53 \\
54\end{array}$ & $\begin{array}{ll}9.74 & 568 \\
9.74 & 587 \\
9.74 & 606 \\
9.74 & 625 \\
9.74 & 644\end{array}$ & $\begin{array}{l}19 \\
19 \\
19 \\
19 \\
18\end{array}$ & $\begin{array}{ll}9.82 & 626 \\
9.82 & 653 \\
9.82 & 681 \\
9.82 & 708 \\
9.82 & 735\end{array}$ & $\begin{array}{l}27 \\
28 \\
27 \\
27\end{array}$ & $\begin{array}{ll}0.17 & 374 \\
0.17 & 347 \\
0.17 & 319 \\
0.17 & 292 \\
0.17 & 265\end{array}$ & 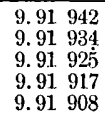 & $\begin{array}{l}8 \\
9 \\
8 \\
9 \\
8\end{array}$ & $\begin{array}{r}\mathbf{1 0} \\
9 \\
8 \\
7 \\
6\end{array}$ & 44 & $\begin{array}{l}40 \\
36 \\
32 \\
28 \\
24\end{array}$ \\
\hline 15 & $\begin{array}{l}40 \\
44 \\
48 \\
52 \\
56\end{array}$ & $\begin{array}{l}55 \\
56 \\
57 \\
58 \\
59\end{array}$ & $\begin{array}{l}9.74662 \\
9.74681 \\
9.74700 \\
9.74719 \\
9.74 \\
\end{array}$ & $\begin{array}{l}19 \\
19 \\
19 \\
18 \\
19\end{array}$ & $\begin{array}{ll}9.82 & 762 \\
9.82 & 790 \\
9.82 & 817 \\
9.82 & 844 \\
9.82 & 871\end{array}$ & $\begin{array}{l}28 \\
27 \\
27 \\
27 \\
28\end{array}$ & $\begin{array}{ll}0.17 & 238 \\
0.17 & 210 \\
0.17 & 183 \\
0.17 & 156 \\
0.17 & 129\end{array}$ & 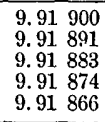 & $\begin{array}{l}9 \\
8 \\
9 \\
8 \\
9\end{array}$ & $\begin{array}{l}5 \\
4 \\
3 \\
2 \\
1\end{array}$ & 44 & $\begin{array}{r}20 \\
16 \\
12 \\
8 \\
4\end{array}$ \\
\hline 16 & 0 & 60 & 9.74756 & & 9.82899 & & 0.17101 & 9.91857 & & 0 & 44 & \\
\hline & & & L. Cos. & d. & I. Cotg. & c. d. & L. Tang. & L. $\operatorname{Sin}$. & d. & 1 & & \\
\hline
\end{tabular}

$33^{\circ}$

$56^{\circ}$ 
Table 19.-Five-place logarithms of circular functions, elc.-Continued.

\section{$2^{\mathrm{h}}$}

\begin{tabular}{|c|c|c|c|c|c|c|c|c|c|c|c|}
\hline m. s. & , & L. $\operatorname{Sin}$. & d. & I. Tang. & c. d. & I. 'Cotg. & L. Cos. & d. & & & \\
\hline $\begin{array}{r}16 \quad 0 \\
4 \\
8 \\
12 \\
16 \\
\end{array}$ & $\begin{array}{l}0 \\
1 \\
2 \\
3 \\
4 \\
\end{array}$ & 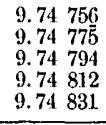 & $\begin{array}{l}19 \\
19 \\
18 \\
19\end{array}$ & 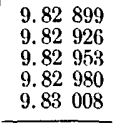 & $\begin{array}{l}27 \\
27 \\
27 \\
28 \\
27\end{array}$ & $\begin{array}{ll}0.17 & 101 \\
0.17 & 074 \\
0.17 & 047 \\
0.17 & 020 \\
0.16 & 992 \\
\end{array}$ & $\begin{array}{l}9.91857 \\
9.91849 \\
9.91 .840 \\
9.91 .832 \\
9.91823 \\
\end{array}$ & $\begin{array}{l}8 \\
9 \\
8 \\
9\end{array}$ & $\begin{array}{l}60 \\
59 \\
58 \\
57 \\
56 \\
\end{array}$ & & $\begin{array}{r}0 \\
56 \\
52 \\
48 \\
44\end{array}$ \\
\hline $\begin{array}{rr}16 & 20 \\
& 2 \\
& 28 \\
& 32 \\
& 30 \\
\end{array}$ & $\begin{array}{l}5 \\
6 \\
7 \\
8 \\
9\end{array}$ & $\begin{array}{l}9.74850 \\
9.74868 \\
9.74887 \\
9.74906 \\
9.74924\end{array}$ & $\begin{array}{l}18 \\
19 \\
19 \\
18 \\
19\end{array}$ & 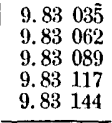 & $\begin{array}{l}27 \\
27 \\
28 \\
27 \\
27\end{array}$ & $\begin{array}{ll}0.16 & 965 \\
0.16 & 938 \\
0.16 & 911 \\
0.16 & 883 \\
0.16 & 856\end{array}$ & $\begin{array}{ll}9.91 & 815 \\
9.91 & 806 \\
9.91 & 798 \\
9.91 & 789 \\
9.91 & 781\end{array}$ & $\begin{array}{l}9 \\
8 \\
9 \\
8\end{array}$ & $\begin{array}{l}55 \\
54 \\
53 \\
52 \\
51 \\
\end{array}$ & 43 & $\begin{array}{l}40 \\
36 \\
32 \\
28 \\
24\end{array}$ \\
\hline $\begin{array}{rr}16 \quad 40 \\
& 44 \\
& 48 \\
& 52 \\
& 50\end{array}$ & $\begin{array}{l}10 \\
11 . \\
12 \\
13 \\
14\end{array}$ & 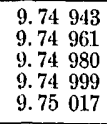 & $\begin{array}{l}18 \\
19 \\
19 \\
18 \\
19\end{array}$ & $\begin{array}{ll}9.83 & 171 \\
9.83 & 198 \\
9.83 & 225 \\
9.83 & 252 \\
9.83 & 280\end{array}$ & $\begin{array}{l}27 \\
27 \\
27 \\
28 \\
27\end{array}$ & $\begin{array}{ll}0.16 & 829 \\
0.16 & 802 \\
0.16 & 775 \\
0.16 & 748 \\
0.16 & 720 \\
\end{array}$ & $\begin{array}{ll}9.91 & 772 \\
9.91 & 763 \\
9.91 & 755 \\
9.91 & 746 \\
9.91 & 738\end{array}$ & $\begin{array}{l}9 \\
8 \\
9 \\
8\end{array}$ & $\begin{array}{l}50 \\
49 \\
48 \\
47 \\
46 \\
\end{array}$ & 43 & $\begin{array}{r}20 \\
16 \\
12 \\
8 \\
4\end{array}$ \\
\hline $\begin{array}{r}17 \quad 0 \\
8 \\
8 \\
12 \\
16 \\
\end{array}$ & $\begin{array}{l}15 \\
16 \\
17 \\
18 \\
19 \\
\end{array}$ & $\begin{array}{lll}9.75 & 036 \\
9.75 & 054 \\
9.75 & 073 \\
9.75 & 091 \\
9.75 & 110 \\
\end{array}$ & $\begin{array}{l}18 \\
19 \\
18 \\
19\end{array}$ & $\begin{array}{ll}9.83 & 307 \\
9.83 & 334 \\
9.83 & 361 \\
9.83 & 388 \\
9.83 & 415 \\
\end{array}$ & $\begin{array}{l}27 \\
27 \\
27 \\
27\end{array}$ & $\begin{array}{ll}0.16 & 693 \\
0.16 & 666 \\
0.16 & 639 \\
0.16 & 612 \\
0.16 & 585 \\
\end{array}$ & $\begin{array}{ll}9.91 & 729 \\
9.91 & 720 \\
9.91 . & 712 \\
9.91 & 703 \\
9.91 & 695 \\
\end{array}$ & $\begin{array}{l}9 \\
8 \\
9 \\
8\end{array}$ & $\begin{array}{l}45 \\
44 \\
43 \\
42 \\
41\end{array}$ & 43 & $\begin{array}{r}0 \\
56 \\
52 \\
48 \\
44\end{array}$ \\
\hline $\begin{array}{ll}17 \quad 20 \\
& 24 \\
& 28 \\
& 32 \\
& 30\end{array}$ & $\begin{array}{l}20 \\
21 \\
22 \\
23 \\
24\end{array}$ & $\begin{array}{lll}9.75 & 128 \\
9.75 & 147 \\
9.75 & 165 \\
9.75 & 184 \\
9.75 & 202\end{array}$ & $\begin{array}{l}19 \\
18 \\
19 \\
18\end{array}$ & $\begin{array}{ll}9.83 & 442 \\
9.83 & 470 \\
9.83 & 497 \\
9.83 & 524 \\
9.83 & 551\end{array}$ & $\begin{array}{l}28 \\
27 \\
27 \\
27\end{array}$ & $\begin{array}{ll}0.16 & 558 \\
0.16 & 530 \\
0.16 & 503 \\
0.16 & 476 \\
0.16 & 449\end{array}$ & $\begin{array}{ll}9.91 & 686 \\
9.91 & 677 \\
9.91 & 669 \\
9.91 & 660 \\
9.91 & 651\end{array}$ & $\begin{array}{l}9 \\
8 \\
9 \\
9\end{array}$ & $\begin{array}{l}\mathbf{4 0} \\
39 \\
38 \\
37 \\
36\end{array}$ & 42 & $\begin{array}{l}40 \\
36 \\
32 \\
28 \\
24\end{array}$ \\
\hline $\begin{array}{r}17 \quad 40 \\
44 \\
48 \\
52 \\
56 \\
\end{array}$ & $\begin{array}{l}25 \\
26 \\
27 \\
28 \\
29 \\
\end{array}$ & $\begin{array}{lll}9.75 & 221 \\
9.75 & 289 \\
9.75 & 258 \\
9.75 & 276 \\
9.75 & 294 \\
\end{array}$ & $\begin{array}{l}18 \\
19 \\
18 \\
18\end{array}$ & $\begin{array}{l}9.83578 \\
9.83605 \\
9.83632 \\
9.83659 \\
9.83686 \\
\end{array}$ & $\begin{array}{l}27 \\
27 \\
27 \\
27\end{array}$ & $\begin{array}{ll}0.16 & 422 \\
0.16 & 395 \\
0.16 & 368 \\
0.16 & 341 \\
0.16 & 314 \\
\end{array}$ & $\begin{array}{ll}9.91 & 643 \\
9.91 & 534 \\
9.91 & 625 \\
9.91 & 617 \\
9.91 & 608 \\
\end{array}$ & $\begin{array}{l}9 \\
9 \\
8 \\
9\end{array}$ & $\begin{array}{l}35 \\
34 \\
33 \\
32 \\
31 \\
\end{array}$ & 42 & $\begin{array}{r}20 \\
16 \\
12 \\
8 \\
4 \\
\end{array}$ \\
\hline $\begin{array}{r}0 \\
4 \\
8 \\
12 \\
16 \\
\end{array}$ & $\begin{array}{l}30 \\
31 \\
32 \\
33 \\
34\end{array}$ & $\begin{array}{ll}9.75 & 313 \\
9.75 & 331 \\
9.75 & 350 \\
9.75 & 368 \\
9.75 & 386 \\
\end{array}$ & $\begin{array}{l}18 \\
19 \\
18 \\
18\end{array}$ & 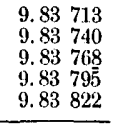 & $\begin{array}{l}27 \\
28 \\
27 \\
27\end{array}$ & $\begin{array}{ll}0.16 & 287 \\
0.16 & 260 \\
0.16 & 232 \\
0.16 & 205 \\
0.16 & 178 \\
\end{array}$ & 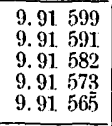 & $\begin{array}{l}8 \\
9 \\
9 \\
8\end{array}$ & $\begin{array}{l}30 \\
29 \\
28 \\
27 \\
26\end{array}$ & 42 & $\begin{array}{r}0 \\
56 \\
52 \\
48 \\
44\end{array}$ \\
\hline $\begin{array}{ll}18 \quad 20 \\
& 24 \\
& 28 \\
& 32 \\
& 36 \\
\end{array}$ & $\begin{array}{l}35 \\
36 \\
37 \\
38 \\
39 \\
\end{array}$ & $\begin{array}{ll}9.75 & 405 \\
9.75 & 423 \\
9.75 & 441 \\
9.75 & 459 \\
9.75 & 478 \\
\end{array}$ & $\begin{array}{l}18 \\
18 \\
18 \\
19\end{array}$ & $\begin{array}{ll}9.83 & 849 \\
9.83 & 876 \\
9.83 & 903 \\
9.83 & 930 \\
9.83 & 957 \\
\end{array}$ & $\begin{array}{l}27 \\
27 \\
27 \\
27\end{array}$ & $\begin{array}{ll}0.16 & 151 \\
0.16 & 124 \\
0.16 & 097 \\
0.16 & 070 \\
0.16 & 043 \\
\end{array}$ & 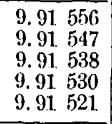 & $\begin{array}{l}9 \\
9 \\
8 \\
9\end{array}$ & $\begin{array}{l}25 \\
24 \\
23 \\
22 \\
21\end{array}$ & 41 & $\begin{array}{l}40 \\
36 \\
32 \\
28 \\
24\end{array}$ \\
\hline $\begin{array}{ll}18 \quad 40 \\
& 44 \\
& 48 \\
& 52 \\
& 56\end{array}$ & $\begin{array}{l}40 \\
41 \\
42 \\
43 \\
44 \\
\end{array}$ & $\begin{array}{ll}9.75 & 496 . \\
9.75 & 51.4 \\
9.75 & 533 \\
9.75 & 551 \\
9.75 & 569 \\
\end{array}$ & $\begin{array}{l}18 \\
19 \\
18 \\
18\end{array}$ & $\begin{array}{ll}9.83 & 984 \\
9.84 & 011 \\
9.84 & 038 \\
9.84 & 065 \\
9.84 & 092\end{array}$ & $\begin{array}{l}27 \\
27 \\
27 \\
27\end{array}$ & 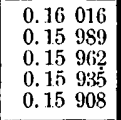 & $\begin{array}{ll}9.91 & 512 \\
9.91 & 504 \\
9.91 & 495 \\
9.91 & 486 \\
9.91 & 477\end{array}$ & $\begin{array}{r}8 \\
9 \\
9 \\
9 \\
8\end{array}$ & $\begin{array}{l}\mathbf{2 0} \\
19 \\
18 \\
17 \\
1.6\end{array}$ & 41 & $\begin{array}{r}20 \\
16 \\
12 \\
8 \\
4\end{array}$ \\
\hline $\begin{array}{r}0 \\
4 \\
8 \\
12 \\
16\end{array}$ & $\begin{array}{l}45 \\
46 \\
47 \\
48 \\
49\end{array}$ & 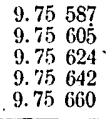 & $\begin{array}{l}18 \\
19 \\
18 \\
18\end{array}$ & $\begin{array}{ll}9.84 & 119 \\
9.84 & 146 \\
9.84 & 173 \\
9.84 & 200 \\
9.84 & 227\end{array}$ & $\begin{array}{l}27 \\
27 \\
27 \\
27\end{array}$ & $\begin{array}{ll}0.15 & 881 \\
0.15 & 854 \\
0.15 & 827 \\
0.15 & 800 \\
0.15 & 773\end{array}$ & 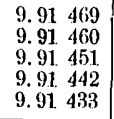 & $\begin{array}{l}9 \\
9 \\
9 \\
9\end{array}$ & $\begin{array}{l}15 \\
14 \\
13 \\
12 \\
11\end{array}$ & 41 & $\begin{array}{l}0 \\
56 \\
52 \\
48 \\
44\end{array}$ \\
\hline $\begin{array}{ll}19 & 20 \\
& 24\end{array}$ & $\begin{array}{l}50 \\
51 \\
52 \\
53 \\
54\end{array}$ & $\begin{array}{l}9.75678 \\
9.75696 \\
9.75714 \\
9.75733 \\
9.75751\end{array}$ & $\begin{array}{l}18 \\
18 \\
19 \\
18\end{array}$ & 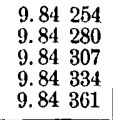 & $\begin{array}{l}26 \\
27 \\
27 \\
27\end{array}$ & 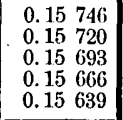 & $\begin{array}{ll}9.91 & 425 \\
9.91 & 416 \\
9.91 & 407 \\
9.91 & 398 \\
9.91 & 389\end{array}$ & $\begin{array}{l}9 \\
9 \\
9 \\
9 \\
8\end{array}$ & $\begin{array}{r}\mathbf{1 0} \\
9 \\
8 \\
7 \\
6\end{array}$ & .40 & $\begin{array}{l}40 \\
36 \\
32 \\
28 \\
24\end{array}$ \\
\hline $\begin{array}{ll}19 \quad 40 \\
& 44 \\
& 48 \\
& 48\end{array}$ & $\begin{array}{l}55 \\
56 \\
57 \\
58 \\
59\end{array}$ & $\begin{array}{ll}9.75 & 769 \\
9.75 & 787 \\
9.75 & 805 \\
9.75 & 823 \\
9.75 & 841\end{array}$ & $\begin{array}{l}18 \\
18 \\
18 \\
18 \\
18\end{array}$ & $\begin{array}{ll}9.84 & 388 \\
9.84 & 415 \\
9.84 & 442 \\
9.84 & 469 \\
9.84 & 496\end{array}$ & $\begin{array}{l}27 \\
27 \\
27 \\
27 \\
27\end{array}$ & $\begin{array}{ll}0.15 & 612 \\
0.15 & 585 \\
0.15 & 558 \\
0.15 & 531 \\
0.15 & 504\end{array}$ & $\begin{array}{ll}9.91 & 381 \\
9.91 & 372 \\
9.91 & 363 \\
9.91 & 354 \\
9.91 & 345\end{array}$ & $\begin{array}{l}9 \\
9 \\
9 \\
9 \\
9\end{array}$ & $\begin{array}{l}5 \\
4 \\
3 \\
2 \\
1\end{array}$ & $\begin{array}{c}40 \\
.\end{array}$ & $\begin{array}{r}20 \\
16 \\
12 \\
8 \\
4\end{array}$ \\
\hline 20 & 60 & 9.75859 & & 9.84523 & & 0.15477 & 9.91336 & & 0 & 40 & 0 \\
\hline & & J. Cos. & d. & L. Cotg. & c. d. & L. Tang. & L. $\operatorname{Sin}$. & d. & ' & $\mathrm{m}$ & . \\
\hline
\end{tabular}


TABLE 19.-Five-place logarithms of circular functions, etc.-Continued.

$2^{\text {h }}$

\begin{tabular}{|c|c|c|c|c|c|c|c|c|c|c|c|c|}
\hline $\mathrm{m}$. & s. & '. & L. Sin. & d. & L. Tang. & c. d. & L. Cotg. & L. Cos. & d. & & \\
\hline 20 & $\begin{array}{r}0 \\
4 \\
8 \\
12 \\
16\end{array}$ & $\begin{array}{l}0 \\
1 \\
2 \\
3 \\
4\end{array}$ & $\begin{array}{ll}9.75 & 859 \\
9.75 & 877 \\
9.75 & 895 \\
9.75 & 913 \\
9.75 & 931\end{array}$ & \multirow{2}{*}{$\begin{array}{l}18 \\
18 \\
18 \\
18 \\
18 \\
18 \\
18 \\
18 \\
18 \\
18\end{array}$} & $\begin{array}{ll}9.84 & 523 \\
9.84 & 550 \\
9.84 & 576 \\
9.84 & 603 \\
9.84 & 630\end{array}$ & $\begin{array}{l}27 \\
26 \\
27 \\
27 \\
27\end{array}$ & $\begin{array}{ll}0.15 & 477 \\
0.15 & 450 \\
0.15 & 424 \\
0.15 & 397 \\
0.15 & 370\end{array}$ & $\begin{array}{ll}9.91 & 336 \\
9.91 & 328 \\
9.91 & 319 \\
9.91 & 310 \\
9.91 & 301\end{array}$ & $\begin{array}{l}8 \\
9 \\
9 \\
9 \\
9\end{array}$ & $\begin{array}{l}60 \\
59 \\
58 \\
57 \\
56\end{array}$ & & $\begin{array}{r}0 \\
56 \\
52 \\
48 \\
44\end{array}$ \\
\hline 20 & $\begin{array}{l}20 \\
24 \\
28 \\
32 \\
36\end{array}$ & $\begin{array}{l}5 \\
6 \\
7 \\
8 \\
9\end{array}$ & $\begin{array}{ll}9.75 & 949 \\
9.75 & 967 \\
9.75 & 985 \\
9.76 & 003 \\
9.76 & 021 .\end{array}$ & & 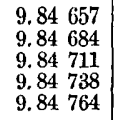 & $\begin{array}{l}27 \\
27 \\
27 \\
26\end{array}$ & $\begin{array}{ll}0.15 & 343 \\
0.15 & 316 \\
0.15 & 289 \\
0.15 & 262 \\
0.15 & 236\end{array}$ & $\begin{array}{ll}9.91 & 292 \\
9.91 & 283 \\
9.91 & 274 \\
9.91 & 266 \\
9.91 & 257\end{array}$ & $\begin{array}{l}9 \\
9 \\
9 \\
8 \\
9\end{array}$ & $\begin{array}{l}55 \\
54 \\
53 \\
52 \\
51\end{array}$ & 39 & $\begin{array}{l}40 \\
36 \\
32 \\
28 \\
24\end{array}$ \\
\hline 20 & $\begin{array}{l}40 \\
44 \\
48 \\
52 \\
56\end{array}$ & $\begin{array}{r}10 \\
11 \\
12 \\
13 \\
14\end{array}$ & $\begin{array}{ll}9.76 & 039 \\
9.76 & 057 \\
9.76 & 075 \\
9.76 & 093 \\
9.76 & 111\end{array}$ & $\begin{array}{r}10 \\
18 \\
18 \\
18 \\
18\end{array}$ & 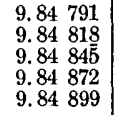 & $\begin{array}{l}27 \\
27 \\
27 \\
27\end{array}$ & $\begin{array}{ll}0.15 & 209 \\
0.15 & 182 \\
0.15 & 155 \\
0.15 & 128 \\
0.15 & 101\end{array}$ & $\begin{array}{ll}9.91 & 248 \\
9.91 & 239 \\
9.91 & 230 \\
9.91 & 221 \\
9.91 & 212\end{array}$ & $\begin{array}{l}\mathbf{9} \\
9 \\
\mathbf{9} \\
\mathbf{9}\end{array}$ & \begin{tabular}{r|}
$\mathbf{5 0}$ \\
49 \\
48 \\
47 \\
46
\end{tabular} & 39 & $\begin{array}{r}20 \\
16 \\
12 \\
8 \\
4\end{array}$ \\
\hline 21 & $\begin{array}{r}0 \\
4 \\
8 \\
12 \\
16\end{array}$ & $\begin{array}{l}15 \\
16 \\
17 \\
18 \\
19\end{array}$ & $\begin{array}{lll}9.76 & 129 \\
9.76 & 146 \\
9.76 & 164 \\
9.76 & 182 \\
9.76 & 200 \\
\end{array}$ & $\begin{array}{l}17 \\
18 \\
18 \\
18\end{array}$ & $\begin{array}{ll}9.84 & 925 \\
9.84 & 952 \\
9.84 & 979 \\
9.85 & 006 \\
9.85 & 033\end{array}$ & $\begin{array}{l}27 \\
27 \\
27 \\
27\end{array}$ & $\begin{array}{ll}0.15 & 075 \\
0.15 & 048 \\
0.15 & 021 \\
0.14 & 994 \\
0.14 & 967\end{array}$ & $\begin{array}{lll}9.91 & 203 \\
9.91 & 194 \\
9.91 & 185 \\
9.91 & 176 \\
9.91 & 167\end{array}$ & $\begin{array}{l}9 \\
9 \\
9 \\
9 \\
9\end{array}$ & \begin{tabular}{l|}
45 \\
44 \\
43 \\
42 \\
41
\end{tabular} & 39 & $\begin{array}{r}0 \\
56 \\
52 \\
48 \\
44\end{array}$ \\
\hline 21 & $\begin{array}{l}20 \\
24 \\
28 \\
32 \\
36\end{array}$ & \begin{tabular}{r|}
$\mathbf{2 0}$ \\
21 \\
22 \\
23 \\
24
\end{tabular} & $\begin{array}{ll}9.76 & 218 \\
9.76 & 236 \\
9.76 & 253 \\
9.76 & 271 \\
9.76 & 289\end{array}$ & $\begin{array}{l}18 \\
17 \\
18 \\
18\end{array}$ & $\begin{array}{ll}\mathbf{9 . 8 5} & 059 \\
9.85 & 086 \\
9.85 & 113 \\
9.85 & 140 \\
\mathbf{9 . 8 5} & 166\end{array}$ & $\begin{array}{l}20 \\
27 \\
27 \\
27 \\
26\end{array}$ & $\begin{array}{ll}0.14 & 941 \\
0.14 & 914 \\
0.14 & 887 \\
0.14 & 860 \\
0.14 & 834\end{array}$ & $\begin{array}{lll}9.91 & 158 \\
9.91 & 149 \\
9.91 & 141 \\
9.91 & 132 \\
9.91 & 123\end{array}$ & $\begin{array}{l}9 \\
8 \\
9 \\
9 \\
0\end{array}$ & \begin{tabular}{l|}
40 \\
39 \\
38 \\
37 \\
36
\end{tabular} & 38 & $\begin{array}{l}40 \\
36 \\
32 \\
28 \\
24\end{array}$ \\
\hline 21 & $\begin{array}{l}40 \\
44 \\
48 \\
52 \\
56 \\
\end{array}$ & $\begin{array}{l}25 \\
26 \\
27 \\
28 \\
29 \\
\end{array}$ & $\begin{array}{ll}9.76 & 307 \\
9.76 & 324 \\
9.76 & 342 \\
9.76 & 360 \\
9.76 & 378 \\
\end{array}$ & $\begin{array}{l}17 \\
18 \\
18 \\
18\end{array}$ & $\begin{array}{ll}9.85 & 193 \\
9.85 & 220 \\
9.85 & 247 \\
9.85 & 273 \\
9.85 & 300 \\
\end{array}$ & $\begin{array}{l}27 \\
27 \\
26 \\
27\end{array}$ & $\begin{array}{ll}0.14 & 807 \\
0.14 & 780 \\
0.14 & 753 \\
0.14 & 727 \\
0.14 & 700\end{array}$ & $\begin{array}{ll}9.91 & 114 \\
9.91 & 105 \\
9.91 & 096 \\
9.91 & 087 \\
9.91 & 078\end{array}$ & $\begin{array}{l}9 \\
9 \\
9 \\
9\end{array}$ & $\begin{array}{l}35 \\
34 \\
33 \\
32 \\
31\end{array}$ & 38 & $\begin{array}{r}20 \\
16 \\
12 \\
8 \\
4\end{array}$ \\
\hline 22 & $\begin{array}{r}0 \\
4 \\
8 \\
12 \\
16\end{array}$ & \begin{tabular}{c|}
$\mathbf{3 0}$ \\
31 \\
32 \\
33 \\
34
\end{tabular} & $\begin{array}{ll}9.76 & 395 \\
9.76 & 413 \\
9.76 & 431 \\
9.76 & 448 \\
9.76 & 466\end{array}$ & $\begin{array}{l}18 \\
18 \\
17 \\
18\end{array}$ & $\begin{array}{ll}9.85 & 327 \\
9.85 & 354 \\
9.85 & 380 \\
9.85 & 407 \\
9.85 & 434\end{array}$ & $\begin{array}{l}27 \\
26 \\
27 \\
27\end{array}$ & $\begin{array}{ll}0.14 & 673 \\
0.14 & 646 \\
0.14 & 620 \\
0.14 & 593 \\
0.14 & 566\end{array}$ & $\begin{array}{lll}9.91 & 069 \\
9.91 & 060 \\
9.91 & 051 \\
9.91 & 042 \\
9.91 & 033\end{array}$ & $\begin{array}{r}9 \\
9 \\
9 \\
9\end{array}$ & $\begin{array}{l}\mathbf{3 0} \\
29 \\
28 \\
27 \\
26\end{array}$ & 38 & $\begin{array}{r}0 \\
56 \\
52 \\
48 \\
44\end{array}$ \\
\hline 22 & $\begin{array}{l}20 \\
24 \\
28 \\
32 \\
36\end{array}$ & $\begin{array}{l}35 \\
36 \\
37 \\
38 \\
39\end{array}$ & $\begin{array}{ll}9.76 & 884 \\
9.76 & 501 \\
9.76 & 519 \\
9.76 & 537 \\
9.76 & 554\end{array}$ & $\begin{array}{l}17 \\
18 \\
18 \\
17\end{array}$ & $\begin{array}{ll}9.85 & 460 \\
9.85 & 487 \\
9.85 & 514 \\
9.85 & 540 \\
9.85 & 567\end{array}$ & $\begin{array}{l}27 \\
27 \\
26 \\
27\end{array}$ & 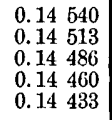 & $\begin{array}{ll}9.91 & 023 \\
9.91 & 014 \\
9.91 & 005 \\
9.90 & 996 \\
9.90 & 987\end{array}$ & $\begin{array}{l}9 \\
9 \\
9 \\
9 \\
0\end{array}$ & \begin{tabular}{l|}
25 \\
24 \\
23 \\
22 \\
21
\end{tabular} & 37 & $\begin{array}{l}40 \\
36 \\
32 \\
28 \\
24\end{array}$ \\
\hline 22 & $\begin{array}{l}40 \\
44 \\
48 \\
52 \\
56\end{array}$ & $\begin{array}{l}40 \\
41 \\
42 \\
43 \\
44\end{array}$ & 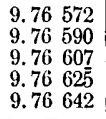 & $\begin{array}{l}18 \\
17 \\
18 \\
17\end{array}$ & $\begin{array}{ll}9.85 & 594 \\
9.85 & 620 \\
9.85 & 647 \\
9.85 & 674 \\
9.85 & 700\end{array}$ & $\begin{array}{l}26 \\
27 \\
27 \\
26\end{array}$ & $\begin{array}{ll}0.14 & 406 \\
0.14 & 380 \\
0.14 & 353 \\
0.14 & 326 \\
0.14 & 300\end{array}$ & $\begin{array}{l}9.90978 \\
9.90969 \\
9.90960 \\
9.90951 \\
9.90942\end{array}$ & $\begin{array}{l}9 \\
9 \\
9 \\
9 \\
0\end{array}$ & $\begin{array}{l}20 \\
19 \\
18 \\
17 \\
16\end{array}$ & 37 & $\begin{array}{r}20 \\
16 \\
12 \\
8 \\
4\end{array}$ \\
\hline 23 & $\begin{array}{r}0 \\
4 \\
8 \\
12 \\
16\end{array}$ & $\begin{array}{l}45 \\
46 \\
47 \\
48 \\
49\end{array}$ & 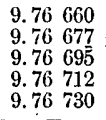 & $\begin{array}{l}17 \\
18 \\
17 \\
18\end{array}$ & 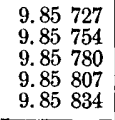 & $\begin{array}{l}27 \\
26 \\
27 \\
27\end{array}$ & $\begin{array}{ll}0.14 & 273 \\
0.14 & 246 \\
0.14 & 220 \\
0.14 & 193 \\
0.14 & 166\end{array}$ & $\begin{array}{l}9.90933 \\
9.90924 \\
9.90915 \\
9.90906 \\
9.90896\end{array}$ & $\begin{array}{r}9 \\
9 \\
9 \\
10\end{array}$ & $\begin{array}{l}15 \\
14 \\
13 \\
12 \\
11\end{array}$ & 37 & $\begin{array}{r}0 \\
56 \\
52 \\
48 \\
44\end{array}$ \\
\hline 23 & $\begin{array}{l}20 \\
24 \\
28 \\
32 \\
36\end{array}$ & $\begin{array}{l}\mathbf{5 0} \\
51 \\
52 \\
53 \\
54\end{array}$ & 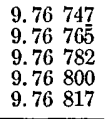 & $\begin{array}{l}18 \\
17 \\
18 \\
17\end{array}$ & 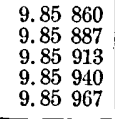 & $\begin{array}{l}27 \\
26 \\
27 \\
27\end{array}$ & $\begin{array}{ll}0.14 & 140 \\
0.14 & 113 \\
0.14 & 087 \\
0.14 & 060 \\
0.14 & 033\end{array}$ & $\begin{array}{ll}9.90 & 887 \\
9.90 & 878 \\
9.90 & 869 \\
9.90 & 860 \\
9.90 & 851\end{array}$ & $\begin{array}{l}9 \\
9 \\
9 \\
9 \\
9\end{array}$ & $\begin{array}{r}10 \\
9 \\
8 \\
7 \\
6\end{array}$ & 36 & $\begin{array}{l}40 \\
36 \\
32 \\
28 \\
24\end{array}$ \\
\hline 23 & $\begin{array}{l}40 \\
44 \\
48 \\
52 \\
56\end{array}$ & $\begin{array}{l}55 \\
56 \\
57 \\
58 \\
59\end{array}$ & $\begin{array}{l}9.76835 \\
9.76852 \\
9.76870 \\
9.76887 \\
9.76904\end{array}$ & $\begin{array}{l}17 \\
18 \\
17 \\
17\end{array}$ & $\begin{array}{ll}9.85 & 993 \\
9.86 & 020 \\
9.86 & 046 \\
9.86 & 073 \\
9.86 & 100\end{array}$ & $\begin{array}{l}27 \\
26 \\
27 \\
27\end{array}$ & $\begin{array}{ll}0.14 & 007 \\
0.13 & 980 \\
0.13 & 954 \\
0.13 & 927 \\
0.13 & 900\end{array}$ & $\begin{array}{ll}9.90 & 842 \\
9.90 & 832 \\
9.90 & 823 \\
9.90 & 814 \\
9.90 & 805\end{array}$ & $\begin{array}{r}10 \\
9 \\
9 \\
9\end{array}$ & $\begin{array}{l}5 \\
4 \\
3 \\
2 \\
1\end{array}$ & 36 & $\begin{array}{r}20 \\
16 \\
12 \\
8 \\
4\end{array}$ \\
\hline 24 & 0 & 60 & 9.76922 & & $9.86 \quad 126$ & & 0.13874 & 9.90796 & & 0 & 36 & 0 \\
\hline & & & L. Cos. & d. & L. Cotg. & c. d. & L. Tang. & L. $\operatorname{Sin}$. & d. & $'$ & $\mathrm{~m}$ & s. \\
\hline
\end{tabular}


TABLe 19.-Five-place logarithms of circular functions, etc.-Continued.

$\mathbf{2}^{\mathrm{h}}$

\begin{tabular}{|c|c|c|c|c|c|c|c|c|c|c|c|c|}
\hline m. & s. & ' & L. Sin. & d. & L. Tang. & c. d. & L. Cotg. & L. Cos. & d. & & & \\
\hline 24 & $\begin{array}{r}0 \\
4 \\
8 \\
12 \\
16 \\
\end{array}$ & $\begin{array}{l}0 \\
1 \\
2 \\
3 \\
4 \\
\end{array}$ & 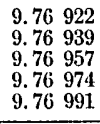 & \multirow{3}{*}{$\begin{array}{l}17 \\
18 \\
17 \\
17 \\
18 \\
17 \\
17 \\
18 \\
17 \\
17 \\
17 \\
18 \\
17 \\
17 \\
17\end{array}$} & $\begin{array}{ll}9.86 & 126 \\
9.86 & 153 \\
9.86 & 179 \\
9.86 & 21) 6 \\
9.86 & 232 \\
\end{array}$ & \multirow{4}{*}{$\begin{array}{l}27 \\
26 \\
27 \\
26 \\
27 \\
20 \\
27 \\
26 \\
27 \\
27 \\
26 \\
27 \\
26 \\
27 \\
20 \\
27 \\
26 \\
20 \\
27 \\
26\end{array}$} & 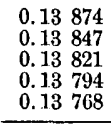 & 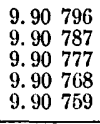 & \multirow{2}{*}{$\begin{array}{r}9 \\
10 \\
9 \\
9 \\
9 \\
9 \\
10 \\
9 \\
9 \\
9\end{array}$} & $\begin{array}{r}60 \\
59 \\
58 \\
57 \\
56 \\
\end{array}$ & 36 & $\begin{array}{r}0 \\
56 \\
52 \\
48 \\
44 \\
\end{array}$ \\
\hline 24 & $\begin{array}{l}20 \\
24 \\
28 \\
32 \\
36\end{array}$ & $\begin{array}{l}5 \\
6 \\
7 \\
8 \\
9\end{array}$ & $\begin{array}{ll}9.77 & 009 \\
9.77 & 026 \\
9.77 & 043 \\
9.77 & 061 \\
9.77 & 078\end{array}$ & & 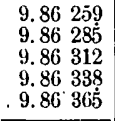 & & 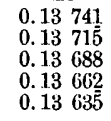 & 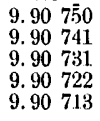 & & $\begin{array}{l}55 \\
54 \\
53 \\
52 \\
51 .\end{array}$ & 35 & $\begin{array}{l}40 \\
36 \\
32 \\
28 \\
24\end{array}$ \\
\hline 24 & $\begin{array}{l}40 \\
44 \\
48 \\
52 \\
56\end{array}$ & $\begin{array}{l}10 \\
11 \\
12 \\
13 \\
14\end{array}$ & $\begin{array}{ll}9.77 & 095 \\
9.77 & 112 \\
9.77 & 130 \\
9.77 & 147 \\
9.77 & 164\end{array}$ & & 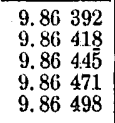 & & $\begin{array}{ll}0.13 & 608 \\
0.13 & 582 \\
0.13 & 555 \\
0.13 & 529 \\
0.13 & 502\end{array}$ & 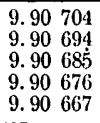 & $\begin{array}{r}10 \\
9 \\
9 \\
9 \\
10\end{array}$ & $\begin{array}{r}50 \\
49 \\
48 \\
47 \\
46\end{array}$ & 35 & $\begin{array}{r}20 \\
16 \\
12 \\
8 \\
4\end{array}$ \\
\hline 25 & $\begin{array}{r}0 \\
4 \\
8 \\
12 \\
16\end{array}$ & $\begin{array}{l}15 \\
16 \\
17 \\
18 \\
19\end{array}$ & $\begin{array}{ll}9.77 & 181 \\
9.77 & 199 \\
9.77 & 216 \\
9.77 & 233 \\
9.77 & 250\end{array}$ & $\begin{array}{l}18 \\
17 \\
17 \\
17 \\
18\end{array}$ & $\begin{array}{ll}9.86 & 524 \\
9.86 & 551 \\
9.86 & 577 \\
9.86 & 603 \\
9.86 & 630\end{array}$ & & $\begin{array}{l}0.13476 \\
0.13449 \\
0.13423 \\
0.13397 \\
0.13370\end{array}$ & 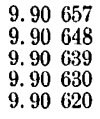 & $\begin{array}{r}9 \\
9 \\
9 \\
10 \\
\quad 9\end{array}$ & $\begin{array}{l}45 \\
44 \\
43 \\
42 \\
41\end{array}$ & 35 & $\begin{array}{r}0 \\
56 \\
52 \\
48 \\
44\end{array}$ \\
\hline 25 & $\begin{array}{l}20 \\
24 \\
28 \\
32 \\
36\end{array}$ & $\begin{array}{l}\mathbf{2 0} \\
21 \\
22 \\
23 \\
24\end{array}$ & $\begin{array}{ll}9.77 & 268 \\
9.77 & 285 \\
9.77 & 302 \\
9.77 & 319 \\
9.77 & 336\end{array}$ & $\begin{array}{l}17 \\
17 \\
17 \\
17 \\
17\end{array}$ & $\begin{array}{ll}9.86 & 656 \\
9.86 & 683 \\
9.86 & 709 \\
9.86 & 736 \\
9.86 & 762\end{array}$ & $\begin{array}{l}27 \\
26 \\
27 \\
26 \\
27\end{array}$ & $\begin{array}{ll}0.13 & 344 \\
0.13 & 317 \\
0.13 & 291 \\
0.13 & 264 \\
0.13 & 238\end{array}$ & 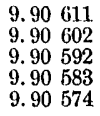 & $\begin{array}{r}9 \\
10 \\
9 \\
9 \\
9\end{array}$ & $\begin{array}{l}40 \\
39 \\
38 \\
37 \\
36\end{array}$ & 34 & $\begin{array}{l}40 \\
36 \\
32 \\
28 \\
24\end{array}$ \\
\hline 25 & $\begin{array}{l}40 \\
44 \\
48 \\
52 \\
56\end{array}$ & $\begin{array}{l}25 \\
26 \\
27 \\
28 \\
29\end{array}$ & 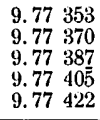 & $\begin{array}{l}17 \\
17 \\
18 \\
17 \\
17\end{array}$ & 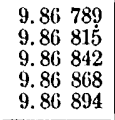 & $\begin{array}{l}26 \\
27 \\
26 \\
26 \\
27\end{array}$ & $\begin{array}{ll}0.13 & 211 \\
0.13 & 185 \\
0.13 & 158 \\
0.13 & 132 \\
0.13 & 106\end{array}$ & $\begin{array}{l}\mathbf{9 . 9 0} 56 \overline{5} \\
\mathbf{9 . 9 0} 555 \\
\mathbf{9 . 9 0} 546 \\
\mathbf{9 . 9 0} 537 \\
\mathbf{9 . 9 0} 527\end{array}$ & $\begin{array}{r}10 \\
9 \\
9 \\
10\end{array}$ & $\begin{array}{l}35 \\
34 \\
33 \\
32 \\
31\end{array}$ & 34 & $\begin{array}{r}20 \\
16 \\
12 \\
8 \\
4\end{array}$ \\
\hline 26 & $\begin{array}{r}0 \\
4 \\
8 \\
12 \\
16\end{array}$ & $\begin{array}{l}30 \\
31 \\
32 \\
33 \\
34\end{array}$ & 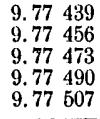 & $\begin{array}{l}17 \\
17 \\
17 \\
17 \\
17\end{array}$ & 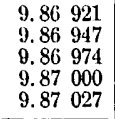 & $\begin{array}{l}26 \\
27 \\
26 \\
27 \\
26\end{array}$ & $\begin{array}{ll}0.13 & 079 \\
0.13 & 053 \\
0.13 & 026 \\
0.13 & 000 \\
0.12 & 973\end{array}$ & 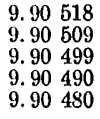 & $\begin{array}{r}9 \\
10 \\
9 \\
10 \\
9\end{array}$ & $\begin{array}{l}\mathbf{3 0} \\
29 \\
28 \\
27 \\
26\end{array}$ & 34 & $\begin{array}{r}0 \\
56 \\
52 \\
48 \\
44\end{array}$ \\
\hline 26 & $\begin{array}{l}20 \\
24 \\
28 \\
32 \\
36\end{array}$ & $\begin{array}{l}35 \\
36 \\
37 \\
38 \\
39 \\
\end{array}$ & $\begin{array}{ll}9.77 & 524 \\
9.77 & 541 \\
9.77 & 558 \\
9.77 & 575 \\
9.77 & 592 \\
\end{array}$ & $\begin{array}{l}17 \\
17 \\
17 \\
17 \\
17\end{array}$ & $\begin{array}{ll}9.87 & 053 \\
9.87 & 079 \\
9.87 & 106 \\
9.87 & 132 \\
9.87 & 158 \\
\end{array}$ & $\begin{array}{l}26 \\
27 \\
26 \\
26 \\
27\end{array}$ & $\begin{array}{l}0.12947 \\
0.12921 \\
0.12894 \\
0.12868 \\
0.12842\end{array}$ & 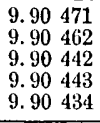 & $\begin{array}{r}9 \\
10 \\
9 \\
9 \\
10\end{array}$ & $\begin{array}{l}25 \\
24 \\
23 \\
22 \\
21 \\
\end{array}$ & 33 & $\begin{array}{l}40 \\
36 \\
32 \\
28 \\
24\end{array}$ \\
\hline 20 & $\begin{array}{l}40 \\
44 \\
48 \\
52 \\
56\end{array}$ & $\begin{array}{r}40 \\
41 \\
42 \\
43 \\
44\end{array}$ & $\begin{array}{l}9.77609 \\
9.77620 \\
9.77643 \\
9.77660 \\
9.77677\end{array}$ & $\begin{array}{l}17 \\
17 \\
17 \\
17 \\
17\end{array}$ & $\begin{array}{ll}9.87 & 185 \\
9.87 & 211 \\
9.87 & 238 \\
9.87 & 264 \\
9.87 & 290\end{array}$ & $\begin{array}{l}26 \\
27 \\
26 \\
26 \\
27\end{array}$ & 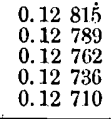 & 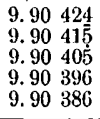 & $\begin{array}{r}9 \\
10 \\
9 \\
10 \\
9\end{array}$ & $\begin{array}{r}20 \\
19 \\
18 \\
17 \\
16\end{array}$ & 33 & $\begin{array}{r}20 \\
16 \\
12 \\
8 \\
4\end{array}$ \\
\hline 27 & $\begin{array}{r}0 \\
4 \\
8 \\
12 \\
16\end{array}$ & $\begin{array}{l}45 \\
46 \\
47 \\
48 \\
49\end{array}$ & $\begin{array}{ll}9.77 & 694 \\
9.77 & 711 \\
9.77 & 728 \\
9.77 & 744 \\
9.77 & 761\end{array}$ & $\begin{array}{l}17 \\
17 \\
16 \\
17 \\
17\end{array}$ & $\begin{array}{ll}9.87 & 317 \\
9.87 & 343 \\
9.87 & 369 \\
9.87 & 396 \\
9.87 & 422\end{array}$ & $\begin{array}{l}26 \\
26 \\
27 \\
26 \\
26\end{array}$ & 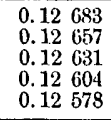 & 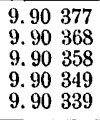 & $\begin{array}{r}9 \\
1.0 \\
9 \\
10 \\
9\end{array}$ & $\begin{array}{l}15 \\
14 \\
13 \\
12 \\
11\end{array}$ & 33 & $\begin{array}{r}0 \\
56 \\
52 \\
48 \\
44\end{array}$ \\
\hline 27 & $\begin{array}{l}20 \\
24 \\
28 \\
32 \\
36 \\
\end{array}$ & $\begin{array}{r}\mathbf{5 0} \\
51 \\
52 \\
53 \\
54 \\
\end{array}$ & $\begin{array}{l}9.77778 \\
9.77795 \\
9.778 .12 \\
9.77829 \\
9.77846 \\
\end{array}$ & $\begin{array}{l}17 \\
17 \\
17 \\
17 \\
16\end{array}$ & $\begin{array}{ll}9.87 & 448 \\
9.87 & 475 \\
9.87 & 501 \\
9.87 & 527 \\
9.87 & 554 \\
\end{array}$ & $\begin{array}{l}27 \\
26 \\
26 \\
27 \\
26\end{array}$ & $\begin{array}{ll}0.12 & 552 \\
0.12 & 525 \\
0.12 & 499 \\
0.12 & 473 \\
0.12 & 446 \\
\end{array}$ & 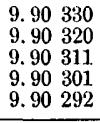 & $\begin{array}{r}10 \\
9 \\
10 \\
9\end{array}$ & $\begin{array}{r}10 \\
9 \\
8 \\
7 \\
6\end{array}$ & 32 & $\begin{array}{l}40 \\
36 \\
32 \\
28 \\
24\end{array}$ \\
\hline 27 & $\begin{array}{l}40 \\
44 \\
48 \\
52 \\
56\end{array}$ & $\begin{array}{l}55 \\
56 \\
57 \\
58 \\
59\end{array}$ & 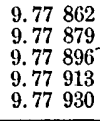 & $\begin{array}{l}17 ! \\
17 \\
17 \\
17 \\
16\end{array}$ & $\begin{array}{l}9.87580 \\
9.87606 \\
9.87633 \\
9.87659 \\
9.87685\end{array}$ & $\begin{array}{l}26 \\
27 \\
26 \\
26 \\
26\end{array}$ & $\begin{array}{ll}0.12 & 420 \\
0.12 & 394 \\
0.12 & 367 \\
0.12 & 341 \\
0.12 & 315\end{array}$ & 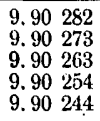 & $\begin{array}{r}9 \\
10 \\
9 \\
10 \\
9\end{array}$ & $\begin{array}{l}5 \\
4 \\
3 \\
2 \\
1\end{array}$ & 32 & $\begin{array}{r}20 \\
16 \\
12 \\
8 \\
4\end{array}$ \\
\hline 28 & 0 & 60 & 9. 77946 & & 9.87711 & & 0.12289 & $9.9023 \overline{5}$ & & 0 & 32 & 0 \\
\hline & & & L. Cos. & d. & I. Cotg. & c. d. & L. Tang. & L. Sin. & d. & ' & $\mathrm{m}$. & s. \\
\hline
\end{tabular}

$36^{\circ}$ 
Taвue 19:- Five-place logarithms of circular functions, ete.-Continued.

$2^{\text {h }}$ $37^{\circ}$

\begin{tabular}{|c|c|c|c|c|c|c|c|c|c|c|c|c|}
\hline $\mathrm{m}$. & s. & ' & L. $\operatorname{Sin}$. & d. & L. Tang. & c. d. & L. Cotg. & Cos. & d. & & & \\
\hline 28 & $\begin{array}{r}0 \\
4 \\
8 \\
12 \\
16\end{array}$ & $\begin{array}{l}0 \\
1 \\
2 \\
3 \\
4\end{array}$ & 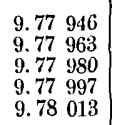 & \multirow{3}{*}{$\begin{array}{l}17 \\
17 \\
17 \\
16 \\
17 \\
17 \\
16 \\
17 \\
17 \\
16 \\
17 \\
17 \\
16 \\
17\end{array}$} & $\begin{array}{l}9.87 \\
911 \\
9.87738 \\
9.87764 \\
9.87790 \\
9.87817\end{array}$ & \multirow{3}{*}{$\begin{array}{l}27 \\
26 \\
26 \\
27 \\
26 \\
26 \\
26 \\
27 \\
26 \\
26 \\
26 \\
27 \\
26 \\
26 \\
26\end{array}$} & \multirow{2}{*}{\begin{tabular}{l|ll}
7 & 0.12 & 289 \\
6 & 0.12 & 262 \\
6 & 0.12236 \\
7 & 0.12 & 210 \\
6 & 0.12 & 183 \\
6 & 0.12 & 157 \\
6 & 0.12 & 131 \\
7 & 0.12 & 105 \\
6 & 0.12 & 078 \\
6 & 0.12 & 052
\end{tabular}} & 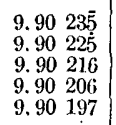 & \multirow{2}{*}{$\begin{array}{r}10 \\
9 \\
10 \\
9 \\
10 \\
9 \\
10 \\
9 \\
10 \\
10\end{array}$} & $\begin{array}{r}60 \\
59 \\
58 \\
57 \\
56\end{array}$ & \multicolumn{2}{|c|}{$\begin{array}{rr}32 & 0 \\
& 56 \\
& 52 \\
& 48 \\
& 44\end{array}$} \\
\hline 28 & $\begin{array}{l}20 \\
24 \\
28 \\
32 \\
36\end{array}$ & $\begin{array}{l}5 \\
6 \\
7 \\
8 \\
9\end{array}$ & $\begin{array}{lll}9.78 & 030 \\
9.78 & 047 \\
9.78 & 063 \\
9.78 & 080 \\
9.78 & 097\end{array}$ & & 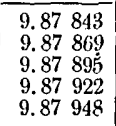 & & & $\begin{array}{lll}9.90 & 187 \\
9.90 & 178 \\
9.90 & 168 \\
9.90 & 159 \\
9.90 & 149\end{array}$ & & $\begin{array}{l}55 \\
54 \\
53 \\
52 \\
51\end{array}$ & \multicolumn{2}{|c|}{$\begin{array}{ll}31 & 40 \\
& 36 \\
& 32 \\
& 28 \\
& 24\end{array}$} \\
\hline 28 & $\begin{array}{l}40 \\
44 \\
48 \\
52 \\
56\end{array}$ & $\begin{array}{l}10 \\
11 \\
12 \\
13 \\
14\end{array}$ & $\begin{array}{lll}9.78 & 113 \\
9.78 & 130 \\
9.78 & 147 \\
9.78 & 163 \\
9.78 & 180\end{array}$ & & $\begin{array}{lll}9.87 & 974 \\
9.88 & 000 \\
9.88 & 027 \\
9.88 & 053 \\
9.88 & 079\end{array}$ & & $\begin{array}{lll}0.12 & 026 \\
0.12 & 000 \\
0.11 & 973 \\
0.11 & 947 \\
0.11 & 921\end{array}$ & $\begin{array}{lll}9.90 & 139 \\
9.90 & 130 \\
9.90 & 120 \\
9.90 & 111 \\
9.90 & 101\end{array}$ & $\begin{array}{r}9 \\
10 \\
9 \\
10 \\
10\end{array}$ & $\begin{array}{l}50 \\
49 \\
48 \\
47 \\
46\end{array}$ & \multicolumn{2}{|c|}{$\begin{array}{rr}31 \quad 20 \\
& 16 \\
& 12 \\
& 8 \\
& 4 \\
\end{array}$} \\
\hline 29 & $\begin{array}{r}0 \\
4 \\
8 \\
12 \\
16\end{array}$ & $\begin{array}{l}15 \\
16 \\
17 \\
18 \\
19\end{array}$ & $\begin{array}{ll}9.78 & 197 \\
9.78 & 213 \\
9.78 & 230 \\
9.78 & 246 \\
9.78 & 263\end{array}$ & \multirow{2}{*}{$\begin{array}{l}16 \\
17 \\
16 \\
17 \\
17 \\
16 \\
16 \\
17 \\
16 \\
17 \\
16\end{array}$} & $\begin{array}{lll}9.88 & 105 \\
9.88 & 131 \\
9.88 & 158 \\
9.88 & 184 \\
9.88 & 210\end{array}$ & $\begin{array}{l}26 \\
27 \\
26 \\
26 \\
26\end{array}$ & $\begin{array}{ll}0.11 & 89 \tilde{5} \\
0.11 & 869 \\
0.11 & 842 \\
0.11 & 816 \\
0.11 & 790\end{array}$ & $\begin{array}{lll}9.90 & 091 \\
9.90 & 082 \\
9.90 & 072 \\
9.90 & 063 \\
9.90 & 053\end{array}$ & $\begin{array}{r}9 \\
10 \\
9 \\
10 \\
10\end{array}$ & $\begin{array}{l}45 \\
44 \\
43 \\
42 \\
41\end{array}$ & \multicolumn{2}{|c|}{$\begin{array}{rr}31 . & 0 \\
& 56 \\
& 52 \\
& 48 \\
& 44\end{array}$} \\
\hline 29 & $\begin{array}{l}20 \\
24 \\
28 \\
32 \\
36\end{array}$ & $\begin{array}{l}20 \\
21 \\
22 \\
23 \\
24\end{array}$ & $\begin{array}{ll}\mathbf{9 . 7 8} & 280 \\
\mathbf{9 . 7 8} & 296 \\
9.78 & 313 \\
9.78 & 329 \\
9.78 & 346\end{array}$ & & $\begin{array}{ll}9.88 & 236 \\
9.88 & 262 \\
9.88 & 289 \\
9.88 & 315 \\
9.88 & 341\end{array}$ & $\begin{array}{l}26 \\
27 \\
26 \\
26 \\
26\end{array}$ & $\begin{array}{ll}0.11 & 764 \\
0.11 & 735 \\
0.11 & 711 \\
0.11 & 685 \\
0.11 & 659\end{array}$ & $\begin{array}{lll}9.90 & 043 \\
9.90 & 034 \\
9.90 & 024 \\
9.90 & 014 \\
9.90 & 005\end{array}$ & $\begin{array}{r}9 \\
10 \\
10 \\
9 \\
10\end{array}$ & $\begin{array}{l}\mathbf{4 0} \\
39 \\
38 \\
37 \\
36\end{array}$ & 30 & $\begin{array}{l}40 \\
36 \\
32 \\
28 \\
24\end{array}$ \\
\hline 29 & $\begin{array}{l}40 \\
44 \\
48 \\
52 \\
56\end{array}$ & $\begin{array}{l}25 \\
26 \\
27 \\
28 \\
29\end{array}$ & $\begin{array}{lll}9.78 & 362 \\
9.78 & 379 \\
9.78 & 395 \\
9.78 & 412 \\
9.78 & 428\end{array}$ & $\begin{array}{l}17 \\
16 \\
17 \\
16 \\
17\end{array}$ & $\begin{array}{ll}9.88 & 367 \\
9.88 & 393 \\
9.88 & 420 \\
9.88 & 446 \\
9.88 & 472\end{array}$ & $\begin{array}{l}26 \\
.27 \\
26 \\
26 \\
26\end{array}$ & $\begin{array}{lll}0.11 & 633 \\
0.11 & 607 \\
0.11 & 580 \\
0.11 & 554 \\
0.11 & 528\end{array}$ & 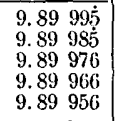 & $\begin{array}{r}10 \\
9 \\
10 \\
10 \\
9\end{array}$ & $\begin{array}{l}35 \\
34 \\
33 \\
32 \\
31 \\
\end{array}$ & 30 & $\begin{array}{r}20 \\
16 \\
12 \\
8 \\
4\end{array}$ \\
\hline 30 & $\begin{array}{r}0 \\
4 \\
8 \\
12 \\
16 \\
\end{array}$ & $\begin{array}{l}\mathbf{3 0} \\
31 \\
32 \\
33 \\
34\end{array}$ & $\begin{array}{lll}9.78 & 445 \\
9.78 & 461 \\
9.78 & 478 \\
9.78 & 494 \\
9.78 & 510 \\
\end{array}$ & $\begin{array}{l}16 \\
17 \\
16 \\
16 \\
17\end{array}$ & $\begin{array}{ll}9.88 & 498 \\
9.88 & 524 \\
9.88 & 550 \\
9.88 & 577 \\
9.88 & 603\end{array}$ & $\begin{array}{l}26 \\
26 \\
27 \\
26 \\
26\end{array}$ & $\begin{array}{lll}0.11 & 502 \\
0.11 & 476 \\
0.11 & 450 \\
0.11 & 423 \\
0.11 & 397\end{array}$ & 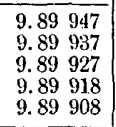 & \begin{tabular}{r|}
10 \\
10 \\
9 \\
10 \\
10
\end{tabular} & $\begin{array}{r}30 \\
29 \\
28 \\
27 \\
26\end{array}$ & \multicolumn{2}{|c|}{$\begin{array}{r}30 \\
56 \\
52 \\
52 \\
48 \\
44\end{array}$} \\
\hline 30 & $\begin{array}{l}20 \\
24 \\
28 \\
32 \\
36\end{array}$ & $\begin{array}{l}35 \\
36 \\
37 \\
38 \\
39\end{array}$ & $\begin{array}{lll}9.78 & 527 \\
9.78 & 543 \\
9.78 & 560 \\
9.78 & 576 \\
9.78 & 592\end{array}$ & $\begin{array}{l}16 \\
17 \\
16 \\
16 \\
17\end{array}$ & 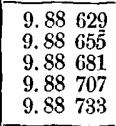 & $\begin{array}{l}26 \\
26 \\
26 \\
26 \\
26\end{array}$ & $\begin{array}{ll}0.11 & 371 \\
0.11 & 345 \\
0.11 & 319 \\
0.11 & 293 \\
0.11 & 267 \\
\end{array}$ & $\begin{array}{llll}9 . & 89 & 898 \\
9.89 & 888 \\
9.89 & 879 \\
9.89 & 869 \\
9.89 & 859 \\
\end{array}$ & $\begin{array}{r}10 \\
9 \\
10 \\
10 \\
10\end{array}$ & $\begin{array}{l}25 \\
24 \\
23 \\
22 \\
21\end{array}$ & 29 & $\begin{array}{l}40 \\
36 \\
32 \\
28 \\
24\end{array}$ \\
\hline 30 & $\begin{array}{l}40 \\
44 \\
48 \\
52 \\
56\end{array}$ & $\begin{array}{l}40 \\
41 \\
42 \\
43 \\
44\end{array}$ & 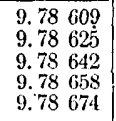 & $\begin{array}{l}16 \\
17 \\
16 \\
16 \\
17 .\end{array}$ & 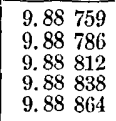 & $\begin{array}{l}27 \\
26 \\
26 \\
26 \\
26\end{array}$ & $\begin{array}{ll}0.11 & 241 \\
0.11 & 214 \\
0.11 & 188 \\
0.11 & 162 \\
0.11 & 136\end{array}$ & 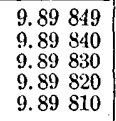 & $\begin{array}{r}9 \\
10 \\
10 \\
10 \\
9\end{array}$ & $\begin{array}{l}\mathbf{2 0} \\
19 \\
18 \\
17 \\
16\end{array}$ & 29 & $\begin{array}{r}20 \\
16 \\
12 \\
8 \\
4\end{array}$ \\
\hline 31 & \begin{tabular}{r|}
0 \\
4 \\
8 \\
12 \\
16 \\
\end{tabular} & $\begin{array}{l}45 \\
46 \\
47 \\
48 \\
49\end{array}$ & $\begin{array}{lll}9.78 & 691 \\
9.78 & 707 \\
\mathbf{9 . 7 8} & 723 \\
\mathbf{9 . 7 8} & 739 \\
\mathbf{9 . 7 8} & 756\end{array}$ & $\begin{array}{l}16 \\
16 \\
16 \\
17 \\
16\end{array}$ & $\begin{array}{l}9.88890 \\
9.88916 \\
9.88942 \\
9.88968 \\
9.88994\end{array}$ & $\begin{array}{l}26 \\
26 \\
26 \\
26 \\
26\end{array}$ & $\begin{array}{lll}0.11 & 110 \\
0.11 & 084 \\
0.11 & 058 \\
0.11 & 032 \\
0.11 & 006\end{array}$ & $\begin{array}{lll}9.89 & 801 \\
9.89 & 791 \\
9.89 & 781 \\
9.89 & 771 \\
9.89 & 761\end{array}$ & $\begin{array}{r}10 \\
10 \\
10 \\
10 \\
9\end{array}$ & $\begin{array}{l}15 \\
14 \\
13 \\
12 \\
11\end{array}$ & \multicolumn{2}{|c|}{$\begin{array}{cr}29 & 0 \\
& 56 \\
& 52 \\
& 48 \\
& 44\end{array}$} \\
\hline 31 & $\begin{array}{l}20 \\
24 \\
28 \\
32 \\
36 \\
\end{array}$ & $\begin{array}{l}50 \\
51 \\
52 \\
53 \\
54\end{array}$ & $\begin{array}{lll}9.78 & 772 \\
9.78 & 788 \\
9.78 & 805 \\
9.78 & 821 \\
9.78 & 837\end{array}$ & \multirow{3}{*}{$\begin{array}{l}16 \\
17 \\
16 \\
16 \\
16 \\
16 \\
17 \\
16 \\
16 \\
16\end{array}$} & $\begin{array}{lll}9.89 & 020 \\
9.89 & 046 \\
9.89 & 073 \\
9.89 & 099 \\
9.89 & 125\end{array}$ & $\begin{array}{l}26 \\
27 \\
26 \\
26 \\
26\end{array}$ & 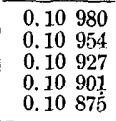 & 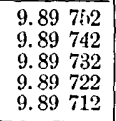 & $\begin{array}{l}10 \\
10 \\
10 \\
10 \\
10\end{array}$ & $\begin{array}{r}10 \\
9 \\
8 \\
7 \\
6\end{array}$ & \multicolumn{2}{|c|}{$\begin{array}{ll}28 & 40 \\
& 36 \\
& 32 \\
& 28 \\
& 24 \\
\end{array}$} \\
\hline 31 & $\begin{array}{l}40 \\
44 \\
48 \\
52 \\
56 \\
\end{array}$ & $\begin{array}{l}55 \\
56 \\
57 \\
58 \\
59\end{array}$ & \begin{tabular}{lll|}
9.78 & 853 \\
9.78 & 869 \\
9.78 & 886 \\
9.78 & 902 \\
9.78 & 918 \\
\end{tabular} & & $\begin{array}{lll}9.89 & 151 \\
9.89 & 177 \\
9.89 & 203 \\
9.89 & 229 \\
9.89 & 255 \\
\end{array}$ & $\begin{array}{l}26 \\
26 \\
26 \\
26 \\
26\end{array}$ & $\begin{array}{l}0.10849 \\
0.10 \quad 823 \\
0.10797 \\
0.10771 \\
0.10745\end{array}$ & 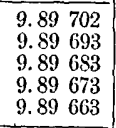 & $\begin{array}{r}9 \\
10 \\
10 \\
10 \\
10\end{array}$ & $\begin{array}{l}5 \\
4 \\
3 \\
2 \\
1\end{array}$ & \multicolumn{2}{|c|}{$\begin{array}{rr}28 \quad 20 \\
& 16 \\
& 12 \\
& 8 \\
& 4\end{array}$} \\
\hline 32 & 0 & 60 & 9.78934 & & 9.89281 & & 0.10719 & 9.89653 & & 0 & 28 & 0 \\
\hline & & & L. Cos. & d. & L. Cotg. & c. d. & L. Tang. & L. Sin. & d. & ' & $\mathrm{m}$. & s. \\
\hline
\end{tabular}


TABLe 19.-Five-place logarithms of circular functions, elc.-Continued.

$2^{\text {h }}$ $38^{\circ}$

\begin{tabular}{|c|c|c|c|c|c|c|c|c|c|c|c|c|}
\hline $\mathrm{m}$. & s. & ' & L. Sin. & d. & L. Tang. & c. d. & I. Cotg. & L. Cos. & d. & & \\
\hline 32 & $\begin{array}{r}0 \\
4 \\
8 \\
12 \\
16\end{array}$ & $\begin{array}{l}\mathbf{0} \\
1 \\
2 \\
3 \\
4\end{array}$ & 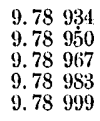 & $\begin{array}{l}16 \\
17 . \\
16 \\
16\end{array}$ & 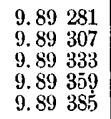 & $\begin{array}{r}26 \\
26 \\
.26 \\
26\end{array}$ & $\begin{array}{l}0.10719 \\
0.10693 \\
0.10667 \\
0.10641 \\
0.10615\end{array}$ & 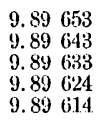 & $\begin{array}{r}10 \\
10 \\
9 \\
10\end{array}$ & $\begin{array}{c}\mathbf{6 0} \\
59 \\
58 \\
57 \\
56\end{array}$ & 28 & $\begin{array}{r}0 \\
56 \\
52 \\
48 \\
44\end{array}$ \\
\hline 32 & $\begin{array}{l}20 \\
24 \\
28 \\
32 \\
36\end{array}$ & $\begin{array}{l}5 \\
6 \\
7 \\
8 \\
9\end{array}$ & $\begin{array}{ll}9.79 & 015 \\
9.79 & 031 \\
9.79 & 047 \\
9.79 & 063 \\
9.79 & 079\end{array}$ & $\begin{array}{l}16 \\
16 \\
16 \\
16\end{array}$ & 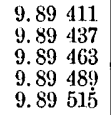 & $\begin{array}{l}26 \\
26 \\
26 \\
26\end{array}$ & 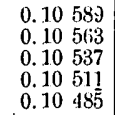 & 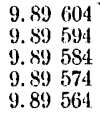 & $\begin{array}{l}10 \\
10 \\
10 \\
10\end{array}$ & $\begin{array}{l}55 \\
54 \\
53 \\
52 \\
51\end{array}$ & 27 & $\begin{array}{l}40 \\
36 \\
32 \\
28 \\
24\end{array}$ \\
\hline 32 & $\begin{array}{l}40 \\
44 \\
48 \\
52 \\
56\end{array}$ & $\begin{array}{l}10 \\
11 \\
12 \\
13 \\
14\end{array}$ & $\begin{array}{ll}9.79 & 095 \\
9.79 & 111 \\
9.79 & 128 \\
9.79 & 144 \\
9.79 & 160\end{array}$ & $\begin{array}{l}16 \\
17 \\
16 \\
16\end{array}$ & $\begin{array}{ll}9.89 & 541 \\
9.89 & 567 \\
9.89 & 593 \\
9.89 & 619 \\
9.89 & 645\end{array}$ & $\begin{array}{l}26 \\
26 \\
26 \\
26\end{array}$ & $\begin{array}{ll}0.10 & 459 \\
0.10 & 433 \\
0.10 & 407 \\
0.10 & 381 \\
0.10 & 355\end{array}$ & 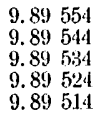 & $\begin{array}{l}10 \\
10 \\
10 \\
10\end{array}$ & $\begin{array}{l}50 \\
49 \\
48 \\
47 \\
46\end{array}$ & 27 & $\begin{array}{r}20 \\
16 \\
12 \\
8 \\
4\end{array}$ \\
\hline 33 & $\begin{array}{r}0 \\
4 \\
8 \\
12 \\
16\end{array}$ & $\begin{array}{l}15 \\
16 \\
17 \\
18 \\
19\end{array}$ & 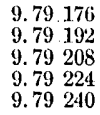 & $\begin{array}{l}16 \\
16 \\
16 \\
16\end{array}$ & 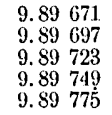 & $\begin{array}{l}20 \\
26 \\
26 \\
26 \\
26\end{array}$ & $\begin{array}{l}0.10329 \\
0.10303 \\
0.10277 \\
0.10251 \\
0.10225\end{array}$ & 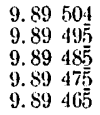 & $\begin{array}{r}10 \\
9 \\
10 \\
10 \\
10\end{array}$ & $\begin{array}{l}45 \\
44 \\
43 \\
42 \\
41\end{array}$ & 27 & $\begin{array}{r}0 \\
56 \\
52 \\
48 \\
44\end{array}$ \\
\hline 33 & $\begin{array}{l}20 \\
24 \\
28 \\
32 \\
36\end{array}$ & $\begin{array}{l}20 \\
21 \\
22 \\
23 \\
24\end{array}$ & $\begin{array}{ll}9.79 & 256 \\
9.79 & 272 \\
9.79 & 288 \\
9.79 & 304 \\
9.79 & 319\end{array}$ & $\begin{array}{l}16 \\
16 \\
16 \\
15\end{array}$ & 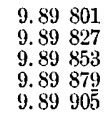 & $\begin{array}{l}26 \\
26 \\
26 \\
26\end{array}$ & $\begin{array}{lll}0.10 & 199 \\
0.10 & 173 \\
0.10 & 147 \\
0.10 & 121 \\
0.10 & 095\end{array}$ & $\begin{array}{ll}9.89 & 45 \overline{5} \\
9.89 & 445 \\
9.89 & 435 \\
9.89 & 425 \\
9.89 & 415\end{array}$ & $\begin{array}{l}10 \\
10 \\
10 \\
10\end{array}$ & $\begin{array}{l}\mathbf{4 0} \\
39 \\
38 \\
37 \\
36\end{array}$ & 26 & $\begin{array}{l}40 \\
36 \\
32 \\
28 \\
24\end{array}$ \\
\hline 33 & $\begin{array}{l}40 \\
44 \\
48 \\
52 \\
56\end{array}$ & $\begin{array}{l}25 \\
26 \\
27 \\
28 \\
29\end{array}$ & 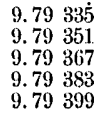 & $\begin{array}{l}16 \\
16 \\
16 \\
16\end{array}$ & 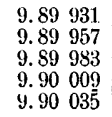 & $\begin{array}{l}26 \\
26 \\
26 \\
26\end{array}$ & $\begin{array}{ll}0.10 & 069 \\
0.10 & 0.43 \\
0.10 & 017 \\
0.09 & 991 \\
0.09 & 965\end{array}$ & $\begin{array}{l}9.89405 \\
9.89395 \\
9.89385 \\
9.89375 \\
9.89364\end{array}$ & $\begin{array}{l}10 \\
10 \\
10 \\
1.1\end{array}$ & $\begin{array}{l}35 \\
34 \\
33 \\
32 \\
31\end{array}$ & 26 & $\begin{array}{r}20 \\
16 \\
12 \\
8 \\
4\end{array}$ \\
\hline 34 & $\begin{array}{r}0 \\
4 \\
8 \\
12 \\
76\end{array}$ & $\begin{array}{l}\mathbf{3 0} \\
31 \\
32 \\
33 \\
34\end{array}$ & 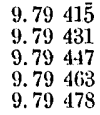 & $\begin{array}{l}16 \\
16 \\
.16 \\
15\end{array}$ & $\begin{array}{ll}9.90 & 061 \\
9.90 & 086 \\
9.90 & 112 \\
9.90 & 138 \\
9.90 & 164\end{array}$ & $\begin{array}{l}25 \\
26 \\
26 \\
26\end{array}$ & $\begin{array}{l}0.09989 \\
0.09914 \\
0.09888 \\
0.09862 \\
0.09836\end{array}$ & 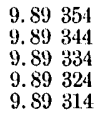 & $\begin{array}{l}10 \\
10 \\
10 \\
10\end{array}$ & $\begin{array}{l}30 \\
29 \\
28 \\
27 \\
26\end{array}$ & 26 & $\begin{array}{r}0 \\
56 \\
52 \\
48 \\
44\end{array}$ \\
\hline 34 & $\begin{array}{l}20 \\
24 \\
28 \\
32 \\
36\end{array}$ & $\begin{array}{l}35 \\
36 \\
37 \\
38 \\
39\end{array}$ & 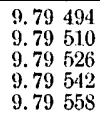 & $\begin{array}{l}16 \\
1.6 \\
1.6 \\
1.6\end{array}$ & 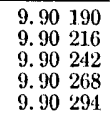 & $\begin{array}{l}26 \\
26 \\
26 \\
26\end{array}$ & $\begin{array}{l}0.09810 \\
0.09784 \\
0.09758 \\
0.09732 \\
0.09\end{array}$ & 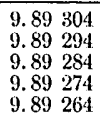 & $\begin{array}{l}10 \\
10 \\
10 \\
10 \\
10\end{array}$ & $\begin{array}{l}25 \\
24 \\
23 \\
22 \\
21 .\end{array}$ & 25 & $\begin{array}{l}40 \\
36 \\
32 \\
28 \\
24\end{array}$ \\
\hline 31 & $\begin{array}{l}40 \\
44 \\
48 \\
52 \\
56\end{array}$ & $\begin{array}{l}40 \\
41 \\
42 \\
43 \\
44\end{array}$ & 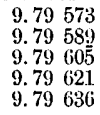 & $\begin{array}{l}16 \\
16 \\
16 \\
15\end{array}$ & 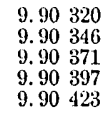 & $\begin{array}{l}26 \\
25 \\
26 \\
26\end{array}$ & $\begin{array}{l}0.09680 \\
0.09654 \\
0.09629 \\
0.09603 \\
0.09577\end{array}$ & $\begin{array}{ll}9.89 & 254 \\
9.89 & 244 \\
9.89 & 233 \\
9.89 & 223 \\
9.89 & 213\end{array}$ & $\begin{array}{l}10 \\
11 \\
10 \\
10\end{array}$ & $\begin{array}{l}\mathbf{2 0} \\
19 \\
18 \\
17 \\
16\end{array}$ & 25 & $\begin{array}{r}20 \\
16 \\
12 \\
8 \\
4\end{array}$ \\
\hline 35 & $\begin{array}{r}0 \\
4 \\
8 \\
12 \\
16\end{array}$ & $\begin{array}{l}45 \\
46 \\
47 \\
48 \\
49\end{array}$ & $\begin{array}{l}9.79652 \\
9.79668 \\
9.79 .684 \\
9.79699 \\
9.79\end{array}$ & $\begin{array}{l}16 \\
16 \\
15 \\
16\end{array}$ & $\begin{array}{ll}9.90 & 449 \\
9.90 & 475 \\
9.90 & 501 \\
9.90 & 527 \\
9.90 & 553\end{array}$ & $\begin{array}{l}26 \\
26 \\
26 \\
26\end{array}$ & 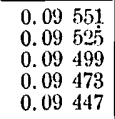 & $\begin{array}{ll}9.89 & 203 \\
9.89 & 193 \\
9.89 & 183 \\
9.89 & 173 \\
9.89 & 162\end{array}$ & $\begin{array}{l}10 \\
10 \\
10 \\
10 \\
11\end{array}$ & $\begin{array}{l}15 \\
14 \\
13 \\
12 \\
11 .\end{array}$ & 25 & $\begin{array}{r}0 \\
56 \\
52 \\
43 \\
44\end{array}$ \\
\hline 35 & $\begin{array}{l}20 \\
24 \\
28 \\
32 \\
36\end{array}$ & $\begin{array}{l}50 \\
51 \\
52 \\
53 \\
54\end{array}$ & 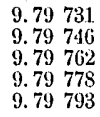 & $\begin{array}{l}15 \\
16 \\
16 \\
15\end{array}$ & $\begin{array}{l}9.90578 \\
9.90604 \\
9.90630 \\
9.90656 \\
9.90682\end{array}$ & $\begin{array}{l}26 \\
26 \\
26 \\
26\end{array}$ & $\begin{array}{l}0.09422 \\
0.09396 \\
0.09370 \\
0.09344 \\
0.09318\end{array}$ & $\begin{array}{ll}9.89 & 152 \\
9.89 & 142 \\
9.89 & 132 \\
9.89 & 122 \\
9.89 & 112\end{array}$ & $\begin{array}{l}10 \\
10 \\
10 \\
10\end{array}$ & $\begin{array}{r}10 \\
9 \\
8 \\
7 \\
6\end{array}$ & 24 & $\begin{array}{l}40 \\
36 \\
32 \\
28 \\
24\end{array}$ \\
\hline 35 & $\begin{array}{l}40 \\
44 \\
48 \\
52 \\
56\end{array}$ & $\begin{array}{l}55 \\
56 \\
57 \\
58 \\
59\end{array}$ & 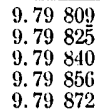 & $\begin{array}{l}16 \\
15 \\
16 \\
16\end{array}$ & 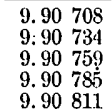 & $\begin{array}{l}26 \\
25 \\
26 \\
26\end{array}$ & $\begin{array}{ll}0.09292 \\
0.09266 \\
0.09241 \\
0.09215 \\
\text { 0.09 } 159\end{array}$ & $\begin{array}{ll}9.89 & 101 \\
9.89 & 091 \\
9.89 & 081 \\
9.89 & 071 \\
9.89 & 060\end{array}$ & $\begin{array}{l}10 \\
10 \\
10 \\
11\end{array}$ & $\begin{array}{l}5 \\
4 \\
3 \\
2 \\
1\end{array}$ & 24 & $\begin{array}{r}20 \\
16 \\
12 \\
8 \\
4\end{array}$ \\
\hline 36 & 0 & 60 & 9.79887 & & 9.90837 & 20 & 0.09163 & $9.890 \dot{50}$ & & 0 & 24 & 0 \\
\hline & & & I. Cos. & d. & L. Cotg. & c. d. & L. 'Tang. & L. Sin. & d. & ' & m. & s. \\
\hline
\end{tabular}


TABLE 19.-Five-place logarithms of circular functions, etc.-Continued.

$2^{\text {h }}$

\begin{tabular}{|c|c|c|c|c|c|c|c|c|c|c|c|c|}
\hline $\mathrm{m}$ & s. & ' & L. Sin. & d. & I. Tang. & c. $\mathrm{d}$. & L. Cotg. & L. Cos. & d. & & & \\
\hline 36 & $\begin{array}{r}0 \\
4 \\
8 \\
12 \\
16\end{array}$ & $\begin{array}{l}\mathbf{0} \\
1 \\
2 \\
3 \\
4\end{array}$ & 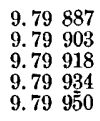 & $\begin{array}{l}16 \\
15 \\
16 \\
16\end{array}$ & 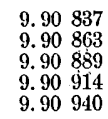 & $\begin{array}{l}26 \\
26 \\
25 \\
26\end{array}$ & $\begin{array}{ll}0.09 & 163 \\
0.09 & 137 \\
0.09 & 111 \\
0.09 & 086 \\
0.09 & 060\end{array}$ & 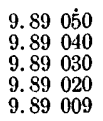 & $\begin{array}{l}10 \\
10 \\
10 \\
11\end{array}$ & $\begin{array}{l}60 \\
59 \\
58 \\
57 \\
56\end{array}$ & & $\begin{array}{r}0 \\
56 \\
52 \\
48 \\
44\end{array}$ \\
\hline 36 & $\begin{array}{l}20 \\
24 \\
28 \\
32 \\
36\end{array}$ & $\begin{array}{l}5 \\
6 \\
7 \\
8 \\
9\end{array}$ & 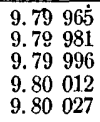 & $\begin{array}{l}16 \\
15 \\
16 \\
15\end{array}$ & $\begin{array}{ll}9.90 & 966 \\
9.90 & 992 \\
9.91 & 018 \\
9.91 & 043 \\
9.91 & 069\end{array}$ & $\begin{array}{l}26 \\
26 \\
25 \\
26\end{array}$ & 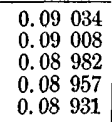 & 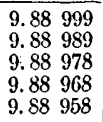 & $\begin{array}{l}10 \\
11 \\
10 \\
10\end{array}$ & $\begin{array}{l}55 \\
54 \\
53 \\
52 \\
51\end{array}$ & 23 & $\begin{array}{l}40 \\
36 \\
32 \\
29 \\
24\end{array}$ \\
\hline 36 & $\begin{array}{l}40 \\
44 \\
48 \\
52 \\
56\end{array}$ & $\begin{array}{l}10 \\
11 \\
12 \\
13 \\
14\end{array}$ & $\begin{array}{lll}9.80 & 043 \\
9.80 & 058 \\
9.80 & 074 \\
9.80 & 089 \\
9.80 & 105\end{array}$ & $\begin{array}{l}15 \\
16 \\
15 \\
16\end{array}$ & $\begin{array}{ll}9.91 & 095 \\
9.91 & 121 \\
9.91 & 147 \\
9.91 & 172 \\
9.91 & 198\end{array}$ & $\begin{array}{l}26 \\
26 \\
25 \\
26\end{array}$ & $\begin{array}{ll}0.08 & 905 \\
0.08 & 879 \\
0.08 & 853 \\
0.08 & 828 \\
0.08 & 802\end{array}$ & 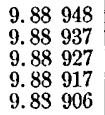 & $\begin{array}{l}10 \\
11 \\
10 \\
10 \\
11\end{array}$ & $\begin{array}{l}50 \\
49 \\
48 \\
47 \\
46\end{array}$ & 23 & $\begin{array}{r}20 \\
16 \\
12 \\
8 \\
4\end{array}$ \\
\hline 37 & $\begin{array}{r}0 \\
4 \\
8 \\
12 \\
16\end{array}$ & $\begin{array}{l}\cdot 15 \\
16 \\
17 \\
18 \\
19\end{array}$ & $\begin{array}{lll}9.80 & 120 \\
9.80 & 136 \\
9.80 & 151 \\
9.80 & 166 \\
9.80 & 1.82\end{array}$ & $\begin{array}{l}16 \\
15 \\
15 \\
16\end{array}$ & $\begin{array}{ll}9.91 & 224 \\
9.91 & 250 \\
9.91 & 276 \\
9.91 & 301 \\
9.91 & 327\end{array}$ & $\begin{array}{l}26 \\
26 \\
25 \\
26\end{array}$ & 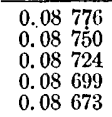 & 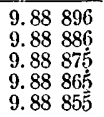 & $\begin{array}{l}10 \\
\mathbf{1 1} \\
10 \\
10\end{array}$ & $\begin{array}{l}45 \\
44 \\
43 \\
42 \\
41\end{array}$ & 23 & $\begin{array}{r}0 \\
56 \\
-52 \\
48 \\
44\end{array}$ \\
\hline 37 & $\begin{array}{l}20 \\
24 \\
28 \\
32 \\
36\end{array}$ & $\begin{array}{r}\mathbf{2 0} \\
21 \\
22 \\
23 \\
24\end{array}$ & $\begin{array}{lll}9.80 & 197 \\
9.80 & 213 \\
9.80 & 228 \\
9.80 & 244 \\
9.80 & 259\end{array}$ & $\begin{array}{l}16 \\
15 \\
16 \\
15\end{array}$ & $\begin{array}{ll}9.91 & 353 \\
9.91 & 379 \\
9.91 & 404 \\
9.91 & 430 \\
9,91 & 456\end{array}$ & $\begin{array}{l}26 \\
25 \\
26 \\
26\end{array}$ & $\begin{array}{ll}0.08 & 647 \\
0.08 & 621 \\
0.08 & 596 \\
0.08 & 570 \\
0.08 & 544\end{array}$ & 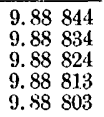 & $\begin{array}{l}10 \\
10 \\
11 \\
10\end{array}$ & $\begin{array}{l}\mathbf{4 0} \\
39 \\
38 \\
37 \\
36\end{array}$ & 22 & $\begin{array}{l}40 \\
36 \\
32 \\
28 \\
24\end{array}$ \\
\hline 37 & $\begin{array}{l}40 \\
44 \\
48 \\
52 \\
56\end{array}$ & $\begin{array}{l}25 \\
26 \\
27 \\
28 \\
29\end{array}$ & $\begin{array}{ll}9.80 & 274 \\
9.80 & 290 \\
9.80 & 305 \\
9.80 & 320 \\
9.80 & 336\end{array}$ & $\begin{array}{l}16 \\
15 \\
15 \\
16\end{array}$ & $\begin{array}{ll}9.91 & 482 \\
9.91 & 507 \\
9.91 & 583 \\
9.91 & 559 \\
9.91 & 585\end{array}$ & $\begin{array}{l}25 \\
26 \\
26 \\
26\end{array}$ & $\begin{array}{ll}0.08 & 518 \\
0.08 & 493 \\
0.08 & 467 \\
0.08 & 441 \\
0.08 & 415\end{array}$ & $\begin{array}{ll}9.88 & 793 \\
9.88 & 782 \\
9.88 & 772 \\
9.88 & 761 \\
9.88 & 751\end{array}$ & $\begin{array}{l}11 \\
10 \\
11 \\
10\end{array}$ & $\begin{array}{l}35 \\
34 \\
33 \\
32 \\
31\end{array}$ & 22 & $\begin{array}{r}20 \\
16 \\
12 \\
8 \\
4\end{array}$ \\
\hline 38 & $\begin{array}{r}0 \\
4 \\
8 \\
12 \\
16\end{array}$ & $\begin{array}{l}\mathbf{3 0} \\
31 \\
32 \\
33 \\
34\end{array}$ & $\begin{array}{lll}9.80 & 351 \\
9.80 & 366 \\
9.80 & 382 \\
9.80 & 397 \\
9.80 & 412\end{array}$ & $\begin{array}{l}15 \\
16 \\
15 \\
15\end{array}$ & $\begin{array}{ll}9.91 & 610 \\
9.91 & 636 \\
9.91 & 662 \\
9.91 & 688 \\
9.91 & 713\end{array}$ & $\begin{array}{l}26 \\
26 \\
26 \\
25\end{array}$ & $\begin{array}{ll}0.08 & 390 \\
0.08 & 364 \\
0.08 & 338 \\
0.08 & 312 \\
0.08 & 287\end{array}$ & 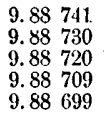 & $\begin{array}{l}11 \\
10 \\
11 \\
10\end{array}$ & $\begin{array}{l}\mathbf{3 0} \\
29 \\
28 \\
27 \\
26\end{array}$ & 22 & $\begin{array}{r}0 \\
56 \\
52 \\
48 \\
44\end{array}$ \\
\hline 38 & $\begin{array}{l}20 \\
24 \\
28 \\
32 \\
36\end{array}$ & $\begin{array}{l}35 \\
36 \\
37 \\
38 \\
39\end{array}$ & 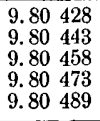 & $\begin{array}{l}15 \\
15 \\
15 \\
16\end{array}$ & $\begin{array}{ll}9.91 & 739 \\
9.91 & 765 \\
9.91 & 791 \\
9.91 & 816 \\
9.91 & 842\end{array}$ & $\begin{array}{l}26 \\
26 \\
25 \\
26\end{array}$ & $\begin{array}{ll}0.08 & 261 \\
0.08 & 235 \\
0.08 & 209 \\
0.08 & 184 \\
0.08 & 158\end{array}$ & $\begin{array}{ll}9.88 & 688 \\
9.88 & 678 \\
9.88 & 668 \\
9.88 & 657 \\
9.88 & 647\end{array}$ & $\begin{array}{l}10 \\
10 \\
11 \\
10\end{array}$ & $\begin{array}{l}25 \\
24 \\
23 \\
22 \\
21\end{array}$ & 21 & $\begin{array}{l}40 \\
36 \\
32 \\
28 \\
24\end{array}$ \\
\hline 38 & $\begin{array}{l}4 \hat{v} \\
44 \\
48 \\
52 \\
56\end{array}$ & $\begin{array}{r}40 \\
41 \\
42 \\
43 \\
44\end{array}$ & 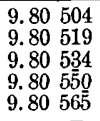 & $\begin{array}{l}15 \\
15 \\
16 \\
15\end{array}$ & $\begin{array}{ll}9.91 & 868 \\
9.91 & 893 \\
9.91 & 919 \\
9.91 & 945 \\
9.91 & 971\end{array}$ & $\begin{array}{l}25 \\
26 \\
26 \\
26\end{array}$ & $\begin{array}{ll}0.08 & 132 \\
0.08 & 107 \\
0.08 & 081 \\
0.08 & 055 \\
0.08 & 029\end{array}$ & 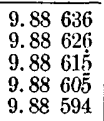 & $\begin{array}{l}10 \\
11 \\
10 \\
11\end{array}$ & $\begin{array}{l}\mathbf{2 0} \\
19 \\
18 \\
17 \\
16\end{array}$ & 21 & $\begin{array}{r}20 \\
16 \\
12 \\
8 \\
4\end{array}$ \\
\hline 39 & $\begin{array}{r}0 \\
4 \\
8 \\
12 \\
16\end{array}$ & $\begin{array}{l}45 \\
46 \\
47 \\
48 \\
49\end{array}$ & 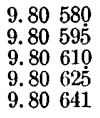 & $\begin{array}{l}15 \\
15 \\
15 \\
16\end{array}$ & $\begin{array}{ll}9.91 & 996 \\
9.92 & 022 \\
9.92 & 048 \\
9.92 & 073 \\
9.92 & 099\end{array}$ & $\begin{array}{l}26 \\
26 \\
25 \\
26\end{array}$ & 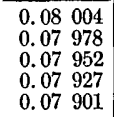 & 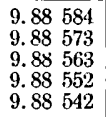 & $\begin{array}{l}11 \\
10 \\
11 \\
10\end{array}$ & $\begin{array}{l}15 \\
14 \\
13 \\
12 \\
11\end{array}$ & 21 & $\begin{array}{r}0 \\
56 \\
52 \\
48 \\
44\end{array}$ \\
\hline 39 & $\begin{array}{l}20 \\
24 \\
28 \\
32 \\
36\end{array}$ & $\begin{array}{c}50 \\
51 \\
52 \\
53 . \\
54\end{array}$ & 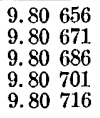 & $\begin{array}{l}15 \\
15 \\
15 \\
15\end{array}$ & $\begin{array}{ll}9.92 & 12 \overline{5} \\
9.92 & 150 \\
9.92 & 176 \\
9.92 & 202 \\
9.92 & 227\end{array}$ & $\begin{array}{l}25 \\
26 \\
26 \\
25\end{array}$ & 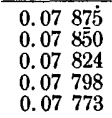 & $\begin{array}{lll}9.88 & 531 \\
9.88 & 521 \\
9.88 & 510 \\
9.88 & 499 \\
9.88 & 489\end{array}$ & $\begin{array}{l}10 \\
11 \\
11 \\
-10\end{array}$ & $\begin{array}{r}10 \\
9 \\
8 \\
7 \\
6\end{array}$ & 20 & $\begin{array}{l}40 \\
36 \\
32 \\
28 \\
24\end{array}$ \\
\hline 39 & $\begin{array}{l}40 \\
44 \\
48 \\
52 \\
56\end{array}$ & $\begin{array}{l}55 \\
56 \\
57 \\
58 \\
59\end{array}$ & 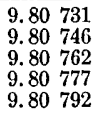 & $\begin{array}{l}15 \\
16 \\
15 \\
15\end{array}$ & $\begin{array}{ll}9.92 & 253 \\
9.92 & 279 \\
9.92 & 304 \\
9.92 & 330 \\
9.92 & 356\end{array}$ & $\begin{array}{l}26 \\
25 \\
26 \\
26\end{array}$ & 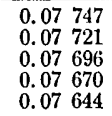 & $\begin{array}{ll}9.88 & 478 \\
9.88 & 468 \\
9.88 & 457 \\
9.88 & 447 \\
9.88 & 436\end{array}$ & $\begin{array}{l}10 \\
11 \\
10 \\
11\end{array}$ & $\begin{array}{l}5 \\
4 \\
3 \\
2 \\
1\end{array}$ & 20 & $\begin{array}{r}20 \\
16 \\
12 \\
8 \\
4\end{array}$ \\
\hline 40 & 0 & 60 & 9.80807 & & 9.92381 & & 0.07619 & $9.88425^{\circ}$ & 11 & 0 & 20 & 0 \\
\hline & & & L. Cos. & d. & L. Cotg. & c. d. & L. Tang. & L. Sin. & d. & ' & $\mathrm{m}$. & s. \\
\hline
\end{tabular}


ТавIE 19.-Five-place logarithms of circular functions, elc.-Continued. $2^{\text {h }}$ $40^{\circ}$

\begin{tabular}{|c|c|c|c|c|c|c|c|c|c|c|c|c|}
\hline $\mathrm{m}$. & s. & 1 & I. Sin. & d. & L. Tang. & c. $d$. & I. Cotg. & I. Cos. & d. & & & \\
\hline 40 & $\begin{array}{r}0 \\
4 \\
5 \\
12 \\
16\end{array}$ & $\begin{array}{l}0 \\
1 \\
2 \\
3 \\
4\end{array}$ & 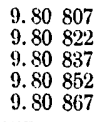 & $\begin{array}{l}15 \\
15 \\
15 \\
15 \\
15\end{array}$ & $\begin{array}{ll}9.92 & 381 . \\
9.92 & 407 \\
9.92 & 433 \\
9.92 & 468 \\
9.92 & 484\end{array}$ & $\begin{array}{l}26 \\
26 \\
25 \\
26 \\
26\end{array}$ & $\begin{array}{ll}0.07 & 619 \\
0.07 & 593 \\
0.07 & 567 \\
0.07 & 542 \\
0.07 & 516\end{array}$ & $\begin{array}{ll}9.88 & 425 \\
9.88 & 415 \\
9.88 & 404 \\
9.88 & 394 \\
9.88 & 383\end{array}$ & $\begin{array}{l}10 \\
11 \\
10 \\
11\end{array}$ & $\begin{array}{r}60 \\
\cdot 59 \\
58 \\
57 \\
56\end{array}$ & 20 & $\begin{array}{r}0 \\
56 \\
52 \\
48 \\
44\end{array}$ \\
\hline 40 & $\begin{array}{l}20 \\
24 \\
28 \\
32 \\
36\end{array}$ & $\begin{array}{l}5 \\
6 \\
7 \\
8 \\
9\end{array}$ & 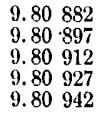 & $\begin{array}{l}15 \\
15 \\
15 \\
15\end{array}$ & $\begin{array}{ll}9.92 & 510 \\
9.92 & 535 \\
9.92 & 561 \\
9.92 & 587 \\
9.92 & 612\end{array}$ & $\begin{array}{l}25 \\
26 \\
26 \\
25\end{array}$ & $\begin{array}{ll}0.07 & 490 \\
0.07 & 465 \\
0.07 & 439 \\
0.07 & 413 \\
0.07 & 388\end{array}$ & $\begin{array}{ll}9.88 & 372 \\
9.88 & 362 \\
9.88 & 351 \\
9.88 & 340 \\
9.88 & 330\end{array}$ & $\begin{array}{l}10 \\
11 \\
11 \\
10 \\
11\end{array}$ & $\begin{array}{l}55 \\
54 \\
53 \\
52 \\
51\end{array}$ & 19 & $\begin{array}{l}40 \\
30 \\
32 \\
28 \\
24\end{array}$ \\
\hline 40 & $\begin{array}{l}40 \\
44 \\
48 \\
52 \\
56\end{array}$ & $\begin{array}{l}10 \\
11 \\
12 \\
13 \\
14\end{array}$ & $\begin{array}{ll}9.80 & 957 \\
9.80 & 972 \\
9.80 & 987 \\
9.81 & 002 \\
9.81 & 017\end{array}$ & $\begin{array}{l}15 \\
15 \\
15 \\
15 \\
15\end{array}$ & $\begin{array}{ll}9.92 & 638 \\
9.92 & 663 \\
9.92 & 689 \\
9.92 & 715 \\
9.92 & 740\end{array}$ & $\begin{array}{l}25 \\
26 \\
26 \\
25 \\
26\end{array}$ & $\begin{array}{ll}0.07 & 362 \\
0.07 & 337 \\
0.07 & 311 . \\
0.07 & 285 \\
0.07 & 260\end{array}$ & $\begin{array}{ll}9.88 & 31.9 \\
9.88 & 308 \\
9.88 & 298 \\
9.88 & 287 \\
9.88 & 276\end{array}$ & $\begin{array}{l}11 \\
10 \\
11 \\
11\end{array}$ & $\begin{array}{l}50 \\
49 \\
48 \\
47 \\
46\end{array}$ & 19 & $\begin{array}{r}20 \\
16 \\
12 \\
8 \\
4\end{array}$ \\
\hline 41. & $\begin{array}{r}0 \\
4 \\
8 \\
12 \\
16\end{array}$ & $\begin{array}{l}15 \\
16 \\
17 \\
18 \\
19\end{array}$ & $\begin{array}{ll}9.81 & 032 \\
9.81 & 047 \\
9.81 & 061 \\
9.81 & 076 \\
9.81 & 091\end{array}$ & $\begin{array}{l}15 \\
14 \\
15 \\
15 \\
15\end{array}$ & $\begin{array}{r}9.92766 \\
-9.92792 \\
9.92817 \\
9.92843 \\
9.92868\end{array}$ & $\begin{array}{l}26 \\
25 \\
26 \\
25 \\
06\end{array}$ & $\begin{array}{ll}0.07 & 234 \\
0.07 & 208 \\
0.07 & 183 \\
0.07 & 157 \\
0.07 & 132\end{array}$ & $\begin{array}{ll}9.88 & 266 \\
9.88 & 25 \overline{5} \\
9.88 & 244 \\
9.88 & 234 \\
9.88 & 223\end{array}$ & $\begin{array}{l}11 \\
11 \\
10 \\
11\end{array}$ & $\begin{array}{l}45 \\
44 \\
43 \\
42 \\
41\end{array}$ & 19 & $\begin{array}{r}0 \\
56 \\
52 \\
48 \\
44\end{array}$ \\
\hline 41 & $\begin{array}{l}20 \\
24 \\
28 \\
32 \\
36\end{array}$ & $\begin{array}{l}\mathbf{2 0} \\
21 \\
22 \\
23 \\
24 \\
\end{array}$ & $\begin{array}{ll}9.81 & 106 \\
9.81 & 121 \\
9.81 & 136 \\
9.81 & 151 \\
9.81 & 166\end{array}$ & $\begin{array}{l}15 \\
15 \\
15 \\
15 \\
14\end{array}$ & 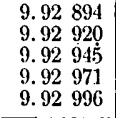 & $\begin{array}{l}26 \\
25 \\
26 \\
25 \\
26\end{array}$ & $\begin{array}{ll}0.07 & 106 \\
0.07 & 080 \\
0.07 & 055 \\
0.07 & 029 \\
0.07 & 004\end{array}$ & $\begin{array}{ll}9.88 & 212 \\
9.88 & 201 \\
9.88 & 191 \\
9.88 & 180 \\
9.88 & 169\end{array}$ & $\begin{array}{l}11 \\
10 \\
11 \\
11\end{array}$ & $\begin{array}{l}\mathbf{4 0} \\
39 \\
38 \\
37 \\
36\end{array}$ & 18 & $\begin{array}{l}40 \\
36 \\
32 \\
28 \\
24\end{array}$ \\
\hline 41 & $\begin{array}{l}40 \\
44 \\
48 \\
52 \\
56\end{array}$ & $\begin{array}{l}25 \\
26 \\
27 \\
28 \\
29\end{array}$ & $\begin{array}{ll}9.81 & 180 \\
9.81 & 195 \\
9.81 & 210 \\
9.81 & 225 \\
9.81 & 240\end{array}$ & $\begin{array}{l}15 \\
15 \\
15 \\
15\end{array}$ & 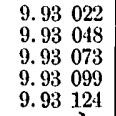 & $\begin{array}{l}26 \\
25 \\
26 \\
25\end{array}$ & 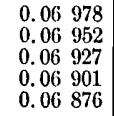 & $\begin{array}{ll}9.88 & 158 \\
9.88 & 148 \\
9.88 & 137 \\
9.88 & 126 \\
9.88 & 115\end{array}$ & $\begin{array}{l}10 \\
11 \\
11 \\
11\end{array}$ & $\begin{array}{l}35 \\
34 \\
33 \\
32 \\
31\end{array}$ & 18 & $\begin{array}{r}20 \\
16 \\
12 \\
8 \\
4\end{array}$ \\
\hline 42 & $\begin{array}{r}0 \\
4 \\
8 \\
12 \\
16\end{array}$ & $\begin{array}{l}30 \\
31 \\
32 \\
33 \\
34\end{array}$ & 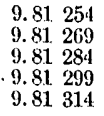 & $\begin{array}{l}15 \\
15 \\
15 \\
15\end{array}$ & $\begin{array}{ll}9.93 & 150 \\
9.93 & 175 \\
9.93 & 201 \\
9.93 & 227 \\
9.93 & 252\end{array}$ & $\begin{array}{l}25 \\
26 \\
26 \\
25\end{array}$ & 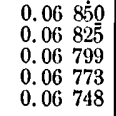 & $\begin{array}{lll}9.88 & 105 \\
\mathbf{9 . 8 8} & 094 \\
\mathbf{9 . 8 8} & 083 \\
\mathbf{9 . 8 8} & 072 \\
\mathbf{9 . 8 8} & 061\end{array}$ & $\begin{array}{l}11 \\
11 . \\
11 \\
11 .\end{array}$ & $\begin{array}{l}30 \\
29 \\
29 \\
27 \\
26\end{array}$ & 18 & $\begin{array}{r}0 \\
56 \\
52 \\
48 \\
44\end{array}$ \\
\hline 42 & $\begin{array}{l}20 \\
24 \\
28 \\
32 \\
36\end{array}$ & $\begin{array}{l}35 \\
36 \\
37 \\
35 \\
39\end{array}$ & $\begin{array}{ll}9.81 & 328 \\
9.81 & 343 \\
9.81 & 358 \\
9.81 & 372 \\
9.81 & 387\end{array}$ & $\begin{array}{l}15 \\
15 \\
14 \\
15\end{array}$ & 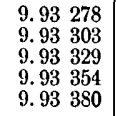 & $\begin{array}{l}25 \\
26 \\
25 \\
26\end{array}$ & 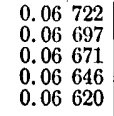 & $\begin{array}{ll}9.88 & 051 \\
9.88 & 040 \\
9.88 & 029 \\
9.88 & 018 \\
9.88 & 007\end{array}$ & $\begin{array}{l}11 \\
11 \\
11 . \\
11 .\end{array}$ & $\begin{array}{l}25 \\
24 \\
23 \\
22 \\
21\end{array}$ & 17 & $\begin{array}{l}40 \\
36 \\
32 \\
28 \\
24\end{array}$ \\
\hline 42 & $\begin{array}{l}40 \\
44 \\
48 \\
52 \\
56\end{array}$ & $\begin{array}{l}40 \\
41 \\
42 \\
43 \\
44\end{array}$ & $\begin{array}{ll}9.81 & 402 \\
9.81 . & 417 \\
9.81 & 431 \\
9.81 . & 446 \\
9.81 & 461\end{array}$ & $\begin{array}{l}15 \\
14 \\
15 \\
15\end{array}$ & $\begin{array}{ll}9.93 & 406 \\
9.93 & 431 \\
9.93 & 457 \\
9.93 & 482 \\
9.93 & 508\end{array}$ & $\begin{array}{l}25 \\
26 \\
25 \\
26\end{array}$ & 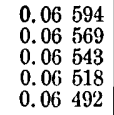 & $\begin{array}{l}9.87996 \\
9.87985 \\
9.87975 \\
9.87964 \\
9.87953\end{array}$ & $\begin{array}{l}11 \\
10 \\
1.1 \\
11\end{array}$ & $\begin{array}{l}\mathbf{2 0} \\
19 \\
18 \\
17 \\
1.6\end{array}$ & 17 & $\begin{array}{r}20 \\
16 \\
12 \\
8 \\
4\end{array}$ \\
\hline 43 & $\begin{array}{r}0 \\
4 \\
8 \\
12 \\
16\end{array}$ & $\begin{array}{l}45 \\
46 \\
47 \\
48 \\
49\end{array}$ & 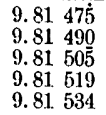 & $\begin{array}{l}15 \\
15 \\
14 \\
15\end{array}$ & 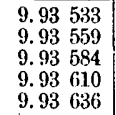 & $\begin{array}{l}26 \\
25 \\
26 \\
26\end{array}$ & 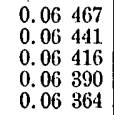 & $\begin{array}{ll}9.87 & 942 \\
9.87 & 931 \\
9.87 & 920 \\
9.87 & 909 \\
9.87 & 898\end{array}$ & $\begin{array}{l}11 \\
11 \\
11 \\
11\end{array}$ & $\begin{array}{l}15 \\
14 \\
13 \\
12 \\
11 .\end{array}$ & 17 & $\begin{array}{r}0 \\
56 \\
52 \\
48 \\
44\end{array}$ \\
\hline 43 & $\begin{array}{l}20 \\
24 \\
28 \\
32 \\
36\end{array}$ & $\begin{array}{l}50 \\
51 \\
52 \\
53 \\
54\end{array}$ & $\begin{array}{ll}9.81 & 549 \\
9.81 & 563 \\
9.81 & 578 \\
9.81 & 592 \\
9.81 & 607\end{array}$ & $\begin{array}{l}14 \\
15 \\
14 \\
16\end{array}$ & 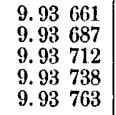 & $\begin{array}{l}26 \\
25 \\
26 \\
25\end{array}$ & 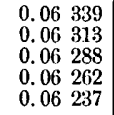 & 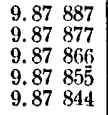 & $\begin{array}{l}10 \\
11 \\
11 . \\
11\end{array}$ & $\begin{array}{r}10 \\
9 \\
8 \\
7 \\
6\end{array}$ & 16 & $\begin{array}{l}40 \\
36 \\
32 \\
28 \\
24\end{array}$ \\
\hline 43 & $\begin{array}{l}40 \\
44 \\
48 \\
52 \\
56\end{array}$ & $\begin{array}{l}55 \\
56 \\
57 \\
58 \\
59\end{array}$ & $\begin{array}{ll}9.81 & 622 \\
9.81 & 636 \\
9.81 & 651 \\
9.81 & 665 \\
9.81 & 680\end{array}$ & $\begin{array}{l}14 \\
15 \\
14 \\
15\end{array}$ & 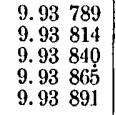 & $\begin{array}{l}25 \\
26 \\
25 \\
26 \\
\end{array}$ & $\begin{array}{ll}0.06 & 211 \\
0.06 & 186 \\
0.06 & 160 \\
0.06 & 135 \\
0.06 & 109\end{array}$ & 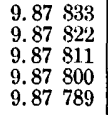 & $\begin{array}{l}11 \\
11 \\
11 \\
11\end{array}$ & $\begin{array}{l}5 \\
4 \\
3 \\
2 \\
1 .\end{array}$ & 16 & $\begin{array}{r}20 \\
16 \\
12 \\
8 \\
4\end{array}$ \\
\hline 44 & 0 & 60 & 9.81694 & & 9.93916 & & 0.06084 & 9.87778 & & 0 & 16 & 0 \\
\hline & & & I. Cos. & d. & L. Cotg. & c. d. & L. Tang. & L. Sin. & d. & ' & $\mathrm{m}$ & $\mathbf{s}$ \\
\hline
\end{tabular}


TABLE 19.-Five-place logarithms of circular functions, etc.-Continued.

$2^{\text {h }}$

\begin{tabular}{|c|c|c|c|c|c|c|c|c|c|c|c|c|}
\hline III. & s. & 1 & L. Sin. & d. & L. Tang. & c. d. & L. Cotg. & L. Cos. & d. & & & \\
\hline 44 & $\begin{array}{r}0 \\
4 \\
5 \\
12 \\
16 \\
\end{array}$ & $\begin{array}{l}0 \\
1 \\
2 \\
3 \\
4 \\
\end{array}$ & $\begin{array}{ll}9.81 & 694 \\
9.81 & 709 \\
9.81 & 723 \\
9.81 & 738 \\
9.81 & 752\end{array}$ & $\begin{array}{l}15 \\
14 \\
15 \\
14\end{array}$ & 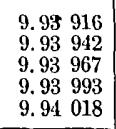 & $\begin{array}{l}26 \\
25 \\
26 \\
25 \\
26\end{array}$ & $\begin{array}{ll}0.06 & 084 \\
0.06 & 058 \\
0.06 & 033 \\
0.06 & 007 \\
0.05 & 982\end{array}$ & 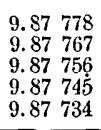 & $\begin{array}{l}11 \\
11 \\
11 . \\
11\end{array}$ & $\begin{array}{r}\mathbf{6 0} \\
59 \\
58 \\
57 \\
56\end{array}$ & 16 & $\begin{array}{r}0 \\
56 \\
52 \\
48 \\
44\end{array}$ \\
\hline 44 & $\begin{array}{l}20 \\
24 \\
28 \\
32 \\
36\end{array}$ & $\begin{array}{l}5 \\
6 \\
7 \\
5 \\
9\end{array}$ & $\begin{array}{ll}9.81 & 767 \\
9.81 & 781 \\
9.81 & 796 \\
9.81 & 810 \\
9.81 & 825\end{array}$ & $\begin{array}{l}10 \\
14 \\
15 \\
14 \\
15\end{array}$ & 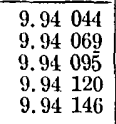 & $\begin{array}{l}25 \\
26 \\
25 \\
26\end{array}$ & $\begin{array}{ll}0.05 & 956 \\
0.05 & 931 \\
0.05 & 905 \\
0.05 & 880 \\
0.05 & 854\end{array}$ & $\begin{array}{ll}9.87 & 723 \\
9.87 & 712 \\
9.87 & 701 \\
9.87 & 690 \\
9.87 & 679\end{array}$ & $\begin{array}{l}11 \\
11 \\
11 \\
11 .\end{array}$ & $\begin{array}{l}55 \\
54 \\
53 \\
52 \\
51\end{array}$ & 15 & $\begin{array}{l}40 \\
36 \\
32 \\
28 \\
24\end{array}$ \\
\hline 44 & $\begin{array}{l}40 \\
44 \\
48 \\
52 \\
56 \\
\end{array}$ & $\begin{array}{l}10 \\
11 \\
12 \\
13 \\
14 \\
\end{array}$ & $\begin{array}{ll}9.81 & 839 \\
9.81 & 854 \\
9.81 & 868 \\
9.81 & 882 \\
9.81 & 897\end{array}$ & $\begin{array}{l}15 \\
14 \\
14 \\
15 \\
14\end{array}$ & $\begin{array}{ll}9.94 & 171 \\
9.94 & 197 \\
9.94 & 222 \\
9.94 & 248 \\
9.94 & 273 \\
\end{array}$ & $\begin{array}{l}26 \\
25 \\
26 \\
25 \\
26\end{array}$ & $\begin{array}{ll}0.05 & 829 \\
0.05 & 803 \\
0.05 & 778 \\
0.05 & 752 \\
0.05 & 727\end{array}$ & 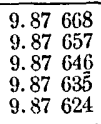 & $\begin{array}{l}11 \\
11 \\
11 . \\
11 \\
11\end{array}$ & $\begin{array}{r}50 \\
49 \\
48 \\
47 \\
46\end{array}$ & 15 & $\begin{array}{r}20 \\
16 \\
12 \\
8 \\
4\end{array}$ \\
\hline 45 & $\begin{array}{r}0 \\
4 \\
5 \\
12 \\
16\end{array}$ & $\begin{array}{l}15 \\
16 \\
17 \\
18 \\
19\end{array}$ & $\begin{array}{ll}9.81 & 911 \\
9.81 & 926 \\
9.81 & 940 \\
9.81 & 955 \\
9.81 & 969\end{array}$ & $\begin{array}{l}15 \\
14 \\
15 \\
14\end{array}$ & $\begin{array}{ll}9.94 & 299 \\
9.94 & 324 \\
9.94 & 350 \\
9.94 & 375 \\
9.94 & 401\end{array}$ & $\begin{array}{l}25 \\
26 \\
25 \\
26\end{array}$ & 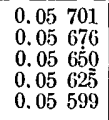 & $\begin{array}{ll}9.87 & 613 \\
9.87 & 601 \\
9.87 & 590 \\
9.87 & 579 \\
9.87 & 568\end{array}$ & $\begin{array}{l}12 \\
11 \\
11 \\
11\end{array}$ & $\begin{array}{l}45 \\
44 \\
43 \\
42 \\
41\end{array}$ & 15 & $\begin{array}{r}0 \\
56 \\
52 \\
48 \\
44\end{array}$ \\
\hline 45 & $\begin{array}{l}20 \\
24 \\
28 \\
32 \\
36\end{array}$ & $\begin{array}{l}\mathbf{2 0} \\
21 . \\
22 \\
23 \\
24\end{array}$ & $\begin{array}{ll}9.81 & 983 \\
9.81 & 998 \\
9.82 & 012 \\
9.82 & 026 \\
9.82 & 041\end{array}$ & $\begin{array}{l}15 \\
14 \\
14 \\
15\end{array}$ & $\begin{array}{ll}9.94 & 426 \\
9.94 & 452 \\
9.94 & 477 \\
9.94 & 503 \\
9.94 & 528\end{array}$ & $\begin{array}{l}26 \\
25 \\
26 \\
25 \\
25\end{array}$ & 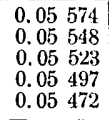 & $\begin{array}{ll}9.87 & 557 \\
9.87 & 546 \\
9.87 & 535 \\
9.87 & 524 \\
9.87 & 513\end{array}$ & $\begin{array}{l}11 \\
11 \\
11 \\
11\end{array}$ & $\begin{array}{r}\mathbf{4 0} \\
39 \\
38 \\
37 \\
36\end{array}$ & 14 & $\begin{array}{l}40 \\
36 \\
32 \\
28 \\
24\end{array}$ \\
\hline 45 & $\begin{array}{l}40 \\
44 \\
48 \\
52 \\
56\end{array}$ & $\begin{array}{l}25 \\
26 \\
27 \\
28 \\
29\end{array}$ & $\begin{array}{lll}9.82 & 05 \overline{5} \\
9.82 & 069 \\
9.82 & 084 \\
9.82 & 098 \\
9.82 & 112\end{array}$ & $\begin{array}{l}14 \\
15 \\
14 \\
14\end{array}$ & $\begin{array}{ll}9.94 & 554 \\
9.94 & 579 \\
9.94 & 604 \\
9.94 & 630 \\
9.94 & 655 \\
\end{array}$ & $\begin{array}{l}25 \\
25 \\
26 \\
25 \\
26\end{array}$ & $\begin{array}{ll}0.05 & 446 \\
0.05 & 421 \\
0.05 & 396 \\
0.05 & 370 \\
0.05 & 345\end{array}$ & $\begin{array}{ll}9.87 & 501 \\
9.87 & 490 \\
9.87 & 479 \\
9.87 & 468 \\
9.87 & 457\end{array}$ & $\begin{array}{l}11 \\
11 \\
11 \\
11 .\end{array}$ & $\begin{array}{l}35 \\
34 \\
33 \\
32 \\
31\end{array}$ & 14 & $\begin{array}{r}20 \\
16 \\
12 \\
8 \\
4\end{array}$ \\
\hline 46 & $\begin{array}{r}0 \\
4 \\
8 \\
12 \\
16\end{array}$ & $\begin{array}{l}30 \\
31 \\
32 \\
33 \\
34\end{array}$ & $\begin{array}{ll}9.82 & 126 \\
9.82 & 141 \\
9.82 & 15 \overline{5} \\
9.82 & 169 \\
9.82 & 184\end{array}$ & $\begin{array}{l}15 \\
14 \\
14 \\
15\end{array}$ & 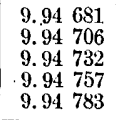 & $\begin{array}{l}25 \\
26 \\
25 \\
26\end{array}$ & $\begin{array}{ll}0.05 & 319 \\
0.05 & 294 \\
0.05 & 268 \\
0.05 & 243 \\
0.05 & 217\end{array}$ & 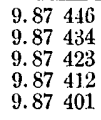 & $\begin{array}{l}12 \\
11 \\
11 \\
\mathrm{J1}\end{array}$ & $\begin{array}{l}30 \\
29 \\
28 \\
27 \\
26\end{array}$ & 14 & $\begin{array}{r}0 \\
56 \\
52 \\
48 \\
44\end{array}$ \\
\hline 46 & $\begin{array}{l}20 \\
24 \\
28 \\
32 \\
36\end{array}$ & $\begin{array}{l}35 \\
36 \\
37 \\
38 \\
39\end{array}$ & $\begin{array}{ll}9.82 & 198 \\
9.82 & 212 \\
9.82 & 226 \\
9.82 & 240 \\
9.82 & 255\end{array}$ & $\begin{array}{l}14 \\
14 \\
14 \\
15\end{array}$ & 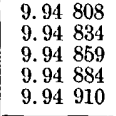 & $\begin{array}{l}26 \\
25 \\
25 \\
26\end{array}$ & $\begin{array}{ll}0.05 & 192 \\
0.05 & 166 \\
0.05 & 141 . \\
0.05 & 116 \\
0.05 & 090\end{array}$ & 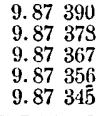 & $\begin{array}{l}12 \\
11 \\
11 \\
11\end{array}$ & $\begin{array}{l}25 \\
24 \\
23 \\
22 \\
21\end{array}$ & 13 & $\begin{array}{l}40 \\
36 \\
32 \\
28 \\
24\end{array}$ \\
\hline 46 & $\begin{array}{l}40 \\
44 \\
48 \\
52 \\
56 \\
\end{array}$ & $\begin{array}{l}40 \\
41 \\
42 \\
43 \\
44 \\
\end{array}$ & $\begin{array}{ll}9.82 & 269 \\
9.82 & 283 \\
9.82 & 297 \\
9.82 & 311 \\
9.82 & 326 \\
\end{array}$ & $\begin{array}{l}14 \\
14 \\
14 \\
15\end{array}$ & $\begin{array}{ll}9.94 & 935 \\
9.94 & 961 \\
9.94 & 986 \\
9.95 & 012 \\
9.95 & 037\end{array}$ & $\begin{array}{l}26 \\
25 \\
26 \\
25 \\
25\end{array}$ & 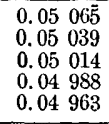 & $\begin{array}{ll}9.87 & 334 \\
9.87 & 322 \\
9.87 & 311 \\
9.87 & 300 \\
9.87 & 288\end{array}$ & $\begin{array}{l}12 \\
11 . \\
11 \\
12 \\
11\end{array}$ & $\begin{array}{l}\mathbf{2 0} \\
19 \\
18 \\
17 \\
16\end{array}$ & 13 & $\begin{array}{r}20 \\
16 \\
12 \\
8 \\
4\end{array}$ \\
\hline 47 & \begin{tabular}{r|}
0 \\
4 \\
8 \\
12 \\
16
\end{tabular} & $\begin{array}{l}45 \\
46 \\
47 \\
48 \\
49\end{array}$ & 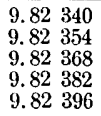 & $\begin{array}{l}14 \\
14 \\
14 \\
14\end{array}$ & $\begin{array}{ll}9.95 & 062 \\
9.95 & 088 \\
9.95 & 113 \\
9.95 & 139 \\
9.95 & 164\end{array}$ & $\begin{array}{l}26 \\
25 \\
26 \\
25\end{array}$ & $\begin{array}{l}0.04938 \\
0.04912 \\
0.04887 \\
0.04861 \\
0.04836\end{array}$ & $\begin{array}{ll}9.87 & 277 \\
9.87 & 266 \\
9.87 & 255 \\
9.87 & 243 \\
9.87 & 232\end{array}$ & $\begin{array}{l}11 \\
11 \\
12 \\
11\end{array}$ & $\begin{array}{l}15 \\
14 \\
13 \\
12 \\
11\end{array}$ & 13 & $\begin{array}{r}0 \\
56 \\
52 \\
48 \\
44\end{array}$ \\
\hline 47 & $\begin{array}{l}20 \\
24 \\
28 \\
32 \\
36\end{array}$ & $\begin{array}{l}50 \\
51 \\
52 \\
53 \\
54\end{array}$ & $\begin{array}{ll}9.82 & 410 \\
9.82 & 424 \\
9.82 & 439 \\
9.82 & 453 \\
9.82 & 467\end{array}$ & $\begin{array}{l}14 \\
15 \\
14 \\
14 \\
14\end{array}$ & $\begin{array}{ll}9.95 & 190 \\
9.95 & 215 \\
9.95 & 240 \\
9.95 & 266 \\
9.95 & 291\end{array}$ & $\begin{array}{l}25 \\
25 \\
26 \\
25\end{array}$ & $\begin{array}{l}0.04810 \\
0.04785 \\
0.04760 \\
0.04734 \\
0.04709\end{array}$ & $\begin{array}{ll}9.87 & 221 \\
9.87 & 209 \\
9.87 & 198 \\
9.87 & 187 \\
9.87 & 175\end{array}$ & $\begin{array}{l}12 \\
11 \\
11 \\
12\end{array}$ & $\begin{array}{r}10 \\
9 \\
8 \\
7 \\
6\end{array}$ & 12 & $\begin{array}{l}40 \\
36 \\
32 \\
28 \\
24\end{array}$ \\
\hline 47 & $\begin{array}{l}40 \\
44 \\
48 \\
52 \\
56\end{array}$ & $\begin{array}{l}55 \\
56 \\
57 \\
58 \\
59\end{array}$ & $\begin{array}{ll}9.82 & 481 \\
9.82 & 495 \\
9.82 & 509 \\
9.82 & 523 \\
9.82 & 537\end{array}$ & $\begin{array}{l}14 \\
14 \\
14 \\
14\end{array}$ & $\begin{array}{ll}9.95 & 317 \\
9.95 & 342 \\
9.95 & 368 . \\
9.95 & 393 \\
9.95 & 418\end{array}$ & $\begin{array}{l}25 \\
26 \\
25 \\
25\end{array}$ & $\begin{array}{l}0.04683 \\
0.04658 \\
0.04632 \\
0.04607 \\
0.04582\end{array}$ & $\begin{array}{ll}9.87 & 164 \\
9.87 & 153 \\
9.87 & 141 \\
9.87 & 130 \\
9.87 & 119\end{array}$ & $\begin{array}{l}11 \\
12 \\
11 \\
11\end{array}$ & $\begin{array}{l}5 \\
4 \\
3 \\
2 \\
1\end{array}$ & 12 & $\begin{array}{r}20 \\
16 \\
12 \\
8 \\
4\end{array}$ \\
\hline 48 & 0 & 60 & 9.82551 & & 9.95444 & & 0.04556 & $9.87 \quad 107$ & & 0 & 12 & 0 \\
\hline & & & L. Cos. . & d. & L. Cotg. & c. d. & L. Tang. & L. Sin. & d. & 1 & $\mathrm{~m}$. & s. \\
\hline
\end{tabular}

$48^{\circ}$ 
Taвье 19.-Five-place logarithms of circular functions, etc.-Continued.

$2^{11}$

\begin{tabular}{|c|c|c|c|c|c|c|c|c|c|c|c|c|}
\hline $\mathrm{m}$. & s. & ' & I. Sin. & d. & I. 'Tung. & c. d. & I. Cotg. & J. Cos. & d. & & & \\
\hline $4 S$ & $\begin{array}{r}0 \\
4 \\
8 \\
12 \\
16\end{array}$ & $\begin{array}{l}0 \\
1 \\
2 \\
3 \\
4\end{array}$ & $\begin{array}{ll}9.82 & 551 \\
9.82 & 565 \\
9.82 & 579 \\
9.82 & 593 \\
9.82 & 607\end{array}$ & \multirow{2}{*}{$\begin{array}{l}14 \\
14 \\
14 \\
14 \\
14 \\
14 \\
14 \\
14 \\
14 \\
14\end{array}$} & 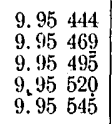 & \multirow{2}{*}{$\begin{array}{l}25 \\
26 \\
25 \\
25 \\
26 \\
25 \\
26 \\
25 \\
25 \\
26\end{array}$} & $\begin{array}{ll}0.04 & 556 \\
0.04 & 531 \\
0.04 & 505 \\
0.04 & 480 \\
0.04 & 45 \overline{5}\end{array}$ & $\begin{array}{ll}9.87 & 107 \\
9.87 & 096 \\
9.87 & 085 \\
9.87 & 073 \\
9.87 & 062\end{array}$ & \multirow{2}{*}{$\begin{array}{l}11 \\
11 \\
12 \\
-1 \\
12 \\
11 \\
11 \\
12 \\
11 \\
12\end{array}$} & $\begin{array}{l}\mathbf{6 0} \\
59 \\
58 \\
57 \\
56\end{array}$ & 12 & $\begin{array}{r}0 \\
56 \\
52 \\
48 \\
44\end{array}$ \\
\hline 48 & $\begin{array}{l}20 \\
24 \\
28 \\
32 \\
36\end{array}$ & $\begin{array}{l}5 \\
6 \\
7 \\
8 \\
9 \\
\end{array}$ & $\begin{array}{lll}9.82 & 621 \\
9.82 & 635 \\
9.82 & 649 \\
9.82 & 663 \\
9.82 & 677\end{array}$ & & $\begin{array}{ll}9.95 & 571 \\
9.95 & 596 \\
9.95 & 622 \\
9.95 & 647 \\
9.95 & 672\end{array}$ & & $\begin{array}{ll}0.04 & 429 \\
0.04 & 404 \\
0.04 & 378 \\
0.04 & 353 \\
0.04 & 328\end{array}$ & $\begin{array}{ll}9.87 & 050 \\
9.87 & 039 \\
9.87 & 028 \\
9.87 & 016 \\
9.87 & 005 \\
\end{array}$ & & $\begin{array}{l}55 \\
54 \\
53 \\
52 \\
51\end{array}$ & 11 & $\begin{array}{l}40 \\
36 \\
32 \\
28 \\
24\end{array}$ \\
\hline 48 & $\begin{array}{l}40 \\
44 \\
48 \\
52 \\
56\end{array}$ & $\begin{array}{l}10 \\
11 \\
12 \\
13 \\
14\end{array}$ & $\begin{array}{ll}9.82 & 691 \\
9.82 & 705 \\
9.82 & 719 \\
9.82 & 733 \\
9.82 & 747\end{array}$ & $\begin{array}{l}14 \\
14 \\
14 \\
14 \\
14\end{array}$ & $\begin{array}{ll}9.95 & 698 \\
9.95 & 723 \\
9.95 & 748 \\
9.95 & 774 \\
9.95 & 799\end{array}$ & $\begin{array}{l}25 \\
25 \\
26 \\
25 \\
26\end{array}$ & $\begin{array}{ll}0.04 & 302 \\
0.04 & 277 \\
0.04 & 252 \\
0.04 & 226 \\
0.04 & 201\end{array}$ & 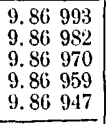 & $\begin{array}{l}11 \\
12 \\
11 \\
12 \\
11\end{array}$ & $\begin{array}{l}50 \\
49 \\
48 \\
47 \\
46\end{array}$ & 11 & $\begin{array}{r}20 \\
16 \\
12 \\
8 \\
4\end{array}$ \\
\hline 49 & $\begin{array}{r}0 \\
4 \\
8 \\
12 \\
16 \\
\end{array}$ & $\begin{array}{l}15 \\
16 \\
17 \\
18 \\
19\end{array}$ & 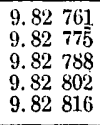 & $\begin{array}{l}14 \\
13 \\
14 . \\
14 \\
14\end{array}$ & 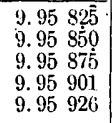 & $\begin{array}{l}25 \\
25 \\
26 \\
25 \\
26\end{array}$ & $\begin{array}{ll}0.04 & 175 \\
0.04 & 150 \\
0.04 & 125 \\
0.04 & 099 \\
0.04 & 074\end{array}$ & 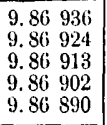 & $\begin{array}{l}12 \\
11 \\
11 \\
12 \\
11\end{array}$ & $\begin{array}{l}45 \\
44 \\
43 \\
42 \\
41\end{array}$ & 11 & $\begin{array}{r}0 \\
56 \\
52 \\
48 \\
44\end{array}$ \\
\hline 49 & $\begin{array}{l}20 \\
24 \\
28 \\
32 \\
36\end{array}$ & $\begin{array}{l}\mathbf{2 0} \\
21 \\
22 \\
23 \\
24\end{array}$ & 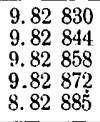 & $\begin{array}{l}14 \\
14 \\
14 \\
13 \\
14\end{array}$ & $\begin{array}{ll}9.95 & 952 \\
9.95 & 977 \\
9.96 & 002 \\
9.96 & 028 \\
9.96 & 053\end{array}$ & $\begin{array}{l}25 \\
25 \\
26 \\
25 \\
25\end{array}$ & $\begin{array}{ll}0.04 & 048 \\
0.04 & 023 \\
0.03 & 998 \\
0.03 & 972 \\
0.03 & 947\end{array}$ & 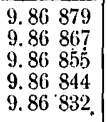 & $\begin{array}{l}12 \\
12 \\
11 \\
12 \\
11\end{array}$ & $\begin{array}{l}40 \\
39 \\
38 \\
37 \\
36\end{array}$ & 10 & $\begin{array}{l}40 \\
36 \\
32 \\
28 \\
24\end{array}$ \\
\hline 49 & $\begin{array}{l}40 \\
44 \\
48 \\
52 \\
56\end{array}$ & $\begin{array}{l}25 \\
26 \\
27 \\
28 \\
29\end{array}$ & 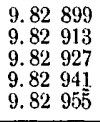 & $\begin{array}{l}14 \\
14 \\
14 \\
14 \\
13\end{array}$ & $\begin{array}{ll}9.96 & 078 \\
9.96 & 104 \\
9.96 & 129 \\
9.96 & 155 \\
.9 .96 & 180\end{array}$ & $\begin{array}{l}26 \\
25 \\
26 \\
25 \\
25\end{array}$ & $\begin{array}{ll}0.03 & 922 \\
0.03 & 896 \\
0.03 & 871 \\
0.03 & 845 \\
0.03 & 820\end{array}$ & 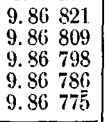 & $\begin{array}{l}12 \\
11 \\
12 \\
11 \\
12\end{array}$ & $\begin{array}{l}35 \\
34 \\
33 \\
32 \\
31\end{array}$ & 10 & $\begin{array}{r}20 \\
16 \\
12 \\
8 \\
4\end{array}$ \\
\hline 50 & $\begin{array}{r}0 \\
4 \\
8 \\
12 \\
16\end{array}$ & $\begin{array}{l}30 \\
31 \\
32 \\
33 \\
34\end{array}$ & 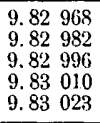 & $\begin{array}{l}14 \\
14 \\
14 \\
13 \\
44\end{array}$ & $\begin{array}{ll}9.96 & 205 \\
9.96 & 231 \\
9.96 & 256 \\
9.96 & 281 \\
9.96 & 307\end{array}$ & $\begin{array}{l}26 \\
25 \\
25 \\
26 \\
25\end{array}$ & $\begin{array}{ll}0.03 & 795 \\
0.03 & 769 \\
0.03 & 744 \\
0.03 & 719 \\
0.03 & 693\end{array}$ & $\begin{array}{ll}9.86 & 763 \\
9.86 & 752 \\
9.86 & 740 \\
9.86 & 728 \\
9.86 & 717 \\
\end{array}$ & $\begin{array}{l}11 \\
12 \\
12 \\
11 \\
12\end{array}$ & $\begin{array}{l}\mathbf{3 0} \\
29 \\
28 \\
27 \\
26\end{array}$ & 10 & $\begin{array}{r}0 \\
56 \\
52 \\
48 \\
44 \\
\end{array}$ \\
\hline 50 & $\begin{array}{l}20 \\
24 \\
28 \\
32 \\
36\end{array}$ & $\begin{array}{l}35 \\
36 \\
37 \\
38 \\
39\end{array}$ & $\begin{array}{ll}9.83 & 037 \\
9.83 & 051 \\
9.83 & 065 \\
9.83 & 078 \\
9.83 & 092\end{array}$ & $\begin{array}{l}14 \\
14 \\
13 \\
14 \\
14\end{array}$ & 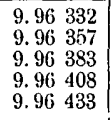 & $\begin{array}{l}25 \\
26 \\
25 \\
25 \\
26\end{array}$ & $\begin{array}{ll}0.03 & 668 \\
0.03 & 643 \\
0.03 & 617 \\
0.03 & 592 \\
0.03 & 567\end{array}$ & 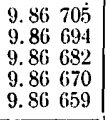 & $\begin{array}{l}11 \\
12 \\
12 \\
11 \\
12\end{array}$ & $\begin{array}{l}25 \\
24 \\
23 \\
22 \\
21\end{array}$ & 9 & $\begin{array}{l}40 \\
36 \\
32 \\
28 \\
24\end{array}$ \\
\hline 50 & $\begin{array}{l}40 \\
44 \\
48 \\
52 \\
56\end{array}$ & $\begin{array}{l}40 \\
41 \\
42 \\
43 \\
44\end{array}$ & $\begin{array}{ll}9.83 & 106 \\
9.83 & 120 \\
9.83 & 133 \\
9.83 & 147 \\
9.83 & 161\end{array}$ & $\begin{array}{l}14 \\
13 \\
14 \\
14 \\
13\end{array}$ & 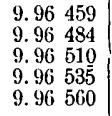 & $\begin{array}{l}25 \\
26 \\
25 \\
25 \\
26\end{array}$ & $\begin{array}{ll}0.03 & 541 \\
0.03 & 516 \\
0.03 & 490 \\
0.03 & 465 \\
0.03 & 440\end{array}$ & 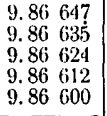 & $\begin{array}{l}12 \\
11 \\
12 \\
12 \\
11\end{array}$ & $\begin{array}{l}20 \\
19 \\
18 \\
17 \\
16\end{array}$ & 9 & $\begin{array}{r}20 \\
16 \\
12 \\
8 \\
4\end{array}$ \\
\hline 51 & $\begin{array}{r}0 \\
4 \\
8 \\
12 \\
16 \\
\end{array}$ & $\begin{array}{l}45 \\
46 \\
47 \\
48 \\
49 \\
\end{array}$ & $\begin{array}{ll}9.83 & 174 \\
9.83 & 188 \\
9.83 & 202 \\
9.83 & 215 \\
9.83 & 229 \\
\end{array}$ & $\begin{array}{l}14 \\
14 \\
13 \\
.14\end{array}$ & 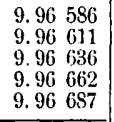 & $\begin{array}{l}25 \\
25 \\
26 \\
25 \\
25\end{array}$ & $\begin{array}{ll}0.03 & 414 \\
0.03 & 389 \\
0.03 & 364 \\
0.03 & 338 \\
0.03 & 313\end{array}$ & 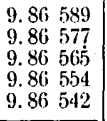 & $\begin{array}{l}12 \\
12 \\
11 \\
12 \\
12\end{array}$ & $\begin{array}{l}15 \\
14 \\
13 \\
12 \\
11\end{array}$ & 9 & $\begin{array}{r}0 \\
50 \\
52 \\
48 \\
44\end{array}$ \\
\hline 51 & $\begin{array}{l}20 \\
24 \\
28 \\
32 \\
36\end{array}$ & $\begin{array}{l}50 \\
51 \\
52 \\
53 \\
54\end{array}$ & $\begin{array}{ll}9.83 & 242 \\
9.83 & 256 \\
9.83 & 270 \\
9.83 & 283 \\
9.83 & 297\end{array}$ & $\begin{array}{l}14 \\
14 \\
13 \\
14 \\
13\end{array}$ & 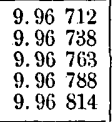 & $\begin{array}{l}26 \\
25 \\
25 \\
26 \\
25\end{array}$ & $\begin{array}{ll}0.03 & 288 \\
0.03 & 262 \\
0.03 & 237 \\
0.03 & 212 \\
0.03 & 186\end{array}$ & $\begin{array}{lll}9.86 & 530 \\
9.86 & 518 \\
9.86 & 507 \\
9.86 & 495 \\
9.86 & 483\end{array}$ & $\begin{array}{l}12 \\
11 \\
12 \\
12 \\
11\end{array}$ & $\begin{array}{r}\mathbf{1 0} \\
.9 \\
8 \\
7 \\
6\end{array}$ & 8 & $\begin{array}{l}40 \\
36 \\
32 \\
28 \\
24\end{array}$ \\
\hline 51. & $\begin{array}{l}40 \\
44 \\
48 \\
52 \\
56\end{array}$ & $\begin{array}{l}55 \\
56 \\
57 \\
58 \\
59\end{array}$ & 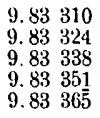 & \multirow[t]{2}{*}{$\begin{array}{l}14 \\
14 \\
13 \\
14 \\
13\end{array}$} & 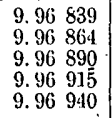 & $\begin{array}{l}25 \\
26 \\
25 \\
25 \\
26\end{array}$ & $\begin{array}{ll}0.03 & 161 \\
0.03 & 136 \\
0.03 & 110 \\
0.03 & 085 \\
0.03 & 060\end{array}$ & $\begin{array}{ll}9.86 & 472 \\
9.86 & 460 \\
9.86 & 448 \\
9.86 & 436 \\
9.86 & 425\end{array}$ & \multirow[t]{2}{*}{$\begin{array}{l}12 \\
12 \\
12 \\
11 \\
12\end{array}$} & $\begin{array}{r}5 \\
4 \\
-3 \\
2 \\
1\end{array}$ & 8 & $\begin{array}{r}20 \\
16 \\
12 \\
8 \\
4\end{array}$ \\
\hline 52 & 0 & 60 & 9.83375 & & 9.96966 & & 0.03034 & 9.86413 & & 0 & 8 & 0 \\
\hline & & & Cos. & d & L. Cotg. & c. $d$. & 1. Tang. & L. Sin. & d. & ' & $\mathrm{m}$ & . \\
\hline
\end{tabular}

Bull. $214-03--11$ 
TABLE 19.-Five-place logarithms of circular functions, etc.-Continued.

$2^{\mathrm{h}}$

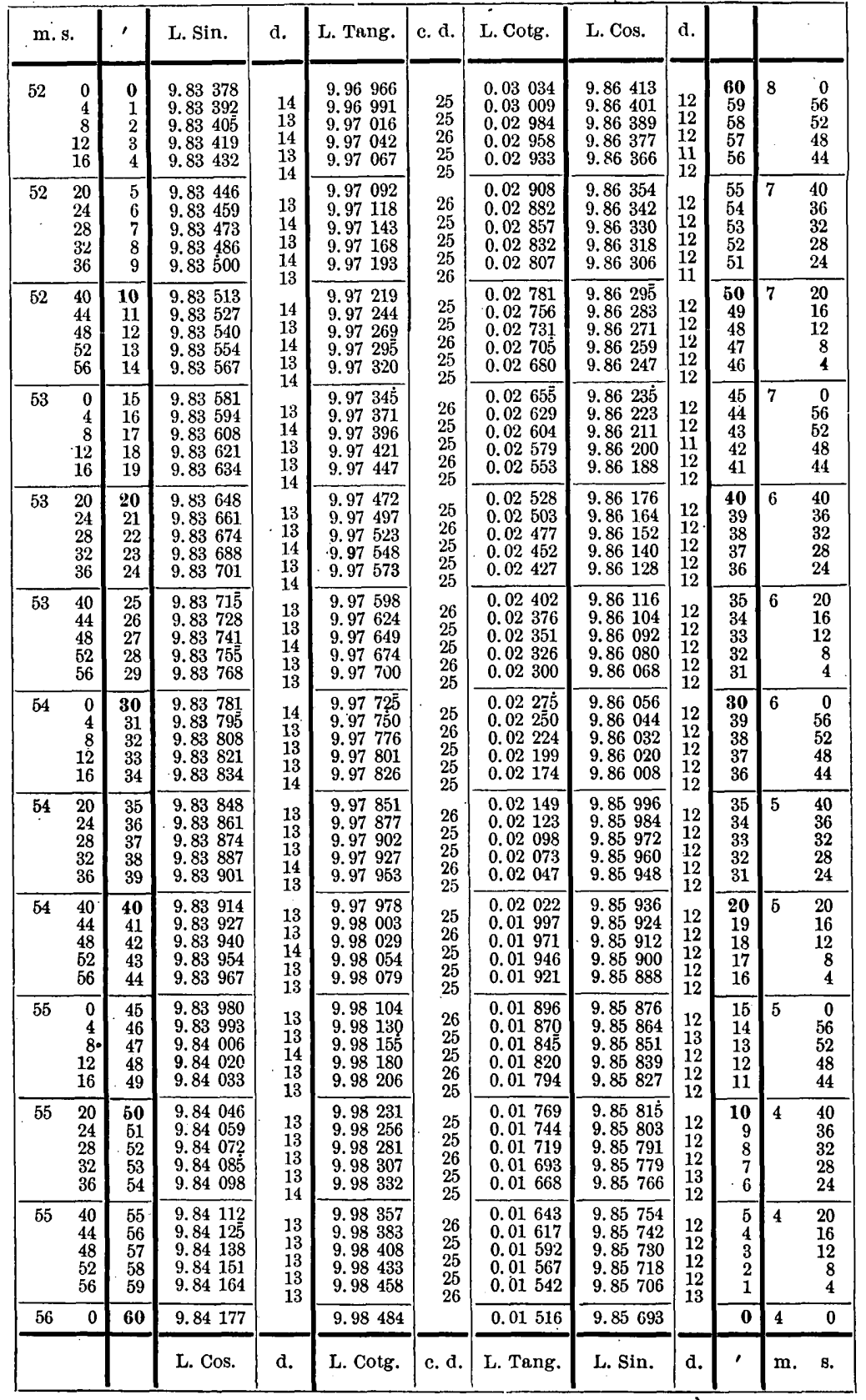

$43^{\circ}$

$46^{\circ}$ 
TaвLE 19. - Five-place logarithms of circular functions, etc.-Continued.

$2^{\mathrm{h}}$

\begin{tabular}{|c|c|c|c|c|c|c|c|c|c|c|c|c|}
\hline $\mathrm{m}$. & s. & 1 & I. Sin. & d. & I. Tang. & c. $\mathrm{d}$. & I. Cotg. & L. Cos. & d. & & & \\
\hline 56 & \begin{tabular}{r|}
0 \\
4 \\
8 \\
12 \\
16 \\
\end{tabular} & $\begin{array}{l}0 \\
1 \\
2 \\
3 \\
4 \\
\end{array}$ & 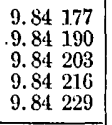 & \multirow{3}{*}{$\begin{array}{l}13 \\
13 \\
13 \\
13 \\
13 \\
13 \\
14 \\
13 \\
13 \\
13 \\
13 \\
13 \\
13 \\
13 \\
13\end{array}$} & $\begin{array}{l}9.98 \quad 484 \\
9.98509 \\
9.98534 \\
9.98560 \\
9.98585\end{array}$ & $\begin{array}{l}25 \\
25 \\
26 \\
25 \\
25\end{array}$ & $\begin{array}{ll}0.01 & 51.6 \\
0.01 & 491 \\
0.01 & 466 \\
0.01 & 440 \\
0.01 & 415\end{array}$ & 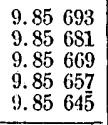 & \multirow{2}{*}{$\begin{array}{l}12 \\
12 \\
12 \\
12 \\
13 \\
12 \\
12 \\
12 \\
13 \\
12\end{array}$} & $\begin{array}{l}\mathbf{6 0} \\
59 \\
58 \\
57 \\
56 \\
\end{array}$ & 4 & $\begin{array}{r}0 \\
56 \\
52 \\
48 \\
44 \\
\end{array}$ \\
\hline 56 & \begin{tabular}{l|}
20 \\
24 \\
28 \\
32 \\
36
\end{tabular} & $\begin{array}{l}5 \\
6 \\
7 \\
8 \\
9\end{array}$ & 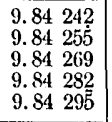 & & 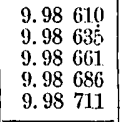 & $\begin{array}{l}25 \\
26 \\
25 \\
25 \\
26\end{array}$ & $\begin{array}{ll}0.01 & 390 \\
0.01 . & 365 \\
0.01 & 339 \\
0.01 & 314 \\
0.01 & 289\end{array}$ & $\begin{array}{ll}9.85 & 632 \\
9.85 & 620 \\
9.85 & 608 \\
9.85 & 596 \\
9.85 & 583\end{array}$ & & $\begin{array}{l}55 \\
54 \\
53 \\
52 \\
51\end{array}$ & 3 & $\begin{array}{l}40 \\
36 \\
32 \\
28 \\
24\end{array}$ \\
\hline 56 & $\begin{array}{l}40 \\
44 \\
48 \\
52 \\
56\end{array}$ & $\begin{array}{l}1.0 \\
11 \\
12 \\
13 \\
14\end{array}$ & 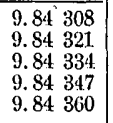 & & $\begin{array}{ll}9.98 & 737 \\
9.98 & 762 \\
9.98 & 787 \\
9.98 & 812 \\
9.98 & 838\end{array}$ & $\begin{array}{l}25 \\
25 \\
25 \\
26 \\
25\end{array}$ & $\begin{array}{ll}0.01 & 263 \\
0.01 & 238 \\
0.01 .213 \\
0.01 & 188 \\
0.01 & 162\end{array}$ & $\begin{array}{ll}9.85 & 571 \\
9.85 & 559 \\
9.85 & 547 \\
9.85 & 534 \\
9.85 & 522\end{array}$ & $\begin{array}{l}12 \\
12 \\
13 \\
12 \\
12\end{array}$ & $\begin{array}{r}50 \\
49 \\
48 \\
47 \\
46 \\
\end{array}$ & 3 & $\begin{array}{r}20 \\
16 \\
12 \\
8 \\
4\end{array}$ \\
\hline 57 & \begin{tabular}{r|}
0 \\
4 \\
8 \\
12 \\
.16 \\
\end{tabular} & $\begin{array}{l}15 \\
16 \\
17 \\
18 \\
19 \\
\end{array}$ & \begin{tabular}{ll|}
9.84 & 373 \\
9.84 & 385 \\
9.84 & 398 \\
9.84 & 411 \\
9.84 & 424 \\
\end{tabular} & $\begin{array}{l}12 \\
13 \\
13 \\
13 \\
13\end{array}$ & 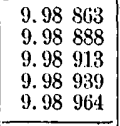 & $\begin{array}{l}25 \\
25 \\
26 \\
25 \\
25\end{array}$ & $\begin{array}{ll}0.01 & 137 \\
0.01 & 112 \\
0.01 & 087 \\
0.01 & 061 \\
0.01 & 036 \\
\end{array}$ & $\begin{array}{ll}9.85 & 510 \\
9.85 & 497 \\
9.85 & 485 \\
9.85 & 473 \\
9.85 & 460 \\
\end{array}$ & $\begin{array}{l}13 \\
12 \\
12 \\
13 \\
12\end{array}$ & $\begin{array}{l}45 \\
44 \\
43 \\
42 \\
41 \\
\end{array}$ & 3 & $\begin{array}{r}0 \\
56 \\
52 \\
48 \\
44 \\
\end{array}$ \\
\hline 57 & $\begin{array}{l}20 \\
24 \\
28 \\
32 \\
36 \\
\end{array}$ & \begin{tabular}{l|}
$\mathbf{4 0}$ \\
21 \\
22 \\
23 \\
24
\end{tabular} & 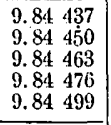 & $\begin{array}{l}13 \\
13 \\
13 \\
13 \\
13\end{array}$ & $\begin{array}{ll}9.98 & 989 \\
9.99 & 015 \\
9.99 & 040 \\
9.99 & 065 \\
9.99 & 090 \\
\end{array}$ & $\begin{array}{l}26 \\
25 \\
25 \\
25 \\
26\end{array}$ & $\begin{array}{ll}0.01 & 011 \\
0.00 & 985 \\
0.00 & 960 \\
0.00 & 935 \\
0.00 & 910 \\
\end{array}$ & $\begin{array}{ll}9.85 & 448 \\
9.85 & 436 \\
9.85 & 423 \\
9.85 & 411 \\
9.85 & 399 \\
\end{array}$ & $\begin{array}{l}12 \\
13 \\
12 \\
12 \\
13\end{array}$ & $\begin{array}{l}40 \\
39 \\
38 \\
37 \\
36 \\
\end{array}$ & 2 & $\begin{array}{l}40 \\
36 \\
32 \\
28 \\
24\end{array}$ \\
\hline 57 & $\begin{array}{l}40 \\
44 \\
48 \\
52 \\
56 \\
\end{array}$ & $\begin{array}{l}25 \\
26 \\
27 \\
28 \\
29\end{array}$ & 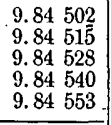 & $\begin{array}{l}13 \\
13 \\
12 \\
13 \\
13\end{array}$ & $\begin{array}{ll}9.99 & 116 \\
9.99 & 141 \\
9.99 & 166 \\
9.99 & 191 \\
9.99 & 217 \\
\end{array}$ & $\begin{array}{l}25 \\
25 \\
25 \\
26 \\
25\end{array}$ & $\begin{array}{ll}0.00 & 884 \\
0.00 & 859 \\
0.00 & 834 \\
0.00 & 809 \\
0.00 & 783 \\
\end{array}$ & 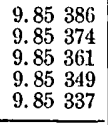 & $\begin{array}{l}12 \\
13 \\
12 \\
12 \\
13\end{array}$ & $\begin{array}{l}35 \\
34 \\
33 \\
32 \\
31 \\
\end{array}$ & 2 & $\begin{array}{r}20 \\
16 \\
12 \\
8 \\
4 \\
\end{array}$ \\
\hline 58 & $\begin{array}{r}0 \\
4 \\
8 \\
12 \\
16 \\
\end{array}$ & $\begin{array}{c}\mathbf{3 0} \\
31 \\
32 \\
33 \\
.34\end{array}$ & $\begin{array}{lll}9.84 & 566 \\
9.84 & 579 \\
9.84 & 592 \\
9.84 & 605 \\
9.84 & 618 \\
\end{array}$ & $\begin{array}{l}13 \\
13 \\
13 \\
13 \\
12\end{array}$ & $\begin{array}{ll}9.99 & 242 \\
9.99 & 267 \\
9.99 & 293 \\
9.99 & 318 \\
9.99 & 343 \\
\end{array}$ & $\begin{array}{l}25 \\
26 \\
25 \\
25 \\
25\end{array}$ & 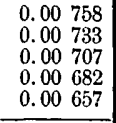 & $\begin{array}{ll}9.85 & 324 \\
9.85 & 312 \\
9.85 & 299 \\
9.85 & 287 \\
9.85 & 274 \\
\end{array}$ & $\begin{array}{l}12 \\
13 \\
12 \\
13 \\
12\end{array}$ & $\begin{array}{l}30 \\
29 \\
28 \\
27 \\
26 \\
\end{array}$ & 2 & $\begin{array}{r}0 \\
56 \\
52 \\
48 \\
44\end{array}$ \\
\hline 58 & $\begin{array}{l}20 \\
24 \\
28 \\
32 \\
36\end{array}$ & $\begin{array}{l}35 \\
36 \\
37 \\
38 \\
39\end{array}$ & 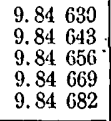 & $\begin{array}{l}13 \\
13 \\
13 \\
13 \\
1.2\end{array}$ & 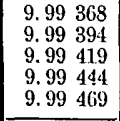 & $\begin{array}{l}26 \\
25 \\
25 \\
25 \\
26\end{array}$ & $\begin{array}{ll}0.00 & 632 \\
0.00 & 606 \\
0.00 & 581 \\
0.00 & 556 \\
0.00 & 531\end{array}$ & $\begin{array}{ll}9.85 & 262 \\
9.85 & 250 \\
9.85 & 237 \\
9.85 & 225 \\
9.85 & 212 \\
\end{array}$ & $\begin{array}{l}12 \\
13 \\
12 \\
13 \\
12\end{array}$ & $\begin{array}{l}25 \\
24 \\
23 \\
22 \\
21\end{array}$ & 1 & $\begin{array}{l}40 \\
36 \\
32 \\
28 \\
24\end{array}$ \\
\hline 58 & $\begin{array}{l}40 \\
44 \\
48 \\
52 \\
56 \\
\end{array}$ & $\begin{array}{l}40 \\
41 \\
42 \\
43 \\
44 \\
\end{array}$ & $\begin{array}{ll}9.84 & 694 \\
9.84 & 707 \\
9.84 & 720 \\
9.84 & 733 \\
9.84 & 745 \\
\end{array}$ & $\begin{array}{l}13 \\
13 \\
13 \\
12 \\
13\end{array}$ & $\begin{array}{ll}9.99 & 495 \\
9.99 & 520 \\
9.99 & 545 \\
9.99 & 570 \\
9.99 & 596\end{array}$ & $\begin{array}{l}25 \\
25 \\
25 \\
26 \\
25\end{array}$ & $\begin{array}{ll}0.00 & 505 \\
0.00 & 480 \\
0.00 & 455 \\
0.00 & 430 \\
0.00 & 404\end{array}$ & $\begin{array}{ll}9.85 & 200 \\
9.85 & 187 \\
9.85 & 175 \\
9.85 & 162 \\
9.85 & 150 \\
\end{array}$ & $\begin{array}{l}13 \\
12 \\
13 \\
12 \\
13\end{array}$ & $\begin{array}{l}20 \\
19 \\
18 \\
17 \\
16\end{array}$ & 1 & $\begin{array}{r}20 \\
16 \\
12 \\
8 \\
4\end{array}$ \\
\hline 59 & $\begin{array}{r}0 \\
4 \\
8 \\
12 \\
16\end{array}$ & $\begin{array}{l}45 \\
46 \\
47 \\
48 \\
49\end{array}$ & 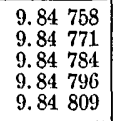 & $\begin{array}{l}13 \\
13 \\
12 \\
13 \\
13\end{array}$ & 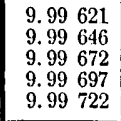 & $\begin{array}{l}25 \\
26 \\
25 \\
25 \\
25\end{array}$ & $\begin{array}{ll}0.00 & 379 \\
0.00 & 354 \\
0.00 & 328 \\
0.00 & 303 \\
0.00 & 278\end{array}$ & $\begin{array}{ll}9.85 & 137 \\
9.85 & 125 \\
9.85 & 112 \\
9.85 & 100 \\
9.85 & 087\end{array}$ & $\begin{array}{l}12 \\
13 \\
12 \\
13 \\
13\end{array}$ & $\begin{array}{l}15 \\
14 \\
13 \\
12 \\
11\end{array}$ & 1 & $\begin{array}{r}0 \\
56 \\
52 \\
48 \\
44\end{array}$ \\
\hline 59 & $\begin{array}{l}20 \\
24 \\
28 \\
32 \\
36\end{array}$ & $\begin{array}{c}\mathbf{5 0} \\
51 \\
52 \\
53 \\
54\end{array}$ & 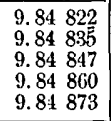 & $\begin{array}{l}13 \\
12 \\
13 \\
13 \\
12\end{array}$ & 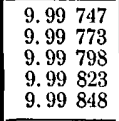 & $\begin{array}{l}26 \\
25 \\
25 \\
25 \\
26\end{array}$ & $\begin{array}{ll}0.00 & 253 \\
0.00 & 227 \\
0.00 & 202 \\
0.00 & 177 \\
0.00 & 152\end{array}$ & $\begin{array}{lll}9.85 & 074 \\
9.85 & 062 \\
9.85 & 049 \\
9.85 & 037 \\
9.85 & 024\end{array}$ & $\begin{array}{l}12 \\
13 \\
12 \\
13 \\
12\end{array}$ & $\begin{array}{r}10 \\
9 \\
8 \\
7 \\
6\end{array}$ & 0 . & $\begin{array}{l}40 \\
36 \\
32 \\
28 \\
24\end{array}$ \\
\hline 59 & $\begin{array}{l}40 \\
44 \\
48 \\
52 \\
56\end{array}$ & $\begin{array}{l}55 \\
56 \\
57 \\
58 \\
59\end{array}$ & 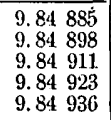 & $\begin{array}{l}13 \\
13 \\
12 \\
13 \\
13\end{array}$ & 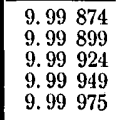 & $\begin{array}{l}25 \\
25 \\
25 \\
26 \\
25\end{array}$ & $\begin{array}{lll}0.00 & 126 \\
0.00 & 101 \\
0.00 & 076 \\
0.00 & 051 \\
0.00 & 025\end{array}$ & 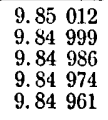 & $\begin{array}{l}13 \\
13 \\
12 \\
13 \\
12\end{array}$ & $\begin{array}{l}5 \\
4 \\
3 \\
2 \\
1\end{array}$ & 0 & $\begin{array}{r}20 \\
16 \\
12 \\
8 \\
4\end{array}$ \\
\hline 60 & 0 & 60 & 9.84949 & & 0.00000 & & 0.00000 & 9.84949 & & 0 & 0 & 0 \\
\hline & & & L. Cos. & d. & L. Cotg. & c. a. & L. Tang. & L. $\operatorname{Sin}$. & d. & ' & $\mathrm{m}$. & s. \\
\hline
\end{tabular}

$45^{\circ}$ 
TABLE 20.-Geodetic position computations.

TABLE OF LOGARITHMS OF FACTORS A, B, C, D, E, F, BASED UPON THE CLARKE SPHEROID OF 1866 AND THE METRIC SYSTEM, BETWEEN LATITUDES, $0^{\circ}$ AND $72^{\circ}$.

[Extracted from reports of the U. S. Coast and Geodetic Survey.]

\section{CONSTANTS.}

$$
\begin{aligned}
& A=\frac{\left(1-e^{2} \sin ^{2} \varphi\right)^{\frac{1}{3}}}{a \operatorname{arc} 1^{\prime \prime}} \\
& \mathrm{B}=\frac{\left(1-e^{2} \sin ^{2} \varphi\right)^{\frac{3}{2}}}{a\left(1-e^{2}\right) \operatorname{arc~} 1^{\prime \prime}} \\
& \mathrm{C}=\frac{\left(1-e^{2} \sin ^{2} \varphi\right)^{2} \tan \varphi}{2 a^{2}\left(1-e^{2}\right) \operatorname{arc} 1^{\prime \prime}} \\
& \mathrm{D}=\frac{\frac{3}{2} e^{2} \sin \varphi \cos \varphi \operatorname{arc} 1^{\prime \prime}}{1-e^{2} \sin ^{2} \phi} \\
& \mathrm{E}=\frac{\left(1+3 \tan ^{2} \varphi\right)\left(1-e^{2} \sin ^{2} \varphi\right)}{6 a^{2}} \\
& \mathrm{~F}=\frac{1}{I^{2}} \sin \varphi \cos ^{2} \phi \operatorname{arc}^{2} 1^{\prime \prime} \\
& \log a=6.80469857 \\
& \log b=6.80322378 \\
& \log e^{2}=7.83050257 \\
& \log \frac{1}{a \operatorname{arc~} 1^{\prime \prime}}=\overline{8} .50972656 \\
& \log \frac{1}{a\left(1-e^{2}\right) \operatorname{arc~} 1^{\prime \prime}}=\overline{8} .51267615 \\
& \log \frac{1}{2 a^{2}\left(1-e^{2}\right) \operatorname{arc} 1^{\prime \prime}}=\overline{1} .4069476 \\
& \log \left(\frac{3}{2} e^{2} \operatorname{arc} 1^{\prime \prime}\right)=\overline{2} .6921687 \\
& \log \frac{1}{6 a^{2}}=\overline{5} .61245 \\
& \log \left(\frac{1}{12} \operatorname{arc}^{2} 1^{\prime \prime}\right)=\overline{8} .29196
\end{aligned}
$$

Ratio adopted in this table is the Clarke value of the meter, namely, 1 meter $=$ 39.370432 inches. 
TABLs 20.-Geodetic position computations-Continued.

IAATITUDE $0^{\circ}$ :

\begin{tabular}{|c|c|c|c|c|c|c|c|}
\hline Lat. & $\log A$ & $\log B$ & & $\log C$ & $\log D$ & $\log \mathrm{E}$ & $\log F$ \\
\hline $\begin{array}{cc}\circ & 1 \\
00 & 00 \\
& 1 \\
& 2 \\
& 3 \\
& 4 \\
& 4\end{array}$ & $\begin{array}{r}\overline{8} .5097266 \\
66 \\
66 \\
66 \\
66\end{array}$ & $\begin{array}{rr}8.512 \quad 6761 \\
61 \\
61 \\
61 \\
61 \\
61\end{array}$ & & $\begin{array}{l}-\infty \\
\overline{7} .8707 \\
8.1717 \\
3477 \\
4727\end{array}$ & $\begin{array}{c}-\infty \\
\overline{9} .156 \\
457 \\
633 \\
758\end{array}$ & $\begin{array}{r}\overline{\overline{5} .6125} \\
5 \\
5 \\
5 \\
5\end{array}$ & $-\infty$ \\
\hline $\begin{array}{r}05 \\
6 \\
7 \\
8 \\
9\end{array}$ & $\begin{array}{l}66 \\
66 \\
66 \\
66 \\
66\end{array}$ & $\begin{array}{l}61 \\
61 \\
61 \\
61 \\
61\end{array}$ & - & $\begin{array}{l}5696 \\
6488 \\
7158 \\
7740 \\
8249\end{array}$ & $\begin{array}{r}855 \\
\overline{9} .934 \\
\overline{0} .001 \\
059 \\
110\end{array}$ & $\begin{array}{l}5 \\
5 \\
5 \\
5 \\
5\end{array}$ & \\
\hline $\begin{array}{l}10 \\
11 \\
12 \\
13 \\
14\end{array}$ & $\begin{array}{r}8.5097266 \\
65 \\
65 \\
65 \\
65 \\
65\end{array}$ & $\begin{array}{r}8.512 \quad 6761 \\
61 \\
61 \\
61 \\
61\end{array}$ & & $\begin{array}{r}8.8707 \\
9121 \\
9499 \\
8.9846 \\
9.0168\end{array}$ & $\begin{array}{r}0.156 \\
197 \\
235 \\
270 \\
302\end{array}$ & $\begin{array}{r}5.6125 \\
5 \\
5 \\
5 \\
5\end{array}$ & \\
\hline $\begin{array}{r}15 \\
16 \\
17 \\
18 \\
\cdot 19\end{array}$ & $\begin{array}{l}65 \\
65 \\
65 \\
65 \\
65\end{array}$ & $\begin{array}{l}61 \\
61 \\
60 \\
60 \\
60\end{array}$ & & $\begin{array}{l}0468 \\
0748 \\
1011 \\
1259 \\
1494\end{array}$ & $\begin{array}{l}332 \\
360 \\
386 \\
411 \\
435\end{array}$ & $\begin{array}{l}5 \\
5 \\
5 \\
5 \\
5\end{array}$ & \\
\hline $\begin{array}{l}20 \\
21 \\
22 \\
23 \\
24\end{array}$ & $\begin{array}{r}8.5097265 \\
65 \\
65 \\
65 \\
65\end{array}$ & $\begin{array}{r}8.5126760 \\
60 \\
60 \\
60 \\
59\end{array}$ & & $\begin{array}{r}9.1717 \\
1929 \\
2131 \\
2324 \\
2509\end{array}$ & $\begin{array}{r}0.457 \\
478 \\
498 \\
518 \\
536\end{array}$ & $\begin{array}{r}\text { ค. } 6125 \\
5 \\
5 \\
5 \\
5\end{array}$ & है. 057 \\
\hline $\begin{array}{l}25 \\
26 \\
27 \\
28 \\
29\end{array}$ & $\begin{array}{l}65 \\
65 \\
65 \\
65 \\
65\end{array}$ & $\begin{array}{l}59 \\
59 \\
59 \\
59 \\
58\end{array}$ & & $\begin{array}{l}2686 \\
2857 \\
3020 \\
3178 \\
3331\end{array}$ & $\begin{array}{l}554 \\
571 \\
587 \\
603 \\
618\end{array}$ & $\begin{array}{l}\mathbf{5} \\
5 \\
5 \\
5 \\
5 \\
\mathbf{5}\end{array}$ & \\
\hline $\begin{array}{l}30 \\
31 \\
32 \\
33 \\
34\end{array}$ & $\begin{array}{r}8.5097265 \\
64 \\
64 \\
64 \\
64\end{array}$ & $\begin{array}{r}8.512 \quad 6758 \\
58 \\
58 \\
57 \\
57\end{array}$ & & $\begin{array}{r}9.3478 \\
3620 \\
3758 \\
9.3892 \\
9.4022\end{array}$ & $\begin{array}{r}0.633 \\
647 \\
661 \\
674 \\
687\end{array}$ & $\begin{array}{r}5.6126 \\
6 \\
6 \\
6 \\
6\end{array}$ & \\
\hline $\begin{array}{l}35 \\
36 \\
37 \\
38 \\
39\end{array}$ & $\begin{array}{l}64 \\
64 \\
64 \\
64 \\
64\end{array}$ & $\begin{array}{l}\mathbf{5 7} \\
\mathbf{5 7} \\
56 \\
56 \\
56\end{array}$ & & $\begin{array}{l}4148 \\
4270 \\
4389 \\
4505 \\
4618\end{array}$ & $\begin{array}{r}700 \\
712 \\
724 \\
-736 \\
747\end{array}$ & $\begin{array}{l}6 \\
6 \\
6 \\
6 \\
6\end{array}$ & \\
\hline $\begin{array}{l}40 \\
41 \\
42 \\
43 \\
44\end{array}$ & $\begin{array}{r}8.5097264 \\
64 \\
64 \\
64 \\
63\end{array}$ & $\begin{array}{r}8.512 \quad 6756 \\
55 \\
55 \\
55 \\
54\end{array}$ & & $\begin{array}{r}9.4728 \\
4835 \\
9.4939 \\
9.5042 \\
5141\end{array}$ & $\begin{array}{r}0.758 \\
769 \\
779 \\
789 \\
799\end{array}$ & 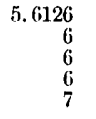 & 6. 358 \\
\hline $\begin{array}{l}45 \\
46 \\
47 \\
48 \\
49\end{array}$ & $\begin{array}{l}63 \\
63 \\
63 \\
63 \\
63\end{array}$ & $\begin{array}{r}54 \\
54 \\
53 \\
53 \\
.53\end{array}$ & - & $\begin{array}{l}5239 \\
5335 \\
5428 \\
5519 \\
5609\end{array}$ & $\begin{array}{l}809 \\
819 \\
828 \\
837 \\
846\end{array}$ & $\begin{array}{l}7 \\
7 \\
7 \\
7 \\
7\end{array}$ & - \\
\hline $\begin{array}{l}50 \\
51 \\
52 \\
53 \\
54\end{array}$ & $\begin{array}{r}8.5097263 \\
63 \\
62 \\
62 \\
62\end{array}$ & $\begin{array}{r}8.512 \quad 6752 \\
52 \\
51 \\
51 \\
51\end{array}$ & & $\begin{array}{r}9.5697 \\
5783 \\
5866 \\
9.5950 \\
9.6031\end{array}$ & $\begin{array}{r}0.855 \\
863 \\
872 \\
880 \\
888\end{array}$ & $\begin{array}{r}5.6127 \\
7 \\
7 \\
7 \\
8\end{array}$ & \\
\hline $\begin{array}{l}55 \\
56 \\
57 \\
58 \\
59\end{array}$ & $\begin{array}{l}62 \\
62 \\
62 \\
61 \\
61\end{array}$ & $\begin{array}{l}50 \\
50 \\
49 \\
49 \\
49\end{array}$ & . & $\begin{array}{l}6111 \\
6189 \\
6266 \\
6341 \\
6416\end{array}$ & $\begin{array}{l}896 \\
904 \\
912 \\
919 \\
927\end{array}$ & $\begin{array}{l}8 \\
8 \\
8 \\
8 \\
8\end{array}$ & \\
\hline 60 & 8.5097261 & 8.5126748 & & 9.6489 & 0.934 & 5.6128 & 6.534 \\
\hline
\end{tabular}


TABLE 20.-Geodetic position computations-Continued.

LATITUDE $1^{\circ}$.

\begin{tabular}{|c|c|c|c|c|c|c|}
\hline Lat. & $\log \mathrm{A}$ & $\log B$ & $\log C$ & $\log \mathrm{D}$ & $\log E$ & $\log F$ \\
\hline $\begin{array}{ccc}\vdots & \circ & 1 \\
\vdots & 1 & 00 \\
& \cdot & 1 \\
& & 2 \\
& & 3 \\
& & 4\end{array}$ & $\begin{array}{r}\overline{8} .5097261 \\
61 \\
61 \\
61 \\
61\end{array}$ & $\begin{array}{r}\overline{8} .512 \quad 6748 \\
48 \\
47 \\
47 \\
46\end{array}$ & $\begin{array}{r}\overline{\overline{9}} .6489 \\
560 \\
631 \\
701 \\
769\end{array}$ & $\begin{array}{r}\overline{0} .934 \\
941 \\
948 \\
955 \\
962\end{array}$ & $\begin{array}{r}5.6128 \\
29 \\
29 \\
29 \\
29\end{array}$ & $\overline{6} .534$ \\
\hline $\begin{array}{r}05 \\
6 \\
7 \\
8 \\
9\end{array}$ & $\begin{array}{l}60 \\
60 \\
60 \\
60 \\
60\end{array}$ & $\begin{array}{l}46 \\
45 \\
45 \\
44 \\
44\end{array}$ & $\begin{array}{r}836 \\
903 \\
9.6968 \\
9.7032 \\
096\end{array}$ & $\begin{array}{r}969 \\
975 \\
982 \\
988 \\
0.995\end{array}$ & $\begin{array}{l}29 \\
29 \\
29 \\
30 \\
30\end{array}$ & \\
\hline $\begin{array}{l}10 \\
11 \\
12 \\
13 \\
14\end{array}$ & $\begin{array}{r}8.509 \quad 7260 \\
59 \\
59 \\
59 \\
59\end{array}$ & $\begin{array}{r}8.512 \quad 6743 \\
43 \\
42 \\
42 \\
41\end{array}$ & $\begin{array}{r}9.7158 \\
220 \\
281 \\
341 \\
400\end{array}$ & $\begin{array}{r}1.001 \\
007 \\
013 \\
019 \\
025\end{array}$ & $\begin{array}{r}5.6130 \\
30 \\
30 \\
30 \\
31\end{array}$ & . \\
\hline $\begin{array}{r}15 \\
16 \\
17 \\
18 \\
.19\end{array}$ & $\begin{array}{r}59 \\
58 \\
-58 \\
58 \\
58\end{array}$ & $\begin{array}{l}41 \\
40 \\
39 \\
39 \\
38\end{array}$ & $\begin{array}{l}458 \\
516 \\
572 \\
628 \\
684\end{array}$ & $\begin{array}{l}031 \\
037 \\
042 \\
048 \\
053\end{array}$ & $\begin{array}{l}31 \\
31 \\
31 \\
31 \\
31\end{array}$ & \\
\hline $\begin{array}{l}20 \\
21 \\
22 \\
23 \\
24\end{array}$ & $\begin{array}{r}8.5097258 \\
57 \\
57 \\
57 \\
5 \quad 57\end{array}$ & $\begin{array}{r}8.51 .26738 \\
37 \\
36 \\
36 \\
35\end{array}$ & $\begin{array}{r}9.7738 \\
792 \\
846 \\
898 \\
9.7950\end{array}$ & $\begin{array}{r}1.059 \\
064 \\
070 \\
075 \\
080\end{array}$ & $\begin{array}{r}5.6132 \\
32 \\
32 \\
32 \\
32\end{array}$ & 6.658 \\
\hline $\begin{array}{l}25 \\
26 \\
27 \\
28 \\
29\end{array}$ & $\begin{array}{l}57 \\
56 \\
56 \\
56 \\
56\end{array}$ & $\begin{array}{l}35 \\
34 \\
33 \\
33 \\
32\end{array}$ & $\begin{array}{r}9.8002 \\
053 \\
103 \\
152 \\
202\end{array}$ & $\begin{array}{l}085 \\
090 \\
095 \\
100 \\
105\end{array}$ & $\begin{array}{l}32 \\
33 \\
33 \\
33 \\
33\end{array}$ & \\
\hline $\begin{array}{l}30 \\
31 \\
32 \\
33 \\
34\end{array}$ & $\begin{array}{rr}8.509 & 7256 \\
& 55 \\
. \quad 55 \\
. \quad 55 \\
& 55\end{array}$ & $\begin{array}{r}8.512 \quad 6731 \\
31 \\
30 \\
29 \\
29\end{array}$ & $\begin{array}{r}9.8250 \\
298 \\
346 \\
-\quad 393 \\
439\end{array}$ & $\begin{array}{r}1.110 \\
115 \\
119 \\
124 \\
129\end{array}$ & $\begin{array}{r}5: 6133 \\
34 \\
34 \\
34 \\
34 \\
. \quad 3\end{array}$ & \\
\hline $\begin{array}{l}35 \\
36 \\
37 \\
38 \\
39\end{array}$ & $\begin{array}{l}54 \\
54 \\
54 \\
54 \\
53\end{array}$ & $\begin{array}{l}28 \\
27 \\
26 \\
26 \\
25\end{array}$ & $\begin{array}{l}485 \\
531 \\
576 \\
620 \\
664\end{array}$ & $\begin{array}{l}133 \\
138 \\
142 \\
147 \\
151\end{array}$ & $\begin{array}{r}34 \\
35 \\
35 \\
35 \\
35\end{array}$ & \\
\hline $\begin{array}{l}40 \\
41 \\
42 \\
43 \\
44\end{array}$ & $\begin{array}{r}8.5097253 \\
53 \\
53 \\
52 \\
52\end{array}$ & $\begin{array}{r}8.512 \quad 6724 \\
23 \\
23 \\
22 \\
21\end{array}$ & $\begin{array}{r}9.8708 \\
751 \\
794 \\
836 \\
878\end{array}$ & $\begin{array}{r}1.156 \\
160 \\
164 \\
168 \\
173\end{array}$ & $\begin{array}{r}5.6136 \\
36 \\
36 \\
36 \\
36\end{array}$ & 6.755 \\
\hline $\begin{array}{l}45 \\
46 \\
47 \\
48 \\
49\end{array}$ & $\begin{array}{l}52 \\
52 \\
51 \\
51 \\
51\end{array}$ & $\begin{array}{l}20 \\
20 \\
19 \\
19 \\
17\end{array}$ & $\begin{array}{r}920 \\
961 \\
9.9002 \\
042 \\
082\end{array}$ & $\begin{array}{l}177 \\
181 \\
185 \\
189 \\
193\end{array}$ & $\begin{array}{l}37 \\
37 \\
37 \\
37 \\
38\end{array}$ & \\
\hline $\begin{array}{l}50 \\
51 \\
52 \\
53 \\
54\end{array}$ & $\begin{array}{r}8.5097251 \\
50 \\
50 \\
50 \\
49\end{array}$ & $\begin{array}{r}8.51 .26716 \\
16 \\
15 \\
14 \\
13\end{array}$ & $\begin{array}{r}9.9122 \\
161 \\
200 \\
239 \\
277\end{array}$ & $\begin{array}{r}1.197 \\
2001 \\
205 \\
209 \\
212\end{array}$ & $\begin{array}{r}5.6138 \\
38 \\
38 \\
.39 \\
39\end{array}$ & \\
\hline $\begin{array}{l}55 \\
56 \\
57 \\
58 \\
59\end{array}$ & $\begin{array}{r}49 \\
\quad 49 \\
.49 \\
48 \\
48\end{array}$ & $\begin{array}{l}12 \\
11 \\
10 \\
10 \\
09\end{array}$ & $\begin{array}{l}315 \\
353 \\
390 \\
427 \\
464\end{array}$ & $\begin{array}{l}216 \\
220 \\
224 \\
2227 \\
231\end{array}$ & $\begin{array}{l}39 \\
39 \\
40 \\
40 \\
40\end{array}$ & - \\
\hline 60 & 8.5097248 & 8.5126708 & 9. ${ }^{9} 500$ & 1.2347 & 5.6140 & 6.834 \\
\hline
\end{tabular}


Table 20.-Geodetic position computations-Continued.

IAATITUDE $2^{\circ}$

\begin{tabular}{|c|c|c|c|c|c|c|c|c|}
\hline Lat. & $\log A$ & $\log \mathrm{B}$ & $\log C$ & $\log \mathbf{D}$ & $\log E$ & \multicolumn{3}{|c|}{$\log \mathbf{F}$} \\
\hline $\begin{array}{rr}\circ & 1 \\
2 & 00 \\
& 1 \\
& 2 \\
& 3 \\
& 4\end{array}$ & $\begin{array}{r}\overline{8} .5097248 \\
47 \\
47 \\
47 \\
47\end{array}$ & $\begin{array}{r}\overline{8} .5126708 \\
07 \\
06 \\
05 \\
01\end{array}$ & $\begin{array}{r}\overline{9 .} 95002 \\
5363 \\
5721 \\
6076 \\
6428\end{array}$ & $\begin{array}{r}\overline{1} .2347 \\
383 \\
419 \\
454 \\
489\end{array}$ & $\begin{array}{r}5.6140 \\
41 \\
41 \\
41 \\
41\end{array}$ & & [0.834 & \\
\hline $\begin{array}{r}05 \\
6 \\
7 \\
8 \\
9\end{array}$ & $\begin{array}{l}46 \\
46 \\
46 \\
45 \\
45\end{array}$ & $\begin{array}{r}03 \\
02 \\
01 \\
6700 \\
6699\end{array}$ & $\begin{array}{r}6777 \\
7123 \\
7467 \\
.7808 \\
8146\end{array}$ & $\begin{array}{l}524 \\
559 \\
593 \\
627 \\
661\end{array}$ & $\begin{array}{r}42 \\
42 \\
42 \\
. \quad 43 \\
43\end{array}$ & & & \\
\hline $\begin{array}{l}10 \\
11 \\
12 \\
13 \\
14\end{array}$ & $\begin{array}{r}8.5097245 \\
44 \\
44 \\
44 \\
43\end{array}$ & $\begin{array}{r}8.5126698 \\
97 \\
97 \\
96 \\
95\end{array}$ & $\begin{array}{r}\overline{9} .98482 \\
8815 \\
9145 \\
9473 \\
\overline{9} .99799\end{array}$ & $\begin{array}{r}1.2694 \\
\cdot \quad 727 \\
760 \\
793 \\
826\end{array}$ & $\begin{array}{r}5.6143 \\
. \quad 43 \\
44 \\
44 \\
. \quad 44\end{array}$ & & & \\
\hline $\begin{array}{l}15 \\
16 \\
17 \\
18 \\
19\end{array}$ & $\begin{array}{l}43 \\
43 \\
42 \\
42 \\
42\end{array}$ & $\begin{array}{l}94 \\
93 \\
91 \\
90 \\
89\end{array}$ & $\begin{array}{r}\overline{0} .00122 \\
0443 \\
0762 \\
1078 \\
1392\end{array}$ & $\begin{array}{r}858 \\
890 \\
922 \\
953 \\
1.2984\end{array}$ & $\begin{array}{r}45 \\
45 \\
45 \\
. \quad 45 \\
46\end{array}$ & & & \\
\hline $\begin{array}{l}20 \\
21 \\
22 \\
23 \\
24\end{array}$ & $\begin{array}{r}8.5097241 \\
41 \\
41 \\
40 \\
40\end{array}$ & $\begin{array}{r}8.512 \quad 6688 \\
87 \\
86 \\
85 \\
84\end{array}$ & $\begin{array}{r}0.01703 \\
2013 \\
2320 \\
2625 \\
2928\end{array}$ & $\begin{array}{r}1.3015 \\
046 \\
077 \\
107 \\
138\end{array}$ & $\begin{array}{r}5.6146 \\
46 \\
-47 \\
47 \\
47\end{array}$ & & 6.901 & \\
\hline $\begin{array}{l}25 \\
26 \\
27 \\
28 \\
29\end{array}$ & $\begin{array}{r}40 \\
-39 \\
39 \\
38 \\
38\end{array}$ & $\begin{array}{l}83 \\
82 \\
81 \\
80 \\
79\end{array}$ & $\begin{array}{l}3229 \\
3528 \\
3825 \\
4119 \\
4412\end{array}$ & $\begin{array}{l}168 \\
197 \\
227 \\
256 \\
285\end{array}$ & $\begin{array}{l}48 \\
48 \\
48 \\
49 \\
49\end{array}$ & & & \\
\hline $\begin{array}{l}30 \\
31 \\
32 \\
33 \\
34\end{array}$ & 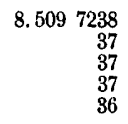 & $\begin{array}{r}8.5126678 \\
76 \\
75 \\
74 \\
. \quad 73\end{array}$ & $\begin{array}{r}0.04703 \\
-\quad 4992 \\
5279 \\
5564 \\
5847\end{array}$ & $\begin{array}{r}1.3314 \\
343 \\
372 \\
400 \\
428\end{array}$ & $\begin{array}{r}5.6149 \\
50 \\
50 \\
50 \\
51\end{array}$ & & & \\
\hline $\begin{array}{l}35 \\
36 \\
37 \\
38 \\
39\end{array}$ & $\begin{array}{r}36 \\
35 \\
35 \\
35 \\
34\end{array}$ & $\begin{array}{l}72 \\
71 \\
70 \\
68 \\
67\end{array}$ & $\begin{array}{l}6129 \\
6408 \\
6686 \\
6962 \\
7237\end{array}$ & $\begin{array}{l}456 \\
484 \\
512 \\
539 \\
567\end{array}$ & $\begin{array}{l}51 \\
51 \\
52 \\
52 \\
52\end{array}$ & & . & : \\
\hline $\begin{array}{l}40 \\
41 \\
42 \\
43 \\
44\end{array}$ & $\begin{array}{r}8.5097234 \\
33 \\
33 \\
33 \\
32\end{array}$ & $\begin{array}{r}8.512 \quad 6666 \\
65 \\
64 \\
62 \\
61\end{array}$ & $\begin{array}{r}0.07509 \\
7780 \\
8050 \\
831.7 \\
8583\end{array}$ & $\begin{array}{r}1.3594 \\
621 \\
648 \\
674 \\
701\end{array}$ & $\begin{array}{r}5.6153 \\
53 \\
53 \\
54 \\
54\end{array}$ & . & $\begin{array}{c}6.959 \\
.\end{array}$ & : \\
\hline $\begin{array}{l}45 \\
46 \\
47 \\
48 \\
49\end{array}$ & $\begin{array}{l}32 \\
31 \\
31 \\
31 \\
30\end{array}$ & $\begin{array}{l}60 \\
59 \\
58 \\
56 \\
55\end{array}$ & $\begin{array}{r}8848 \\
9111 \\
9372 \\
9631 \\
.0 .09890\end{array}$ & $\begin{array}{r}727 \\
753 \\
779 \\
\times \quad 805 \\
831\end{array}$ & $\begin{array}{l}54 \\
55 \\
55 \\
56 \\
56\end{array}$ & - & & $\begin{array}{l}\vdots \\
\vdots \\
\vdots\end{array}$ \\
\hline $\begin{array}{l}50 \\
51 \\
52 \\
53 \\
54\end{array}$ & $\begin{array}{r}8.5097230 \\
29 \\
29 \\
29 \\
29\end{array}$ & $\begin{array}{r}8.5126654 \\
52 \\
. \quad 51 \\
. \quad 50 \\
\\
49\end{array}$ & $\begin{array}{r}0.10146 \\
0401 \\
0655 \\
0907 \\
1158\end{array}$ & $\begin{array}{r}\text { 1. } 3856 \\
882 \\
907 \\
932 \\
957\end{array}$ & $\begin{array}{r}5.6156 \\
57 \\
57 \\
57 \\
58\end{array}$ & & & $\begin{array}{l}\vdots \\
\vdots \\
\vdots\end{array}$ \\
\hline $\begin{array}{l}55 \\
56 \\
57 \\
58 \\
59\end{array}$ & $\begin{array}{l}28 \\
27 \\
27 \\
26 \\
26\end{array}$ & $\begin{array}{l}47 \\
46 \\
45 \\
43 \\
42\end{array}$ & $\begin{array}{r}1407 \\
1655 \\
1902 \\
2147 \\
2390\end{array}$ & $\begin{array}{r}1.3982 \\
1.4007 \\
.031 \\
055 \\
080\end{array}$ & $\begin{array}{l}58 \\
59 \\
59 \\
59 \\
60\end{array}$ & & , & : \\
\hline 60 & 8.5097225 & 8.5126641 & 0.12633 & 1.4104 & 5.6160 & & 7.010 & \\
\hline
\end{tabular}


TABLe 20.-Geodetic position computations-Continued.

LATITUDE $3^{\circ}$.

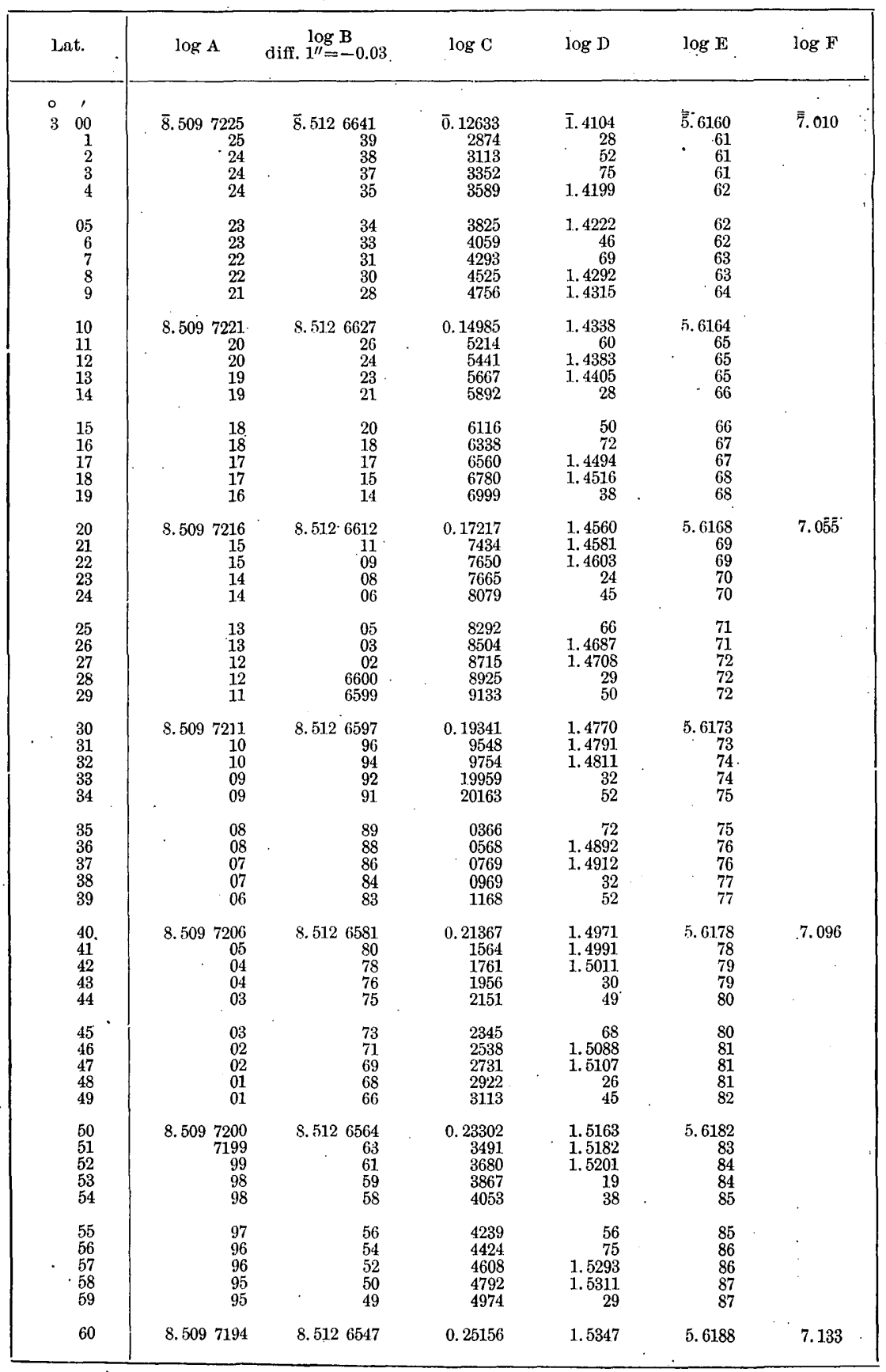


TA Bre 20.-Geodetic position computations-Continued.

LATITUDE $4^{\circ}$

\begin{tabular}{|c|c|c|c|c|c|c|}
\hline Trat. & $\log A$ & $\begin{array}{c}\log B \\
\text { diff. } 1^{\prime \prime}=-0.04\end{array}$ & $\log C$ & $\log \mathrm{D}$ & $\log E$ & $\log \mathrm{F}$ \\
\hline 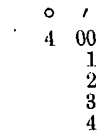 & $\begin{array}{r}\overline{8} .5097194 \\
93 \\
93 \\
92 \\
92\end{array}$ & $\begin{array}{r}\overline{8} .5126547 \\
45 \\
43 \\
42 \\
40\end{array}$ & $\begin{array}{r}\overline{0} .25156 \\
5337 \\
5518 \\
5697 \\
5876\end{array}$ & $\begin{array}{r}1.5347 \\
65 \\
1.5383 \\
1.5401 \\
18\end{array}$ & $\begin{array}{r}5.6188 \\
88 \\
89 \\
89 \\
90\end{array}$ & 7.133 \\
\hline $\begin{array}{r}05 \\
6 \\
7 \\
8 \\
9\end{array}$ & $\begin{array}{r}91 \\
91 \\
90 \\
89 \\
89\end{array}$ & $\begin{array}{l}38 \\
36 \\
34 \\
32 \\
31\end{array}$ & $\begin{array}{l}6055 \\
6232 \\
6409 \\
6585 \\
6760\end{array}$ & $\begin{array}{r}36 \\
54 \\
71 \\
1.5489 \\
1.5506\end{array}$ & $\begin{array}{l}90 \\
91 \\
91 \\
92 \\
92\end{array}$ & \\
\hline $\begin{array}{l}10 \\
11 \\
12 \\
13 \\
14\end{array}$ & $\begin{array}{r}8.5097188 \\
87 \\
87 \\
86 \\
86\end{array}$ & $\begin{array}{r}8.5126529 \\
27 \\
25 \\
23 \\
21\end{array}$ & $\begin{array}{r}0.26935 \\
7109 \\
7282 \\
7455 \\
7627\end{array}$ & $\begin{array}{r}1.5523 \\
40 \\
58 \\
75 \\
1.5592\end{array}$ & $\begin{array}{r}5.6193 \\
93 \\
94 \\
95 \\
95\end{array}$ & \\
\hline $\begin{array}{l}15 \\
16 \\
17 \\
18 \\
19\end{array}$ & $\begin{array}{l}85 \\
84 \\
84 \\
83 \\
82\end{array}$ & $\begin{array}{l}19 \\
17 \\
16 \\
14 \\
12\end{array}$ & $\begin{array}{l}7798 \\
7968 \\
8138 \\
8308 \\
8476\end{array}$ & $\begin{array}{r}1.5609 \\
25 \\
42 \\
59 \\
76\end{array}$ & $\begin{array}{r}96 \\
96 \\
97 \\
97 \\
98\end{array}$ & . \\
\hline $\begin{array}{l}20 \\
21 \\
22 \\
23 \\
24\end{array}$ & $\begin{array}{r}8.5097 .182 \\
81 \\
8 \quad 80 \\
80 \\
79\end{array}$ & $\begin{array}{r}8.512 \quad 6510 \\
08 \\
06 \\
04 \\
02\end{array}$ & $\begin{array}{r}0.28644 \\
8812 \\
8978 \\
9144 \\
9310\end{array}$ & $\begin{array}{r}1.5692 \\
1.5709 \\
25 \\
42 \\
58\end{array}$ & $\begin{array}{r}5.6199 \\
5.6199 \\
5.6200 \\
00 \\
01\end{array}$ & $\begin{array}{l}7.168 \\
.\end{array}$ \\
\hline $\begin{array}{l}25 \\
26 \\
27 \\
28 \\
29\end{array}$ & $\begin{array}{r}78 \\
78 \\
. \quad 77 \\
. \quad 76 \\
. \quad 76\end{array}$ & $\begin{array}{r}6500 \\
6498 \\
96 \\
94 \\
92\end{array}$ & $\begin{array}{r}9475 \\
9639 \\
9802 \\
0.29965 \\
0.30128\end{array}$ & $\begin{array}{r}74 \\
1.5791 \\
1.5807 \\
23 \\
39\end{array}$ & $\begin{array}{l}01 \\
02 \\
03 \\
03 \\
04\end{array}$ & . \\
\hline $\begin{array}{l}30 \\
31 \\
32 \\
33 \\
34\end{array}$ & $\begin{array}{r}8.5097175 \\
. \quad 74 \\
. \quad 74 \\
73 \\
72\end{array}$ & $\begin{array}{r}8.512 \quad 6490 \\
88 \\
86 \\
84 \\
82\end{array}$ & $\begin{array}{r}0.30290 \\
0451 \\
0611 \\
0771 \\
0931\end{array}$ & $\begin{array}{r}1.5855 \\
71 \\
1.5887 \\
1.5902 \\
18\end{array}$ & $\begin{array}{r}5.6204 \\
05 \\
05 \\
06 \\
07\end{array}$ & i. \\
\hline $\begin{array}{l}35 \\
36 \\
37 \\
38 \\
39\end{array}$ & $\begin{array}{l}72 \\
71 \\
70 \\
70 \\
69\end{array}$ & $\begin{array}{l}80 \\
78 \\
76 \\
74 \\
72\end{array}$ & $\begin{array}{l}1090 \\
1248 \\
1406 \\
1563 \\
1719\end{array}$ & $\begin{array}{r}34 \\
50 \\
65 \\
81 \\
1.5996\end{array}$ & $\begin{array}{l}07 \\
08 \\
08 \\
09 \\
10\end{array}$ & : \\
\hline $\begin{array}{l}40 \\
41 \\
42 \\
43 \\
44\end{array}$ & $\begin{array}{r}8.5097169 \\
67 \\
67 \\
66 \\
66\end{array}$ & $\begin{array}{r}8.5126470 \\
68 \\
65 \\
63 \\
61\end{array}$ & $\begin{array}{r}0.31875 \\
2031 \\
2186 \\
2340 \\
2494\end{array}$ & $\begin{array}{r}1.6011 \\
27 \\
42 \\
57 \\
73\end{array}$ & $\begin{array}{r}5.6210 \\
11 \\
12 \\
12 \\
13\end{array}$ & 7.200 \\
\hline $\begin{array}{l}40 \\
46 \\
47 \\
48 \\
49\end{array}$ & $\begin{array}{r}65 \\
64 \\
63 \\
63 \\
62\end{array}$ & $\begin{array}{l}59 \\
57 \\
55 \\
53 \\
51\end{array}$ & $\begin{array}{l}2647 \\
2800 \\
2953 \\
3104 \\
3255\end{array}$ & $\begin{array}{r}1.6088 \\
1.6103 \\
18 \\
33 \\
48\end{array}$ & $\begin{array}{l}13 \\
14 \\
15 \\
15 \\
16\end{array}$ & \\
\hline $\begin{array}{l}50 \\
51 \\
52 \\
53 \\
54\end{array}$ & $\begin{array}{r}8.5097161 \\
60 \\
60 \\
59 \\
58\end{array}$ & $\begin{array}{r}8.5126448 \\
46 \\
44 \\
42 \\
40\end{array}$ & $\begin{array}{r}0.33406 \\
.3556 \\
3706 \\
3855 \\
4004\end{array}$ & $\begin{array}{r}1.6163 \\
77 \\
1.6192 \\
1.6207 \\
21\end{array}$ & $\begin{array}{r}5.6216 \\
17 \\
18 \\
18 \\
19\end{array}$ & \\
\hline $\begin{array}{l}55 \\
56 \\
57 \\
58 \\
59\end{array}$ & $\begin{array}{l}57 \\
57 \\
56 \\
55 \\
55\end{array}$ & $\begin{array}{r}38 \\
35 \\
33 \\
31 \\
29\end{array}$ & $\begin{array}{l}4152 \\
4300 \\
4447 \\
4594 \\
4740\end{array}$ & $\begin{array}{r}36 \\
51 \\
65 \\
80 \\
1.6294\end{array}$ & $\begin{array}{l}20 \\
20 \\
21 \\
22 \\
22\end{array}$ & . \\
\hline 60 & 8.5097154 & 8.5126427 & 0.34885 . & 1.6308 & 5.6223 & 7. 229 \\
\hline
\end{tabular}


TABLE 20.-Geodetic position computations-Continued.

LATITUDE $5^{\circ}$.

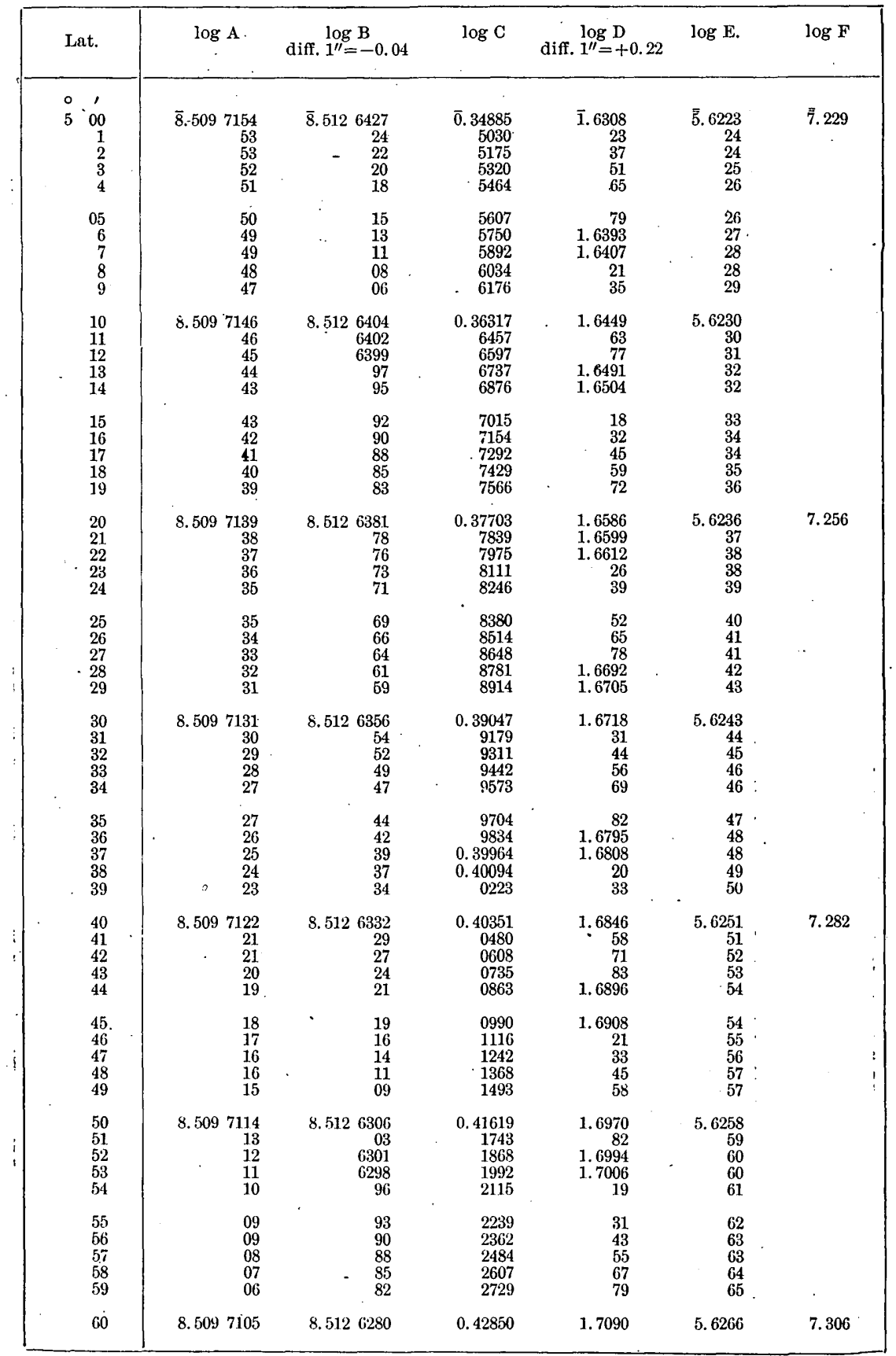


TABLA 20.-Geodetic position computations-Continued.

LATITUDE $6^{\circ}$.

\begin{tabular}{|c|c|c|c|c|c|c|}
\hline Lat. & $\begin{array}{c}\log \mathrm{A} \\
\text { diff. } 1^{\prime \prime}=-0.02\end{array}$ & $\begin{array}{c}\log B \\
\text { diff. } 1^{\prime \prime}=-0.05\end{array}$ & $\log C$ & $\underset{\text { diff. }}{\log { }^{\prime \prime}=+}+\underset{+0.18}{D}$ & $\log E$ & $\log F$ \\
\hline $\begin{array}{cc}0 & 1 \\
6 & 00 \\
& 1 \\
& \\
& 2 \\
& 3 \\
& 4\end{array}$ & $\begin{array}{r}\overline{8} .5097105 \\
04 \\
03 \\
02 \\
01\end{array}$ & $\begin{array}{r}\overline{8} .512 \quad 6280 \\
77 \\
74 \\
72 \\
69\end{array}$ & $\begin{array}{r}\overline{0} .42850 \\
2972 \\
-\quad 3093 \\
-\quad 3213 \\
3334\end{array}$ & $\begin{array}{r}1.7090 \\
7102 \\
14 \\
26 \\
38\end{array}$ & $\begin{array}{r}\text { J. } 6266 \\
67 \\
67 \\
68 \\
69\end{array}$ & 7.306 \\
\hline $\begin{array}{r}05 \\
6 \\
7 \\
8 \\
9\end{array}$ & $\begin{array}{r}01 \\
7100 \\
7099 \\
98 \\
97\end{array}$ & $\begin{array}{l}66 \\
64 \\
61 \\
58 \\
55\end{array}$ & $\begin{array}{l}3454 \\
3573 \\
3693 \\
3812 \\
3931\end{array}$ & $\begin{array}{r}50 \\
61 \\
73 \\
85 \\
1.7196\end{array}$ & $\begin{array}{l}70 \\
70 \\
71 \\
72 \\
73\end{array}$ & . \\
\hline $\begin{array}{l}10 \\
11 \\
12 \\
13 \\
14\end{array}$ & $\begin{array}{r}8.5097096 \\
95 \\
94 \\
93 \\
92\end{array}$ & $\begin{array}{r}8.512 \quad 6253 \\
50 \\
\quad 47 \\
44 \\
42\end{array}$ & $\begin{array}{r}0.44049 \\
4167 \\
4285 \\
4402 \\
4519\end{array}$ & $\begin{array}{r}1.7208 \\
19 \\
31 \\
42 \\
54\end{array}$ & $\begin{array}{r}5.6274 \\
74 \\
75 \\
76 \\
77\end{array}$ & \\
\hline $\begin{array}{l}15 \\
16 \\
17 \\
18 \\
19\end{array}$ & $\begin{array}{l}91 \\
91 \\
90 \\
89 \\
88\end{array}$ & $\begin{array}{l}39 \\
36 \\
33 \\
31 \\
28\end{array}$ & $\begin{array}{l}4636 \\
4753 \\
4869 \\
4985 \\
5101\end{array}$ & $\begin{array}{r}65 \\
76 \\
88 \\
1.7299 \\
\quad 1.7310\end{array}$ & $\begin{array}{l}78 \\
78 \\
79 \\
80 \\
81\end{array}$ & \\
\hline $\begin{array}{l}20 \\
21 \\
22 \\
23 \\
24\end{array}$ & $\begin{array}{r}8.5097087 \\
86 \\
85 \\
84 \\
83\end{array}$ & $\begin{array}{r}8.512 \quad 6225 \\
22 \\
19 \\
16 \\
14\end{array}$ & $\begin{array}{r}0.45216 \\
5331 \\
5446 \\
5560 \\
5674\end{array}$ & $\begin{array}{r}1.7322 \\
33 \\
44 \\
55 \\
66\end{array}$ & $\begin{array}{r}5.6282 \\
83 \\
83 \\
84 \\
85\end{array}$ & 7. 329 \\
\hline $\begin{array}{l}25 \\
26 \\
27 \\
28 \\
29\end{array}$ & $\begin{array}{l}82 \\
81 \\
80 \\
79 \\
78\end{array}$ & $\begin{array}{r}11 \\
08 \\
05 \\
6202 \\
6199\end{array}$ & $\begin{array}{r}5788 \\
5902 \\
6015 \\
6128 \\
6241\end{array}$ & $\begin{array}{r}78 \\
1.7389 \\
1.7400 \\
. \quad 11 \\
22\end{array}$ & $\begin{array}{l}86 \\
87 \\
88 \\
88 \\
89\end{array}$ & \\
\hline $\begin{array}{l}30 \\
31 \\
32 \\
33 \\
34\end{array}$ & $\begin{array}{r}8.5097077 \\
76 \\
75 \\
74 \\
73\end{array}$ & $\begin{array}{r}8: 512 \quad 6196 \\
94 \\
91 \\
88 \\
85\end{array}$ & $\begin{array}{r}0.46353 \\
6465 \\
6577 \\
6689 \\
6800\end{array}$ & $\begin{array}{r}1.7433 \\
44 \\
54 \\
65 \\
76\end{array}$ & $\begin{array}{r}5.6290 \\
91 \\
92 \\
93 \\
93\end{array}$ & \\
\hline $\begin{array}{l}35 \\
36 \\
37 \\
38 \\
39\end{array}$ & $\begin{array}{l}72 \\
71 \\
70 \\
70 \\
69\end{array}$ & $\begin{array}{l}82 \\
79 \\
76 \\
73 \\
70\end{array}$ & $\begin{array}{l}6911 \\
7022 \\
7132 \\
7742 \\
7352\end{array}$ & $\begin{array}{r}87 \\
1.7498 \\
1.7508 \\
19 \\
30\end{array}$ & $\begin{array}{l}94 \\
95 \\
96 \\
97 \\
98\end{array}$ & \\
\hline $\begin{array}{r}40 \\
41 \\
42 \\
43 \\
.44\end{array}$ & $\begin{array}{rr}8.5097068 \\
6 \\
66 \\
65 \\
65 \\
64\end{array}$ & $\begin{array}{r}8.5126167 \\
64 \\
61 \\
58 \\
55\end{array}$ & $\begin{array}{r}0.47462 \\
7571 \\
7681 \\
7789 \\
7898\end{array}$ & $\begin{array}{r}1.7541 \\
51 \\
62 \\
73 \\
83\end{array}$ & $\begin{array}{r}5.6299 \\
5.6299 \\
5.6300 \\
01 \\
02\end{array}$ & 7.351 \\
\hline $\begin{array}{l}45 \\
46 \\
47 \\
48 \\
49\end{array}$ & $\begin{array}{l}63 \\
62 \\
61 \\
60 \\
59\end{array}$ & $\begin{array}{l}52 \\
49 \\
46 \\
43 \\
40\end{array}$ & $\begin{array}{l}8006 \\
8114 \\
8222 \\
8330 \\
8437\end{array}$ & $\begin{array}{r}1.7594 \\
1.7604 \\
15 \\
25 \\
\mathbf{3 6}\end{array}$ & $\begin{array}{l}03 \\
04 \\
05 \\
06 \\
06\end{array}$ & \\
\hline $\begin{array}{l}50 . \\
51 \\
52 \\
53 \\
54\end{array}$ & $\begin{array}{r}8.5097058 \\
57 \\
56 \\
55 \\
53\end{array}$ & $\begin{array}{r}\text { s. } 5126137 \\
34 \\
31 \\
28 \\
25\end{array}$ & $\begin{array}{r}0.48544 \\
8651 \\
8757 \\
8864 \\
8970\end{array}$ & $\begin{array}{r}1.7646 \\
56 \\
67 \\
77 \\
87\end{array}$ & $\begin{array}{r}5.6207 \\
08 \\
09 \\
10 \\
11\end{array}$ & \\
\hline $\begin{array}{l}55 \\
56 \\
57 \\
58 \\
59\end{array}$ & $\begin{array}{l}52 \\
51 \\
50 \\
49 \\
48\end{array}$ & $\begin{array}{l}22 \\
19 \\
16 \\
13 \\
10\end{array}$ & $\begin{array}{l}9075 \\
9181 \\
9286 \\
9391 \\
9496\end{array}$ & $\begin{array}{r}1.7698 \\
1.7708 \\
18 \\
28 \\
38\end{array}$ & $\begin{array}{l}12 \\
13 \\
13 \\
1.4 \\
15\end{array}$ & \\
\hline 60 & 8.5097047 & 8. 51.26107 & 0.49600 & 1.7749 & 5.6216 & 7.371 \\
\hline
\end{tabular}


TaBLe 20.-Geodetic position computations-Continned.

LATITUDE $\overline{\bar{\gamma}}^{\circ}$.

\begin{tabular}{|c|c|c|c|c|c|c|}
\hline Lat. & $\begin{array}{c}\log \mathrm{A} \\
\text { diff. } 1^{\prime \prime}=-0.02\end{array}$ & $\begin{array}{c}\log \mathrm{B} \\
\text { diff. } 1^{\prime \prime}=-0.06\end{array}$ & $\log \mathrm{C}$ & $\begin{array}{c}\log D \\
\text { diff. } 1^{\prime \prime}=+0.16\end{array}$ & $\log \mathbf{E}$ & $\log \mathrm{F}$ \\
\hline $\begin{array}{rc}\circ & 1 \\
7 & 00 \\
& 1 \\
& 2 \\
& 3 \\
& \\
& 4\end{array}$ & $\begin{array}{r}\overline{8} .509 \quad 7047 \\
46 \\
45 \\
44 \\
43\end{array}$ & $\begin{array}{r}\overline{8} .512 \quad 6107 \\
03 \\
6100 \\
6097 \\
94\end{array}$ & $\begin{array}{r}\overline{0} .49600 \\
705 \\
809 \\
0.49913 \\
0.50016\end{array}$ & $\begin{array}{r}\text { I. } 7749 \\
59 \\
69 \\
79 \\
89\end{array}$ & $\begin{array}{r}\overline{\overline{5}} .6316 \\
17 \\
18 \\
19 \\
20\end{array}$ & $\overline{7} .371$ \\
\hline $\begin{array}{r}05 \\
6 \\
7 \\
8 \\
9\end{array}$ & $\begin{array}{l}42 \\
41 \\
40 \\
39 \\
38\end{array}$ & $\begin{array}{l}91 . \\
88 \\
85 \\
82 \\
78\end{array}$ & $\begin{array}{r}119 \\
222 \\
325 \\
428 \\
530\end{array}$ & $\begin{array}{r}1.7799 \\
1.7809 \\
19 \\
29 \\
39\end{array}$ & $\begin{array}{l}21 \\
22 \\
23 \\
23 \\
24\end{array}$ & \\
\hline $\begin{array}{l}10 \\
11 \\
12 \\
13 \\
1.4\end{array}$ & $\begin{array}{r}8.5097037 \\
36 \\
35 . \\
34 \\
33\end{array}$ & $\begin{array}{r}8.5126075 \\
72 \\
69 \\
66 \\
62\end{array}$ & $\begin{array}{r}0.50632 \\
734 \\
836 \\
0.50937 \\
0.51 .039\end{array}$ & $\begin{array}{r}1.7849 \\
59 \\
68 \\
78 \\
88\end{array}$ & $\begin{array}{r}5.6325 \\
26 \\
27 \\
28 \\
29\end{array}$ & \\
\hline $\begin{array}{l}1.5 \\
16 \\
1.7 \\
18 \\
19\end{array}$ & $\begin{array}{l}32 \\
30 \\
29 \\
28 \\
27\end{array}$ & $\begin{array}{l}59 \\
56 \\
53 \\
50 \\
46\end{array}$ & $\begin{array}{l}140 \\
240 \\
341 \\
441 \\
541\end{array}$ & $\begin{array}{r}1.7898 \\
1.7908 \\
17 \\
27 \\
37\end{array}$ & $\begin{array}{l}30 \\
31 \\
32 \\
33 \\
34\end{array}$ & \\
\hline $\begin{array}{l}20 \\
21 \\
22 \\
23 \\
24\end{array}$ & $\begin{array}{r}8.5097026 \\
25 \\
24 \\
23 \\
22\end{array}$ & $\begin{array}{r}8.512 \quad 6043 \\
40 \\
37 \\
33 \\
30\end{array}$ & $\begin{array}{r}0.51641 \\
741 \\
840 \\
0.51939 \\
0.52038\end{array}$ & $\begin{array}{r}1.7946 \\
56 \\
66 \\
75 \\
85\end{array}$ & $\begin{array}{r}5.6335 \\
36 \\
37 \\
37 \\
.38\end{array}$ & 7. 391 \\
\hline $\begin{array}{l}25 \\
26 \\
27 \\
28 \\
29\end{array}$ & $\begin{array}{l}21 \\
20 \\
19 \\
17 \\
16\end{array}$ & $\begin{array}{r}27 \\
23 \\
20 \\
. \quad 17 \\
14\end{array}$ & $\begin{array}{l}137 \\
236 \\
334 \\
432 \\
530\end{array}$ & $\begin{array}{r}1.7994 \\
1.8004 \\
13 \\
23 \\
32\end{array}$ & $\begin{array}{l}39 \\
40 \\
41 \\
42 \\
43\end{array}$ & \\
\hline $\begin{array}{r}30 \\
-\quad 31 \\
32 \\
35 \\
34\end{array}$ & $\begin{array}{r}8.5097015 \\
14 \\
13 \\
12 \\
11\end{array}$ & $\begin{array}{r}8.512 \times 6010 \\
07 \\
04 \\
6000 \\
5997\end{array}$ & $\begin{array}{r}0.52628 \\
725 \\
822 \\
0.52919 \\
0.53016\end{array}$ & $\begin{array}{r}1.8042 \\
51 \\
61 \\
70 \\
79\end{array}$ & $\begin{array}{r}5.6344 \\
45 \\
46 \\
47 \\
48\end{array}$ & \\
\hline $\begin{array}{l}35 \\
36 \\
37 \\
38 \\
39\end{array}$ & $\begin{array}{l}10 \\
09 \\
07 \\
06 \\
05\end{array}$ & $\begin{array}{l}94 \\
90 \\
87 \\
83 \\
80\end{array}$ & $\begin{array}{l}113 \\
209 \\
306 \\
402 \\
497\end{array}$ & $\begin{array}{r}89 \\
1.8098 \\
1.8107 \\
17 \\
26\end{array}$ & $\begin{array}{l}49 \\
50 \\
51 \\
52 \\
53\end{array}$ & \\
\hline $\begin{array}{l}40 \\
41 \\
42 \\
43 \\
44\end{array}$ & $\begin{array}{r}8.5097004 \\
03 \\
02 \\
01 \\
7000\end{array}$ & $\begin{array}{r}8.5125977 \\
73 \\
. \quad 70 \\
66 \\
63\end{array}$ & $\begin{array}{r}0.53593 \\
688 \\
784 \\
879 \\
0.53973\end{array}$ & $\begin{array}{r}1.8135 \\
44 \\
53 \\
63 \\
72\end{array}$ & $\begin{array}{r}5.6354 \\
+55 \\
56 \\
57 \\
58\end{array}$ & 7.409 \\
\hline $\begin{array}{l}45 \\
46 \\
47 \\
48 \\
49\end{array}$ & $\begin{array}{r}6998 \\
97 \\
96 \\
95 \\
94\end{array}$ & $\begin{array}{l}60 \\
56 \\
53 \\
49 \\
46\end{array}$ & $\begin{array}{r}0.54068 \\
162 \\
257 \\
351 \\
444\end{array}$ & $\begin{array}{r}81 \\
90 \\
1.8199 \\
1.8208 \\
17\end{array}$ & $\begin{array}{l}59 \\
60 \\
61 \\
62 \\
63\end{array}$ & \\
\hline $\begin{array}{l}50 \\
51 \\
52 \\
53 \\
54\end{array}$ & $\begin{array}{r}8.509 \quad 6993 \\
\dot{y l} \\
90 \\
89 \\
88\end{array}$ & $\begin{array}{r}8.512 \quad 5942 \\
39 \\
35 \\
32 \\
28\end{array}$ & $\begin{array}{r}0.545338 \\
631 \\
725 \\
818 \\
0.54911\end{array}$ & $\begin{array}{r}1.8226 \\
35 \\
44 \\
53 \\
62\end{array}$ & $\begin{array}{r}5.6364 \\
65 \\
66 \\
67 \\
68\end{array}$ & $\therefore$ \\
\hline $\begin{array}{l}55 \\
56 \\
57 \\
58 \\
59\end{array}$ & $\begin{array}{l}87 \\
86 \\
84 \\
83 \\
82\end{array}$ & $\begin{array}{l}25 \\
21 \\
18 \\
14 \\
11\end{array}$ & $\begin{array}{r}0.55003 \\
096 \\
188 \\
280 \\
372\end{array}$ & $\begin{array}{r}71 \\
80 \\
89 \\
1.8298 \\
1.8307\end{array}$ & $\begin{array}{l}69 \\
70 \\
71 \\
72 \\
73\end{array}$ & \\
\hline 60 & 8. 5096981 & 8.5125907 & 0.55464 & 1.8315 & 5.6374 & 7.427 \\
\hline
\end{tabular}


TABLe 20.-Geodetic position compulations-Continued.

LATITUDE $\mathrm{S}^{\circ}$.

\begin{tabular}{|c|c|c|c|c|c|c|}
\hline Lat. & $\begin{array}{c}\log \Lambda \\
\text { diff. } 1^{\prime \prime}=-0.02\end{array}$ & diff. $1^{\log B}=-0.06$ & $\log C$ & $\begin{array}{c}\log D \\
\text { diff. } 1^{\prime \prime}=+0.14\end{array}$ & $\begin{array}{l}\log \mathrm{E} \\
\text { diff. } \mathrm{x}^{\prime \prime}=+0.02\end{array}$ & $\log F$ \\
\hline $\begin{array}{rc}\circ & 1 \\
8 & 00 \\
& \\
& 1 \\
& 2 \\
& 3 \\
& 4\end{array}$ & $\begin{array}{r}5.509 \quad 6981 \\
80 \\
79 \\
77 \\
76\end{array}$ & $\begin{array}{r}8.512 \quad 5907 \\
04 \\
5900 \\
5897 \\
93\end{array}$ & $\begin{array}{r}\overline{0} .55464 \\
555 \\
646 \\
738 \\
829\end{array}$ & $\begin{array}{r}1.8315 \\
24 \\
33 \\
42 \\
50\end{array}$ & $\begin{array}{r}5.6374 \\
75 \\
76 \\
77 \\
78\end{array}$ & 7.427 \\
\hline $\begin{array}{r}05 \\
6 \\
\cdot \quad 7 \\
8 \\
9\end{array}$ & $\begin{array}{l}75 \\
74 \\
73 \\
71 \\
70\end{array}$ & $\begin{array}{l}90 \\
86 \\
82 \\
79 \\
75\end{array}$ & $\begin{array}{r}0.55919 \\
0.56010 \\
100 \\
191 \\
281\end{array}$ & $\begin{array}{r}59 \\
68 \\
77 \\
85 \\
1.8394\end{array}$ & $\begin{array}{l}79 \\
80 \\
81 \\
82 \\
83\end{array}$ & \\
\hline $\begin{array}{l}10 \\
11 \\
12 \\
13 \\
14\end{array}$ & $\begin{array}{r}8.509 \quad 6969 \\
68 \\
67 \\
65 \\
64\end{array}$ & $\begin{array}{r}8.512 \quad 5872 \\
68 \\
64 \\
61 \\
\quad 57\end{array}$ & $\begin{array}{r}0.56371 \\
460 \\
550 \\
639 \\
728\end{array}$ & $\begin{array}{r}1.8403 \\
.12 \\
20 \\
28 \\
37\end{array}$ & $\begin{array}{r}5.6384 \\
85 \\
86 \\
87 \\
88\end{array}$ & \\
\hline $\begin{array}{l}15 \\
16 \\
17 \\
18 \\
19\end{array}$ & $\begin{array}{l}63 \\
62 \\
61 \\
59 \\
58\end{array}$ & $\begin{array}{l}54 \\
50 \\
46 \\
43 \\
39\end{array}$ & $\begin{array}{r}817 \\
906 \\
0.56995 \\
0.57083 \\
172\end{array}$ & $\begin{array}{l}45 \\
54 \\
62 \\
71 \\
79\end{array}$ & $\begin{array}{l}90^{\circ} \\
91 \\
92 \\
93 \\
94\end{array}$ & \\
\hline $\begin{array}{l}20 \\
21 \\
22 \\
23 \\
24\end{array}$ & $\begin{array}{r}8.509 \quad 6957 \\
56 \\
54 \\
53 \\
\tilde{6} 2\end{array}$ & $\begin{array}{r}8.512 \quad 5835 \\
32 \\
28 \\
24 \\
20\end{array}$ & $\begin{array}{r}0.5: 260 \\
348 \\
436 \\
523 \\
611\end{array}$ & $\begin{array}{r}1.8<88 \\
1.8496 \\
1.8605 \\
13 \\
21\end{array}$ & ร. 1 $\begin{array}{r}95 \\
96 \\
97 \\
98 \\
99\end{array}$ & 7.444 \\
\hline $\begin{array}{l}25 \\
26 \\
27 \\
28 \\
29\end{array}$ & $\begin{array}{l}51 \\
49 \\
48 \\
47 \\
46\end{array}$ & $\begin{array}{r}17 \\
13 \\
09 \\
06 \\
5802\end{array}$ & $\begin{array}{r}698 \\
785 \\
872 \\
0.57959 \\
0.58045\end{array}$ & $\begin{array}{l}30 \\
38 \\
46 \\
55 \\
63\end{array}$ & $\begin{array}{r}\text { 5. } 6400 \\
\text { 5. } 6401 \\
02 . \\
03 \\
04\end{array}$ & \\
\hline $\begin{array}{l}30 \\
31 \\
32 \\
33 \\
34\end{array}$ & $\begin{array}{r}8.5096945 \\
43 \\
42 \\
41 \\
39\end{array}$ & $\begin{array}{r}8.5125798 \\
94 \\
91 \\
87 \\
83\end{array}$ & $\begin{array}{r}0.5813 .2 \\
218 \\
304 \\
390 \\
476\end{array}$ & $\begin{array}{r}1.8571 \\
. \quad 80 \\
88 \\
1.8596 \\
1.8604\end{array}$ & $\begin{array}{r}5.6406 \\
07 \\
08 \\
09 \\
10\end{array}$ & \\
\hline $\begin{array}{l}35 \\
36 \\
37 \\
38 \\
39\end{array}$ & $\begin{array}{l}38 \\
37 \\
36 \\
34 \\
33\end{array}$ & $\begin{array}{l}79 \\
75 \\
72 \\
68 \\
64\end{array}$ & $\begin{array}{l}562 \\
647 \\
732 \\
818 \\
903\end{array}$ & $\begin{array}{r}13 \\
21 \\
\quad 29 \\
-\quad 37 \\
45\end{array}$ & $\begin{array}{l}11 \\
12 \\
13 \\
14 \\
15\end{array}$ & \\
\hline $\begin{array}{l}40 \\
41 \\
42 \\
43 \\
44\end{array}$ & $\begin{array}{r}8.509 \quad 6932 \\
31 \\
29 \\
28 \\
27\end{array}$ & $\begin{array}{r}8.512 \quad 5760 \\
56 \\
53 \\
49 \\
45\end{array}$ & $\begin{array}{r}0.58987 \\
0.59072 \\
157 \\
241 \\
325\end{array}$ & $\begin{array}{r}1.8653 \\
61 \\
69 \\
77 \\
85\end{array}$ & $\begin{array}{r}5.6416 \\
18 \\
19 \\
20 \\
21\end{array}$ & 7. 461 \\
\hline $\begin{array}{l}45 \\
46 \\
47 \\
48 \\
49\end{array}$ & $\begin{array}{l}25 \\
24 \\
23 \\
22 \\
20\end{array}$ & $\begin{array}{l}41 \\
37 \\
33 \\
29 \\
26\end{array}$ & $\begin{array}{r}409 \\
-493 \\
\cdot \quad 577 \\
660 \\
744\end{array}$ & $\begin{array}{r}1.8693 \\
1.8701 \\
09 \\
17 \\
25\end{array}$ & $\begin{array}{l}22 \\
23 \\
24 \\
25 \\
26\end{array}$ & \\
\hline $\begin{array}{l}50 \\
51 \\
52 \\
53 \\
54\end{array}$ & $\begin{array}{r}8.5096919 \\
18 \\
16 \\
15 \\
. \quad 14\end{array}$ & $\begin{array}{r}8.512 \quad 5722 \\
18 \\
14 \\
10 \\
06\end{array}$ & $\begin{array}{r}0.59827 \\
910 \\
0.59993 \\
0.60076 \\
159\end{array}$ & $\begin{array}{r}1.8733 \\
41 \\
49 \\
57 \\
65\end{array}$ & $\begin{array}{r}5.6428 \\
29 \\
30 \\
31 \\
32\end{array}$ & \\
\hline $\begin{array}{l}55 \\
56 \\
57 \\
58 \\
59\end{array}$ & $\begin{array}{l}12 \\
11 \\
10 \\
09 \\
07\end{array}$ & $\begin{array}{r}5702 \\
5698 \\
94 \\
90 \\
86\end{array}$ & $\begin{array}{r}241 \\
324 \\
406 \\
488 \\
570\end{array}$ & $\begin{array}{r}73 \\
81 \\
89 \\
1.8796 \\
1.8804\end{array}$ & $\begin{array}{l}33 \\
34 \\
35 \\
37 \\
38\end{array}$ & \\
\hline 60 & 8.5096906 & $8.512 \quad 3682$ & 0.60652 & 1.8812 & 5. 6439 & 7.476 \\
\hline
\end{tabular}


TABLE 20.-Geodetic position computaiions-Continued.

LATTTUDE $9^{\circ}$.

\begin{tabular}{|c|c|c|c|c|c|c|}
\hline Lat. & $\begin{array}{c}\log \mathrm{A} \\
\text { diff. } 1^{\prime \prime}=-0.02\end{array}$ & diff. $1^{\prime \prime}=-0.07$ & $\log \mathrm{C}$ & $\log _{\mathrm{D}} \mathrm{l}$ & $\begin{array}{c}\log \mathbf{E} \\
\text { diff. } 1^{\prime \prime}=+0.02\end{array}$ & $\log \mathbf{F}$ \\
\hline \begin{tabular}{cc}
$\circ$ & \multicolumn{1}{c}{} \\
9 & 00. \\
& 1 \\
& 2 \\
& 3 \\
& 4
\end{tabular} & $\begin{array}{r}\overline{8} .5096906 \\
05 \\
03 \\
02 \\
6901\end{array}$ & $\begin{array}{r}\overline{8} .5125682 \\
78 \\
74 \\
70 \\
66\end{array}$ & $\begin{array}{r}0.60652 \\
733 \\
815 \\
896 \\
0.60977\end{array}$ & $\begin{array}{r}\overline{1 .} 8812 \\
20 \\
27 \\
35 \\
43\end{array}$ & $\begin{array}{r}\overline{\overline{0}} .6439 \\
40 \\
41 \\
42 \\
44\end{array}$ & 7.476 \\
\hline $\begin{array}{r}05 \\
6 \\
7 \\
8 \\
9\end{array}$ & $\begin{array}{r}6899 \\
98 \\
97 \\
95 \\
94\end{array}$ & $\begin{array}{l}62 \\
58 \\
54 \\
50 \\
46\end{array}$ & $\begin{array}{r}0.61058 \\
139 \\
220 \\
301 \\
881\end{array}$ & $\begin{array}{l}51 \\
58 \\
66 \\
74 \\
81\end{array}$ & $\begin{array}{l}45 \\
46 \\
47 \\
48 \\
49\end{array}$ & \\
\hline $\begin{array}{l}10 \\
11 \\
12 \\
13 \\
14\end{array}$ & $\begin{array}{r}8.5096893 \\
91 \\
90 \\
89 \\
87\end{array}$ & $\begin{array}{r}8.5125642 \\
38 \\
34 \\
. \quad 30 \\
26\end{array}$ & $\begin{array}{r}0.61461 \\
542 \\
622 \\
702 \\
781\end{array}$ & $\begin{array}{r}1.8889 \\
1.8897 \\
1.8904 \\
12 \\
19\end{array}$ & $\begin{array}{r}5450 \\
52 \\
53 \\
54 \\
55\end{array}$ & \\
\hline $\begin{array}{l}15 \\
16 \\
17 \\
18 \\
19\end{array}$ & $\begin{array}{l}86 \\
84 \\
83 \\
82 \\
80\end{array}$ & $\begin{array}{l}22 \\
18 \\
14 \\
10 \\
06\end{array}$ & $\begin{array}{r}861 \\
0.61941 \\
0.62020 \\
099 \\
178\end{array}$ & $\begin{array}{l}27 \\
34 \\
42 \\
50 \\
57\end{array}$ & $\begin{array}{l}56 \\
57 \\
59 \\
60 \\
61 .\end{array}$ & \\
\hline $\begin{array}{l}20 \\
21 \\
22 \\
23 \\
24\end{array}$ & $\begin{array}{r}8.5096879 \\
78 \\
76 \\
75 \\
74\end{array}$ & $\begin{array}{r}8.5125602 \\
5598 \\
93 \\
89 \\
85\end{array}$ & $\begin{array}{r}0.62257 \\
336 \\
415 \\
493 \\
572\end{array}$ & $\begin{array}{r}1.8964 \\
72 \\
79 \\
87 \\
1.8994\end{array}$ & $\begin{array}{r}5.6462 \\
63 \\
65 \\
66 \\
67\end{array}$ & 7.490 \\
\hline $\begin{array}{l}25 \\
26 \\
27 \\
28 \\
29\end{array}$ & $\begin{array}{l}72 \\
71 \\
69 \\
68 \\
67\end{array}$ & $\begin{array}{l}81 \\
77 \\
73 \\
69 \\
64\end{array}$ & $\begin{array}{r}650 \\
728 \\
806 \\
884 \\
0.62962\end{array}$ & $\begin{array}{r}1.9002 \\
09 \\
17 \\
24 \\
31\end{array}$ & $\begin{array}{l}68 \\
69 \\
70 \\
72 \\
73\end{array}$ & \\
\hline $\begin{array}{l}30 \\
31 \\
32 \\
33 \\
34\end{array}$ & $\begin{array}{r}8.5096865 \\
64 \\
62 \\
61 \\
60\end{array}$ & $\begin{array}{r}8.5125560 \\
56 \\
52 \\
48 \\
43\end{array}$ & $\begin{array}{r}0.63039 \\
117 \\
194 \\
271 \\
349\end{array}$ & $\begin{array}{r}\text { 1. } 9039 \\
46 \\
53 \\
61 \\
68\end{array}$ & $\begin{array}{r}5.6474 \\
75 \\
76 \\
78 \\
79\end{array}$ & 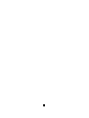 \\
\hline $\begin{array}{l}\mathbf{3 5} \\
36 \\
37 \\
38 \\
39\end{array}$ & $\begin{array}{l}58 \\
57 \\
55 \\
54 \\
53\end{array}$ & $\begin{array}{l}39 \\
35 \\
31 \\
27 \\
22\end{array}$ & $\begin{array}{r}426 \\
502 \\
579 \\
656 \\
732\end{array}$ & $\begin{array}{r}75 \\
82 \\
90 \\
1.9097 \\
1.9104\end{array}$ & $\begin{array}{l}80 \\
81 \\
83 \\
84 \\
85\end{array}$ & \\
\hline $\begin{array}{l}40 \\
41 \\
42 \\
43 \\
44\end{array}$ & $\begin{array}{r}8.5096851 \\
50 \\
48 \\
47 \\
45\end{array}$ & $\begin{array}{r}8.5125518 \\
14 \\
10 \\
05 \\
5501\end{array}$ & $\begin{array}{r}0.63808 \\
885 \\
0.63961 \\
0.64037 \\
112\end{array}$ & $\begin{array}{r}1.9111 \\
19 \\
26 \\
33 \\
40\end{array}$ & $\begin{array}{r}5.6486 \\
87 \\
89 \\
90 \\
91\end{array}$ & 7.505 \\
\hline $\begin{array}{l}45 \\
46 \\
47 \\
48 \\
49\end{array}$ & $\begin{array}{l}44 \\
43 \\
41 \\
40 \\
38\end{array}$ & $\begin{array}{r}5497 \\
92 \\
88 \\
84 \\
80\end{array}$ & $\begin{array}{r}188 \\
264 \\
339 \\
415 \\
490\end{array}$ & $\begin{array}{l}47 \\
54 \\
61 \\
69 \\
76\end{array}$ & $\begin{array}{l}92 \\
94 \\
95 \\
96 \\
97\end{array}$ & \\
\hline $\begin{array}{r}50 \\
51 \\
52 \\
53 \\
54\end{array}$ & $\begin{array}{r}8.5096837 \\
35 \\
34 \\
33 \\
31\end{array}$ & $\begin{array}{r}8.5125475 \\
71 \\
67 \\
62 \\
58\end{array}$ & $\begin{array}{r}0.64565 \\
640 \\
715 \\
789 \\
864\end{array}$ & $\begin{array}{r}1.9188 \\
90 \\
1.9197 \\
1.9204 \\
11\end{array}$ & $\begin{array}{r}5.6498 \\
5.6500 \\
01 \\
02 \\
03\end{array}$ & . \\
\hline $\begin{array}{l}55 \\
56 \\
57 \\
58 \\
59\end{array}$ & $\begin{array}{r}30 \\
-\quad 28 \\
27 \\
25 \\
24\end{array}$ & $\begin{array}{l}54 \\
49 \\
45 \\
40 \\
36\end{array}$ & $\begin{array}{r}0.64938 \\
0.65013 \\
087 \\
161 \\
235\end{array}$ & $\begin{array}{l}18 \\
25 \\
32 \\
39 \\
46\end{array}$ & $\begin{array}{l}05 \\
06 \\
07 \\
08 \\
10\end{array}$ & . \\
\hline 60 & 8.5096822 & 8.5125432 & 0.65309 & 1.9253 & 5. 6411 & 7.518 \\
\hline
\end{tabular}


TaвLe 20.-Geodetic position computations-Continued.

LATITUDE $10^{\circ}$.

\begin{tabular}{|c|c|c|c|c|c|c|c|}
\hline \multicolumn{2}{|c|}{ Lat. } & diff. $1^{\prime \prime}=-0.08$ & $\begin{array}{l}\log B \\
\text { diff. } 1^{\prime \prime}=-0.08\end{array}$ & $\log C$ & $\begin{array}{c}\log \mathrm{D} \\
\operatorname{diff.} 1^{\prime \prime}=+0.11\end{array}$ & $\begin{array}{c}\log \mathrm{E} \\
\text { diff. } 1^{\prime \prime}=+0.02\end{array}$ & $\log F$ \\
\hline$\stackrel{\circ}{10}$ & $\begin{array}{r}1 \\
00 \\
1 \\
2 \\
3 \\
4\end{array}$ & $\begin{array}{r}\overline{8} .5096822 \\
21 \\
19 \\
18 \\
17\end{array}$ & $\begin{array}{r}\overline{8} .512 \quad 5432 \\
27 \\
23 \\
19 \\
14\end{array}$ & $\begin{array}{r}0.65309 \\
383 \\
456 \\
530 \\
603\end{array}$ & $\begin{array}{r}\text { 1. } 9253 \\
60 \\
67 \\
74 \\
80\end{array}$ & $\begin{array}{r}5.6511 \\
12 \\
13 \\
15 \\
16\end{array}$ & \multirow[t]{2}{*}{7.518} \\
\hline & $\begin{array}{r}05 \\
6 \\
7 \\
8 \\
9\end{array}$ & $\begin{array}{r}15 \\
14 \\
12 \\
11 \\
9\end{array}$ & $\begin{array}{r}10 \\
05 \\
5401 \\
5396 \\
92\end{array}$ & $\begin{array}{r}677 \\
750 \\
823 \\
896 \\
0.65968\end{array}$ & $\begin{array}{r}87 \\
1.9294 \\
1.9301 \\
08 \\
15\end{array}$ & $\begin{array}{l}17 \\
18 \\
20 \\
21 \\
22\end{array}$ & \\
\hline & $\begin{array}{l}10 . \\
11 \\
12 \\
13 \\
14\end{array}$ & $\begin{array}{r}8.5096808 \\
06 \\
05 \\
03 \\
02\end{array}$ & $\begin{array}{r}8.512 \quad 5388 \\
83 \\
79 \\
74 \\
70\end{array}$ & $\begin{array}{r}0.66041 \\
114 \\
186 \\
259 \\
331\end{array}$ & $\begin{array}{r}1.9322 \\
28 \\
35 \\
42 \\
49\end{array}$ & $\begin{array}{r}5.6524 \\
25 \\
26 \\
27 \\
29\end{array}$ & \\
\hline & $\begin{array}{l}1.5 \\
16 \\
17 \\
18 \\
19\end{array}$ & $\begin{array}{r}6800 \\
6799 \\
97 \\
96 \\
94\end{array}$ & $\begin{array}{l}65 \\
61 \\
56 \\
52 \\
47\end{array}$ & $\begin{array}{l}403 \\
475 \\
547 \\
619 \\
691\end{array}$ & $\begin{array}{l}56 \\
62 \\
69 \\
76 \\
82\end{array}$ & $\begin{array}{l}30 \\
31 \\
33 \\
34 \\
35\end{array}$ & \\
\hline & $\begin{array}{l}20 \\
21 \\
22 \\
23 \\
24\end{array}$ & $\begin{array}{r}8.509 \quad 6793 \\
91 \\
90 \\
88 \\
87\end{array}$ & $\begin{array}{r}8.5125313 \\
38 \\
33 \\
29 \\
24\end{array}$ & $\begin{array}{r}0.66762 \\
834 \\
905 \\
0.66976 \\
0.67047\end{array}$ & $\begin{array}{r}1.9389 \\
1.9396 \\
1.9403 \\
09 \\
16\end{array}$ & $\begin{array}{r}5.6536 \\
38 \\
39 \\
40 \\
42\end{array}$ & 7.532 \\
\hline & $\begin{array}{l}25 \\
26 \\
27 \\
28 \\
29\end{array}$ & $\begin{array}{r}85 \\
84 \\
82 \\
81 \\
79\end{array}$ & $\begin{array}{r}20 \\
15 \\
11 \\
06 \\
5302\end{array}$ & $\begin{array}{l}118 \\
189 \\
260 \\
331 \\
401\end{array}$ & $\begin{array}{l}23 \\
29 \\
36 \\
42 \\
49\end{array}$ & $\begin{array}{l}43 \\
44 \\
46 \\
47 \\
48\end{array}$ & \\
\hline & $\begin{array}{l}30 \\
31 \\
32 \\
33 \\
34\end{array}$ & $\begin{array}{r}8.5096777 \\
76 \\
74 \\
73 \\
71\end{array}$ & $\begin{array}{r}\text { S. } 512 \quad 5297 \\
92 \\
88 \\
83 \\
79\end{array}$ & $\begin{array}{r}0.67472 \\
542 \\
613 \\
683 \\
753\end{array}$ & $\begin{array}{r}1.9456 \\
62 \\
69 \\
75 \\
82\end{array}$ & $\begin{array}{r}5.6549 \\
51 \\
52 \\
53 \\
55\end{array}$ & $\cdot$ \\
\hline & $\begin{array}{l}35 \\
36 \\
37 \\
38 \\
39\end{array}$ & $\begin{array}{r}70 \\
\cdot \quad 68 \\
67 \\
65 \\
64\end{array}$ & $\begin{array}{l}74 \\
69 \\
65 \\
60 \\
55\end{array}$ & $\begin{array}{r}823 \\
893 \\
0.67962 \\
0.68032 \\
102\end{array}$ & $\begin{array}{r}88 \\
1.9495 \\
1.9501 \\
08 \\
14\end{array}$ & $\begin{array}{l}56 \\
57 \\
59 \\
60 \\
61\end{array}$ & \\
\hline & $\begin{array}{l}40 \\
41 \\
42 \\
43 \\
44\end{array}$ & $\begin{array}{r}8.5096762 \\
60 \\
59 \\
57 \\
56\end{array}$ & $\begin{array}{r}8.5125251 \\
46 \\
41 \\
37 \\
32\end{array}$ & $\begin{array}{r}0.68171 \\
240 \\
310 \\
379 \\
448\end{array}$ & $\begin{array}{r}1.9521 \\
27 \\
34 \\
40 \\
47\end{array}$ & $\begin{array}{r}5.6563 \\
64 \\
65 \\
67 \\
68\end{array}$ & 7.544 \\
\hline & $\begin{array}{l}45 \\
46 \\
47 \\
48 \\
49\end{array}$ & $\begin{array}{l}54 \\
53 \\
51 \\
50 \\
48\end{array}$ & $\begin{array}{l}27 \\
23 \\
18 \\
13 \\
08\end{array}$ & $\begin{array}{l}517 \\
586 \\
654 \\
723 \\
791 .\end{array}$ & $\begin{array}{l}53 \\
60 \\
66 \\
72 \\
79\end{array}$ & $\begin{array}{l}69 \\
71 \\
72 \\
73 \\
75\end{array}$ & \\
\hline & $\begin{array}{l}50 \\
51 \\
52 \\
53 \\
54\end{array}$ & $\begin{array}{r}8.509 \quad 6746 \\
45 \\
43 \\
42 \\
40\end{array}$ & $\begin{array}{r}8.512 \quad 5204 \\
5199 \\
94 \\
89 \\
85\end{array}$ & $\begin{array}{r}0.68860 \\
928 \\
0.68996 \\
0.69064 \\
132\end{array}$ & $\begin{array}{r}1.9585 \\
91 \\
1.9598 \\
1.9604 \\
10\end{array}$ & $\begin{array}{r}5.6576 \\
78 \\
79 \\
80 \\
-\quad 82\end{array}$ & \\
\hline & $\begin{array}{l}55 \\
56 \\
57 \\
58 \\
59\end{array}$ & $\begin{array}{l}38 \\
37 \\
35 \\
34 \\
32\end{array}$ & $\begin{array}{l}80 \\
75 \\
70 \\
66 \\
61\end{array}$ & $\begin{array}{l}200 \\
268 \\
336 \\
404 \\
471\end{array}$ & $\begin{array}{l}17 \\
23 \\
29 \\
36 \\
42\end{array}$ & $\begin{array}{l}83 \\
84 \\
86 \\
87 \\
88\end{array}$ & \\
\hline & 60 & 8. 5096730 & 8.5125156 & 0.69539 & 1.9648 & 5.6590 & 7.556 \\
\hline
\end{tabular}


Table 20.-Geodetic position computations-Continued.

LATITUDE $11^{\circ}$.

\begin{tabular}{|c|c|c|c|c|c|c|}
\hline Lat. & $\operatorname{diff.} 1^{\prime \prime \prime}=-0.03$ & $\begin{array}{c}\log B \\
\text { diff. } 1^{\prime \prime}=-0.08\end{array}$ & $\log \mathrm{C}$ & $\begin{array}{c}\log \mathrm{D} \\
\text { diff. } 1^{\prime \prime}=+0.10\end{array}$ & $\begin{array}{c}\log \mathrm{E} \\
\text { diff. } 1^{\prime \prime}=+0.02\end{array}$ & $\log \mathrm{F}$ \\
\hline $\begin{array}{cc}0 & , \\
11 & 00 \\
& 1 \\
& 2 \\
& 3 \\
& 4\end{array}$ & $\begin{array}{r}5.5096730 \\
29 \\
27 \\
26 \\
24\end{array}$ & $\begin{array}{r}\overline{8} .5125156 \\
51 \\
46 \\
\quad 41 \\
\quad 37\end{array}$ & $\begin{array}{r}\overline{0} .69539 \\
606 \\
673 \\
740 \\
807\end{array}$ & $\begin{array}{r}\overline{1} .9648 \\
54 \\
61 \\
67 \\
73\end{array}$ & $\begin{array}{r}\overline{\overline{5}} .6590 \\
91 \\
. \quad 93 \\
. \quad 94 \\
95\end{array}$ & $\overline{\overline{7}} .556$. \\
\hline $\begin{array}{r}05 \\
6 \\
7 \\
8 \\
9\end{array}$ & $\begin{array}{l}22 \\
21 \\
19 \\
18 \\
16\end{array}$ & $\begin{array}{l}32 \\
27 \\
22 \\
17 \\
12\end{array}$ & $\begin{array}{r}874 \\
0.69941 \\
0.70008 \\
074 \\
141\end{array}$ & $\begin{array}{r}79 \\
86 \\
92 \\
1.9698 \\
1.9704\end{array}$ & $\begin{array}{r}97 \\
98 \\
5.6599 \\
5.6601 \\
02\end{array}$ & \\
\hline $\begin{array}{l}10 \\
11 \\
12 \\
13 \\
14\end{array}$ & $\begin{array}{r}8.5096714 \\
13 \\
11 \\
09 \\
08\end{array}$ & $\begin{array}{r}8.5125108 \\
5103 \\
5098 \\
5093 \\
88\end{array}$ & $\begin{array}{r}0.70208 \\
274 \\
340 \\
406 \\
473\end{array}$ & $\begin{array}{r}1.9710 \\
16 \\
23 \\
29 \\
35\end{array}$ & $\begin{array}{r}5.6604 \\
05 \\
06 \\
08 \\
09\end{array}$ & \\
\hline $\begin{array}{l}15 \\
16 \\
17 \\
18 \\
19\end{array}$ & $\begin{array}{r}06 \\
. \quad 05 \\
\therefore \quad 03 \\
. \quad 6700\end{array}$ & $\begin{array}{l}83 \\
78 \\
73 \\
68 \\
63\end{array}$ & $\begin{array}{l}539 \\
604 \\
670 \\
736 \\
802\end{array}$ & $\begin{array}{l}41 \\
47 \\
53 \\
59 \\
65\end{array}$ & $\begin{array}{l}11 \\
12 \\
13 \\
15 \\
16\end{array}$ & \\
\hline $\begin{array}{l}20 \\
21 \\
22 \\
23 \\
24\end{array}$ & $\begin{array}{r}8.5096698 \\
96 \\
95 \\
93 \\
91\end{array}$ & $\begin{array}{r}8.5125058 \\
53 \\
49 \\
44 \\
39\end{array}$ & $\begin{array}{r}0.70867 \\
933 \\
0.70998 \\
0.71063 \\
128\end{array}$ & $\begin{array}{r}1.9771 \\
77 \\
83 \\
89 \\
1.9795\end{array}$ & $\begin{array}{r}5.6618 \\
19 \\
20 \\
22 \\
23\end{array}$ & 7.568 \\
\hline $\begin{array}{l}25 \\
26 \\
27 \\
28 \\
29\end{array}$ & $\begin{array}{l}90 \\
88 \\
86 \\
85 \\
83\end{array}$ & $\begin{array}{l}34 \\
29 \\
24 \\
19 \\
14\end{array}$ & $\begin{array}{l}194 \\
259 \\
323 \\
388 \\
453\end{array}$ & $\begin{array}{r}1.9801 . \\
07 \\
13 \\
19 \\
25\end{array}$ & $\begin{array}{l}25 \\
26 \\
27 \\
29 \\
30\end{array}$ & . \\
\hline $\begin{array}{l}30 \\
31 \\
32 \\
33 \\
34\end{array}$ & $\begin{array}{r}8.509 \quad 6681 \\
80 \\
\quad 78 \\
\quad \quad 76 \\
\end{array}$ & $\begin{array}{r}8.5125009 \\
04 \\
4999 \\
94 \\
89\end{array}$ & $\begin{array}{r}0.71518 \\
582 \\
647 \\
711 \\
\quad 775\end{array}$ & $\begin{array}{r}1.9831 \\
37 \\
43 \\
49 \\
55\end{array}$ & $\begin{array}{r}5.6632 \\
33 \\
.35 \\
36 \\
37\end{array}$ & . \\
\hline $\begin{array}{l}35 \\
36 \\
37 \\
38 \\
39\end{array}$ & 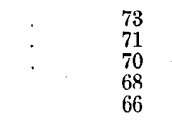 & $\begin{array}{r}83 \\
78 \\
73 \\
68 \\
63\end{array}$ & $\begin{array}{r}840 \\
904 \\
0.71968 \\
0.72032 \\
095\end{array}$ & $\begin{array}{r}61 \\
67 \\
73 \\
79 \\
85\end{array}$ & $\begin{array}{l}39 \\
40 \\
42 \\
43 \\
45\end{array}$ & \\
\hline $\begin{array}{l}40 \\
41 \\
42 \\
43 \\
44\end{array}$ & $\begin{array}{r}8.509 \quad 6665 \\
63 \\
61 \\
59 \\
58\end{array}$ & $\begin{array}{r}8.512 \quad 4958 \\
53 \\
48 \\
43 \\
38 \\
38\end{array}$ & $\begin{array}{r}0.72159 \\
223 \\
286 \\
350 \\
413\end{array}$ & $\begin{array}{r}1.9890 \\
1.9896 \\
1.9902 \\
.08 \\
14\end{array}$ & $\begin{array}{r}5.6646 \\
47 \\
49 \\
50 \\
52\end{array}$ & 7.580 \\
\hline $\begin{array}{l}45 \\
46 \\
47 \\
48 \\
49\end{array}$ & $\begin{array}{l}56 \\
54 \\
53 \\
51 \\
49\end{array}$ & $\begin{array}{l}33 \\
28 \\
22 \\
17 \\
12\end{array}$ & $\begin{array}{l}477 \\
540 \\
603 \\
666 \\
729\end{array}$ & $\begin{array}{l}20 \\
25 \\
31 \\
37 \\
43\end{array}$ & $\begin{array}{l}53 \\
55 \\
56 \\
58 \\
59\end{array}$ & \\
\hline $\begin{array}{l}50 \\
51 \\
52 \\
53 \\
54\end{array}$ & $\begin{array}{rr}8.509647 \\
\ldots \quad 46 \\
\quad 44 \\
-\quad 41\end{array}$ & $\begin{array}{r}8.5124907 \\
4902 \\
4897 \\
92 \\
86\end{array}$ & $\begin{array}{r}0.72792 \\
855 \\
918 \\
0.72980 \\
0.73043\end{array}$ & $\begin{array}{r}1.9949 \\
54 \\
60 \\
66 \\
72\end{array}$ & $\begin{array}{r}5.6661 \\
62 \\
64 \\
65 \\
66\end{array}$ & \\
\hline $\begin{array}{l}55 \\
56 \\
57 \\
58 \\
\mathbf{5 9}\end{array}$ & $\begin{array}{l}39 \\
37 \\
35 \\
34 \\
32\end{array}$ & $\begin{array}{r}81 \\
76 \\
71 \\
66 \\
60\end{array}$ & $\begin{array}{l}106 \\
168 \\
230 \\
293 \\
355\end{array}$ & $\begin{array}{r}77 \\
83 \\
89 \\
94 \\
1.9900\end{array}$ & $\begin{array}{l}68 \\
69 \\
71 \\
72 \\
74\end{array}$ & . \\
\hline 60 & 8.5096630 & 8.5124855 & 0.73417 & 2.0006 & 5.6675 & 7.591 \\
\hline
\end{tabular}


Table 20.-Geodetic position compulations-Continued.

LA'TITUDE $12^{\circ}$.

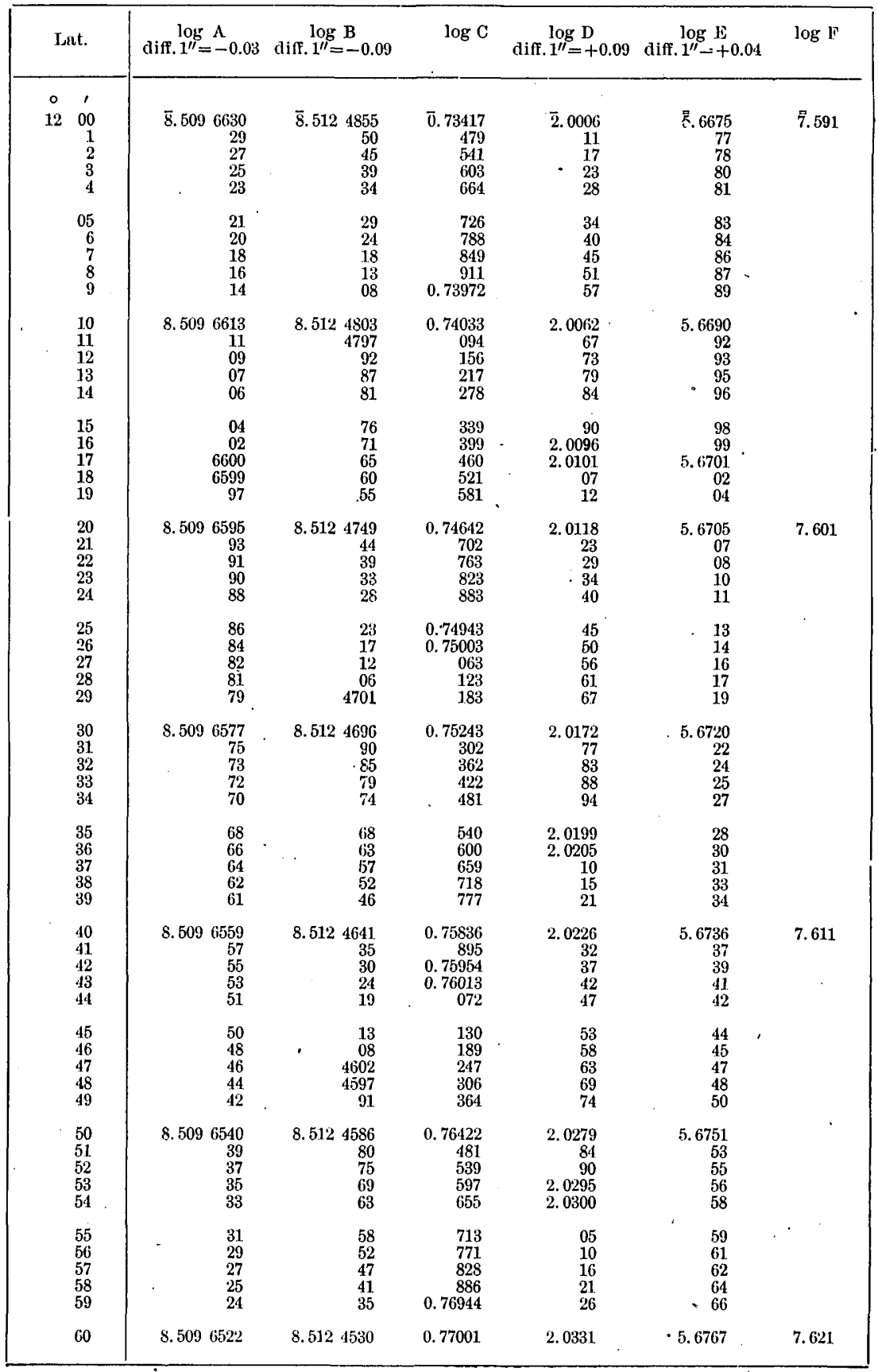

Bull. $214-03-12$ 
TABLe 20.-Geodetic position computations-Continued.

LATITUDE $13^{\circ}$.

\begin{tabular}{|c|c|c|c|c|c|c|}
\hline Lat. & $\begin{array}{c}\log \mathrm{A} \\
\text { diff. } 1^{\prime \prime}=-0.03\end{array}$ & $\begin{array}{c}\log B \\
\text { diff. } 1^{\prime \prime}=-0.10\end{array}$ & $\begin{array}{c}\log \mathrm{C} \\
\text { diff. } 1^{\prime \prime}=+0.93\end{array}$ & $\begin{array}{l}\log \mathrm{D} \\
\operatorname{diff.} 1^{\prime \prime}=+0.08\end{array}$ & $\begin{array}{l}\log \mathrm{E} \\
\text { diff. } 1^{\prime \prime}=+0.03\end{array}$ & $\log \mathrm{F}$ \\
\hline $\begin{array}{cc}\circ & , \\
13 & 00 \\
& 1 \\
& 2 \\
& 3 \\
& 4\end{array}$ & $\begin{array}{r}8.5096522 \\
20 \\
18 \\
16 \\
14\end{array}$ & $\begin{array}{r}8.5124530 \\
24 \\
19 \\
13 \\
07\end{array}$ & $\begin{array}{r}\overline{0} .77001 \\
059 \\
116 \\
174 \\
231\end{array}$ & $\begin{array}{r}\overline{2} .0331 \\
36 \\
42 \\
47 \\
52\end{array}$ & $\begin{array}{r}\overline{5} .6767 \\
69 \\
70 \\
72 \\
74\end{array}$ & $\overline{\overline{7}} .(i 21$. \\
\hline $\begin{array}{r}05 \\
6 \\
7 \\
8 \\
9\end{array}$ & $\begin{array}{l}12 \\
10 \\
09 \\
07 \\
05\end{array}$ & $\begin{array}{r}4502 \\
4496 \\
90 \\
85 \\
79\end{array}$ & $\begin{array}{l}288 \\
346 \\
403 \\
460 \\
517\end{array}$ & $\begin{array}{l}57 \\
62 \\
67 \\
73 \\
78\end{array}$ & $\begin{array}{l}75 \\
77 \\
78 \\
80 \\
82\end{array}$ & \\
\hline $\begin{array}{l}10 \\
11 \\
12 \\
13 \\
14\end{array}$ & $\begin{array}{r}8.5096503 \\
6501 \\
6499 \\
97 \\
95\end{array}$ & $\begin{array}{r}8.5124473 \\
67 \\
62 \\
56 \\
50\end{array}$ & $\begin{array}{r}0.77574 \\
630 \\
687 \\
744 \\
801\end{array}$ & $\begin{array}{r}2.0383 \\
88 \\
93 \\
93 \\
2.0398 \\
2.0403\end{array}$ & $\begin{array}{r}5.6783 \\
85 \\
86 \\
.88 \\
90\end{array}$ & \\
\hline $\begin{array}{l}15 \\
16 \\
17 \\
18 \\
19\end{array}$ & $\begin{array}{l}93 \\
91 \\
90 \\
88 \\
86\end{array}$ & $\begin{array}{l}45 \\
39 \\
33 \\
27 \\
22\end{array}$ & $\begin{array}{r}857 \\
914 \\
=\quad 77970 \\
0.78027 \\
083\end{array}$ & $\begin{array}{l}08 \\
13 \\
18 \\
23 \\
28\end{array}$ & $\begin{array}{l}91 \\
93 \\
94 \\
96 \\
98\end{array}$ & \\
\hline $\begin{array}{l}20 \\
21 \\
22 \\
23 \\
24\end{array}$ & $\begin{array}{r}8.509 \quad 6484 \\
82 \\
80 \\
78 \\
76\end{array}$ & $\begin{array}{r}8.5124416 \\
10 \\
4404 \\
4399 \\
93\end{array}$ & $\begin{array}{r}0.78139 \\
195 \\
251 \\
307 \\
363\end{array}$ & $\begin{array}{r}2.0433 \\
38 \\
44 \\
49 \\
54\end{array}$ & $\begin{array}{r}5.6799 \\
5.6801 \\
03 \\
04 \\
06\end{array}$ & 7. 631 \\
\hline $\begin{array}{l}25 \\
26 \\
27 \\
28 \\
29\end{array}$ & $\begin{array}{l}74 \\
72 \\
70 \\
68 \\
66\end{array}$ & $\begin{array}{l}87 \\
81 \\
76 \\
70 \\
64\end{array}$ & $\begin{array}{r}.419 \\
475 \\
531 \\
587 \\
642\end{array}$ & $\begin{array}{l}59 \\
644 \\
69 \\
74 \\
78\end{array}$ & $\begin{array}{l}07 \\
09 \\
11 \\
12 \\
14\end{array}$ & \\
\hline $\begin{array}{l}30 \\
31 \\
32 \\
33 \\
34\end{array}$ & $\begin{array}{r}8.5096464 \\
63 \\
-61 \\
59 \\
57\end{array}$ & $\begin{array}{r}8.512 \quad 4358 \\
52 \\
: 46 \\
41 \\
.35\end{array}$ & $\begin{array}{r}0.78698 \\
754 \\
909 \\
865 \\
920\end{array}$ & $\begin{array}{r}2.0483 \\
-\quad 88 \\
93 \\
2.0498 \\
2.0503\end{array}$ & $\begin{array}{r}5.6816 \\
17 \\
19 \\
20 \\
22\end{array}$ & \\
\hline $\begin{array}{l}35 \\
36 \\
37 \\
38 \\
39\end{array}$ & $\begin{array}{l}55 \\
53 \\
51 \\
49 \\
47\end{array}$ & $\begin{array}{r}29 \\
23 \\
17 \\
11 \\
4305\end{array}$ & $\begin{array}{r}0.78975 \\
0.79030 \\
086 \\
141 \\
196\end{array}$ & $\begin{array}{l}08 \\
13 \\
18 \\
23 \\
28\end{array}$ & $\begin{array}{l}24 \\
25 \\
27 \\
29 \\
30\end{array}$ & \\
\hline $\begin{array}{l}40 \\
41 \\
42 \\
43 \\
44\end{array}$ & $\begin{array}{r}8.509 \quad 6445 \\
43 \\
41 \\
39 \\
37\end{array}$ & $\begin{array}{r}8.5124299 \\
94 \\
88 \\
\quad 82 \\
76\end{array}$ & $\begin{array}{r}0.79251 \\
306 \\
360 \\
415 \\
470\end{array}$ & $\begin{array}{r}2.0533 \\
38 \\
42 \\
47 \\
52\end{array}$ & $\begin{array}{r}\text { 5. } 6832 \\
34 \\
35 \\
37 \\
39\end{array}$ & 7. 640 \\
\hline $\begin{array}{r}45 \\
46 \\
47 \\
-\quad 48 \\
49\end{array}$ & $\begin{array}{r}.35 \\
\times \quad 33 \\
31 \\
\therefore \quad 29 \\
\therefore \quad 27\end{array}$ & $\begin{array}{l}70 \\
64 \\
58 \\
52 \\
46\end{array}$ & $\begin{array}{l}525 \\
579 \\
634 \\
588 \\
743\end{array}$ & $\begin{array}{l}57 \\
62 \\
67 \\
72 \\
76\end{array}$ & $\begin{array}{l}40 \\
42 \\
44 \\
45 \\
47\end{array}$ & \\
\hline $\begin{array}{l}50 \\
51 \\
52 \\
53 \\
54\end{array}$ & $\begin{array}{r}8.5096425 \\
23 \\
21 \\
19 \\
17\end{array}$ & $\begin{array}{r}8.5124240 \\
34 \\
28 \\
22 \\
16\end{array}$ & $\begin{array}{r}0.79797 \\
851 \\
905 \\
0.79960 \\
0.80014\end{array}$ & $\begin{array}{r}2.0581 \\
86 \\
91 \\
2.0596 \\
2.0601\end{array}$ & $\begin{array}{r}5.6849 \\
50 \\
52 \\
54 \\
55\end{array}$ & \\
\hline $\begin{array}{l}55 \\
56 \\
57 \\
58 \\
59\end{array}$ & $\begin{array}{l}15 \\
13 \\
11 \\
09 \\
07\end{array}$ & $\begin{array}{r}10 \\
4204 \\
4198 \\
92 \\
86\end{array}$ & $\begin{array}{l}068 \\
122 \\
176 \\
230 \\
284\end{array}$ & $\begin{array}{l}05 \\
10 \\
15 \\
20 \\
24\end{array}$ & $\begin{array}{l}57 \\
59 \\
60 \\
62 \\
64\end{array}$ & \\
\hline 60 & 8. 5096405 . & S. 5124180 & 0.80337 & 2.0629 & 5.6865 & 7.649 \\
\hline
\end{tabular}


TABLE 20.-Geodetic position compututions-Continued.

LATITUDE $14^{\circ}$.

\begin{tabular}{|c|c|c|c|c|c|c|}
\hline Lat. & $\begin{array}{c}\log A \\
\operatorname{dint} 1^{\prime \prime}=-0.03\end{array}$ & $\begin{array}{c}\log B \\
\text { diff. } 1^{\prime \prime}=-0.10\end{array}$ & $\begin{array}{c}\log \mathrm{C} \\
\text { diff. } 1^{\prime \prime}=+0.87\end{array}$ & $\begin{array}{c}\log \mathrm{D} \\
\text { diff. } 1^{\prime \prime}=+0.08\end{array}$ & $\begin{array}{c}\log \mathrm{E} \\
\text { diff. } 1^{\prime \prime}=+0.03\end{array}$ & $\log F$ \\
\hline $\begin{array}{cc}0 & 1 \\
1.1 & 00 \\
& 1 \\
& 2 \\
& 3 \\
& 3 \\
& 4\end{array}$ & $\begin{array}{r}\overline{5} .50904 \\
03 \\
6401 \\
63899 \\
97\end{array}$ & $\begin{array}{r}5.512+4180 \\
74 \\
68 \\
62 \\
56\end{array}$ & $\begin{array}{r}\overline{0} .80337 \\
391 \\
445 \\
498 \\
552\end{array}$ & $\begin{array}{r}\overline{2} .0629 \\
34 \\
39 \\
43 \\
48\end{array}$ & $\begin{array}{r}5.6865 \\
67 \\
69 \\
71 \\
72\end{array}$ & $\overline{\overline{7}} .649$ \\
\hline $\begin{array}{r}05 \\
6 \\
7 \\
8 \\
9\end{array}$ & $\begin{array}{l}95 \\
93 \\
91 \\
89 \\
57\end{array}$ & $\begin{array}{l}50 \\
44 \\
38 \\
32 \\
26\end{array}$ & $\begin{array}{l}605 \\
659 \\
712 \\
765 \\
319\end{array}$ & $\begin{array}{l}53 \\
58 \\
62 \\
67 \\
72\end{array}$ & $\begin{array}{l}74 \\
76 \\
77 \\
79 \\
81\end{array}$ & \\
\hline $\begin{array}{r}10 \\
11 \\
112 \\
13 \\
11\end{array}$ & $\begin{array}{r}8.509 \\
6385 \\
. \quad 81 \\
\quad 79 \\
77\end{array}$ & $\begin{array}{r}8.5124120 \\
14 \\
08 \\
4101 \\
4095\end{array}$ & $\begin{array}{r}0.80872 \\
925 \\
0.80978 \\
0.81031 \\
.084\end{array}$ & $\begin{array}{r}2.0676 \\
81 \\
86 \\
90 \\
2.0695\end{array}$ & $\begin{array}{r}5.6882 \\
84 \\
86 \\
88 \\
89\end{array}$ & \\
\hline $\begin{array}{l}15 \\
16 \\
17 \\
18 \\
19\end{array}$ & $\begin{array}{l}75 \\
73 \\
71 \\
69 \\
67\end{array}$ & $\begin{array}{l}89 \\
83 \\
77 \\
71 \\
65\end{array}$ & $\begin{array}{l}137 \\
190 \\
243 \\
295 \\
348\end{array}$ & $\begin{array}{r}2.0700 \\
04 \\
09 \\
.14 \\
18\end{array}$ & $\begin{array}{l}91 \\
93 \\
94 \\
96 \\
98\end{array}$ & \\
\hline $\begin{array}{l}20 \\
21 \\
22 \\
23 \\
24\end{array}$ & $\begin{array}{r}8.5090365 \\
63 \\
61 \\
58 \\
56\end{array}$ & $\begin{array}{r}8.5124059 \\
52 \\
46 \\
40 \\
34\end{array}$ & $\begin{array}{r}0.81401 \\
453 \\
506 \\
558 \\
611\end{array}$ & $\begin{array}{r}2.0723 \\
28 \\
32 \\
36 \\
41\end{array}$ & $\begin{array}{r}5.6900 \\
01 \\
03 \\
05 \\
06\end{array}$ & 7.658 \\
\hline $\begin{array}{l}25 \\
26 \\
27 \\
28 \\
29\end{array}$ & $\begin{array}{l}54 \\
52 \\
50 \\
48 \\
46\end{array}$ & $\begin{array}{r}28 \\
21 \\
15 \\
09 \\
4003\end{array}$ & $\begin{array}{r}663 \\
715 \\
767 \\
920 \\
872\end{array}$ & $\begin{array}{l}46 \\
51 \\
55 \\
60 \\
64\end{array}$ & $\begin{array}{l}08 \\
10 \\
12 \\
13 \\
15\end{array}$ & \\
\hline $\begin{array}{l}30 \\
31 . \\
32 \\
33 \\
34\end{array}$ & $\begin{array}{r}8.509 \quad 6344 \\
42 \\
40 \\
38 \\
36\end{array}$ & $\begin{array}{r}8.5123997 \\
90 \\
84 \\
78 \\
72\end{array}$ & $\begin{array}{r}0 . \$ 1924 \\
0.81976 \\
0 . \$ 2028 \\
080 \\
1.31\end{array}$ & $\begin{array}{r}2.0769 \\
73 \\
78 \\
83 \\
87\end{array}$ & $\begin{array}{r}5.6917 \\
19 \\
20 \\
22 \\
24\end{array}$ & \\
\hline $\begin{array}{l}35 \\
36 \\
37 \\
38 \\
39\end{array}$ & $\begin{array}{l}34 \\
32 \\
29 \\
27 \\
25\end{array}$ & $\begin{array}{l}65 \\
59 \\
53 \\
47 \\
40\end{array}$ & $\begin{array}{l}183 \\
235 \\
287 \\
338 \\
390\end{array}$ & $\begin{array}{r}92 \\
2.0796 \\
2.0801 \\
05 \\
10\end{array}$ & $\begin{array}{l}26 \\
27 \\
29 \\
31 \\
33\end{array}$ & \\
\hline $\begin{array}{r}40 \\
41 \\
+42 \\
43 \\
44\end{array}$ & $\begin{array}{r}8.509 \quad 6323 \\
\quad 21 \\
\quad 19 \\
17 \\
15\end{array}$ & $\begin{array}{r}8.5123984 \\
28 \\
-\quad 15 \\
-\quad 15 \\
09\end{array}$ & $\begin{array}{r}0.82441 . \\
493 \\
544 \\
596 \\
647\end{array}$ & $\begin{array}{r}2.0814 \\
19 \\
23 \\
28 \\
32\end{array}$ & $\begin{array}{r}5.6934 \\
36 \\
38 \\
40 \\
41\end{array}$ & 7.667 \\
\hline $\begin{array}{l}45 \\
46 \\
47 \\
48 \\
49\end{array}$ & $\begin{array}{l}13 \\
1.1 \\
08 \\
06 \\
0.4\end{array}$ & $\begin{array}{r}3903 \\
3896 \\
90 \\
84 \\
77\end{array}$ & $\begin{array}{l}698 \\
749 \\
800 \\
852 \\
903\end{array}$ & $\begin{array}{r}37 \\
41 \\
46 \\
50 \\
54\end{array}$ & $\begin{array}{r}43 \\
45 \\
47 \\
\cdot \quad 48 \\
50\end{array}$ & \\
\hline $\begin{array}{l}50 \\
51 . \\
52 \\
53 \\
54\end{array}$ & $\begin{array}{r}8.5096302 \\
6300 \\
6298 \\
.96 \\
94\end{array}$ & $\begin{array}{r}8.5123871 \\
65 \\
58 \\
52 \\
45\end{array}$ & $\begin{array}{r}0.82954 \\
0.83005 \\
055 \\
106 \\
157\end{array}$ & $\begin{array}{r}2.0859 \\
63 \\
68 \\
72 \\
77\end{array}$ & 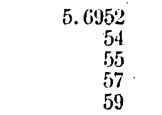 & \\
\hline $\begin{array}{l}55 \\
56 \\
57 \\
56 \\
59\end{array}$ & $\begin{array}{l}92 \\
89 \\
87 \\
85 \\
83\end{array}$ & $\begin{array}{l}39 \\
33 \\
26 \\
20 \\
1.3\end{array}$ & $\begin{array}{l}208 \\
258 \\
309 \\
360 \\
410\end{array}$ & $\begin{array}{r}81 \\
85 \\
90 \\
94 \\
2.0899\end{array}$ & $\begin{array}{l}61 . \\
63 \\
64 \\
66 \\
68\end{array}$ & \\
\hline 60 & 8. 5096281. & 8.5123807 & 0.83461 & 2.0903 & 5.6970 & 7.675 \\
\hline
\end{tabular}


Table 20.-Geodetic position computations-Continued.

LATITUDE $15^{\circ}$.

\begin{tabular}{|c|c|c|c|c|c|c|}
\hline Lat. & $\begin{array}{c}\log A \\
\text { diff. } 1^{\prime \prime}=-0.04\end{array}$ & $\begin{array}{c}\log B \\
\text { diff. } 1^{\prime \prime}=-0.11\end{array}$ & $\begin{array}{c}\log \mathrm{C} \\
\text { diff. } 1^{\prime \prime}=+0.82\end{array}$ & $\begin{array}{c}\log \mathrm{D} \\
\text { diff. } 1^{\prime \prime}=+0.07\end{array}$ & $\begin{array}{c}\log \mathrm{E} \\
\text { diff. } 1^{\prime \prime}=+0.03\end{array}$ & $\log \mathrm{F}$ \\
\hline $\begin{array}{rc}0 & 1 \\
15 & 00 \\
& 1 \\
& 2 \\
& 3 \\
& 4\end{array}$ & $\begin{array}{r}\overline{8} .509 \quad 6281 \\
79 \\
77 \\
74 \\
72\end{array}$ & $\begin{array}{r}\overline{8} .5123807 \\
3801 \\
3794 \\
88 \\
.81\end{array}$ & $\begin{array}{r}\overline{0} .83461 \\
511 \\
561 \\
612 \\
662\end{array}$ & $\begin{array}{r}\overline{2} .0903 \\
07 \\
12 \\
16 \\
21\end{array}$ & $\begin{array}{r}\overline{5} .6970 \\
72 \\
73 \\
75 \\
77\end{array}$ & $\overline{7} .675$ \\
\hline $\begin{array}{r}05 \\
6 \\
7 \\
8 \\
9\end{array}$ & $\begin{array}{l}70 \\
68 \\
66 \\
64 \\
62\end{array}$ & $\begin{array}{l}75 \\
68 \\
62 \\
56 \\
49\end{array}$ & $\begin{array}{l}712 \\
762 \\
813 \\
863 \\
913\end{array}$ & $\begin{array}{l}25 \\
29 \\
34 \\
38 \\
42\end{array}$ & $\begin{array}{l}79 \\
80 \\
82 \\
84 \\
86\end{array}$ & \\
\hline $\begin{array}{l}10 \\
11 \\
12 \\
13 \\
14\end{array}$ & $\begin{array}{r}8.509 \quad 6259 \\
57 \\
55 \\
53 \\
51\end{array}$ & $\begin{array}{r}8.512 \quad 3743 \\
36 \\
30 \\
23 \\
17\end{array}$ & $\begin{array}{r}0.83963 \\
0.84012 \\
062 \\
112 \\
162\end{array}$ & $\begin{array}{r}2.0947 \\
51 \\
55 \\
59 \\
64\end{array}$ & $\begin{array}{r}5.6988 \\
89 \\
91 \\
93 \\
95\end{array}$ & \\
\hline $\begin{array}{l}15 \\
16 \\
17 \\
18 \\
19\end{array}$ & $\begin{array}{l}49 \\
46 \\
44 \\
42 \\
40\end{array}$ & $\begin{array}{r}10 \\
3704 \\
3697 \\
91 \\
84\end{array}$ & $\begin{array}{l}212 \\
261 \\
311 \\
361 \\
410\end{array}$ & $\begin{array}{l}68 \\
72 \\
77 \\
81 \\
85\end{array}$ & $\begin{array}{r}97 \\
5.6999 \\
5.7000 \\
02 \\
04\end{array}$ & \\
\hline $\begin{array}{l}20 \\
21 \\
22 \\
23 \\
24\end{array}$ & $\begin{array}{r}8.5096238 \\
35 \\
33 \\
31 \\
29\end{array}$ & $\begin{array}{r}8.5123677 \\
71 \\
64 \\
58 \\
51\end{array}$ & $\begin{array}{r}0.84460 \\
509 \\
558 \\
608 \\
657\end{array}$ & $\begin{array}{r}2.0990 \\
94 \\
2.0998 \\
2.1002 \\
07\end{array}$ & $\begin{array}{r}5.7006 \\
08 \\
09 \\
11 \\
1.3\end{array}$ & 7.683 \\
\hline $\begin{array}{l}25 \\
26 \\
27 \\
28 \\
29\end{array}$ & $\begin{array}{l}27 \\
24 \\
22 \\
20 \\
18\end{array}$ & $\begin{array}{r}45 \\
38 \\
31 \\
25 \\
\quad 18\end{array}$ & $\begin{array}{l}706 \\
755 \\
804 \\
854 \\
903\end{array}$ & $\begin{array}{l}11 \\
15 \\
19 \\
23 \\
28\end{array}$ & $\begin{array}{l}15 \\
17 \\
19 \\
20 \\
22\end{array}$ & \\
\hline $\begin{array}{l}30 \\
31 \\
32 \\
33 \\
34\end{array}$ & $\begin{array}{r}8.5096216 \\
14 \\
11 \\
09 \\
07\end{array}$ & $\begin{array}{r}8.5123612 \\
3605 \\
3598 \\
92 \\
85\end{array}$ & $\begin{array}{r}0.84952 \\
0.85001 \\
049 \\
098 \\
147\end{array}$ & $\begin{array}{r}2.1032 \\
36 \\
40 \\
44 \\
49\end{array}$ & $\begin{array}{r}5.7024 \\
26 \\
28 \\
30 \\
31\end{array}$ & \\
\hline $\begin{array}{l}35 \\
36 \\
37 \\
38 \\
39\end{array}$ & $\begin{array}{r}05 \\
02 \\
6200 \\
6198 \\
96\end{array}$ & $\begin{array}{l}79 \\
72 \\
65 \\
59 \\
52\end{array}$ & $\begin{array}{l}196 \\
245 \\
293 \\
342 \\
390\end{array}$ & $\begin{array}{l}53 \\
57 \\
61 \\
65 \\
69\end{array}$ & $\begin{array}{l}33 \\
35 \\
37 \\
39 \\
41\end{array}$ & \\
\hline $\begin{array}{l}40 \\
41 \\
42 \\
43 \\
44\end{array}$ & $\begin{array}{r}8.5096194 \\
91 \\
\quad 89 \\
87 \\
85\end{array}$ & $\begin{array}{r}8.512 \quad 3545 \\
39 \\
32 \\
25 \\
19\end{array}$ & $\begin{array}{r}0.85439 \\
487 \\
. \quad 536 \\
. \quad 584 \\
633\end{array}$ & $\begin{array}{r}2.1074 \\
78 \\
82 \\
86 \\
90\end{array}$ & $\begin{array}{r}5.7042 \\
44 \\
46 \\
48 \\
50\end{array}$ & 7.691 \\
\hline $\begin{array}{l}45 \\
46 \\
47 \\
48 \\
49\end{array}$ & $\begin{array}{l}82 \\
80 \\
78 \\
76 \\
73\end{array}$ & $\begin{array}{r}12 \\
3505 \\
3498 \\
92 \\
85\end{array}$ & $\begin{array}{l}681 \\
729 \\
777 \\
825 \\
874\end{array}$ & $\begin{array}{r}94 \\
2.1099 \\
2.1103 \\
67 \\
11\end{array}$ & $\begin{array}{l}52 \\
54 \\
55 \\
57 \\
59\end{array}$ & . \\
\hline $\begin{array}{l}50 \\
51 \\
52 \\
53 \\
54\end{array}$ & $\begin{array}{r}8.509 \quad 6171 \\
69 \\
67 \\
64 \\
62\end{array}$ & $\begin{array}{r}8.5123478 \\
71 \\
65 \\
58 \\
51\end{array}$ & $\begin{array}{r}0.85922 \\
0.85970 \\
0.86018 \\
066 \\
113\end{array}$ & $\begin{array}{r}2.1115 \\
19 \\
23 \\
27 \\
31\end{array}$ & $\begin{array}{r}5.7061 \\
63 \\
65 \\
67 \\
69\end{array}$ & \\
\hline $\begin{array}{l}55 \\
56 \\
57 \\
58 \\
59\end{array}$ & $\begin{array}{l}60 \\
58 \\
55 \\
53 \\
51\end{array}$ & $\begin{array}{r}44 \\
38 \\
31 \\
24 \\
17\end{array}$ & $\begin{array}{l}161 \\
209 \\
257 \\
304 \\
352\end{array}$ & $\begin{array}{l}35 \\
39 \\
44 \\
48 \\
52\end{array}$ & $\begin{array}{r}70 \\
72 \\
74 \\
76 \\
\cdot \quad 78\end{array}$ & \\
\hline 60 & 8.5096149 & 8.5123411 & 0.86400 & 2.1156 & 5.7080 & 7. 698 \\
\hline
\end{tabular}


Table 20.-Geodetic position computations-Continued.

LATITUDE $16^{\circ}$.

\begin{tabular}{|c|c|c|c|c|c|c|}
\hline Lat. & diff. $1^{\prime \prime}=-0.04$ & $\begin{array}{c}\log B \\
\text { diff. } 1^{\prime \prime}=-0.12\end{array}$ & $\begin{array}{c}\log C \\
\text { diff. } 1^{\prime \prime}=+0.77\end{array}$ & $\begin{array}{c}\log \mathrm{D} \\
\text { diff. } 1^{\prime \prime}=+0.06\end{array}$ & $\begin{array}{c}\log E \\
\text { diff. } 1^{\prime \prime}=+0.03\end{array}$ & $\log F$ \\
\hline $\begin{array}{cc}\circ & 1 \\
16 & 00 \\
& 1 \\
& 2 \\
& 3 \\
& 4\end{array}$ & $\begin{array}{r}\overline{8} .509 \quad 61.49 \\
46 \\
44 \\
42 \\
40\end{array}$ & $\begin{array}{r}\overline{8} .5123411 \\
3404 \\
3397 \\
.90 \\
83\end{array}$ & $\begin{array}{r}\overline{0} .86400 \\
447 \\
495 \\
542 \\
590\end{array}$ & $\begin{array}{r}\overline{2} .1156 \\
60 \\
64 \\
68 \\
72\end{array}$ & $\begin{array}{r}\overline{5} .7080 \\
82 \\
84 \\
85 \\
87\end{array}$ & 7. 698 \\
\hline $\begin{array}{r}05 \\
6 \\
7 \\
8 \\
8 \\
9\end{array}$ & $\begin{array}{r}37 \\
35 \\
33 \\
30 \\
28\end{array}$ & $\begin{array}{l}76 \\
70 \\
63 \\
56 \\
49\end{array}$ & $\begin{array}{l}637 \\
684 \\
732 \\
779 \\
826\end{array}$ & $\begin{array}{l}76 \\
80 \\
84 \\
88 \\
92\end{array}$ & $\begin{array}{l}89 \\
91 \\
93 \\
95 \\
97\end{array}$ & . \\
\hline $\begin{array}{l}10 \\
11 \\
12 \\
13 \\
14\end{array}$ & $\begin{array}{r}8.5096126 \\
24 \\
21 \\
19 \\
17\end{array}$ & $\begin{array}{r}8.512 \quad 3342 \\
35 \\
28 \\
22 \\
15\end{array}$ & $\begin{array}{r}0.86873 \\
921 \\
0.86968 \\
0.87015 \\
062\end{array}$ & $\begin{array}{r}2.1196 \\
2.1200 \\
04 \\
08 \\
12\end{array}$ & $\begin{array}{r}5.7099 \\
5.7101 \\
03 \\
04 \\
06\end{array}$ & - \\
\hline $\begin{array}{l}15 \\
16 \\
17 \\
18 \\
19\end{array}$ & $\begin{array}{r}14 \\
12 \\
10 \\
08 \\
05\end{array}$ & $\begin{array}{r}08 \\
3301 \\
3294 \\
87 \\
80\end{array}$ & $\begin{array}{l}109 \\
156 \\
202 \\
249 \\
296\end{array}$ & $\begin{array}{l}16 \\
20 \\
24 \\
28 \\
32\end{array}$ & $\begin{array}{l}08 \\
10 \\
12 \\
14 \\
16\end{array}$ & \\
\hline $\begin{array}{l}20 \\
21 \\
22 \\
23 \\
24\end{array}$ & $\begin{array}{r}8.5096103 \\
6101 \\
6098 \\
96 \\
94\end{array}$ & $\begin{array}{r}8.5123273 \\
66 \\
-59 \\
-52 \\
45\end{array}$ & $\begin{array}{r}0.87343 \\
389 \\
436 \\
483 \\
529\end{array}$ & $\begin{array}{r}2.1236 \\
40 \\
44 \\
47 \\
51\end{array}$ & $\begin{array}{r}5.7118 \\
20 \\
22 \\
24 \\
25\end{array}$ & 7.705 \\
\hline $\begin{array}{l}25 \\
26 \\
27 \\
28 \\
29\end{array}$ & $\begin{array}{l}91 \\
89 \\
87 \\
84 \\
82\end{array}$ & $\begin{array}{r}39 \\
32 \\
25 \\
. \quad 18 \\
.11\end{array}$ & $\begin{array}{l}576 \\
622 \\
669 \\
715 \\
761\end{array}$ & $\begin{array}{l}55 \\
59 \\
63 \\
67 \\
71\end{array}$ & $\begin{array}{l}27 . \\
29 \\
31 \\
33 \\
35\end{array}$ & \\
\hline $\begin{array}{l}30 \\
31 \\
32 \\
33 \\
34\end{array}$ & $\begin{array}{r}8.509 \quad 6080 \\
. \quad 77 \\
75 \\
73 \\
70\end{array}$ & $\begin{array}{r}8.5123204 \\
3197 \\
90 \\
83 \\
76\end{array}$ & $\begin{array}{r}0.87808 \\
854 \\
900 \\
947 \\
0.87993\end{array}$ & $\begin{array}{r}2.1275 \\
79 \\
83 \\
87 \\
90\end{array}$ & $\begin{array}{r}5.7137 \\
39 \\
41 \\
43 \\
45\end{array}$ & \\
\hline $\begin{array}{l}35 \\
36 \\
37 \\
38 \\
39\end{array}$ & $\begin{array}{l}68 \\
66 \\
63 \\
61 \\
59\end{array}$ & $\begin{array}{l}69 \\
62 \\
55 \\
43 \\
41\end{array}$ & $\begin{array}{r}0.88039 \\
\cdot \quad 085 \\
131 \\
177 \\
223\end{array}$ & $\begin{array}{r}94 \\
2.1298 \\
2.1302 \\
06 \\
10\end{array}$ & $\begin{array}{r}47 \\
49 \\
51 \\
52 \\
54\end{array}$ & \\
\hline $\begin{array}{l}40 \\
41 \\
42 \\
43 \\
44\end{array}$ & $\begin{array}{r}8.5096056 \\
54 \\
52 \\
49 \\
47\end{array}$ & $\begin{array}{r}8.51231 .83 \\
26 \\
19 \\
12 \\
3105\end{array}$ & $\begin{array}{r}0.88269 \\
315 \\
360 \\
406 \\
452\end{array}$ & $\begin{array}{r}2.1314 \\
17 \\
21 \\
25 \\
29\end{array}$ & $\begin{array}{r}5.7156 \\
58 \\
60 \\
62 \\
64\end{array}$ & 7.712 \\
\hline $\begin{array}{l}45 \\
46 \\
47 \\
48 \\
49\end{array}$ & $\begin{array}{l}45 \\
42 \\
40 \\
37 \\
35\end{array}$ & $\begin{array}{r}3098 \\
91 \\
84 \\
77 \\
70\end{array}$ & $\begin{array}{l}498 \\
543 \\
589 \\
634 \\
680\end{array}$ & $\begin{array}{l}33 \\
37 \\
40 \\
44 \\
48\end{array}$ & $\begin{array}{l}66 \\
68 \\
70 \\
72 \\
74\end{array}$ & \\
\hline $\begin{array}{l}50 \\
51 \\
52 \\
53 \\
54\end{array}$ & $\begin{array}{r}8.509 \quad 6033 \\
30 \\
28 \\
20 \\
23\end{array}$ & $\begin{array}{r}8.5123063 \\
56 \\
48 \\
41 \\
34\end{array}$ & $\begin{array}{r}0.88726 \\
771 \\
816 \\
862 \\
907\end{array}$ & $\begin{array}{r}2.1352 \\
56 \\
59 \\
63 \\
67\end{array}$ & $\begin{array}{r}5.7176 \\
78 \\
80 \\
82 \\
84\end{array}$ & \\
\hline $\begin{array}{l}55 \\
56 \\
57 \\
58 \\
59\end{array}$ & $\begin{array}{l}21 . \\
18 \\
16 \\
14 \\
11\end{array}$ & $\begin{array}{r}27 \\
20 \\
13 \\
3006 \\
2998\end{array}$ & $\begin{array}{r}952 \\
0.88998 \\
0.89043 \\
088 \\
133\end{array}$ & $\begin{array}{l}71 \\
74 \\
78 \\
82 \\
86\end{array}$ & $\begin{array}{l}86 \\
88 \\
90 \\
92 \\
94\end{array}$ & \\
\hline 60 & 8.5096009 & 8. 5122991 & 0.89178 & 2.1390 & 5.7196 & 7.719 \\
\hline
\end{tabular}


TABLE 20.-Geodetic position computations-Continued.

LATITUDE $17^{\circ}$.

\begin{tabular}{|c|c|c|c|c|c|c|}
\hline Lat. & $\begin{array}{c}\log A \\
\operatorname{diff.} 1^{\prime \prime}=-0.04\end{array}$ & $\begin{array}{c}\log \mathrm{B} \\
\text { diff. } 1^{\prime \prime}=-0.12\end{array}$ & $\begin{array}{c}\log \mathrm{C} \\
\text { diff. } 1^{\prime \prime}=-0.73\end{array}$ & $\begin{array}{c}\log \mathrm{D} \\
\operatorname{diff} .1^{\prime \prime}=+0.06\end{array}$ & $\begin{array}{c}\log \mathrm{E} \\
\text { diff. } 1^{\prime \prime}=+0.03\end{array}$ & $\log \mathbf{F}$ \\
\hline $\begin{array}{rr}\circ & 1 \\
17 & 00 \\
-\quad 1 \\
& 1 \\
& 3 \\
& 3 \\
& 4\end{array}$ & $\begin{array}{r}\overline{8} .5096009 \\
06 \\
04 \\
6002 \\
5999\end{array}$ & $\begin{array}{r}\overline{8} .512 \quad 2991 \\
84 \\
77 \\
70 \\
62\end{array}$ & $\begin{array}{r}\overline{0} .89178 \\
223 \\
268 \\
313 \\
358\end{array}$ & $\begin{array}{r}2.1390 \\
93 \\
2.1397 \\
2.1401 \\
04\end{array}$ & $\begin{array}{r}\overline{\overline{5}} .71 .96 \\
97 \\
99 \\
5.7201 \\
03\end{array}$ & 7.719 \\
\hline $\begin{array}{r}05 \\
6 \\
7 \\
8 \\
9\end{array}$ & $\begin{array}{l}97 \\
94 \\
92 \\
90 \\
87\end{array}$ & $\begin{array}{l}55 . \\
48 \\
41 \\
34 \\
26\end{array}$ & $\begin{array}{l}403 \\
448 \\
493 \\
538 \\
583\end{array}$ & $\begin{array}{l}08 \\
12 \\
16 \\
19 \\
23\end{array}$ & $\begin{array}{l}05 \\
07 \\
09 \\
11 \\
13\end{array}$ & . \\
\hline $\begin{array}{l}10 \\
11 \\
12 \\
13 \\
14\end{array}$ & $\begin{array}{r}8.5095985 \\
82 \\
80 \\
78 \\
75\end{array}$ & $\begin{array}{r}8.512 \quad 2919 \\
12 \\
2905 \\
2897 \\
90\end{array}$ & $\begin{array}{r}0.89627 \\
672 \\
717 \\
761 \\
806\end{array}$ & $\begin{array}{r}2.1427 \\
30 \\
34 \\
38 \\
42\end{array}$ & $\begin{array}{r}5.7215 \\
17 \\
19 \\
21 \\
23\end{array}$ & 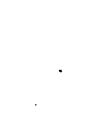 \\
\hline $\begin{array}{l}15 \\
16 \\
17 \\
18 \\
19\end{array}$ & $\begin{array}{l}73 \\
70 \\
68 \\
65 \\
63\end{array}$ & $\begin{array}{l}83 \\
76 \\
68 \\
61 \\
54\end{array}$ & $\begin{array}{r}850 \\
895 \\
939 \\
0.89984 \\
0.90028\end{array}$ & $\begin{array}{l}45 \\
49 \\
53 \\
56 \\
60\end{array}$ & $\begin{array}{l}25 \\
27 \\
29 \\
31 \\
33\end{array}$ & \\
\hline $\begin{array}{l}20 \\
21 \\
22 \\
23 \\
24\end{array}$ & $\begin{array}{r}8.5095961 \\
58 \\
56 \\
53 \\
51\end{array}$ & $\begin{array}{r}8.512 \quad 2846 \\
39 \\
32 \\
24 \\
17\end{array}$ & $\begin{array}{r}0.90072 \\
117 \\
161 \\
205 \\
249\end{array}$ & $\begin{array}{r}2.1464 \\
67 \\
71 \\
75 \\
78\end{array}$ & $\begin{array}{r}5.7235 \\
37 \\
39 \\
41 \\
43\end{array}$ & 7.720 \\
\hline $\begin{array}{l}25 \\
26 \\
27 \\
28 \\
29\end{array}$ & $\begin{array}{l}48 \\
46 \\
44 \\
41 \\
39\end{array}$ & $\begin{array}{r}10 \\
2802 \\
2795 \\
88 \\
80\end{array}$ & $\begin{array}{l}294 \\
338 \\
382 \\
426 \\
470\end{array}$ & $\begin{array}{r}82 \\
85 \\
89 \\
93 \\
2.1496\end{array}$ & $\begin{array}{l}45 \\
47 \\
49 \\
51 \\
53\end{array}$ & \\
\hline $\begin{array}{l}30 \\
31 \\
32 \\
33 \\
34\end{array}$ & $\begin{array}{r}8.5095936 \\
34 \\
31 \\
29 \\
26\end{array}$ & $\begin{array}{r}8.512 \quad 2773 \\
66 \\
58 \\
51 \\
44\end{array}$ & $\begin{array}{r}0.90514 \\
558 \\
602 \\
646 \\
.689\end{array}$ & $\begin{array}{r}2.1500 \\
04 \\
07 \\
11 \\
14\end{array}$ & $\begin{array}{r}5.7255 \\
57 \\
59 \\
61 \\
64\end{array}$ & \\
\hline $\begin{array}{l}35 \\
36 \\
37 \\
38 \\
39\end{array}$ & $\begin{array}{l}24 \\
21 \\
19 \\
16 \\
14\end{array}$ & $\begin{array}{r}36 \\
29 \\
21 \\
14 \\
2707\end{array}$ & $\begin{array}{l}733 \\
777 \\
821 \\
864 \\
908\end{array}$ & $\begin{array}{l}18 \\
22 \\
25 \\
29 \\
32\end{array}$ & $\begin{array}{l}66 \\
68 \\
70 \\
72 \\
74\end{array}$ & \\
\hline $\begin{array}{l}40 \\
41 \\
42 \\
43 \\
44\end{array}$ & $\begin{array}{r}8.5095912 \\
09 \\
07 \\
04 \\
5902\end{array}$ & $\begin{array}{r}8.512 \quad 2699 \\
92 \\
84 \\
77 \\
69\end{array}$ & $\begin{array}{r}0.90952 \\
0.90995 \\
0.91039 \\
082 \\
126\end{array}$ & $\begin{array}{r}2.1536 \\
\cdot \quad 39 \\
43 \\
47 \\
50\end{array}$ & $\begin{array}{r}5.7276 \\
78 \\
80 \\
82 \\
84\end{array}$ & 7. 732 \\
\hline $\begin{array}{l}45 \\
46 \\
47 \\
48 \\
49\end{array}$ & $\begin{array}{r}5899 \\
97 \\
94 \\
92 \\
.89\end{array}$ & $\begin{array}{l}62 \\
55 \\
47 \\
40 \\
32\end{array}$ & $\begin{array}{l}169 \\
212 \\
256 \\
299 \\
342\end{array}$ & $\begin{array}{l}54 \\
57 \\
61 \\
64 \\
68\end{array}$ & $\begin{array}{r}86 \\
88 \\
90 \\
92 \\
. \quad 94\end{array}$ & \\
\hline $\begin{array}{l}50 \\
51 \\
52 \\
53 \\
54\end{array}$ & $\begin{array}{r}8.5095887 \\
84 \\
82 \\
79 \\
77\end{array}$ & $\begin{array}{r}8.5122625 \\
17 \\
10 \\
-2602 \\
2595\end{array}$ & $\begin{array}{r}0.91386 \\
429 \\
472 \\
515 \\
558\end{array}$ & $\begin{array}{r}2.1571 \\
75 \\
78 \\
82 \\
85\end{array}$ & $\begin{array}{r}5.7296 \\
5.7298 \\
5.7300 \\
-\quad 02 \\
04\end{array}$ & \\
\hline $\begin{array}{l}55 \\
56 \\
57 \\
58 \\
59\end{array}$ & $\begin{array}{l}74 \\
72 \\
69 \\
67 \\
64\end{array}$ & $\begin{array}{l}87 \\
80 \\
72 \\
65 \\
57\end{array}$ & $\begin{array}{l}601 \\
644 \\
687 \\
730 \\
773\end{array}$ & $\begin{array}{r}89 \\
92 \\
96 \\
2.1599 \\
2.1603\end{array}$ & $\begin{array}{l}06 \\
08 \\
11 \\
13 \\
15\end{array}$ & \\
\hline 60 & 8.5095862 & 8.5122550 & 0.91816 & 2.1606 & 5.7317 & 7.738 \\
\hline
\end{tabular}


TABLE 20.-Geodetic position computations-Continued.

LATITUDE $18^{\circ}$.

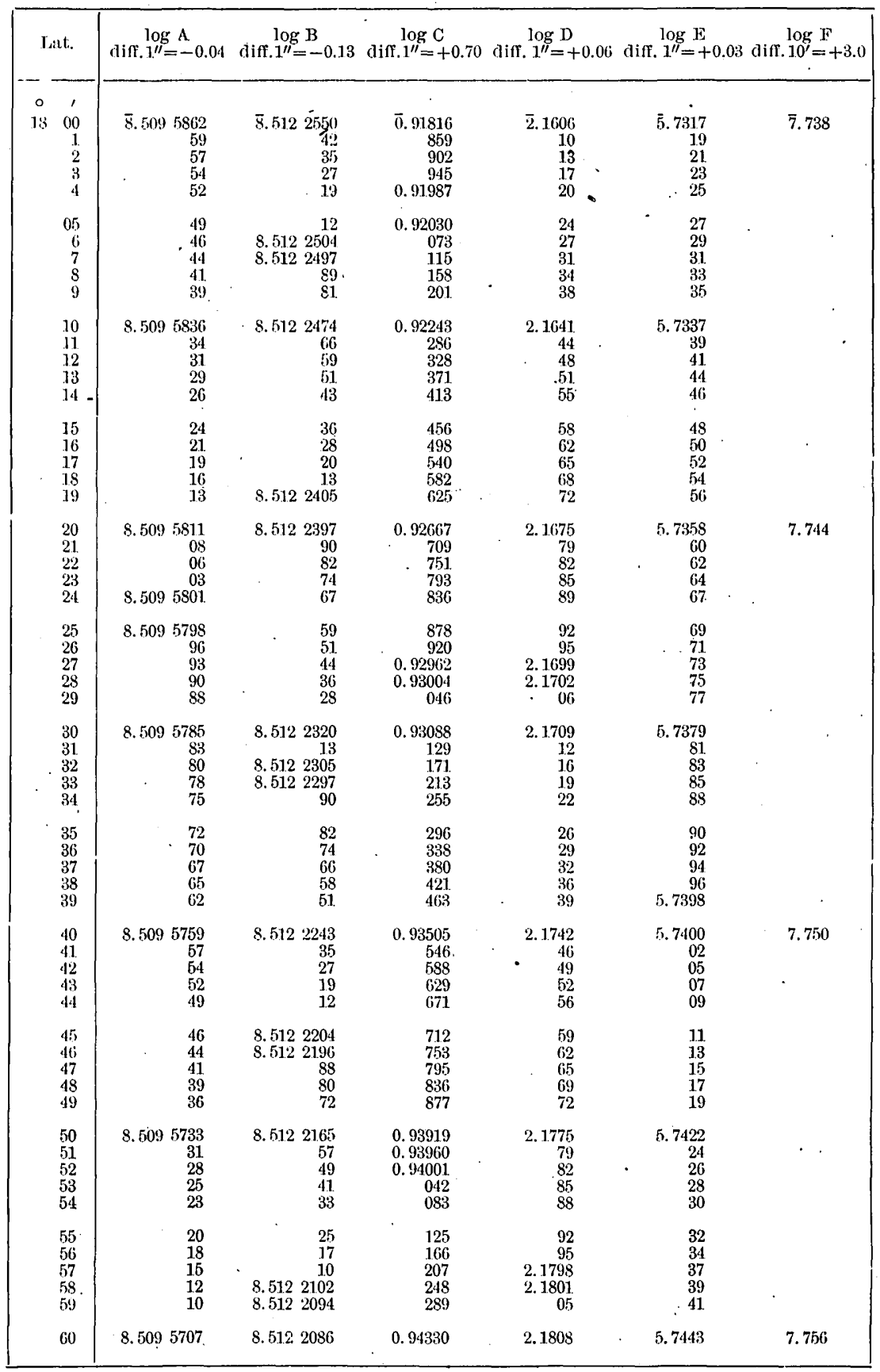


TABLE 20.-Geodetic vosition computations-Continued.

LATITUDE $19^{\circ}$.

\begin{tabular}{|c|c|c|c|c|c|c|}
\hline $\begin{array}{l}\text { Lat. } \\
0 \quad,\end{array}$ & \multicolumn{6}{|c|}{ 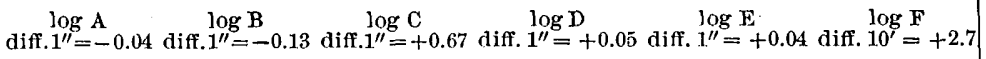 } \\
\hline $\begin{array}{cc}\circ & 1 \\
19 & 00 \\
& 1 \\
& 2 \\
& 3 \\
& 4\end{array}$ & $\begin{array}{r}8.5095707 \\
0.509 \\
8.5702 \\
8.509 \quad 5699 \\
96\end{array}$ & $\begin{array}{r}8.5122086 \\
78 \\
70 \\
62 \\
\quad 54\end{array}$ & $\begin{array}{r}0.94330 \\
370 \\
411 \\
452 \\
493\end{array}$ & 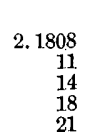 & $\begin{array}{r}5.7443 \\
45 \\
47 \\
49 \\
52\end{array}$ & 7.756 \\
\hline $\begin{array}{r}05 \\
6 \\
7 \\
8 \\
9\end{array}$ & $\begin{array}{l}94 \\
91 \\
89 \\
86 \\
83\end{array}$ & $\begin{array}{l}46 \\
38 \\
30 \\
22 \\
14\end{array}$ & $\begin{array}{r}534 \\
575 \\
615 \\
656 \\
697\end{array}$ & $\begin{array}{r}24 \\
27 \\
30 \\
34 \\
37\end{array}$ & $\begin{array}{l}54 \\
56 \\
58 \\
60 \\
62\end{array}$ & \\
\hline $\begin{array}{l}10 \\
11 \\
12 \\
13 \\
14\end{array}$ & $\begin{array}{r}8.509 \quad 5681 \\
78 \\
75 \\
73 \\
70\end{array}$ & $\begin{array}{r}8.5122006 \\
8.5121999 \\
91 \\
83 \\
75\end{array}$ & $\begin{array}{r}0.94737 \\
778 \\
819 \\
859 \\
900\end{array}$ & $\begin{array}{r}2.1840 \\
43 \\
46 \\
50 \\
53\end{array}$ & $\begin{array}{r}5.7464 \\
67 \\
69 \\
71 \\
73\end{array}$ & \\
\hline $\begin{array}{l}15 \\
16 \\
17 \\
18 \\
19\end{array}$ & $\begin{array}{l}67 \\
65 \\
62 \\
59 \\
57\end{array}$ & $\begin{array}{l}67 \\
59 \\
51 \\
43 \\
35\end{array}$ & $\begin{array}{r}940 \\
0.94981 \\
0.95021 \\
061 \\
.102\end{array}$ & $\begin{array}{l}56 \\
59 \\
62 \\
66 \\
69\end{array}$ & $\begin{array}{l}75 \\
78 \\
80 \\
82 \\
84\end{array}$ & \\
\hline $\begin{array}{l}20 \\
21 \\
22 \\
23 \\
24\end{array}$ & $\begin{array}{r}8.5095654 \\
52 \\
49 \\
46 \\
43\end{array}$ & $\begin{array}{r}8.5121 .927 \\
19 \\
11 \\
8.5121903 \\
8.5121895\end{array}$ & $\begin{array}{r}0.95142 \\
182 \\
223 \\
263 \\
303\end{array}$ & $\begin{array}{r}2.1872 \\
75 \\
78 \\
81 \\
34\end{array}$ & $\begin{array}{r}5.7486 \\
88 \\
91 \\
93 \\
95\end{array}$ & 7.761 \\
\hline $\begin{array}{l}25 \\
26 \\
27 \\
28 \\
29\end{array}$ & $\begin{array}{l}41 \\
38 \\
35 \\
33 \\
30\end{array}$ & $\begin{array}{l}87 \\
79 \\
71 \\
63 \\
55\end{array}$ & $\begin{array}{l}344 \\
384 \\
424 \\
464 \\
504\end{array}$ & $\begin{array}{r}88 \\
91 \\
94 \\
2.1897 \\
2.1900\end{array}$ & $\begin{array}{r}97 \\
5.7499 \\
5.7501 \\
04 \\
06\end{array}$ & - \\
\hline $\begin{array}{l}30 \\
31 \\
32 \\
33 \\
34\end{array}$ & $\begin{array}{r}8.509 \quad 5627 \\
25 \\
22 \\
19 \\
16\end{array}$ & $\begin{array}{r}8.512 \quad 1847 \\
38 \\
30 \\
22 \\
14\end{array}$ & $\begin{array}{r}0.95544 \\
584 \\
624 \\
664 \\
704\end{array}$ & $\begin{array}{r}2.1903 \\
07 \\
10 \\
13 \\
16\end{array}$ & $\begin{array}{r}5.7508 \\
\therefore \quad 10 \\
12 \\
15 \\
17\end{array}$ & \\
\hline $\begin{array}{l}35 \\
36 \\
37 \\
38 \\
39\end{array}$ & $\begin{array}{l}14 \\
11 \\
08 \\
06 \\
03\end{array}$ & $\begin{array}{r}8.5121806 \\
8.5121798 \\
90 \\
82 \\
74\end{array}$ & $\begin{array}{l}744 \\
784 \\
824 \\
863 \\
903\end{array}$ & $\begin{array}{l}19 \\
22 \\
25 \\
28 \\
31\end{array}$ & $\begin{array}{l}19 \\
21 \\
23 \\
26 \\
28\end{array}$ & \\
\hline $\begin{array}{l}40 \\
41 \\
42 \\
43 \\
44\end{array}$ & $\begin{array}{r}8.5095600 \\
8.5095598 \\
95 \\
92 \\
89\end{array}$ & $\begin{array}{r}8.5121766 \\
57 \\
49 \\
41 \\
33\end{array}$ & $\begin{array}{r}0.95943 \\
0.95983 \\
0.96022 \\
062 \\
102\end{array}$ & $\begin{array}{r}2.1934 \\
38 \\
41 \\
-\quad 44 \\
47\end{array}$ & $\begin{array}{r}5.7530 \\
32 \\
34 \\
37 \\
39\end{array}$ & 7.767 \\
\hline $\begin{array}{l}45 \\
46 \\
47 \\
48 \\
49\end{array}$ & $\begin{array}{l}87 \\
84 \\
81 \\
78 \\
76\end{array}$ & $\begin{array}{r}25 \\
17 \\
08 \\
8.5121700 \\
8.5121692\end{array}$ & $\begin{array}{l}142 \\
181 \\
221 \\
260 \\
300\end{array}$ & $\begin{array}{l}50 \\
53 \\
56 \\
59 \\
62\end{array}$ & $\begin{array}{l}41 \\
43 \\
46 \\
48 \\
50\end{array}$ & \\
\hline $\begin{array}{l}50 \\
51 \\
52 \\
53 \\
54\end{array}$ & $\begin{array}{r}8.5095573 \\
70 \\
68 \\
65 \\
62\end{array}$ & $\begin{array}{rr}8.5121684 \\
\\
: \quad 65 \\
& 59 \\
& 51\end{array}$ & $\begin{array}{r}0.96339 \\
379 \\
418 \\
457 \\
497\end{array}$ & $\begin{array}{r}2.1965 \\
68 \\
71 \\
74 \\
77\end{array}$ & $\begin{array}{r}5.7552 \\
54 \\
57 \\
59 \\
61\end{array}$ & \\
\hline $\begin{array}{l}55 \\
56 \\
57 \\
58 \\
59\end{array}$ & $\begin{array}{l}59 \\
57 \\
54 \\
51 \\
48\end{array}$ & $\begin{array}{r}43 \\
34 \\
\cdot \quad 26 \\
18 \\
10\end{array}$ & $\begin{array}{l}536 \\
575 \\
615 \\
654 \\
693\end{array}$ & $\begin{array}{l}80 \\
83 \\
86 \\
89 \\
92\end{array}$ & $\begin{array}{l}63 \\
65 \\
68 \\
70 \\
72\end{array}$ & \\
\hline 60 & 8.5095546 & 8. 5121602 & 0.96733 & 2. 1996 & 5.7574 & 7. 772 \\
\hline
\end{tabular}


TARLA 20.-Geodetic position computations-Continued.

LATITUDE $20^{\circ}$.

\begin{tabular}{|c|c|c|c|c|c|c|}
\hline Lat. & $\begin{array}{c}\log A \\
\text { diff. } 1^{\prime \prime}=-0.05\end{array}$ & $\begin{array}{c}\log \mathrm{B} \\
\text { diff. } 1^{\prime \prime}=-0.14\end{array}$ & $\begin{array}{c}\log C \\
\text { diff. } 1^{\prime \prime}=+0.64\end{array}$ & $\begin{array}{c}\log \mathrm{D} \\
\text { diff. } 1^{\prime \prime}=+0.05\end{array}$ & $\begin{array}{c}\log \mathbf{E} \\
\operatorname{diff} .1^{\prime \prime}=+0.04\end{array}$ & $\begin{array}{c}\log \mathrm{F} \\
\text { diff. } 10^{\prime}=+2.5\end{array}$ \\
\hline $0 \quad 1$ & & & & & & \\
\hline $\begin{array}{lr}20 & 00 \\
& 1 \\
& 2 \\
& \\
& 3 \\
& 4\end{array}$ & $\begin{array}{r}8.5095546 \\
43 \\
40 \\
37 \\
35\end{array}$ & $\begin{array}{r}8.5121602 \\
8.5121593 \\
85 \\
77 \\
68\end{array}$ & $\begin{array}{r}0.96733 \\
772 \\
811 \\
850 \\
889\end{array}$ & $\begin{array}{r}2.1996 \\
2.1999 \\
2.2002 \\
05 \\
08\end{array}$ & $\begin{array}{r}5.7574 \\
77 \\
79 \\
81 \\
83\end{array}$ & 7.772 \\
\hline $\begin{array}{r}05 \\
6 \\
7 \\
8 \\
9\end{array}$ & $\begin{array}{l}32 \\
29 \\
26 \\
24 \\
21\end{array}$ & $\begin{array}{l}60 \\
52 \\
44 \\
35 \\
27\end{array}$ & $\begin{array}{r}928 \\
0.96967 \\
0.97006 \\
046 \\
084\end{array}$ & $\begin{array}{l}11 \\
14 \\
17 \\
20 \\
23\end{array}$ & $\begin{array}{l}86 \\
88 \\
90 \\
92 \\
94\end{array}$ & \\
\hline $\begin{array}{l}10 \\
11 \\
12 \\
13 \\
14\end{array}$ & $\begin{array}{r}8.5095518 \\
15 \\
12 \\
10 \\
07\end{array}$ & $\begin{array}{rr}8.512 & 1519 \\
& 10 \\
8.512 & 1502 \\
8.51 .21494 \\
85\end{array}$ & $\begin{array}{r}0.97123 \\
162 \\
201 \\
240 \\
279\end{array}$ & $\begin{array}{r}2.2026 \\
28 \\
31 \\
34 \\
37\end{array}$ & $\begin{array}{r}5.7597 \\
5.7599 \\
5.7601 \\
03 \\
06\end{array}$ & - \\
\hline $\begin{array}{l}15 \\
16 \\
17 \\
18 \\
19\end{array}$ & $\begin{array}{r}04 \\
8.5095501 \\
8.5095499 \\
96 \\
93\end{array}$ & $\begin{array}{l}77 \\
69 \\
60 \\
52 \\
44\end{array}$ & $\begin{array}{l}318 \\
356 \\
395 \\
434 \\
472\end{array}$ & $\begin{array}{l}40 \\
43 \\
46 \\
49 \\
52\end{array}$ & $\begin{array}{l}08 \\
10 \\
12 \\
15 \\
17\end{array}$ & . \\
\hline $\begin{array}{l}20 \\
21 \\
22 \\
23 \\
24\end{array}$ & $\begin{array}{r}8.509 \quad 5490 \\
87 \\
85 \\
82 \\
79\end{array}$ & $\begin{array}{r}8.5121435 \\
27 \\
18 \\
10 \\
8.5121402\end{array}$ & $\begin{array}{r}0.97511 \\
550 \\
588 \\
627 \\
666\end{array}$ & $\begin{array}{r}2.2055 \\
58 \\
61 \\
64 \\
67\end{array}$ & $\begin{array}{r}5.7619 \\
21 \\
24 \\
20 \\
28\end{array}$ & 7.777 \\
\hline $\begin{array}{l}25 \\
26 \\
27 \\
28 \\
29\end{array}$ & $\begin{array}{l}76 \\
73 \\
71 \\
68 \\
65\end{array}$ & $\begin{array}{r}8.5121393 \\
85 \\
76 \\
68 \\
60\end{array}$ & $\begin{array}{l}704 \\
743 \\
781 \\
819 \\
858\end{array}$ & $\begin{array}{l}70 \\
73 \\
76 \\
79 \\
81\end{array}$ & $\begin{array}{r}30 \\
33 \\
\quad 35 \\
-\quad 37 \\
40\end{array}$ & \\
\hline $\begin{array}{l}30 \\
31 \\
32 \\
33 \\
34\end{array}$ & $\begin{array}{r}8.5095462 \\
59 \\
57 \\
54 \\
51\end{array}$ & $\begin{array}{r}8.512 \quad 1351 \\
43 \\
34 \\
36 \\
26 \\
17\end{array}$ & $\begin{array}{r}0.97896 \\
935 \\
0.97973 \\
0.98011 \\
050\end{array}$ & $\begin{array}{r}2.2084 \\
87 \\
90 \\
93 \\
96\end{array}$ & $\begin{array}{r}5.7642 \\
44 \\
46 \\
49 \\
51\end{array}$ & \\
\hline $\begin{array}{l}35 \\
36 \\
37 \\
88 \\
39\end{array}$ & $\begin{array}{l}48 \\
45 \\
42 \\
40 \\
37\end{array}$ & $\begin{array}{r}09 \\
8.5121301 \\
8.5121292 \\
. \quad 84 \\
\quad 75\end{array}$ & $\begin{array}{l}088 \\
126 \\
164 \\
208 \\
241\end{array}$ & $\begin{array}{r}2.2099 \\
2.2102 \\
05 \\
08 \\
10\end{array}$ & $\begin{array}{l}53 \\
55 \\
58 \\
60 \\
62\end{array}$ & \\
\hline $\begin{array}{l}40 \\
41 \\
42 \\
43 \\
44\end{array}$ & $\begin{array}{r}8.5095434 \\
31 \\
28 \\
25 \\
23\end{array}$ & $\begin{array}{r}8.5121267 \\
58 \\
50 \\
41 \\
33\end{array}$ & $\begin{array}{r}0.98279 \\
317 \\
355 \\
393 \\
431\end{array}$ & $\begin{array}{r}2.2113 \\
16 \\
19 \\
22 \\
25\end{array}$ & $\begin{array}{r}5.7664 \\
67 \\
69 \\
71 \\
74 \\
74\end{array}$ & 7.782 \\
\hline $\begin{array}{l}45 \\
46 \\
47 \\
48 \\
49\end{array}$ & $\begin{array}{l}20 \\
17 \\
14 \\
11 \\
08\end{array}$ & $\begin{array}{r}24 \\
16 \\
8.5121207 \\
8.6121199 \\
90\end{array}$ & $\begin{array}{l}469 \\
507 \\
545 \\
583 \\
621\end{array}$ & $\begin{array}{l}28 \\
31 \\
33 \\
36 \\
\mathbf{3 9}\end{array}$ & $\begin{array}{l}76 \\
78 \\
81 \\
83 \\
85\end{array}$ & $\cdot$ \\
\hline $\begin{array}{l}50 \\
51 \\
52 \\
53 \\
54\end{array}$ & $\begin{array}{r}8.5095406 \\
03 \\
8.5095400 \\
8.5095397 \\
94\end{array}$ & $\begin{array}{r}8.5121182 \\
73 \\
64 \\
6 \quad 56 \\
\quad 47\end{array}$ & $\begin{array}{r}0.98659 \\
697 \\
735 \\
773 \\
811\end{array}$ & $\begin{array}{r}2.2142 \\
45 \\
48 \\
50 \\
53\end{array}$ & $\begin{array}{r}\text { 5. } 7688 \\
90 \\
92 \\
94 \\
97\end{array}$ & . \\
\hline $\begin{array}{l}55 \\
56 \\
67 \\
58 \\
59\end{array}$ & $\begin{array}{l}91 \\
88 \\
86 \\
83 \\
80\end{array}$ & $\begin{array}{r}39 \\
30 \\
21 \\
13 \\
8.51211 .04\end{array}$ & $\begin{array}{r}848 \\
886 \\
.924 \\
962 \\
0.98999\end{array}$ & $\begin{array}{l}56 \\
59 \\
62 \\
65 \\
67\end{array}$ & $\begin{array}{r}5.7699 \\
5.7701 \\
04 \\
06 \\
08\end{array}$ & . \\
\hline 60 & 8.5096377 & 8.5121096 & 0.99037 & 2.2170 & 5.7711 & 7.787: \\
\hline
\end{tabular}


TABLe 20.-Geodetic position computations-Continued.

LATITUDE $21^{\circ}$.

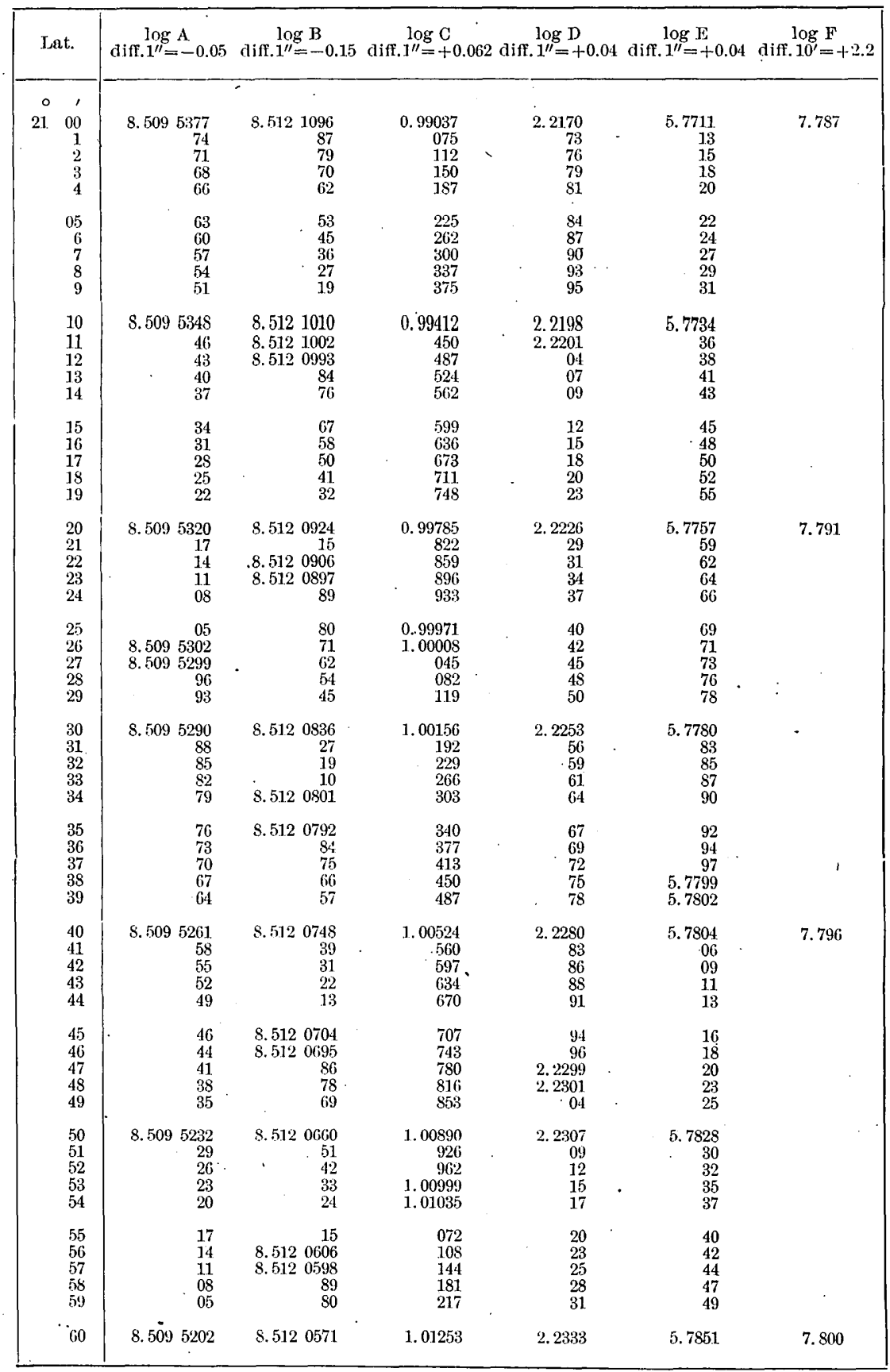


TABLE 20.-Geodetic position computations-Continued.

LATITUDE $22^{\circ}$.

\begin{tabular}{|c|c|c|c|c|c|c|}
\hline Lat. & $\begin{array}{l}\log A \\
\operatorname{diff} .1^{\prime \prime}=-0.05\end{array}$ & $\begin{array}{c}\log B \\
\text { diff. } 1^{\prime \prime}=-0.15\end{array}$ & $\begin{array}{c}\log \mathrm{C} \\
\text { iff. } 1^{\prime \prime}=+0.59\end{array}$ & $\begin{array}{c}\log \mathrm{D} \\
\operatorname{diffi} 1^{\prime \prime}=+0.04\end{array}$ & $\begin{array}{c}\log \mathrm{E} \\
\text { diff. } 1^{\prime \prime}=+0.04\end{array}$ & $\begin{array}{c}\log F \\
\text { diff. } 10^{\prime}=+2.0\end{array}$ \\
\hline 0,1 & & & & & $\cdot$ & \\
\hline $\begin{array}{rr}22 & 00 \\
& 1 \\
& 2 \\
& 3 \\
& 4\end{array}$ & $\begin{array}{r}8.5095202 \\
8.5095199 \\
96 \\
93 \\
90\end{array}$ & $\begin{array}{r}8.512 \quad 0571 \\
62 \\
53 \\
44 \\
35\end{array}$ & $\begin{array}{r}\text { 1. } 01253 \\
289 \\
326 \\
362 \\
398\end{array}$ & $\begin{array}{r}2.2333 \\
36 \\
38 \\
41 \\
44\end{array}$ & $\begin{array}{r}5.7851 \\
54 \\
56 \\
59 \\
61\end{array}$ & 7.800 \\
\hline $\begin{array}{r}05 \\
6 \\
7 \\
8 \\
9\end{array}$ & $\begin{array}{r}57 \\
. \quad 84 \\
81 \\
78 \\
75\end{array}$ & $\begin{array}{r}26 \\
17 \\
8.5120508 \\
8.5120499 \\
90\end{array}$ & $\begin{array}{l}434 \\
470 \\
506 \\
542 \\
578\end{array}$ & $\begin{array}{l}46 \\
49 \\
51 \\
.54 \\
57\end{array}$ & $\begin{array}{l}63 \\
66 \\
68 \\
71 \\
73\end{array}$ & \\
\hline $\begin{array}{l}10 \\
11 \\
12 \\
13 \\
14\end{array}$ & $\begin{array}{r}8.509 .5172 \\
69 \\
66 \\
63 \\
60\end{array}$ & $\begin{array}{r}8.512 \quad 0481 \\
72 \\
63 \\
54 \\
45\end{array}$ & $\begin{array}{r}1.01615 \\
651 \\
687 \\
723 \\
759\end{array}$ & $\begin{array}{r}2.2359 \\
62 \\
64 \\
67 \\
70\end{array}$ & $\begin{array}{r}5.7875 \\
78 \\
80 \\
83 \\
85\end{array}$ & . \\
\hline $\begin{array}{l}15 \\
-\quad 16 \\
17 \\
18 \\
19\end{array}$ & $\begin{array}{l}57 \\
54 \\
51 \\
48 \\
45\end{array}$ & $\begin{array}{r}36 \\
27 \\
18 \\
09 \\
8.5120400\end{array}$ & $\begin{array}{l}794 \\
830 \\
866 \\
902 \\
938\end{array}$ & $\begin{array}{r}72 \\
-\quad 75 \\
\quad \quad 87 \\
\quad 80 \\
\end{array}$ & $\begin{array}{l}87 \\
90 \\
92 \\
95 \\
97\end{array}$ & . \\
\hline $\begin{array}{l}20 \\
21 \\
22 \\
23 \\
24\end{array}$ & $\begin{array}{r}8.509 \quad 5142 \\
39 \\
36 \\
33 \\
30\end{array}$ & $\begin{array}{r}8.512 \quad 0391 \\
82 \\
73 \\
64 \\
55\end{array}$ & $\begin{array}{r}1.01974 \\
1.02010 \\
045 \\
081 \\
117 .\end{array}$ & $\begin{array}{r}2.2385 \\
88 \\
90 \\
93 \\
95\end{array}$ & $\begin{array}{r}5.7899 \\
5.7902 \\
04 \\
07 \\
09\end{array}$ & 7.804 \\
\hline $\begin{array}{r}25 \\
26 \\
.27 \\
28 \\
29\end{array}$ & $\begin{array}{l}27 \\
24 \\
21 \\
18 \\
15\end{array}$ & $\begin{array}{l}46 \\
37 \\
28 \\
19 \\
10\end{array}$ & $\begin{array}{l}153 \\
188 \\
224 \\
260 \\
295\end{array}$ & $\begin{array}{r}2.2398 \\
2.2400 \\
03 \\
06 \\
08\end{array}$ & $\begin{array}{l}11 \\
14 \\
16 \\
19 \\
21\end{array}$ & \\
\hline $\begin{array}{l}30 \\
31 . \\
32 \\
33 \\
34\end{array}$ & $\begin{array}{r}8.5095112 \\
09 \\
06 \\
03 \\
8.50951 .00\end{array}$ & $\begin{array}{r}8.5120301 \\
8.5120292 \\
83 \\
73 \\
64\end{array}$ & $\begin{array}{r}1.02331 \\
367 \\
402 \\
438 \\
473\end{array}$ & $\begin{array}{r}2.211 . \\
13 \\
16 \\
18 \\
21\end{array}$ & $\begin{array}{r}5: 7924 \\
26 \\
28 \\
31 \\
33\end{array}$ & \\
\hline $\begin{array}{l}35 \\
36 \\
37 \\
38 \\
39\end{array}$ & $\begin{array}{r}8.509 \quad 5097 \\
94 \\
91 \\
88 \\
85\end{array}$ & $\begin{array}{l}55 \\
46 \\
37 \\
28 \\
19\end{array}$ & $\begin{array}{l}509 \\
544 \\
580 \\
615 \\
651\end{array}$ & $\begin{array}{l}23 \\
26 \\
28 \\
31 \\
33\end{array}$ & $\begin{array}{l}36 \\
38 \\
41 \\
43 \\
45\end{array}$ & \\
\hline $\begin{array}{l}40 \\
41 \\
42 \\
43 \\
44\end{array}$ & $\begin{array}{r}8.5095082 \\
79 \\
76 \\
72 \\
69\end{array}$ & $\begin{array}{rr}8.512 & 0210 \\
8.512 & 0200 \\
8.512 & 0191 \\
. \quad & 82 \\
& 73\end{array}$ & $\begin{array}{r}\text { 1.. } 02686 \\
721 \\
757 \\
792 \\
828\end{array}$ & $\begin{array}{r}2.2436 \\
\cdot \quad 38 \\
41 \\
43 \\
46 .\end{array}$ & $\begin{array}{r}5.7948 \\
50 \\
53 \\
55 \\
58\end{array}$ & 7.808 \\
\hline $\begin{array}{l}45 \\
46 \\
47 \\
48 \\
49\end{array}$ & $\begin{array}{l}66 \\
63 \\
60 \\
57 \\
64\end{array}$ & $\begin{array}{l}64 \\
55 \\
46 \\
36 \\
27\end{array}$ & $\begin{array}{r}863 \\
. \quad 898 \\
933 \\
1.02969 \\
1.03004\end{array}$ & $\begin{array}{l}48 \\
51 \\
53 \\
56 \\
58\end{array}$ & $\begin{array}{l}60 \\
62 \\
65 \\
67 \\
70\end{array}$ & \\
\hline $\begin{array}{l}50 \\
51 \\
52 \\
53 \\
54\end{array}$ & $\begin{array}{rr}8.509 & 5051 \\
\cdot & 48 \\
& 45 \\
& 42 \\
& 39\end{array}$ & $\begin{array}{r}8.5120118 \\
09 \\
8.5120100 \\
8.5120090 \\
81 .\end{array}$ & $\begin{array}{r}1.03039 \\
074 \\
109 \\
145 \\
180\end{array}$ & $\begin{array}{r}2.2461 \\
63 \\
66 \\
68 \\
70\end{array}$ & ธ. $\begin{array}{r}7972 \\
75 \\
77 \\
80 \\
82\end{array}$ & \\
\hline $\begin{array}{l}55 \\
56 \\
57 \\
58 \\
59\end{array}$ & $\begin{array}{l}30 \\
33 \\
30 \\
27 \\
23\end{array}$ & $\begin{array}{l}72 \\
63 \\
54 \\
44 \\
35\end{array}$ & $\begin{array}{l}215 \\
250 \\
285 \\
320 \\
355\end{array}$ & $\begin{array}{l}73 \\
75 \\
78 \\
80 \\
83\end{array}$ & $\begin{array}{l}84 \\
87 \\
89 \\
92 \\
94\end{array}$ & \\
\hline 60 & $8.5095 \mathrm{C} 20$ & 8.6120026 & 1.08390 & 2.2485 & 5. 7997 & 7.812 \\
\hline
\end{tabular}


TABLE 20.-Geodetic position computations-Continued

LATITUDE $23^{\circ}$.

\begin{tabular}{|c|c|c|c|c|c|c|c|}
\hline Lat. & & $\begin{array}{c}\log A \\
\text { diff. } 1^{\prime \prime}=-0.05\end{array}$ & $\begin{array}{c}\log B \\
\text { diff. } 1^{\prime \prime}=-0.16\end{array}$ & $\begin{array}{c}\log \mathrm{C} \\
\text { diff. } 1^{\prime \prime}=+0.57\end{array}$ & $\begin{array}{c}\log \mathrm{D} \\
\text { diff. } 1^{\prime \prime}=+0.04\end{array}$ & $\begin{array}{c}\log \mathrm{E} \\
\text { diff. } 1^{\prime \prime}=+0.04\end{array}$ & diff. $10^{\prime}=+1.8$ \\
\hline$\circ$ & , & & & & & & \\
\hline 230 & $\begin{array}{r}00 \\
1 \\
2 \\
3 \\
4\end{array}$ & $\begin{array}{r}\text { 8. } 5095020 \\
17 \\
14 \\
11 \\
08\end{array}$ & $\begin{array}{r}8.5120026 \\
17 \\
8.5120008 \\
8.511 \quad 9998 \\
89\end{array}$ & $\begin{array}{r}\text { 1. } 03390 \\
425 \\
460 \\
49 \overline{5} \\
530\end{array}$ & $\begin{array}{r}2.2485 \\
88 \\
90 \\
93 \\
95\end{array}$ & $\begin{array}{r}\text { 5. } 7997 \\
\text { 5. } 7999 \\
\text { 5. } 8002 \\
04 \\
07\end{array}$ & 7.812 \\
\hline & $\begin{array}{r}05 \\
6 \\
7 \\
8 \\
9\end{array}$ & $\begin{array}{r}05 \\
8.5095002 \\
8.509 \\
4999 \\
96 \\
93\end{array}$ & $\begin{array}{l}80 \\
71 \\
61 \\
52 \\
43\end{array}$ & $\begin{array}{l}565 \\
600 \\
634 \\
669 \\
704\end{array}$ & $\begin{array}{r}2.2497 \\
2.2500 \\
02 \\
05 \\
07\end{array}$ & $\begin{array}{l}09 \\
12 \\
14 \\
16 \\
19\end{array}$ & . \\
\hline & $\begin{array}{l}10 \\
11 \\
12 \\
13 \\
14\end{array}$ & $\begin{array}{r}8.5094990 \\
87 \\
83 \\
80 \\
77\end{array}$ & $\begin{array}{r}8.511 .9934 \\
24 \\
15 \\
8.5119906 \\
8.5119896\end{array}$ & $\begin{array}{r}1.03739 \\
774 \\
809 \\
843 \\
878\end{array}$ & $\begin{array}{r}2.2510 \\
12 \\
14 \\
17 \\
19\end{array}$ & $\begin{array}{r}5.8021 \\
24 \\
26 \\
29 \\
31\end{array}$ & . \\
\hline $\begin{array}{r}1 \\
1 \\
1 \\
1 \\
1\end{array}$ & $\begin{array}{l}15 \\
16 \\
17 \\
18 \\
19\end{array}$ & $\begin{array}{l}74 \\
71 \\
68 \\
65 \\
62\end{array}$ & $\begin{array}{r}87 \\
78 \\
68 \\
59 \\
50\end{array}$ & $\begin{array}{r}913 \\
947 \\
\text { 1. } 03982 \\
1.04017 \\
052\end{array}$ & $\begin{array}{l}22 \\
24 \\
26 \\
29 \\
31\end{array}$ & $\begin{array}{l}34 \\
36 \\
39 \\
41 \\
44\end{array}$ & $:$ \\
\hline & $\begin{array}{l}20 \\
21 \\
22 \\
23 \\
24\end{array}$ & $\begin{array}{r}8.509 \quad 4959 \\
55 \\
52 \\
49 \\
46\end{array}$ & $\begin{array}{r}8.5119840 \\
31 \\
22 \\
12 \\
8.5119803\end{array}$ & $\begin{array}{r}1.04086 \\
121 \\
155 \\
190 \\
224\end{array}$ & $\begin{array}{r}2.2534 \\
36 \\
38 \\
41 \\
43\end{array}$ & $\begin{array}{r}5.8046 \\
49 \\
51 \\
54 \\
56\end{array}$ & 7.816 \\
\hline & $\begin{array}{l}25 \\
26 \\
27 \\
28 \\
29\end{array}$ & $\begin{array}{l}43 \\
40 \\
37 \\
34 \\
31\end{array}$ & $\begin{array}{r}8.511 .9794 \\
84 \\
75 \\
66 \\
56\end{array}$ & $\begin{array}{l}259 \\
293 \\
328 \\
362 \\
397\end{array}$ & $\begin{array}{l}45 \\
48 \\
50 \\
53 \\
55\end{array}$ & $\begin{array}{l}59 \\
61 \\
64 \\
66 \\
69\end{array}$ & \\
\hline & $\begin{array}{l}30 \\
31 \\
32 \\
33 \\
34\end{array}$ & $\begin{array}{r}8.5094927 \\
24 \\
21 \\
18 \\
15\end{array}$ & $\begin{array}{r}8.5119747 \\
37 \\
28 \\
19 \\
09\end{array}$ & $\begin{array}{r}1.04431 \\
466 \\
500 \\
534 \\
569\end{array}$ & $\begin{array}{r}2.2557 \\
60 \\
62 \\
64 \\
67\end{array}$ & $\begin{array}{r}5.8071 \\
74 \\
76 \\
79 \\
81\end{array}$ & . \\
\hline & $\begin{array}{l}35 \\
36 \\
37 \\
38 \\
39\end{array}$ & $\begin{array}{cr}. & 12 \\
& 09 \\
& 05 \\
8.509 & 4902 \\
8.509 \quad 4899\end{array}$ & $\begin{array}{rr}8.511 & 9700 \\
8.511 & 9690 \\
& 81 \\
71 \\
62\end{array}$ & $\begin{array}{l}603 \\
637 \\
672 \\
706 \\
740\end{array}$ & $\begin{array}{l}69 \\
71 \\
74 \\
76 \\
78\end{array}$ & $\begin{array}{l}84 \\
86 \\
89 \\
91 \\
93\end{array}$ & \\
\hline $\begin{array}{r}4 \\
4 \\
4 \\
4 \\
4\end{array}$ & $\begin{array}{l}40 \\
41 \\
42 \\
43 \\
44\end{array}$ & $\begin{array}{r}8.5094896 \\
93 \\
90 \\
87 \\
83\end{array}$ & $\begin{array}{r}8.511 .9653 \\
43 \\
34 \\
24 \\
. \quad 15\end{array}$ & $\begin{array}{r}1.04775 \\
809 \\
843 \\
877 \\
911\end{array}$ & $\begin{array}{r}2.2581 \\
83 \\
85 \\
88 \\
90\end{array}$ & $\begin{array}{r}5.8096 \\
5.8099 \\
5.8101 \\
04 \\
06\end{array}$ & 7.819 \\
\hline $\begin{array}{l}4 \\
4 \\
4 \\
4 \\
4\end{array}$ & $\begin{array}{l}45 \\
46 \\
47 \\
48 \\
49\end{array}$ & $\begin{array}{l}80 \\
77 \\
74 \\
71 \\
68\end{array}$ & $\begin{array}{rr}8.511 & 9605 \\
8.511 & 9596 \\
& 86 \\
& 77 \\
& 67\end{array}$ & $\begin{array}{r}945 \\
1.04980 \\
1.05014 \\
048 \\
082\end{array}$ & $\begin{array}{r}92 \\
95 \\
97 \\
2.2599 \\
2.2601\end{array}$ & $\begin{array}{l}09 \\
11 \\
14 \\
16 \\
19\end{array}$ & \\
\hline & $\begin{array}{l}50 \\
51 \\
52 \\
53 \\
54\end{array}$ & $\begin{array}{r}8.509 \quad 4865 \\
61 \\
58 \\
55 \\
52\end{array}$ & $\begin{array}{r}8.511 \quad 9558 \\
48 \\
\quad 39 \\
\\
\quad 29 \\
20\end{array}$ & $\begin{array}{r}1.05116 \\
150 \\
184 \\
218 \\
252\end{array}$ & $\begin{array}{r}2.2604 \\
06 \\
09 \\
11 \\
13\end{array}$ & $\begin{array}{r}5.8121 \\
24 \\
26 \\
29 \\
31\end{array}$ & 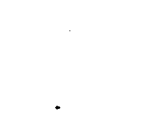 \\
\hline & $\begin{array}{l}55 \\
56 \\
57 \\
58 \\
59\end{array}$ & $\begin{array}{l}49 \\
45 \\
42 \\
39 \\
36\end{array}$ & $\begin{array}{rr} & 10 \\
8.511 & 9501 \\
8.511 & 9491 \\
& 82 \\
& 72\end{array}$ & $\begin{array}{l}286 \\
320 \\
354 \\
388 \\
422\end{array}$ & $\begin{array}{l}16 \\
18 \\
20 \\
23 \\
25\end{array}$ & $\begin{array}{l}34 \\
36 \\
39 \\
41 \\
44\end{array}$ & . \\
\hline & 60 & 8.5094833 & 8.5119463 & 1.05456 & 2.2627 & 5.8146 & 7.823 \\
\hline
\end{tabular}


TARLE 20.-Geodetic position computations-Continued.

LATITUDE $24^{\circ}$.

\begin{tabular}{|c|c|c|c|c|c|c|}
\hline Litt. & $\begin{array}{c}\log A \\
\text { diff. } 1^{\prime \prime}=-0.05\end{array}$ & $\begin{array}{l}\log B \\
\text { diff. } 1^{\prime \prime}=-0.16\end{array}$ & $\begin{array}{c}\log C \\
\text { diff. } 1^{\prime \prime}=+0.56\end{array}$ & (i) diff. $1^{\prime \prime}=+0.04$ & $\begin{array}{c}\log \mathrm{E} \\
\text { diff. } 1^{\prime \prime}=+0.04\end{array}$ & $\begin{array}{c}\log \mathrm{F} \\
\text { diff. } 10^{\prime}=+1.6\end{array}$ \\
\hline $\begin{array}{cc}\circ & 1 \\
24 & 00 \\
& 1 \\
& 1 \\
& 3 \\
& \\
& 4\end{array}$ & $\begin{array}{r}8.5094833 \\
30 \\
26 \\
23 \\
20\end{array}$ & $\begin{array}{r}8.5119463 \\
53 \\
\cdot 44 \\
34 \\
. \quad 24\end{array}$ & $\begin{array}{r}1.05456 \\
490 \\
523 \\
557 \\
591\end{array}$ & $\begin{array}{r}2,2627 \\
29 \\
31 \\
34 \\
36\end{array}$ & $\begin{array}{r}5.8146 \\
49 \\
51 \\
54 \\
57\end{array}$ & 7.823 \\
\hline $\begin{array}{r}05 \\
6 \\
7 \\
8 \\
9\end{array}$ & $\begin{array}{l}17 \\
14 \\
10 \\
07 \\
04\end{array}$ & $\begin{array}{r}15 \\
8.5119405 \\
8.5119396 \\
86 \\
77\end{array}$ & $\begin{array}{l}625 \\
658 \\
692 \\
776 \\
760\end{array}$ & $\begin{array}{l}\dot{5} 8 \\
41 \\
43 \\
45 \\
47\end{array}$ & $\begin{array}{l}69 \\
62 \\
64 \\
67 \\
69\end{array}$ & \\
\hline $\begin{array}{l}10 \\
11 \\
12 \\
13 \\
14\end{array}$ & $\begin{array}{r}8.5094801 \\
8.5094798 \\
94 \\
91 \\
88\end{array}$ & $\begin{array}{r}8.5119367 \\
58 \\
48 \\
38 \\
29\end{array}$ & $\begin{array}{r}1.05794 \\
827 \\
861 \\
894 \\
\quad 928\end{array}$ & $\begin{array}{r}2.2650 \\
52 \\
54 \\
56 \\
59\end{array}$ & $\begin{array}{r}5.8172 \\
74 \\
77 \\
79 \\
82\end{array}$ & \\
\hline $\begin{array}{l}15 \\
16 \\
17 \\
18 \\
19\end{array}$ & $\begin{array}{l}85 \\
82 \\
78 \\
75 \\
72\end{array}$ & $\begin{array}{r}19 \\
09 \\
\text { 8.511 } 9300 \\
8.5119290 \\
81\end{array}$ & $\begin{array}{r}962 \\
1.05995 \\
1.06029 \\
062 \\
096\end{array}$ & $\begin{array}{l}61 \\
63 \\
65 \\
68 \\
70\end{array}$ & $\begin{array}{l}85 \\
87 \\
90 \\
92 \\
95\end{array}$ & \\
\hline $\begin{array}{l}20 \\
21 \\
22 \\
23 \\
24\end{array}$ & $\begin{array}{r}8.5094769 \\
66 \\
62 \\
59 \\
56\end{array}$ & $\begin{array}{r}8.5119271 \\
61 \\
52 \\
42 \\
32\end{array}$ & $\begin{array}{r}1.06130 \\
163 \\
197 \\
230 \\
263\end{array}$ & $\begin{array}{r}2.2672 \\
74 \\
77 \\
79 \\
81\end{array}$ & $\begin{array}{r}5.8197 \\
5.8200 \\
02 \\
05 \\
07\end{array}$ & 7.826 \\
\hline $\begin{array}{l}25 \\
26 \\
27 \\
28 \\
29\end{array}$ & $\begin{array}{l}53 \\
50 \\
46 \\
43 \\
40\end{array}$ & $\begin{array}{r}23 \\
13 \\
8.5119203 \\
8.5119194 \\
84\end{array}$ & $\begin{array}{l}297 \\
330 \\
364 \\
397 \\
431\end{array}$ & $\begin{array}{l}83 \\
85 \\
88 \\
90 \\
92\end{array}$ & $\begin{array}{l}10 \\
13 \\
15 \\
18 \\
20\end{array}$ & \\
\hline $\begin{array}{l}30 \\
31 \\
32 \\
33 \\
34\end{array}$ & $\begin{array}{r}8.509 \quad 437 \\
\cdot \quad 33 \\
\quad 30 \\
. \quad 27 \\
24\end{array}$ & $\begin{array}{r}8.511 .9174 \\
\cdot \quad 65 \\
55 \\
45 \\
35\end{array}$ & $\begin{array}{r}1.06464 \\
497 \\
530 \\
564 \\
597\end{array}$ & $\begin{array}{r}2.2694 \\
96 \\
2.2699 \\
2.2701 \\
03\end{array}$ & $\begin{array}{r}5.8223 \\
25 \\
28 \\
31 \\
33\end{array}$ & \\
\hline $\begin{array}{l}35 \\
36 \\
37 \\
38 \\
39\end{array}$ & $\begin{array}{l}20 \\
17 \\
14 \\
11 . \\
07\end{array}$ & $\begin{array}{rr} & 26 \\
16 \\
8.511 & 91.06 \\
8.511 & 9096 \\
& 87\end{array}$ & $\begin{array}{l}630 \\
664 \\
697 \\
730 \\
763\end{array}$ & $\begin{array}{l}05 \\
07 \\
10 \\
12 \\
14\end{array}$ & $\begin{array}{l}36 \\
38 \\
41 \\
43 \\
46\end{array}$ & \\
\hline $\begin{array}{l}40 \\
41 \\
42 \\
43 \\
44\end{array}$ & $\begin{array}{r}8.5094704 \\
8.5094701 \\
\text { S.509 } 4698 \\
94 \\
91\end{array}$ & $\begin{array}{r}8.5119077 \\
67 \\
\quad 58 \\
48 \\
38\end{array}$ & $\begin{array}{r}1.06797 \\
830 \\
863 \\
896 \\
929\end{array}$ & $\begin{array}{r}2.2716 \\
18 \\
20 \\
23 \\
25\end{array}$ & $\begin{array}{r}5.8249 \\
51 \\
54 \\
56 \\
59\end{array}$ & 7.829 \\
\hline $\begin{array}{l}45 \\
46 \\
47 \\
48 \\
49\end{array}$ & $\begin{array}{l}88 \\
85 \\
81 \\
78 \\
75\end{array}$ & $\begin{array}{rr}\cdot & 28 \\
18 \\
8.5119009 \\
8.511 \quad 8999 \\
\quad 89\end{array}$ & $\begin{array}{r}962 \\
1.06995 \\
1.07028 \\
061 \\
095\end{array}$ & $\begin{array}{l}27 \\
29 \\
31 \\
33 \\
36\end{array}$ & $\begin{array}{l}61 \\
64 \\
67 \\
69 \\
72\end{array}$ & \\
\hline $\begin{array}{l}50 \\
51 \\
52 \\
53 \\
54\end{array}$ & $\begin{array}{r}8.5094672 \\
-\quad 68 \\
65 \\
62 \\
59\end{array}$ & $\begin{array}{r}8.5118979 \\
70 \\
60 \\
50 \\
40\end{array}$ & $\begin{array}{r}1.07128 \\
161 \\
194 \\
226 \\
259\end{array}$ & $\begin{array}{r}2.2738 \\
40 \\
42 \\
44 \\
46\end{array}$ & $\begin{array}{r}5.8274 \\
\cdot 77 \\
80 \\
82 \\
85\end{array}$ & i \\
\hline $\begin{array}{l}55 \\
56 \\
57 \\
58 \\
59\end{array}$ & $\begin{array}{l}55 \\
52 \\
49 \\
45 \\
42\end{array}$ & $\begin{array}{r}30 \\
21 \\
11 \\
8.5118901 \\
8.5118891\end{array}$ & $\begin{array}{l}292 \\
325 \\
358 \\
391 \\
424\end{array}$ & $\begin{array}{l}49 \\
51 \\
53 \\
55 \\
57\end{array}$ & $\begin{array}{r}87 \\
90 \\
92 \\
95 \\
5.8298\end{array}$ & $\vdots$ \\
\hline 60 & 8.5094639 & 8.5118881 & 1.07457 & 2. 2759 & 5.8300 & 7.832 \\
\hline
\end{tabular}


Tarle 20.-Geodetic position compulations-Continued.

LATITUDE $25^{\circ}$.

\begin{tabular}{|c|c|c|c|c|c|c|}
\hline Lat. & $\begin{array}{c}\log A \\
\text { diff. } 1^{\prime \prime}=-0.06\end{array}$ & $\begin{array}{l}\log B \\
\text { diff. } 1^{\prime \prime}=-0.16\end{array}$ & $\begin{array}{c}\log \mathrm{C} \\
\text { iiff. } \mathbf{1}^{\prime \prime}=-0.54\end{array}$ & $\begin{array}{c}\log \mathrm{n} \\
\text { diff. } 1^{\prime \prime}=+0.0\end{array}$ & $\begin{array}{c}\log \mathrm{IE} \\
\text { diff. } 1^{\prime \prime}=+0.04\end{array}$ & $\begin{array}{c}\log F \\
\text { diff. } 10^{\prime}=+1 \cdot 5\end{array}$ \\
\hline $0 \quad 1$ & & & & & & \\
\hline $\begin{array}{rr}25 & 00 \\
& 1 \\
& 2 \\
& 3 \\
& 4\end{array}$ & $\begin{array}{r}8.509 \quad 1639 \\
36 \\
32 \\
\quad 29 \\
26\end{array}$ & $\begin{array}{rr}8.511 & 8881 \\
\ddots & 71 \\
& 62 \\
& 52 \\
& 42\end{array}$ & $\begin{array}{r}1.07457 \\
490 \\
523 \\
555 \\
588\end{array}$ & $\begin{array}{r}2.2759 \\
61 \\
63 \\
66 \\
68\end{array}$ & $\begin{array}{r}5.8300 \\
03 \\
05 \\
08 \\
11\end{array}$ & $7.83 \%$ \\
\hline $\begin{array}{r}05 \\
6 \\
7 \\
8 \\
9\end{array}$ & $\begin{array}{l}23 \\
19 \\
16 \\
13 \\
09\end{array}$ & $\begin{array}{r}32 \\
22 \\
12 \\
8.5118802 \\
8.5118793\end{array}$ & $\begin{array}{l}621 \\
654 \\
687 \\
719 \\
752\end{array}$ & $\begin{array}{l}70 \\
72 \\
74 \\
76 \\
78\end{array}$ & $\begin{array}{l}13 \\
16 \\
18 \\
21 \\
24 .\end{array}$ & . \\
\hline $\begin{array}{l}10 \\
11 \\
12 \\
13 \\
14\end{array}$ & $\begin{array}{r}8.5094606 \\
003 \\
8.5094600 \\
8.5094596 \\
993\end{array}$ & $\begin{array}{r}8.511 .8783 \\
73 \\
63 \\
53 \\
43\end{array}$ & $\begin{array}{r}1.07785 \\
817 \\
850 \\
883 \\
915\end{array}$ & $\begin{array}{r}2.2780 \\
82 \\
85 \\
87 \\
89\end{array}$ & 5. $\begin{array}{r}8326 \\
29 \\
32 \\
34 \\
37\end{array}$ & 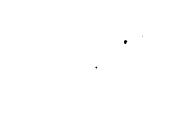 \\
\hline $\begin{array}{l}1.5 \\
16 \\
1.7 \\
1.8 \\
19\end{array}$ & $\begin{array}{l}90 \\
86 \\
83 \\
80 \\
76\end{array}$ & $\begin{array}{r}33 \\
23 \\
13 \\
\text { 8.511. } 8704 \\
8.511 .8694\end{array}$ & $\begin{array}{r}948 \\
1.07981 \\
1.08013 \\
046 \\
078\end{array}$ & $\begin{array}{r}91 \\
93 \\
95 \\
97 \\
2.2799\end{array}$ & $\begin{array}{l}39 \\
42 \\
45 \\
47 \\
50\end{array}$ & \\
\hline $\begin{array}{l}20 \\
21 . \\
22 \\
23 \\
24\end{array}$ & $\begin{array}{r}8.509 \quad 4573 \\
70 \\
66 \\
63 \\
60\end{array}$ & $\begin{array}{r}\text { 8.511. } 8684 \\
74 \\
64 \\
54 \\
44\end{array}$ & $\begin{array}{r}1.08111 \\
143 \\
176 \\
208 \\
241\end{array}$ & $\begin{array}{r}2.2801 \\
03 \\
05 \\
07 \\
10\end{array}$ & 5. $\begin{array}{r}8352 \\
55 \\
59 \\
60 \\
63\end{array}$ & 7. 835 \\
\hline $\begin{array}{l}25 \\
26 \\
27 \\
28 \\
29\end{array}$ & $\begin{array}{l}56 \\
53 \\
50 \\
46 \\
43\end{array}$ & $\begin{array}{r}34 \\
24 \\
14 \\
8.511 .8604 \\
8.511 \quad 8594\end{array}$ & $\begin{array}{l}273 \\
306 \\
338 \\
370 \\
403\end{array}$ & $\begin{array}{l}12 \\
14 \\
1.6 \\
18 \\
20\end{array}$ & $\begin{array}{l}66 \\
68 \\
71 \\
-73 \\
76\end{array}$ & . \\
\hline $\begin{array}{l}30 \\
31 \\
32 \\
33 \\
34\end{array}$ & $\begin{array}{r}8.5094540 \\
37 \\
33 \\
30 \\
26\end{array}$ & $\begin{array}{r}8.511 .8584 \\
-\quad 74 \\
64 \\
54 \\
44\end{array}$ & $\begin{array}{r}1.08435 \\
468 \\
500 \\
532 \\
565\end{array}$ & $\begin{array}{r}2.2822 \\
24 \\
26 \\
28 \\
30\end{array}$ & $\begin{array}{r}5.8379 \\
81 \\
84 \\
87 \\
89\end{array}$ & \\
\hline $\begin{array}{l}35 \\
36 \\
37 \\
38 \\
39\end{array}$ & $\begin{array}{r}23 \\
20 \\
17 \\
13 \\
10\end{array}$ & $\begin{array}{r}34 \\
24 \\
14 \\
8.5118504 \\
8.51119494\end{array}$ & $\begin{array}{l}597- \\
629 \\
662 \\
694 \\
726\end{array}$ & $\begin{array}{l}32 \\
34 \\
36 \\
38 \\
40\end{array}$ & $\begin{array}{r}92 \\
94 \\
5.8397 \\
5.8400 \\
02\end{array}$ & . \\
\hline $\begin{array}{l}40 \\
41 \\
42 \\
43 \\
44\end{array}$ & $\begin{array}{r}8.5094507 \\
03 \\
8.5094500 \\
8.5094496 \\
93\end{array}$ & $\begin{array}{r}8.5118484 \\
74 \\
64 \\
54 \\
. \quad 44\end{array}$ & $\begin{array}{r}1.08758 \\
791 \\
823 \\
855 \\
. \quad 887\end{array}$ & $\begin{array}{r}2.2842 \\
44 \\
46 \\
48 \\
50\end{array}$ & $\begin{array}{r}5.8405 \\
08 \\
.10 \\
13 \\
16\end{array}$ & 7.838 \\
\hline $\begin{array}{l}45 \\
46 \\
47 \\
49 \\
49\end{array}$ & $\begin{array}{l}90 \\
86 \\
83 \\
80 \\
76\end{array}$ & $\begin{array}{r}34 \\
24 \\
14 \\
8.5118404 \\
\text { S.511 } 8393\end{array}$ & $\begin{array}{r}919 \\
951 \\
1.08984 \\
1.09016 \\
048\end{array}$ & $\begin{array}{l}52 \\
54 \\
56 \\
58 \\
60\end{array}$ & $\begin{array}{l}18 \\
21 \\
24 \\
26 \\
29\end{array}$ & \\
\hline $\begin{array}{l}50 \\
51 \\
52 \\
53 \\
54\end{array}$ & $\begin{array}{r}8.509 \quad 4473 \\
70 \\
66 \\
63 \\
60\end{array}$ & $\begin{array}{r}8.511 \quad 8383 \\
73 \\
63 \\
53 \\
43\end{array}$ & $\begin{array}{r}1.09080 \\
112 \\
1.44 \\
176 \\
208\end{array}$ & $\begin{array}{r}2.2862 \\
64 \\
66 \\
68 \\
70\end{array}$ & $\begin{array}{r}5.8431 \\
34 \\
37 \\
39 \\
42\end{array}$ & \\
\hline $\begin{array}{l}55 \\
56 \\
57 \\
58 \\
59\end{array}$ & $\begin{array}{l}56 \\
53 \\
50 \\
46 \\
43\end{array}$ & $\begin{array}{r}33 \\
23 \\
13 \\
8.5118303 \\
8.5118293\end{array}$ & $\begin{array}{l}240 \\
272 \\
304 \\
336 \\
368\end{array}$ & $\begin{array}{l}72 \\
74 \\
76 \\
78 \\
80\end{array}$ & $\begin{array}{r}45 \\
47 \\
50 \\
53 \\
.55\end{array}$ & \\
\hline 60 & 8.5094439. & 8.511 8283 & 1. 09400 & 2.2882 & $5.845 \mathrm{~S}$ & 7. 841 \\
\hline
\end{tabular}


TABLe 20.-Geodetic position computations-Continued.

IATTTUDE $26^{\circ}$.

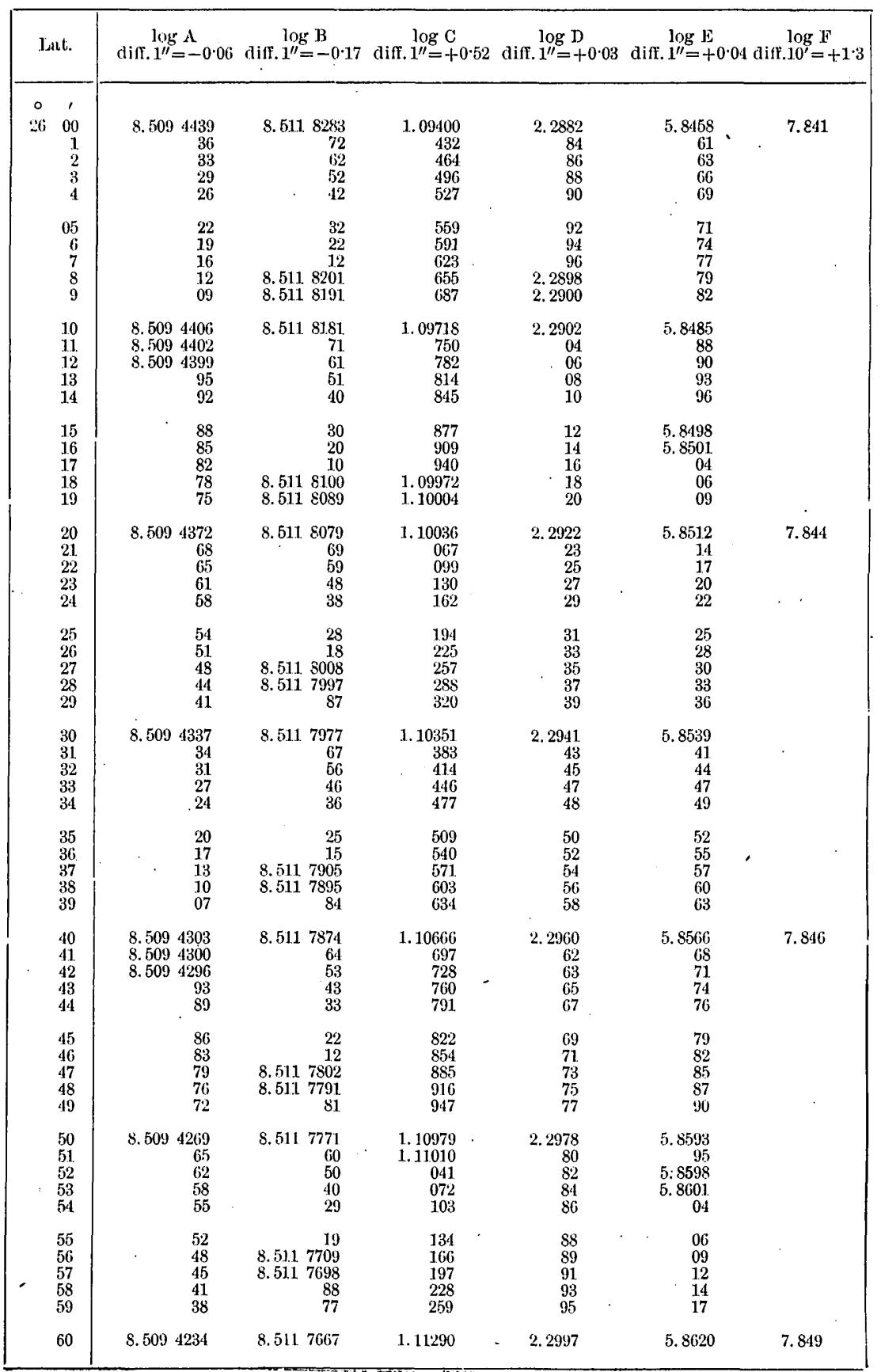


TabLe 20.-Geodetic position computations-Continued.

LATITUDE $27^{\circ}$.

\begin{tabular}{|c|c|c|c|c|c|c|}
\hline Lat. & diff. $1^{\prime \prime}=-0.06$ & $\begin{array}{c}\log B \\
\text { diff. } 1^{\prime \prime}=-0.18\end{array}$ & $\begin{array}{c}\log C \\
\operatorname{diff} .1^{\prime \prime}=+0.51\end{array}$ & $\begin{array}{c}\log \mathrm{D} \\
\text { diff. } 1^{\prime \prime}=+0.03\end{array}$ & $\operatorname{diff}^{\log 1^{\prime \prime}=+0.05}$ & $\begin{array}{c}\log F \\
\text { diff. } 10^{\prime}=+1.1\end{array}$ \\
\hline $\begin{array}{cc}\circ & , \\
27 & 00 \\
& 1 \\
& 2 \\
& 3 \\
& 4\end{array}$ & $\begin{array}{r}8.509 \quad 4234 \\
31 \\
27 \\
\quad 24 \\
20\end{array}$ & $\begin{array}{r}8.5117667 \\
57 \\
46 \\
36 \\
25\end{array}$ & $\begin{array}{r}1.11290 \\
321 \\
352 \\
383 \\
414\end{array}$ & $\begin{array}{r}2.2997 \\
2.2999 \\
2.3001 \\
03 \\
04\end{array}$ & $\begin{array}{r}5.8620 \\
23 \\
25 \\
28 \\
31\end{array}$ & 7. 849 \\
\hline $\begin{array}{r}05 \\
6 \\
7 \\
8 \\
9\end{array}$ & $\begin{array}{l}17 \\
13 \\
10 \\
06 \\
03\end{array}$ & $\begin{array}{rr}15 \\
8.511 & 7605 \\
8.511 & 7594 \\
8 & 84 \\
. \quad 73\end{array}$ & $\begin{array}{l}\mathbf{4 4 5} \\
\mathbf{4 7 6} \\
507 \\
538 \\
569\end{array}$ & $\begin{array}{l}06 \\
08 \\
10 \\
12 \\
14\end{array}$ & $\begin{array}{l}34 \\
36 \\
39 \\
42 \\
44\end{array}$ & \\
\hline $\begin{array}{l}10 \\
11 \\
12 \\
13 \\
14\end{array}$ & $\begin{array}{r}8.5094200 \\
8.5094196 \\
93 \\
89 \\
86\end{array}$ & $\begin{array}{r}8.511 \quad 7563 \\
53 \\
42 \\
32 \\
21\end{array}$ & $\begin{array}{r}1.11600 \\
631 \\
662 \\
693 \\
724\end{array}$ & $\begin{array}{r}2.3015 \\
17 \\
19 \\
21 \\
23\end{array}$ & $\begin{array}{r}5.8647 \\
.50 \\
53 \\
55 \\
58\end{array}$ & \\
\hline $\begin{array}{l}15 \\
16 \\
17 \\
18 \\
19\end{array}$ & $\begin{array}{r}82 \\
79 \\
75 \\
72 \\
68\end{array}$ & $\begin{array}{r}11 \\
8.5117500 \\
8.5117490 \\
79 \\
69\end{array}$ & $\begin{array}{l}755 \\
786 \\
817 \\
848 \\
878\end{array}$ & $\begin{array}{l}24 \\
26 \\
28 \\
30 \\
32\end{array}$ & $\begin{array}{l}61 \\
64 \\
66 \\
69 \\
72\end{array}$ & \\
\hline $\begin{array}{l}20 \\
21 \\
22 \\
23 \\
24\end{array}$ & $\begin{array}{r}8.5094165 \\
61 \\
58 \\
54 \\
51\end{array}$ & $\begin{array}{r}5.5117458 \\
48 \\
37 \\
27 \\
16\end{array}$ & $\begin{array}{r}1.11909 \\
940 \\
1.11971 \\
1.12002 \\
032\end{array}$ & $\begin{array}{r}2.3033 \\
35 \\
37 \\
39 \\
41\end{array}$ & $\begin{array}{r}5.8675 \\
77 \\
80 \\
83 \\
86\end{array}$ & 7.851 \\
\hline $\begin{array}{l}25 \\
26 \\
27 \\
28 \\
29\end{array}$ & $\begin{array}{l}47 \\
44 \\
40 \\
37 \\
33\end{array}$ & $\begin{array}{r}8.5117406 \\
8.5117395 \\
85 \\
74 \\
64\end{array}$ & $\begin{array}{l}063 \\
094 \\
125 \\
156 \\
186\end{array}$ & $\begin{array}{l}42 \\
44 \\
46 \\
48 \\
50\end{array}$ & $\begin{array}{r}88 \\
91 \\
94 \\
-\quad 97 \\
5.8699\end{array}$ & \\
\hline $\begin{array}{l}30 \\
31 \\
32 \\
33 \\
34\end{array}$ & $\begin{array}{r}8.509 \quad 4130 \\
26 \\
23 \\
19 \\
16\end{array}$ & $\begin{array}{r}8.5117353 \\
43 \\
32 \\
22 \\
11\end{array}$ & $\begin{array}{r}1.12217 \\
248 \\
278 \\
309 \\
340\end{array}$ & $\begin{array}{r}2.3051 \\
53 \\
55 \\
57 \\
58\end{array}$ & $\begin{array}{r}5.8702 \\
05 \\
08 \\
10 \\
13\end{array}$ & \\
\hline $\begin{array}{l}35 \\
36 \\
37 \\
38 \\
39\end{array}$ & $\begin{array}{rr} & 12 \\
& 08 \\
& 05 \\
8.5094101 \\
8.5094098\end{array}$ & $\begin{array}{r}8.5117301 \\
8.5117290 \\
80 \\
69 \\
58\end{array}$ & $\begin{array}{l}370 \\
401 \\
432 \\
462 \\
493\end{array}$ & $\begin{array}{l}60 \\
62 \\
64 \\
65 \\
67\end{array}$ & $\begin{array}{l}16 \\
19 \\
22 \\
24 \\
27\end{array}$ & \\
\hline $\begin{array}{l}40 \\
41 \\
42 \\
43 \\
44\end{array}$ & $\begin{array}{r}8.509 \quad 4094 \\
91 \\
87 \\
84 \\
80\end{array}$ & $\begin{array}{r}8.5117248 \\
37 \\
27 \\
16 \\
8.5117206\end{array}$ & $\begin{array}{r}1.12523 \\
554 \\
584 \\
615 \\
\mathbf{6 4 6}\end{array}$ & $\begin{array}{r}2.3069 \\
\times 70 \\
72 \\
74 \\
76\end{array}$ & $\begin{array}{r}5.8730 \\
33 \\
35 \\
38 \\
41\end{array}$ & 7.853 \\
\hline $\begin{array}{l}45 \\
46 \\
47 \\
48 \\
49\end{array}$ & $\begin{array}{l}77 \\
73 \\
70 \\
66 \\
63\end{array}$ & $\begin{array}{r}8.511 .7195 \\
84 \\
74 \\
63 \\
53\end{array}$ & $\begin{array}{l}676 \\
707 \\
737 \\
768 \\
798\end{array}$ & $\begin{array}{l}78 \\
79 \\
81 \\
83 \\
85\end{array}$ & $\begin{array}{l}44 \\
46 \\
49 \\
52 \\
55\end{array}$ & \\
\hline $\begin{array}{l}50 \\
51 \\
52 \\
53 \\
54\end{array}$ & $\begin{array}{r}8.509 \quad 4059 \\
56 \\
52 \\
49 \\
45\end{array}$ & $\begin{array}{r}8.5117142 \\
31 \\
21 \\
10 \\
8.5117100\end{array}$ & $\begin{array}{r}1.12829 \\
859 \\
889 \\
920 \\
950\end{array}$ & $\begin{array}{r}2.3086 \\
88 \\
90 \\
91 \\
93\end{array}$ & $\begin{array}{r}5.8757 \\
60 \\
63 \\
66 \\
69\end{array}$ & 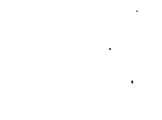 \\
\hline $\begin{array}{l}55 \\
56 \\
57 \\
58 \\
59\end{array}$ & $\begin{array}{l}41 \\
38 \\
34 \\
31 \\
27\end{array}$ & $\begin{array}{r}8.5117089 \\
78 \\
68 \\
57 \\
46\end{array}$ & $\begin{array}{r}1.12981 \\
1.13011 \\
041 \\
072 \\
102\end{array}$ & $\begin{array}{r}95 \\
97 \\
2.3099 \\
2.3100 \\
02\end{array}$ & $\begin{array}{l}72 \\
74 \\
77 \\
80 \\
83\end{array}$ & \\
\hline 60 & 8.5094024 & 8.5117036 & 1.13132 & 2.3104 & 5.8785 & 7.855 \\
\hline
\end{tabular}


TABle 20.-Geodetic position computations-Continued.

LATITUDE $28^{\circ}$.

\begin{tabular}{|c|c|c|c|c|c|c|}
\hline Lat. & $\begin{array}{c}\log A \\
\text { diff. } 1^{\prime \prime}=-0.06\end{array}$ & $\begin{array}{c}\log \mathrm{B} \\
\text { diff. } 1^{\prime \prime}=-0.18\end{array}$ & $\begin{array}{c}\log C \\
\text { diff. } 1^{\prime \prime}=+0.50\end{array}$ & $\begin{array}{c}\log \mathrm{D} \\
\text { diff. } 1^{\prime \prime}=+0.03\end{array}$ & $\begin{array}{c}\log \mathrm{E} \\
\text { diff. } 1^{\prime \prime}=+0.05\end{array}$ & 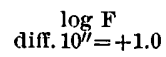 \\
\hline $\begin{array}{cc}0 & , \\
28 & 00 \\
& 1 \\
& 2 \\
& 2 \\
& 3 \\
& 4\end{array}$ & 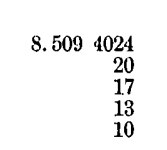 & $\begin{array}{r}8.5117036 \\
25 \\
14 \\
8.5117004 \\
8.5116993\end{array}$ & $\begin{array}{r}1.13132 \\
163 \\
193 \\
223 \\
254\end{array}$ & $\begin{array}{r}2.3104 \\
05 \\
07 \\
09 \\
10\end{array}$ & $\begin{array}{r}5.8785 \\
88 \\
91 \\
94 \\
97\end{array}$ & 7.855 \\
\hline $\begin{array}{r}05 \\
6 \\
7 \\
8 \\
9\end{array}$ & $\begin{array}{r}06 \\
8.5094002 \\
8.509 \quad 3999 \\
95 \\
92\end{array}$ & $\begin{array}{l}82 \\
72 \\
61 \\
50 \\
40\end{array}$ & $\begin{array}{r}284 \\
314 \\
345 \\
375 \\
405\end{array}$ & $\begin{array}{l}12 \\
14 \\
16 \\
17 \\
19\end{array}$ & $\begin{array}{r}5.8799 \\
5.8802 \\
05 \\
08 \\
11\end{array}$ & \\
\hline $\begin{array}{l}10 \\
11 . \\
12 \\
13 \\
14\end{array}$ & $\begin{array}{r}8.5093988 \\
85 \\
81 \\
78 \\
74\end{array}$ & $\begin{array}{rr}8.511 & 6929 \\
18 \\
8.511 & 6908 \\
8.511 & 6897 \\
86\end{array}$ & $\begin{array}{r}1.13435 \\
465 \\
496 \\
526 \\
556\end{array}$ & $\begin{array}{r}2.3121 \\
22 \\
24 \\
26 \\
27\end{array}$ & $\begin{array}{r}5.8813 \\
16 \\
19 \\
22 \\
25\end{array}$ & \\
\hline $\begin{array}{l}15 \\
16 \\
17 \\
18 \\
19\end{array}$ & $\begin{array}{l}70 \\
67 \\
63 \\
60 \\
56\end{array}$ & $\begin{array}{l}75 \\
65 \\
54 \\
43 \\
33\end{array}$ & $\begin{array}{r}586 \\
616 \\
646 \\
677 \\
707\end{array}$ & $\begin{array}{l}29 \\
31 \\
32 \\
34 \\
36\end{array}$ & $\begin{array}{l}27 \\
30 \\
33 \\
36 \\
39\end{array}$ & \\
\hline $\begin{array}{l}20 \\
21 \\
22 \\
23 \\
24\end{array}$ & $\begin{array}{r}8.5093952 \\
49 \\
45 \\
42 \\
38\end{array}$ & $\begin{array}{r}8.5116822 \\
11 \\
8.5116800 \\
8.5116790 \\
79\end{array}$ & $\begin{array}{r}1.13737 \\
767 \\
797 \\
827 \\
857\end{array}$ & $\begin{array}{r}2.3137 \\
39 \\
41 \\
42 \\
44\end{array}$ & $\begin{array}{r}5.8841 \\
44 \\
47 \\
50 \\
53\end{array}$ & 7.857 \\
\hline $\begin{array}{l}25 \\
26 \\
27 \\
28 \\
29\end{array}$ & $\begin{array}{l}35 \\
31 \\
27 \\
24 \\
20\end{array}$ & $\begin{array}{l}68 \\
57 \\
47 \\
36 \\
25\end{array}$ & $\begin{array}{r}887 \\
917 \\
947 \\
1.13977 \\
1.14007\end{array}$ & $\begin{array}{l}46 \\
.47 \\
49 \\
51 \\
52\end{array}$ & $\begin{array}{r}55 \\
58 \\
61 \\
. \quad 64 \\
67\end{array}$ & \\
\hline $\begin{array}{l}30 \\
31 \\
32 \\
33 \\
34\end{array}$ & $\begin{array}{r}8.5093917 \\
13 \\
09 \\
06 \\
0.509 \quad 3902\end{array}$ & $\begin{array}{r}8.5116714 \\
8.5116704 \\
8.5116693 \\
82 \\
71\end{array}$ & $\begin{array}{r}1.14037 \\
067 \\
097 \\
127 \\
157\end{array}$ & $\begin{array}{r}2.3154 \\
56 \\
57 \\
59 \\
61\end{array}$ & $\begin{array}{r}5.8870 \\
72 \\
75 \\
78 \\
81\end{array}$ & \\
\hline $\begin{array}{l}35 \\
36 \\
37 \\
38 \\
39\end{array}$ & $\begin{array}{r}8.5093899 \\
95 \\
92 \\
88 \\
84\end{array}$ & $\begin{array}{l}61 \\
50 \\
39 \\
28 \\
17\end{array}$ & $\begin{array}{l}187 \\
217 \\
247 \\
277 \\
307\end{array}$ & $\begin{array}{l}62 \\
64 \\
65 \\
67 \\
69\end{array}$ & $\begin{array}{l}84 \\
87 \\
89 \\
92 \\
95\end{array}$ & $:$ \\
\hline $\begin{array}{l}40 \\
41 \\
42 \\
43 \\
44\end{array}$ & $\begin{array}{r}8.5093881 \\
77 \\
73 \\
70 \\
66\end{array}$ & $\begin{array}{r}8.5116607 \\
8.5116596 \\
85 \\
74 \\
63\end{array}$ & $\begin{array}{r}1.14337 \\
366 \\
396 \\
426 \\
456\end{array}$ & $\begin{array}{r}2.3170 \\
72 \\
74 \\
75 \\
77\end{array}$ & $\begin{array}{r}5.8898 \\
5.8901 \\
04 \\
06 \\
09\end{array}$ & $\begin{array}{c}7.859 \\
\vdots\end{array}$ \\
\hline $\begin{array}{l}45 \\
46 \\
47 \\
48 \\
49\end{array}$ & $\begin{array}{l}63 \\
59 \\
55 \\
52 \\
48\end{array}$ & $\begin{array}{r}52 \\
42 \\
31 \\
. \quad 20 \\
8.511 \quad 6509\end{array}$ & $\begin{array}{l}486 \\
516 \\
545 \\
575 \\
605\end{array}$ & $\begin{array}{l}78 \\
80 \\
82 \\
83 \\
85\end{array}$ & $\begin{array}{l}12 \\
15 \\
18 \\
21 \\
23\end{array}$ & : \\
\hline $\begin{array}{l}50 \\
51 \\
52 \\
53 \\
54\end{array}$ & $\begin{array}{r}8.509 \quad 3845 \\
41 \\
37 \\
34 \\
30\end{array}$ & $\begin{array}{r}8.511 .6498 \\
87 \\
76 \\
66 \\
55\end{array}$ & $\begin{array}{r}1.14635 \\
664 \\
694 \\
724 \\
754\end{array}$ & $\begin{array}{r}2.3187 \\
88 \\
90 \\
91 \\
93\end{array}$ & $\begin{array}{r}5.8926 \\
29 \\
32 \\
35 \\
3 \times\end{array}$ & $\begin{array}{c}\because \\
\because \\
\because\end{array}$ \\
\hline $\begin{array}{l}55 \\
56 \\
57 \\
58 \\
59\end{array}$ & $\begin{array}{l}26 \\
23 \\
19 \\
16 \\
12\end{array}$ & $\begin{array}{r}44 \\
33 \\
22 \\
11 \\
8.511 \quad 6400\end{array}$ & $\begin{array}{l}783 \\
813 \\
843 \\
872 \\
902\end{array}$ & $\begin{array}{r}95 \\
96 \\
98 \\
2.3199 \\
2.3201\end{array}$ & $\begin{array}{l}40 \\
43 \\
46 \\
49 \\
52\end{array}$ & \\
\hline 60 & 8.5093808 & 8.5116389 & 1.14932 & 2.3203 & 5.8955 & 7.861 \\
\hline
\end{tabular}

Bull. $214-03-13$ 
TABLE 20.-Geodetic position computations-Continued.

LATITUDE $29^{\circ}$.

\begin{tabular}{|c|c|c|c|c|c|c|}
\hline Lat. & $\begin{array}{c}\operatorname{lrg} A \\
\text { diff. } 1^{\prime \prime}=-0.06\end{array}$ & \multirow[t]{2}{*}{$\begin{array}{c}\log B \\
\text { diff. } 1^{\prime \prime}=-0.18\end{array}$} & $\begin{array}{c}\log \mathrm{C} \\
\text { diff. } 1^{\prime \prime}=+0.49\end{array}$ & $\begin{array}{c}\log \mathbf{D} \\
\operatorname{diff} .1^{\prime \prime}=+0.03\end{array}$ & \multirow[t]{2}{*}{$\begin{array}{c}\log 1 \\
\text { diff. } 1^{\prime \prime}=+0.05\end{array}$} & $\begin{array}{c}\log \mathbf{F} \\
\text { dift. } 10^{\prime}=+0.8\end{array}$ \\
\hline $0 \quad 1$ & & & - & & & · \\
\hline $25 \cdot 00$ & 8.5093808 & 8. 5116389 & 1. 14932 & 2. 3203 & 5.8955 & 7.861 \\
\hline 1 & 05 & & 961 & 04 & 58 & \\
\hline 2 & 8.5093801 & 68 & 1. 14991 & 06 & 60 & \\
\hline 3 & 8. 5093797 & 57 & 1. 15021 & 07 & 63 & \\
\hline 4 & 94 & 46 & 050 & 09 & 66 & \\
\hline 05 & . 90 & 35 & 080 & 10 & 69 & \\
\hline 6 & 86 & 24 & 109 & 12 & 72 & \\
\hline 7 & 83 & 13 & 139 & 14 & 75 & \\
\hline 8 & 79 & 8.5116302 & 168 & 15 & 78 & \\
\hline 9 & 76 & $8.511,6291$ & 198 & 17 & 80 & \\
\hline 10 & 8.5093772 & 8.511. 6280 & 1. 15228 & 2.3218 & 5.8983 & \\
\hline 11 & 68 & 69 & 257 & 20 & & \\
\hline 12 & & & 287 & 21 & 89 & \\
\hline 13 & 61 & 47 & 316 & 23 & 92 & . \\
\hline . 14 & 57 & 36 & 346 & 25 & · 95 & \\
\hline 15 & 54 & 26 & 375 & 26 & 5.8998 & \\
\hline 16 & 50 & 15 & 405 & 28 & 5. 9000 & \\
\hline 17 & 46 & 8.5116204 & 434 & 29 & 03 & . \\
\hline 18 & 43 & 8.5116193 & 464 & 31 & 06 & \\
\hline 19 & 39 & 82 & 493 & 32 & 09 & \\
\hline 20 & 8. 5093735 & 8.5116171 & 1.15522 & 2.3234 & 5. 9012 & 7.863 \\
\hline 21 & & & 552 & 35 & 15 & \\
\hline 22 & 28 & 49 & 581 & 37 & 18 & \\
\hline 23 & 24 & 38 & 611. & $\cdot 38$ & 21 & \\
\hline 24 & 21 & 27 & 640 & 40 & 23 & \\
\hline 25 & 17 & 16 & 670 & 42 & 26 & \\
\hline 26 & - 13 & 8.5116105 & 699 & 43 & 29 & \\
\hline 27 & 10 & 8.5116094 & 728 & 45 & 32 & \\
\hline 28 & 06 & 83 & 758 & 46 & 35 & \\
\hline 29 & 8.5093702 & 72 & 787 & 48 & 38 & \\
\hline 30 & 8.5093699 & 8.5116061 & 1.15816 & 2.3249 & 5.9041 & \\
\hline 31 & 95 & 50 & 846 & 51 & 43 & \\
\hline 32 & 91 & 39 & 875 & 52 & 46 & \\
\hline 33 & 88 & 28 & 904 & 54 & 49 & \\
\hline 34 & 84 & 17 & 934 & 55 & 52 & \\
\hline 35 & 80 & 8.5116006 & 963 & 57 & 55 & \\
\hline 36 & 77 & 8.5115995 & 1. 15992 & 58 & 58 & \\
\hline 37 & 73 & 84 & 1.16021 & 60 & 61 & \\
\hline 38 & 69 & 73 & 051. & 61 & 64 & \\
\hline 39 & 66 & 61 & 080 & 63 & 67 & \\
\hline 40 & 8. 5093662 & 8.5115950 & 1. 16109 & 2. 3264 & 5. 9069 & 7.864 \\
\hline .41 & 58 & & 138 & 66 & & \\
\hline 42 & 55 & & 167 & 67 & 75 & \\
\hline 43 & 51 & & 197 & 69 & & . \\
\hline 44 & 47 & 8.5115906 & 226 & 70 & 81 & \\
\hline 45 & 44 & 8.5115895 & 255 & 72 & 84 & \\
\hline 46 & 40 & & 284 & 73 & 87 & \\
\hline 47 & 36 & 73 & 313 & 75 & 90 & . \\
\hline 48 & 33 & & 343 & 76 & 93 & \\
\hline 49 & 29 & & 372 & 78 & 96 & \\
\hline 50 & 8.5093625 & 8.5115840 & 1. 16401 . & 2.3279 & 5.9098 & \\
\hline 51 & 21 & 29 & .430 & 81 & 5. 9101 & \\
\hline 52 & 18 & - 18 & 459 & 82 & 04 & \\
\hline 53 & 14 & 8.511.5806 & 488 & 84 & 07 & \\
\hline 54 & 10 & 8.5115795 & 517 & 85 & 10 & \\
\hline 55 & 07 & 84 & 546 & 87 & 13 & \\
\hline 56 & 8. 5093603 & 73 & 575 & 88 & & \\
\hline 57 & 8. 5093599 & 62 & 604 & 90 & 19 & \\
\hline 58 & 96 & 51 & 633 & 91 & 22 & \\
\hline 59 & 92 & 40 & 663 & 93 & 25 & \\
\hline 60 & 8.5093588 & 8.5115729 & 1.16692 & 2.3294 & 5.9127 & 7.866 \\
\hline
\end{tabular}


TABLe 20.-Geodetic position computations-Continued.

IATTITUDE $30^{\circ}$.

\begin{tabular}{|c|c|c|c|c|c|c|}
\hline Lat. & 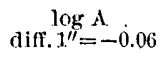 & $\begin{array}{c}\log B \\
\text { diff. } 1^{\prime \prime}=-0.19\end{array}$ & $\begin{array}{c}\log \mathrm{C} \\
\text { diff. } 1^{\prime \prime}=+0.48\end{array}$ & $\begin{array}{l}\log \mathrm{D} \\
\text { diff. } 1^{\prime \prime}=+-0.02\end{array}$ & diff. $1^{\prime \prime}=+0.05$ & $\begin{array}{c}\log \mathrm{F} \\
\text { diff. } 10^{\prime}=-+0.7\end{array}$ \\
\hline $\begin{array}{cc}\circ & , \\
30 & 00 \\
& 1 . \\
& 2 \\
& 3 \\
& 4\end{array}$ & $\begin{array}{r}8.5093588 \\
84 \\
81 \\
77 \\
73\end{array}$ & $\begin{array}{rr}8.511 . & 5729 \\
& 18 \\
\text { S.511 } & 5706 \\
8.511 & 5695 \\
84\end{array}$ & $\begin{array}{r}1.16692 \\
721 . \\
750 \\
778 \\
807\end{array}$ & $\begin{array}{r}2.3294 \\
96 \\
97 \\
2.3298 \\
2.3300\end{array}$ & $\begin{array}{r}5.9127 \\
30 \\
33 \\
30 \\
39\end{array}$ & 7.866 \\
\hline $\begin{array}{r}05 \\
6 \\
7 \\
8 \\
9\end{array}$ & $\begin{array}{l}69 \\
66 \\
62 \\
58 \\
55\end{array}$ & $\begin{array}{r}73 \\
62 \\
51 \\
40 \\
28\end{array}$ & $\begin{array}{l}836 \\
865 \\
894 \\
923 \\
952\end{array}$ & $\begin{array}{l}01 \\
03 \\
04 \\
06 \\
07\end{array}$ & $\begin{array}{l}42 \\
45 \\
48 \\
51 \\
54\end{array}$ & \\
\hline $\begin{array}{l}10 \\
11 \\
12 \\
13 \\
14\end{array}$ & $\begin{array}{r}8.50935 \mathrm{I} \\
47 \\
43 \\
40 \\
36\end{array}$ & $\begin{array}{r}8.5115617 \\
8.5115606 \\
\text { S.511 } 5595 \\
84 \\
73\end{array}$ & $\begin{array}{r}1.16981 \\
1.17010 \\
039 \\
068 \\
097\end{array}$ & $\begin{array}{r}2.3309 \\
10 \\
12 \\
13 \\
14\end{array}$ & $\begin{array}{r}5.9157 \\
59 \\
62 \\
65 \\
68\end{array}$ & \\
\hline $\begin{array}{l}15 \\
16 \\
17 \\
18 \\
19\end{array}$ & $\begin{array}{r}32 \\
29 \\
25 \\
+\quad 21 \\
17\end{array}$ & $\begin{array}{l}61 \\
50 \\
39 \\
28 \\
17\end{array}$ & $\begin{array}{l}126 \\
155 \\
184 \\
212 \\
241\end{array}$ & $\begin{array}{l}16 \\
17 \\
18 \\
20 \\
22\end{array}$ & $\begin{array}{l}71 . \\
74 \\
77 \\
80 \\
83\end{array}$ & . \\
\hline $\begin{array}{l}20 \\
21 \\
22 \\
23 \\
24\end{array}$ & $\begin{array}{r}8.5093514 \\
10 \\
06 \\
8.5093502 \\
8.5093499\end{array}$ & $\begin{array}{r}\text { S.511. } 5505 \\
\text { S.511 } 5494 \\
83 \\
72 \\
61\end{array}$ & $\begin{array}{r}1.17270 \\
299 \\
328 \\
357 \\
385\end{array}$ & $\begin{array}{r}2.3323 \\
24 \\
26 \\
27 \\
29\end{array}$ & $\begin{array}{r}5.9186 \\
89 \\
92 \\
95 \\
5.9198\end{array}$ & 7.867 \\
\hline $\begin{array}{l}25 \\
26 \\
27 \\
28 \\
29\end{array}$ & $\begin{array}{l}95 \\
91 \\
88 \\
84 \\
80\end{array}$ & $\begin{array}{r}49 \\
38 \\
27 \\
16 \\
8.511 \quad 5404\end{array}$ & $\begin{array}{l}414 \\
443 \\
472 \\
500 \\
529\end{array}$ & $\begin{array}{l}30 \\
32 \\
33 \\
34 \\
36\end{array}$ & $\begin{array}{r}5.9200 \\
03 \\
06 \\
09 \\
12\end{array}$ & \\
\hline $\begin{array}{l}30 \\
31 \\
32 \\
33 \\
34\end{array}$ & $\begin{array}{r}\text { S. } 5093476 \\
72 \\
69 \\
65 \\
6.1\end{array}$ & $\begin{array}{r}8.5115393 \\
82 \\
71 \\
59 \\
48\end{array}$ & $\begin{array}{r}1.17558 \\
587 \\
615 \\
644 \\
673\end{array}$ & $\begin{array}{r}2.3337 \\
39 \\
40 \\
41 \\
43\end{array}$ & $\begin{array}{r}5.921 .5 \\
18 \\
21 \\
24 \\
27\end{array}$ & \\
\hline $\begin{array}{l}35 \\
36 \\
37 \\
38 \\
39\end{array}$ & $\begin{array}{l}57 \\
54 \\
50 \\
46 \\
42\end{array}$ & $\begin{array}{r}37 \\
26 \\
14 \\
\text { 8.511 } 5303 \\
\text { 8.511 } 5292\end{array}$ & $\begin{array}{l}701 \\
730 \\
759 \\
788 \\
816\end{array}$ & $\begin{array}{l}44 \\
46 \\
47 \\
48 \\
50\end{array}$ & $\begin{array}{l}30 \\
33 \\
36 \\
39 \\
42\end{array}$ & \\
\hline $\begin{array}{r}40 \\
41 \\
42 \\
43 \\
44\end{array}$ & $\begin{array}{r}8.509 \quad 3439 \\
35 \\
31 \\
27 \\
24\end{array}$ & $\begin{array}{r}8.5115281 \\
69 \\
58 \\
47 \\
35\end{array}$ & $\begin{array}{r}1.17845 \\
874 \\
902 \\
931 \\
959\end{array}$ & $\begin{array}{r}2.3351 \\
53 \\
54 \\
55 \\
57\end{array}$ & $\begin{array}{r}5.9245 \\
48 \\
51 \\
53 \\
56\end{array}$ & 7.869 \\
\hline $\begin{array}{l}45 \\
46 \\
47 \\
48 \\
49\end{array}$ & $\begin{array}{l}20 \\
16 \\
12 \\
09 . \\
05\end{array}$ & $\begin{array}{rr} & 24 \\
\text { S. } 511 . & 1.32 \\
\text { 8.511 } 5190 \\
5190 \\
79\end{array}$ & $\begin{array}{r}1.17988 \\
1.18017 \\
045 \\
074 \\
102\end{array}$ & $\begin{array}{l}58 \\
59 \\
61 \\
62 \\
64\end{array}$ & $\begin{array}{r}59 \\
62 \\
65 \\
-\quad 68 \\
71\end{array}$ & \\
\hline $\begin{array}{l}50 \\
51 \\
52 \\
53 \\
54\end{array}$ & $\begin{array}{r}8.5093401 \\
8.5093397 \\
94 \\
90 \\
86\end{array}$ & $\begin{array}{r}8.511 .5168 \\
56 \\
45 \\
34 \\
22\end{array}$ & $\begin{array}{r}1.18131 \\
160 \\
188 \\
217 \\
245\end{array}$ & $\begin{array}{r}2.3365 \\
66 \\
68 \\
69 \\
70\end{array}$ & $\begin{array}{r}5.9274 \\
77 \\
80 \\
83 \\
86\end{array}$ & \\
\hline $\begin{array}{l}55 \\
56 \\
67 \\
58 \\
59\end{array}$ & $\begin{array}{l}82 \\
78 \\
75 \\
71 \\
67\end{array}$ & $\begin{array}{r}11 \\
8.511 .5100 \\
8.511 .5088 \\
77 \\
66\end{array}$ & $\begin{array}{l}274 \\
302 \\
331 \\
359 \\
388\end{array}$ & $\begin{array}{l}72 \\
73 \\
74 \\
76 \\
77\end{array}$ & $\begin{array}{r}89 \\
92 \\
95 \\
\text { 5. } 9298 \\
\text { 5. } 9301\end{array}$ & \\
\hline 60 & S. 5093363 & S.511 5054 & 1.18416 & 2.3379 & 5.930 .4 & 7.870 \\
\hline
\end{tabular}


TABLE 20.-Geodetic position computations-Continued.

LATITUDE $31^{\circ}$.

\begin{tabular}{|c|c|c|c|c|c|c|}
\hline Lat. & diff. $1^{\prime \prime}=-0.06$ & $\begin{array}{c}\log B \\
\text { diff. } 1^{\prime \prime}=-0.19\end{array}$ & $\begin{array}{c}\log \mathrm{C} \\
\text { diff. } 1^{\prime \prime}=+0.47\end{array}$ & $\begin{array}{c}\log \mathrm{D} \\
\text { diff. } 1^{\prime \prime}=+0.02\end{array}$ & diff. $1^{\prime \prime}=+0.05$ & $\begin{array}{c}\log \mathbf{F} \\
\text { diff. } 10^{\prime}=+0.5\end{array}$ \\
\hline $0 \quad 1$ & & & & . & & \\
\hline $\begin{array}{rr}31 & 00 \\
& 1 \\
& 2 \\
& 3 \\
& 4\end{array}$ & $\begin{array}{r}8.509 \quad 3363 \\
60 \\
56 \\
52 \\
48\end{array}$ & $\begin{array}{r}8.511 \text { = } 544 \\
43 \\
32 \\
20 \\
8.5115009\end{array}$ & $\begin{array}{r}1.18416 \\
445 \\
473 \\
501 \\
530\end{array}$ & $\begin{array}{r}2.3379 \\
80 \\
81 \\
83 \\
84\end{array}$ & $\begin{array}{r}5.9304 \\
07 \\
10 \\
13 \\
16\end{array}$ & 7.870 \\
\hline $\begin{array}{r}05 \\
6 \\
7 \\
8 \\
9\end{array}$ & $\begin{array}{l}44 \\
41 \\
37 \\
33 \\
29\end{array}$ & $\begin{array}{r}8.5114998 \\
86 \\
75 \\
64 \\
52\end{array}$ & $\begin{array}{l}558 \\
587 \\
615 \\
.643 \\
672\end{array}$ & $\begin{array}{r}85 \\
87 \\
88 \\
-\quad 89 \\
91\end{array}$ & $\begin{array}{l}19 \\
22 \\
25 \\
28 \\
31\end{array}$ & \\
\hline $\begin{array}{l}10 \\
11 \\
12 \\
13 \\
14\end{array}$ & $\begin{array}{r}8.5093325 \\
22 \\
18 \\
14 \\
10\end{array}$ & $\begin{array}{r}8.5114941 \\
29 \\
18 \\
8.5114907 \\
8.5114895\end{array}$ & $\begin{array}{r}1.18700 \\
729 \\
757 \\
785 \\
813\end{array}$ & $\begin{array}{r}2.3392 \\
93 \\
95 \\
96 \\
97\end{array}$ & $\begin{array}{r}5.9334 \\
37 \\
39 \\
42 \\
45\end{array}$ & \\
\hline $\begin{array}{l}15 \\
16 \\
17 \\
18 \\
19\end{array}$ & $\begin{array}{r}06 \\
8.5093303 \\
8.5053299 \\
95 \\
91\end{array}$ & $\begin{array}{l}84 \\
72 \\
61 \\
50 \\
38\end{array}$ & $\begin{array}{l}842 \\
870 \\
898 \\
927 \\
955\end{array}$ & $\begin{array}{r}\text { 2. } 3399 \\
2.3400 \\
01 \\
03 \\
04\end{array}$ & $\begin{array}{l}48 \\
51 \\
54 \\
57 \\
60\end{array}$ & ' \\
\hline $\begin{array}{l}20 \\
21 \\
22 \\
23 \\
24\end{array}$ & $\begin{array}{r}8.509 \quad 3287 \\
. \quad 84 \\
80 \\
76 \\
72\end{array}$ & $\begin{array}{rr}8.511 & 4827 \\
. & 15 \\
8.511 & 4804 \\
8.511 & 4793 \\
& 81\end{array}$ & $\begin{array}{r}1.18983 \\
1.19012 \\
040 \\
068 \\
096\end{array}$ & $\begin{array}{r}2.3405 \\
06 \\
08 \\
09 \\
10\end{array}$ & $\begin{array}{r}5.9363 \\
66 \\
69 \\
72 \\
75\end{array}$ & 7.871 \\
\hline $\begin{array}{l}25 \\
26 \\
27 \\
28 \\
29\end{array}$ & $\begin{array}{l}68 \\
65 \\
61 \\
57 \\
53\end{array}$ & $\begin{array}{l}70 \\
58 \\
47 \\
35 \\
24\end{array}$ & $\begin{array}{l}125 \\
153 \\
181 \\
209 \\
238\end{array}$ & $\begin{array}{l}12 \\
13 \\
14 \\
16 \\
17\end{array}$ & $\begin{array}{l}78 \\
81 \\
84 \\
87 \\
90\end{array}$ & \\
\hline $\begin{array}{l}30 \\
31 \\
32 \\
33 \\
34\end{array}$ & $\begin{array}{r}8.509 \quad 3249 \\
46 \\
42 \\
38 \\
34\end{array}$ & $\begin{array}{r}8.5114713 \\
8.5114701 \\
8.5114690 \\
78 \\
67\end{array}$ & $\begin{array}{r}1.19266 \\
294 \\
322 \\
351 \\
379\end{array}$ & $\begin{array}{r}2.3418 \\
\cdot \quad 20 \\
21 \\
22 \\
23\end{array}$ & $\begin{array}{r}5.9393 \\
96 \\
5.9399 \\
5.9402 \\
05\end{array}$ & \\
\hline $\begin{array}{l}35 \\
36 \\
37 \\
38 \\
39\end{array}$ & $\begin{array}{l}30 \\
26 \\
23 \\
19 \\
15\end{array}$ & $\begin{array}{r}55 \\
44 \\
32 \\
21 \\
8.511 \quad 4609\end{array}$ & $\begin{array}{l}407 \\
435 \\
463 \\
491 \\
520\end{array}$ & $\begin{array}{l}25 \\
26 \\
27 \\
29 \\
30\end{array}$ & $\begin{array}{l}08 \\
11 \\
1.4 \\
17 \\
20\end{array}$ & . \\
\hline $\begin{array}{l}40 \\
41 \\
42 \\
43 \\
44\end{array}$ & $\begin{array}{r}8.5093211 \\
07 \\
03 \\
8.5093200 \\
8.5093196\end{array}$ & $\begin{array}{r}8.5114598 \\
86 \\
75 \\
.63 \\
. \quad 52\end{array}$ & $\begin{array}{r}1.19548 \\
576 \\
604 \\
632 \\
660\end{array}$ & $\begin{array}{r}2.3431 \\
32 \\
34 \\
35 \\
36\end{array}$ & $\begin{array}{r}\text { 5. } 9423 \\
26 \\
29 \\
32 \\
35\end{array}$ & 7.872 \\
\hline $\begin{array}{l}45 \\
46 \\
47 \\
48 \\
49\end{array}$ & $\begin{array}{l}92 \\
88 \\
84 \\
81 \\
77\end{array}$ & $\begin{array}{r}40 \\
29 \\
17 \\
8.5114506 \\
8.5114494\end{array}$ & $\begin{array}{l}688 \\
716 \\
744 \\
772 \\
800\end{array}$ & $\begin{array}{l}37 \\
39 \\
40 \\
41 \\
43\end{array}$ & $\begin{array}{l}38 \\
41 \\
44 \\
47 \\
50\end{array}$ & . \\
\hline $\begin{array}{l}50 \\
51 \\
52 \\
53 \\
54\end{array}$ & $\begin{array}{r}8.5093173 \\
.69 \\
65 \\
61 \\
\quad 57\end{array}$ & $\begin{array}{r}8.5114483 \\
71 \\
60 \\
48 \\
37\end{array}$ & $\begin{array}{r}1.19828 \\
856 \\
884 \\
912 \\
940\end{array}$ & $\begin{array}{r}2.3444 \\
45 \\
46 \\
48 \\
49\end{array}$ & $\begin{array}{r}5.9453 \\
56 \\
59 \\
62 \\
65\end{array}$ & \\
\hline $\begin{array}{l}55 \\
56 \\
57 \\
58 \\
59\end{array}$ & $\begin{array}{r}54 \\
50 \\
46 \\
42 \\
38\end{array}$ & $\begin{array}{r}25 \\
14 \\
8.5114402 \\
8.5114391 \\
79\end{array}$ & $\begin{array}{r}968 \\
1.19996 \\
1.20024 \\
052 \\
080\end{array}$ & $\begin{array}{l}50 \\
51 \\
53 \\
54 \\
55\end{array}$ & $\begin{array}{l}68 \\
72 \\
75 \\
78 \\
81\end{array}$ & \\
\hline 60 & 8.5093134 & 8.5114368 & 1. 20108 & 2.3456 & 5.9484 & 7.873 \\
\hline
\end{tabular}


TABte 20.-Geodetic position computations-Continued.

LATITUDE $32^{\circ}$.

\begin{tabular}{|c|c|c|c|c|c|c|}
\hline Lut. & $\begin{array}{c}\log A \\
\text { diff. } 1^{\prime \prime}=-0.06\end{array}$ & $\begin{array}{c}\log B \\
\text { diff. } 1^{\prime \prime}=-0.19\end{array}$ & $\begin{array}{c}\log C \\
\text { dift. } 1^{\prime \prime}=+0.46^{\circ}\end{array}$ & $\begin{array}{c}\log \mathrm{D} \\
\text { diff. } 1^{\prime \prime}=+0.02\end{array}$ & $\begin{array}{c}\log \mathbf{E} \\
\text { diff. } 1^{\prime \prime}=+0.05\end{array}$ & $\begin{array}{c}\log \mathbf{F} \\
\text { diff. } 10^{\prime}=+0.3\end{array}$ \\
\hline $\begin{array}{cc}\circ & 1 \\
82 & 00 \\
& 1 \\
& 2 \\
& 3 \\
& 4\end{array}$ & $\begin{array}{r}8.509 \quad 3134 \\
31 \\
27 \\
23 \\
19\end{array}$ & $\begin{array}{r}8.5114368 \\
56 \\
44 \\
33 \\
21\end{array}$ & $\begin{array}{r}1.20108 \\
136 \\
\cdot \quad 164 \\
192 \\
220\end{array}$ & $\begin{array}{r}2.3456 \\
57 \\
59 \\
60 \\
61\end{array}$ & $\begin{array}{r}5.9484 \\
87 \\
90 \\
93 \\
96\end{array}$ & 7.873 \\
\hline $\begin{array}{r}05 \\
6 \\
7 \\
8 \\
9\end{array}$ & $\begin{array}{r}15 \\
11 \\
07 \\
04 \\
8.509 \quad 3100\end{array}$ & $\begin{array}{r}8.5114310 \\
8.511429 \varepsilon \\
87 \\
75 \\
63\end{array}$ & $\begin{array}{l}248 \\
276 \\
304 \\
332 \\
360\end{array}$ & $\begin{array}{r}62 \\
64 \\
65 \\
66 \\
67\end{array}$ & $\begin{array}{r}5.9499 \\
5.9502 \\
05 \\
08 \\
11\end{array}$ & \\
\hline $\begin{array}{l}10 \\
11 . \\
12 \\
13 \\
1.1\end{array}$ & $\begin{array}{r}\text { S. } 509 \quad 3096 \\
92 \\
88 \\
84 \\
80\end{array}$ & $\begin{array}{r}8.5114252 \\
40 \\
29 \\
17 \\
8.5114205\end{array}$ & $\begin{array}{r}1.20387 \\
415 \\
443 \\
471 \\
499\end{array}$ & $\begin{array}{r}2.3469 \\
70 \\
71 \\
72 \\
73\end{array}$ & 5. $\begin{array}{r}9514 \\
17 \\
20 \\
23 \\
26\end{array}$ & \\
\hline $\begin{array}{l}15 \\
16 \\
17 \\
18 \\
19\end{array}$ & $\begin{array}{l}76 \\
73 \\
69 \\
65 \\
61 .\end{array}$ & $\begin{array}{r}8.511+194 \\
82 \\
71 \\
59 \\
47\end{array}$ & $\begin{array}{l}527 \\
555 \\
582 \\
610 \\
638\end{array}$ & $\begin{array}{l}75 \\
76 \\
77 \\
78 \\
79\end{array}$ & $\begin{array}{l}29 \\
32 \\
35 \\
38 \\
41\end{array}$ & \\
\hline $\begin{array}{r}20 \\
21 \\
\therefore \quad 22 \\
23 \\
24\end{array}$ & $\begin{array}{r}8.509 \quad 3057 \\
53 \\
49 \\
46 \\
42\end{array}$ & $\begin{array}{r}8.5114136 \\
24 \\
13 \\
8.5114101 \\
8.511 \\
4089\end{array}$ & $\begin{array}{r}1.20666 \\
694 \\
722 \\
749 \\
777\end{array}$ & $\begin{array}{r}2.3481 \\
82 \\
83 \\
84 \\
85\end{array}$ & $\begin{array}{r}5.9544 \\
47 \\
50 \\
53 \\
56\end{array}$ & 7.874 \\
\hline $\begin{array}{l}25 \\
26 \\
27 \\
28 \\
29\end{array}$ & $\begin{array}{l}38 \\
34 \\
30 \\
26 \\
22\end{array}$ & $\begin{array}{l}78 \\
66 \\
54 \\
48 \\
31\end{array}$ & $\begin{array}{l}805 \\
833 \\
860 \\
888 \\
916\end{array}$ & $\begin{array}{l}87 \\
88 \\
89 \\
90 \\
91\end{array}$ & $\begin{array}{l}60 \\
63 \\
66 \\
69 \\
72\end{array}$ & \\
\hline $\begin{array}{l}30 \\
31 \\
32 \\
33 \\
34\end{array}$ & $\begin{array}{r}8.5093018 \\
15 \\
11 \\
07 \\
8.609 \quad 3003\end{array}$ & $\begin{array}{r}8.5114020 \\
8.5114008 \\
8.511 .3996 \\
86 \\
78 .\end{array}$ & $\begin{array}{r}1.20944 \\
971 \\
1.20999 \\
1.21027 \\
054\end{array}$ & $\begin{array}{r}2.3493 \\
94 \\
95 \\
96 \\
97\end{array}$ & $\begin{array}{r}5.9575 \\
78 \\
81 \\
84 \\
87\end{array}$ & \\
\hline $\begin{array}{l}35 \\
36 \\
37 \\
38 \\
39\end{array}$ & $\begin{array}{r}8.5092999 \\
95 \\
91 \\
87 \\
83\end{array}$ & $\begin{array}{l}61 . \\
50 \\
38 \\
26 \\
16\end{array}$ & $\begin{array}{l}082 \\
110 \\
137 \\
165 \\
193\end{array}$ & $\begin{array}{r}2.3499 \\
2.3500 \\
01 \\
02 \\
03\end{array}$ & $\begin{array}{r}90 \\
93 \\
96 \\
5.9599 \\
5.9602\end{array}$ & . \\
\hline $\begin{array}{l}40 \\
41 \\
42 \\
43 \\
44\end{array}$ & $\begin{array}{r}8.509 \quad 2980 \\
76 \\
72 \\
68 \\
64\end{array}$ & $\begin{array}{r}8.511390 \% \\
8.5113891 \\
79 \\
68 \\
56\end{array}$ & $\begin{array}{r}1.21220 \\
\cdot \quad 248 \\
276 \\
303 \\
331\end{array}$ & $\begin{array}{r}2.3504 \\
06 \\
07 \\
08 \\
09\end{array}$ & $\begin{array}{r}5.9605 \\
08 \\
11 \\
15 \\
18\end{array}$ & 7.875 \\
\hline $\begin{array}{l}45 \\
46 \\
47 \\
48 \\
49\end{array}$ & $\begin{array}{l}60 \\
56 \\
52 \\
48 \\
44\end{array}$ & $\begin{array}{r}44 \\
3 \% \\
21 \\
8.5113809 \\
8.5113798\end{array}$ & $\begin{array}{l}358 \\
386 \\
414 \\
441 \\
469\end{array}$ & $\begin{array}{l}10 \\
11 \\
13 \\
14 \\
15\end{array}$ & $\begin{array}{l}21 \\
24 \\
27 \\
30 \\
33\end{array}$ & . \\
\hline $\begin{array}{l}50 \\
51 \\
52 \\
53 \\
54\end{array}$ & $\begin{array}{r}8.509 \quad 2940 \\
-37 \\
33 \\
29 \\
25\end{array}$ & $\begin{array}{r}8.5113780 \\
74 \\
63 \\
51 \\
39\end{array}$ & $\begin{array}{r}1.21496 \\
524 \\
551 \\
579 \\
607\end{array}$ & $\begin{array}{r}2.3516 \\
17 \\
18 \\
19 \\
21\end{array}$ & $\begin{array}{r}5.9636 \\
\cdot \quad 39 \\
42 \\
45 \\
48\end{array}$ & \\
\hline $\begin{array}{l}55 \\
56 \\
57 \\
58 \\
59\end{array}$ & $\begin{array}{l}21 . \\
17 \\
13 \\
09 \\
05\end{array}$. & $\begin{array}{r}27 \\
16 \\
8.5113704 \\
8.5113692 \\
80\end{array}$ & $\begin{array}{l}.634 \\
662 \\
689 \\
717 \\
744\end{array}$ & $\begin{array}{l}22 \\
23 \\
24 \\
25 \\
26\end{array}$ & $\begin{array}{l}51 \\
64 \\
68 \\
61 \\
64\end{array}$ & . \\
\hline 60 & 8.5092901 & 8.511 366! & 1.21772 & 2.3527 & 5.9667 & 7.875 \\
\hline
\end{tabular}


TABLE 20.-Geodetic position computations-Continued.

LATITUDE $33^{\circ}$.

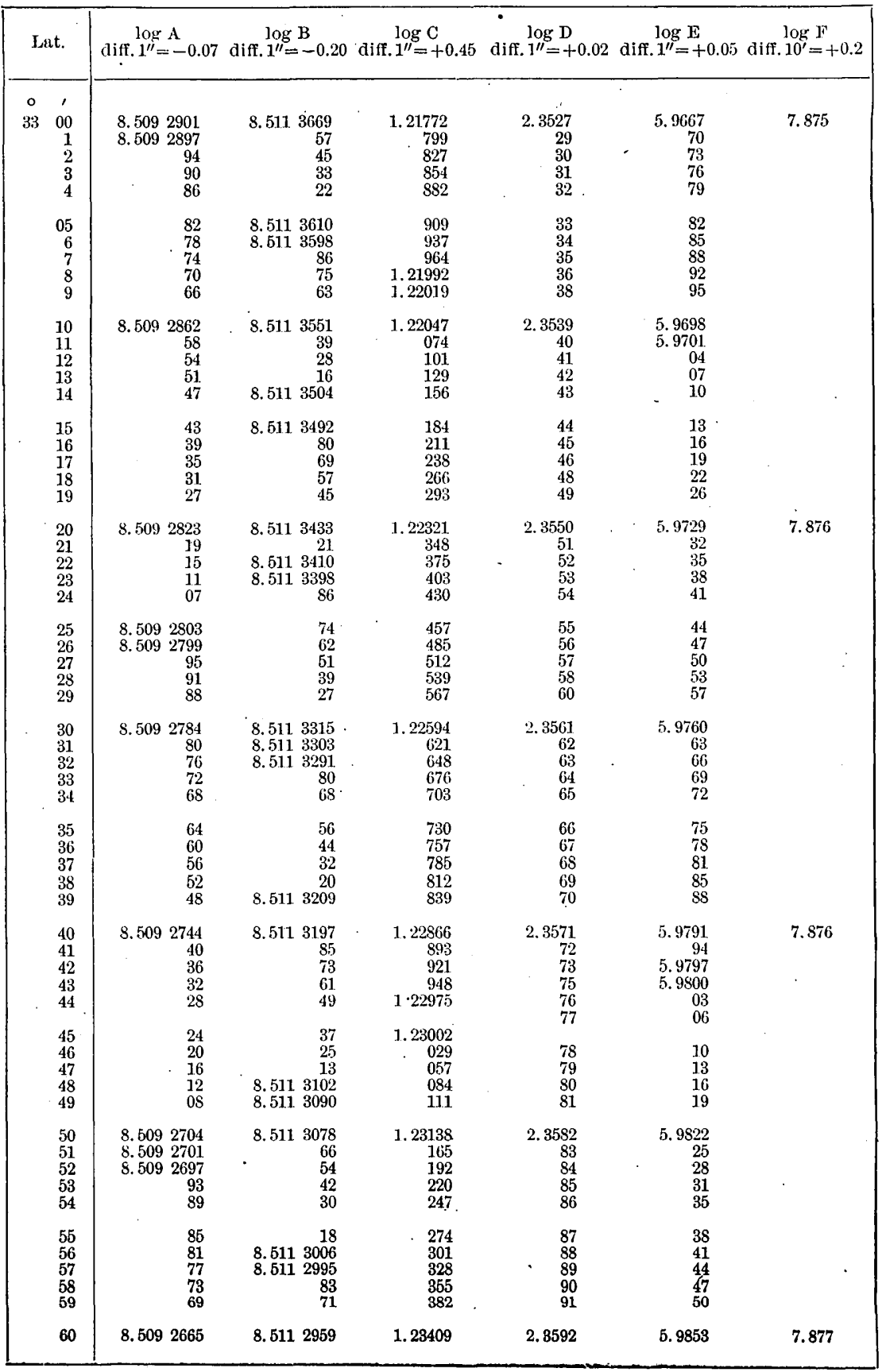


TABLE 20.-Geodetic position computations-Continued.

LATITUDE $34^{\circ}$.

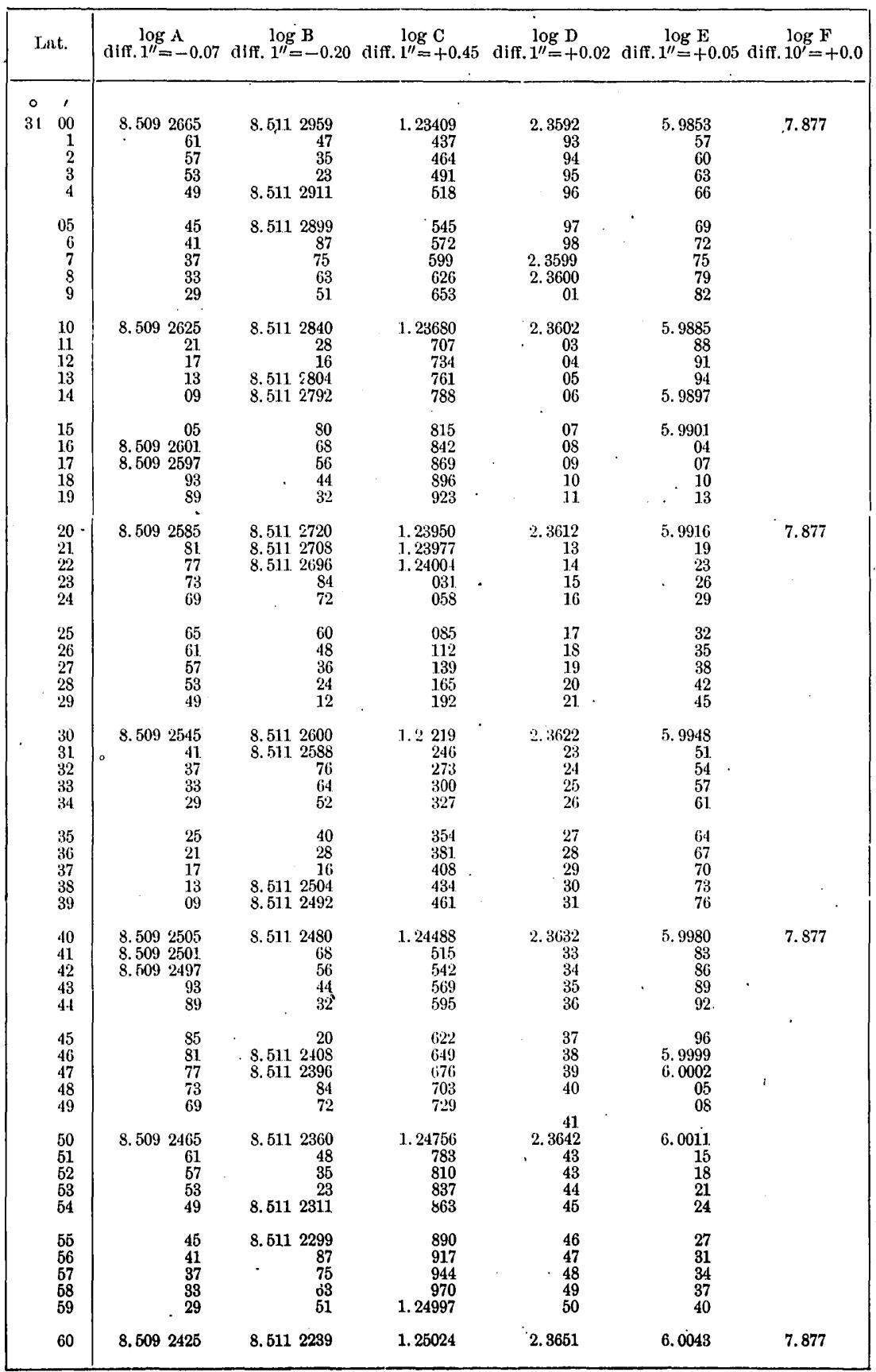


TABLE 20.-Geodetic position computations-Continued.

LATITUDE $35^{\circ}$.

\begin{tabular}{|c|c|c|c|c|c|c|c|}
\hline \multicolumn{2}{|c|}{ Lat. } & \multirow[t]{2}{*}{$\begin{array}{c}\log A \\
\text { diff. } 1^{\prime \prime}=-0.07\end{array}$} & \multirow[t]{2}{*}{$\begin{array}{c}\log B \\
\text { diff. } 1^{\prime \prime}=-0.20\end{array}$} & \multirow[t]{2}{*}{$\begin{array}{c}\log C \\
\text { diff, } 1^{\prime \prime}=+0.44\end{array}$} & \multirow[t]{2}{*}{$\begin{array}{c}\log \mathrm{D} \\
\text { diff. } 1^{\prime \prime}=+0.01\end{array}$} & \multirow[t]{2}{*}{$\begin{array}{c}\log \mathrm{E} \\
\text { diff. } 1^{\prime \prime}=+0.05\end{array}$} & \multirow[t]{2}{*}{ diff. $10^{\prime}=+0.0$} \\
\hline$\circ$ & 1 & & & & & & \\
\hline 35 & $\begin{array}{r}00 \\
1 \\
2 \\
3 \\
4\end{array}$ & $\begin{array}{r}8.5092425 \\
21 \\
17 \\
13 \\
09\end{array}$ & $\begin{array}{rr}8.511 & 2239 \\
& 27 \\
15 \\
8.511 & 2203 \\
8.511 & 2191\end{array}$ & $\begin{array}{r}1.25024 \\
050 \\
077 \\
104 \\
131\end{array}$ & $\begin{array}{r}2.3651 \\
52 \\
53 \\
54 \\
55\end{array}$ & $\begin{array}{r}6.0043 \\
47 \\
50 \\
53 \\
56\end{array}$ & 7.877 \\
\hline & $\begin{array}{r}05 \\
6 \\
7 \\
8 \\
9\end{array}$ & $\begin{array}{r}05 \\
8.5092401 \\
8.5092396 \\
92 \\
88\end{array}$ & $\begin{array}{l}78 \\
66 \\
54 \\
42 \\
30\end{array}$ & $\begin{array}{l}157 \\
184 \\
211 \\
237 \\
264\end{array}$ & $\begin{array}{l}56 \\
56 \\
57 \\
58 \\
59\end{array}$ & $\begin{array}{l}59 \\
63 \\
66 \\
69 \\
72 \\
\end{array}$ & \\
\hline & $\begin{array}{l}10 \\
11 \\
12 . \\
13 \\
14\end{array}$ & $\begin{array}{r}8.5092384 \\
80 \\
76 \\
72 \\
68\end{array}$ & $\begin{array}{rr}8.511 & 2118 \\
8.511 & 2106 \\
8.511 & 2094 \\
& 82 \\
& 70\end{array}$ & $\begin{array}{r}\text { 1. } 25291 \\
317 \\
344 \\
371 \\
397\end{array}$ & $\begin{array}{r}2.3660 \\
61 \\
62 \\
63 \\
64\end{array}$ & $\begin{array}{r}6.0075 \\
79 \\
82 \\
85 \\
88\end{array}$ & \\
\hline & $\begin{array}{l}15 \\
16 \\
17 \\
18 \\
19\end{array}$ & $\begin{array}{l}64 \\
60 \\
56 \\
52 \\
48 .\end{array}$ & $\begin{array}{r}57 \\
45 \\
33 \\
21 \\
21 \\
8.5112009\end{array}$ & $\begin{array}{r}424 \\
451 \\
477 \\
504 \\
531\end{array}$ & $\begin{array}{l}65 \\
66 \\
66 \\
67 \\
68\end{array}$ & $\begin{array}{r}91 \\
95 \\
6.0098 \\
6.0101 \\
04\end{array}$ & \\
\hline & $\begin{array}{l}20 \\
21 \\
22 \\
23 \\
24\end{array}$ & $\begin{array}{r}8.5092344 \\
40 \\
36 \\
32 \\
28\end{array}$ & $\begin{array}{r}8.5111997 \\
85 \\
72 \\
60 \\
48\end{array}$ & $\begin{array}{r}\text { 1. } 25557 \\
584 \\
610 \\
637 \\
664\end{array}$ & $\begin{array}{r}2.3669 \\
70 \\
71 \\
72 \\
73\end{array}$ & $\begin{array}{r}6.0107 \\
11 . \\
14 \\
17 \\
. \quad 20\end{array}$ & 7.877 \\
\hline & $\begin{array}{l}25 \\
26 \\
27 \\
28 \\
29\end{array}$ & $\begin{array}{l}24 \\
20 \\
16 \\
12 \\
05\end{array}$ & $\begin{array}{r}36 \\
24 \\
12 \\
8.5111900 \\
8.5111887\end{array}$ & $\begin{array}{l}690 \\
717 \\
743 \\
770 \\
796\end{array}$ & $\begin{array}{l}74 \\
75 \\
75 \\
76 \\
77\end{array}$ & $\begin{array}{l}23 \\
27 \\
30 \\
33 \\
36\end{array}$ & \\
\hline & $\begin{array}{l}30 \\
31 \\
32 \\
33 \\
34\end{array}$ & $\begin{array}{r}8.5092304 \\
8.5092300 \\
\times 8.5092296 \\
92 \\
\quad 87\end{array}$ & $\begin{array}{r}8.511 .1875 \\
63 \\
51 \\
39 \\
27\end{array}$ & $\begin{array}{r}1.25823 \\
850 \\
876 \\
903 \\
929\end{array}$ & $\begin{array}{r}2.3678 \\
79 \\
80 \\
81 \\
82\end{array}$ & $\begin{array}{r}6.0140 \\
43 \\
46 \\
49 \\
52\end{array}$ & . \\
\hline & $\begin{array}{l}35 \\
36 \\
37 \\
38 \\
39\end{array}$ & $\begin{array}{l}83 \\
79 \\
75 \\
71 \\
67\end{array}$ & $\begin{array}{rr} & 15 \\
8.511 & 1802 \\
8.511 . & 1790 \\
78 \\
66\end{array}$ & $\begin{array}{r}956 \\
1.25982 \\
1.26009 \\
035 \\
062\end{array}$ & $\begin{array}{l}82 \\
83 \\
84 \\
85 \\
86\end{array}$ & $\begin{array}{l}56 \\
59 \\
62 \\
65 \\
69\end{array}$ & \\
\hline & $\begin{array}{l}40 . \\
41 \\
42 \\
43 \\
44\end{array}$ & $\begin{array}{r}8.5092263 \\
59 \\
55 \\
51 \\
47\end{array}$ & $\begin{array}{r}8.5111754 \\
41 \\
29 \\
17 \\
8.5111705\end{array}$ & $\begin{array}{r}1.26088 \\
115 \\
141 \\
168 \\
194\end{array}$ & $\begin{array}{r}2.3097 \\
88 \\
88 \\
89 \\
90\end{array}$ & $\begin{array}{r}6.0172 \\
75 \\
78 \\
81 \\
85\end{array}$ & 7.877 \\
\hline & $\begin{array}{l}45 \\
46 \\
47 \\
48 \\
49\end{array}$ & $\begin{array}{l}43 \\
39 \\
35 \\
31 \\
27\end{array}$ & $\begin{array}{r}8.5111693 \\
80 \\
68 \\
56 \\
44\end{array}$ & $\begin{array}{l}221 \\
247 \\
274 \\
300 \\
327\end{array}$ & $\begin{array}{l}91 \\
92 \\
93 \\
94 \\
94\end{array}$ & $\begin{array}{r}88 \\
91 \\
94 \\
6.0198 \\
6.0201\end{array}$ & \\
\hline & $\begin{array}{l}50 \\
51 \\
52 \\
53 \\
54\end{array}$ & $\begin{array}{r}8.5092222 \\
18 \\
14 \\
10 \\
06\end{array}$ & $\begin{array}{rr}8.511 & 1632 \\
& 20 \\
8.511 & 1607 \\
8.511 & 1595 \\
& 83\end{array}$ & $\begin{array}{r}1.26353 \\
380 \\
406 \\
432 \\
459\end{array}$ & $\begin{array}{r}2.3695 \\
96 \\
97 \\
98 \\
99\end{array}$ & $\begin{array}{r}6.0204 \\
07 \\
11 \\
14 \\
17\end{array}$ & \\
\hline . & $\begin{array}{l}55 \\
56 \\
57 \\
58 \\
59\end{array}$ & $\begin{array}{rr}8.509 & 2202 \\
8.509 & 2198 \\
& 94 \\
& 90 \\
& 86\end{array}$ & $\begin{array}{l}71 \\
58 \\
46 \\
34 \\
22\end{array}$ & $\begin{array}{l}485 \\
512 \\
538 \\
565 \\
\text { b91 }\end{array}$ & $\begin{array}{r}2.3699 \\
2.3700 \\
01 \\
02 \\
03\end{array}$ & $\begin{array}{l}20 \\
24 \\
27 \\
30 \\
33\end{array}$ & \\
\hline & 60 & 8.5092182 & 8. 5111510 & 1. 26617 & 2.3704 & 6.0237 & 7.877 \\
\hline
\end{tabular}


TABLe 20.-Geodetic position computations-Continued.

LATITUDE $36^{\circ}$.

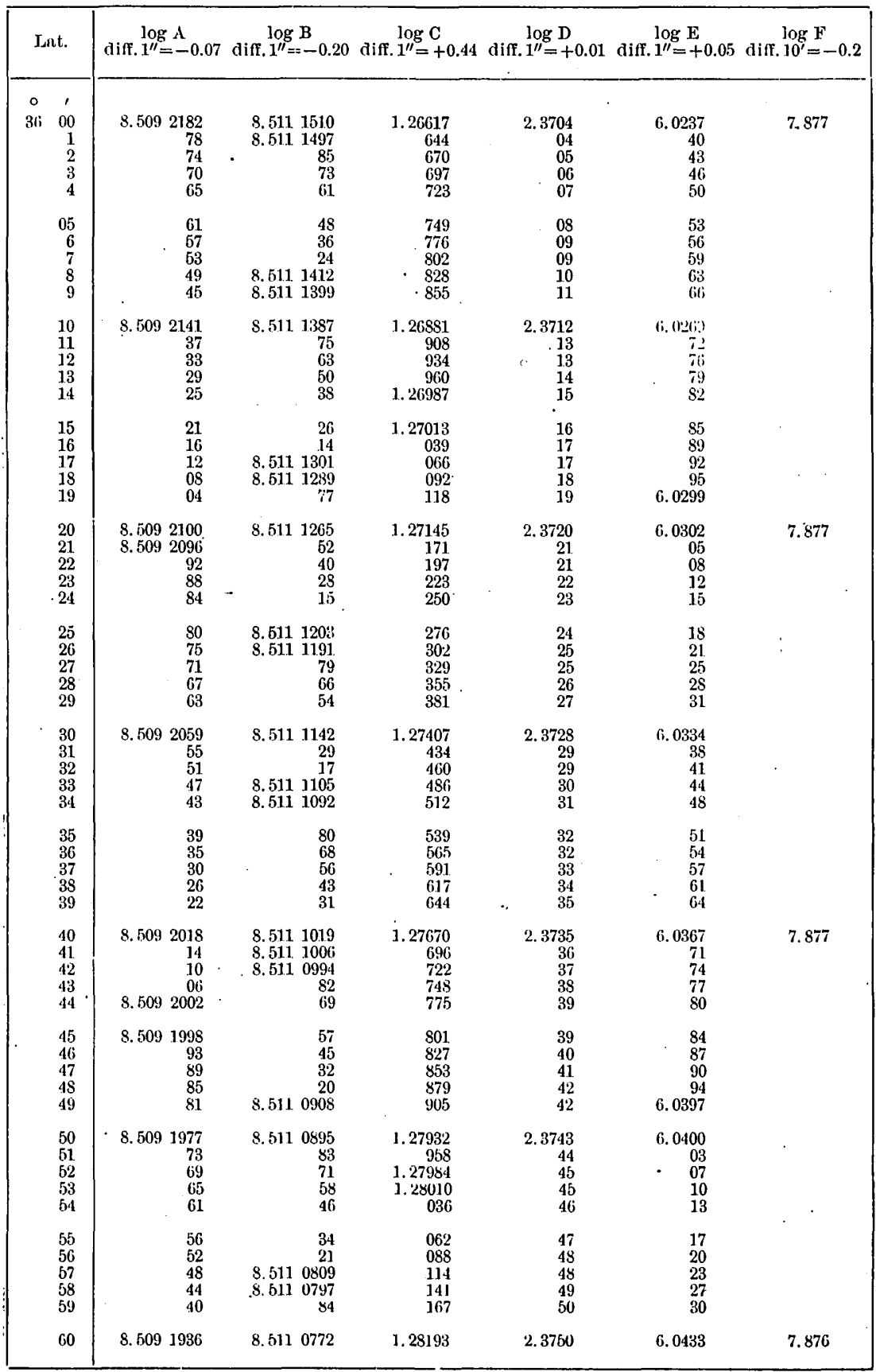


Table 20.-Geodetic position computations-Continued.

LATITUDE $37^{\circ}$.

\begin{tabular}{|c|c|c|c|c|c|c|c|}
\hline \multicolumn{2}{|c|}{ Lat. } & $\begin{array}{c}\log A \\
\operatorname{diff} .1^{\prime \prime}=-0.07\end{array}$ & $\begin{array}{c}\log B \\
\operatorname{diff} .1^{\prime \prime}=-0.21\end{array}$ & $\begin{array}{c}\log C \\
\text { diff. } 1^{\prime \prime}=+0.43\end{array}$ & $\begin{array}{c}\log \mathrm{D} \\
\operatorname{diff.} 1^{\prime \prime}=+0.01\end{array}$ & $\begin{array}{c}\log \mathrm{E} \\
\text { diff. } 1^{\prime \prime}=+0.06\end{array}$ & $\begin{array}{c}\log . \mathrm{F} \\
\text { diff. } 10^{\prime}=-0.3\end{array}$ \\
\hline 0 & , & & . & & & & \\
\hline $\begin{array}{ll}37 & 0\end{array}$ & $\begin{array}{r}00 \\
1 \\
2 \\
3 \\
4\end{array}$ & $\begin{array}{r}8.5091936 \\
32 \\
28 \\
23 \\
19\end{array}$ & $\begin{array}{r}8.5110772 \\
60 \\
47 \\
35 \\
22\end{array}$ & $\begin{array}{r}1.28193 \\
219 \\
245 \\
271 \\
297\end{array}$ & $\begin{array}{r}2.3750 \\
51 \\
52 \\
53 \\
53\end{array}$ & $\begin{array}{r}6.0433 \\
. \quad 37 \\
40 \\
43 \\
46\end{array}$ & 7.876 \\
\hline & $\begin{array}{r}05 \\
6 \\
7 \\
8 \\
9\end{array}$ & $\begin{array}{rr} & 15 \\
11 \\
07 \\
85.09 & 1903 \\
85.09 & 1899\end{array}$ & $\begin{array}{rr}8.511 & 0710 \\
8.511 & 0698 \\
85 \\
73 \\
61\end{array}$ & $\begin{array}{l}324 \\
350 \\
376 \\
402 \\
428\end{array}$ & $\begin{array}{l}54 \\
55 \\
56 \\
56 \\
57\end{array}$ & $\begin{array}{l}50 \\
53 \\
56 \\
60 \\
63\end{array}$ & . \\
\hline & $\begin{array}{l}10 \\
11 \\
12 \\
13 \\
14\end{array}$ & $\begin{array}{r}8.5091895 \\
90 \\
86 \\
82 \\
78\end{array}$ & $\begin{array}{rr}8.511 & 0648 \\
& 36 \\
23 \\
8.511 & 0611 \\
8.511 & 0599\end{array}$ & $\begin{array}{r}1.28454 \\
480 \\
506 \\
532 \\
558\end{array}$ & $\begin{array}{r}2.3758 \\
59 \\
59 \\
60 \\
61\end{array}$ & $\begin{array}{r}6.0466 \\
70 \\
73 \\
76 \\
80\end{array}$ & \\
\hline & $\begin{array}{l}15 \\
16 \\
17 \\
18 \\
19\end{array}$ & $\begin{array}{l}74 \\
70 \\
66 \\
62 \\
57 .\end{array}$ & $\begin{array}{r}86 \\
74 \\
61 \\
. \quad 49 \\
37\end{array}$ & $\begin{array}{l}584 \\
610 \\
636 \\
662 \\
688\end{array}$ & $\begin{array}{l}61 \\
62 \\
63 \\
73 \\
64\end{array}$ & $\begin{array}{l}83 \\
86 \\
89 \\
93 \\
96\end{array}$ & \\
\hline & $\begin{array}{l}20 \\
21 \\
22 \\
23 \\
24\end{array}$ & $\begin{array}{r}8.509 \quad 1853 \\
49 \\
45 \\
41 \\
37\end{array}$ & $\begin{array}{rr}8.511 & 0524 \\
& 12 \\
8.511 & 0500 \\
8.511 & 0487 \\
& 75\end{array}$ & $\begin{array}{r}1.28715 \\
741 \\
767 \\
793 \\
819\end{array}$ & $\begin{array}{r}2.3765 \\
66 \\
66 \\
67 \\
68\end{array}$ & $\begin{array}{r}6.0499 \\
6.0503 \\
06 \\
09 \\
13\end{array}$ & 7.876 \\
\hline & $\begin{array}{l}25 \\
26 \\
27 \\
28 \\
29\end{array}$ & $\begin{array}{l}38 \\
28 \\
24 \\
20 \\
16\end{array}$ & $\begin{array}{l}62 \\
50 \\
37 \\
25 \\
13\end{array}$ & $\begin{array}{l}845 \\
871 \\
897 \\
923 \\
949\end{array}$ & $\begin{array}{l}68 \\
69 \\
70 \\
70 \\
71\end{array}$ & $\begin{array}{l}16 \\
19 \\
23 \\
26 \\
29\end{array}$ & \\
\hline & $\begin{array}{l}30 \\
31 \\
32 \\
33 \\
34\end{array}$ & $\begin{array}{rr}8.509 & 1812 \\
08 \\
04 \\
0.509 \\
8800 \\
8.509 & 1795\end{array}$ & $\begin{array}{rr}8.511 & 0400 \\
8.511 & 0388 \\
75 \\
63 \\
51\end{array}$ & $\begin{array}{r}1.28975 \\
1.29001 \\
027 \\
053 \\
079\end{array}$ & $\begin{array}{r}2.3772 \\
72 \\
73 \\
74 \\
74\end{array}$ & $\begin{array}{r}6.0533 \\
36 \\
39 \\
43 \\
46\end{array}$ & \\
\hline & $\begin{array}{l}35 \\
36 \\
37 \\
38 \\
39\end{array}$ & $\begin{array}{l}91 \\
87 \\
83 \\
79 \\
75\end{array}$ & $\begin{array}{rr} & 38 \\
. \quad & 26 \\
& 13 \\
8.511 & 0301 \\
8.511 & 0288\end{array}$ & $\begin{array}{l}104 \\
130 \\
156 \\
182 \\
208\end{array}$ & $\begin{array}{l}75 \\
76 \\
76 \\
77 \\
78\end{array}$ & $\begin{array}{l}49 \\
53 \\
56 \\
59 \\
68\end{array}$ & . \\
\hline & $\begin{array}{l}40 \\
41 \\
42 \\
43 \\
44\end{array}$ & 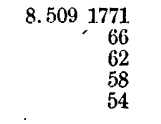 & $\begin{array}{r}8.5110276 \\
64 \\
51 \\
39 \\
\\
26\end{array}$ & $\begin{array}{r}1.29234 \\
260 \\
286 \\
312 \\
338\end{array}$ & $\begin{array}{r}2.3779 \\
79 \\
80 \\
81 \\
81\end{array}$ & $\begin{array}{r}6.0566 \\
69 \\
73 \\
76 \\
.79\end{array}$ & $\begin{array}{l}7.875 \\
.\end{array}$ \\
\hline & $\begin{array}{l}45 \\
46 \\
47 \\
48 \\
49\end{array}$ & $\begin{array}{l}50 \\
46 \\
41 \\
37 \\
33\end{array}$ & $\begin{array}{rr} & 14 \\
8.512 & 0201 \\
8.511 & 0189 \\
76 \\
64\end{array}$ & $\begin{array}{l}364 \\
390 \\
416 \\
442 \\
468\end{array}$ & $\begin{array}{l}82 \\
82 \\
83 \\
84 \\
84\end{array}$ & $\begin{array}{r}83 \\
86 \\
89 \\
93 \\
6.0596\end{array}$ & \\
\hline & $\begin{array}{l}50 \\
51 \\
52 \\
53 \\
54\end{array}$ & $\begin{array}{r}8.5091729 \\
25 \\
21 \\
16 \\
12\end{array}$ & $\begin{array}{r}8.5110151 \\
39 \\
26 \\
14 \\
8.5110102\end{array}$ & $\begin{array}{r}1.29494 \\
520 \\
546 \\
571 \\
597\end{array}$ & $\begin{array}{r}2.3785 \\
86 \\
86 \\
87 \\
88\end{array}$ & $\begin{array}{r}6.0600 \\
03 \\
06 \\
10 \\
13\end{array}$ & \\
\hline & $\begin{array}{l}55 \\
56 \\
57 \\
58 \\
59\end{array}$ & $\begin{array}{r}08 \\
04 \\
8.509 \\
1700 \\
8.509 \quad 1696 \\
92\end{array}$ & $\begin{array}{r}8.5110089 \\
77 \\
64 \\
52 \\
39\end{array}$ & $\begin{array}{l}623 \\
649 \\
675 \\
701 \\
727\end{array}$ & $\begin{array}{l}88 \\
89 \\
90 \\
90 \\
91\end{array}$ & $\begin{array}{l}16 \\
20 \\
23 \\
26 \\
30\end{array}$ & \\
\hline & 60 & 8.5091687 & 8.5110027 & 1.29753 & 2.8792 & 6.0633 & 7.874 \\
\hline
\end{tabular}


TABLE 20.-Greodetic position computations-Continued.

IATITUDE $38^{\circ}$.

\begin{tabular}{|c|c|c|c|c|c|c|}
\hline Lat. & $\begin{array}{c}\log A \\
\text { diff. } 1^{\prime \prime}=-0.07\end{array}$ & $\begin{array}{c}\log B \\
\text { diff. } 1^{\prime \prime}=-0.21\end{array}$ & $\begin{array}{c}\log \mathrm{C} \\
\text { diff. } 1^{\prime \prime}=+0.43\end{array}$ & $\begin{array}{c}\log \mathrm{D} \\
\text { diff. } 1^{\prime \prime}=+0.01\end{array}$ & $\begin{array}{c}\log \mathrm{E} \\
\text { diff. } 1^{\prime \prime}=+0.06\end{array}$ & $\begin{array}{c}\log F \\
\text { diff. } 10^{\prime}=-0.4\end{array}$ \\
\hline , & & & & & & \\
\hline 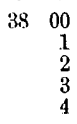 & $\begin{array}{r}8.5091687 \\
83 \\
79 \\
75 \\
71\end{array}$ & $\begin{array}{r}8.5110027 \\
14 \\
8.5110002 \\
8.5109989 \\
77\end{array}$ & $\begin{array}{r}1.29753 \\
778 \\
804 \\
830 \\
856\end{array}$ & $\begin{array}{r}2.3792 \\
92 \\
93 \\
93 \\
94\end{array}$ & $\begin{array}{r}6.0633 \\
. \quad 36 \\
40 \\
43 \\
47\end{array}$ & 7.874 \\
\hline $\begin{array}{r}05 \\
6 \\
7 \\
8 \\
9\end{array}$ & $\begin{array}{l}67 \\
62 \\
58 \\
54 \\
50\end{array}$ & $\begin{array}{l}64 \\
52 \\
39 \\
27 \\
14\end{array}$ & $\begin{array}{r}882 \\
908 \\
934 \\
959 \\
1.29985\end{array}$ & $\begin{array}{l}95 \\
95 \\
96 \\
97 \\
97\end{array}$ & $\begin{array}{l}50 \\
53 \\
57 \\
60 \\
63\end{array}$ & , \\
\hline $\begin{array}{l}10 \\
11 \\
12 \\
13 \\
14\end{array}$ & $\begin{array}{r}8.5091 .646 \\
42 \\
37 \\
33 \\
29\end{array}$ & $\begin{array}{r}8.5109902 \\
8.5109889 \\
77 \\
64 \\
52\end{array}$ & $\begin{array}{r}1.30011 \\
037 \\
063 \\
089 \\
114\end{array}$ & $\begin{array}{r}2.3798 \\
2.3799 \\
2.3800 \\
00 \\
01 .\end{array}$ & $\begin{array}{r}6.0667 \\
70 \\
73 \\
77 \\
80\end{array}$ & \\
\hline $\begin{array}{l}15 \\
16 \\
17 \\
18 \\
19\end{array}$ & $\begin{array}{l}25 \\
21 \\
17 \\
.12 \\
08\end{array}$ & $\begin{array}{r}39 \\
27 \\
14 \\
14 \\
8.5109802 \\
8.5109789\end{array}$ & $\begin{array}{l}140 \\
166 \\
192 \\
218 \\
243\end{array}$ & $\begin{array}{l}01 \\
02 \\
02 \\
03 \\
03 \\
03\end{array}$ & $\begin{array}{r}84 \\
87 \\
90 \\
94 \\
\text { 6. } 0697\end{array}$ & - \\
\hline $\begin{array}{l}20 \\
21 \\
22 \\
23 \\
24\end{array}$ & $\begin{array}{rr}8.509 & 1604 \\
8.509 & 1600 \\
\text { 8. } 509 & 1596 \\
. & 92 \\
& 87\end{array}$ & $\begin{array}{r}8.5109777 \\
64 \\
52 \\
39 \\
27\end{array}$ & $\begin{array}{r}1.30269 \\
295 \\
321 \\
347 \\
372\end{array}$ & $\begin{array}{r}2.3804 \\
05 \\
05 \\
06 \\
06\end{array}$ & $\begin{array}{r}\text { 6. } 0701 \\
04 \\
.07 \\
11 \\
14\end{array}$ & 7.874 \\
\hline $\begin{array}{l}25 \\
26 \\
27 \\
28 \\
29\end{array}$ & $\begin{array}{l}83 \\
79 \\
75 \\
71 \\
66\end{array}$ & $\begin{array}{r}14 \\
8.5109701 \\
8.5109689 \\
77 \\
64\end{array}$ & $\begin{array}{l}398 \\
424 \\
450 \\
476 \\
501\end{array}$ & $\begin{array}{r}. \quad 07 \\
.08 \\
08 \\
0 \quad 09 \\
. \quad 09\end{array}$ & $\begin{array}{r}17 \\
21 \\
24 \\
. \quad 28 \\
31\end{array}$ & \\
\hline $\begin{array}{l}30 \\
31 \\
32 \\
33 \\
34\end{array}$ & $\begin{array}{r}8.5091562 \\
58 \\
54 \\
50 \\
46\end{array}$ & $\begin{array}{r}8.5109652 \\
39 \\
27 \\
14 \\
8.5109601\end{array}$ & $\begin{array}{r}1.30527 \\
553 \\
579 \\
604 \\
630\end{array}$ & $\begin{array}{r}2.3810 \\
11 \\
11 \\
12 \\
12\end{array}$ & $\begin{array}{r}6.0734 \\
+\quad 38 \\
41 \\
44 \\
48\end{array}$ & \\
\hline $\begin{array}{l}35 \\
36 \\
37 \\
38 \\
39\end{array}$ & $\begin{array}{l}41 \\
37 \\
33 \\
29 \\
25\end{array}$ & $\begin{array}{r}8.5109589 \\
76 \\
64 \\
51 \\
39\end{array}$ & $\begin{array}{l}656 \\
682 \\
707 \\
733 \\
759\end{array}$ & $\begin{array}{l}13 \\
14 \\
14 \\
15 \\
15\end{array}$ & $\begin{array}{l}51 \\
55 \\
58 \\
61 \\
65\end{array}$ & . \\
\hline $\begin{array}{l}40 \\
41 \\
42 \\
43 \\
44\end{array}$ & $\begin{array}{r}8.509 .1521 \\
16 \\
12 \\
.08 \\
04\end{array}$ & $\begin{array}{r}8.5109526 \\
14 \\
8.5109501 \\
8.5109488 \\
76\end{array}$ & $\begin{array}{r}1.30785 \\
810 \\
836 \\
862 \\
887\end{array}$ & $\begin{array}{r}2.3816 \\
16 \\
17 \\
18 \\
18\end{array}$ & $\begin{array}{r}6.0768 \\
72 \\
75 \\
78 \\
82\end{array}$ & 7.873 \\
\hline $\begin{array}{l}45 \\
46 \\
47 \\
48 \\
49\end{array}$ & $\begin{array}{r}8.5091500 \\
8.509 \quad 1495 \\
91 \\
87 \\
83\end{array}$ & $\begin{array}{l}63 \\
51 \\
38 \\
26 \\
13\end{array}$ & $\begin{array}{r}913 \\
939 \\
965 \\
1.30990 \\
1.31016\end{array}$ & $\begin{array}{l}19 \\
19 \\
20 \\
20 \\
21\end{array}$ & $\begin{array}{r}\mathrm{S5} \\
89 \\
92 \\
95 \\
6.0799\end{array}$ & . \\
\hline $\begin{array}{l}50 \\
51 \\
62 \\
53 \\
54\end{array}$ & $\begin{array}{r}8.5091479 \\
75 \\
70 \\
66 \\
62\end{array}$ & $\begin{array}{r}8.5109401 \\
8.5109388 \\
76 \\
63 \\
50\end{array}$ & $\begin{array}{r}1.31042 \\
067 \\
093 \\
119 \\
144\end{array}$ & $\begin{array}{r}2.3822 \\
22 \\
23 \\
23 \\
24 \\
24\end{array}$ & $\begin{array}{r}6.0802 \\
06 \\
09 \\
13 \\
16\end{array}$ & \\
\hline $\begin{array}{l}65 \\
56 \\
67 \\
58 \\
59\end{array}$ & $\begin{array}{l}58 \\
53 \\
49 \\
45 \\
41\end{array}$ & $\begin{array}{r}38 \\
25 \\
13 \\
8.5109300 \\
8.5109287\end{array}$ & $\begin{array}{r}170 \\
196 \\
221 \\
247 \\
273\end{array}$ & $\begin{array}{l}24 \\
25 \\
25 \\
26 \\
27\end{array}$ & $\begin{array}{l}19 \\
23 \\
26 \\
30 \\
33\end{array}$ & . \\
\hline 60 & 8. 5091437 & 8.5109275 & 1. 31299 & 2.3827 & 6.0836 & 7.872 \\
\hline
\end{tabular}


TABLE 20.-Geodetic position computations-Continued.

LATITUDE $39^{\circ}$.

\begin{tabular}{|c|c|c|c|c|c|c|}
\hline Lat. & $\begin{array}{c}\log A \\
\text { diff. } 1^{\prime \prime}=-0.07\end{array}$ & $\begin{array}{c}\log B \\
\text { diff. } 1^{\prime \prime}=-0.21\end{array}$ & $\begin{array}{c}\log \mathrm{C} \\
\text { diff. } 1^{\prime \prime}=+0,43\end{array}$ & $\begin{array}{c}\log \mathrm{D} \\
\text { diff. } 1^{\prime \prime}=+0.01\end{array}$ & $\begin{array}{c}\log \mathrm{E} \\
\operatorname{diff} 1^{\prime \prime}=+0.06\end{array}$ & $\begin{array}{c}\log F \\
\text { diff. } 10^{\prime}=-0.5\end{array}$ \\
\hline , & & & & & & \\
\hline $\begin{array}{rr}39 & 00 \\
& 1 \\
& 2 \\
& 3 \\
& 4\end{array}$ & $\begin{array}{r}8.509 \quad 1437 \\
33 \\
28 \\
24 \\
20\end{array}$ & $\begin{array}{r}8.5109275 \\
62 \\
50 \\
.37 \\
25\end{array}$ & $\begin{array}{r}\text { T. } 31299 \\
324 \\
350 \\
375 \\
401\end{array}$ & $\begin{array}{r}2.3827 \\
28 \\
28 \\
29 \\
29\end{array}$ & $\begin{array}{r}6.0836 \\
40 \\
43 \\
47 \\
50\end{array}$ & 7.872 \\
\hline $\begin{array}{r}05 \\
6 \\
7 \\
8 \\
9\end{array}$ & $\begin{array}{r}16 \\
12 \\
07 \\
8.5091403 \\
8.5091399\end{array}$ & $\begin{array}{r}8.5109212 \\
8.5109199 \\
87 \\
74 \\
62\end{array}$ & $\begin{array}{l}427 \\
452 \\
478 \\
504 \\
529\end{array}$ & $\begin{array}{l}30 \\
30 \\
31 \\
31 \\
32\end{array}$ & $\begin{array}{l}53 \\
57 \\
60 \\
64 \\
67\end{array}$ & \\
\hline $\begin{array}{l}10 \\
11 \\
12 \\
13 \\
14\end{array}$ & $\begin{array}{r}8.509 \quad 1395 \\
91 \\
86 \\
82 \\
78\end{array}$ & $\begin{array}{r}8.5109149 \\
36 \\
24 \\
8.5109111 \\
8.5109098\end{array}$ & $\begin{array}{r}1.31555 \\
581 \\
606 \\
632 \\
658\end{array}$ & 2. $\begin{array}{r}3832 \\
33 \\
33 \\
34 \\
35\end{array}$ & $\begin{array}{r}6.0871 \\
74 \\
77 \\
81 \\
84\end{array}$ & \\
\hline $\begin{array}{l}15 \\
16 \\
17 \\
18 \\
19\end{array}$ & $\begin{array}{l}74 \\
70 \\
65 \\
61 \\
57\end{array}$ & $\begin{array}{l}86 \\
73 \\
61 \\
48 \\
36\end{array}$ & $\begin{array}{l}683 \\
709 \\
734 \\
760 \\
786\end{array}$ & $\begin{array}{l}35 \\
36 \\
36 \\
37 \\
37\end{array}$ & $\begin{array}{r}88 \\
91 \\
95 \\
6.0898 \\
6.0902\end{array}$ & - \\
\hline $\begin{array}{l}20 \\
21 \\
22 \\
23 \\
24\end{array}$ & $\begin{array}{r}8.509 \quad 1353 \\
49 \\
44 \\
40 \\
36\end{array}$ & $\begin{array}{r}8.5109023 \\
8.5109010 \\
8.510 .8998 \\
85 \\
73\end{array}$ & $\begin{array}{r}1.31811 \\
837 \\
862 \\
888 \\
913\end{array}$ & $\begin{array}{r}2.3838 \\
38 \\
39 \\
39 \\
40\end{array}$ & 6. $\begin{array}{r}0905 \\
08 \\
12 \\
15 \\
19\end{array}$ & 7.871 \\
\hline $\begin{array}{l}25 \\
26 \\
27 \\
28 \\
29\end{array}$ & $\begin{array}{l}32 \\
28 \\
23 \\
19 \\
15\end{array}$ & $\begin{array}{r}60 \\
47 \\
35 \\
22 \\
8.510 \quad 8909\end{array}$ & $\begin{array}{r}939 \\
965 \\
1.31990 \\
1.32016 \\
\quad 041\end{array}$ & $\begin{array}{l}40 \\
41 \\
41 \\
42 \\
42\end{array}$ & $\begin{array}{l}22 \\
26 \\
29 \\
32 \\
36\end{array}$ & \\
\hline $\begin{array}{l}30 \\
31 \\
32 \\
33 \\
34\end{array}$ & $\begin{array}{r}8.5091311 \\
07 \\
0.509 \\
8.502 \\
8.509 \\
1298 \\
94\end{array}$ & $\begin{array}{r}8.510 \quad 8897 \\
84 \\
72 \\
59 \\
46\end{array}$ & $\begin{array}{r}1.32067 \\
092 \\
11.8 \\
144 \\
169\end{array}$ & $\begin{array}{r}2.3843 \\
43 \\
44 \\
44 \\
45\end{array}$ & $\begin{array}{r}6.0939 \\
43 \\
46 \\
50 \\
53\end{array}$ & \\
\hline $\begin{array}{l}35 \\
36 \\
37 \\
38 \\
39\end{array}$ & $\begin{array}{l}90 \\
86 \\
81 \\
77 \\
73\end{array}$ & $\begin{array}{r}34 \\
21 \\
8.510 \quad 8808 \\
8.5108796 \\
8: 3\end{array}$ & $\begin{array}{l}195 \\
220 \\
246 \\
271 \\
297\end{array}$ & $\begin{array}{l}45 \\
46 \\
46 \\
47 \\
47\end{array}$ & $\begin{array}{l}57 \\
60 \\
63 \\
67 \\
70\end{array}$ & \\
\hline $\begin{array}{l}40 \\
41 \\
42 \\
43 \\
44\end{array}$ & $\begin{array}{r}8.5091269 \\
64 \\
60 \\
56 \\
52\end{array}$ & $\begin{array}{r}8.510 \quad 8771 \\
58 \\
45 \\
33 \\
20\end{array}$ & $\begin{array}{r}1.32323 \\
348 \\
374 \\
399 \\
425\end{array}$ & $\begin{array}{r}2.3848 \\
48 \\
49 \\
49 \\
50\end{array}$ & $\begin{array}{r}6.0974 \\
77 \\
81 \\
84 \\
88\end{array}$ & 7.870 \\
\hline $\begin{array}{l}45 \\
46 \\
47 \\
48 \\
49\end{array}$ & $\begin{array}{l}48 \\
43 \\
39 \\
35 \\
31\end{array}$ & $\begin{array}{r}8.510 \quad 8707 \\
8.510 \quad 8695 \\
82 \\
69 \\
57\end{array}$ & $\begin{array}{l}450 \\
476 \\
501 \\
527 \\
552\end{array}$ & $\begin{array}{l}50 \\
51 \\
51 \\
52 \\
52\end{array}$ & $\begin{array}{r}91 \\
95 \\
6.0998 \\
6.1002 \\
05\end{array}$ & . \\
\hline $\begin{array}{l}50 \\
51 \\
52 \\
53 \\
54\end{array}$ & $\begin{array}{r}8.5091227 \\
22 \\
18 \\
14 \\
10\end{array}$ & $\begin{array}{r}8.5108644 \\
31 \\
19 \\
8.5108606 \\
8.5108593\end{array}$ & $\begin{array}{r}1.32578 \\
603 \\
629 \\
654 \\
680\end{array}$ & $\begin{array}{r}2.3852 \\
53 \\
53 \\
54 \\
54\end{array}$ & 6. $\begin{array}{r}1008 \\
12 \\
15 \\
19 \\
22\end{array}$ & . \\
\hline $\begin{array}{l}55 \\
56 \\
57 \\
58 \\
59\end{array}$ & $\begin{array}{r}06 \\
8.5091201 \\
8.5091197 \\
93 \\
89\end{array}$ & $\begin{array}{l}81 \\
68 \\
55 \\
43 \\
30\end{array}$ & $\begin{array}{l}705 \\
731 \\
756 \\
782 \\
807\end{array}$ & $\begin{array}{l}55 \\
5 \\
55 \\
56 \\
56 \\
57\end{array}$ & $\begin{array}{l}26 \\
29 \\
33 \\
36 \\
40\end{array}$ & . \\
\hline 60 & 8.5091184 & 8.5108517 & 1.32833 & 2.3857 & 6. 1043 & 7.869 \\
\hline
\end{tabular}


TABLe 20.-Geodelic position compulations-Continued.

LATITUDE $40^{\circ}$.

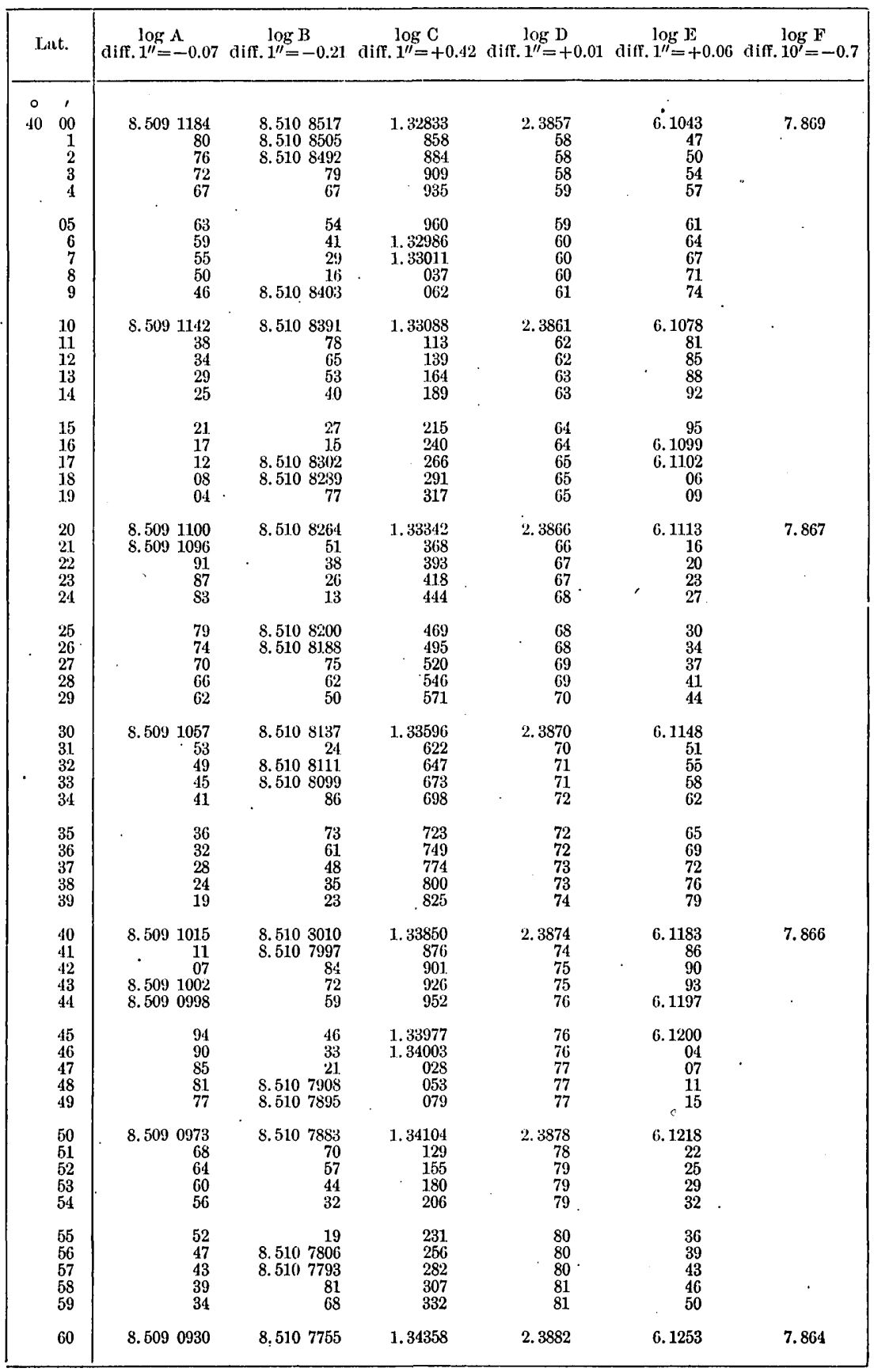


TABLE 20.-Geodetic position computations-Continued.

IATITUDE $41^{\circ}$.

\begin{tabular}{|c|c|c|c|c|c|c|c|}
\hline Lat. & & $\begin{array}{c}\log A \\
\text { diff. } 1^{\prime \prime}=-0.07\end{array}$ & 7. diff. $1^{\prime \prime \prime}=-0.21$ & $\begin{array}{c}\log \mathrm{C} \\
\text { diff. } 1^{\prime \prime}=+0.42\end{array}$ & $\begin{array}{c}\log \mathrm{D} \\
\text { diff. } 1^{\prime \prime}=+0.01\end{array}$ & $\begin{array}{c}\log \mathrm{E} \\
\text { diff. } 1^{\prime \prime}=+0.06\end{array}$ & $\begin{array}{c}\log \mathrm{F} \\
\text { dift. } 10^{\prime}=-0 . S\end{array}$ \\
\hline 0 & , & & & & . & & \\
\hline $\begin{array}{l}41.0 \\
.\end{array}$ & $\begin{array}{r}00 \\
1 \\
2 \\
3 \\
4\end{array}$ & $\begin{array}{r}8.5090930 \\
26 \\
22 \\
18 \\
-\quad 13\end{array}$ & $\begin{array}{r}8.5107755 \\
42 \\
30 \\
17 \\
8.510 \quad 7704\end{array}$ & $\begin{array}{r}1.34358 \\
383 \\
408 \\
434 \\
459\end{array}$ & $\begin{array}{r}2.3882 \\
82 \\
82 \\
83 \\
.83\end{array}$ & $\begin{array}{r}6.1253 \\
57 \\
60 \\
64 \\
67\end{array}$ & 7.864 \\
\hline & $\begin{array}{l}05 \\
6 \\
7 \\
8 \\
9\end{array}$ & $\begin{array}{cr}. & 09 \\
& 05 \\
8.509 & 0900 \\
8.5090896 \\
92\end{array}$ & $\begin{array}{r}8.510 \quad 7691 \\
79 \\
66 \\
53 \\
40\end{array}$ & $\begin{array}{l}484 \\
510 \\
535 \\
560 \\
586\end{array}$ & $\begin{array}{l}83 \\
84 \\
84 \\
84 \\
85\end{array}$ & $\begin{array}{l}71 \\
75 \\
78 \\
82 \\
85\end{array}$ & \\
\hline & $\begin{array}{l}10 \\
11 \\
12 \\
13 \\
14\end{array}$ & $\begin{array}{r}8.5090888 \\
83 \\
79 \\
75 \\
71\end{array}$ & $\begin{array}{rr}8.510 & 7628 \\
1.5 \\
8.510 & 7602 \\
8.510 & 7590\end{array}$ & $\begin{array}{r}\text { 1. } 34611 \\
636 \\
662 \\
687 \\
712\end{array}$ & $\begin{array}{r}2.3885 \\
85 \\
86 \\
86 \\
87\end{array}$ & $\begin{array}{r}6.1289 \\
92 \\
96 \\
6.1299 \\
6.1303\end{array}$ & . \\
\hline & $\begin{array}{l}15 \\
16 \\
17 \\
18 \\
19\end{array}$ & $\begin{array}{l}67 \\
62 \\
58 \\
54 \\
49\end{array}$ & $\begin{array}{l}64 \\
51 \\
39 \\
26 \\
13\end{array}$ & $\begin{array}{l}738 \\
763 \\
788 \\
814 \\
839\end{array}$ & $\begin{array}{l}87 \\
87 \\
88 \\
88 \\
88\end{array}$ & $\begin{array}{l}06 \\
10 \\
14 \\
17 \\
21\end{array}$ & . \\
\hline $\begin{array}{l}2 \\
2 \\
2 \\
2 \\
2\end{array}$ & $\begin{array}{l}20 \\
21 \\
22 \\
23 \\
24\end{array}$ & $\begin{array}{r}8.5090845 \\
41 \\
37 \\
32 \\
28\end{array}$ & $\begin{array}{r}8.5107500 \\
8.5107488 \\
75 \\
62 \\
49\end{array}$ & $\begin{array}{r}\text { 1. } 34864 \\
890 \\
915 \\
940 \\
965\end{array}$ & $\begin{array}{r}2.3889 \\
89 \\
89 \\
90 \\
90\end{array}$ & $\begin{array}{r}6.1324 \\
28 \\
31 \\
35 \\
38\end{array}$ & 7.863 \\
\hline $\begin{array}{l}2 \\
2 \\
2 \\
2 \\
2\end{array}$ & $\begin{array}{l}25 \\
26 \\
27 \\
28 \\
29\end{array}$ & $\begin{array}{l}24 \\
20 \\
15 \\
11 \\
07\end{array}$ & $\begin{array}{rr}36 \\
24 \\
8.5107411 \\
8.5107398 \\
85\end{array}$ & $\begin{array}{r}\text { 1. } 34991 \\
\text { 1. } 35016 \\
041 \\
066 \\
092\end{array}$ & $\begin{array}{l}90 \\
91 \\
91 \\
91 \\
91\end{array}$ & $\begin{array}{l}42 \\
46 \\
49 \\
53 \\
56\end{array}$ & . \\
\hline $\begin{array}{l}3 \\
3 \\
3 \\
3 \\
3\end{array}$ & $\begin{array}{l}30 \\
31 \\
32 \\
33 \\
34\end{array}$ & $\begin{array}{r}8.5090803 \\
\text { S. } 5090798 \\
94 \\
90 \\
86\end{array}$ & $\begin{array}{r}8.510 \quad 7373 \\
60 \\
47 \\
34 \\
22\end{array}$ & $\begin{array}{r}1.35117 \\
142 \\
168 \\
193 \\
218\end{array}$ & $\begin{array}{r}2.3592 \\
92 \\
92 \\
93 \\
93\end{array}$ & 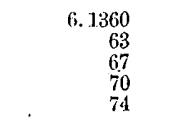 & ' \\
\hline & $\begin{array}{l}35 \\
36 \\
37 \\
38 \\
39\end{array}$ & $\begin{array}{r}81 \\
77 \\
73 \\
69 \\
64\end{array}$ & $\begin{array}{r}8.510 \\
8.510 \\
72909 \\
83 \\
70 \\
\\
58\end{array}$ & $\begin{array}{l}243 \\
269 \\
294 \\
319 \\
345\end{array}$ & $\begin{array}{l}93 \\
94 \\
94 \\
94 \\
95\end{array}$ & $\begin{array}{l}78 \\
81 \\
85 \\
85 \\
92\end{array}$ & . \\
\hline $\begin{array}{l}4 \\
4 \\
4 \\
4 \\
4\end{array}$ & $\begin{array}{l}40 \\
41 \\
42 \\
43 \\
44\end{array}$ & $\begin{array}{r}8.5090760 \\
56 \\
52 \\
47 \\
43\end{array}$ & $\begin{array}{r}8.5107245 \\
32 \\
19 \\
8.5107207 \\
8.5107194\end{array}$ & $\begin{array}{r}1.35370 \\
395 \\
420 \\
446 \\
471\end{array}$ & $\begin{array}{r}2.3895 \\
95 \\
96 \\
96 \\
. \quad 96\end{array}$ & $\begin{array}{r}6.1395 \\
6.1399 \\
6.1403 \\
06 \\
10\end{array}$ & 7.861 \\
\hline $\begin{array}{l}4 \\
4 \\
4 \\
4 \\
4\end{array}$ & $\begin{array}{l}45 \\
46 \\
47 \\
48 \\
49\end{array}$ & $\begin{array}{l}39 \\
35 \\
30 \\
26 \\
22\end{array}$ & $\begin{array}{l}81 \\
68 \\
55 \\
43 \\
30\end{array}$ & $\begin{array}{l}496 \\
522 \\
547 \\
572 \\
597\end{array}$ & $\begin{array}{l}97 \\
97 \\
97 \\
97 \\
98\end{array}$ & $\begin{array}{l}13 \\
17 \\
20 \\
24 \\
28\end{array}$ & \\
\hline & $\begin{array}{l}50 \\
51 \\
52 \\
53 \\
54\end{array}$ & $\begin{array}{r}8.5090718 \\
13 \\
09 \\
05 \\
8.5090700\end{array}$ & $\begin{array}{rr}8.510 & 7117 \\
8.510 & 7104 \\
\text { S. } 510 & 7091 \\
& 79 \\
& 66\end{array}$ & $\begin{array}{r}\text { 1. } 35623 \\
648 \\
673 \\
698 \\
723\end{array}$ & $\begin{array}{r}2.3898 \\
98 \\
.98 \\
99 \\
.99\end{array}$ & $\begin{array}{r}\text { 6.1431 } \\
35 \\
38 \\
42 \\
46\end{array}$ & \\
\hline $\begin{array}{l}5 \\
5 \\
5 \\
5 \\
. \quad 5\end{array}$ & $\begin{array}{l}55 \\
56 \\
57 \\
58 \\
59\end{array}$ & $\begin{array}{r}8.5090696 \\
92 \\
88 \\
83 \\
.79\end{array}$ & $\begin{array}{r}53 \\
40 \\
27 \\
15 \\
8.5107002\end{array}$ & $\begin{array}{l}749 \\
774 \\
799 \\
824 \\
850\end{array}$ & $\begin{array}{r}2.3899 \\
2.3900 \\
00 \\
00 \\
00\end{array}$ & $\begin{array}{l}49 \\
53 \\
56 \\
60 \\
63\end{array}$ & \\
\hline & 60 & 8.5090675 & 8.5106989 & 1. 35875 & 2.3901 & 6.1467 & 7.860 \\
\hline
\end{tabular}


TABLE 20.-Geodetic position computations-Continued.

LATITUDE $42^{\circ}$.

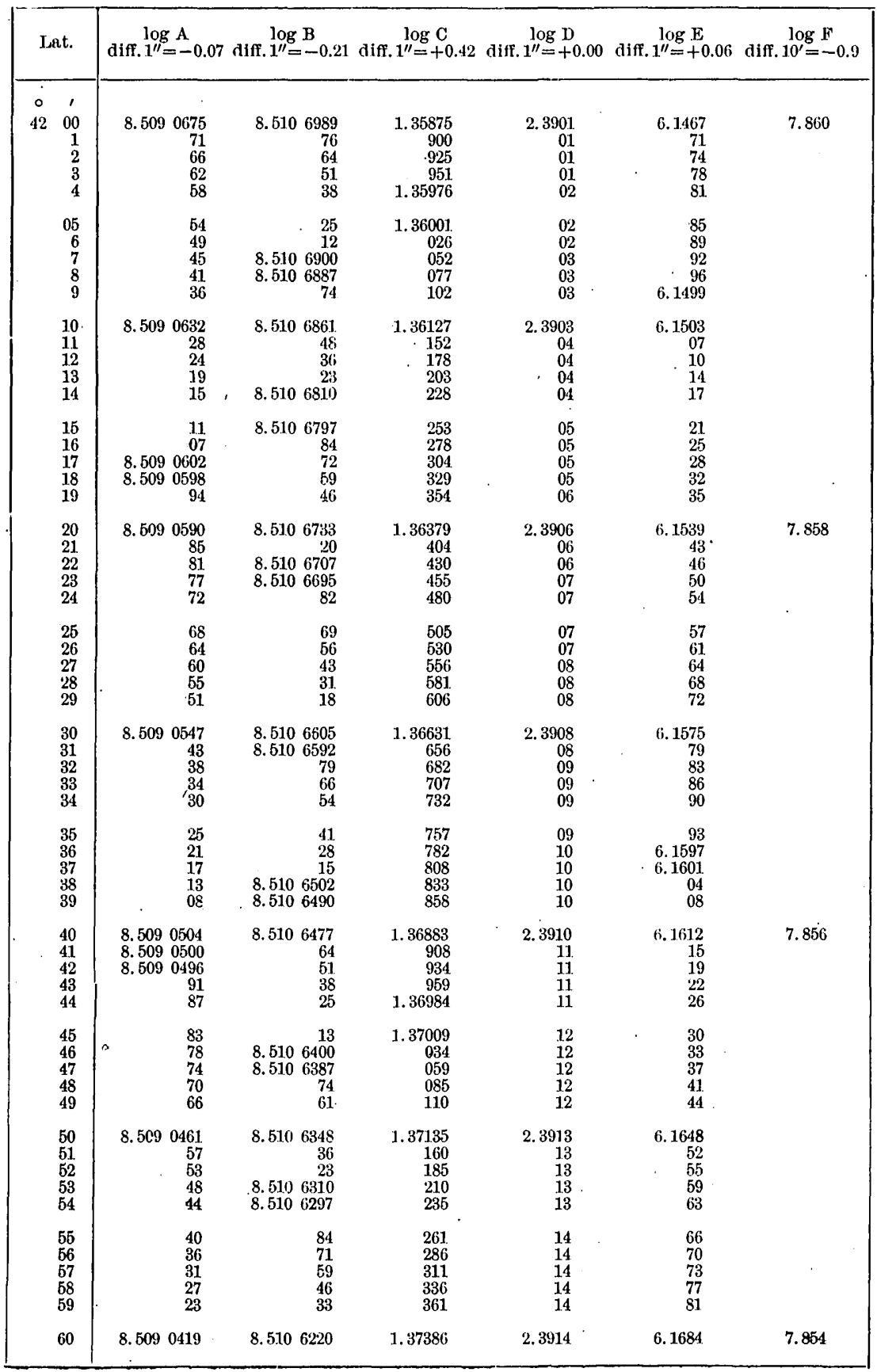


TABLE 20.-Geodetic position computations-Continued.

LATITUDE $43^{\circ}$.

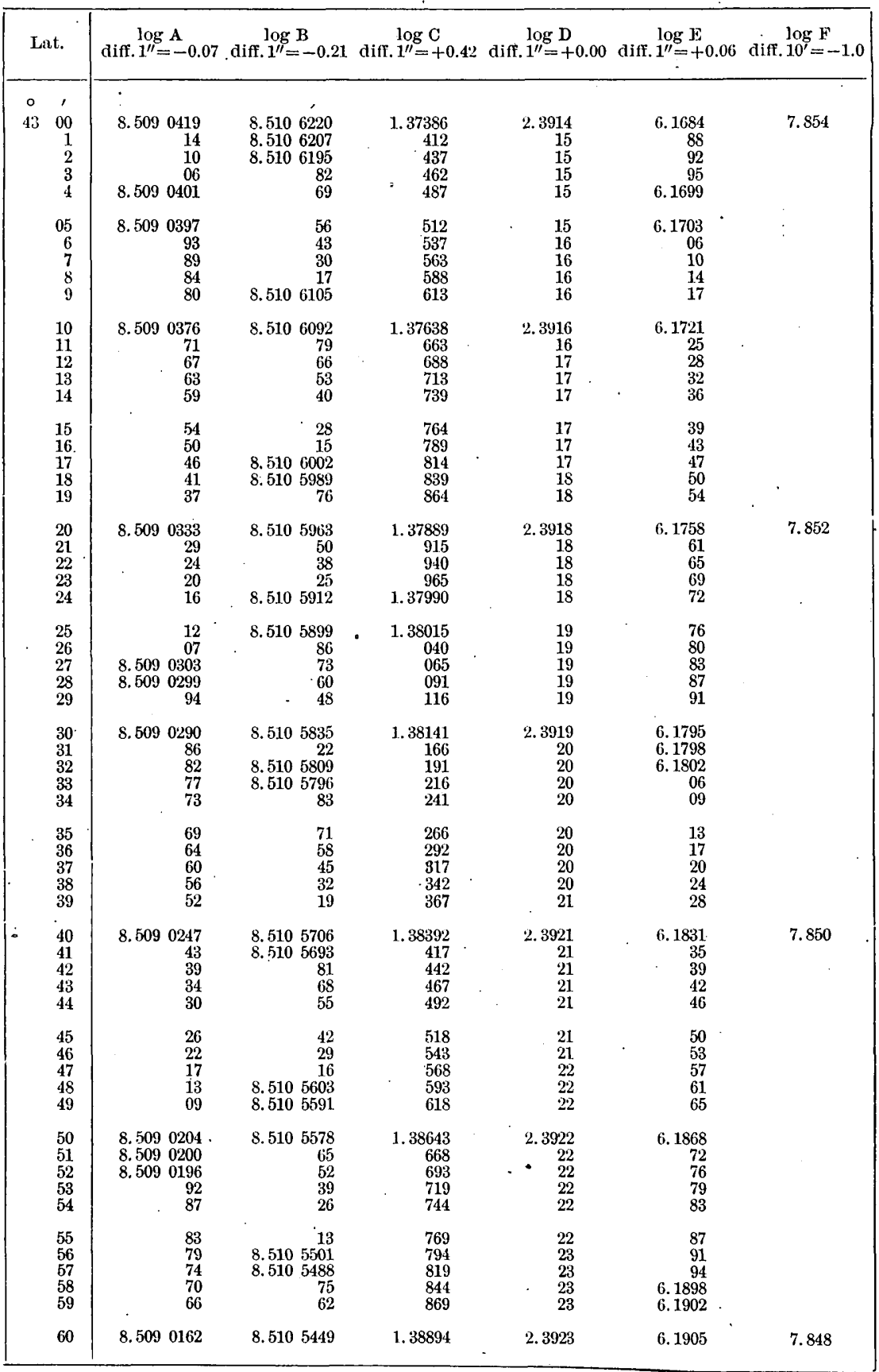


TABLE 20.-Geodetic position computations-Continued.

LATITUDE $44^{\circ}$.

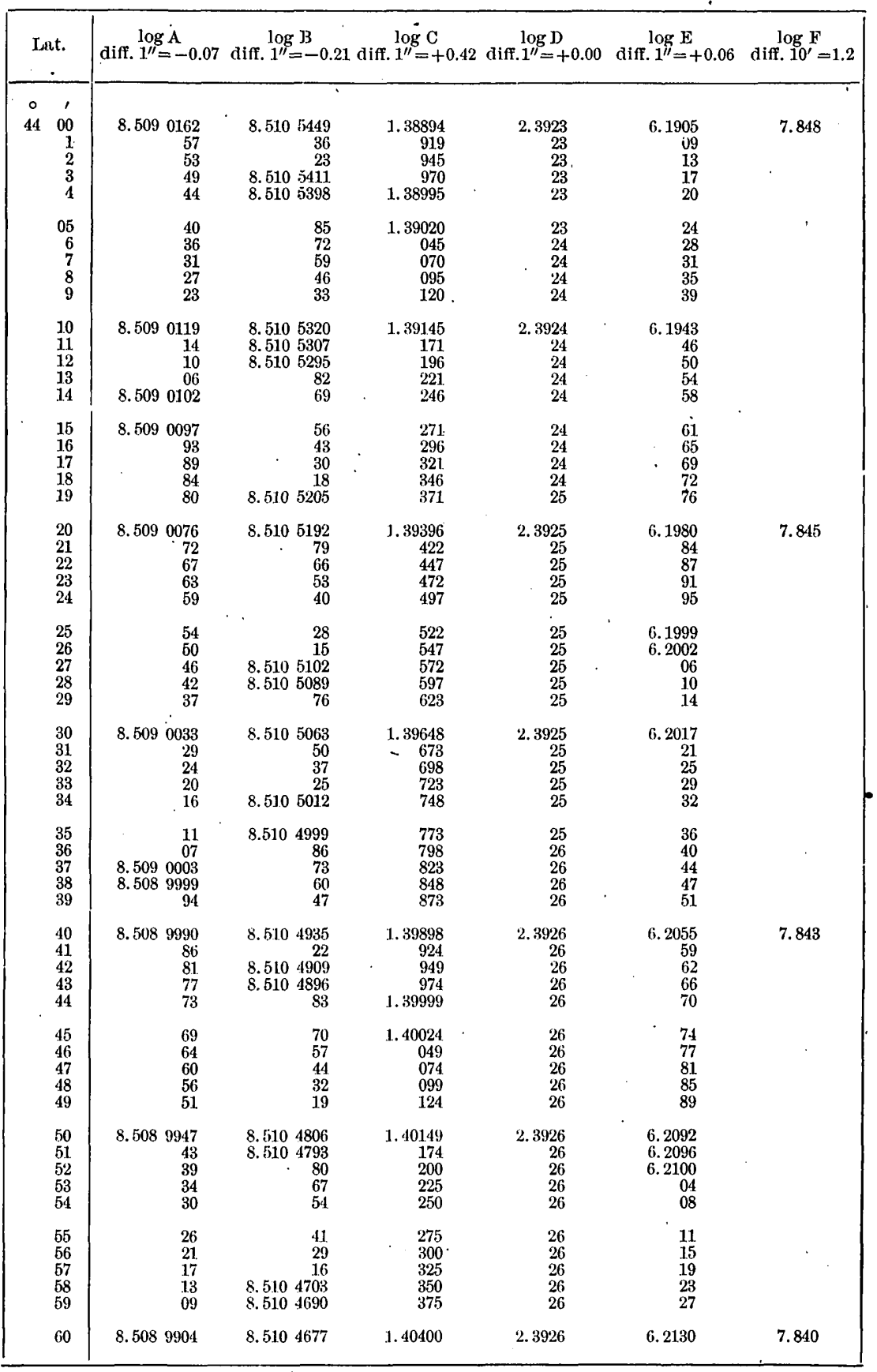

Bull. $214-03-14$ 
TABle 20.-Geodetic position computations-Continued.

LATITUDE $45^{\circ}$.

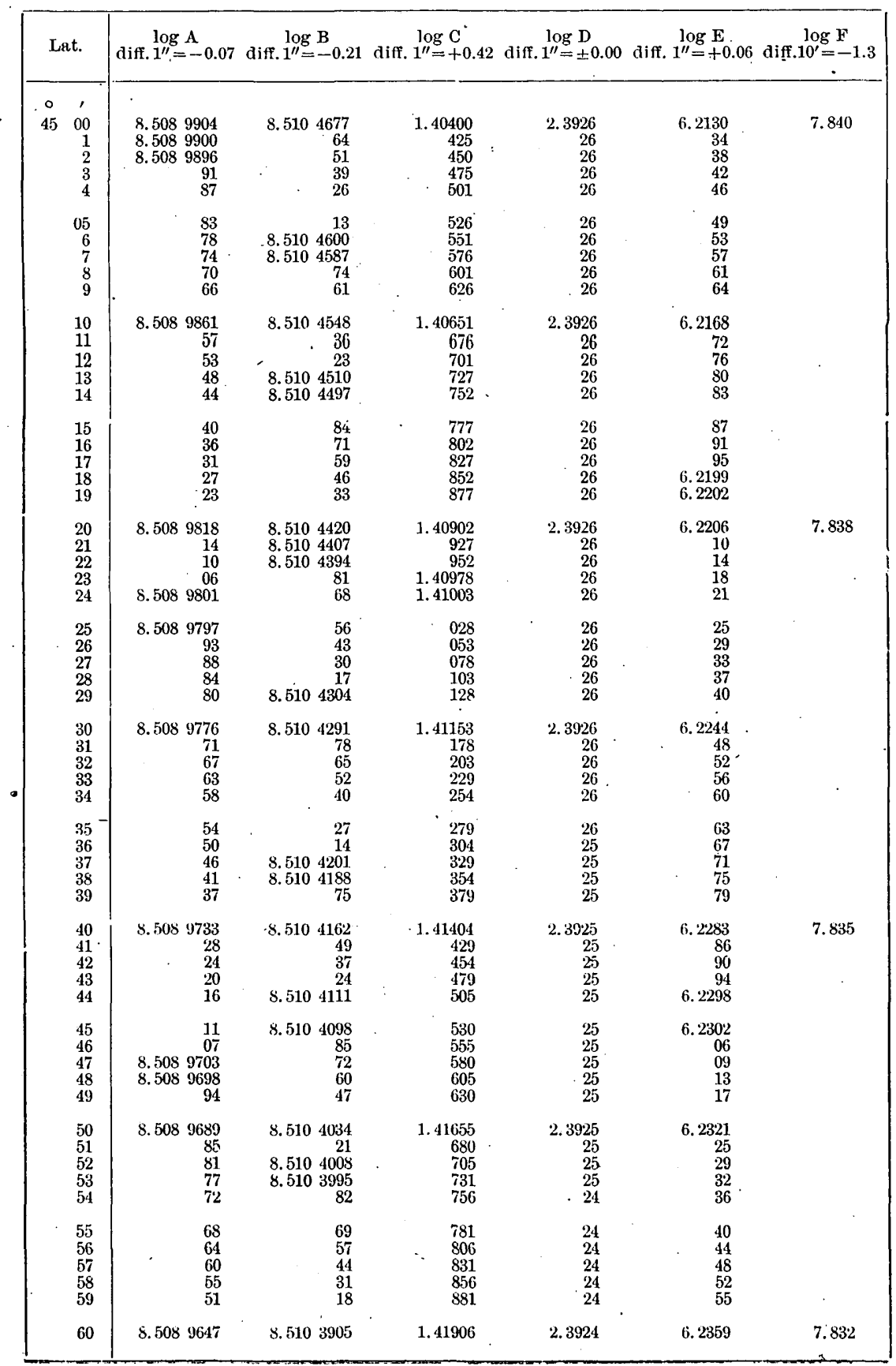


TABLE 20.-Geodetic position computations-Continued.

LATITUDE $46^{\circ}$.

\begin{tabular}{|c|c|c|c|c|c|c|}
\hline Lat. & $\begin{array}{c}\log A \\
\text { diff. } 1^{\prime \prime}=-0.07\end{array}$ & $\begin{array}{c}\log B \\
\text { diff. } 1^{\prime \prime}=-0.21\end{array}$ & $\begin{array}{c}\log C \\
\text { diff. } 1^{\prime \prime}=+0.42\end{array}$ & $\log \mathrm{D}$ & $\begin{array}{c}\log \mathrm{E} \\
\text { diff. } 1^{\prime \prime}=+0.06\end{array}$ & $\begin{array}{c}\log \mathrm{F} \\
\text {;iff. } 10^{\prime}=-1.4\end{array}$ \\
\hline 01 & & & & & & \\
\hline $\begin{array}{rr}46 & 00 \\
& 1 \\
& 2 \\
& 3 \\
& 4\end{array}$ & $\begin{array}{r}8.5 C 89647 \\
43 \\
-\quad 38 \\
34 \\
30\end{array}$ & $\begin{array}{r}8.5103905 \\
8.5103892 \\
79 \\
67 \\
54\end{array}$ & $\begin{array}{r}1.41906 \\
931 \\
957 \\
1.41982 \\
1.42007\end{array}$ & $\begin{array}{r}2.3924 \\
24 \\
24 \\
24 \\
24\end{array}$ & $\begin{array}{r}6.2359 \\
63 \\
67 \\
71 \\
75\end{array}$ & 7.832 \\
\hline $\begin{array}{r}05 \\
6 \\
7 \\
8 \\
9\end{array}$ & $\begin{array}{l}25 \\
21 \\
17 \\
13 \\
08\end{array}$ & $\begin{array}{r}41 \\
28 \\
15 \\
8.5103802 \\
8.5103789\end{array}$ & $\begin{array}{l}032 \\
057 \\
082 \\
107 \\
132\end{array}$ & $\begin{array}{l}24 \\
23 \\
23 \\
23 \\
23\end{array}$ & $\begin{array}{l}79 \\
82 \\
86 \\
90 \\
94\end{array}$ & \\
\hline $\begin{array}{l}10 \\
11 \\
12 \\
13 \\
14\end{array}$ & $\begin{array}{r}8.5089604 \\
8.5089600 \\
8.5089595 \\
91 \\
87\end{array}$ & $\begin{array}{r}8.5103776 \\
64 \\
51 \\
38 \\
25\end{array}$ & $\begin{array}{r}1.42157 \\
183 \\
208 \\
. \quad 233 \\
258\end{array}$ & $\begin{array}{r}2.3923 \\
23 \\
23 \\
23 \\
23\end{array}$ & $\begin{array}{r}6.2398 \\
6.2402 \\
06 \\
09 \\
13\end{array}$ & \\
\hline $\begin{array}{l}15 \\
16 \\
17 \\
18 \\
19\end{array}$ & $\begin{array}{l}83 \\
78 \\
74 \\
70 \\
65\end{array}$ & $\begin{array}{r}8.5103712 \\
\text { S. } 5103699 \\
86 \\
74 \\
61\end{array}$ & $\begin{array}{l}283 \\
308 \\
333 \\
358 \\
384\end{array}$ & $\begin{array}{l}23 \\
23 \\
22 \\
22 \\
22\end{array}$ & $\begin{array}{l}17 \\
21 \\
25 \\
29 \\
33\end{array}$ & • \\
\hline $\begin{array}{l}20 \\
21 \\
22 \\
23 \\
24\end{array}$ & $\begin{array}{r}8.5059561 \\
57 \\
53 \\
48 \\
44\end{array}$ & $\begin{array}{r}8.5103648 \\
35 \\
22 \\
8.5103609 \\
8.5103596\end{array}$ & $\begin{array}{r}\text { 1. } 42409 \\
434 \\
459 \\
484 \\
509\end{array}$ & $\begin{array}{r}2.3922 \\
22 \\
22 \\
22 \\
22\end{array}$ & $\begin{array}{r}6.2436 \\
40 \\
44 \\
48 \\
52\end{array}$ & 7. $830^{\circ}$ \\
\hline $\begin{array}{l}25 \\
26 \\
27 \\
28 \\
29\end{array}$ & $\begin{array}{l}40 \\
35 \\
31 \\
27 \\
23\end{array}$ & $\begin{array}{l}84 \\
71 \\
58 \\
45 \\
32\end{array}$ & $\begin{array}{l}534 \\
559 \\
584 \\
610 \\
635\end{array}$ & $\begin{array}{l}22 \\
21 \\
21 \\
21 \\
21\end{array}$ & $\begin{array}{l}56 \\
60 \\
64 \\
67 \\
71\end{array}$ &. \\
\hline $\begin{array}{l}30 \\
31 \\
32 \\
33 \\
34\end{array}$ & $\begin{array}{r}8.5089518 \\
.14 \\
10 \\
05 \\
8.5089501\end{array}$ & $\begin{array}{r}8.5103519 \\
8.5103506 \\
8.5103494 \\
81 \\
68\end{array}$ & $\begin{array}{r}1.42660 \\
685 \\
710 \\
735 \\
760\end{array}$ & $\begin{array}{r}2.3921 . \\
21 \\
21 \\
21 \\
20\end{array}$ & $\begin{array}{r}6.2475 \\
79 \\
.83 \\
87 \\
91\end{array}$ & . \\
\hline $\begin{array}{l}35 \\
36 \\
37 \\
38 \\
39\end{array}$ & $\begin{array}{r}8.508 \quad 9497 \\
93 \\
88 \\
84 \\
80\end{array}$ & $\begin{array}{r}55 \\
42 \\
29 \\
17 \\
8.510 \quad 3104\end{array}$ & $\begin{array}{l}786 \\
811 \\
836 \\
861 \\
886\end{array}$ & $\begin{array}{l}20 \\
20 \\
20 \\
20 \\
20\end{array}$ & $\begin{array}{r}95 \\
6.2499 \\
6.2502 \\
06 \\
10\end{array}$ & . \\
\hline $\begin{array}{l}40 \\
41 \\
42 \\
43 \\
44\end{array}$ & $\begin{array}{r}\text { s.508 9475 } \\
71 \\
67 \\
63 \\
58\end{array}$ & $\begin{array}{r}8.5103391 \\
78 \\
65 \\
652 \\
39\end{array}$ & $\begin{array}{r}1.42911 \\
936 \\
961 \\
1.42987 \\
1.43012\end{array}$ & ¿. $\begin{array}{r}3 y 20 \\
19 \\
19 \\
19 \\
19\end{array}$ & 6. $\begin{array}{r}2514 \\
18 \\
22 \\
26 \\
30\end{array}$ & 7.827 \\
\hline $\begin{array}{l}45 \\
46 \\
47 \\
48 \\
49\end{array}$ & $\begin{array}{l}54 \\
50 \\
45 \\
41 \\
37\end{array}$ & $\begin{array}{r}27 \\
14 \\
8.5103301 \\
8.5103288 \\
\\
75\end{array}$ & $\begin{array}{l}037 \\
062 \\
087 \\
112 \\
137\end{array}$ & $\begin{array}{l}19 \\
19 \\
19 \\
18 \\
18\end{array}$ & $\begin{array}{l}34 \\
38 \\
41 \\
45 \\
49\end{array}$ & \\
\hline $\begin{array}{l}50 \\
51 \\
52 \\
53 \\
54\end{array}$ & $\begin{array}{r}8.5089433 \\
28 \\
24 \\
20 \\
16\end{array}$ & $\begin{array}{r}8.5103262 \\
49 \\
37 \\
24 \\
8.5103211\end{array}$ & $\begin{array}{r}1.43163 \\
188 \\
213 \\
238 \\
263\end{array}$ & $\begin{array}{r}2.3918 \\
18 \\
18 \\
18 \\
18\end{array}$ & 6. $\begin{array}{r}2553 \\
57 \\
61 \\
65 \\
69\end{array}$ & \\
\hline $\begin{array}{l}55 \\
56 \\
57 \\
58 \\
59\end{array}$ & $\begin{array}{r}11 \\
07 \\
\text { 8. } 5089403 \\
\text { S. } 505 \quad 9398 \\
94\end{array}$ & $\begin{array}{rr}8.510 \quad 31.98 \\
. \quad 85 \\
7.2 \\
60 \\
47 \\
4\end{array}$ & $\begin{array}{l}288 \\
31.4 \\
339 \\
364 \\
389\end{array}$ & $\begin{array}{l}17 \\
17 \\
17 \\
17 \\
17\end{array}$ & $\begin{array}{l}73 \\
77 \\
81 \\
84 \\
88\end{array}$ & . \\
\hline 60 & 8.5089390 & 8.5103134 & 1.43414 & 2.3917 & 6. 2592 & 7.824 \\
\hline
\end{tabular}


TaBle 20.-Geodetic posilion computations-Continued.

LATITUDE $47^{\circ}$.

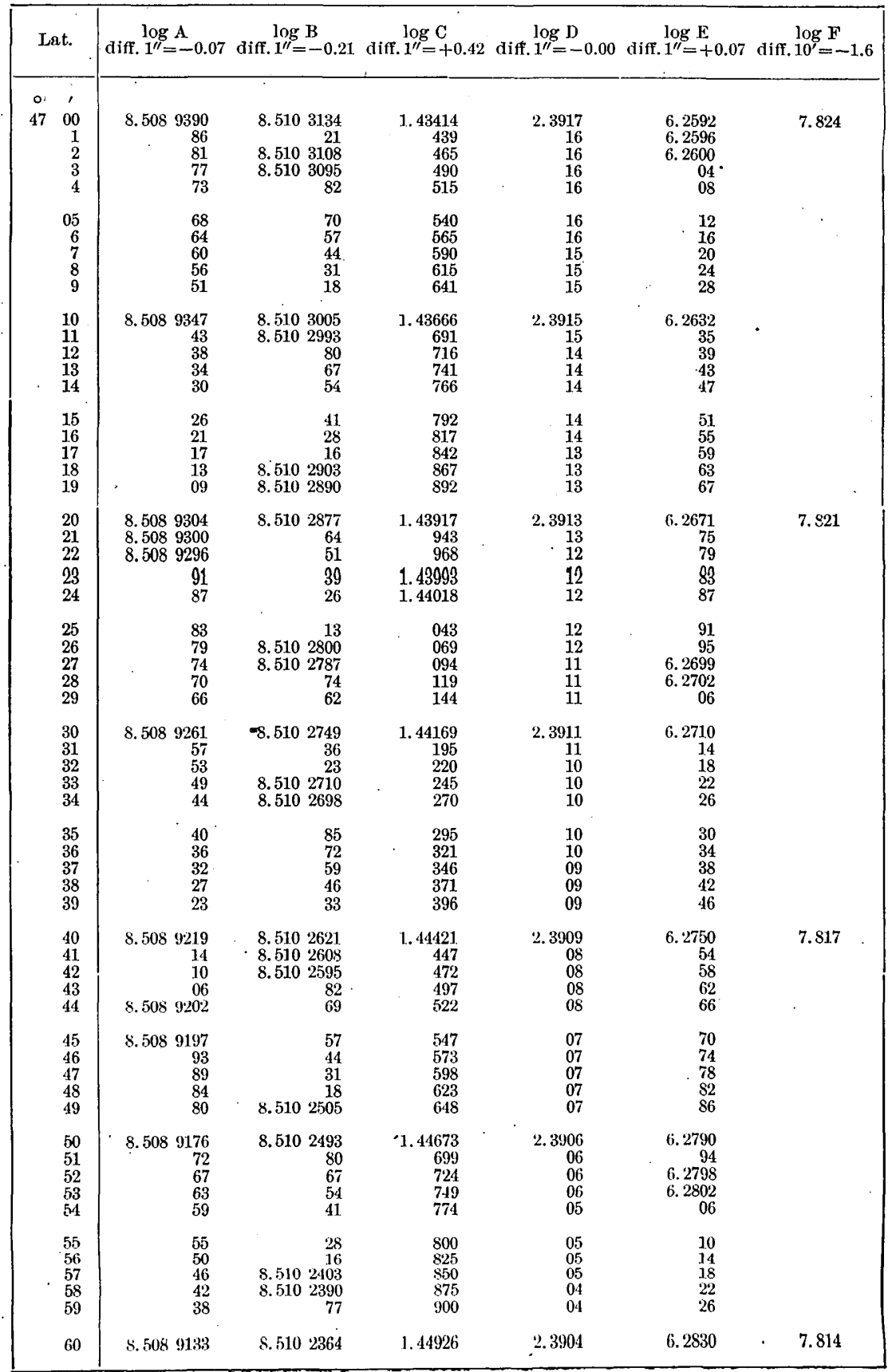


TABL: 20.-Geodetic position computations-Continued.

LATITUDE $48^{\circ}$.

\begin{tabular}{|c|c|c|c|c|c|c|}
\hline Lat. & diff. $1^{\prime \prime}=-0.07$ & $\begin{array}{c}\log B \\
\text { diff. } 1^{\prime \prime}=-0.2\end{array}$ & $\begin{array}{c}\log \mathrm{C} \\
\text { liff. } 1^{\prime \prime}=+0.42\end{array}$ & $\begin{array}{c}\log \mathrm{D} \\
\operatorname{diff} 1^{\prime \prime}=-0.00\end{array}$ & 0 diff. $1^{\prime \prime}=+0.07$ & diff. $\log _{0} F=-1.7$ \\
\hline $\begin{array}{cc}\circ & 1 \\
48 & 00 \\
& 1 \\
& \\
& 2 \\
& 3 \\
& 4\end{array}$ & $\begin{array}{r}8.5089133 \\
29 \\
25 \\
20 \\
16\end{array}$ & $\begin{array}{r}8.510 \quad 2364 \\
52 \\
39 \\
26 \\
13\end{array}$ & $\begin{array}{r}1.44926 \\
951 \\
1.44976 \\
1.45001 \\
027\end{array}$ & $\begin{array}{r}2.3904 \\
04 \\
03 \\
03 \\
03\end{array}$ & $\begin{array}{r}6.2830 \\
34 \\
38 \\
42 \\
. \quad 46\end{array}$ & 7.814 \\
\hline $\begin{array}{r}05 \\
6 \\
7 \\
8 \\
9\end{array}$ & $\begin{array}{r}12 \\
08 \\
\text { 8.508 } 9103 \\
8.5089099 \\
95\end{array}$ & $\begin{array}{r}8.51 .02300 \\
8.51 .02288 \\
75 \\
62 \\
49\end{array}$ & $\begin{array}{l}052 \\
077 \\
102 \\
128 \\
153\end{array}$ & $\begin{array}{l}02 \\
02 \\
02 \\
02 \\
01\end{array}$ & $\begin{array}{l}50 \\
54 \\
58 \\
62 \\
66\end{array}$ & \\
\hline $\begin{array}{l}10 \\
11 \\
12 \\
13 \\
14\end{array}$ & $\begin{array}{r}8.5089091 \\
86 \\
82 \\
78 \\
74\end{array}$ & $\begin{array}{r}8.5102236 \\
24 \\
8.5102211 \\
8.51021 .98 \\
85\end{array}$ & $\begin{array}{r}1.45178 \\
203 \\
229 \\
254 \\
279\end{array}$ & $\begin{array}{r}2.3901 . \\
01 . \\
01 \\
00 \\
00\end{array}$ & $\begin{array}{r}6.2870 \\
74 \\
78 \\
82 \\
86\end{array}$ & 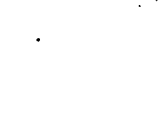 \\
\hline $\begin{array}{l}15 \\
.16 \\
17 \\
18 \\
19\end{array}$ & $\begin{array}{l}69 \\
65 \\
61 \\
57 \\
52\end{array}$ & $\begin{array}{l}72 \\
60 \\
47 \\
34 \\
21\end{array}$ & $\begin{array}{l}304 \\
330 \\
355 \\
380 \\
406\end{array}$ & $\begin{array}{r}2.3900 \\
2.3899 \\
99 \\
99 \\
99\end{array}$ & $\begin{array}{r}90 \\
94 \\
6.2898 \\
6.2902 \\
06\end{array}$ & \\
\hline $\begin{array}{l}20 \\
21 \\
22 \\
23 \\
24\end{array}$ & $\begin{array}{r}8.508 \quad 9048 \\
44 \\
39 \\
35 \\
35 \\
: 31\end{array}$ & $\begin{array}{r}8.5102108 \\
8.5102096 \\
83 \\
70 \\
57\end{array}$ & $\begin{array}{r}1.45431 \\
456 \\
481 \\
507 \\
532\end{array}$ & $\begin{array}{r}2.3898 \\
98 \\
98 \\
97 \\
97\end{array}$ & $\begin{array}{r}6.2910 \\
14 \\
18 \\
22 \\
26\end{array}$ & 7.811 \\
\hline $\begin{array}{l}25 \\
26 \\
27 \\
28 \\
29\end{array}$ & $\begin{array}{l}27 \\
22 \\
18 \\
14 \\
10\end{array}$ & $\begin{array}{r}45 \\
32 \\
19 \\
8.5102006 \\
8.5101993\end{array}$ & $\begin{array}{l}557 \\
582 \\
608 \\
633 \\
658\end{array}$ & $\begin{array}{l}97 \\
97 \\
96 \\
96 \\
96\end{array}$ & $\begin{array}{l}30 \\
34 \\
38 \\
42 \\
46\end{array}$ & \\
\hline $\begin{array}{l}30 \\
31 \\
32 \\
33 \\
34\end{array}$ & $\begin{array}{r}8.5089005 \\
8.5089001 \\
8.508 \quad 8997 \\
93 \\
88\end{array}$ & $\begin{array}{r}8.510 \quad 1981 . \\
68 \\
55 \\
42 \\
30\end{array}$ & $\begin{array}{r}1.45683 \\
709 \\
734 \\
759 \\
785\end{array}$ & $\begin{array}{r}2.3895 \\
95 \\
95 \\
95 \\
94\end{array}$ & $\begin{array}{r}6.2950 \\
54 \\
58 \\
62 \\
66\end{array}$ & \\
\hline $\begin{array}{l}35 \\
36 \\
37 \\
38 \\
39\end{array}$ & $\begin{array}{l}84 \\
80 \\
76 \\
71 \\
67\end{array}$ & $\begin{array}{r}17 \\
8.5101904 \\
8.510 \quad 1891 \\
78 \\
66\end{array}$ & $\begin{array}{l}810 \\
835 \\
861 \\
886 \\
911\end{array}$ & $\begin{array}{l}94 \\
94 \\
93 \\
93 \\
93\end{array}$ & $\begin{array}{r}70 \\
74 \\
78 \\
78 \\
82 \\
86\end{array}$ & \\
\hline $\begin{array}{l}40 \\
41 \\
42 \\
43 \\
44\end{array}$ & $\begin{array}{r}8.508 \quad 8963 \\
59 \\
54 \\
50 \\
46\end{array}$ & $\begin{array}{r}8.5101853 \\
40 \\
27 \\
15 \\
8.510180^{0}\end{array}$ & $\begin{array}{r}1.45937 \\
962 \\
1.45987 \\
1.46012 \\
038\end{array}$ & $\begin{array}{r}2.3892 \\
92 \\
92 \\
91 \\
91\end{array}$ & $\begin{array}{r}6.2990 \\
94 \\
6.2998 \\
6.3002 \\
06\end{array}$ & 7.807 \\
\hline $\begin{array}{l}45 \\
46 \\
47 \\
48 \\
49\end{array}$ & $\begin{array}{l}41 \\
37 \\
33 \\
29 \\
24\end{array}$ & $\begin{array}{r}8.5101789 \\
76 \\
64 \\
51 \\
38\end{array}$ & $\begin{array}{l}063 \\
088 \\
114 \\
139 \\
164\end{array}$ & $\begin{array}{l}91 \\
90 \\
90 \\
90 \\
89\end{array}$ & $\begin{array}{l}10 \\
15 \\
19 \\
23 \\
27\end{array}$ & \\
\hline $\begin{array}{l}50 \\
51 \\
52 \\
53 \\
54\end{array}$ & $\begin{array}{r}8.508 \quad 8920 \\
16 \\
12 \\
08 \\
8.508 \quad 8903\end{array}$ & $\begin{array}{r}8.5101725 \\
13 \\
8.5101700 \\
8.5101687 \\
74\end{array}$ & $\begin{array}{r}1.46190 \\
215 \\
240 \\
266 \\
291\end{array}$ & $\begin{array}{r}2.3889 \\
89 \\
88 \\
88 \\
88\end{array}$ & $\begin{array}{r}6.3031 \\
35 \\
39 \\
43 \\
47\end{array}$ & . \\
\hline $\begin{array}{l}55 \\
56 \\
57 \\
58 \\
59\end{array}$ & $\begin{array}{r}8.508 \quad 8899 \\
95 \\
\quad 90 \\
86 \\
82\end{array}$ & $\begin{array}{r}62 \\
49 \\
36 \\
.23 \\
8.510 \quad 1610\end{array}$ & $\begin{array}{l}316 \\
342 \\
367 \\
392 \\
418\end{array}$ & $\begin{array}{l}87 \\
87 \\
87 \\
86 \\
86\end{array}$ & $\begin{array}{l}51 \\
55 \\
59 \\
63 \\
67\end{array}$ & \\
\hline 60 & 8.5088878 & 8.5101598 & 1.46443 & 2.3886 & 6.3071 & 7.804 \\
\hline
\end{tabular}


TABLE 20.-Geodetic position compulations-Continued.

ILATITUDE $49^{\circ}$.

\begin{tabular}{|c|c|c|c|c|c|c|}
\hline Lat. & $\begin{array}{c}\log A \\
\text { diff. } 1^{\prime \prime}=-0.07\end{array}$ & $\begin{array}{c}\log B \\
\text { diff. } 1^{\prime \prime}=-0.21\end{array}$ & $\begin{array}{c}\log \mathrm{C} \\
\text { diff. } 1^{\prime \prime}=+0.42\end{array}$ & diff. $1^{\prime \prime}=-0.01$ & $\begin{array}{c}\log \mathrm{E} \\
\text { diff. } 1^{\prime \prime}=+0.07\end{array}$ & $\begin{array}{c}\log F \\
\text { diff. } 10^{\prime}=-1.9\end{array}$ \\
\hline $0 .$, & & . & & & & , \\
\hline $\begin{array}{rr}49 & 00 \\
& 1 \\
& 2 \\
& 3 \\
& 4\end{array}$ & $\begin{array}{r}8.508 \quad 8878 \\
73 \\
69 \\
65 \\
61\end{array}$ & $\begin{array}{r}8.510 \quad 1598 \\
.85 \\
72 \\
59 \\
47\end{array}$ & $\begin{array}{r}1.46443 \\
468 \\
494 \\
519 \\
544\end{array}$ & $\begin{array}{r}2.3886 \\
85 \\
85 \\
85 \\
84\end{array}$ & $\begin{array}{r}6.3071 \\
75 \\
79 \\
84 \\
88\end{array}$ & 7.804 \\
\hline $\begin{array}{r}05 \\
6 \\
7 \\
8 \\
9\end{array}$ & $\begin{array}{r}57 \\
52 \\
48 \\
-\quad 44 \\
39\end{array}$ & $\begin{array}{r}34 \\
21 \\
8.5101508 \\
8.510 \quad 1496 \\
83\end{array}$ & $\begin{array}{l}570 \\
595 \\
621 \\
646 \\
671\end{array}$ & $\begin{array}{l}84 \\
84 \\
83 \\
83 \\
83\end{array}$ & $\begin{array}{r}92 \\
6.3096 \\
6.3100 \\
04 \\
08\end{array}$ & \\
\hline $\begin{array}{l}10 \\
11 \\
12 \\
13 \\
14\end{array}$ & $\begin{array}{r}8.508 \quad 8835 \\
\cdot \quad 31 \\
27 \\
\quad 23 \\
18\end{array}$ & $\begin{array}{r}8.510 \quad 1470 \\
58 \\
45 \\
32 \\
19\end{array}$ & $\begin{array}{r}1.46696 \\
722 \\
747 \\
773 \\
798\end{array}$ & $\begin{array}{r}2.3882 \\
82 \\
81 \\
. \quad 81 \\
81\end{array}$ & $\begin{array}{r}6.3112 \\
16 \\
20 \\
24 \\
28\end{array}$ & \\
\hline $\begin{array}{l}15 \\
16 \\
17 \\
18 \\
19\end{array}$ & $\begin{array}{r}14 \\
10 \\
06 \\
8.508 \quad 8801 \\
8.508 \quad 8797\end{array}$ & $\begin{array}{r}8.5101407 \\
8.5101394 \\
81 \\
68 \\
56\end{array}$ & $\begin{array}{r}824 \\
849 \\
.874 \\
899 \\
925\end{array}$ & $\begin{array}{l}80 \\
80 \\
80 \\
79 \\
79\end{array}$ & $\begin{array}{l}32 \\
37 \\
41 \\
45 \\
49\end{array}$ & . \\
\hline $\begin{array}{l}20 \\
21 \\
22 \\
23 \\
24\end{array}$ & $\begin{array}{r}8.508 \quad 8793 \\
89 \\
84 \\
80 \\
76\end{array}$ & $\begin{array}{r}8.5101343 \\
30 \\
17 \\
8.5101305 \\
8.510 \quad 1292\end{array}$ & $\begin{array}{r}1.46950 \\
1.46976 \\
1.47001 \\
026 \\
052\end{array}$ & $\begin{array}{r}2.3878 \\
78 \\
78 \\
77 \\
77\end{array}$ & $\begin{array}{r}6.3153 \\
57 \\
61 \\
65 \\
69\end{array}$ & 7.800 \\
\hline $\begin{array}{l}25 \\
26 \\
27 \\
28 \\
29\end{array}$ & $\begin{array}{l}72 \\
67 \\
63 \\
59 \\
55\end{array}$ & $\begin{array}{l}79 \\
67 \\
54 \\
41 \\
28\end{array}$ & $\begin{array}{l}077 \\
103 \\
128 \\
153 \\
179\end{array}$ & $\begin{array}{l}77 \\
76 \\
76 \\
75 \\
75\end{array}$ & $\begin{array}{l}73 \\
78 \\
82 \\
86 \\
90\end{array}$ & \\
\hline $\begin{array}{l}30 \\
31 \\
32 \\
33 \\
34\end{array}$ & $\begin{array}{r}8.508 \quad 8750 \\
46 \\
42 \\
\quad 38 \\
33\end{array}$ & $\begin{array}{r}8.5101216 \\
8.5101203 \\
8.5101190 \\
78 \\
65\end{array}$ & $\begin{array}{r}1.47204 \\
230 \\
255 \\
281 \\
306\end{array}$ & $\begin{array}{r}2.3875 \\
74 \\
74 \\
73 \\
73\end{array}$ & $\begin{array}{r}6.3194 \\
6.3198 \\
6.3202 \\
06 \\
10\end{array}$ & . \\
\hline $\begin{array}{l}35 \\
36 \\
37 \\
38 \\
39\end{array}$ & $\begin{array}{r}29 \\
25 \\
21 \\
16 \\
12\end{array}$ & $\begin{array}{r}52 \\
39 \\
27 \\
14 \\
8.5101101\end{array}$ & $\begin{array}{l}331 \\
357 \\
382 \\
408 \\
433\end{array}$ & $\begin{array}{l}73 \\
72 \\
72 \\
71 \\
71\end{array}$ & $\begin{array}{l}15 \\
19 \\
23 \\
27 \\
31\end{array}$ & \\
\hline $\begin{array}{l}40 \\
41 \\
42 \\
43 \\
44\end{array}$ & $\begin{array}{r}8.5088708 \\
04 \\
8.5088700 \\
8.5088695 \\
91\end{array}$ & $\begin{array}{r}8.5101088 \\
76 \\
63 \\
50 \\
38\end{array}$ & $\begin{array}{r}1.47459 \\
484 \\
509 \\
535 \\
560\end{array}$ & $\begin{array}{r}2.3871 \\
70 \\
70 \\
69 \\
69\end{array}$ & $\begin{array}{r}6.3235 \\
39 \\
43 \\
47 \\
52\end{array}$ & 7.796 \\
\hline $\begin{array}{l}45 \\
46 \\
47 \\
48 \\
49\end{array}$ & $\begin{array}{l}87 \\
83 \\
78 \\
74 \\
70\end{array}$ & $\begin{array}{r}25 \\
12 \\
8.5101000 \\
8.5100987 \\
74\end{array}$ & $\begin{array}{l}586 \\
611 \\
637 \\
662 \\
688\end{array}$ & $\begin{array}{l}69 \\
68 \\
68 \\
67 \\
67\end{array}$ & $\begin{array}{l}56 \\
60 \\
64 \\
68 \\
72\end{array}$ & \\
\hline $\begin{array}{l}50 \\
51 \\
52 \\
53 \\
54\end{array}$ & $\begin{array}{r}8.508 \quad 8666 \\
61 \\
57 \\
53 \\
49\end{array}$ & $\begin{array}{r}8.510 \quad 0962 \\
49 \\
36 \\
23 \\
23 \\
8.510 \quad 0911\end{array}$ & $\begin{array}{r}1.47713 \\
738 \\
764 \\
789 \\
815\end{array}$ & $\begin{array}{r}2.3866 \\
66 \\
66 \\
65 \\
65\end{array}$ & $\begin{array}{r}6.3276 \\
81 \\
85 \\
89 \\
93\end{array}$ & \\
\hline $\begin{array}{l}55 \\
56 \\
57 \\
58 \\
59\end{array}$ & $\begin{array}{l}45 \\
40 \\
36 \\
32 \\
28\end{array}$ & $\begin{array}{r}8.510 \quad 0898 \\
85 \\
73 \\
60 \\
48\end{array}$ & $\begin{array}{l}840 \\
866 \\
891 \\
917 \\
942\end{array}$ & $\begin{array}{l}\mathbf{6 4} \\
\mathbf{6 4} \\
63 \\
63 \\
63\end{array}$ & $\begin{array}{r}6.3297 \\
6.3301 \\
05 \\
09 \\
14\end{array}$ & \\
\hline 60 & 8.5088623 & 8.5100835 & 1.47968 & $2.3862^{\circ}$ & 6.3318 & 7.792 \\
\hline
\end{tabular}


Table 20.-Geodetic position computations-Continued.

LATITUDE $50^{\circ}$.

\begin{tabular}{|c|c|c|c|c|c|c|}
\hline Lat. & diff, $1^{\prime \prime}=-0.07$ & diff. $1^{\prime \prime}=-0.21$ & $\begin{array}{c}\log \mathrm{C} \\
\text { diff. } 1^{\prime \prime}=+0.43\end{array}$ & $\operatorname{diff} 1^{\prime \prime}=-0.01$ & $\begin{array}{c}\log \mathrm{E} \\
\text { diff. } 1^{\prime \prime}=+0.07\end{array}$ & $\begin{array}{c}\log F \\
\text { diff. } 10^{\prime}=-2.0\end{array}$ \\
\hline \begin{tabular}{rr}
$\circ$ & 1 \\
\hdashline 0 & 00 \\
& 1 \\
& 2 \\
& 3 \\
& 4
\end{tabular} & $\begin{array}{r}8.508 \quad 8623 \\
19 \\
15 \\
11 \\
. \\
06\end{array}$ & $\begin{array}{rr}8.510 & 0835 \\
22 \\
8.510 & 0809 \\
8.510 & 0797 \\
84\end{array}$ & $\begin{array}{r}1.47968 \\
1.47993 \\
1.48019 \\
044 \\
670\end{array}$ & $\begin{array}{r}2.3862 \\
62 \\
61 \\
-\quad 61 \\
60\end{array}$ & $\begin{array}{r}6.3318 \\
22 \\
26 \\
30 \\
34\end{array}$ & 7. 792 \\
\hline $\begin{array}{r}05 \\
6 \\
7 \\
8 \\
9\end{array}$ & $\begin{array}{r}8.5088602 \\
8.5088598 \\
94 \\
90 \\
85\end{array}$ & $\begin{array}{l}71 \\
59 \\
46 \\
33 \\
21\end{array}$ & $\begin{array}{l}095 \\
121 \\
146 \\
172 \\
197\end{array}$ & $\begin{array}{l}60 \\
60 \\
59 \\
59 \\
58\end{array}$ & $\begin{array}{l}39 \\
43 \\
47 \\
51 \\
55\end{array}$ & . \\
\hline $\begin{array}{l}10 \\
11 \\
12 \\
13 \\
14\end{array}$ & $\begin{array}{r}8.5058581 \\
77 \\
73 \\
68 \\
64\end{array}$ & $\begin{array}{r}8.5100708 \\
8.5100695 \\
83 \\
70 \\
57\end{array}$ & $\begin{array}{r}1.48223 \\
248 \\
274 \\
299 \\
325\end{array}$ & $\begin{array}{r}2.3858 \\
57 \\
57 \\
56 \\
56\end{array}$ & $\begin{array}{r}6.3359 \\
63 \\
68 \\
72 \\
76\end{array}$ & - \\
\hline $\begin{array}{l}15 \\
16 \\
17 \\
18 \\
19\end{array}$ & $\begin{array}{l}60 \\
56 \\
52 \\
47 \\
43\end{array}$ & $\begin{array}{r}45 \\
32 \\
19 \\
8.5100607 \\
8.5100594\end{array}$ & $\begin{array}{l}350 \\
376 \\
401 \\
427 \\
452\end{array}$ & $\begin{array}{l}55 \\
55 \\
55 \\
54 \\
54\end{array}$ & $\begin{array}{r}80 \\
84 \\
88 \\
93 \\
6.3397\end{array}$ & \\
\hline $\begin{array}{l}20 \\
21 \\
22 \\
23 \\
24\end{array}$ & $\begin{array}{r}8.508 \quad 8539 \\
35 \\
30 \\
26 \\
22\end{array}$ & $\begin{array}{r}8.5100581 \\
69 \\
66 \\
43 \\
31\end{array}$ & $\begin{array}{r}1.48478 \\
504 \\
529 \\
555 \\
580\end{array}$ & $\begin{array}{r}2.3853 \\
53 \\
52 \\
52 \\
51\end{array}$ & $\begin{array}{r}6.3401 \\
05 \\
09 \\
14 \\
18\end{array}$ & 7.788 \\
\hline $\begin{array}{l}25 \\
26 \\
27 \\
28 \\
29\end{array}$ & $\begin{array}{r}18 \\
14 \\
09 \\
05 \\
8.508 \quad 3501\end{array}$ & $\begin{array}{r}18 \\
8.510 \\
8005 \\
8.5100493 \\
80 \\
67\end{array}$ & $\begin{array}{r}606 \\
631 \\
657 \\
\times \quad 682 \\
708\end{array}$ & $\begin{array}{l}51 \\
50 \\
50 \\
49 \\
49\end{array}$ & $\begin{array}{r}22 \\
26 \\
30 \\
34 \\
39\end{array}$ & $\cdot$ \\
\hline $\begin{array}{l}30 \\
31 \\
32 \\
33 \\
34\end{array}$ & $\begin{array}{r}8.508 \quad 8497 \\
93 \\
88 \\
84 \\
80\end{array}$ & $\begin{array}{r}8.5100455 \\
42 \\
29 \\
29 \\
17 \\
8.5100404\end{array}$ & $\begin{array}{r}1.48734 \\
759 \\
785 \\
810 \\
836\end{array}$ & $\begin{array}{r}2.3848 \\
48 \\
47 \\
47 \\
46\end{array}$ & 6. $\begin{array}{r}3443 \\
47 \\
51 \\
55 \\
60\end{array}$ & \\
\hline $\begin{array}{l}35 \\
36 \\
37 \\
38 \\
39\end{array}$ & $\begin{array}{l}76 \\
71 \\
67 \\
63 \\
59\end{array}$ & $\begin{array}{r}8.510 \quad 0392 \\
79 \\
66 \\
\\
54 \\
\quad 41\end{array}$ & $\begin{array}{l}861 \\
887 \\
913 \\
938 \\
964\end{array}$ & $\begin{array}{l}46 \\
45 \\
45 \\
44 \\
44\end{array}$ & $\begin{array}{l}64 \\
68 \\
72 \\
76 \\
81\end{array}$ & \\
\hline $\begin{array}{l}40 \\
41 \\
42 \\
43 \\
44\end{array}$ & $\begin{array}{r}8.508 \quad 8455 \\
50 \\
46 \\
42 \\
38\end{array}$ & $\begin{array}{rr}8510 & 0328 \\
& 16 \\
8510 & 0303 \\
8.510 & 0291 \\
& 78\end{array}$ & $\begin{array}{r}\text { 1. } 48989 \\
\text { 1. } 49015 \\
041 \\
066 \\
092\end{array}$ & $\begin{array}{r}2.3843 \\
43 \\
42 \\
42 \\
41\end{array}$ & $\begin{array}{r}\text { 6. } 3485 \\
89 \\
93 \\
6.3497 \\
6.3502\end{array}$ & 7.784 \\
\hline $\begin{array}{l}45 \\
46 \\
47 \\
48 \\
49\end{array}$ & $\begin{array}{l}34 \\
29 \\
25 \\
21 \\
17\end{array}$ & $\begin{array}{l}65 \\
53 \\
40 \\
27 \\
15\end{array}$ & $\begin{array}{l}117 \\
143 \\
169 \\
194 \\
220\end{array}$ & $\begin{array}{l}41 \\
40 \\
40 \\
39 \\
39\end{array}$ & $\begin{array}{l}06 \\
10 \\
14 \\
18 \\
23\end{array}$ & \\
\hline $\begin{array}{l}50 \\
51 \\
52 \\
53 \\
54\end{array}$ & $\begin{array}{r}8.5088413 \\
08 \\
04 \\
8.5088400 \\
8.5088396\end{array}$ & $\begin{array}{rr}8.510 & 0202 \\
8.510 & 0190 \\
& 77 \\
& 64 \\
& 52\end{array}$ & i. $\begin{array}{r}49246 \\
271 \\
297 \\
322 \\
348\end{array}$ & 2. $\begin{array}{r}3838 \\
38 \\
37 \\
37 \\
36\end{array}$ & $\begin{array}{r}6.3527 \\
31 \\
35 \\
40 \\
44\end{array}$ & $n$ \\
\hline $\begin{array}{l}55 \\
56 \\
57 \\
58 \\
59\end{array}$ & $\begin{array}{l}92 \\
87 \\
83 \\
79 \\
75\end{array}$ & $\begin{array}{r}39 \\
27 \\
14 \\
8.5100101 \\
8.51000 \$ 9\end{array}$ & $\begin{array}{l}374 \\
399 \\
425 \\
451 \\
476\end{array}$ & $\begin{array}{l}36 \\
35 \\
35 \\
34 \\
34\end{array}$ & $\begin{array}{l}48 \\
52 \\
56 \\
61 \\
65\end{array}$ & \\
\hline 60 & 8.5088871 & $8.51000 \% 6$ & 1.49502 & 2.3833 & 6.3569 & 7.780 \\
\hline
\end{tabular}


TabLe 20.-Geodetic position computations-Continued.

IAATITUDE $51^{\circ}$.

\begin{tabular}{|c|c|c|c|c|c|c|}
\hline Lat. & $\begin{array}{c}\log A \\
\operatorname{diff} .1^{\prime \prime}=-0.07\end{array}$ & $\begin{array}{c}\log B \\
\text { diff, } 1^{\prime \prime}=-0.21 \text {. }\end{array}$ & $\begin{array}{c}\log \mathrm{C} \\
\text { diff. } 1^{\prime \prime}=+0.43\end{array}$ & $\begin{array}{c}\log \mathrm{D} \\
\operatorname{diff} .1^{\prime \prime}=-0.0 t\end{array}$ & diff, $1^{\prime \prime}=+0.07$ & $\begin{array}{c}\log F \\
\text { diff. } 10^{\prime}=-2,2\end{array}$ \\
\hline , & & . & & & & \\
\hline $\begin{array}{r}51 \quad 00 \\
1 \\
2 \\
3 \\
4\end{array}$ & $\begin{array}{r}8.5088371 \\
66 \\
62 \\
58 \\
54\end{array}$ & $\begin{array}{r}8.510 \quad 0076 \\
64 \\
51 \\
38 \\
26\end{array}$ & $\begin{array}{r}49502 \\
528 \\
553 \\
579 \\
605\end{array}$ & $\begin{array}{r}2.3833 \\
33 \\
32 \\
32 \\
31\end{array}$ & $\begin{array}{r}6.3569 \\
73 \\
78 \\
82 \\
86\end{array}$ & 7.780 \\
\hline $\begin{array}{r}05 \\
6 \\
7 \\
8 \\
9\end{array}$ & $\begin{array}{l}50 \\
45 \\
41 \\
37 \\
33\end{array}$ & $\begin{array}{rr} & 13 \\
8.510 & 0001 \\
8.509 & 9988 \\
& 75 \\
& 63\end{array}$ & $\begin{array}{l}630 \\
656 \\
682 \\
707 \\
733\end{array}$ & $\begin{array}{l}31 \\
30 \\
29 \\
29 \\
28\end{array}$ & $\begin{array}{r}90 \\
95 \\
6.3599 \\
6.3603 \\
07\end{array}$ & \\
\hline $\begin{array}{l}10 \\
11 \\
12 \\
13 \\
.14\end{array}$ & $\begin{array}{r}8.5088329 \\
24 \\
20 \\
16 \\
12\end{array}$ & $\begin{array}{r}8.5099950 \\
38 \\
25 \\
13 \\
8.5099900\end{array}$ & $\begin{array}{r}1.47759 \\
785 \\
810 \\
836 \\
862\end{array}$ & $\begin{array}{r}2.3828 \\
27 \\
27 \\
26 \\
26\end{array}$ & $\begin{array}{r}6.3612 \\
16 \\
20 \\
24 \\
28\end{array}$ & \\
\hline $\begin{array}{l}15 \\
16 \\
17 \\
18 \\
19\end{array}$ & $\begin{array}{rr} & 68 \\
8.508 & 8303 \\
8.508 & 8299 \\
95 \\
91\end{array}$ & $\begin{array}{r}8.5099887 \\
75 \\
62 \\
50 \\
37\end{array}$ & $\begin{array}{r}887 \\
913 \\
939 \\
965 \\
1.49990\end{array}$ & $\begin{array}{l}25 \\
25 \\
24 \\
23 \\
23\end{array}$ & $\begin{array}{l}33 \\
37 \\
41 \\
45 \\
50\end{array}$ & . \\
\hline $\begin{array}{l}20 \\
21 \\
22 \\
23 \\
24\end{array}$ & $\begin{array}{r}8.5088287 \\
82 \\
78 \\
74 \\
70\end{array}$ & 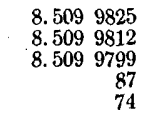 & $\begin{array}{r}1.50016 \\
042 \\
067 \\
093 \\
119\end{array}$ & $\begin{array}{r}2.3822 \\
22 \\
21 \\
21 \\
20\end{array}$ & $\begin{array}{r}6.3654 \\
58 \\
63 \\
67 \\
71\end{array}$ & 7.776 \\
\hline $\begin{array}{l}25 \\
26 \\
27 \\
28 \\
29\end{array}$ & $\begin{array}{l}66 \\
62 \\
57 \\
53 \\
49\end{array}$ & $\begin{array}{r}62 \\
49 \\
37 \\
24 \\
8.509 \quad 9711\end{array}$ & $\begin{array}{l}145 \\
170 \\
196 \\
222 \\
248\end{array}$ & $\begin{array}{l}20 \\
19 \\
18 \\
18 \\
17\end{array}$ & $\begin{array}{l}75 \\
80 \\
84 \\
88 \\
92\end{array}$ & . \\
\hline $\begin{array}{l}30 \\
31 \\
32 \\
33 \\
34\end{array}$ & $\begin{array}{r}8.508 \quad 8245 \\
41 \\
36 \\
32 \\
28\end{array}$ & $\begin{array}{r}8.5099699 \\
86 \\
74 \\
61 \\
49\end{array}$ & $\begin{array}{r}1.50273 \\
299 \\
325 \\
351 \\
376\end{array}$ & $\begin{array}{r}2.3817 \\
16 \\
16 \\
15 \\
14\end{array}$ & $\begin{array}{r}6.3697 \\
6.3701 \\
05 \\
10 \\
14\end{array}$ & · \\
\hline $\begin{array}{l}35 \\
36 \\
\mathbf{3 7} \\
38 \\
\mathbf{3 9}\end{array}$ & $\begin{array}{l}24 \\
20 \\
16 \\
11 \\
07\end{array}$ & $\begin{array}{rr} & 36 \\
& 24 \\
8.509 & 9611 \\
8.509 & 9599 \\
86\end{array}$ & $\begin{array}{l}402 \\
428 \\
454 \\
480 \\
505\end{array}$ & $\begin{array}{l}14 \\
13 \\
13 \\
12 \\
11\end{array}$ & $\begin{array}{l}18 \\
22 \\
27 \\
31 \\
35\end{array}$ & \\
\hline $\begin{array}{l}40 \\
41 \\
42 \\
43 \\
44\end{array}$ & $\begin{array}{rr}8.508 & 8203 \\
8.508 & 8199 \\
. & 95 \\
. & 90 \\
& 86\end{array}$ & $\begin{array}{r}8.5099574 \\
61 \\
48 \\
36 \\
23\end{array}$ & $\begin{array}{r}\text { 1. } 50531 \\
557 \\
583 \\
609 \\
634\end{array}$ & $\begin{array}{r}2.3811 \\
10 \\
10 \\
09 \\
08\end{array}$ & $\begin{array}{r}6.3740 \\
44 \\
48 \\
52 \\
57\end{array}$ & 7. 772 \\
\hline $\begin{array}{l}45 \\
46 \\
47 \\
48 \\
49\end{array}$ & $\begin{array}{r}82 \\
78 \\
74 \\
70 \\
65\end{array}$ & $\begin{array}{r}8.5099511 \\
8.5099498 \\
86 \\
73 \\
61\end{array}$ & $\begin{array}{r}660 \\
686 \\
712 \\
738 \\
.764\end{array}$ & $\begin{array}{l}08 \\
07 \\
07 \\
06 \\
05\end{array}$ & $\begin{array}{l}61 \\
65 \\
70 \\
74 \\
78\end{array}$ & . \\
\hline $\begin{array}{l}50 \\
51 \\
52 \\
53 \\
54\end{array}$ & $\begin{array}{r}8.508 \quad 8161 \\
57 \\
53 \\
49 \\
45\end{array}$ & $\begin{array}{r}8.5099448 \\
36 \\
23 \\
8.5099411 \\
8.5099398\end{array}$ & $\begin{array}{r}1.50789 \\
815 \\
841 \\
867 \\
893\end{array}$ & $\begin{array}{r}2.3805 \\
04 \\
04 \\
03 \\
02\end{array}$ & $\begin{array}{r}6.3782 \\
87 \\
91 \\
6.3795 \\
6.3800\end{array}$ & \\
\hline $\begin{array}{l}55 \\
56 \\
57 \\
58 \\
59\end{array}$ & $\begin{array}{l}40 \\
36 \\
32 \\
28 \\
24\end{array}$ & $\begin{array}{l}86 \\
73 \\
61 \\
48 \\
36\end{array}$ & $\begin{array}{r}919 \\
944 \\
970 \\
1.50996 \\
1.51022\end{array}$ & $\begin{array}{r}02 \\
01 \\
01 \\
2.3800 \\
2.3799\end{array}$ & $\begin{array}{l}04 \\
08 \\
13 \\
17 \\
21\end{array}$ & . \\
\hline .60 & 8.5088120 & 8.5099323 & 1. 51048 & 2.3799 & 6.3826 & 7.767 \\
\hline
\end{tabular}


Tabra 20.-Geodelic position computations-Continued.

LATI'TUDE $52^{\circ}$.

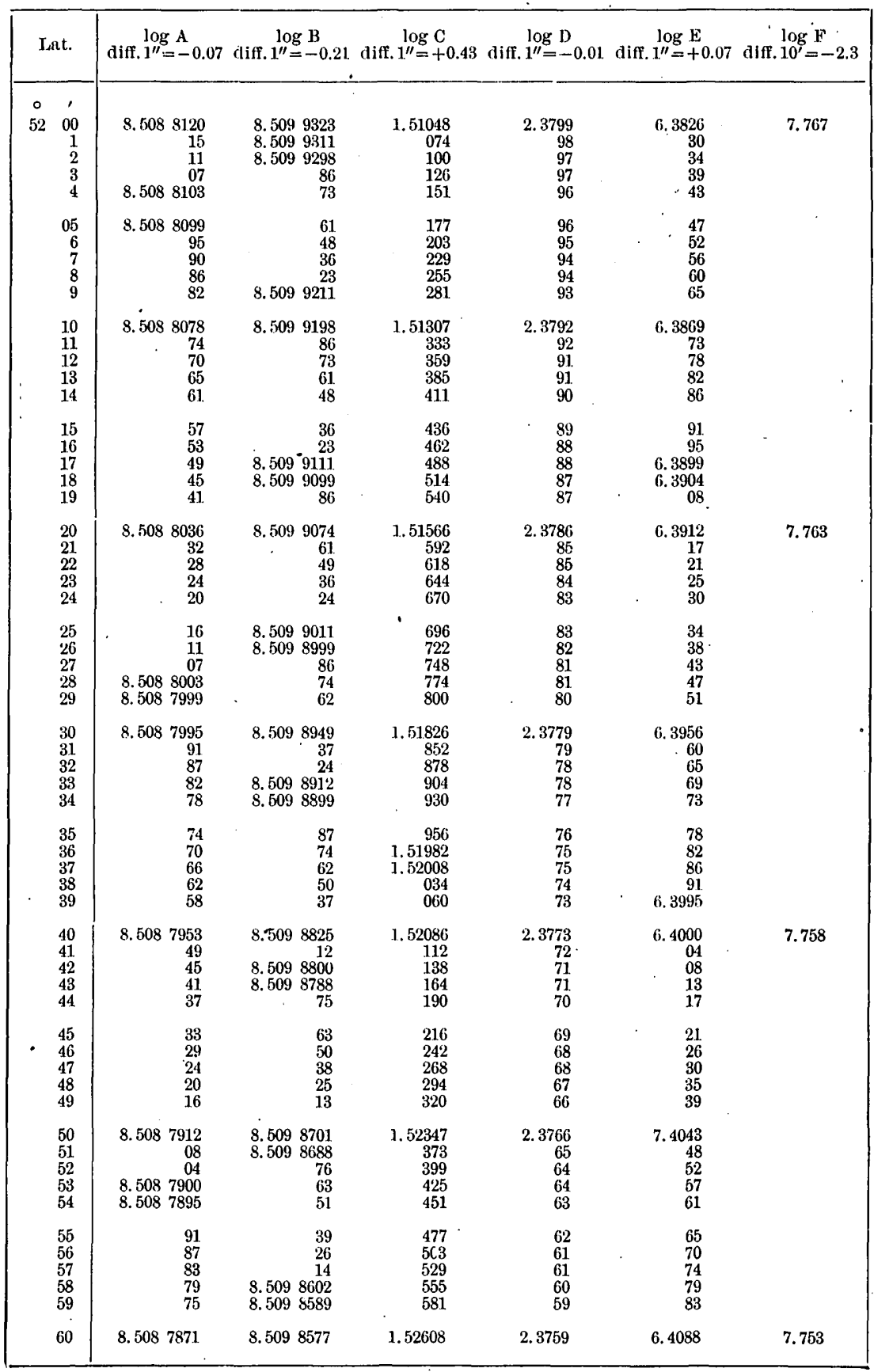


TABre 20.-Geodetic position computations-Continued.

LATITUDE $53^{\circ}$.

\begin{tabular}{|c|c|c|c|c|c|c|}
\hline Lat. & $\begin{array}{c}\log A \\
\text { diff. } 1^{\prime \prime}=-0.07\end{array}$ & $\begin{array}{c}\log B \\
\text { diff. } 1^{\prime \prime}=-0.21\end{array}$ & $\begin{array}{c}\log \mathrm{C} \\
\text { diff, } 1^{\prime \prime}=+0.44\end{array}$ & $\begin{array}{c}\log \mathrm{D} \\
\text { diff. } 1^{\prime \prime}=-0.01\end{array}$ & $\begin{array}{c}\log E \\
\text { diff. } 1^{\prime \prime}=+0.07\end{array}$ & $\begin{array}{c}\log F \\
\text { diff. } 10^{\prime}=-2.5\end{array}$ \\
\hline , & & & & & & \\
\hline $\begin{array}{rr}53 & 00 \\
& 1 \\
. \quad 2 \\
& 3 \\
& 4\end{array}$ & $\begin{array}{r}8.5087871 \\
\cdot \quad 67 \\
\quad 62 \\
\quad 58 \\
54\end{array}$ & $\begin{array}{r}8.509 \quad 8577 \\
64 \\
\quad 52 \\
40 \\
27\end{array}$ & $\begin{array}{r}1.52608 \\
634 \\
660 \\
686 \\
712\end{array}$ & $\begin{array}{r}2.3759 \\
58 \\
57 \\
56 \\
56\end{array}$ & $\begin{array}{r}6.4088 \\
92 \\
6.4096 \\
6.4101 \\
05\end{array}$ & 7.753 \\
\hline $\begin{array}{r}05 \\
6 \\
7 \\
8 \\
9\end{array}$ & $\begin{array}{l}50 \\
46 \\
42 \\
38 \\
34\end{array}$ & $\begin{array}{r}15 \\
8.5098502 \\
8.5098490 \\
78 \\
65\end{array}$ & $\begin{array}{r}738 \\
764 \\
790 \\
817 \\
843\end{array}$ & $\begin{array}{l}55 \\
54 \\
53 \\
53 \\
52\end{array}$ & $\begin{array}{l}10 \\
14 \\
18 \\
23 \\
27\end{array}$ & \\
\hline $\begin{array}{l}10 \\
11 \\
12 \\
13 \\
14\end{array}$ & $\begin{array}{r}8.508 \quad 7829 \\
25 \\
21 \\
17 \\
13\end{array}$ & $\begin{array}{r}8.5098453 \\
41 \\
28 \\
16 \\
8.5098404\end{array}$ & $\begin{array}{r}1.52869 \\
895 \\
921 \\
947 \\
1.52974\end{array}$ & $\begin{array}{r}2.3751 \\
51 \\
50 \\
49 \\
48\end{array}$ & $\begin{array}{r}6.4132 \\
36 \\
41 \\
45 \\
49\end{array}$ & \\
\hline $\begin{array}{l}15 \\
16 \\
17 \\
18 \\
19\end{array}$ & $\begin{array}{r}09 \\
. \quad 05 \\
8.5087801 \\
8.508 \quad 7797 \\
92\end{array}$ & $\begin{array}{r}8.5098391 \\
79 \\
67 \\
54 \\
42\end{array}$ & $\begin{array}{r}1.53000 \\
026 \\
052 \\
078 \\
105\end{array}$ & $\begin{array}{l}48 \\
47 \\
46 \\
45 \\
45\end{array}$ & $\begin{array}{l}54 \\
58 \\
63 \\
67 \\
72\end{array}$ & \\
\hline $\begin{array}{l}20 \\
21 \\
22 \\
23 \\
24\end{array}$ & $\begin{array}{r}8.5087788 \\
84 \\
80 \\
76 \\
72\end{array}$ & $\begin{array}{r}8.5098329 \\
17 \\
8.5098305 \\
8.5098292 \\
80\end{array}$ & $\begin{array}{r}1.53131 \\
157 \\
183 \\
209 \\
236\end{array}$ & $\begin{array}{r}2.3744 \\
43 \\
42 \\
42 \\
41\end{array}$ & $\begin{array}{r}6.4176 \\
80 \\
85 \\
89 \\
94\end{array}$ & 7.748 \\
\hline $\begin{array}{l}25 \\
26 \\
27 \\
28 \\
29\end{array}$ & $\begin{array}{l}68 \\
64 \\
60 \\
55 \\
51\end{array}$ & $\begin{array}{l}68 \\
55 \\
43 \\
31 \\
18\end{array}$ & $\begin{array}{l}262 \\
288 \\
314 \\
341 \\
367\end{array}$ & $\begin{array}{r}40 \\
-39 \\
39 \\
38 \\
37\end{array}$ & $\begin{array}{r}6.4198 \\
6.4203 \\
07 \\
12 \\
16\end{array}$ & \\
\hline $\begin{array}{l}30 \\
31 \\
32 \\
33 \\
34\end{array}$ & $\begin{array}{r}8.508 \quad 7747 \\
43 \\
39 \\
35 \\
31\end{array}$ & $\begin{array}{r}8.5098206 \\
8.5098194 \\
82 \\
69 \\
57\end{array}$ & $\begin{array}{r}1.53393 \\
419 \\
446 \\
472 \\
498\end{array}$ & $\begin{array}{r}2.3736 \\
36 \\
35 \\
34 \\
33\end{array}$ & $\begin{array}{r}6.4221 \\
25 \\
29 \\
34 \\
38\end{array}$ & \\
\hline $\begin{array}{l}35 \\
36 \\
37 \\
38 \\
39\end{array}$ & $\begin{array}{l}27 \\
23 \\
18 \\
14 \\
10\end{array}$ & $\begin{array}{r}45 \\
32 \\
20 \\
8.5098108 \\
8.5098095\end{array}$ & $\begin{array}{l}524 \\
551 \\
577 \\
603 \\
630\end{array}$ & $\begin{array}{l}33 \\
32 \\
31 \\
30 \\
29\end{array}$ & $\begin{array}{l}43 \\
47 \\
52 \\
56 \\
61\end{array}$ & . \\
\hline $\begin{array}{l}40 \\
41 \\
42 \\
43 \\
44\end{array}$ & $\begin{array}{r}8.508 .7706 \\
8.508 \\
8.508 \\
7698 \\
94 \\
90\end{array}$ & $\begin{array}{r}8.5098083 \\
71 \\
58 \\
46 \\
34\end{array}$ & $\begin{array}{r}1.53656 \\
682 \\
709 \\
735 \\
761\end{array}$ & $\begin{array}{r}2.3729 \\
28 \\
27 \\
26 \\
26\end{array}$ & $\begin{array}{r}6.4265 \\
70 \\
74 \\
79 \\
83\end{array}$ & 7.743 \\
\hline $\begin{array}{l}45 \\
46 \\
47 \\
48 \\
49\end{array}$ & $\begin{array}{l}86 \\
82 \\
77 \\
73 \\
69\end{array}$ & $\begin{array}{r}22 \\
8.5098009 \\
8.5097997 \\
85 \\
72\end{array}$ & $\begin{array}{l}788 \\
814 \\
840 \\
867 \\
893\end{array}$ & $\begin{array}{l}25 \\
24 \\
23 \\
22 \\
22\end{array}$ & $\begin{array}{r}88 \\
92 \\
6.4297 \\
6.4301 \\
06\end{array}$ & \\
\hline $\begin{array}{l}50 \\
51 \\
52 \\
53 \\
54\end{array}$ & $\begin{array}{r}8.508 \quad 7665 \\
61 \\
57 \\
53 \\
49\end{array}$ & $\begin{array}{r}8.5097960 \\
48 \\
36 \\
23 \\
8.5097911\end{array}$ & $\begin{array}{r}1.53919 \\
946 \\
972 \\
1.53998 \\
1.540^{2} 25\end{array}$ & $\begin{array}{r}2.3721 \\
20 \\
19 \\
18 \\
18\end{array}$ & $\begin{array}{r}6.4310 \\
15 \\
19 \\
24 \\
28\end{array}$ & \\
\hline $\begin{array}{l}55 \\
56 \\
57 \\
58 \\
59\end{array}$ & $\begin{array}{l}45 \\
41 \\
37 \\
32 \\
28\end{array}$ & $\begin{array}{r}8.5097899 \\
87 \\
74 \\
62 \\
50\end{array}$ & $\begin{array}{l}051 \\
077 \\
104 \\
130 \\
157\end{array}$ & $\begin{array}{l}17 \\
16 \\
15 \\
14 \\
14\end{array}$ & $\begin{array}{l}33 \\
37 \\
42 \\
46 \\
51\end{array}$ & \\
\hline 60 & 8.5087624 & 8. 5097838 & 1.54183 & 2.3713 & 6.4355 & 7.738 \\
\hline
\end{tabular}


TABLE 20.-Cieodetic position computations-Continued.

IATITUDE $54^{\circ}$.

\begin{tabular}{|c|c|c|c|c|c|c|}
\hline Lat. & diff. $1^{\prime \prime}=-0.0$ & liff. $1^{\prime \prime}=-0.20$ & iff. $\begin{array}{l}\log \mathrm{C} \\
.^{\prime \prime}=+0.4\end{array}$ & $\begin{array}{c}\log D \\
\text { diff. } 1^{\prime \prime}=-0.01\end{array}$ & diff. $1^{\prime \prime}=+0.0 \mathrm{~s}$ & diff. $10^{\prime}=-2.6$ \\
\hline $\begin{array}{rr}\circ & , \\
64 & 00 \\
\cdot \quad 1 \\
\cdot \\
\\
\\
& 3 \\
& 4\end{array}$ & $\begin{array}{r}8.5087624 \\
20 \\
16 \\
12 \\
08\end{array}$ & $\begin{array}{r}8.5097838^{\circ} \\
25 \\
13 \\
8.5097801 \\
8.5097789\end{array}$ & $\begin{array}{r}1.54183 \\
209 \\
236 \\
262 \\
288\end{array}$ & $\begin{array}{r}2.3713 \\
12 \\
11 \\
10 \\
09\end{array}$ & $\begin{array}{r}6.4355 \\
60 \\
64 \\
69 \\
.73\end{array}$ & 7. 738 \\
\hline $\begin{array}{r}05 \\
6 \\
7 \\
8 \\
9\end{array}$ & $\begin{array}{r}04 \\
8.5087600 \\
8.508 \quad 7596 \\
92 \\
88\end{array}$ & $\begin{array}{l}76 \\
64 \\
52 \\
40 \\
27\end{array}$ & $\begin{array}{r}315 \\
341 \\
368 \\
394 \\
421\end{array}$ & $\begin{array}{l}09 \\
08 \\
07 \\
06 \\
05\end{array}$ & $\begin{array}{r}78 \\
82 \\
87 \\
91 \\
6.4396\end{array}$ & \\
\hline $\begin{array}{l}10 \\
11 \\
12 \\
13 \\
14\end{array}$ & $\begin{array}{r}8.508 \quad 7584 \\
79 \\
75 \\
71 \\
67\end{array}$ & $\begin{array}{rr}8.509 & 7715 \\
8.509 & 7703 \\
8.509 & 7691 . \\
& 78 \\
& 66\end{array}$ & $\begin{array}{r}1.54447 \\
474 \\
500 \\
527 \\
553\end{array}$ & $\begin{array}{r}2.3705 \\
04 \\
03 \\
02 \\
01\end{array}$ & $\begin{array}{r}\text { 6. } 4400 \\
05 \\
09 \\
14 \\
18\end{array}$ & \\
\hline $\begin{array}{l}15 \\
16 \\
17 \\
18 \\
19\end{array}$ & $\begin{array}{l}63 \\
59 \\
55 \\
51 \\
47\end{array}$ & $\begin{array}{r}54 \\
42 \\
30 \\
17 \\
8.509 \quad 7605\end{array}$ & $\begin{array}{l}580 \\
606 \\
633 \\
659 \\
686\end{array}$ & $\begin{array}{r}00 \\
2.3700 \\
2.3699 \\
98 \\
97\end{array}$ & $\begin{array}{l}23 \\
28 \\
32 \\
37 \\
41\end{array}$ & \\
\hline $\begin{array}{l}20 \\
21 \\
22 \\
23 \\
24\end{array}$ & $\begin{array}{r}8.508 \quad 7543 \\
39 \\
35 \\
31 \\
27\end{array}$ & $\begin{array}{r}8.5097593 \\
81 \\
69 \\
56 \\
44\end{array}$ & $\begin{array}{r}1.54712 \\
739 \\
765 \\
792 \\
818\end{array}$ & $\begin{array}{r}2.3696 \\
95 \\
94 \\
94 \\
93\end{array}$ & $\begin{array}{r}6.4446 \\
50 \\
65 \\
59 \\
64\end{array}$ & 7.733 \\
\hline $\begin{array}{l}25 \\
26 \\
27 \\
28 \\
29\end{array}$ & $\begin{array}{l}22 \\
18 \\
14 \\
10 \\
06\end{array}$ & $\begin{array}{r}32 \\
20 \\
8.5097508 \\
8.5097495 \\
83\end{array}$ & $\begin{array}{l}845 \\
871 \\
898 \\
924 \\
951\end{array}$ & $\begin{array}{l}92 \\
91 \\
90 \\
89 \\
88\end{array}$ & $\begin{array}{l}68 \\
73 \\
78 \\
82 \\
87\end{array}$ & \\
\hline $\begin{array}{l}30 \\
31 \\
32 \\
33 \\
34\end{array}$ & $\begin{array}{r}8.5087502 \\
8.508 \quad 7498 \\
94 \\
90 \\
96\end{array}$ & $\begin{array}{r}8.5097471 \\
.59 \\
47 \\
34 \\
22\end{array}$ & $\begin{array}{r}1.54977 \\
1.55004 \\
031 \\
.057 \\
084\end{array}$ & $\begin{array}{r}2.3688 \\
87 \\
.86 \\
85 \\
84\end{array}$ & $\begin{array}{r}6.4491 \\
6.4496 \\
6.4500 \\
05 \\
09\end{array}$ & \\
\hline $\begin{array}{l}35 \\
36 \\
37 \\
38 \\
39\end{array}$ & $\begin{array}{l}82 \\
78 \\
74 \\
70 \\
66\end{array}$ & $\begin{array}{r}8.5097410 \\
8.5097398 \\
86 \\
74 \\
61\end{array}$ & $\begin{array}{l}110 \\
137 \\
163 \\
190 \\
217\end{array}$ & $\begin{array}{l}83 \\
82 \\
82 \\
81 \\
80\end{array}$ & $\begin{array}{l}14 \\
19 \\
23 \\
28 \\
32\end{array}$ & \\
\hline $\begin{array}{l}40 \\
41 \\
42 \\
43 \\
44\end{array}$ & $\begin{array}{r}8.508 \quad 7462 \\
58 \\
53 \\
49 \\
45\end{array}$ & $\begin{array}{r}8.5097349 \\
37 \\
25 \\
13 \\
8.5097301\end{array}$ & $\begin{array}{r}1.55243 \\
270 \\
297 \\
323 \\
350\end{array}$ & $\begin{array}{r}2.3679 \\
78 \\
77 \\
76 \\
75\end{array}$ & 6. $\begin{array}{r}4537 \\
41 \\
46 \\
51 \\
55\end{array}$ & 7.728 \\
\hline $\begin{array}{l}45 \\
46 \\
47 \\
48 \\
49\end{array}$ & $\begin{array}{l}41 \\
37 \\
33 \\
29 \\
25\end{array}$ & $\begin{array}{r}8.5097289 \\
76 \\
64 \\
52 \\
40\end{array}$ & $\begin{array}{l}376 \\
403 \\
430 \\
456 \\
483\end{array}$ & $\begin{array}{l}74 \\
74 \\
73 \\
72 \\
71\end{array}$ & $\begin{array}{l}60 \\
64 \\
69 \\
74 \\
78\end{array}$ & . \\
\hline $\begin{array}{l}50 \\
51 \\
52 \\
53 \\
54\end{array}$ & $\begin{array}{rr}8.508 \quad 7421 \\
17 \\
13 \\
09 \\
05\end{array}$ & $\begin{array}{r}8.5097228 \\
16 \\
8.5097204 \\
8.5097191 \\
79\end{array}$ & $\begin{array}{r}\text { 1. } 55510 \\
536 \\
563 \\
590 \\
616\end{array}$ & $\begin{array}{r}2.3670 \\
69 \\
\cdot \quad 68 \\
67 \\
66\end{array}$ & $\begin{array}{r}6.4583 \\
87 \\
92 \\
6.4597 \\
6.4601\end{array}$ & . \\
\hline $\begin{array}{l}55 \\
56 \\
57 \\
58 \\
59\end{array}$ & $\begin{array}{r}8.508 \quad 7401 \\
8.508 \quad 7397 \\
93 \\
89 \\
85 \\
85\end{array}$ & $\begin{array}{l}67 \\
55 \\
43 \\
31 \\
19\end{array}$ & $\begin{array}{l}643 \\
670 \\
696 \\
723 \\
750\end{array}$ & $\begin{array}{l}66 \\
65 \\
64 \\
63 \\
62\end{array}$ & $\begin{array}{l}06 \\
10 \\
15 \\
20 \\
24\end{array}$ & \\
\hline 60 & 8.508 7381 & 8.5097107 & 1.55777 & 2.3661 & 6.4629 & 7.723 \\
\hline
\end{tabular}


TABLE 20.-Geodetic position computations-Continued.

IATITUDE $55^{\circ}$.

\begin{tabular}{|c|c|c|c|c|c|c|c|}
\hline \multicolumn{2}{|c|}{ Lat. } & $\log _{\mathrm{diff} .} \mathbf{1}^{\prime \prime}=-0.07$ & diff. $1^{\prime \prime}=-0.20$ & \multirow[t]{2}{*}{ diff. $1^{\prime \prime \prime}=+0.45$} & \multirow[t]{2}{*}{$\begin{array}{c}\log \mathrm{D} \\
\text { diff. } 1^{\prime \prime}=-0.02\end{array}$} & $\frac{\log \mathrm{E}}{\text { diff. } 1^{\prime \prime}-+0.08}$ & \multirow[t]{2}{*}{$\begin{array}{c}\log \mathrm{F} \\
\text { diff. } 10^{\prime}=-2.8\end{array}$} \\
\hline$\circ$ & , & & & & & . & \\
\hline 55 & $\begin{array}{l}00 \\
1 \\
2 \\
3 \\
4\end{array}$ & $\begin{array}{r}8.5087381 \\
77 \\
73 \\
69 \\
65\end{array}$ & $\begin{array}{r}8.5097107 \\
8.509 .7095 \\
82 \\
. \quad 70 \\
58\end{array}$ & $\begin{array}{r}1.55777 \\
803 \\
830 \\
857 \\
884\end{array}$ & $\begin{array}{r}2.3661 \\
60 \\
59 \\
58 \\
57\end{array}$ & $\begin{array}{r}6.4629 \\
33 \\
38 \\
43 \\
47\end{array}$ & 7.723 \\
\hline & $\begin{array}{r}05 \\
6 \\
7 \\
8 \\
9\end{array}$ & $\begin{array}{r}61 \\
56 \\
. \quad 52 \\
48 \\
. \quad 44\end{array}$ & $\begin{array}{r}46 \\
34 \\
22 \\
8.5097010 \\
8.5096998\end{array}$ & $\begin{array}{r}910 \\
937 \\
964 \\
1.55991 \\
1.56017\end{array}$ & $\begin{array}{l}56 \\
56 \\
55 \\
54 \\
53\end{array}$ & $\begin{array}{l}52 \\
57 \\
61 \\
66 \\
70\end{array}$ & $\cdot$ \\
\hline & $\begin{array}{l}10 \\
11 \\
12 \\
13 \\
14\end{array}$ & $\begin{array}{r}8.5087340 \\
36 \\
32 \\
28 \\
24\end{array}$ & $\begin{array}{r}8.509 \quad 6986 \\
74 \\
62 \\
49 \\
37\end{array}$ & $\begin{array}{r}1.56044 \\
071 \\
098 \\
125 \\
151\end{array}$ & $\begin{array}{r}2.3652 \\
51 \\
50 \\
49 \\
48\end{array}$ & $\begin{array}{r}6.4675 \\
80 \\
84 \\
89 \\
94\end{array}$ & \\
\hline & $\begin{array}{l}15 \\
16 \\
17 \\
18 \\
19\end{array}$ & $\begin{array}{r}20 \\
16 \\
12 \\
08 \\
04\end{array}$ & $\begin{array}{rr} & 25 \\
13 \\
8.509 & 6901 \\
8.509 & 6889 \\
& 77\end{array}$ & $\begin{array}{l}178 \\
205 \\
232 \\
259 \\
286\end{array}$ & $\begin{array}{l}47 \\
46 \\
45 \\
44 \\
43\end{array}$ & $\begin{array}{r}6.4698 \\
6.4703 \\
08 \\
12 \\
17\end{array}$ & \\
\hline & $\begin{array}{l}20 \\
21 \\
22 \\
23 \\
24\end{array}$ & $\begin{array}{r}8.5087300 \\
8.508 \quad 7296 \\
92 \\
88 \\
84\end{array}$ & $\begin{array}{r}8.5096865 \\
53 \\
41 \\
29 \\
17\end{array}$ & $\begin{array}{r}1.56312 \\
339 \\
366 \\
393 \\
420\end{array}$ & $\begin{array}{r}2.3642 \\
42 \\
41 \\
40 \\
. \quad 39\end{array}$ & $\begin{array}{r}\text { 6. } 4721 \\
26 \\
31 \\
35 \\
40\end{array}$ & 7.717 \\
\hline & $\begin{array}{l}25 \\
26 \\
27 \\
28 \\
29\end{array}$ & $\begin{array}{l}80 \\
76 \\
72 \\
68 \\
64\end{array}$ & $\begin{array}{r}8.5096805 \\
8.5096793 \\
81 \\
69 \\
57\end{array}$ & $\begin{array}{l}447 \\
474 \\
500 \\
527 \\
564\end{array}$ & $\begin{array}{l}38 \\
37 \\
36 \\
35 \\
34\end{array}$ & $\begin{array}{l}45 \\
49 \\
54 \\
59 \\
63\end{array}$ & \\
\hline & $\begin{array}{l}30 \\
31 \\
32 \\
33 \\
34\end{array}$ & $\begin{array}{r}8.5087260 \\
56 \\
52 \\
48 \\
44\end{array}$ & $\begin{array}{r}8.5096745 \\
33 \\
21 \\
8.5096709 \\
8.5096696\end{array}$ & $\begin{array}{r}1.56581 \\
608 \\
635 \\
662 \\
689\end{array}$ & $\begin{array}{r}2.3633 \\
32 \\
31 \\
30 \\
.29\end{array}$ & $\begin{array}{r}6.4768 \\
73 \\
77 \\
82 \\
87\end{array}$ & . \\
\hline & $\begin{array}{l}35 \\
\mathbf{3 6} \\
\mathbf{3 7} \\
38 \\
39\end{array}$ & $\begin{array}{l}40 \\
36 \\
32 \\
28 \\
24\end{array}$ & $\begin{array}{l}84 \\
72 \\
60 \\
48 \\
36\end{array}$ & $\begin{array}{l}716 \\
743 \\
770 \\
797 \\
823\end{array}$ & $\begin{array}{l}28 \\
27 \\
26 \\
25 \\
24\end{array}$ & $\begin{array}{r}91 \\
6.4796 \\
6.4801 \\
05 \\
10\end{array}$ & . \\
\hline & $\begin{array}{l}40 \\
41 \\
42 \\
43 \\
44\end{array}$ & $\begin{array}{r}8.508 \quad 7220 \\
\mathbf{1 6} \\
12 \\
08 \\
04\end{array}$ & $\begin{array}{rr}8.509 & 6624^{\prime} \\
& 12 \\
8.509 & 6600 \\
8.509 & 6588 \\
& 76\end{array}$ & $\begin{array}{r}1.58550 \\
877 \\
904 \\
931 \\
958\end{array}$ & $\begin{array}{r}2.3623 \\
22 \\
21 \\
20 \\
19\end{array}$ & $\begin{array}{r}\text { 6. } 4815 \\
20 \\
24 \\
29 \\
34\end{array}$ & $\begin{array}{l}7.711 \\
\end{array}$ \\
\hline & $\begin{array}{l}45 \\
46 \\
47 \\
48 \\
49\end{array}$ & $\begin{array}{r}8.5087200 \\
8.5087196 \\
92 \\
88 \\
84\end{array}$ & $\begin{array}{l}64 \\
52 \\
40 \\
28 \\
16\end{array}$ & $\begin{array}{r}1.56985 \\
1.57012 \\
039 \\
. \quad 066 \\
093\end{array}$ & $\begin{array}{l}18 \\
17 \\
16 \\
15 \\
14\end{array}$ & $\begin{array}{l}38 \\
43 \\
48 \\
52 \\
57\end{array}$ & \\
\hline & $\begin{array}{l}50 \\
51 \\
52 \\
53 \\
54\end{array}$ & $\begin{array}{r}8.5087180 \\
76 \\
72 \\
68 \\
64\end{array}$ & $\begin{array}{r}8.5096505 \\
8.5096493 \\
81 \\
69 \\
57\end{array}$ & $\begin{array}{r}1.57120 \\
147 \\
174 \\
201 \\
229\end{array}$ & 2. $\begin{array}{r}613 \\
12 \\
11 \\
10 \\
09\end{array}$ & $\begin{array}{r}6.4862 \\
66 \\
71 \\
76 \\
81\end{array}$ & \\
\hline & $\begin{array}{l}55 \\
56 \\
57 \\
58 \\
59\end{array}$ & $\begin{array}{l}60 \\
56 \\
52 \\
48 \\
44\end{array}$ & $\begin{array}{rr} & 45 \\
. \quad 33 \\
& 21 \\
8.509 & 6409 \\
8.509 & 6397\end{array}$ & $\begin{array}{l}256 \\
283 \\
310 \\
337 \\
364\end{array}$ & $\begin{array}{l}08 \\
07 \\
06 \\
05 \\
04\end{array}$ & $\begin{array}{r}85 \\
90 \\
6.4895 \\
6.4900 \\
04\end{array}$ & \\
\hline & 60 & $8.508 \cdot 7140$ & 8.5096385 & 1. 57391 & 2. 3603 & 6.4909 & 7.706 \\
\hline
\end{tabular}


TABLE 20.-Geodetic position computations-Continued.

LATITUDE $56^{\circ}$.

\begin{tabular}{|c|c|c|c|c|c|c|}
\hline Lat. & $\begin{array}{c}\log A \\
\text { diff. } 1^{\prime \prime}=-0.07\end{array}$ & $\begin{array}{c}\log B \\
\text { diff. } 1^{\prime \prime}=-0.20\end{array}$ & $\begin{array}{c}\log C \\
\text { diff. } 1^{\prime \prime}=+0.45\end{array}$ & $\begin{array}{c}\log \mathrm{D} \\
\text { diff. } 1^{\prime \prime}=-0.02\end{array}$ & $\begin{array}{c}\log \mathrm{E} \\
\text { diff. } 1^{\prime \prime}=+0.08\end{array}$ & $\begin{array}{c}\log \mathrm{F} \\
\text { diff. } 10^{\prime}=-3.0\end{array}$ \\
\hline $\begin{array}{cc}\circ & 1 \\
56 & 00 \\
& 1 \\
& 2 \\
& 3 \\
& 3\end{array}$ & $\begin{array}{r}8.5087140 \\
36 \\
32 \\
. \quad 28 \\
24\end{array}$ & $\begin{array}{r}8.5096385 \\
73 \\
61 \\
49 \\
37\end{array}$ & $\begin{array}{r}1.57391 \\
418 \\
445 \\
472 \\
499\end{array}$ & $\begin{array}{r}2.3603 \\
02 \\
01 \\
2.3600 \\
2.3599\end{array}$ & $\begin{array}{r}6.4909 \\
14 \\
18 \\
23 \\
28\end{array}$ & 7.706 \\
\hline $\begin{array}{r}05 \\
6 \\
7 \\
8 \\
9\end{array}$ & $\begin{array}{l}20 \\
16 \\
12 \\
08 \\
04\end{array}$ & $\begin{array}{r}25 \\
13 \\
8.509 \quad 6301 \\
8.509 \quad 6289 \\
77\end{array}$ & $\begin{array}{l}526 \\
554 \\
581 . \\
608 \\
635\end{array}$ & $\begin{array}{l}98 \\
97 \\
96 \\
95 \\
94\end{array}$ & $\begin{array}{l}33 \\
37 \\
42 \\
47 \\
52\end{array}$ & \\
\hline $\begin{array}{l}10 \\
11 \\
1.2 \\
13 \\
14\end{array}$ & $\begin{array}{r}8.5087100 \\
8.508 \quad 7096 \\
92 \\
88 \\
84\end{array}$ & $\begin{array}{r}8.509 \quad 6266 \\
54 \\
42 \\
30 \\
18\end{array}$ & $\begin{array}{r}1.57662 \\
689 \\
717 \\
744 \\
771\end{array}$ & $\begin{array}{r}2.3593 \\
92 \\
91 \\
90 \\
-\quad 89\end{array}$ & $\begin{array}{r}6.4956 \\
61 \\
66 \\
71 \\
75\end{array}$ & \\
\hline $\begin{array}{l}15 \\
16 \\
17 \\
18 \\
19\end{array}$ & $\begin{array}{l}80 \\
76 \\
72 \\
69 \\
65\end{array}$ & $\begin{array}{r}8.5096206 \\
8.50961 .94 \\
82 \\
70 \\
58\end{array}$ & $\begin{array}{l}798 \\
825 \\
852 \\
880 \\
907\end{array}$ & $\begin{array}{l}88 \\
87 \\
86 \\
85 \\
84\end{array}$ & $\begin{array}{r}80 \\
85 \\
90 \\
94 \\
6.4999\end{array}$ & . \\
\hline $\begin{array}{l}20 \\
21 \\
22 \\
23 \\
24\end{array}$ & $\begin{array}{r}8.508 \quad 7061 \\
57 \\
53 \\
49 \\
45\end{array}$ & $\begin{array}{r}8.5096147 \\
35 \\
23 \\
8.5096111 \\
8.509 .6099\end{array}$ & $\begin{array}{r}1.57 y 34 \\
961 \\
1.57989 \\
1.58016 \\
043\end{array}$ & $\begin{array}{r}2.3583 \\
82 \\
81 \\
80 \\
78\end{array}$ & 6. $\begin{array}{r}5004 \\
09 \\
13 \\
18 \\
23\end{array}$ & 7. 700 \\
\hline $\begin{array}{l}25 \\
26 \\
27 \\
28 \\
29\end{array}$ & $\begin{array}{l}41 \\
37 \\
33 \\
29 \\
25\end{array}$ & $\begin{array}{l}87 \\
75 \\
63 \\
51 \\
40\end{array}$ & $\begin{array}{l}070 \\
098 \\
125 \\
152 \\
179\end{array}$ & $\begin{array}{l}77 \\
76 \\
75 \\
74 \\
73\end{array}$ & $\begin{array}{l}28 \\
32 \\
37 \\
42 \\
47\end{array}$ & \\
\hline $\begin{array}{l}30 \\
31 \\
32 \\
33 \\
34\end{array}$ & $\begin{array}{r}8.5087021 \\
17 \\
13 \\
09 \\
05\end{array}$ & $\begin{array}{r}8.5096028 \\
16 \\
8.5096004 \\
8.5095992 \\
80\end{array}$ & $\begin{array}{r}\text { 1. } 58207 \\
234 \\
261 \\
289 \\
316\end{array}$ & $\begin{array}{r}2.3572 \\
71 \\
70 \\
\quad \quad 69 \\
68\end{array}$ & $\begin{array}{r}6.5052 \\
56 \\
61 \\
66 \\
71\end{array}$ & \\
\hline $\begin{array}{l}35 \\
36 \\
37 \\
38 \\
39\end{array}$ & $\begin{array}{r}8.5087001 \\
8.5086997 \\
93 \\
89 \\
86\end{array}$ & $\begin{array}{r}68 \\
57 \\
45 \\
-\quad 33 \\
21\end{array}$ & $\begin{array}{l}343 \\
371 \\
398 \\
425 \\
\mathbf{4 5 3}\end{array}$ & $\begin{array}{l}67 \\
66 \\
65 \\
64 \\
62\end{array}$ & $\begin{array}{l}75 \\
80 \\
85 \\
90 \\
95\end{array}$ & . \\
\hline $\begin{array}{l}40 \\
41 \\
42 \\
43 \\
44\end{array}$ & $\begin{array}{r}8.508 \quad 6982 \\
78 \\
74 \\
70 \\
66\end{array}$ & $\begin{array}{r}8.5095909 \\
8.5095897 \\
86 \\
74 \\
62\end{array}$ & $\begin{array}{r}1.58480 \\
507 \\
535 \\
562 \\
589\end{array}$ & $\begin{array}{r}2.3561 \\
60 \\
59 \\
58 \\
57\end{array}$ & $\begin{array}{r}6.5099 \\
6.5104 \\
09 \\
14 \\
19\end{array}$ & 7.694 \\
\hline $\begin{array}{l}45 \\
46 \\
47 \\
48 \\
49\end{array}$ & $\begin{array}{l}62 \\
58 \\
54 . \\
50 \\
46\end{array}$ & $\begin{array}{r}50 \\
38 \\
27 \\
15 \\
15 \\
85095303\end{array}$ & $\begin{array}{l}617 \\
644 \\
672 \\
699 \\
726\end{array}$ & $\begin{array}{l}56 \\
55 \\
54 \\
53 \\
52\end{array}$ & $\begin{array}{l}24 \\
28 \\
33 \\
38 \\
43\end{array}$ & \\
\hline $\begin{array}{l}50 \\
61 \\
52 \\
53 \\
54\end{array}$ & $\begin{array}{r}8.508 \quad 6942 \\
38 \\
34 \\
30 \\
26\end{array}$ & $\begin{array}{r}8.509 \quad 5791 \\
79 \\
67 \\
56 \\
44\end{array}$ & $\begin{array}{r}1.58754 \\
781 \\
809 \\
836 \\
864\end{array}$ & $\begin{array}{r}2.3550 \\
49 \\
48 \\
47 \\
46\end{array}$ & $\begin{array}{r}6.5148 \\
52 \\
57 \\
62 \\
67\end{array}$ & \\
\hline $\begin{array}{l}55 \\
56 \\
57 \\
58 \\
59\end{array}$ & $\begin{array}{l}23 \\
19 \\
15 \\
11 \\
07\end{array}$ & $\begin{array}{r}32 \\
20 \\
8.5095709 \\
8.5095697 \\
35\end{array}$ & $\begin{array}{r}891 \\
919 \\
946 \\
1.58974 \\
1.59001\end{array}$ & $\begin{array}{l}45 \\
44 \\
43 \\
42 \\
41\end{array}$ & $\begin{array}{l}72 \\
77 \\
81 . \\
86 \\
91 .\end{array}$ & \\
\hline (i0 & 8. 5086903 & 8.5095673 & 1.59028 & 2.3539 & 6.5196 & 7.688 \\
\hline
\end{tabular}


TABLE 20.-Geodetic position computations-Continued.

LATITUDE $57^{\circ}$.

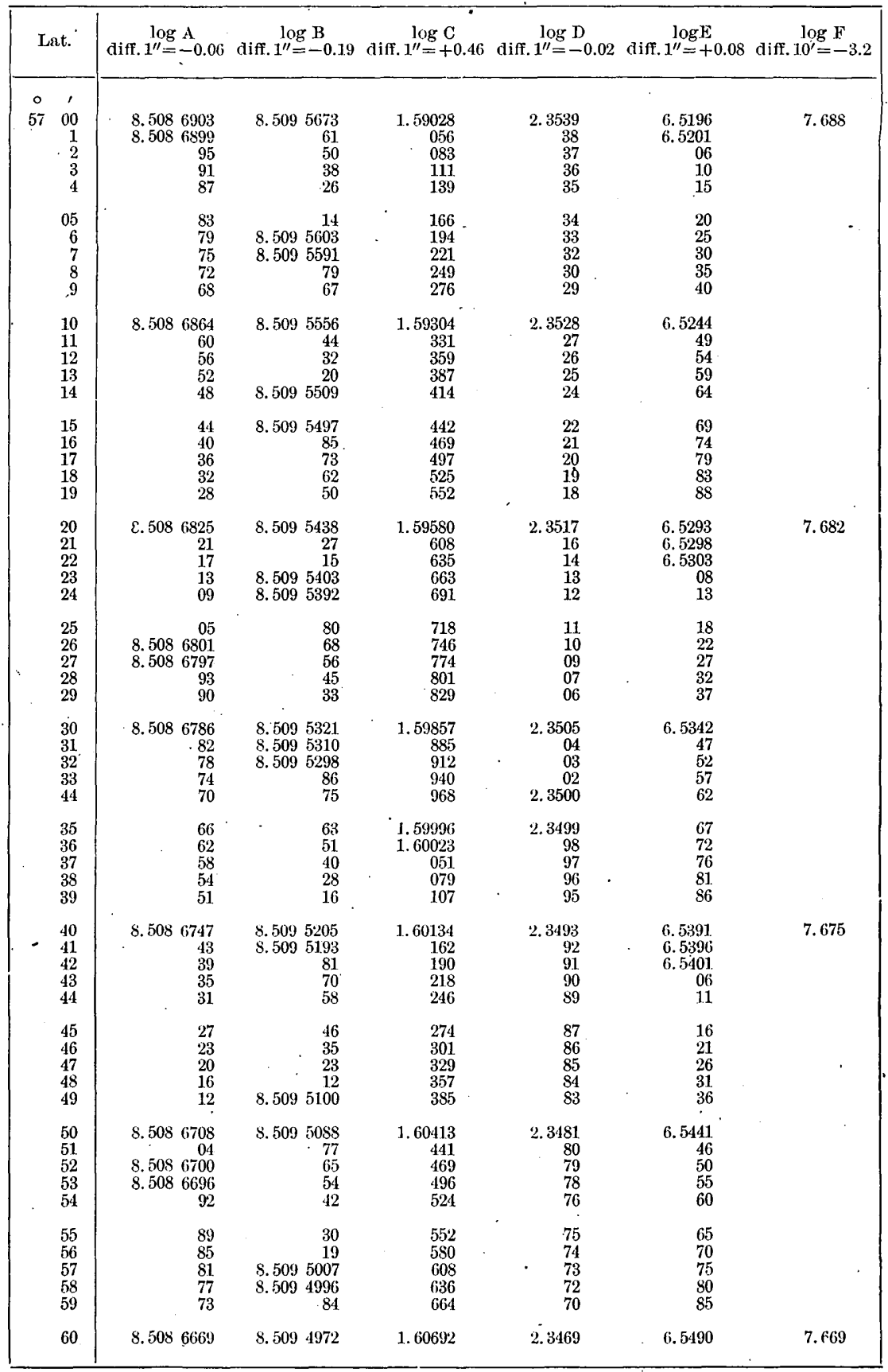


Table 20.-Geodetic position computations-Continued.

LAIITUDE $58^{\circ}$.

\begin{tabular}{|c|c|c|c|c|c|c|}
\hline Isat. & $\begin{array}{c}\log A \\
\operatorname{diff} .1^{\prime \prime}=-0.06\end{array}$ & $\begin{array}{c}\log B \\
\text { diff. } 1^{\prime \prime}=-0.19\end{array}$ & diff. $x^{\prime \prime \prime}=+0.47$ & $\begin{array}{c}\log \mathrm{D} \\
\operatorname{diff} .1^{\prime \prime}=-0.02\end{array}$ & $\begin{array}{c}\log \mathbf{E} \\
\text { diff. } 1^{\prime \prime}=+0.08\end{array}$ & $\begin{array}{c}\log F \\
\text { diff. } 10^{\prime}=-3.3\end{array}$ \\
\hline $\begin{array}{cc}\circ & 1 \\
58 & 00 \\
& 1 \\
& 2 \\
& 3 \\
& 4\end{array}$ & $\begin{array}{r}8.508 \quad 6669 \\
65 \\
62 \\
58 \\
54\end{array}$ & $\begin{array}{r}8.509 \quad 4972 \\
61 \\
49 \\
38 \\
26\end{array}$ & $\begin{array}{r}1.60692 \\
720 \\
748 \\
776 \\
804\end{array}$ & $\begin{array}{r}2.3469 \\
68 \\
67 \\
66 \\
64\end{array}$ & $\begin{array}{r}6.5490 \\
6.5495 \\
6.5500 \\
05 \\
10\end{array}$ & 7. 669 \\
\hline $\begin{array}{r}05 \\
6 \\
7 \\
8 \\
9\end{array}$ & $\begin{array}{l}50 \\
46 \\
42 \\
38 \\
35\end{array}$ & $\begin{array}{r}14 \\
8.5094903 \\
8.5094891 \\
80 \\
68\end{array}$ & $\begin{array}{l}832 \\
860 \\
888 \\
916 \\
944\end{array}$ & $\begin{array}{l}63 \\
62 \\
61 \\
59 \\
58\end{array}$ & $\begin{array}{l}15 \\
20 \\
25 \\
30 \\
35\end{array}$ & \\
\hline $\begin{array}{l}10 \\
11 \\
12 \\
1.3 \\
14\end{array}$ & $\begin{array}{r}8.508 \quad 6631 \\
27 \\
23 \\
19 \\
15\end{array}$ & $\begin{array}{r}8.5094857 \\
45 \\
33 \\
22 \\
8.5094810\end{array}$ & $\begin{array}{r}1.60972 \\
1.61000 \\
028 \\
056 \\
084\end{array}$ & $\begin{array}{r}2.3457 \\
56 \\
54 \\
53 \\
52\end{array}$ & $\begin{array}{r}6.5540 \\
45 \\
50 \\
55 \\
60\end{array}$ & \\
\hline $\begin{array}{l}15 \\
16 \\
17 \\
18 \\
19\end{array}$ & $\begin{array}{r}11 \\
08 \\
04 \\
8.5086600 \\
8.5086596\end{array}$ & $\begin{array}{r}8.509 \quad 4799 \\
87 \\
76 \\
64 \\
53\end{array}$ & $\begin{array}{l}112 \\
140 \\
168 \\
197 \\
225\end{array}$ & $\begin{array}{l}51 \\
49 \\
48 \\
47 \\
46\end{array}$ & $\begin{array}{l}65 \\
70 \\
75 \\
80 \\
85\end{array}$ & \\
\hline $\begin{array}{l}20 \\
21 \\
22 \\
23 \\
24\end{array}$ & $\begin{array}{r}8.508 \quad 6592 \\
88 \\
85 \\
81 \\
77\end{array}$ & $\begin{array}{r}8.5094741 \\
30 \\
18 \\
8.5094707 \\
8.5094695\end{array}$ & $\begin{array}{r}1.61253 \\
281 \\
309 \\
337 \\
365\end{array}$ & $\begin{array}{r}2.3444 \\
43 \\
42 \\
41 \\
39\end{array}$ & $\begin{array}{r}6.5590 \\
6.5595 \\
6.5600 \\
05 \\
10\end{array}$ & 7.662 \\
\hline $\begin{array}{l}25 \\
26 \\
27 \\
28 \\
29\end{array}$ & $\begin{array}{l}73 \\
69 \\
65 \\
62 \\
58\end{array}$ & $\begin{array}{l}84 \\
72 \\
61 \\
49 \\
38\end{array}$ & $\begin{array}{l}393 \\
422 \\
450 \\
478 \\
506\end{array}$ & $\begin{array}{l}38 \\
37 \\
35 \\
34 \\
33\end{array}$ & $\begin{array}{l}15 \\
20 \\
25 \\
30 \\
35\end{array}$ & . \\
\hline $\begin{array}{l}30 \\
31 \\
32 \\
33 \\
34\end{array}$ & $\begin{array}{r}8.508 \quad 6554 \\
50 \\
46 \\
42 \\
39\end{array}$ & $\begin{array}{r}8.5094626 \\
15 \\
8.5094603 \\
8.5094592 \\
. \quad 80\end{array}$ & $\begin{array}{r}1.61534 \\
563 \\
591 \\
619 \\
647\end{array}$ & $\begin{array}{r}2.3432 \\
30 \\
29 \\
28 \\
26\end{array}$ & $\begin{array}{r}6.5640 \\
45 \\
50 \\
55 \\
60\end{array}$ & \\
\hline $\begin{array}{l}35 \\
36 \\
37 \\
38 \\
39\end{array}$ & $\begin{array}{l}35 \\
31 \\
27 \\
23 \\
20\end{array}$ & $\begin{array}{l}69 \\
57 \\
46 \\
35 \\
23\end{array}$ & $\begin{array}{r}675 \\
704 \\
732 \\
-\quad 760 \\
789\end{array}$ & $\begin{array}{l}25 \\
24 \\
23 \\
21 \\
20\end{array}$ & $\begin{array}{l}65 \\
70 \\
75 \\
80 \\
86\end{array}$ & \\
\hline $\begin{array}{l}40 \\
41 . \\
42 \\
43 \\
44\end{array}$ & $\begin{array}{r}8.508 \quad 6516 \\
12 \\
08 \\
04 \\
8.508 \quad 6500\end{array}$ & $\begin{array}{r}8.5094512 \\
8.5094500 \\
8.5094489 \\
77 \\
66\end{array}$ & $\begin{array}{r}1.61817 \\
845 \\
873 \\
902 \\
930\end{array}$ & $\begin{array}{r}2.3419 \\
17 \\
16 \\
15 \\
14\end{array}$ & $\begin{array}{r}6.5691 \\
6.5696 \\
6.5701 . \\
06 \\
11\end{array}$ & 7.656 \\
\hline $\begin{array}{l}45 \\
46 \\
47 \\
48 \\
49\end{array}$ & $\begin{array}{r}8.508 \quad 6497 \\
93 \\
89 \\
85 \\
81\end{array}$ & $\begin{array}{r}54 \\
43 \\
32 \\
20 \\
8.509 \quad 4409\end{array}$ & $\begin{array}{r}958 \\
1.61987 \\
1.62015 \\
043 \\
072\end{array}$ & $\begin{array}{r}12 \\
11 . \\
10 \\
08 \\
07\end{array}$ & $\begin{array}{l}16 \\
21 \\
26 \\
31 \\
36\end{array}$ & \\
\hline $\begin{array}{l}50 \\
51 \\
52 \\
53 \\
54\end{array}$ & $\begin{array}{r}8.508 \quad 6478 \\
74 \\
70 \\
66 \\
62\end{array}$ & $\begin{array}{rr}8.509 & 4397 \\
. \quad 86 \\
\cdot \quad 74 \\
\\
63 \\
52\end{array}$ & $\begin{array}{r}1.62100 \\
129 \\
1.57 \\
185 \\
214\end{array}$ & $\begin{array}{r}2.3406 \\
04 \\
03 \\
02 \\
2.3400\end{array}$ & $\begin{array}{r}6.5741 \\
46 \\
51 \\
56 \\
62\end{array}$ & \\
\hline $\begin{array}{l}55 \\
56 \\
57 \\
58 \\
59\end{array}$ & $\begin{array}{r}59 \\
55 \\
51 \\
47 \\
43\end{array}$ & $\begin{array}{r}40 \\
29 \\
17 \\
8.5094306 \\
8.5094295\end{array}$ & $\begin{array}{l}242 \\
271 \\
299 \\
327 \\
356\end{array}$ & $\begin{array}{r}2.3399 \\
98 \\
96 \\
95 \\
94\end{array}$ & $\begin{array}{l}67 \\
72 \\
77 \\
82 \\
87\end{array}$ & \\
\hline 60 & 8.5086440 & 8. 5094283 & 1.62384 & 2. 3392 & (6. 5792 & 7.649 \\
\hline
\end{tabular}


TABLE 20.-Geodetic position computations-Continued.

IATITUDE $59^{\circ}$.

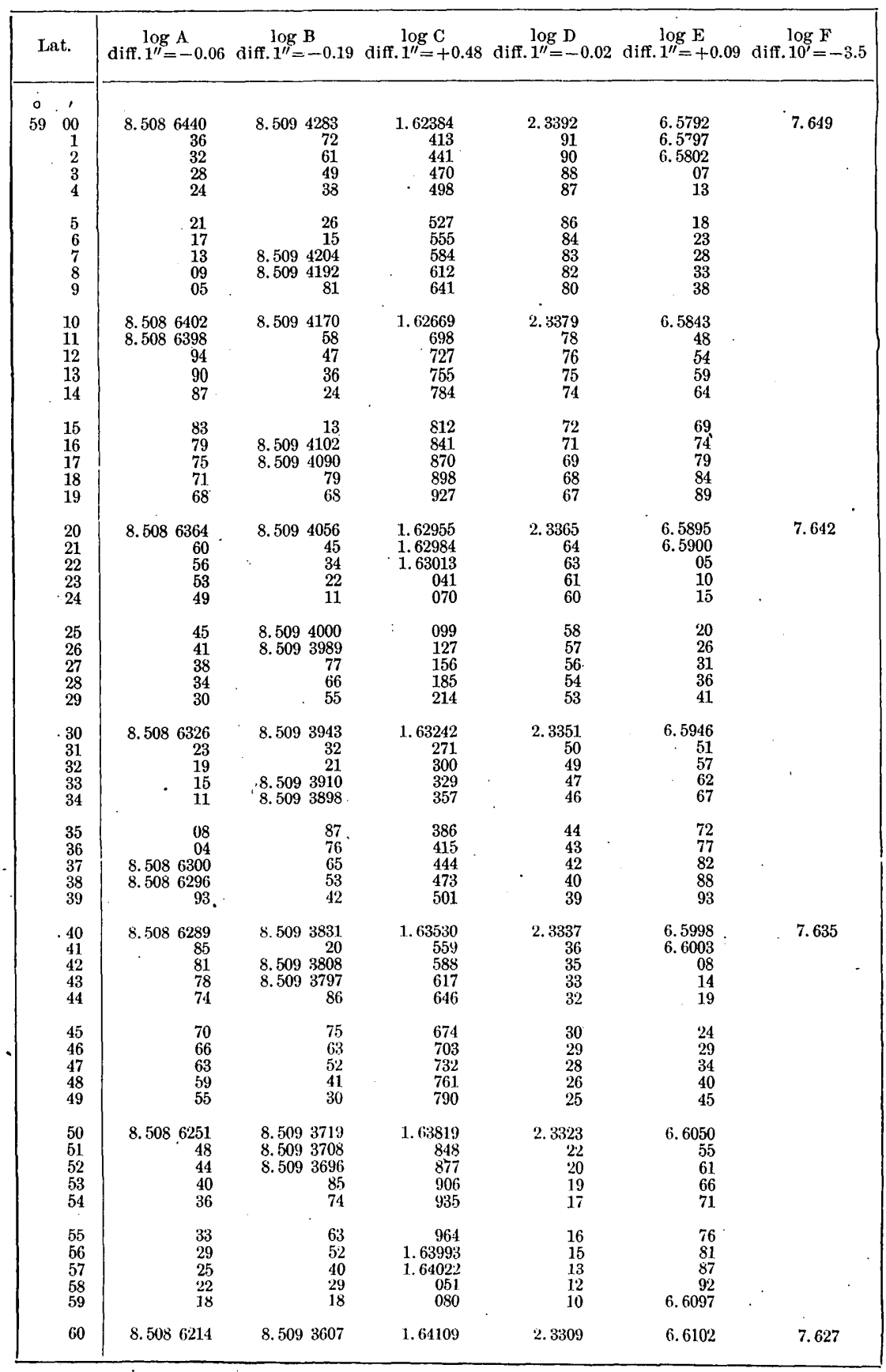


TABLE 20.-Geodetic position computations-Continued.

LATITUDE $60^{\circ}$.

\begin{tabular}{|c|c|c|c|c|c|c|}
\hline Lat. & diff. $\begin{array}{l}\log A \\
1^{\prime \prime}=-0.06\end{array}$ & $\begin{array}{c}\log \mathrm{I} \\
\text { diff. } 1^{\prime \prime}=-0 . \mathrm{i}\end{array}$ & c diff. $1^{\prime \prime}=+0.49$ & $\begin{array}{c}\log \mathrm{D} \\
\text { diff. } 1^{\prime \prime}=-0.03\end{array}$ & $\begin{array}{c}\log \mathbf{E} \\
\text { diff. } 1^{\prime \prime}=+0.09\end{array}$ & 9 diff. $10^{\prime}=-3.7$ \\
\hline $\begin{array}{cc}0 & 1 \\
60 & 00 \\
& 1 \\
& 2 \\
& 2 \\
& 3 \\
& 4\end{array}$ & $\begin{array}{rr}8.508 & 6214 \\
. \quad 10 \\
8.500 & 07 \\
8.508 & 6199\end{array}$ & $\begin{array}{r}8.5093607 \\
8.5093596 \\
85 \\
73 \\
62\end{array}$ & $\begin{array}{r}1.64109 \\
138 \\
167 \\
196 \\
225\end{array}$ & $\begin{array}{r}2.3309 \\
07 \\
06 \\
04 \\
03\end{array}$ & $\begin{array}{r}6.6102 \\
08 \\
13 \\
18 \\
\quad 23\end{array}$ & 7.627 \\
\hline $\begin{array}{r}05 \\
6 \\
7 \\
8 \\
9\end{array}$ & $\begin{array}{l}96 \\
92 \\
88 \\
84 \\
81\end{array}$ & $\begin{array}{r}51 \\
40 \\
29 \\
18 \\
8.509 \quad 3507\end{array}$ & $\begin{array}{l}254 \\
283 \\
312 \\
341 \\
370\end{array}$ & $\begin{array}{r}02 \\
2.3300 \\
2.3299 \\
97 \\
96\end{array}$ & $\begin{array}{l}29 \\
34 \\
39 \\
44 \\
50\end{array}$ & \\
\hline $\begin{array}{l}10 \\
11 \\
12 \\
13 \\
14\end{array}$ & $\begin{array}{r}8.508 \quad 6177 \\
73 \\
70 \\
66 \\
62\end{array}$ & $\begin{array}{r}8.5093495 \\
84 \\
73 \\
62 \\
51\end{array}$ & $\begin{array}{r}1.64400 \\
429 \\
458 \\
487 \\
516\end{array}$ & $\begin{array}{r}2.3294 \\
93 \\
91 \\
90 \\
88\end{array}$ & $\begin{array}{r}6.6155 \\
60 \\
66 \\
71 \\
76\end{array}$ & \\
\hline $\begin{array}{l}15 \\
16 \\
17 \\
18 \\
19\end{array}$ & $\begin{array}{l}58 \\
55 \\
51 \\
47 \\
44\end{array}$ & $\begin{array}{r}40 \\
29 \\
18 \\
\text { 8. } 5093407 \\
8.5093395\end{array}$ & 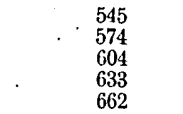 & $\begin{array}{l}87 \\
85 \\
84 \\
82 \\
81\end{array}$ & $\begin{array}{r}81 \\
87 \\
92 \\
6.6197 \\
6.6203\end{array}$ & . \\
\hline $\begin{array}{l}20 \\
21 \\
22 \\
23 \\
24\end{array}$ & $\begin{array}{r}8.508 \quad 6140 \\
36 \\
33 \\
29 \\
-\quad 25\end{array}$ & $\begin{array}{r}8.5093384 \\
73 \\
62 \\
51 \\
40\end{array}$ & $\begin{array}{r}1.64691 \\
720 \\
750 \\
779 \\
808\end{array}$ & $\begin{array}{r}2.3279 \\
78 \\
76 \\
75 \\
73\end{array}$ & $\begin{array}{r}6.6208 \\
13 \\
18 \\
24 \\
.29\end{array}$ & 7.620 \\
\hline $\begin{array}{l}25 \\
26 \\
27 \\
28 \\
29\end{array}$ & $\begin{array}{r}21 \\
18 \\
14 \\
10 \\
07\end{array}$ & $\begin{array}{r}29 \\
18 \\
8.5093307 \\
8.5093296 \\
85\end{array}$ & $\begin{array}{l}838 \\
867 \\
890 \\
925 \\
955\end{array}$ & $\begin{array}{l}72 \\
70 \\
69 \\
67 \\
66\end{array}$ & $\begin{array}{r}34 \\
40 \\
.45 \\
50 \\
56\end{array}$ & \\
\hline $\begin{array}{l}30 \\
31 \\
32 \\
33 \\
34\end{array}$ & $\begin{array}{r}8.5086103 \\
8.508 \quad 6099 \\
96 \\
92 \\
88\end{array}$ & $\begin{array}{r}8.5093274 \\
63 \\
52 \\
40 \\
29\end{array}$ & $\begin{array}{r}1.64984 \\
1.65013 \\
043 \\
072 \\
101 .\end{array}$ & $\begin{array}{r}2.3264 \\
63 \\
61 \\
60 \\
58\end{array}$ & $\begin{array}{r}6.6261 \\
66 \\
72 \\
77 \\
82\end{array}$ & \\
\hline $\begin{array}{l}35 \\
36 \\
37 \\
38 \\
39\end{array}$ & $\begin{array}{l}85 \\
81 \\
77 \\
74 \\
70\end{array}$ & $\begin{array}{r}18 \\
8.5093207 \\
8.5093196 \\
85 \\
74\end{array}$ & $\begin{array}{l}131 \\
160 \\
190 \\
219 \\
248\end{array}$ & $\begin{array}{l}57 \\
55 \\
54 \\
52 \\
51\end{array}$ & $\begin{array}{r}87 \\
93 \\
6.6298 \\
6.6304 \\
09\end{array}$ & \\
\hline $\begin{array}{l}40 \\
41 \\
42 \\
43 \\
.44\end{array}$ & $\begin{array}{r}8.508 \quad 6066 \\
63 \\
59 \\
55 \\
52\end{array}$ & $\begin{array}{r}8.50963 \\
52 \\
41 \\
-\quad 30 \\
\quad 19\end{array}$ & $\begin{array}{r}1.65278 \\
307 \\
337 \\
366 \\
396\end{array}$ & $\begin{array}{r}2.3249 \\
48 \\
46 \\
45 \\
43\end{array}$ & $\begin{array}{r}6.6314 \\
20 \\
25 \\
30 \\
36\end{array}$ & 7.613 \\
\hline $\begin{array}{l}45 \\
46 \\
47 \\
48 \\
49\end{array}$ & $\begin{array}{l}48 \\
44 \\
41 \\
37 \\
33\end{array}$ & $\begin{array}{r}\text { 8. } 5093108 \\
\text { 8. 509 } 3097 \\
86 \\
75 . \\
64\end{array}$ & $\begin{array}{l}425 \\
455 \\
484 \\
514 \\
543\end{array}$ & $\begin{array}{l}41 \\
40 \\
38 \\
37 \\
35\end{array}$ & $\begin{array}{l}41 \\
46 \\
52 \\
57 \\
62\end{array}$ & \\
\hline $\begin{array}{l}50 \\
51 \\
52 \\
53 \\
54\end{array}$ & $\begin{array}{r}8.508 \quad 6030 \\
26 \\
22 \\
19 \\
-\quad 15\end{array}$ & $\begin{array}{r}8.5093053 \\
.42 \\
31 \\
20 \\
8.509 \quad 3010\end{array}$ & $\begin{array}{r}1.65573 \\
602 \\
632 \\
661 \\
691\end{array}$ & $\begin{array}{r}2.3234 \\
32 \\
31 \\
29 \\
28\end{array}$ & 6. $\begin{array}{r}6368 \\
73 \\
79 \\
84 \\
89\end{array}$ & \\
\hline $\begin{array}{l}55 \\
56 \\
57 \\
58 \\
59\end{array}$ & $\begin{array}{r}11 \\
08 \\
04 \\
8.5086000 \\
8.5085997\end{array}$ & $\begin{array}{r}8.5092999 \\
88 \\
77 \\
66 \\
55\end{array}$ & $\begin{array}{l}721 \\
750 \\
780 \\
809 \\
839\end{array}$ & $\begin{array}{l}26 \\
24 \\
23 \\
21 \\
20\end{array}$ & $\begin{array}{r}6.6395 \\
6.6400 \\
05 \\
11 \\
16\end{array}$ & \\
\hline 60 & 8. 5085993 & 8.509 2944 & 1.65869 & 2.3218 & 6.6422 & 7.605 \\
\hline
\end{tabular}

Bull. 214-03-15 
TABLE 20.-Geodetic nosition computations-Continued.

LATITUDE $61^{\circ}$.

\begin{tabular}{|c|c|c|c|c|c|c|}
\hline Lat. & $\begin{array}{c}\log A \\
\text { diff. } \mathbf{1}^{\prime \prime}=-0.06\end{array}$ & $\begin{array}{c}\log B \\
\text { diff. } 1^{\prime \prime}=-0.18\end{array}$ & $\begin{array}{c}\log C \\
\text { diff. } 1^{\prime \prime}=+0.50\end{array}$ & $\begin{array}{c}\log \mathrm{D} \\
\text { diff. } 1^{\prime \prime}=-0.03\end{array}$ & $\begin{array}{c}\log \mathbf{E} \\
\text { diff. } 1^{\prime \prime}=+0.09\end{array}$ & $\begin{array}{c}\log \mathrm{F} \\
\operatorname{diff} .10^{\prime}=-4.0\end{array}$ \\
\hline $0^{\circ}$, & & & & & & . \\
\hline $\begin{array}{cc}61 \quad 00 \\
-\quad 1 \\
-\quad 2 \\
& 3 \\
& 4\end{array}$ & $\begin{array}{r}8.5085993 \\
89 \\
86 \\
82 \\
79\end{array}$ & $\begin{array}{r}8.5092944 \\
33 \\
22 \\
11 \\
5.5092900\end{array}$ & $\begin{array}{r}1.65869 \\
898 \\
928 \\
958 \\
1.65987\end{array}$ & $\begin{array}{r}2.3218 \\
-\quad 17 \\
15 \\
13 \\
12\end{array}$ & 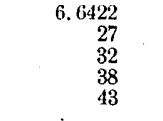 & 7.605 \\
\hline $\begin{array}{r}05 \\
6 \\
7 \\
8 \\
9 \\
9\end{array}$ & $\begin{array}{l}75 \\
71 \\
68 \\
64 \\
60\end{array}$ & $\begin{array}{r}8.5092889 \\
78 \\
67 \\
56 \\
46\end{array}$ & $\begin{array}{r}1.66017 \\
047 \\
076 \\
106 \\
136\end{array}$ & $\begin{array}{r}10 \\
09 \\
-\quad 07 \\
06 \\
04\end{array}$ & $\begin{array}{l}48 \\
54 \\
59 \\
65 \\
70\end{array}$ & \\
\hline $\begin{array}{l}10 \\
11 \\
12 \\
13 \\
14\end{array}$ & $\begin{array}{r}8.5085957 \\
53 \\
49 \\
46 \\
42\end{array}$ & $\begin{array}{r}8.5092835 \\
24 \\
13 \\
\text { S. } 5092802 \\
8.5092791\end{array}$ & $\begin{array}{r}1.66166 \\
195 \\
225 \\
255 \\
285\end{array}$ & $\begin{array}{r}2.3202 \\
2.3201 \\
2.3199 \\
98 \\
96\end{array}$ & $\begin{array}{r}6.6476 \\
81 \\
87 \\
92 \\
6.6497\end{array}$ & \\
\hline $\begin{array}{l}15 \\
16 \\
17 \\
18 \\
19\end{array}$ & $\begin{array}{l}39 \\
35 \\
31 \\
28 \\
24\end{array}$ & $\begin{array}{l}80 \\
69 \\
58 \\
48 \\
37\end{array}$ & $\begin{array}{l}315 \\
344 \\
374 \\
404 \\
434\end{array}$ & $\begin{array}{l}94 \\
93 \\
91 \\
90 \\
88\end{array}$ & $\begin{array}{r}6.6503 \\
08 \\
14 \\
19 \\
25\end{array}$ & \\
\hline $\begin{array}{l}20 \\
21 . \\
22 \\
23 \\
24\end{array}$ & $\begin{array}{r}8.5085920 \\
17 \\
.13 \\
10 \\
. \quad 06\end{array}$ & $\begin{array}{r}8.5092726 \\
15 \\
8.5092704 \\
8.5092693 \\
83\end{array}$ & $\begin{array}{r}1.66464 \\
-\quad 494 \\
524 \\
553 \\
583\end{array}$ & $\begin{array}{r}2.3186 \\
85 \\
83 \\
81 \\
80\end{array}$ & $\begin{array}{r}6.6530 \\
36 \\
41 \\
46 \\
52\end{array}$ & 7.597 \\
\hline $\begin{array}{l}25 \\
26 \\
27 \\
28 \\
29\end{array}$ & $\begin{array}{r}8.508 \quad 5902 \\
8.508 \quad 5899 \\
95 \\
92 \\
88\end{array}$ & $\begin{array}{l}72 \\
61 \\
50 \\
39 \\
28\end{array}$ & $\begin{array}{l}613 \\
643 \\
673 \\
703 \\
733\end{array}$ & $\begin{array}{l}78 \\
77 \\
75 \\
73 \\
72\end{array}$ & $\begin{array}{l}57 \\
63 \\
68 \\
74 \\
79\end{array}$ & \\
\hline $\begin{array}{l}30 \\
31 \\
32 \\
33 \\
34\end{array}$ & $\begin{array}{r}8.5085884 \\
81 \\
77 \\
. \quad 74 \\
70\end{array}$ & $\begin{array}{r}8.5092618 \\
8.5092607 \\
8.5092596 \\
85 \\
74\end{array}$ & $\begin{array}{r}1.66763 \\
793 \\
823 \\
.853 \\
883\end{array}$ & $\begin{array}{r}2.3170 \\
68 \\
67 \\
65 \\
64\end{array}$ & $\begin{array}{r}6.6585 \\
90 \\
6.6596 \\
6.6601 \\
07\end{array}$ & \\
\hline $\begin{array}{l}35 \\
36 \\
37 \\
38 \\
39\end{array}$ & $\begin{array}{l}66 \\
63 \\
59 \\
56 \\
52\end{array}$ & $\begin{array}{l}64 \\
53 \\
42 \\
31 \\
20\end{array}$ & $\begin{array}{r}913 \\
943 \\
1.66973 \\
1.67003 \\
033\end{array}$ & $\begin{array}{l}62 \\
60 \\
58 \\
57 \\
55\end{array}$ & $\begin{array}{l}12 \\
18 \\
23 \\
29 \\
34\end{array}$ & . \\
\hline $\begin{array}{l}40 \\
41 \\
42 \\
43 \\
44\end{array}$ & $\begin{array}{r}8.5085848 \\
45 \\
41 \\
38 \\
34\end{array}$ & $\begin{array}{r}8.5092510 \\
8.5092499 \\
88 \\
77 \\
67\end{array}$ & $\begin{array}{r}1.67063 \\
094 \\
124 \\
154 \\
184\end{array}$ & $\begin{array}{r}2.3154 \\
52 \\
50 \\
49 \\
47\end{array}$ & $\begin{array}{r}6.6640 \\
45 \\
51 \\
56 \\
62\end{array}$ & 7.589 \\
\hline $\begin{array}{l}45 \\
46 \\
47 \\
48 \\
49\end{array}$ & $\begin{array}{l}30 \\
27 \\
23 \\
20 \\
16\end{array}$ & $\begin{array}{l}56 \\
45 \\
34 \\
24 \\
13\end{array}$ & $\begin{array}{l}214 \\
244 \\
274 \\
305 \\
335\end{array}$ & $\begin{array}{l}45 \\
44 \\
42 \\
40 \\
39\end{array}$ & $\begin{array}{l}67 \\
73 \\
78 \\
84 \\
89\end{array}$ & 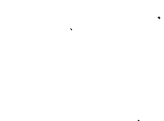 \\
\hline $\begin{array}{l}50 \\
51 \\
52 \\
53 \\
54\end{array}$ & $\begin{array}{r}8.5085813 \\
09 \\
05 \\
8.5085802 \\
8.5085798\end{array}$ & $\begin{array}{r}8.5092402 \\
8.5092391 \\
81 \\
70 \\
59\end{array}$ & $\begin{array}{r}1.67365 \\
395 \\
425 \\
456 \\
486\end{array}$ & $\begin{array}{r}2.3137 \\
35 \\
34 \\
32 \\
30\end{array}$ & $\begin{array}{r}6.6695 \\
6.6700 \\
06 \\
12 \\
17\end{array}$ & \\
\hline $\begin{array}{l}55 \\
56 \\
57 \\
58 \\
59\end{array}$ & $\begin{array}{l}95 \\
91 \\
88 \\
84 \\
80\end{array}$ & $\begin{array}{r}49 \\
38 \\
27 \\
16 \\
8.509 \quad 2306\end{array}$ & $\begin{array}{l}516 \\
547 \\
577 \\
607 \\
637\end{array}$ & $\begin{array}{l}29 \\
27 \\
25 \\
23 \\
22\end{array}$ & $\begin{array}{l}23 \\
28 \\
34 \\
39 \\
45\end{array}$ & \\
\hline 60 & 8.5085777 & 8.5092295 & 1.67668 & 2.3120 & 6.6750 & 7.581 \\
\hline
\end{tabular}


TABLe 20.-Geodetic position computations-Continued.

LATITUDE $62^{\circ}$.

\begin{tabular}{|c|c|c|c|c|c|c|}
\hline Lut. & $\operatorname{diff} .1^{\prime \prime}=-0.06$ & $\begin{array}{c}\log B \\
\text { diff. } 1^{\prime \prime}=-0.18\end{array}$ & $\begin{array}{c}\log \mathrm{C} \\
\text { ditf. } 1^{\prime \prime}=+0.51\end{array}$ & $\begin{array}{c}\log \mathrm{D} \\
\text { diff. } 1^{\prime \prime}=-0.03\end{array}$ & $\begin{array}{c}\log \mathrm{E} \\
\text { diff. } 1^{\prime \prime}=+0.09\end{array}$ & $\begin{array}{c}\log \mathrm{F} \\
\text { diff. } 10^{\prime}=-4.2\end{array}$ \\
\hline 0 & & & & & & \\
\hline 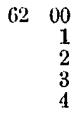 & $\begin{array}{r}8.5085777 \\
73 \\
70 \\
66 \\
63\end{array}$ & $\begin{array}{r}8.509 \quad 2295 \\
\cdot \quad 84 \\
74 \\
\cdot \quad 63 \\
52\end{array}$ & $\begin{array}{r}1.67668 \\
698 \\
728 \\
759 \\
789\end{array}$ & $\begin{array}{r}2.3120 \\
18 \\
17 \\
. \quad 15 \\
13\end{array}$ & $\begin{array}{r}6.6750 \\
56 \\
61 \\
67 \\
73\end{array}$ & 7.581 \\
\hline $\begin{array}{r}05^{\circ} \\
6 \\
7 \\
8 \\
0\end{array}$ & $\begin{array}{l}59 \\
55 \\
52 \\
48 \\
45\end{array}$ & $\begin{array}{r}42 \\
31 \\
20 \\
8.5092210 \\
8.5092199\end{array}$ & $\begin{array}{l}820 \\
850 \\
880 \\
911 \\
941\end{array}$ & $\begin{array}{l}1.2 \\
1.0 \\
08 \\
06 \\
05\end{array}$ & $\begin{array}{r}78 \\
84 \\
89 \\
6.6795 \\
6.6801\end{array}$ & \\
\hline $\begin{array}{l}10 \\
11 \\
1.2 \\
13 \\
14\end{array}$ & $\begin{array}{r}8.5085741 \\
38 \\
34 \\
30 \\
27\end{array}$ & $\begin{array}{r}8.5092188 \\
78 \\
67 \\
56 \\
46\end{array}$ & $\begin{array}{r}1.67972 \\
1.68002 \\
033 \\
063 \\
094\end{array}$ & $\begin{array}{r}2.3103 \\
01 \\
2.3100 \\
2.3098 \\
96\end{array}$ & $\begin{array}{r}6.6806 \\
12 \\
17 \\
23 \\
29\end{array}$ & \\
\hline $\begin{array}{l}15 \\
16 \\
17 \\
18 \\
19\end{array}$ & $\begin{array}{l}24 \\
20 \\
16 \\
13 \\
09\end{array}$ & $\begin{array}{r}35 \\
25 \\
14 \\
8.50921 .03 \\
8.5092093\end{array}$ & $\begin{array}{l}124 \\
155 \\
185 \\
216 \\
246\end{array}$ & $\begin{array}{l}94 \\
93 \\
91 \\
89 \\
87\end{array}$ & $\begin{array}{r}34 \\
40 \\
+\quad 45 \\
51 \\
57\end{array}$ & . \\
\hline $\begin{array}{l}20 \\
21 \\
22 \\
23 \\
24\end{array}$ & $\begin{array}{r}8.5085706 \\
8.5085702 \\
8.508 \quad 5699 \\
95 \\
92\end{array}$ & $\begin{array}{r}8.509 \quad 2082 \\
71 \\
61 \\
50 \\
40\end{array}$ & $\begin{array}{r}1.68277 \\
307 \\
338 \\
369 \\
399\end{array}$ & $\begin{array}{r}2.3086 \\
84 \\
82 \\
80 \\
79\end{array}$ & $\begin{array}{r}6.6862 \\
68 \\
73 \\
79 \\
85\end{array}$ & 7.573 \\
\hline $\begin{array}{l}25 \\
26 \\
27 \\
28 \\
29\end{array}$ & $\begin{array}{l}88 \\
85 \\
81 . \\
78 \\
74\end{array}$ & $\begin{array}{r}29 \\
19 \\
\text { S.509 2008 } \\
8.5091997 \\
87\end{array}$ & $\begin{array}{l}430 \\
461 \\
491 \\
522 \\
553\end{array}$ & $\begin{array}{l}77 \\
75 \\
74 \\
72 \\
70\end{array}$ & $\begin{array}{r}90 \\
6.6896 \\
6.6902 \\
07 \\
13\end{array}$ & \\
\hline $\begin{array}{l}30 \\
31 \\
32 \\
33 \\
34\end{array}$ & $\begin{array}{r}8.5085671 \\
67 \\
64 \\
60 \\
56\end{array}$ & $\begin{array}{r}8.5091976 \\
66 \\
55 \\
45 \\
34\end{array}$ & $\begin{array}{r}1.68583 \\
614 \\
645 \\
675 \\
706\end{array}$ & $\begin{array}{r}2.3068 \\
66 \\
65 \\
63 \\
61\end{array}$ & $\begin{array}{r}6.6919 \\
24 \\
30 \\
36 \\
41\end{array}$ & \\
\hline $\begin{array}{l}35 \\
36 \\
37 \\
38 \\
39\end{array}$ & $\begin{array}{l}53 \\
49 \\
46 \\
42 \\
39\end{array}$ & $\begin{array}{rr} & 23 \\
13 \\
8.5091902 \\
\text { 8.509 } 1892 \\
81\end{array}$ & $\begin{array}{l}737 \\
768 \\
799 \\
829 \\
860\end{array}$ & $\begin{array}{l}59 \\
58 \\
56 \\
54 \\
52\end{array}$ & $\begin{array}{l}47 \\
53 \\
58 \\
64 \\
70\end{array}$ & \\
\hline $\begin{array}{l}40 \\
41 \\
42 \\
43 \\
44\end{array}$ & $\begin{array}{r}8.508 \quad 5635 \\
32 \\
28 \\
25 \\
. \quad 21\end{array}$ & $\begin{array}{r}8.5091871 \\
60 \\
50 \\
39 \\
29\end{array}$ & $\begin{array}{r}1.68891 \\
922 \\
953 \\
1.68984 \\
1.69014\end{array}$ & $\begin{array}{r}2.3050 \\
49 \\
47 \\
45 \\
43\end{array}$ & $\begin{array}{r}\text { 6. } 6975 \\
81 \\
87 \\
92 \\
6.6998\end{array}$ & 7.564 \\
\hline $\begin{array}{r}45 \\
46 \\
47 \\
48 \\
49\end{array}$ & $\begin{array}{l}18 \\
14 \\
11 \\
07 \\
04\end{array}$ & $\begin{array}{r}18 \\
8.5091808 \\
8.5091797 \\
87 \\
76\end{array}$ & $\begin{array}{l}045 \\
076 \\
107 \\
138 \\
169\end{array}$ & $\begin{array}{l}42 \\
40 \\
38 \\
36 \\
34\end{array}$ & $\begin{array}{r}6.7004 \\
09 \\
15 \\
21 \\
26\end{array}$ & \\
\hline $\begin{array}{l}50 \\
51 \\
62 \\
53 \\
54\end{array}$ & $\begin{array}{r}8.5085600 \\
\text { S.508 5597 } \\
93 \\
90 \\
86\end{array}$ & $\begin{array}{r}8.5091766 \\
55 \\
45 \\
34 \\
24\end{array}$ & $\begin{array}{r}1.69200 \\
231 \\
262 \\
293 \\
324\end{array}$ & $\begin{array}{r}2.3033 \\
31 \\
29 \\
27 \\
25\end{array}$ & $\begin{array}{r}6.7032 \\
38 \\
44 \\
49 \\
55\end{array}$ & - \\
\hline $\begin{array}{l}55 \\
56 \\
57 \\
58 \\
59\end{array}$ & $\begin{array}{r}83 \\
80 \\
76 \\
73 \\
69\end{array}$ & $\begin{array}{r}14 \\
8.5091703 \\
8.5091693 \\
82 \\
72\end{array}$ & $\begin{array}{r}355 \\
386 \\
417 \\
448 \\
479\end{array}$ & $\begin{array}{l}23 \\
22 \\
20 \\
18 \\
16\end{array}$ & $\begin{array}{l}61 \\
67 \\
72 \\
78 \\
84\end{array}$ & \\
\hline 60 & 8.5085566 & 8. 5091661 & 1. 69510 & 2.3014 & 6.7089 & 7.556 \\
\hline
\end{tabular}


TABLe 20.-Geodetic position computations-Continued.

LATITUDE $63^{\circ}$.

\begin{tabular}{|c|c|c|c|c|c|c|}
\hline Lat. & diff. $1^{\prime \prime}=-0.06$ & $\begin{array}{c}\log B \\
\text { diff. } 1^{\prime \prime}=-0.17\end{array}$ & $\begin{array}{c}\log C \\
\text { diff. } 1^{\prime \prime}=+0.52\end{array}$ & $\begin{array}{c}\log \mathrm{D} \\
\text { diff. } 1^{\prime \prime}=-0.03\end{array}$ & $\begin{array}{c}\log \mathrm{E} \\
\operatorname{diff} .1^{\prime \prime}=+0.10\end{array}$ & $\begin{array}{c}\log F \\
\text { diff. } 10^{\prime}=-4.5\end{array}$ \\
\hline $0 \quad 1$ & & & . & & & \\
\hline $\begin{array}{cc}63 \quad 00 \\
1 \\
\\
4 \\
\\
4 \\
\\
4\end{array}$ & $\begin{array}{r}8.5085566 \\
62 \\
5 \quad 59 \\
55 \\
52\end{array}$ & $\begin{array}{r}8.5091661 \\
51 \\
40 \\
30 \\
20\end{array}$ & $\begin{array}{r}1.69510 \\
541 \\
572 \\
603 \\
635\end{array}$ & $\begin{array}{r}2.3014 \\
13 \\
11 \\
09 \\
07\end{array}$ & $\begin{array}{r}6.7089 \\
6.7095 \\
6.7101 \\
07 \\
12\end{array}$ & 7.556 \\
\hline $\begin{array}{r}05 \\
6 \\
7 \\
8 \\
9\end{array}$ & $\begin{array}{l}48 \\
45 \\
41 \\
38 \\
34\end{array}$ & $\begin{array}{r}8.5091609 \\
8.5091599 \\
88 \\
78 \\
68\end{array}$ & $\begin{array}{l}666 \\
697 \\
728 \\
759 \\
791\end{array}$ & $\begin{array}{r}05 \\
03 \\
02 \\
2.3000 \\
2.2998\end{array}$ & $\begin{array}{l}18 \\
24 \\
30 \\
35 \\
41\end{array}$ & . \\
\hline $\begin{array}{l}10 \\
11 \\
12 \\
13 \\
14\end{array}$ & $\begin{array}{r}8.5085531 \\
27 \\
24 \\
20 \\
17\end{array}$ & $\begin{array}{r}8.5091557 \\
47 \\
36 \\
26 \\
16\end{array}$ & $\begin{array}{r}1.69822 \\
853 \\
884 \\
915 \\
947\end{array}$ & $\begin{array}{r}2.2996 \\
94 \\
92 \\
90 \\
89\end{array}$ & $\begin{array}{r}6.7147 \\
53 \\
59 \\
64 \\
70\end{array}$ & \\
\hline $\begin{array}{l}15 \\
16 \\
17 \\
18 \\
19\end{array}$ & $\begin{array}{r}14 \\
10 \\
07 \\
03 \\
8.508 \quad 5500\end{array}$ & $\begin{array}{r}8.5091505 \\
8.5091495 \\
85 \\
74 \\
64\end{array}$ & $\begin{array}{r}1.69978 \\
1.70009 \\
. \quad 041 \\
072 \\
103\end{array}$ & $\begin{array}{l}87 \\
85 \\
83 \\
81 \\
79\end{array}$ & $\begin{array}{r}76 \\
82 \\
88 \\
93 \\
6.7199\end{array}$ & \\
\hline $\begin{array}{l}20 \\
21 \\
22 \\
23 \\
24\end{array}$ & $\begin{array}{r}8.508 .5496 \\
93 \\
89 \\
86 \\
83\end{array}$ & $\begin{array}{r}8.5091454 \\
43 \\
33 \\
23 \\
12\end{array}$ & $\begin{array}{r}1.70135 \\
166 \\
197 \\
229 \\
260\end{array}$ & $\begin{array}{r}2.2977 \\
75 \\
74 \\
72 \\
70\end{array}$ & 6. $\begin{array}{r}7205 \\
11 \\
17 \\
22 \\
28\end{array}$ & 7.547 \\
\hline $\begin{array}{l}25 \\
26 \\
27 \\
28 \\
29\end{array}$ & $\begin{array}{l}79 \\
76 \\
72 \\
69 \\
65\end{array}$ & $\begin{array}{r}8.5091402 \\
8.5091392 \\
81 \\
71 \\
61\end{array}$ & $\begin{array}{l}292 \\
323 \\
355 \\
386 \\
417\end{array}$ & $\begin{array}{l}68 \\
66 \\
64 \\
62 \\
60\end{array}$ & $\begin{array}{l}34 \\
40 \\
46 \\
51 \\
57\end{array}$ & \\
\hline $\begin{array}{l}30 \\
31 \\
32 \\
33 \\
34\end{array}$ & $\begin{array}{r}8.508 \quad 5462 \\
58 \\
55 \\
52 \\
48\end{array}$ & $\begin{array}{r}8.509 .1350 \\
40 \\
30 \\
19 \\
8.5091309\end{array}$ & $\begin{array}{r}1.70449 \\
480 \\
512 \\
544 \\
575\end{array}$ & $\begin{array}{r}2.2958 \\
57 \\
\cdot \quad 55 \\
53 \\
51\end{array}$ & $\begin{array}{r}6.7263 \\
69 \\
75 \\
81 \\
86\end{array}$ & - \\
\hline $\begin{array}{l}35 \\
36 \\
37 \\
38 \\
39\end{array}$ & $\begin{array}{r}45 \\
-41 \\
\mathbf{4 8} \\
34 \\
31\end{array}$ & $\begin{array}{r}8.5091299 \\
89 \\
78 \\
68 \\
58\end{array}$ & $\begin{array}{l}607 \\
638 \\
670 \\
701 \\
733\end{array}$ & $\begin{array}{l}49 \\
47 \\
45 \\
43 \\
41\end{array}$ & $\begin{array}{r}92 \\
6.7298 \\
6.7304 \\
\cdot \quad 10 \\
16\end{array}$ & \\
\hline $\begin{array}{l}40 \\
41 \\
42 \\
43 \\
44\end{array}$ & $\begin{array}{rr}8.508 \quad 5428 \\
24 \\
21 \\
17 \\
14\end{array}$ & $\begin{array}{r}8.5091248 \\
37 \\
27 \\
17 \\
8.5091207\end{array}$ & $\begin{array}{r}1.70765 \\
796 \\
828 \\
860 \\
891\end{array}$ & $\begin{array}{r}2.2939 \\
37 \\
36 \\
34 \\
32\end{array}$ & $\begin{array}{r}6.7322 \\
\cdot \quad 28 \\
33 \\
39 \\
45\end{array}$ & 7.538 \\
\hline $\begin{array}{l}45 \\
46 \\
47 \\
48 \\
49\end{array}$ & $\begin{array}{r}11 \\
07 \\
.04 \\
8.5085400 \\
8.5085397\end{array}$ & $\begin{array}{r}8.5091196 \\
86 \\
76 \\
66 \\
55\end{array}$ & $\begin{array}{r}923 \\
955 \\
1.70986 \\
1.71018 \\
050\end{array}$ & $\begin{array}{r}30 \\
28 \\
. \quad 26 \\
24 \\
22\end{array}$ & $\begin{array}{l}51 \\
57 \\
63 \\
69 \\
75\end{array}$ & \\
\hline $\begin{array}{r}50 \\
51 \\
52 \\
. \quad 53 \\
54\end{array}$ & $\begin{array}{r}8.5085394 \\
90 \\
87 \\
83 \\
. \quad 80\end{array}$ & $\begin{array}{r}8.5091145 \\
35 \\
25 \\
15 \\
8.5091104 .\end{array}$ & $\begin{array}{r}1.71082 \\
114 \\
145 \\
177 \\
209\end{array}$ & $\begin{array}{r}2.2920 \\
18 \\
16 \\
14 \\
12\end{array}$ & $\begin{array}{r}6.7381 \\
86 \\
92 \\
6.7398 \\
6.7404\end{array}$ & . \\
\hline $\begin{array}{l}55 \\
56 \\
57 \\
58 \\
59\end{array}$ & $\begin{array}{l}77 \\
73 \\
70 \\
66 \\
63\end{array}$ & $\begin{array}{r}8.5091094 \\
84 \\
74 \\
64 \\
64\end{array}$ & $\begin{array}{l}241 \\
273 \\
305 \\
337 \\
368\end{array}$ & $\begin{array}{l}10 \\
08 \\
06 \\
04 \\
02\end{array}$ & $\begin{array}{l}10 \\
16 \\
22 \\
28 \\
34\end{array}$ & \\
\hline 60 & 8.5085360 & 8.5091043 & 1.71400 & 2.2901 & $6.7440^{\circ}$ & 7.529 \\
\hline
\end{tabular}


Tabse 20.-Geodetic position computations-Continued.

IJATITUDE $64^{\circ}$.

\begin{tabular}{|c|c|c|c|c|c|c|}
\hline Lat. & \multicolumn{2}{|l|}{$\begin{array}{c}\log A \\
\text { diff. } 1^{\prime \prime}=-0.06\end{array}$} & $\begin{array}{c}\log \mathrm{C} \\
\text { diff. } 1^{\prime \prime}=+0.5\end{array}$ & $\begin{array}{c}\log \mathrm{D} \\
\text { diff. } 1^{\prime \prime}=-0.03\end{array}$ & $\begin{array}{l}\log \mathrm{E} \\
\text { diff. } 1^{\prime \prime}=+0.10\end{array}$ & diff. $10^{\prime}=-4.7$ \\
\hline $0 \quad 1$ & & & & & & \\
\hline $64 \begin{array}{r}00 \\
1 \\
\\
\\
\\
\\
\\
\\
3 \\
4\end{array}$ & $\begin{array}{r}8.5085360 \\
56 \\
53 \\
49 \\
\quad 46\end{array}$ & $\begin{array}{r}8.5091043 \\
33 \\
23 \\
13 \\
8.509 \quad 1003\end{array}$ & $\begin{array}{r}1.71400 \\
432 \\
464 \\
496 \\
528\end{array}$ & $\begin{array}{r}2.2901 \\
2.2899 \\
97 \\
95 \\
93\end{array}$ & $\begin{array}{r}6.7440 \\
46 \\
52 \\
58 \\
63\end{array}$ & 7.529 \\
\hline $\begin{array}{r}05 \\
6 \\
7 \\
8 \\
9\end{array}$ & $\begin{array}{l}43 \\
39 \\
36 \\
33 \\
29\end{array}$ & $\begin{array}{r}8.5090993 \\
82 \\
72 \\
62 \\
52\end{array}$ & $\begin{array}{l}560 \\
592 \\
624 \\
650 \\
688\end{array}$ & $\begin{array}{l}91 \\
89 \\
87 \\
85 \\
83\end{array}$ & $\begin{array}{l}69 \\
75 \\
81 \\
87 \\
93\end{array}$ & . \\
\hline $\begin{array}{l}10 \\
11 \\
12 \\
13 \\
14\end{array}$ & $\begin{array}{r}8.508 \quad 5326 \\
22 \\
19 \\
16 \\
12\end{array}$ & $\begin{array}{r}8.509 \cdot 0942 \\
32 \\
22 \\
12 \\
8.5090902\end{array}$ & $\begin{array}{r}1.71720 \\
752 \\
785 \\
817 \\
849\end{array}$ & $\begin{array}{r}2.2881 \\
79 \\
77 \\
75 \\
73\end{array}$ & $\begin{array}{r}6.7499 \\
6.7505 \\
11 \\
17 \\
23\end{array}$ & . \\
\hline $\begin{array}{l}15 \\
16 \\
17 \\
18 \\
19\end{array}$ & $\begin{array}{r}09 \\
06 \\
8.5085302 \\
8.508 \quad 5299 \\
96\end{array}$ & $\begin{array}{rr}8.5090891 \\
& 81 \\
& 71 \\
& 61 \\
\end{array}$ & $\begin{array}{r}881 \\
913 \\
945 \\
1.71977 \\
1.72010\end{array}$ & $\begin{array}{l}71 \\
69 \\
67 \\
65 \\
63\end{array}$ & $\begin{array}{l}29 \\
35 \\
41 . \\
47 \\
53\end{array}$ & - \\
\hline $\begin{array}{l}20 \\
21 \\
22 \\
23 \\
24\end{array}$ & $\begin{array}{r}8.508 \quad 5292 \\
89 \\
85 \\
82 \\
79\end{array}$ & $\begin{array}{r}8.5090841 \\
31 \\
21 \\
11 \\
8.5090801\end{array}$ & $\begin{array}{r}1.72042 \\
074 \\
106 \\
139 \\
171\end{array}$ & $\begin{array}{r}2.2861 . \\
59 \\
57 \\
55 \\
53\end{array}$ & $\begin{array}{r}6.7559 \\
65 \\
71 \\
77 \\
83\end{array}$ & $\begin{array}{l}7.520 \\
.\end{array}$ \\
\hline $\begin{array}{l}25 \\
26 \\
27 \\
28 \\
29\end{array}$ & $\begin{array}{r}75 \\
72 \\
69 \\
65 \\
62\end{array}$ & $\begin{array}{r}8.51) 90791 \\
81 \\
71 \\
61 \\
51\end{array}$ & $\begin{array}{l}203 \\
235 \\
268 \\
300 \\
332\end{array}$ & $\begin{array}{r}51 \\
49 \\
47 \\
45 \\
. \quad 42\end{array}$ & $\begin{array}{r}89 \\
6.7595 \\
6.7601 \\
07 \\
13\end{array}$ & \\
\hline $\begin{array}{l}30 \\
31 . \\
32 \\
33 \\
34\end{array}$ & $\begin{array}{r}8.508 \quad 5259 \\
55 \\
52 \\
49 \\
45\end{array}$ & $\begin{array}{r}8.5090741 \\
31 \\
21 \\
11 \\
8.509 \cdot 0701\end{array}$ & $\begin{array}{r}1.72365 \\
397 \\
430 \\
462 \\
495\end{array}$ & $\begin{array}{r}2.2840 \\
38 \\
36 \\
34 \\
32\end{array}$ & $\begin{array}{r}6.7619 \\
25 \\
31 \\
37 \\
43\end{array}$ & \\
\hline $\begin{array}{l}35 \\
36 \\
37 \\
38 \\
39\end{array}$ & $\begin{array}{l}42 \\
39 \\
35 \\
32 \\
0_{29}^{32}\end{array}$ & $\begin{array}{r}8.5090691 \\
81 . \\
71 \\
61 \\
51\end{array}$ & $\begin{array}{l}527 \\
559 \\
592 \\
624 \\
657\end{array}$ & $\begin{array}{r}30 \\
28 \\
26 \\
24 \\
22\end{array}$ & $\begin{array}{l}49 \\
56 \\
62 \\
68 \\
74\end{array}$ & . \\
\hline $\begin{array}{l}40 \\
41 \\
42 \\
43 \\
44\end{array}$ & $\begin{array}{r}8.508 \quad 5225 \\
22 \\
19 \\
15 \\
12\end{array}$ & $\begin{array}{r}8.5090641 \\
31 \\
21 \\
11 \\
8.5090601\end{array}$ & $\begin{array}{r}1.72689 \\
722 \\
755 \\
787 \\
820\end{array}$ & $\begin{array}{r}2.2820 \\
18 \\
16 \\
14 \\
12\end{array}$ & $\begin{array}{r}6.7680 \\
86 \\
92 \\
6.7698 \\
6.7704\end{array}$ & 7.511 \\
\hline $\begin{array}{l}45 \\
46 \\
47 \\
48 \\
49\end{array}$ & $\begin{array}{r}09 \\
05 \\
8.5085202 \\
8.50851 .99 \\
95\end{array}$ & $\begin{array}{r}8.5090591 \\
81 \\
71 \\
61 \\
61 .\end{array}$ & $\begin{array}{r}852 \\
885 \\
918 \\
950 \\
1.72983\end{array}$ & $\begin{array}{r}10 \\
07 \\
05 \\
03 \\
2.2801\end{array}$ & $\begin{array}{l}10 \\
16 \\
22 \\
28 \\
35\end{array}$ & \\
\hline $\begin{array}{l}50 \\
51 \\
52 \\
53 \\
54\end{array}$ & $\begin{array}{r}8.5085192 \\
89 \\
86 \\
82 \\
79\end{array}$ & $\begin{array}{r}8.5090541 \\
31 \\
21 \\
11 \\
8.5090501\end{array}$ & $\begin{array}{r}1.73016 \\
048 \\
081 \\
114 \\
146\end{array}$ & $\begin{array}{r}2.2799 \\
97 \\
95 \\
93 \\
91\end{array}$ & (i. $\begin{array}{r}7741 \\
47 \\
53 \\
59 \\
65\end{array}$ & \\
\hline $\begin{array}{l}55 \\
56 \\
57 \\
58 \\
59\end{array}$ & $\begin{array}{l}76 \\
72 \\
69 \\
66 \\
62\end{array}$ & $\begin{array}{r}8.5090491 \\
82 \\
72 \\
62 \\
52\end{array}$ & $\begin{array}{l}179 \\
212 \\
245 \\
278 \\
310\end{array}$ & $\begin{array}{l}89 \\
87 \\
84 \\
82 \\
80\end{array}$ & $\begin{array}{r}71 . \\
77 \\
84 \\
90 \\
6.7796\end{array}$ & \\
\hline 60 & 8. 5085159 & 8. 6090442 & 1.73343 & 2.2778 & 6.7802 & 7.501 \\
\hline
\end{tabular}


TABLE 20.-Geodetic position computations-Continued.

LATITUDE $65^{\circ}$.

\begin{tabular}{|c|c|c|c|c|c|c|}
\hline Lat. & $\operatorname{diff.} .^{\prime \prime \prime}=-0.05$ & $\begin{array}{c}\log B \\
\text { diff. } 1^{\prime \prime}=-0.16\end{array}$ & diff. $1^{\prime \prime}=+0.56$ & $\begin{array}{c}\log \mathrm{D} \\
\text { diff. } 1^{\prime \prime}=-0.04\end{array}$ & $\operatorname{diff} .1^{\prime \prime}=+0.10$ & diff. $10^{\prime}=-5.0$ \\
\hline $0 \quad 1$ & & & & & & . \\
\hline $\begin{array}{rr}65 & 00 \\
& 1 \\
& 2 \\
& 3 \\
& 4\end{array}$ & $\begin{array}{r}8.5085159 \\
56 \\
-\quad 52 \\
49 \\
46\end{array}$ & $\begin{array}{r}8.5090442 \\
32 \\
22 \\
12 \\
8.5090402\end{array}$ & $\begin{array}{r}1.73343 \\
376 \\
409 \\
442 \\
475\end{array}$ & $\begin{array}{r}2.2778 \\
76 \\
74 \\
72 \\
70\end{array}$ & $\begin{array}{r}6.7802 \\
08 \\
14 \\
20 \\
27\end{array}$ & 7.501. \\
\hline $\begin{array}{r}05 \\
6 \\
7 \\
8 \\
9\end{array}$ & $\begin{array}{r}43 \\
39 \\
36 \\
33 \\
30\end{array}$ & $\begin{array}{r}8.5090393 \\
83 \\
73 \\
63 \\
53\end{array}$ & $\begin{array}{l}508 \\
541 \\
574 \\
607 \\
640\end{array}$ & $\begin{array}{r}68 \\
65 \\
63 \\
61 \\
61 \\
\quad 59\end{array}$ & $\begin{array}{l}33 \\
39 \\
45 \\
51 \\
57\end{array}$ & \\
\hline $\begin{array}{l}10 \\
11 \\
12 \\
13 \\
14\end{array}$ & $\begin{array}{r}8.508 \quad 5126 \\
23 \\
20 \\
17 \\
13\end{array}$ & $\begin{array}{r}8.5090344 \\
34 \\
24 \\
14 \\
8.5090304\end{array}$ & $\begin{array}{r}1.73673 \\
706 \\
739 \\
772 \\
805\end{array}$ & $\begin{array}{r}2.2757 \\
55 \\
53 \\
.50 \\
48\end{array}$ & $\begin{array}{r}6.7864 \\
70 \\
76 \\
82 \\
88\end{array}$ & \\
\hline $\begin{array}{l}15 \\
16 \\
17 \\
18 \\
19\end{array}$ & $\begin{array}{r}10 \\
07 \\
03 \\
8.5085100 \\
8.5085097\end{array}$ & $\begin{array}{r}8.5090295 \\
. \quad 85 \\
75 \\
65 \\
55\end{array}$ & $\begin{array}{r}838 \\
871 . \\
904 \\
937 \\
\text { 1. } 73970\end{array}$ & $\begin{array}{l}46 \\
44 \\
42 \\
40 \\
38\end{array}$ & $\begin{array}{r}6.7895 \\
6.7901 \\
07 \\
13 \\
19\end{array}$ & - \\
\hline $\begin{array}{l}20 \\
21 \\
22 \\
23 \\
24\end{array}$ & $\begin{array}{r}8.508 \quad 5094 \\
90 \\
87 \\
84 \\
81\end{array}$ & $\begin{array}{r}8.5090245 \\
36 \\
26 \\
16 \\
8.5090206\end{array}$ & $\begin{array}{r}1.74004 \\
037 \\
070 \\
103 \\
136\end{array}$ & $\begin{array}{r}2.2735 \\
33 \\
31 \\
29 \\
27\end{array}$ & $\begin{array}{r}6.7926 \\
32 \\
38 \\
44 \\
51\end{array}$ & 7.491 \\
\hline $\begin{array}{l}25 \\
26 \\
27 \\
28 \\
29\end{array}$ & $\begin{array}{l}77 \\
74 \\
71 \\
68 \\
64\end{array}$ & $\begin{array}{r}8.5090197 \\
87 \\
77 \\
67 \\
57\end{array}$ & $\begin{array}{l}170 \\
203 \\
236 \\
270 \\
303\end{array}$ & $\begin{array}{l}24 \\
22 \\
20 \\
18 \\
.16\end{array}$ & $\begin{array}{l}57 \\
63 \\
69 \\
76 \\
82\end{array}$ & \\
\hline $\begin{array}{r}30 \\
31 \\
32 \\
33 \\
34\end{array}$ & $\begin{array}{r}8.508 \quad 5061 \\
58 \\
54 \\
51 \\
48\end{array}$ & $\begin{array}{r}8.50901 .48 \\
38 \\
28 \\
18 \\
8.50901 .09\end{array}$ & $\begin{array}{r}1.74336 \\
370 \\
403 \\
436 \\
. \quad 470\end{array}$ & $\begin{array}{r}2714 \\
11 \\
09 \\
07 \\
05\end{array}$ & $\begin{array}{r}6.7988 \\
6.7994 \\
6.8001 \\
07 \\
1.3\end{array}$ & \\
\hline $\begin{array}{l}35 \\
36 \\
37 \\
38 \\
39\end{array}$ & $\begin{array}{l}45 \\
41 \\
38 \\
35 \\
32\end{array}$ & $\begin{array}{r}8.5090099 \\
89 \\
80 \\
70 \\
60\end{array}$ & $\begin{array}{l}503 \\
537 \\
570 \\
604 \\
637\end{array}$ & $\begin{array}{r}03 \\
2.2700 \\
2.2698 \\
96 \\
94\end{array}$ & $\begin{array}{l}19 \\
26 \\
32 \\
38 \\
44\end{array}$ & , \\
\hline $\begin{array}{l}40 \\
41 \\
42 \\
43 \\
44\end{array}$ & $\begin{array}{r}8.5085029 \\
25 \\
22 \\
19 \\
16\end{array}$ & $\begin{array}{r}8.509 \quad 0051 \\
41 \\
31 \\
22 \\
. \quad 12\end{array}$ & $\begin{array}{r}1.74670 \\
704 \\
738 \\
771 \\
805\end{array}$ & $\begin{array}{r}2.2692 \\
89 \\
87 \\
85 \\
83\end{array}$ & fi. $\begin{array}{r}8051 \\
57 \\
63 \\
70 \\
76\end{array}$ & 7.481 \\
\hline $\begin{array}{l}45 \\
46 \\
47 \\
48 \\
49\end{array}$ & $\begin{array}{r}13 \\
09 \\
06 \\
03 \\
8.508 \quad 5000\end{array}$ & $\begin{array}{r}8.5090002 \\
8.5089993 \\
83 \\
73 \\
64\end{array}$ & $\begin{array}{r}838 \\
872 \\
906 \\
939 \\
1.74973\end{array}$ & $\begin{array}{l}80 \\
78 \\
76 \\
74 \\
72\end{array}$ & $\begin{array}{r}82 \\
89 \\
6.8095 \\
6.8101 \\
07\end{array}$ & \\
\hline $\begin{array}{l}50 \\
51 \\
52 \\
53 \\
54\end{array}$ & $\begin{array}{r}8.508 \quad 4996 \\
93 \\
90 \\
87 \\
84\end{array}$ & $\begin{array}{r}8.5089954 \\
44 \\
35 \\
25 \\
15\end{array}$ & $\begin{array}{r}\text { 1. } 75007 \\
040 \\
074 \\
108 \\
142\end{array}$ & $\begin{array}{r}2.2669 \\
67 \\
65 \\
63 \\
60\end{array}$ & $\begin{array}{r}6.8114 \\
20 \\
. \quad 27 \\
33 \\
39\end{array}$ & \\
\hline $\begin{array}{l}55 \\
56 \\
57 \\
58 \\
59\end{array}$ & $\begin{array}{l}80 \\
77 \\
.74 \\
71 \\
68\end{array}$ & $\begin{array}{r}8.5089906 \\
8.5089896 \\
87 \\
77 \\
67\end{array}$ & $\begin{array}{l}175 \\
209 \\
243 \\
277 \\
311\end{array}$ & $\begin{array}{l}58 \\
56 \\
53 \\
51 \\
49\end{array}$ & $\begin{array}{l}46 \\
52 \\
58 \\
65 \\
71\end{array}$ & , \\
\hline 60 . & 8.5084964 & 8.508 9858 & 1. 75344 & 2.2647 & 6.8177 & 7.471 \\
\hline
\end{tabular}


TA Rt. 20.-Geodetic position computations-Continued.

LATTTUDE $66^{\circ}$

\begin{tabular}{|c|c|c|c|c|c|c|}
\hline Lat. & $\begin{array}{c}\log \mathrm{A} \\
\text { diff. } 1^{\prime \prime}=-0.05\end{array}$ & $\operatorname{diff} 1^{\log B}=-0.16$ & $\begin{array}{c}\log \mathrm{C} \\
\text { diff. } 1^{\prime \prime}=+0.5\end{array}$ & $\begin{array}{c}\log \mathrm{D} \\
\text { diff. } 1^{\prime \prime}=-0.04\end{array}$ & $\begin{array}{c}\log \mathrm{E} \\
\text { diff. } 1^{\prime \prime=}=0.11\end{array}$ & $\begin{array}{c}\log F \\
\text { diff. } 10^{\prime}=-5.3\end{array}$ \\
\hline $\begin{array}{rr}0 & 1 \\
66 & 00 \\
& 1 \\
& 2 \\
& 3 \\
& 4\end{array}$ & $\begin{array}{r}8.5084964 \\
61 \\
58 \\
55 \\
52\end{array}$ & $\begin{array}{r}8.5089858 \\
48 \\
39 \\
29 \\
20\end{array}$ & $\begin{array}{r}1.75344 \\
378 \\
412 \\
446 \\
.480\end{array}$ & $\begin{array}{r}2.2647 \\
44 \\
42 \\
40 \\
38\end{array}$ & $\begin{array}{r}6.8177 \\
\quad \quad 84 \\
\times \quad 90 \\
6.8196 \\
6.8203\end{array}$ & 7.471 \\
\hline $\begin{array}{r}05 \\
6 \\
7 \\
8 \\
9\end{array}$ & $\begin{array}{l}48 \\
45 \\
42 \\
39 \\
36\end{array}$ & $\begin{array}{r}10 \\
8.5089801 \\
8.5089791 \\
82 \\
72\end{array}$ & $\begin{array}{l}514 \\
548 \\
.582 \\
616 \\
650\end{array}$ & $\begin{array}{l}35 \\
33 \\
31 \\
28 \\
26\end{array}$ & $\begin{array}{l}09 \\
16 \\
22 \\
28 \\
35\end{array}$ & \\
\hline $\begin{array}{l}10 \\
11 \\
12 \\
13 \\
14\end{array}$ & $\begin{array}{r}8.5084933 \\
29 \\
26 \\
23 \\
20 \\
2\end{array}$ & $\begin{array}{r}8.5089762 \\
: 53 \\
43 \\
34 \\
24\end{array}$ & $\begin{array}{r}.1 .75684 \\
718 \\
752 \\
786 \\
820\end{array}$ & $\begin{array}{r}2.2624 \\
22 \\
19 \\
17 \\
15\end{array}$ & $\begin{array}{r}\text { 6. } 8241 \\
48 \\
54 \\
61 \\
67\end{array}$ & \\
\hline $\begin{array}{l}15 \\
16 \\
17 \\
18 \\
19\end{array}$ & $\begin{array}{l}17 \\
.13 \\
10 \\
07 \\
04\end{array}$ & $\begin{array}{r}14 \\
8.5089705 \\
3.5089696 \\
86 \\
77\end{array}$ & $\begin{array}{r}854 \\
889 \\
923 \\
957^{\circ} \\
1.75991\end{array}$ & $\begin{array}{l}12 \\
10 \\
08 \\
05 \\
03\end{array}$ & $\begin{array}{r}73 \\
80 \\
\cdot \quad 86 \\
93 \\
6.8299\end{array}$ & \\
\hline $\begin{array}{l}20 \\
21 \\
22 \\
23 \\
24\end{array}$ & $\begin{array}{r}8.5084901 \\
\text { 8. } 5084898 \\
95 \\
91 \\
88\end{array}$ & $\begin{array}{r}3.5089607 \\
58 \\
48 \\
39 \\
. \quad 29\end{array}$ & $\begin{array}{r}1.76025 \\
060 \\
094 \\
128 \\
163\end{array}$ & $\begin{array}{r}2.2601 \\
\text { 2. } 2598 \\
\quad 96 \\
94 \\
91\end{array}$ & $\begin{array}{r}6.8300 \\
12 \\
19 \\
25 \\
31\end{array}$ & 7.461 \\
\hline $\begin{array}{l}25 \\
26 \\
27 \\
28 \\
29\end{array}$ & $\begin{array}{l}85 \\
82 \\
79 \\
76 \\
73\end{array}$ & $\begin{array}{rr}20 \\
11 \\
\text { 8. } 5089601 \\
8.508 \quad 9592 \\
\quad 82\end{array}$ & $\begin{array}{l}197 \\
231 \\
266 \\
300 \\
334\end{array}$ & $\begin{array}{l}89 \\
87 \\
84 \\
82 \\
80\end{array}$ & $\begin{array}{l}38 \\
44 \\
51 \\
57 \\
64\end{array}$ & \\
\hline $\begin{array}{l}30 \\
31 \\
32 \\
33 \\
34\end{array}$ & $\begin{array}{r}8.5084869 \\
66 \\
63 \\
60 \\
57\end{array}$ & $\begin{array}{r}8.5089573 \\
63 \\
54 \\
44 \\
35\end{array}$ & $\begin{array}{r}1.76369 \\
403 \\
438 \\
472 \\
507\end{array}$ & $\begin{array}{r}2.2578 \\
75 \\
73 \\
70 \\
68\end{array}$ & $\begin{array}{r}6: 8370 \\
77 \\
83 \\
90 \\
6.8396\end{array}$ & \\
\hline $\begin{array}{l}35 \\
36 \\
37 \\
38 \\
39\end{array}$ & $\begin{array}{l}54 \\
50 \\
47 \\
44 \\
41\end{array}$ & $\begin{array}{rr} & 25 \\
& 16 \\
8.508 & 9507 \\
8.508 & 9497 \\
. \quad 88\end{array}$ & $\begin{array}{l}541 \\
576 \\
610 \\
645 \\
679\end{array}$ & $\begin{array}{l}66 \\
63 \\
61 \\
59 \\
56\end{array}$ & $\begin{array}{r}6.8403 \\
09 \\
16 \\
22 \\
29\end{array}$ & \\
\hline $\begin{array}{l}40 \\
41 \\
42 \\
43 \\
44\end{array}$ & $\begin{array}{r}8.5084838 \\
35 \\
32 \\
29 \\
26\end{array}$ & $\begin{array}{r}8.508 \quad 9478 \\
69 \\
\quad 60 \\
\quad \quad 51 \\
\quad 41\end{array}$ & $\begin{array}{r}1.76714 \\
749 \\
783 \\
818 \\
853\end{array}$ & $\begin{array}{r}2.2554 \\
51 \\
49 \\
47 \\
44\end{array}$ & $\begin{array}{r}6.8436 \\
. \quad 42 \\
49 \\
55 \\
62\end{array}$ & 7.450 \\
\hline $\begin{array}{l}45 \\
46 \\
47 \\
48 \\
49\end{array}$ & $\begin{array}{l}22 \\
19 \\
16 \\
13 \\
10\end{array}$ & $\begin{array}{r}32 \\
23 \\
13 \\
8.5089404 \\
8.5089395\end{array}$ & $\begin{array}{r}887 \\
922 \\
957 \\
1.76991 \\
1.77026\end{array}$ & $\begin{array}{l}42 \\
39 \\
37 \\
35 \\
32\end{array}$ & $\begin{array}{r}68 \\
75 \\
81 . \\
88 \\
6.8495\end{array}$ & \\
\hline $\begin{array}{l}50 \\
51 \\
52 \\
53 \\
54\end{array}$ & $\begin{array}{r}8.5084807 \\
04 \\
8.5084801 \\
8.508 \quad 4797 \\
94\end{array}$ & $\begin{array}{r}8.5089385 \\
76 \\
66 \\
57 \\
48\end{array}$ & $\begin{array}{r}1.77061 \\
096 \\
131 \\
166 \\
200\end{array}$ & $\begin{array}{r}2.2530 \\
27 \\
25 \\
23 \\
20\end{array}$ & $\begin{array}{r}6.8501 \\
08 \\
14 \\
21 \\
27\end{array}$ & - \\
\hline $\begin{array}{l}55 \\
56 \\
57 \\
58 \\
59\end{array}$ & $\begin{array}{r}91 \\
88 \\
-.85 \\
82 \\
79\end{array}$ & $\begin{array}{r}38 \\
29 \\
20 \\
10 \\
8.5089301\end{array}$ & $\begin{array}{l}235 \\
270 \\
305 \\
340 \\
375\end{array}$ & $\begin{array}{l}18 \\
15 \\
\cdot 13 \\
11 \\
08\end{array}$ & $\begin{array}{l}34 \\
41 \\
47 \\
54 \\
60\end{array}$ & \\
\hline 60 & 8.5084776 & 8. 5089292 & 1.77410 & 2.2500 & 6.8567 & 7.440 \\
\hline
\end{tabular}


TaBle 20.-Geodetic position computations-Continued.

IAATITUDE $67^{\circ}$

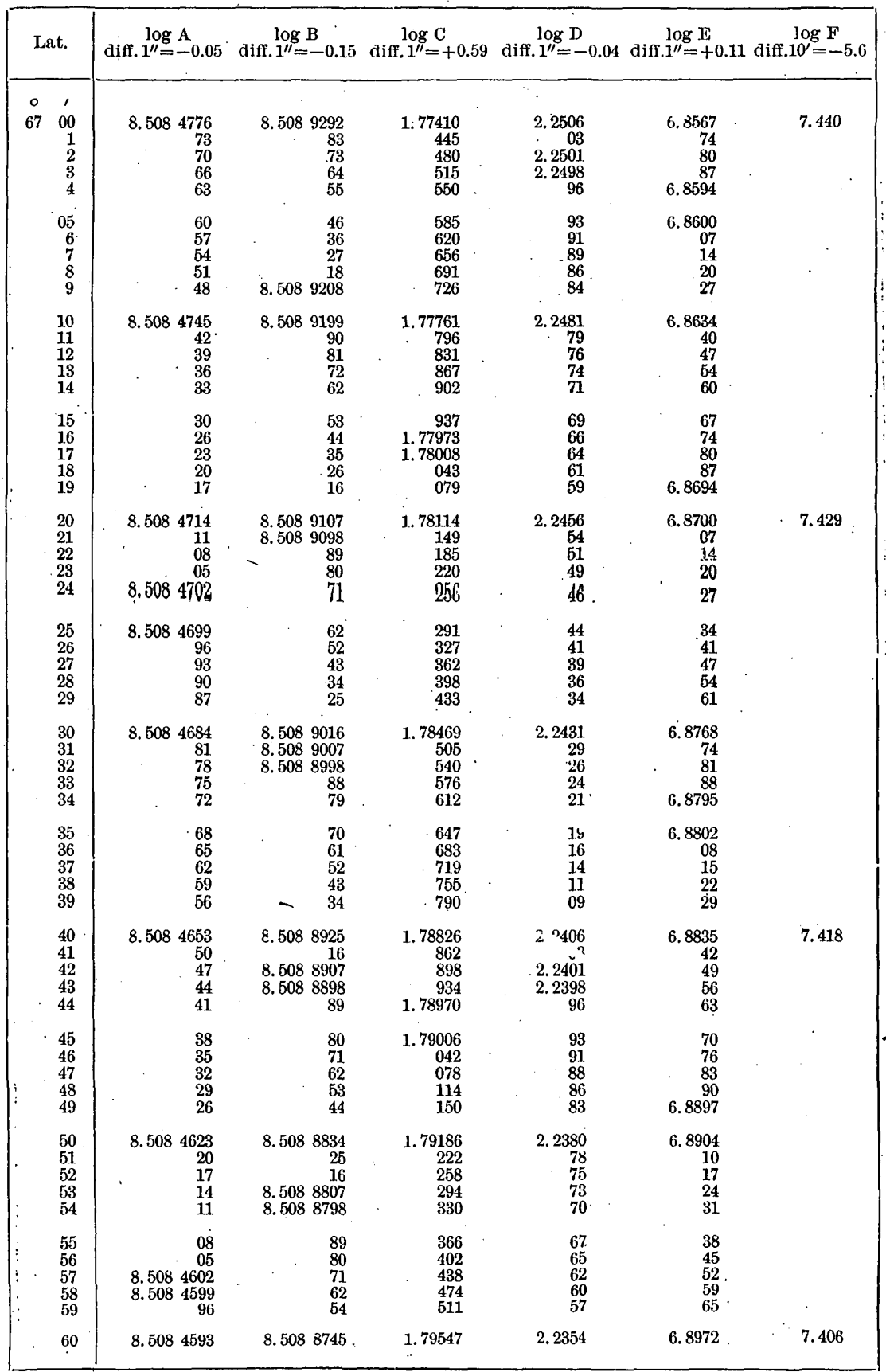


TaBta 20.-Geodetic position computations-Continned.

LATITUDE $68^{\circ}$.

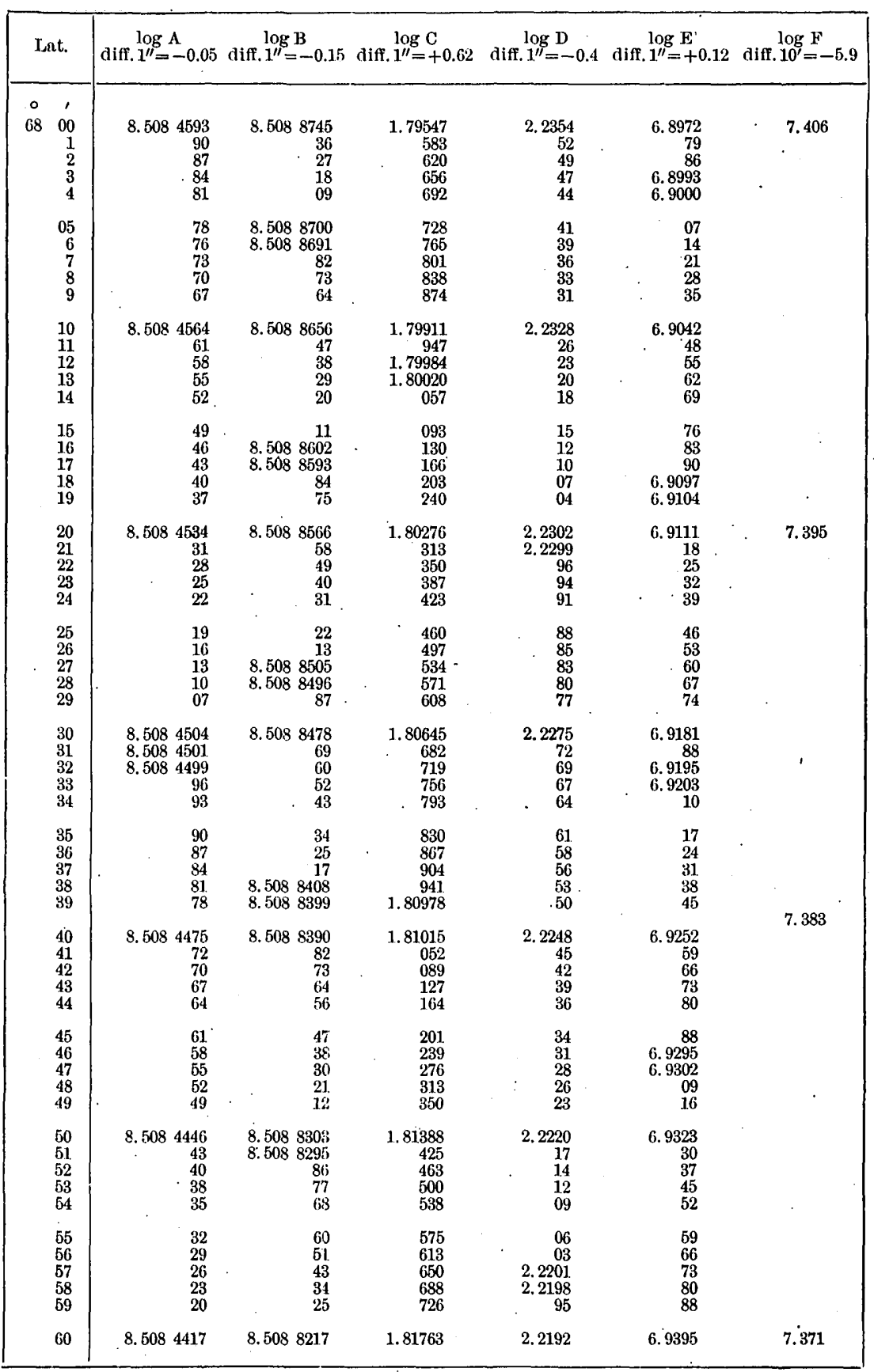


TABre 20.-Geodetic position computations-Continued.

LATITUDE $69^{\circ}$.

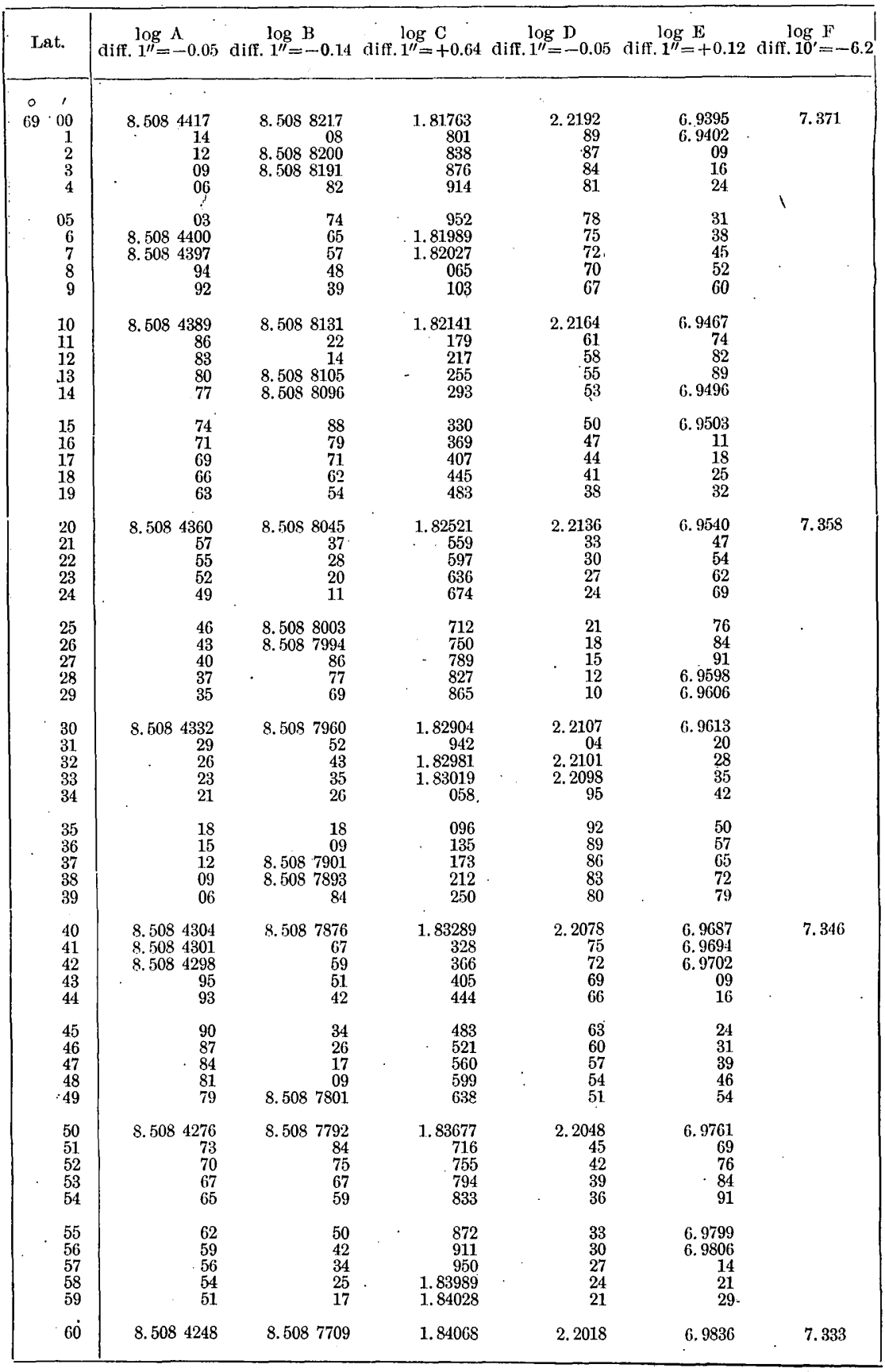


Тавце 20.-Geodetic position computations-Continned.

LATITUDE $70^{\circ}$.

\begin{tabular}{|c|c|c|c|c|c|c|}
\hline Lat. & diff. $1^{\log A}=-0.0$ & liff. $1^{\prime \prime}=-0.14$ & diff. $1^{\log C}=+0.67$ & $\begin{array}{c}\log \mathrm{D} \\
\text { diff, } 1^{\prime \prime}=-0.05\end{array}$ & $\begin{array}{c}\log \mathbf{E} \\
\text { diff. } 1^{\prime \prime}=+0.13\end{array}$ & $\begin{array}{c}\log F \\
\text { diff. } 10^{\prime}=-6.7\end{array}$ \\
\hline 0, & & & - & & & \\
\hline $\begin{array}{rr}70 \quad 00 \\
& 1 . \\
& 2 \\
& 3 \\
& 4\end{array}$ & $\begin{array}{r}8.508 \quad 4248 \\
45 \\
43 \\
40 \\
37\end{array}$ & $\begin{array}{r}8.5087709 \\
8.5087701 \\
8.5087692 \\
84 \\
76\end{array}$ & $\begin{array}{r}1.84068 \\
107 \\
146 \\
185 \\
225\end{array}$ & $\begin{array}{r}2.2018 \\
15 \\
12 \\
09 \\
06\end{array}$ & $\begin{array}{r}6.9836 \\
44 \\
. \quad 51 \\
59 \\
60\end{array}$ & 7.333 \\
\hline $\begin{array}{r}05 \\
6 \\
7 \\
8 \\
9\end{array}$ & $\begin{array}{r}34 \\
32 \\
29 \\
. \quad 26 \\
\quad 23\end{array}$ & $\begin{array}{l}68 \\
59 \\
51 \\
43 \\
35\end{array}$ & $\begin{array}{l}.264 \\
303 \\
343 \\
382 \\
421\end{array}$ & $\begin{array}{r}03 \\
2.2000 \\
2.1 .997 \\
94 \\
91\end{array}$ & $\begin{array}{r}74 \\
81 \\
89 \\
6.9896 \\
\text { 6. } 9904\end{array}$ & \\
\hline $\begin{array}{r}10 \\
11 \\
12 \\
.13 \\
14\end{array}$ & $\begin{array}{r}8.508 \quad 4221 \\
1.8 \\
15 \\
12 \\
10\end{array}$ & $\begin{array}{r}8.5087626 \\
18 \\
10 \\
10 \\
8.508 \quad 7602 \\
8.5087594\end{array}$ & $\begin{array}{r}1.84461 \\
. \quad 500 \\
540 \\
579 \\
619\end{array}$ & 2. $\begin{array}{r}1988 \\
85 \\
82 \\
79 \\
76\end{array}$ & $\begin{array}{r}6.9912 \\
19 \\
27 \\
34 \\
42\end{array}$ & $\therefore$ \\
\hline $\begin{array}{l}15 \\
16 \\
17 \\
18 \\
19\end{array}$ & $\begin{array}{r}07 \\
04 \\
8.5084201 \\
8.508 \quad 41.99 \\
96\end{array}$ & $\begin{array}{l}86 \\
78 \\
69 \\
61 \\
52\end{array}$ & $\begin{array}{l}658 \\
698 \\
738 \\
778 \\
817\end{array}$ & $\begin{array}{c}73 \\
70 \\
66 \\
63 \\
60\end{array}$ & $\begin{array}{l}50 \\
57 \\
65 \\
73 \\
80\end{array}$ & . \\
\hline $\begin{array}{l}20 \\
21 \\
22 \\
23 \\
24\end{array}$ & $\begin{array}{r}8.50841 .93 \\
90 \\
88 \\
85 \\
82\end{array}$ & $\begin{array}{r}8.5087544 \\
36 \\
. \quad 23 \\
20 \\
12\end{array}$ & $\begin{array}{r}1.84857 \\
897 \\
937 \\
1.84976 \\
1.85016\end{array}$ & $\begin{array}{r}2.1957 \\
54 \\
51 \\
48 \\
45\end{array}$ & $\begin{array}{r}6.9988 \\
6.9995 \\
7.0003 \\
11 \\
18\end{array}$ & 7.320 \\
\hline $\begin{array}{l}25 \\
26 \\
27 \\
28 \\
29\end{array}$ & $\begin{array}{l}80 \\
77 \\
74 \\
71 \\
69\end{array}$ & $\begin{array}{r}8.508 \quad 7504 \\
\text { 8.508 7495 } \\
87 \\
79 \\
71\end{array}$ & $\begin{array}{l}056 \\
096 \\
136 \\
176 \\
\mathbf{2 1 6}\end{array}$ & $\begin{array}{r}42 \\
39 \\
36 \\
33 \\
29\end{array}$ & $\begin{array}{r}26 \\
34 \\
41 \\
49 \\
57\end{array}$ & \\
\hline $\begin{array}{l}30 \\
31 \\
32 \\
33 \\
34\end{array}$ & $\begin{array}{r}8.508 \quad 4166 \\
63 \\
60 \\
58 \\
55\end{array}$ & $\begin{array}{r}8.5087462 \\
54 \\
46 \\
38 \\
30\end{array}$ & $\begin{array}{r}1.85256 \\
296 \\
336 \\
376 \\
416\end{array}$ & $\begin{array}{r}2.1926 \\
23 \\
20 \\
17 \\
14\end{array}$ & $\begin{array}{r}7.0064 \\
72 \\
80 \\
88 \\
7.0095\end{array}$ & 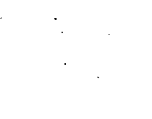 \\
\hline $\begin{array}{l}35 \\
36 \\
37 \\
38 \\
39\end{array}$ & $\begin{array}{l}52 \\
50 \\
47 \\
44 \\
42\end{array}$ & $\begin{array}{r}22 \\
14 \\
8.508 \quad 7401 \\
8.508 \quad 7398 \\
90\end{array}$ & $\begin{array}{l}456 \\
497 \\
537 \\
577 \\
618\end{array}$ & $\begin{array}{r}11 \\
08 \\
04 \\
2.1901 \\
2.1898\end{array}$ & $\begin{array}{r}7.0103 \\
11 \\
19 \\
26 \\
34\end{array}$ & . \\
\hline $\begin{array}{l}40 \\
41 \\
42 \\
43 \\
44\end{array}$ & $\begin{array}{r}8.508 \quad 4139 \\
36 \\
34 \\
31 \\
28\end{array}$ & $\begin{array}{r}8.5087382 \\
74 \\
66 \\
58 \\
50 \\
5 .\end{array}$ & $\begin{array}{r}1.85658 \\
698 \\
739 \\
. \quad 779 \\
819\end{array}$ & $\begin{array}{r}2.1895 \\
92 \\
89 \\
85 \\
82\end{array}$ & $\begin{array}{r}7.0142 \\
50 \\
57 \\
65 \\
73\end{array}$ & 7.307 \\
\hline $\begin{array}{l}45 \\
46 \\
47 \\
48 \\
49\end{array}$ & $\begin{array}{l}26 \\
23 \\
20 \\
1.8 \\
1.5\end{array}$ & $\begin{array}{l}42 \\
34 \\
26 \\
18 \\
10\end{array}$ & $\begin{array}{r}860 \\
900 \\
941 \\
1.85981 \\
1.86022\end{array}$ & $\begin{array}{r}79 \\
76 \\
73 \\
70 \\
.66\end{array}$ & $\begin{array}{r}81 \\
88 \\
7.0196 \\
7.0204 \\
12\end{array}$ & . \\
\hline $\begin{array}{l}50 \\
51 \\
52 \\
53 \\
54\end{array}$ & $\begin{array}{r}8.508 \quad 41112 \\
10 \\
07 \\
04 \\
8.508 \quad 4101\end{array}$ & $\begin{array}{r}8.5087302 \\
8.508 \quad 7294 \\
84 \\
77 \\
69\end{array}$ & $\begin{array}{r}1.86063 \\
103 \\
144 \\
185 \\
225\end{array}$ & $\begin{array}{r}2.1863 \\
.60 \\
57 \\
54 \\
50\end{array}$ & $\begin{array}{r}7.0220 \\
27 \\
35 \\
43 \\
51\end{array}$ & 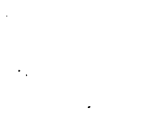 \\
\hline $\begin{array}{l}55 \\
56 \\
57 \\
58 \\
59\end{array}$ & $\begin{array}{r}8.508 \quad 4099 \\
96 \\
93 \\
91 \\
88\end{array}$ & $\begin{array}{l}61 \\
53 \\
45 \\
38 \\
30\end{array}$ & $\begin{array}{r}266 \\
307 \\
348 \\
389 \\
430\end{array}$ & $\begin{array}{l}47 \\
44 \\
41 \\
38 \\
34\end{array}$ & $\begin{array}{r}59 \\
-\quad 67 \\
75 \\
82 \\
90\end{array}$ & - \\
\hline 60 & $8.508 \cdot 4086$ & 8.508 7222 & 1.86470 & 2.1831 & 7.0298 & . 7.293 \\
\hline
\end{tabular}


TABLE 20.-Geodetic position computations-Continued.

LATITUDE $71^{\circ}$.

\begin{tabular}{|c|c|c|c|c|c|c|}
\hline Lat. & diff. $1^{\prime \prime}=-0.04$ & diff. $1^{\prime \prime}=-0.13$ & diff. $1^{\prime \prime}=+0.70$. & diff. $1^{\prime \prime}=-0.05$ & $\begin{array}{c}\log \mathrm{E} \\
\text { diff. } 1^{\prime \prime}=+0.13\end{array}$ & diff. $10^{\prime \prime}=-7.2$ \\
\hline , & & & - & & & \\
\hline $\begin{array}{rr}71 & 00 \\
& 1 \\
& 2 \\
& 3 \\
& 4\end{array}$ & $\begin{array}{r}8.508 \quad 4086 \\
83 \\
80 \\
\quad 78 \\
75\end{array}$ & $\begin{array}{r}8.5087222 \\
14 \\
8.5087206 \\
8.5087198 \\
90\end{array}$ & $\begin{array}{r}1.86470 \\
511 \\
552 \\
593 \\
634\end{array}$ & $\begin{array}{r}2.1831 \\
28 \\
25 \\
21 \\
18\end{array}$ & $\begin{array}{r}7.0298 \\
7.0306 \\
14 \\
22 \\
30\end{array}$ & 7.293 \\
\hline $\begin{array}{r}05 \\
6 \\
7 \\
8 \\
9\end{array}$ & $\begin{array}{r}72 \\
70 \\
67 \\
64 \\
62\end{array}$ & $\begin{array}{r}82 \\
74 \\
66 \\
58 \\
50\end{array}$ & $\begin{array}{l}675 \\
717 \\
758 \\
799 \\
840\end{array}$ & $\begin{array}{r}15 \\
12 \\
08 \\
05 \\
2.1802\end{array}$ & $\begin{array}{l}38 \\
46 \\
54 \\
62 \\
70\end{array}$ & \\
\hline $\begin{array}{l}10 \\
11 \\
12 \\
13 \\
14\end{array}$ & $\begin{array}{r}8.508 \quad 4059 \\
57 \\
54 \\
51 \\
49\end{array}$ & $\begin{array}{r}8.5087142 \\
.34 \\
27 \\
19 \\
11\end{array}$ & $\begin{array}{r}1.86881 \\
923 \\
1.86964 \\
1.87005 \\
046\end{array}$ & $\begin{array}{r}2.1799 \\
95 \\
92 \\
89 \\
86\end{array}$ & $\begin{array}{r}7.0378 \\
85 \\
7.0393 \\
7.0401 \\
09\end{array}$ & - \\
\hline $\begin{array}{l}15 \\
16 \\
17 \\
18 \\
19\end{array}$ & $\begin{array}{l}46 \\
43 \\
41 \\
38 \\
36\end{array}$ & $\begin{array}{r}8.5087103 \\
8.508 \quad 7095 \\
87 \\
79 \\
72\end{array}$ & $\begin{array}{l}088 \\
129 \\
171 \\
212 \\
254\end{array}$ & $\begin{array}{l}82 \\
79 \\
76 \\
72 \\
69\end{array}$ & $\begin{array}{l}17 \\
25 \\
33 \\
41 \\
49\end{array}$ & . \\
\hline $\begin{array}{l}20 \\
21 \\
22 \\
23 \\
24\end{array}$ & $\begin{array}{r}8.508 \quad 4033 \\
30 \\
28 \\
25 \\
23\end{array}$ & $\begin{array}{r}8.508 \quad 7064 \\
56 \\
48 \\
40 \\
53\end{array}$ & $\begin{array}{r}1.87295 \\
337 \\
378 \\
420 \\
462\end{array}$ & $\begin{array}{r}2.1766 \\
62 \\
59 \\
56 \\
52\end{array}$ & $\begin{array}{r}7.0457 \\
65 \\
73 \\
82 \\
90\end{array}$ & $\begin{array}{r}7.279 \\
.\end{array}$ \\
\hline $\begin{array}{l}25 \\
26 \\
27 \\
28 \\
29\end{array}$ & $\begin{array}{l}20 \\
17 \\
15 \\
12 \\
10\end{array}$ & $\begin{array}{r}25 \\
17 \\
09 \\
8.5087002 \\
8.5086994\end{array}$ & $\begin{array}{l}503 \\
545 \\
587 \\
629 \\
671\end{array}$ & $\begin{array}{l}49 \\
46 \\
42 \\
39 \\
36\end{array}$ & $\begin{array}{r}7.0498 \\
7.0506 \\
14 \\
22 \\
30\end{array}$ & \\
\hline $\begin{array}{l}30 \\
31 \\
32 \\
33 \\
34\end{array}$ & $\begin{array}{r}8.5084007 \\
05 \\
8.5084002 \\
8.508 \quad 3999 \\
97\end{array}$ & $\begin{array}{r}8.5086986 \\
78 \\
71 \\
63 \\
55\end{array}$ & $\begin{array}{r}1.87712 \\
754 \\
.796 \\
838 \\
880\end{array}$ & $\begin{array}{r}2.1732 \\
29 \\
26 \\
22 \\
19\end{array}$ & $\begin{array}{r}7.0538 \\
46 \\
54 \\
62 \\
70\end{array}$ & \\
\hline $\begin{array}{l}35 \\
36 \\
37 \\
38 \\
39\end{array}$ & $\begin{array}{l}94 \\
92 \\
89 \\
86 \\
84\end{array}$ & $\begin{array}{l}47 \\
40 \\
32 \\
24 \\
16\end{array}$ & $\begin{array}{r}922 \\
1.87964 \\
1.88006 \\
049 \\
091\end{array}$ & $\begin{array}{r}16 \\
12 \\
09 \\
06 \\
2.1702\end{array}$ & $\begin{array}{r}79 \\
87 \\
7.0595 \\
7.0603 \\
11\end{array}$ & . \\
\hline $\begin{array}{l}40 \\
41 \\
42 \\
43 \\
44\end{array}$ & $\begin{array}{r}8.5083981 \\
79 \\
76 \\
74 \\
. \quad 71\end{array}$ & $\begin{array}{r}8.5086908 \\
8.5086901 \\
8.5086893 \\
85 \\
78\end{array}$ & $\begin{array}{r}1.88133 \\
175 \\
217 \\
260 \\
302\end{array}$ & $\begin{array}{r}2.1699 \\
95 \\
92 \\
89 \\
85\end{array}$ & $\begin{array}{r}7.0619 \\
27 \\
.36 \\
44 \\
52\end{array}$ & 7.265 \\
\hline $\begin{array}{l}45 \\
.46 \\
47 \\
48 \\
49\end{array}$ & $\begin{array}{l}68 \\
66 \\
63 \\
61 \\
58\end{array}$ & $\begin{array}{r}70 \\
62 \\
55 \\
47 \\
40\end{array}$ & $\begin{array}{l}344 \\
387 \\
429 \\
472 \\
514\end{array}$ & $\begin{array}{l}82 \\
78 \\
75 \\
72 \\
68\end{array}$ & $\begin{array}{r}60 \\
68 \\
77 \\
85 \\
7.0693\end{array}$ & \\
\hline $\begin{array}{l}50 \\
51 \\
52 \\
53 \\
54\end{array}$ & $\begin{array}{r}8.5083956 \\
53 \\
51 \\
48 \\
46\end{array}$ & $\begin{array}{r}8.5086832 \\
24 \\
17 \\
.09 \\
8.5086802\end{array}$ & $\begin{array}{r}1.88557 \\
599 \\
642 \\
685 \\
727\end{array}$ & $\begin{array}{r}2.1665 \\
61 \\
58 \\
54 \\
51\end{array}$ & $\begin{array}{r}7.0701 \\
09 \\
18 \\
26 \\
34\end{array}$ & . \\
\hline $\begin{array}{l}55 \\
56 \\
57 \\
58 \\
59\end{array}$ & $\begin{array}{l}43 \\
41 \\
38 \\
36 \\
33\end{array}$ & $\begin{array}{r}8.508 \quad 6794 \\
86 \\
79 \\
71 \\
64\end{array}$ & $\begin{array}{l}770 \\
813 \\
855 \\
898 \\
941\end{array}$ & $\begin{array}{r}48 \\
44 \\
-\quad 41 \\
37 \\
34\end{array}$ & $\begin{array}{l}42 \\
51 \\
59 \\
67 \\
75\end{array}$ & $\cdot$ \\
\hline 60 & 8.5083930 & 8.5086756 & 1.88984 & 2.1630 & 7.0784 & 7.250 \\
\hline
\end{tabular}


Table of values of $\log \sec \frac{1}{2}(\cdot \Delta \phi)$.

\begin{tabular}{|c|c|c|c|c|c|c|c|c|c|}
\hline$\Delta \phi$ & $\log _{(\Delta \phi)} \sec \frac{1}{(2)}$ & $\Delta \varphi$ & $\log _{(\Delta \Phi)} \sec \frac{1}{x}$ & $\Delta \phi$ & $\log _{(\Delta \varphi)} \sec \frac{1}{\Delta}$ & $\Delta \varphi$ & $\log _{(\Delta \phi)} \sec \frac{1}{9}$ & $\Delta \phi$ & $\underset{(\Delta \Phi)}{\log \sec \frac{1}{6}}$ \\
\hline $\begin{array}{r}1 \\
10 \\
11 \\
1.2 \\
13 \\
14\end{array}$ & $\begin{array}{r}0.000000 \\
1 \\
1 \\
1 \\
1 \\
1\end{array}$ & $\begin{array}{l}, \\
28 \\
29 \\
30 \\
31 \\
32\end{array}$ & $\begin{array}{rr}0.000004 \\
. \quad 4 \\
\quad 4 \\
. \quad 4 \\
\quad 4\end{array}$ & $\begin{array}{r}\prime \\
46 \\
47 \\
48 \\
49 \\
50\end{array}$ & $\begin{array}{r}0.000010 \\
10 \\
11 \\
11 \\
11\end{array}$ & $\begin{array}{r}\prime \\
64 \\
65 \\
66 \\
67 \\
68\end{array}$ & $\begin{array}{r}0.000019 \\
19 \\
20 \\
21 \\
21\end{array}$ & $\begin{array}{r} \\
82 \\
83 \\
84 \\
85 \\
86\end{array}$ & $\begin{array}{r}0.000 \quad 031 \\
32 \\
32 \\
33 \\
34\end{array}$ \\
\hline $\begin{array}{l}15 \\
16 \\
17 \\
18 \\
19\end{array}$ & $\begin{array}{l}1 \\
1 \\
1 \\
1 \\
2\end{array}$ & $\begin{array}{l}33 \\
34 \\
35 \\
36 \\
37\end{array}$ & $\begin{array}{l}5 \\
5 \\
6 \\
6 \\
6\end{array}$ & $\begin{array}{l}51 \\
52 \\
53 \\
54 \\
55\end{array}$ & $\begin{array}{l}12 \\
12 \\
13 \\
13 \\
14\end{array}$ & $\begin{array}{l}69 \\
70 \\
71 \\
72 \\
73\end{array}$ & $\begin{array}{l}22 \\
22 \\
23 \\
24 \\
24\end{array}$ & $\begin{array}{l}87 \\
88 \\
89 \\
90 \\
91\end{array}$ & $\begin{array}{l}35 \\
36 \\
36 \\
37 \\
38\end{array}$ \\
\hline $\begin{array}{l}20 \\
21 \\
22 \\
23 \\
24\end{array}$ & $\begin{array}{r}2 \\
2 \\
2 \\
2 \\
2 \\
3\end{array}$ & $\begin{array}{l}38 \\
39 \\
40 \\
41 \\
42\end{array}$ & $\begin{array}{l}7 \\
7 \\
7 \\
8 \\
8\end{array}$ & $\begin{array}{l}56 \\
57 \\
58 \\
59 \\
60\end{array}$ & $\begin{array}{l}14 \\
15 \\
15 \\
16 \\
16\end{array}$ & $\begin{array}{l}74 \\
75 \\
76 \\
77 \\
78\end{array}$ & $\begin{array}{l}25 \\
26 \\
26 \\
27 \\
28\end{array}$ & $\begin{array}{l}92 \\
93 \\
94 \\
95 \\
96\end{array}$ & $\begin{array}{r}39 \\
. \quad 40 \\
41 \\
41 \\
42\end{array}$ \\
\hline $\begin{array}{l}25 \\
26 \\
27\end{array}$ & $\begin{array}{l}3 \\
3 \\
3\end{array}$ & $\begin{array}{l}43 \\
44 \\
45\end{array}$ & $\begin{array}{l}8 \\
9 \\
9\end{array}$ & $\begin{array}{l}61 \\
62 \\
63\end{array}$ & $\begin{array}{l}17 \\
18 \\
18\end{array}$ & $\begin{array}{l}79 \\
80 \\
81\end{array}$ & $\begin{array}{l}29 \\
29 \\
30\end{array}$ & $\begin{array}{l}97 \\
98 \\
99\end{array}$ & $\begin{array}{l}43 \\
44 \\
45\end{array}$ \\
\hline
\end{tabular}

\section{To convert:}

Meters to feet.

$\begin{array}{lrllll}1 & = & 3.280 & 833 & 1 & 1 \\ 2 & 6.561 & 667 & 2 & 0.304 & 8006 \\ 3 & 9.842 & 500 & 3 & 0.909 & 0012 \\ 4 & 13.123 & 333 & 4 & 1.219 & 4018 \\ 5 & 16.404 & 166 & 5 & 1.524 & 0030 \\ 6 & 19.685 & 000 & 6 & 1.828 & 8037 \\ 7 & 22.965 & 833 & 7 & 2.133 & 6048 \\ 8 & 26.246 & 666 & 8 & 2.438 & 4049 \\ 9 & 29.527 & 500 & 9 & 2.743 & 2055\end{array}$

To convert:

Kilometers to stat- Statute miles to ute miles. kilometers.

\begin{tabular}{|c|c|c|}
\hline & \\
\hline $1=0.6213699$ & $1=$ & 1. 609347 \\
\hline 1.2427399 & & 3. 218694 \\
\hline 1. 864.1098 & & $4.828 \quad 042$ \\
\hline $2.485 \quad 4798$ & 4 & 6.437389 \\
\hline 3.1068497 & 5 & 46736 \\
\hline 3. 7282196 & 6 & 56083 \\
\hline 4.3495896 & 7 & 11.265430 \\
\hline 4.9709595 & 8 & 12.874778 \\
\hline 23295 & 9 & 14.484 \\
\hline
\end{tabular}


Table of corrections to longitude for difference in arc and sine.

\begin{tabular}{|c|c|c|c|c|c|c|c|c|c|}
\hline $\log s(-)$ & \multicolumn{2}{|c|}{$\begin{array}{l}\log \text { dif- } \\
\text { ference. }\end{array}$} & $\log \Delta \lambda(t)$ & $\log s(-)$ & $\begin{array}{l}\log \text { dif- } \\
\text { ference. }\end{array}$ & $\log \Delta \lambda(+)$ & $\log s(-)$ & $\begin{array}{l}\log \text { dif- } \\
\text { ference. }\end{array}$ & $\log \Delta \lambda(t)$ \\
\hline $\begin{array}{r}3.876 \\
4.026 \\
4.114 \\
4.177 \\
4.225\end{array}$ & 0.000 & $\begin{array}{r}0001 \\
02 \\
03 \\
04 \\
05\end{array}$ & $\begin{array}{l}2.385 \\
2.535 \\
2.623 \\
2.686 \\
2.734\end{array}$ & $\begin{array}{r}4.871 \\
4.882 \\
4.892 \\
4.903 \\
4.913\end{array}$ & $\begin{array}{r}0.0000098 \\
103 \\
108 \\
114 \\
11.9\end{array}$ & $\begin{array}{l}3.380 \\
3.391 \\
3.401 \\
3.412 \\
3.422\end{array}$ & $\begin{array}{l}5.172 \\
5.178 \\
5.183 \\
5.188 \\
5.193\end{array}$ & $\begin{array}{r}0.000 \quad 0392 \\
402 \\
412 \\
422 \\
433\end{array}$ & $\begin{array}{l}3.681 \\
3.687 \\
3.692 \\
3.697 \\
3.702\end{array}$ \\
\hline $\begin{array}{l}4.265 \\
4.298 \\
4.327 \\
4.353 \\
4.376\end{array}$ & . & $\begin{array}{l}06 \\
07 \\
08 \\
09 \\
10\end{array}$ & $\begin{array}{l}2.774 \\
2.807 \\
2.836 \\
2.862 \\
2.885\end{array}$ & $\begin{array}{l}4.922 \\
4.932 \\
4.941 \\
4.950 \\
4.959\end{array}$ & $\begin{array}{l}124 \\
130 \\
136 \\
142 \\
147\end{array}$ & $\begin{array}{l}3.431 \\
3.441 \\
3.450 \\
3.459 \\
3.468\end{array}$ & $\begin{array}{l}5.199 \\
5.204 \\
5.209 \\
5.214 \\
5.219\end{array}$ & $\begin{array}{l}443 \\
453 \\
464 \\
474 \\
486\end{array}$ & $\begin{array}{l}3.708 \\
3.713 \\
3.718 \\
3.723 \\
3.728\end{array}$ \\
\hline $\begin{array}{l}4.396 \\
4.415 \\
4.433 \\
4.449 \\
4.464\end{array}$ & & $\begin{array}{l}11 \\
12 \\
13 \\
14 \\
15\end{array}$ & $\begin{array}{l}2.905 \\
2.924 \\
2.942 \\
2.958 \\
2.973\end{array}$ & $\begin{array}{l}4.968 \\
4.976 \\
4.985 \\
4.993 \\
5.002\end{array}$ & $\begin{array}{l}153 \\
160 \\
166 \\
172 \\
179\end{array}$ & $\begin{array}{l}3.477 \\
3.485 \\
3.494 \\
3.502 \\
3.511\end{array}$ & $\begin{array}{l}5.223 \\
5.228 \\
5.233 \\
5.238 \\
5.242\end{array}$ & $\begin{array}{l}497 \\
508 \\
519 \\
530 \\
541\end{array}$ & $\begin{array}{l}3.732 \\
3.737 \\
3.742 \\
3.747 \\
3.751\end{array}$ \\
\hline $\begin{array}{l}4.478 \\
4.491 \\
4.503 \\
4.526 \\
4.548\end{array}$ & & $\begin{array}{l}16 \\
17 \\
18 \\
20 \\
23\end{array}$ & $\begin{array}{l}2.987 \\
3.000 \\
3.012 \\
3.035 \\
3.057\end{array}$ & $\begin{array}{l}5.010 \\
5.017 \\
5.025 \\
5.033 \\
5.040\end{array}$ & $\begin{array}{l}186 \\
192 \\
199 \\
206 \\
213\end{array}$ & $\begin{array}{l}3.519 \\
3.526 \\
3.534 \\
3.542 \\
3.549\end{array}$ & $\begin{array}{l}5.247 \\
5.251 \\
5.256 \\
5.260 \\
5.265\end{array}$ & $\begin{array}{l}553 \\
565 \\
577 \\
588 \\
600\end{array}$ & $\begin{array}{l}3.756 \\
3.760 \\
3.765 \\
3.769 \\
3.774\end{array}$ \\
\hline $\begin{array}{l}4.570 \\
4.591 \\
4.612 \\
4.631 \\
4.649\end{array}$ & & $\begin{array}{l}25 \\
27 \\
30 \\
33 \\
36\end{array}$ & $\begin{array}{l}3.079 \\
3.100 \\
3.121 \\
3.140 \\
3.158\end{array}$ & $\begin{array}{l}5.047 \\
5.054 \\
5.062 \\
5.068 \\
5.075\end{array}$ & $\begin{array}{l}221 \\
228 \\
236 \\
243 \\
251\end{array}$ & $\begin{array}{l}3.556 \\
3.563 \\
3.571 \\
3.577 \\
3.584\end{array}$ & $\begin{array}{l}5.269 \\
5.273 \\
5.278 \\
5.282 \\
5.286\end{array}$ & $\begin{array}{l}613 \\
625 \\
637 \\
650 \\
663\end{array}$ & $\begin{array}{l}3.778 \\
3.782 \\
3.787 \\
3.791 \\
3.795\end{array}$ \\
\hline $\begin{array}{l}4.667 \\
4.684 \\
4.701 \\
4.716 \\
4.732\end{array}$ & & $\begin{array}{l}39 \\
42 \\
45 \\
48 \\
52\end{array}$ & $\begin{array}{l}3.176 \\
3.193 \\
3.210 \\
3.225 \\
3.241\end{array}$ & $\begin{array}{l}5.082 \\
5.088 \\
5.095 \\
5.102 \\
5.108\end{array}$ & $\begin{array}{r}259 \\
267 \\
275 \\
-\quad 284 \\
292\end{array}$ & $\begin{array}{l}3.591 \\
3.597 \\
3.604 \\
3.611 \\
3.617\end{array}$ & $\begin{array}{l}5.290 \\
5.294 \\
5.299 \\
5.303 \\
5.307\end{array}$ & $\begin{array}{l}674 \\
687 \\
702 \\
716 \\
729\end{array}$ & $\begin{array}{l}3.799 \\
3.803 \\
3.808 \\
3.812 \\
3.816\end{array}$ \\
\hline $\begin{array}{l}4.746 \\
4.761 \\
4.774 \\
4.788 \\
4.801\end{array}$ & & $\begin{array}{l}56 \\
59 \\
63 \\
67 \\
71\end{array}$ & $\begin{array}{l}3.255 \\
3.270 \\
3.283 \\
3.297 \\
3.310\end{array}$ & $\begin{array}{r}5.114 \\
5.120 \\
5.126 \\
5.132 \\
5.138\end{array}$ & $\begin{array}{l}300 \\
309 \\
318 \\
327 \\
336\end{array}$ & $\begin{array}{l}3.623 \\
3.629 \\
3.635 \\
3.641 \\
3.647\end{array}$ & $\begin{array}{l}5.311 . \\
5.315 \\
5.319 \\
5.323 \\
5.327\end{array}$ & $\begin{array}{l}743 \\
757 \\
771 \\
785 \\
800\end{array}$ & $\begin{array}{l}3.820 \\
3.824 \\
3.828 \\
3.832 \\
3.836\end{array}$ \\
\hline $\begin{array}{l}4.813 \\
4.825 \\
4.834 \\
4.849 \\
4.860\end{array}$ & . & $\begin{array}{l}75 \\
80 \\
84 \\
89 \\
94\end{array}$ & $\begin{array}{l}3.322 \\
3.334 \\
3.343 \\
3.358 \\
3.369\end{array}$ & $\begin{array}{l}5.144 \\
5.1 .50 \\
5.156 \\
5.161 \\
5.167\end{array}$ & $\begin{array}{l}345 \\
354 \\
364 \\
373 \\
383\end{array}$ & $\begin{array}{l}3.653 \\
3.659 \\
3.665 \\
3.670 \\
3.676\end{array}$ & $\begin{array}{l}5.331 . \\
5.335 \\
5.339 \\
5.343 \\
5.347\end{array}$ & $\begin{array}{r}814 \\
829 \\
845 \\
861 \\
877\end{array}$ & $\begin{array}{l}3.840 \\
3.844 \\
3.848 \\
3.852 \\
3.856\end{array}$ \\
\hline
\end{tabular}

INVERSE SOLUTION.

Having Latitudes and Longrtudes of Two Pontrs to Conpute Azimuthe and Distances.

The following example shows the method of performing the operation. The northernmost point should be used as the initial position, then all signs for (I), (II), and (III) are +, and for (IV) -. The value of $\Delta \lambda$ may be either + or - , but this sign need only be used in determining in which quadrant the azimuth angle $\alpha$ falls, i. e., the sign of $\tan \alpha(12)$. An inspection of a rough plat of the positions will also determine this. The correction to $\Delta \lambda$ is found from a distance scaled off from the plat, and need not be very close. In (8) the term $(\mathrm{I}+\mathrm{II})^{2}$ is the square of the difference of latitude $\Delta \varphi$ in seconds. Since (IV) is always small, $\log (\mathrm{I})$ in (8) may be taken as $\log$ of $\Delta \phi$ from (1). If $\cos \alpha$ is smaller than $\sin \alpha$, find $s$ from $\log 5 \cos \alpha$ in (11). As a check on the work compute the second 
position, using distance and azimuth found as above. The order of solution is shown by figures in parentheses. The cosines of latitudes are proportional to the intercepted parallels.
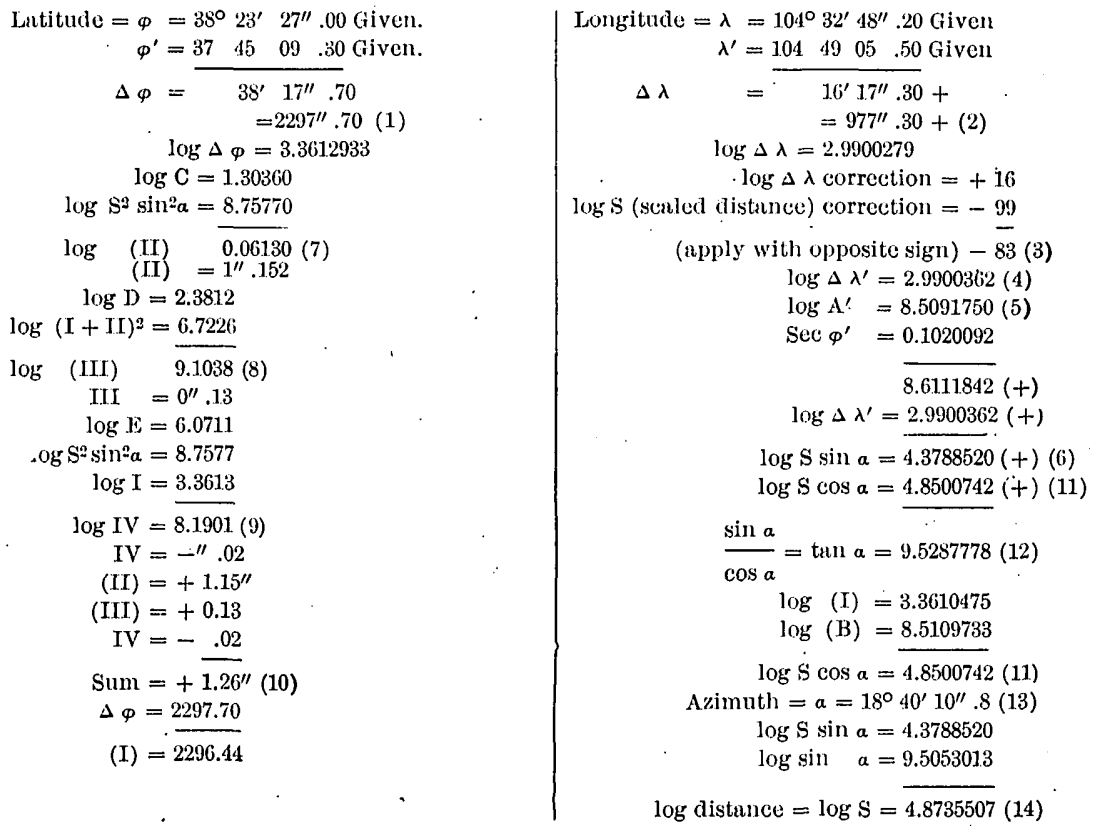
TABLE 21.-Log $m$, for use in computing spherical excess.

[Computed for the Clarke spheroid of 1866.]

\begin{tabular}{|c|c|c|c|c|c|c|c|}
\hline \multicolumn{2}{|c|}{ Lat. } & \multirow{2}{*}{$\log \mathrm{m}}$. & \multicolumn{2}{|c|}{ Lat. } & \multirow[t]{2}{*}{ Log $\mathrm{m}$. } & Lat. & \multirow[t]{2}{*}{$\log \mathrm{m}}$. \\
\hline$\circ$ & , & & 0 & , & & $\circ$ & \\
\hline 0 & 00 & 1. 40695 & 25 & 00 & 1.40590 & $50 \quad 00$ & 1. 40349 \\
\hline 0 & 30 & 1.40695 & & 30 & 1. 40586 & $50 \quad 30$ & 1. 40344 \\
\hline 1 & 00 & 1.40695 & 26 & 00 & 1. 40582 & 5100 & 1.40339 \\
\hline 1 & 30 & 1. 40694 & 26 & 30 & 1.40578 & $51-30$ & 1. 40334 \\
\hline & 00 & $1: 40694$ & & 00 & 1. 40573 & 52.00 & 1. 40329 \\
\hline 2 & 30 & 1. 40694 & 27 & 30 & 1. 40569 & $52 \quad 30$ & 1. 40324 \\
\hline 3 & 00 & 1.40693 & 28 & 00 & 1.40565 & $\begin{array}{ll}53 & 00\end{array}$ & 1.40319 \\
\hline 3 & 30 & 1. 40693 & 28 & 30 & 1. 40560 & $53 \quad 30$ & 1. 40314 \\
\hline 4 & 00 & 1.40692 & 29 & 00 & 1. 40556 & $54 \quad 00$ & 1. 40309 \\
\hline 4 & 30 & 1. 40691 & 29 & 30 & 1. 40552 & $54 \quad 30$ & 1. 40304 \\
\hline 5 & 00 & 1. 40690 & 30 & 00 & 1. 40548 & $55 \quad 00$ & 1. 40299 \\
\hline 5 & 30 & 1. 40689 & 30 & 30 & 1. 40544 & $55 \quad 30$ & 1. 40295 \\
\hline 6 & 00 & 1. 40688 & 31 & 00 & 1. 40539 & $\begin{array}{ll}56 & 00\end{array}$ & 1. 40290 \\
\hline 6 & 30 & 1.40687 & 31 & 30 & 1. 40534 & $56 \quad 30$ & 1.40285 \\
\hline 7 & 00 & 1.40686 & 32 & .00 & 1.40530 & $\begin{array}{ll}57 & 00\end{array}$ & 1.40280 \\
\hline 7 & 30 & 1.40685 & $\cdot 32$ & 30 & 1. 40525 & $57 \quad 30$ & 1. 40276 \\
\hline 8 & 00 & 1. 40683 & 33 & 00 & 1. 40520 & $\begin{array}{ll}58 & 00\end{array}$ & 1. 40271 \\
\hline 8 & 30 & 1.40682 & 33 & 30 & 1. 40516 & $58 \quad 30$ & 1. 40266 \\
\hline 9 & 00 & 1.40680 & 34 & 00 & 1.40511 & $\begin{array}{ll}59 & 00\end{array}$ & 1. 40262 \\
\hline 9 & 30 & 1. 40679 & 34 & 30 & I. 40506 & $59 \quad 30$ & 1. 40257 \\
\hline 10 & 00 & 1. 40677 . & 35 & 00 & 1. 40501 & $\begin{array}{ll}60 & 00\end{array}$ & 1. 40253 \\
\hline 10 & 30 & 1. 40675 & 35 & 30 & 1.40496 & $\begin{array}{ll}60 & 30\end{array}$ & 1. 40249 \\
\hline 11 & 00 & 1. 40673 & 36 & 00 & 1.40491 & $\begin{array}{ll}61 & 00\end{array}$ & 1.40244 \\
\hline 11 & 30 & 1. 40671 & 36 & 30 & 1. 40486 & $61 \quad 30$ & 1.40240 \\
\hline 12 & 00 & 1.40669 & 37 & 00 & 1. 40482 & $\begin{array}{ll}62 & 00\end{array}$ & 1.40235 \\
\hline 12 & 30 & 1. 40667 & 37 . & 30 & 1. 40477 & $\begin{array}{ll}62 & 30\end{array}$ & 1. 40231 \\
\hline 13 & 00 & 1. 40665 & 38 & 00 & 1. 40472 & $\begin{array}{ll}63 & 00\end{array}$ & 1.40227 \\
\hline 13 & 30 & 1. 40663 & 38 & 30 & 1. 40467 & $63 \quad 30$ & 1.40223 \\
\hline 14 & 00 . & 1. 40660 & 39 & 00 & 1. 40462 & $\begin{array}{ll}64 & 00\end{array}$ & 1. 40219 \\
\hline 14 & 30 & 1. 40658 & 39 & 30 & 1. 40457 & $64 \quad 30$ & 1.40215 \\
\hline 15 & 00 & 1. 40655 & 40 & 00 & 1. 40452 & $65 \quad 00$ & 1.40210 \\
\hline 15 & 30 & 1. 40653 & 40 & 30 & 1. 40446 & $65 \quad 30$ & 1. 40207 \\
\hline 16 & 00 & 1. 40650 & 41 & 00 & 1.40441 & $\begin{array}{ll}66 & 00\end{array}$ & 1.40203 \\
\hline 16 & 30 & 1.40647 & 41 & 30 & 1. 40436 & $\begin{array}{ll}66 & 30\end{array}$ & 1. 40199 \\
\hline 17 & 00 & 1. 40644 & 42 & 00 & 1. 40431 & $67 \quad 00$ & 1. 40195 \\
\hline 17 & 30 & 1. 40642 & 42 & 30 & 1.40426 & & 1.40192 \\
\hline 18 & 00 & 1.40639 & 43 & 00 & 1. 40421 & $68 \quad 00$ & 1.40188 \\
\hline 18 & 30 & 1.40636 & 43 & 30 & 1. 40416 & $\begin{array}{ll}68 & 30\end{array}$ & 1. 40185 \\
\hline 19 & 00 & 1.40632 & 44 & 00 & 1.40411 & 69.00 & 1. 40181 \\
\hline 19 & 30 & 1. 40629 & 44 & 30 & 1.40406 & $69 \quad 30$ & 1. 40178 \\
\hline 20 & 00 & 1. 40626 & 45 & 00 & 1.40400 & $\begin{array}{ll}70 & 00\end{array}$ & 1. 40174 \\
\hline 20 & 30 & 1. 40623 & 45 & 30 & 1. 40395 & $\begin{array}{ll}70 & 30\end{array}$ & 1. 40171 \\
\hline 21 & 00 & 1.40619 & 46 & 00 & 1. 40390 & $71 \quad 00$ & 1. 40168 \\
\hline 21 & 30 & 1. 40616 & 46 & 30 & 1. 40385 & $71 \quad 30$ & 1. 40164 \\
\hline 22 & 00 & 1. 40612 & 47 & 00 & 1. 40380 & $\begin{array}{ll}72 & 00\end{array}$ & 1. 40161 \\
\hline 22 & 30 & 1. 40608 & 47 & 30 & 1.40375 & & \\
\hline 23 & 00 & 1.40605 & 48 & 00 & 1.40369 & & \\
\hline 23 & 30 & 1. 40601 & 48 & 30 & 1. 40364 & & \\
\hline 24 & 00 & 1.40597 & 49 & 00 & 1. 40359 & & \\
\hline 24 & 30 & 1. 40594 & 49 & 30 & 1. 40354 & & \\
\hline
\end{tabular}


APPROXIMATE SPHERICAL EXCESS.

This may be obtained by dividing the area of the triangle in square miles by 75.5 .

TABLE 22.-Mean refraction.

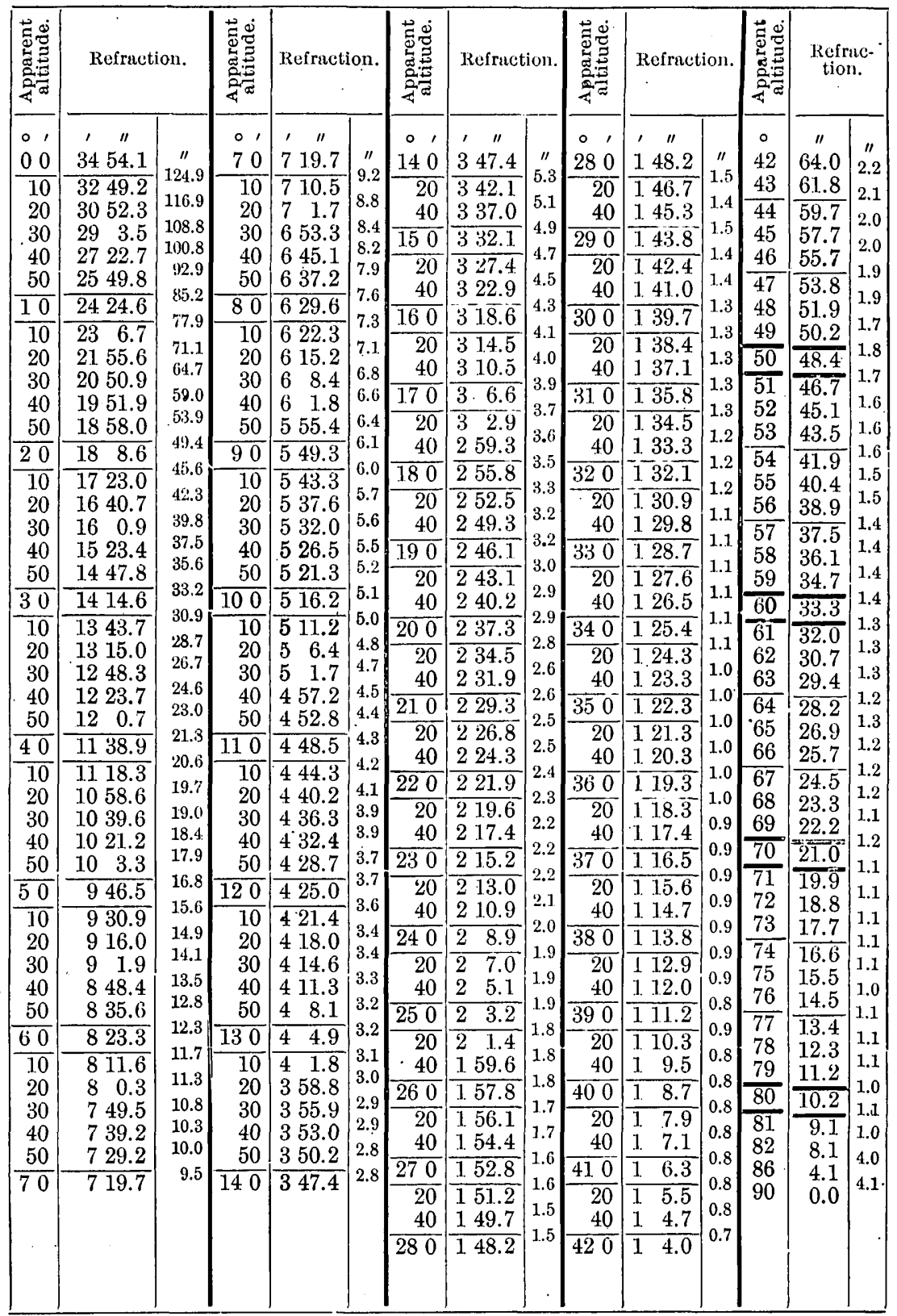

Bull. 214--03-16 
Table 23.-Corrections for curvature and refraction, in feet=0.5\%4 (distance, miles) ${ }^{2}$.

[Difference in feet between the apparent and true level at distances varying from 1 to 66 miles.]

\begin{tabular}{|c|c|c|c|c|c|c|c|}
\hline \multirow[b]{2}{*}{$\begin{array}{c}\text { Distance, } \\
\text { miles. }\end{array}$} & \multicolumn{3}{|c|}{ Difference in feet for- } & \multirow[b]{2}{*}{$\begin{array}{c}\text { Distance, } \\
\text { miles. }\end{array}$} & \multicolumn{3}{|c|}{ Difference in feet for- } \\
\hline & Curvature. & $\begin{array}{c}\text { Refrac- } \\
\text { tion. }\end{array}$ & $\begin{array}{l}\stackrel{c}{c} \\
\text { Curvature } \\
\text { and } \\
\text { refraction. }\end{array}$ & & Curvature. & $\begin{array}{c}\text { Refrac- } \\
\text { tion. }\end{array}$ & $\begin{array}{l}\text { Curvature } \\
\text { and } \\
\text { refraction. }\end{array}$ \\
\hline 1 & 0.7 & 0.1 & 0.6 & 34 & 771.3 & 108.0 & 663.3 \\
\hline .2 & 2.7 & 0.4 & 2.3 & 35 & 817.4 & 114.4 & 703.0 \\
\hline 3 & 6.0 & 0.8 & 5.2 & 36 & 864.8 & 121.1 & 743.7 \\
\hline 4 & 10.7 & 1.5 & 9.2 & 37 & 913.5 & 127.9 & 785.6 \\
\hline 5 & 16.7 & 2.3 & 14.4 & 38 & 963.5 & 134.9 & 828.6 \\
\hline 6 & 24.0 & 3.4 & 20.6 & 39 & $1,014.9$ & 142.1 & 872.8 \\
\hline 7 & 32.7 & 4.6 & 28.1 & 40 & $1,067.6$ & 1.49 .5 & 918.1 \\
\hline 8 & 427. & 6.0 & 36.7 & 41 & $1,121.7$ & 157.0 & 964.7 \\
\hline 9 & 54.0 & 7.6 & 46.4 & 42 & $1,177.0$ & 1.64 .8 & $1,012.2$ \\
\hline 10 & 66.7 & 9.3 & 57.4 & 43 & $1,233.7$ & 172.7 & $1,061.0$ \\
\hline 11 & 80.7 & 11.3 & 69.4 & 44 & $1,291.8$ & 180.8 & $1,111.0$ \\
\hline 12 & 96.1 & 13.4 & 82.7 & 45 & $1,351,2$ & 189.2 & $1,162.0$ \\
\hline 13 & 112.8 & 15.8 & 97.0 & 46 & $1,411.9$ & 197.7 & $1,214.2$ \\
\hline 14 & 130.8 & 18.3 & 112.5 & 47 & $1,474.0$ & 206.3 & $1,267.7$ \\
\hline 15 & 150.1 & 21.0 & 129.1 & 48 & 1, 537.3 & 2152 & $1,322.1$ \\
\hline 16. & 170.8 & 23.9 & 146.9 & 49 & $1,602.0$ & 224.3 & $1,377.7$ \\
\hline 17. & 192.8. & 27.0 & 165.8 & 50 & $1,668.1$ & 233.5 & - $1,434.6$ \\
\hline 18 & 216.2 & 30.3 & 185.9 & 51 & $1,735.5$ & 243.0 & $1,492.5$ \\
\hline 19 & 240.9 & 33.7 & 207.2 & 52 & $1,804.2$ & 252.6 & $1,551.6$ \\
\hline 20 & 266.9 & 37.4 & 229.5 & 53 & $1,874.3$ & 262.4 & $1,611.9$ \\
\hline 21 & 294.3 & 41.2 & 253.1 & 54 & $1,945.7$ & 272.4 & $1,673.3$ \\
\hline 22 & 322.9 & 45.2 & 277.7 & 55 & $2,018.4$ & 282.6 & $1,735.8$ \\
\hline 23 & 353.0 & 49.4 & 303.6 & 56 & $2,092.5$ & 292.9 & $1,799.6$ \\
\hline 24 & 384.3 & 53.8 & .330 .5 & 57 & $2,167.9$ & 303.5 & $1,864.4$ \\
\hline 25 & 417.0 & 58.4 & 358.6 & 58 & $2,244.6$ & 314.2 & $1,930.4$ \\
\hline 26 & 451.1 . & 63.1 & 388.0 & 59 & $2,322.7$ & 325: 2 & $1,997.5$ \\
\hline 27 & 486.4 & 68.1 & 418.3 & 60 & $2,402.1$ & 336.3 & $2,065.8$ \\
\hline 28 & 523.1 & 73.2 & 449.9 & 61. & $2,482.8$ & 347.6 & $2,135.2$ \\
\hline 29 & 561.2 & 78.6 & 482.6 & 62 & $2,564.9$ & 359.1 & $2,205.8$ \\
\hline 30 & 600.5 & 84.1 & 516.4 & 63 & $2,648.3$ & 370.8 & $2,277.5$ \\
\hline 31 & 641.2 & 89.8 & 551.4 & 64 & $2,733.0$ & 382.6 & $2,350.4$ \\
\hline 32 & 683.3 & 95.7 & 587.6 & 65 & $2,819.1$ & 394.7 & $2,424.4$ \\
\hline 33 & 726.6 & 101.7 & 624.9 & 66 & $2,906.5$ & 406.9 & $2,499.6$ \\
\hline
\end{tabular}


TABLE 24.-For obtaining differences of altitude for any minute up to 15 degrees. and for any distance.

[Prepared by Arthur P. Davis.]

\section{EXPLANATION OF TABLE.}

The left-hand column is the minutes of the vertical angle, the degrees being denoted by the large number at top of page. The boldface figures at top of column is the distance in miles. Numbers in the body of the table denote the difference of elevation corresponding to the angle on the left and the distance at top. The correction for curvature, refraction, and height of instrument is always plus; it therefore increases the difference of level for angles of elevation, and is subtracted from the difference of level for angles of depression.

Example.-Required the difference of altitude corresponding to a vertical angle of $+9^{\circ} 18^{\prime}$ at a distance of 3.628 miles. On page 253 the tabular number corresponding to $9^{\circ} 18^{\prime}$ and-

A distance of 3 miles is

................................................ 2,594

For a distance of 6 miles is 5,188 -for 0.6 is therefore . . . . . . . . . . . . . 519

For a distance of 2 miles is $1,729-$ for 0.02 is therefore . . . . . . . . . . . . . 17

For a distance of 8 miles is 6,917 -for 0.008 is therefore ................ 7

Correction for curvature, refraction, and height of instrument for 3.6 miles is.$+ \quad 12$

Total difference of altitude ................................ 
$\mathrm{T}_{\triangle \mathrm{BLE}}$ 24.-For obtaining differences of altitude for any minute, etc.-Continued.

$0^{\circ}$.

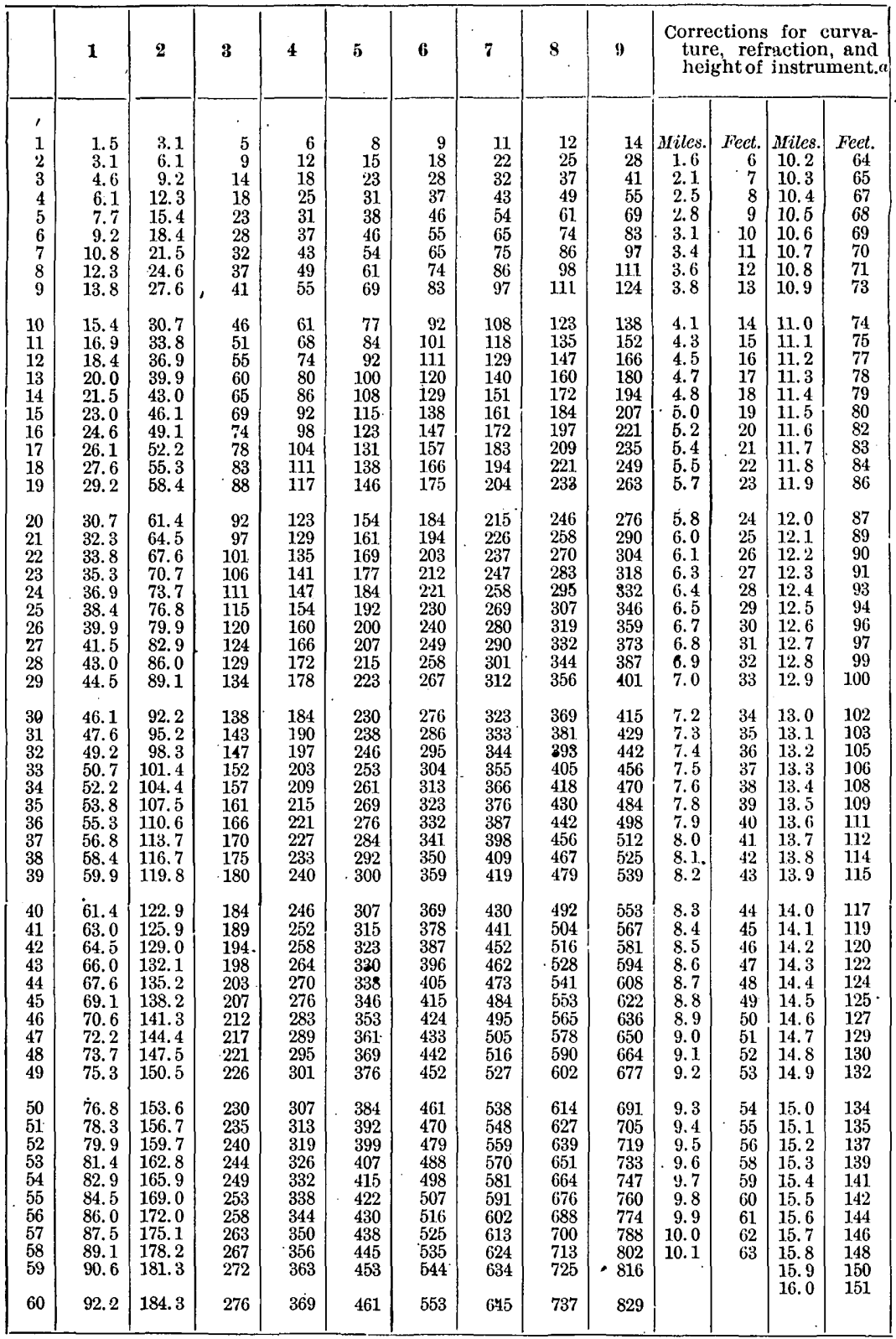

$a$ For all distances under 1.6 miles the correction may be taken as +5 feet. Height of instrument is assumed 4.5 feet. 
TABLE 24.-For obtaining differences of altitude for any minute, etc.-Continued.

$1^{\circ}$.

\begin{tabular}{|c|c|c|c|c|c|c|c|c|c|c|c|c|c|}
\hline & 1 & I & 3 & 4 & 5 & (j) & 7 & 8 & () & \multicolumn{4}{|c|}{$\begin{array}{l}\text { Corrections for curva- } \\
\text { ture, refraction, and } \\
\text { height of instrument. }\end{array}$} \\
\hline 0 & 92.2 & 184.3 & 276 & 369 & 461. & 553 & 645 & 737 & 829 & Afiles. & Feet. & ariles. & Feet. \\
\hline 1 & 93.7. & 187.4 & 281 & 375 & 468 & 562 & 650 & 750 & 843 & 16.1 & 153 & 22.1 & 285 \\
\hline 2 & 95.2 & 190.5 & 286 & 381 & 476 & 571. & 667 & 762 & 857 & 16.2 & 155 & 22.2 & 287 \\
\hline 3 & 96.8 & 193. 5 & 290 & 387 & 484 & 581 & 677 & 774 & 871 & 16.3 & 157 & 22.3 & 290 \\
\hline 4 & 98.3 & 196.6 & 295 & 393 & 492 & 590 & 688 & 786 & 885 & 16.4 & 159 & 22.4 & 293 \\
\hline 5 & 99.8 & 199.7 & 300 & 399 & 499 & 599 & 699 & 799 & 899 & 16.5 & 161 & 22.5 & 295 \\
\hline 6 & 101.4 & 202.8 & 304 & 406 & 507 & 608 & 71.0 & 811. & 912 & 16.6 & 163 & 22.6 & 298 \\
\hline 7 & 102.9 & 205.8 & 309 & 412 & 515 & 618 & 720 & 823 & 926 & 16.7 & 165 & 22.7 & 300 \\
\hline 8 & 104.4 & 208. 9 & 313 & 418 & 522 & 627 & 731 & 836 & 940 & 16.8 & 167 & 22.8 & 303 \\
\hline 9 & 106.0 & 212.0 & 318 & 424 & 530 & 636 & 742 & 848 & 954 & 16.9 & 168 & 22.9 & 306 \\
\hline 10 & 107.5 & 215.1 & 323 & 430 & 538 & 645 & 753 & 860 & 968 & 17.0 & 170 & 23.0 & 308 \\
\hline 11. & 109.1 & 218. 1 & 327 & 436 & 545 & 654 & 763 & 873 & 982 & 17.1 & 172 & 23.1 & 311 \\
\hline 12 & 110.6 & 221.2 & 332 & 442 & 553 & 664 & 774 & 885 & 995 & 17.2 & 174 & 23.2 & 313 \\
\hline 13 & 112.1 & 224.3 & 336 & 449 & 561 & 673 & 785 & 897 & 1,009 & 17.3 & 176 & 23.3 & 316 \\
\hline 14 & 113.7 & 227.3 & 341 & 455 & 568 & 682 & 796 & 909 & 1,023 & 17.4 & 178 & 23.4 & 31.9 \\
\hline 15 & 115.2 & 230.4 & 346 & 461 & 576 & 691. & 806 & 922 & 1,037 & 17.5 & 180 & 23.5 & 321 \\
\hline 16 & 116.7 & 233.5 & 350 & 467 & 584 & 700 & 817 & 934 & 1,051 & 17.6 & 182 & 23.6 & 324 \\
\hline 17 & 118.3 & 236.6 & 355 & 473 & 591. & 710 & 828 & 946 & 1,065 & 17.7 & 184 & 23.7 & 327 \\
\hline 18 & 119.8 & 239.6 & 359 & 479 & 599 & 719 & 839 & 959 & 1,078 & 17.8 & 186 & 23.8 & 330 \\
\hline .19 & 121.4 & 242.7 & 364 & 485 & 607 & 728 & 849 & 971 & 1,092 & 17.9 & 188 & 23.9 & 332 \\
\hline 20 & 122. 9 & 245.8 & 369 & 492 & 614 & 737 & 860 & 983 & 1,106 & 18.0 & 190 & 24.0 & 335 \\
\hline 21 & 124.4 & 248.9 & 373 & 498 & 622 & 747 & 871. & 995 & 1,120 & 18.1 & 193 & 24.1 & 338 \\
\hline 22 & 126.0 & 251.9 & 378 & 504 & 630 & 756 & 882 & 1,008 & 1,134 & 18.2 & 195 & 24.2 & 341. \\
\hline 23 & 127.5 & 255.0 & 383 & 510 & 638 & 765 & 893 & 1,020 & 1,148 & 18.3 & 197 & 24.3 & 343 \\
\hline 24 & 129.0 & 258.1 & 387 & 516 & 645 & 774 & 903 & 1,032 & 1,161 & 18.4 & 199 & 24.4 & 346 \\
\hline 25 & 130.6 & 261.2 & 392 & 522 & 653 & 783 & 914 & 1,045 & 1,175 & 18.5 & 201. & 24.5 & 349 \\
\hline 26 & 132.1 & 264.2 & 396 & 528 & 661 & 793 & 925 & 1,057 & 1,189 & 18.6 & 203 & 24.6 & 352 \\
\hline 27 & 133.6 & 267.3 & 401 & 535 & 668 & 802 & 936 & 1,069 & 1,203 & 18.7 & 205 & 24.7 & 355 \\
\hline 28 & 135.2 & 270.4 & 406 & 541 & 676 & 811 & 946 & 1,082 & 1,217 & 18.8 & 207 & 24.8 & 358 \\
\hline 29 & 136.7 & 278.5 & 410 & 547 & 684 & 820 & 957 & 1,094 & 1,231 & 18.9 & 210 & 24.9 & 360 . \\
\hline 30 & 138.3 & 276.5 & 415 & 553 & 691. & 830 & 968 & 1,106 & 1,244 & 19.0 & 21.2 & 25.0 & 363 \\
\hline 31. & 139.8 & 279.6 & 419 & 559 & 699 & 839 & 979 & 1,118 & 1,258 & 19.1 & 214 & 25.1 & 366 \\
\hline 32 & 141.3 & 282.7 & $424^{\circ}$ & 565 & 707. & 848 & 989 & $1,131$. & 1,272 & 19.2 & 216 & 25.2 & 369 \\
\hline 33 & 142.9 & 285.7 & 429 & 571 & 714 & 857 & 1,000 & 1,143 & 1,286 & 19.3 & 218 & 25.3 & 372 \\
\hline 34 & 144.4 & 289.8 & 433 & 578 & 722 & 866 & 1,011 & 1,155 & 1,300 & 19.4 & 221 & 25.4 & 375 \\
\hline 35 & 146.0 & 291.9 & 438 & 584 & 730 & 876 & 1,022 & 1,168 & 1,314 & 19.5 & 223 & 25.5 & 378 \\
\hline 36 & 147.5 & 295.0 & 442 & 590 & .737 & 885 & 1,032 & 1,180 & 1,327 & 19.6 & 225 & 25.6 & 381. \\
\hline 37 & 149.0 & 298.0 & 447 & 596 & 745 & 894 & 1,043 & 1,192 & $1,341$. & 19.7 & 227 & 25.7 & 384 \\
\hline 38 & 150.6 & 301.1 . & 452 & 602 & 753 & 903 & 1., 054 & 1,204 & 1,355 & 19.8 & 230 & 25.8 & 387 \\
\hline 39 & 152. 1 & 304.2 & 456 & 608 & 760 & 913 & 1,065 & 1,217 & 1,369 & 19.9 & 232 & 25.9 & 390 \\
\hline 40 & 153.6 & 307.3 & 461 & 615 & 768 & 922 & 1,075 & 1,229 & $.1,383$ & 20.0 & 234 & 26.0 & 393 \\
\hline 41 & 155.2 & 310.3 & 466 & 6 & 776 & 931 & 1,086 & 1,241 & 1,397 & 20 & 236 & & 399 \\
\hline 42 & 156.7 & 31.3 .4 & 470 & 427 & 784 & 940 & 1,097 & 1,254 & 1,410 & 20.2 & 239 & 26.4 & 405 \\
\hline 43 & 158.2 & 316.5 & 475 & (633 & 791 & 949 & 1,108 & 1,266 & 1,424 & 20.3 & 241 & 26.6 & 411. \\
\hline 44 & 159.8 & 319.6 & 479 & (639 & 799 & 959 & 1,118 & i., 278 & 1,438 & 20.4 & 243 & 26.8 & 417 \\
\hline 45 & 161.3 & 322.6 & 484 & 645 & 807 & 968 & 1,129 & 1,291 & 1,452 & 20.5 & 246 & 27.0 & 423 \\
\hline 46 & 162.9 & 32 & 489 & 6.51 & 814 & 977 & 1,140 & 1,303 & 1,466 & 20.6 & 248 & 27. 2 & 429 \\
\hline 47 & 164.4 & 328.8 & 493 & ti58 & 822 & 986 & 1,151 & 1,315 & 1,480 & 20.7 & 250 & 27.4 & 435 \\
\hline 48 & 165.9 & 331.9 & 498 & 664 & 830 & 996 & 1,162 & 1,327 & 1,493 & 20.8 & 253 & 27.6 & 442 \\
\hline 49 & 167.5 & 334.9 & 502 & 670 & 837 & 1,005 & 1,172 & 1,340 & 1,567 & 20.9 & 255 & 27.8 & 448 \\
\hline 50 & 169.0 & 338.0 & 507 & 676 & 845 & 1,014 & $1 ;, 183$ & 1,352 & $1,521$. & 21.0 & 258 & 28.0 & 455 \\
\hline 51 & 170.6 & 341.1. & 512 & 682 & 853 & 1,023 & 1,194 & 1,364 & 1,535 & 21.1 & 260 & 28.2 & 461 \\
\hline 52 & 172.1 & 344.2 & 516 & 688 & 860 & 1,032 & 1,205 & 1,377 & 1,549 & 21.2 & 262 & 28.4 & 467 \\
\hline 53 & 173.6 & 347.2 & 521 & 694 & 868 & 1,042 & 1,215 & $1,38: 1$ & 1,563 & 21.3 & 265 & 28.6 & 474 \\
\hline 54 & 175.2 & 350.3 & 525 & 701 & 876 & 1,051 & 1,226 & 1,401 & 1,576 & 21.4 & 267 & 28.8 & 480 \\
\hline 55 & 176.7 & 353.4 & 530 & 707 & 883 & 1,060 & 1,237 & 1,414 & 1,590 & 21.5 & 270 & 29.0 & 487 \\
\hline 56 & 178.2 & 356.5 & 535 & 713 & 891 & 1,069 & 1,248 & 1,426 & 1,604 & 21.6 & 272 & 29.2 & 494 \\
\hline 57 & 179.8 & 359.5 & 539 & 719 & 899 & 1,079 & 1,258 & 1,438 & 1,618 & 21.7 & 275 & 29.4 & 501. \\
\hline 58 & 181.3 & 362.6 & 544 & 725 & 907 & 1,088 & 1,269 & 1,450 & 1,632 & 21.8 & 277 & 29.6 & 507 \\
\hline 59 & 182.8 & 365.7 & 549 & 731 & 914 & 1,097 & 1,280 & 1,465 & 1,643 & 21.9 & 280 & 29.8 & 514 \\
\hline 60 & 184.4 & 368.8 & 553 & $7: 38$ & 922 & 1,106 & 1,291 & 1,475 & 1,659 & 22.0 & 282 & 30.0 & 521. \\
\hline
\end{tabular}

$a$ For all distances under 1.6 miles the correction may be taken as +5 feet. Height of instrument is assumed 4.5 feet. 
TABLe 24.-For obtaining differences of altitude for any minute, etc.-Continued.

$2^{\circ}$.

\begin{tabular}{|c|c|c|c|c|c|c|c|c|c|c|c|c|c|}
\hline & 1. & 2 & 3 & 4 & 5 & 6 & 7 & 8 & $\mathbf{9}$ & \multicolumn{4}{|c|}{$\begin{array}{l}\text { Corrections for curva } \\
\text { ture, refraction, and } \\
\text { height of instrument. }\end{array}$} \\
\hline 0 & $184: 4$ & 368.8 & 553 & 738 & 922 & 1.106 & 1.291 & 1475 & 1,659 & & & & \\
\hline 1 & $\begin{array}{l}18.4 \\
185.9\end{array}$ & $\begin{array}{l}50.0 \\
371.8\end{array}$ & $\begin{array}{l}500 \\
558\end{array}$ & 744 & 930 & $\begin{array}{l}1,100 \\
1,116\end{array}$ & 1,301 & $\begin{array}{l}1,487 \\
1,487\end{array}$ & 1,673 & Niles & Ficet. & Miles. & Feet. \\
\hline 2 & 187.5 & 374.9 & 562 & 750 & 937 & 1,125 & 1,312 & 1., 500 & 1,687 & 1.6 & 6 & 10.2 & 64 \\
\hline 3 & 189.0 & 378.0 & 567 & 756 & 945 & 1,134 & 1,323 & 1,512 & 1,701 & 2.1 & 7 & 10.3 & 65 \\
\hline 4 & 190.5 & 381.1. & 572 & 762 & 953 & 1,143 & 1,334 & 1,524 & 1,715 & 2.5 & 8 & 10.4 & 67 \\
\hline 5 & 192.1 & 384.1 & 576 & 768 & 960 & 1,152 & 1,344 & 1,537 & 1,729 & 2.8 & 9 & 10.5 & 68 \\
\hline 6 & 193.6 & 387.2 & 581 & 774 & 968 & 1,162 & 1,355 & 1,549 & 1,742 & 3.1 & 10 & 10.6 & 69 \\
\hline 7 & 195.1 & 390.3 & 585 & 781 & 976 & 1,171 & 1,366 & 1,561 & 1,756 & 3.4 & 11. & 10.7 & 70 \\
\hline 8 & 196.7 & 393.4 & 590 & 787 & 983 & 1,180 & 1,377 & 1,573 & 1,770 & 3. 6 & 12 & 10.8 & 71 \\
\hline 9 & 198.2 & 396.4 & 595 & 793 & 991 & 1,189 & 1,388 & 1,586 & 1,784 & 3.8 & 13 & 10.9 & 73 \\
\hline 10 & 199.8 & 399.5 & 599 & 799 & 999 & 1,199 & 1,398 & 1,598 & 1,798 & 4.1 & 14 & 11.0 & 74 \\
\hline 11 & 201.3 & 402.6 & 604 & 805 & 1,006 & 1,208 & 1,409 & 1,610 & 1,812 & 4.3 & 15 & 11. 1 & 75 \\
\hline 12 & 202.8 & 405.7 & 609 & 811. & 1,014 & 1,217 & 1,420 & 1,623 & 1,826 & 4.5 & 16 & 11.2 & 77 \\
\hline 13 & 204.4 & 408.8 & 613 & 818 & 1,022 & 1,226 & 1,431 & 1,635 & 1,839 & 4.7 & 17 & 11.3 & 78 \\
\hline 14 & 203.9 & 411.8 & 618 & 824 & 1,030 & 1,235 & 1,441 & 1,647 & 1,853 & 4.8 & 18 & 11.4 & 79 \\
\hline 15 & 207.5 & 414.9 & 622 & 830 & 1,037 & 1,245 & 1,452 & 1,660 & 1,867 & 5.0 & 19 & 11.5 & 80 \\
\hline 16 & 209.0 & 418.0 & 627 & 836 & 1,045 & 1,254 & 1, 463 & 1,672 & 1,881 & 5.2 & 20 & 11.6 & 82 \\
\hline 17 & 210.5 & 421.1 & 632 & 842 & 1,053 & ], 263 & 1,474 & 1,684 & 1,895 & 5.4 & 21 & $11 . ;$ & 83 \\
\hline 18 & 212.1 & 424.1 & 636 & 848 & 1,060 & 1,272 & 1,484 & 1,697 & 1,909 & 5.5 & 22 & 11.8 & 84 \\
\hline 19 & 213.6 & 427.2 & 641 & 854 & 1,068 & 1,282 & 1,495 & 1,709 & 1, 932 & 5.7 & 23 & 11.9 & 86 \\
\hline 20 & 215.1 & 430.3 & 645 & 861. & 1,076 & 1,291 & 1,506 & 1,721 & 1,936 & 5.8 & 24 & 12.0 & 87 \\
\hline 21 & 216.7 & 433.4 & 650 & 867 & 1,083 & 1,300 & 1,517 & 1,733 & 1,950 & 6.0 & 25 & 12.1 & 89 \\
\hline 22 & 218.2 & 436.4 & 655 & 873 & 1,091 & 1,309 & 1,528 & 1,746 & 1,964 & 6.1 & 26 & 12.2 & 90 \\
\hline 23 & 219.8 & 439.5 & $65^{\circ}$ & 879 & 1,099 & 1,319 & 1, 538 & 1,758 & $\begin{array}{l}1,978 \\
1,90\end{array}$ & 6.3 & 27 & 12.3 & 91 \\
\hline 24 & 221.3 & 442.6 & 664 & 885 & 1,106 & 1,328 & 1,549 & 1,770 & 1,992 & 6.4 & 28 & 12.4 & 93 \\
\hline 25 & 222.8 & 445.7 & 669 & 891. & 1,114 & 1,337 & 1,560 & 1,783 & 2,006 & 6.5 & 29 & 12.5 & 94 \\
\hline 26 & 224.4 & 448.7 & 673 & 897 & 1,122 & 1,346 & 1,571 & 1,795 & 2,019 & 6.7 & 30 & 12.6 & 96 \\
\hline 27 & 225.9 & 451.8 & 678 & 904 & 1,130 & 1,355 & 1,581 & 1,807 & 2,033 & 6.8 & 31 & 12.7 & 97 \\
\hline 28 & 227.5 & 454.9 & 682 & 910 & 1,137 & 3,365 & 1,592 & 1,820 & 2,047 & 6.9 & 32 & 12.8 & 99 \\
\hline 29 & 229.0 & 458.0 & 687 & 916 & 1,145 & 1,374 & 1,603 & 1,832 & 2,061 & 7.0 & 33 & 12.9 & 100 \\
\hline 30 & 230.5 & 461.1 & 692 & 922 & 1,153 & 1,383 & 1,614 & 1,844 & 2,075 & 7.2 & 34 & 13.0 & 102 \\
\hline 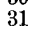 & 232.1 & $4 t$ & 696 & 928 & $\begin{array}{l}1,160 \\
0\end{array}$ & $\begin{array}{l}1,392 \\
1,392\end{array}$ & 1,624 & $\begin{array}{l}1,857 \\
1,857\end{array}$ & 2,089 & 7.3 & 35 & 13.1 & 103 \\
\hline 32 & 233.6 & 467.2 & 701. & 934 & 1,168 & 1,402 & 1,635 & 1,869 & 2,102 & 7.4 & 36 & 13.2 & 105 \\
\hline 33 & 23 & 470.3 & 7 & 941 & 1,176 & 1,411 & 1,646 & 1,881 & 2,116 & 7.5 & 37 & 13.3 & 106 \\
\hline 34 & 236.7 & 47 & 7 & 947 & 1, & 1,420 & 1,657 & 1,893 & 2,130 & 7.6 & 38 & 13.4 & 108 \\
\hline 3 & 238.2 & $\begin{array}{r}776.4 \\
\end{array}$ & 715 & 953 & 1,191 & $\begin{array}{l}1,429 \\
\end{array}$ & 1,668 & $\begin{array}{l}1,906 \\
1,90\end{array}$ & $2,1.44$ & $\because$. & 39 & 13.5 & 109 \\
\hline 36 & 239.8 & 479.5 & 71 & 959 & 1,199 & 1,439 & 1,678 & 1,918 & 2,158 & 7. & 40 & 13.6 & 111 \\
\hline 37 & 24 & & 7 & 9 & 1, & 1,448 & 1 & $\hat{1}$, & $.2,172$ & 8. & 41 & 13.7 & 112 \\
\hline 38 & 242.8 & 48 & 7 & 971 & 1,214 & 1,457 & 1,700 & 1,943 & 2,186 & 8. & 42 & \begin{tabular}{|l|l}
13.8 \\
13.8
\end{tabular} & 114 \\
\hline 39 & 244.4 & 488.8 & 733 & 978 & 1,222 & 1,466 & 1,711 & ], 955 & 2,199 & 8.2 & 43 & 13.9 & 115 \\
\hline 40 & 245.9 & 491.8 & 738 & 984 & 1,230 & 1,476 & 1,7 & 1,967 & 2,2 & 8. & 44 & 14. & 117 \\
\hline 41 & 24 & & 7 & & & , & & & & & 45 & & 119 \\
\hline 42 & 249.0 & 497.0 & 747 & 996 & 1,245 & 1,494 & 1,743 & 1., 992 & 2,241 & 8.5 & 46 & 14.2 & 120 \\
\hline 43 & 250 & 501.1 & 752 & 1,002 & 1,253 & 1,503 & 1,754 & 2,004 & 2,255 & 8.6 & 47 & 14.3 & 122 \\
\hline 44 & 25 & & 7 & 1 & 1 , & & 1, & 2, & & 8.7 & 48 & 14 & 124 \\
\hline 45 & 253.6 & 50 & 7 & 1 & i, & 2 & 1, & 2, & & 8.8 & 49 & 14.5 & .125 \\
\hline 46 & 255.2 & 510.3 & 765 & 1,021 & 1,276 & 1,531 & 1,786 & 2,041 & 2,296 & 8.9 & 50 & 14.6 & .127 \\
\hline 47 & 256.7 & 51 & 770 & 1 & 1, & & 1., 797 & 2,054 & & $\because$ & 1. & 14.7 & 129 \\
\hline 48 & 258.2 & 51 & 775 & 1,033 & 1 , & 1,549 & 1,8 & 2,066 & 2,324 & 9.1 & 52 & 14.8 & 130 \\
\hline 49 & 259.8 & 519.5 & 779 & 1,039 & 1,299 & 1,559 & 1,818 & 2,078 & 2,338 & 9.2 & 53 & 14.9 & 132 \\
\hline 50 & 261.3 & 522. & 784 & 1,0 & 1,307 & 1,568 & 1,8 & 2,0 & 2,3 & 9.3 & 54 & 15 & 134 \\
\hline 51 & & & 78 & & 1,314 & 1,577 & 1 & & & 9. & 5 & 15.1 & 135 \\
\hline 52 & 26 & 52 & 793 & 1,058 & 1,322 & 1,586 & $1,851$. & 2,115 & 2,380 & 9.5 & 56 & 15.2 & 137 \\
\hline 53 & 26 & 53 & 79 & 1,064 & 1,330 & $\begin{array}{l}1,596 \\
\text { (1) }\end{array}$ & 1,862 & 2,127 & 2,393 & 9.6 & 58 & 15.3 & 139 \\
\hline 54 & 267.5 & & 80 & 1,070 & & 1,605 & 1,872 & 2,140 & 2,407 & 9.7 & 59 & 15.4 & 141. \\
\hline 55 & 269.0 & 53 & 80 & $1,0 \div 6$ & 1,345 & 1,614 & 1,8 & 2,152 & 2,421 . & 9.8 & 60 & 15.5 & 142 \\
\hline 56 & 270.6 & 541.1 . & 812 & 1,082 & 1,353 & 1,623 & 1,894 & 2,164 & 2,435 & 9.9 & 61. & 15.6 & 144 \\
\hline 57 & 272.1 & & 816 & 1,088 & 1,360 & ], 633 & 1,905 & 2,177 & 2,449 & 10.0 & 52 & 15.7 & 146 \\
\hline 58 & 273.6 & 547.3 & 821 & 1,095 & 1,368 & 1,642 & 1,915 & 2,189 & 2,463 & 10.1 & 63 & 15.8 & 1.48 \\
\hline 5 & 275.2 & 550.3 & 826 & 1,101 & 1,376 & 1,651 & 1,926 & 2,201 & 2,477 & & & 15.9 & 150 \\
\hline 60 & 276.7 & $55 \dot{3} .4$ & 830 & 1,107 & 1,384 & 1,660 & $1,937^{\circ}$ & 2,214 & 2,490 & & & & \\
\hline
\end{tabular}

a For all distances under 1.6 miles the correction may be taken as +5 feet. Height of instrument is assumed 4.5 feet. 
TaBtw 24.-For oblaining differences of altitude for any minute, etc.-Continued.

$3^{\circ}$.

\begin{tabular}{|c|c|c|c|c|c|c|c|c|c|c|c|c|}
\hline & 1 & 2 & 3 & 4 & j & (i & 7 & s & 9 & \multicolumn{3}{|c|}{$\begin{array}{l}\text { Corrections for curva- } \\
\text { ture, refraction, and } \\
\text { heightof instrument. } a\end{array}$} \\
\hline ' & & & & & & & & & & & & \\
\hline 0 & 276.7 & 553.4 & 830 & 1,107 & 1,384 & 1,660 & 1,937 & 2,214 & 2,490 & Niles. & Feet. & \\
\hline$\frac{1}{2}$ & $\begin{array}{l}270.8 \\
279.8\end{array}$ & $\begin{array}{l}500.0 \\
559.6\end{array}$ & 839 & $\begin{array}{l}1,110 \\
1,119\end{array}$ & $\begin{array}{l}1,391 \\
1,399\end{array}$ & 1,670 & $\begin{array}{l}, 948 \\
1.959\end{array}$ & $\begin{array}{l}2,220 \\
2,238\end{array}$ & 2,004 & 10.1 & $\begin{array}{l}63 \\
64\end{array}$ & \\
\hline 3 & 281.3 & 562.7 & 844 & 1., 125 & 1,407 & 1,688 & 1,969 & 2,251 & 2,532 & 10.3 & 65 & \\
\hline 4 & 282.9 & 565.7 & 849 & 1,131 & 1., 414 & 1,697 & 1,980 & 2,263 & 2,546 & 10.4 & 67 & \\
\hline 5 & 284.4 & 568.8 & 853 & 1,138 & 1,422 & 1,706 & 1,991 & 2,275 & 2,560 & 10.5 & 68 & \\
\hline 6 & 286.0 & 571.9 & 858 & 1,144 & 1,430 & 1,716 & 2,002 & 2,288 & 2,574 & 10.6 & 69 & \\
\hline 7 & 237.5 & 575.0 & 862 & 1,150 & 1,437 & 1,725 & 2,012 & 2,300 & 2,587 & 10.7 & 70 & \\
\hline 8 & 289.0 & 578.1 & 867 & 1,156 & 1,445 & 1,734 & 2,023 & 2,312 & 2,601 & 10.8 & 71 & \\
\hline 9 & 290.6 & 581.2 & 872 & 1,162 & 1,453 & 1,743 & 2,034 & 2,325 & 2,615 & 10.9 & 73 & \\
\hline 10 & 292.1 . & 584.2 & 870 & 1,168 & 1,461 & 1,753 & $2,04 \pi$ & 2,337 & 2,629 & 11.0 & 75 & \\
\hline 11 & 293.7 & 5873 & 881 & 1,175 & 1,468 & 1,762 & 2,056 & 2,349 & $\overrightarrow{2}, 643$ & 11.2 & 77 & \\
\hline 12 & 295.2 & 590.4 & 886 & 1,181 & 1,576 & 1,771 & 2,066 & 2,362 & 2,657 & 11.4 & 79 & \\
\hline 13 & 296.7 & 593.5 & 890 & 1,187 & 1,484 & 1,780 & 2,077 & 2,373 & 2,671 & 11.6 & 82 & \\
\hline 14 & 298.3 & 596.6 & 895 & 1,193 & 1,491 & 1,790 & 2,088 & 2,386 & 2,685 & 11.8 & 84 & \\
\hline 15 & 299.8 & 599.6 & 899 & 1., 199 & ., 499 & 1,799 & 2,099 & 2,399 & 2,698 & 12.0 & 87 & \\
\hline 16 & 301.4 & 602.7 & $904:$ & 1,205 & ., 507 & 1. 808 & 2,110 & $2,411$. & 2,712 & 12.2 & 90 & \\
\hline 17 & 302.9 & 605.8 & 909 & 1., 212 & 1,515 & 1,817 & 2,120 & 2,423 & 2,726 & 12.4 & 93 & \\
\hline 18 & 304.4 & 608.9 & 913 & 1,218 & 1,522 & 1,827 & 2,131 & 2,436 & 2,740 & 12.6 & 96 & \\
\hline 19 & 306.0 & 612.0 & 918 & 1,224 & 1,530 & 1,836 & 2,142 & 2,448 & 2,754 & 12.8 & 99 & \\
\hline 20 & $3 c 7.5$ & 615.0 & 923 & 1,230 & J., 538 & 1,845 & 2,153 & 2,460 & 2,768 & 13.0 & 102 & \\
\hline 21. & 309.1 & 618.1 & 927 & 1,236 & J., 545 & 1,854 & 2,163 & 2,473 & 2,782 & 13.2 & 105 & \\
\hline 22 & 310.6 & 621.2 & 932 & 1,242 & 1., 553 & 1,864 & 2,174 & 2,485 & $2,79 \overline{0}$ & 13.4 & 108 & \\
\hline 28 & 312.1 & 624.3 & 936 & 1,249 & $\begin{array}{l}1,5000 \\
1.561 .\end{array}$ & 1,873 & 2,185 & 2,497 & 2,809 & 13.6 & 111 & \\
\hline 24 & 33.3 .7 & 627.4 & 941 & 1,255 & 1., 568 & 1,882 & 2,196 & 2,510 & 2,823 & 13.8 & 11.4 & \\
\hline 25 & 315.2 & 930.5 & 946 & 1,261 & 3,576 & $1,891$. & 2,207 & 2,522 & 2,837 & 14.0 & 117 & \\
\hline 26 & 316.8 & 633.5 & 950 & 1,267 & i, 584 & 1,901 & 2,217 & 2,534 & 2,851 & 14.2 & 120 & \\
\hline 27 & 318.3 & (636. 6 & 955 & 1,273 & $\begin{array}{l}1,592 \\
3,5\end{array}$ & 1,910 & 2,228 & 2,547 & 2,865 & 14.4 & 124 & \\
\hline 28 & 319.9 & 639.7 & 960 & 1,279 & 1,599 & 1,919 & 2,240 & 2,559 & 2,879 & 14.6 & 127 & \\
\hline 29 & 321.4 & 642.7 & 964 & 1,286 & 1,607 & 1,928 & 2,250 & 2,571 & 2,893 & 14.8 & 130 & \\
\hline 30 & 322.9 & 645.9 & 969 & 1.,292 & 1,615 & 1,938 & 2,261 & 2.584 & 2,906 & 15.0 & 132 & \\
\hline 31 & 324.5 & 649.0 & 973 & 1,298 & 1,022 & 1,947 & 2,271 & 2,596 & 2,920 & 15.2 & 137 & \\
\hline 32 & 326.0 & 652.0 & 978 & 1,304 & 1,630 & 1,956 & 2,282 & 2,608 & 2,934 & 15.4 & 141 & \\
\hline 33 & 327.6 & 655.1. & 983 & 1,310 & 1,638 & 1., 965 & 2,293 & 2,621 & 2,948 & 15.6 & 144 & \\
\hline 34 & 329.1 & 658.2 & 987 & 1,316 & 3,646 & 1,975 & 2,304 & 2,633 & 2,962 & 15.8 & 148 & \\
\hline 35 & 330.6 & 661.3 & 992 & 1,323 & 1,653 & 1,984 & 2,315 & 2,645 & 2,976 & .16 .0 & 151 & \\
\hline 36 & 332.2 & 664.4 & 997 & 1,329 & $1,661$. & 1, 993 & 2,325 & 2,658 & 2,990 & 16.2 & 153 & \\
\hline 37 & 333.7 & 667.5 & 1,001 & 1,335 & 1,669 & 2,002 & 2,336 & 2,670 & 3,004 & 36.4 & 159 & \\
\hline 38 & 335.3 & 670.5 & 1,006 & $1,341$. & j, 676 & 2,012 & 2,347 & 2,682 & 3,017 & 16.6 & 163 & \\
\hline 39 & 336.8 & 673.6 & 1,010 & 1,347 & 1,684 & 2,021 & 2,358 & 2,695 & 3,031 & 16.8 & 167 & \\
\hline 40 & 338.4 & 676.7 & 1,015 & 1,353 & 1,692 & 2,030 & 2,369 & 2,707 & 3,045 & 17.0 & 170 & \\
\hline 41. & 339.9 & $679 . \mathrm{s}$ & 1,020 & 1, 360 & 1,700 & 2,039 & 2,379 & 2,719 & 3,059 & 17.2 & 174 & \\
\hline 42 & 341.4 & 682.9 & 1,024 & 1,366 & 1., 707 & 2,049 & 2,390 & 2,732 & 3,073 & 17.4 & 178 & \\
\hline 43 & 343.0 & 686.0 & 1,029 & 1,372 & 1,715 & 2,058 & 2,401 & 2,744 & $.3,087$ & 17.6 & 182 & \\
\hline 44 & 344.5 & 689.1 & 1,034 & 1,378 & 1,723 & 2,067 & 2,412 & 2,756 & 3,101 & 17.8 & 186 & \\
\hline 45 & 346.1 & 692.1. & 1,038 & 1,384 & 1,730 & 2,076 & 2,422 & 2,769 & 3,115 & 18.0 & 190 & \\
\hline 46 & 347.6 & 695.2 & 1,043 & 1,390 & 1,738 & 2,086 & 2,433 & 2,781 & 3,129 & 18.2 & 195 & \\
\hline 47 & 349.2 & (69). 3 & 1,047 & 1,397 & $\begin{array}{l}1,746 \\
\end{array}$ & 2,095 & 2,444 & 2,793 & 3,142 & 18.4 & 199 & \\
\hline 48 & 350.7 & 701.4 & 1,052 & 1,403 & 1,753 & 2,104 & 2,455 & 2,806 & 3,156 & 18.6 & 203 & \\
\hline 49 & 352.2 & 701.5 & 1,057 & 1,409 & 1,761 & 2,113 & 2,466 & 2,818 & 3,170 & 18. 8 & 207 & \\
\hline 50 & 353.8 & 707.6 & 1,061 & 1,415 & 1,769 & 2,123 & 2,476 & 2,830 & 3,184 & 19.0 & 212 & \\
\hline 51 & 355.3 & 710.7 & 1,066 & 1,421 & 1,777 & 2,132 & 2,487 & 2,843 & 3,198 & 19.2 & 216 & \\
\hline 52 & 356.9 & 71.3. 7 & 1,071 & 1., 427 & 1,784 & $2,141$. & 2,498 & $2 ; 855$ & 3,212 & 19. 4 & 221 & \\
\hline 53 & 358.4 & 716.8 & 1,075 & 1, 434 & 1,792 & 2,150 & 2,509 & 2,867 & 3,226 & 19.6 & 225 & \\
\hline 54 & 360.0 & 719.9 & 1,080 & 1,440 & 1,800 & 2,160 & 2,520 & 2,880 & 3,240 & 19.8 & 230 & \\
\hline 55 & 361.5 & 723.0 & 1,085 & 1., 446 & 1,807 & 2,169 & 2,530 & 2,892 & 3,253 & 20.0 & 234 & \\
\hline 56 & 363.0 & 726.1 & 1,089 & 1,452 & 1,815 & 2,178 & 2,541 & 2,904 & 3,267 & 21.0 & 258 & \\
\hline 57 & 364.6 & 729.2 & 1., 094 & $\mathrm{i}, 458$ & 1., 823 & 2,188 & 2,552 & 2,917 & 3,281 . & 22.0 & 282 & \\
\hline 58 & 366.1 & 732.3 & 1, 098 . & 1,465 & 1,831 & 2,197 & 2,563 & 2,929 & 3,295 & 23.0 & 308 & \\
\hline 59 & 367.7 & 735.3 & 1., 103 & 1., 471 & 1,838 & 2,206 & 2,574 & 2,941 & 3,309 & 24.0 & 335 & \\
\hline 60 & 369.2 & 738.4 & 1., 108 & 1., 477 & 1,846 & 2,215 & 2,584 & 2,954 & 3,323 & 25.0 & 363 & \\
\hline
\end{tabular}

a For all distances under 1.6 miles the correction may be taken as +5 feet. Height of instrument is assumed 4.5 feet. 
TABLE 24.-For obtaining differences of altitude for any minute, elc.-Continued.

$4^{\circ}$

\begin{tabular}{|c|c|c|c|c|c|c|c|c|c|c|c|c|c|}
\hline & 1 & 2 & 3 & 4 & 5 & 6 & 7 & 8 & 9 & \multicolumn{4}{|c|}{$\begin{array}{l}\text { Corrections for curva- } \\
\text { ture, refraction, and } \\
\text { height of instrument.a }\end{array}$} \\
\hline & & & & & & & & & & & & & \\
\hline 0 & 369.2 & 738 & 1,108 & 1,477 & 1,846 & 2,215 & 2,584 & 2,954 & 3,323 & & & & \\
\hline 1 & 370.8 & 742 & 1,112 & 1,483 & 1,854 & 2,225 & 2,595 & 2,966 & 3,337 & Miles. & Feet. & Miles. & Feet. \\
\hline 2 & 372.3 & 745 & 1,117 & 1,489 & 1,862 & 2,234 & 2,606 & 2,978 & 3,351 & 1.6 & 6 & 10.2 & 64 \\
\hline 3 & 373.8 & 748 & 1,122 & 1,495 & 1,869 & 2,243 & 2,617 & 2,991 & 3,365 & ì. 1 & 7 & 10.3 & 65 \\
\hline 4 & 375.4 & 751 & 1,126 & 1,502 & 1,877 & 2,252 & 2,628 & 3,003 & 3,378 & 2.5 & 8 & 10.4 & 67 \\
\hline 5 & 376.9 & 754 & 1,131 & 1,508 & 1,885 & 2,262 & 2,639 & 3,015 & 3,392 & 2.8 & 9 & 10.5 & 68 \\
\hline 6 & 378.5 & $75 \overline{7}$ & $\overrightarrow{1}, 135$ & 1,514 & 1,892 & 2,271 & 2,649 & 3,028 & 3,406 & 3.1 & 10 & 10.6 & 69 \\
\hline 7 & 380.0 & 760 & 1,140 & 1,520 & 1,900 & 2,280 & 2,660 & 3,040 & 3,420 & 8.4 & 11 & 10.7 & 70 \\
\hline 8 & 381.6 & 763 & 1,145 & 1,526 & 1,908 & 2,289 & 2,671 & 3,053 & 3,434 & 3.6 & 12 & 10.8 & 71 \\
\hline 9 & 383.1 & 766 & 1,149 & 1,532 & 1,916 & 2,299 & 2,682 & 3,065 & 3,448 & 3.5 & 13 & 10.9 & 73 \\
\hline 10 & 384.7 & 769 & 1,154 & 1,539 & 1,923 & 2,308 & 2,693 & 3,077 & 3,462 & 4.1 & 14 & 11.0 & 74 \\
\hline 11 & 386.2 & 772 & 1,159 & 1,545 & 1,931 & 2,317 & 2,703 & 3,090 & 3,476 & 4.3 & 15 & 11.1 & 75 \\
\hline 12 & 387.7 & 775 & 1,163 & 1,551 & 1,939 & 2,326 & 2,714 & 3,102 & 3,490 & 4.5 & 16 & 11.2 & 77 \\
\hline 13 & 389.3 & 779 & 1,168 & 1,557 & 1,946 & 2,336 & 2,725 & 3,114 & 3,504 & 4.7 & 17 & 11.3 & 78 \\
\hline 14 & 390.8 & 782 & 1,172 & 1,563 & 1,954 & 2,345 & 2,736 & 3,127 & 3,517 & 4.8 & 18 & 11.4 & 79 \\
\hline 15 & 392.4 & 785 & 1,177 & 1,569 & 1,962 & 2,354 & 2,747 & 3,139 & 3,531 & 5.0 & 19 & 11.5 & $\$ 0$ \\
\hline 16 & 393.9 & 788 & 1,182 & 1,576 & 1,970 & 2,363 & 2,757 & 3,151 & 3,545 & 5.2 & 20 & 11.6 & 82 \\
\hline 17 & 395.5 & 791 & 1,186 & 1,582 & 1,977 & 2,373 & 2,768 & 3,164 & 3,559 & 5.4 & 21. & 11.7 & 83 \\
\hline 18 & 397.0 & 794 & 1,191 & 1,588 & 1,985 & 2,382 & 2,779 & 3,176 & 3.573 & 5.5 & 22 & 11.8 & 84 \\
\hline 19 & 398.6 & 797 & 1,196 & 1,594 & 1,993 & 2,391 & 2,790 & 3,188 & 3,587 & 5.7 & 23 & 11.9 & 86 \\
\hline 20 & 400.1 & 800 & 1,200 & 1,600 & 2,000 & 2,401 & 2,801 & 3,201 & 3,601 & 5.8 & 24 & 12.0 & 87 \\
\hline 21 & 401.6 & 803 & 1,205 & 1,607 & 2,008 & 2,410 & 2,811 & 3,213 & 3,615 & 6.0 & 25 & 12.1 & 89 \\
\hline 22 . & 403.2 & 806 & 1,210 & 1,613 & 2,016 & 2,419 & 2,822 & 3,225 & 3,629 & 6.1 & 26 & 12.2 & 90 \\
\hline 23 & 404.7 & 809 & 1,214 & 1,619 & 2,024 & 2,428 & 2,833 & 3,238 & 3,643 & 6.3 & 27 & 12.3 & 91 \\
\hline 24 & 406.3 & 813 & 1,219 & 1,625 & 2,031 & 2,438 & 2,844 & 3,250 & 3,656 & (6. 4 & 28 & 12.4 & 93 \\
\hline 25 & 407.8 & 816 & 1,223 & 1,631 & 2,039 & 2,447 & 2,855 & 3,263 & 3,670 & 6.5 & 29 & 12.5 & 94 \\
\hline 26 & 409.4 & 819 & 1,228 & 1,637 & 2,047 & 2,456 & 2,866 & 3,275 & 3,684 & 6.7 & 30 & 12.6 & 96 \\
\hline 27 & 410.9 & 822 & 1,233 & 1,644 & 2,055 & 2,465 & 2,876 & 3,287 & 3,698 & 6.8 & 31 & 12.7 & 97 \\
\hline 28 & 412.5 & 825 & 1,237 & 1,650 & 2,062 & 2,475 & 2,887 & 3,300 & 3,712 & 6.9 & 32 & 12.8 & 99 \\
\hline 29 & 414.0 & 828 & 1,242 & 1,656 & 2,070 & 2,484 & 2,898 & 3,312 & 3,726 & 7.0 & 33 & 12.9 & 100 \\
\hline 30 & 415.5 & 831 & 1,247 & 1,662 & 2,078 & 2,493 & 2,909 & 3,324 & 3,740 & 7. 2 & 34 & 13.0 & 102 \\
\hline 31 & 417.1 & 834 & 1,251 & 1,668 & 2,085 & 2,503 & 2,920 & 3,337 & 3,754 & 7.3 & 35 & 13.1 & 103 \\
\hline 32 & 418.6 & 837 & 1,256 & 1,675 & 2,093 & 2,512 & 2,930 & 3,349 & 3,768 & 7.4 & 36 & 13.2 & 105 \\
\hline 33 & 420.2 & 840 & 1,261 & 1,681 & 2,101 & 2,521 & 2,941 & 3,361 & 3,782 & 7.5 & 37 & 13.3 & 106 \\
\hline 34 & 421.7 & 843 & 1,265 & 1,687 & 2,109 & 2,530 & 2,952 & 3,374 & 3,796 & 7.6 & 38 & 13.4 & 108 \\
\hline 35 & 423.3 & 847 & 1,270 & 1,693 & 2,116 & 2,540 & 2,963 & 3,386 & 3,809 & 7.8 & 39 & 13.5 & 109 \\
\hline 36 & 424.8 & 850 & 1,274 & 1,699 & 2,124 & 2,549 & 2,974 & 3,399 & 3,823 & 7.9 & 40 & 13.6 & 111 \\
\hline 37 & 426.4 & 853 & 1,279 & 1,705 & 2,132 & 2,558 & 2,985 & 3,411 & 3,837 & 8.0 & 41 & 13.7 & 112 \\
\hline 38 & 427.9 & 856 & 1,284 & 1,712 & 2,140 & 2,567 & 2,995 & 3,423 & 3,851 & 8.1 & 42 & 13.8 & 114 \\
\hline 39 & 429.5 & 859 & 1,288 & 1,718 & 2,147 & 2,577 & 3,006 & 3,436 & 3,865 & 8.2 & 43 & 13.9 & 115 \\
\hline 40 & 431.0 & 862 & 1,293 & 1,724 & 2,155 & 2,586 & 3,017 & 3,448 & 3,879 & 8.3 & 44 & 14.0 & 117 \\
\hline 41 & 432. & 865 & 1,298 & 1,730 & 2,163 & 2,595 & 3,028 & 3,460 & 3,993 & 8.4 & 45 & 14.1 & 119 \\
\hline 42 & 434.1 & 868 & 1,302 & 1,736 & 2,170 & 2,605 & 3,039 & 3,473 & 3,907 & 8.5 & 46 & 14.2 & 120 \\
\hline 43 & 435.6 & 871 & 1,307 & 1,743 & 2,178 & 2,614 & 3,049 & 3,485 & 3,921 & 8.6 & 47 & 14.3 & 122 \\
\hline 44 & 437.2 & 874 & 1,312 & 1,749 & 2,186 & 2,623 & 3,060 & 3,498 & 3,935 & 8.7 & 48 & 14.4 & 124 \\
\hline 45 & 488.7 & 877 & 1,316 & 1,755 & 2,194 & 2,632 & 3,071 . & 3,510 & 3,949 & 8.8 & 49 & 14.5 & 125 \\
\hline 46 & 440.3 & 881 & 1,321 & 1., 761 & 2,201 & 2,642 & 3,082 & 3,522 & 3,963 & 8.9 & 50 & 14.6 & 127 \\
\hline 47 & 441.8 & 884 & 1,325 & 1,767 & 2,209 & 2,651 . & 3,093 & 3,535 & 3,976 & 9.0 & 51. & 14.7 & 129 \\
\hline 48 & 443.4 & 897 & 1,330 & 1,773 & 2,217 & 2,660 & 3,104 & 3,547 & 3,990 & 9.1 & 52 & 14.8 & 130 \\
\hline 49 & 444.9 & 890 & 1,335 & 1,780 & 2,225 & 2,669 & 3,113 & 3,558 & 4,003 & 9.2 & 53 & 14.9 & 132 \\
\hline 50 & 446.5 & 893 & 1, 339 & 1,786 & 2,232 & 2,679 & 25 & 3,572 & 4, & 9.3 & 54 & 15.0 & 134 \\
\hline 51 & 448.0 & 896 & 1,344 & 1,792 & 2,240 & 2,688 & 3,136 & 3,584 & 4,032 & 9.4 & 55 & 15.1 & 135 \\
\hline 52 & 449.6 & 899 & 1,349 & 1,798 & 2,248 & 2,697 & 3,147 & 3,596 & 4,046 & 9.5 & 56 & 15.2 & 137 \\
\hline 53 & 451.1 & 902 & 1,353 & 1,804 & 2,256 & 2,707 & 3,158 & 3,609 & 4,060 & 9.6 & 58 & 15.3 & 139 \\
\hline 54 & 452.7 & 905 & 1,358 & 1,811 & 2,263 & 2,716 & 3,169 & 3,621 . & 4,074 & 9.7 & 59 & 15.4 & 141 \\
\hline 55 & 454.2 & 908 & 1,363 & 1,817 & 2,271 & 2,725 & 3,179 & 3,634 & 4,088 & 9.8 . & 60 & 15.5 & 142 \\
\hline 56 & 455.8 & 912 & 1,367 & 1,823 & 2,279 & 2,735 . & 3,190 & 3,646 & 4,102 & 9.9 & 61 & 15.6 & 144 \\
\hline 57 & 457. 3 & 915 & 1,372 & 1,829 & 2,286 & 2,744 & 3,201 & 3,658 & 4,116 & 10.0 & 62 & 15.7 & 146 \\
\hline 58 & 458.8 & 918 & 1,377 & 1,835 & 2,294 & 2,753 & 3,212 & 3,671 & 4,130 & 10.1 & 63 & 15.8 & 148 \\
\hline 59 & 460.4 & 921 & 1,381 & 1,842 & 2,302 & 2,762 & 3,223 & 3,683 & 4,144 & & & 15.9 & 150 \\
\hline 60 & 461.9 & 924 & 1,386 & 1,848 & 2,310 & 2,772 & 3,234 & 3,696 & 4,157 & & & & \\
\hline
\end{tabular}

$a$ For all distances under 1.6 miles the correction may be taken as +5 feet. Height of instrument is assumed 4.5 feet. 
TABLe 24.-For obtaining differences of altitude for any minute, etc.-Continued.

$5^{\circ}$

\begin{tabular}{|c|c|c|c|c|c|c|c|c|c|c|c|c|c|}
\hline & 1 & 2 & 3 & 4 & 5 & (i) & $\boldsymbol{7}$ & 8 & 9 & \multicolumn{4}{|c|}{$\begin{array}{l}\text { Corrections for curva- } \\
\text { ture, refraction, and } \\
\text { height of instrument.a }\end{array}$} \\
\hline 0 & 4619 & (924 & 1386 & 1848 & 2.310 & 2.772 & 3.234 & 3,00 & 4.157 & & & & \\
\hline 1 . & $\begin{array}{l}463.5 \\
4\end{array}$ & 927 & $\begin{array}{l}1,300 \\
1,390\end{array}$ & $\begin{array}{l}1,840 \\
1,854\end{array}$ & $\begin{array}{l}2,310 \\
2,317\end{array}$ & $\begin{array}{l}2,781 \\
\text { 2. }\end{array}$ & $\begin{array}{l}0,254 \\
3,244\end{array}$ & $\begin{array}{r}3,090 \\
3,708\end{array}$ & 4,171. & Iriles. & Feet. & Mfiles. & Feet. \\
\hline 2 & 465.0 & 930 & 1,395 & 1,860 & 2,325 & 2,790 & 3,255 & 3,720 & 4,185 & 1.6 & 6 & 10.2 & 64 \\
\hline 3 & 466.6 & 933 & 1,400 & 1,866 & 2,333 & 2,800 & 3,266 & 3,733 & 4,199 & 2.1 & 7 & 10.3 & 65 \\
\hline 4 & 468.1 & 936 & 1,405 & 1,873 & $2,341$. & 2,809 & 3,277 & 3,745 & 4,213 & 2.5 & 8 & 10.4 & 67 \\
\hline 5 & 469.7 & 939 & 1,409 & 1,879 & 2,348 & 2,818 & 3,288 & 3,757 & 4,227 & 2.8 & 9 & 10.5 & 68 \\
\hline 0 & 471.2 & 942 & 1,414 & 1,385 & 2,356 & 2,827 & 3,299 & 3,770 & 4,241 & 3.1 & 10 & 10.6 & 69 \\
\hline 7 & 472.8 & 946 & 1,419 & $1,391$. & 2,364 & 2,837 & 3,309 & 3,782 & 4,255 & 3.4 & 11 & 10.7 & 70 \\
\hline 8 & 474.3 & 949 & 1, 423 & 1,397 & 2,372 & 2,846 & 3,320 & 3,795 & 4,269 & 3.6 & 12 & 10.8 & 71 \\
\hline 9 & 475.9 & 952 & 1,428 & 1,904 & 2,379 & 2,855 & 3,331 & 3,807 & 4,283 & 3.8 & 13 & 10.9 & 73 \\
\hline 10 & 477.4 & 955 & 1,432 & 1,910 & 2,387 & 2,865 & 3,342 & 3,819 & 4,297 & 4.1 & 14 & 11.0 & 74 \\
\hline 11 & 479.0 & 958 & 1,437 & 1,916 & 2,395 & 2,874 & 3,353 & 3,832 & 4,311 & 4.3 & 15 & 11.1 & .75 \\
\hline 12 & 480.5 & 961 & 1,442 & 1,922 & 2,403 & 2,883 & 3,364 & 3,844 & 4,325 & 4.5 & 16 & 11.2 & 77 \\
\hline 13 & 482.1 & 964 & 1,447 & 1,928 & 2,410 & 2,892 & 3,375 & 3,857 & 4,339 & 4.7 & 17 & 11.3 & 78 \\
\hline 14 & 483.6 & 967 & 1,451 & 1,935 & 2,418 & 2,902 & 3,385 & 3,869 & 4,353 & 4.8 & 18 & 11.4 & 79 \\
\hline 15 & 485.2 & 970 & 1,456 & 1,941 & 2,426 & 2,911 & 3,396 & 3,881 & 4,367 & 5.0 & 19 & 11.5 & 80 \\
\hline 16 & 486.7 & 973 & 1,461 & 1,947 & 2,434 & 2,920 & 3,407 & 3,894 & 4,381 & 5.2 & 20 & 11.6 & 82 \\
\hline 17 & 488.3 & 976 & 1,465 & 1,953 & 2,441 & 2,930 & 3,418 & 3,906 & 4,394 & 5.4 & 21 & 11.7 & 83 \\
\hline 18 & 489.8 & 980 & 1,470 & 1,959 & 2,449 & 2,939 & 3,429 & 3,919 & 4,408 & 5.5 & 22 & 11.8 & 84 \\
\hline 19 & 491.3 & 983 & 1,475 & 1,966 & 2,457 & 2,948 & 3,440 & 3,931 & 4,422 & 5.7 & 23 & 11.9 & 86 \\
\hline 20 & 492.9 & 986 & 1,479 & 1,972 & 2,465 & 2,958 & 3,450 & 3,943 & 436 & 5.8 & 24 & 1.2 .0 & 87 \\
\hline 21 & 494.5 & 989 & 1,483 & 1,978 & 2,472 & 2,967 & $3,46 \mathrm{I}$ & 3,956 & 4,450 & 6.0 & 25 & 12.1 & 89 \\
\hline 22 & 496.0 & 992 & 1,488 & 1,984 & 2,480 & 2,976 & 3,472 & 3,968 & 4,464 & 6.1 & 26 & 12.2 & 90 \\
\hline 23 & 497.6 & 995 & 1,493 & 1,990 & 2,488 & 2,985 & 3,483 & 3,981 & 4,478 & 6.3 & 27 & 12.3 & 91 . \\
\hline 2.4 & 499.1 & 998 & 1,498 & 1,996 . & 2,496 & 2,995 & 3,494 & 3,993 & 4,492 & 6.4 & 28 & 12.4 & 93 \\
\hline 25 & 500.7 & 1,001 & 1,502 & 2,003 & 2,503 & 3,004 & 3,505 & 4,005 & 4,506 & 6.5 & 29 & 12.5 & 94 \\
\hline 26 & 502.2 & 1,004 & 1,507 & 2,009 & 2,511 & 3,013 & 3,515 & 4,018 & 4,520 & 6. & 30 & 12.6 & 96 \\
\hline 27 & 503.8 & 1,007 & 1,512 & 2,015 & 2,519 & 3,023 & 3,526 & $4 ; 030$ & 4,534 & 6. & 31 & 12.7 & 97 \\
\hline 28 & 505.3 & 1,010 & 1,516 & $2,0.21$ & 2,527 & 3,032 & 3,537 & 4,042 & 4,548 & 6.9 & 32 & 12.8 & 99 \\
\hline 29 & 506.9 & 1,014 & 1,521 & 2,027 & 2,534 & 3,041 & 3,548 & 4,055 & 4,562 & 7.0 & 33 & 12.9 & 100 \\
\hline 30 & 508.4 & 1,017 & 1,525 & 2,084 & 2,542 & 3,050 & 3,559 & 4,067 & 4,576 & 7. & 34 & 13 & 102 \\
\hline 31 & 510.0 & 1,020 & 1,530 & 2,040 & 2,550 & 3,060 & 3,570 & 4,080 & 4,590 & 7.3 & 35 & 13.1 & 103 \\
\hline 32 & 511.5 & 1,023 & 1,535 & 2,046 & 2,558 & 3,069 & 3,581 & 4,092 & 4,604 & 7. & 36 & 13.2 & 105 \\
\hline 33 & 513.0 & 1,026 & $\begin{array}{l}1,539 \\
\text {. }\end{array}$ & 2,052 & 2,565 & 3,078 & 3,591 & 4,105 & 4,618 & 7. & 37 & 13.3 & 106 \\
\hline 34 & 514.6 & 1,029 & 1,544 & 2,058 & 2,573 & 3,088 & 3,602 & 4,117 & 4,632 & 7. & 38 & 13.4 & 108 \\
\hline 35 & 516.2 & 1,032 & 1,549 & 2,065 & 2,581 & 3,097 & 3,613 & $\begin{array}{l}4,129 \\
\end{array}$ & 4,645 & 7.8 & 39 & 13.5 & 109 \\
\hline 36 & 517.7 & 1,035 & 1,553 & 2,071 & 2, & 3,106 & 3,624 & 4,142 & 4,659 & 7. & 40 & 13.6 & 111. \\
\hline 37 & 519.3 & ], 039 & 1,558 & 2,077 & 2,596 & 3,116 & 3,635 & 4,154 & 4,673 & 8. & 41 & 13.7 & 112 \\
\hline 38 & 520.8 & 1,042 & 1,563 & 2,083 & 2,604 & 3,125 & 3,646 & 4,167 & 4,687 & 8. & 42 & 13.8 & 114 \\
\hline 39 & 522.4 & 1,045 & 1,568 & 2,089 & 2,612 & 3,134 & 3,657 & 4,179 & 4,701 & 8.2 & 43 & 13.9 & 115 \\
\hline 40 & 523.9 & 1,048 & 1,572 & 2,095 & 2,620 & 3,1 & $3,667^{\circ}$ & 4,191 & 4,7 & 8. & 44 & 14 & 117 \\
\hline 41 & 525.5 & 1,051 & 1,576 & 2,102 & & 3,1 & & 4,204 & & 8. & 45 & & 119 \\
\hline 42 & 527.0 & 1,054 & 1,581 & 2,108 & 2,635 & 3,162 & 3,689 & 4,216 & 4,743 & 8.5 & 46 & 14.2 & 120 \\
\hline 43 & 528 & 1,057 & 1 & 2,114 & 2 & 3,172 & 3,700 & 4,229 & 4,757 & 8. & 47 & 1.4 .3 & 122 \\
\hline 44 & 530 & 1, & $1,591$. & 2.121 & 2,651 & 3,181 & 3,711 & 4,241 & 4,771 & 8. & 48 & 14.4 & 124 \\
\hline 45 & 531.7 & 1,0 & $\begin{array}{l}1,595 \\
1,595\end{array}$ & 2,127 & 2,0 & 3,1 & 3,722 & 4, 253 & & 8. & $\begin{array}{l}49 \\
49\end{array}$ & 14.5 & 125 \\
\hline 46 & 533.2 & 1,066 & 1,600 & 2,13 & 2,666 & 3,199 & 3,733 & 4,266 & 4,799 & 8. & 50 & 14.6 & 127 \\
\hline 47 & 534.8 & 1,070 & 1,605 & 2,139 & 2,674 & 3,2 & 3,743 & 4,278 & & 9. & 51. & 14.7 & 129 \\
\hline 48 & 536.3 & ]., 073 & 1,609 & 2,145 & 2,682 & 3,218 & 3 , & 4,291 & 4,827 & 9. & 52 & 14.8 & 130 \\
\hline 49 & 537.9 & 1,076 & 1,614 & 2,154 & 2,689 & 3,227 & 3,765 & 4,303 & 4,841 & 9.2 & 53 & 14.9 & 132 \\
\hline 50 & 539.4 & 1,079 & 1,618 & 2,1 & 2,0 & 3,23 & 3,776 & 4,315 & 4,8 & 9.3 & 54 & 15.0 & 134 \\
\hline & & & & & & & & 4, & & 9. & 5.5 & & 135 \\
\hline 02 & 542.5 & 1,085 & 1,628 & 2,170 & 2,713 & 3,25 & 3,798 & 4,340 & & 9.5 & 56 & 15.2 & 137 \\
\hline 53 & 544.1 & 1,088 & 1,632 & 2,176 & 2,7 & 3,265 & 3,809 & $\begin{array}{l}4,353 \\
\end{array}$ & 4,897 & 9.6 & 58 & 15.3 & 139 \\
\hline 54 & 545.6 & 1,091 & 1,637 & 2,183 & 2,728 & 3,274 & 3,819 & 4,365 & $4,911$. & 9.7 & 59 & 15.4 & 141 \\
\hline & 547.2 & 1,094 & 1,642 & 2,189 & 2,736 & 3,283 & 3,830 & 4,378 & & 9.8 & 60 & 15.5 & 142 \\
\hline & 548.7 & 1,097 & 1,646 & 2,195 & 2,743 & 3,292 & 3,841 . & 4,390 & $\begin{array}{r}4,939 \\
\end{array}$ & 9.9 & 61 & 15.6 & 144 \\
\hline & 550.3 & 1,101 & 1,651 & $2,201$. & 2,752 & 3,302 & 3,852 & 4,402 & 4,953 & 10.0 & 62 & 15.7 & 146 \\
\hline 58 & 551.8 & 1,104 & 1,656 & 2,207 & 2,759 & 3,311 & 3,863 & 4,415 & 4,967 & 10.1 & 63 & 15.8 & 148 \\
\hline 59 & 553.4 & 1,107 & 1,661 & 2,214 & 2,767 & 3,320 & 3,874 & 4,427 & 4,981 & & & & 150 \\
\hline 60 & 555.0 & $\cdot 1,1.10$ & 1,665 & 2,220 & 2,775 & 3,330 & 3,885 & 4,440 & 4,995 & & & 10.0 & ].51 \\
\hline
\end{tabular}

a For all distances under 1.6 miles the correction may be taken as +5 feet. Height of instrument is assumed 4.5 feet. 
TABLE 24.-For obtaining differences of altitude for any minute, etc.-Continued.

$6^{\circ}$

\begin{tabular}{|c|c|c|c|c|c|c|c|c|c|c|c|c|c|}
\hline & 1 & 2 & 3 & 4 & 5 & 6 & 7 & 8 & 9 & \multicolumn{4}{|c|}{$\begin{array}{l}\text { Corrections for curva- } \\
\text { ture, refraction and } \\
\text { height of instrument.a }\end{array}$} \\
\hline & & & & & & & & & & & 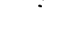 & & \\
\hline $\begin{array}{l}0 \\
1\end{array}$ & $\begin{array}{l}555.0 \\
556.5\end{array}$ & $\begin{array}{l}1,110 \\
1,113\end{array}$ & $\begin{array}{l}1,665 \\
1,670\end{array}$ & $\begin{array}{l}2,220 \\
2,226\end{array}$ & $\begin{array}{l}2,775 \\
2,783\end{array}$ & $\begin{array}{l}3,330 \\
3,339\end{array}$ & $\begin{array}{l}3,855 \\
3,896\end{array}$ & $\begin{array}{l}4,440 \\
4,452\end{array}$ & $\begin{array}{l}4,995 \\
5,009\end{array}$ & Wiles & & & \\
\hline 2 & 558.1 & 1,116 & 1,674 & 2,232 & 2,790 & 3,348 & $\begin{array}{l}3,890 \\
3,906\end{array}$ & $\begin{array}{l}4,402 \\
4,464\end{array}$ & $\begin{array}{l}5 \\
5,023\end{array}$ & $\begin{array}{l}\text { Miles. } \\
1.6\end{array}$ & $\begin{array}{r}\text { reec. } \\
6\end{array}$ & 10.2 & $\begin{array}{c}\text { Feet. } \\
64\end{array}$ \\
\hline 3 & 559.6 & 1,119 & 1,679 & 2,238 & 2,798 & 3,358 & 3,917 & 4,477 & 5,037 & 2.1 & 7 & 10.3 & 65 \\
\hline 4 & 561.2 & 1,122 & 1,684 & 2,245 & 2,806 & 3,367 & 3,928 & 4,489 & 5,050 & 2.5 & 8 & 10.4 & 67 \\
\hline 5 & 562.7 & 1,125 & 1,688 & 2,251 & 2,814 & 3,370 & 3,939 & 4,502 & 5,064 & 2.8 & 9 & 10.5 & 68 \\
\hline 6 & 564.3 & 1,129 & 1,693 & 2,257 & 2,821 & 3,386 & 3,950 & 4,514 & 5,078 & 3.1 & 10 & 10.6 & 69 \\
\hline 7 & 565.8 & 1,132 & 1, 697 & 2,263 & 2,829 & 3,395 & 3,961 & 4,527 & 5,092 & 3.4 & 11 & 10.7 & 70 \\
\hline $\begin{array}{l}8 \\
9\end{array}$ & $\begin{array}{l}567.4 \\
568.9\end{array}$ & $\begin{array}{l}1,135 \\
1,138\end{array}$ & $\begin{array}{l}1,702 \\
1,707\end{array}$ & $\begin{array}{l}2,270 \\
2,276\end{array}$ & $\begin{array}{l}2,837 \\
2,845\end{array}$ & $\begin{array}{l}3,404 \\
3,414\end{array}$ & $\begin{array}{l}3,972 \\
3,983\end{array}$ & $\begin{array}{l}4,539 \\
4,551\end{array}$ & $\begin{array}{l}5,106 \\
5,120\end{array}$ & $\begin{array}{l}3.6 \\
3.8\end{array}$ & $\begin{array}{l}12 \\
13\end{array}$ & $\begin{array}{l}10.8 \\
10.9\end{array}$ & $\begin{array}{l}71 \\
73\end{array}$ \\
\hline 10 & 570.5 & 141 & 71 & 2,282 & 2,852 & 423 & 15 & 564 & 5,134 & 4.1 & 14 & 11.0 & 74 \\
\hline & 572.0 & 1,144 & 1,716 & 2,288 & 2,860 & 3,432 & 4,004 & 4,576 & 5,148 & 4.3 & 15 & 11.1 & 75 \\
\hline 12 & 573.6 & 1,147 & 1,721 . & 2,294 & 2,868 & 3,442 & 4,015 & 4,589 & 5,162 & 4.5 & 16 & 11.2 & 77 \\
\hline 13 & 575.2 & 1,150 & 1,725 & 2,301 & 2,876 & 3,451 & 4,026 & 4,601 & 5,176 & 4.7 & 17 & 11.3 & 78 \\
\hline 14 & 576.7 & 1,153 & 1,730 & 2,307 & 2,884 & 3,460 & 4,037 & 4,614 & 5,190 & 4.8 & 18 & 11.4 & 79 \\
\hline 15 & 578.3 & 1,15 & 1,735 & 2,313 & 2,891 & 3,470 & 4,048 & 4,626 & 5,204 & 5.0 & 19 & 11.5 & 80 \\
\hline 16 & 579.8 & 1,160 & 1,739 & 2,319 & 2,899 & 3,479 & 4,059 & 4,639 & 5,218 & 5.2 & 20 & 11.6 & 82 \\
\hline 17 & 581.4 & 1,163 & 1,744 & 2,325 & 2,907 & 3,488 & 4,070 & 4,651 & 5,232 & 5.4 & 21. & 11.7 & 83 \\
\hline 18 & $\begin{array}{l}582.9 \\
584\end{array}$ & 1,166 & 1,749 & 2,332 & 2,915 & 3,498 & 4,080 & 4,663 & 5,246 & 5.5 & 22 & 11.8 & 84 \\
\hline 19 & 584.5 & 1,169 & 1,753 & 2,338 & 2,922 & 3,507 & 4,091 & 4,676 & 5,260 & 5.7 & 23 & 11.9 & 86 \\
\hline 20 & 586.0 & 1,172 & 1,758 & 2,344 & 2,930 & 3,516 & $4,10^{\circ}$ & 4,688 & 5,274 & 5.8 & 24 & 12.0 & 87 \\
\hline 21 & 587. & 1,175 & 1,763 & 2,350 & 2,938 & 3,526 & 4,113 & 4,701 & 5,2 & 6.0 & 25 & 12.1. & 89 \\
\hline 22 & 589.1 & 1,178 & 1,767 & 2,357 & 2,946 & 3,535 & 4,124 & 4,713 & 5,302 & 6.1 & 26 & 12.2 & 90 \\
\hline 23 & 5 & $1,181$. & 1,77 & 363 & 2,953 & 3,544 & 4,135 & 4,726 & 5,316 & 6.3 & 27 & 12.3 & 91. \\
\hline 24 & 592.2 & 1,185 & 1,777 & 2,369 & 2,961 & 3,554 & 4,146 & 4,738 & 5,330 & 6.4 & 28 & 12 & 93 \\
\hline 25 & 593.8 & 1,188 & 1,781 & 2,375 & 2,969 & 3,563 & 4,157 & 4,750 & 5,344 & 6.5 & 29 & 12.5 & 94 \\
\hline 26 & 595.4 & 1,191 & 1,786 & 2,381 & 2,977 & 3,572 & 4,1 & 4,7 & 5,358 & 6.7 & 30 & 12.6 & 96 \\
\hline & & 1,19 & 1,7 & 388 & 2,985 & 3,581 & 4,1 & 4,775 & 5,372 & 6.8 & 31 & & 97 \\
\hline 28 & 598.5 & 1,197 & 1,795 & 2,394 & 2,992 & 3,591 & 4,189 & 4,788 & 5,386 & 6.9 & 32 & 12. & 99 \\
\hline 29 & 600.0 & 1,200 . & 1,800 & 2,400 & 3,000 & 3,600 & 4,200 & 4,800 & 5,400 & 7.0 & 33 & 12.9 & 100 \\
\hline 30 & 60 & 1,203 & 1, & 406 & 3,008 & 3,609 & 4,211 & 4,813 & 5,414 & 7.2 & 34 & 13 & 102 \\
\hline 31 & & & & 3 & a & 3,619 & 2 & & & & & & 103 \\
\hline 32 & .7 & 1,209 & 1,814 & 2,419 & 3,023 & 3,628 & 4,233 & 4,838 & 5,442 & 7.4 & 6 & 13.2 & 105 \\
\hline 33 & & 1,213 & 1,819 & 2,425 & 3,031 & 3,637 & 4,244 & 4,850 & 5,456 & 7.5 & 7 & 13.3 & 106 \\
\hline 34 & & & & 1 & 3,0 & 3,6 & 4,2 & 4,8 & & & 8 & & 108 \\
\hline 35 & .4 & 9 & 1,828 & 437 & 3,047 & 3,656 & 4,266 & 10 & 5,4 & 7. & 9 & 13 & 109 \\
\hline 36 & & 1,222 & 1,83 & 2,444 & 3,055 & 3,666 & 4,2 & 4 & 5,498 & 7.9 & 40 & 13.6 & 111. \\
\hline 37 & & 0 & 1,8 & 450 & 3,062 & 3,675 & 4,287 & 4,8 & 5,512 & & 41 & 13.7 & 112 \\
\hline 38 & 614.0 & 1,228 & 1,842 & 2,456 & 3,070 & 3,684 & 4,298 & 4,912 & 5,526 & 8.1 & 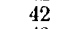 & 13 & 114 \\
\hline 39 & 615.5 & 1,231 & 1,847 & 2,462 & 3,078 & 3,694 & 4,309 & 4,925 & 5,540 & 8.2 & 43 & 13.9 & 115 \\
\hline 40 & 617.2 & 1,234 & 1,851 & 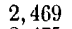 & 36 & 3,7 & 4,320 & 4, & 5 , & 8. & 4 & 14 & 117 \\
\hline 41 & & & 1 & & 3,094 & 3,7 & 4,3 & 4,8 & & & 45 & & 11.9 \\
\hline 42 & 62 & 1 & 1,8 & 481 & 3,101 & 3,722 & 4,3 & 4,962 & 5,5 & 8.5 & 6 & 14 & 120 \\
\hline 43 & & 1 , & 1 & 109 & 3, & 3,7 & 4,3 & 4,975 & & & 7 & & 122 \\
\hline 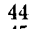 & & 1 , & & 2 & & 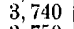 & 4,364 & 4,9 & & & 8 & 14 & 124 \\
\hline 45 & 62 & 1 , & $1, c$ & 0 &, & 3,7 & 4,374 & 4,9 & & & 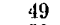 & & 125 \\
\hline 46 & & 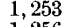 & 1,87 & 506 & 3,132 & 3,759 & 4,385 & 5,0 & 5,6 & 8. & 50 & 14 & 127 \\
\hline 47 & & 1,256 & & & & & 4,3 & & & 9. & 1. & & 129 \\
\hline & & 1 , & 1., 889 & & 3,148 & 3,778 & 4,407 & 5,037 & 5,667 & 9. & 2 & 14 & 130 \\
\hline 49 & 631.2 & 1,262 & 1,894 & 2,525 & 3,156 & 3,787 & 4,418 & 5,049 & 5,681 & 9.2 & 53 & 14.9 & 132 \\
\hline 50 & 632.7 & 1 , & 1,898 & 2,531 & 3 & 3, & 4,4 & 5 & 5,6 & 9. & 54 & 15.0 & 13 \\
\hline & & & & & & & & & & & & & 135 \\
\hline & 8 & 272 & 1,9 & 2,5 & 3,179 & $3,81.5$ & 4,451 & 5 , & & 9. & 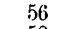 & 15 & 137 \\
\hline 53 & 637.4 & 1,275 & 1,9 & 2,55 & 3,187 & 3,824 & 4,462 & 5,0 & 5,7 & 9. & 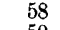 & 15.3 & 139 \\
\hline & & & 19 & & 3,195 & 3,8 & 4,473 & 5,112 & 5,7 & 9.7 & 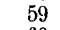 & 15.4 & 141. \\
\hline & & 1 & & 2,5 & & & 4,4 & 5, & & 9 . & 0 & 15 & 142 \\
\hline 56 & 64 & 1, & 1,9 & 2,568 & 3,210 & 3,8 & 4,494 & 5,1 & 5,779 & 9.9 & + & 15.6 & 144 \\
\hline 57 & & 1,287 & 19 & 2,575 & 3,218 & 3,8 & 4,505 & 5,149 & 5,793 & 10.0 & 62 & 15.7 & 146 \\
\hline & & 1.9 & 36 & 2,581 & 3,2 & 3,8 & 4,5 & 5,161 & 5,8 & 10.1 & 63 & 15 & 148 \\
\hline 59 & 646.7 & 1,293 & 1,940 & 2,587 & 3,234 & 3,880 & 4,527 & 5,174 & 5,821 & & & 15.9 & 150 \\
\hline 60 & 648.3 & 1,297 & 1,945 & $2,59 \ddot{3}$ & 3,242 & 3,890 & 4,538 & 5,186 & 5,835 & & & & \\
\hline
\end{tabular}

$a$ For all distances under 1.6 miles the correction may be taken as +5 feet. Height of instrument is assumed 4.5 feet. 
TAвtв 24.-For obtaining differences of altitude for any minute, etc.-Continued.

$7^{\circ}$ 운

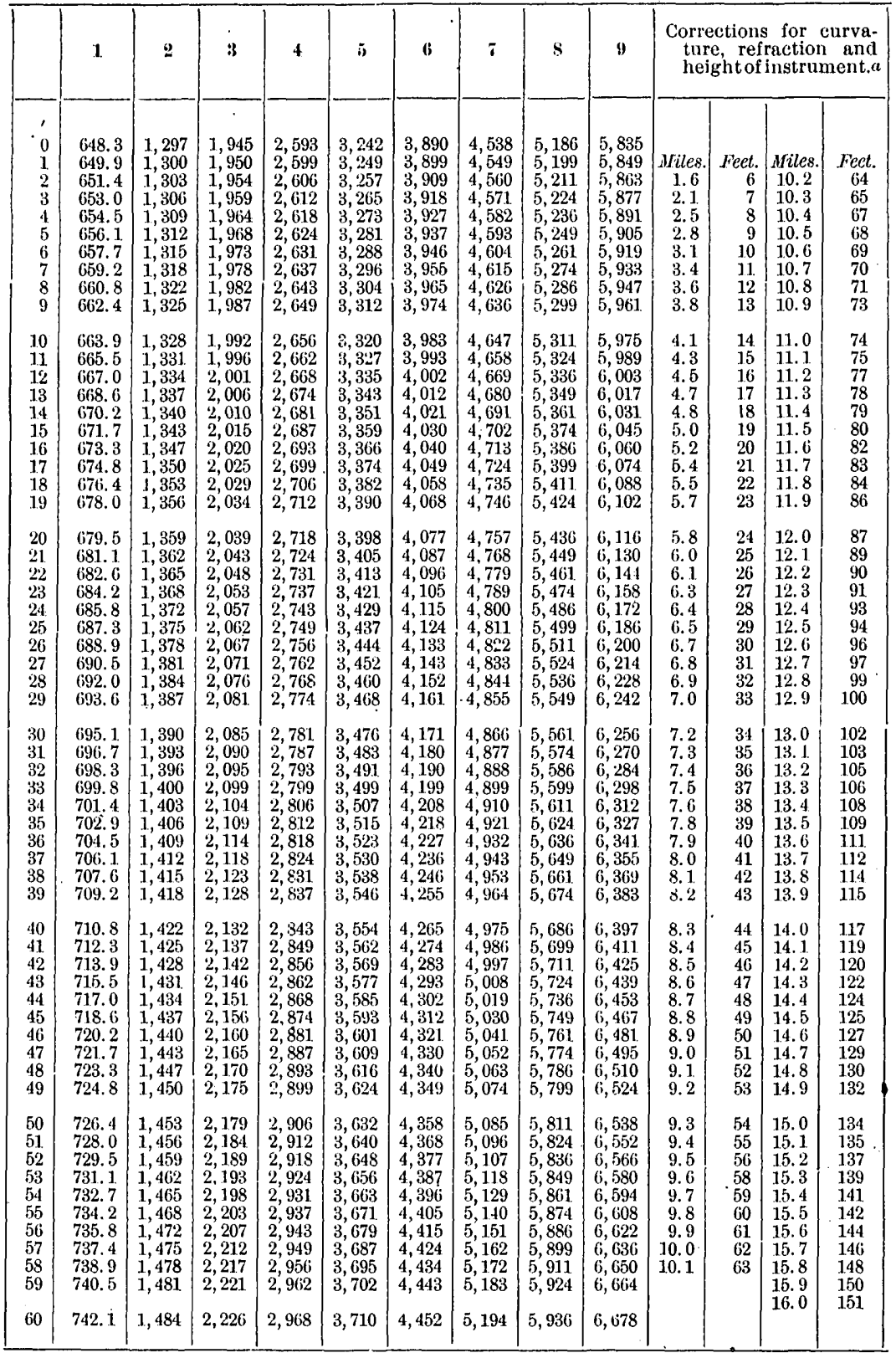

a For all distances under 1.6 miles the correction may be taken us +5 feet. Height of instrument is assumed 4.5 feet. 
TABLE 24.-For obtaining differences of altitude for any minute, etc.-Cọtinued.

$8^{\circ}$

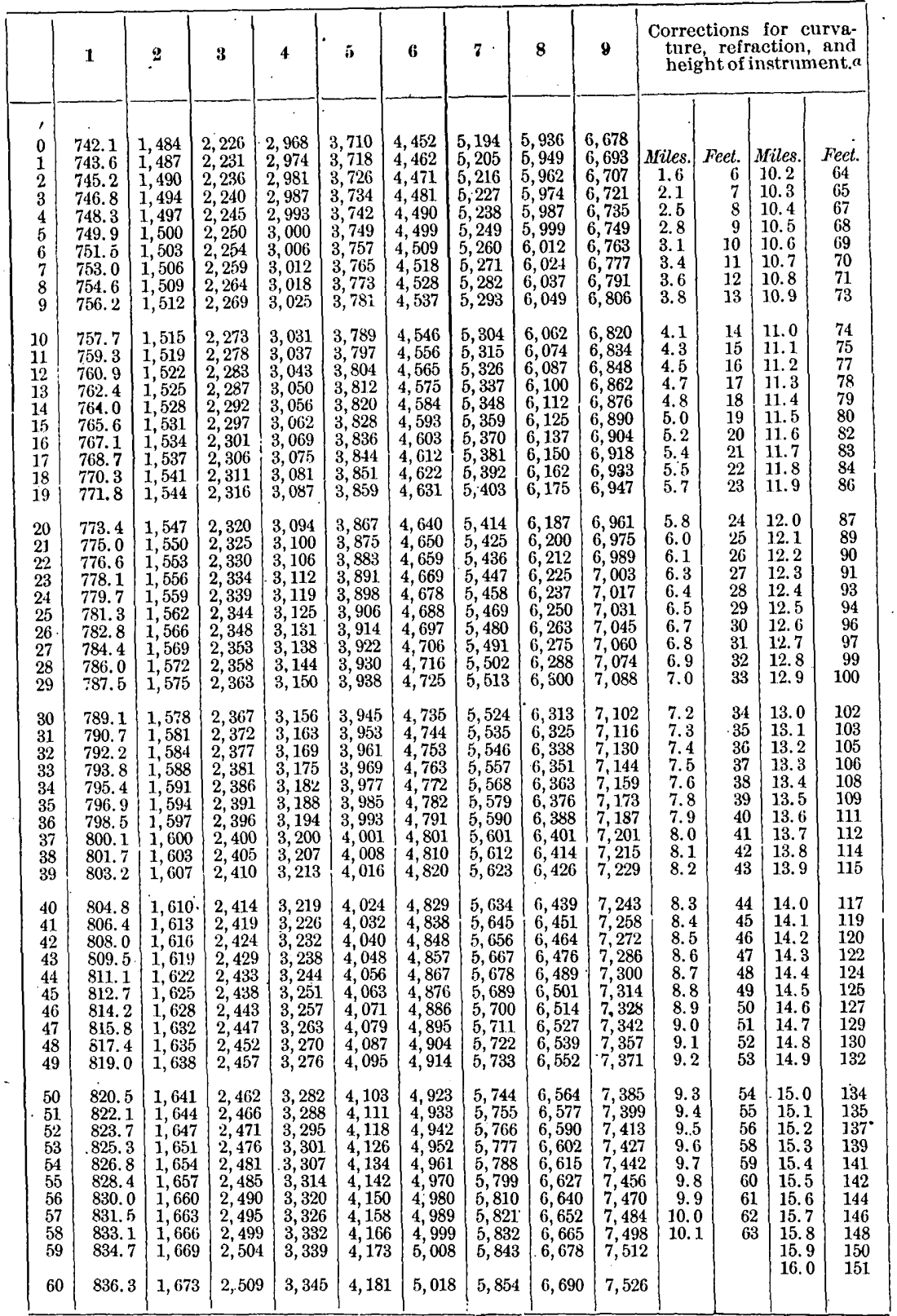

a For all distances under 1.6 miles the correction may be taken as +5 feet. Height of instrument is assumed 4.5 feet. 
'TABLE 24.-For obtaining differences of altitude for any minute, etc.-Continued.

$9^{\circ}$

\begin{tabular}{|c|c|c|c|c|c|c|c|c|c|c|c|c|c|}
\hline & 1 & 2 & 3 & 4 & 5) & 6 & 7 & 8 & 9 & \multicolumn{4}{|c|}{$\begin{array}{l}\text { Corrections for curva- } \\
\text { ture, refraction, and } \\
\text { height of instrument.a. }\end{array}$} \\
\hline 1 & - & & & & & & & & & & & & \\
\hline 0 & 836.3 & 1,673 & 2,509 & 3,345 & 4,181 & 5,018 & 5,854 & 6,690 & 7,526 & & & & \\
\hline 1 & 837.8 & 1,676 & 2,514 & 3,351 & 4,189 & 5,027 & 5,865 & 6,703 & 7,541 & Miles. & Feet. & ifiles. & Feet. \\
\hline 2 & 839.4 & 1,679 & 2,518 & 3,358 & 4,197 & 5,037 & 5,876 & 6,715 & 7,555 & 1.6 & 6 & 10.2 & 64 \\
\hline 3 & 841.0 & 1,682 & 2,523 & 3,364 & 4,205 & 5,046 & 5,887 & 6,728 & 7,569 & 2.1 & 7 & 10.3 & 65 \\
\hline 4 & 842.6 & 1,685 & 2,528 & 3,370 & 4,213 & 5,055 & 5,898 & 6,741 & 7,583 & 2.5 & 8 & 10.4 & 67 \\
\hline 5 & 844.2 & 1,688 & 2,532 & 3,377 & 4. 221 & 5,065 & 5,909 & 6,753 & 7,597 & 2.8 & 9 & 10.5 & 68 \\
\hline 6 & 845.7 & 1,691 & 2,537 & 3,388 & 4,229 & 5,074 & 5,920 & 6,766 & 7,612 & 3.1 & 10 & 10.6 & 69 \\
\hline 7 & 847.8 & 1,695 & 2,542 & 3,389 & 4,237 & 5,084 & 5,931 & 6,778 & 7,626 & 3.4 & 11 & 10.7 & 70 \\
\hline 8 & 848.9 & 1,698 & 2,547 & 3,396 & 4,244 & 5,093 & 5,942 & 6,791 & 7,640 & 3.6 & 12 & 10.8 & 71 \\
\hline 9 & 850.5 & 1,701 & 2,551 & $3,40 \%$ & 4,252 & 5,103 & 5,953 & 6,804 & 7,654 & 3.8 & 13 & 10.9 & 73 \\
\hline 10 & 852.0 & 1,704 & 2,556 & 3,408 & 4,260 & 5,112 & 5,964 & 6,816 & 7,668 & 4.1 & 14 & 11.0 & 74 \\
\hline 11. & 853.6 & 1,707 & 2,561 & 3,414 & 4,268 & 5,122 & 5,975 & 6,829 & 7,683 & 4.3 & 15 & 11.1 & 75 \\
\hline 12 & 855.2 & 1,710 & 2,566 & $3,42 \mathrm{~L}$ & 4,276 & 5,131 & 5,986 & 6,842 & 7,697 & 4.5 & 16 & 11.2 & 77 \\
\hline i3 & 856.8 & 1,714 & 2,570 & 3,427 & 4,284 & 5,141 & 5,997 & 6,854 & 7,711 & 4.7 & 17 & 11.3 & 78 \\
\hline 14 & 858.3 & 1.717 & 2,575 & 3,433 & 4,292 & 5,150 & 6,008 & 6,867 & 7,725 & 4.8 & 18 & 11.4 & 79 \\
\hline 15 & 859.9 & 1,720 & 2,580 & 3,440 & 4,300 & 5,160 & $6,0^{\circ} 20$ & 6,879 & 7,739 & 5.0 & 19 & 11.5 & 80 \\
\hline 16 & 861.5 & 1,723 & 2,585 & 3,446 & 4,308 & 5,169 & 6,031 & 6,892 & 7,754 & 5.2 & 20 & 11.6 & 82 \\
\hline 17 & 863.1 & 1,726 & 2,589 & 3,452 & 4,315 & 5,179 & 6,042 & 6,905 & 7,768 & 5.4 & 21. & 11.7 & 83 \\
\hline 18 & 864.7 & 1,729 & 2,594 & 3,450 & 4,323 & 5,188 & 6,053 & 6,917 & 7,782 & 5. 5 & 22 & 11.8 & 84 \\
\hline 19 & 866.2 & 1,732 & 2,599 & 3,465 & 4,331 & 5,197 & 6,064 & 6,930 & 7,796 & 5.7 & 23 & 11.9 & 86 \\
\hline 20 & 867.8 & 1,736 & 2,603 & $3,47 \mathrm{I}$ & 4,339 & 5,207 & 6,075 & 6,943 & 7,810 & 5.8 & 24 & 12.0 & 87 \\
\hline 21 & 869.4 & 1,739 & 2,608 & 3,478 & 4,347 & 5,216 & 6,086 & 6,955 & 7,825 & 6.0 & 25 & 12.1 & 89 \\
\hline 22 & 871.0 & 1,742 & 2,613 & 3,484 & 4,355 & 5,226 & 6,097 & 6,968 & 7,839 & 6.1 . & 26 & 12.2 & 90 \\
\hline .23 & 972.5 & 1,745 & 2,618, & 3,490 & 4,363 & 5,235 & 6,108 & 6,980 & 7,853 & 6.3 & 27 & 12.3 & 91. \\
\hline 24 & 874.1 & 1,748 & 2,622 & $3 ; 496$ & 4,371 & 5,245 & 6.119 & 6,998 & 7,867 & 6.4 & 28 & 12.4 & 93 \\
\hline 25 & 875.7 & 1,751 & 2,627 & $3,5(1) 3$ & 4,379 & 5,254 & 6,130 & 7,006 & 7,881 & 6.5 & 29 & 12.5 & 94 \\
\hline 26 & 877.3 & 1,755 & 2,632 & 3,509 & 4,386 & 5,264 & 6,141 & 7,018 & 7,896 & 6.7 & 30 & 12.6 & 96 \\
\hline 27 & 878.8 & 1,758 & 2,637 & 3,515 & 4,394 & 5,273 & 6,152 & 7,031 & 7,910 & 6.8 & 31 & 12.7 & 97 \\
\hline 28 & 880.4 & 1,761 & 2,641 & 3,522 & 4,402 & 5,283 & 6,163 & 7,043 & 7,924 & 6.9 & 32 & 12.8 & 99 \\
\hline 29 & 882.0 & 1,764 & 2,646 & 3,528 & 4,410 & $5,29^{\prime}$ & 6,174 & 7,056 & 7,938 & 7.0 & 33 & 12.9 & 100 \\
\hline 30 & 883.6 & 1,767 & 2,651 & 3,534 & 4,418 & $5,302^{\circ}$ & 6,185 & 7,068 & 7,952 & 7.2 & 34 & 13.0 & 102 \\
\hline 31 & 885.2 & 1,770 & 2,656 & 3,541 & 4,426 & 5,311 & 6,196 & 7,081 & 7,967 & 7.3 & 35 & 13.1 & 103 \\
\hline 32 & 886.7 & 1,774 & 2,660 & 3,547 & 4,434 & 5,320 & 6,207 & 7,094 & 7,981 & 7.4 & 36 & 13.2 & 105 \\
\hline 33 & 888.3 & 1,777 & 2,665 & 3,553 & 4,442 & 5,330 & 6,218 & 7,107 & 7,995 & 7.5 & 37 & 13.3 & 106 \\
\hline 34 & 889.9 & 1,780 & 2,670 & 3,560 & 4,450 & 5,339 & 6,229 & 7,119 & 8,009 & 7. 6 & 38 & 13.4 & 108 \\
\hline 35 & 891.5 & 1,783 & 2,674 & 3,566 & 4,457 & 5,349 & 6,240 & 7,132 & 8,023 & 7.8 & 39 & 13.5 & 109 \\
\hline 36 & 893.1 & 1,786 & 2,679 & 3,572 & 4,465 & 5,358 & 6,252 & 7,145 & 8,038 & 7.9 & 40 & 13.6 & 111 \\
\hline 37 & 894.6 & 1,789 & 2,684 & 3,579 & 4,473 & 5,368 & 6,263 & 7,157 & 8,052 & 8.0 & 41. & 13.7 & 112 \\
\hline 38 & 896.2 & 1,792 & 2,689 & 3,685 & 4,481 & 5,377 & 6,274 & 7,170 & 8,066 & 8.1. & 42 & 13.8 & 114 \\
\hline 39 & 897.8 & 1,796 & 2,693 & 3,591 & 4,489 & 5,387 & 6,285 & 7,183 & 8,080 & 8.2 & 43 & 13.9 & 115 \\
\hline 40 & 899.4 & 1,799 & 2,698 & 3,598 & 4,497 & 5,396 & 6,296 & 7,195 & 8,095 & 8.3 & 44 & 14.0 & 117 \\
\hline 41 & 901.0 & 1,802 & 2,703 & 3,604 & 4,505 & 5,406 & 6,307 & 7,208 & 8,109 & 8.4 & 45 & 14.1 & 119 \\
\hline 42 & 902.5 & 1,805 & 2,708 & 3,610 & 4,513 & 5,415 & 6,318 & 7,220 & 8,123 & 8.5 & 46 & 14.2 & 120 \\
\hline 43 & 904.1 & 1,808 & 2,712 & 3,617 & 4,521 & 5,425 & 6,329 & 7,233 & 8,137 & 8.6 & 47 & 14.3 & 122 \\
\hline 44 & 905.7 & 1,811 & 2,717 & 3,623 & 4,529 & 5,434 & 6,340 & 7,246 & 8,151 & 8.7 & 48 & 14.4 & 124 \\
\hline 45 & 907.3 & 1,814 & 2,722 & 3,629 & 4,537 & 5,444 & 6,351 & 7,258 & 8,166 & 8.8 & 49 & 14.5 & 125 \\
\hline 46 & 908.9 & 1,818 & 2,727 & 3,636 & 4,544 & 5,453 & 6,362 & 7,271 & 8,180 & 8.9 & 50 & 14.6 & 127 \\
\hline 47 & 910.5 & 1,821 & 2,731 & 3,642 & 4,552 & 5,463 & 6,373 & 7,284 & 8,194 & 9.0 & 51 & 14.7 & 129 \\
\hline 48 & 912.0 & 1,824 & 2,736 & 3,648 & 4,560 & 5,472 & 6,384 & 7,296 & 8,208 & 9.1 & 52 & 14.8 & 130 \\
\hline 49 & 913.6 & 1,827 & 2,741 & 3,654 & 4,568 & 5,482 & 6,395 & 7,309 & 8,223 & 9.2 & 53 & $" 14.9$ & 132 \\
\hline 50 & 91.5 .2 & 1,830 & 2,746 & 3,661 & 4,576 & 5,491 & 6,406 & 7,322 & 8,237 & 9.3 & 54 & 15.0 & $\cdot 134$ \\
\hline 5. & 916.8 & 1,833 & 2,750 & 3,667 & 4,584 & 5,501 & 6,417 & 7,334 & 8,251 & 9.4 & 55 & 15.1 & 135 \\
\hline 52 & 918.4 & 1,837 & 2,755 & 3,673 & 4,592 & 5,510 & 6,429 & 7,347 & 8,265 & 9.5 & 56 & 15.2 & '137 \\
\hline 53 & 919.9 & 1,840 & 2,760 & 3,680 & 4,600 & 5,520 & 6,440 & 7,360 & 8,279 & 9.6 & 58 & 15.3 & 139 \\
\hline 54 & 921.5 & 1,843 & 2,765 & 3,686 & 4,608 & 5,529 & 6,451 & 7,372 & 8,294 & 9.7 & 59 & 15.4 & 141 \\
\hline 55 & 923.1 & 1,846 & 2,769 & 3,692 & 4,616 & 5,539 & 6,462 & 7,385 & 8,308 & 9.8 & 60 & 15.5 & 142 \\
\hline 56 & 924.7 & 1,849 & 2,774 & 3,699 & 4,623 & 5,548 & 6,473 & 7,397 & 8,322 & 9.9 & 61 & 15.6 & 144 \\
\hline 57 & 926.3 & 1,852 & 2,779 & 3,705 & 4,631 & 5,558 & 6,484 & 7,410 & 8,336 & $10.0^{\circ}$ & 62 & 15.7 & 146 \\
\hline 58 & 927.8 & 1,855 & 2,784 & 3,711 & 4,639 & 5,567 & 6,495 & 7,423 & 8,351 & 10.1 & 63 & 15.8 & 148 \\
\hline 59 & 929.4 & 1,859 & 2,788 & 3,718 & 4,647 & 5,577 & 6,506 & 7,435 & 8,365 & & & 15.9 & 150 \\
\hline 60 & 931.0 & 1,862 & 2,793 & 3,724 & 4,655 & 5,586 & $6 ;, 517$ & 7,448 & 8,379 & & & & \\
\hline
\end{tabular}

a For all distances under 1.6 miles the correction may be taken as +5 feet. Height of instrument is assumed 4.5 feet. 
TABLE 24.-For obtaining differences of altitude for any minute, etc.-Continued.

$10^{\circ}$

\begin{tabular}{|c|c|c|c|c|c|c|c|c|c|c|c|c|c|}
\hline & $\mathbf{1}$ & 2 & 3 & 4 & 5 & 6 & 7 & 8 & 9 & \multicolumn{4}{|c|}{$\begin{array}{l}\text { Corrections for curva- } \\
\text { ture, refraction and } \\
\text { height of instrument. }\end{array}$} \\
\hline & & & & & & & & & & & & & \\
\hline 0 & 931.0 & 1,862 & 2,793 & 3,724 & 4,655 & 5,586 & 6,517 & 7,448 & $8 ; 379$ & & & & \\
\hline 1 & 932.6 & 1,865 & 2,798 & 3,730 & 4,663 & 5,596 & 6,528 & 7,461 & 8,393 & Miles. & Feet. & Miles. & Feet. \\
\hline 3 & $\begin{array}{l}934.2 \\
935.8\end{array}$ & $\begin{array}{l}1,868 \\
1,872\end{array}$ & $\begin{array}{l}2,803 \\
2,807\end{array}$ & $\begin{array}{l}3,737 \\
3,743\end{array}$ & $\begin{array}{l}4,671 \\
4,679\end{array}$ & $\begin{array}{l}5,605 \\
5,615\end{array}$ & $\begin{array}{l}6,539 \\
6,550\end{array}$ & $\begin{array}{l}7,473 \\
7,486\end{array}$ & $\begin{array}{l}8,408 \\
8,422\end{array}$ & $\begin{array}{l}1.6 \\
2.1\end{array}$ & $\begin{array}{l}6 \\
7\end{array}$ & \begin{tabular}{|l|}
10.2 \\
10.3
\end{tabular} & $\begin{array}{l}64 \\
65\end{array}$ \\
\hline 4 & 937.4 & 1,875 & 2,812 & 3,749 & 4,687 & 5,624 & 6,561 & 7,499 & 8,436 & 2.5 & 8 & 10.4 & 67 \\
\hline 5 & 938.9 & 1,878 & 2,817 & 3,756 & 4,695 & 5,634 & 6,573 & 7,512 & 8,450 & 2.8 & 9 & 10.5 & 68 \\
\hline 6 & 940.5 & 1,881 & 2,822 & 3,762 & 4,703 & 5,643 & $6,5 \times 4$ & 7,524 & 8,465 & 3.1 & 10 & 10.6 & 69 \\
\hline 7 & 942.1 & 1,884 & 2,826 & 3,768 & 4,711 & 5,653 & 6,595 & 7,537 & 8,479 & 3.4 & 11 & 10.7 & 70 \\
\hline 8 & 943.7 & 1,887 & 2,831 & 3,775 & 4,718 & 5,662 & 6,606 & 7,550 & 8,493 & 3.6 & 12 & 10.8 & 71 \\
\hline 9 & 945.3 & 1,891 & 2,836 & 3,781 & 4,726 & 5,672 & 6,617 & 7,562 & 8,508 & 3.8 & 13 & 10.9 & 73 \\
\hline 10 & 946.9 & 1,894 & 2,841 & $3 ; 787$ & 4,734 & 5,681 & 6,628 & 7,575 & 8,522 & 4.1 & 14 & 11.0 & 74 \\
\hline 11 & 948.5 & 1,897 & 2,845 & 3,794 & $\begin{array}{l}4,742 \\
\end{array}$ & 5,691 & 6,639 & 7,588 & 8,536 & 4.3 & 15 & 11.. 1 & 75 \\
\hline 12 & 950.0 & 1,900 & 2,850 & 3,800 & 4,750 & 5,700 & 6,650 & 7,600 & 8,550 & 4.5 & 16 & 11.2 & 77 \\
\hline 13 & 951.6 & 1, 903 & 2,855 & 3,807 & 4,758 & 5,710 & 6,661 & 7,613 & 8,565 & 4.7 & 17 & 11.3 & 78 \\
\hline 14 & 953.2 & $.1,906$ & 2,860 & 3,813 & 4,766 & 5,719 & 6,672 & 7,626 & 8,579 & 4.8 & 18 & 11.4 & 79 \\
\hline 15 & 954.8 & 1,910 & 2,864 & 3,819 & 4,774 & 5,729 & 6,684 & 7,638 & 8,593 & 5.0 & 19 & 11.5 & 80 \\
\hline 16 & 956.4 & 1,913 & 2,869 & 3,826 & 4,782 & 5,738 & 6,695 & 7,651 & 8,607 & 5.2 & 20 & 11.6 & 82 \\
\hline 17 & 958.0 & 1,916 & 2,874 & 3,832 & 4,790 & 5,748 & 6,706 & 7,664 & 8,622 & 5.4 & 21. & 11.7 & 86 \\
\hline 18 & 959.6 & 1,919 & 2,879 & 3,838 & 4,798 & 5,757 & 6,217 & 7,676 & 8,636 & 5.5 & 22 & 11.8 & 84 \\
\hline 19 & 961.1 & 1,922 & 2,883 & 3,845 & 4,806 & 5,767 & 6,728 & 7,689 & 8,650 & 5.7 & 23 & 11.9 & 86 \\
\hline 20 & 962.7 & 1,926 & 2,888 & 3,851 & 4,814 & 5,776 & 6,739 & 7,702 & 8,665 & 5.8 & 24 & 12.0 & 87 \\
\hline & 964 & 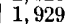 & 2,893 & 857 & & & & 7,715 & 8,679 & 6.0 & 25 & 12.1. & 89 \\
\hline 22 & 965.9 & 1,932 & 2,898 & 3,864 & 4,830 & 5,795 & 6,751 & $\ddot{7}, 727$ & 8,693 & 6.1 & 26 & 12.2 & 90 \\
\hline 23 & 967.5 & 1,935 & $2,90^{\circ}$ & 3,870 & 4,837 & 5,805 & 6,772 & 7,740 & 8,707 & 6.3 & 27 & 12.3 & 91. \\
\hline 24 & 969.1 & 1,938 & 2,907 & 3,876 & 4,845 & 5,814 & 6,784 & 7,753 & 8,722 & 6.4 & 28 & 12.4 & 93 \\
\hline 25 & 970.7 & 1,941 & 2,912 & 3,883 & 4,853 & 5,824 & 6,795 & 7,765 & 8,736 & 6.5 & 29 & 12.5 & 94 \\
\hline 26 & 972.2 & 1., 944 & 2,917 & 3,889 & 4,861 & 5,833 & 6,806 & 7,778 & 8,750 & 6.7 & 30 & 12.6 & 96 \\
\hline 27 & 973.8 & 1,948 & 2,921 & 3,895 & $\begin{array}{l}1,869 \\
\end{array}$ & 5,843 & 6,817 & 7,791 & 8,764 & 6.8 & 31 & 12.7 & 97 \\
\hline 28 & 975.4 & 1,951 & 2,926 & 3,902 & 4877 & 5,853 & 6,828 & 7,803 & 8,779 & 6.9 & 32 & 12.8 & 99 \\
\hline 29 & 977.0 & 1,954 & 2,931 & 3,908 & 4,885 & 5,862 & 6,839 & 7,816 & 8,793 & 7.0 & 33 & 12.9 & 100 \\
\hline 30 & 978.6 & 1,957 & 2,936 & 3,914 & 4,893 & 5,872 & 6,850 & 7,829 & 8,8 & 7.2 & 34 & 13.0 & 1.02 \\
\hline 31 & 980.2 & 1,960 & 2,941 & 3,921 & & & 6,861 & 7,8 & & 7. & 5 & 13. 1 & 103 \\
\hline 32 & 981.8 & 1,964 & 2,9 & 3,927 & 4, & $b$, & & 7, & & 7. & 36 & 13.2 & 105 \\
\hline 33 & 983.4 & 1, 967 & 2,950 & 3,933 & 4,917 & 5,900 & 6,884 & 7,867 & 8,850 & 7.5 & 37 & 13. 3 & 106 \\
\hline 34 & 985.0 & 1,970 & 2,955 & 3,940 & 4,925 & 5,910 & 6,895 & 7,880 & 8,865 & 7. & 38 & 13.4 & 1.08 \\
\hline 35 & 3.5 & 1,973 & 2,960 & $3 ; 946$ & 4,933 & 5, & & 7,8 & 8,879 & 7. & 39 & 13.5 & 109 \\
\hline 36 & 8.1 & 1,976 & 2,964 & 3,953 & 4, & 5, & & 7, & & 7. & 40 & 13.6 & 111 \\
\hline 37 & 989.7 & 1,980 & 2,969 & 3,959 & 4,949 & 5,938 & 6,928 & 7,918 & 8, & 8. & 41. & 13 & 112 \\
\hline 38 & 991.3 & 1,983 & 2,974 & 3,965 & 4,957 & 5,948 & 6,939 & 7,931 & 8,922 & 8. & 42 & 13.8 & 114 \\
\hline 39 & 992.9 & 1,986 & 2,979 & 3,972 & 4,965 & 5,957 & 6,950 & 7,943 & 8,936 & 8.2 & 43 & 13.9 & 11.5 \\
\hline 40 & 994.5 & 1,989 & 2,984 & 3,978 & 4,973 & 5,967 & 6,962 & 7,956 & 8,951 & 8.3 & 44 & 14.0 & 117 \\
\hline 41. & 3.1 & 2 & & 3,984 & & 5 & & 7 & 8 & 8. & 45 & & 119 \\
\hline 42 & 7.7 & $\overrightarrow{1}$ & 2, & 3,991 & 4, & 5 & 6,984 & 7,981 & 8, & 8.5 & 46 & 14.2 & 120 \\
\hline 43 & 999.3 & 1,999 & 2,9 & 3,997 & 4, & 5,996 & 6,995 & 7,994 & & & 47 & 14.4 & 122 \\
\hline 44 & $1,000.9$ & 2,002 & 3,003 & 4,003 & 5,004 & 6,005 & 7,006 & 8,007 & 9,008 & 8. & 48 & 14.3 & 124 \\
\hline 45 & & 2,00 & 3,007 & 4,010 & 5,0 & 6,0 & 7,017 & 8,020 & 9. & 8.8 & 49 & 14.5 & 125 \\
\hline 46 & $1,004.0$ & 2,008 & 3,012 & 4,016 & $5 ; 0$ & 6, & 7,028 & 8, & & 8.9 & 50 & 14.6 & 127 \\
\hline 47 & $1,005.6$ & 2,01 & 3,017 & 4,023 & 5,028 & 6,034 & 7,039 & 8,045 & 9, & 9.0 & 51 & 14.7 & 129 \\
\hline 48 & $1,007.2$ & 2,014 & 3,022 & 4,029 & 5,036 & 6., 043 & 7,051 & 8,058 & 9,065 & 9. & 52 & 14.8 & 130 \\
\hline 49 & $1,008.8$ & 2,018 & 3,026 & 4,035 & 5,044 & 6,053 & 7,062 & 8,071 . & 9,079 & 9.2 & 53 & 14.9 & 132 \\
\hline 50 & & 2 , & & 4,042 & 5 & 6 , & 7,073 & 8,0 & & 9.3 & 54 & & \\
\hline 51 & & 2,02 & 3, & 4,048 & 5,0 & 6,072 & 7,084 & $\begin{array}{l}0,000 \\
8,096\end{array}$ & 9, & 9.4 & 55 & 15.1 & 135 \\
\hline 52 & 1 & 2,02 & 3,041 & 4,054 & 5,0 & 6,0 & 7,095 & 8,109 & $9, \mathbf{1 2 2}$ & 9. & 56 & 15.2 & 137 \\
\hline 53 & & 2,030 & 3,1 & 4,061 & $5, c$ & 6,091 & 7, & 8, & 9 & 9. & 58 & 15. 3 & 139 \\
\hline$=$ & & 2, & 3,050 & 4,067 & 5,0 & 6,101 & 7,117 & 8,1 & 9 & 9. & 59 & 15.4 & 141 \\
\hline 55 & 1,01 & 2,0 & 3,0 & 4,073 & 5,0 & 6,110 & 7,129 & 8,1 & 9,165 & 9. & 60 & 15.5 & 142 \\
\hline 56 & 20.0 & 2,040 & 3,060 & 4,080 & 5,100 & 6,120 & 7,140 & 8,160 & 9,180 & 9.9 & 61 & 15.6 & 144 \\
\hline 57 & $1,021.5$ & 2,043 & 3,065 & 4,086 & 5,108 & 6,129 & 7,151 & 8,172 & 9,194 & 10.0 & 62 & 15.7 & 146 \\
\hline & & & & 4,093 & 5,1 & 6,139 & 7,162 & 8,18 & 9,208 & 10.1 & 63 & 15.8 & 14 \\
\hline 5 & $1,024.7$ & 2,049 & 3,074 & 4,099 & 5,12 & 6,148 & 7,173 & 8,198 & 9,223 & & & 1.5. 9 & 150 \\
\hline 60 & $1,026.3$ & 2,053 & 3,079 & 4,105 & 5,132 & 6,158 & 7,184 & 8,211 & \begin{tabular}{|l|}
9,237 \\
\end{tabular} & & & & \\
\hline
\end{tabular}

a For all distances under 1.6 miles the correction may be taken as +5 feet. Height of instrument is assumed 4.5 feet. 
TABLE 24.-For obtaining differences of altitude for any minute, etc.-Continued.

$11^{\circ}$

\begin{tabular}{|c|c|c|c|c|c|c|c|c|c|c|c|c|c|}
\hline & 1 & 2 & 3 & 4 & 5 & 6 & 7 & 8 & 9 & \multicolumn{4}{|c|}{$\begin{array}{l}\text { Corrections for curva- } \\
\text { ture, refraction, and } \\
\text { height of instrument.a }\end{array}$} \\
\hline 0 & $1,020.3$ & 2,053 & 3.079 & 4.105 & 5.132 & 6.158 & 7.184 & 8211 & 9.237 & & & & \\
\hline 1 & $\begin{array}{l}1,020.0 \\
1,027.9\end{array}$ & $\begin{array}{l}2,060 \\
2,056\end{array}$ & $\begin{array}{l}3,084 \\
3,084\end{array}$ & $\begin{array}{l}4,100 \\
4,112\end{array}$ & $\begin{array}{l}0,1,02 \\
5,140\end{array}$ & $\begin{array}{l}0,108 \\
6,168\end{array}$ & $\begin{array}{l}7,104 \\
7,195\end{array}$ & $\begin{array}{l}8,211 \\
8,223\end{array}$ & $\begin{array}{l}9,251 \\
9,251\end{array}$ & Miles. & Feet. & Miles. & Fect. \\
\hline 2 & $1,029.5$ & 2,059 & 3,089 & 4,118 & 5,148 & 6,177 & 7,207 & 8,236 & 9,266 & 1.6 & 6 & 10.2 & 64 \\
\hline 3 & $1,031.1$ & 2,062 & 3,093 & 4,124 & 5,156 & $6,1.87$ & 7,218 & 8,249 & 9,280 & 2.1 & 7 & 10.3 & 65 \\
\hline 4 & $1,032.7$ & 2,065 & 3,098 & 4,131 & 5,164 & 6,196 & 7,229 & 8,262 & 9,294 & 2.5 & 8 & 10.4 & 67 \\
\hline 6 & $1,034.3$ & 2,069 & 3,103 & 4,137 & 5,172 & 6,206 & 7,240 & 8,275 & 9,309 & 2.8 & 9 & 10.5 & 68 \\
\hline 6 & 1,036 & 2,072 & 3,108 & 4,144 & 5,180 & 6,215 & 7,251 & 8,287 & 9,323 & 3.1 & 10 & 10.6 & 69 \\
\hline 7 & 1,038 & 2,075 & 3,113 & 4,150 & 5,188 & 6,225 & 7,263 & 8,300 & 9,338 & 3.4 & 11 & 10.7 & 70 \\
\hline 8 & 1,039 & 2,078 & 3,117 & 4,156 & 5,190 & 6,235 & 7,274 & 8,313 & 9,352 & 3.6 & 12 & 10.8 & 71 \\
\hline 9 & 1,041 & 2,081 & 3,122 & 4,163 & 5,204 & 6,244 & 7,285 & 8,326 & 9,366 & 3.8 & 13 & 10.9 & 73 \\
\hline 10 & 1,042 & 2,085 & 3,127 & 4,169 & 5,212 & 6,254 & 7,296 & 8,338 & 9,381 & $4 . i$ & 14 & 1.1 .0 & 74 \\
\hline 11. & 1,044 & 2,088 & 3,132 & 4,176 & 5,219 & 6,263 & 7,307 & 8,351 & 9,395 & 4.3 & 15 & 11.1 & 75 \\
\hline 12 & 1,046 & 2,091 & 3,136 & 4,182 & 5,227 & 6,273 & 7,318 & 8,364 & 9,409 & 4.5 & 16 & 11.2 & 77 \\
\hline 13 & 1,047 & 2,094 & 3,141 & 4,188 & 5,235 & 6,283 & 7,330 & 8,377 & 9,424 & 4.7 & 17 & 11.3 & 78 \\
\hline 14 & 1, 049 & 2,097 & 3,146 & 4,195 & 5,243 & 6,292 & 7,341 & 8,390 & 9,438 & 4.8 & 18 & 11.4 & 79 \\
\hline 15 & 1,050 & 2,101 & 3,151 & $4,201$. & 5,251 & 6,302 & 7,352 & 8,402 & 9,453 & 5.0 & 19 & 11.5 & 80 \\
\hline 16 & 1,052 & 2,104 & 3,156 & 4,208 & 5,259 & $6,311$. & 7,363 & 8,415 & 9,467 & 5.2 & 20 & 11.6 & 82 \\
\hline 17 & 1,053 & 2,107 . & 3,160 & 4,214 & 5,267 & 6i, 321 & 7,374 & 8,428 & $8,481$. & 5.4 & 21 & 11.7 & 83 \\
\hline 18 & 1,055 & $2,110^{\circ}$ & 3,165 & 4,220 & 5,275 & 6,330 & 7,386 & 8,441 & 9,496 & 5.5 & 22 & 11.8 & 84 \\
\hline 19 & 1,057 & 2,113 & 3,170 & 4,227 & 5,283 & 6,340 & 7,397 & 8,453 & 9,510 & 5.7 & 23 & 11.9 & 86 \\
\hline 20 & 1,058 & 2,117 & 3,175 & 4,233 & 5,291 . & 6,350 & 7,408 & 8,466 & 9,524 & 5.8 & 24 & 12.0 & 87 \\
\hline 21 & 1,060 & 2,120 & $3,1.80$ & 4,239 & 5,299 & 6,359 & 7,419 & 8,479 & 9,539 & 6.0 & 25 & 12.1 & 89 \\
\hline 22 & 1,061 & 2,123 & 3,184 & 4,246 & 5,307 & 6,369 & 7,430 & 8,492 & 9,553 & 6.1 & 26 & 12.2 & 90 \\
\hline 23 & 1,063 & 2,126 & 3,189 & 4,252 & 5,315 & 6,378 & 7,441 & 8,504 & 9,568 & $6 . \overline{3}$ & 27 & 12.3 & 91 \\
\hline 24 & 1,065 & 2,129 & 3,194 & 4,259 & 5,323 & 6,388 & 7,453 & 8,517 & 9,582 & 6.4 & 28 & 12.4 & 93 \\
\hline 25 & .1, 066 & 2,133 . & 3,199 & 4,265 & 5,331 . & 6,398 & 7,464 & 8,530 & 9,596 & 6.5 & 29 & 12.5 & 94 \\
\hline 26 & 1,068 & 2,13 & 3,204 & 4, 271 & 5,339 & 6,407 & 7,475 & 8,543 & 9,611 & 6. & 30 & 12.6 & 96 \\
\hline 27 & 1,069 & 2,139 & 3,208 & 4,278 & 5,347 & 6,417 & 7,486 & 8,556 & 9,625 & 6.8 & 31. & 12.7 & 97 \\
\hline 28 & 1,071 & 2,142 & 3,213 & 4,284 & 5,355 & 6,426 & 7,497 & 8,568 & 9,639 & 6.9 & 32 & 12.8 & 99 \\
\hline 29 & 1,073 & 2,145 & 3,218 & 4,291 & 5,363 & 6,436 & 7,509 & 8,581 & 9,654 & 7.0 & 33 & 12.9 & 100 \\
\hline 30 & 1,074 & 2,148 & 3,223 & 4,297 & 5,371 & 6,445 & 7,520 & 8,594 & 9,668 & 7.2 & 34 & 13.0 & 102 \\
\hline 31. & 1,076 & 2,152 & 3,227 & 4,303 & 5,379 & 6,4 & 7 , & 8,607 & 9,682 & 7. & 35 & 13.1 & 103 \\
\hline 32 & 1,077 & 2,156 & 3,2 & 4,310 & 5,387 & 6,465 & 7,542 & 8,619 & 9,697 & 7. & 36 & 13.2 & 105 \\
\hline 33 & 1,079 & 2,158 & 3,237 & 4,316 & 5,395 & 6,474 & 7,553 & 8,632 & 9,711 & 7. & 37 & 13.3 & 106 \\
\hline 34 & 1,081 & 2,161 & 3,242 & 4,323 & 5,403 & 6,484 & 7,564 & 8,645 & 9,726 & 7. & .38 & 13.4 & 108 \\
\hline 35 & 1,082 & 2,164 & 3,247 & 4,329 & 5,411 & 6,493 & 7,576 & 8,658 & 9,740 & 7. & 39 & 13.5 & 109 \\
\hline 36 & 1,084 & 2,16 & 3,25 & 4,3 & 5,4 & 6,5 & 7,5 & 8,671 & 9 , & 7. & 40 & 13.6 & 111 \\
\hline 37 & 1,085 & 2,171 & 3,256 & 4,342 & 5,427 & 6,513 & 7,598 & 8,683 & 9,769 & 8. & 41 & 13.7 & 112 \\
\hline 38 & 1., 087 & 2,174 & 3,261 & 4,348 & 5,435 & 6,522 & 7,609 & 8,696 & 9,783 & 8. & 42 & 13.8 & .114 \\
\hline 39 & 1, 089 & 2,177 & 3,266 & 4,355 & 5,443 & 6,532 & 7,621 & 8,709 & 9,798 & 8.2 & 43 & 13.9 & 115 \\
\hline 40 & 1,090 & 2,181 & 3,271 & 4 , & 5,45 & 6,542 & 7,632 & 0 & 9,8 & 8. 3 & 44 & 14.0 & 117 \\
\hline 41 & 1, 092 & 2 , & $w_{i}$ & 4 & 5,4 & 6, & 7,6 & 8,735 & 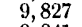 & & 4 & 14.1 & 119 \\
\hline 42 & 1,093 & 2,187 & 3,2 & 4, & 5,4 & 6 , & 7,654 & 8, & 9 & 8. & 46 & 14.2 & 120 \\
\hline 43 & 1,095 & 2,190 & 3,2 & 4,3 & 5,4 & 6,5 & 7,6 & 8, & 9, & $\delta$. & 47 & 14.3 & 122 \\
\hline 44 & 1. 097 & 2,193 & 3,290 & 4,387 & 5,483 & 6,580 & 7,677 & 8,773 & 9,870 & 8. & 48 & 14.4 & 1.24 \\
\hline 45 & 1,0 & 2,19 & 3,29 & 4,393 & 5,4 & 6,5 & 7,688 & 8,786 & 9,884 & 8. & 4 & 14.5 & 125 \\
\hline 46 & 1,100 & 2, & 3,30 & 4, & 5 , & 6,5 & 7,699 & 8,7 & & 8. & 5 & & 1.27 \\
\hline 47 & 1,101 & & 3,3 & 4,406 & 5,5 & 6 & 7,710 & 8,8 & 9, & 9. & 51 & 14.7 & 129 \\
\hline 48 & 1, & 2,206 & 3,309 & 4,412 & 5,515 & 6,6 & 7,721 & 8,8 & 9,928 & 9. & 52 & 14.8 & 130 \\
\hline 49 & 1,105 & 2,209 & 3,314 & 4,419 & 5,523 & 6,628 & 7,733 & 8,837 & 9,942 & 9.2 & 53 & 14.9 & 132 \\
\hline 50 & 1 & 2,213 & 3,31 & 4,425 & 5 , & 6 , & 7 , & 8 & 9, & 9. & 54 & 15 & 134 \\
\hline 51. & & & 3,324 & 4,431 & & 6,6 & 7,755 & 8,8 & 9, & & & 15.1 & $135^{\circ}$ \\
\hline 52 & 1,109 & 2,219 & 3,32 & 4,438 & 5,547 & 6,657 & 7,766 & 8,876 & 9,9 & 9. & 56 & 15.2 & 137 \\
\hline 53 & 1,111 & 2,22 & 3,3 & 4,444 & & 6,6 & 7,778 & & 0 & 9. & 5 & 15.3 & 139 \\
\hline 54 & 1,113 & 2,225 & 3,3 & 4,451 & $\tilde{b}, 563$ & 6,676 & 7,789 & 8,901 & 10,0 & 9. & 59 & 15.4 & 141. \\
\hline 55 & 1,114 & 2,229 & 3,343 & 4,4 & 5,5 & 6,6 & 7,800 & 8,9 & 10, & 9 & t & 15.5 & 142 \\
\hline 56 & 1,116 & 2,232 & 3,34 & 4,464 & 5,579 & 6,695 & 7,811 & 8,927 & 10,043 & 9.9 & 61 & 15.6 & 144 \\
\hline 57 & 1,117 & 2,235 & 3,35 & 4,470 & 5,5 & 6,7 & 7,8 & 8,940 & 10,057 & 10.0 & 62 & 15.7 & 146 \\
\hline 58 & 1,119 & 2,238 & 3,357 & 4,476 & 5, 595 & 6,715 & 7,834 & 8,953 & 10,072 & 10.1 & 63 & 15.8 & 148 \\
\hline 50 & 1,121 & 2,241 & 3,362 & 4,483 & 5,603 & 6,724 & 7,845 & 8,966 & 10,086 & & & 15.9 & 150 \\
\hline 60 & 1,122 & 2,245 & 3,367 & 4,489 & 5,611 & 6,734 & 7,856 & 8,978 & 10,101 & & & & \\
\hline
\end{tabular}

a For all distunces.under 1.6 miles the correction may be taken as +5 feet: Height of instrument is assumed 4.5 feet. 
TABLE 24.-For obtaining differences of altitude for any minute, etc.-Continued.

$12 \circ$.

\begin{tabular}{|c|c|c|c|c|c|c|c|c|c|c|c|c|c|}
\hline & 1 & 2 & 3 & 4 & 5 & 6 & 7 & 8 & 9 & \multicolumn{4}{|c|}{$\begin{array}{l}\text { Corrections for curva- } \\
\text { ture, refraction, and } \\
\text { height of instrument. }\end{array}$} \\
\hline & & & & & & & & & & & & & \\
\hline 0 & 1,122 & 2,245 & 3,367 & 4,489 & 5,612 & 6,734 & 7,856 & 8,978 & 10,101 & & & & \\
\hline 1 & 1,124 & 2,248 & 3,372 & 4,496 & 5,620 & 6,743 & 7,867 & 8,991 & 10,115 & Miles & Feet. & Miles. & Feet. \\
\hline 3 & $\begin{array}{l}1,120 \\
1,127\end{array}$ & $\begin{array}{l}2,251 \\
2,254\end{array}$ & $\begin{array}{l}3,377 \\
3,381\end{array}$ & $\begin{array}{l}4,502 \\
4,508\end{array}$ & $\begin{array}{l}5,628 \\
5,636\end{array}$ & $\begin{array}{l}6,753 \\
6,763\end{array}$ & $\begin{array}{l}7,879 \\
7,890\end{array}$ & $\begin{array}{l}9,004 \\
9,017\end{array}$ & $\begin{array}{l}10,130 \\
10,144\end{array}$ & $\begin{array}{l}1.6 \\
2.1\end{array}$ & $\begin{array}{l}0 \\
7\end{array}$ & \begin{tabular}{|l|}
10.2 \\
10.3
\end{tabular} & $\begin{array}{l}64 \\
65\end{array}$ \\
\hline 4 & 1,129 & 2,257 & 3,386 & 4,515 & 5,644 & 6,772 & 7,901 & 9.030 & 10,159 & 2.5 & 8 & 10.4 & 67 \\
\hline 5 & 1,130 & 2,261 & 3,391 & 4,521 & 5,652 & 6,782 & 7,912 & 9,043 & 10,173 & 2.8 & 9 & 10.5 & 68 \\
\hline 6 & 1,132 & 2,264 & 3,396 & 4,528 & 5,660 & 6,792 & 7,924 & 9,056 & 10,188 & 3. 1 & 10 & 10.6 & 69 \\
\hline 7 & 1,134 & 2,267 & 3,401 & 4,534 & 5,668 & 6,801 & 7,935 & 9,068 & 10,202 & 3.4 & 11 & 10.7 & 70 \\
\hline 8 & 1,135 & 2,270 & 3,405 & 4,541 & 5,676 & 6,811 & 7,946 & 9,081 & 10,216 & 3.6 & 12 & 10.8 & 71 \\
\hline 9 & 1,137 & 2,274 & 3,410 & 4,547 & 5,684 & 6,821 & 7,957 & 9,094 & 10,231 & 3.8 & 13 & 10.9 & 73 \\
\hline 10 & 1,138 & 2,277 & 3,415 & 4,554 & 5,692 & 6,830 & 7,969 & 9,107 & 10,245 & 4.1 & 14 & 11.0 & 74 \\
\hline 11 & 1,140 & 2,280 & 3,420 & 4,560 & 5,700 & 6,840 & 7,980 & 9,120 & 10,260 & 4.3 & 15 & 11.1 & 75 \\
\hline 12 & 1,142 & 2,283 & 3,425 & 4,566 & 5,708 & 6,850 & 7,991 & 9,133 & 10,274 & 4.5 & 16 & 11.2 & 77 \\
\hline 13 & 1,143 & 2,286 & 3,430 & 4,573 & 5,716 & 6,859 & 8,002 & 9,146 & 10,289 & 4.7 & 17 & 11.3 & 78 \\
\hline 14 & 1,145 & 2,290 & 3,434 & 4,579 & 5,724 & 6,869 & 8,014 & 9,158 & 10,303 & 4.8 & 18 & 11.4 & 79 \\
\hline 1.5 & 1,146 & 2,293 & 3,439 & 4,586 & 5,732 & 6,879 & 8,025 & 9,171 & 10,318 & 5.0 & 19 & 11.5 & 80 \\
\hline 16 & 1,148 & 2,296 & 3,444 & 4,592 & 5,740 & 6,888 & 8,036 & 9,184 & 10,332 & 5.2 & 20 & 11.6 & $\delta 2$ \\
\hline 17 & 1,150 & 2,299 & 3,449 & $\begin{array}{l}4,599 \\
4,0\end{array}$ & 5,748 & 6,898 & 8,047 & 9,197 & $10,347$. & 5.4 & 21 & 11.7 & 83 \\
\hline 18 & 1,151 & 2,302 & 3,454 & 4,605 & 5,756 & 6,907 & 8,059 & 9,210 & 10,361 & 5.5 & 22 & 11.8 & 84 \\
\hline 19 & 1,153 & 2,306 & 3,459 & 4,611 & 5,764 & 6,917 & 8,070 & 9,223 & 10,376 & 5.7 & 23 & 11.9 & 86 \\
\hline 20 & 1,154 & 2,309 & 3,463 . & 4,618 & 5,772 & 6,927 & 8,081 & 9,236 & 10 & 5.8 & 24 & 12.0 & 87 \\
\hline 21 & 1,156 & 2,312 & 3,468 & 4,010 & 5,780 & 6,936 & 8,0 & 9,249 & & 6. & 25 & 12.1 & 89 \\
\hline 22 & 1,158 & 2,315 & 3,473 & 4,631 & 5,788 & 6,946 & 8,104 & 9,261 & 19 & 6. & 26 & 12.2 & 90 \\
\hline 23 & 1,159 & $\overrightarrow{2}, 319$ & 3,478 & 4,637 & 5,796 & 6,956 & 8,115 & 9,274 & 10,434 & 6.3 & 27 & 12.3 & 91 \\
\hline 24 & 1,161 & 2,322 & 3,483 & 4,644 & 5,804 & 6,965 & 8,126 & 9,287 & 10,448 & 6.4 & 28 & 12.4 & 93 \\
\hline 25 & 1,163 & 2,325 & 3,487 & 4,650 & 5,812 & 6,975 & 8,138 & 9,300 & 10,463 & 6.5 & 29 & 12.5 & 94 \\
\hline 26 & 1,16 & 2,328 & 3,492 & 4,656 & 5,821 & 6,985 & 8, & 9,313 & 10,477 & 6. & 30 & 12.6 & 96 \\
\hline 27 & 1,166 & 2,331 & 3,497 & 4,663 & 5,829 & 6,994 & 8,160 & 9,326 & 10,491 & 6.8 & 31 & 12.7 & 97 \\
\hline 28 & 1,167 & 2,335 & 3,502 & 4,669 & 5,837 & 7,004 & 8,171 & 9,339 & 10,506 & 6.9 & 32 & 12.8 & 99 \\
\hline 29 & 1,169 & 2,338 & 3,507 & 4,676 & 5,845 & 7,014 & 8,183 & 9,351 & 10,520 & 7.0 & 33 & 12.9 & 100 \\
\hline 30 & 1,171 & 2,3 & 3 & 4 & 5,853 & 7,023 & 8,194 & 9,364 & 10,535 & 7.2 & 34 & 0 & 102 \\
\hline 31 & 1,172 & 2,344 & 3,516 & $4 ; 689$ & 5,861 & 7,033 & 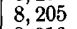 & 9,377 & 10 & 7. & 35 & 13.1 & 103 \\
\hline 32 & 1,174 & 2,348 & 3, & 4,695 & 5,869 & 7,043 & 8 & 9,390 & & 7. & 36 & 13.2 & 105 \\
\hline 33 & 1,175 & 2,351 & 3 & 4,702 & 5,877 & 7,052 & 8 & 9,403 & & 7. & 37 & 13.3 & 106 \\
\hline 34 & 1,177 & 2,354 & 3,531 & 4,708 & 5,885 & 7,062 & 8,239 & 9,416 & 10,593 & 7. & 38 & 13.4 & 108 \\
\hline 35 & 1,179 & 2,357 & 3,536 & 4,714 & 5,893 & 7,072 & 8 & 9,429 & & 7. & 39 & 13.5 & 109 \\
\hline 36 & 1,180 & 0 & 3 & 4,7 & 5,901 & 7,0 & 8 & 9,442 & 10 & 7. & 40 & 13.6 & 111 \\
\hline 37 & 1, & 2 & 3 & 4,727 & 5,909 & 7,091 & 8 & 9,455 & 10 & $\because$ & 41 & 13.7 & 112 \\
\hline 38 & 1,183 & 2,367 & 3,550 & 4,734 & 5,917 & 7,101 & 8,284 & 9,468 & 10,651 & 8. & 42 & 13.8 & 114 \\
\hline 39 & 1,185 & 2,370 & 3,555 & 4,740 & 5,925 & 7,110 & $8 ; 296$ & 9,481 & 10,666 & 8.2 & 43 & 13.9 & 115 \\
\hline 40 & 1,18 & 2,373 & 3, & 4,7 & 5,9 & 7,120 & 8 & 9 & 10 & 8. & 44 & $1 s$ & 117 \\
\hline 41 & $1 ; 188$ & 2,377 & 3 & 4,753 & 5,942 & 7,130 & 8,318 & 9,506 & 10 & 8. & 45 & 14.1 & 119 \\
\hline 42 & 90 & 2,380 & 3,5 & 4,760 & 5,950 & 7,1 & 8 & 9,519 & & 8. & 46 & 14.2 & 120 \\
\hline 43 & 1 & 2, & 3, & 4,766 & 5,958 & 7,149 & 8 & 9, & & 8. & 47 & 14.3 & 122 \\
\hline 44 & 1,1 & 2,386 & 3,57 & 4,773 & 5,966 & 7,159 & 8,352 & 9,545 & & 8. & 48 & 14.4 & 124 \\
\hline 45 & 1,195 & $\overrightarrow{2}, 390$ & 3,584 & 4,779 & 5,974 & 7,169 & 8,363 & 9,558 & 10,753 & 8. & 49 & 14.5 & 125 \\
\hline 46 & 1 , & 2, & & 4 & 5, & 7,178 & 8, & 9,571 & & 8. & 50 & & 127 \\
\hline 47 & & 2 & 3 & 4, & 5,990 & 7,188 & & 9,584 & & 9. & 51 & & 129 \\
\hline 48 & 1,20 & & 3,5 & 4,798 & 5,998 & 7,198 & 8,397 & 9,597 & & 9. & 52 & 14.8 & 130 \\
\hline 49 & 1,201 & 2,402 & 3,604 & 4,805 & 6,006 & 7,207 & 8,409 & 9,610 & 10,811 & 9.2 & 53 & 14.9 & 132 \\
\hline 50 & 1,20 & 2,4 & 3, & 4,8 & 6,0 & 7,2 & 8,4 & 9,6 & 10 & 9. & 54 & 15 & $1:$ \\
\hline 00 & 1,2 & & & & & & & & & & & & \\
\hline 52 & & 2,4 & 3,6 & 4,8 & 6,030 & 7,236 & 8,4 & 9,648 & & 9. & 56 & 15.2 & 1 \\
\hline .53 & 1,208 & 2,415 & 3,6 & 4,831 & 6,038 & 7,246 & 8,454 & 9,661 . & & 9. & 58 & 15.3 & 139 \\
\hline 54 & 1,209 & 2,419 & 3,6 & 4,8 & 6,046 & 7,256 & 8,465 & 9,674 & & 9. & 59 & 15.4 & 141. \\
\hline 55 & 1,211 & $\begin{array}{l}2,422 \\
2,42\end{array}$ & 3,6 & $\begin{array}{l}1,8 \\
4,8\end{array}$ & 6,055 & 7,265 & 8,476 & 9 & & 9. & 60 & 15.5 & 142 \\
\hline 56 & 1,2 & 2,4 & 3,6 & 4,8 & 6,0 & 7,275 & 8,4 & 9,700 & 10 & 9. & 01 & 15.6 & 144 \\
\hline 57 & 1,214 & 2,428 & $\begin{array}{l}0,0 \\
3,6\end{array}$ & $\begin{array}{l}4,857 \\
4\end{array}$ & 6,071 & 7,2 & $\begin{array}{l}8,499^{\circ} \\
\end{array}$ & 9,713 & 10,927 & 10.0 & 62 & 15.7 & 146 \\
\hline 58 & 1,216 & 2,431 & 3,647 & 4,863 & 6,079 & 7,294 & 8,510 & 9,726 & 10,942 & 10.1 & 63 & 15.8 & 148 \\
\hline 59 & 1,217 & $2, \pm 35$ & 3,652 & 4,869 & 6,087 & 7,304 & $8,521$. & 9,739 & 10,456 & & & 15.9 & 150 \\
\hline 60 & 1,219 & 2,438 & 3,657 & 4,876 & 6,095 & 7,314 & 8,533 & 9,752 & 10,971 & & & & \\
\hline
\end{tabular}

$a$ For all distances under 1.6 miles the correction may be taken as +5 feet. Height of instrument is assumed 4.5 feet. 
TABLE 24.-For oblaining differences of allitude for any minute, etc.-Continued.

$13^{\circ}$.

\begin{tabular}{|c|c|c|c|c|c|c|c|c|c|c|c|c|c|}
\hline & 1 & $g$ & 3 & 4 & $\check{\boldsymbol{b}}$ & 6 & 7 & 8 & 9 & \multicolumn{4}{|c|}{$\begin{array}{l}\text { Corrections for curva- } \\
\text { ture, refraction, and } \\
\text { height of instrument.a }\end{array}$} \\
\hline & & & & & & & & & & & & & \\
\hline 0 & $1,21.9$ & 2,438 & 3,657 & 4,876 & 6,095 & 7,314 & 8,533 & 9,752 & 10,971 & & & & \\
\hline 1 & 1,221 & 2,441 & 3,662 & 4,882 & 6,103 & 7,324 & 8,544 & 9,765 & 10,985 & Miles. & Feet. & Miles. & Feet. \\
\hline 2 & 1,222 & 2,444 & 3,667 & 4,889 & 6,111 & 7,333 & 8,556 & 9,778 & 11,000 & 1.6 & 6 & 10.2 & 64 \\
\hline 3 & 1,224 & 2,448 & 3,672 & 4,895 & 6,119 & 7,343 & 8,567 & 9,791 & 11,015 & 2.1 & 7 & 10.3 & 65 \\
\hline 4 & 1,225 & 2,451 & 3,676 & 4,902 & 6,127 & 7,353 & 8,578 & 9,804 & 11,029 & 2.5 & 8 & 10.4 & 67 \\
\hline 5 & 1,227 & 2,454 & 3,681 . & 4,908 & 6,135 & 7,362 & 8,590 & 9,817 & 11,044 & 2.8 & 9 & 10.5 & 68 \\
\hline 6 & 1,229 & 2,457 & 3,686 & 4,915 & 6,143 & 7,372 & 8,601 & 9,830 & 11,058 & 3.1 & 10 & 10.6 & 69 \\
\hline 7 & 1. 230 & 2,461 & 3,691 & 4,921 . & 6,152 & 7,382 & 8,612 & 9,843 & 11,073 & 3.4 & 11 & 10.7 & 70 \\
\hline 8 & 1,232 & 2,464 & 3,696 & 4,928 & $6,1.60$ & 7,392 & 8,624 & 9,855 & 11,087 & 3.6 & 12 & 10.8 & 71 \\
\hline 9 & 1,234 & 2,467 & 3,701 & 4,934 & 6,168 & 7,401 & 8,635 & 9,868 & 11,102 & 3.8 & 13 & 10.9 & 73 \\
\hline 10 & 1,235 & 2,470 & 3,706 & 4,941 & 6,176 & 7,411 & 8,646 & 9,881 & 11,117 & 4.1 & 14 & 11.0 & 74 \\
\hline 11 & $\begin{array}{l}1,230 \\
\text {. }\end{array}$ & 2,474 & 3,710 & 4,947 & 6,184 & 7,421 & 8,658 & 9,894 & 11,131 & 4.3 & 15 & 11.1 & 75 \\
\hline 12 & 1,238 & 2,477 & 3,715 & 4,954 & 6,192 & 7,430 & 8,669 & 9,907 & 11,146 & 4.5 & 16 & 11.2 & 77 \\
\hline 13 & 1,240 & 2,480 & 3,720 & 4,960 & 6,200 & 7,440 & 8,680 & 9,920 & 11,160 & 4.7 & 17 & 11.3 & 78 \\
\hline 14 & 1., 243 & 2,483 & 3,725 & 4,967 & 6,208 & 7,450 & 8,692 & 9,933 & 11,175 & 4.8 & 18 & 11.4 & 79 \\
\hline 15 & 1,243 & 2,487 & 3,730 & 4,973 & 6,216 & 7,460 & 8,703 & 9,946 & 11,190 & 5.0 & 19 & 11.5 & 80 \\
\hline 16 & 1,245 & 2,490 & 3,735 & 4,980 & 6,224 & 7,469 & 8,714 & 9,959 & 11,204 & 5.2 & 20 & 11.6 & 82 \\
\hline 17 & 1,247 & 2,493 & 3,740 & 4,986 & (i, 233 & 7,479 & 8,726 & 9,972 & 11,219 & $5 . \overline{4}$ & 21. & 11.7 & \\
\hline 18 & 1,248 & 2,496 & 3,744 & 4,993 & 6,241 & 7,489 & 8,737 & 9,985 & 11,233 & $5 . \overline{5}$ & 22 & 11.8 & 84 \\
\hline 19 & 1,250 & 2,500 & 3,749 & 4,999 & 6,249 & 7,499 & 8,748 & 9,998 & 11,248 & 5.7 & 23 & 11.9 & 86 \\
\hline 20 & 1,251 & 2,503 & 3,754 & 5,006 & 6,257 & 7,508 & 8,760 & 10,011 & 11,262 & 5.8 & 24 & 12.0 & 87 \\
\hline 21 & 1,253 & 2,506 & 3,759 & 5,012 & 6,265 & 7,518 & 8,771 & 10,024 & 11,277 & 6.0 & 25 & 12.1 & 89 \\
\hline 22 & 1,255 & 2,509 & 3,764 & 5,019 & 6,273 & 7,528 & 8,782 & 10,037 & 11,292 & 6.1 & 26 & 12.2 & 90 \\
\hline 23 & 1,256 & $.2,513$ & 3,769 & 5,025 & 6,281 & 7,537 & 8,794 & 10,050 & 11,306 & 6.3 & 27 & 12.3 & 91 \\
\hline t) & 1,258 & 2,516 & 3,7 & 5,032 & 6,289 & 7,5 & 8,8 & 10,063 & 11,321 & 6. & 28 & 12. & 93 \\
\hline 25 & 1,260 & 2,519 & 3,779 & 5,038 & 6,297 & 7,557 & 8,816 & 10,076 & 11,336 & 6.5 & 29 & 12.5 & 94 \\
\hline 26 & 1,261 & 2,522 & 3,783 & 5,044 & 6,306 & 7,567 & 8,828 & 10,089 & 11,350 & 6.7 & 30 & 12.6 & 96 \\
\hline 27 & 1,263 & 2,625 & 3,788 & 5,051 & 6,314 & 7,576 & 8,839 & 10,102 & 11,365 & 6.8 & 31 & $\mid 12.7$ & 97 \\
\hline & 1,26 & 2,529 & 3,7 & 5,057 & 6,322 & 7, & 8,8 & 10,115 & 11,379 & 6. & 32 & 12. & 99 \\
\hline 29 & 1,266 & 2,532 & 3,798 & 5,064 & 6,330 & 7,596 & 8,862 & 10,128 & 11,394 & 7.0 & 33 & 12.9 & 100 \\
\hline 30 & 1,268 & 2 & 3,8 & 5,070 & 6,338 & 7,606 & 8,873 & 10 & 11 & $7 .:$ & 34 & 13.0 & 102 \\
\hline & 1 & & 3 & 5,077 & 6 & 7,615 & & 10 & 3 & 7. & 35 & 13.1 & 103 \\
\hline 32 & 1,271 & 2,542 & 3,813 & 5,083 & 6,354 & 7,625 & 8,896 & 10,167 & 11,438 & 7.4 & 36 & \begin{tabular}{|l|}
13.2 \\
\end{tabular} & 105 \\
\hline 33 & 1,273 & 2,545 & 3,817 . & 5,090 & 6,362 & 7,635 & 8,907 & 10,180 & 11,452 & 7.5 & 37 & 13.3 & 106 \\
\hline 34 & 1,27 & 2,548 & 3,822 & 5,096 & 6,371 & 7,6 & 8,919 & 10, & 111, & 7. & 38 & 13. & 108 \\
\hline 35 & 1,276 & 2,551 & 3,827 & 5,103 & 6,379 & 7,654 & 8,930 & 10,206 & 11,482 & 7. & 39 & $13 . \hat{5}$ & 109 \\
\hline 36 & 1,277 & 2,555 & 3,832 & 5,109 & 6,387 & 7,664 & 8,942 & 10,219 & 11,496 & 7.9 & 40 & 13.6 & 111. \\
\hline 37 & 1,279 & 2,558 & 3,837 & 5,116 & (i, 395 & 7,674 & 8,953 & 10,232 & 11,511 & 8.0 & 41 & 13.7 & 112 \\
\hline 38 & 1,281 & 2,0 & 3,8 & 5,122 & 6,403 & 7,684 & 8,9 & 10 & 11,526 & 8. & 42 & 13.8 & 114 \\
\hline 39 & 1,282 & 2,565 & 3,847 & 5,129 & 6,411 & 7,693 & 8,976 & 10,258 & 11,540 & 8.2 & 43 & 13.9 & 115 \\
\hline 40 & 1 & 2 & 3,8 & 5 & 6 , & 7, & 8,987 & 10 & 11,555 & 8 & 44 & 14 & 117 \\
\hline & & & & & & & & & & & & & 119 \\
\hline 42 & 1,287 & 2,574 & 3,861 . & 6,149 & 6,436 & $\pi, 723$ & 9,010 & 10,297 & 11 , & 8.5 & 46 & 14. & 120 \\
\hline 43 & 1,28 & 2,578 & 3,8 & 5,155 & 6,4 & 7,7 & 0 & 10,310 & 11 , & 8.6 & 47 & 14.3 & 122 \\
\hline 44 & 1,290 & 2,6 & 3,871 . & 5,1 & 6,452 & 7,7 & 9 & & 11 , & 8. & 48 & 14 & 124 \\
\hline & 1 & 0 & 3,8 & & & 7,7 & & & & & & 14 & 125 \\
\hline 46 & 1, & 2,587 & 3,881 & 5,1 & 6,468 & 7,7 & 9,0 & 10,349 & 11 , & 8. & 50 & 14. & 127 \\
\hline 47 & 1,2 & 2,591 & 3,8 & 5,181 & 6 , & 7,7 & 9,0 & 10,362 & 111, & 9.0 & 51 & 14.7 & 120 \\
\hline 48 & 1., & 2 & 3, & 5, & & 7,781 & 9,078 & 10 , & 11,672 & 9. & 52 & 14.8 & 130 \\
\hline 49 & 1,299 & 2,597 & 3,896 & 194 & 6,493 & 7,791 & 9,090 & 10,388 & 11,687 & 9.2 & 53 & 14.9 & 132 \\
\hline 50 & 1,300 & 2,600 & 3.900 & 5,201 & 6,501 & 7,801 & 9,101 & 10,401 & 11,701 & 9.3 & 54 & 15.0 & 134 \\
\hline 51 & 1 & & 3,9 & & & & & & & 9. & 50 & & 135 \\
\hline 52 & 303 & 2,6 & 3,9 & 5,214 & 6,517 & 7,8 & 9,1 & 10 & 11, & 9. & 56 & 15.2 & 137 \\
\hline 5 & 1,305 & 2,6 & 3,9 & & & 7,8 & 9,1 & 10 & & 9. & . & 15. & 139 \\
\hline 54 & 1 & 2,613 & 3,920 & 5,227 . & 6 , & 7,840 & 9,147 & 10,453 & 11,760 & 9. & 59 & 15.4 & 141 \\
\hline 65 & 1,308 & 2,617 & 3,9 & 5,2 & & 7,8 & 9,158 & 10,466 & 11,775 & 9.8 & 60 & 15.5 & 142 \\
\hline 50 & & 2,620 & 3,9 & 5,2 & 6,5 & 7,8 & 9 & 10, & 11 , & 9.9 & 61. & 15.6 & 144 \\
\hline 67 & & & 3,9 & & & & 9,1 & & 11, & 10. & $0^{\circ}$ & 15. & 7 \\
\hline 58 & 1,313 & 2,626 & 3,940 & 5,253 & 6,566 & 7,879 & 9,192 & 10,506 & 11,819 & 10.1 & 63 & 15.8 & 148 \\
\hline 59 & 1,315 & 2,630 & 3,944 & 5,259 & 6,574 & 7,889 & 9,204 & 10,519 & 11,833 & & & 15.9 & 150 \\
\hline 60 & 1,316 & 2,633 & 3,949 & 5,266 & 6,582 & 7,899 & 9,215 & 10,532 & 11,848 & & & & \\
\hline
\end{tabular}

a For all distances under 1.6 miles the correction may be taken as +5 feet. Height of instrument is assumed 4.5 feet.

Bull. 214-03-17 
TABLE 24.-For obtaining differences of altitude for any minute, etc.-Continued.

$14^{\circ}$

\begin{tabular}{|c|c|c|c|c|c|c|c|c|c|c|c|c|c|}
\hline & 1 & 2 & 3 & 4 & 5 & 6 & 7 & 8 & 9 & \multicolumn{4}{|c|}{$\begin{array}{l}\text { Corrections for curva- } \\
\text { ture, ref raction, and } \\
\text { height of instrument. }\end{array}$} \\
\hline '́ & & & 3049 & & 6582 & 7899 & 0215 & 10532 & & & & & \\
\hline 1 & 1,318 & $\begin{array}{l}2,053 \\
2 ; 636\end{array}$ & $\begin{array}{l}0,949 \\
3,954\end{array}$ & $\begin{array}{l}5,200 \\
5,272\end{array}$ & $\begin{array}{l}0,002 \\
6,590\end{array}$ & 7,909 & 9,227 & 10,052 & $\begin{array}{l}11 \\
11,840 \\
\end{array}$ & Miles & Feet. & Miles. & Feet. \\
\hline 2 & 1., 320 & 2,639 & 3,959 & 5,279 & 6,599 & 7,918 & 9,238 & 10,558 & 11,877 & 1.6 & 6 & 10.2 & 64 \\
\hline 3 & 1,321 & 2,643 & 3,964 & 5,285 & 6,607 & 7,928 & 9,249 & 10,571 & 11,892 & 2.1 & 7 & 10.3 & 65 \\
\hline 4 & 1,323 & 2,646 & 3,969 & 5,292 & 6,615 & 7,938 & 9,261 . & 10,584 & 11,907 & 2.5 & 8 & 10.4 & 67 \\
\hline 5 & 1,325 & 2,649 & 3,974 & 5,298 & 6,623 & 7,948 & 9,272 & 10,597 & 11,923 & 2.8 & 9 & 10.5 & 68 \\
\hline 6 & 1,326 & 2,653 & 3,979 & 5,305 & 6,631 . & 7,957 & 9,284 & 10,610 & 11,936 & 3.1 & 10 & 10.6 & 69 \\
\hline 7 & 1,328 & 2,656 & 3,984 & 5,312 & 6,639 & 7,967 & 9,295 & 10,623 & $11 ., 951$ & 3.4 & 11 & 10.7 & 70 \\
\hline 8 & 1,330 & $\overrightarrow{2}, 659$ & 3,989 & 5,318 & 6,648 & 7,977 & 9,307 & 10,636 & 11,966 & 3.6 & 12 & 10.8 & 71. \\
\hline 9 & 1,331 & 2,662 & 3,993 & 5,325 & 6,656 & 7,987 & 9,318 & 10,649 & 11,980 & 3.8 & 13 & 10.9 & 73 \\
\hline 10 & 1,333 & 2,666 & 3,998 & 5,331 & 6,664 & 7,997 & 9,329 & 10,662 & 11,995 & 4. 1. & 14 & 11.0 & 74 \\
\hline 11 & 1,334 & 2,669 & 4,003 & 5,338 & 6,672 & 8,006 & 9,341 & 10,675 & 12,010 & 4.3 & 15 & 11.1 . & 75 \\
\hline 12 & 1,336 & 2,672 & 4,008 & 5,344 & 6,680 & 8,016 & 9,352 & 10,688 & 12,024 & 4.5 & 16 & 11.2 & 77 \\
\hline 13 & 1,338 & 2,675 & 4,013 & $5,351$. & 6,688 & 8,026 & 9,364 & 10,701 & 12,039 & 4.7 & 17 & 11.3 & 78 \\
\hline 14 & 1,339 & 2,679 & 4,018 & 5,357 & 6,697 & 8,036 & 9,375 & 10,715 & 12,054 & 4. 8 & 18 & 11.4 & 79 \\
\hline 15 & 1,341 . & 2,682 & 4,023 & 5,364 & 6,705 & 8,046 & 9,387 & 10,728 & 12,069 & 5.0 & 19 & 11.5 & 80 \\
\hline 16 & 1,343 & 2,685 & 4,028 & 5,370 & 6,713 & 8,056 & 9,398 & 10,741 & 12,083 & 5.2 & 20 & 11.6 & 82 \\
\hline 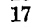 & 1,344 & 2,688 & 4,033 & 5,377 & 6,721 & 8,065 & 9,410 & 10,754 & 12,098 & 5.4 & 21 & 11.7 & 83 \\
\hline 18 & 1,346 & 2,692 & 4,038 & 5,383 & 6,729 & 8,075 & 9,421 & 10,767 & 12,113 & 5.5 & 22 & 11.8 & 84 \\
\hline 19 & 1,348 & 2,695 & 4,042 & 5,390 & 6,737 & 8,085 & $y, 432$ & 10,780 & $1.2,127$ & 5.7 & 23 & 11.9 & 86 \\
\hline 20 & 1,349 & 2,698 & 4,047 & 5,397 & 6,746 & 8,095 & 9,444 & 10,793 & 42 & 5.8 & 24 & 12.0 & 87 \\
\hline & & & 4,052 & 5 & & 8,105 & 9,455 & 10 & 12,157 & 6.0 & 25 & 12.1 & 89 \\
\hline 22 & 1,352 & 2,705 & 4,057 & 5,410 & 6,762 & 8,114 & 9,467 & 10,819 & 12,172 & 6.1 & 26 & 12.2 & 90 \\
\hline 23 & 1,354 & 2,708 & 4,062 & 5,416 & 6,770 & 8,124 & 9,478 & 10,832 & 12,186 & 6.3 & 27 & 12.3 & 91 \\
\hline 24 & 1,356 & 2,711 & 4,067 & 5,423 & 6,778 & 8,134 & 9,490 & 10,845 & 12,201 & 6.4 & 28 & 12.4 & 93 \\
\hline 25 & 1,357 & 2,715 & 4,072 & 5,429 & 6,787 & 8,144 & 9,501 & 10,859 & 12,216 & 6.5 & 29 & 12.5 & 94 \\
\hline 2 & 1,359 & 2,718 & 4,077 & 5,436 & 6,795 & 8,154 & 9,513 & 10,872 & 12,231 & 6. & 30 & 12.6 & 96 \\
\hline 27 & 1,361 & 2,721 & 4,082 & 5,442 & 6,803 & 8,164 & 9,524 & 10,885 & 12,245 & 6. & 31 & 12.7 & 97 \\
\hline 28 & 1,362 & 2,724 & 4,087 & 5,449 & 6,811 & 8,173 & 9,536 & 10,898 & 12,260 & 6.9 & 32 & 12.8 & 99 \\
\hline 29 & 1,364 & 2,728 & 4,092 & 5,455 & 6,819 & 8,183 & 9,547 & 10,911 & 12,275 & 7.0 & 33 & 12.9 & 1.00 \\
\hline 30 & 1 & 2,7 & 4,097 & 5,462 & 6,828 & 8,193 & 9,559 & 10,924 & 12,290 & 7.2 & 34 & 13.0 & 102 \\
\hline $\begin{array}{l}\text { so } \\
31 .\end{array}$ & $\begin{array}{l}1,360 \\
1,367\end{array}$ & 2,734 & 4,101 & 5,469 & 836 & 8,203 & 9,570 & 10,937 & 12,304 & 7 & 35 & 13.1 & 103 \\
\hline 32 & 1,369 & 2,738 & 4,106 & 5,475 & 14 & 8,213 & 9 & 10, & 12 & 7. & 36 & 13.2 & 105 \\
\hline 33 & 1,370 & 11 & 4,111 & 5,482 & 2 & 8,223 & 9,593 & 10 , & 12 & 7. & 37 & 13.3 & 106 \\
\hline 34 & 1,372 & 2,744 & 4,116 & 5,488 & 6,860 & 8,232 & 9,604 & 10 & 12, & 7. & 38 & 13.4 & 108 \\
\hline 35 & 1,374 & 2,747 & 4,121 & 5,495 & 6,868 & 8,242 & 9,616 & 10,990 & 12,363 & 7. & 39 & 13.5 & 109 \\
\hline 36 & 1,375 & 2,751 & 4,126 & $5,601$. & 6,877 & 8,252 & 9,627 & 11, & 12, & 7. & 40 & & 111 \\
\hline 3 & 1,377 & 2 & 4,1 & 5,5 & 6 & 8,262 & 9,639 & 11,016 & 12, & 8. & 41 & & 112 \\
\hline 38 & 1,379 & 2,757 & 4,136 & 5,514 & 6,893 & 8,272 & 9,650 & 11,029 & 12,408 & 8. & 42 & 13.8 & 114 \\
\hline 39 & 1,380 & 2,761 & 4,141 & 5,521 & 6,901 & 8,282 & 9,662 & 11,042 & 12,422 & 8.2 & 43 & 13.9 & · 115 \\
\hline 40 & 1,382 & 2,764 & 6 & 5,528 & 6,910 & 8,29 & 9,673 & 11, & 12, & 8 . & 44 & 1 & 11 \\
\hline 41 & 1,384 & & & 5,5 & & & & & & & 45 & & 119 \\
\hline 42 & 1,385 & 2,770 & 4,156 & 5,541 & 6,926 & 8,311 & 9,696 & 11,081 & 12,467 & 8. & 46 & 14.2 & 120 \\
\hline 43 & 1,387 & 2,774 & 4,160 & 5,547 & 6,934 & 8,321 & 9,708 & 11 , & & 8 & 47 & 14.3 & 122 \\
\hline 44 & 1, & 2,777 & 4,1 & 5,554 & 6,942 & 8,331 & 9,719 & & 12 , & 8. & 48 & 14.4 & .124 \\
\hline 45 & $\mathbf{1}, 390$ & 2,780 & 4,170 & 5,560 & 6,951 & 8,341 & 9,731 & 11,121 & 12,511 & 8. & 49 & 14.5 & 125 \\
\hline 46 & 1,3 & 2 & 4,1 & 5,5 & 6,959 & 8,351 & 9,742 & & & 8 & 50 & & 127 \\
\hline 47 & 1,393 & 2,787 & 4 & 5,574 & 6, & 8,3 & 9 & & & & 51 & & 129 \\
\hline 48 & 1,395 & 2, & 4,185 & 5 & 6,975 & 8,370 & 9,765 & & & 9. & 52 & 14.8 & 130 \\
\hline 49 & 1,397 & 2 & 4,190 & 5 & 6,983 & 8 & 9,777 & 11,173 & 12,570 & 9.2 & 53 & 14.9 & 132 \\
\hline 50 & 1,398 & 2,797 & 4,1 & 5,593 & 6,992 & 8,390 & 9,788 & 11,187 & 12, & 9. & 54 & 15.0 & 134 \\
\hline 51 & 1 & & & 5 & & 8, & 9,800 & & & & 55 & & \\
\hline 52 & 1,402 & & & 5, & & 8, & 9 & 11 , & & 9. & 56 & 15.2 & \\
\hline 53 & 1,403 & 2,807 & 4,210 & 5,6 & 7,016 & 8, & & 11,226 & & 9 & 00 & 15. 3 & 139 \\
\hline 54 & $\overrightarrow{1}, 405$ & 2,810 & 4,215 & 5,620 & 7,024 & 8,429 & 9,834 & 11,239 & 12,644 & 9 . & 59 & 15.4 & 141 \\
\hline 55 & 1,407 & 2,813 & 4,220 & 5,626 & 7,033 & 8,439 & $9,8+6$ & 11,202 & 12,659 & 9.8 & 60 & 15.5 & 142 \\
\hline 56 & 1,408 & 2,816 & 4,225 & 5,633 & 7,041 & 8,449 & 9,857 & 11,266 & 12,674 & 9.9 & 61 & 15.6 & 144 \\
\hline 57 & 1,410 & 2,820 & 4,230 & 5,639 & 7,049 & 8,459 & 9,869 & $11,2^{79}$ & 12,689 & 10.0 & 62 & 15.7 & 146 \\
\hline 58 & 1,411 & 2,823 & 4,234 & 5,646 & 7,057 & 8,469 & 9,880 & 11,292 & 12,703 & 10.1 & 63 & 15.8 & 148 \\
\hline 5 & 1,413 & 2,826 & 4,239 & 5,653 & 7,066 & 8,479 & 9,892 & 11,305 & $12 ; 718$ & & & 15.9 & 150 \\
\hline 60 & 1,415 & 2,830 & 4,244 & 5,659 & 7,074 & 8,489 & 9,903 & 11,318 & 12,733 & & & & \\
\hline
\end{tabular}

a For all distances under 1.6 miles the correction may be taken as +5 feet. Height of instrument is assumed 4.5 feet. 
ТАвте 25.--Horizonta' distances and elevations from stadia readings.

This is a most generally useful stadia table for rods reading 1 foot to the 100 feet and with angles up to $30^{\circ}$. 'The values of other' measures than those given in the table are obtained by multiplying the quantities under the proper vertical angle by stadia readings in hundreds of units. The quantity representing the focal distance is very small and is given at the bottom of each page for focal lengths between threefourths and 1 f feet and is represented as a coustant equal to $c$. For ordinary work it is not necessary to take the latter into account. The direct use of the table involves a multiplication for each result obtained.

Example.-Let rod intercept be 3.25 feet, and the angle of inclination be $5^{\circ} 35^{\prime}$. Then the distance on the horizontal would be

$$
d=325 \text { feet. }
$$

If we accept the focal distance $f+c$ as 1.25 feet, we have from the tables

$$
d^{\prime}=3.25 \text { feet } \times 99.05+1.24=323.15 \text { feet }
$$

and

$$
h=3.25 \text { feet } \times 9.68+0.11=31.57 \text { feet. }
$$


TABLE 25.-Horizontal distances and elevations from stadia readings.

\begin{tabular}{|c|c|c|c|c|c|c|c|c|}
\hline \multirow[b]{2}{*}{ Minutes. } & \multicolumn{2}{|c|}{$0^{\circ}$. } & \multicolumn{2}{|c|}{10} & \multicolumn{2}{|c|}{$2^{\circ}$} & \multicolumn{2}{|c|}{$3^{\circ}$} \\
\hline & $\begin{array}{c}\text { Horizon- } \\
\text { tal dis- } \\
\text { tance. }\end{array}$ & $\begin{array}{l}\text { Difference } \\
\text { of eleva- } \\
\text { tion. }\end{array}$ & $\begin{array}{l}\text { Horizon- } \\
\text { tal dis- } \\
\text { tance. }\end{array}$ & $\begin{array}{l}\text { Difference } \\
\text { of eleva- } \\
\text { tion. }\end{array}$ & $\begin{array}{l}\text { Horizon- } \\
\text { tal dis- } \\
\text { tance. }\end{array}$ & $\begin{array}{l}\text { Difference } \\
\text { of eleva- } \\
\text { tion. }\end{array}$ & $\begin{array}{l}\text { Horizon- } \\
\text { tal dis- } \\
\text { tance. }\end{array}$ & $\begin{array}{c}\text { Difference } \\
\text { of eleva- } \\
\text { tion. }\end{array}$ \\
\hline 0 & 100.00 & 0.00 & 99.97 & 1. 74 & 99.88 & 3.49 & 99.73 & 5.23 \\
\hline 2 & 100.00 & 0.06 & 99.97 & 1.80 & 99.87 & 3.55 & 99.72 & 5.28 \\
\hline 4 & 100.00 & 0.12 & 99.97 & 1. 86 & 99.87 & 3.60 & 99.71 & 5. 34 \\
\hline 6 & 100.00 & 0.17 & 99.96 & 1.92 & 99.87 & 3. 66 & 99.71 & 5. 40 \\
\hline 8 & 100.00 & 0.23 & 99.96 & 1. 98 & 99.86 & 3.72 & 99.70 & 5.46 \\
\hline 10 & 100.00 & 0.29 & 99.96 & 2.04 & 99.86 & 3.78 & 99.69 & 5.52 \\
\hline 12 & 100.00 & 0.35 & 99.96 & 2.09 & 99.85 & 3.84 & 99.69 & 5.57 \\
\hline 14 & 100.00 & 0.41 & 99.95 & 2.15 & 99.85 & 3.90 & 99.68 & 5.63 \\
\hline 16 & 100.00 & 0.47 & 99.95 & 2.21 & 99.84 & 3.95 & 99.68 & 5.69 \\
\hline 18 & 100.00 & 0.52 & 99.95 & 2.27 & 99.84 & 4. 01 & 99.67 & 5.75 \\
\hline 20 & 100.00 & 0.58 & 99.95 & 2. 33 & 99.83 & 4.07 & 99.66 & 5.80 \\
\hline 22 & 100.00 & 0.64 & 99.94 & 2. 38 & 99.83 & 4. 13 & 99.66 & 5. 86 \\
\hline 24 & 100.00 & 0.70 & 99.94 & 2.44 & 99.82 & 4. 18 & 99.65 & 5.92 \\
\hline 26 & 99.99 & 0.76 & 99.94 & 2.50 & 99.82 & 4. 24 & 99.64 & 5.98 \\
\hline 28 & 99.99 & 0.81 & 99.93 & 2.56 & 99.81 & 4. 30 & 99.63 & 6.04 \\
\hline 30 & 99.99 & 0.87 & 99.93 & 2.62 & 99.81 & 4. 36 & 99.63 & 6.09 \\
\hline 32 & 99.99 & 0.93 & 99.93 & 2.67 & 99.80 & 4.42 & 99.62 & 6.15 \\
\hline 34 & 99.99 & 0.99 & 99.93 & 2.73 & 99.80 & 4. 48 & 99.62 & 6.21 \\
\hline 36 & 99.99 & 1.05 & 99.92 & 2.79 & 99.79 & 4.53 & 99.61 & 6.27 \\
\hline 38 & 99.99 & 1.11 & 99.92 & 2.85 & 99.79 & 4.59 & 99.60 & 6.33 \\
\hline 40 & 99.99 & 1. 16 & 99.92 & 2. 91 & 99.78 & 4.65 & 99.59 & 6.38 \\
\hline 42 & 99.99 & 1.. 22 & 99. 91 . & 2.97 & 99.78 & 4. 71 & 99.59 & 6.44 \\
\hline 44 & 99.98 & 1. 28 & 99.91 & 3.02 & 99.77 & 4. 76 & 99.58 & 6.50 \\
\hline 46 & 99.98 & 1.34 & 99.90 & 3.08 & 99.77 & 4.82 & 99.57 & 6.56 \\
\hline 48 & 99.98 & 1.40 & 90.90 & 3. 14 & 99.76 & 4. 88 . & 99.56 & 6. 61 \\
\hline 50 & 99.98 & 1.45 & 99.90 & 3. 20 & 99.76 & 4. 94 & 99.56 & 6. 67 \\
\hline 52 & 99.98 & 1.51 & 99.89 & 3.26 & 99.75 & 4.99 & 99.55 & 6.73 \\
\hline 54 & 99.98 & 1.57 & 99.89 & 3.31 & 99.74 & 5.05 & 99.54 & 6.78 \\
\hline 56 & 99.97 & 1.63 & 99.89 & 3.37 & 99.74 & 5.11 & 99.53 & 6.84 \\
\hline 58 & 99.97 & 1. 69 & 99.88 & 3. 43 & 99.73 & 5.17 & 99.52 & 9.90 \\
\hline 60 & 99.97 & 1. 74 & 99.88 & 3. 49 & 99.73 & 5.23 & 99.51 & 6.96 \\
\hline$c=0.75$ & 0.75 & 0.01 & 0.75 & 0.02 & 0.75 & 0.03 & 0.75 & 0.05 \\
\hline$c=1.00$ & 1.00 & 0.01 & 1.00 & 0.03 & 1.00 & 0.04 & 1.00 & 0.06 \\
\hline$c=1.25$ & 1.25 & 0.02 & $\because 1.25$ & 0.03 & 1.25 & 0.05 & 1. 25 & 0.08 \\
\hline
\end{tabular}


TABLE 25.-Horizontal distances and elevations from stadia readings-Continued.

\begin{tabular}{|c|c|c|c|c|c|c|c|c|}
\hline \multirow[b]{2}{*}{ Minutes. } & \multicolumn{2}{|c|}{$4^{\circ}$. } & \multicolumn{2}{|c|}{$5^{\circ}$. } & \multicolumn{2}{|c|}{$6^{\circ}}$. & \multicolumn{2}{|c|}{$7^{\circ}$. } \\
\hline & $\begin{array}{c}\text { Horizon- } \\
\text { tal dis- } \\
\text { tances. }\end{array}$ & $\begin{array}{c}\text { Difference } \\
\text { of eleva- } \\
\text { tion. }\end{array}$ & $\begin{array}{l}\text { Horizon- } \\
\text { tal dis- } \\
\text { tances. }\end{array}$ & $\begin{array}{c}\text { Difference } \\
\text { of eleva- } \\
\text { tion. }\end{array}$ & $\begin{array}{l}\text { Horizon- } \\
\text { tal dis- } \\
\text { tances. }\end{array}$ & $\begin{array}{c}\text { Difference } \\
\text { of eleva- } \\
\text { tion. }\end{array}$ & $\begin{array}{l}\text { Horizon- } \\
\text { tal dis- } \\
\text { tances. }\end{array}$ & $\begin{array}{l}\text { Differenec } \\
\text { of eleval- } \\
\text { tion. }\end{array}$ \\
\hline 0 & 99.51 & 6.96 & 99.24 & 8.68 & 98.91 & 1.0. 40 & 98.51. & 12.10 \\
\hline 2 & 99.51 & 7.02 & 99.23 & 8. 74 & 98.90 & 10. 45 & 98.50 & 12.15 \\
\hline 4 & 99.50 & 7.07 & 99.22 & 8.80 & 98.88 & 10.51 & 98.48 & 12.21 \\
\hline 6 & 99.49 & 7.13 & 99.21 & 8.85 & 98.87 & 10.57 & 98.47 & 12.26 \\
\hline 8 & 99.48 & 7.19 & 99.20 & 8.91. & 98.86 & 10.62 & 98.46 & 12.32 \\
\hline 10 & 99.47 & 7.25 & 99.19 & 8.97 & 98.85 & 10.68 & 98.44 & 12.38 \\
\hline 12 & 99.46 & 7.30 & 99.18 & 9.03 & 98.83 & 10.74 & 98.43 & 12.43 \\
\hline 14 & 99.46 & 7. 36 & 99.17 & 9.08 & 98.82 & 10.79 & 98.41 & 12.49 \\
\hline 16 & 99.45 & 7. 42 & 99.16 & 9.14 & 98.81 & 10.85 & 98.40 & 12.55 \\
\hline 18 & 99.44 & 7.48 & 99.15 & 9.20 & 98.80 & 10.91 & 98.39 & 12.60 \\
\hline 20 & 99.43 & 7.53 & 99.14 & 9.25 & 98.78 & 10.96 & 98.37 & 12.66 \\
\hline 22 & 99.42 & 7.59 & 99.13 & 9.31 & 98.77 & 11.02 & 98.36 & 12.72 \\
\hline 24 & 99.41. & 7.65 & 99.11 & 9.37 & 98.76 & 11.08 & 98.34 & 12.77 \\
\hline 26 & 99.40 & 7.71 & 99.10 & 9.43 & 98.74 & 11.13 & 98.33 & 12.83 \\
\hline 28 & 99.39 & 7.76 & 99. 09 & 9.48 & 98.73 & 11. 19 & 98.31 & 12.88 \\
\hline 30 & 99.38 & 7.82 & 99.08 & 9.54 & 98.72 & 11.25 & 98.29 & 12.94 \\
\hline 32 & 99.33 & 7.88 & 99.07 & 9.60 & 98.71 & 11. 30 & 98.28 & 13.00 \\
\hline 34 & 99.37 & 7.94 & 99.06 & 9.65 & 98.69 & 11.36 & 98.27 & 13.05 \\
\hline 36 & 99.36 & 7.99 & 99.05 & 9.71 & 98.68 & 11. 42 & 98.25 & 13.11 \\
\hline 38 & 99.35 & 8.05 & 99.04 & 9.77 & 98.67 & 11. 47 & 98.24 & 13. 17 \\
\hline 40 & 99.34 & 8.11 & 99.03 & 9.83 & 98.65 & 11.. 53 & 98.22 & 13.22 \\
\hline 42 & 99.33 & 8.17 & 99.01. & 9.88 & 98.64 & 11.59 & 98.20 & 13. 28 , \\
\hline 44 & 99.32 & 8.2 & 99.00 & 9.9 & 98.63 & 11.64 & 98.19 & 13.33 \\
\hline 46 & 99.31 & 8.28 & 98.99 & 10.00 & 98.61 & 11. 70 & 98.17 & 13.39 \\
\hline 48 & 99.30 & 8.34 & 98.98 & 10.05 & 98.60 & 11.76 & 98.16 & 13.45 \\
\hline 50 & 99.29 & 8.40 & 98.97 & 10.11 & 98.58 & 11.81 & 98.14 & 13.50 \\
\hline 52 & 99.28 & 8.45 & 98.96 & 10. 17 & 98.57 & 11.87 & 98.13 & 13.56 \\
\hline 54 & 99.27 & 8.51 & 98.94 & 10.22 & 98.56 & 11.93 & 98.11 & 1.3. 61. \\
\hline 56 & 99. 26 & 8.5 & 98.93 & 10.28 & 98.54 & 11.98 & 98.10 & 13.67 \\
\hline 58 & 99.25 & 8.63 & 98.92 & 10.34 & 98.53 & 12.04 . & 98.08 & 13.73 \\
\hline 60 & 99.24 & 8.68 & 98.91 & 10.40 & 98.51 & $12.10^{\circ}$ & 98.06 & 13.78 \\
\hline$c=0.75$ & 0.75 & 0.06 & 0.75 & 0.07 & 0.75 & 0.08 & 0.74 & 0.10 \\
\hline$c=1.00$ & 1.00 & 0.08 & 0.99 & 0.09 & 0.99 & 0.11 & 0.99 & 0.13 \\
\hline$c=1.25$ & 1. 25 & 0.10 & 1. 24 & 0.11 & 1.24 & 0.14 & 1. 24 & 0.16 \\
\hline
\end{tabular}


TABLe 25.-Forizontal distances and elevations from stadia readings-Continued.

\begin{tabular}{|c|c|c|c|c|c|c|c|c|}
\hline \multirow[b]{2}{*}{ Minutes. } & \multicolumn{2}{|c|}{$8^{\circ}}$. & \multicolumn{2}{|c|}{$9^{\circ}$. } & \multicolumn{2}{|c|}{$10^{\circ}$. } & \multicolumn{2}{|c|}{$11^{\circ}$} \\
\hline & $\begin{array}{l}\text { Horizon- } \\
\text { tal dis- } \\
\text { tances. }\end{array}$ & $\begin{array}{c}\text { Difference } \\
\text { of eleva- } \\
\text { tion. }\end{array}$ & $\begin{array}{l}\text { Horizon- } \\
\text { tal dis- } \\
\text { tances. }\end{array}$ & $\begin{array}{l}\text { Difference } \\
\text { of elev't- } \\
\text { tion. }\end{array}$ & $\begin{array}{l}\text { Horizon- } \\
\text { tal dis- } \\
\text { tinces. }\end{array}$ & $\begin{array}{c}\text { Difference } \\
\text { of elevit- } \\
\text { tion. }\end{array}$ & $\begin{array}{l}\text { Horizon- } \\
\text { tal dis- } \\
\text { tinnces. }\end{array}$ & $\begin{array}{l}\text { Difference } \\
\text { of eleva- } \\
\text { tion. }\end{array}$ \\
\hline 0 & 98.06 & ه13. 78 & 97.55 & 15.45 & 96.98 & 17.10 & 96.36 & 18.73 \\
\hline 2 & 98.05 & 13.84 & 97.53 & 15.51 & 96.96 & 17.16 & 96.34 & 18.78 \\
\hline 4 & 98.03 & 13.89 & 97.52 & 15.56 & 96.94 & 17.21 & 96.32 & 18.84 \\
\hline 6 & 98.01. & 13.95 & 97.50 & 15.62 & 96.92 & 17.26 & 96.29 & 18.89 \\
\hline 8 & 98.00 & 14.01 & 97.48 & 15.67 & 96.90 & 17.32 & 96.27 & 18.95 \\
\hline 10 & 97.98 & 14.06 & 97.46 & 15. 73 & 96.88 & 17.37 & 96.25 & 19.00 \\
\hline 12 & 97.97 & 14. 12 & 97.44 & 15.78 & 96.86 & 17.43 & 96.23 & $19.0 \dot{5}$ \\
\hline 14 & 97.95 & 14. 17 & 97.43 & 15.84 & 96.84 & 17.48 & 96.21 & 19. 11 \\
\hline 16 & 97.93 & 14.28 & 97.41 . & 15.89 & 96.82 & 17.54 & 96.18 & 19. 16 \\
\hline 18 & 97.92 & 14. 28 & 97.39 & 15.95 & 96.80 & 17.59 & 96.16 & 19.21 \\
\hline 20 & 97.90 & 1.4. 34 & 97.37 & 16.00 & 96.78 & 17.65 & 96.14 & 19.27 \\
\hline 22 & 97.88 & 14. 40 & 97.35 & $16.06^{\circ}$ & 96.76 & 17. 70 & 96.12 & 19.82 \\
\hline 24 & 97.87 & 14.45 & 97.33 & 16.11 & 96.74 & 17.76 & 96.09 & 19.38 \\
\hline 26 & 97.85 & 14.51 & 97.31 & 16.17 & 96.72 & 17.81 & 96.07 & 19.43 \\
\hline 28 & 97.83 & 14.56 & 97.29 & 16.22 & 96.70 & 17.86 & 96.05 & 19.48 \\
\hline 30 & 97.82 & 14. 62 & 97.28 & 16.28 & 96.68 & 17.92 & 96.03 & 19.54 \\
\hline 32 & 97.80 & 14.67 & 97.26 & 16.3 & 96.66 & 17.9 & 96.00 & 19.59 \\
\hline 34 & 97.78 & 14.73 & 97.24 & 16. & 96.64 & 18.03 & 95.98 & 19.64 \\
\hline 36 & 97.76 & 14. 79 & 97.22 & 16. 44 & 96.62 & 18.08 & 95.96 & 19.70 \\
\hline 38 & 97.75 & 14.84 & 97.20 & 16.50 & 96.60 & 18.14 & 95.93 & 19.75 \\
\hline 40 & 97.73 & 14.90 & 97.18 & 16.55 & 96.57 & 18. 19 & 95.91 & 19.80 \\
\hline 42 & 97.71 & 14.95 & 97.16 & 16.61 & 96.55 & 18. 24 & 95.89 & 19. 86 \\
\hline 44 & 97.69 & 15. 01 . & 97.14 & 16.66 & 96.53 & 18.30 & 95.86 & 19.91 \\
\hline 46 & 97.68 & 15.06 & 97.12 & $\overline{16} 6.72$ & 96.51 & 18.35 & 95.84 & 19.96 \\
\hline 48 & 97.66 & 15. 12 & 97.10 & 16.77 & 96.49 & 18. 41 & 95.82 & 20.02 \\
\hline 50 & 97.64 & 15.17 & 97.08 & 16. 83 & 96.47 & 18.46 & 95.79 & 20.07 \\
\hline 52 & 97.62 & 15.23 & 97.06 & 16.88 & 96.45 & 18. 51 & 95.77 & 20.12 \\
\hline 54 & 97.61 & 15.28 & 97.04 & 16. & 96.42 & 18.57 & 95.75 & 20.18 \\
\hline 56 & 97.59 & 15.34 & 97.02 & 16.99 . & 96.40 & 18.62 & 95.72 & 20.23 \\
\hline 58 & 97.57 & 15.40 & 97.00 & 17.05 & 96.38 & 18. 68 & 95.70 & 20.28 \\
\hline 60 & 97.55 & 15.45 & 96.98 & 17.10 & 96.36 & 18.73 & 95.68 & 20.34 \\
\hline$c=0.75$ & 0.74 & 0.11 & 0.74 & 0.12 & 0.74 & 0.14 & 0.73 & 0.15 \\
\hline$c=1.00$ & 0.99 & 0.15 & 0.99 & 0.16 & 0.98 & 0.18 & 0,98 & 0.20 \\
\hline$c=1.25$ & 1.23 & 0.18 & $1.23^{\circ}$ & 0.21 & 1.. 23 & 0.23 & 1.22 & 0.25 \\
\hline
\end{tabular}


Tавье 25.-Horizontal distances and elevations from stadia readings-Continued.

\begin{tabular}{|c|c|c|c|c|c|c|c|c|}
\hline \multirow[b]{2}{*}{ Minutes. } & \multicolumn{2}{|c|}{$12^{\circ}$. } & \multicolumn{2}{|c|}{$13^{\circ}$} & \multicolumn{2}{|c|}{$14^{\circ}}$. & \multicolumn{2}{|c|}{$15^{\circ}$. } \\
\hline & $\begin{array}{l}\text { Horizon- } \\
\text { tal dis- } \\
\text { tances. }\end{array}$ & $\begin{array}{l}\text { Difference } \\
\text { of eleva- } \\
\text { tion. }\end{array}$ & $\begin{array}{l}\text { Horizon- } \\
\text { tal dis- } \\
\text { tances. }\end{array}$ & $\begin{array}{l}\text { Differenec } \\
\text { of eleva- } \\
\text { tion. }\end{array}$ & $\begin{array}{l}\text { Horizon- } \\
\text { tal dis- } \\
\text { tances. }\end{array}$ & $\begin{array}{c}\text { Difference } \\
\text { of eleval- } \\
\text { tion. }\end{array}$ & $\begin{array}{c}\text { Horizon- } \\
\text { tal dis- } \\
\text { tances. }\end{array}$ & $\begin{array}{c}\text { Difference } \\
\text { of eleva- } \\
\text { tion. }\end{array}$ \\
\hline 0 & 95.68 & 20.34 & 94.94 & 21. 92 & 94.15 & 23.47 & 93.30 & 25.00 \\
\hline 2 & 95.65 & 20.39 & 94.91 & 21.97 & 94.12 & 23.52 & 93.27 & 25.05 \\
\hline 4. & 95.63 & 20.44 & 94.89 & 22.02 & 94. 09 & 23.58 & 93.24 & 25. 10 \\
\hline 6 & 95.61. & 20.50 & 94.86 & 22.08 & 94.07 & 23. 63 & 93.21 & 25.15 \\
\hline 8 & 95.58 & 20.55 & 94.84 & 22.13 & 94.04 & 23.68 & 93.18 & 25.20 \\
\hline 10 & 95.56 & 20.60 & 94.81. & 22.18 & 94.01 & 23.73 & 93.16 & 25.25 \\
\hline 12 & 95.53 & 20.66 & 94.79 & 22.23 & 93.98 & 23.78 & 93.13 & 25.30 \\
\hline 14. & 95.51 & 20.71 & 94.76 & 22. 28 & 93.95 & 23.83 & 93.10 & 25.35 \\
\hline 16 & 95.49 & 20.76 & 94.73 & 22.34 & 93.93 & 23.88 & 93.07 & 25.40 \\
\hline 18 & 95.46 & 20.81 & 94.71 & 22.39 & 93.90 & 23.93 & 93.04 & 25.45 \\
\hline 20 & 95.44 & 20.87 & 94.68 & 22.44 & 93.87 & 23.99 & 93.01 & 25.50 \\
\hline 22 & 95.41. & 20.92 & 94.66 & 22.49 & 93.84 & 24.04 & 92.98 & 25.55 \\
\hline 24 & 95.39 & 20.97 & 94.63 & 22.54 & 93. 81 & 24.09 & 92.95 & 25.60 \\
\hline 26 & 95.36 & 21.03 & 94.60 & 22.60 & 93.79 & 24.14 & 92.92 & 25.65 \\
\hline 28 & 95.34 & 21.08 & 94.58 & 22.65 & 93.76 & 24,19 & 92.89 & 25.70 \\
\hline 30 & 95.32 . & 21.13 & 94.55 & 22.70 & 93.73 & 24.24 & 92.86 & 25.75 \\
\hline 32 & 95.29 & 21.18 & 94.52 & 22.75 & 93.70 & 24.29 & 92.83 & 25.80 \\
\hline 34 & 95.27 & 21.24 & 94.50 & 22.80 & 93.67 & 24.34 & 92.80 & 25.85 \\
\hline 36 & 95.24 & 21. 29 & 94.47 & 22.85 & 93.65 & 24.39 & 92.77 & 25.90 \\
\hline 38 & $95.2 \%$ & 21.34 & 94.44 & 22.91 & 93.62 & 24.44 & 92.74 & 25.95 \\
\hline 40. & 95.19 & 21.39 & 94.42 & 22.96 & 93.59 & 24.49 & 92.71 & 26.00 \\
\hline 42 & 95.17 & 21.45 & 94.39 & 23.01. & 93.56 & 24.55 & 92.68 & 26.05 \\
\hline 44 & 95.14 & 21. 50 & 94.36 & 23. 06 & 93.53 & 24.60 & 92.65 & 26.10 \\
\hline 46 & 95.12 & 21.55 & 94.34 & 23.11 & 93.50 & 24.65 & 92.62 & 26.15 \\
\hline 48 & 95.09 & 21.60 & 94.31. & 23. 16 & 93.47 & 24.70 & 92.59 & 26.20 \\
\hline 50 & 95.07 & 21. 66 & 94.28 & 23.22 & 93.45 & 24.75 & 92.56 & 26.25 \\
\hline 52 & 95.04 & 21. 71. & 94.26 & 23.27 & 93.42 & 24.80 & 92.53 & 26.30 \\
\hline 54 & 95.02 & 21.76 & 94.23 & 23. 32 & 93.39 & 24.85 & 92.49 & 26.35 \\
\hline 56 & 94.99 & 21.81 & 94.20 & 23.37 & 93.36 & 24.90 & 92.46 & 26.40 \\
\hline 58 & 94.97 & 21.87 & 94.17 & 23.42 & 93.33 & 24.95 & 92.43 & 26.45 \\
\hline 60 & 94.94 & 21.92 & 94.15 & 23.47 & 93.30 & 25.00 & 92.40 & 26.50 \\
\hline$\dot{c}=0.75$ & 0.73 & 0.16 & 0.73 & 0.17 & 0.73 & 0.19 & 0.72 & 0.20 \\
\hline$c=1.00$ & 0.98 & 0.22 & 0.97 & 0.23 & 0.97 & 0.25 & 0.96 & 0.27 \\
\hline$c=1.25$ & 1. 22 & 0.27 & 1. 21. & 0.29 & 1.21 & 0.31 & 1. 20 & 0.34 \\
\hline
\end{tabular}


TABLE 25.-Horizontal distances and elevations from stadia readings-Continued.

\begin{tabular}{|c|c|c|c|c|c|c|c|c|}
\hline \multirow[b]{2}{*}{ Minutes. } & \multicolumn{2}{|c|}{$16^{\circ}$. } & \multicolumn{2}{|c|}{$17^{\circ}$. } & \multicolumn{2}{|c|}{$18^{\circ}$. } & \multicolumn{2}{|c|}{$19^{\circ}$. } \\
\hline & $\begin{array}{l}\text { Horizon- } \\
\text { tal dis- } \\
\text { tances. }\end{array}$ & $\begin{array}{l}\text { Difference } \\
\text { of eleva- } \\
\text { tion. }\end{array}$ & $\begin{array}{l}\text { Horizon- } \\
\text { tal dis- } \\
\text { tances. }\end{array}$ & $\begin{array}{l}\text { Difference } \\
\text { of eleva- } \\
\text { tion. }\end{array}$ & $\begin{array}{l}\text { Horizon- } \\
\text { tal dis- } \\
\text { tances. }\end{array}$ & $\begin{array}{l}\text { Difference } \\
\text { of eleva- } \\
\text { tion. }\end{array}$ & $\begin{array}{l}\text { Horizon- } \\
\text { tal dis- } \\
\text { tances. }\end{array}$ & $\begin{array}{l}\text { Difference } \\
\text { of eleval- } \\
\text { tion. }\end{array}$ \\
\hline 0 & 92.40 & 26.50 & 91.45 & 27.96 & 90.45 & 29.39 & 89.40 & 30.78 \\
\hline 2 & 92.37 & 26.55 & 91.42 & 28.01 & 90.42 & 29.44 & 89.36 & 30.83 \\
\hline 4 & 92.34 & 26.59 & 91.39 & 28.06 & 90.38 & 29.48 & 89.33 & 30.87 \\
\hline 6 & 92.31 & 26.64 & 91.35 & 28.10 & 90.35 & 29.53 & 89.29 & 30.92 \\
\hline 8 & 92.28 & 26.69 & 91.32 & 28.15 & 90.31 & 29.58 & 89.26 & 30.97 \\
\hline 10 & 92.25 & 26.74 & 91.29 & 28.20 & 90.23 & 29.62 & 89.22 & 31.01 \\
\hline 12 & 92.22 & 26.79 & 91.26 & 28.25 & 90.24 & 29.67 & 89.18 & 31.06 \\
\hline 14 & 92.19 & 26.84 & 91.22 & 28. 30 & 90.21 & 29.72 & 89.15 & 31.10 \\
\hline 16 & 92.15 & 26.89 & 91.19 & 28.34 & 90.18 & 29.76 & 89.11 & 31.1 .5 \\
\hline 18 & 92.12 & 26.94 & 91.16 & 28. 39 & 90.14 & 29.81 & 89.08 & 31.1 .9 \\
\hline 20 & 92.09 & 26.99 & 91. 12 & 28.44 & 90.11 & 29.86 & 89.04 & 31.24 \\
\hline 22 & 92.06 & 27.04 & 91.09 & 28,49 & 90.07 & 29.90 & 89.00 & 31.28 \\
\hline 24 & 92.03 & 27.09 & 91.06 & 28.54 & 90.04 & 29.95 & 88.96 & 31.33 \\
\hline 26 & 92.00 & 27.13 & 91.02 & 28.58 & 90.00 & 30.00 & 88.93 & 31.38 \\
\hline 28 & 91.97 & 27.18 & 90.99 & 28.63 & 89.97 & 30.04 & 88.89 & 31.42 \\
\hline 30 & 91.93 & 27.23 & 90.96 & 28.68 & 89.93 & 30.09 & 88.86 & 31.47 \\
\hline 32 & 91.90 & 27.28 & 90.92 & 28.73 & 89.90 & 30.14 & 88.82 & 31.51 \\
\hline 34 & 91.87 & 27.33 & 90.89 & 28.77 & 89.86 & 30. & 88.78 & 31.56 \\
\hline 36 & 91.84 & 27.38 & 90.86 & 28.82 & 89.83 & 30.23 & 88.75 & 31.60 \\
\hline 38 & 91.81 & 27.43 & 90.82 & 28.87 & 89.79 & 30.28 & 88.71 & 31.65 \\
\hline 40 & 91.77 & 27.48 & 90.79 & 28.92 & 89.76 & 30.32 & 88.67 & 31.69 \\
\hline 42 & 91.74 & 27.52 & 90.76 & 28.96 & 89.72 & 30.3 & 88.64 & 31.74 \\
\hline & 81.71 & & & & & & & 31.78 \\
\hline 46 & 91.68 & 27.62 & 90.69 & 29.06 & 89.65 & 30.46 & 88.56 & 31.83 \\
\hline 18 & 91.65 & 27.67 & 90.66 & 29.11 & 89.61 . & 30.51 & 88.53 & 31.87 \\
\hline 50 & $91 . .^{\prime} 61$ & 27.72 & 90.62 & 29.15 & 89.58 & 30.55 & 88.49 & 31.92 \\
\hline 52 & 91.58 & 27.77 & 90.59 & 29.20 & 89.54 & 30.60 & 88.45 & 31.96 \\
\hline 54 & 91.55 & 27.81 & 90.55 & 29. & 89.51 . & 30. & 88.41 & 32.01 \\
\hline 56 & 91.52 & 27.86 & 90.52 & 29.30 & 89.47 & 30.69 & 88.38 & 32.05 \\
\hline 58 & 91.48 & 27.91 & 90.48 & 29.34 & 89.44 & 30.74 & 88.34 & 32.09 \\
\hline 60 & 91.45 & 27.96 & 90.45 & 29.39 & 89.40 & 30.78 & 88.30 & 32.14 \\
\hline$c=0.75$ & 0.72 & 0.21 & 0.72 & 0.23 & 0.71 & 0.24 & 0.71 & 0.25 \\
\hline$c=1.00$ & 0.86 & 0.28 & 0.95 & 0.30 & 0.95 & 0.32 & 0.94 & 0.33 \\
\hline$c=1.25$ & 1. 20 & 0.35 & 1.19 & 0.38 & 1.19 & 0.40 & 1.18 & 0.42 \\
\hline
\end{tabular}


TABLE 25.-Horizontal distances and elevations from stadia readings-Continued.

\begin{tabular}{|c|c|c|c|c|c|c|c|c|}
\hline \multirow{2}{*}{ Minutes. } & \multicolumn{2}{|c|}{$20^{\circ}$. } & \multicolumn{2}{|c|}{$21^{\circ}$. } & \multicolumn{2}{|c|}{$22^{\circ}$. } & \multicolumn{2}{|c|}{$23^{\circ}$. } \\
\hline & $\begin{array}{c}\text { Horizon- } \\
\text { tal dis- } \\
\text { tances. }\end{array}$ & $\begin{array}{c}\text { Difference } \\
\text { of eleva- } \\
\text { tion. }\end{array}$ & $\begin{array}{l}\text { Horizon- } \\
\text { tal dis- } \\
\text { tances. }\end{array}$ & $\begin{array}{c}\text { Difference } \\
\text { of eleva- } \\
\text { tion. }\end{array}$ & $\begin{array}{c}\text { Horizon- } \\
\text { tal dis- } \\
\text { tances. }\end{array}$ & $\begin{array}{c}\text { Difference } \\
\text { of eleva- } \\
\text { tion. }\end{array}$ & $\begin{array}{c}\text { Horizon- } \\
\text { tal dis- } \\
\text { tances. }\end{array}$ & $\begin{array}{c}\text { Difference } \\
\text { of eleva- } \\
\text { tion. . }\end{array}$ \\
\hline 0 & 88.30 & 32.14 & 87.16 & 33.46 & 85.97 & 34.73 & 84.73 & 35.97 \\
\hline 2 & 88. 26 & 32.18 & 87.12 & 33.50 & 85.93 & 34.77 & 84.69 & 36.01 \\
\hline 4 & 88.23 & 32.23 & 87.08 & 33.54 & 85.89 & 34.82 & 84.65 & 36.05 \\
\hline 6 & 88. 19 & 32.27 & 87.04 & 33.59 & 85.85 & 34.86 & 84.61 & 36.09 \\
\hline 8 & 88.15 & 32.32 & .87 .00 & 33.63 & 85.80 & 34.90 & 84.57 & 36.13 \\
\hline 10 & 88.11 & 32.36 & 86.96 & 33.67 & 85.76 & 34.94 & 84.52 & 36.17 \\
\hline 12 & 88.08 & 32.41 & 86.92 & 33.72 & 85.72 & 34.98 & 84.48 & 36.21 \\
\hline 14 & 88.04 & 32.45 & 86.88 & 33.76 & 85.68 & 35.02 & 84.44 & 36.25 \\
\hline 16 & 88.00 & 32.49 & 86.84 & 33.80 & 85.64 & 35.07 & 84.40 & 36.29 \\
\hline 18 & 87. 96 & 32.54 & 86. 80 & 33.84 & 85.60 & 35.11 & 84. 35 & 36.33 \\
\hline 20 & 87.93 & 32.58 & 86.77 & 33.89 & 85.56 & 35.15 & 84.31 & 36.37 \\
\hline 22 & 87.89 & 32.63 & 86.73 & 33.93 & 85.52 & 35.19 & 84.27 & 36.41 \\
\hline 24 & 87.85 & 32.67 & 86.69 & 33.97 & 85.48 & 35.23 & 84.23 & 36.45 \\
\hline 26 & 87.81 & 32.72 & 86.65 & 34.01 & 85.44 & 35.27 & 84.18 & 36.49 \\
\hline 28 & 87.77 & 32.76 & $86: 61$ & 34.06 & 85.40 & 35.31 & 84.14 & 36.53 \\
\hline 30 & 87. 74 & 32.80 & 86.57 & 34.10 & 85.36 & 35.36 & 84.10 & 36.57 \\
\hline 32 & 87.70 & 32.85 & 86.53 & 34.14 & 85.31 & 35.40 & 84.06 & 36.61 \\
\hline 34 & 87.66 & 32.89 & 86.49 & 34. 18 & 85.27 & 35.44 & 84.01 . & 36.65 \\
\hline 36 & 87.62 & 32.93 & 86.45 & 34.23 & 85.23 & 35.48 & 83.97 & 36.69 \\
\hline 38 & 87.58 & 32.98 & 86.41 & 34.27 & 85. 19 & 35.52 & 83.93 & 36.73 \\
\hline 40 & 87.54 & 33.02 & 86.37 & 34.31 & 85.15 & 35.56 & 83.89 & 36.77 \\
\hline 42 & 87.51 & 33.07 & $86: 33$ & 34.35 & 85.11 & 35.60 & 83.84 & 36.80 \\
\hline 44 & 87.47 & 33.11 & 86.29 & 34.40 & 85.07 & 35.64 & 83.80 & 36.84 \\
\hline 46 & 87.43 & 33.15 & 86.25 & 34.44 & 85.02 & 35.68 & 83.76 & 36.88 \\
\hline 48 & 87.39 & 33.20 & 86. 21 & 34.48 & 84.98 & 35.72 & 83. 72 & 36.92 \\
\hline 50 & 87.35 & 33.24 & 86.17 & 34.52 & 84.94 & 35.76 & 83.67 & 36.96 \\
\hline 52 & 87.31 & 33.28 & 86.13 & 34.57 & 84.90 & 35.80 & 83.63 & 37.00 \\
\hline 54. & 87.27 & 33.33 & 86.09 & 34.6 & 84.86 & 35.85 & 83.59 & 37.04 \\
\hline 56 & 87.24 & 33.37 & 86.05 & 34.6 & 84.82 & 35.89 & 83.54 & 37.08 \\
\hline 58 & 87.20 & 33.41 & 86.01 & 34.6 & 84.77 & 35.93 & 83.50 & 37.12 \\
\hline ? & 87.16 & .33 .46 & 85.97 & 34.73 & 84.73 & 35.97 & 83.46 & 37.16 \\
\hline$c=0.75$ & 0.70 & 0.26 & 0.70 & 0.27 & 0.69 & 0.29 & 0.69 & 0.30 \\
\hline$c=1.00$ & 0.94 & 0.35 & 0.93 & 0.37 & 0.92 & 0.38 & 0.92 & 0.40 \\
\hline$c=1.25$ & 1. 17 & 0.44 & 1. 16 & 0.46 & 1.15 & 0.48 & 1.15 & 0.50 \\
\hline
\end{tabular}


TABLe 25.-Horizontal distances and elevations from stadia readings-Continued.

\begin{tabular}{|c|c|c|c|c|c|c|c|c|}
\hline \multirow[b]{2}{*}{ Minutes. } & \multicolumn{2}{|c|}{$24^{\circ}$. } & \multicolumn{2}{|c|}{$25^{\circ}$. } & \multicolumn{2}{|c|}{$26^{\circ}$. } & \multicolumn{2}{|c|}{$27^{\circ}$. } \\
\hline & $\begin{array}{l}\text { Horizon- } \\
\text { tal dis- } \\
\text { tances. }\end{array}$ & $\begin{array}{l}\text { Difference } \\
\text { of eleva- } \\
\text { tion. }\end{array}$ & $\begin{array}{l}\text { Horizon- } \\
\text { tal dis- } \\
\text { tances. }\end{array}$ & $\begin{array}{c}\text { Difference } \\
\text { of eleva- } \\
\text { tion. }\end{array}$ & $\begin{array}{c}\text { Horizon- } \\
\text { tal dis- } \\
\text { tances. }\end{array}$ & $\begin{array}{c}\text { Difference } \\
\text { of eleva- } \\
\text { tion. }\end{array}$ & $\begin{array}{c}\text { Horizon- } \\
\text { tal dis- } \\
\text { tances. }\end{array}$ & $\begin{array}{l}\text { Difference } \\
\text { of eleva- } \\
\text { tion. }\end{array}$ \\
\hline 0 & 83.46 & 37.16 & 82.14 & 38.30 & 80.78 & 39.40 & 79. 39 & 40.45 \\
\hline 2 & 83.41 . & 37.20 & 82. 09 & 38.34 & 80.74 & 39.44 & 79.34 & 40.49 \\
\hline 4 & 83.37 & 37.23 & 82.05 & 38.38 & 80.69 & 39.47 & 79.30 & 40.52 \\
\hline 6 & 83. 33 & 37.27 & 82.01 & 38.41 & 80.65 & 39.51 & 79.25 . & 40.55 \\
\hline 8 & 83.28 & 37.31 & 81.96 & 38.45 & 80.60 & 39.54 & 79.20 & 40.59 \\
\hline 10 & 83.24 & 37.35 & 81.92 & 38.49 & 80.55 & 39.58 & 79.15 & 40.62 \\
\hline 12 & 83.20 & 37.39 & 81.87 & 38.53 & 80.51 & 39.61 & 79.11. & 40.66 \\
\hline 14 & 83.15 & 37.43 & 81.83 & 38.56 & 80.46 & 39.65 & 79.06 & 40.69 \\
\hline 16 & 83. 11 & 37.47 & $81.78^{\circ}$ & 38.60 . & 80.41 & 39.69 & $79: 01$ & 40.72 \\
\hline 18 & 83.07 & 37.51 & 81.74 & 38.64 & 80.37 & 39.72 & 78. 96 & 40.76 \\
\hline 20 & 83.02 & 37.54 & 81.69 & 38.67 & 80.32 & 39.76 & 78.92 & 40.79 \\
\hline 22 & 82.98 & 37.58 & 81.65 & 38.71 & 80.28 & 39.79 & 78.87 & 40.82 \\
\hline 24 & 82.93 & 37.6 & 81.60 & 38.75 & 80.23 & 39.83 & 78.82 & 40.86 \\
\hline 26 & 82.89 & 37.66 & 81.56 & 38.78 & 80.18 & 39.86 & 78.77 & 40.89 \\
\hline 28 & 82.85 & 37.70 & 81.51. & 38.62 & 80.14 & 39.90 & 78.73 & 40.92 \\
\hline 30 & 82.80 & 37.74 & 81.47 & 38.86 & 80.09 & 39.93 & 78.68 & 40.96 \\
\hline 32 & 82.76 & 37.77 & 81.42 & 38.89 & 80.04 & 39.97 & 78.63 & 40.99 \\
\hline 34 & 82.72 & 37.81 & 81.38 & 38.93 & 80.00 & 40.00 & 78.58 & 41.02 \\
\hline 36 & 82.67 & 37.85 & 81.33 & 38.97 & 79.95 & 40.04 & 78.54 & 41.06 \\
\hline 38 & 82.63 & 37.89 & 81.28 & 39.00 & 79.90 & 40.07 & 78.49 & 41.09 \\
\hline 40 & 82.58 & 37.93 & 81.24 & 39.04 & 79.86 & 40.11 & 78.44 & 41. 12 \\
\hline 42 & 82.54 & 37.96 & 81.19 & 39.08 & 79.81 & 40.14 & 78. 39 & 41. 16 \\
\hline 44 & 82.49 & 38.00 & 81.15 & 39.11 & 79.76 & 40.18 & 78.34 & 41. 19 \\
\hline 46 & 82.45 & 38.04 & 81.10 & 39.15 & 79.72 & 40.21 & 78. 30 & 41.22 \\
\hline 43 & 82.41 & 38.08 & 81.06 & 39.18 & 79.67 & 40.24 & 78.25 & 41.26 \\
\hline 50 & 82.36 & 38.11 & 81.01 & 39.22 & 79.62 & 40.28 & 78.20 & 41. 29 \\
\hline & 82.32 & 38.15 & 80.97 & $39.26^{\circ}$ & 79.58 & 40.31 & 78.15 & 41. 32 \\
\hline 54 & 82.27 & 38.19 & 80.92 & 39.29 & 79.53 & 40.35 & 78.10 & 41.35 \\
\hline 56 & 82.23 & $38: 23$ & 80.87 & 39.33 & 79.48 & 40.38 & 78.06 & 41.39 \\
\hline 58 & 82.18 & 38.26 & 80.83 & 39.36 & 79.44 & 40.42 & 78.01. & 41.42 \\
\hline 60 & 82.14 & 38.30 & 80.78 & 39.40 & 79.39 & 40.45 & 77.96 & 41.45 \\
\hline$c=0.75$ & 0.68 & 0.31 & 0.68 & 0.32 & 0.67 & 0.33 & 0.66 & 0.35 \\
\hline$c=1.00$ & 0.91 & 0.41 & 0.90 & 0.43 & 0.89 & 0.45 & 0.89 & 0.46 \\
\hline$c=1.25$ & .1 .14 & 0.52 & 1. 13 & 0.54 & 1.12 & 0.56 & 1.11 & 0.58 \\
\hline
\end{tabular}


TABte 25.-Horizontal distances and elevations from stadia readings-Continued.

\begin{tabular}{|c|c|c|c|c|c|c|}
\hline \multirow[b]{2}{*}{ Minutes. } & \multicolumn{2}{|c|}{$28^{\circ}}$. & \multicolumn{2}{|c|}{$29^{\circ}}$. & \multicolumn{2}{|r|}{$30^{\circ}$. } \\
\hline & $\begin{array}{l}\text { Horizon- } \\
\text { tal dis- } \\
\text { tances. }\end{array}$ & $\begin{array}{c}\text { Difference } \\
\text { of eleva- } \\
\text { tions. }\end{array}$ & $\begin{array}{l}\text { Horizon- } \\
\text { tal dis- } \\
\text { tances. }\end{array}$ & $\begin{array}{l}\text { Difference } \\
\text { of eleva- } \\
\text { tions. }\end{array}$ & $\begin{array}{l}\text { Horizon- } \\
\text { tal dis- } \\
\text { tances. }\end{array}$ & $\begin{array}{c}\text { Difference } \\
\text { of elevil- } \\
\text { tions. }\end{array}$ \\
\hline 0 & 77.96 & 41.45 & 76.50 & 42.40 & 75.00 & 43.30 \\
\hline 2 & 77.91 & 41.48 & 76.45 & 42.43 & 74.95 & 43.33 \\
\hline 4 & 77.86 & 41.52 & 76.40 & 42.46 & 74.90 & 43.36 \\
\hline 6 & 77.81 . & 41.55 & 76.35 & 42.49 & 74.85 & 43.39 \\
\hline 8 & 77.77 & 41.58 & 76. 30 & 42.53 & 74.80 & 43.42 \\
\hline 10 & 77.72 & 41. 61 & 76.25 & 42.56 & 74.75 & 43.45 \\
\hline 12 & 77.67 & 41. 65 & 76. 20 & 42.59 & 74.70 & 43.47 \\
\hline 14 & 77.62 & 41.68 & 76.15 & 42.62 & 74.65 & 43.50 \\
\hline 16 & 77.57 & 41. 71 & 76. 10 & 42.65 & 74.60 & 43.53 \\
\hline 18 & 77.52 & 41.74 & 76.05 & 42.68 & 74.55 & 43.56 \\
\hline 20 & 77.48 & 41.77 & 76.00 & 42.71 & 74.49 & 43.59 \\
\hline 22 & 77.42 & 41. 81. & 75. 95 & 42.74 & 74.44 & 43. 62 \\
\hline 24 & 77.38 & 41.84 & 75.90 & 42.77 & 74.39 & 43.65 \\
\hline 26 & 77. 33 & 41. 87 & 75.85 & 42.80 & 74. 34 & 43.67 \\
\hline 28 & 77.28 & 41.90 & 75.80 & 42.83 & 74.29 & 43.70 \\
\hline 30 & 77.23 & 41.93 & 75.75 & 42.86 & 74.24 & 43.73 \\
\hline 32 & 77.18 & 41.97 & 75.70 & 42.89 & 74. 19 & 43. 76 \\
\hline 34. & 77.13 & 42.0 & 75.65 & 42.9 & 74.14 & 43. 79 \\
\hline 36 & 77.09 & 42.03 & 75. 60 & 42.9 & 74.09 & 43.82 \\
\hline 38 & 77.04 & 42.06 & 75.55 & 42.98 & 74.04 & 43.84 \\
\hline 40 & 76.99 & 42.09 & 75. 50 & 43.01 & 73.99 & 43.87 \\
\hline 42 & 76.94 & 42.12 & $75: 45$ & 43.04 & 73.93 & 43.90 \\
\hline 44 & .76 .89 & 42.15 & 75.40 & 43.07 & 73.88 & 43.93 \\
\hline 46 & 76.84 & 42.19 & 73.35 & 43.10 & 73.83 & 43.95 \\
\hline 48 & 76.79 & 42.22 & 75. 30 & 43.13 & 73.78 & 43.98 \\
\hline 50 & 76. 74 & 42.25 & 75.25 & 43. 16 & 73.73 & 44.01 \\
\hline 52 & 76.69 & 42.2 & 75.20 & 43.18 & 73.68 & 44.04 \\
\hline 54 & 76.64 & 42.3 & 75.15 & 43.2 & 73.63 & 44. 07 \\
\hline 56 & 76.59 & 42.34 & 75.10 & 43.2 & 73.58 & 44.09 \\
\hline 58 & 76.55 & 42.37 & 75.05 & 43.27 & 73.52 & 44. 12 \\
\hline 60 & 76.50 & 42.40 & 75.00 & 43.30 & 73.47 & 44.15 \\
\hline$c=0.75$ & 0.66 & 0.36 & 0.65 & 0.37 & 0.65 & 0.38 \\
\hline$c=1.00$ & 0.88 & 0.48 & 0.87 & 0.49 & 0.86 & 0.51 \\
\hline$c=1.25$ & 1. 10 & 0.60 & 1.09 & 0.62 & 1.08 & 0.64 \\
\hline
\end{tabular}


TABLE 26.-For converting metric into United States measures.

LINEAR.

\begin{tabular}{|cc|rr|rr|rr|}
\hline Meters. & Inches. & Meters. & Feet. & Meters. & Yards. & $\begin{array}{c}\text { Kilo- } \\
\text { meters. }\end{array}$ & Miles. \\
\hline 1 & 39.3700 & 1 & 3.280833 & 1 & 1.093611 & 1 & 0.62137 \\
2 & 78.7400 & 2 & 6.561667 & 2 & 2.187222 & 2 & 1.24274 \\
3 & 118.1100 & 3 & 9.842500 & 3 & 3.280833 & 3 & 1.83411 \\
4 & 157.4800 & 4 & 13.123333 & 4 & 4.374444 & 4 & 2.48548 \\
5 & 196.8500 & 5 & 16.404166 & 5 & 5.468056 & 5 & 3.10685 \\
6 & 236.2200 & 6 & 19.685000 & 6 & 6.561667 & 6 & 3.72822 \\
7 & 275.5900 & 7 & 22.965833 & 7 & 7.655278 & 7 & 4.34959 \\
8 & 314.9600 & 8 & 26.246666 & 8 & 8.748889 & 8 & 4.97096 \\
9 & 354.3300 & 9 & 29.527500 & 9 & 9.842500 & 9 & 5.59233 \\
\hline
\end{tabular}

SQUARE.

\begin{tabular}{|cl|rr|rr|rr|}
\hline $\begin{array}{c}\text { Square } \\
\text { centi- } \\
\text { meters. }\end{array}$ & $\begin{array}{c}\text { Square } \\
\text { inches. }\end{array}$ & $\begin{array}{l}\text { Square } \\
\text { meters. }\end{array}$ & $\begin{array}{c}\text { Square } \\
\text { feet. }\end{array}$ & $\begin{array}{l}\text { Square } \\
\text { meters. }\end{array}$ & $\begin{array}{c}\text { Square } \\
\text { yards. }\end{array}$ & $\begin{array}{r}\text { Hec- } \\
\text { tares. }\end{array}$ & Acres. \\
\hline 1 & 0.1550 & 1 & 10.764 & 1 & 1.196 & 1 & 2.471 \\
2 & 0.3100 & 2 & 21.528 & 2 & 2.392 & 2 & 4.942 \\
3 & 0.4650 & 3 & 32.292 & 3 & 3.588 & 3 & 7.413 \\
4 & 0.6200 & 4 & 43.055 & 4 & 4.784 & 4 & 9.884 \\
5 & 0.7750 & 5 & 53.819 & 5 & 5.980 & 5 & 12.355 \\
6 & 0.9300 & 6 & 64.583 & 6 & 7.176 & 6 & 14.826 \\
7 & 1.0850 & 7 & 75.347 & 7 & 8.372 & 7 & 17.297 \\
8 & 1.2400 & 8 & 86.111 & 8 & 9.568 & 8 & 19.768 \\
9 & 1.3950 & 9 & 96.875 & 9 & 10.764 & 9 & 22.239 \\
\hline
\end{tabular}


TaвLE 27.-For converting United States measures into metric.

LINEAR.

\begin{tabular}{|cc|cc|cc|cc|}
\hline Inches. & $\begin{array}{c}\text { Milli- } \\
\text { meters. }\end{array}$ & Feet. & Meters. & Yards. & Meters. & Miles. & $\begin{array}{c}\text { Kilo- } \\
\text { meters. }\end{array}$ \\
\cline { 1 - 3 } 1 & 25.4001 & 1. & 0.304801 & 1 & 0.914402 & 1 & 1.60935 \\
2 & 50.8001 & 2 & 0.609601 & 2 & 1.828804 & 2 & 3.21869 \\
3 & 76.2002 & 3 & 0.914402 & 3 & 2.743205 & 3 & 4.82804 \\
4 & 101.6002 & 4 & 1.219202 & 4 & 3.657607 & 4 & 6.43739 \\
5 & 127.0003 & 5 & 1.524003 & 5 & 4.572009 & 5 & 8.04674 \\
6 & 152.4003 & 6 & 1.828804 & 6 & 5.486411. & 6 & 9.65608 \\
7 & 177.8004 & 7 & 2.133604 & 7 & 6.40081 .3 & 7 & 11.26543 \\
8 & 203.2004 & 8 & 2.438405 & 8 & 7.31521 .5 & 8 & 12.87478 \\
9 & 228.6005 & 9 & 2.743205 & 9 & 8.229616 & 9 & 14.48412 \\
\hline
\end{tabular}

SQUARE.

\begin{tabular}{|cc|cc|cc|cc|}
\hline $\begin{array}{c}\text { Square } \\
\text { inches. }\end{array}$ & $\begin{array}{c}\text { Square } \\
\text { centi- } \\
\text { meters. }\end{array}$ & $\begin{array}{l}\text { Square } \\
\text { leet. }\end{array}$ & $\begin{array}{c}\text { Square } \\
\text { deci- } \\
\text { meters. }\end{array}$ & $\begin{array}{l}\text { Square } \\
\text { yards. }\end{array}$ & $\begin{array}{c}\text { Square } \\
\text { meters. }\end{array}$ & Acres. & $\begin{array}{c}\text { Hec- } \\
\text { tares. }\end{array}$ \\
\cline { 1 - 5 } 1 & 6.452 & 1 & 9.290 & 1 & 0.836 & 1 & 0.4047 \\
2 & 12.903 & 2 & 18.581 & 2 & 1.672 & 2 & 0.8094 \\
3 & 19.355 & 3 & 27.871 & 3 & 2.508 & 3 & 1.2141 \\
4 & 25.807 & 4 & 37.161 & 4 & 3.344 & 4 & 1.6187 \\
5 & 32.258 & 5 & 46.452 & 5 & 4.181 & 5 & 2.0234 \\
6 & 38.710 & 6 & 55.742 & 6 & 5.017 & 6 & 2.4281 \\
7 & 45.161 & 7 & 65.032 & 7 & 5.853 & 7 & 2.8328 \\
8 & 51.613 & 8 & 74.323 & 8 & 6.689 & 8 & 3.2375 \\
9 & 58.065 & 9 & 83.613 & 9 & 7.525 & 9 & 3.6422 \\
\hline
\end{tabular}


TABLE 28.-For interconversion of miles and logarithms of meters, for distances from 10 to 100 miles.

[Prepared by S. S. Gannett.]

The value adopted for the meter is 39.3700 inches. Distances between triangulation stations are given in logarithms of meters, but for general use distances in imiles are most frequently desired.

The following examples illustrate use of the table:

To find the number of miles corresponding to log. distance in meters 4.56857

Next lower log. in table is for 23.00 miles 4. 56838

Difference

Corresponding to tabular difference for 0.01 mile.

Hence distance required is 23.01 miles.

For distances less than 10 miles proceed as above; first adding 1 to the characteristic of the given logarithm and afterwards dividing the corresponding number of miles by 10 . Example:

Having given the log. 3.84062 , which is less than any given in the table, and therefore for a distance less than 10 miles, adding 1 to the characteristic of the logarithm gives 4.84062 , which corresponds to a distance of 43.05 miles. Hence the distance sought is $\frac{43.05}{10}=4.305$ miles.

To change-

Log. of miles to log. of meters.

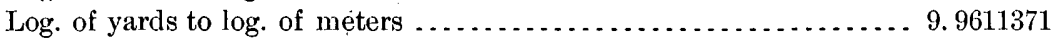

Log. of feet to $\log$. of meters . . . . . . . . . . . . . . . . . . . . . . . . . . . . 9.4840158

Log. of inches to log. of meters . . . . . . . . . . . . . . . . . . . . . . . . . . . . 8. 4048346

Log. of meters to $\log$. of miles . . . . . . . . . . . . . . . . . . . . . . . 6. 6. 7933502

Log. of meters to log. of yards . . . . . . . . . . . . . . . . . . . . . . . . . . 0.0388629

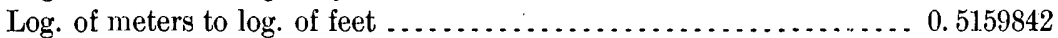

Log. of meters to log. of inches.

TABLE 28.-For interconversion of miles and logarithms of melers.

\begin{tabular}{|c|c|c|c|c|c|c|c|c|}
\hline Miles. & Log. meters. & $\begin{array}{l}\text { Diff. } \log \text {. } \\
.01 \text { mile. }\end{array}$ & Miles. & Log. meters. & $\begin{array}{l}\text { Diff. } \log \text {. } \\
\text {.01 mile. }\end{array}$ & Miles. & Log. meters. & $\begin{array}{l}\text { Diff. } \log . \\
.01 \text { mile. }\end{array}$ \\
\hline 10.00 & 4. 20665 & 43 & 10.50 & 4. 22784 & 41 & 11.00 & 4. 24804 & 39 \\
\hline .05 & 4. 20882 & & .55 & 4.22990 & & .05 & 4. 25001 & \\
\hline .10 & 4. 21097 & & 60 & 4. 23196 & & .10 & 4. 25197 & . \\
\hline .15 & 4. 21312 & & .65 & 4. 23400 & & 15 & 4. 25393 & \\
\hline .20 & $4: 21525$ & 42 & .70 & 4. 23603 & & .20 & 4. 25587 & \\
\hline .25 & 4. 21737 & & .75 & 4. 23806 & 40 & .25 & 4. 25780 & \\
\hline .30 & 4. 21949 & & .80 & 4. 24007 & & .30 & 4. 25973 & 38 \\
\hline .35 & 4. 22159 & & .85 & 4. 24208 & & .35 & 4. 26165 & \\
\hline .40 & 4. 22368 & & .90 & 4. 24408 & & .40 & 4. 26355 & \\
\hline .45 & 4. 22577 & 41 & .95 & 4. 24606 . & & .45 & 4. 26545 & \\
\hline
\end{tabular}


TABLe 28.-For interconversion of miles and logarithms of meters-Continued.

\begin{tabular}{|c|c|c|c|c|c|c|c|c|}
\hline Miles: & Log. meters. & $\begin{array}{l}\text { Diff. log. } \\
.01 \text { mile. }\end{array}$ & Miles. & Log. meters. & $\begin{array}{l}\text { Diff. log. } \\
\text {.01. mile. }\end{array}$ & Miles. & Log. meters. & $\begin{array}{l}\text { Diff. } \log \\
.01 \text { mile }\end{array}$ \\
\hline 11.50 & 4. 26735 & 38 & 14.00 & 4. 35278 & 31. & 16.50 & 4. 42413 & 26 \\
\hline .55 & 4. 26923 & & .05 & 4. 35433 & & .55 & 4. 42545 & \\
\hline .60 & 4. 27111 & 37 & .10 & 4. 35587 & & .60 & 4. 42676 & \\
\hline .65 & 4. 27298 & & .15 & 4. 35741 & & .65 & 4. 42806 & \\
\hline .70 & 4. 27484 & & .20 & 4. 35894 & & .70 & 4. 42937 & \\
\hline .75 & 4. 27669 & & .25 & 4. 36047 & 30 & .75 & 4. 43067 & \\
\hline .80 & 4. 27853 & & .30 & 4. 36199 & & .80 & 4. 43196 & \\
\hline. .85 & 4. 28087 & & .35 & 4. 36350 & & .85 & 4. 43325 & \\
\hline .90 & 4. 28220 & 36 & .40 & 4. 36501 & & .90 & 4. 43454 & \\
\hline .95 & 4. 28402 & & .45 & 4. 36652 & & .95 & 4. 43582 & \\
\hline 12.00 & 4. 28583 & & .50 & 4.36802 & & 17.00 & 4. 43710 & 25 \\
\hline .05 & 4. 28764 & & .55 & 4. 36951 & & .05 & 4. 43837 & \\
\hline .10 & 4. 28944 & & .60 & 4. 37100 & & .10 & 4. 43964 & \\
\hline .15 & 4. 29123 & & .65 & 4. 37249 & & .15 & 4. 44091 & \\
\hline .20 & 4. 29301 & . & .70 & 4. 37397 & 29 & .20 & 4. 44218 & \\
\hline .25 & 4. 29479 & 35 & .75 & 4. 37544 & & .25 & 4. 44344 & \\
\hline .30 & 4. 29656 & & .80 & 4. 37691 & & .30 & 4. 44470 & \\
\hline .35 & 4. 29832 & & .85 & 4. 37838 & & .35 & 4. 44595 & \\
\hline .40 & 4. 30007 & & .90 & 4. 37984 & & .40 & 4. 44720 & \\
\hline .45 & 4. 30182 & & .95 & 4. 38129 & & .45 & 4. 44845 & \\
\hline .50 & 4. 30356 & & 15.00 & 4. 38274 & & .50 & 4. 44969 & \\
\hline .55 & 4. 30529 & & .05 & 4. 39419 & & .55 & 4. 45093 & \\
\hline .60 & 4. 30702 & 34 & .10 & 4. 38563 & & .60 & 4. 45216 & \\
\hline .65 & 4. 30874 & & .15 & 4. 38706 & & .65 & 4. 45339 & . \\
\hline .70 & 4. 31046 & & .20 & 4. 38849 & & .70 & 4. 45462 & \\
\hline .75 & 4. 31216 & & .25 & 4. 38992 & 28 & .75 & 4. 45585 & 24 \\
\hline .80 & 4. 31386 & & .30 & 4. 39134 & & .80 & 4. 45707 & \\
\hline .85 & 4. 31555 & & .35 & 4. 39276 & & .85 & 4. 45829 & \\
\hline .90 & 4. 31724 & & .40 & 4. 39417 & & .90 & 4. 45950 & \\
\hline 95 & 4. 31892 & 33 & .45 & 4. 39558 & & .95 & 4. 46071 & \\
\hline 13.00 & 4. 32059 & & .50 & 4. 39698 & & 18. 00 & 4. 46192 & \\
\hline .05 & 4. 32226 & & .55 & 4. 39838 & & .05 & 4. 46313 & \\
\hline .10 & 4. 32392 & & .60 & 4. 39977 & & .10 & 4. 46433 & \\
\hline .15 & 4. 32558 & & .65 & 4. 40116 & & .15 & 4. 46553 & \\
\hline .20 & 4. 32722 & & .70 & 4. 40255 & 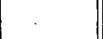 & .20 & 4. 46672 & \\
\hline .25 & 4. 32887 & & .75 & 4. 40393 & & .25 & 4. 46791 & \\
\hline .30 & 4. 33050 & & .80 & 4. 40531 & 27 & .30 & 4. 46910 & \\
\hline .35 & 4. 33213 & 32 & .85 & 4. 40668 & & .35 & 4. 47029 & \\
\hline .40 & 4. 33375 & & .90 & 4. 40805 & & .40 & 4. 47147 & \\
\hline .45 & 4. 33537 & & .95 & 4. 40941 & & .45 & 4. 47265 & 23 \\
\hline .50 & 4. 33698 & & 16.00 & 4. 41077 & & .50 & 4. 47382 & \\
\hline .55 & 4. 33859 & & .05 & 4. 41213 & & .55 & 4. 47499 & \\
\hline .60 & 4. 34019 & & .10 & 4. 41348 & & .60 & 4. 47616 & \\
\hline .65 & 4. 34178 & & .15 & 4. 41482 & & .65 & 4. 47733 & \\
\hline .70 & 4. 34337 & & .20 & 4. 41616 & & .70 & 4. 47849 & \\
\hline .75 & 4. 34495 & & .25 & 4. 41750 & & .75 & 4. 47965 & \\
\hline .80 & 4. 34653 & 31 & .30 & 4. 41884 & & .80 & 4. 48081 & \\
\hline .85 & 4. 34810 & & .35 & 4. 42017 & 26 & .85 & 4. 48196 & \\
\hline .90 & 4. 34966 & & .40 & 4. 42149 & & .90 & 4. 48311 & \\
\hline .95 & 4. 35122 & & .45 & 4. 42282 & & .95 & 4. 48426 & \\
\hline
\end{tabular}


TABLE 28.-For interconversion of miles and logarithms of meters-Continued.

\begin{tabular}{|c|c|c|c|c|c|c|c|c|}
\hline Miles. & Log. meters. & $\begin{array}{l}\text { Diff. log. } \\
.01 \text { mile. }\end{array}$ & Miles. & Log. meters. & $\begin{array}{l}\text { Diff. log. } \\
.01 \text { mile. }\end{array}$ & Miles. & Log. meters. & $\begin{array}{l}\text { Diff. log. } \\
.01 \text { mile. }\end{array}$ \\
\hline 19.00 & 4. 48540 & 23 & 21.50 & 4. 53909 & 20 & 24.00 & 4. 58686 & 18 \\
\hline .05 & 4. 48654 & & .55 & 4.54010 & & .05 & 4.58777 & \\
\hline .10 & 4. 48768 & & .60 & 4.54110 & & .10 & 4. 58867 & \\
\hline .15 & 4. 48882 & & .65 & 4.54211 & & .15 & 4. 58957 & \\
\hline .20 & 4. 48995 & & .70 & 4. 54311 & & .20 & 4. 59047 & \\
\hline .25 & 4. 49108 & & .75 & 4.54411 & & .25 & 4. 59136 & \\
\hline .30 & 4. 49221 . & 22 & .80 & 4. 54511 & & .30 & 4.59226 & \\
\hline .35 & 4. 49333 & & .85 & 4. 54610 & & .35 & 4.59315 & \\
\hline .40 & 4. 49445 & & .90 & 4.54709 & & .40 & 4. 59404 & \\
\hline .45 & 4. 49557 & & .95 & 4. 54808 & & .45 & 4.59493 & \\
\hline .50 & 4. 49669 & & 22.00 & 4.54907 & & .50 & 4.59582 & \\
\hline .55 & 4. 49780 & & .05 & 4.55006 & & .55 & 4.59670 & \\
\hline .60 & 4. 49891 & & .10 & 4. 55104 & & .60 & 4.59759 & \\
\hline .65 & 4. 50001 & & .15 & 4.55202 & & .65 & 4. 59847 & \\
\hline .70 & 4. 50112 & & .20 & 4.55300 & . & .70 & 4. 59935 & \\
\hline .75 & 4. 50222 & & .25 & 4. 55398 & 19 & .75 & 4. 60023 & \\
\hline .80 & 4. 50332 & & .30 & 4. 55495 & & .80 & 4. 60110 & \\
\hline .85 & 4. 50441 & & .35 & 4. 55593 & & .85 & 4. 60198 & \\
\hline .90 & 4.50550 & . & .40 & 4. 55690 & & .90 & 4. 60285 & 17 \\
\hline .95 & 4. 50659 & & .45 & 4. 55787 & & .95 & 4. 60372 & \\
\hline 20.00 & 4. 50768 & & .50 & 4. 55883 & & 25.00 & 4. 60459 & \\
\hline .05 & 4. 50876 & & .55 & 4. 55980 & & .05 & 4. 60546 & \\
\hline .10 & 4. 50985 & & .60 & 4. 56076 & & .10 & 4. 60632 & \\
\hline .15 & 4. 51093 & & .65 & 4.56172 & & .15 & 4. 60719 & \\
\hline .20 & 4. 51200 & & .70 & 4. 56268 & & .20 & 4. 60805 & \\
\hline .25 & 4. 51308 & 21 & .75 & 4. 56363 & & .25 & 4. 608: 1 & \\
\hline .30 & 4. 51415 & & .80 & 4. 56459 & & .30 & 4. 60977 & \\
\hline .35 & 4. 51521 & & .85 & 4. 56554 & & .35 & 4. 61063 & \\
\hline .40 & 4. 51628 & & .90 & 4. 56649 & & .40 & 4. 61148 & \\
\hline .45 & 4. 51734 & & .95 & 4. 56743 & & .45 & 4. 61234 & \\
\hline .50 & 4.51840 & & 23.00 & 4. 56838 & & .50 & 4. 61319 & \\
\hline .55 & 4. 51946 & & .05 & 4. 56932 & & .55 & 4. 61404 & \\
\hline .60 & 4. 52052 & & .10 & $4.570^{\circ} 6$ & & .60 & 4. 61489 & \\
\hline .65 & 4. 52157 & & .15 & 4. 57120 & & .65 & 4. 61574 & \\
\hline .70 & 4. 52262 & & .20 & 4.57214 & & $.70^{\circ}$ & 4. 61658 & \\
\hline .75 & 4. 52367 & & .25 & 4. 57307 & & .75 & 4. 61743 & \\
\hline .80 & 4. 52471 & & .30 & 4. 57401 & & .80 & 4. 61827 & \\
\hline .85 & 4.52576 & & .35 & 4. 57494 & & .85 & 4. 61911 & \\
\hline .90 & 4. 52680 & & .40 & 4. 57587 & 18 & .90 & 4. 61995 & \\
\hline .95 & 4. 52783 & & .45 & 4. 57679 & & .95 & 4. 62079 & \\
\hline 21.00 & 4. 52887 & & .50 & 4. 57772 & . & 26.00 & 4. 62162 & \\
\hline .05 & 4. 52990 & & .55 & 4. 57864 & & .05 & 4. 62246 & \\
\hline .10 & 4. 53093 & & .60 & 4. 57956 & & .10 & 4. 62329 & \\
\hline .15 & 4. 53196 & & .65 & 4.58048 & & .15 & 4. 62412 & \\
\hline .20 & 4. 53299 & 20 & .70 & 4. 58140 & & .20 & 4. 62495 & \\
\hline .25 & 4. 53401 & & .75 & 4. 58231 & & .25 & 4. 62578 & \\
\hline .30 & 4.53503 & & .80 & 4. 58323 & & .30 & 4. 62661 & 16 \\
\hline .35 & 4. 53605 & & .85 & 4. 58414 & & .35 & 4. 62743 & \\
\hline .40 & 4. 53706 & & .90 & 4.58505 & & .40 & 4. 62825 & \\
\hline .45 & 4. 53808 & & .95 & 4. 58596 & & .45 & 4. 62908 & \\
\hline
\end{tabular}


ТАвце 28. - For interconversion of miles and logarithms of meters-Continued.

\begin{tabular}{|c|c|c|c|c|c|c|c|c|}
\hline Miles. & Log. meters. & $\begin{array}{l}\text { Diff. log. } \\
.01 \text { mile. }\end{array}$ & Miles. & Log. meters. & $\begin{array}{l}\text { Diff, log. } \\
.01 \text { mile. }\end{array}$ & Miles. & Log. meters. & $\begin{array}{l}\text { Diff. log. } \\
.01 \text { mile. }\end{array}$ \\
\hline $\begin{array}{r}26.50 \\
.55 \\
.60 \\
.65 \\
.70\end{array}$ & $\begin{array}{l}4.62990 \\
4.63071 \\
4.63153 \\
4.63235 \\
4.63316\end{array}$ & 16 & $\begin{array}{r}29.00 \\
.05 \\
.10 \\
.15 \\
.20\end{array}$ & $\begin{array}{l}4.66905 \\
4.66980 \\
4.67054 \\
4.67129 \\
4.67203\end{array}$ & 15 & $\begin{array}{r}31.50 \\
.55 \\
.60 \\
.65 \\
.70\end{array}$ & $\begin{array}{l}4.70496 \\
4.70565 \\
4.70634 \\
4.70702 \\
4.70771\end{array}$ & 14 \\
\hline $\begin{array}{l}.75 \\
.80 \\
.85 \\
.90 \\
.95\end{array}$ & $\begin{array}{l}\text { 4. } 63397 \\
\text { 4. } 63479 \\
\text { 4. } 63559 \\
\text { 4. } 63640 \\
4.63721\end{array}$ & & $\begin{array}{l}.25 \\
.30 \\
.35 \\
.40 \\
.45\end{array}$ & $\begin{array}{l}4.67278 \\
4.67352 \\
4.67426 \\
4.67500 \\
4.67573\end{array}$ & & $\begin{array}{r}.75 \\
.80 \\
.85 \\
.90 \\
.95\end{array}$ & $\begin{array}{l}\text { 4. } 70839 \\
\text { 4. } 70908 \\
\text { 4. } 70976 \\
\text { 4. } 71044 \\
\text { 4. } 71112\end{array}$ & 3 \\
\hline $\begin{array}{r}27.00 \\
.05 \\
.10 \\
.15 \\
.20\end{array}$ & $\begin{array}{l}\text { 4. } 63801 \\
\text { 4. } 63882 \\
\text { 4. } 63962 \\
\text { 4. } 64042 \\
\text { 4. } 64122\end{array}$ & & $\begin{array}{l}.50 \\
.55 \\
.60 \\
.65 \\
.70\end{array}$ & $\begin{array}{l}\text { 4. } 67647 \\
4.67721 . \\
\text { 4. } 67794 \\
\text { 4. } 67867 \\
4.67941\end{array}$ & & $\begin{array}{r}32.00 \\
.05 \\
.10 \\
.15 \\
.20\end{array}$ & $\begin{array}{l}4.71180 \\
4.71248 \\
4.71315 \\
4.71383 \\
4.71451\end{array}$ & 13 \\
\hline $\begin{array}{l}.25 \\
.30 \\
.35 \\
.40 \\
.45\end{array}$ & $\begin{array}{l}\text { 4. } 64202 \\
\text { 4. } 64281 \\
\text { 4. } 64361 \\
\text { 4. } 64440 \\
4.64519\end{array}$ & & $\begin{array}{l}.75 \\
.80 \\
.85 \\
.90 \\
.95\end{array}$ & $\begin{array}{l}4.68014 \\
4.68087 \\
4.68159 \\
4.68232 \\
4.68305\end{array}$ & & $\begin{array}{l}.25 \\
.30 \\
.35 \\
.40 \\
.45\end{array}$ & $\begin{array}{l}\text { 4. } 71518 \\
\text { 4. } 71585 \\
\text { 4. } 71652 \\
\text { 4. } 71719 \\
\text { 4. } 71787\end{array}$ & \\
\hline $\begin{array}{l}.50 \\
.55 \\
.60 \\
.65 \\
.70\end{array}$ & $\begin{array}{l}4.64598 \\
4.64677 \\
4.64756 \\
4.64835 \\
4.64913\end{array}$ & - & $\begin{array}{r}30.00 \\
.05 \\
.10 \\
.15 \\
.20\end{array}$ & $\begin{array}{l}\text { 4. } 68377 \\
\text { 4. } 68449 \\
\text { 4. } 68522 \\
\text { 4. } 68594 \\
\text { 4. } 68666\end{array}$ & 14 & $\begin{array}{l}.50 \\
.55 \\
.60 \\
.65 \\
.70\end{array}$ & $\begin{array}{l}\text { 4. } 71853 \\
\text { 4. } 71920 \\
\text { 4. } 71987 \\
\text { 4. } 72053 \\
\text { 4. } 72120\end{array}$ & \\
\hline $\begin{array}{r}.75 \\
-.80 \\
.85 \\
.90 \\
.95\end{array}$ & $\begin{array}{l}4.64991 \\
4.65069 \\
4.65147 \\
4.65225 \\
4.65303\end{array}$ & & $\begin{array}{l}.25 \\
.30 \\
.35 \\
.40 \\
.45\end{array}$ & $\begin{array}{l}4.68737 \\
4.68809 \\
4.68881 \\
4.68952 \\
4.69024\end{array}$ & & $\begin{array}{l}.75 \\
.80 \\
.85 \\
.90 \\
.95\end{array}$ & $\begin{array}{l}\text { 4. } 72186 \\
\text { 4. } 72252 \\
\text { 4. } 72319 \\
\text { 4. } 72385 \\
\text { 4. } 72451\end{array}$ & \\
\hline $\begin{array}{r}28.00 \\
.05 \\
.10 \\
.15 \\
.20\end{array}$ & $\begin{array}{l}4.65381 \\
4.65458 \\
4.65536 \\
4.65613 \\
4.65690\end{array}$ & 15 & $\begin{array}{l}.50 \\
.55 \\
.60 \\
.65 \\
.70\end{array}$ & $\begin{array}{l}4.69095 \\
4.69166 \\
4.69237 \\
4.69308 \\
4.69379\end{array}$ & & $\begin{array}{r}33.00 \\
.05 \\
.10 \\
.15 \\
.20\end{array}$ & $\begin{array}{l}\text { 4. } 72516 \\
\text { 4. } 72582 \\
\text { 4. } 72648 \\
\text { 4. } 72713 \\
4.72779\end{array}$ & · \\
\hline $\begin{array}{l}.25 \\
.30 \\
.35 \\
.40 \\
.45\end{array}$ & $\begin{array}{l}\text { 4. } 65767 \\
\text { 4. } 65844 \\
\text { 4. } 65920 \\
4.65997 \\
4.66073\end{array}$ & & $\begin{array}{l}.75 \\
.80 \\
.85 \\
.90 \\
.95\end{array}$ & $\begin{array}{l}4.69449 \\
4.69520 \\
4.69590 \\
4.69661 \\
4.69731\end{array}$ & & $\begin{array}{l}.25 \\
.30 \\
.35 \\
.40 \\
.45\end{array}$ & $\begin{array}{l}\text { 4. } 72844 \\
\text { 4. } 72909 \\
\text { 4. } 72975 \\
\text { 4. } 73040 \\
\text { 4. } 73105\end{array}$ & \\
\hline $\begin{array}{l}.50 \\
.55 \\
.60 \\
.65 \\
.70\end{array}$ & $\begin{array}{l}4.66149 \\
4.66226 \\
4.66302 \\
4.66377 \\
4.66453\end{array}$ & & $\begin{array}{r}31.00 \\
.05 \\
.10 \\
.15 \\
.20\end{array}$ & $\begin{array}{l}\text { 4. } 69801 \\
\text { 4. } 69871 \\
\text { 4. } 69941 \\
\text { 4. } 70011 \\
\text { 4. } 70081\end{array}$ & - & $\begin{array}{l}.50 \\
.55 \\
.60 \\
.65 \\
.70\end{array}$ & $\begin{array}{l}\text { 4. } 73169 \\
\text { 4. } 73234 \\
\text { 4. } 73299 \\
\text { 4. } 73363 \\
\text { 4. } 73428\end{array}$ & \\
\hline $\begin{array}{l}.75 \\
.80 \\
.85 \\
.90 \\
.95\end{array}$ & $\begin{array}{l}4.66529 \\
4.66604 \\
4.66680 \\
4.66755 \\
4.66830\end{array}$ & & $\begin{array}{l}.25 \\
.30 \\
.35 \\
.40 \\
.45\end{array}$ & $\begin{array}{l}\text { 4. } 70150 \\
\text { 4. } 70219 \\
\text { 4. } 70289 \\
\text { 4. } 70358 \\
\text { 4. } 70427\end{array}$ & . & $\begin{array}{l}.75 \\
.80 \\
.85 \\
.90 \\
.95\end{array}$ & $\begin{array}{l}\text { 4. } 73492 \\
\text { 4. } 73557 \\
\text { 4. } 73621 \\
\text { 4. } 73685 \\
\text { 4. } 73749\end{array}$ & \\
\hline
\end{tabular}

Bull, 214-03-1S 
TABLE 28.-For interconversion of miles and logarithms of meters-Continued.

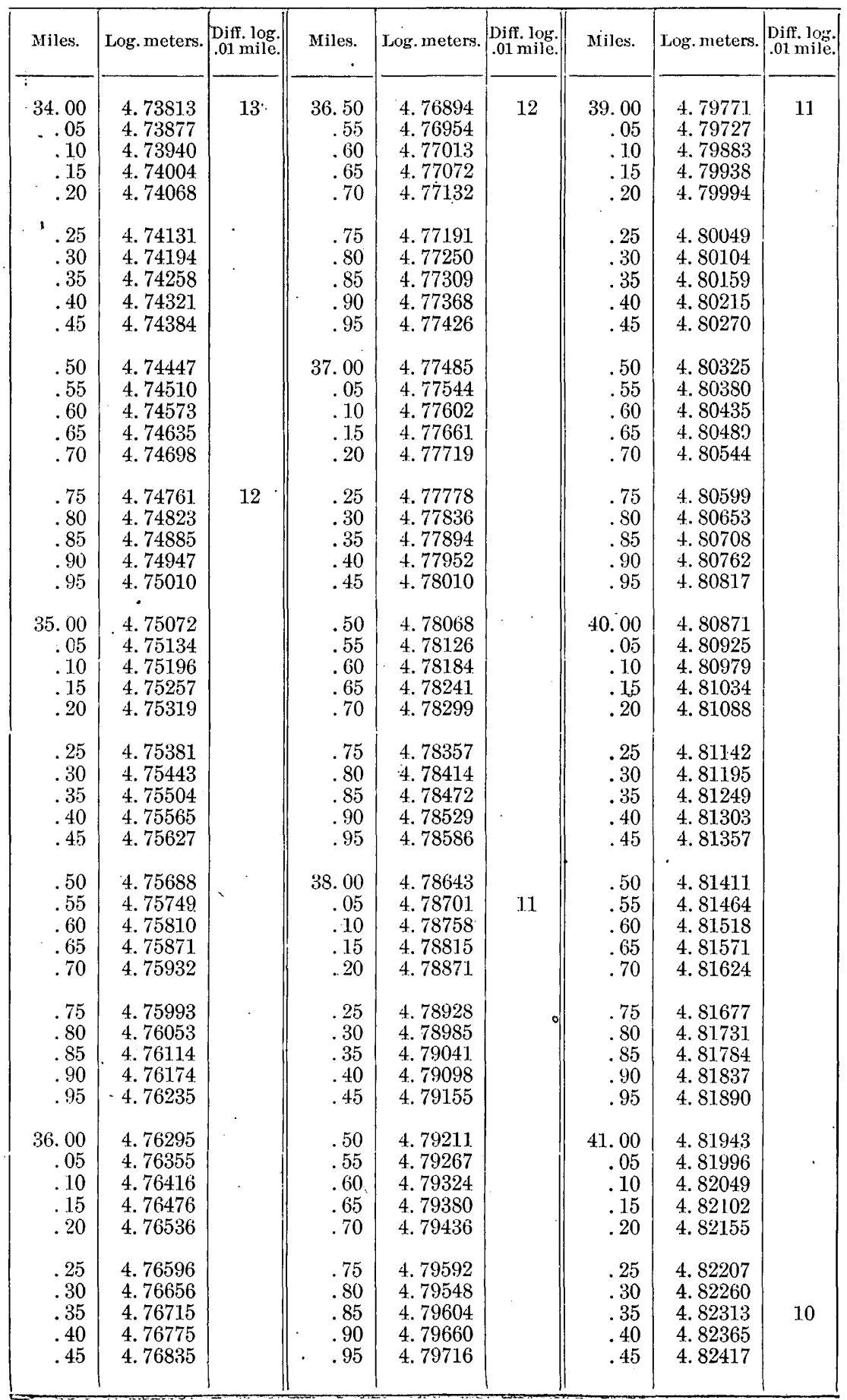


TAвLE 28. - For interconversion of miles and logarithms of meters-Continued.

\begin{tabular}{|c|c|c|c|c|c|c|c|c|}
\hline Miles. & logr. meters. & $\begin{array}{l}\text { Diff. logr. } \\
.01 \text { mile. }\end{array}$ & Miles. & Log. meters. & $\begin{array}{l}\text { Diff. log. } \\
.01 \text { mile. }\end{array}$ & Miles. & Log. meters. & $\begin{array}{l}\text { Dif: log. } \\
.01 \text { mile. }\end{array}$ \\
\hline 41.50 & 4.82470 & 10 & 44.00 & 4. 85010 & 10 & 46.50 & 4. 87410 & 9 \\
\hline .55 & 4. 82522 & & .05 & 4.85060 & & .55 & 4. 87457 & \\
\hline .60 & 4.82574 & & .10 & 4. 851.09 & & 60 & 4. 87504 & \\
\hline 65 & 4.82627 & & .15 & 4.85158 & & .65 & 4.87550 & \\
\hline .70 & 4. 82679 & & .20 & 4. 85207 & & .70 & 4.87597 & \\
\hline .75 & 4. $\$ 2731$ & & .25 & 4. 85256 & & .75 & 4.87643 & \\
\hline .80 & 4. 82783 & & .30 & 4. 85305 & • & .80 & 4. 87690 & \\
\hline .85 & 4. 82835 & & .35 & 4. 85354 & $\cdot$ & .85 & 4. 87736 & \\
\hline .90 & 4. 82886 & & .40 & 4. 85403 & & $.90^{\circ}$ & 4. 87782 & \\
\hline .95 & 4. 82938 & & .45 & 4. 85452 & . & .95 & 4. 87829 & \\
\hline 42.00 & 4. $\$ 2990$ & . & .50 & 4. $\$ 5501$ & . & 47.00 & 4.87875 & \\
\hline .05 & 4. 83042 & & .55 & 4.85550 & & .05 & 4. 87921 . & \\
\hline 10 & 4. 83093 & & .60 & 4.85599 & & .10 & 4. 87967 & \\
\hline .15 & 4. 83145 & . & .65 & 4. 85647 & & .15 & 4. 88013 & \\
\hline .20 & 4. 83196 & & .70 & 4.85696 & . & .20 & 4. 88059 & \\
\hline .25 & 4. 83248 & $\cdot$ & .75 & 4. 85744 & & .25 & 4. 88105 & \\
\hline .30 & 4. 83299 & & .80 & 4.85793 & & .30 & 4. 88151 . & \\
\hline .35 & 4. 83350 & & .85 & 4. 85841 & & .35 & 4. 88197 & \\
\hline .40 & 4. 83402 & & .90 & 4.85890 & & .40 & 4.88243 & \\
\hline .45 & 4. 83453 & & .95 & 4. 85938 & & .45 & 4. 88289 & \\
\hline .50 & 4. 83504 & & 45.00 & 4. 85986 & & .50 & 4. 88334 & \\
\hline .55 & 4. 83555 & & .05 & 4. 86035 & & .55 & 4. 88380 & \\
\hline .60 & 4. 83606 & & .10 & 4. 86083 & & .60 & 4.88326 & \\
\hline .65 & 4. 83657 & & .15 & 4. 86131 & & .65 & 4. 88471 & \\
\hline .70 & 4. 83708 & & .20 & 4. 86179 & & .70 & 4. 88517 & \\
\hline .75 & 4. 83759 & & .25 & 4. 86227 & & .75 & 4. 885662 & \\
\hline .80 & 4. 83809 & & .30 & 4. 86275 & & .80 & 4. 88608 & \\
\hline .85 & 4. 83860 & & .35 & 4. 86323 & & .85 & 4. 88653 & \\
\hline .90 & 4. 83911 . & & .40 & 4. 86371 & & .90 & 4. 88699 & \\
\hline .95 & 4. 83961 & . & .45 & 4. 86418 & & .95 & 4. 88744 & \\
\hline 43.00 & 4. 84012 & & .50 & 4. 86466 & & 48.00 & 4. $\$ 8789$ & \\
\hline .05 & 4.84062 & & .55 & 4. 86514 & & .05 & 4. 88834 & \\
\hline 1.0 & 4. 84113 & & .60 & 4. 86561 & & .10 & 4. 88879 & \\
\hline .15 & 4. 84163 & & .65 & 4. 86609 & & .15 。 & 4. 88925 & . \\
\hline .20 & 4. 84213 & & .70 & 4. 86657 & & .20 & 4. 88970 & \\
\hline .25 & 4. 84264 & & .75 & 4. 86704 & & .25 & 4. 89015 & \\
\hline .30 & 4. 84314 & & .80 & 4. 86751 & & .30 & 4. 89060 & \\
\hline .35 & 4. 84364 & & .85 & 4. 86799 & & .35 & 4. 89105 & \\
\hline .40 & 4. $8+414$ & & .90 & 4. 86846 & & .40 & 4. 89149 & \\
\hline .45 & 4. 84464 & & .95 & 4. 86894 & & .45 & 4. 89194 & \\
\hline .50 & 4. 84514 & & 46.00 & 4. 86941 & 9 & .50 & 4. 89239 & \\
\hline .55 & 4. 84564 & & .05 & 4. 86988 & & .55 & 4. 89284 & \\
\hline .60 & 4.84614 & & .10 & 4. 87035 & & .60 & 4. 89329 & \\
\hline .65 & 4. 84663 & & .15 & 4. 87082 & & .65 & 4. 89373 & \\
\hline .70 & 4. 84713 & & .20 & 4. 87129 & & .70 & 4. 89418 & \\
\hline .75 & 4. 84763 & & .25 & 4. 87176 & & .75 & 4. 89462 & \\
\hline .80 & 4. 84812 & & .30 & 4. 87223 & & .80 & 4. 89507 & \\
\hline .85 & 4. 84862 & & .35 & 4. 87270 & & .85 & 4. 89551 . & \\
\hline .90 & 4. 84911 & & .40 & 4. 87317 & & .90 & 4. 89596 & \\
\hline .95 & 4. 84961 & & .45 & 4. 87364 & & .95 & 4. 89640 & \\
\hline
\end{tabular}


TABLE 28.-For interconversion of miles and logarithms of meters-Continued.'

\begin{tabular}{|c|c|c|c|c|c|c|c|c|}
\hline Miles. & Log. meters. & $\begin{array}{l}\text { Diff. } \log \text {. } \\
.01 \text { mile. }\end{array}$ & Miles. & Log. meters. & $\begin{array}{l}\text { Diff. } \log . \\
.01 \text { mile. }\end{array}$ & Miles. & Log. meters. & $\begin{array}{l}\text { Diff. log. } \\
.01 \text { mile. }\end{array}$ \\
\hline $\begin{array}{r}49.00 \\
.05 \\
.10 \\
.15 \\
.20\end{array}$ & $\begin{array}{l}4.89685 \\
4.89729 \\
4.89773 \\
4.89817 \\
4.89861\end{array}$ & 9 & $\begin{array}{r}51.50 \\
.55 \\
.60 \\
.65 \\
.70\end{array}$ & $\begin{array}{l}4.91846 \\
4.91888 \\
4.91930 \\
4.91972 \\
4.92014\end{array}$ & 8 & $\begin{array}{r}54.00 \\
.05 \\
.10 \\
.15 \\
.20\end{array}$ & $\begin{array}{l}4.93904 \\
4.93945 \\
4.93985 \\
4.94025 \\
4.94065\end{array}$ & 8 \\
\hline $\begin{array}{l}.25 \\
.30 \\
.35 \\
.40 \\
.45\end{array}$ & $\begin{array}{l}4.89906 \\
4.89950 \\
4.89994 \\
4.90038 \\
4.90082\end{array}$ & & $\begin{array}{r}.75 \\
. .80 \\
.85 \\
.90 \\
.95\end{array}$ & $\begin{array}{l}4.92056 \\
4.92098 \\
4.92140 \\
4.92182 \\
4.92224\end{array}$ & & $\begin{array}{l}.25 \\
.30 \\
.35 \\
.40 \\
.45\end{array}$ & $\begin{array}{l}4.94105 \\
4.94145 \\
4.94185 \\
4.94225 \\
4.94265\end{array}$ & \\
\hline $\begin{array}{l}.50 \\
.55 \\
.60 \\
.65 \\
.70\end{array}$ & $\begin{array}{l}4.90125 \\
4.90169 \\
4.90213 \\
4.90257 \\
4.90301\end{array}$ & & $\begin{array}{r}52.00 \\
.05 \\
.10 \\
.15 \\
.20\end{array}$ & $\begin{array}{l}4.92265 \\
4.92307 \\
4.92349 \\
4.92390 \\
4.92432\end{array}$ & & $\begin{array}{l}.50 \\
.55 \\
.60 \\
.65 \\
.70\end{array}$ & $\begin{array}{l}4.94305 \\
4.94345 \\
\text { 4. } 94384 \\
\text { 4. } 94424 \\
4.94464\end{array}$ & \\
\hline $\begin{array}{l}.75 \\
.80 \\
.85 \\
.90 \\
.95\end{array}$ & $\begin{array}{l}\text { 4. } 90344 \\
\text { 4. } 90388 \\
\text { 4. } 90431 \\
\text { 4. } 90475 \\
\text { 4. } 90519\end{array}$ & & $\begin{array}{l}.25 \\
.30 \\
.35 \\
.40 \\
.45\end{array}$ & $\begin{array}{l}4.92474 \\
4.92515 \\
4.92557 \\
4.92598 \\
4.92639\end{array}$ & & $\begin{array}{l}.75 \\
.80 \\
.85 \\
.90 \\
.95\end{array}$ & $\begin{array}{l}\text { 4. } 94503 \\
\text { 4. } 94543 \\
\text { 4. } 94583 \\
\text { 4. } 94622 \\
\text { 4. } 94662\end{array}$ & 。 \\
\hline $\begin{array}{r}50.00 \\
.05 \\
.10 \\
.15 \\
.20\end{array}$ & $\begin{array}{l}\text { 4. } 90562 \\
\text { 4. } 90605 \\
\text { 4. } 90649 \\
4.90692 \\
4.90735\end{array}$ & & $\begin{array}{l}.50 \\
.55 \\
.60 \\
.65 \\
.70\end{array}$ & $\begin{array}{l}4.92681 \\
4.92722 \\
4.92764 \\
4.92805 \\
4.92846\end{array}$ & & $\begin{array}{r}55.00 \\
.05 \\
.10 \\
.15 \\
.20\end{array}$ & $\begin{array}{l}4.94701 \\
4.94741 \\
4.94780 \\
4.94820 \\
4.94859\end{array}$ & \\
\hline $\begin{array}{l}.25 \\
.30 \\
.35 \\
.40 \\
.45\end{array}$ & $\begin{array}{l}4.90779 \\
4.90822 \\
4.90865 \\
4.90908 \\
4.90951\end{array}$ & & $\begin{array}{l}.75 \\
.80 \\
.85 \\
.90 \\
.95\end{array}$ & $\begin{array}{l}4.92887 \\
4.92928 \\
4.92969 \\
4.93011 \\
4.93052\end{array}$ & & $\begin{array}{l}.25 \\
.30 \\
.35 \\
.40 \\
.45\end{array}$ & $\begin{array}{l}\text { 4. } 94898 \\
4.94937 \\
4.94977 \\
4.95016 \\
4.95055\end{array}$ & \\
\hline $\begin{array}{l}.50 \\
.55 \\
.60 \\
.65 \\
.70\end{array}$ & $\begin{array}{l}4.90994 \\
4.91037 \\
4.91080 \\
4.91123 \\
4.91166\end{array}$ & & $\begin{array}{r}53.00 \\
.05 \\
.10 \\
.15 \\
.20\end{array}$ & $\begin{array}{l}4.93093 \\
4.93133 \\
4.93175 \\
4.93215 \\
4.93256\end{array}$ & & $\begin{array}{l}.50 \\
.55 \\
.60 \\
.65 \\
.70\end{array}$ & \begin{tabular}{|l|}
4.95094 \\
4.951 .33 \\
4.95172 \\
4.95212 \\
4.95251
\end{tabular} & .. \\
\hline $\begin{array}{l}.75 \\
.80 \\
.85 \\
.90 \\
.95\end{array}$ & $\begin{array}{l}4.91209 \\
4.91251 \\
4.91294 \\
4.91337 \\
4.91379\end{array}$ & & $\begin{array}{l}.25 \\
.30 \\
.35 \\
.40 \\
.45\end{array}$ & $\begin{array}{l}4.93297 \\
4.93338 \\
4.93378 \\
4.93419 \\
4.93460\end{array}$ & & $\begin{array}{l}.75 \\
.80 \\
.85 \\
.90 \\
.95\end{array}$ & $\begin{array}{l}\text { 4. } 95289 \\
\text { 4. } 95328 \\
\text { 4. } 95367 \\
4.95406 \\
4.95445\end{array}$ & \\
\hline $\begin{array}{r}51.00 \\
.05 \\
.10 \\
.15 \\
.20\end{array}$ & $\begin{array}{l}4.91422 \\
4.91465 \\
4.91507 \\
4.91550 \\
4.91592\end{array}$ & & $\begin{array}{l}.50 \\
.55 \\
.60 \\
.65 \\
.70\end{array}$ & $\begin{array}{l}4.93500 \\
4.93541 \\
4.93581 \\
4.93622 \\
4.93662\end{array}$ & & $\begin{array}{r}56.00 \\
.05 \\
.10 \\
.15 \\
.20\end{array}$ & $\begin{array}{l}\text { 4. } 95484 \\
\text { 4. } 95523 \\
\text { 4. } 95561 \\
\text { 4. } 95600 \\
\text { 4. } 95639\end{array}$ & \\
\hline $\begin{array}{l}.25 \\
.30 \\
.35 \\
.40 \\
.45\end{array}$ & $\begin{array}{l}\text { 4. } 91634 \\
4.91677 \\
4.91719 \\
4.91761 \\
4.91803\end{array}$ & 8 & $\begin{array}{l}.75 \\
.80 \\
.85 \\
.90 \\
.95\end{array}$ & $\begin{array}{l}\text { 4. } 93703 \\
\text { 4. } 93743 \\
\text { 4. } 93784 \\
\text { 4. } 93824 \\
\text { 4. } 93864\end{array}$ & & $\begin{array}{l}.25 \\
.30 \\
.35 \\
.40 \\
.45\end{array}$ & $\begin{array}{l}\text { 4. } 95677 \\
4.95716 \\
4.95754 \\
4.95793 \\
4.95831\end{array}$ & \\
\hline
\end{tabular}


TABLE 28.-For interconversion of miles and logarithms of meter's-Continued.

\begin{tabular}{|c|c|c|c|c|c|c|c|c|}
\hline Miles. & Log. meters. & $\begin{array}{l}\text { Diff. } \log . \\
.01 \text { mile. }\end{array}$ & Miles. & Log. meters. & $\begin{array}{l}\text { Diff. log. } \\
.01 \text { mile. }\end{array}$ & Miles. & Log. meters. & $\begin{array}{l}\text { Diff. } \log \text {. } \\
.01 \text { mile. }\end{array}$ \\
\hline 56.50 & 4.95870 & 8 & 59.00 & 4. 97750 & 7 & 61.50 & 4. 99553 & 7 \\
\hline .55 & 4.95908 & & .05 & 4. 97787 & & .55 & 4. 99588 & \\
\hline 60 & 4. 95947 & & .10 & 4. 97824 & • & 60 & 4. 99623 & \\
\hline .65 & 4.95985 & & .15 & 4. 97861 & & 65 & 4. 99658 & \\
\hline .70 & 4.96023 & & .20 & 4. 97897 & & .70 & 4. 99693 & \\
\hline .75 & 4. 96062 & & .25 & 4. 97934 & & .75 & 4. 99729 & \\
\hline .80 & 4.96100 & & .30 & 4. 97971 & 10 & .80 & 4. 99764 & \\
\hline .85 & 4. 96138 & & .35 & 4. 98007 & 10 & .85 & 4. 99799 & \\
\hline .90 & 4. 96176 & & .40 & 4. 98044 & & .90 & 4. 99834 & \\
\hline .95 & 4. 96214 & & .45 & 4.98080 & & .95 & 4. 99869 & \\
\hline 57.00 & 4. 96253 & & .50 & 4. 98117 & & 62.00 & 4. 99904 & \\
\hline .05 & 4. 96291 & & .55 & 4.98153 & & .05 & 4. 99939 & \\
\hline .10 & 4. 96329 & & .60 & 4. 98190 & & .10 & 4. 99974 & \\
\hline .15 & 4. 96367 & & .65 & 4. 98226 & & .15 & 5.00009 & \\
\hline .20 & 4. 96405 & & .70 & 4. 98262 & & .20 & 5.00044 & \\
\hline .25 & 4.96443 & & .75 & 4. 98299 & & .25 & 5. 00079 & \\
\hline .30 & 4. 96481 & & .80 & 4. 98335 & & .30 & 5.00114 & \\
\hline .35 & 4. 96518 & & .85 & 4. 98371 & & .35 & 5. 00149 & \\
\hline .40 & 4. 96556. & & .90 & 4. 98408 & & .40 & 5.00183 & \\
\hline .45 & 4. 96594 & & .95 & 4. 98444 & & .45 & 5.00218 & \\
\hline .50 & 4. 96632 & & 60.00 & 4. 98480 & & .50 & 5. 00253 & \\
\hline .55 & 4. 96669 & & .05 & 4. 98516 & & .55 & 5. 00288 & \\
\hline 60 & 4. 96707 & & .10 & 4. 98552 & & .60 & 5. 00322 & . \\
\hline .65 & 4. 96745 & & .15 & 4. 98589 & & .65 & 5. 00357 & \\
\hline .70 & 4. 96783 & & .20 & 4. 98625 & & .70 & 5. 00392 & \\
\hline .75 & 4. 96820 & & .25 & 4. 98661 & & .75 & 5. 00426 & \\
\hline .80 & - 4.96858 & 7 & .30 & 4. 98697 & & .80 & 5.00461 & \\
\hline .85 & 4. 96895 & & .35 & 4. 98733 & & .85 & 5. 00495 & \\
\hline .90 & 4. 96933 & & .40 & 4. 98769 & & .90 & 5.00530 & \\
\hline .95 & 4. 96970 & & .45 & 4. 98805 & & .95 & 5.00565 & \\
\hline 58.00 & 4. 97008 & & .50 & 4. 98841 & & 63.00 & 5.00599 & \\
\hline .05 & 4. 97045 & & .55 & 4. 98876 & & .05 & 5.00633 & \\
\hline .10 & 4. 97083 & & .60 & 4. 98912 & & .10 & 5. 00668 & \\
\hline .15 & 4. 97120 & & .65 & 4. 98948 & & .15 & -5.00702 & \\
\hline .20 & 4. 97157 & & .70 & 4. 98984 & & .20 & 5. 00737 & \\
\hline .25 & 4. 97195 & & .75 & 4. 99020 & & .25 & 5. 00771 & \\
\hline .30 & 4. 97232 & & .80 & 4. 99055 & & .30 & 5.00805 & \\
\hline .35 & 4. 97269 & & .85 & 4. 99091 . & & .35 & 5.00840 & \\
\hline .40 & 4. 97306 & & .90 & 4. 99127 & & .40 & 5.00874 & \\
\hline .45 & 4.97343 & & .95 & 4. 99162 & & .45 & 5. 00908 & \\
\hline .50 & 4. 97381. & & 61.00 & 4. 99198 & & .50 & 5. 00942 & \\
\hline .55 & 4.97418 & & .05 & 4. 99234 & & .55 & 5. 00977 & \\
\hline .60 & 4. 97455 & & .10 & 4. 99269 & . & .60 & 5.01011 & \\
\hline .65 & 4.97492 & & .15 & 4. 99305 & & .65 & Б. 01045 & \\
\hline .70 & 4. 97529 & & .20 & 4. 99340 & & .70 & 5.01079 & \\
\hline • .75 & 4. 97566 & & .25 & 4. 99376 & & .75 & 5. 01113 & \\
\hline .80 & 4. 97603 & & .30 & 4. 99411 & & .80 & 5.01147 & \\
\hline .85 & 4. 97640 & & .35 & 4. 99447 & & .85 & 5.01181 & \\
\hline .90 & 4. 97677 & & .40 & 4. 99482 & & .90 & 5.01215 & . \\
\hline .95 & 4. 97713 & & .45 & 4. 99517 & & .95 & 5.01249 & \\
\hline
\end{tabular}


TABLE 28.-For interconversion of miles and logarithms of meters-Continued.

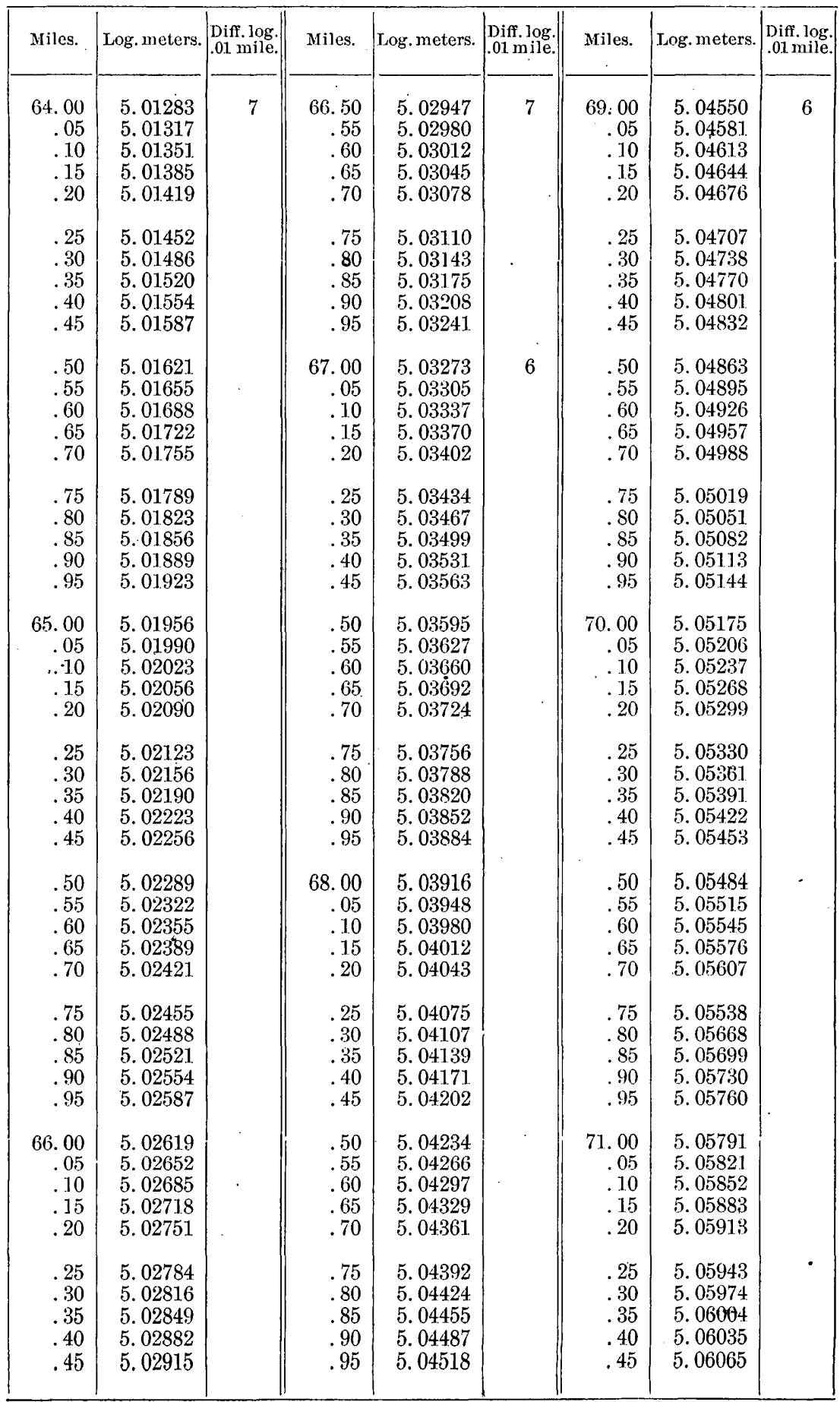


Tавте 28.-For interconversion of miles and logarithms of meters-Continued.

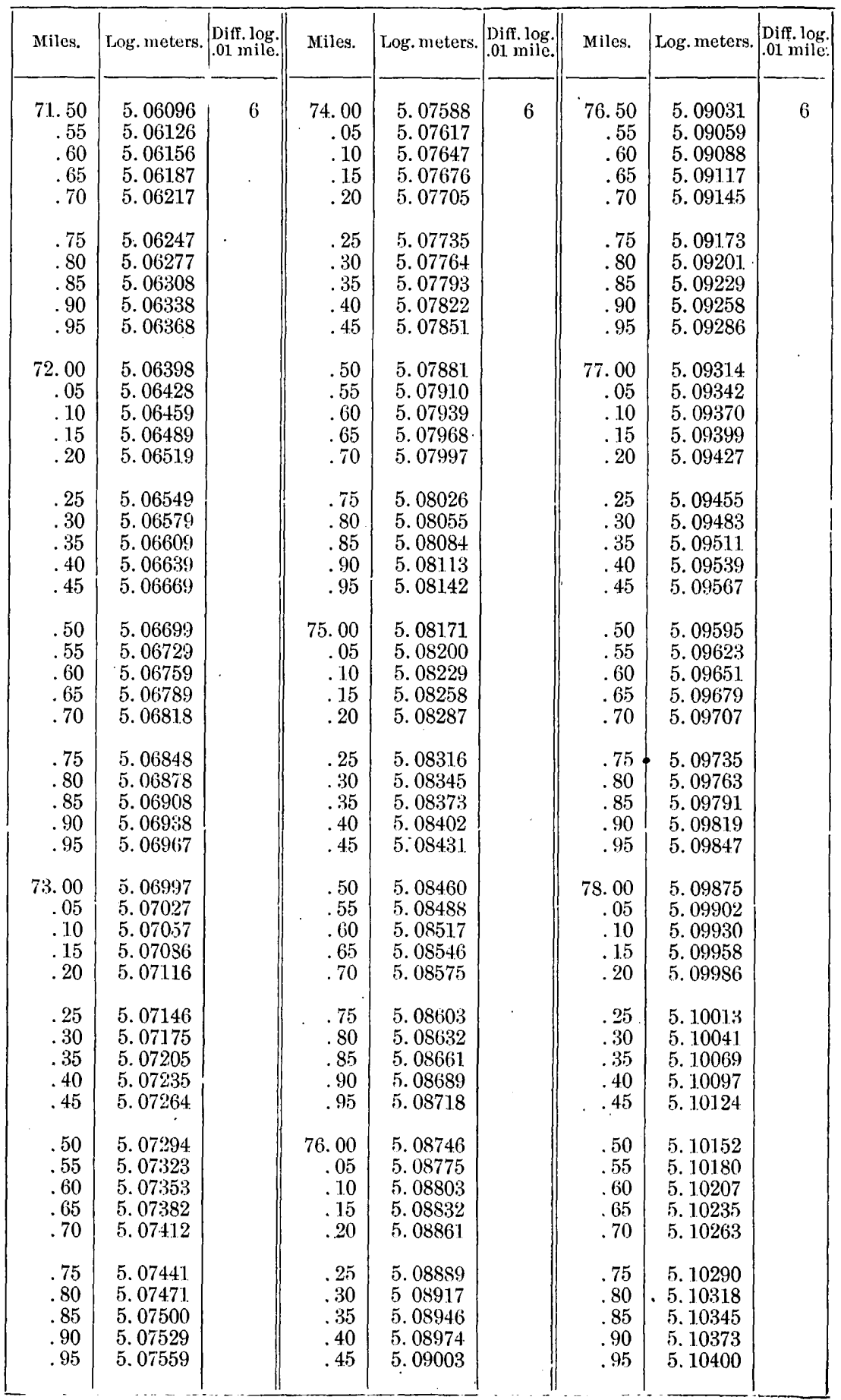


TABLE 28.-For interconversion of miles and logarithms of meters-.-Continued.

\begin{tabular}{|c|c|c|c|c|c|c|c|c|}
\hline Miles. & Log. meters. & Diff. $\log$. & Miles. & Log. meters. & Diff. log. & Miles. & Log. meters. & $\begin{array}{l}\text { Diff. log. } \\
.01 \text { mile. }\end{array}$ \\
\hline 79.00 & $\dot{5} .10428$ & 5 & 81.50 & 5.11781 & 5 & 84.00 & 5. 13093 & 5 \\
\hline $\begin{array}{r}.05 \\
.05\end{array}$ & 5.10455 & & $\begin{array}{r}.1 .00 \\
.55\end{array}$ & 5.11807 & & $\begin{array}{r}07.05 \\
.05\end{array}$ & 5. 13119 & \\
\hline .10 & 5. 10483 & & .60 & 5. 11834 & & .10 & 5. 13145 & \\
\hline .15 & 5. 10510 & & .65 & 5. 11861 & & .15 & 5. 13170 & \\
\hline .20 & 5. 10537 & & .70 & 5.11887 & & .20 & 5.13196 & \\
\hline .25 & 5. 10565 & & .75 & 5. 11913 & & .25 & 5. 13222 & \\
\hline .30 & 5. 10592 & & .80 & 5. 11940 & & .30 & 5. 13248 & \\
\hline .35 & 5. 10620 & & .85 & 5. 11967 & & .35 & 5. 13273 & \\
\hline .40 & 5. 10647 & & .90 & 5. 11993 & & .10 & 5. 13299 & \\
\hline .45 & 5. 10674 & & .95 & 5. 12020 & & .45 & 5. 13325 & \\
\hline-.50 & 5. 10702 & & 82.00 & 5. 12046 & & .50 & 5. 13351 & \\
\hline .55 & 5. 10729 & & $\begin{array}{r}.05 \\
. .05\end{array}$ & 5. 12073 & & .55 & 5. 13376 & \\
\hline .60 & 5. 10756 & & 10 & 5. 12099 & & .60 & 5. 13402 & \\
\hline .65 & 5. 10784 & & .15 & 5. 12126 & & .65 & 5. 13428 & \\
\hline .70 & 5. 10811 & & .20 & 5.12152 & & .70 & 5. 13453 & \\
\hline .75 & 5.10838 & & .25 & 5. 12179 & & .75 & 5. 13479 & \\
\hline .80 & 5. 10865 & & .30 & 5. 12205 & & .80 & 5. 13505 & \\
\hline .85 & 5. 10893 & & .35 & 5. 12231 & & .85 & 5. 13530 & \\
\hline .90 & 5. 10920 & & .40 & 5. 12258 & & .90 & 5. 13556 & \\
\hline .95 & 5. 10947 & & .45 & 5. 12284 & & .95 & 5. 13581 & \\
\hline 80.00 & 5. 10974 & & .50 & 5. 12310 & & -85.00 & 5. 13607 & \\
\hline .05 & 5. 11001 & & .55 & 5. 12337 & & .05 & 5. 13632 & \\
\hline 10 & 5. 11028 & & .60 & 5. 12363 & & .10 & 5. 13658 & \\
\hline .15 & 5. 11055 & & .65 & 5. 12389 & & .15 & 5. 13683 & \\
\hline .20 & 5. 11082 & & .70 & 5. 12416 & & .20 & 5. 13709 & \\
\hline .25 & 5. 11109 & & .75 & 5: 12442 & & .25 & 5.13734 & \\
\hline .30 & 5. 11137 & & .80 & 5. 12468 & & .30 & 5. 13760 & \\
\hline .35 & 5. 11164 & & .85 & 5. 12494 & & .35 & 5. 13785 & \\
\hline .40 & 5. 11191 & & $: \quad .90$ & 5. 12521 & & .40 & 5. 13811 & \\
\hline .45 & 5. 11218 & & .95 & 5. 12547 & & .45 & 5. 13836 & \\
\hline .50 & 5. 11245 & & 83.00 & 5. 12573 & & .50 & 5. 13862 & \\
\hline .55 & 5. 11272 & & .05 & 5. 12599 & & .55 & 5. 13887 & \\
\hline .60 & 5. 11299 & & .10 & 5. 12625 & & .60 & 5. 13912 & \\
\hline .65 & 5. 11325 & & .15 & 5. 12651 & & .65 & 5. 13938 & \\
\hline .70 & 5. 11352 & & .20 & 5. 12677 & & .70 & 5. 13963 & \\
\hline .75 & 5. 11379 & & .25 & 5. 12703 & & .75 & 5. 13988 & \\
\hline .80 & 5. 11406 & t. & .30 & 5. 12729 & & .80 & 5. 14014 & \\
\hline .85 & 5. 11433 & & .35 & 5. 12756 & & .85 & 5. 14039 & \\
\hline .90 & 5. 11460 & & .40 & 5. 12782 & . & .90 & 5. 14064 & \\
\hline$\because 95$ & 5. 11487 & & .45 & 5. 12808 & & .95 & 5. 14090 & \\
\hline 81.00 & 5. 11513 & & .50 & 5. 12834 & & 86.00 & 5. 14115 & \\
\hline .05 & 5.11540 & & .55 & 5. 12860 & & .05 & 5. 14140 & \\
\hline .10 & 5.11567 & & .60 & อ. 12886 & & .10 & 5.14165 & \\
\hline .15 & 5.11594 & & .65 & 5. 12912 & & .15 & 5. 14191 & \\
\hline .20 & 5. 11621 & & .70 & 5. 12937 & & .20 & 5. 14216 & \\
\hline .25 & 5.11647 & & .75 & 5. 12963 & & .25 & 5. 14241 & \\
\hline .30 & 5. 11674 & & .80 & 5. 12989 & & .30 & 5. 14266 & \\
\hline .35 & 5. 11701 & & .85 & 5. 13015 & & .35 & 5. 14291 & \\
\hline .40 & 5. 11727 & & .90 & 5. 13041 & & .40 & 5. 14316 & \\
\hline .45 & 5. 11754 & & .95 & 5. 13067 & & .45 & 5. 14341 & \\
\hline
\end{tabular}


ТАвте 28.-For interconversion of miles and logarithms of meters-Continued.

\begin{tabular}{|c|c|c|c|c|c|c|c|c|}
\hline Miles. & Log. meters. & $\begin{array}{l}\text { Diff. log. } \\
01 \text { mile. }\end{array}$ & Miles. & Log. meters. & $\begin{array}{l}\text { Diff. log. } \\
.01 \text { mile. }\end{array}$ & Miles. & Log. meters. & $\begin{array}{l}\text { Diff. log. } \\
.01 \text { mile. }\end{array}$ \\
\hline 86.50 & 5. 14367 & 5 & $\$ 9.00$ & 5. 15604 & 5 & 91.50 & 5. 16807 & 5 \\
\hline .55 & 5. 14392 & & .05 & 5. 15628 & & .55 & 5.16831 & \\
\hline .60 & 5. 14417 & & .10 & 5. 15653 & & .60 & 5. 16855 & \\
\hline .05 & 5. 14442 & & .15 & 5. 15677 & & .65 & 5. 16878 & \\
\hline .70 & 5. 14467 & & .20 & 5. 15701 & & .70 & 5. 16902 & \\
\hline .75 & 5. 14492 & & .25 & 5.15726 & & .75 & 5. 16926 & \\
\hline .80 & 5. 14517 & & .30 & 5.15750 & & .80 & 5.16949 & \\
\hline .85 & 5. 14542 & & .35 & 5.15775 & & .85 & 5. 16973 & \\
\hline .90 & 5.14567 & & .40 & 5. 15799 & & .90 & 5. 16997 & \\
\hline .95 & 5.14592 & & .45 & 5. 15823 & & .95 & 5.17020 & \\
\hline 87.00 & 5. 14617 & & .50 & 5. 15847 & • & 92.00 & 5.17044 & \\
\hline .05 & 5. $146+2$ & & .55 & 5. 15872 & & .05 & 5. 17067 & \\
\hline .10 & 5. 14667 & & .60 & 5.15896 & & .10 & 5. 17091 & \\
\hline .15 & 5. 14692 & & .65 & 5. 15920 & 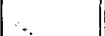 & .15 & 5. 17115 & \\
\hline .20 & 5. 14717 & & .70 & 5. 15944 & $*$ & .20 & 5. 17138 & \\
\hline .25 & 5. 14741 & & .75 & 5.15968 & & .25 & 5. 17162 & \\
\hline$\therefore 30$ & 5. 14766 & & .80 & 5. 15993 & & .30 & 5.17285 & \\
\hline .35 & 5. 14791 & & .85 & 5. 16017 & & .35 & 5. 17209 & \\
\hline .40 & 5. 14816 & & .90 & 5.16041 & & .40 & 5. 17232 & \\
\hline .45 & 5. 14841 & & .95 & 5. 16065 & & .45 & 5. 17256 & \\
\hline .50 & 5. 14866 & & 90.00 & 5. 16089 & & .50 & 5. 17279 & \\
\hline .55 & 5. 14891 & & .05 & 5. 16113 & & .55 & 5. 17303 & \\
\hline .60 & 5. 14915 & & .10 & 5. 16137 & • & .60 & 5. 17326 & \\
\hline .65 & 5. 14940 & & .15 & 5.16162 & 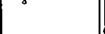 & .65 & 5. 17349 & \\
\hline .70 & 5. 14965 & & .20 & 5.16186 & & .70 & 5.17373 & \\
\hline .75 & 5. 14990 & & .25 & 5.16210 & & .75 & 5. 17396 & \\
\hline .80 & 5. 15014 & & .30 & 5. 16234 & & .80 & 5. 17420 & \\
\hline .85 & 5. 15039 & & .35 & 5.16258 & & .85 & 5. 17443 & \\
\hline .90 & 5. 15064 & & .40 & 5. 16282 & & .90 & 5. 17467 & \\
\hline .95 & 5. 15089 & & .45 & 5.16306 & & .95 & 5. 17490 & \\
\hline 88.00 & 5. 15113 & &.$\quad .50$ & 5.16330 & & 93.00 & 5. 17513 & \\
\hline .05 & 5.15138 & & .55 & 5. 16354 & & .05 & 5. 17537 & \\
\hline .10 & 5. 15163 & & .60 & 5. 16378 & & .10 & 5. 17560 & \\
\hline .15 & 5. 15187 & & .65 & 5. 16402 & & .15 & 5. 17583 & \\
\hline .20 & 5. 15212 & & .70 & 5. 16426 & & .20 & 5. 17607 & \\
\hline .25 & 5. 15237 & & .75 & 5.16450 & & .25 & 5. 17630 & \\
\hline .30 & 5. 15261 & & .80 & 5. 16474 & & .30 & 5. 17653 & \\
\hline .35 & 5. 15286 & & .85 & 5. 16497 & & .35 & $5: 17676$ & \\
\hline .40 & 5. 15310 & & .90 & 5. 16521 & & .40 & 5. 17700 & \\
\hline .45 & 5. 15335 & & .95 & 5. 16545 & & .45 & 5. 17723 & \\
\hline .50 & 5. 15359 & & 91.00 & 5. 16569 & & .50 & 5. 17746 & \\
\hline .55 & 5. 15384 & & .05 & 5. 16593 & & .55 & 5. 17769 & \\
\hline .60 & 5. 15408 & & .10 & 5.16617 & & .60 & 5. 17793 & \\
\hline .65 & 5. 15433 & & .15 & 5. 16641 & & .65 & 5. 17816 & \\
\hline .70 & 5. 15457 & & .20 & 5. 16665 & & .70 & 5. 17839 & \\
\hline .75 & 5. 15482 & & .25 & 5.16688 & & .75 & 5. 17862 & \\
\hline .80 & 5. 15506 & & .30 & 5. 16712 & & .80 & 5. 17885 & \\
\hline .85 & 5. 15531 & & .35 & 5.16736 & & .85 & 5. 17908 & \\
\hline .90 & 5. 15555 & & .40 & 5.16760 & & .90 & 5. 17932 & \\
\hline .95 & 5. 15580 & . & .45 & 5. 16783 & & .95 & 5. 17955 & \\
\hline
\end{tabular}


TABLE 28.-For interconversion of miles and logarithms of meters-Continued.

\begin{tabular}{|c|c|c|c|c|c|c|c|c|}
\hline Miles. & Log.meters. & $\begin{array}{l}\text { Diff .log. } \\
01 \text { mile. }\end{array}$ & Miles. & Log. meters. & $\begin{array}{l}\text { Diff. log. } \\
.01 \text { mile }\end{array}$ & Miles. & Log.meters. & $\begin{array}{l}\text { Diff. log. } \\
01 \text { mile. }\end{array}$ \\
\hline $\begin{array}{r}84.00 \\
.05 \\
.10 \\
.15 \\
.20\end{array}$ & $\begin{array}{l}\text { 5. } 17978 \\
5.18001 \\
5.18024 \\
5.18047 \\
5.18170\end{array}$ & 5 & $\begin{array}{r}96.00 \\
.05 \\
.10 \\
.15 \\
.20\end{array}$ & $\begin{array}{l}5.18892 \\
5.18915 \\
5.18937 \\
5.18960 \\
5.18983\end{array}$ & 5 & $\begin{array}{r}98.00 \\
.05 \\
.10 \\
.15 \\
.20\end{array}$ & $\begin{array}{l}\text { 5. } 19788 \\
\text { 5. } 19810 \\
\text { 5. } 19832 \\
\text { 5. } 19854 \\
\text { 5. } 19876\end{array}$ & 4 \\
\hline $\begin{array}{l}.25 \\
.30 \\
.35 \\
.40 \\
.45\end{array}$ & $\begin{array}{l}\text { 5. } 18193 \\
5.18116 \\
5.18139 \\
5.18162 \\
5.18185\end{array}$ & & $\begin{array}{l}.25 \\
.30 \\
.35 \\
.40 \\
.45\end{array}$ & $\begin{array}{l}5.19005 \\
5.19028 \\
5.19050 \\
5.19073 \\
5.19095\end{array}$ & & $\begin{array}{l}.25 \\
.30 \\
.35 \\
.40 \\
.45\end{array}$ & $\begin{array}{l}5.19898 \\
5.19920 \\
5.19942 \\
5.19965 \\
5.19987\end{array}$ & \\
\hline $\begin{array}{l}.50 \\
.55 \\
.60 \\
.65 \\
.70\end{array}$ & $\begin{array}{l}5.18208 \\
5.18231 \\
5.18254 \\
5.18277 \\
5.18300\end{array}$ & & $\begin{array}{l}.50 \\
.55 \\
.60 \\
.65 \\
.70\end{array}$ & $\begin{array}{l}\text { 5. } 19118 \\
5.19140 \\
5.19163 \\
5.19185 \\
5.19208\end{array}$ & & $\begin{array}{l}.50 \\
.55 \\
.60 \\
.65 \\
.70\end{array}$ & $\begin{array}{l}5.20009 \\
5.20031 \\
5.20053 \\
5.20075 \\
5.20097\end{array}$ & \\
\hline $\begin{array}{l}.75 \\
.80 \\
.85 \\
.90 \\
.95\end{array}$ & $\begin{array}{l}\text { 5. } 18323 \\
5.18346 \\
5.18369 \\
5.18392 \\
5.18415\end{array}$ & & $\begin{array}{l}.75 \\
.80 \\
.85 \\
.90 \\
.95\end{array}$ & $\begin{array}{l}5.19230 \\
5.19253 \\
5.19275 \\
5.19297 \\
5.19320\end{array}$ & & $\begin{array}{l}.75 \\
.80 \\
.85 \\
.90 \\
.95\end{array}$ & $\begin{array}{l}\text { 5. } 20119 \\
5.20141 \\
5.20163 \\
5.20185 \\
5.20207\end{array}$ & \\
\hline $\begin{array}{r}95.00 \\
.05 \\
.10 \\
.15 \\
.20\end{array}$ & $\begin{array}{l}5.18437 \\
5.18460 \\
5.18483 \\
5.18506 \\
5.18529\end{array}$ & & $\begin{array}{r}97.00 \\
.05 \\
.10 \\
.15 \\
.20\end{array}$ & $\begin{array}{l}5.19342 \\
5.19365 \\
5.19387 \\
5.19409 \\
5.19432\end{array}$ & 4 & $\begin{array}{r}99.00 \\
.05 \\
.10 \\
.15 \\
.20\end{array}$ & $\begin{array}{l}\text { 5. } 20229 \\
5.20250 \\
5.20272 \\
5.20294 \\
5.20316\end{array}$ & \\
\hline $\begin{array}{l}.25 \\
.30 \\
.35 \\
.40 \\
.45\end{array}$ & $\begin{array}{l}5.18551 \\
5.18574 \\
5.18597 \\
5.18620 \\
5.18643\end{array}$ & & $\begin{array}{l}.25 \\
.30 \\
.35 \\
.40 \\
.45\end{array}$ & $\begin{array}{l}\text { 5. } 19454 \\
5.19476 \\
5.19499 \\
5.19521 \\
5.19543\end{array}$ & & $\begin{array}{l}.25 \\
.30 \\
.35 \\
.40 \\
.45\end{array}$ & $\begin{array}{l}5.20338 \\
5.20360 \\
5.20382 \\
5.20404 \\
5.20425\end{array}$ & \\
\hline $\begin{array}{l}.50 \\
.55 \\
.60 \\
.65 \\
.70\end{array}$ & $\begin{array}{l}5.18665 \\
5.18688 \\
5.18711 \\
5.18733 \\
5.18756\end{array}$ & & $\begin{array}{l}.50 \\
.55 \\
.60 \\
.65 \\
.70\end{array}$ & $\begin{array}{l}5.19565 \\
5.19588 \\
5.19610 \\
5.19632 \\
5.19655\end{array}$ & . & $\begin{array}{l}.50 \\
.55 \\
.60 \\
.65 \\
.70\end{array}$ & $\begin{array}{l}\text { 5. } 20447 \\
\text { 5. } 20469 \\
5.20491 \\
5.20513 \\
\text { 5. } 20535\end{array}$ & \\
\hline $\begin{array}{l}.75 \\
.80 \\
.85 \\
.90 \\
.95\end{array}$ & $\begin{array}{l}\text { 5. } 18779 \\
5.18802 \\
5.18824 \\
5.18847 \\
5.18869\end{array}$ & & $\begin{array}{l}.75 \\
.80 \\
.85 \\
.90 \\
.95\end{array}$ & $\begin{array}{l}5.19677 \\
5.19699 \\
5.19721 \\
5.19743 \\
5.19765\end{array}$ & & $\begin{array}{l}.75 \\
.80 \\
.85 \\
.90 \\
.95\end{array}$ & $\begin{array}{l}5.20556 \\
5.20578 \\
5.20600 \\
5.20621 \\
5.20643\end{array}$ & \\
\hline
\end{tabular}

\section{CON VENIEN'T EQUIVALENTS.}

1 acre $=209$ feet square, nearly.

1 acre $=43,560$ square feet $=4,840$ square yards.

1 statute mile $=1,760$ yards $=5,280$ feet $=63,360$ inches.

1 cubic foot $=7.48$ gallons $=0.804$ bushel.

1 cubic foot of water weighs 62.4 pounds.

1. wine gallon $=8.34$ pounds water.

1 wine gallon $=231$ cubic inches.

1 avoirdupois pound $=7,000$ grains.

1 troy pound $=5,760$ grains. 
1. meter $=39.37$ inches. Log. 1.5951654 .

1. meter $=3.28083$ feet. $\quad$ Log. 0.5159842 .

1 meter $=1.093611$ yards. $\quad$ Log. 0.0388629 .

1 meter $=0.00062137$ mile. Log. 6.7933502 .

1 kilometer $=3,281$ feet $=$ five-eighths mile, nearly.

1 cubic meter $=35.314$ cubic feet $=1.308$ yards.

1 liter $=1.0567$ quarts.

$1 \mathrm{gram}=15.43$ grains.

1 kilogram $=2.2046$ avoirdupois pounds.

1 tonneau (metric ton) $=2,204.6$ pounds.

1 c:nbic meter per minute $=0.5886$ second-foot.

1 second-foot $=50$ California miner's inches.

1 second-foot $=40$ Arizona miner's inches.

1 second-foot $=449$ gallons per minute.

1 second-foot for one day $=1.9835$ acre-feet.

1 second-foot for one day $=646,272$ United States gallons.

1 second-foot $=$ about one acre-inch per hour.

1 acre-foot $=325,850$ gallons.

$1,000,000$ gallons $=3.07$ acre-feet.

$1,000,000$ cubic feet $=22.95$ acre-feet.

$1,000,000$ gallons per 24 hours $=1.55$ second-feet.

1 horse power $=550$ foot-pounds per second.

1 horse power $=76$ kilogrammeters per second.

1 horse pow $\cdot=746$ watts.

1 horse power $=1$ second-foot water falling 8.8 feet.

1 second-foot falling 10 feet $=1.135$ horse power.

1 foot per second $=1.077$ kilometers per hour.

1 foot per second $=0.68$ miles per hour.

1 inch $=2.5+$ centimeters.

1 foot $=0.3048$ meters.

1 yard $=0.91 .44$ meters.

1 mile $=1.60935$ kilometers.

1. square yard $=0.836$ sovare meters.

1 acre $=0.4047$ hectares.

1 syuare mile $=259$ hectares.

1 square mile $=2.59$ square kilometers.

1 cubic foot $=0.0283$ cubic meters.

1 abic yard $=0.76+6$ cubic meters.

1 gallon $=3.7854$ liters.

1. pound $=0.4536$ kilograms.

1 atmosphere $=$ about $\left\{\begin{array}{l}15 \text { pounds per square inch. } \\ 1 \text { ton per square foot. } \\ 1 \text { kilo per square centimeter. }\end{array}\right.$

Acceleration of gravity $=32.16$ feet per second.

To change miles to inches on map:

Scale 1: 125000,1 mile $=0.50688$ inches. Log. $=\$ .7049052$.

Scale 1:90000, 1 mile $=0.70400$ inches. Log. $=\$ .8475727$.

Scale 1:62500, 1 mile $=1.01376$ inches. $\quad$ Log. $=\emptyset .00599352$.

Scale 1:45000, 1. mile $=1.40800$ inches. Log. $=\$ .1486027$.

To change log. of meters to log. of inches on map:

Scale 1:125000 add 6.4982552.

Scale 1: 90000 add 6.6409228 .

Scale 1:62500 add 6.7992853 .

Scale 1:45000 add 6.9419528 . 


\section{CONSTANTS.}

Basis of natural logarithms

$\mathrm{e}=2.7182818285$

Log.

Modulus of Briggs's logarithms ...........m $=0.4342944819$

0.4342944819

Radius of the circle in seconds............ $=206264.8062$

9. $6377843113-10$

Radius of the circle in minutes

3437.74677

5. 3144251332

Radius of the circle in degrees.

57.2957795

3. 5362738828

Circumference of the circle in seconds

1296000

1. 7581226824

Circumference of the circle in minutes

21600

6. 1126050015

Circumference of the circle in degrees ......... 360

Circumference of the circle for the diameter. =

4. 3344537512

2. 5563025008

$$
=3.1415926536
$$

0.0000000000

0.4971498727

\section{ASTRONOMICAL CONSTAN'SS (HARKNESS).}

Sidereal year $=365.2563578$ mean solar days.

Sidereal day $=23^{\mathrm{h}} 56^{\mathrm{m}} 4 .^{\mathrm{s}} 100$ mean solar time.

Mean solar day $=24^{\mathrm{h}} 3^{\mathrm{m}} 56.5546$ sidereal time.

Mean distance of the earth from the sun $=92800000$ miles.

PHYSICAL CONSTANTS.

Velocity of light (Harkness) $=186337$ miles per second $=299878 \mathrm{~km}$. per second. Velocity of sound through dry air $=1090 \sqrt{1+0.00367 t^{\circ} \mathrm{C}}$. feet per second.

\section{LINEAR EXPANSIONS OF PRINCIPAL METALS IN MICRONS PER METRE (OR MILLIONTHS PER UNIT LENGTH).}

\begin{tabular}{|c|c|c|}
\hline Name of metal. & $\begin{array}{c}\text { Expansion } \\
\text { per } \\
\text { degree C. }\end{array}$ & $\begin{array}{c}\text { Expansion } \\
\text { per } \\
\text { degree } \mathrm{F} .\end{array}$ \\
\hline Aluminum... & 20 & 11.1 \\
\hline Brass.......... & 19 & 10.5 \\
\hline Copper....... & 17 & 9.4 \\
\hline Glass........... & 9 & 5.0 \\
\hline Gold $\ldots \ldots \ldots \ldots \ldots$ & 15 & 8.3 \\
\hline Iron, cast.......... & 11 & 6.1 \\
\hline Iron, wrought...... & 12 & 6.7 \\
\hline Lead $\ldots \ldots \ldots \ldots \ldots \ldots \ldots \ldots$ & 28 & 15.5 \\
\hline Platinum ........ & 9 & 5.0 \\
\hline Platinum-iridium & 8. 7 & 4.8 \\
\hline Silver .......... & 19 & 10.5 \\
\hline Steel, hard....... & 12 & 6.7 \\
\hline 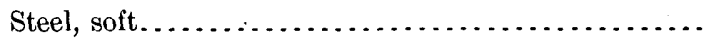 & 11 & 6.1 \\
\hline Tin $\ldots \ldots \ldots \ldots \ldots \ldots$ & 19 & 10.5 \\
\hline Zinc................................... & 29 & 16.1 \\
\hline
\end{tabular}




\section{PUBLICATIONS OF UNITED STATES GEOLOGICAL SURVEY.}

[Bulletin No. 214.]

The publications of the United States Geological Survey consist of (1) Annual Reports, (2) Monographs, (3) Professional Papers, (4) Bulletins, (5) Mineral Resources, (6) Water-Supply and Irrigation Papers, (7) Topographic Atlas of United States-folios and separate sheets thereof, (8) Geologic Atlas of Unitëd States-folios thereof. The classes numbered 2,7 , and 8 are sold at cost of publication; the others are distributed free. A circular giving complete lists may be had on application.

The Bulletins, Professional Papérs, and Water-Supply Papers treat of a variety of subjects, and the total number issued is large. They have therefore been classified into the following series: A, Economic geology; B, Descriptive geology; C, Systematic geology and paleontology; D, Petrography and mineralogy; E, Chemistry and physics; F, Geography; G, Miscellaneous; H, Forestry; I, Irrigation; J, Water storage; K, Pumping water; L, Quality of water; M, General hydrographic investigations; $\mathrm{N}$, Water power; $\mathrm{O}$, Underground waters; $\mathrm{P}$, Hydrographic progress reports. This bulletin is the thirty-fourth in Series F, the complete list of which follows (all are bulletins thus far):

\section{SERIES F, GEOGRAPHY.}

5. Dictionary of altitudes in United States, by Henry Gannett. 1884. 325 pp. (Out of stock; see Bulletin 160.)

6. Elevations in Dominion of Canada, by J. W. Spencer. 1884. 43 pp.

13. Boundaries of United States and of the several States and Territories, with historical sketch of territorial changes, by Henry Gannett. 1885. $135 \mathrm{pp}$. (Out of stock; seo Bulletin 171.)

48. On form and position of sea level, by R. S. Woodward. $1888.88 \mathrm{pp}$. (Out of stock.)

49. Latitudes and longitudes of certain points in Missouri, Kansas, and New Mexico, by R. S Woodward. $1889.133 \mathrm{pp}$.

50. Formulas and tables to facilitate the construction and use of maps, by R. S. Woodward. 1889. $124 \mathrm{pp.} \mathrm{(Out} \mathrm{of} \mathrm{stock.)}$

70.- Report on astronomical work of 1889 and 1890 , by R. S. Woodward. $1890.79 \mathrm{pp}$.

72. Altitudes between Lake Superior and Rocky Mountains, by Warren Upham. 1891. 229.pp.

76. Dictionary of altitudes in United States (second edition), by Henry Gannett. 1891. 393 pp. (Out of stock; see Bulletin 160.)

115. Geographic dictionary of Rhode Island, by Henry Gannett. 1894. $31 \mathrm{pp.}$

116. Geographic dictionary of Massačhusetts, by Henry Gannett. 1894. 126 pp.

117. Geographic dictionary of Connecticut, by Henry Gannett. $1894.67 \mathrm{pp}$ :

118. Geographic dictionary of New Jersey, by Henry Gannett. 1894. 131. pp.

122. Results of primary triangulation, by Henry Gannett. $1894.412 \mathrm{pp}$., $17 \mathrm{pls}$.

123. Dictionary of geographic positions, by Henry Gannett.' 1895.183 pp., 1 map.

154. Gazetteer of Kansas, by Henry Gannett. 1898. 246 pp., 6 pls.

160. Dictionary of altitudes in United States (third edition), by Henry Gannett. 1899. $775 \mathrm{pp}$. (Out of stock.)

166. Gazetteer of Utah, by Henry Gannett. 1900. 43 pp., 1 map.

169. Altitudes in Alaska, by Henry Gannett. 1900. $13 \mathrm{pp.}$

170. Survey of boundary line between Idaho and Montana from international boundary to crest of Bitterroot Mountains, by R. U. Goode. $1900.67 \mathrm{pp} ., 14 \mathrm{pls}$.

171. Boundaries of United States and of the several States and Territories, with outline of history of all important changes of territory (second edition), by Henry Gannett. 1900. $142 \mathrm{pp} ., 53 \mathrm{pls}$.

174. Survey of northwestern boundary of United States, 1857-1861, by Marcus Baker. 1900. $78 \mathrm{pp} ., 1 \mathrm{pl}$.

175. Triangulation and spirit leveling in Indian Territory, by C. H. Fitch. 1900.141 pp., 1 pl.

181. Results of primary triangulation and primary traverse, fiscal year 1900-01, by H. M. Wilson, J. H. Renshawe, E. M. Douglas, and R. U. Goode. 1901.240 pp., 1 map.

183. Gazetteer of Porto Rico, by Henry Gannett. 1901. 51 pp. 
185. Restits of spirit leveling, fiscal year 1900-01, by H. M. Wilson, J. H. Renshawc, E. M. Douglas, and R. U. Goode. 1901. 2219 pp.

187. Geographic dictionary of Alaska, by Marcus Baker. 1901. 446 pp.

190. Gazetteer of Texas, by Henry Gannett. 1902. $162 \mathrm{pp} ., 8 \mathrm{pls}$.

192. Gazetteer of Cuba, by Henry Gannett. 1902. 113 pp., 8 pls.

194. Northwest boundary of Texas, by Marcus Baker. $1902.51 \mathrm{pp} ., 1 \mathrm{pl}$.

196. Topographic development of the Klamath Mountains, by J. S. Diller. 1902. 69 pp., 13 pls.

197. The origin of certain place names in the United States, by Henry Gannets. 1902. '281) pp. (Out of stock.)

201. Results of primary triangulation and primary traverse, fiscal year 1901-'02, by H. M. Wilson, J. H. Renshawe, E. M. Douglas, and R. U. Goode. 1902. 164 pp., 1 pl.

214. Geographic tables and formulas, compiled by S. S. Gannett. $1903.284 \mathrm{pp.}$

Correspondence should be addressed to

The DIRECTOR,

United States Geological. Survey,

JUNE, 1903.

Washington, D. C. 